\title{
Biosensors
}

\section{Fundamentals and}

Applications

Edited by

ANTONY P. F. TURNER

ISAO KARUBE

and

GEORGE S. WILSON

\section{OXFORD SCIENCE PUBLICATIONS}




\section{BIOSENSORS}

Fundamentals and Applications

EDITED BY

ANTHONY P.F. TURNER

Cranfield Institute of Technology

ISAO KARUBE

University of Tokyo

and

GEORGE S. WILSON

University of Arizona

OXFORD NEW YORK TOKYO

OXFORD UNIVERSITY PRESS 
Oxford University Press, Walton Street, Oxford OX2 6DP

Oxford New York Toronto

Delhi Bombay Calcutta Madras Karachi

Petaling Jaya Singapore Hong Kong Tokyo

Nairobi Dar es Salaam Cape Town

Melbourne Auckland

and associated companies in

Berlin Ibadan

Oxford is a trade mark of Oxford University Press

Published in the United States

by Oxford University Press, New York

(c) The various contributors listed on pp, xiii-xvi, 1987. except chapter 34 (5) HMSO, 1987

First published 1987

First published as paperback (with corrections) 1989

All rights reserved. No part of this publication may be reproduced, stored in a retrieval system, or transmitted, in any form or by any means, electronic, mechanical, photocopying, recording, or otherwise, without the prior permission of Oxford University Press

This book is sold subject to the condition that it shall not, by way of trade or otherwise, be lent, re-sold, hired out or otherwise circulated without the publisher's prior consent in any form of binding or cover other than that in which it is published and without a similar condition including this condition being imposed on the subsequent purchaser.

British Library Cataloguing in Publication Data

Biosensors: fundamentals and applications.

$$
\text { 1. Biosensors }
$$

1. Turner, Anthony P.F. II. Karube, Isao III. Wilson, George S.

$$
547 \quad R 857 . B 54
$$

ISBN $0-19-854724-2$

ISBN 0-19-854745-5 (Pbk)

Library of Congress Cataloging in Publication Data

Biosensors: fundamentals and applications. Includes index.

I. Biosensors. I. Turner, Anthony P.F.

II. Karube, Isao, 1942- * III. Wilson, George S.

R857.B54B56 $1986 \quad 681.761 \quad 86-21811$

ISBN 0-19-854724-2

ISBN 0-19-854745-5 (Pbk)

Printed in Great Britain by

St Edmundsbury Press, Bury St Edmunds, Suffolk 


\section{Preface}

A biosensor is a device incorporating a biological sensing element either intimately connected to or integrated within a transducer. The usual aim is to produce a digital electronic signal which is proportional to the concentration of a specific chemical or set of chemicals. The apparently alien marriage of two contrasting disciplines combines the specificity and sensitivity of biological systems with the computing power of the microprocessor. This emerging technology crosses many traditional academic delineations and offers a powerful new tool which threatens to radically alter our attitude to analytical science.

The modern concept of a biosensor owes much to the ideas of Leland C. Clark Jr. and co-workers, 1962 et seq. (see Chapter 1). They proposed that enzymes could be immobilized at electrochemical detectors to form 'enzyme electrodes' which would expand the analyte range of the base sensor. A wealth of work ensued, elucidating a myriad of permutations on this theme until slowly the horizons were expanded. The present picture is summarized in Table 1.

All the possible combinations of sensing element and transducer (Table 1) have not yet been explored in true biosensor configurations. This, together with the breadth of multidisciplinary knowledge required to grasp essential concepts, has led the editors to include a few chapters in this monograph falling outside the strict definition given above. In particular, some techniques capable of closely monitoring biological systems have been

Table 1 Components that may be used to construct a biosensor

\begin{tabular}{ll}
\hline Biological elements & Transducers \\
\hline Organisms & Potentiometric \\
Tissues & Amperometric \\
Cells & Conductimetric \\
Organelles & Impedimetric \\
Membranes & Optical \\
Enzymes & Calorimetric \\
Enzyme components & Acoustic \\
Receptors & Mechanical \\
Antibodies & 'Molecular' electronic \\
Nucleic acids & \\
Organic molecules & \\
\hline
\end{tabular}


examined in an attempt to pave the way for future developments in this evolving science.

Biosensors will develop as a result of effort on several fronts. Configurations reported to date have relied largely on effecting novel partnerships between independent approaches which were in themselves conventional. Increasingly, attention will have to be paid to engineering both the components and the whole device to meet specific requirements. New biochemical reactions will either be unearthed or will have to be engineered using genetic manipulation or chemical techniques. These will be designed with a suitable detector in mind rather than relying on fortuitous availability from prior investigations. New materials for constructing transducers or effecting links between the components of a sensor is an exciting avenue for research. In addition, the plummetting price of some hardware such as lasers will make available low cost versions of sophisticated laboratory instrumentation. The construction of the sensor as a whole should not be neglected. The fundamental properties of the device must be understood both in terms of its constituents and in the complexities of their interrelationships in order to optimize critical criteria such as response time, selectivity, and stability. Immobilization technologies and new membrane materials may profoundly affect the end performance of a particular sensor.

The study of biosensors has been motivated by a strong practical instinct with clear applications always in sight. Much of the impetus of work has come from medical requirements. Instant analysis of clinical samples has an obvious appeal to physicians and patients alike, although some national health services are finding it difficult to incorporate this philosophy. Perhaps more exciting is the possibility of continuous in vivo monitoring of metabolites, drugs, and proteins using miniature, highly portable systems. The cardinal clinical example is the glucose sensor for diabetes which has become a classic subject for study in the field of biosensors. In this condition there is a requirement for both in vitro and in vivo monitoring with the possibility of managing the disease in a totally automated fashion via the insulin infusion pump. Implantable sensors share the hurdles facing other applications with the additional serious problem of the need for biocompatibility.

In recent years there has been a growing appreciation of the other possible uses of biosensors. Clinical research is spinning off into related veterinary areas and animal husbandry. The food industry is increasingly concerned about quality and has long recognized the value of rapid methods for estimating shelf life, deterioration, and contamination. The rise of biotechnology has stimulated investigations into fermentation monitoring and control with possibilities for process control also arising. Concern for both the industrial and natural environment has led to research on sensors for pollutants such as carbon monoxide and herbicides; while the ubiquitous 
military interest is focusing on specialized needs including biological and chemical defence.

The aim of this book is to provide the first advanced and comprehensive treatise on the subject of biosensors. A multiauthor approach was chosen to do full justice to the individualistic approaches that have characterized the history of the area. The contentious and fluid nature of some aspects of the field are expounded by their main proponents; each argument is made in a clear and concise style amenable to scientists of many disciplines with the overall conclusion being left to the reader. It is uncertain exactly which direction will predominate in the next decade, but there is little doubt that the biosensor will impinge on the lives of an increasing number and wide range of scientists.

Cranfield

A.P.F.T.

June 1986 



\section{Contents}

\section{The biological component}

1 The enzyme electrode LELAND C, CLARK, JR.

2 Micro-organism based sensors ISAO KARUBE

3 Biosensors based on plant and animal tissue MARK A. ARNOLD and GARRY A. RECHNITZ

4 New approaches to electrochemical immunoassays 60 MONIKA J. GREEN

5 The diagnosis of human genetic diseases JOHNM. OLD and KAY E. DAVIES

6 Immobilization of the biological component of biosensors

S. A. BARKER

7 Genetic engineering $P, J, W A R N E R$

8 Protein engineering and its potential application to biosensors ANTHONY E. G. CASS and ENDA KENNY

\section{Bioelectrochemistry}

(a) Potentiometric sensors

9 Ion-selective electrodes and biosensors based on ISEs

S. S. KUAN and G. G. GUILBAULT

10 Potentiometric biosensors based on redox electrodes

LEMUEL B. WINGARD JR, and JAMES CASTNER

(b) Amperometric sensors

11 Fundamentals of amperometric sensors GEORGE S. WILSON

12 Amperometric enzyme electrodes: theory and experiment W. JOHN ALBERY and DEREK H. CRASTON 
13 The use of electrochemical methods in the study of modified electrodes

P. N. BARTLETT

14 Cyclic voltammetry studies of enzymatic reactions

for developing mediated biosensors

GRAHAM DAVIS

15 The realization of electron transfer from biological molecules to electrodes

M. F. CARDOSI and A. P.F. TURNER

16 The construction of mediated amperometric biosensors W. J. ASTON

17 Redox-mediated electrochemistry of whole micro-organisms: from fuel cells to biosensors

H. PETER BENNETTO, JONATHAN BOX, GERARD M.

DELANEY, JEREMY R. MASON, SIBEL D. ROLLER, JOHNL.

STIRLING, and CHRISTOPHER F. THURSTON

18 Application of enzyme-based amperometric biosensors to the analysis of 'real' samples

FRIEDER W. SCHELLER, DOROTHEA PFEIFFER, FLORIAN

SCHUBERT, REINHARD RENNEBERG, and DIETER KIRSTEIN

19 Compensated enzyme-electrodes for in situ process control SVEN-OLOF ENFORS

20 In vivo chemical sensors and biosensors in clinical medicine DENZIL J. CLAREMONT and JOHN C, PICKUP

21 Thin-film micro-electrodes for in vivo electrochemical analysis O. PROHASKA

22 The design and development of in vivo glucose sensors for an artificial endocrine pancreas

GILBERTO D. VELHO, GERARD REACH, and DANIEL $R$.

THÉVENOT

23 Needle-type glucose sensor and its clinical applications

MOTOAKI SHICHIRI, RYUZO KAWAMORI, and YOSHIMITSU YAMASAKI

(c) Analysis of electrical impedance

24 The principles of potential of electrical admittance spectroscopy: an introduction

DOUGLAS B. KELL

(d) Silicon-based sensors 
25 Micro-biosensors based on silicon fabrication technology ISAO KARUBE

26 Chemically sensitive field-effect transistors GARYF. BLACKBURN

27 Biosensors based on semiconductor gas sensors BENGT DANIELSSON and FREDRIK WINQUIST

28 Principles and potential of piezo-electric transducers and acoustical techniques

DAVID J. CLARKE, BARRIE C. BLAKE-COLEMAN, and MICHAEL R, CALDER

\section{Calorimetry}

29 Theory and applications of calorimetric sensors

BENGT DANIELSSON and KLAUS MOSBACH

\section{Photometry}

30 Optical sensors based on immobilized reagents W. RUDOLF SEITZ

31 Potential applications of bioluminescence and chemiluminescence in biosensors F. MCCAPRA

32 Design of fibre-optic biosensors based on bioreceptors JEROME S. SCHULTZ

33 IRS devices for optical immunoassays RANALD M. SUTHERLAND and CLAUS DÄHNE

34 Laser light scattering and related techniques 679 ROBERT J. G. CARR, ROBERT G.W. BROWN, JOHN G. RARITY, and DAVID J. CLARKE

\section{Applications of microprocessors}

35 The use of microprocessors for the evaluation of the 705 analytical performance of enzyme-based sensors DANIEL R. THÉVENOT, THIERRY TALLAGRAND, and ROBERT STERNBERG 
37. Exploiting biosensors JAMES MCCANN

Index 


\section{Contributors}

W, JOHN ALBERY Department of Chemistry, Imperial College, London SW7 2AY, UK

K.G.M.M. ALBERTI The Medical School, University of Newcastle, Newcastle upon Tyne, UK

MARK A. ARNOLD Department of Chemistry, University of Iowa, Iowa City, Iowa 52242, USA

W.J. ASTON Genetics International (UK) Inc., 38 Nuffield Way, Abingdon, Oxfordshire OX14 1RL, UK

S.A. BARKER Department of Chemistry, University of Birmingham, Birmingham, UK

P.N. BARTLETT Department of Chemistry, University of Warwick, Coventry CV4 7AL, UK

H. PETER BENNETTO Bioelectrochemistry and Biosensors Group, King's College, University of London, Kensington Campus, Campden Hill Road, London W8 7AH, UK

BARRIE C. BLAKE-COLEMAN Biosensor Group, Microbial Technology Laboratory, Centre for Applied Microbiology and Research, Porton Down, Wiltshire, SP4 0JG, UK

GARYF. BLACKBURN Fundamental Research Laboratory, GTE Laboratories Inc., 40 Sylvan Road, Waltham, MA 02254, USA

JONATHAN BOX Bioelectrochemistry and Biosensors Group, King's College, University of London, Kensington Campus, Campden Hill Road, London W8 7AH, UK

ROBERT G.W. BROWN Royal Signals and Radar Establishment, Malvern, Worcs. WR14 3PS, UK

MICHAEL R. CALDER Biosensor Group, Microbial Technology Laboratory, Centre for Applied Microbiology and Research, Porton Down, Wiltshire, SP4 0JG, UK

M.F. CARDOSI Bioelectronics Divisions, Biotechnology Centre, Cranfield Institute of Technology, Cranfield, Bedfordshire, MK43 OAL, UK ROBERT J.G. CARR Biosensor Group, Microbial Technology Laboratory, Centre for Applied Microbiology and Research, Porton Down, Wiltshire, SP4 0JG, UK

ANTHONY E. G. CASS Centre for Biotechnology, Imperial College of 
Science and Technology, London SW7 2AZ, UK

JAMES CASTNER Biomedical Division, E. I. du Pont de Nemours Co., Wilmington, Delaware, 19898, USA

DENZIL J. CLAREMONT Division of Chemical Pathology, United Medical and Dental Schools, Guy's Hospital Campus, London SE1 9RT, UK LELAND C. CLARK, JR. Children's Hospital Research Foundation, Elland and Bethesda Avenues, Cincinnati, Ohio 45229, USA

DAVID J. CLARKE Biosensor Group, Microbial Technology Laboratory, Centre for Applied Microbiology and Research, Porton Down, Wiltshire SP4 0JG, UK

DEREK H. CRASTON Department of Chemistry, Imperial College, London SW7 2AY, UK

CLAUS DÄHNE Battelle Geneva Research Centres, 7 Route de Drize, 1227 Carouge/Geneva, Switzerland

BENGT DANIELSSON Pure and Applied Biochemistry, Chemical Centre, University of Lund, P.O. Box 124, S-221 00 Lund, Sweden

$K A Y E$. DAVIES Nuffield Department of Clinical Medicine, John Radcliffe Hospital, Oxford OX3 9DU, UK

GRAHAM DAVIS Integrated Ionics Inc., 2235 State Route 130, Dayton, New Jersey 08810, USA

GERARD M. DELANEY Bioelectrochemistry and Biosensors Group, King's College, University of London, Kensington Campus, Campden Hill Road, London W8 7AH, UK

SVEN-OLOF ENFORS Department of Biochemistry and Biotechnology, The Royal Institute of Technology, S-100 44 Stockholm, Sweden

MONIKA J. GREEN Genetics International (UK), Inc., 11 Nuffield Way, Abingdon, Oxfordshire OX14 1RL, UK

G. G. GUILBAULT Department of Chemistry, and University of New Orleans, New Orleans, Louisiana 70148, USA

P.D. HOME The Medical School, University of Newcastle, Newcastle upon Tyne, UK

ISAO KARUBE Research Centre for Advanced Science and Technology, University of Tokyo, 4-6-1 Komaba, Meguro-ku, Tokyo 153, Japan

RYUZO KAWAMORI First Department of Medicine, Osaka University Medical School, 1-1-50 Fukushima, Fukushima-ku, Osaka 553, Japan

DOUGLAS B. KELL Department of Botany and Microbiology, University College of Wales, Aberystwyth, Dyfed SY23 3DA, UK

ENDA KENNY Delta Biotechnology Ltd., Castle Court, 59 Castle Court, Boulevard, Nottingham NG7 1FO, UK

DIETER KIRSTEIN Central Institute of Molecular Biology of the 
Academy of Sciences of the GDR, Robert-Róssle-Str, 10, 1115 Berlin, GDR S. S. KUAN Mycotoxin Research Center, Food and Drug Administration, 4298 Elysian Fields Avenue, New Orleans, Louisiana 70122, USA

JEREMYR.MASON Bioelectrochemistry and Biosensors Group, King's College, University of London, Kensington Campus, Campden Hill Road, London W8 7AH, UK

KLAUS MOSBACH Pure and Applied Biochemistry, Chemical Centre, University of Lund, P.O. Box 124, S-221 00 Lund, Sweden

JAMES MCCANN Genetics International (UK) Inc., 11 Nuffield Way, Abingdon, Oxfordshire OX14 1RL, UK

FRANK MCCAPRA The University of Sussex, School of Chemistry and Molecular Sciences, Falmer, Brighton BN1 9QJ, UK

JOHNM, OLD Nuffield Department of Clinical Medicine, John Radcliffe Hospital, Oxford OX3 9DU, UK

DOROTHEA PFEIFFER Central Institute of Molecular Biology of the Academy of Sciences of the GDR, Robert-Rössle-Str. 10, 1115 Berlin, GDR JOHN C. PICKUP Division of Chemical Pathology, United Medical and Dental Schools, Guy's Hospital Campus, London SE1 9RT, UK

O. PROHASKA Case Western Reserve University, Department of Biomedical Engineering, 501 Wichender Building, Cleveland, Ohio 44106, USA

JOHN G. RARITY Royal Signals and Radar Establishment, Malvern, Worcs. WR14 3PS, UK

GERARD REACH Unité de Recherches sur le Diabète et la Nutrition chez l'Enfant, INSERM U-290, Hopital Saint Lazare, 107 rue du Fanbourg Saint Denis, 75010 Paris, France

GARRYA. RECHNITZ Department of Chemistry, University of Hawaii, Honolulu, Hawaii 96822 , USA

REINHARD RENNEBERG Central Institute of Molecular Biology of the Academy of Sciences of the GDR, Robert Rössle-Str. 10, 1115 Berlin, GDR SIBEL D. ROLLER Leatherhead Food R.A., Randalls Road, Surrey KT22 7RY, UK

FRIEDER W. SCHELLER Central Institute of Molecular Biology of the Academy of Sciences of the GDR, Robert-Rössle-Str. 10, 1115 Berlin, GDR FLORIAN SCHUBERT Central Institute of Molecular Biology of the Academy of Sciences of the GDR, Robert-Rössle-Str. 10, 1115 Berlin, GDR

JEROMES. SCHULTZ Head of Biotechnology, University of Pittsburgh, 518 Scaife Hall, PA 15261, USA

W, RUDOLF SEITZ Department of Chemistry, University of New Hampshire, Durham, NH 03824, USA 
MOTOAKI SHICHIRI Department of Metabolic Medicine, Kumanoto University Medical School, 1-1-1 Honjo, Kumanoto, Japan

ROBERT STERNBERG Laboratoire de Bioélectrochimie et d'Analyse du Milieu, U.E.R, de Sciences, U.A. 329 du C.N.R.S., Université Paris-Val de Marne, Ave. du Général de Gaulle, 94010 Créteil Cedex, France

JOHN L. STIRLING Bioelectrochemistry and Biosensors Group, King's College, University of London, Kensington Campus, Campden Hill Road, London W8 7AH, UK

RANALDM. SUTHERLAND Battelle Geneva Research Centres, 7 Route de Drize, 1227 Carouge/Geneva, Switzerland

THIERRY TALLAGRAND Laboratoire de Bioelectrochimie et d'Analyse du Milieu, U.E.R. de Sciences, U.A. 329 du C.N.R.S., Universite Paris-Val de Marne, Ave du Général de Gaulle, 94010 Créteil Cedex, France DANIEL R. THÉVENOT Laboratoire de Bioélectrochimie et d'Analyse du Milieu, U.E.R. de Sciences, U.A. 329 du C.N.R.S., Université Paris-VaI de Marne, Ave. du Général de Gaulle, 94010 Créteil Cedex, France

CHRISTOPHER F, THURSTON Bioelectrochemistry and Biosensors Group, King's College, University of London, Kensington Campus, Campden Hill Road, London W8 7AH, UK

A.P.F. TURNER Bioelectronics Division, Biotechnology Centre, Cranfield Institute of Technology, Cranfield, Beds. MK43 0AL, UK

GILBERTOD. VELHO Unité de Recherches sur le Diabète et la Nutrition chez l'enfant, INSERM U-290, Hopital Saint Lazare, 107 rue du Fanbourg Saint Denis, 75010 Paris, France

P.J. WARNER Biotechnology Centre, Cranfield Institute of Technology, Cranfield, Beds. MK43 OAL, UK

GEORGE S. WILSON Department of Chemistry, University of Arizona, Tucson, Arizona 85721, USA

LEMUEL B. WINGARD, JR. Department of Pharmacology, School of Medicine, University of Pittsburgh, Pittsburgh, Pennsylvania 15261, USA FREDRIK WINQUIST University of Lund, PO Box 124, S-22100 Lund, Sweden

YOSHIMITSU YAMASAKI First Department of Medicine, Osaka University Medical School, 1-1-50 Fukushima, Fukushima-ku, Osaka 553, Japan 
The biological component 



\section{1 \\ The enzyme electrode}

LELAND C. CLARK, JR.

Since life itself depends upon almost incomprehensibly balanced enzymemediated substrate-specific transfer of electrons, it may not be surprising that means to measure the vital biochemical cellular processes would involve sensors composed of the same substances. Like advances in the past, progress in the future will depend upon increased understanding and control of enzymes, probably synthesis of enzymes, a more sophisticated control of electron transfer, and a close interaction between electrochemistry and the physiology of living systems.

The key discovery by the Buchner brothers, that fermentation proceeded in the filtered juice of yeast cells destroyed by grinding with sand, was followed in 1926 by Sumner's success in crystallizing urease thus demonstrating that enzymes were merely proteins. Before that, their supposedly magic role was believed to be intrinsically intertwined in the life process. Most thought enzymes should be treated as highly perishable, like fresh eggs, and stored in the cold until they could be measured or used.

By looking at enzymes as specific chemical transducers, translating an analyte into a substance capable of being detected by a chemically or physically sensitive detector, a new class of sensors, intrinsically responsive to biological compounds, has been conceived and developed. Combinations of enzymes, such as esterases, dehydrogenases, and oxidases, and of detectors, such as polarographic, conductimetric, potentiometric, acoustic, and optical, offer promise to expand the selectivity, sensitivity, and versatility of these detectors. The first enzyme electrodes relied on enzymes physically entrapped on or very near the sensor's surface. Later on, chemical immobilization, insolubilization, or fixation techniques were adopted. Coenzymes have also been physically and chemically affixed. Insolubilization as a means of enzyme life extension may have the advantage of avoiding the complications of colloid osmotic forces, especially when analyte-permeable membranes are used in conjunction with an enzyme electrode. Ideally, enzyme-based biosensors should, like the revolutionary blood gas and $\mathrm{pH}$ electrodes, work directly in undiluted whole blood.

Glucose and lactate electro-enzymatic systems are being widely used in biomedicine, especially where rapid on-the-spot analysis of small samples of blood are desired. Intravascular biosensors may find use in continuous monitoring of blood in paediatric and coronary intensive care units. The 


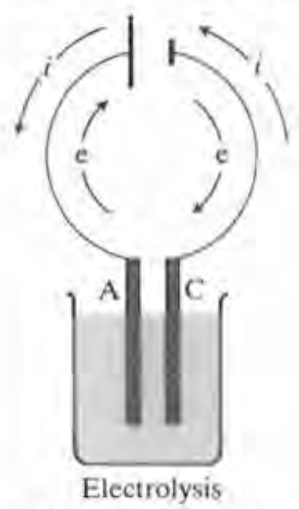

Fig. 1.1 Electrolysis. At the anode (A) electrons are removed from the substrate and oxidation occurs. At the cathode $(\mathrm{C})$ electrons (e) are added and reduction occurs. The applied potential controls the kind of reactions to some extent. Current $(i)$, by convention, flows in the opposite direction.

future of implanted sensors, for example glucose electrodes, to control insulin pumps and lactate sensors to control cardiac pacemakers and defibrillators, depends largely upon finding means of stabilizing the needed enzymes when used at body temperature in contact with body fluids. A bright future for biosensors in biology and medicine seems intrinsic to their very nature.

In the evolutional time scale of biosensors, we are just leaving the stage where we climbed down at dusk to hunt and entering the time when we cultivated the land in open sunshine. Enzymes are being harnessed for industrial use, in the analytical research laboratory, and in clinical monitoring. In a random selection of ten issues of Analytical Electrochemistry (CA Selects) for 1985, out of about 1500 abstracts, around 600 deal with enzyme electrodes.

Polarography depends upon electrolysis (Fig. 1.1). The beginnings of the polarographic enzyme electrode can be found in the dropping mercury electrode (Fig. 1.2) of Heyrovsky (1960), for in the years between the two wars he elucidated the electrochemical nature of the interface between this metal, the applied potential, and the chemical reactions. This was worked out in air-free solutions because oxygen was a considerable nuisance to polarography, as this new science was called, using the mercury electrode. There has been a continuous effort by chemists to increase the versatility and specificity of this electrochemical method. From an analytical viewpoint, the salient advantages of the dropping mercury electrode were its theoretical appeal, its reproducibility and its analytical capabilities, especially in pretreated samples in the laboratory. The workable but complex instrumentation required to adapt mercury polarography to the measurement of enzymes is shown in Fig. 1.3. But because liquid mercury was potentially toxic and cumbersome to use in biology the wonderful advantages inherent in a shiny new stirred 


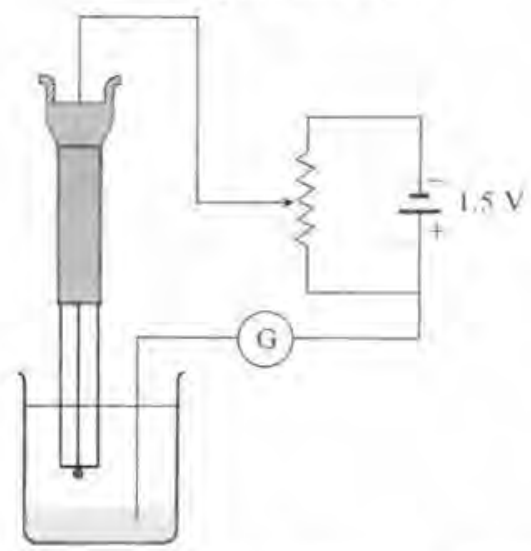

Fig. 1.2 The Heyrovsky dropping mercury electrode (Heyrovsky 1960). It is most useful in the range of +0.4 to $-2.6 \mathrm{~V}$ applied voltage. Platinum electrodes can be used between +0.9 and $-0.8 \mathrm{~V}$.

mercury electrode every few seconds could not be used in many situations. For the many gains to be made in physiology by oxygen measurement, biologically inert solid anode and cathode surfaces seemed vastly preferable. Many kinds of solid electrodes were (and are still being) tested. Platinum was usually selected, partly because it could be sealed into and insulated with glass, But this solid unrenewable unstirred platinum surface was easily contaminated by the myriad of substances present in blood and living tissues. These problems have been reviewed by Davies (1962). Clark's cellophanecovered platinum oxygen cathode (Clark et al, 1953) overcame many of these problems but it was not until both platinum cathode and silver reference electrode were placed in their own electrically conductive micro-environment behind an electrically non-conductive gas-only permeable polyethylene membrane (Clark 1956) that a new, reproducible way to measure oxygen tension in tissues, liquids, and gases resulted. Because oxygen was the main concern in biology, and not the quantitation of a number of ions and organic substances, the potential could be held constant when oxygen was measured with a platinum electrode. Many biosensors combine the Clark oxygen electrode measurements with enzymes fastened on the electrode's membrane.

The cathode largely performs irreversible reduction of oxygen and the anode performs irreversible oxidation of its substrate, let's say, hydrogen peroxide and ascorbic acid. Unlike optical methods, though, the applied potential determines, as Heyrovsky showed, the kind of chemistry, while the current shows the amount. Thus the pressure of electrons controls and the flow measures,

Biosensors, meaning sensors which incorporate biological material in their structure (Fig. 1.4) were first described in 1962 at a New York Academy of Sciences symposium (Clark and Lyons 1962). In that presentation the use of 


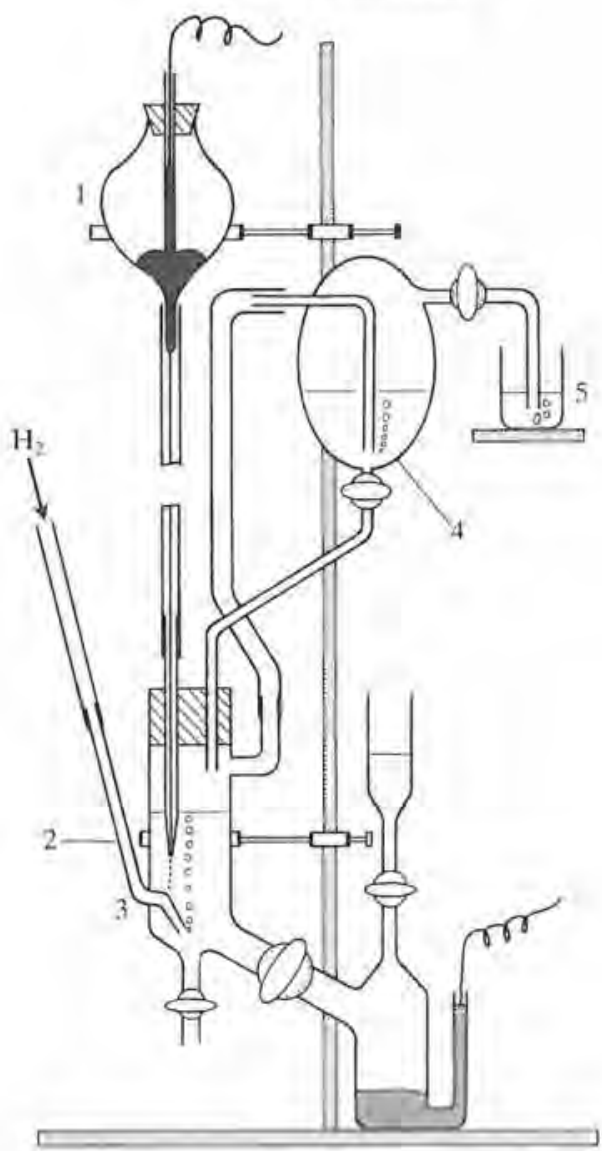

Fig. 1.3 Apparatus for following enzyme action. (From Knoblock, E. (1944). Chem. Listy 38, 193.)

enzyme transducers as membrane-enclosed sandwiches was described to make electrochemical sensors $(\mathrm{pH}$, polarographic, potentiometric, or conductimetric) more intelligent. They became specific for certain substrates by detection of a product of an enzyme-catalysed reaction or a drop in a substance used in the reaction. For example, the combination of glucose oxidase with a Clark $\mathrm{pO}_{2}$ electrode to measure glucose by detecting the drop in oxygen when glucose was converted to gluconic acid and hydrogen peroxide was described.

So basically, there are two kinds of polarographic enzyme electrode sensors. In one, the analyte consumes oxygen in the presence of an enzyme and the measurement depends upon a change in oxygen tension. In the other, the enzyme converts the analyte to a substance to which the sensor is sensitive. The purpose of the enzyme is to transduce the substance being 


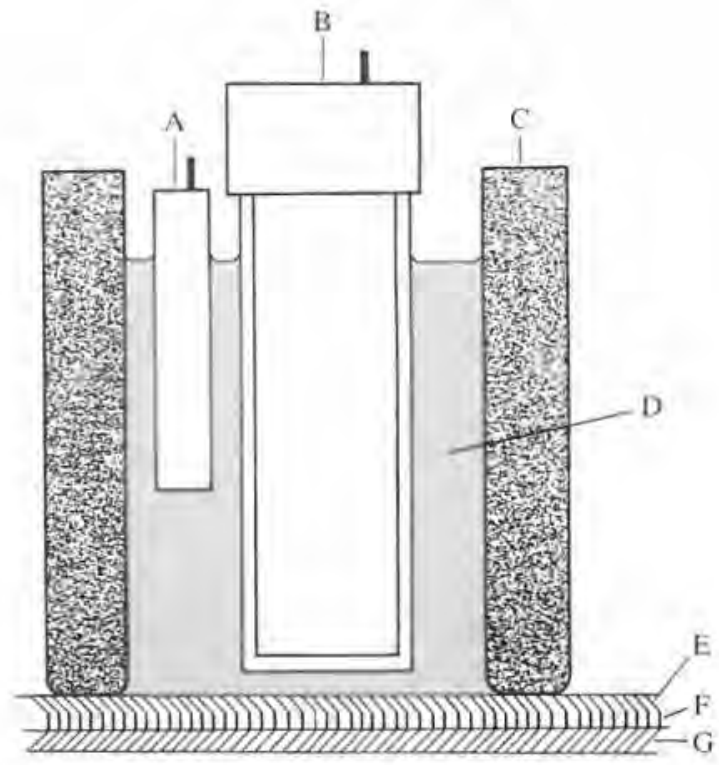

Fig. 1.4 The first enzyme electrode (Clark and Lyons 1962).

measured, from one to which the sensor is not responsive, to one to which it is responsive. For this reason, the enzyme layer was originally referred to as a transducer. Perbaps the enzyme-formed substance, the product of interest, should be called a 'transformate'. The substrate, the analyte, would be changed to the 'transformate', in the milieu of either the 'catholyte' or the 'anolyte' in the enzyme layer.

If the classical amperometric oxygen electrode polarity is reversed so that the anode is about $0.6 \mathrm{~V}$ positive, the electrode is completely insensitive to oxygen but responds to hydrogen peroxide which is oxidized to water. The platinum anode also oxidizes ascorbic acid but not many other substances are found in animal juices in sufficient quantity to effect a current anywhere near the corresponding cathodic current for the oxygen electrode. The sensitivity of the anode to hydrogen peroxide was intriguing but since catalase is nearly everywhere it seemed that a biosensor to measure peroxide would be next to worthless, except perhaps to measure catalase or peroxidase. Heretofore, proteins were regarded as something which contaminated the platinum surface. My first use of platinum cathodes, for example, was motivated by the perceived need to keep blood proteins and cells away from the platinum surface. I suppose it was thinking about how to keep catalase away from the platinum anode that began the process that ended up by using the same membrane to keep catalase away, and since all enzymes are big protein molecules, to keep other enzymes near the platinum, at the same time. In the first enzyme electrode, the enzyme was shown as a sandwich, because one was 
still nervous about contaminating the platinum surface with proteins and coenzymes. But I also added enzyme to the electrolyte forming the path from the anode to the cathode, and the electrode worked well to measure glucose.

By 1963 I was working primarily with anodic polarography to measure $\mathrm{H}_{2} \mathrm{O}_{2}$ formed by reactions catalysed by the oxygen oxidoreductases. With the peroxide sensor, whole blood could be used, eliminating the need for centrifugation and permitting continuous monitoring of substrates such as glucose in vivo or in a flowing stream in vitro. When using oxygen electrodes the red cells and their oxygen-carrying haemoglobin had to be removed and only the serum or plasma used.

By 1965 or so, I was measuring glucose in the diluted blood of postoperative patients with a hand-held electrode in a beaker. I was still measuring lactate by the Barker-Summerson method and wishing that somehow a lactate oxidase that generated peroxide could be found. A patent applied for in 1965 (Clark 1970) covered the use of one or more enzymes to convert various substrates ultimately to hydrogen peroxide. It described the use of two electrodes to enable subtraction of current from an electrode without enzyme from current of an electrode with enzyme to eliminate interfering currents. By 19691 had convinced the Yellow Springs Instrument Company to undertake the development of a dedicated glucose analyser for the direct measurement of glucose in 25 microlitre samples of whole blood. And by 1974 the Model 23 YSI analyser, after some faltering steps, appeared on the market.

It must be kept in mind that both the enzyme used and the polarized electrode itself generate products, by-products if you will, which are not necessarily wanted and which may impede the desired reaction. For example phenol, when oxidized, produces a black shiny deposit on the electrode surface which alters its chemical reactivity and may also produce an electrically insulating layer. It is also important that the by-products must not affect the activity of the enzyme. It is best when the products of the reaction are water soluble and can diffuse away. In the case of the glucose oxidasebased anode, the products are hydrogen peroxide, gluconolactone, gluconate, water, and oxygen, all of which are very soluble and diffusable.

One solution to interfering polarographically active substances in the sample, is to construct membranes which prevent the unwanted substances from reaching the platinum surface. This necessitates two membranes, the inner one preventing permeation of any unwanted substance, the outer one permitting passage of the substrate into the enzyme layer, and usually the interfering substance as well. A number of such proprietary combination membranes have been developed and several are on the market. The YSI glucose and lactate electrodes, for example, use a cellulose acetate and polycarbonate Nuclepore combination.

The advance of polarographic techniques from the early two-electrode to three-electrode systems, to pulse polarography, to differential pulse 
polarography, and so on, has increased sensitivity and accuracy. It is important to keep in mind that the application of potentials in various schema not only affects the electrochemistry on the surface of the electrode but may also influence the nature and activity of the enzyme used. Signal amplification by enzyme recycling offers new avenues of increasing the sensitivity of enzyme based sensors (Scheller et al. 1985).

Of the many substrates that have been measured by the use of oxygen oxidoreductases are included glucose, lactate, pyruvate, galactose, alcohol, cholesterol, glycerol, hypoxanthine, xanthine, oxalate, and fructose. The ingenious use of ferrocene to mediate electron transfer in an oxidase-based sensor to measure glucose is worthy of note (Cass et al, 1984; Aston, this volume, Chapter 16; Cardosi and Turner, this volume, Chapter 15).

New oxygen oxidoreductases, found in primitive forms of life such as fungi, are being discovered each year, but many more dehydrogenases have been found, usually in higher life forms of plants and animals. Racine et al. (1975) have coupled ferrocyanide/ferricyanide with lactic dehydrogenase in a lactate sensor.

The biosensor measurement of activity of a large number of enzymes in blood is also possible. Measurement of 'cardiac' enzymes, such as aspartate aminotransferase, creatine kinase, and creatine kinase MB, has proven clinically useful in judging infarct size. Stat measurement of these enzymes, together with lactate and glucose, could prove to be valuable in deciding therapeutic courses for patients with potentially lethal arrhythmias in the prehospitalization phase of acute myocardial infarction. Stat amylase measurements in pediatric patients may be valuable.

Rapid glucose measurement capabilities should be a part of every paediatric unit. Such round the clock availability of glucose measurements, among other things, could protect children from dangerous hypoglycaemia before surgery. Lactate and, possibly, pyruvate measurements should be available. Paediatric residents and nurses should learn to calibrate biosensorbased instrumentation so that glucose and lactate can be run at the same time or on the same samples used for blood gases and $\mathrm{pH}$.

Conjunctival oxygen tension measurements have been pioneered by Fatt. The unique character of the palpebral circulation which make continuous $\mathrm{pO}_{2}$ (as well as $\mathrm{CO}_{2}$ and, perhaps, $\mathrm{pH}$ ) monitoring possible could prove to be of value for glucose monitoring. A biosensor under the eyelid may be between 'invasive' and 'non-invasive'. Perhaps continuous recording of conjunctival lactate will become possible. Continuous monitoring of blood glucose would be valuable in labour and delivery by pregnant diabetics.

Enzyme micro-electrodes have a promising future. For the measurement of cellular intermediary metabolites it is difficult to imagine a method which could be more specific and elegant, Silver (1976) was the first to measure intracellular glucose. The Austrian scientists (Geibel et al. 1984) have measured volume fluxes and glucose in isolated perfused tubule segments 
using a platinum galactose oxidase electrode having a tip diameter of $15-30 \mu \mathrm{m}$. The galactose oxidase electrode is sensitive to raffinose, used in the latter studies, as well as to galactose, glycerol, fructose, and dihydroxyacetone. The nature of this enzyme electrode is related to internal solution potential in an interesting way (Johnson et al. 1982). There are several interesting ways to couple oxidase activity to other substrates (Hopkin 1985).

For use in undiluted blood or for implanted electrodes it must be remembered that the oxygen oxidoreductases ('oxidases') require oxygen and often their rate of oxidation of the substrate is a function of the $\mathrm{pO}_{2}$. Without oxygen they will not normally function because the transformate will not be generated by the enzyme. Enfors (1983; Cleland and Enfors, 1983) has provided oxygen by anodic generation of oxygen in the enzyme layer, measuring the fermentation analyte according to the oxygen current required to keep the oxygen pressure steady (Fig. 1.5). The need for in situ fermentation biosensors parallel those for surgical implantation. Clark and Sachs (1968) had previously used a similar oxystat principle but the oxygen was added from a saturated solution by a servo system. Recently, it has been shown (Clark et al. 1987) that sufficient gaseous oxygen can be supplied from an implanted Silastic drum to make an integral glucose sensor which is glucose dependent and essentially $\mathrm{pO}_{2}$ independent. Gough and Leypoldt (1981) have described means of supplying oxygen to glucose sensors.

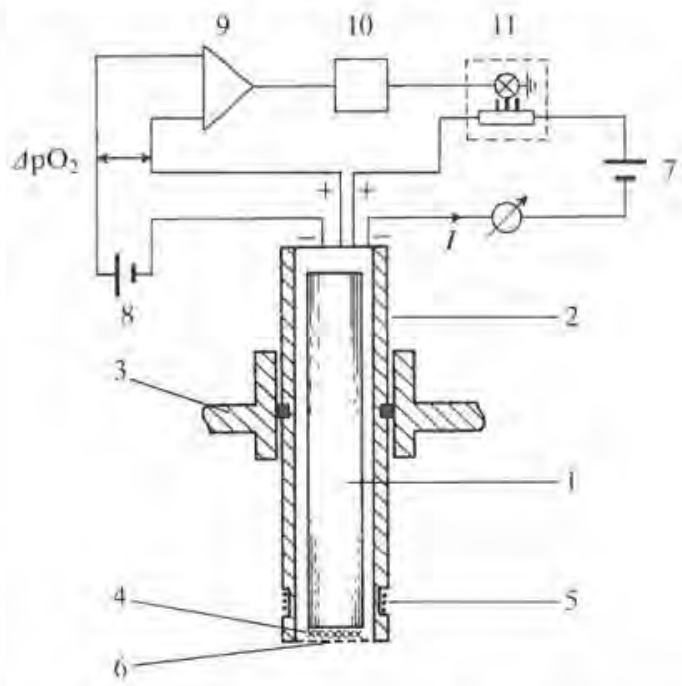

Fig. 1.5 Oxidase-based electrode supplied with electrolytic oxygen (from Enfors 1983).

1. Oxygen electrode; 2, electrode housing; 3, fermenter lid; 4, Pt-gauze with immobilized enzymes; 5, Pt-coil (cathode); 6, semipermeable membrane; 7 , electrolysis voltage source; 8 , reference voltage; 9 , differential amplifier; 10 , PIDcontroller; 11 , electrolysis current controller; $I$, electrolysis current. 


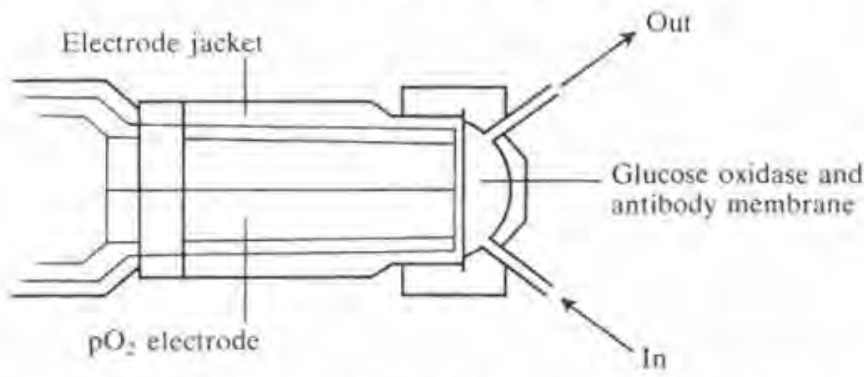

Fig. 1.6 Immunosensor based on the Clark $\mathrm{pO}_{2}$ electrode (from Boitieux, J. L. et al. (1984). Clinica Chimica Acta 136, 19).

Various combinations of antibody membrane and enzyme electrodes may provide new automatable sensors for antigens. Such biosensors, coupled perhaps with sensors for 'liver enzymes', could provide rapid, reliable means of screening blood supplies. Figure 1.6 is based upon the measurement of oxygen consumption in the presence of glucose oxidase and glucose for quantitation of hepatitis B surface antigen antibodies. Other electroenzymatic methods for immunological research have been published and many more can be expected (Green, this volume, Chapter 4).

Enzyme electrodes seem particularly well suited for instrumentation for physicians' offices and home monitoring, since such biosensors could be readily mass produced from relatively inexpensive and stable components. An inexpensive device to monitor blood alcohol, perhaps through the skin, could be devised. Emergency medicine has specialized needs where rapid results can be life saving.

But perhaps the greatest future for enzyme electrodes will be as biosensors in or on the body. Sensors, as for lactate and glucose, would be made exceedingly small and incorporated in intravascular catheters for monitoring critically ill patients (Clark et al. 1988; Clark and Duggan 1982). The importance of blood lactate as a measure of the adequacy of tissue oxygenation, or cardiac output, cannot be overemphasized. There is evidence, too, that a high maternal lactate during labour may have a deleterious effect on the newborn. Hypoxanthine may prove to be a valuable integrator of hypoxia. Implantable glucose sensors will almost certainly be devised capable of controlling insulin pumps (Clark et al. 1988). This use in diabetes alone would justify the enormous effort in combining enzymes and electrochemistry.

\section{References}

Cass, A. E. G., Davis, G., Francis, G. D., Hill, H. A. O., Aston, W. J., Higgins, I. J., Plotkin, E. V., Scott, L. D. L. and Turner, A.P. F. (1984). Ferrocene-mediated enzyme electrode for amperometric determination of glucose. Anal. Chem. 56, $667-71$. 
Clark, L. C.. Jr. (1956). Monitor and control of blood and tissue oxygen tensions. Trans. Am. Soc. Artif. Intern. Organs 2, 41-8.

(1970). Membrane polarographic electrode system and method with electrochemical compensation. U.S. Patent No. 3539455.

Clark, L. C., Jr, and Duggan, C.A. (1982). Implanted electroenzymatic glucose sensors. Diabetes Care 5, 174-80.

Clark, L. C., Jr. and Lyons, C. (1962). Electrode systems for continuous monitoring in cardiovascular surgery. Ann. NY Acad. Sci. 102, 29-45.

Clark, L. C., Jr. and Sachs, G. (1968). Bioelectrodes for tissue metabolism. Ann. NY Acad. Sci. 148, 133-53.

Clark, L. C., Jr., Noyes, L. K., Grooms, T. A. and Moore M. S. (1984). Rapid micromeasurement of lactate in whole blood. Crit. Care Med, 12, 461-4.

Clark, L. C., Jr,, Wolf, R., Granger, D. and Taylor, Z. (1953). Continuous recording of blood oxygen tensions by polarography. J. Appl. Physiol. 6, 189-93.

- (1987). Design and long-term performance of surgically implanted electroenzymatic glucose sensors. Ann. NY Acad. Sci. 501, 534-537.

Clark, L. C., Jr., Noyes, L. K., Spokane, R. B., Sudan, R. and Miller, M. L. (1988). Long term implantation of voltammetric oxidase/peroxide glucose sensors in the rat peritoneum. In Methods in Enzymology (Immobilized Enzymes and Cells) (ed. K. Mosbach), Vol. 137. Academic Press, San Diego.

Cleland, N. and Enfors, S.-O. (1983). Control of glucose-fed batch cultivation of $E$. coli by means of an oxygen stabilized enzyme electrode. Eur. J. Appl. Microbiol. Biotechnol. 18, 141-7.

Davies, P.W. (1962). The oxygen cathode. In Physical techniques in biological research (ed. W. L. Nastuk), Vol. 4, pp. 137-79. Academic Press, New York.

Enfors, S-O. (1983). Oxygen stabilized enzyme electrode. U.S. Patent No. 4374013.

Geibel, J., Volkl, H. and Lang, F. (1984). A microelectrode for continuous recording of volume fluxes in isolated perfused tubule segments. Pflugers Arch. 400, 388-92.

Gough, D. A. and Leypoldt, J. K. (1981). Theoretical aspects of enzyme electrode design. Appl. Biochem. and Bioeng, 3, 175-206.

Heyrovsky, J. (1960). Trends in polarography. Nobel Laureate Lecture, Science 132, 123-30.

Hopkin, T. R., (1985). A multipurpose enzyme sensor based on alcohol oxidase. Am. Biotechnol. Lab., Sept/Oct. 13.

Johnson, I.M., Halsall, H. B. and Heineman, W. R. (1982). Galactose oxidase enzyme electrode with internal solutions potential control. Anal, Chem. 54, 1394-9.

Racine, P., Engelhardt, R., Higelin, J. C. and Mindt, W. (1975). An instrument for the rapid determination of L-lactate in biological fluids. Med. Instrum. 9, 11-14.

Scheller, F., Renneberg, R, and Schubert, F. (1985). Coupled enzyme reactions. In Intelligent sensors. Engineering Foundation VIIIth Int. Conf. on Enzyme Eng., Helsingor, Denmark, Sept. 22-27, 1985. Prog, and Abstracts p. 49.

Silver, 1. A. (1976). An ultra micro glucose electrode. In Ion and enzyme electrodes in biology and medicine (eds. M. Kessler, L.C. Clark, Jr,, D. W. Lubbers, I. A. Silver, and W. Simon), pp. 189-92. Urban and Schwarzenberg, Munchen. 


\title{
2 \\ Micro-organism based sensors
}

\author{
ISAO KARUBE
}

\subsection{Introduction}

The industrial application of biochemical and microbiological processes in fields such as the production of pharmaceuticals, food manufacturing, wastewater treatment, and energy production is on the increase. Fermentation plays a very important role in such biotechnological processes, therefore a monitoring of raw materials, cell population, and products is necessary to achieve an effective system. Spectrophotometry and chromatography can be used for the determination of organic compounds, but they are not suitable for on-line measurement. Electrochemical determination of such compounds has distinct advantages: for example, samples can be measured over a wide concentration range without pretreatment and do not need to be optically clear. Recently, many biosensors have been developed for the determination of organic compounds, Enzyme sensors are highly specific for the substrate of interest, but the enzymes employed are generally expensive and unstable. Microbial sensors are composed of immobilized micro-organisms and an electrochemical device and are suitable for the on-line control of biochemical processes (Chang 1977; Guilbault 1976; Aizawa et al, 1974; Satoh et al. $1977 a, b)$. The microbial sensors developed by the author involve the assimilation of organic compounds by the micro-organisms, change in respiratory activity, or the production of electroactive metabolites; these being monitored directly by an electrochemical device. This chapter describes several microbial sensors currently being developed in Japan.

\subsection{Assimilable sugar sensor}

In the cultivation of micro-organisms in canc molasses, which contains various sugars, determination of the total assimilable sugars in the broth is important for the control of the fermentation process. For example, catabolite repression occurs at high sugar concentration, causing an inhibition of cell growth. Reduced sugars and sucrose in culture broths are determined by the ferricyanide method (Technicon Industrial Systems 1972). This method, however, is not completely reliable because unassimilable substances can interfere with the determination.

Assimilation of organic compounds by micro-organisms can be deter- 


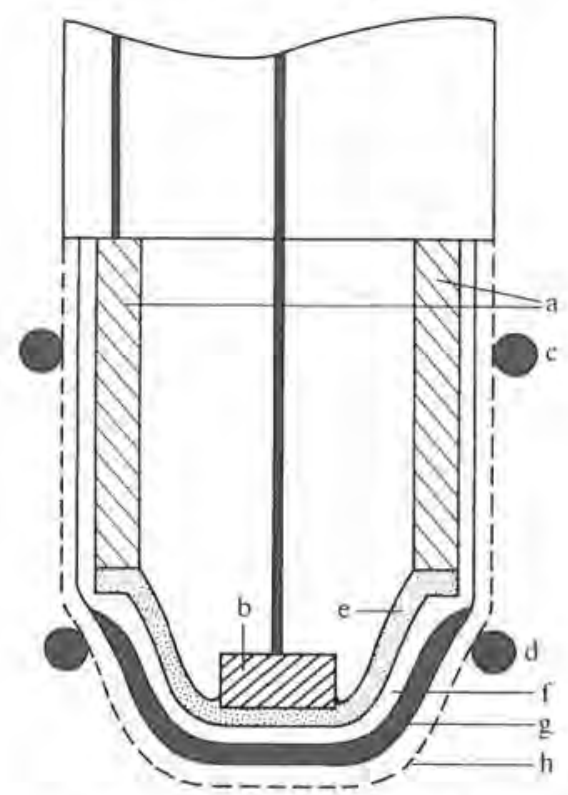

Fig. 2.1 Scheme of the microbial electrode for total assimilable sugars. a, Silver anode; b, platinum cathode; c, d, rubber rings; e, electrolyte gel; f, Teflon membrane; $\mathrm{g}$, micro-organisms retained on nylon net; $\mathrm{h}$, cellophane membrane.

mined from their respiratory activity, which can be measured directly using an oxygen electrode.

A microbial sensor consisting of immobilized living whole cells of Brevibacterium lactofermentum and an oxygen electrode was constructed for the continuous determination of total assimilable sugars (glucose, fructose, and sucrose) in a fermentation broth (Hikuma et al. 1980 b), Brevibacterium lactofermentum was immobilized in a strip of nylon net $(1 \mathrm{~cm} \times 1 \mathrm{~cm}, 20$ mesh) and attached to the oxygen electrode (Figs. 2.1 and 2.2). Total assimilable sugars were estimated from oxygen consumption by the immobilized micro-organisms. Addition of a glucose aliquot to the sensor system resulted in an increased oxygen consumption by the micro-organisms. This lowered the dissolved oxygen concentration of the solution causing the electrode current to decrease markedly with time until steady state was reached. The response time was 10 min using a steady-state determination and $1 \mathrm{~min}$ by the pulse method. A linear relationship was found between the decrease in current and the concentration of glucose $(1 \mathrm{~mm})$, fructose $(1 \mathrm{~mm})$, and sucrose $(0.8 \mathrm{~mm})$ respectively. Sensitivity of the microbial sensor to glucose, fructose, and sucrose existed in a ratio of 1.00:0.80:0.92. The decrease in current was reproducible to within $2 \%$ of the relative standard deviation when a sample solution containing glucose $(0.8 \mathrm{~mm})$ was employed for the 


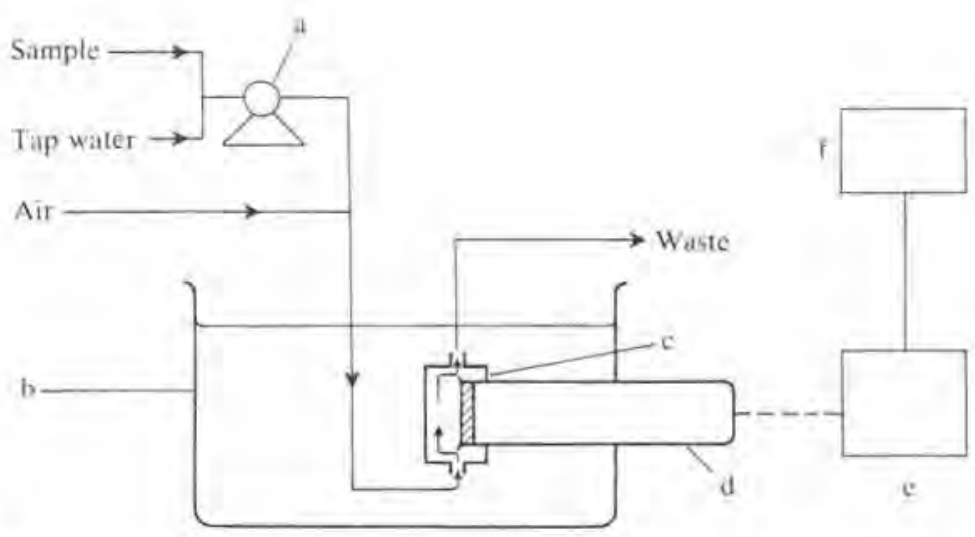

Fig, 2.2 Schematic diagram of the sensor system. a, Peristaltic pump; b, water bath; c, flow cell; $d$, microbial electrode; $e$, transmitter; $f$, recorder.

experiments. Total assimilable sugar was calculated by a summation of the responses of glucose, fructose, and sucrose, the difference between the observed and calculated concentrations being within $8 \%$. The microbial sensor was applied in a fermentation broth for glutamic acid production, where it operated reliably for more than ten days and 960 assays.

\subsection{Glucose sensor}

A microbial sensor consisting of immobilized whole cells of Pseudomonas fluorescens and an oxygen electrode was developed for the determination of glucose (Hikuma et al. 1980b; Karube et al. 1979b) (Fig. 2.3).

The microbial sensor was inserted into a sample solution and the sample solution was saturated with dissolved oxygen and stirred magnetically while measurements were taken.

Figure 2.4 shows typical response curves of the sensor. The theory of this sensor was the same as that of the assimilable sugar sensor. The steady-state current was attained within $10 \mathrm{~min}$ at $30^{\circ} \mathrm{C}$. The exact time depended on the concentration of glucose added.

When the sensor was removed from the sample and placed in a glucose-free solution, the current of the microbial sensor gradually increased and returned to the initial level within $15 \mathrm{~min}$ at $30^{\circ} \mathrm{C}$.

The sensor responded slightly to fructose, galactose, mannose, and saccharose, but no response was observed in the case of amino acids. Therefore, the selectivity of the microbial sensor for glucose was considered satisfactory. 


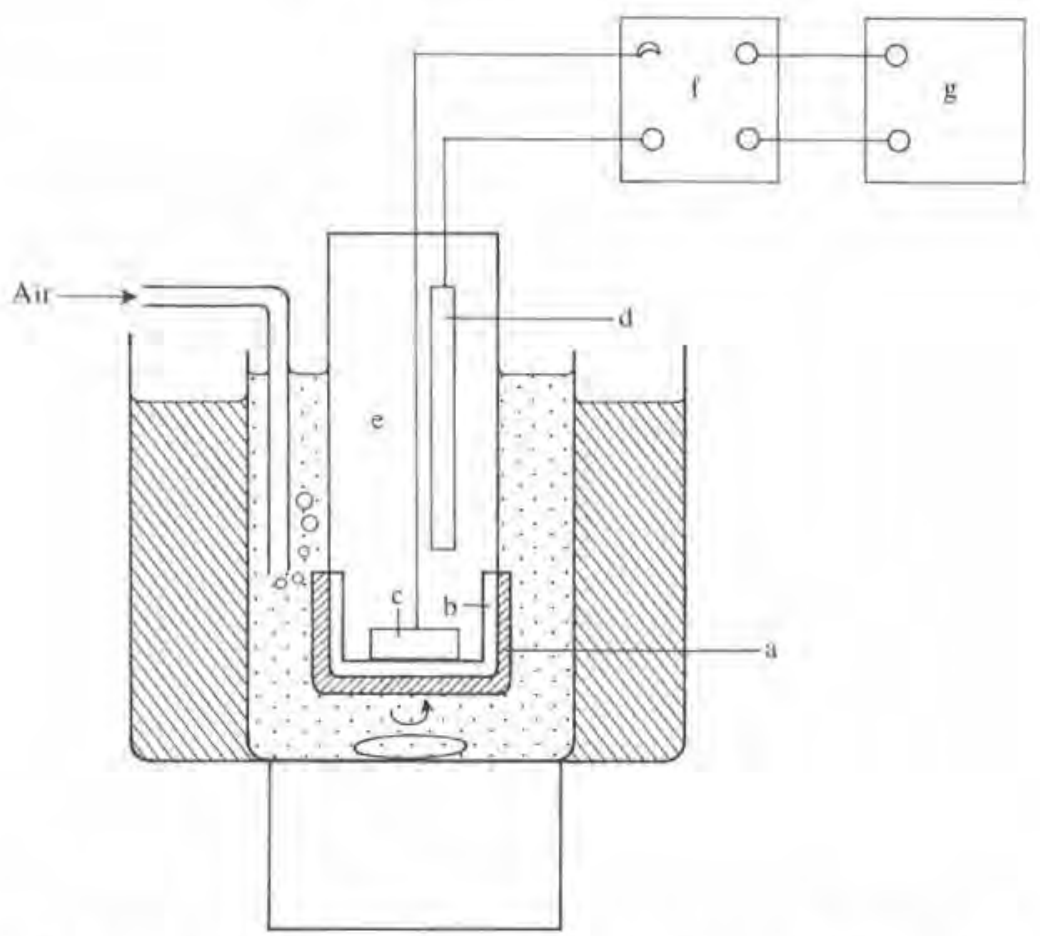

Fig. 2.3 Scheme of the microbial electrode sensor for glucose. a, Bacterial collagen membrane; $b$, Teflon membrane; c, platinum cathode; $d$, lead anode; e, electrolyte $(\mathrm{KOH}) ; \mathrm{f}$, ammeter; $\mathrm{g}$, recorder.

A linear relationship was observed between the current and the concentration of glucose below $20 \mathrm{mg} \mathrm{1}^{-1}$ by a steady-state determination and the minimum detectable concentration of glucose was $2 \mathrm{mg} \mathrm{l}^{-1}$. The current was reproducible to within $\pm 6 \%$ when a sample solution containing $10 \mathrm{mg} \mathrm{I}^{-1}$ of glucose was employed. The standard deviation was $6.5 \mathrm{mg} \mathrm{l}^{-1}$ over 20 experiments.

The microbial glucose sensor was applied to molasses broth and glucose was determined with an average relative error of $\pm 10 \%$. The concentration of glucose was also determined by an enzymatic method (Karube et al. 1979b) for comparison, which gave correlation with the electrochemical method.

The re-usability of the microbial sensor was examined. No decrease in current output was observed over a two-week period and 150 assays.

\subsection{Acetic acid sensor}

When micro-organisms are grown on acetic acid as the carbon source, excess acetic acid inhibits growth and hence, the optimal concentration must be 


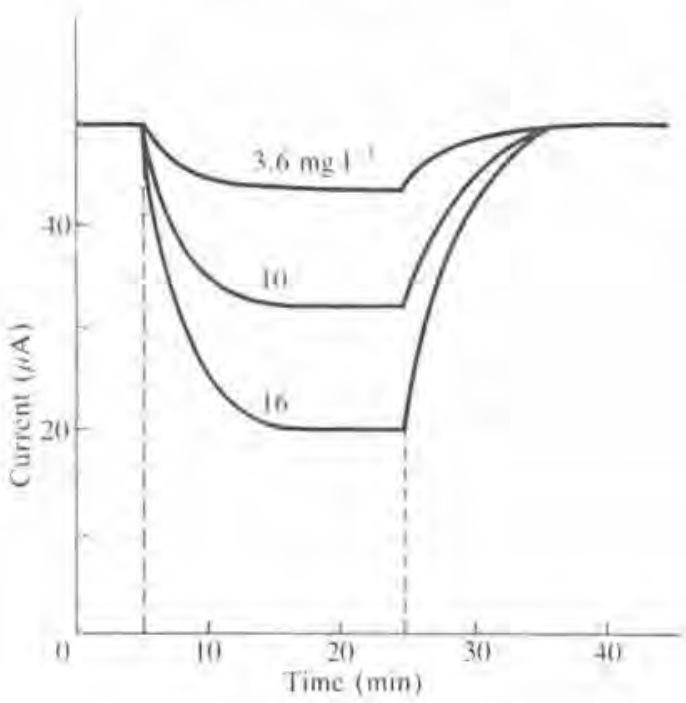

Fig. 2.4 Response curve of the microbial electrode sensor.

maintained by on-line monitoring. A microbial sensor comprising of immobilized yeast (Trichosporon brassicae), a gas-permeable Teflon membrane, and an oxygen electrode has been investigated for the continuous determination of acetic acid in fermentation broths (Hikuma et al. 1979a).

A porous membrane bearing the immobilized $T$. brassicae was attached to the surface of the oxygen electrode Teflon membrane and covered with a second gas-permeable Teflon membrane: thus the micro-organisms were trapped between the two porous membranes. The microbial sensor system consisted of a jacketed flow cell, a magnetic stirrer, a peristaltic pump, an automatic sampler, and a current recorder.

The principle of this microbial sensor was similar to that described above. The sample was kept at a $\mathrm{pH}$ well below the $\mathrm{pK}$ value for acetic acid $(4.75$ at $30^{\circ} \mathrm{C}$ ) because acetate ions cannot pass through the membrane. Figure 2.5 shows the response curves obtained for acetic acid concentrations of 18,36 , 54 , and $72 \mathrm{mg} \mathrm{1}^{-1}$.

The calibration graphs obtained showed linear relationships between the

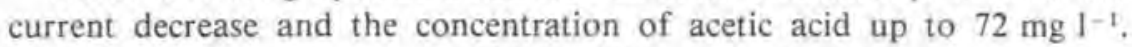
The minimum concentration which could be determined was $5 \mathrm{mg} \mathrm{I}^{-1}$. The current difference was reproducible within $\pm 6 \%$ for an acetic acid sample containing $54 \mathrm{mg} \mathrm{I}^{-1}$ and the standard deviation was $1.6 \mathrm{mg} \mathrm{l}^{-1}$ over 20 experiments.

With regard to the selectivity of the microbial sensor for acetic acid, it did not respond to volatile compounds such as formic acid and methanol, or to 


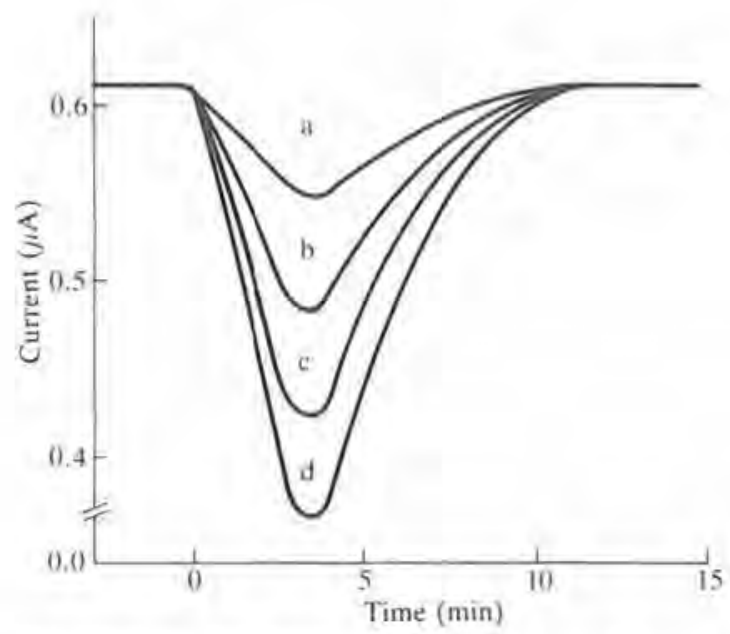

Fig. 2.5 Response curve of the acetic acid sensor. Sample solution $(2.4 \mathrm{ml})$ was passed into the flow cell for $3 \mathrm{~min}$. Acetic acid concentrations were a, $18 \mathrm{mg} \mathrm{I}^{-1}$; b, $36 \mathrm{mg} \mathrm{l}^{-1} ; \mathrm{c}, 54 \mathrm{mg} \mathrm{I}^{-1} ; \mathrm{d}, 72 \mathrm{mg} \mathrm{l}^{-1}$.

involatile nutrients such as glucose and phosphate ions. Although Trichosporon brassicae does utilize propionic acid, $n$-butyric acid, and ethanol, these are generally not present in fermentations, or are present in concentrations too low to affect the measurement of acetic acid.

The concentration of acetic acid in a fermentation broth for glutamic acid production was determined by the microbial sensor and also by gas chromatography: good agreement between the two was achieved, the regression coefficient being 1.04 for 26 experiments. The current output $(0.29-0.25 \mu \mathrm{A})$ of the sensor was constant (within $\pm 10 \%$ of the original value) for more than three weeks and 1500 assays. This microbial sensor for acetic acid is now commercialized in Japan.

\subsection{Alcohol sensor}

On-line measurements of methanol and ethanol concentrations in culture broths are necessary in the fermentation industries. In the cultivation of micro-organisms using alcohols as a carbon source, the concentration of alcohols must be maintained at the optimal level in order to avoid substrate inhibition. It is well known that many micro-organisms utilize alcohols as carbon sources. Therefore it is possible to construct a microbial sensor for alcohol using alcohol-utilizing micro-organisms (Hikuma et al. 1979b).

The ethanol sensor consisted of immobilized Trichosporon brassicae and an oxygen electrode. The immobilization of the cells and electrode 
construction was the same as for the glucose sensor.

A long time is required for the determination using the steady-state method. Therefore, the pulse method was employed for the determination, providing a response within six minutes. A linear relationship was observed between the current decrease and the ethanol concentration up to a maximum concentration of $22.5 \mathrm{mg} \mathrm{l}^{-1}$ and minimum concentration of $2 \mathrm{mg} \mathrm{l}^{-1}$. The current difference was reproducible to within $\pm 6 \%$ of the relative error when a sample solution containing $16.5 \mathrm{mg}$ ethanol $1^{-1}$ was employed. The standard deviation was $0.5 \mathrm{mg} \mathrm{l}^{-1}$ over 40 experiments.

The sensor did not respond to volatile compounds such as methanol, formic acid, acetic acid, propionic acid, and other non-volatiles such as carbohydrates, amino acids, and ions (Table 2,1). As the microbial sensor was covered with a gas-permeable membrane, only volatile compounds can penetrate through the membrane.

Table 2.1 Response of the microbial electrode sensor to various compounds

\begin{tabular}{lllll}
\hline $\begin{array}{llll}\text { Immobilized } \\
\text { micro-organisms }\end{array}$ & Composition of sample* & $\begin{array}{l}\text { Current } \\
\text { decrease }(\mu \mathrm{A})\end{array}$ & Remarks \\
\hline $\begin{array}{l}\text { Trichosporon } \\
\text { brassicae }\end{array}$ & Ethanol & $30 \mathrm{ppm}$ & 0.13 & \\
& Methanol & $30 \mathrm{ppm}$ & 0 & \\
& Acetic acid & $100 \mathrm{ppm}$ & 0 & $\mathrm{pH}>6$ \\
& Formic acid & $100 \mathrm{ppm}$ & 0 & \\
& Propionic acid & $100 \mathrm{ppm}$ & 0 & \\
& Glucose & $1 \%$ & 0 & \\
& Saccharose & $\mathrm{I} \%$ & 0 & \\
& $\mathrm{KH}_{2} \mathrm{PO}_{4}$ & $5 \%$ & 0 & \\
\hline
\end{tabular}

* Original concentration, diluted 2.8 times in the flow cell.

The selectivity of the microbial sensor for ethanol was therefore found to be satisfactory.

The microbial ethanol sensor was applied to yeast fermentation broths. The concentration of ethanol was also determined by gas chromatography, which gave satisfactory comparative results to the microbial sensor. The correlation coefficient was 0.98 over 20 experiments. The current output of the sensor in an ethanol concentration of $5.5-22.3 \mathrm{mg} \mathrm{l}^{-1}$ remained almost constant for more than three weeks and 2100 assays. This ethanol sensor is now commercialized in Japan.

An unidentified bacterium AJ3993 was also employed for a methanol sensor using the same configuration as for the ethanol sensor. The methanol concentration had a linear relationship to the current decrease up to $25 \mathrm{mg} \mathrm{1}^{-1}$. 


\subsection{Formic acid sensor}

Formic acid commonly occurs as an intermediate of cellular metabolism and is found in culture media, urine, blood, and gastric juices, also being a product of many chemical reactions. Selective spectrophotometric enzyme assays involving formate dehydrogenase, malate dehydrogenase, and tetrahydrofolic acid synthetase are not suitable for on-line monitoring.

Several anaerobic bacteria such as Escherichia coli, Citrobacter freundii, and Rhodospirillum rubrum produce hydrogen from formic acid. The reactions are summarized as follows:

$$
\begin{aligned}
& \text { Formic acid } \longrightarrow \text { Ferredoxin }{ }_{\text {reduced }}+\mathrm{CO}_{2} \\
& \text { Ferredoxin }_{\text {reduced }} \stackrel{\text { Hydrogenase }}{\longrightarrow} \text { Ferredoxin }{ }_{\text {oxidized }}+\mathrm{H}_{2} \\
& \text { Cytochrome } c_{\text {reduced }} \stackrel{\text { Hydrogenase }}{\longrightarrow} \text { Cytochrome } c_{\text {oxidized }}+\mathrm{H}_{2}
\end{aligned}
$$

Therefore, determination of formic acid is possible by using $C$. freundii and a fuel cell-type electrode. The principle of this microbial sensor is illustrated in Fig. 2.6. Such a specific microbial sensor, comprising immobilized C. freundii, two gas-permeable Teflon membranes and a fuel-cell-type electrode has been investigated (Matsunaga et al. 1980). A linear relationship was obtained between the steady state current and the formic acid concentration up to a maximum of $1000 \mathrm{mg} \mathrm{I}^{-1}$ and a minimum $10 \mathrm{mg} \mathrm{l}^{-1}$. The currents were reproducible with an average relative error of $\pm 5 \%$ at a concentration of $200 \mathrm{mg} \mathrm{l}^{-1}$. The standard deviation was $3.4 \mathrm{mg} \mathrm{1}^{-1}$ over 30 experiments. This sensor did not respond to non-volatile nutrients such as glucose, pyruvic acid, and phosphate ions. Although volatile compounds such as acetic acid, propionic acid, n-butyric acid, methanol, and ethanol can

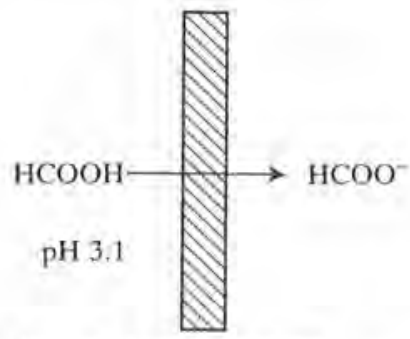

Porous Teflon membrane (gas and volatile-acid permeatble)

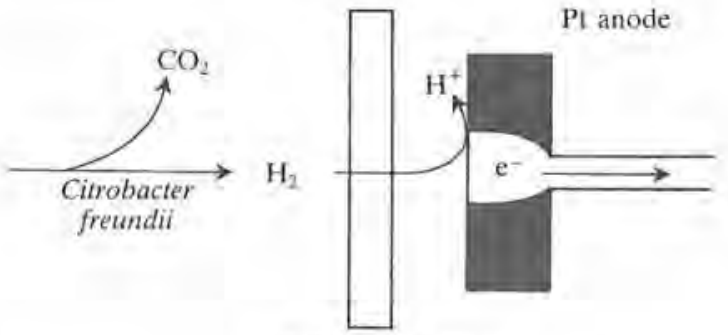

Teflon membrane (gas permeablc)

Fig. 2.6 Principle of the microbial sensor. 
permeate through the porous Teflon membrane, no current was obtained with these compounds because $C$. freundii cannot utilize them for $\mathrm{H}_{2}$ production. The microbial sensor, and also gas chromatography, were used to determine the formic acid concentration in Aeromonas formicans culture medium. Good agreement was obtained between these methods, the regression coefficient being 0.98 for ten experiments. To study the stability of the immobilized $C$. freundii in the sensor, it was stored in $0.1 \mathrm{M}$ phosphate buffer at $5^{\circ} \mathrm{C}$ and used for formic acid $\left(200 \mathrm{mg} 1^{-1}\right)$ determination over fiveday intervals. The current output obtained from each assay remained constant for 20 days.

\subsection{Methane sensor}

World-wide interest has arisen in the production of methane by fermentation of biomass. Methane is an attractive energy source and a main component of the natural gases used for fuel. Rapid methods for the detection and determination of methane in air are required, for example in the field of coal mining, because it forms an explosive mixture with air $(5-14 \%)$. A methane sensor consisting of immobilized methane-oxidizing bacteria and an oxygen electrode has been developed (Karube et al. 1982).

The system is comprised of two oxygen electrodes, two reactors, an electrometer, and a recorder (Fig. 2.7). The reactors both contain culture media, one with and one without the immobilized bacterium Methylomonas flagellata. The electrodes were fixed inside custom-made Teflon flowthrough cells, connected using glass and Teflon tubing. Two vacuum pumps were used, one to evacuate the gas-sample tube and the other to transport the sample gas through the system. The flow rate of the sample gas through the reactors was controlled $\left(80 \mathrm{ml} \mathrm{min}^{-1}\right)$ using glass valves. A cotton filter removed other micro-organisms in the gas samples, preventing contamination of the reactors and gas lines. The latter were designed to maintain symmetry between the measuring and reference flows.

When sample gas containing methane was passed into the reactor, methane was assimilated by the immobilized micro-organisms with consumption of oxygen causing the current from the oxygen electrode to decrease to a minimum steady state, As the system contained two oxygen electrodes, the maximum difference between the currents depended on the concentration of methane in the sample gas. When pure air was again passed through the reactors, the current returned to its initial level within $60 \mathrm{~s}$. The response time for the determination of methane was less than $60 \mathrm{~s}$, and the total time required for methane assay was two minutes.

Calibration graphs for the system were perfectly linear for methane concentrations in the range $0-6.6 \mathrm{mmol}$, the current difference ranging from 0 to $0.35 \mu \mathrm{A}$ and the minimum determinable concentration being $5 \mu \mathrm{mol}$. 


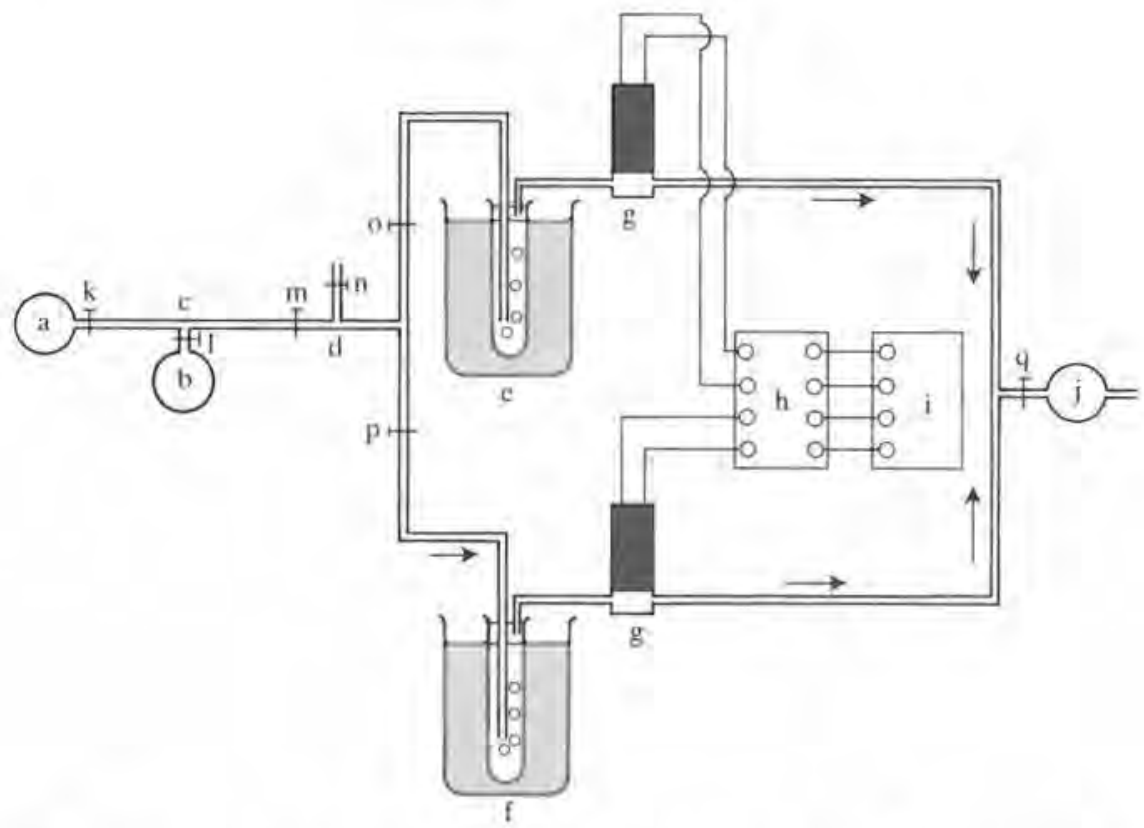

Fig. 2.7 Diagram of microbial sensor system for methane. a, Vacuum pump; b, sample gas bag; c, gas sample line; d, cotton filter; e, control reactor; f, methaneoxidizing bacteria reactor; $g$, oxygen electrode; $h$, amplifier; $i$, recorder; $j$, vacuum pump; k-q, glass stopcocks.

The current difference of a $0.66 \mathrm{mmol}$ sample was reproducible to within $\pm 5 \%$ (S.D. $9.40 \mathrm{nA}$ ) over 25 experiments.

Analysis by conventional gas chromatography, over the range $0.2-3.5$ mmol methane in air, gave a good correlation with the electrochemical method (correlation coefficient 0.97 ). The minimum measurable concentration of methane is $3 \mathrm{mmol}$ by gas chromatography using a flame ionization detector, and $5 \mathrm{mmol}$ with the microbial sensor. The sensor employing $M$. flagellata therefore warrants further development for rapid on-line determination of methane.

\subsection{Glutamic acid sensor}

Glutamic acid is produced by a fermentation process (being used as a seasoning for foods). A rapid and automatic method was required for determination of its concentration. Enzyme-based auto-analysers can be used, but enzyme costs are prohibitive. The selectivity of this sensor to various amino acids was examined. The sensor responded to glutamic acid and glutamine 
and very slightly to some other amino acids. The response to glutamine can be decreased, if necessary, using acetone-treated $E$. coli. The microbial sensor did not respond under anaerobic conditions to organic substances such as glucose $\left(7800 \mathrm{mg}^{-1}\right)$ and acetic acid $\left(9200 \mathrm{mg} \mathrm{t}^{-1}\right)$ and the influence of inorganic ions on the response was negligible.

When this sensor was used to determine known concentrations of glutamic acid in a fermentation broth, satisfactory recovery data were obtained $(99-103 \%)$ which were in good agreement with auto-analyser determinations. The sensor was considered to be highly selective, stable, and reproducible.

Glutamate decarboxylase catalyses the decarboxylation of glutamic acid producing carbon dioxide and amine, but the enzyme is expensive and unstable. Certain micro-organisms, however, contain glutamate decarboxylase.

Consequently a microbial sensor for glutamic acid has therefore been devised incorporating immobilized Escherichia coli (as a source of glutamate decarboxylase activity) in conjunction with a $\mathrm{CO}_{2}$-sensing electrode (Hikuma et al. 1980c). Preliminary experiments have shown that $E$. coli does not evolve carbon dioxide under anaerobic conditions in the absence of glutamic acid. Any carbon dioxide produced by these bacteria under such conditions results from the glutamate decarboxylase reaction. Nitrogen gas was passed through the flow cell in order to remove any dissolved oxygen from the buffer and sample solution. When a sample solution containing glutamic acid was injected into the system, glutamic acid permeated through the membrane to the immobilized micro-organisms and was metabolized to produce carbon dioxide. The enzyme reaction was carried out at $\mathrm{pH} 4.4$, which was sufficiently below the $\mathrm{p} k_{\mathrm{a}}$ value $\left(6.34\right.$ at $\left.25^{\circ} \mathrm{C}\right)$ of carbon dioxide. As a result, the potential of the $\mathrm{CO}_{2}$-sensing electrode increased with time. The assay can be performed using an injection period of 1-3 min and measuring the maximum potential, with little loss of sensitivity.

A plot of the maximum potential vs, the logarithm of the glutamic acid concentration was linear in the range $100-800 \mathrm{mg} \mathrm{I}^{-1}$. When replicates of a glutamic acid solution $\left(400 \mathrm{mg}^{-1}\right)$ were measured, the standard deviation was $1.2 \mathrm{mg} \mathrm{l}^{-1}$ (20 experiments).

\subsection{Cephalosporin sensor}

Antibiotics are usually determined by microbioassay based on a turbidimetric or titrimetric method, but these methods require complicated procedures and are not suitable for a rapid determination.

It was found that Citrobacter freundii produced cephalosporinase, which catalyses the following reaction of cephalosporin with liberation of hydrogen ions: 
<smiles>[R1]Cc1[cH+]cc(C(NC([R])=O)C(=O)[O-])[nH]c1C(=O)O</smiles>

Cephalosporinase is, however, very unstable and as a result it is difficult to utilize it in the preparation of an enzyme sensor. Immobilized whole cells of Citrobacter freundii were employed for the cephalosporin sensor, being immobilized in a collagen membrane. This bacteria-collagen membrane was then inserted into a membrane reactor.

The system used for continuous determination of cephalosporins is illustrated in Fig. 2.8. The reactor is a membrane type with a spacer located in the centre. The $\mathrm{pH}$ change caused by the enzymatic reaction was measured using a combined glass electrode and displayed on a recorder.

Sample solutions containing various amounts of cephalosporins were inserted into the reactor, causing the electrode potential difference to increase with time until a maximum was reached. The minimum response time depended on the flow rate and activity of the bacteria-collagen membrane. For a flow rate of $2 \mathrm{ml} \mathrm{min}^{-1}$, the maximum potential difference was reached after $10 \mathrm{~min}$.

A linear relationship was obtained between the logarithm of the
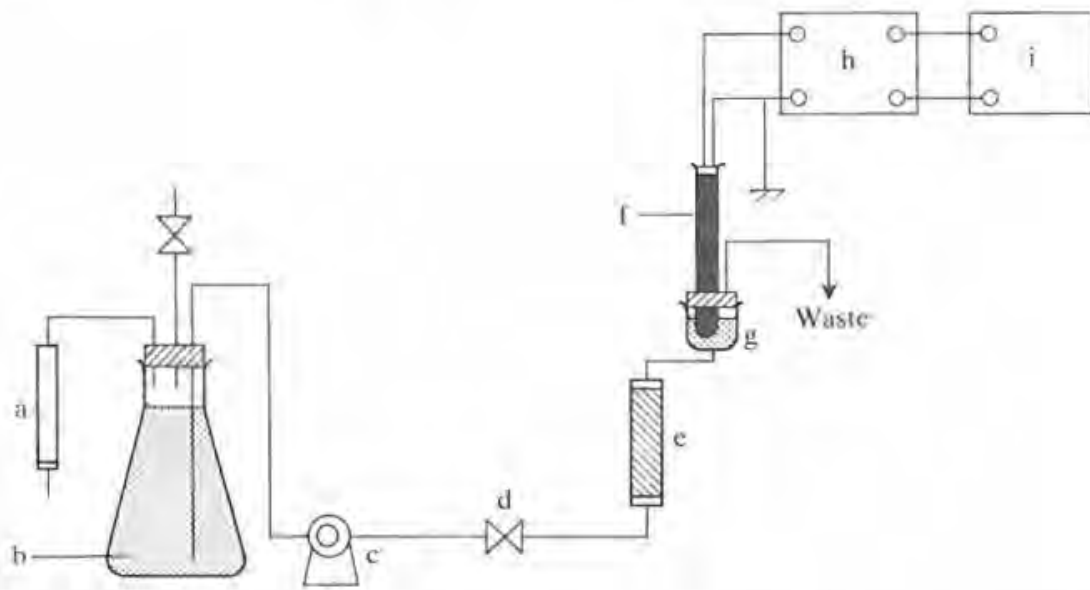

Fig. 2.8 Immobilized whole-cell-based flow-type sensor for cephlosporins. a, Soda lime; b, buffer reservoir; c, peristaltic pump; d, sample inlet; e, immobilized whole cell reactor; $f$, combined glass electrode; $g$, sensing chamber; h, amplifier; i, recorder. 
cephalosporin concentration and the maximum potential difference. 7-Phenyl-acetylamidodesacetoxy-sporanic acid (phenyl-acetyl-7ADCA), cephaloridine, cephalothin, and cephalosporin $C$ were determined by the cephalosporin sensor.

The stability of the microbial sensor was examined with a solution containing $125 \mu \mathrm{g} \mathrm{ml}^{-1}$ of phenyl-acetyl-7ADCA. The cephalosporin determination was carried out several times a day, giving no change in the observed potential difference response after one week.

The system was applied to the determination of cephalosporin C in a broth of Cephalosporium acremonium, and was compared with a method based on high-pressure liquid chromatography (HPLC). The relative error of the determination by the microbial system was $8 \%$. Accordingly, the method is suitable for continuous analysis of cephalosporins in fermentation broths.

\subsection{BOD sensor}

The biochemical oxygen demand (BOD) is one of the most widely used tests in the measurement of organic pollution. The conventional BOD test requires, however, a five-day incubation period. Therefore, a more rapid and reproducible method is required for assessing BOD.

Trichosporon cutaneum, which is used for wastewater treatment, was used for the BOD sensor (Karube et al. 1977). The sensor configuration is the same as previously described.

Phosphate buffer solution $(0.01 \mathrm{M}, \mathrm{pH} 7)$ saturated with dissolved oxygen was transferred to the flow cell at a flow rate of $1 \mathrm{ml} \mathrm{min}^{-1}$. When the current reached a steady-state value, a sample was injected into the flow cell at a rate of $0.2 \mathrm{ml} \mathrm{min}^{-1}$.

The steady-state current was dependent on the BOD of the sample solution. Then the current of the microbial sensor gradually returned to the initial level. The response time of the microbial sensor (time required for the current to reach steady state) depended on the nature of the sample solution used.

A linear relationship was observed between the current difference (between initial and steady-state currents) and the five-day BOD assay of the standard solution (glucose and glutamate solution) up to $60 \mathrm{mg} \mathrm{I}^{-1}$. The minimum measurable BOD was $3 \mathrm{mg} \mathrm{l}^{-1}$. The current was reproducible within $\pm 6 \%$ of the relative error when a BOD of $40 \mathrm{mg} \mathrm{1}^{-1}$ was employed over ten experiments.

The microbial sensor was applied to the estimation of 5-day BOD for untreated wastewaters from a fermentation factory. The 5-day BOD of the wastewaters was determined using a JIS method (Japanese Industrial Standard Committee), Good correlation was obtained between BOD estimated by the microbial sensor and those determined by the JIS method. The regression coefficient was 1.2 over 17 experiments and the ratios (BOD estimated by the 


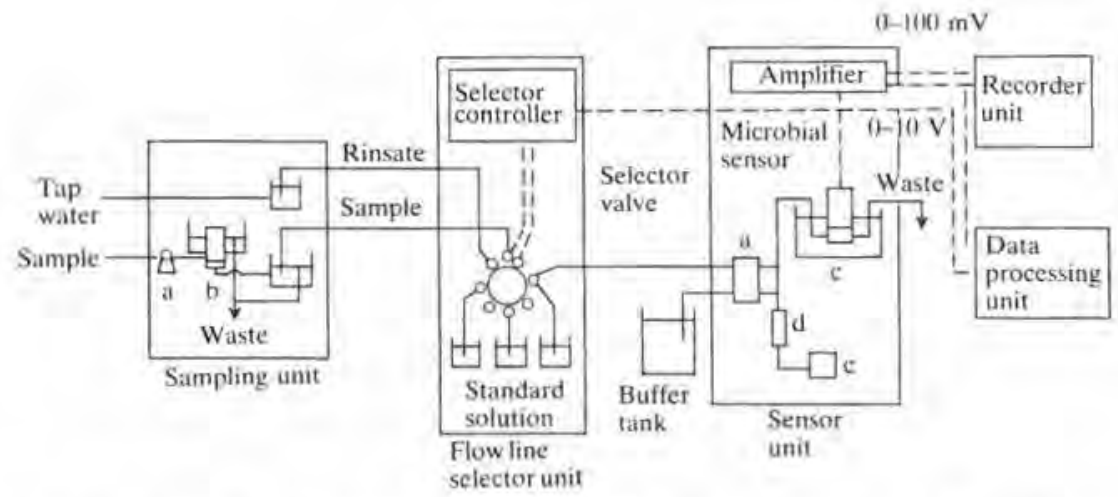

Fig. 2.9 Schematic diagram of continual measuring system for BOD. a, pump; b, filter; c, incubator; d, flow meter; e, air pump.

microbial sensor/5-day BOD determined by JIS method) were in the range from 0.85 to 1.36 . This variation might have been caused by a change in composition of the organic wastewater. The BOD of various kinds of untreated industrial wastewaters were estimated by the sensor, and the response was found to depend on compounds present in the wastewaters. The BOD sensor system shown in Fig. 2.9 has now been commercialized in Japan.

\subsection{Ammonia sensor}

The determination of ammonia is important in clinical and industrial process analysis. Several ammonia sensors based on potentiometry have been developed for the determination of ammonia, but interference by metal ions and volatile amines can occur. Therefore, an ammonia sensor based on amperometry is desirable for the determination of ammonia.

Nitrifying bacteria utilize ammonia as the sole energy source, the respiratory consumption of oxygen being as follows:

$$
\begin{aligned}
& \mathrm{NH}_{3}+1.5 \mathrm{O}_{2} \stackrel{\text { Nitrosomonas } \mathrm{sp} .}{\longrightarrow} \mathrm{NO}_{2}^{-}+\mathrm{H}_{2} \mathrm{O}+\mathrm{H}^{+} \\
& \mathrm{NO}_{2}^{-}+0.5 \mathrm{O}_{2} \stackrel{\text { Nitrobacter sp. }}{\longrightarrow} \mathrm{NO}_{3}^{-}
\end{aligned}
$$

Therefore, oxygen uptake by the bacteria can be determined directly by immobilizing the bacteria to an oxygen electrode.

An ammonia sensor consisted of immobilized bacteria, a gas-permeable Teflon membrane, and an oxygen electrode (Hikuma et al 1980a; Okada et al. 1983). A linear relationship was observed between the current decrease and the ammonia concentration up to a maximum concentration of 
$42 \mathrm{mg} \mathrm{l}^{-1}$, the minimum determinable concentration being $0.1 \mathrm{mg} \mathrm{1}^{-1}$. The current decrease was reproducible to within $\pm 4 \%$ of the relative error when a sample solution containing $21 \mathrm{mg} \mathrm{1}^{-1}$ of ammonium hydroxide was employed. The standard deviation was $0.7 \mathrm{mg} \mathrm{dl}^{-1}$ over 20 experiments.

The sensitivity of the microbial sensor was approximately equal to that of a glass electrode.

The sensor did not respond to volatile compounds such as acetic acid, ethanol and amines or non-volatile nutrients such as glucose, amino acids, and metal ions. The current output of the sensor was stable for more than ten days and 200 assays.

The determination of ammonia in human urine was performed using the microbial sensor and a conventional method. Good comparative results were obtained between ammonia concentrations determined by both methods, the microbial sensor possessing good long-term stability.

\subsection{Other microbial sensors}

Various other microbial sensors have been developed by our group (Karube et al. 1979a; Matsunaga et al. 1978a, $b ; 1979)$. The characteristics of these microbial sensors are summarized in Table 2.2 (see over). 
Table 2.2 Characteristics of micro-organism-based sensors

\begin{tabular}{|c|c|c|c|c|}
\hline Sensor & Immobilized micro-organisms & Device & $\begin{array}{l}\text { Response } \\
\text { time (min) }\end{array}$ & $\begin{array}{l}\text { Range } \\
\left(\mathrm{mg} \mathrm{dm}^{-3}\right)\end{array}$ \\
\hline Assimilable sugars & Brevibacterium lactofermentum & $\mathrm{O}_{2}$-probe & 10 & $10-200$ \\
\hline Glucose & Pseudomonas fluorescens & $\mathrm{O}_{2}$-probe & 10 & $2-2 \times 10$ \\
\hline Acetic acid & Trichosporon brassicae & $\mathrm{O}_{2}$-probe & 10 & $3-60$ \\
\hline Ethanol & Trichosporon brassicae & $\mathrm{O}_{2}$-probe & 10 & $2-25$ \\
\hline Methanol & Unidentified bacteria & $\mathrm{O}_{2}$-probe & 10 & $5-2 \times 10$ \\
\hline Formic acid & Citrobacter freundii & fuel cell & 30 & $10-10^{3}$ \\
\hline Methane & Methylomonas flagellata & $\mathrm{O}_{2}$-probe & 2 & $0-6.6^{a}$ \\
\hline Glutamic acid & Escherichia coli & $\mathrm{CO}_{2}$-probe & 5 & $8-800$ \\
\hline Cephalosporin & Citrobacter freundii & pH electrode & 10 & $10^{2}-5 \times 10^{2}$ \\
\hline BOD & Trichosporon cutaneum & $\mathrm{O}_{2}$-probe & 15 & $3-60$ \\
\hline Lysine & Escherichia coli & $\mathrm{CO}_{2}$-probe & 5 & $10-10^{2}$ \\
\hline Ammonia & Nitrifying bacteria & $\mathrm{O}_{2}$-probe & 10 & $0.05-1$ \\
\hline Nitrogen dioxide & Nitrifying bacteria & $\mathrm{O}_{2}$-probe & 3 & $0.51-255^{b}$ \\
\hline Nystatin & Saccharomyces cerevisiae & $\mathrm{O}_{2}$-probe & 1(h) & $0.5-54^{c}$ \\
\hline Nicotinic acid & Lactobacillus arabinosis & pH electrode & I(h) & $10^{-5}-5$ \\
\hline Vitamin $\mathrm{B}_{1}$ & Lactobacillus fermenti & fuel cell & $6(\mathrm{~h})$ & $10^{-3}-10^{-2}$ \\
\hline Cell population & - & fuel cell & 15 & $10^{8}-10^{9 d}$ \\
\hline Mutagen & Bacillus subtilis $\mathrm{Rec}^{-}$ & $\mathrm{O}_{2}$-probe & I(h) & $1.6-2.8 \times 10^{3}$ \\
\hline
\end{tabular}

${ }^{a}$ mmol, ${ }^{b} \mathrm{ppm}, \quad$ U Units $\mathrm{cm}^{-3},{ }^{d}$ Number $\mathrm{cm}^{-3}$ 


\section{References}

Aizawa, M., Karube, I. and Suzuki, S, (1974). A specific bioelectrochemical sensor for hydrogen peroxide. Anal. Chim. Acta 69, 431.

Chang, T. M.S. (ed.)(1977). Biomedical applications of immobilized enzymes and proteins, Vol. 2. Plenum, New York.

Guilbault, G. G. (1976). Handbook of enzymatic methods of analysis, Marcel Dekker, New York.

Hikuma, M., Kubo, T., Yasuda, T., Karube, I. and Suzuki, S. (1979a). Amperometric determination of acetic acid with immobilized Trichosporon brassicae. Anal. Chim, Acta 109, 33.

- $(1979 b)$. Microbial electrode sensor for alcohols. Biotechnol. Bioeng. 21, 1845 . $(1980 a)$. Ammonia electrode with immobilized nitrifying bacteria. Anal. Chem. 52,1020 .

- Obana, H., Yasuda, T., Karube, I., and Suzuki, S. (1980b). Amperometric determination of total assimilable sugars in fermentation broths with use of immobilized whole cells. Enzyme Microb. Technol, 2, 234.

- (1980c). A potentiometric microbial sensor based on immobilized Escherichia coli for glutamic acid. Anal. Chim. Acta 116, 61 .

Karube, I. (1984). Microbial sensor for screening mutagens. Trends in Anal. Chem. 3. 40.

Karube, I., Matsunaga, T. and Suzuki, S. (1979a). Microbioassay of nystatin with a yeast electrode. Anal. Chim. Acta 109, 39.

- Mitsuda, S. and Suzuki, S. (1979b). Glucose sensor using immobilized whole cells of Pseudomonas fluorecens. Europ. J. Appl, Microbiol. Biotechnol. 7, 343.

Okada, T. and Suzuki, S. (1982). A methane gas sensor based on oxidizing bacteria. Anal. Chim. Acta 135, 61.

— Mitsuda, S., Matsunaga, T, and Suzuki, S. (1977). A rapid method for estimation of BOD by using immobilized microbial cells. J. Ferment. Technol. 55, 243.

Matsunaga, T., Karube, 1. and Suzuki, S. (1978a). Rapid determination of nicotinic acid by immobilized Lactobacillus arabinosus, Anal. Chim. Acta 99, 233.

- (1978b). Electrochemical microbioassay of vitamin B1. Anal. Chim. Acta 98, 25. (1979). Electrode system for the determination of microbial population. Appl. Envir. Microb. 37, 117.

(1980). A specific microbial sensor for formic acid. Europ. J. Microbiol. Biotechnol. 10, 235.

Okada, T., Karube, I. and Suzuki, S. (1983). $\mathrm{NO}_{2}$ sensor which use immobilized nitrite oxidizing bacteria. Biotechnol. Bioeng. 25, 1641.

Satoh, I., Karube, 1. and Suzuki, S. (1977a). Continuous neutral lipid determination with lipase-collagen membrane reactor. J. Solid-Phase Biochem, 2, 1.

(1977b). Enzyme electrode for free cholesterol, Biotechnol, Bioeng. 19, 1095.

Technicon Industrial Systems, No. 142-71 A, (1972). 


\title{
Biosensors based on plant and animal tissue
}

\author{
MARK A. ARNOLD and GARRY A. RECHNITZ
}

Tissue materials from plant and mammalian sources have been successfully employed as the biocatalytic component for the construction of biosensors. This class of biocatalytic materials simply maintains the enzyme of interest in its natural environment which results in a considerable stabilization of the desired enzymatic activity. In many cases, tissue-based biosensors have been found to have much improved useful lifetimes in comparison to the corresponding isolated enzyme-based biosensor. In addition, tissue materials have been shown to provide sufficiently high specific activities for the construction of certain biosensors where isolated enzymes have failed. These advantages have been obtained without sacrificing overall selectivity in most cases. For those situations where interfering processes are present in the tissue material, a selectivity enhancement strategy has been developed. In this chapter the relative merits of tissue-based biocatalysts are presented by considering individually each tissue biosensor that has been developed. Moreover, several biosensors based on related types of biocatalytic materials such as subcellular fractions of mammalian cells are reviewed. Finally, several possible models for the transport of substrate and product into, within, and out from the immobilized tissue cells are proposed for the first time.

From an historical point of view, tissue biosensors were introduced after the development of isolated enzyme and bacterial biosensors (Arnold 1983; Arnold and Meyerhoff 1984; Guilbault 1984; Meyerhoff and Fraticelli 1982; Rechnitz 1981). The concept of employing a whole section of mammalian tissue as a biocatalytic layer was first demonstrated with the fabrication of an arginine sensor (Rechnitz 1978). In this first case, a thin slice of bovine liver and an aliquot of the enzyme urease were co-immobilized at the surface of an ammonia gas-sensing probe. The reactions catalysed at the sensor tip are shown below.

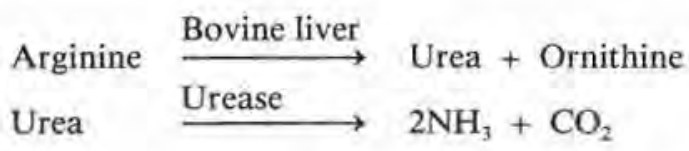

This first bovine liver probe opened the way for the development of several practical tissue biosensors. 
Table 3.1 Biosensors based on tissues and related materials

\begin{tabular}{|c|c|c|}
\hline Substrate & Biocatalytic material & Sensing element \\
\hline Glutamine & Porcine kidney cells & $\mathrm{NH}_{3}$-sensor \\
\hline Adenosine & $\begin{array}{l}\text { Mouse small-intestine } \\
\text { mucosal cells }\end{array}$ & $\mathrm{NH}_{3}$-sensor \\
\hline Adenosine 5 -monophosphate & Rabbit muscle & $\mathrm{NH}_{3}$-sensor \\
\hline Adenosine $5^{\prime}$-monophosphate & $\begin{array}{l}\text { Rabbit muscle acetonc } \\
\text { powder }\end{array}$ & $\mathrm{NH}_{3}$-sensor \\
\hline Guanine & Rabbit liver & $\mathrm{NH}_{3}$-sensor \\
\hline Hydrogen peroxide & Bovine liver & $\mathrm{O}_{2}$-sensor \\
\hline Glutamate & Yellow squash & $\mathrm{CO}_{2}$-sensor \\
\hline Pyruvate & Corn kernel & $\mathrm{CO}_{2}$-sensor \\
\hline Urea & Jack bean meal & $\mathrm{NH}_{3}$-sensor \\
\hline Phosphate/fluoride & $\begin{array}{l}\text { Potato tuber/glucose } \\
\text { oxidase }\end{array}$ & $\mathrm{O}_{2}$-sensor \\
\hline Dopamine & Banana pulp & $\mathrm{O}_{2}$-sensor \\
\hline Tyrosine & Sugar beet & $\mathrm{O}_{2}$-sensor \\
\hline Cysteine & Cucumber leaf & $\mathrm{NH}_{3}$-sensor \\
\hline Glutamine & $\begin{array}{l}\text { Porcine kidney } \\
\text { mitochondria }\end{array}$ & $\mathrm{NH}_{3}$-sensor \\
\hline
\end{tabular}

Table 3.1 summarizes the tissue biosensors and related systems which have been reported since the first bovine-liver-based arginine electrode. Related systems include the use of sub-cellular fractions, mammalian organ acetone powders, plant seed meals, and entire plant leaves and fruits as the biocatalytic component. The next section of this chapter will detail these biosensor systems individually with a particular interest in enhancements of the analytical response owing to the biocatalyst of interest.

\subsection{Glutamine biosensor}

A thin slice of porcine kidney cortex cells has been immobilized at the surface of an ammonia gas-sensing probe. A high concentration of the enzyme glutaminase, which catalyses the reaction shown below, is known to be located within these cells. By utilizing this biocatalytic activity in combination with an ammonia probe, a sensor for glutamine can be constructed.

$$
\text { Glutamine }+\mathrm{H}_{2} \mathrm{O} \rightleftharpoons \text { Glutamate }+\mathrm{NH}_{3}
$$

Immobilization of the porcine kidney is accomplished by physically retaining the tissue material using a monofilament mesh of nylon with a pore size of $149 \mu \mathrm{m}$. To protect the gas-permeable membrane of the ammonia probe from the various cellular components of the tissue slice, a thin cellulose 


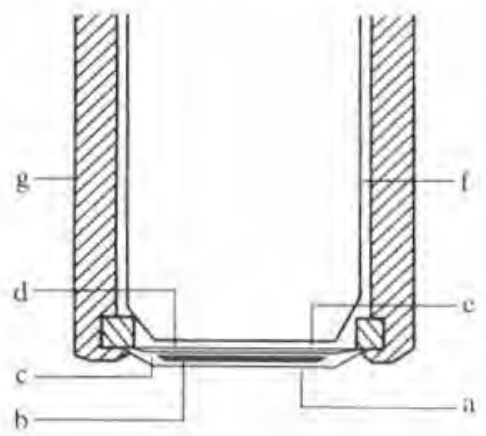

Fig. 3.1 Configuration of porcine kidney biosensor for glutamine. a, Nylon support membrane; b, porcine kidney slice; c, inner dialysis membrane; d, Teflon gaspermeable membrane; e, internal electrolyte; $f$, combination glass $\mathrm{pH}$ electrode; and $\mathrm{g}$, outer body (components $\mathrm{d}-\mathrm{g}$ represent the ammonia gas sensor).

diacetate membrane is positioned between the kidney section and the Teflon membrane. Figure 3.1 shows the arrangement of layers used for this biosensor.

As glutamine from the bulk solution interacts with the immobilized bio-

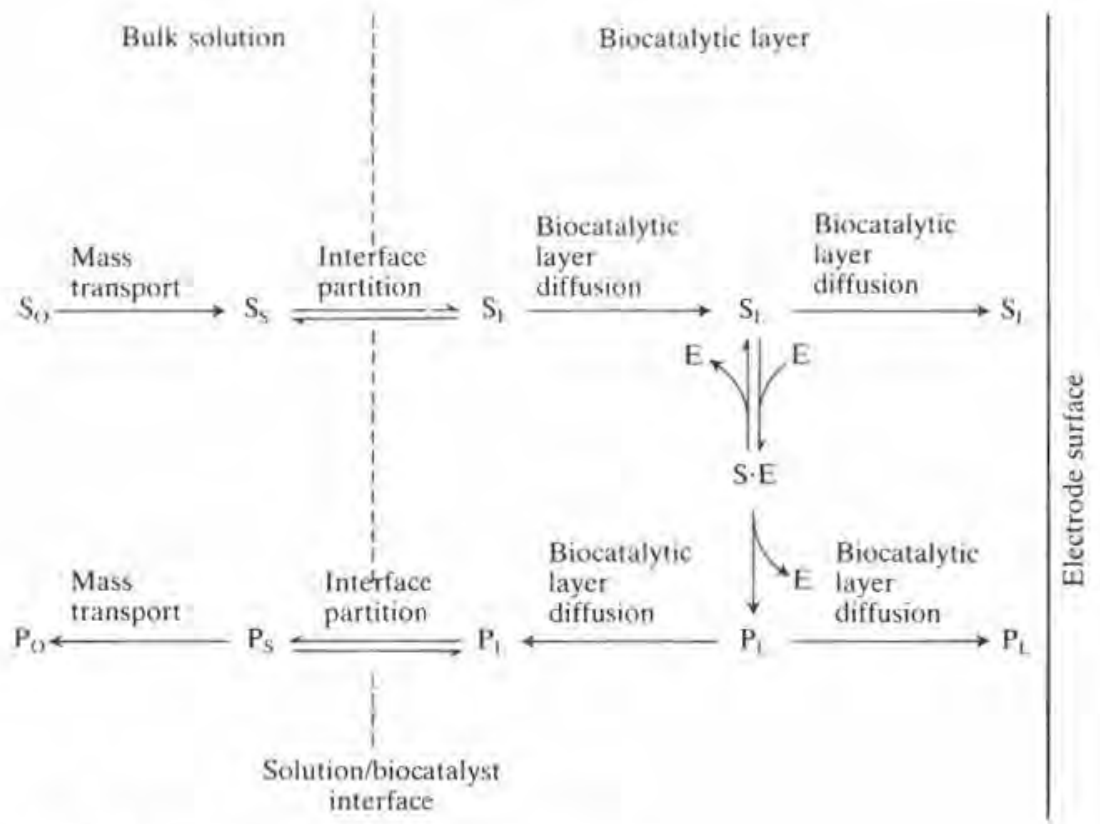

Fig. 3.2 Kinetic processes in biosensor response. 
catalytic layer, ammonia is generated by the action of glutaminase. A steadystate concentration of ammonia is eventually reached as the production of ammonia is counterbalanced by the depletion of ammonia at the probe surface. Simple diffusion of ammonia back into the bulk solution is mainly responsible for ammonia depletion (Arnold and Rechnitz 1982b, Carr and Bowers 1980). Figure 3.2 shows schematically the various kinetic processes which contribute to the sensor response. Because the ammonia probe is a potentiometric device, the resulting sensor response is related to bulk glutamine concentrations in a logarithmic fashion.

Figure 3.3 presents a typical glutamine calibration curve. For the porcine kidney glutamine biosensor, a typical response slope of $50 \mathrm{mV}$ per concentration decade is observed over a linear range which extends from approximately 0.1 to $10 \mathrm{~mm}$. A detection limit, as defined by IUPAC recommendations (IUPAC Analytical Chemistry Division 1976), of $0.01 \mathrm{~mm}$ has been established. Measured response times range from 7 to 5 minutes over the linear range of the sensor using a $0.1 \mathrm{M}$ phosphate, $\mathrm{pH} 7.8$ buffer. Longer response times are obtained at lower glutamine concentrations as is expected for this type of sensor (Carr and Bowers 1980; Kobos 1980).

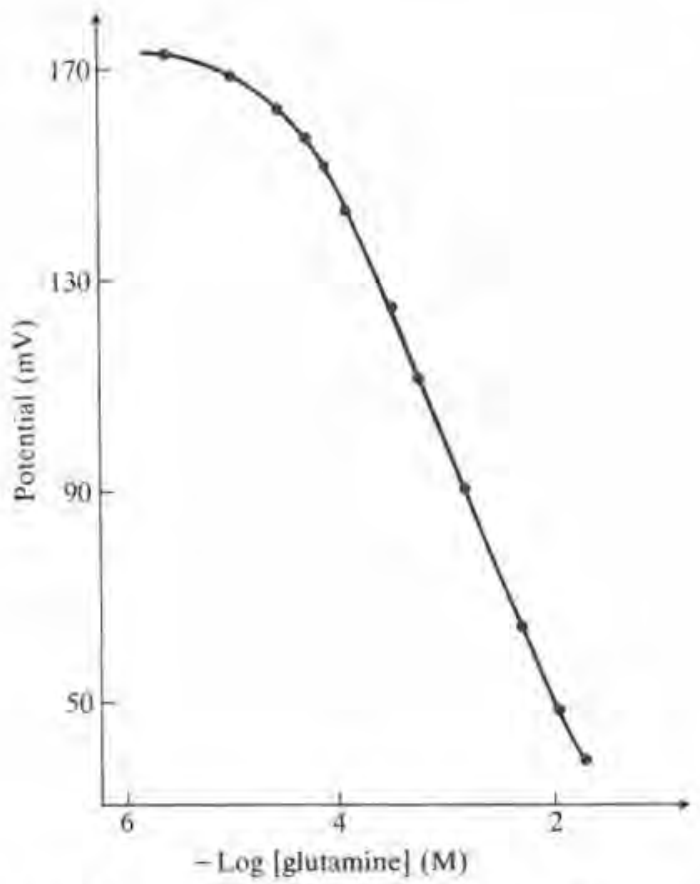

Fig. 3,3 Glutamine calibration curve using porcine kidney biosensor, 
Of course, the selectivity of a biosensor based on whole tissue cells must be considered in detail owing to the large number of suspected biocatalytic activities within these cells. The selectivity properties of the porcine kidney biosensor have been examined and found to be suitable for glutamine determinations in complex biological matrixes. The following compounds have been specifically tested as possible interferents but no significant response has been observed: urea, L-alanine, L-arginine, L-histidine, L-valine, L-serine, L-glutamate, L-asparagine, L-aspartate, D-alanine, D-aspartate, glycine, and creatinine. Sensor response to various D-amino acids and glycine has been examined because high concentrations of $\mathrm{D}$-amino acid oxidase are known to be located in porcine kidney cells (Dixon and Kleppe 1965). This enzyme catalyses the oxidative deamination of several D-amino acids in the presence of oxygen and water. Under the specified operating conditions of the glutamine biosensor, however, no response to the tested D-amino acids has been detected. Most likely this lack of interfering activity is due to the absence of flavin adenine dinucleotide (FAD) in the buffer system (Guilbault and Hrabankova 1971).

In order to obtain such a high degree of selectivity, it is necessary to include an anti-microbial agent in order to prohibit bacterial contamination of the tissue slice. Without the addition of a suitable agent, bacterial growth on the tissue material results in unwanted biocatalytic activities at the probe surface which drastically alters the sensor's selectivity pattern. For the glutamine sensors, $0.02 \%$ sodium azide is added as a preservative.

Selectivity of the tissue-based glutamine biosensor has been further established by application of the sensor to the quantification of glutamine in cerebrospinal fluid (CSF) control samples (Arnold and Rechnitz 1980a). After treatment of CSF samples with a cation exchanger to remove background ammonia-nitrogen which would interfer with the biosensor response, glutamine measurements can be made with good precision and accuracy over the clinically important range. For these glutamine measurements, however, iodoacetamide must be added to the working buffer to prohibit an interfering process which involves glucose. Apparently, glycolysis within the kidney cells generates an acid from glucose which alters the potential of the $\mathrm{pH}$-sensitive ammonia probe. Iodoacetamide is an established inhibitor of glycolysis and has been found to be effective in suppressing the glucose response of the glutamine biosensor. Glutamine determination in CSF samples can be made over the concentration range of $2.2 \times 10^{-5}$ to $1.29 \times 10^{-3} \mathrm{M}$ with an average relative standard deviation of $5.6 \%$. Such a sensor might prove valuable in studies involving Reye's syndrome where elevated glutamine levels have been proposed as a diagnostic tool.

An interesting feature of the tissue-based glutamine biosensor is its extended lifetime in comparison with that of a similar biosensor based on the 


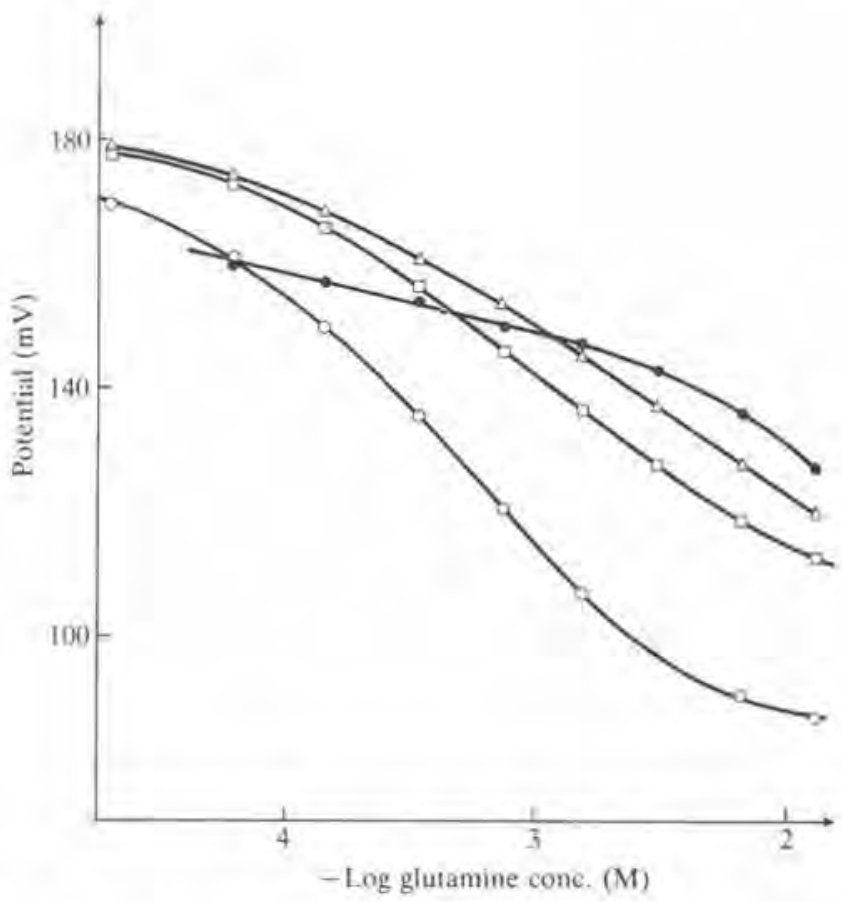

Fig. 3.4 Response curves for enzyme-based glutamine biosensor. $\bigcirc$, Day one; $\square$, day two; $\triangle$, day three; and $\bullet$, day four.

isolated enzyme. In the case of the isolated enzyme system, glutaminase is immobilized at the ammonia sensor surface using a thin cellulose diacetate membrane. Calibration curves with the enzyme system have been found to degrade quite rapidly after sensor construction. Figure 3.4 shows several glutamine response curves using isolated glutaminase as the biocatalytic component. It can be seen that in a matter of days the response is essentially unusable owing to the loss of a large portion of the enzymatic activity. On the other hand, the tissue glutamine sensor has been found to be usable for up to 28 days with essentially no change in the steady-state and dynamic response properties of the biosensor (Rechnitz et al. 1979). This tremendous enhancement in the useful lifetime of the sensor has been attributed to a stabilization of the enzymatic activity by maintaining the enzyme in the tissue matrix. We are simply taking advantage of the optimal environment that nature has provided for the use of this particular enzyme. For these lifetime studies, biosensors were simply stored between use in the working buffer at room temperature; hence, no elaborate conditions or equipment are required for effective storage of the tissue biosensor. 
Table 3.2 Response characteristics of glutamine biosensors

\begin{tabular}{lllll}
\hline & Enzyme & Mitochondria & Bacteria & Tissue \\
\hline Slope (mV/decade) & $33-41$ & 53 & 49 & 50 \\
Detection limit (M) & $6.0 \times 10^{-5}$ & $2.2 \times 10^{-5}$ & $5.6 \times 10^{-5}$ & $2.0 \times 10^{-5}$ \\
Linear range (mM) & $0.15-3.3$ & $0.11-5.5$ & $0.1-10$ & $0.064-5.2$ \\
Response time (min) & $4-5$ & $6-7$ & 5 & $5-7$ \\
Lifetime (days) & 1 & $10^{a}$ & $20^{a}$ & $30^{a}$ \\
\hline
\end{tabular}

${ }^{a}$ Minimum value

The glutamine biosensor is unique in the sense that several types of biocatalytic materials have been employed and examined for its construction. Specifically, the isolated enzyme glutaminase, mitochondria from porcine kidney cells (see below), whole sections of porcine kidney tissue, and intact bacterial cells of the strain Sarcina flava have been studied as possible biocatalytic materials and they have been directly compared in order to determine their relative merits (Arnold and Rechnitz 1980b).

Tables 3.2 and 3.3 summarize the most important properties of glutamine sensors based on each type of biocatalytic material. Table 3.2 lists the various

Table 3.3 Operation and preparation requirements for glutamine biosensors

\begin{tabular}{|c|c|c|}
\hline Electrode type & Operating medium & Preparation needs \\
\hline Enzyme electrode & $\begin{array}{l}0.1 \text { M phosphate buffer, } \\
\text { pH } 7.8,0.02 \% \mathrm{NaN}_{3}\end{array}$ & $\begin{array}{l}\text { Enzyme suspension held } \\
\text { between dialysis membranes; } \\
\text { store at room temperature in } \\
\text { buffer. }\end{array}$ \\
\hline Tissue electrode & $\begin{array}{l}\text { 0.I M phosphate buffer, } \\
\text { pH } 7.8,0.02 \% \mathrm{NaN}_{3}\end{array}$ & $\begin{array}{l}\text { Tissue sliced and supported on } \\
\text { nylon net; store in working } \\
\text { buffer at room temp. }\end{array}$ \\
\hline $\begin{array}{l}\text { Mitochondrial } \\
\text { electrode }\end{array}$ & 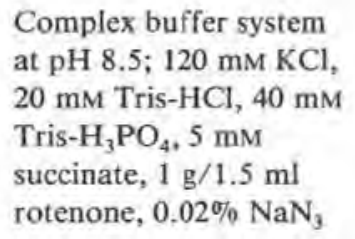 & $\begin{array}{l}\text { Isolation of mitochondrial } \\
\text { fraction, suspension of } \\
\text { fraction between dialysis } \\
\text { membranes. Condition in } \\
\text { working buffer. }\end{array}$ \\
\hline Bacterial electrode & $\begin{array}{l}0.1 \mathrm{~m} \text { Tris- } \mathrm{HCl}, 0.01 \mathrm{M} \\
\mathrm{MnCl}_{2}, \mathrm{pH} 7.5\end{array}$ & $\begin{array}{l}\text { Culture bacterial strain under } \\
\text { sterile conditions; washed cell } \\
\text { suspension held between } \\
\text { dialysis membranes. }\end{array}$ \\
\hline
\end{tabular}


analytical response characteristics of the sensors and the operation and preparation requirements are summarized in Table 3.3. These sensor properties have been attained under optimal conditions for the particular biosensor of interest.

From Table 3.2 it can be seen that values for the respective sensitivities, linear ranges, limits of detection, and response times are similar except for the poorly reproducible enzyme sensor. Hence, these response properties offer no compelling basis for selecting one type of biocatalyst over the other. From consideration of sensor lifetime (see Table 3.2), however, partial differentation of the biocatalyst types can be made. The isolated enzyme has simply too short a useful lifetime to be considered practical. The mitochondrial, bacterial, and tissue biosensors possess significantly longer lifetimes than the isolated enzyme because the enzymatic activity is housed in its natural environment.

After consideration of the preparation and operation requirements for the bacterial and mitochondrial biosensors, it becomes evident that the tissue biosensor is the system of choice for glutamine measurements. The bacterial sensor must be constructed under sterile conditions and the purity of the cell line must be maintained to ensure proper response properties. In addition, mitochondrial fractions must be isolated and maintained for consistent operation of the mitochondrial biosensor. Although isolation of mitochondria is relatively easy in comparison with purification of an enzyme, the required procedure is much more involved then that required to obtain a thin section of porcine kidney tissue. The bacterial biosensor might be the system of choice in cases where a phosphate buffer is unsuitable.

From this comparison study, it can be concluded that the biocatalytic material of choice for glutamine measurements is the porcine kidney slice. However, this conclusion can not be generalized to other types of biosensor systems. Indeed, more comparisons of this type are needed before any general statements can be made concerning biocatalytic materials.

\subsection{Adenosine biosensor}

Whereas the glutamine biosensor based on a slice of porcine kidney tissue has been demonstrated to possess a high degree of selectivity for glutamine over other biomolecules, it must be realized that tissue biosensors will rarely be highly selective for a given substrate because most tissue materials contain numerous enzymes and are capable of sustaining multiple metabolic pathways. Indeed, a biosensor for adenosine which involves the immobilization of mouse small-intestinal mucosal cells at the surface of an ammonia gas-sensing probe displays a considerable response to adenosine-base nucleotides. A selectivity enhancement strategy has been developed for such tissue systems. This strategy involves experimental identification of the interfering 


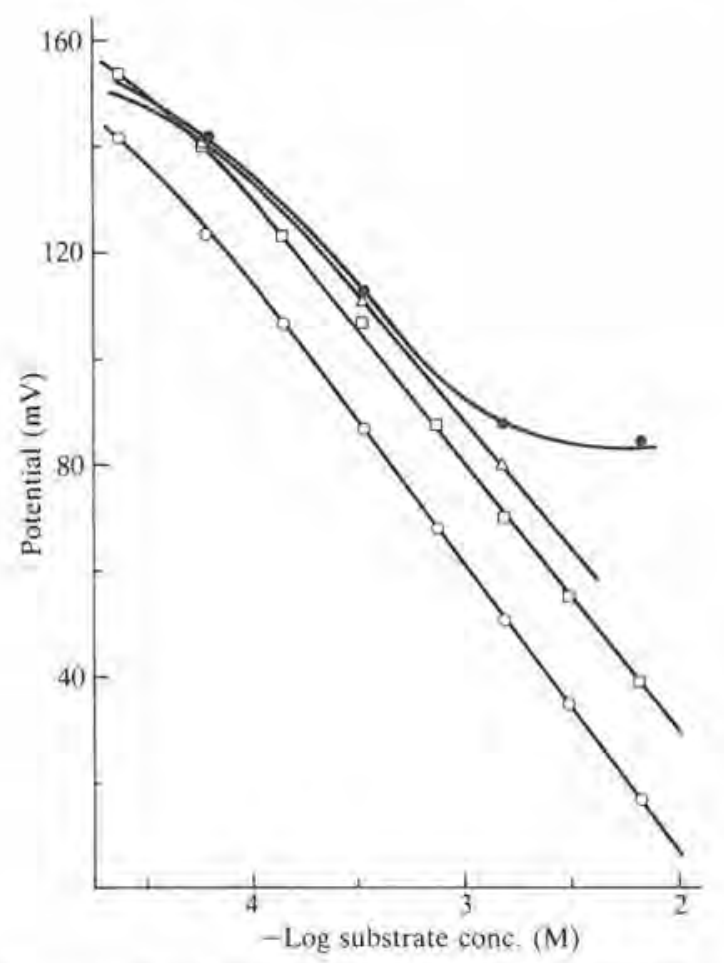

Fig. 3.5 Initial response of mouse small-intestine biosensor. $\bigcirc$, Adenosine; $\square, \mathrm{AMP} ; \triangle, \mathrm{ADP}$; and $\odot$, ATP.

pathway and selective repression of a key enzymatic activity within this pathway. This selectivity exhancement strategy is demonstrated with the mouse small intestine-based adenosine biosensor.

Adenosine electrodes are constructed by maintaining a suspension of mouse small-intestine mucosal cells at the surface of an ammonia gas-sensing probe. This immobilization is accomplished by entrapping the cells in a bovine serum albumin (BSA)-glutaraldehyde matrix on the gas-permeable membrane of the ammonia probe (Arnold and Rechnitz 1981a). The activity of the enzyme adenosine deaminase is employed.

Figure 3.5 shows the response observed for adenosine, adenosine monophosphate (AMP), adenosine diphosphate (ADP), and adenosine triphosphate (ATP), using the mouse small-intestine biosensor. These response curves are obtained using a working buffer of $0.2 \mathrm{M}$ Tris- $\mathrm{HC} 1$ and $0.02 \%$ sodium azide at $\mathrm{pH} 8.2$. It can be seen that adenosine-containing nucleotides elicit a significant response which would severely interfere with adenosine measurements. To enhance the sensor selectivity, it is desirable to 
Table 3.4 Possible interfering schemes for adenosine sensor

Scheme I:

$$
\mathrm{AXP}+\mathrm{H}_{2} \mathrm{O} \stackrel{\begin{array}{c}
\text { Non-specific } \\
\text { deamination }
\end{array}}{\longrightarrow} \mathrm{IXP}+\mathrm{NH}_{3}
$$

Scheme II:

$$
\text { AMP }+\mathrm{H}_{2} \mathrm{O} \stackrel{\text { AMP }}{\stackrel{\text { deaminase }}{\longrightarrow}} \mathrm{IMP}+\mathrm{NH}_{3}
$$

Scheme III:

$$
\text { AMP }+\mathrm{H}_{2} \mathrm{O} \stackrel{\begin{array}{c}
\text { Alkaline } \\
\text { phosphatase }
\end{array}}{\stackrel{\text { Adenosine }}{\text { deaminase }}} \text { Adenosine }+ \text { Phosphate }
$$

optimize the adenosine-deaminating activity and to repress the nucleotidedeaminating activity. The most effective method of repressing this activity depends on the metabolic pathway responsible for the interfering deamination.

Reports of the presence of AMP deaminase (Dixon and Webb 1964) and alkaline phosphatase (Conway and Cooke 1939) in mouse small-intestinal mucosal cells have led to three possible schemes for the interfering activity which are shown in Table 3.4. Scheme I represents the case where a single, non-specific enzyme is present that catalyses the deamination of AMP, ADP, and ATP. Scheme II represents the situation where three specific deaminating enzymes are present for each of the substrates. Finally, Scheme III involves the combined activities of alkaline phosphatase and adenosine deaminase, in which the adenosine-containing nucleotides are converted to adenosine via the first enzyme and then deaminated by the second enzyme which results in the interference.

By examining the effects of various activators and inhibitors on the interfering activity, attempts can be made to distinguish which, if any, of the above mentioned schemes accurately describes the interfering activity. Reports have shown that phosphate ions inhibit the enzymatic activity of both alkaline phosphatase (Fernley and Walker 1967) and AMP deaminase (Ronca-Testoni et al. 1970). Also glycerophosphate is known to inhibit AMP deaminase (Sammons et al. 1970) and it is acted upon by alkaline phosphatase (Macfarlane et al. 1934). In the latter case, substrate specificity is such that at high concentrations of glycerophosphate the action of alkaline phosphatase on AMP is negligible. Figure 3.6 shows the ef fect of phosphate and glycerophosphate on the AMP and adenosine-deaminating activities of 


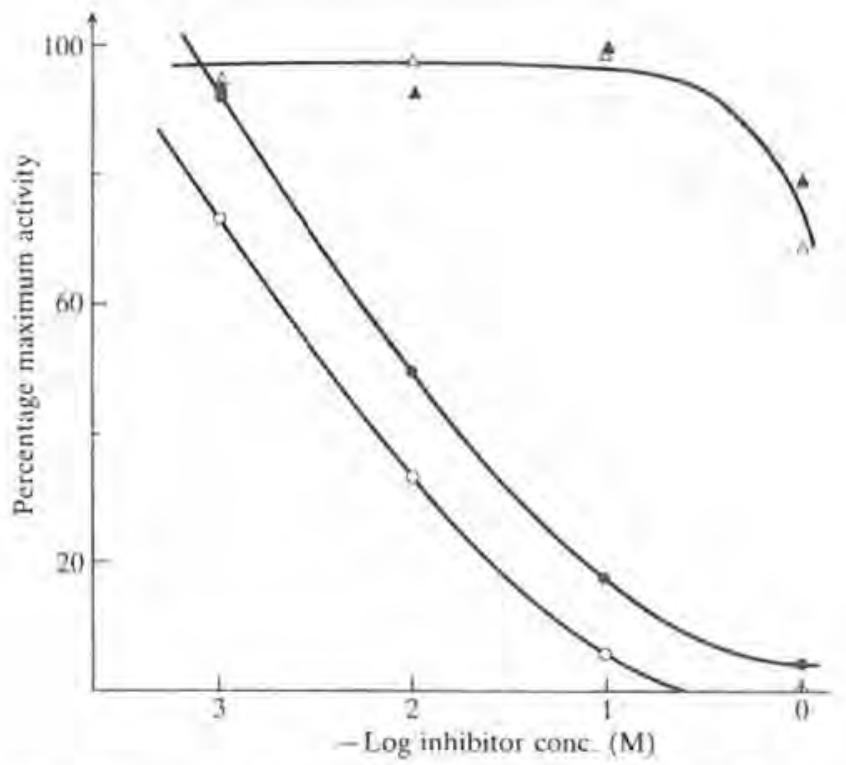

Fig. 3.6 Effect of glycerophosphate (solid characters) and phosphate (open characters) on the deamination of $0, \Theta, A M P$ and $\Delta, \Delta$, adenosine.

the tissue-based adenosine electrode. It can be seen that high concentrations of each substance result in a drastic decrease in the AMP-deaminating activity with only a slight decrease in the adenosine-deaminating activity.

It is known that AMP deaminase is dependent on the presence of potassium ions for its activity (Zielke and Suelter 1960). A study of the effect of potassium ions on the mouse small-intestinal mucosal cell deamination of AMP reveals that there is no such dependence in terms of the interfering activity. On the other hand, the activity of intestinal alkaline phosphatase is reported to be stereospecifically inhibited by L-phenylalanine (Fishman $e t a l$. 1963; Ghosh and Fishman 1966). The effect of L-phenylalanine on the response for AMP reveals an inhibition of the AMP-deaminating activity at a concentration of $0.1 \mathrm{ML}$-phenylalanine. As a result, virtually no response for AMP is detectable by the electrode system in the presence of L-phenylalanine.

The reported $\mathrm{pH}$ optima for the enzymes under consideration are approximately 6.5 for AMP deaminase (Conway and Cooke 1939) and from 7.0 to 9.0 for alkaline phosphatase (Kay 1932). The $\mathrm{pH}$ optimum for the AMP deaminating activity of the mouse small-intestinal mucosal cells is 8.2 which is within the optimum $\mathrm{pH}$ range of alkaline phosphatase. A pH range from 9.0 to 9.4 has been found to be optimal for the adenosine-deaminating activity.

High activity of AMP deaminase is commonly found in muscle tissues (Conway and Cooke 1939); whereas, alkaline phosphatase is found in high levels in intestinal mucosal cells of various mammalian species (Kay 1932). 
Previously reported distribution patterns of these enzymes and the results presented above, including the inhibitory effects of glycerophosphate, phosphate, and $\mathrm{L}$-phenylalanine, the lack of activation by potassium ions, and the $\mathrm{pH}$ optimum of the interfering activity, provide strong evidence that the coupling of alkaline phosphatase and adenosine deaminase is principally responsible for the interfering activity of the mucosal cell biocatalytic layer.

On the basis of the above results, a buffer system containing phosphate ions at $\mathrm{pH} 9.0$ should effectively repress the interfering activity of the tissuebased adenosine electrode while optimizing the desired biocatalytic activity. Figure 3.7 shows the selectivity for adenosine which is obtained with a buffer system of $0.1 \mathrm{M}$ Tris- $\mathrm{HCl}, 0.2 \mathrm{M} \mathrm{K}_{2} \mathrm{HPO}_{4}$, and $0.02 \% \mathrm{NaN}_{3}$ at $\mathrm{pH} 9.0$. Under these conditions no response is observed for AMP, ADP, or ATP. Other possible interferents which have been tested and found to give no response include 3'-AMP, cAMP, adenine, guanosine, and guanine.

Although selectivity for adenosine is not an inherent characteristic of the tissue-based adenosine electrode, it is possible to enhance the selectivity of

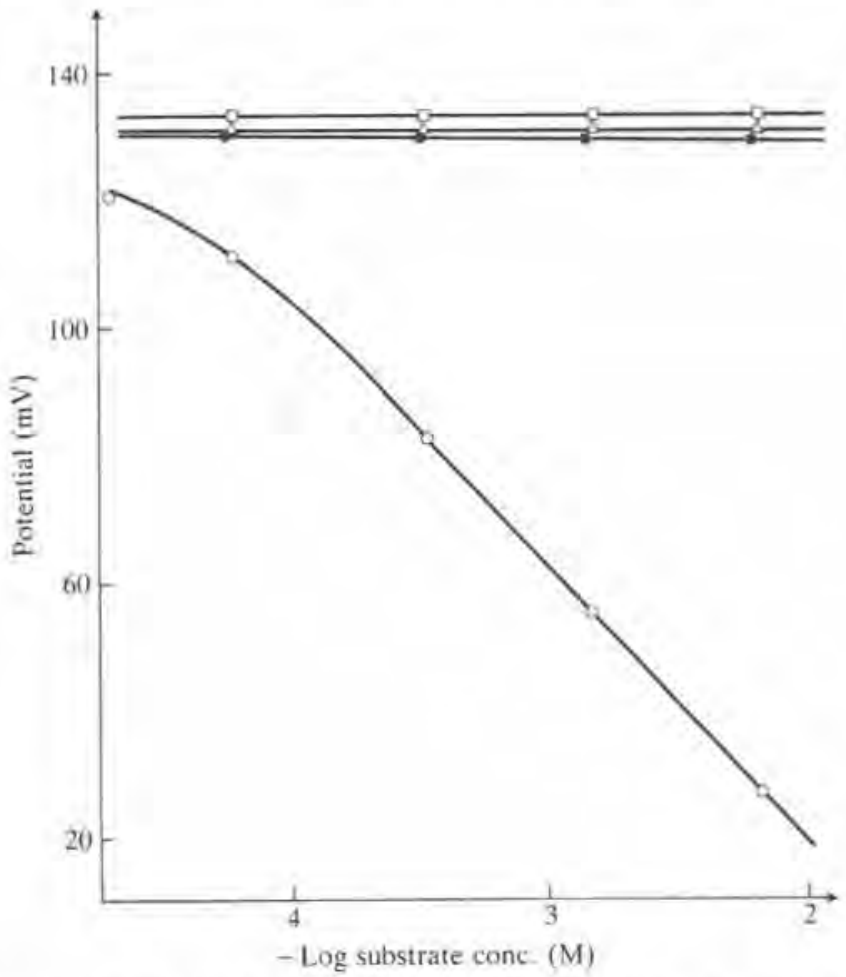

Fig. 3.7 Response of mouse small-intestine biosensor after selectivity enhancement. 0 , Adenosine; $\square$, AMP; $\triangle$, ADP; and $\bullet$, ATP. 
the electrode system by determining the metabolic pathway responsible for the interfering activity and by repressing this activity with an effective inhibitor. This technique of enhancing selectivity has proven valuable in the development of other tissue-based membrane electrodes.

\subsection{AMP biosensor}

Aside from the enhancement of biosensor lifetimes, tissue materials have been demonstrated to provide a large concentration of a particular biocatalytic activity. Because of the restricted surface area of the ammonia gas-sensing probe, limited amounts of an enzyme preparation can be immobilized at the sensor surface. Therefore, if the specific activity of the enzyme preparation is too low, small amounts of immobilized enzyme will result which leads to poor analytical response characteristics. An example of this low enzyme concentration effect is the AMP enzyme electrode (Papastathopoulos and Rechnitz 1976). The isolated enzyme for this sensor is commonly available only at low specific activities and, as a result, AMP biosensors with low slopes and short useful lifetimes are obtained. By employing a thin layer of rabbit muscle tissue, however, an AMP biosensor with considerable improvement in slope and lifetime is possible. Improvement in response can be directly attributed to a five-fold increase in the amount of biocatalytic activity at the probe surface,

Both the enzyme and tissue biosensors for AMP use the catalysed reaction shown below at the surface of an ammonia gas-sensing probe.

$$
\mathrm{AMP}+\mathrm{H}_{2} \mathrm{O} \rightleftharpoons \mathrm{IMP}+\mathrm{NH}_{3}
$$

The isolated enzyme is immobilized at the probe surface using a cellulose diacetate membrane and the tissue biosensor holds a thin section of rabbit muscle tissue at the probe surface with a $37 \mu$ m nylon mesh. AMP biosensors of both types are stored at room temperature between use in a working buffer which consists of $0.1 \mathrm{M}$ Tris- $\mathrm{HCl}, 0.1 \mathrm{M} \mathrm{KCl}$, and $0.02 \%$ sodium azide at $\mathrm{pH}$ 7.5. The tissue biosensor must be conditioned for 2 to 4 hours after construction to remove background ammonia.

Various experimental parameters have been optimized with respect to the response of the tissue-based AMP sensor. These parameters include $\mathrm{pH}$, potassium concentration, temperature, and tissue thickness. Optimal conditions have been found to be $\mathrm{pH} 7.5$ with $0.1 \mathrm{M}$ potassium at $25^{\circ} \mathrm{C}$. The effect of tissue thickness has been examined and the results are presented in Fig. 3.8. Increases in tissue thickness result in longer electrode response times with unusable response times being observed when the tissue thickness is greater than $0.81 \mathrm{~mm}$. On the other hand, tissue slices less than $0.5 \mathrm{~mm}$ are difficult to handle and reproduce. For these reasons, tissue thicknesses ranging from 0.5 to $0.8 \mathrm{~mm}$ are typically used for sensor construction and 


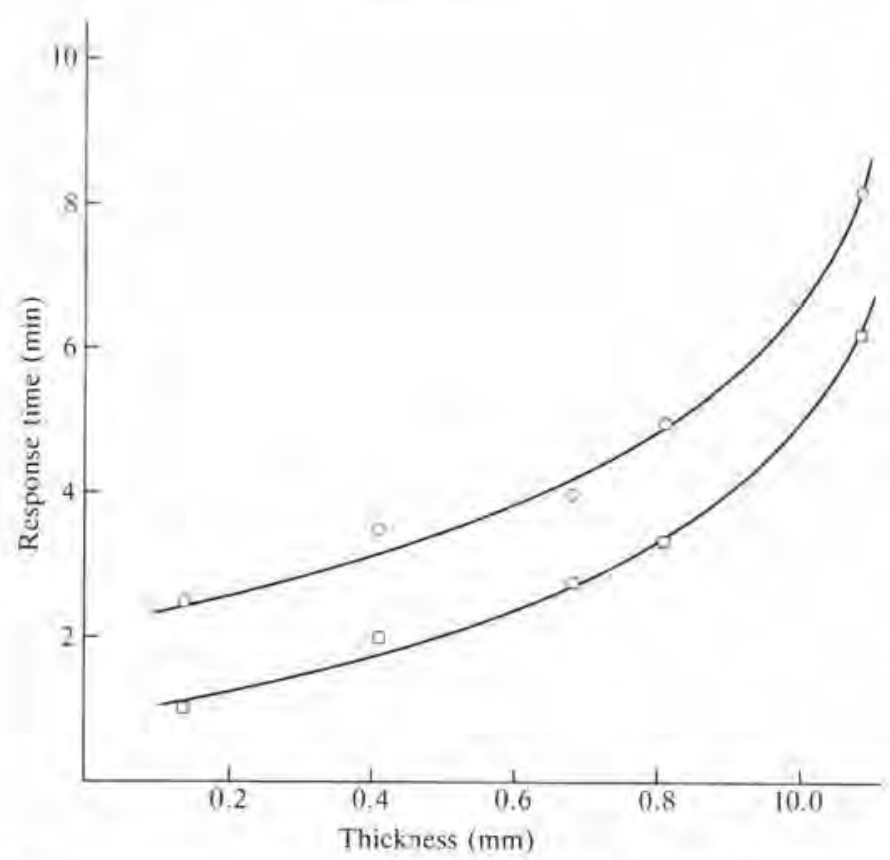

Fig. 3.8 Effect of tissue thickness on biosensor response time, Change in substrate concentration; 0 , from 0.14 to $0.34 \mathrm{~mm}$ and $\square$, from 3.0 to $6.6 \mathrm{~mm}$.

can be conveniently prepared with a sharp razor blade.

A $0.5 \mathrm{~mm}$ rabbit muscle slice contains approximately five international units (IU) of AMP-deaminating activity (Arnold and Rechnitz 1981b). This compares to only $0.1 \mathrm{IU}$ of activity from a comparable volume $(25 \mu \mathrm{l})$ of the commercially available enzyme. This small amount of activity results in enzyme biosensors with poor analytical response. In fact, before immobilization the isolated enzyme must be concentrated using a 16-hour filtration process which results in $0.9 \mathrm{IU}$ of activity at the electrode surface (Papastathopoulos and Rechnitz 1976). Even after this concentration procedure, nearly five times greater activity can be supplied using the tissue slice. Table 3.5 summarizes the response characteristics for AMP sensors based upon each of these biocatalytic materials. Higher amounts of activity using the tissue section results in excellent response characteristics including a slope of $58 \mathrm{mV}$ per concentration decade and a lifetime of at least 28 days. In contrast, the enzyme system displays a slope of only $46 \mathrm{mV}$ per concentration decade and a lifetime of just four days. This study shows the effectiveness of using tissue slices over isolated enzymes in situations where the latter have insufficient biocatalytic specific activity.

In certain cases, it is difficult to locate a reliable supplier of a particular 
Table 3.5 Comparison of AMP biosensor response characteristics

\begin{tabular}{llll}
\hline $\begin{array}{l}\text { Response } \\
\text { characteristic }\end{array}$ & $\begin{array}{l}\text { Isolated } \\
\text { enzyme }\end{array}$ & $\begin{array}{l}\text { Tissue } \\
\text { slice }\end{array}$ & $\begin{array}{l}\text { Acetone } \\
\text { powder }\end{array}$ \\
\hline Slope $(\mathrm{mV} /$ Decade) & 46 & 58 & 57 \\
Linear range $\left(\times 10^{4}, \mathrm{M}\right)$ & $0.8-150$ & $1.4-100$ & $3.3-130$ \\
Detection limit $\left(\times 10^{5}, \mathrm{M}\right)$ & 6.0 & 4.8 & 4.0 \\
Response time $(\mathrm{min})$ & $2-6$ & $2.5-8.5$ & $2.5-8.0$ \\
Lifetime (days) & 4 & 28 & 25 \\
\hline
\end{tabular}

mammalian species in order to obtain a specific tissue material. In these cases, it might be more convenient to employ an acetone powder of the desired tissue material as the biocatalytic component. The first such attempt has been reported in the construction of an AMP biosensor in which a slurry of rabbit muscle acetone powder is physically retained at the surface of an ammonia probe (Arnold and Fiocchi 1984).

The rabbit muscle acetone powder slurry is prepared by dispensing $300 \mu \mathrm{l}$ of a $0.1 \mathrm{M}$ Tris- $\mathrm{HCl}, 0.1 \mathrm{M} \mathrm{KCl}, 0.02 \%$ sodium azide, $\mathrm{pH} 7.9$ buffer into a $1 \mathrm{ml}$ plastic vial. A $100 \mathrm{mg}$ portion of frozen acetone powder is added to this vial and the mixture is agitated on a vortex mixer for thirty seconds. This treatment produces a homogeneous slurry of the biocatalyst of which the desired amount (generally $10 \mathrm{mg}$ ) is placed on the Teflon membrane of the ammonia probe. A cellulose diacetate membrane is placed over the slurry and the electrode cap is screwed in position which holds the slurry in place. The finished biosensor must be allowed to condition overnight in the previously mentioned buffer solution to remove background ammonia from the biocatalytic layer.

Table 3.5 summarizes the response characteristics of the rabbit-muscle acetone-powder biosensor for AMP. In comparison to the other AMP biosensors, the acetone-powder system matches very closely the response characteristics of the tissue biosensor. Both are superior to the isolated enzyme case, particularly with respect to slope and lifetime. Because the acetone powder is generally more readily available at lower cost it is most likely the biocatalytic material of choice for many AMP measurements.

\subsection{Guanine biosensor}

As more tissue-type biosensing probes are developed, the need for an effective optimization strategy for tissue biocatalysts becomes increasingly evident. Such a strategy has been proposed in which the biochemical processes and membrane phases involved in biosensor response are specifically considered (Arnold and Rechnitz 1982a). This strategy is illustrated through the optimi- 
zation of a guanine biosensor which employs a section of rabbit liver with an ammonia-gas sensor. The important biocatalytic activity is shown below.

\section{Guanine $+\mathrm{H}_{2} \mathrm{O} \rightleftharpoons$ Xanthine $+\mathrm{NH}_{3}$}

Guanine biosensors are prepared by placing a $0.5 \mathrm{~mm}$ slice of rabbit liver between two cellulose dialysis membranes. The liver section is then positioned on the gas-permeable membrane of the ammonia probe and the sensor is assembled as normal. Freshly prepared sensors are conditioned overnight in a pH 8.0,0.2 $\mathrm{M}$ borate buffer containing $0.02 \%$ sodium azide. Guanine sensors are stored in this buffer at room temperature between measurements.

Optimization of a tissue biosensor must include experimental characterizations of various sensor parameters. Typically, the method of tissue-slice immobilization, effects of $\mathrm{pH}$, activators, and inhibitors, biosensor lifetime, and overall selectivity must be considered in detail. Several methods of tissueslice immobilization are available based either on physical retainment with an appropriate membrane or entrappment in a crosslinked protein matrix. Many tissue materials can be effectively immobilized with relative ease using a mesh of nylon owing to the connective properties of most tissue sections. For the guanine biosensor, the rabbit liver is held at the electrode surface using a cellulose diacetate membrane because this liver material does not have the required mechanical integrity to permit the use of a large pore nylon mesh.

The effects of $\mathrm{pH}$ and activator as well as inhibitor concentrations on a tissue biosensor are generally important to consider. Biocatalytic activities and gas-sensing membrane electrodes are both dependent on solution $\mathrm{pH}$. In addition, activators can often be added to enhance a desired activity and inhibitors can be employed to suppress an interfering activity. A convenient method for studying these effects is the initial rate measurement where the rate of product generation at the start of a biocatalysed reaction is directly related to the effective biocatalytic activity (Guilbault er al. 1968). Although this method can only supply relative information, it is quick and convenient for comparisons of various solution conditions. It is important to realize, especially during $\mathrm{pH}$ studies, that the initial rate of a biosensing probe is a function of both the catalytic and the sensing components. Therefore, measurements of this type allow for a simultaneous optimization of conditions with respect to each. For the guanine sensor, maximal rate of ammonia production is obtained using $\mathrm{pH} 9.5$; however, based on biocatalyst stability and electrode lifetime considerations, $\mathrm{pH} 8.0$ is more practical even though only $50 \%$ of the maximal activity is obtained.

The useful lifetime of a biosensor is frequently limited by the stability of the biocatalytic component (Arnold 1982a). For this reason, addition of stabilizing agents and optimization of storage conditions must be considered 


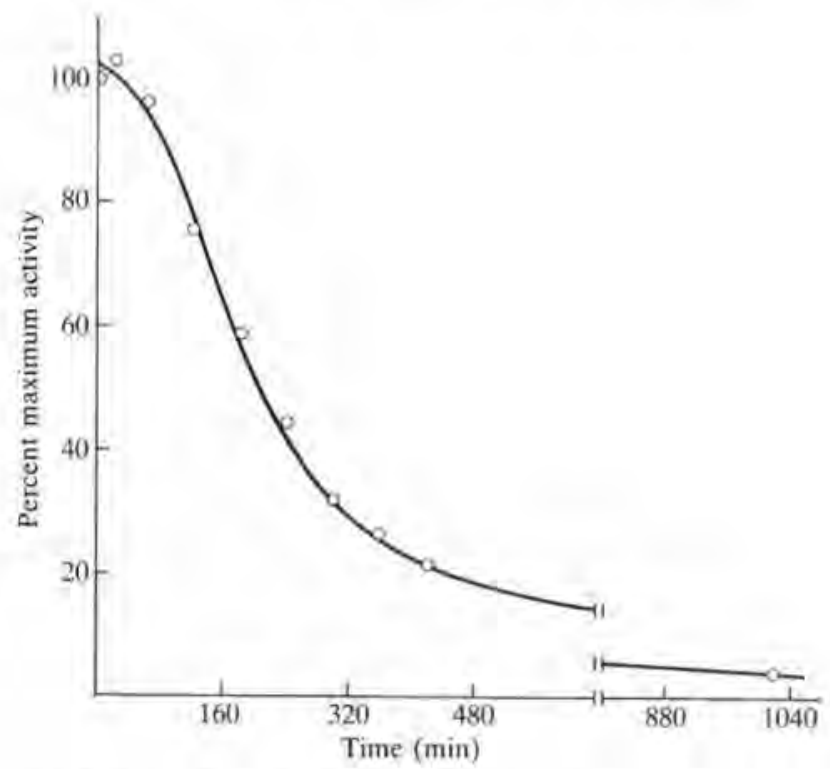

Fig. 3.9 Instability of guanase activity at pH 9.5.

for maximal lifetimes. The importance of proper storage conditions is exemplified nicely by the rabbit-liver guanine sensor where the optimal $\mathrm{pH}$ for action of the biocatalyst is not the $\mathrm{pH}$ optimum for biocatalyst stability. Figure 3.9 shows the instability of the guanase activity at $\mathrm{pH} 9.5$ which is the optimal $\mathrm{pH}$ for the catalysed reaction. The rapid decline in activity clearly indicates that biosensors stored at this $\mathrm{pH}$ would be short lived. Similar results are obtained at $\mathrm{pH} 9.0$ and 8.5 , but a stable biosensor results when a pH 8.0 storage solution is used.

Besides the stability of the immobilized tissue material, the lifetime of the bulk organ from which numerous biosensors can be prepared must be considered. Table 3.6 summarizes the storage conditions and minimal lifetime of several tissue materials. Excellent storage times and inherent low cost can make tissue slices the most economical type of biocatalyst for the construction of many biosensing probes.

Of course, any optimization scheme for tissue-based biosensors must give special attention to selectivity, because of the numerous metabolic paths typically found in tissue materials. The main type of interfering activities which are likely to occur include the generation of the measured product from a substrate other than the principal substrate and the utilization of a substrate whose reaction changes the $\mathrm{pH}$ at the electrode surface. The first of these is more common and more difficult to eliminate, A strategy for eliminating this type of interference has been reported (see above; Arnold 
Table 3.6 Storage conditions of selected bulk organs

\begin{tabular}{llll}
\hline Substrate & $\begin{array}{l}\text { Biocatalytic } \\
\text { material }\end{array}$ & $\begin{array}{l}\text { Storage } \\
\text { conditions }\end{array}$ & $\begin{array}{l}\text { Lifetime } \\
\text { (months) }\end{array}$ \\
\hline Glutamine & Porcine kidney & $-25^{\circ} \mathrm{C}$ & 6 \\
AMP & Rabbit muscle & $-25^{\circ} \mathrm{C}$ & 7 \\
Guanine & Rabbit liver & $-25^{\circ} \mathrm{C}$ & 7 \\
Adenosine & $\begin{array}{l}\text { Mouse small } \\
\text { intestine }\end{array}$ & $-25^{\circ} \mathrm{C}$ in & 2 \\
Glutamate & Yellow squash & $100 \%$ glycerol & \\
Pyruvate & Corn kernel & $+4{ }^{\circ} \mathrm{C}$ & 1 \\
\hline
\end{tabular}

${ }^{a}$ Minimum values

and Rechnitz 1981a) and involves determining the specific enzyme or enzymes responsible for the interfering activity followed by repression of that activity with the use of specific inhibitors. A major concern in this strategy is to ensure that the added inhibitor has no adverse effect on the desired biocatalytic activity. The second type of interference is best minimized by employing a working solution with high buffer capacity.

Selectivity for the mouse-liver guanine biosensor has been found to be excellent in the presence of $1 \mathrm{~mm}$ magnesium (II) to inhibit adenosine deaminase and in the absence of phosphate to prohibit the action of guanosine phosphorylase (Arnold and Rechnitz 1982a). The resulting sensor displays no response to millimolar concentrations of inosine, adenine, GMP, IMP, creatinine, creatine, asparagine, serine, urea, glutamine, glutamate, ornithine, threonine, lysine, valine, glycine, and arginine.

The combination of fine-tuning the biochemical process and the appropriate selection of membrane materials, tissue thickness, and immobilization process represent the essential elements of the best optimization strategy currently available for tissue biosensors.

\subsection{Hydrogen peroxide biosensor}

Up to this point each of the tissue sections of interest have been coupled with a potentiometric ammonia membrane electrode. The first amperometricbased tissue biosensor has been reported by Mascini et al. (1982) where a slice of bovine liver has been immobilized on an oxygen-sensitive probe for the measurement of hydrogen peroxide. This liver contains a large concentration of the enzyme catalase which catalyses the following reaction:

$$
2 \mathrm{H}_{2} \mathrm{O}_{2} \longrightarrow \mathrm{O}_{2}+2 \mathrm{H}_{2} \mathrm{O}
$$

Oxygen production is monitored amperometrically. 
Tissue-based peroxide sensors are prepared using a $0.1 \mathrm{~mm}$ thick section of bovine liver which is held at the oxygen electrode by a nylon mesh. Livers from a variety of species have been found to be suitable and measurements are obtained in a nitrogen-purged buffer of $0.05 \mathrm{M}$ phosphate and $0.2 \%$ sodium azide at $\mathrm{pH}$ 6.8. A conditioning period of two hours is required after construction.

Hydrogen peroxide response curves reveal a linear response from the bovine liver biosensor down to the $10 \mu \mathrm{M}$ level. Curves are linear for approximately one order of magnitude and response as well as recovery times are quite fast, being less than two minutes at moderate concentrations. This sensor displays remarkable selectivity for hydrogen peroxide with large concentrations of likely interferents such as glucose, alcohol, L-amino acids, and lactate giving no response.

In comparison to an isolated enzyme biosensor, the tissue sensor displays less susceptibility to changes in solution $\mathrm{pH}$ and temperature. Moreover, the tissue system is show to have a considerably longer lifetime than the isolated enzyme system, presumably, due to a stabilizing effect of the tissue matrix.

\subsection{Glutamate biosensor}

The first tissue biosensor involving a slice of plant material has been reported for glutamate measurements (Kuriyama and Rechnitz 1981). A thin layer of yellow squash is immobilized at the surface of a carbon dioxide gas-sensing probe. The reaction shown below is catalysed by the action of glutamate decarboxylase which is know to be present in high concentrations in yellow squash.

\section{Glutamate $\rightleftharpoons$ 4-Aminobutyrate $+\mathrm{CO}_{2}$}

The section of squash material is immobilized by entrapment in a BSA-glutaraldehyde matrix and $0.002 \%$ chlorhexidine diacetate is used as the preservative.

Optimal response of the yellow-squash glutamate biosensor requires a 0.1 M phosphate, pH 5.5 buffer which includes $40 \%$ glycerol and $0.3 \mathrm{~mm}$ pyridoxal-5'-phosphate (PLP). Under these conditions a Nernstian response to glutamate is obtained from $4.4 \times 10^{-4}$ to $4.7 \times 10^{-3} \mathrm{M}$ with a slope of $48 \mathrm{mV}$ per concentration decade and a detection limit of $2 \times 10^{-4} \mathrm{M}$. Response times in the range of ten minutes are common, and the probe remains active over a seven day period. Most importantly, the selectivity for this sensor is excellent with no response to a wide variety of other biologically important compounds (Kuriyama and Rechnitz 1981).

Overall the tissue glutamate biosensor compares favorably to the isolatedenzyme system which demonstrates the feasibility of employing plant materials as the biocatalytic component. 


\subsection{Pyruvate biosensor}

As with certain mammalian tissue-based biosensing electrodes, a plant tissue pyruvate biosensor shows an extended lifetime over the corresponding isolated-enzyme system (Kuriyama et al. 1983). This enhancement in sensor lifetime is achieved with no loss of selectivity and is attributed to stabilization of the biocatalytic activity by the plant tissue matrix.

Corn kernels are known to possess high concentrations of the enzyme pyruvate decarboxylase which catalyses the following reaction:

$$
\text { Pyruvate }+\mathrm{H}_{2} \mathrm{O} \rightleftharpoons \text { Acetaldehyde }+\mathrm{CO}_{2}
$$

By coupling this biocatalytic activity with a carbon dioxide gas sensor, pyruvate measurements can be made. Pyruvate sensors have been prepared by physically retaining a thin layer of corn kernel at the carbon dioxide electrode surface with a common dialysis membrane and by entrappment in a BSA-glutaraldehyde matrix. Similar immobilization schemes have been used in the fabrication of pyruvate sensors based on the isolated enzyme.

Response characteristics for the corn kernel tissue and isolated enzyme pyruvate sensors are tabulated in Table 3.7. The corn kernel biocatalyst results in superior response with respect to slope, linear range, and limit of detection. The enzyme system, on the other hand, displays better response times. An attempt to quicken the response time of the tissue electrode has been made by fractionating the corn kernel and immobilizing only the active components. Unfortunately, this strategy is not effective in this case because the biocatalytic activity is evenly distributed throughout much of the kernel.

As summarized in Table 3.7, the useful lifetime of the corn kernel electrode is significantly better than that for the isolated-enzyme-based sensor. In fact, the response slope of the enzyme system continually declines from about 35 to $12 \mathrm{mV}$ per concentration decade over a three-day period; whereas, the tissue system shows minimal changes over a seven-day period. This extended lifetime is achieved without sacrificing selectivity, as the tissue system shows no response to a wide variety of compounds tested as possible interferents (Kuriyama et al. 1983).

Table 3.7 Response characteristics of pyruvate biosensors

\begin{tabular}{lll}
\hline & Enzyme & Corn kernel \\
\hline Slope (mV/decade) & 35 & 47 \\
Detection limit $(\mathrm{M})$ & $1.6 \times 10^{-4}$ & $8.0 \times 10^{-5}$ \\
Linear range $(\mathrm{mM})$ & $0.74-4.3$ & $0.25-3.0$ \\
Response time $(\mathrm{min})$ & $4-10$ & $10-25$ \\
Lifetime (days) & 1 & 7 \\
\hline
\end{tabular}




\subsection{Urea biosensor}

A biosensor for the measurement of urea has been reported in which a layer of jack bean meal is employed as the biocatalytic component (Arnold and Glazier 1984). This meal naturally contains a high amount of the enzyme urease which catalyses the following reaction:

$$
\text { Urea }+\mathrm{H}_{2} \mathrm{O} \longrightarrow 2 \mathrm{NH}_{3}+\mathrm{CO}_{2}
$$

This biocatalytic material has been found to be an effective alternative to the isolated-enzyme system.

Jack-bean-meal urea biosensors are prepared by removing the outer layer of the whole jack bean and pulverizing the treated seed with a mortar and pestle. Generally, $7 \mathrm{mg}$ of the freshly ground meal is placed on the surface of an ammonia-gas sensor and a paste is made by adding a small volume of buffer ( $0.2 \mathrm{M}$ Tris- $\mathrm{HCl}, \mathrm{pH} 8.5,0.1 \mathrm{~mm}$ EDTA). After the paste is spread evenly over the membrane, glutaraldehyde is added to crosslink the proteins which results in a stable biocatalytic layer. Urea response curves are obtained in the Tris- $\mathrm{HCl}$, EDTA buffer at $25^{\circ} \mathrm{C}$ and biosensors are conveniently stored in this same buffer at room temperature.

Response characteristics for the bean-meal urea sensor compare favorably with an isolated-enzyme-based urea sensor. Table 3.8 summarizes the important response characteristics for each of these sensors. Moreover, the bean meal has been found to possess an impressive selectivity for urea over a wide range of tested possible interferents (Arnold and Glazier 1984). The jack bean meal has the advantages of lower cost and more convenient storage conditions over the isolated enzyme. Purified urease is moderately expensive and must be stored at or below freezing temperatures. The bean meal, on the other hand, is considerably less expensive and can be effectively stored at room temperature. Overall, jack bean meal is a suitable alternative to purified urease for the construction of urea biosensors.

Table 3.8 Comparison of jack bean meal and urease biosensors

\begin{tabular}{lll}
\hline Response characteristic & Jack bean meal & Urease \\
\hline Slope $(\mathrm{mV} /$ decade $)$ & 58 & 55 \\
Linear range $\left(\times 10^{5}, \mathrm{M}\right)$ & $3,4-150$ & $3.0-500$ \\
Detection limit $\left(\times 10^{6}, \mathrm{M}\right)$ & 2.1 & 1.0 \\
Response time $(\mathrm{min})$ & $1-5$ & $1-5$ \\
Lifetime (days) & 94 & 60 \\
\hline
\end{tabular}

\subsection{Phosphate-fluoride sensor}

A hybrid biosensor based on a thin layer of potato tuber and a solution of 
glucose oxidase has been described for the quantification of phosphate and/or fluoride. The following reactions are catalysed at the surface of an oxygen sensor where the potato tuber provides the enzymatic activity of acid phosphatase:

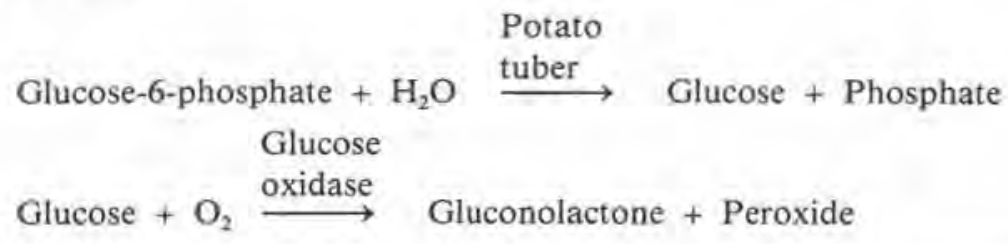

A steady-state current is attained from a set glucose-6-phosphate concentration in the external solution. Phosphate and fluoride are well-known inhibitors of acid phosphatase (Schubert et al. 1984); therefore, the addition of either anion will slow the rate of glucose generation which can be measured as a decrease in the consumption of oxygen. Typically, a steady-state current is established, corresponding to a particular rate of oxygen consumption. The sample or standard containing either phosphate or fluoride is then added and the increase in current is determined from a first derivative recording of a current $v s$. time curve.

Because phosphate is a competitive inhibitor of acid phosphatase, a linear relationship is observed between phosphate concentrations and the reciprocal of the maximum rate of current change. A linear range has been reported from $25 \mu \mathrm{M}$ to $1.5 \mathrm{mM}$ phosphate. On the other hand, fluoride calibration curves are not linear because of the complicated nature of the noncompetitive type of inhibition this anion imposes on acid phosphatase. A workable curve from $0.2 \mathrm{~mm}$ to $6 \mathrm{mM}$ fluoride can be obtained, however. Measuring times for both anions are in the order of five minutes and the hybrid sensor remains active for at least 24 days when stored at $4{ }^{\circ} \mathrm{C}$ between measurements.

As one might expect for a sensor dependent on inhibition of an enzymatic activity, the potato-based hybrid biosensor suffers from interferences by a variety of acid phosphatase inhibitors. Compounds such as nitrate, borate, molybdate, and organic phosphates are the most noteworthy. Also, glucose and glucose-6-phosphate must be considered interferents as their presence can alter the rate of oxygen production. With appropriate sample pretreatment, however, accurate measurements of both phosphate and fluoride concentrations can be obtained.

\subsection{Dopamine biosensor}

A tissue biosensor with selective response to dopamine has been developed in which a thin layer of banana pulp is physically immobilized at the surface of an amperometric oxygen electrode (Sidwell and Rechnitz 1985). Figure 3.10 


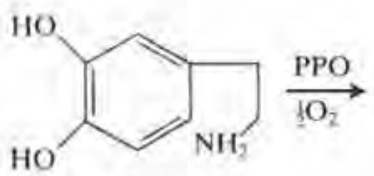

Dopamine

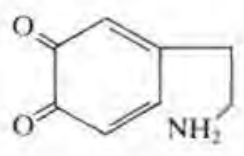

Dopamine quinone<smiles>O=C1C=c2cc[nH]c2=CC1=O</smiles>

Indole-5,6-quinone<smiles>O=C1C=C2CCNC2=CC1=O</smiles>

2,3-Dihydroindole5,6-quinone

$\downarrow$ Slow

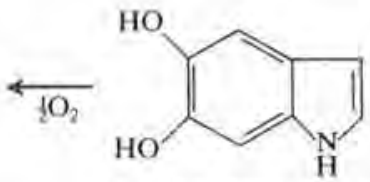

5,6-Dihydroxyindole

Fig. 3.10 Reactions in the catalytic oxidation of dopamine, PPO represents polyphenol oxidase.

shows the reactions that occur at the electrode surface. Oxygen consumption during this reaction sequence is monitored and the resulting current is related to dopamine concentrations.

Calibration curves based on steady-state currents have been obtained using a $0.1 \mathrm{M}$, pH 6.5 phosphate buffer at $25^{\circ} \mathrm{C}$. These response curves are linear over a dopamine concentration range from 0.2 to $1.2 \mathrm{mM}$ and sensor response times are on the order of one to three minutes. These electrode response characteristics remain unchanged for at least one week when the electrode is stored in buffer.

The development of this dopamine sensor suggests that by careful selection of appropriate plant materials, tissue sensors for catacholamine neurotransmitters are possible.

\subsection{Tyrosine biosensor}

An amperometric tyrosine biosensor has been reported in which a slice of sugar beet (Beta vulgaris altissima) is immobilized at the surface of an oxygen sensing probe (Schubert et al. 1983) This sensor employs the activity of tyrosinase which is located within the beet structure and oxygen consumption is monitored. The beet slice is maintained on the probe surface using a common dialysis membrane and response curves are obtained using a $\mathrm{pH} 7.0$ phosphate buffer at $25^{\circ} \mathrm{C}$.

Response to tyrosine is linear from 0.1 to $0.4 \mathrm{~mm}$ and a useful response up to $0.9 \mathrm{~mm}$ is achieved. The sensor is stable for at least eight days and its response times are in the order of several minutes, Selectivity is marginal for 
tyrosine with other materials eliciting a significant response such as dihydroxyphenylalanine, 2,4-dichlorophenol and p-chlorophenol.

\subsection{Cysteine biosensor}

Yet another type of plant material has been demonstrated to be applicable for the construction of biosensors. Modified cucumber leaves have been immobilized at the surface of an ammonia sensor for the measurement of cysteine. Plant leaves offer a particularly attractive structural arrangement for possible use as biocatalysts. Many leaves have a multilayer structure consisting of a waxy coating (cuticle) at the outer surface, a layer of epidermal cells, followed by a third layer (spongy mesophyll) directly under the epidermis, with the same arrangement repeated in reverse on the other side of the leaf. The cuticle is hydrophobic in nature but permits the passage of gases; gas exchange takes place through small surface openings called stomata. The spongy mesophyll layer is the most active in metabolic processes involving gases. For the construction of biocatalytic membrane electrodes, the cuticle can be detached from either the upper or lower epidermal layer and the remaining leaf structure fixed at the surface of a gas-sensing potentiometric electrode with the exposed epidermal layer contacting the sample and the gas-permeable waxy cuticle facing the internal elements of the sensor.

The principle has been demonstrated (Smit and Rechnitz 1984) with the use of cucumber leaves at an $\mathrm{NH}_{3}$ sensor to construct a probe for L-cysteine. Such leaves have biocatalytic activity involving the enzyme L-cysteine desulphhydrolase according to

$$
\text { L-Cysteine }+\mathrm{H}_{2} \mathrm{O} \longrightarrow \text { Pyruvate }+\mathrm{H}_{2} \mathrm{~S}+\mathrm{NH}_{3}
$$

Thus, sensors can be constructed using either $\mathrm{NH}_{3}$ or $\mathrm{H}_{2} \mathrm{~S}$ gas-sensing electrodes, but the $\mathrm{NH}_{3}$ case is preferable on chemical grounds.

The technique is quite simple. Cucumber plants (Cucumis saturis) are grown from seed in Fertilite seed starter soil. Mature leaves are detached when needed and soaked in water for 45 minutes; this soaking softens the cuticle and permits ready removal to expose the biochemically active epidermis. This procedure is necessary because the substrate, L-cysteine, cannot readily diffuse through the waxy cuticle layer. Leaf discs are then cut to fit the gas-sensor tip and held there with a dialysis membrane. Such a biosensor gives a response to $\mathrm{L}$-cysteine in $\mathrm{pH} 7.6$ phosphate buffer between approximately $10^{-3}$ to $10^{-5} \mathrm{M}$ with a slope of about $35 \mathrm{mV}$ per decade. The relatively poor slope and fairly long response times of this sensor show that further development is needed, but the long useful lifetime (up to four weeks) and extremely low cost of the biocatalyst suggest that leaf materials could be attractive alternatives to immobilized enzymes or cells. 


\subsection{Mitochondria-based biosensor}

Besides whole slices of mammalian tissue, effective biosensors can be prepared by fractionating the tissue cells and immobilizing just the subcellular component that is richest in the biocatalytic activity of interest. Such a strategy might prove fruitful to increase the amount of immobilized activity or to improve sensor selectivity by eliminating an interfering activity which is present in another compartment of the cell. Indeed, subcellular fractions have been demonstrated to be useful as analytical reagents with the use of rat liver microsomes for the determination of thyroxine (Meyerhoff and Rechnitz 1979). The first successful subcellular-based biosensor has been developed for glutamine measurements in which the mitochondrial fraction from porcine kidney cortex cells is immobilized at an ammonia-gas sensor (Arnold and Rechnitz 1980b). Two isozymes of glutaminase are known to exist in mitochondria (Crompton et al. 1973), and this activity is utilized in the glutamine probe.

The mitochondrial fraction of porcine kidney cells is isolated according to a standard procedure involving differential centrifugation (Johnson and Lardy 1967). The glutamine sensor is prepared by immobilizing the resulting mitochondrial fraction with a common cellulose diacetate-type dialysis membrane. Completed biosensors are stored in a buffer composed of $0.120 \mathrm{M}$ potassium chloride, $0.02 \mathrm{M}$ Tris-chloride, $0.04 \mathrm{M}$ Tris-phosphate, $0.005 \mathrm{M}$ succinate, $1 \mu \mathrm{g} / 1.5 \mathrm{ml}$ rotenone, and $0.02 \%$ sodium azide at $\mathrm{pH} 8.5$. Sensors are stored and operated at room temperature.

Table 3.2 summarizes the analytical response characteristics which are obtained from the mitochondria electrode. These response characteristics compare very well with the tissue and bacterial electrode systems and are superior to the isolated-enzyme case. Measurements show that the selectivity for the mitochondria-based glucose sensor is very good (Arnold and Rechnitz 1980b).

Successful fabrication of this mitochondria biosensor demonstrates that subcellular materials can be effective biocatalytic components. Although it is not necessary in the case of a glutamine biosensor, subcellular fractions may be useful in improving sensor response and selectivity when the entire tissue section lacks the necessary properties.

\subsection{Mechanism of tissue biosensor response}

The response mechanism for plant and animal tissue electrodes has not yet been determined. In fact, no fundamental studies have been reported concerning the transport mechanism of substrate and product molecules within a tissue slice biocatalytic layer. Determination of the transport mechanism involved is important for the overall development of tissue materials as analytical reagents. 
Because tissue cells serve to house the enzyme of interest, the substrate must be transported within the biocatalytic layer in such a way as to make contact with the enzyme. It is reasonable that substrate must be transported into the immobilized cells before contact with the enzyme. Moreover, transport of the electrode-measurable product from the cells must be considered. To further complicate matters, the principal enzyme can be located within a specific subcellular organelle which requires additional mechanisms for the transport of substrate and product into, within, and from the organelle of interest. In the light of experimental (Arnold and Rechnitz 1982b) and theoretical (Carr and Bowers 1980) observations concerning the dependency of biosensor response characteristics on substrate diffusion processes within the biocatalytic layer, the transport mechanisms in question have important implications with respect to the analytical properties of tissue-based biocatalysts.

Several models can be proposed to describe the interaction between substrate and enzyme within a tissue slice biocatalyst. Figure 3.11 shows these various models schematically.

Model I represents the case where cells on the outer surface of the tissue slice completely break down structurally releasing the principal enzyme at the sensor surface. As the electrode ages, cellular debris diffuses away from the electrode surface which exposes a fresh layer of cells and generates a fresh supply of enzyme. This model eliminates the complications of substrate entrance into the cells. Of course the rate of enzyme release must be constant to be consistent with the excellent reproducibility observed for tissue electrodes and it must be slow to ensure sufficient amount of enzyme over extended periods (i.e. 30 days).

The structural integrity of porcine kidney tissue cells has been estimated in preliminary studies (Arnold 1982b). For this study a slice of porcine kidney was suspended in a small volume of a phosphate buffer $(0.1 \mathrm{M}, \mathrm{pH} 7.8)$ with $0.02 \%$ sodium azide. After this material was incubated overnight at room temperature, the solution was centrifuged and the cells collected. The

I

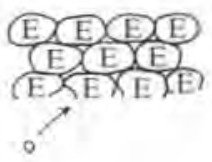

11

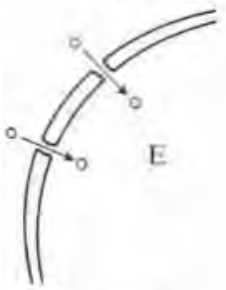

Cell membrane

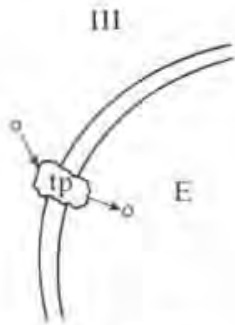

Cell membrane

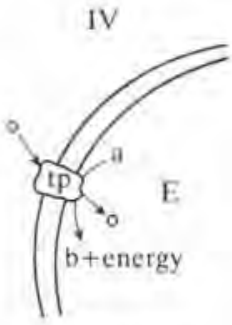

Cell membrane

Fig. 3.11 Schematic representations of proposed models for substrate-enzyme interaction, $o$, Substrate, E, active enzyme; and, tp, transport protein. 
resulting supernatant was analysed for glutaminase activity which is the enzymatic activity that is employed in the glutamine biosensor. A small amount of glutaminase activity was found in this supernatant. The resulting pellet of kidney cells was resuspended in fresh buffer. This mixture was again incubated at room temperature overnight and centrifuged as before. The second time, no glutaminase activity was found in the supernatant, but considerable activity was found in the kidney cells themselves.

The above-mentioned results, suggest that the integrity of the cells is maintained at least during initial use of kidney cells as a biocatalyst. The small amount of glutaminase in the first supernatant is most likely from cell fragments generated during the initial cutting of the tissue slice from the bulk organ.

Model II presents the possible case where the immobilized cells break down sufficiently to allow free diffusion of substrate and product molecules in and out of the tissue cells but not so completely that the principal enzyme can diffuse away from the electrode surface. Recent research in the area of permeabilized cells show how such channels can develop in the outer membranes of both prokaryotic and eukaryotic cells by treatments such as osmotic shock and freeze-thaw cycling (Felix 1982). It might be the case that such channels develop in immobilized tissue slices during electrode construction either by water washing of the tissue layer or by thawing of the previously frozen tissue material.

Models III and IV represent the cases where the immobilized tissue cells remain intact at the electrode surface. Model III requires that presence of transport proteins specific for the substrate of interest to aid in taking this substrate across the cell membrane. Besides requiring a transport protein, model IV requires a sources of energy from within the tissue cell to aid in the transport process. The requirement of an energy source for model IV renders this model quite unlikely, since cell viability in these systems is very seriously in doubt.

At this point, little information is available concerning any of these proposed mechanisms. We offer these models as a starting point for investigations into the response mechanism of tissue-based sensors.

As is presented in this chapter, considerable progress has been made in the advancement of tissue-based biosensing probes. Future research in this area looks promising with the development of new probes for different biomolecules. The application of novel tissue classes, such as insects and aquatic plants, have yet to be investigated. Moreover, fundamental studies concerning the structure of tissue-slice biocatalytic layers are most certainly needed. Finally, the application of tissue biocatalysts with other types of analytical transducers must be considered. Indeed, the major advantages of providing stable and large amounts of a desired biocatalytic activity should benefit other types of biocatalysis-based analyses. 


\section{References}

Arnold, M. A. (1983). An introduction to biocatalytic membrane electrodes. Amer. Lab, 15, 34-40.

- (1982a). Tissue-based biocatalytic membrane electrodes. Ph.D. Dissertation, University of Delaware, Section I.

- (1982b). Tissue-based biocatalytic membrane electrodes. Ph.D. Dissertation, University of Delaware, Section II.

- and Fiocchi, J.A. (1984). Rabbit muscle acetone powder as biocatalyst for adenosine 5'-monophosphate biosensor. Anal, Lett. 17, 2091-109.

- and Glazier, S. A. (1984). Jack bean meal as biocatalyst for urea biosensors. Biotech. Lett. 6, 313-18.

- and Meyerhoff, M.E. (1984). Ion-selective electrodes. Anal. Chem. 56, 20R-48R.

- and Rechnitz, G. A. (1980a), Determination of glutamine in cerebrospinal fluid with a tissue-based membrane electrode. Anal. Chim. Acta. 113, 351-4.

- (1980b). Comparison of bacterial, mitochondrial, tissue and enzyme biocatalysts for glutamine selective membrane electrodes. Anal. Chem. 52, 1170-4.

- $(1981 a)$. Selectivity enhancement of a tissue-based adenosine sensing membrane electrode. Anal. Chem. 53, 515-8.

- $(1981 b)$. High activity membrane electrode for adenosine 5 -monophosphate using rabbit muscle tissue as biocatalyst, Anal. Chem. 53, 1837-42.

(1982a). Optimization of a tissue-based membrane electrode for guanine. Anal. Chem. 54, 777-82.

- $(1982 b)$. Substrate consumption by biocatalytic potentiometric membrane electrodes. Anal. Chem. 54, 2315-17.

Carr, P. W. and Bowers, L. D. (1980). Immobilized enzymes in analytical and clinical chemistry. Wiley, New York.

Conway, E. J. and Cooke, R. (1939). The deaminases of adenosine and adenylic acid in blood and tissues, Biochem. J. 33, 479-92.

Crompton, M., McGivan, J, D, and Chappei, J, B. (1973). The intramitochondrial location of the glutaminase isoenzymes in pig kidney. Biochem. J. 132, 27-34.

Dixon, M. and Kleppe, K. (1965). D-amino acid oxidase; II. specificity, competitive inhibition, and reaction sequence. Biochim. Biophys. Acta 96, 368-82.

— and Webb, E. C. (1964). Enzymes (2nd edn.). Academic Press, New York.

Felix, H. (1982). Permeabilized cells. Anal. Biochem. 120, 211-34.

Fernley, H. H. and Walker, P. G. (1967). Studies on alkaline phosphatase; inhibition by phosphate derivatives and the substrate specificity. Biochem. J. 104, $1011-18$.

Fishman, W. H., Green, S. and Inglis, N. I. (1963). L-phenylalanine: an organ specific, stereospecific inhibitor of human intestinal alkaline phosphatase. Nature (London) 198, 685-86.

Ghosh, N. K. and Fishman, W. H. (1966), On the mechanism of inhibition of intestial alkaline phosphatase by L-phenylalanine; 1. Kinetic studies. J. Biol. Chem. 241, 2516-22.

Guilbault, G, G. (1984). Analytical uses of immobilized enzymes. Marcel Dekker, New York. 
and Hrabankova, E. (1971). New enzyme electrode probes for D-amino acids and asparagine. Anal. Chim. Acta 56, 285-90.

- Smith, R. K. and Montalvo, J. G., Jr. (1968). Use of ion selective electrodes in enzymic analysis; cation electrodes for deaminase enzyme systems. Anal. Chem. 41, 600-605.

IUPAC Analytical Chemistry Division (1976). Recommendations for nomenclature of ion-selective electrodes. Pure Appl. Chem. 48, 127-32.

Johnson, D. and Lardy, H. (1967). Isolation of liver or kidney mitochondria. In Methods in Enzymology (eds. R. W. Estabrook and M. E. Pullman), Vol. X. Academic Press, New York.

Kay, H. D. (1932). Phosphatase in growth and disease of bone, Physiol. Rev. 12, $384-442$.

Kobos, R. K. (1980). Potentiometric enzyme methods In Ion-selective electrodes in analytical chemistry (ed. M. Freiser), Vol. II, Chapter 1. Plenum, New York.

Kuriyama, S. and Rechnitz, G. A. (1981). Plant tissue-based biocatalytic membrane electrode for glutamate. Anal. Chim. Acta 131, 91-6.

— Arnold, M. A. and Rechnitz, G. A. (1983). Improved membrane electrode using plant tissue as biocatalyst. J. Membr. Sci. 12, 269-78.

Macfarlane, M. G., Patterson, L. M. B. and Robison, R. (1934). The phosphatase activity of animal tissue. Biochem. J. 28, 720-24.

Mascini, M., Jannelle, M. and Palleschi, G. (1982). A liver tissue-based electrochemical sensor for hydrogen peroxide. Anal. Chim. Acta 138, 65-9.

Meyerhoff, M. E. and Fraticelli, Y.M. (1982), Ion-selective electrodes. Anal. Chem. 54, 27R-44R.

— and Rechnitz, G. A. (1979). Microsomal thyroxine measurements with iodide selective membrane electrode. Anal, Lett. 12, 1339-46,

Papastathopoulos, D.S. and Rechnitz, G. A. (1976). Highly selective enzyme electrode for 5'-adenosine monophosphate, Anal. Chem. 48, 862-4.

Rechnitz, G.A. (1978). Biochemical electrodes uses tissue slice. Chem. Eng. News 56 (Oct. 9), 16.

- (1981). Bioselective membrane electrode probes. Science 214, 287-91.

- (1988). Bioselective membrane electrodes using tissue materials as biocatalysts.

In Methods in enzymology (ed. K. Mosbach), Vol. 137. Academic Press, San Diego.

- Arnold, M. A. and Meyerhoff, M. E. (1979). Bio-selective membrane electrode using tissue slices. Nature (London) 278, 466-7.

Ronca-Testoni, S. Raggi, A. and Ronca, G. (1970). Muscle AMP aminohydrolase; III. A comparative study on the regulatory properties of skeletal muscle enzyme from various sources. Biochem. Biophys. Acta 198, 101-12.

Sammons, D. W., Henry, H. and Chilson, D. P. (1970). Effect of salts on inhibition of chicken muscle adenosine monophosphate deaminase by phosphate esters and inorganic phosphate. J. Biol. Chem. 245, 2109-13.

Schubert, F., Rennebarg, R., Scheller, F. W. and Kirstein, L. (1984). Plant tissue hybride electrode for determination of phosphate and fluoride. Anal. Chem. 56, 1677-82.

Wallenberger, U. and Scheller, F, (1983). Plant tissue-based amperometric tyrosine electrode. Biotech. Lett. 5, 239-42. 
Sidwell, J.S. and Rechnitz, G.A. (1985). Bananatrode - an electrochemical biosensor for dopamine. Biotech. Lett. 7, 419-22.

Smit, N, and Rechnitz, G. A. (1984). Leaf-based biocatalytic membrane electrodes. Biotech. Lett. 6, 209-14.

Valle-Vega, P., Young, C. T. and Swaisgood, H. E. (1980). Arginase-urease electrode for determination of arginine and peanut maturity. J. Food. Sci. 45, 1026-30.

Zielke, C. L. and Suelter, C. H. (1960). Purine, purine nucleoside, and purine nucleotide aminohydrolases. The enzymes (3rd edn; ed. P. D. Boyer), Vol, 4, Chapter 3. Academic Press, New York. 


\section{New approaches to electrochemical immunoassays}

MONIKA J. GREEN

When Yalow and Berson $(1959,1960)$ published their work on the detection of plasma insulin by radioimmunoassay they provided a revolutionary new method for the accurate and specific measurement of low levels of hormones, enzymes, drugs, viruses, tumour antigens, bacterial antigens, and many proteins and organic substances that had hitherto been difficult or impossible to detect.

Immunoassays are fundamentally simple and are based on the interaction of the analyte or ligand $(\mathrm{Ag})$ in question with its specific binding partner or antibody $(\mathrm{Ab})$ (eqn 4.1)

$$
\mathrm{Ab}+\mathrm{Ag} \rightleftharpoons \mathrm{AbAg}
$$

to form an antibody/antigen ( $\mathrm{AbAg}$ ) complex. An equilibrium is reached and the equilibrium or affinity constant $K$ is defined as

$$
K=[\mathrm{AgAb}] /[\mathrm{Ag}][\mathrm{Ab}] \text {. }
$$

So for a fixed concentration of antibody, the ratio of bound-to-free antigen at equilibrium is quantitatively related to the total amount of ligand present. This forms the basis for all immunoassays. Thus if a fixed amount of labelled antigen is introduced into the assay the concentration of the unknown antigen can then be determined. Unknown concentrations of antibodies can be determined by using labelled antibodies. 'Labelling' agents such as radioisotopes, enzymes, red cells, fluorescent probes, chemiluminescent probes, or metal tags may be used to label either an antibody or an antigen. In radioimmunoassay (RIA) and enzyme immunoassays (EIA) the antigen is labelled. In immunoradiometric (IRMA) and immunoenzymometric assays (IEMA) antibodies are labelled. Most immunoassay techniques require a separation step to discriminate between the bound and unbound labelled antigen or labelled antibodies; this makes the technique somewhat cumbersome and time consuming.

The growing trend away from radioimmunoassays and the increasing number of enzyme-linked immunoassays, coupled with the wide range of low detection limits of electroanalytical methods, has resulted in a proliferation 
of papers in the last decade trying to link immunoassays to electrochemical means of detection. Amperometric and potentiometric methods have both been used with varying degrees of success. This review will limit itself to these methods. The differences between these two electrochemical techniques is clearly defined elsewhere in this book, (Kuan and Guilbault, Chapter 9; Wilson, Chapter 11) and it should suffice for the distinction to be made that amperometric assays measure current and a linear relationship exists between the current and the concentration of electroactive species that is either oxidized or reduced at the electrode; potentiometric assays measure the change in potential and a logarithmic relationship exists between potential and concentration with an idealized change in potential of $59 / n \mathrm{mV}$ per decade.

Current published electrochemical immunoassays can be further classified (see Fig. 4.1).

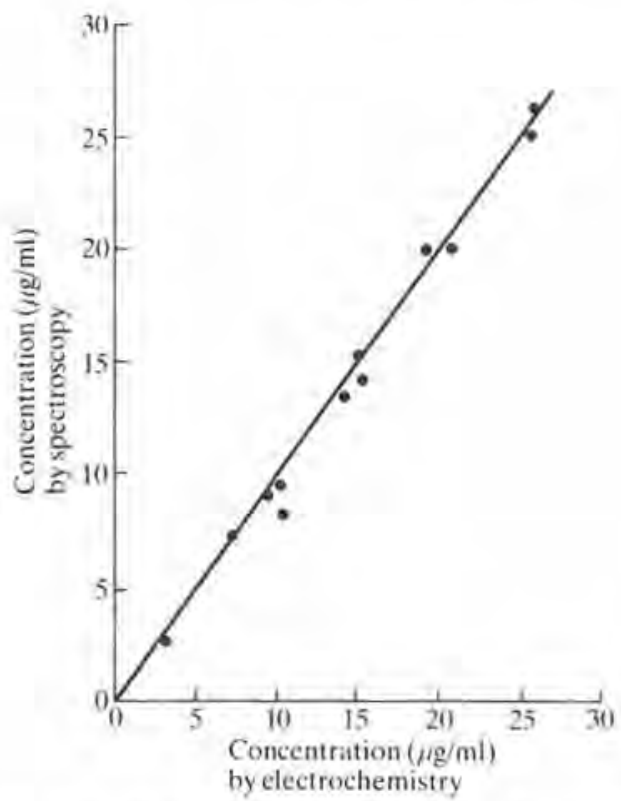

Fig. 4.1 Correlation plot of spectroscopic assay results against electrochemical results for serum samples from patients on phenytoin maintenance therapy (from Eggers et al, 1982).

a) Amperometric immunoassays developed around the Clark electrode. These employ enzymes that can either consume or produce oxygen in the presence of suitable substrates.

b) Amperometric enzyme immunoassays that employ both an enzyme 
and electrochemically detect products of that enzyme.

c) Amperometric immunoassays that do not employ enzymes but utilize either an antibody or antigen that is labelled with an electroactive species.

d) Potentiometric immunoassays that are based on a change in potential that occurs when either an antibody or antigen is immobilized on an electrode and its specific binding partner binds to it.

e) Potentiometric immunoassays where the method of detection is a more conventional potentiometric electrode, e.g. ion-selective electrode, $\mathrm{CO}_{2}$ electrode, ammonia electrode, or $\mathrm{pH}$ electrode.

Examples of all five types of immunoassays will be given below.

\subsection{Amperometric immunoassays based on the Clark electrode}

The use of the Clark oxygen electrode to detect either the loss or formation of oxygen as a consequence of an enzymic reaction was an interesting progression for enzyme immunoassays. The two most commonly used enzyme labels were glucose oxidase and catalase.

Aizawa et al. in 1979 constructed an enzyme-linked immunoassay to monitor human chorionic gonadotrophin (hCG) using catalase-labelled hCG. They immobilized an antibody on to a pre-cast cellulose membrane, the membrane was then placed over the Teflon membrane of the oxygen electrode.

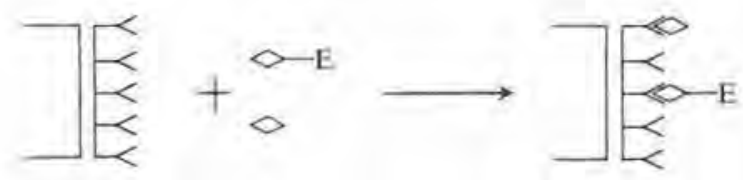

$$
2 \mathrm{H}_{2} \mathrm{O}_{2} \longrightarrow 2 \mathrm{H}_{2} \mathrm{O}+\mathrm{O}_{2}
$$

Both labelled and non-labelled hCG were allowed to compete for the antibody in the membrane. The membrane was washed to remove bound from free hCG - and the electrode exposed to hydrogen peroxide solution. The hydrogen peroxide solution in the presence of catalase disproportionates to yield oxygen and water. The rate of increase in oxygen tension is monitored, a calibration plot suggests that the sensor can monitor between 0.02 and 100 $\mathrm{IU} \mathrm{ml} \mathrm{m}^{-1}$ of hCG. Unfortunately using one antibody the assay was prone to cross reactivity due to luteinizing hormone. However the existence of good monoclonal antibodies against the $\alpha$ and $\beta$ subunits of hCG and the use of a sandwich-type ELISA assay - using, not labelled hCG but, labelled second antibody, could form the basis of an improved biosensor for hCG. Another Japanese group (Itagaki et al. 1983) have more recently used catalase labels in 
an amperometric assay for theophylline - the principles of this assay were similar to the assay described for hCG.

In comparison to the Japanese group Renneberg and co-workers (Renneberg et al. 1983) have concentrated on the consumption of oxygen rather than its production, using a less than simple enzyme linked immunoassay for Factor VIII-related antigen. They have adapted a glucose oxidase (GOD) electrode (Updike and Hicks 1967) to monitor glucose produced by an antibody labelled with alkaline phosphatase (AP)(eqns 4.5 and 4.6),

$$
\begin{aligned}
& \text { Glucose-6-phosphate }+\mathrm{H}_{2} \mathrm{O} \stackrel{\text { AP }}{\longrightarrow} \text { Glucose }+ \text { Phosphate } \\
& \text { Glucose }+\mathrm{O}_{2} \stackrel{\text { GOD }}{\longrightarrow} \text { Gluconolactone }+\mathrm{H}_{2} \mathrm{O}_{2}
\end{aligned}
$$

The assay appears somewhat cumbersome to perform and requires reasonably expensive reagents.

\subsection{Amperometric enzyme-linked immunoassays}

A more elegant use of alkaline phosphatase as a label has been demonstrated by Doyle et al. $(1982,1984)$. For their model antigen they used human serum orosomucoid which is a small glycoprotein (molecular weight 41000 daltons), implicated in various malignant conditions (Doyle et al. 1984) and believed to be related to carcinoembryonic antigen. This protein was labelled with alkaline phosphatase. A competitive reaction was allowed to occur between antibody against the orosomucoid protein immobilized on the surface of a cuvette and the enzyme-labelled protein - after a suitable time period the cuvette was washed and substrate solution was added.

$$
\text { phenylphosphate }+\mathrm{H}_{2} \mathrm{O} \stackrel{\mathrm{AP}}{\longrightarrow} \mathrm{H}_{3} \mathrm{PO}_{4}+\bigcirc-\mathrm{OH}
$$

The substrate for the enzyme, phenyl phosphate, is electrochemically inactive; the product, phenol, is electrochemically active and detected at a carbon-paste electrode by liquid chromatography electrochemistry. The disadvantage of the assay is that it is somewhat slow to perform, requiring an initial $12 \mathrm{~h}$ incubation period for the competitive assay to occur and a further hour for enough phenol to be generated to be detected. On the other hand the assay is extremely sensitive and capable of detecting $1 \mathrm{ng} \mathrm{ml}^{-1}$ of sample.

Some of the more commonly used enzyme-linked immunoassays employ antigens labelled with dehydrogenases and measure the formation of NADH spectrophotometrically. The direct electrochemical oxidation of NADH has been studied and Eggers et al. (1982) have monitored NADH formed electrochemically rather than spectroscopically. They adapted a commercially available kit to monitor phenytoin, a small drug, using flow-injection analysis. When great care was taken to protect against electrode fouling by serum 
proteins, good agreement with routine clinical laboratory procedures was obtained for serum samples (see Fig. 4.1).

\subsection{Amperometric immunoassays utilizing antigens labelled with electroactive species}

In 1979 two groups published papers on novel homogenous electrochemical immunoassays using antigens labelled with an electroactive species. In both cases the model antigen used was small. Weber and Purdy (1979) labelled morphine with ferrocene. They described their assay as a voltammetric immunoassay and monitored the oxidation of their ferrocene-morphine conjugate in the presence and absence of the antibody against morphine. This formed the basis of a homogenous assay for morphine. In the presence of the antibody the oxidation current due to the ferrocene-morphine conjugate is reduced. They were able to show that codeine was able to displace the morphine conjugate from the antibody and increase the oxidation current. Weber and Purdy worked at $+500 \mathrm{mV}$ vs. SCE and were therefore prone to oxidizing other species apart from their labelled drug; they did not have interference due to the reduction of oxygen. Heineman et al. (1979) demonstrated their homogenous immunoassay by labelling an antigen, oestriol, with mercuric acetate. A homogenous competitive immunoassay was performed. Separation of free estriol from estriol bound to the antibody was unnecessary as estriol is not electroactive in the potential range used. Furthermore, the reduction of antibody-bound labelled antigen occurs at a more negative potential to free labelled antigen. The reduction of the mercuric-labelled estriol occurs at $-300 \mathrm{mV} v s$. SCE and will be prone to oxygen interference if the samples are not carefully degassed. This may result in insensitivity at low concentrations of antigen. Heineman (Wehmeyer et al. 1982) continued using estriol as a model system rendering estriol electroactive by nitrating it. Estriol, when nitrated in the 2 and 4 positions, was electroactive with reduction waves at $-422 \mathrm{mV}$ and $-481 \mathrm{mV}$ against a silver/silver chloride reference electrode, and this compound was used in a homogenous immunoassay. These homogenous assays using small electroactive antigens can not be readily extended to larger proteins.

A heterogenous voltammetric immunoassay has been devised by Doyle et al. (1982) for larger molecules using human serum albumin modified by diethylenetriaminepentaacetic acid (DTPA) and labelled with indium $\left(\mathrm{In}^{3 *}\right)$ as a model. Indium may not appear to be an obvious metal to use. However it is not normally found in detectable levels in biological fluids so background levels would be low and it forms an extremely tight complex with DTPA $\left(K_{\mathrm{f}}=10^{29}\right)$. The assay is a competitive assay using a fixed amount of antibody.

The free and bound antigens are separated and the indium label is released 
from the DTPA complex by lowering the $\mathrm{pH}$. The metal is detected using anodic pulse stripping voltammetry. This method is potentially a very sensitive method.

\subsection{Potentiometric immunoassays}

A great deal of interest has been expressed in this area since Janata published his observations in 1975 . These were based on the premise that proteins in aqueous solutions were polyelectrolytes and hence, as an antibody is a protein, its electrical charge will be affected on binding an antigen. The potential difference between an electrode on to which antibody has been immobilized and a reference electrode will depend on the concentration of the free antigen, assuming that the antibody binding site is free to participate in the interaction with the antigen. Janata's model system used concanavalin A, a lectin, immobilized on the electrode surface. A potential change was observed on addition of a polysaccharide, unfortunately a similar response was observed using a platinum wire without concanavalin $\mathrm{A}$. The response of an ovalbumin electrode to its antibody was of the order of $2 \mathrm{mV}$ with reference to an electrode which contained immobilized serum proteins. Unfortunately these potentiometric electrodes are rather prone to nonspecific binding and little success has been reported in their use. A similar system was examined using a titanium wire on to which human chorionic gonadotrophin ( $\mathrm{hCG}$ ) or antibody against hCG were immobilized (Yamamoto et al. (1978). These also showed small $(<5 \mathrm{mV})$ changes in potential on addition of either the respective antibody or antigen. This lack of sensitivity in measuring a direct potential difference between the working electrode and the reference electrode compared with amperometric immunoassays using enzyme labels was well demonstrated by Aizawa et al. (1982) when they compared different immunoassays for human serum albumin. They used similar membranes containing antibodies against human serum albumin and found that as expected both membranes were equally selective. The use of the amperometric enzyme-linked immunoassay was far superior in sensitivity which is one of the prime issues in immunoassays.

\subsection{Potentiometric electrode-linked immunoassays}

Although the observations made on potentiometric immunoassays as originated by Janata have shown them to be insensitive, a considerable amount of work has been published by Rechnitz and co-workers linking immunoassays of conventional carbon dioxide, ammonia, and potassium (ion-selective) potentiometric electrodes. Earlier work involved the use of iodide and fluoride electrodes. In one example (Gebauer and Rechnitz 1982), the lack of sensitivity is alleviated by the use of enzyme labels using membrane 
electrodes. The antigens were labelled with asparginase, adenosine deaminase, or urease. Asparaginase appeared to be the best label to use as both urease and adenosine deaminase activity were affected by coupling to a hapten. An immunoassay was demonstrated for cortisol which could be used with diluted human blood or plasma. This assay was a heterogenous immunoassay using anti-rabbit IgG antibody bound to agarose beads to separate the free from the bound label,

Fortunately potentiometric enzyme-linked immunoassays need not all be heterogenous in nature. Homogenous assays are more convenient to use than heterogenous assays as they require less manipulation. Fonong and Rechnitz (1984) described a homogenous potentiometric enzyme immunoassay for human immunoglobulin $\mathrm{G}$ using a $\mathrm{CO}_{2}$ electrode. An enzyme-labelled conjugate of $\mathrm{IgG}$ is formed, and enzymatic activity is inhibited on binding antibody against IgG. The enzyme label used is slightly unusual, it is chloroperoxidase. This is a haem-containing enzyme that catalyses a number of halogenations of organic molecules in the presence of hydrogen peroxide and the suitable halide e.g. it will brominate $\beta$-ketoadipic acid in the presence of hydrogen peroxide and sodium bromide. It is the inhibition of the formation of $\mathrm{CO}_{2}$ that is exploited in this assay (eqn 4.8).

$$
\begin{aligned}
& \left.{ }^{-} \mathrm{OOCCH}_{2} \mathrm{CH}_{2} \mathrm{Cl}=\mathrm{O}\right) \mathrm{CH}_{2} \mathrm{COO}^{-}+\mathrm{Br}^{-}+\mathrm{H}_{2} \mathrm{O}_{2}+2 \mathrm{H}^{+} \longrightarrow \\
& \left.-\mathrm{OOCCH}_{2} \mathrm{CH}_{2} \mathrm{Cl}=\mathrm{O}\right) \mathrm{CH}_{2} \mathrm{Br}+2 \mathrm{H}_{2} \mathrm{O}+\mathrm{CO}_{2}
\end{aligned}
$$

The assay requires a 30 minute incubation period prior to addition of substrates and is sensitive to $\mathrm{IgG}$ in the $\mu \mathrm{g} \mathrm{ml}$ - range. This is considerably lower than the clinical range of $\mathrm{IgG}\left(8-14 \mathrm{mg} \mathrm{ml}^{-}\right)$and so samples would require dilution prior to assay, which would also reduce any matrix effects due to serum.

Ion-selective electrodes can also be adapted for use in immunoassays. Indeed a potentiometric ionophore modulation immunoassay (PIMIA) has been described by Keating and Rechnitz (1984) for the measurement of antibodies (see Fig. 4.2a). In this assay the corresponding antigen is coupled to an ionophore for potassium e.g. cis-dibenzo-18-crown-6 or benzo-15-crown-5. The ionophore antigen conjugate is then immobilized in a polyvinyl-chloride film containing a plasticizer and mounted on to a conventional ion-selective electrode. The electrode is then exposed to a constant concentration of potassium and a constant background potential is observed. Addition of antibody results in binding to the antigen portion of the conjugate present at

Fig. 4.2 (a) A pictorial representation of the potentiometric ionophore-modulation immunoassay

(a) PVC membrane containing digoxin-ionophore conjugate,

(b) $10 \mathrm{mM} \mathrm{KCl}$ solution.

(c) Plasticizer. 

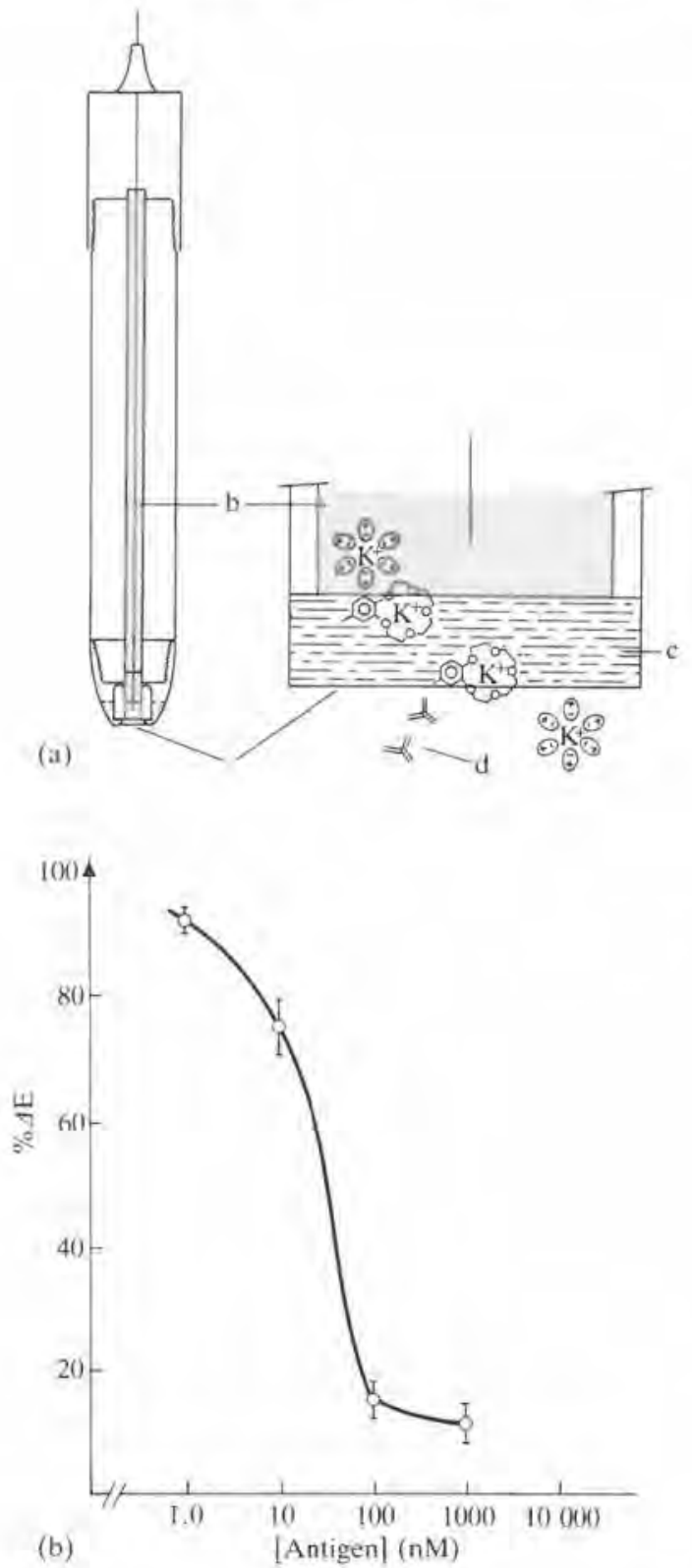

(d) Digoxin antibodies.

(b) Competitive binding curve for digoxin working at a constant antibody concentration (from Keating and Rechnitz 1984). 
the membrane solution interface and a potential change occurs which is proportional to the antibody concentration. This PIMIA technique has been used to detect antibodies against digoxin and in a conventional immunoassay to detect digoxin (see Fig. 4,2b). The main disadvantage of this assay is that the sample requires extensive dialysis to remove any interfering ions, as the electrode must operate at fixed potassium concentrations. This results in relatively large sample volumes ( $1-3 \mathrm{ml}$ sera) and lengthy dialysis resulting in a long wait before results are obtained.

An alternative assay for digoxin (Keating and Rechnitz 1985) utilizes the $\mathrm{CO}_{2}$ electrode. Polystyrene beads coated with digoxin are used and a competitive immunoassay is performed using antibodies against digoxin labelled with horse-radish peroxidase. The principle of the assay is described in Fig. 4.3a. The beads can be spun down and the enzyme-catalysed formation of $\mathrm{CO}_{2}$ from pyrogallol and hydrogen peroxide detected. In this heterogenous enzyme immunoassay there is no inhibition of enzyme activity and increasing the concentration of free digoxin will reduce the rate of $\mathrm{CO}_{2}$ production as monitored potentiometrically. The assay is sensitive to picomolar concentrations of digoxin (see Fig. 4.3b), unfortunately the assay is still slow.

Thus, in summary, the area of electrochemical immunoassays is still quite young. The most promising results in both amperometric and potentiometric assays appear to be in the assays which have been specifically designed for electrochemical detection and not in the adaptation of existing enzyme immunoassays to electrochemical detection. This is well illustrated in cases c and e (section 4.3 and 4.5) i.e. using antigens tagged with electroactive species or using enzyme-linked immunoassays and $\mathrm{CO}_{2}$ or $\mathrm{NH}_{3}$ electrodes. Although it is possible to demonstrate immunosensors in the laboratory environment there may be considerable difficulties in commercializing novel immunosensors.

\section{References}

Aizawa, M., Morioka, A., Suzuki, S. and Nagamura. Y. (1979). Enzyme immunosensor, III Amperometric determination of human chorionic gonadotropin by membrane bound antibody. Anal. Biochem. 94, 22-8.

- and Suzuki, S. (1982). Chemical amplification in biosensors. Jap. J. App. Phys. 21 Supplement 21-1,219-23.

Doyle, M.J., Halsall, H.B. and Heineman, W. R. (1982). Heterogenous immunoassay for serum proteins by differential pulse anodic stripping voltammetry. Anal. Chem. 54, 2318-22.

- (1984). Enzyme-linked immunoabsorbent assay with electrochemical detection for $a_{1}$-acid glycoprotein. Anal. Chem. 56, 2355-60.

Eggers, H. M., Halsall, H. B. and Heineman, W. R. (1982). Enzyme immunoassay with flow amperometric detection of NADH. Clin. Chem. 28, 1848-51. 
Digoxin-BSA coated bead

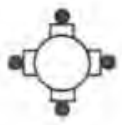

- Digoxin

HRP-labelled inti-digoxin

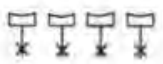

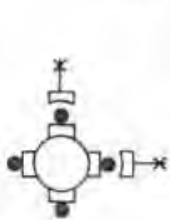

$\downarrow$

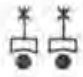

Centrifuge<smiles>CC(C)C</smiles>

Wash

它它

(a)

$\downarrow$ Substrate

$\mathrm{CO}$.

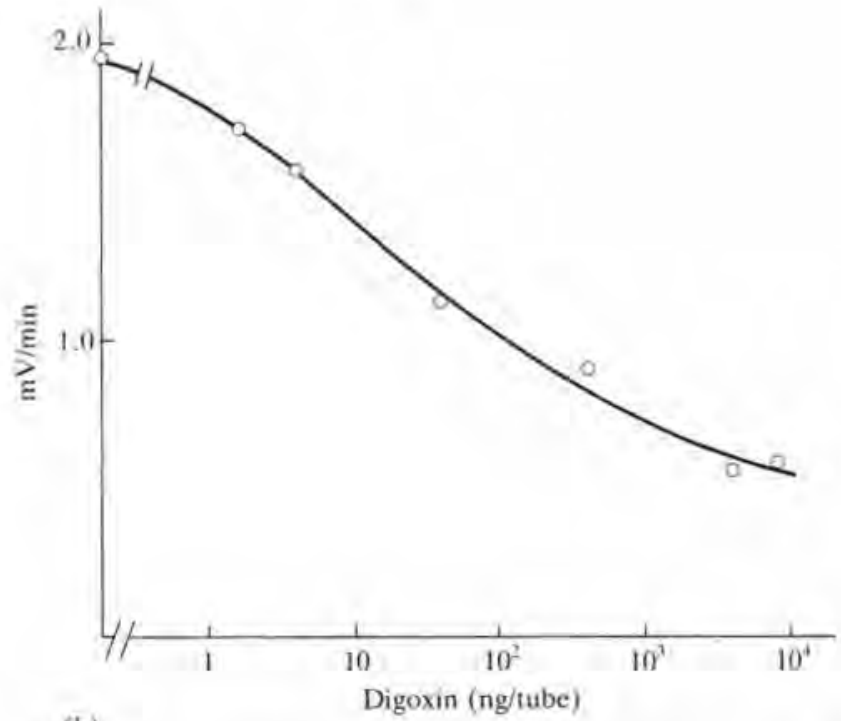

(b)

Fig. 4.3 (a) Schematic representation of the enzyme immunoassay. (b) Typical binding curve for the digoxin enzyme immunoassay (from Keating and Rechnitz 1985). 
Fonong, T. and Rechnitz, G. A. (1984). Homogeneous potentiometric enzyme immunoassay for human immunoglobulin G. Anal. Chem. 56, 2586-90.

Gebauer, C. R, and Rechnitz, G. A. (1982). Deaminating enzyme labels for enzyme immunoassays, Anal. Biochem, 124, 338-48.

Heineman, W. R., Anderson, C. W. and Halsall, B. H. (1979). Immunoassay by differential pulse polarography. Science 204, 865-6.

Itagaki, H. Hakoda, Y., Suzuki, Y, and Haga, M. (1983). Drug sensor; an enzyme immunoelectrode for theophylline. Chem. Pharm. Bull. 31 1283-8.

Janata, J. (1975). An immunoelectrode. JACS. 97, 2914-6.

Keating, M.Y. and Rechnitz, G.A. (1984). Potentiometric digoxin antibody measurements with antigen ionophore based membrane electrodes. Anal. Chem. $56,801-6$.

- (1985). Potentiometric enzyme immunoassay for digoxin using polystyrene beads. Anal. Letts. 18 (B1), 1-10.

Renneberg, R., Shlossler, W. and Scheller, F. (1983). Amperometric enzyme sensorBased enzyme immunoassay for factor VIII related antigen. Anal. Letts. 16, 1279-89.

Updike, S. J. and Hicks, G. P. (1967). Nature (London) 214, 986.

Weber, S. G. and Purdy, W. C. (1979). Homogenous voltammetric immunoassay: a preliminary study. Anal. Letts, 12, 1-9.

Wehmeyer, K. R., Halsall, H. B. and Heineman, W. R. (1982). Electrochemical investigation of hapten-antibody interactions by differential pulse polarography. Clin. Chem. 28, 1968-72.

Yamamoto, N., Nagasawa, Y., Sawai, M., Sudo, T. and Tsubomura, H. (1978). Potentiometric investigations of antigen-antibody and enzyme-enzyme inhibitor reactions using chemically modified metal electrodes. J. Immunol. Methods 22, 309-17.

Yalow, R. S. and Berson, S. A. (1960). Immunoassay of endogenous plasma insulin in man. J. Clin. Invest. 39, 1157-75.

(1959). Assay of plasma insulin in human subjects by immunological methods. Nature, London 184, 1648-9. 


\title{
5 \\ The diagnosis of human genetic diseases
}

\author{
JOHN M. OLD and KAY E. DAVIES
}

\subsection{Introduction}

Human genetic diseases are an important international health problem. For example, the World Health Organization have estimated that there are over 200 million carriers for the haemoglobinopathies and 200 to 300 thousand severely affected homozygotes or compound heterozygotes are born annually. In Caucasian populations, 1 in 20 individuals are carriers for cystic fibrosis (CF), a recessive disorder of the exocrine glands resulting in the death of affected individuals in their late teens or early twenties. In some genetic diseases where the defective protein is characterized (e.g. $\beta$-globin in $\beta$-thalassaemia), biochemical tests for the absence of the gene product can be carried out after fetal blood sampling. However, this approach is not generally applicable because a disease does not always result in the alteration of a protein in fetal blood. In these cases gene-specific probes are valuable because their use is independent of the cell type being investigated.

DNA recombinant technology permits the clinical phenotype to be related directly to changes in the DNA sequence of the corresponding gene and its surrounding controlling regions. For example, many different DNA base changes have been reported which result in $\beta$-thalassaemia (Orkin and Kazazian 1984), They affect various stages of gene expression: prevention or reduction in the transcription of the DNA into precursor mRNA, incorrect processing of the precursor mRNA to mature mRNA, or premature termination of protein translation. In sickle cell anaemia a $\beta$-globin gene product is produced but with a functionally important amino acid change resulting from a GAG to GTG change in the DNA sequence.

For most genetic disorders, the basic defect is not understood. For example, the molecular basis of the most common recessive condition, cystic fibrosis, remains unknown, Molecular biology is now being applied to the localization of the defect to chromosomal regions such that antenatal diagnosis and carrier exclusion can be performed before the identification of the precise mutation. DNA probes are used as markers for the inheritance of the chromosomal region carrying the mutation and thus do not depend on a detailed knowledge of the altered DNA sequence (Botstein et al. 1980). 


\subsection{Technology}

\subsubsection{DNA probes}

DNA is a double-stranded helical molecule which can be split into its two component complementary strands by treatment with alkali. Under the right experimental conditions, the two strands can be made to stick together again (reassociate by hydrogen bonding between the bases), a process which forms the basis of all DNA hybridization techniques. Such techniques involve a small amount of a pure DNA probe which is labelled and made single stranded so that it can seek out and hybridize to its complementary sequence in a larger amount of target DNA. The probes are usually labelled with radioactive nucleotides so that the resulting double-stranded hybrids can be identified amongst the much larger amount of unlabelled target DNA. Although non-radioactive methods of labelling probes are now being intensively developed, for example, labelling with biotin-dUTP (Leary et al. 1983), none of those methods have yet proved to be as sensitive as the conventional method of ${ }^{32} \mathrm{P}$-labelling.

The first DNA probes for hybridization experiments were made from mRNA molecules by the enzyme reverse transcriptase. This enzyme copies the mRNA molecule, synthesizing a single-stranded DNA molecule with a DNA sequence complementary to it, i.e. the sequence that can hybridize back to the mRNA sequence to form a double-stranded hybrid molecule. These copy DNA probes (cDNAs) were labelled by the inclusion of a radioactive nucleotide during the synthesis and used in simple DNA solution-hybridization experiments. Because reticulocytes are a good source of almost pure globin mRNA, the first genes to be studied in this way were the globin genes using partially purified $\alpha, \beta$, and $\gamma$-cDNA probes. Such experiments led to the discovery that $\alpha$-thalassaemia was due to a gene deletion and to the first prenatal diagnosis of a genetic disease by molecular biology when, in 1976, Kan et al. showed that a fetus affected by $\alpha$-thalassaemia could be diagnosed by a reduced hybridization of an $\alpha$-cDNA probe to amniocyte DNA.

Although much knowledge was gained by such solution hybridization experiments, cDNA probes were found to be too impure to be useful as probes for the next development in molecular biology, the DNA blotting technique introduced by Southern in 1975. This technique (described later) became one of the most important methods in molecular biology after developments in recombinant DNA technology permitted the isolation of pure globin cDNA probes. In 1978, Wilson et al, reported the first preparation of pure human $\alpha, \beta$, and $\gamma$-cDNA probes by inserting double-stranded cDNA molecules into plasmids and isolating the recombinant plasmids from single colonies of infected host bacteria.

The final development in plasmid probe technology followed the successful cloning of human DNA in bacteriophages. In 1978, Maniatis and his 
colleagues reported the first construction of a human gene library (Lawn et al. 1978) from which overlapping DNA fragments spanning the $\alpha$ and $\beta$ globin gene clusters were quickly isolated. Smaller DNA fragments containing individual globin genes were prepared from the cloned DNA and inserted into plasmids to make genomic DNA plasmid probes.

Genomic DNA probes are usually only part of a gene, containing both coding and non-coding sequences, whereas $\mathrm{CDNA}$ probes are complementary to the whole or most of the coding sequence of the gene. As the DNA sequence of most genes is very large (in contrast to the globin genes), cDNA probes generally hybridize to a larger region of DNA and thus to more fragments than genomic DNA probes. This can be very useful when searching for polymorphic restriction enzyme sites, but for the detection of genetic disorders and prenatal diagnosis, the presence of many bands on the autoradiograph can interfere with the interpretation of the result. Therefore genomic DNA probes are preferred and in some cases, very small genomic DNA fragments often have to be used. These are prepared by digesting the genomic DNA plasmid with restriction enzymes and isolating the required DNA fragment by gel electrophoresis and electroelution from the agarose.

The most recent development in probe technology is the development of short synthetic oligonucleotide probes. These are single-stranded DNA molecules of around 20 base pairs in length, chemically synthesized in pairs in which the sequence of one probe differs from the other by only one nucleotide. One of the probes is complementary to the normal DNA sequence and the other to the same DNA sequence containing a point mutation. Such a pair of probes will hybridize differentially to normal and mutant genomic DNA and therefore the presence and absence of hybridization can be used as a diagnostic method to detect genetic disorders resulting from point mutations, such as $\beta$-thalassaemia (Orkin et al. 1983).

\subsubsection{Labelling of probes}

5.2.2.1 Nick translation Nick translation is the most widely used method for labelling either plasmid probes or DNA fragments. This process depends on the enzyme Escherichia coli DNA polymerase 1, which is a DNA repair enzyme, and the enzyme DNAase 1 which introduces nicks into the doublestranded plasmid DNA. Once a nick is introduced, the DNA polymerase 1 removes the existing nucleotide in front of the nick and adds a new deoxyribonucleoside $5^{\prime}$-triphosphate to the free $3^{\prime}-\mathrm{OH}$ terminus on the other side. One radioactive nucleotide (usually $\mathrm{dCTP}$ ) is added to the reaction mixture which contains the enzymes, buffers, plasmid (50-100 $\mu \mathrm{g})$ and cold dATP, dGTP, and dTTP. One can make up these components, following the procedure first described by Rigby et al. (1977), or use one of the commercially available nick-translation kits. The reaction is carried out at $16{ }^{\circ} \mathrm{C}$ for one hour in the presence of $50 \mu \mathrm{l} \alpha^{32} \mathrm{P}-\mathrm{dCTP}, 3000 \mathrm{Ci} / \mathrm{mmol}$ (PB 
10205, Amersham International) after which the reaction is stopped by the addition of EDTA and then the labelled DNA probe is separated from the unincorporated dCTP by either passing down a Sephadex G-100 column in a $1 \mathrm{ml}$ disposable pipette or by the method of centrifugation through Sephadex. The yield is usually measured by counting an aliquot in a scintillation counter and then the probe is boiled for five minutes to make it singlestranded and quickly cooled or frozen if it is not immediately required for hybridization.

\subsubsection{Hexanucleotide priming A recently introduced alternative method} of labelling DNA fragments to a high specific activity is to use a hexanucleotide primer kit (Feinberg and Vogelstein 1983). The advantage of this method over nick translation is that the DNA fragment does not have to be isolated from the gel matrix after gel electrophoresis. The plasmid probe is digested to produce the required DNA fragment and electrophoresed in a low-melting-point agarose gel. The DNA fragment is visualized in the usual way by staining with ethidium bromide, cut out of the gel and boiled for 5 minutes to melt the agarose and make the fragment single-stranded. A mixture of hexanucleotide primers is added, together with $\left[\alpha-{ }^{32} \mathrm{P}\right] \mathrm{dCTP}$ (3000 $\mathrm{Ci} /$ mole), cold dGTP, dATP, and dTTP the enzyme Klenow fragment. The primers hybridize to the fragment to form double-stranded templates which are extended by the Klenow fragment enzyme to make a double-stranded labelled probe. The probe is recovered in the same way as for the nick-translation reaction.

5.2.2.3 End labelling Oligonucleotide probes are labelled differently to plasmid probes and are required at a higher specific activity. The single stranded probes are labelled at their 5 ' ends with ${ }^{32} \mathrm{P}$ by phosphorylation with polynucleotide kinase in the presence of $\left[\alpha^{-32} \mathrm{P}\right] \mathrm{ATP}$. The labelled oligonucleotides are separated from both unlabelled probe and free [ $\left.\alpha-{ }^{32} \mathrm{P}\right] \mathrm{ATP}$ by either homochromotography on thin layer diethylaminoethyl cellulose plates or by polyacrylamide gel electrophoresis (Conner et al. 1983).

\subsubsection{Restriction enzyme analysis}

This technique was introduced in 1975 by Southern and therefore is widely referred to as the 'Southern' blotting method. There have been many modifications and improvements of the original method and therefore there are now many variations of the basic technique. The method used in our laboratory for the detection of the haemoglobinopathies has been described in extensive detail elsewhere (Old and Higgs 1983) and only a simple outline of the procedure is presented here.

The first step is to cleave a sample of genomic DNA with a restriction endonuclease. About 90 restriction endonucleases have been discovered to 
date, all of which cut DNA at DNA sequence-specific sites generating fragments of reproducible size. The digested DNA sample (usually 5 or $10 \mu \mathrm{g}$ ) is then loaded into a well of an $0.8 \%$ agarose gel and subjected to electrophoresis in a horizontal gel apparatus. DNA fragments are negatively charged and migrate towards the anode according to their size, the smallest fragments travelling the furthest. The gel is soaked in ethidium bromide solution so that the DNA can be visualized and photographed under ultraviolet light and then soaked in alkali to make the DNA fragments single-stranded. After neutralization in strong buffer, the gel is set up for the transfer of the DNA fragments out of the gel onto a nitrocellulose or nylon filter. This is achieved by laying the filter on top of the gel and covering the filter with dry paper towels which blot the fragments out of the gel in the same positions to which they had migrated. The fragments are fixed permanently to the filter by baking (if nitrocellulose filters are used) and then the filter (Southern blot) is ready for hybridization to a radioactively labelled DNA probe.

The hybridization reaction is usually carried out in a polythene bag, made by sandwiching the filter between two sheets of thick polythene and heatsealing three sides. the fourth side is heat-sealed after a few millilitres of presoak buffer have been added and the filter is then incubated at the temperature of hybridization for a short period, a step which is necessary to reduce the background hybridization signal to a minimum. After presoaking, the buffer is squeezed out of the bag, 1-2 $\mathrm{ml}$ of hybridization buffer plus the single-stranded ${ }^{32} \mathrm{P}$ labelled probe added, and the bag resealed. When the hybridization reaction is complete, the filter is removed from the bag and washed under stringent conditions $\left(0.1 \times \mathrm{SSC}, 0.1 \% \mathrm{SDS}\right.$ at $\left.65^{\circ} \mathrm{C}\right)$ to remove all the unhybridized probe. The filter is then finally subjected to autoradiography to localize the complementary DNA fragments which have hybridized to the probe. These appear as dark bands on the developed X-ray film. By running marker DNA fragments of known molecular weight, the sizes of the hybridizing fragments can be estimated by the distance they have travelled in the agarose gel.

\subsection{DNA probes in detecting human genetic disease}

\subsubsection{Carrier detection in genetic disease}

The haemoglobinopathies are the most extensively studied group of genetic disorders and many methods of carrier detection based on the technique of restriction enzyme have been developed. The different approaches can be divided into those that detect the genetic disorder directly and the indirect approach of using linked DNA polymorphisms. The direct approaches usually require the isolation of gene-specific probes and the determination of the molecular basis of the genetic disorder. Even then, in many cases, a direct approach may not be possible and an indirect method has to be used. For 
genetic disorders such as Duchenne muscular dystrophy, where the mutant gene is, as yet, unidentified, the indirect method is the only approach for carrier detection and prenatal diagnosis (Harper et al. 1983; Pembrey et al. 1984).

5.3.1.1 Direct detection If a genetic disorder results from a deletion of DNA sequence, genomic DNA probes or CDNA probes can be used to detect the defect directly. If a large deletion is involved, removing all the DNA sequence complementary to the appropriate DNA probe, the probe will fail to hybridize to DNA from an affected individual on a Southern blot. For example, $\alpha^{\circ}$-thalassaemia results from DNA deletions which remove both of the $\alpha$-globin genes and the homozygous condition can be diagnosed by the absence of any $\alpha$-globin gene fragments hybridizing to an $\alpha$-gene probe. However the detection of heterozygotes for such gene deletions is not so straightforward as the probe will hybridize to DNA fragments from the normal chromosome giving an identical band pattern to that from normal DNA, except that the intensity of the bands on the autoradiograph will be only a half that from normal DNA (provided that equal amounts of normal and heterozygous DNA are compared). However, detecting dosage differences on a Southern blot is often technically difficult. For genetic disorders located on the $\mathrm{X}$ chromosome, the absence of hybridization of a specific probe can be used to detect males affected by a gene deletion (Old et al. 1984) but again, the detection of females carrying the disorder depends on detecting only half the hybridization signal generated by an equal amount of normal DNA.

For small gene deletions, or in cases where the DNA probe sequence spans the start or end of the gene deletion, the disorder can be detected by the identification of a characteristic abnormal DNA fragment on a Southern blot. An individual carrying the disorder will have both normal and abnormal bands and an affected individual only abnormal bands. $\alpha^{\circ}$-Thalassaemia can be detected this way by using a $\zeta$-globin gene probe which hybridizes to DNA sequences adjacent to the start of the DNA deletion. Small deletions involving only a few hundred base pairs can be detected by the presence of an abnormal band which is smaller than the normal restriction fragment. A type of $\beta^{\circ}$-thalassaemia found only in Asian Indians can be detected in this manner (Fig. 5.1).

Genetic disorders which result from DNA deletions or insertions of just a few nucleotides cannot be detected by the above method because the resolution between bands on a Southern blot is not good enough. Also many genetic disorders are caused by point mutations which often do not cause any change of length of restriction fragments. However, if the mutation either creates or abolishes a restriction enzyme recognition site, the mutation will be detectable directly by restriction enzyme analysis, provided that the required DNA 


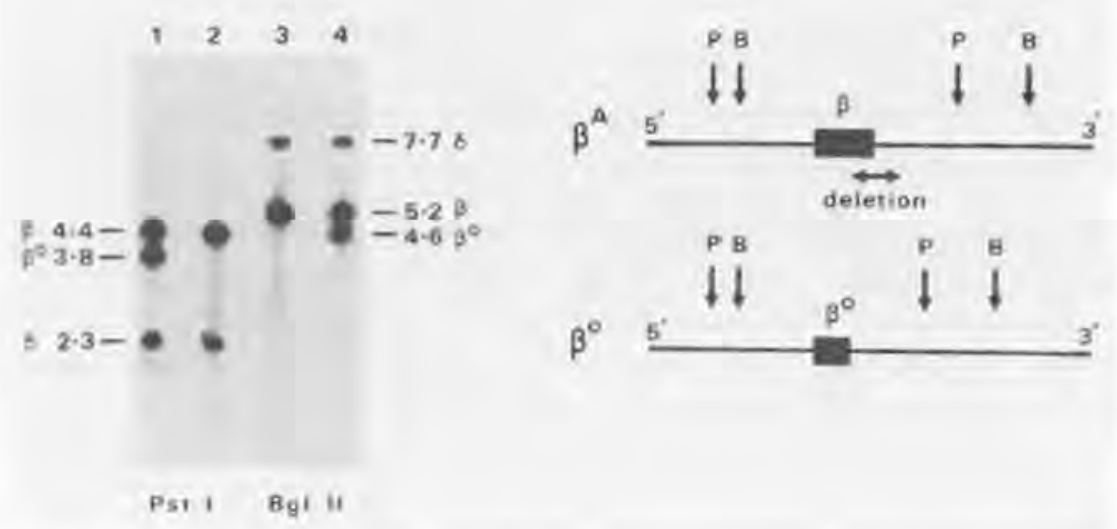

Fig. 5.1 The direct detection of a type of $\beta^{\circ}$-thalassaemia which is caused by a deletion of 617 base pairs at the $3^{\prime}$ end of the $\beta$-globin gene. The $\beta^{\circ}$ gene can be detected directly by using Pst I (P) or Bgl II (B), which have recognition sites on either side of the normal and mutant $\beta$ genes, and therefore the $\beta^{\circ}$ gene DNA fragments are $0.6 \mathrm{~kb}$ smaller than the normal ones. The autoradiograph shows normal DNA (tracks 2 and 3) and DNA from an individual heterozygous for this type of $\beta$-thalassaemia (tracks 1 and 4 ). DNA fragments containing cross-hybridizing $\delta$-globin gene fragments are also observed.

fragments are sufficiently large enough to be detected on a Southern blot (fragments smaller than 500 base pairs are not so easily detected). Sickle cell anaemia is a good example of such a genetic disorder. It results from a single base change of A to T in codon 6 of the $\beta$-globin gene and this mutation abolishes three slightly different recognition sites present in the normal $\beta$-gene sequence. The first two to be discovered, Mnl I and Dde I, produced DNA fragments too small to be detected easily and therefore were not used in preference to the previously established method of linkage analysis using the nearby Hpa I polymorphic site (see later). However the third enzyme site to be discovered at the sickle cell mutation locus, Mst II, generated easily detectable DNA fragments and this approach is now the standard method of prenatal diagnosis even though the sickle point mutation has been shown to be directly detectable by yet another method, the oligonucleotide probe technique.

Oligonucleotide probes are synthetic single-stranded DNA molecules of approximately 20 bases in length which can be used to detect single point mutations. A pair of such oligonucleotide probes are required, one complementary to the normal DNA sequence and the other to the mutant sequence. The probes are hybridized to restriction enzyme digested DNA, 
either on a Southern blot or in a dried-down agarose gel, and then the filter or gel washed under such conditions that the mismatched DNA hybrids are destabilized in relation to the perfectly matched hybrids and thus removed from the filter. This approach has been used to detect sickle cell anaemia, $\beta$-thalassaemia, and $\alpha-1$ antitrypsin deficiency (Conner et al. 1983; Orkin et al. 1983; Kidd et al. 1983). Although $\beta$-thalassaemia has been shown to be caused by at least thirty different point mutations, only a few mutations are found in any particular population and in some populations, such as in Sardinia, one mutation accounts for the vast majority of cases of $\beta$-thalassaemia and such a population has proved very suitable for the use of oligonucleotide probes for prenatal diagnosis of $\beta$-thalassaemia. However for genetic disorders with a high frequency of new mutations such as haemophilia and Duchenne muscular dystrophy, the oligonucleotide approach is not suitable and an indirect approach using linked DNA polymorphisms has to be used.

5.3.1.2 Indirect detection The discovery that natural variations in DNA sequence occur randomly throughout the genome has led to the most applicable method for the carrier detection of genetic disorders. This approach depends on demonstrating linkage of a DNA polymorphism to the mutant gene under study. DNA polymorphisms occur because variations in DNA sequence result in the loss of an existing restriction enzyme recognition site or the creation of a new one. A polymorphic restriction site will produce DNA fragments of different lengths in different people depending on whether the site is present or absent. The polymorphic fragments are inherited in a simple Mendelian fashion and can be used as markers for chromosomes carrying either normal or mutant genes, provided that the polymorphism is sufficiently close to the mutant gene so that the chance of DNA recombination between the polymorphic site and the mutant gene is very low (Kan and Dozy 1978; Botstein et al. 1980). Such DNA polymorphisms are referred to as restriction fragment length polymorphisms (RFLPs), and an example is shown in Fig. 5.2.

More than 17 different polymorphic restriction sites have been discovered in the $\beta$-globin gene cluster since the first report by Kan and Dozy in 1978 of a Hpa I polymorphic site and its usefulness for diagnosing sickle cell anaemia by linkage analysis. Many of these polymorphic sites have proved very useful for the detection and prenatal diagnosis of $\beta$-thalassaemia, $\mathrm{HbS}, \mathrm{HbE}$, and $\mathrm{HbC}$, even though such studies require the investigation of DNA from not just the couple at risk but also from previously born children and/or grandparents and lateral relatives in order to assign the linkage of RFLPs (as the majority of RFLPs do not exist in linkage disequilibrium). Many other genetic disorders can now be diagnosed by using linked RFLPs, even in cases where the biochemical defect is unknown such as Duchenne muscular 
12

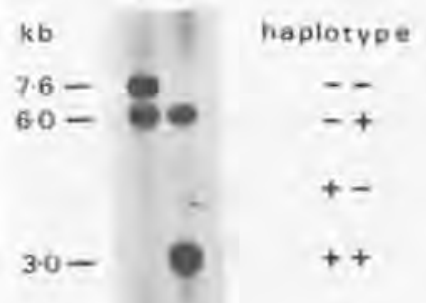

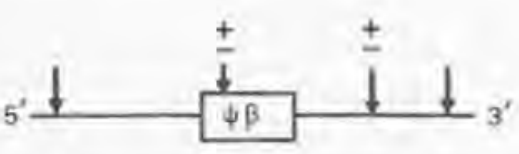

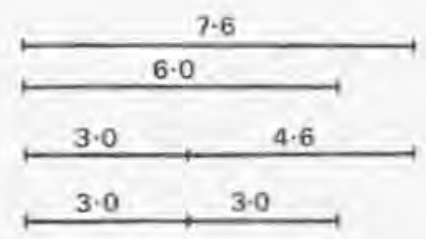

Sample 1 is $--1-+$

Sample 2 is $-+1++$

Fig. 5.2 A restriction fragment length polymorphism used for diagnosing $\beta$ thalassaemia by linkage analysis. The diagram shows the location of two Hind II polymorphic sites $( \pm)$ in and near the $\beta$-globin pseudogene $(\psi \beta)$. Four polymorphic fragments can be detected with a $\psi \beta$-gene probe, depending on the combination of the presence of absence of each site (haplotype). The haplotypes of two normal individuals are shown.

dystrophy (for a review, see Davies (1986)). A list of genetic disorders for which DNA probes are available is shown in Table 5.1.

\subsubsection{Prenatal diagnosis}

5.3.2.1 Source of fetal DNA Fetal DNA for DNA analysis can be obtained by either amniocentesis or chorionic villus sampling. Amniocentesis is a well established procedure which is usually carried out at 15-16 weeks' gestation. As soon as the techniques for the detection of haemoglobinopathies by restriction enzyme analysis were developed in 1978, prenatal diagnosis was shown to be possible using fetal DNA from cultured amniocytes. A large amount of fetal DNA $(20-45 \mu \mathrm{g})$ is obtained from a flask of confluent amniocytes but it takes 2-3 weeks for the cells to grow to confluency. Alternatively DNA can be prepared from amniotic fluid cells without culturing, but a large amount of amniotic fluid is required to obtain enough live cells to provide sufficient DNA for a diagnosis (5 or $10 \mu \mathrm{g}$ ). This is obtained from $40 \mathrm{ml}$ of amniotic fluid, which has been found by the authors to yield from $3 \mu \mathrm{g}$ to $25 \mu \mathrm{g}$ DNA, with an average yield of $14 \mu \mathrm{g}$.

Fetal DNA can also be obtained from a chorionic villi sample which is obtained at 9-11 weeks' gestation and permits a first trimester diagnosis. Such a diagnosis has many major advantages over the midtrimester approach 
Table 5.1 Diagnosis of disease using DNA probes

\begin{tabular}{|c|c|}
\hline Disease & Gene probe \\
\hline Haemoglobinopathies & $\alpha, \beta, \gamma$-Globin (Old 1986a) \\
\hline Collagen disorders & $\begin{array}{c}\text { Collagen (Prockop and Kivirikko 1984) } \\
\text { (Sykes 1983) }\end{array}$ \\
\hline Dwarfism & $\begin{array}{l}\text { Human growth hormone (Moore et al, } \\
\text { 1982) }\end{array}$ \\
\hline Emphysema & $\alpha-1$ Antitrypsin (Kidd et al. 1983) \\
\hline Lesch-Nyhan syndrome & HPRT (Yang et al, 1984) \\
\hline Phenylketonuria & $\begin{array}{l}\text { Phenylalanine hydroxylase (Woo et al. } \\
\text { 1983) }\end{array}$ \\
\hline Christmas disease & Factor IX (Choo et al. 1982) \\
\hline Haemophilia A & Factor VIII \\
\hline $\begin{array}{l}\text { Glucose-6-phosphate } \\
\text { dehydrogenase deficiency }\end{array}$ & G-6PD (Persico et al. 1981) \\
\hline $\begin{array}{l}\text { Ornithine transcarbamylase } \\
\text { deficiency }\end{array}$ & OTC (Old et al, 1984) \\
\hline Antithrombin-3 deficiency & Antithrombin-3 (Bock and Levitan 1983) \\
\hline $\begin{array}{l}\text { Cholesterol metabolism/ } \\
\text { heart disease }\end{array}$ & $\begin{array}{l}\text { LDL receptor (Russell et al. 1983), } \\
\text { HMG co-reductase (Chin et al. 1982), } \\
\text { Apolipoproteins (Tolleshaug et al. 1983) }\end{array}$ \\
\hline Duchenne muscular dystrophy & Linked RFLPs (Bakker et al. 1985) \\
\hline Huntington's chorea & Linked RFLP (Gusella et al. 1983) \\
\hline Cystic fibrosis & Linked RFLPs \\
\hline
\end{tabular}

using amniotic fluid DNA, but the risks to the fetus from the chorionic villus sampling procedure have not yet been fully evaluated. Chorionic villi are a superb source of DNA and an average-sized sample will yield between 25-40 $\mu \mathrm{g}$ of DNA, more than enough DNA for a prenatal diagnosis and therefore allowing in many cases a second and third restriction enzyme site polymorphism to be studied to confirm the diagnosis.

5.3.2.2 A practical example A first trimester diagnosis of $\beta$-thalassaemia by linkage analysis of a restriction fragment length polymorphism is shown in Fig. 5.3. DNA from the father, mother, and their normal child was analysed first to determine if a prenatal diagnosis was possible using RFLPs. A complete diagnosis was shown to be feasible using a polymorphic Ava II site in the $\beta$-globin pseudogene $(\psi \beta)$ approximately 15 kilobases $(\mathrm{kb})$ from the $\beta$-thalassaemia mutation in the $\beta$-globin gene, and a prenatal diagnosis was subsequently performed using DNA from a chorionic villus sample.

Figure 5.3 shows the band pattern obtained by digestion of DNA with Ava II and hybridization of the Southern blot to a ${ }^{32} \mathrm{P}$ labelled plasmid containing the human $\beta$-globin DNA sequence. If the Ava II site is present $(+)$ on both 


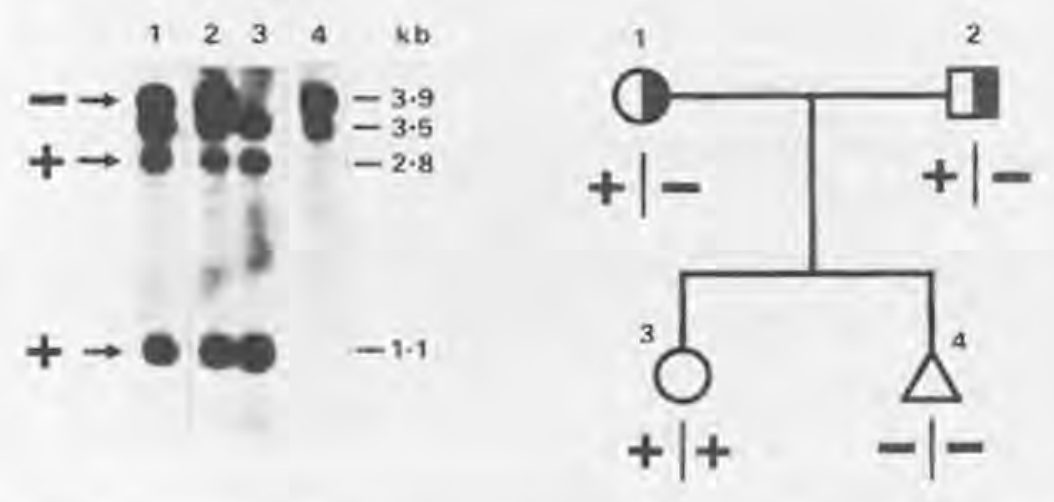

Fig. 5.3 First trimester diagnosis of $\beta$-thalassaemia by linkage analysis using an Ava II polymorphic site in the $\beta$-globin pseudogene. The autoradiograph shows that in tracks 1 and 2 the mother and father, both heterozygous for $\beta$-thalassaemia (halfshaded symbols) were $+f-$; in track 3 the normal child (open circle) was $+1+$; in track 4 the fetus (triangle) was $-/-$, and therefore diagnosed as homozygous for $\beta$ thalassaemia.

chromosomes, three fragments containing $\beta$-globin gene sequences are detected; with sizes of $1.1 \mathrm{~kb}, 2.8 \mathrm{~kb}$, and $3.5 \mathrm{~kb}$. If the site is missing (-) on both chromosomes, only two fragments are observed, a $3.5 \mathrm{~kb}$ and a $3.9 \mathrm{~kb}$ fragment. Therefore the $3.5 \mathrm{~kb}$ is a constant band and the $3.9 \mathrm{~kb}$ fragment results from the sum of the two smaller fragments. An individual heterozygous for the polymorphic site has all four bands, as seen in the DNA from the father and mother in tracks 1 and 2. DNA from the normal child in track 3 contained only the $1.1 \mathrm{~kb}, 2.8 \mathrm{~kb}$, and $3.5 \mathrm{~kb}$ fragments indicating that the polymorphic site was present $(+)$ on both of the normal chromosomes in the child and therefore in each of the parents (assuming that DNA recombination has not taken place between the polymorphic site and the $\beta$ globin gene). Thus the polymorphic site is absent on both the chromosomes carrying the $\beta$-thalassaemia gene in this family and can be used as a marker for prenatal diagnosis. DNA from a chorionic villus sample was than analysed (Old 1986b) and found to contain only the $3.5 \mathrm{~kb}$ and $3.9 \mathrm{~kb}$ bands (track 4). Therefore both fetal chromosomes carried the (-) RFLP indicating that it had inherited both $\beta$-thalassaemia genes and was affected.

\subsection{Future prospects for non-radiometric detection}

Clearly the method of choice at the monent for labelling DNA probes is to use ${ }^{32} \mathrm{P}$-nucleotides of the highest specific activity. There are many disadvantages to ${ }^{32} \mathrm{P}$-labelling such as the potential health hazard of radioactivity, but 
perhaps the most important disadvantage is that the half-life of ${ }^{32} \mathrm{P}$ is only 14 days and therefore probes have to be labelled fresh every one to two weeks. The use of non-radioactive methods of labelling DNA probes will overcome many of these disadvantages and should permit the development of diagnostic kits for detection of DNA sequences of abnormal genes, viruses, micro-organisms, etc.

Although many different methods of non-radioactive DNA labelling are being developed, two approaches have already met with limited success and are being marketed at the time of writing. The first approach is to substitute the incorporation of ${ }^{32} \mathrm{P}$-nucleotide into the DNA probe with a chemicallymodified nucleotide such as a biotinylated dUTP, an analogue of dTTP. The modified nucleotides are incorporated in the standard way e.g. by nick-translation but at a slower rate. The hybridized biotinylated DNA probe is then detected by its interaction with biotin-binding proteins such as avidin or streptavidin complexed with colour-producing enzymes such as horse-radish peroxidase, acid phosphatase, or alkaline phosphatase or by a double antibody system using an anti-biotin antibody followed by a fluorescent secondary antibody. The second approach is to chemically modify the DNA probe in a way which does not affect its hybridization properties, such as by sulphonation. The modified DNA probe is then detected with a specific monoclonal antibody and then by a secondary antibody conjugated to peroxidase or alkaline phosphatase. Kits for DNA labelling by the two approaches are on the market (Enzo Biochem Inc. and Orgenics Ltd.) but the sensitivity of these systems is still not quite sufficient for restriction enzyme analysis of DNA in which the detection of less than $1 \mathrm{pg}$ of single copy gene sequences is the goal. However such systems will detect 5-10 pg of DNA by dot blotting and therefore can be used in genetic engineering experiments for Southern blotting, plaque and colony hybridization for detection of viral DNA in cells and in tissue sections of clinical samples, and for in situ hybridization of DNA probes to chromosomes.

\section{Acknowledgements}

We would like to thank Rachel Kitt for patiently typing the manuscript. We are grateful to The Medical Research Council, The Muscular Dystrophy Group of Great Britain, and The Muscular Dystrophy Association of America for financial support.

\section{References}

Bakker, E., Hofker, M. H., Goorl, N., Mandel, J. L., Davies, K. E., Kunkel, L. M., Willard, H. F., Fenton, W. A., Sandkuyl, L., Majoor-Krakauer, D., Van Essen, A., Jahoda, M., Sachs, E. S., Van Ommen, G. J. B, and Pearson, P. L. (1985). 
Prenatal diagnosis and carrier detection of Duchenne muscular dystrophy with closely linked RFLPs. Lancet 1, 655-8.

Bock, S. C. and Levitan, D. J. (1983). Characterisation of an unusual DNA length polymorphism 5 ' to the human antithrombin III gene. Nucl. Acids Res. 11, 8569-82.

Botstein, D., White, R. L., Scolnick, M. H. and Davis, R. W. (1980). Construction of a genetic linkage map in man using restriction fragment length polymorphisms. Am. J. Hum. Genet. 32, 314-31.

Chin, D. J., Luskey, K. L., Faust, J.R., MacDonald, R. J., Brown, M.S. and Goldstein, J.L. (1982). Molecular cloning of a 3-hydroxyl-methylglutamyl coenzyme A reductase and evidence for regulation of its mRNA. Proc. Natl. Acad. Sci, USA 79, 7704-8.

Choo, K. H., Gould, K. G., Rees, D. J. G, and Brownlee, G. G, (1982). Molecular cloning of the gene for human anti-haemophilic factor IX. Nature 299, 178-80.

Conner, B. J., Reyes, A. A., Morin, C., Itakura, K., Teplitz, R. L. and Wallace, R, B. (1983). Detection of sickle cell $\beta^{5}$-globin allele by hybridization with synthetic oligonucleotides. Proc. Natl. Acad. Sci. USA 80, 278-82.

Davies, K. E. (1985). Molecular genetics of the human X chromosome, J. Med. Genet. 22, 243-9.

Feinberg, A. P. and Vogelstein, B. (1983). A technique for radiolabelling DNA restriction endonuclease fragments to high specific activity. Anal. Biochem. 132, 6-13.

Gusella, J.F., Wexler, M. S., Conneally, P. M., Naylor, S. L., Anderson, M.A., Tanzi, R.E., Watkins, P.C., Ottina, K., Wallace, M.R., Sakaguchi, A.Y., Young, A. B., Shoulson, I., Bonilla, E. and Martin, J. B. (1983). A polymorphic DNA marker genetically linked to Huntington's disease. Nature 306, 234-9.

Harper, P.S., O'Brien, T., Murray, J.M., Davies, K.E., Pearson, P. L. and Williamson, R. (1983). The use of linked DNA polymorphisms for genotype prediction in families with Duchenne muscular dystrophy. J. Med. Genet. 20, 252-4.

Kan, Y. W. and Dozy, A. M. (1978). Polymorphism of DNA sequence adjacent to human $\beta$-globin structural gene: Relationship to sickle mutation. Proc. Natl, Acad. Sci. USA 75, 5631-5.

- Golbus, M. S. and Dozy, A. M. (1976). Prenatal diagnosis of $\alpha$-thalassaemia: Clinical application of molecular hybridization. N. Engl. J. Med, 295, 1165-7.

Kidd, V J., Wallace. R. B., Itakura, K. and Woo, S. L. C. (1983). $\alpha_{1}$-antitrypsin deficiency detection by direct analysis of the mutation in the gene. Nature 304, 230-4.

Lawn, R. M., Fritsch, E. F., Parker, R. C., Blake, G. and Maniatis, T. (1978). The isolation and characterization of linked $\delta$ and $\beta$-globin genes from a cloned library of human DNA. Cell 15, 1157-74.

Leary, J. J., Brigati, D. J. and Ward, D. C. (1983). Rapid and sensitive colorimetric method for visualising biotin-labelled DNA probes hybridised to DNA or RNA immobilised on nitrocellulose: bio-blots. Proc. Natl. Acad. Sci. USA 80, 4045-9.

Moore, D. D., Conkling, M.E. and Goodman, H.M. (1982). Human growth hormone: multigene family. Cell 29, 285-286,

Old. J. M. (1986a). Prenatal diagnosis of the haemoglobinopathies. In Genetic disorders of the fetus (2nd edn., ed. A. Milunksy). pp. 599-624. Plenum, New York. (1986b). Fetal DNA analysis. In Genetic analysis of human diseases: a practical 
approach (ed. K, E. Davies). IRL Press, Oxford.

- and Higgs, D. R. (1983). Gene analysis. Methods in hematology, In The Thalassaemias (ed. D.J, Weatherall), Vol. 6, pp. 74-102. Churchill Livingstone, Edinburgh.

— Briand, P. L., Purvis-Smith, S., Howard, N. J., Wilcken, B., Hammond, J., Pearson, P., Cathelineau, L., Williamson, R. and Davies, K. E., (1984). Prenatal diagnosis of OTC deficiency by direct gene analysis. Lancet 1, 73-5.

Orkin, S. H. and Kazazian, H. H. (1984). The mutation and polymorphism of the human $\beta$-globin gene and its surrounding DNA. Ann. Rev. Genet. 18, 131-71.

- Markham, A. F, and Kazazian, H. H. (1983). Direct detection of the common Mediterranean $\beta$-thalassaemia gene with synthetic DNA probes: an alternative approach for prenatal diagnosis, J. Clin. Invest. 71, 775-9.

Pembrey, M. E, Davies, K. E., Winter, R. M., Elles, R. G., Williamson, R., Fazzoni, T. A. and Walker, C. (1984). The clinical use of DNA markers linked to the gene for Duchenne muscular dystrophy. Arch. Dis. Child. 59, 208-16.

Persico, M. G., Toniolo, C., Nobile, C., D'Urso, M. and Luzzatto, L. (1981). cDNA sequences of human glucose 6-phosphate dehydrogenase cloned in pBR322. Nature 294, 778-80.

Prockop, D. J, and Kivirikko, K. I. (1984). Heritable diseases of collagen. N. Engl. J. Med. 311, 376-86.

Rigby, P.J. W., Dieckmann, M., Rhodes, C, and Berg, P. (1977). Labelling deoxyribonucleic acid to high specific activity in vitro by nick-translation with DNA polymerase, J. Mol. Biol. 113, 237-51.

Russell, D. W., Yamamoto, T., Schneider, W. J., Slaughter, C. J., Brown, M. S. and Goldstein, J.L. (1983). cDNA cloning of the bovine low density lipoprotein receptor: feedback regulation of a receptor mRNA. Proc. Natl, Acad, Sci, USA 80, 7501-5.

Southern, E. M. (1975). Detection of specific sequences among DNA fragments separated by gel electrophoresis. J. Mol, Biol. 98, 503-17.

Sykes, B. (1983). A high frequency Hind III restriction site polymorphism with a collagen gene. Disease Markers 1, 141-6.

Tolleshaug, H., Hobgood, K. K., Brown. M. S, and Goldstein, J. L. (1983). The LDL receptor locus in familial hypercholesterolaemia: multiple mutations disrupt transport and processing of a membrane receptor. Cell 32, 941-51.

Wilson, J.T., Wilson, L.B., DeRiel, J.K., Villa-Komaroff, L., Efstratiadis, A., Forget, B. G. and Weissman, S. M. (1978). Insertion of synthetic copies of human globin genes into bacterial plasmids. Nucl, Acids Res. 5, 563-81.

Woo, S. L. C. , Lidsky, A. S., Guttler, F., Chandra, T. and Robson, K. J. H. (1983). Cloned human phenylalanine hydroxylase gene allows prenatal diagnosis and carrier detection of classical phenylketonuria. Nature 306, 151-5.

Yang, T. P., Patel, P. I., Chinault, A C., Stout, J. T., Jackson, L. G., Hilderbrand, B. M. and Caskey, D. T. (1984). Molecular evidence for new mutation at the hprt locus in Lesch-Nyhan patient. Nature 310, 412-4. 


\title{
Immobilization of the biological component of biosensors
}

\author{
S. A. BARKER
}

\subsection{Applications to sensors}

If we pose the problem: 'what is required of immobilization methods in biosensors?', then adaptability, reliability, and an option to bond the biological component to the sensor via molecules that conduct electrons figure high on the list. Although only minute quantities of the biological component (e.g. enzyme or antibody) are required, the purer it is the more reliable it will be. Obviously it must not contain either other substances which might interfere with the assay or other enzymes which catalyse reactions producing products detectable by the electrode chosen. Other elements of reliability require (1) a high degree of specificity to be exhibited by the biological component; (2) a good stability to the temperature, ionic strength, $\mathrm{pH}$, redox potential, and chemical composition to be encountered within the sample environment; (3) an inbuilt device or devices that limit contamination, biodegradation of the biological component, and/or its mode of attachment; and (4) where the user is a patient then infection must be avoided often by a disposable component approach.

Adaptability includes immobilization methods applicable to enzymes, multiple enzymes and cofactors, micro-organisms, antibodies, lectins, and other immunoreaction components as well as organelles, tissue slices, and liposomes. The test is whether these biological components retain activity and stability when attached to the support matrix in the biosensor.

There is often a need for electrons to pass from an enzyme-based biological component to the amplifier or microprocessor component. Ferrocene represents only the first of many potential ways of solving this problem (see Chapter 15). The cell in its natural state provides many examples of such transmission, e.g. cytochromes which are haemoproteins whose principal biological function is electron and/or hydrogen transport by valency change of their haem iron. The key development in the Yellow Springs Instrument Company sensor (Grooms et al. 1980; Chapter I) was a device to prevent interference from other electroactive species in blood. This was accomplished by a cellulose acetate membrane which is used with a Nucleopore polycarbonate membrane to form an enzyme sandwich. When such a membrane 
had only bovine serum albumin between the layers and no enzyme, virtually no current was recorded in fresh whole blood, plasma, or serum and it was insensitive to uric acid, ascorbic acid, bilirubin, molecular oxygen, and many drugs.

\subsection{Introduction}

In the immobilization of the biological component of the sensor we are particularly concerned with those methods which are applicable generally to a range of surfaces. This permits the choice of support surface to be as wide as possible and ensures that no refabrication of the support is required. Thus many surfaces have hydroxyl groups attached to them whether adjacent to carbon, silicon, or other atoms. Hence a method such as surface treatment with titanium tetrachloride, washing with water, and contacting with the biological component is of very wide applicability and provides a titanium sandwich chelate between support and biological component which is nonbiodegradable and resistant to a wide range of physiological $\mathrm{pHs}$ within which biological components will exhibit their activity. It has the additional advantage that it can also be applied to support surfaces having $-\mathrm{NH}_{2}$ and other groups amenable as ligands to the titanium atom. The initial surface treatment with titanium tetrachloride is the activation step and the activated surface can be dried at this stage if required. Once this surface is washed with water and the chloride ligands thereby replaced, it cannot be dried and should be immediately contacted with the biological component (Barker et al. 1971). Perhaps overriding all other considerations is the necessity for the biological component to exhibit maximum activity in its immobilized micro-environment i.e, to exhibit unit activity comparable with that in solution. Many early procedures gave dismal efficiences of $1-5 \%$ compared with the greater than $50 \%$ achievable by the titanium procedure with many enzymes.

Under some circumstances it may be desirable to re-plate the surface with the biological component. This can always be achieved by the titanium procedure. Other advantages often eagerly sought are (a) an ability for the biologically active component to operate at a wider $\mathrm{pH}$ range than in solution or at a different $\mathrm{pH}$ range than in solution, (b) attainment of greater stability by the immobilization procedure, (c) an ability to dispense with its coenzyme, or (d) the ability to co-immobilize more than one biologically active component. No one method can yet provide all these but typically the titanium procedure affords (a), (b), and (d) with many enzymes.

Where $\mathrm{pH}$ changes are required, then the micro-environment created on the surface of the sensor after immobilization can often act as an insoluble 'buffer'. Thus free amine groups enable the enzyme range to be extended downwards while free carboxyl groups permit its upward extension but never by more than $2 \mathrm{pH}$ units. 
True mating of the sensor and the biological component is essential and has recently been achieved (Higgins et al. 1983; Chapters 15 and 16) by the use of mediator sandwich molecules between the sensor and biological component (e.g. with ferrocene). This approach has the additional advantage of enabling the enzyme quinoprotein glucose dehydrogenase to be used thus dispensing with the need for coenzyme (D'Costa et al. 1986). Here the nature of the bonding between sensor support (carbon-felt electrodes) and enzyme are probably in the nature of electron transition complexes. The famous example of this taught to students is the interaction between benzene and hexafluorobenzene. Thus the aromatic or hetero-aromatic residues in protein enzymes, antigens, or antibodies would be the expected target of such bonding.

Overloading of the surface support with the biological component should be avoided, since, while activity increases with loading initially, this can decrease with high loading because of restricted access particularly where the biological component is interacting with another macromolecule. One method for partially overcoming this is to have a porous surface on the support (Kennedy et at. 1973). Much success has been gained by this ploy with immobilized glucamylase acting on starch or immobilized trypsin acting on casein. This feature is particularly important in immunobiosensors where interaction is often between antibody and an antigen macromolecule.

In biosensors it is vital that leakage of the biological components does not occur to any extent during use of the biosensor. Methods of entrapment of enzymes or other biological components would be suspect in this aspect except where the biologically active component is a microbial cell.

\subsection{Immobilization procedures}

Entrapment in a gel matrix is a favoured method of immobilization particularly for enzymes having small substrates which then have greater ease of access than a large substrate. Numerous matrices have been employed but recently the most favoured have been alginate and gelatin /collagen/crosslinked protein. With alginate, cross-linking the linear chains with $\mathrm{Ca} \cdots$ ions is generally employed. The $\mathrm{Na}$ alginate can be sterilized by autoclaving at $121{ }^{\circ} \mathrm{C}$ for 15 minutes and then, after mixing with the biological component, e.g. Aspergillus cells (Kuek and Armitage 1985), injected into $0.1 \mathrm{M} \mathrm{CaCl}_{2}$ and the beads allowed to harden for 30 minutes. Observation after prolonged use showed that loss of surface calcium alginate was lowest where agitation was least vigorous. Improvement of inulinase stability was noted for calcium alginate immobilized Kluyveromyces marxianus cells following surface treatment with hardening agents. Stability doubled after glutaraldehyde treatment and was increased six fold for hexamethylenediamine/glutaraldehyde 
or polyethyleneimine/glutaraldehyde compared with unhardened cells (Bajpai and Margaritis 1985).

With the need to produce surface coatings, simple procedures like those employed for immobilizing Arthrobacter simplex cells on glass are much sought after (e.g. Fig. 6.1). Here the support was treated with colloidal particles of hydrous alumina or alternatively the cells were pretreated with aluminium ions (Mozes and Rouxhet 1984) Both glass beads of glass wool could be employed with a single layer comprising $3 \times 10^{7}-7 \times 10^{7}$ cells $\mathrm{cm}^{-2}$ for cortisol-prednisolone transformation.

Mere precipitation of lipase (triacylglycerol acylhydrolase) from solution with chilled acetone was sufficient to immobilize the enzyme on diatomaceous earth, Hyflo Supercel giving the highest inter-esterification activity (Wisdom et al. 1984) when employed in an organic phase of petroleum spirit.

Attachment of glucamylase to porous silica was achieved via pretreatment with titanium tetrachloride and then reaction of the dried support with 1 , 6-diaminohexane in carbon tetrachloride. After washing, contacting with glutaraldehyde was used to anchor the enzyme to the surface (Cabral et al. 1984).

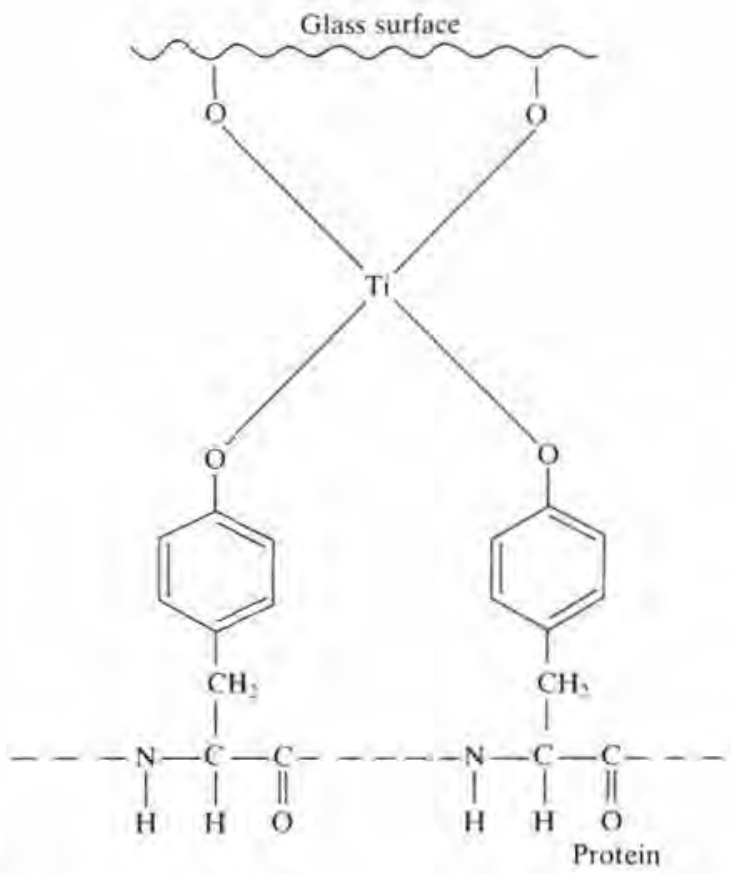

Fig. 6.1 Proposed enzyme-glass complex. 
Table 6.1 Immobilization procedures used in enzyme electrodes and thermistors

\begin{tabular}{|c|c|c|}
\hline Procedure & Enzyme & Reference \\
\hline $\begin{array}{l}\text { 1. Irreversible adsorption } \\
\text { onto graphite }\end{array}$ & D-Glucose oxidase & Ikeda et al. (1984) \\
\hline $\begin{array}{l}\text { 2. Co-immobilization with } \\
\text { FAD on Teflon-bonded } \\
\text { carbon black }\end{array}$ & D-Glucose oxidase & Sonawat et al. (1984) \\
\hline $\begin{array}{l}\text { 3. Glutaraldehyde } \\
\text { mediated cross-linking } \\
\text { with bovine serum } \\
\text { albumin onto a Pd-Pd } \\
\text { O electrode }\end{array}$ & Urease & Szuminsky et aI. (1984) \\
\hline $\begin{array}{l}\text { 4. Glutaraldehyde } \\
\text { mediated cross-linking } \\
\text { with bovine serum } \\
\text { albumin onto platinum }\end{array}$ & D-Glucose oxidase & Wingard et al, (1984) \\
\hline $\begin{array}{l}\text { 5. Dimethylsuberimidate } \\
\text { mediated reaction with } \\
\text { insoluble collagen }\end{array}$ & D-Glucose oxidase & Ngo and Lenhoff (1983) \\
\hline $\begin{array}{l}\text { 6. Entrapment in a gelatin } \\
\text { support }\end{array}$ & L-Lysine oxidase & Romette et al. (1983) \\
\hline 7. Co-immobilization & $\begin{array}{l}\text { D-Glucose oxidase } \\
\text { and catalase }\end{array}$ & $\begin{array}{l}\text { Cleland and Enfors } \\
\text { (1983) }\end{array}$ \\
\hline $\begin{array}{l}\text { 8. Co-immobilization via } \\
\text { glutaraldehyde mediated } \\
\text { cross-linking with } \\
\text { bovine serum albumin } \\
\text { on } \mathrm{Pt} \text { foil }\end{array}$ & $\begin{array}{l}\text { D-Glucose oxidase } \\
\text { and catalase }\end{array}$ & Wingard et al. (1983) \\
\hline $\begin{array}{l}\text { 9. Adsorption on } \mathrm{CaCO}_{3} \\
\text { particles }\end{array}$ & $\begin{array}{l}\text { Nitrite-oxidising } \\
\text { bacteria }\end{array}$ & Okada et al. (1983) \\
\hline $\begin{array}{l}\text { 10. Urease immobilized } \\
\text { membrane dipped in } \\
\text { ethylene diamine or } \\
\text { polylysine }\end{array}$ & Urease & Tokinaga et al. (1984) \\
\hline $\begin{array}{l}\text { 11. Glutaraldehyde for } \\
\text { cross-linking the enzyme } \\
\text { with a porous } \\
\text { polycarbonate film. }\end{array}$ & Glucose oxidase & Matsushita (1984a) \\
\hline $\begin{array}{l}\text { 12. Trapped between } \mathrm{Pt} \\
\text { plate and polyethylene } \\
\text { phthalate film } \\
\text { subsequently irradiated }\end{array}$ & Glucose oxidase & Matsushita (1984b) \\
\hline $\begin{array}{l}\text { 13. Direct O-alkylation of } \\
\text { Nylon } 6\end{array}$ & Urease & $\begin{array}{l}\text { Begum and Mottolo } \\
\text { (1984) }\end{array}$ \\
\hline
\end{tabular}


Table 6.1 Continued

Procedure
14. Entrapment between
cellophane dialysis
membrance and
$\mathrm{NH}_{3}$-gas-permeable
membrane
15. Co-immobilization on
activated collagen strip
supports

16. Co-immobilization by adsorption on medium porosity glassy carbon

17. Reaction with concanvalin A prereacted with cyanogen bromide activated agarose

18. Glutaraldehyde mediated reaction with controlled pore glass

19. Glutaraldehyde mediated reaction with gelatin onto a $\mathrm{CO}_{2}$-gassensitive electrode

20. Glutaraldehyde mediated coimmobilization on Clark type electrode

21. Immobilization via coating enzyme loaded on $p$-benzoquinone-C paste electrode with a nitrocellulose film

22. Co-immobilization on unwoven nylon cloth of enzyme with electron acceptor $\mathrm{K}$ ferricyanide

23. Enzyme on membrane over a platinum electrode

24. Co-immobilization on arylaminated controlled pore glass

Enzyme Reference

Asparaginase

Nikolelis (1984)

Luciferase and

Blum and Coulet (1984)

FMN-oxido reductase

FAD and

glucose oxidase

Miyawaki and Wingard

(1984)

Ascorbic acid oxidase, Mattiasson and

$\beta$-fructofuranosidase in Danielsson (1982) enzyme thermistor

$\beta$-D-Galactosidase, D-

Mattiasson and

galactose oxidase in

Danielsson (1982)

enzyme thermistor

L-Lysine

Tran et al. (1983)

decarboxylase

Alcohol oxidase, Vicrdyuyn et al. (1983) catalase

Glucose oxidase

Ikeda et al. (1985)

Glucose oxidase

Kawaguri et al. (1984)

Uricase or glucose

Tokyo Elec. (1985)

oxidase

$\beta$-Fructofuranosidase

Masoom and Townshend /mutarotase 
Table 6.1 Continued

\begin{tabular}{|c|c|c|}
\hline Procedure & Enzyme & Reference \\
\hline $\begin{array}{l}\text { 25. Enzyme immobilized at } \\
\text { electrode surface with } \\
\text { glutaraldehyde }\end{array}$ & Adenosine deaminase & $\begin{array}{l}\text { Bradley and Rechnitz } \\
\text { (1985) }\end{array}$ \\
\hline $\begin{array}{l}\text { 26. Enzyme immobilized on } \\
\text { a porous polycarbonate } \\
\text { membrane }\end{array}$ & Alcohol oxidase & Clark et al. (1984a) \\
\hline $\begin{array}{l}\text { 27. Enzyme immobilized } \\
\text { between two membranes } \\
\text { of cellulose ester using } \\
\text { buffered glutaraldehyde }\end{array}$ & Oxalate oxidase & Clark et al. (1984b) \\
\hline $\begin{array}{l}\text { 28. Enzyme immobilized on } \\
\text { porous side of a } \\
\text { cellulose acetate } \\
\text { membrane }\end{array}$ & $\mathrm{L}(+)$ Lactate oxidase & Tsuchida et al. (1985) \\
\hline
\end{tabular}

Yeast cells (negatively charged surface) can adhere to glass (negatively charged surface) or polycarbonate without the use of chemical reagents. Only storage of the cells in pure water (Van Haecht et al. 1984) is required prior to adhesion. During this phase the cells are starved and the cell wall modified in such a way as to promote adhesion.

Radiation-mediated grafting of polyacrolein onto poly (methyl methacrylate) microspheres was shown to activate the particles for subsequent chymotrypsin immobilization at pH 8.3 (Clark et al. 1984).

If dextran is periodate oxidized and the product reacted with glycine and then sodium borohydride, coupling with $\mathrm{N}^{6-}(\mathrm{N}-$ ( 2 aminoethyl) propionamide)-NAD ${ }^{*}$ affords a dextran bound co-enzyme NAD ${ }^{+}$derivative (coupling agent 1-ethyl-3-(3-dimethylamino propyl)- carbodiimide) with an activity almost equal to that of free $\mathrm{NAD}^{+}$for the $\mathrm{ADH}$ - and $\mathrm{LDH}$-catalysed reactions (Adachi et al. 1984).

Genetics International (1984a) used ferrocene absorbed on an electrode as the support for their glucose oxidase electrode. Earlier (1984b) they stipulated mediator compounds comprising at least two organic rings on electrodes of carbon particle paste or solid carbon.

The above examples serve to illustrate the four main approaches to enzyme immobilization (Fig. 6.2).

1. Physical adsorption at a solid surface

2. Entrapment in polymeric gel or within microcapsules.

3. Cross-linking by means of bifunctional reagents often in combination with 1 or 2 .

4. Covalent binding to a reactive insoluble support. 


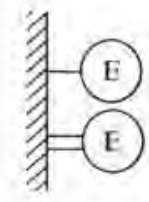

Covalent binding

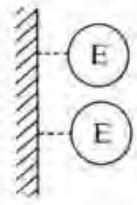

Adsorption

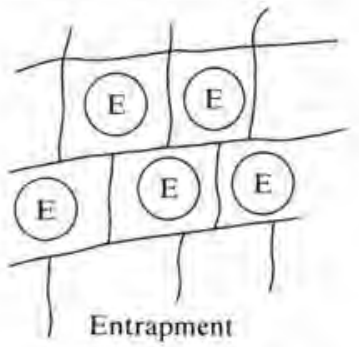

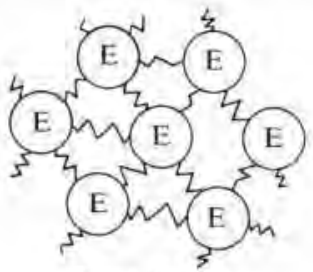

Cross-linking

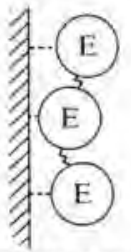

Adsorption-cross-linking

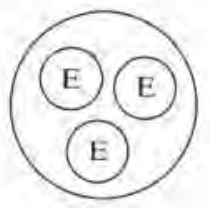

Micro-encapsulation

Fig. 6.2 Methods of enzyme immobilization.

\subsubsection{Adsorption}

Substances such as alumina, charcoal, clay, cellulose, kaolinite, collodion, silica gel, glass, hydroxyapatite, and collagen are known to adsorb enzymes. Obviously this list can be extended to ion exchangers such as DEAE cellulose, CM-cellulose, DEAE-Sephadex, Dowex 50, and a variety of phenolic resins. The great advantage of adsorption is that usually no reagents are required and only a minimum of activation or 'clean-up' steps. Adsorption tends to be less disruptive to enzyme protein than chemical methods of attachment ( 3 and 4 above). Binding forces are due to hydrogen bonds, multiple salt linkages, Van der Wall's forces, and the formation, where appropriate, of electron 
Table 6.2 Immobilization procedures in other devices

\begin{tabular}{|c|c|c|}
\hline Procedure & Objective & Reference \\
\hline $\begin{array}{l}\text { 1. Reaction of concanavalin } \\
\text { with cyanogen-bromide- } \\
\text { activated agarose }\end{array}$ & $\begin{array}{l}\text { Topographic probe for } \\
\text { protein-protein } \\
\text { interactions with D-galacto- } \\
\text { syltransferase }\end{array}$ & $\begin{array}{l}\text { Wong et al. } \\
(1983)\end{array}$ \\
\hline $\begin{array}{l}\text { 2. Reaction with activated } \\
\text { thiol-agarose and } \\
\text { anti-(immunoglobulin G) } \\
\text { antibody via } \\
\text { thiol-disulphide } \\
\text { interchange }\end{array}$ & $\begin{array}{l}\text { Development of a screening } \\
\text { method for detection of } \\
\text { monoclonal antibodies in } \\
\text { growing cultures }\end{array}$ & $\begin{array}{l}\text { Kimura et al. } \\
\text { (1984) }\end{array}$ \\
\hline $\begin{array}{l}\text { 3. Glucose oxidase used as a } \\
\text { label in enzyme } \\
\text { immunoassay - active film } \\
\text { with enzyme coated directly } \\
\text { on the gas-selective } \\
\text { membrane of the } \mathrm{pO}_{2} \\
\text { electrode }\end{array}$ & $\begin{array}{l}\text { A computerized automatic } \\
\text { system for determination } \\
\text { of hepatitis B surface } \\
\text { antigen ( } \mathrm{H} \mathrm{Bs} \mathrm{Ag} \text { ) in } \\
\text { biological fluids }\end{array}$ & $\begin{array}{l}\text { Romette and } \\
\text { Boitieux (1984) }\end{array}$ \\
\hline $\begin{array}{l}\text { 4. Immobilization of } \\
\text { immunoglobulins on silica } \\
\text { surfaces wherein reactive } \\
\text { groups introduced by } \\
\text { chemical vapor deposition } \\
\text { of silane. Also compared } \\
\text { with IgG immobilized by } \\
\text { thiol-disulphide exchange }\end{array}$ & Use in an immunosensor & $\begin{array}{l}\text { Jonsson et al. } \\
\text { (1985) }\end{array}$ \\
\hline
\end{tabular}

Immunosensors are biosensors that embody antibodies as their selective binding components. They include enzyme immunosensors, optical-fiber-based fluoresence immunosensors, piezoelectric systems, immunoFET systems (field effect transistors), optical systems, evanescent wave systems, surface plasmon resonance devices, and conformation sensitive transducers and have been reviewed by North (1985).

transition complexes. Unfortunately, except for the last named, the binding forces are more susceptible to change in $\mathrm{pH}$, temperature, ionic strength, or even the presence of the enzyme substrate.

\subsubsection{Entrapment}

If a polymeric gel is prepared in a solution containing an enzyme, the enzyme becomes trapped within the forming gel matrix. With due attention to the degree of cross-linking employed, this method of preparation can be applied to any enzyme since the protein molecule is trapped within a three 


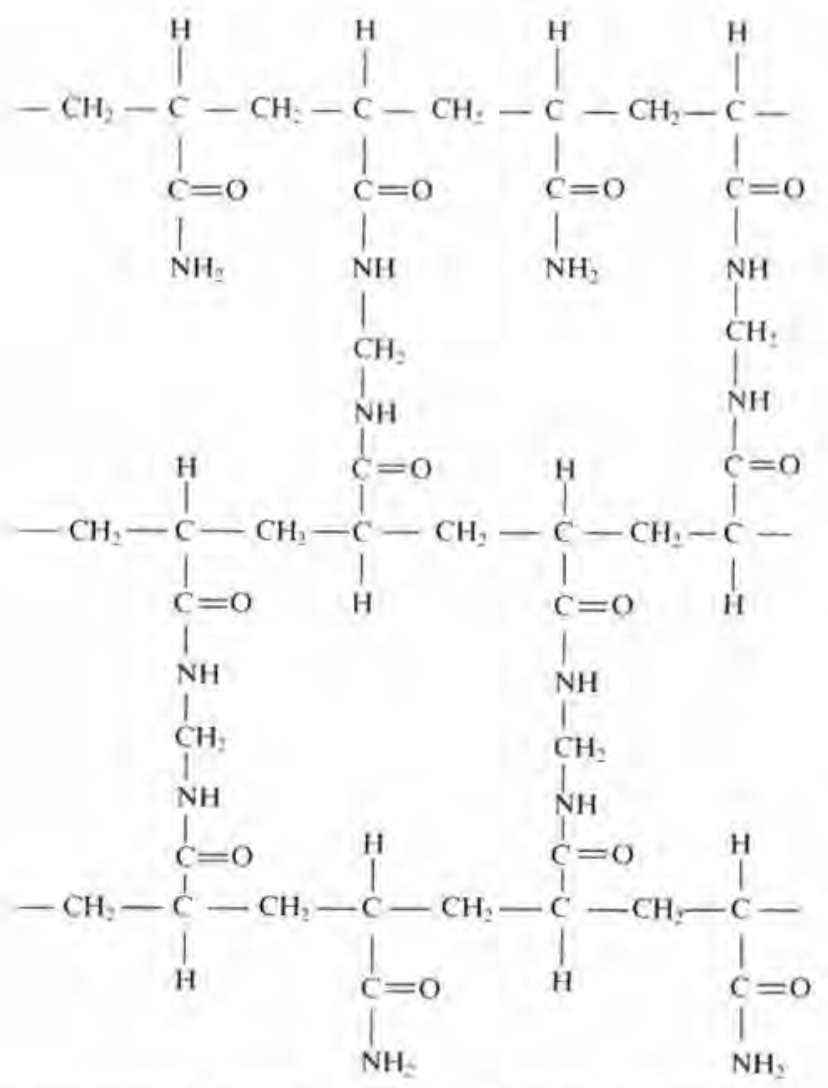

Fig. 6.3 Major structural features of the acrylamide $N, N$-methylene bis-acrylamide copolymer.

dimensional lattice (see Fig. 6.3 - the structure of a polyacrylamide gel used in the early developments of an enzyme electorde). Besides the example already given starch gels, nylon, and silastic gels can be employed.

Unfortunately this method suffers from two major drawbacks, (1) large diffusional barriers to the transport of substrate and product leading to reaction retardation particularly with high-molecular-weight substrates, e.g. ribonuclease, trypsin, and dextranase, and (2) continuous loss of enzyme activity since some pore sizes permit escape of the enzyme. Nevertheless, cross-linking entrapped protein with glutaraldehyde can often overcome the latter problem.

\subsubsection{Cross-linking}

Bifunctional agents that induce intermolecular cross-linking (see Fig. 6.4) can bind enzymes to solid supports. Cross-linking an enzyme to itself is both 
<smiles>O=CCCCC=O</smiles>

Glutaraldehyde<smiles>CCN1CC=C(c2cccc([O-])c2)O1</smiles>

$\mathrm{N}$-ethyl-5-phenylisoxazolium 3'-sulphonate (Woodward reagent $\mathrm{K}$ )

$$
\mathrm{O}=\mathrm{C}=\mathrm{N}-\left(\mathrm{CH}_{2}\right)_{6}-\mathrm{N}=\mathrm{C}=\mathrm{O}
$$

Hexamethylene diisocyanate

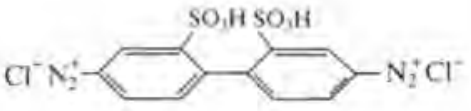

Bisdiazobenzidine-2.2disulphonic acid

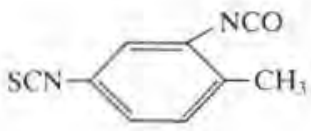

Toluene-2-isocyanate 4 -isothiocyanate

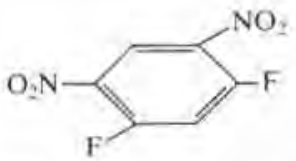

1,5-Difluoro-2,4-dinitrobenzene

Fig. 6.4 Some common bifunctional reagents for cross-linking protein.

expensive and inefficient as some of the protein material will inevitably be acting mainly as a support resulting in relatively low enzymic activity. Other disadvantages include diffusional limitations and lack of rigidity or mechanical strength. However bifunctional reagents are widely used in stabilizing physically adsorbed enzymes by cross-linking. Glutaraldehyde will of ten react with the lysine amino groups in an enzyme and in the case of trypsin will largely prevent self-digestion at the peptide bond adjacent to the point of attachment. Such reactions must be optimized and the $\mathrm{pH}$ values for the most rapid insolublization of lysozyme and papain are nearly the same as their isoelectric points.

\subsubsection{Covalent bonding}

Common reactions for covalent bonding are shown in Fig. 6.5. Covalent bonding between the enzyme and support matrix is accomplished through functional gropus in the enzyme which are not essential for its catalytic activity. Use is made of nucleophilic functional groups present in amino acid 
a. The cyanogen bromide technique

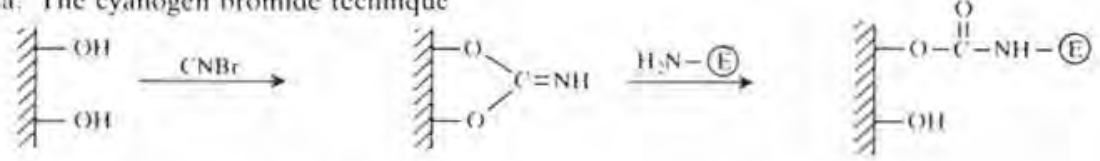

b. The Carbodi-Imide method<smiles>[R]N=C=N[R]</smiles>

c. Via acyl groups by treatment of hydrazides with nitrous acid

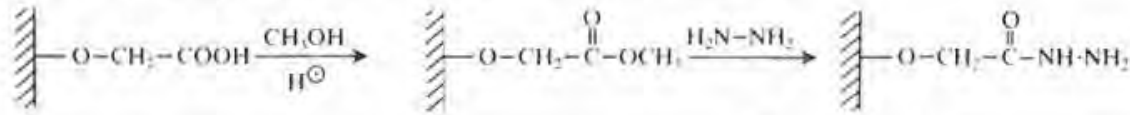

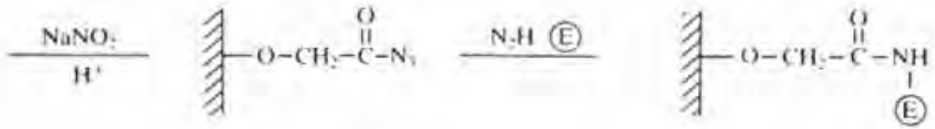

d. Coupling using cyanuric chloride

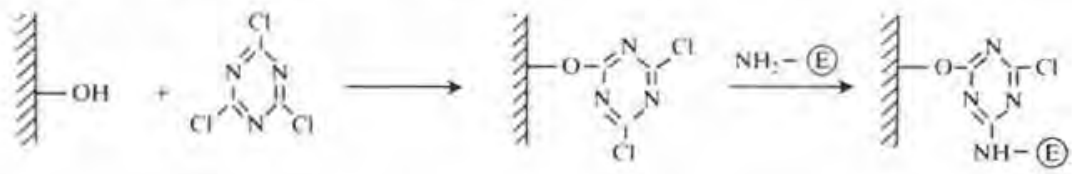

e. Coupling through diazonium groups from aromatic amino groups<smiles>C#Cc1ccc(N)cc1</smiles><smiles>[C+]Cc1ccc(O)cc1</smiles>

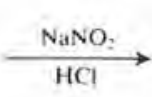<smiles>[B]Cc1ccc(O)c(N=Nc2ccc(C(C)(F)F)cc2)c1</smiles>

f. Coupling via thiol groups
$\not{\gamma} \mathrm{SH}$
$\frac{\text { Oxidation }}{\text { (E) }-\mathrm{SH}}$
$\lambda_{-5-s-E}$

Fig. 6.5 Some common reactions used for covalent binding. 
side chains of proteins for coupling. These are as diverse as amino, carboxylic acid, hydroxyl, phenolic, imidazole, and thiol groups. Coupling preferably takes place at low temperature, low ionic strength, and within the physiological $\mathrm{pH}$ range. Often coupling is done in the presence of the enzyme substrate to protect its active site. Covalent bonding has the great advantage that the enzyme is unlikely to be released during use from the optimum support matrix chosen (e.g for porosity, non-biodegradability, etc). The diversity of methods permits the avoidance of the active site in the process of linking. The efficiency of coupling should always be studied particularly the relative efficiency of a given amount of the enzyme acting in solution compared with its activity in the immobilized state. It is a useful parameter to determine choice of coupling procedure and support before the whole sensor is assembled. Mosbach's (1978) review article affords a good starting point for those new to the field and in particular covers the important area of immobilization of co-enzymes and their recycling. Specific examples of their application in enzyme electrodes are given.

\section{References}

Adachi, S., Ogata, M., Tobata, H. and Hashimoto, K. (1984). Effects of molecular weight of dextran and NAD density on coenzyme activity bound to dextran. Enz. Microb. Technol. 6, 259-62.

Bajpai, P. and Margaritis, A. (1985). Improvement of inulinase stability of calcium alginate immobilized K, marxianus cells. Enz. Microb. Technol, 7, 34-6.

Barker, S. A., Emery, A. N., Novais, J. W. (1971). Enzyme reactors for industry, Proc. Biochem. 6 (Oct), 11-13.

Begum, K. D, and Mottolo, H. A., (1984). Nylon shavings enzyme reactor for batch determination of urea. Anal. Biochem. 142, 1-6.

Blum, L. J., and Coulet, P. R., (1984). Coimmobilisation of luciferase and FMN oxidoreductase in an enzyme electrode. Anal. Chim. Acta 161, 355-8.

Bradley, C. R. and Rechnitz, G.A. (1985). Immobilisation barrier effects on the dynamic response characteristics of potentiometric adenosine deaminase enzyme electrodes - membrance thickness effect etc. Anal, Chem. 57, 1401-4.

Cabral, J. M. S., Cardosa, J. P., Novais, J. M. and Kennedy, J. F. (1984). A simple kinetic model for the hydrolysis of $\alpha$-D-glucans using glucamylase immobilised on porous silica. Enz. Microb. Technol. 6, 365-70.

Clark, D. S., Bailey, J. E., Yen, R. and Rembaum, A. (1984). Enzyme immobilisation on grafted polymeric microspheres. Enz. Microb. Technol. 6, 317-20.

- Noyes, L. K., Grooms, T. A. and Moore, P.E. (1984a). Direct rapid electroenzymatic sensor for measuring alcohol in whole blood and fermentation productsenzyme electrode using Hansenula polymorpha immobilized alcohol oxidase. Ann. N. Y. Acad. Sci. 434, 515-19.

(1984b). Oxalate sensing enzyme electrode using immobilised barley seedling oxalate oxidase. Ann. N. Y, Acad. Sci. 434, 512-14.

Cleland, N. and Enfors, S. O. (1983). Control of glucose fed batch cultivations of 
$E$. coli by means of an oxygen controlled stabilized enzyme electrode containing immobilised glucose oxidase and catalase. Eur. I. Appl. Microbiol. Biotechnol. 18, 141-7.

D'Costa, E. J., Higgins, I. J., and Turner, A. P. F. (1986). Quinoprotein glucose dehydrogenase and its application in an amperometric glucose sensor. Biosensors 2, 71-89.

Genetics International (1984a). Analytical equipment and sensor electrodes therefore. European Patent 127958.

- (1984b). Measurement of enzyme catalysed reactions. European Patent 125137.

Grooms, T. A., Clark, L. C. and Weiner, B. J. (1980). The design of peroxide enzyme membrane polarographic sensors for clinical and industrial analysis. In Enzyme engineering (eds. H. H. Weetall and G. R. Royer), Vol. 5, 217-29. Plenum Press, New York.

Higgins, I. J., Hill H. A. O. and Plotkin, E. V. (1983). Measurement of enzyme catalysed reactions. European Patent 125137. 14th Nov.

Ikeda, T., Hamada, H., Miki, K. and Senda, M. (1985). Glucose oxidase immobilised benzoquinone-carbon paste electrode as a glucose sensor. Agric. Biol. Chem. (Japan) 49, 541-3.

Katasho, I., Kamei, M., and Senda, M. (1984). Electrocatalysis with a glucose oxidase immobilized graphite electrode, Agric. Biol. Chem. (Japan) 48, 1969-76. Jonsson, U., Malmqvist, M. and Ronnberg, I. (1985). Immobilization of immuno globulins in silica surfaces: stability for use as immunosensor and in affinity chromatography, Biochem. J. 227, 363-71.

Kawaguri, M., Nankai, S., Iijima, T. (1984). Biosensor. PCT Int. App. WO 8403562. 13 Sept.

Kennedy, J.F, Barker, S. A. and Rosevear, A. (1973). Preparation of water insoluble trans-2, 3-cyclic carbonate derivative of macroporous cellulose and its use as a matrix for enzyme immobilisation. J. Chem. Soc, Perkin, 2293-9.

Kimura, S., Hayano, T. and Kato, K. (1984). Properties and applications to immunoassay of monoclonal antibodies to neuron specific $\gamma \gamma$ enolase. Biochem. Biophys. Acta 799, 252-9.

Kuek, C, and Armitage, T, M. (1985). Scanning electronmicroscopic examination of calcium alginate beads immobilizing growing mycelia. Enz. Microb. Technol. 7 , 121-5.

Masoom, M. and Townshend, A. (1985). Simultaneous determination of sucrose and glucose in mixtures by flow injection analysis with immobilized enzymes invertase/mutarotase column and glucose oxidase column. Anal. Chim. Acta 171, 185-94.

Matsushita (1984a). Biosensor. Japan Kokai Tokkyo Koho JP 59 67, 452 17th April. - (1984b). Biosensor. Japan Patent 011896, 4th August.

Mattiassin, B. and Danielsson, B. (1982). Calorimetric analysis of sugars and sugar derivatives with aid of an enzyme thermistor. Carb. Res, 102, 273-82.

Miyawaki, O. and Wingard, L. B. (1984). FAD and glucose oxidase immobilised on carbon. Ann. N. Y. Acad. Sci. 434, 520-2.

Mosbach, K. (1978). Immobilized coenzymes in ligand affinity chromatography and their use as active coenzymes. Advances in Enzymology 46, 205-78.

Mozes, N. and Rouxhet, P. G. (1984) Dehydrogenation of cortisol by Arthrobacter simplex immobilized as supported monolayer. Enz. Microb. Technol. 6, 497-502. 
Ngo, T. T. and Lenhoff, H.M. (1983). Amperometric assay for collagenase. Amplification by use of GOD conjugated to insoluble collagen. Appl. Biochem. Biotechnol, 8, 407-14.

Nikolelis, D. P. (1984). Construction of an immobilised asparaginase sensor and determination of asparagine in blood serum. Anal. Chim. Acta 161, 343-8.

North, J.R. (1985). Immunosensors: antibody based biosensors - comparison of construction methods and devices. U.K. Trends Biolechnol. 3, 180-6.

Okada, T., Karube, 1. and Suziki S. (1983). $\mathrm{NO}_{2}$ sensor which uses immobilized nitrite oxidising bacteria. Biotechol. Bioeng. 25, 1641-51.

Romette, J. L. and Boitieux J.L. (1984). Oxidase enzyme: enzyme and immunoenzyme sensor - computerized automatic system development: glucose oxidase application. Ann. N. Y. Acad. Sci. 434, 533-5.

— Yang, J.S., Kusakabe, H. and Thomas, D. (1983). Enzyme electrode for specific determination of L-lysine. Biotechnol, Bioeng. 25, 2557-66.

Sonawat, H. M., Phadke, R. S. and Govil, G. (1984). Covalent immobilization of FAD and glucose oxidase on carbon electrodes studies using cyclic voltammetry. Biotechnol. Bioeng. 26. 1066-70.

Szuminsky, N. J., Chen, A.K, and Liu, C. C. (1984). A miniature Palladium Palladium oxide enzyme electrode for urea determination. Biotechnol. Bioeng. 26, $642-5$.

Tokinaga, D., Kobayashi, T., Katori, A. and Karasawa, Y. (1984). Urease immobilised urea electrode and process for preparing the same U.S. Patent 4,476 , 005. Oct. 9th. Hitachi Ltd,

Tokyo Elec. (1985). Enzyme electrode containing immobilised glucose oxidase or uricase with enzyme store for supplementation JP 224055. 24th June.

Tran, N. D., Romette, J. L. and Thomas, D. (1983). An enzyme electrode for specific determination of L-lysine. A real time sensor. Biotech. Bioeng. 25, 329-40.

Tsuchida, T., Takasugi, H., Yoda, K. Takizawa, K., and Kobayashi, S. (1985). Application of L-lactate electrode for clinical analysis and monitoring of tissue culture medium - an enzyme electode for L-lactic acid determination. Biotechnol. Bioeng. 27, 837-41.

Verdyuyn, C., Van Dijken, J. P., and Scheffers, W, A. (1983). A simple sensitive and accurate alcohol electrode. Biotechnol. Bioeng. 25, 1049-55.

Van Haecht, J. L., De Bremaeker, M. and Rouxhet, P. G. (1984). Immobilization of yeast by adhesion to a support without use of a chemical agent. Enzym. Microbiol. Technol. 6, 221-7.

Wingard, L. B., Cantin, L. A. and Castner, J. F. (1983). Effect of enzyme matrix composition on potentiometric response to glucose using glucose oxidase immobilised on Platinum. Biochim. Biophys, Acta 748, 21-7

— Castner, J. F., Yao, S. J., Wolfson, S. K., Drash, A. L. and Liu, C. C. (1984). Immobilised glucose oxidase in the potentiometric detection of glucose. Appl. Biochem. Biotechnol. 9, 95-104.

Wisdom, R. A., Dunnill, P., Lilly M.D. and McCrae, A. (1984). Enzyme interesterification of fats: factors influencing the choice of support for immobilised lipase. Enzym. Microbiol. Technol. 6, 443-6.

Wong, S.S. Malorie, T.E. and Lee, T. K. (1983). Use of Concanavalin A as a Topographical Probe for Protein-Protein interaction. Lactose Synthase. Biochim. Biophys. Acta 745, 90-6. 


\title{
7 \\ Genetic engineering
}

\author{
P.J. WARNER
}

\subsection{Introduction}

This chapter is concerned with the impact genetic manipulation is likely to have on sensor technology. During the last ten years, four discoveries made during the nineteen seventies have revolutionized genetics. These discoveries were restriction endonucleases, plasmids (initially in bacteria), nucleic acid hybridization, and methods for sequencing DNA. They have allowed genes from diverse origins to be transferred to and expressed in different organisms, usually bacterial cells. The state of the art of recombinant DNA technology is outlined briefly below.

How, then, can use be made of this technology in the development of sensors? The purpose of this chapter is to answer this question, with the use of specific examples, where appropriate.

Recombinant DNA technology will have a ro!e in improvement of the biological component of enzyme-based and whole-cell biosensors. This will allow both improvement of these sensors for existing roles, for example by optimizing the enzymes employed, and also permit their applications to be broadened. It may also be instrumental in reducing cost through providing a readily available source of an enzyme that would otherwise be difficult to obtain in quantity.

\subsection{Recombinant DNA technology}

It is outside the scope of this book to provide the reader with a detailed account of methods of genetic engineering. The reader will however find that an excellent introduction is given by Old and Primrose (1985). Only a brief account of the techniques involved is given here.

\subsubsection{Molecular cloning}

An outline of a typical molecular cloning experiment is given in Fig. 7.1. Firstly it involves the isolation of the nucleic acid encoding the functions of interest from its natural host. In bacteria and other prokaryotes the DNA of interest can often be isolated directly. For higher plants and animals the procedure is slightly different because the DNA includes sequences, known 


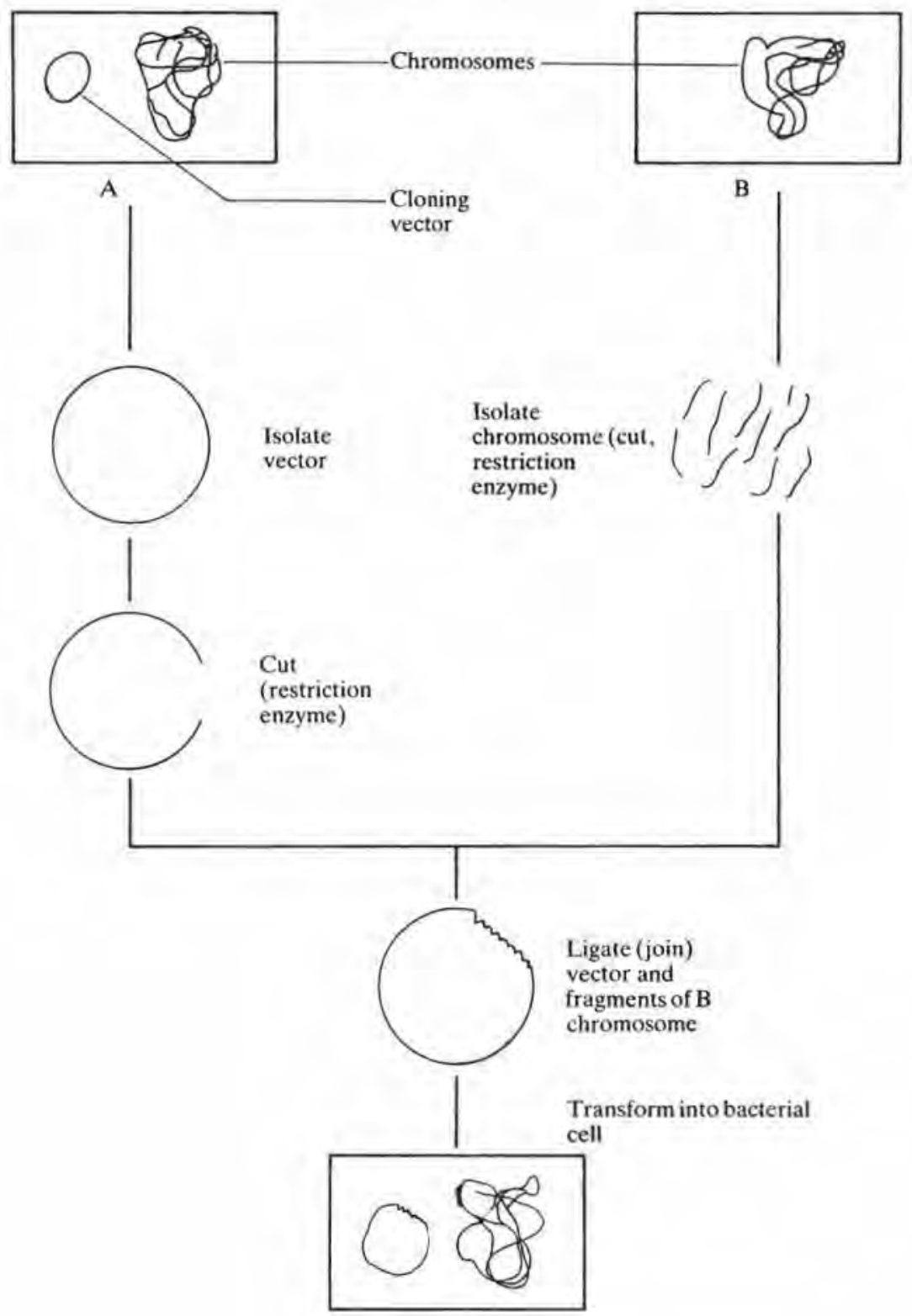

Bacterium contains recombinant plasmid

Fig. 7.1 An example of a cloning experiment showing the role of the cloning vector. 
(a)

1 Prokaryotic chromosome

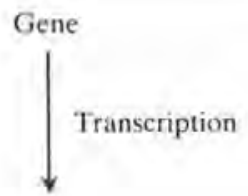

2 Messenger RNA

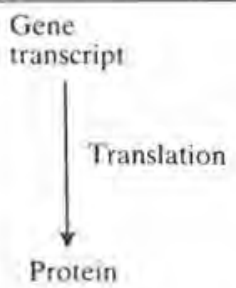

(b)

I Eukaryotic chromosome

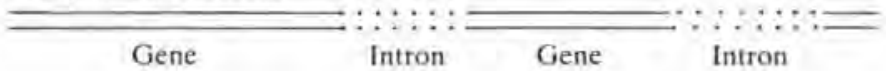

Transcription

2 Messenger RNA

$\begin{array}{lll}\begin{array}{l}\text { Gene } \\ \text { transcript }\end{array} & \begin{array}{c}\text { Intron } \\ \text { transcript }\end{array} & \text { Intron } \\ & \downarrow \text { Splicing }\end{array}$

3 Spliced messenger RNA

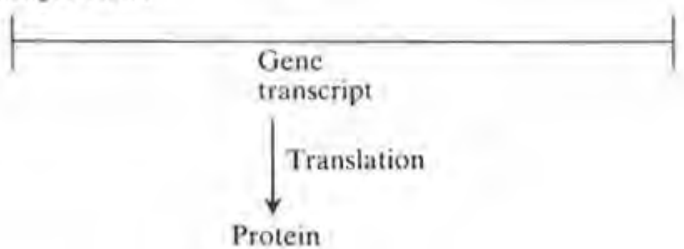

Fig. 7.2 The flow of genetic information in (a) prokaryotes and (b) eukaryotes. In the former, the DNA is transcribed to messenger RNA (mRNA) and translated to give the protein. In eukaryotes the genes include intervening sequences (introns), which are 
as introns. These are intervening sequences within genes, which are subjected to elimination from the messenger RNA (a process known as splicing) after transcription, but before translation (Fig. 7.2). In order that the genes of interest can be expressed in prokaryotes it is necessary to eliminate these (Fig. 7.3). To facilitate this, the messenger RNA (mRNA) is isolated. This is achieved by using cells in which the product of the gene of interest is likely to be induced; that is conditions under which the mRNA of interest is a substantial proportion of the total mRNA present. Messenger RNA can be readily isolated from eukaryotes, because unlike other types of RNA, its ends consist of poly-adenine (polyA) tails, which will associate with poly-thymine (poly $\mathrm{T}$ ) in a suitable affinity column. Once isolated, the mRNA is subjected to reverse transcription in vitro (using a viral enzyme called reverse transcriptase), which produces DNA which does not include introns. This is known as complimentary DNA (cDNA).

Once a DNA fragment likely to be expressed in a bacterium has been obtained the following procedure is followed. The DNA is then cut using restriction endonucleases (Kessler et al. 1985). These are enzymes which cut DNA wherever a specific recognition sequence is found, to leave short overhangs on either fragment. A small autonomously replicating piece of DNA termed a plasmid is similarly treated and then the two fragments of DNA from diverse origins are joined using an enzyme termed DNA ligase. The recombined molecule is then transferred to a bacterium. This is usually achieved by genetic transformation, in which under certain conditions the bacterium will take up the naked DNA, which is then replicated. We now have a recombinant molecule maintained within a new host. The plasmids used as cloning vectors have selectable genetic markers such as antibiotic resistance, and so their presence can be selected for, after transformation. If the foreign DNA is cloned into the middle of one of these genes, the recombinants will have then lost one of these markers, unlike molecules which have just recircularized after they have been cut by restriction endonuclease. They will therefore be recognizable as organisms sensitive to one particular antibiotic. This procedure is termed insertional inactivation, and is shown in Fig. 7.4. The 'foreign genes' may or may not be expressed; this means that the bacterium may or may not display the characteristics encoded by the cloned genes. If the latter is the case, the genes of interest can be re-cloned into plasmids specially designed to promote expression of cloned genes. As an alternative to a bacterial plasmid, some bacteriophages (bacterial viruses)

faithfully transcribed. The components of the genes themselves are known as exons. The introns are removed from the mRNA before transcription. Therefore if the DNA is cloned directly into prokaryotes the introns will not be recognized and will remain in the mRNA at translation, preventing production of an active protein. This problem has been overcome by use of the strategy shown in Fig. 7.3. 
I Isolate spliced messenger RNA

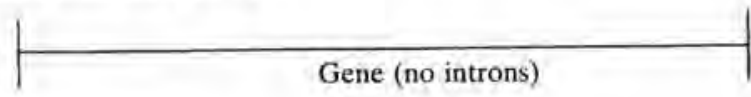

Reverse transcriptase

2 Double-stranded DNA

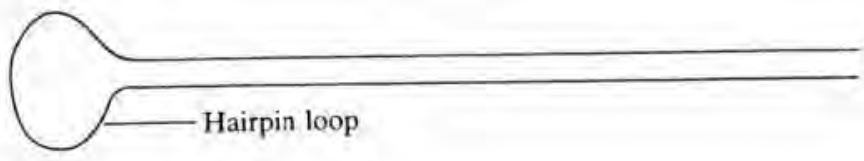

S1 nuclease

3 Double-stranded DNA (complementary DNA)

Fig. 7.3 Molecular cloning of eukaryotic DNA. The messenger RNA encoding the required product is isolated first, because it contains no introns (Fig. 7.2). This is then treated with a viral enzyme called reverse transcriptase, which generates an intron-free DNA sequence. A double-stranded sequence is typically obtained which includes single-stranded regions at one end, which are removed by a single-stranded-specific DNA degrading enzyme (S1 nuclease). This leaves a DNA sequence, known as complementary DNA (cDNA) suitable for cloning in a prokaryotic host (bacterium). 

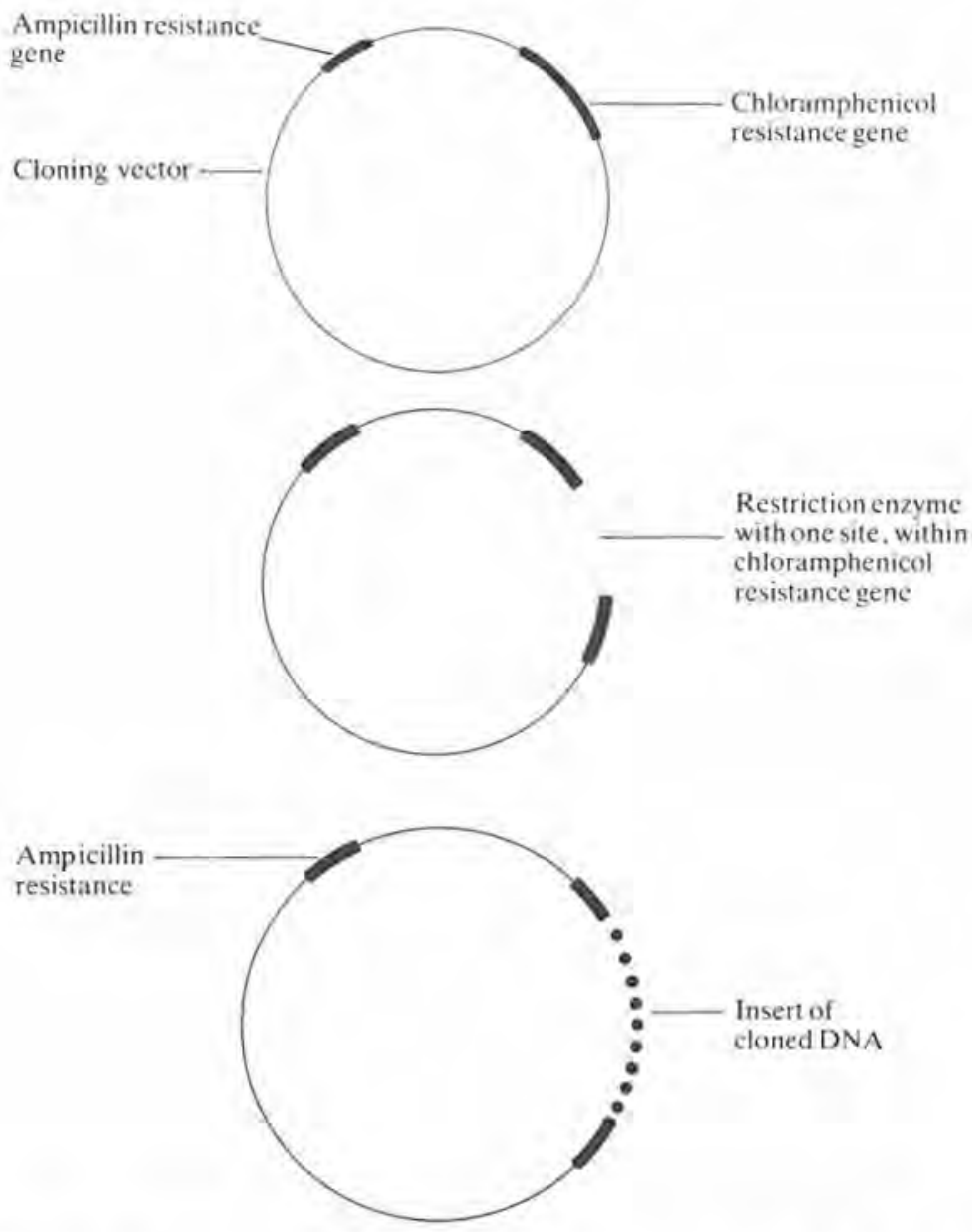

Fig. 7.4 Use of insertional inactivation to identify recombinant molecules. A unique site on the plasmid for a particular restriction enzyme lies within the chloramphenicol resistance gene and it is here that the foreign DNA is inserted. Organisms which have acquired recombinant molecules will be recognized, since they are chloramphenicol sensitive (the insertion has interfered with the structural integrity of the chloramphenicol resistance gene) but retain ampicillin resistance. 


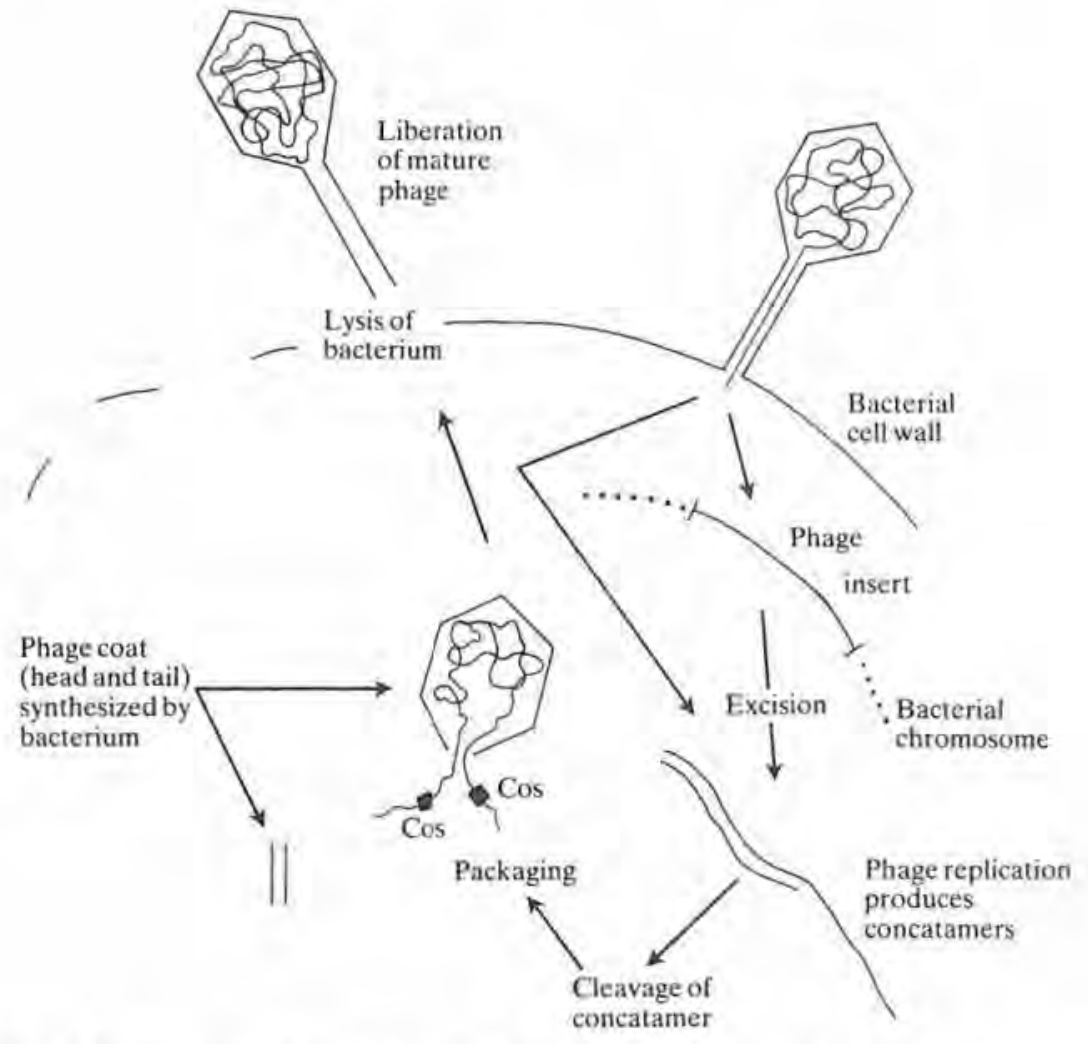

Fig. 7.5 The life cycle of bacteriophage lambda. On infecting the host bacterium, the phage can either undergo the lytic cycle or become incorporated into the bacterial chromosome, with which it replicates (the lysogenic state). At some stage in the future the phage may once again become lytic-this process can be induced by irradiation of lysogens with UV light. The lytic cycle is a vegetative process, at the end of which the host cell lyses, releasing large numbers of phage. The 'cos' sites are responsible for packaging replicated DNA into phage coats.

have also been developed for use as cloning vectors. The life cycle of bacteriophage lambda of Escherichia coli, the best-known example of a phage-cloning vector from which many cloning vectors have been developed (Brammar 1982), is shown in Fig. 7.5. The techniques used are similar to those for cloning using plasmids, but recombinants are usually isolated as plaques of bacteriophage, rather than intact bacterial cells. Plaques are circular regions of clearing in a lawn of bacteria grown on a plate, resulting from infection of a single bacterium with one phage, which has lysed to provide many thousand copies of itself, which then infect surrounding cells. 
Whilst Escherichia coli is the best-developed system to use for DNA cloning, similar systems are being developed for a range of bacteria, both Grampositive and Gram-negative. These include Pseudomonas, Bacillus, and Streptomyces. The above cloning techniques also are possible in yeasts and similar experiments are becoming possible in cells from higher animals and plants.

\subsubsection{Nucleic acid hybridization}

This topic will not be dealt with in any detail here, since the technology has been described elsewhere in this volume (Chapter 5). I shall merely remind the reader that the technique allows the similarity of fragments of two pieces of DNA (southern blotting: Southern 1975) and pieces of RNA and DNA (northern blotting: Adams et al, 1979) to be established. The relevance of the technique to this chapter is discussed below.

\subsubsection{DNA sequencing}

DNA sequencing technology has been available for about the last eight years. Two methods have been developed, a chemical method (Maxam and Gilbert 1980 ) and a method which relies on inhibition of the polymerase reaction leading to a halt being placed on DNA synthesis at specific points (Sanger $e t$ al. 1977).

7.2.3.1 Maxam and Gilbert method In this method a defined fragment of DNA generated by restriction endonuclease digestion is used. The sequence is labelled at one end with ${ }^{32}$ phosphate. Reagents are then used which alter one of the four bases present in DNA such that it can be removed and the strand can be cleaved, using certain chemical reagents (Table 7.1). If only partial reaction is allowed to occur a set of end labelled fragments of different lengths is generated. Each of the reactions detailed in Table 7.1 are used and the sequence information is then determined on a high-resolution polyacrylamide gel.

Table 7.1 Reagents used in Maxam and Gilbert sequencing

\begin{tabular}{llll}
\hline Base specificity & Base reaction & $\begin{array}{l}\text { Altered base } \\
\text { removal }\end{array}$ & Strand cleavage \\
\hline $\begin{array}{l}\text { Guanine } \\
\text { Guanine + adenine }\end{array}$ & $\begin{array}{l}\text { dimethyl sulphate } \\
\text { acid }\end{array}$ & $\begin{array}{l}\text { piperidine } \\
\text { acid-catalysed } \\
\text { depurination }\end{array}$ & $\begin{array}{l}\text { piperidine } \\
\text { piperidine }\end{array}$ \\
$\begin{array}{l}\text { Thymine + cytosine } \\
\text { Cytosine }\end{array}$ & $\begin{array}{l}\text { hydrazine } \\
\text { hydrazine }+\mathrm{NaCl}\end{array}$ & $\begin{array}{l}\text { piperidine } \\
\text { piperidine }\end{array}$ & $\begin{array}{l}\text { piperidine } \\
\text { piperidine } \\
\text { piperidine }\end{array}$ \\
\hline
\end{tabular}


7.2.3.2 Sanger sequencing This is the most widely used method of DNA sequencing, since it generally allows larger rragments of DNA to be analysed, with less chance of error. The DNA to be sequenced is first cloned into a single-stranded bacteriophage vector which can be used in E. coli. Phage M13 and its derivatives are most commonly used (Messing and Vieira 1982). The single-stranded DNA is then used as a substrate for DNA polymerase which faithfully copies the first strand. However, if the normal deoxynucleoside phosphate is substituted by the dideoxynucleoside phosphate, although this analogue can be incorporated, it will halt further activity of the polymerase, thus inhibiting any further chain elongation. Initiation of the process is achieved using a chemically synthesized universal nucleotide primer. The reaction is carried out in the presence of the four dNTPs one of which is labelled with ${ }^{32} \mathrm{P}$, and there are four reaction mixes, each with a low concentration of the analogues. One therefore ends up with a mixture of radiolabelled DNA fragments, with one end in common, but of variable length and a base-specific opposite end. The DNA from each mix, after incubation, is converted to the single-stranded form and electrophoresed on a polyacrylamide gel. The sequence can be read directly from the gel. This method allows between 250 and 500 base pairs to be sequenced in one set of reactions and the data is then analysed, frequently with the assistance of a computer.

\subsubsection{Site-specific mutagenesis}

Once the sequence of a piece of DNA has been determined, the technology now exists to make site-specific changes to the sequence, so that the effect of specific amino acid changes in a protein can be investigated (DalbadieMcFarland et al. 1982; Zoller and Smith 1982). This technique is known as site-specific mutagenesis. Single-stranded DNA is again the substrate and a synthetic oligonucleotide, in which one base pair has been changed and hence a specific mutation with known effect introduced, is used as the primer. DNA polymerase is used to reform a duplex and the resulting molecule (plasmid) with two slightly different strands, is transformed into a bacterial host. Mutant clones may, in some cases, be able to be phenotypically selected or screened for, but otherwise they can be detected by differences in their ability to hybridize with the synthetic oligonucleotide. This technique is the cornerstone of DNA-mediated protein engineering and a more thorough account of this can be found in this volume (Chapter 8).

Above I have attempted to outline some of the techniques used in genetic engineering which may be applicable to the development of biosensors. I shall now move on to describe the type of improvements we can expect to see them bring about in this field. 


\subsection{Applications to sensor technology}

\subsubsection{Improved yield of enzyme}

An obvious application of recombinant DNA technology is for cases when the enzyme to be used in a biosensor is present in small amounts or is difficult to isolate. An example of the latter problem in our laboratory has been the isolation of glucose dehydrogenase (GDH) (Duine et al, 1982). This enzyme is of potential use in a glucose sensor, since it would show greater sensitivity than glucose sensors based on glucose oxidase (GOD), and unlike GOD sensors, would have no requirement for oxygen (Turner and Pickup 1985; D'Costa et al. 1986). The source of GDH for our studies has been the bacterium Acinetobacter calcoaceticus. The yield of the enzyme from this organism has, however, been poor (Ameyama et al. 1981) since it appears to be present at very low levels. Its role in this organism is unclear, but it is probably concerned with energy metabolism. Glucose dehydrogenase consists of an apoenzyme and also a cofactor, PQQ, which serves as a cofactor for several other dehydrogenase enzymes in a range of organisms. Molecular cloning of the genes which encode the apoenzyme, and expression in an alternative organism, such as $E$. coli, would allow easier isolation of GDH. It offers the ability to improve production of the enzyme in three ways:

1. The gene encoding GDH can be cloned into a multi-copy plasmid vector. These plasmids are present in up to 50 copies per cell and hence the gene of interest is present in many copies and a large amount of the product may be produced.

2. The gene of interest can be cloned into a plasmid such that it is close to a strong promoter (for the host organism). A promoter is the site at which ribosomes bind for translation of messenger RNA into protein and so the use of a so-called expression vector of this type permits efficient transcription and translation which will lead to greater production of the protein. A number of plasmids have been engineered such that they contain sites for restriction enzymes which allow insertion of foreign DNA 'downstream' of an efficient promoter.

3. For some systems plasmids known as secretion vectors have been developed and these enable the product of the cloned gene to be secreted into the culture supernatant. This has obvious advantages when one is concerned with isolation and purification of an enzyme.

The cofactor PQQ has been found to be associated with several enzymes, incluing methanol dehydrogenase, the enzyme which allows methylotrophic bacteria to oxidize methanol (Duine and Frank 1981). It can be readily isolated from these organisms and hence cofactor isolation is not a problem. Furthermore the PQQ from these alternative sources, when combined with the GDH apoenzyme, has been shown to result in an active holoenzyme. It is 
therefore only necessary, in this instance, to clone the gene which encodes the apoenzyme.

Above I have used an enzyme from a prokaryotic organism as an example. However, this technique is particularly appropriate to sensors which would employ enzymes from higher animals and plants. Often such enzymes are expensive to produce, because they are present at low concentrations and because the growth of cells from higher organisms under laboratory culture is technically difficult and relatively slow.

\subsubsection{Improvement of enzyme properties}

A second application of molecular biology to sensor development relies on modern specific methods of mutagenesis which allow one to undertake enzyme engineering (see Chapter 8 ). This will permit alteration to the active site of an enzyme, so that it may have a faster turnover rate for a particular substrate. Alternatively the range of substrates attacked by an enzyme may be modified by this process. In this way the enzyme would be made more suitable for the task in hand. A prerequisite for this kind of work is a knowledge of the three-dimensional structure of the enzyme. This is usually determined by X-ray crystallography and will be necessary, in order that a prediction of the region of the protein which forms the active site can be made. One can than make specific base-pair changes in the DNA, which result in a change of a specific amino acid residue in the protein chain. The first stage in this procedure would be to clone the gene of interest on to a small plasmid, and transform the clone into $E$. coli. Using the procedures outlined above it would then be possible to sequence this gene and subsequently make specific base-pair changes to the DNA sequence. These changes could then be examined to determine the effect on the properties of the enzyme. This technique would be applicable to almost any enzyme for which sufficient structural information is available.

\subsubsection{Genetic manipulation of whole organisms for use in sensors}

The use of whole organism sensors has been implicated for certain applications in which it is difficult to use cell-free enzyme systems. An example of this is the use of whole organisms of a methanotrophic bacterium in a sensor designed to detect methane (Chapter 2). The sensor uses the enzyme methane monooxygenase, which oxidizes methane to methanol (Dalton et al. 1984). In this case it has so far not been possible to use the enzyme in a cell-free system, because it has been difficult to obtain a pure stable preparation of the enzyme in vitro and because other components of the bacterial cell are required to maintain its activity. These include the presence of co-enzymes and the ability of the host organism's metabolism to generate sufficient reducing power. Although attempts have been made to provide reducing power for the enzyme electrochemically, no immediate solution to either problem is likely, 
and the alternative is to use immobilized whole organisms rather than the required enzyme alone. However these whole organism systems can be subjected to genetic manipulation and some examples follow. The level of the enzyme required for the sensor can be increased such that it is a higher proportion of the cell protein. Another possibility is to provide the organism with an improved uptake system for the substrate, so that it can be brought into contact with the enzyme more readily and thus improve the response and sensitivity of the sensor. It may also be possible to alter the cellular location of the enzyme of interest, so that, for example in the case of bacteria, it is located in the periplasmic space and again the substrate can gain access more readily. A final possibility involves modification of the genes encoding the cell wall of the organisms employed, so that they attach more readily to the surface of the sensor, or show improved electron transfer to it. This idea is at present somewhat speculative, since it is difficult to envisage the specific genetic changes that would be necessary. As with protein engineering, this application requires prior knowledge of the physiology and genetics of the particular organism and is therefore probably best suited to species which are well characterized in these ways, or to well-characterized organisms to which the genes of interest have been introduced and expressed by genetic manipulation.

\section{7,4 Conclusions}

It is without doubt that genetic manipulation will have a role to play in the development of biosensors, as it is doing in many other areas of biotechnology. However in this field it seems likely that once the commercial exploitation of these devices has been realized, that biosensors whose performance results from the application of genetic manipulation will be of importance in the development of a second generation of sensing devices, financed by the profits obtained from those shortly to be marketed.

\section{References}

Adams, S. L., Alwine, J.C., De Crombugghe, B. and Postan, I. (1979). Use of recombinant plasmids to characterise collagen RNAs in normal and transformed chick embryo fibroblasts. J. Biol. Chem. 254, 4935-8.

Ameyama, M., Shinagawa, E., Matsushita, K. and Adachi, O. (1981). D-Glucose dehydrogenase of Gluconobacter suboxydans. Solubilisation, purification and characterization. Agri. Biol. Chem, 45, 851-61.

Barth, P. T. (1979). RP4 and R300B as wide host range plasmid cloning vehicles. In Plasmids of medical, environmental, and commercial importance (eds. K. N. Timmis and A. Puhler). Elsevier, North Holland.

Bolivar, F. (1979). Molecular cloning vectors derived from the Col El type plasmid, pMBI. Life Sciences 25, 807-17. 
Brammar, W. J. (1982). Vectors based on bacteriophage lambda. In Genetic engineering (ed. R. Williamson), Vol 3, 53-80. Academic Press, New York.

Dalbadie-McFarland, G., Gohen, L. W., Riggs, A. D., Morin, C., Itakura, K. and Richards, J.H. (1982). Oligonucleotide-directed mutagenesis as a general and powerful method for studies of protein functions. Proc. Natl. Acad. Sci. USA 79, 6409-13.

Dalton, H., Prior, S. D., Leak, D. J. and Stanley, S. H. (1984). Regulation and

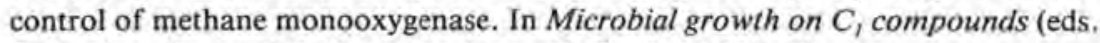
R. L. Crawford and R.S. Hanson) pp. 75-82. ASM, Washington.

D'Costa, E. J., Higgins, I. J. and Turner, A. P. F. (1986). Quinoprotein glucose dehydrogenase and its application in an amperometric glucose sensor. Biosensors 2 (accepted).

Duine, J,A. and Frank J. (1981). Methanol dehydrogenase: A quinoprotein, In

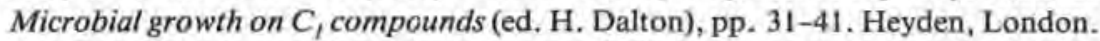

Duine, J. A., Frank, J. and Van der Meer, R. (1982). Different forms of quinoprotein aldolase-(glucose-) dehydrogenase, Archives of Microbiology 31, 27-31.

Kessler, C., Neumaier, T.S. and Wolf, W. (1985). Recognition sequences of restriction endonucleases and methylases - a review, Gene 33, I-102.

Maxam A.M. and Gilbert, W. (1980). Sequencing end-labelled DNA with base specific chemical cleavages. In Nucleic acids, Part 1. Methods in Enzymology 65, (eds. L. Grosman and K. Moldave) pp. 499-560. Acad. Press, New York.

Messing, J. and Vieira, J. (1982). A new pair of M13 vectors for selecting either DNA strand of double digest restriction fragments. Gene 19, 269-76.

Old, R. W. and Primrose, S, B. (1985). Principles of genetic engineering (3rd edn). Blackwell Scientific, Oxford.

Sanger, F., Nicklen, S, and Coulson, A. R. (1977). DNA sequencing with chain terminating inhibitors. Proc. Natl. Acad. Sci. USA 74, 5463-7.

Southern, E. M. (1975). Detection of specific sequences among DNA fragments separated by gel electrophoresis, J, Mol. Biol. 98, 503-17.

Turner, A. P.F. and Pickup, J. C. (1985). Diabetes mellitus: Biosensors for research and management. Biosensors 1, 85-115.

Zoller, M. J. and Smith, M. (1982). Oligonucleotide directed mutagenesis using M13 derived vectors: An efficient and general procedure for the production of point mutations in any fragment of DNA. Nucleic Acids Res. 10, 6487-500. 


\title{
8 \\ Protein engineering and its potential application to biosensors
}

\author{
ANTHONY E. G. CASS and ENDA KENNY
}

\subsection{Introduction}

In this chapter on the potential application of protein engineering to biosensors we take protein engineering in its broad sense to imply the modification of the covalent structure of a protein by addition, substitution, or deletion of groups such that the chemical properties of the molecule are different from those found in the native state. Chemical properties included in this definition could range from catalysis of a completely different reaction by an enzyme through a shift in substrate specificity to an increase in the lifetime of the native biological activity under a particular set of conditions.

The mechanisms that can be used to effect such changes vary from modifying the nucleotide sequence of the DNA that codes for the protein of interest so that a defined change in amino acid sequence results; through to the incorporation of the native enzyme into the final biosensor in such a way that the properties of the enzyme are modified by its interaction with the other components of the device. Before we consider the kinds of changes that might be useful in adapting proteins to biosensor applications it might be appropriate to consider some of the relevant properties of proteins that affect their performance in biosensors.

The kinetic parameters $k_{\text {cat }}$ and $K_{\mathrm{M}}$ are obviously important in determining the properties of the sensor. In general, biosensors contain relatively high loadings of enzyme activity and hence a high $k_{\text {cat }}$ (turnover number) means that only small amounts (in milligrams) of enzyme are needed. In the specific, but important, case of membrane sensors a high $k_{\text {cat }}$ would tend to result in a device limited by membrane transport and thus to one relatively insensitive to variations in enzyme loading or loss of enzyme activity.

When we turn to the Michaelis constant $K_{\mathrm{M}}$, its effect on the sensor appears in the linear range of the device. Ideally there should be a linear relationship between the output signal and the analyte concentration and in general terms this is reflected in the $K_{\mathrm{M}}$ (or apparent $K_{\mathrm{M}}$ ) of the enzyme. Inspection of the Michaelis-Menten equation shows that this condition holds for concentrations up to about $0.2 K_{\mathrm{M}}$ although other factors such as immobilization or the 
presence of a membrane will also be important. A parameter derived from the above two is the specificity constant, $k_{\text {cat }} / K_{\mathrm{M}}$, of the enzyme and it is generally desirable to have a high value for the analyte of interest compared with other potential substrates.

The effect of temperature on the performance of the biosensor is often composed of several different and competing processes. As in any chemical reaction the rate of the enzymatic reaction will increase with increasing temperature and thus the response of the device will also be temperature dependent, the magnitude of this effect reflecting the activation energy of the rate-limiting step. Enzymes also undergo thermal inactivation and the rate of this will depend on the activation energy for the unfolding of the protein and the temperature. Finally, even if the enzyme is stable the device may show a biphasic response if there is a change in rate-determining step. In addition to temperature there are a number of other causes of protein denaturation that can lead to loss of activity, and an increase in the general 'robustness' of the biological component would enhance the practical application of biosensors.

$\mathrm{pH}$ is another environmental variable that affects the response of biosensors when the enzyme's activity is controlled by ionizing groups. A shift in $\mathrm{pH}$ optimum and a broadening of the optimum range may be necessary to make the sensor compatible with the analytical matrix. Proteins are also denatured by extremes of $\mathrm{pH}$ and this may be particularly important in food and drink analyses where the samples are often rather acidic.

Finally where the biological component needs to be immobilized it may be that the residues involved in immobilization are also important for activity and an alternative method is needed. Table 8.1 summarizes some of the potential targets for protein engineering for biosensors.

In this chapter we will survey the many different approaches to modifying proteins starting at the level of DNA sequence and working upwards. The examples we discuss will not be concerned with biosensors per se but they will serve to illustrate some of the results that have been achieved in altering the properties of proteins.

At the outset it should be made clear that the engineering, i.e. directed modification, of proteins can generally only be made within the framework

Table 8.1 Some potential targets for protein engineering for biosensors

1. Improved turnover number.

2. Shift in or removal of $\mathrm{pH}$ dependence.

3. Change in linear response range to substrate concentration.

4. Improved stability during storage and operation.

5. Reduction in susceptibility to interfering substances.

6. Widening or narrowing of substrate specificity.

7. Change in cofactor requirement. 
of a detailed body of information about the protein. Ideally this would include a three-dimensional structure at sufficient resolution to locate the individual residues. Unfortunately, for many proteins used in biosensors this kind of information is either absent or incomplete; for example, probably the most widely used enzyme in this area is glucose oxidase yet despite being readily available in a highly purified form in substantial quantities we still know remarkably little about the molecule. There is no published amino acid sequence and no crystal structure. The kinetic properties of the enzyme are well understood (Bright and Porter 1975) but there is no structural skeleton on which to 'hang' this information. Lack of knowledge at this level means that we are often handicapped in being able to truly engineer molecules or even to rationalize the effects of changes made empirically.

With this caveat in mind we nonetheless feel that the engineering of protein molecules for biosensor applications is sufficiently important to warrant discussion. After all protein molecules evolved to fulfill a specific biological need and not to provide the components of man-made devices. Although some of the biological properties can be exploited to our own ends, it is not surprising that others are more or less incompatible with the final application.

Bearing these points in mind we shall now commence with our survey of the ways and means of protein engineering.

\subsection{Modification at the DNA level}

Since the early 1970s when it was first shown that DNA could be cleaved and rejoined in vitro recombinant DNA technology has become an important tool in molecular biology (Cohen et al. 1973). The techniques of genetic engineering now allow us to isolate, propagate, and sequence any gene of interest be it from plant, animal, or micro-organism. The knowledge of the DNA sequence of the gene then allows us to derive the amino acid sequence of the protein that it codes for, and conversely we can create nucleotide sequences corresponding to any desired polypeptide chain. In the case of fairly small peptides the chemical synthesis of the gene in vitro has been achieved (Edge et al. 1981) and hence there is no theoretical barrier to the construction of genes encoding novel activities. The difficulties arise in the effective synthesis of long polynucleotides in reasonable yield, free from by-products of similar sequence, and in actually designing the polypeptide sequence that has the desired activity. We still know very little about the determinants of protein folding. Accordingly, at present, we are limited to the modification of existing proteins by introducing base changes at specific locations in the gene.

The advantages of effecting these changes at the level of DNA rather than protein are several. Firstly, because DNA is the genetic material and is 
faithfully replicated during growth only a single manipulation is required. Modification at the level of protein will need repeated application as stocks are consumed. Secondly a single genetic change leads to a single product whereas chemical modification of the protein often leads to mixtures of products. Finally any amino acid may be changed by genetic manipulation whereas there may be steric or reactivity barriers to chemically modifying residues in the protein. In its current state in vitro mutagenesis aims to effect point mutations at one or a limited number of sites in the gene and to observe the effect of this on the resulting protein.

Chemical mutagenesis has been used to change cytosine to thymine by treatment with sodium bisulphite where, under appropriate conditions, this can be targeted to a specific region of the DNA (Shortle and Nathans 1978). Techniques have also been developed that render relatively specific regions in a DNA sequence single stranded which, after resynthesizing the gap, force a mismatched or misincorporated base to be included (Shortle et al. 1982). In these methods, however, the position of the point of mutation cannot be exactly predetermined and thus a number of mutants must be screened to find an appropriate one. Oligonucleotide-directed mutagenesis has circumvented these problems and has opened the way to introducing specific point mutations into DNA.

In 1978 two reports demonstrated how precise changes in DNA could be effected with oligonucleotides by introducing mutations into the genome of the bacteriophage $\phi \mathrm{X} 174$. This phage's DNA exists as a single-stranded form in the mature virus particle but upon infection converts to a double-stranded or replicative form (RF) prior to the generation of new phages. Hutchinson et al. (1978) and Razin et al. (1978) used synthetic oligonucleotides complementary to the viral $(+)$ strand as primers for the in vitro synthesis of the opposite (-) strand, forming a closed circular RF molecule incorporating the priming oligonucleotide. If the primers contained a mismatched base the RF DNA still formed satisfactorily but now the progeny were of two types containing either the wild-type DNA or that from the mismatched primer. The latter were detected by screening for the mutant gene. Subsequently several other mutations were introduced into $\phi \mathrm{X} 174$ using this method (Gillam and Smith 1979).

Since these initial observations two developments have tremendously accelerated the use of oligonucleotide-directed mutagenesis. The first has been in the synthesis of the oligonucleotide primers (Sproat and Gait 1984). Automated, semi-automated, and manual solid-phase synthetic protocols are available and the protected, activated precursors are supplied by a number of chemical companies, thus the production of primers is now a routine process. The second advance has been the standardization of the methodology using M13 bacteriophage cloning vectors (Zoller and Smith 1983). M13 is like $\phi \mathrm{X} 174$ in possessing both single- and double-stranded forms thus enabling 


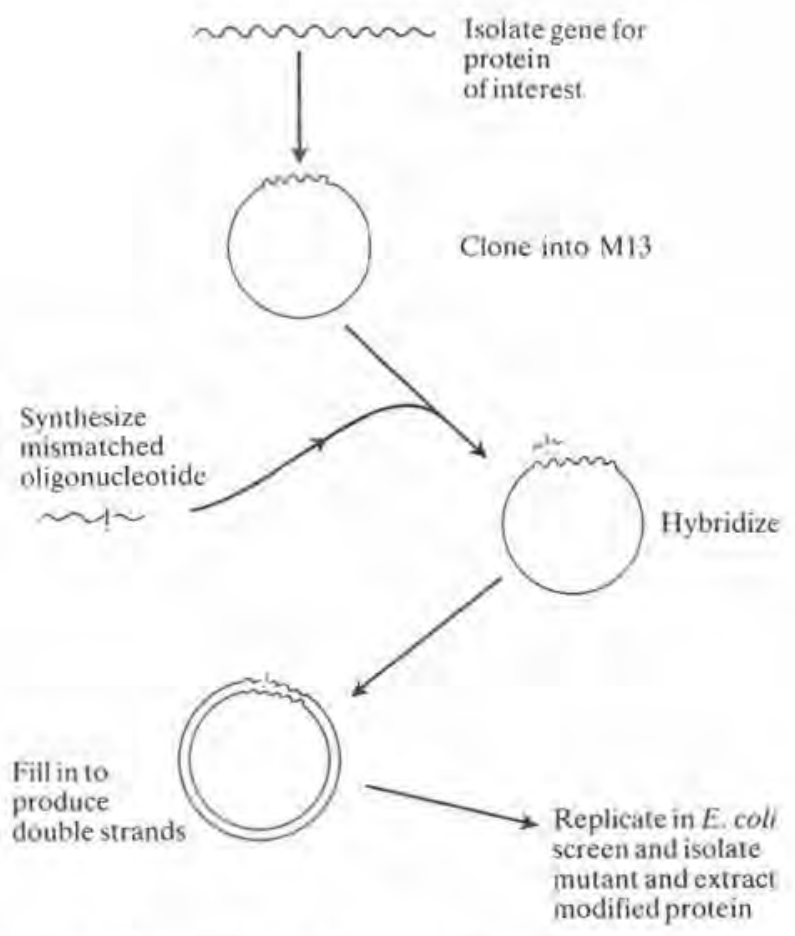

Fig. 8.1 Scheme for oligonucleotide-directed mutagenesis. The basic steps involved in using this method in the bacteriophage M13 are shown. For more details on the methodology the reader should consult the references given in the text.

the cloning of the gene of interest in the RF and the mutagenesis in the singlestranded form. M13 is of particular use in this regard as it has been specifically developed as a cloning vehicle for the isolation of DNA fragments for sequencing by the chain-termination method of Sanger et al. (1977) and there are several unique cloning sites (Messing 1983). The scheme for mutagenesis in M13 is shown in Fig. 8.1.

Wallace et al. (1981) showed that the mutagenic oligonucleotide could also be used as a hybridization probe to screen for the mutant DNA. To do this the oligonucleotide is radioactively labelled with ${ }^{32} \mathrm{P}$ and as it is obviously more homologous to the mutant than wild-type DNA it will hybridize more strongly to the former. Mutant clones can then be identified by autoradiography.

Once the mutated DNA has been identified it can be excised from the RF, recloned into a suitable host/vector system for maximizing expression, and the resulting protein isolated. Multiple application of this method can be used to introduce a number of changes. 
Within the framework of the above strategy a number of enzymes have been modified and the properties of the mutant enzymes investigated (Ackers and Smith 1985).

Tyrosyl tRNA synthetase is one of the most intensively studied examples as both its structure and kinetics are well understood (Fersht et al. 1984). The enzyme catalyses the aminoacylation of tRNA ${ }^{\text {tyr }}$ by tyrosine in a two step reaction:

Enzyme + Tyrosine + ATP $\longrightarrow$ Enzyme-adenyltyrosine + PPi

Enzyme.adenyltyrosine + tRNA $\longrightarrow$ Enzyme + Tyrosyl.tRNA + AMP

Initial results (Winter et al. 1982) showed that when cysteine-35 was altered to a serine there was a 4.5 -fold increase in the $K_{\mathrm{M}}$ for ATP. Similarly the introduction of a glycine residue at this position also resulted in an enzyme of lower activity (Wilkinson et al. 1983). It is known from the crystallographic work that cysteine-35 makes a hydrogen-bond contact with the ribose ring of ATP and so it is not surprising that the introduction of a glycine residue results in a lower activity. At first sight the results with serine seem odd as it might be expected that the stronger hydrogen-bonding ability of the hydroxyl as compared to the sulphydryl group would result in enhanced binding. However in the unliganded enzyme the hydrogen bonding is to a water molecule, and the different steric requirement for sulphydryl versus hydroxyl groups means that in the mutant enzyme the binding of ATP results in the exchange of a strong hydrogen bond by a much weaker one with the observed consequences for binding and catalysis (Wilkinson et al. 1983).

The introduction of different residues into the enzyme does not necessarily result in the loss of some of the catalytic activity, for example the replacement of threonine- 51 by a proline resulted in both a decrease in $K_{\mathrm{M}}$ and an increase in $k_{\text {cat }}$ leading to an overall increase in $k_{\text {cat }} / K_{\mathrm{M}}$ of 25 fold (Wilkinson et al, 1984). In attempting to understand and predict the effects of amino acid replacements in mutant enzymes it is necessarily to be able to distinguish between the direct interaction of the residue with substrate and concomitant structural changes that alter contacts elsewhere in the molecule. The result described above in replacing a threonine residue by a proline is a case in point. One approach used in trying to resolve this point has been to study the effects of double mutants. If the effect of the two mutations is the same as the sum of their individual contributions then it is likely that the role of the amino acid residues is purely a local one, Conversely any deviation from this implies a more diffuse role; Carter et al. (1984) have termed this 'coupling' and have shown that in the threonine/proline case the enhanced activity is due to a structural change, improving the contact of histidine-48 with ATP. This interaction of the histidine residue with ATP has been further probed by introducing mutations into position 48 . When the histidine is replaced by an asparagine the resulting enzyme is as active as the wild type, in contrast the 
introduction of a glutamine residue gives a much less active product (Lowe et al. 1985). This result implies that the histidine residue is hydrogen bonded through its nitrogen atom and rules out any electrostatic interaction.

Further fine-structure analysis of the mutants at position 51 has been carried out by replacing the threonine with alanine, cysteine, or proline (Fersht et al. 1985a). Detailed kinetic analyses of the mutants has shown that each exhibits a maximum activity depending on the ATP concentration. As it is known that this residue is variable in tyrosyl tRNA synthetases from different sources it appears that the different enzymes have evolved to maximize their activity at the prevailing intracellular ATP concentration.

Recently the role of hydrogen bonds in the specificity of tyrosyl tRNA synthetase has been probed by comparing the properties of a number of mutants of the enzyme (Fersht et al. 1985b). By systematically varying the residues the authors were able to assess the contribution of hydrogen bonds to the binding of and specificity for ATP and tyrosine. The conclusions from this work are that removal of a residue that hydrogen bonds to an uncharged group on the substrate only destabilizes the latter's binding energy by $2-6 \mathrm{~kJ} \mathrm{~mol}^{-1}$ whilst if the hydrogen bond is to a charged group then a much larger effect, up to $16 \mathrm{~kJ} \mathrm{~mol}^{-1}$, is observed. In a later paper Wells and Fersht (1985) were able to show that hydrogen bonds are also important in the preferential binding of the transition state of the substrate over the ground state.

Although most of the mutations that have been made in this enzyme have been at the active site the interaction between the subunits has also been investigated. Native tyrosyl tRNA synthetase is a homodimer that exhibits half of the sites' reactivity, and the crystal structure reveals that phenylalanine 164 is important in subunit contacts. If this residue is replaced by an aspartate then steady-state kinetics, active-site titration, and equilibrium binding studies reveal that at high $\mathrm{pH}$, in the absence of tyrosine or ATP as ligands, and at low enzyme concentration the dimer dissociates (Jones et al. 1985). The authors propose that it is the ionization of the aspartate residue that induces dissociation whilst the ligands only bind appreciably to the dimer. tRNA has no effect on the monomer/dimer equilibrium suggesting that it shows no differential binding to either form.

$\beta$-Lactamase catalyses the hydrolysis of penicillins and is the basis of a number of sensors for $\beta$-lactam antibiotics. The gene for this enzyme is a common marker and is carried by the vector pBR322 (Bolivar et al. 1977). Dalbadie-McFarland et al. (1982) used a doubly mismatched oligonucleotide to invert the serine/threonine dipeptide at the active site resulting in the loss of activity. If the active-site serine is replaced by cysteine the mutant protein is still active though at a lower level than the wild type, and is sensitive to inhibition by sulphydryl reagents (Sigal et al. 1982). Thiol lactamase does however have some favourable properties compared to the native enzyme, it 
is more resistant to trypsin and is not inhibited by boric acid (Sigal et al. 1984).

An important goal in improving enzymes is to try and increase the temperature stability and one approach to this is to introduce intramolecular cross-links. This has been attempted with dihydrofolate reductase and with T4 lysozyme. Preliminary results suggest that disulphide bridges can be mutated into both these proteins. In the case of the dihydrofolate reductase proline- 39 was replaced by a cysteine and it appears that a disulphide bridge was formed between this residue and the cysteine at position 85 leading to a dependence of the activity on oxidation state (Villafranca et al. 1983). T4 lysozyme had isoleucine- 3 replaced by a cysteine and peptide mapping revealed that the newly introduced residue formed a disulphide with cysteine-97. Neither the amino acid replacement nor the formation of the bridge affected the activity though there was an effect on the initial rate of thermal denaturation with the mutant showing a slower decay in activity than the wild type. Interestingly though, the difference appears to be due not to the disulphide bridge per se but rather is a function of the thiol group of cysteine-54 (Perry and Wetzel 1984).

The $\mathrm{pH}$ optimum of an enzyme can be shifted by the mutagenesis of surface charged groups as illustrated by the protease subtilisin. Replacement of a surface aspartate residue by serine resulted in a shift in the activity linked $\mathrm{p} K_{\mathrm{a}}$ from 7.17 in the wild-type enzyme to 6.88 in the mutant (Thomas et al. 1985). As expected for an electrostatic effect the shift was only observed at low ionic strength. Another protease recently investigated by oligonucleotide mutagenesis is carboxypeptidase A (CPA). In this example the aim was to probe the postulated role of tyrosine-248 in catalysis (Gardell et al. 1985). A mutant enzyme with this residue replaced by phenylalanine has essentially the same $k_{\text {cat }}$ as the wild type but exhibits a higher $K_{\mathrm{M}}(6$ fold $)$ and a much higher inhibitor constant with potato CPA inhibitor ( 70 fold).

These examples serve to illustrate how we are beginning to be able to modify the sequences and hence properties of proteins by manipulation of their genes. Undoubtedly at present the lack of both structural data and the relevant cloned genes that is the major impediment to the application of this technique to biosensors. The power of the method along with the opportunity to obtain large amounts of material by cloning for overproduction will mean that genetic engineering will have as large an impact in the area of biosensors as it has already had in many other areas of biotechnology (see also Chapter 7).

\subsection{Modification of the polypeptide chain}

There are many reagents that are available to modify the various reactive side chains found in proteins and some of these are collected in Table 8.2. 
Table 8.2 Typical chemical modifications on proteins

\begin{tabular}{|c|c|}
\hline Residue & Modifiers \\
\hline Lysine & $\begin{array}{l}\text { Arylating agents e.g. trinitrobenzenesulphonate } \\
\text { Acid anhydrides e.g. maleic, succinic } \\
\text { Isoureas and imidates }\end{array}$ \\
\hline Arginine & $\alpha, \beta$-Diketones \\
\hline Histidine & $\begin{array}{l}\text { Diethylpyrocarbonate } \\
\text { Alkylating agents e.g. chloroketones }\end{array}$ \\
\hline Cysteine & $\begin{array}{l}\text { Alkylating agents e.g. iodoacetate, } N \text {-ethylmaleimide, } \\
\text { Ethyleneimine }\end{array}$ \\
\hline Tyrosine & $\begin{array}{l}\text { Tetranitromethane } \\
\text { Acylating agents e.g. acetic anhydride, acetylimidazol } \\
\text { Iodinating agents e.g. iodide + lactoperoxidase }\end{array}$ \\
\hline $\begin{array}{l}\text { Glutamic and } \\
\text { aspartic acids }\end{array}$ & $\begin{array}{l}\text { Carbodimides }+ \text { amines } \\
\text { Diazo-compounds }\end{array}$ \\
\hline
\end{tabular}

Chemical-modification studies have been of importance in elucidating the structure and mechanism of many proteins and for labelling the protein with 'reporter' groups. At best these reagents are selective for a particular amino acid residue, and in favourable cases environmental factors may render one or two residues of a particular type significantly more or less reactive than the rest. Targeting the reagent to the active site can be achieved by using a close structural analogue of the substrate that also carries the modifying group. A further elegant refinement of this approach is to 'uncover' the reactive group during catalysis and thus produce a suicide substrate. In most of these modifications the resulting protein is completely devoid of biological activity so this approach is less than suitable for our needs! However there have also been less drastic modifications and we will now consider some of these.

\subsection{Modifications that increase the activity}

These modifications are relatively rare, as might be expected, and at first sight it may appear strange that the activity of an enzyme subject to millions of years of evolutionary pressure can be improved upon. However it must be remembered that the enzyme has evolved to work in a specific cellular environment and thus improvements in activity can occur under conditions that are rather different from this. As an illustration of this the work of Plapp (1970) on alcohol dehydrogenase showed that modification of the lysine residues with methylpicolinimidate

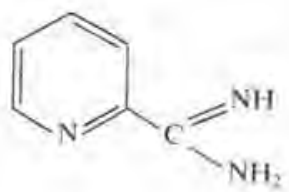


resulted in a derivative 19 times more active than the native enzyme when assayed at high substrate concentration. After modification the enzyme still showed an ordered bi-bi mechanism characteristic of the native enzyme in which co-enzyme dissociation is rate limiting in both directions. It is this observation that provides a clue to the rationale for the observed effects. The Michaelis and product inhibition constants for the modified enzyme are some 12-53 times greater than in the native form whereas the turnover numbers are 12-30 times larger. Apparently the enhanced activity at high substrate concentration is due to an increase in the off-rates for the co-enzymes. When the substrate concentrations are decreased the native and modified species approach one another in activity.

Another example of a modified enzyme with improved activity is provided by dihydrofolate reductase from chicken liver, although in this case the molecular basis for the change is not understood. A variety of reagents that react with cysteine-11 cause a 5-10-fold increase in activity (Barbehenn and Kaufman 1980 and references therein) with the physiological substrates NADPH and dihydrofolate. In this case though a price has to be paid for the improvement in terms of a much reduced thermal stability of the modified enzyme. Finally we also mention the enhancement in ATPase activity of 14S and $30 \mathrm{~S}$ dynein after modification of the amino groups with trinitrobenzenesulphonate (Shimizu 1979).

\subsubsection{Modifications that alter surface properties}

The examples discussed in the previous section although of interest, and in some cases capable of post hoc rationalization, were not predicted and hence are of little use in attempting to 'engineer' a protein in a specific fashion. One class of chemical modification that can be predicted based upon some knowledge of the protein and a little judicious physical chemistry is that which attempts to alter the surface properties of the molecule. Amongst these kinds of modifications the simplest to perform and interpret are those based upon altering the surface charge. Typical changes are shown in Fig. 8.2 where the charge is either removed or reversed. Using this type of chemistry the $\mathrm{p} K_{u}$ of chymotrypsin was shifted $\pm 1 \mathrm{pH}$ unit from the native value of 7 (Valenzuela and Bender 1971). As expected the conversion of a positive charge to negative increased the $\mathrm{p} K_{a}$ whilst a negative to positive decreased it. The succinylated chymotrypsin also showed an increased $k_{\text {cat }}$ and decreased $K_{\mathrm{M}}$ for hydrolysis of ester substrates whilst the enzyme modified with ethylenediamine showed no change in $k_{\text {cat }}$. In the latter case $K_{\mathrm{M}}$ was found to be strongly $\mathrm{pH}-$ dependent; unlike the native enzyme where $K_{\mathrm{M}}$ did not vary with $\mathrm{pH}$.

Acetylation of trypsin also led to an enhancement of activity although in this case it was the $O$-acetylation of an exposed tyrosine residue that is critical (Spomer and Wotton 1971). Modification of the lysine residues with acetyl groups had no effect. The cause of the improved activity was ascribed to an 


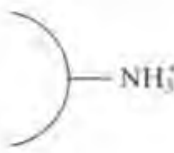

Succinic anhydride
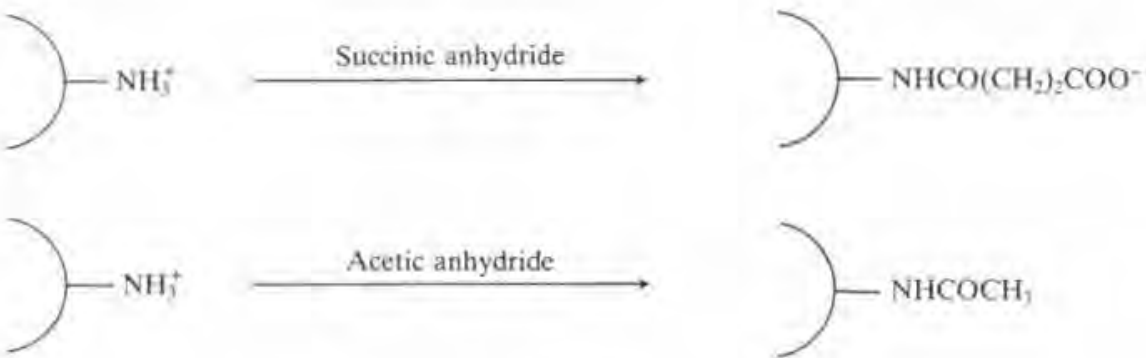

Acetic anhydride
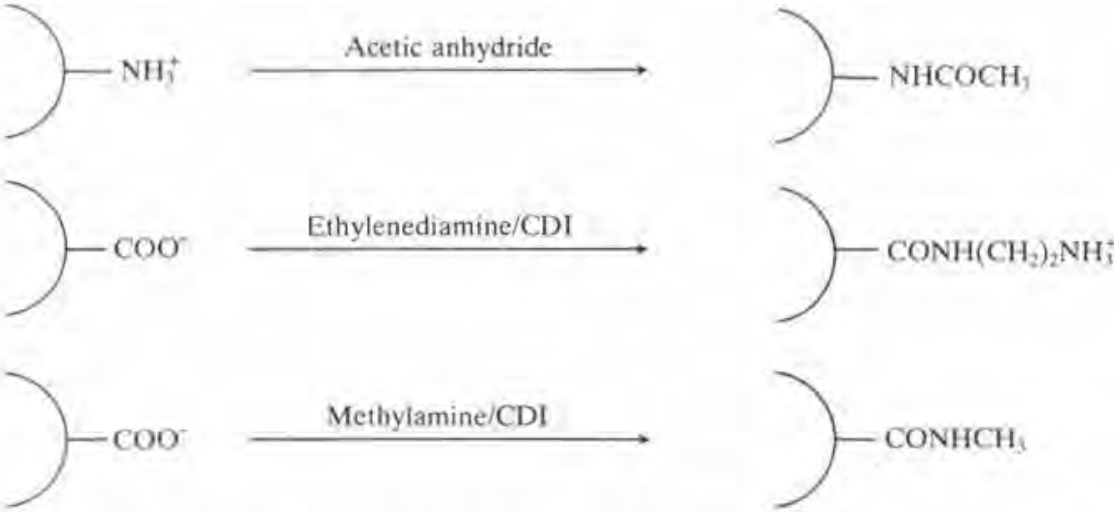

Fig. 8.2 Some typical chemistries for neutralizing or reversing the charge of surface amino or carboxyl groups in proteins. CDI is a water-soluble carbodiimide.

increased rate of formation of the acyl enzyme in ester and amide hydrolyses.

Sometimes the attachment of charged groups can be used as an alternative to the addition of cations to the reaction medium as in the example of plastocyanin. The reaction of this blue copper electron-transfer protein with $\mathrm{P} 700^{+}$ in the chloroplast usually requires the presence of magnesium ions to 'screen' the negative charges of the carboxyl groups on the plastocyanin. Burkey and Gross (1982) were able to achieve the same effect and also to shift the midpoint potential by $>+40 \mathrm{mV}$ by modifying the protein with ethylenediamine.

Even when the surface charge is maintained, favourable effects can sometimes be achieved as shown by the work of Cupo et al. (1980). These authors converted the lysine residues to homoarginine by treating several proteins (chymotrypsin, ribonuclease, lysozyme, $\alpha$-lactalbumin, cytochrome $c$, carbonic anhydrase, and bovine serum albumin) with $O$-methylisourea:<smiles>COC(=N)N</smiles>

It was argued that the bulky guanidino group was less likely than the amino group to move into the hydrophobic regions of the protein and so the 


$$
\begin{aligned}
& \mathrm{CH}_{3}-\mathrm{O}-\left(\mathrm{CH}_{2}\right)_{2}-\mathrm{O}-\left(\mathrm{CH}_{2}\right)_{2}-\mathrm{O}-\left(\mathrm{CH}_{2}\right)_{2}-\mathrm{O}-\left(\mathrm{CH}_{2}\right)_{2} \mathrm{CO}-- \\
& \mathrm{CH}_{3} \mathrm{CH}_{2}\left(\mathrm{CH}_{2}\right)_{2}-\mathrm{O}-\left(\mathrm{CH}_{2}\right)_{2}-\mathrm{O}-\left(\mathrm{CH}_{2}\right)_{2}-\mathrm{O}-\left(\mathrm{CH}_{2}\right)_{2} \mathrm{CO}-- \\
& \mathrm{CH}_{3} \mathrm{CH}_{2}\left(\mathrm{CH}_{2}\right)_{2} \mathrm{CH}_{2}\left(\mathrm{CH}_{2}\right)_{2}-\mathrm{O}-\left(\mathrm{CH}_{2}\right)_{2}-\mathrm{O}-\left(\mathrm{CH}_{2}\right)_{2} \mathrm{CO}- \\
& \mathrm{CH}_{3} \mathrm{CH}_{2}\left(\mathrm{CH}_{2}\right)_{2} \mathrm{CH}_{2}\left(\mathrm{CH}_{2}\right)_{2} \mathrm{CH}_{2}\left(\mathrm{CH}_{2}\right)_{2}-\mathrm{O}-\left(\mathrm{CH}_{2}\right)_{2} \mathrm{CO}-- \\
& \mathrm{CH}_{3} \mathrm{CH}_{2}\left(\mathrm{CH}_{2}\right)_{2} \mathrm{CH}_{2}\left(\mathrm{CH}_{2}\right)_{2} \mathrm{CH}_{2}\left(\mathrm{CH}_{2}\right)_{2} \mathrm{CH}_{2}\left(\mathrm{CH}_{2}\right)_{2} \mathrm{CO}-
\end{aligned}
$$

Fig. 8.3 Various groups attached to the surface lysines of thermolysin, by Urabe et al. (1978). The hydrophobicity of the modifying groups increases from I to $\mathrm{V}$ and has a differential effect on the thermal stability of the resulting derivatives.

substitution of the latter by the former should stabilize the structure. Exchange of tritium out of the protein into water was indeed consistent with a less flexible structure for the modified proteins.

A considerable improvement in the thermal stability of thermolysin was achieved by acylating 6-7 of the amino groups of lysine residues with longchain fatty acids containing different numbers of ether groups (Urabe et al. 1978 ) as shown in Fig. 8.3. The hydrophobic nature of the chain decreases from V to I and derivatives of I had essentially full activity ( $>90 \%$ of the native enzyme) and showed a much improved thermal stability. In contrast derivatives of $\mathrm{V}$ were insoluble in water.

Stabilization of the protein may also be achieved by coupling it to a watersoluble polymer as for example in the case of adenosine deaminase (Rosemeyer et al. 1982). Attachment of the enzyme to cyanogen-bromide-activated dextran resulted in a derivative that was only $13 \%$ as active as the native material with an increased $K_{\mathrm{M}}$ and a decreased $k_{\text {cat }}$. However the half time for loss of activity at $60^{\circ} \mathrm{C}$ was increased from 1 to 2 hours.

\subsubsection{Modifications that alter specificity}

Changes in the substrate specificity of an enzyme can be bought about by a variety of modifications and these have been recently reviewed by Kaiser et al. (1985).

Proteases often have an associated esterase activity and when carboxypeptidase $\mathbf{A}$ is acetylated at 2 of its tyrosines there is an apparent 6 -fold enhancement in its esterase action with concomitant virtually complete abolition of proteolytic activity. Pancreatic ribonucleases (RNase) has been subjected to many chemical modifications and one interesting one involves the dimerization of the enzyme using the cross-linking agent dimethylsuberimidate. The native enzyme is a monomer with little activity towards doublestranded RNA but after cross-linking the product shows an enhanced (78-440 fold) hydrolytic action with the double-stranded form. Esterase activity has also been introduced into this enzymes by cross-linking in the presence of the inhibitor indolepropionic acid. Introduction of activity under these conditions is assumed to arise by cross-linking in an unusual conformation of the enzyme. 


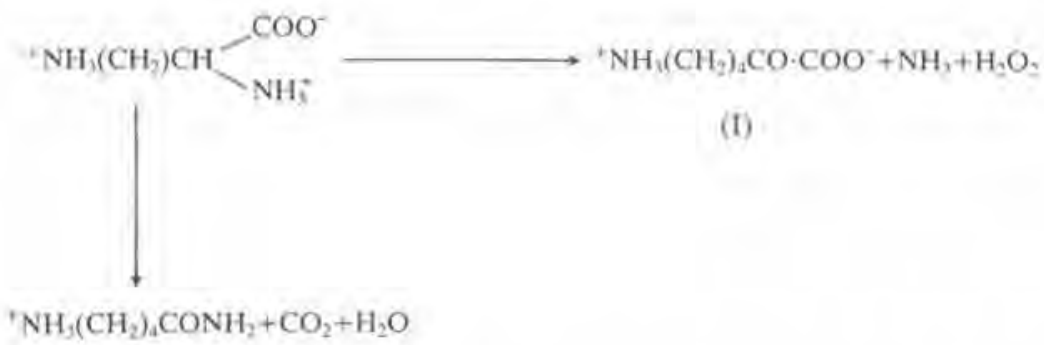

(II)

Fig. 8.4 The reactions of lysine monooxygenase acting as either an oxidase (1) or a monooxygenase (II). The latter is the physiological reaction but the former reaction may be induced by modifying the lysine residues in the enzyme.

Enzymes involved in oxidative reactions can often have their specificity altered under appropriate conditions; for example interconversion of monooxygenase and oxidase reactions of lysine monooxygenase can be achieved by treatment of the enzyme with agents that react with the thiol groups as shown in Fig. 8.4. In the case of monoamine oxidase the type of reaction remains the same but the modification results in a loss of activity with the natural substrate tyramine and an enhanced activity with histamine. Flavoprotein dehydrogenases can, on modification, have their acceptor specificity relaxed so that the range of compounds reducible by these enzymes is widened from $\mathrm{NAD}(\mathrm{P})^{+}$to include dyes and dioxygen. In effect the enzymes have been converted from dehydrogenases to oxidases, an examples of this is xanthine dehydrogenase/oxidase (Kaminski and Jezewska 1979). Glutathione reductase catalyses the reaction

$$
\mathrm{GSSG}+\mathrm{NADPH}+\mathrm{H}^{+} \longrightarrow 2 \mathrm{GSH}+\mathrm{NADP}^{+}
$$

and modifying the lysine residues with trinitrobenzenesulphonate results in a protein with substantial NADPH oxidase activity and no glutathione reductase activity (Carlberg and Mannervik 1980).

\subsubsection{Co-enzyme attachment}

One interesting and potentially useful modification of an enzyme is to attach a normally freely diffusing co-enzyme to the polypeptide chain. This has been described for the combination of an NAD analogue and alcohol dehydrogenase (Mansson et al. 1978). The resulting conjugate contained approximately one co-enzyme per active site and had some $20 \%$ of the activity of the enzyme/soluble co-enzyme combination. In a second publication (Mansson et al. 1979) the authors further demonstrated that this conjugate could be linked to the turnover of a second (lactate) dehydrogenase. This type of approach could obviously be extended to ADP/ATP, pterin, and folate- 
dependent enzyme systems. It has the great advantage that biosensors utilizing these would be independent of added co-enzymes.

\subsubsection{New enzyme activities}

The possibility of introducing a completely new activity into a protein has been achieved in a few instances. The strategy here is to attempt to enhance the weak catalytic activity of an organic molecule by covalently attaching it to a protein chain to take advantage of the latter's ability to bind the substrate. One of the best examples of this approach is flavopapain (Kaiser et al. 1980). Papain is a plant protease that contains a catalytically active thiol group. Treating papain with bromo-isoalloxazines results in the covalent attachment of the redox group to the thiol (Levine et al. 1977) as shown in Fig. 8.5. These molecules are devoid of proteolytic activity but now have oxidase activity. A derivative of papain made by alkylating the active site cysteine residue showed a low activity in oxidizing dithiols (Fried and Kaiser 1981) and dihydronicotinamides (Levine et al. 1977, Levine and Kaiser 1978). Only modest enhancements were observed for dithiol oxidations compared with the isoalloxazine moiety alone; typical rate enhancements were 4-17 fold with $k_{\text {cat }} / K_{\mathrm{M}}$ values of $4-21 \mathrm{M}^{-1} \mathrm{~s}^{-1}$ (Fried and Kaiser 1981). Model building studies suggested that another derivative should be a better catalyst in dihydronicotinamide oxidations and this indeed proved to be so. Rate enhancements compared with the uncomplexed group ranged from 4 to 621 depending upon the dihydronicotinamide and for the best substrate the value of $k_{\text {cat }} / K_{\mathrm{M}}$ was $5.7 \times 10^{5} \mathrm{M}^{-1} \mathrm{~S}^{-1}$ a value comparable with native flavoenzymes (Levine and Kaiser 1978). In addition the derivative did show some typical enzymatic properties including saturation kinetics and a stereochemical preference for the 4A hydrogen atom in the oxidation of NADH (Levine and Kaiser 1980).

Modifications at the active site are not the only way that new enzyme activities can be generated. Margalit et al. (1983) labelled three surface histidine residues in myoglobin with pentammineruthenium groups. After

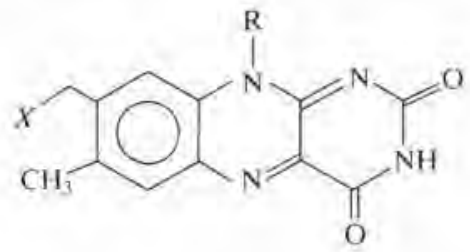

(I)

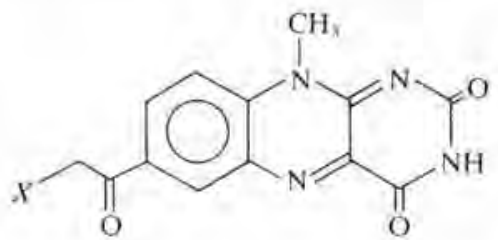

(II)

Fig. 8.5 Two flavin derivatives used in the semi-synthesis of flavopapain. $X$ is $\mathrm{Br}$ or S-Papain.

The derivative (I) (where $X=\mathrm{S}$-Papain) is a much less active enzyme than (II). 
modification the haem centre exhibited stronger anion binding. More interestingly the haem also had good oxygen-dependent ascorbate and durohydroquinone oxidase activities.

\subsubsection{Semi-synthesis}

The use of semi-synthesis of substitute proteins with naturally occurring amino acids has been superseded by the use of oligonucleotide-directed mutagenesis (vide supra). Chemical methods may still be appropriate when substitution by an unusual amino acid is desired. The review by Chaiken (1981) and the book by Offord (1979) cover this area.

\subsubsection{Modification by immobilization}

The immobilization of proteins on many different supports using a wide variety of chemistries has been extensively reviewed in the books by Chibata (1978), Zaborsky (1973), and Mosbach (1976), see also Chapter 6. Analytical applications have been described by Carr and Bowers (1980). In the space available we cannot do justice to all of the work that has been carried out and the reader is urged to consult the sources cited above for specific details and references to the original literature. In this section we will just point out some of the typical effects that immobilization can have.

8.3.7.1 Apparent activity Often the apparent activity of an enzyme is reduced after immobilization. This may be due to a number of factors; sometimes the immobilization chemistry leads to modification of an active-site residue that is important for catalytic function. Such a process might be anticipated as many active-site residues have an enhanced reactivity, although they may also be sterically protected. If this type of inactivation does occur then either an alternative coupling chemistry should be sought or conditions chosen to suppress reaction at the active site, e.g. by performing the immobilization in the presence of substrate, product, or inhibitor.

Even when the immobilization does not directly interfere with the enzyme-substrate reaction the nature of the support may give rise to diffusional barriers. For example in the work of Schaefer and Wilson (1983) on the reactivity of cytochrome $c$ immobilized on a bead-formed agarose support the authors found that although they could preserve much of the activity of the cytochrome $c$ with both oxidase and, to a lesser extent, reductase, only the cytochrome bound on the surface of the bead reacted. The submitochondrial particles used as the substrate were excluded from the majority of the cytochrome $c$ which was inside the porous beads. Similarly it has been found that immobilized hydrolases show a higher apparent activity with small substrates than with macromolecules. In general bead-formed supports show less diffusional restriction than fibrous ones and response times for reactions occurring at planar surfaces tend to be faster than those in porous materials. 
In contrast to these observations, under some circumstances, particularly with coupled-enzyme systems, the immobilized preparation may show an apparently higher overall activity than the soluble one. Under these conditions substrate channeling occurs and the co-immobilization results in a shorter diffusion path between the enzymes, or expressed another way, a higher local concentration of the intermediate product results in an apparently increased activity.

Careful choice of support and coupling chemistry are therefore needed to optimize activity and response time. Also the choice of immobilization method must be compatible with the final configuration and ease of manufacture of the sensor. Finally even if the support and coupling reactions are appropriate the very act of immobilization may reduce the conformational mobility and hence activity of the protein.

8,3.7.2 Stability There is a general feeling that immobilization leads to an improvement in stability of the bound protein towards physical and chemical stresses. Chibata (1978) reports that of 50 enzyme immobilizations $60 \%$ showed an increase in stability, $16 \%$ showed a decrease, and $24 \%$ were unaffected. However having said that, it must be appreciated that stability towards thermal denaturation, proteolytic degradation, $\mathrm{pH}$ extremes, or chemical denaturants may each reflect quite different aspects of protein unfolding. Similarly operational and storage stabilities may rely on quite different physical or chemical processes. At present the best approach is again empirical and it may be necessary to identify the causes of activity loss in order to guide an appropriate immobilization strategy.

\subsubsection{Partitioning Although it is often necessary for the immobilization} matrix to be simply an inert support for the active biological material there may be instances were a more active role can also be assumed. Under such circumstances the matrix can provide a micro-environment that is different from the bulk solution. This effect is closely akin to the protein surface modifications discussed earlier in this chapter. A positively charged matrix will tend to exclude protons so an enzyme in this matrix will exhibit an apparent optimum $\mathrm{pH}$ lower than its normal value. Similarly a hydrophobic substrate will tend to partition into a hydrophobic matrix and thus lower the apparent $K_{\mathrm{M}}$ for that substrate. Conversely the matrix may be designed to exclude interfering substances present in the analyte mixture. Such effects are relatively easy to predict and are likely to become more important as attempts are made to produce biosensors to fulfill more stringent operational criteria.

Finally in this section on immobilization we should mention co-enzyme immobilization (Mosbach 1976). Biosensors using freely diffusing coenzymes suffer from a potential problem due to loss of the co-enzyme from 
the sensor. One solution to this is to immobilize the co-enzyme to a support, either soluble or insoluble, in order to retain it.

\subsection{Conclusions}

In this chapter we have sought to describe the many different ways that are available for altering the properties of proteins and thus render them potentially more suitable for application in biosensors. The most powerful and versatile of these techniques is undoubtedly that of oligonucleotide-directed mutagenesis; however the amount of background information necessary to use it in specific applications is substantial and may not be available for the particular enzyme of interest. Indeed it is true to say that at present none of the important enzymes in biosensors is being subjected to this technique. Tailoring surface properties is another approach that should be widely applicable and is capable of predictive use even in the absense of crystallographic data.

We can summarize this chapter by confidently predicting that the engineering of proteins for biosensors, though far less developed than for other applications at present, is likely to become important in the next generation of these devices,

\section{References}

Ackers, G.K. and Smith, F. R. (1985). Effects of site specific amino acid modification of protein interaction and biological function. Ann. Rev. Biochem. 54, 597-629.

Barbehenn, E. K. and Kaufman, B. T. (1980). Alteration of the properties of chicken liver dihydrofolate reductase as a result of modification by tetrathionate. J. Biol. Chem. 255, 1978-84.

Bolivar, F., Rodriguez, R. L., Greene, P. J. Betlach, M. C., Heyner, H. L., Boyer, H. W., Crosa, J. H, and Falkow, S. (1977). Construction and characterisation of new cloning vehicles. II. A multipurpose cloning system. Gene 2, 95-113.

Bright, H. J. and Porter, D. J. T. (1975). Flavoprotein oxidases The enzymes XII, 421-511.

Burkey, K.O. and Gross, E. L. (1982). Chemical modification of spinach plastocyanin: separation and characterization of four different forms. Biochemistry (USA) 21, 5886-90.

Carlberg, I. and Mannervik, B. (1980). Oxidative activity of glutathione reductase effected by $2,4,6$,-trinitrobenzenesulfonate. FEBS Lett. 115, 265-8,

Carr, P. W. and Bowers, L. D. (1980). Immobilised enzymes in analytical and clinical chemistry. Wiley, New York.

Carter, P. J., Winter, G., Wilkinson, A. J. and Fersht, A. R. (1984). The use of double mutants to detect structural changes in the active site of the tyrosyl-tRNA synthetase (Bacillus stearothermophilus). Cell 38, 835-40. 
Chaiken, I. M. (1981), Semi-synthetic peptides and proteins. Crif. Rev. Biochem. 11. 255-301.

Chibata, I. (1978). Immobilised enzymes. Wiley, New York.

Cohen, S. N., Chang, A. C. Y., Boyer, H. W. and Helling, R. B. (1973). Construction of biologically functional bacterial plasmids in vitro. Proc. Natl. Acad. Sci. (USA) 70, 3240-4.

Cupo, P., El-Deiry, W., Whitney, P. L. and Awad, W. M. (1980). Stabilization of proteins by guanidination. J. Biol. Chem. 255, 10828-33.

Dalbadie-McFarland, G., Cohen, L. W., Riggs, A. D., Morin, D., Itakura, K. and Richards, J.H. (1982). Oligonucleotide-directed mutagenesis as a general and powerful method for studies of protein function. Proc. Natl. Acad. Sci. (USA) 79, 6409-13.

Edge, M.D., Greene, A. R., Heathcliffe, G. R., Meacock, P. A., Schuck, W., Scanlon, P. B., Atkinson, T. C., Newton, C. R. and Markham, A. F. (1981). Total synthesis of a human leukocyte interferon gene. Nature 292, 756-62.

Fersht, A. R., Shi, J. P., Wilkinson, A. J., Blow, D. M., Carter, P., Waye, M. M. Y, and Winter, G.P. (1984). Analysis of enzyme structure and activity by protein engineering. Angew. Chem. (Int. Ed.) 23, 467-73.

- Wilkinson, A. J., Carter, P. J. and Winter, G. (1985a) Fine structure-activity analysis of mutations at position 51 of tyrosyl t-RNA synthetase. Biochemistry (USA) 24, 5858-61.

—- Shi, J. P., Knill-Jones, J., Lowe, D. M., Wilkinson, A. J., Blow, D. M., Brick, P., Carter, P., Waye, M. M. Y. and Winter, G. (1985b). Hydrogen bonding and biological specificity analyzed by protein engineering. Nature 314, 235-8.

Fried, H. E. and Kaiser, E. T. (1981). Oxidation of dithiols by flavopapain. J. Amer. Chem. Soc. 103, 182-4.

Gardell, S. J., Craik, C. S., Hilvert, D., Urdea, M. S. and Rutter, W. J. (1985). Sitedirected mutagenesis shows that tyrosine 248 of carboxypeptidase A does not play a crucial role in catalysis. Nature $317,551-5$.

Gillam, S. and Smith, M. (1979). Site-specific mutagenesis using synthetic oligodeoxyribonucleotide primers: I, Optimum conditions and minimum oligodeoxyribonucleotide length. Gene 8, 81-97.

Hutchinson, C. A., Phillips, S., Edgell, M. H., Gillam, S., Jahnke, P. and Smith, M. (1978). Mutagenesis at a specific position in a DNA sequence. J. Biol. Chem. 253, $6551-60$.

Jones, H. D., McMillan, A. J., Fersht, A. R. and Winter, G. (1985). Reversible dissociation of dimeric tyrosyl-tRNA synthetase by mutagenesis at the subunit interface. Biochemistry (USA) 24, 5852-7.

Kaiser, E.T., Levine, H.L., Otsuki, T. and Fried, H.E. (1980). Studies on the mechanism of action and stereochemical behaviour of semisynthetic model enzymes. Adv. Chem. Ser. 191, 35-48.

- Lawrence, D.S. and Rokita, S.E. (1985). The chemical modification of enzymatic specificity. Ann. Rev. Biochem. 54, 565-95.

Kaminski, Z. W. and Jezewska, M. M. (1979). Intermediate dehydrogenase-oxidase form of xanthine oxidoreductase in rat liver. Biochem. J. 181, 177-82.

Levine, H. L., Nakagawa, Y. and Kaiser, E.T. (1977). Flavapapain: Synthesis and properties of semisynthetic enzymes. Biochem. Biophys. Res. Commun. 76, 64-70. 
Levine, H. L, and Kaiser, E,T. (1978). Oxidation of Dihydonicotinamides by Flavopapain. J. Amer. Chem. Soc. 100, 7670-7.

- (1980). Stereospecificity in the oxidation of NADH by flavopapain. J. Amer. Chem. Soc. 102, 343-5.

Lowe. D. M., Fersht, A. R., Wilkinson, A. J., Carter, P. and Winter, G. (1985). Probing histidine-substrate interactions in tyrosyl-tRNA synthetase using asparagine and glutamine replacements. Biochemistry (USA) 24, 5106-9.

Mansson, M.-O., Larrsson, P.-O. and Mosbach, K. (1978). Covalent binding of an NAD analogue to liver alcohol dehydrogenase resulting in an enzyme-coenzyme complex not requiring exogenous coenzyme for activity. Eur. J. Biochem. 86, 455-63.

- (1979). Recycling by a second enzyme of NAD covalently bound to alcohol dehydrogenase. FEBS Lett 98, 309-13.

Margalit, R., Pecht, I. and Gray, H. B. (1983). Oxidation-reduction catalytic activity of a petaamineruthenium (III) derivative of sperm whale myoglobin. J. Amer. Chem. Soc. 105, 301-2.

Messing, J. (1983). New M13 vectors for cloning. Meth. Enzymol. 101, 20-78.

Mosbach, K. (1976), Immobilised enzymes, Meth. Enzymol. XLIV.

Offord, R. E. (1979). Semisynthetic proteins. John Wiley, Chichester.

Perry, L. J. and Wetzel, R. (1984). Disulfide bond engineered into T4 lysozyme: Stabilization of the protein towards thermal inactivation. Science 226, 555-7.

Plapp, B. V. (1970). Enhancement of the activity of horse liver alcohol dehydrogenase by modification of amino groups at the actives sites. J. Biol. Chem. 245, 1727-35.

Razin, A., Hirose, T. Itakawa, K. and Riggs, A. D. (1978). Efficient correction of a mutation by use of chemically synthesised DNA. Proc. Natl. Acad. Sci. (USA) 75, 4268-75.

Rosemeyer, H., Kornig, E. and Seela, F. (1982). Adenosine deaminase covalently linked to soluble dextran. The effect of immobilisation on thermodynamic and kinetic parameters, Eur, J. Biochem. 122, 375-80.

Sanger, F., Nicklen, S. and Coulson, A. R. (1977), DNA sequencing with chain terminating inhibitors. Proc. Natl. Acad. Sci. (USA) 74, 5463-7.

Schaefer, M. A. and Wilson, G.S. (1983). Spectral and electron transfer properties of sepharose 6MB-immobilized cytochrome c. J. Biol. Chem. 258, 12835-41.

Shimizu, T. (1979). Enhancement of $14 \mathrm{~S}$ and $30 \mathrm{~S}$ dynein adenosine triphosphatase activities by modification of amino groups with trinitrobenzenesulfonate. A comparison with modification of SH groups. J. Biochem. (Tokyo) 85, 1412.

Shortle, D, and Nathans, D. (1978). Local mutagenesis: a method for generating viral mutants with base substitutions in preselected regions of the viral geonome. Proc. Natl. Acad. Sci. (USA) 75, 2170-4.

_-, Grisafi, P., Benkovic, S. J. and Botstein, D. (1982). Gap misrepair mutagenesis: efficient site directed induction of transition, transversion and frameshift mutations in vitro. Proc. Natl. Acad. Sci. (USA) 79, 1588-92.

Sigal, I. S., Harwood, B. G. and Arentzen, R. (1982). Thiol- $\beta$-lactamase: Replacement of the active site serine of RTEM $\beta$-lactamase by a cysteine residue. Proc. Natl. Acad. Sci. (USA) 79, 7157-60.

—- DeGrado, W. F., Thomas, B. J. and Petteway, S. R. (1984). Purification and properties of thiol $\beta$-lactamase. J. Biol. Chem, 259, 5327-32. 
Slama, J.T., Orugati, S. R. and Kaiser, E. T. (1981). Semisynthetic enzymes. Synthesis of a new flavopapain with high catalytic efficiency. J. Amer. Chem. Soc. 103, 6211-3.

Spomer, W. E. and Wotton, J.F. (1971). The hyrolysis of $\alpha^{\prime}-N$-benzoyl-Largininamide catalysed by trypsin and acetyltrypsin. Dependence on pH. Biochim. Biophys. Acta 235, 164-171.

Sproat, B.S. and Gait, M. J. (1984). Solid-phase synthesis of oligodeoxyribonucleotides by the phosphotriester method. In Oligonucleotide synthesis - a practical approach (ed. M. J, Gait) pp, 83-115. IRL Press, Oxford.

Thomas, P. G., Russell, A. J. and Fersht, A. R. (1985). Tailoring the pH dependence of enzyme catalysis using protein engineering. Nature 318, 375-6.

Urabe, 1., Yamamoto, M., Yamada, Y. and Okada, H. (1978). Effect of hydrophobicity of acyl groups on the activity and stability of acylated termolysin. Biochim. Biophys. Acta 524, 435-41.

Valenzuela, P. and Bender, M.L. (1971). Kinetic properties of succinylated and ethylenediamine-amidated $\delta$-chymotrypsins. Biochim. Biophys. Acta 250, 538-48.

Villafranca, J.E., Howell, E.E., Voet, D.H., Strobel, M.S., Ogden, R.C., Abelson, J.N. and Kraut, J. (1983). Directed mutagenesis of dihydrofolate reductase. Science $222,782-8$.

Wallace, R. B., Schold, M., Johnson, M. J., Dembek, P. and Itakura, K. (1981). Oligonucleotide directed mutagenesis of the human $\alpha$-globin gene: a general method for producing point mutations in cloned DNA. Nucleic Acids Res. 9, 3647-56.

Wells, T. N. C. and Fersht, A. R. (1985). Hydrogen bonding in enzymatic catalysis analyzed by protein engineering. Nature 316, 656-7.

Wilkinson, A.J., Fersht, A. R., Blow, D. M. and Winter, G. (1983). Site-directed mutagenesis as a probe of enzyme structure and catalysis: tyrosyl-tRNA synthetase cysteine 35 to glycine 35 mutation. Biochemistry (USA) 22, 3581-6.

—, Carter, P. and Winter, G. (1984). A large increase in enzyme-substrate affinity by protein engineering. Nature 307, 187-8.

Winter, G., Fersht, A. R., Wilkinson, A. J., Zoller, M. and Smith, M. (1982). Redesigning enzyme structure by site-directed mutagenesis: Tyrosyl t-RNA synthetase and ATP binding. Nature 299, 756-8.

Zaborsky, O. R. (1973). Immobilised enzymes. CRC Press, Ohio.

Zoller, M. J. and Smith, M. (1983). Oligonucleotide-directed mutagenesis of DNA fragments cloned into M13 vectors. Meth. Enzymol. 100, 468-500. 


\section{Bioelectrochemistry \\ (a) Potentiometric sensors}





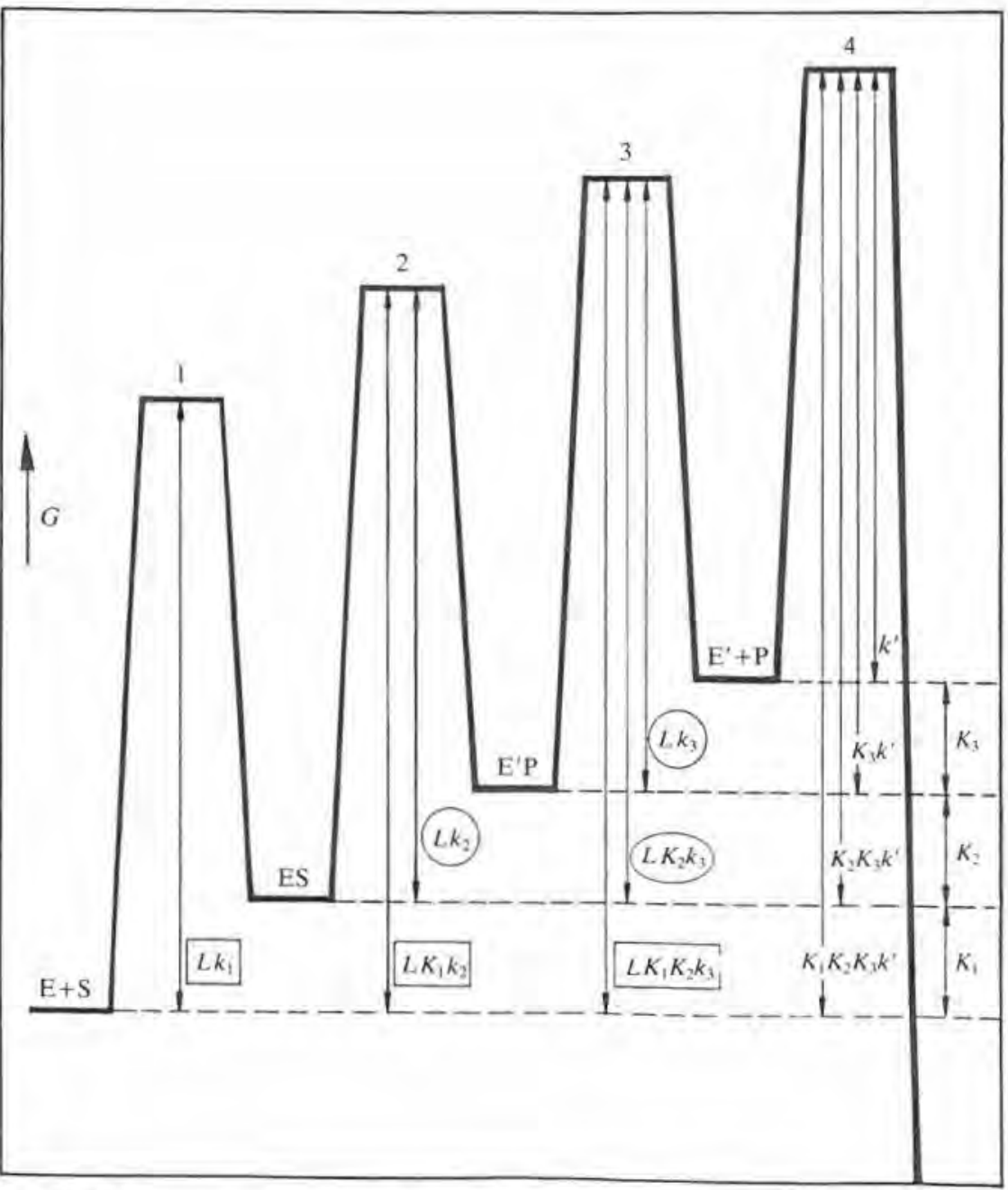

Fig. 12.2 Schematic free-energy profile illustrating the free-energy differences associated with each of the ten possible rate-limiting terms in eqns 12.8-12.10. The three terms that make up $k_{\text {cat }}$ in eqn 12,9 are circled and the three terms that make up $k_{\text {cat }} / K_{\mathrm{M}}$ in eqn 12.10 are boxed. The four terms in the bottom row where the reactants are $\mathrm{E}+\mathrm{S}$ make up the $s_{\infty}$ terms in eqn 12.8. The rest of the terms are found in the first term of eqn 12.8 .

would expect from the external concentration $s_{\infty}$. This effect is described by the first bracket which reduces the significance of the $k_{\text {cat }}$ term.

Secondly, the simple term $k^{\prime-1}$ will be dominant if the electrode kinetics are rate limiting and if nearly all the enzyme is converted to $E^{\prime}$; these conditions 


\subsection{The steady-state equation}

In the steady state we can write for the flux, $j$, (usually measured in $\mathrm{mol} \mathrm{cm}-2$ $\mathrm{s}^{-1}$ ):

$$
\begin{aligned}
j & =k_{\mathrm{S}}^{\prime}\left(s_{\infty}-s_{0}\right) \\
& =L\left[k_{1} s_{0} e-k_{-1} e s\right] \\
& =L\left[k_{2} e s-k_{-2} e^{\prime} p\right] \\
& =L\left[k_{3} e^{\prime} p-k_{-3} p_{\infty} e^{\prime}\right] \\
& =k_{\mathrm{P}}^{\prime}\left(p_{0}-p_{\infty}\right) \\
& =k^{\prime} e^{\prime} .
\end{aligned}
$$

We also have that the total concentration of enzyme, $e_{\Sigma}$, is given by

$$
e_{\Sigma}=e+e s+e^{\prime} p+e^{\prime} .
$$

Elimination of the six unknowns, $s_{0}, p_{0}$, and the four enzyme concentrations, between equs 12.1 to 12.7 gives:

$$
\begin{aligned}
\frac{e_{\Sigma}}{j}= & \left\{1-\frac{j}{k_{\mathrm{S}}^{\prime} s_{\infty}}\right\}\left\{\frac{1}{L k_{\mathrm{cat}}}+\frac{1}{k^{\prime}}+\frac{K_{3}^{-1}\left(1+K_{2}^{-1}\right)}{k^{\prime}}\left[\mathrm{p}_{\infty}+\frac{j}{k_{\mathrm{p}}^{\prime}}\right]\right\} \\
& \left.+\frac{1}{s_{\infty}} \frac{K_{\mathrm{M}}}{L k_{\mathrm{cat}}}+\frac{K_{1}^{-1} K_{2}^{-1} K_{3}^{-1}}{k^{\prime}}\left[p_{\infty}+\frac{j}{k_{\mathrm{p}}^{\prime}}\right]\right\}+\frac{\mathrm{e}_{\mathrm{\Sigma}}}{k_{\mathrm{S}}^{\prime} s_{\infty}}
\end{aligned}
$$

where

$$
\frac{1}{k_{\text {cat }}}=\frac{1}{k_{2}}+\frac{1}{K_{2} k_{3}}+\frac{1}{k_{3}}
$$

and

$$
\frac{K_{\mathrm{M}}}{k_{\text {cat }}}=\frac{1}{k_{1}}+\frac{1}{K_{1} k_{2}}+\frac{1}{K_{1} K_{2} k_{3}} .
$$

These expressions for $k_{\text {cat }}$ and $K_{\mathrm{M}} / k_{\text {cat }}$ have been discussed by Albery and Knowles (1976). The free energy diagram in Fig. 12.2 shows how each term in eqns 12.8 to 12.10 corresponds to a possible rate limiting free energy difference in the enzyme kinetics. The advantage of the reciprocal expressions in eqns 12.8 to 12.10 is that the different possible rate limiting processes are separated in this way (Albery and Knowles 1976). We now discuss the various terms in eqn 12.8.

First there are two terms which include $L$. These terms can only be dominant if the enzyme kinetics are rate limiting; the first of these terms with $k_{\text {cat }}$ corresponds to the saturated enzyme and the second terms with $K_{\mathrm{M}} / k_{\text {eat }}$ to the unsaturated enzyme. If the flux, $j$, becomes close to the limit imposed by transport through the membrane, $j \approx k_{\mathrm{S}}^{\prime} s_{\infty}$, then the concentration polarization means that the enzyme inside the membrane is less saturated than one 


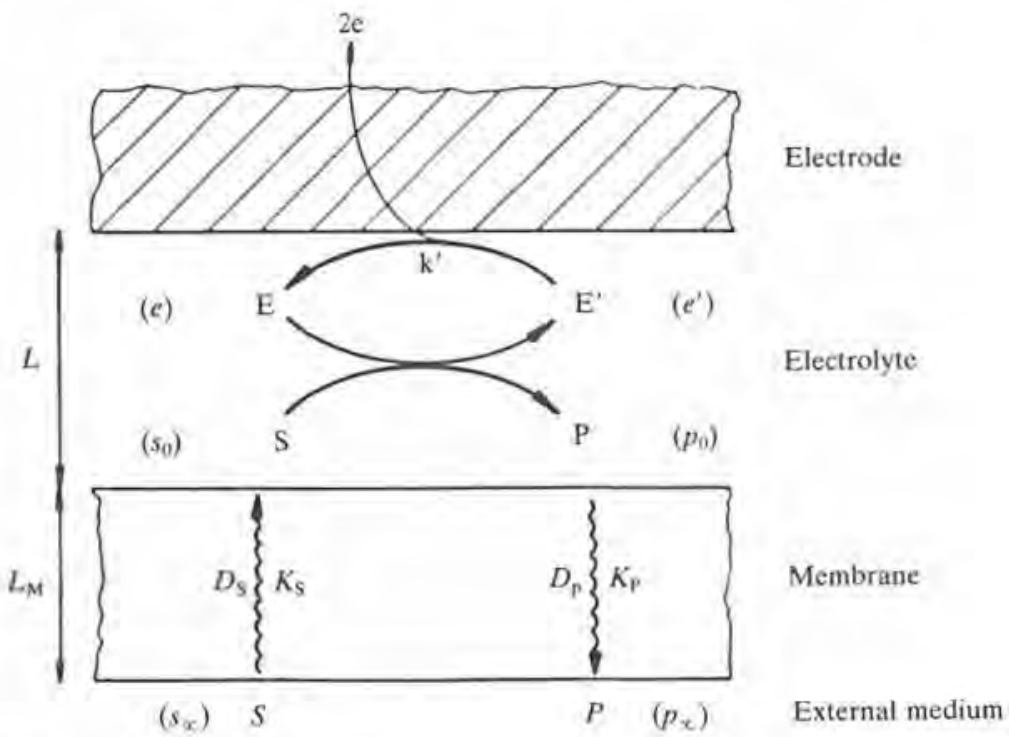

Fig. 12.1 The enzyme electrode.

For each step in the above scheme we write:

$$
K_{n}=k_{n} / k_{-n}
$$

and also

$$
K_{\mathrm{TD}}=K_{1} K_{2} K_{3}
$$

where $K_{\mathrm{TD}}$ describes the overall equilibrium between $\mathrm{S}+\mathrm{E}$ and $\mathrm{P}+\mathrm{E}^{\prime}$.

The transport of $\mathrm{S}$ and $\mathrm{P}$ through the membrane is described by the masstransfer rate constants (Albery 1975) $k_{\mathrm{S}}^{\prime}$ and $k_{\mathrm{p}}^{\prime}$ where

$$
k_{\mathrm{x}}^{\prime}=D_{X} K_{X} / L_{\mathrm{M}} \text {. }
$$

$X$ is either S or P, $D_{X}$ is the diffusion coefficient, $K_{X}$ the partition coefficient of $X$ in the membrane, and $L_{M}$ is the thickness of the membrane. The electrode reaction, described by $k^{*}$, is assumed to be irreversible. All the primed rate constants are heterogeneous rate constants, usually measured in $\mathrm{cm} \mathrm{s}^{-1}$. Lower case letters are used to denote the concentrations of the different species, and for $\mathrm{S}$ and $\mathrm{P}$ the subscripts $\infty$ and 0 refer to the concentrations outside and inside the membrane respectively. We assume that the electrolyte layer behind the membrane is so thin ( $\sim$ a few microns) that there is no concentration polarization in this layer. This case occurs most frequently in practice. We have considered elsewhere the effects of concentration polarization (Albery and Bartlett 1985). 
Glucose + GOD/FAD $\longrightarrow$ Gluconolactone + GOD/FADH ${ }_{2}$

Electrode

$$
\mathrm{GOD} / \mathrm{FADH}_{2} \longrightarrow \mathrm{GOD} / \mathrm{FAD}+2 \mathrm{H}^{+}+2 \mathrm{e}
$$

This reaction scheme is the simplest possible. Examples of this third generation of enzyme electrodes have been recently reported (Albery and Bartlett 1985; Albery et al, 1985). Following on from the work of Kulys and co-workers (Kulys et al, 1980; Cenas and Kulys 1981), it was found that the oxidation of the enzyme could be achieved using conducting organic salts such as NMP* ${ }^{*}$ TCNQ $^{-}$as electrode materials (see also Chapter 15).<smiles>C[n+]1c2ccccc2nc2ccccc21</smiles><smiles>N#CC(C#N)=c1ccc(=C(C#N)C#N)cc1</smiles>

$\mathrm{NMP}^{+}$

TCNQ

In developing enzyme electrodes it is important to be able to identify the rate-limiting step that determines the overall performance of the device. This step could be transport of the substrate through the membrane, reaction of the substrate with the enzyme, transport of the product back through the membrane, or regeneration of the enzyme. We start therefore by developing a model for the enzyme electrode. Since the third-generation electrode has the simplest reaction scheme and we wish to avoid unnecessary algebra, we will develop the model for this type of electrode. We will later extend the treatment to include second-generation electrodes. We will present a simple diagnostic plot of experimental data which identifies the rate-limiting step.

\subsection{The model}

Figure 12.1 illustrates the third-generation enzyme electrode and the kinetic scheme. As regards the enzyme kinetics we assume the following model for a one substrate one product enzyme, which converts substrate S to product $\mathrm{P}$, and which in the course of this conversion is itself converted from E to $E^{\prime}$ :

$$
\mathrm{S}+\mathrm{E} \underset{k_{-1}}{\stackrel{k_{1}}{\rightleftarrows}} \mathrm{ES} \underset{k_{-2}}{\stackrel{k_{2}}{\rightleftarrows}} \mathrm{E}^{\prime} \mathrm{P} \underset{k_{-3}}{\stackrel{k_{3}}{\rightleftarrows}} \mathrm{E}^{\prime}+\mathrm{P}
$$




\title{
Amperometric enzyme electrodes: theory and experiment
}

\author{
W. JOHN ALBERY and DEREK H. CRASTON
}

\subsection{Introduction}

Amperometric enzyme electrodes combine the advantages of the specificity of the enzyme for recognizing particular target molecules with the direct transduction of the rate of reaction into a current. The first generation of devices of this sort, for instance the glucose electrode (Chapter 1; Clark and Lyons 1962; Guilbault and Lubrano 1973), relied on the natural enzymatic reaction:

$$
\text { Glućose }+\mathrm{O}_{2} \stackrel{\text { GOD }}{\longrightarrow} \text { Gluconolactone }+\mathrm{H}_{2} \mathrm{O}_{2}
$$

where GOD is glucose oxidase [EC 1.1.3.4]. The electrode is merely used to measure the concentration of either the natural substrate $\mathrm{O}_{2}$ or the product $\mathrm{H}_{2} \mathrm{O}_{2}$. This has to be a fairly complicated device with two membranes; furthermore the response of the device is affected by the ambient concentration of $\mathrm{O}_{2}$.

More recently second generation systems have been developed (Chapters 15 and 16; Cass et al. 1984a, b) in which the enzyme performs the first redox reaction with its substrate, but is then reoxidized by a mediator as opposed to oxygen; the mediator in its turn is oxidized by the electrode:

$$
\begin{aligned}
\text { Glucose }+\mathrm{GOD} / \mathrm{FAD} & \longrightarrow \text { Gluconolactone + GOD/FADH } \\
\text { GOD/FADH } & \longrightarrow \mathrm{F}+\mathrm{FOD}_{2}+\mathrm{FAD}+2 \mathrm{M}^{\prime}+2 \mathrm{H}^{*}
\end{aligned}
$$

Electrode

$$
2 \mathrm{M}^{\prime} \longrightarrow 2 \mathrm{M}+2 \mathrm{e}
$$

In this scheme FAD represents a flavin redox centre in glucose oxidase and the mediator, $\mathrm{M} / \mathrm{M}^{i}$, has been assumed to be a one-electron couple. Hill, Higgins, and co-workers (Cass et al. 1984a, b) have shown that various ferrocene ferrocinium couples are efficient mediators; other species such as, $\left[\mathrm{Fe}(\mathrm{CN})_{6}\right]^{3-}$ and $\mathrm{N}$-methylphenazinium $\left(\mathrm{NMP}^{+}\right)$are also mediators.

An even simpler and more direct method is to have no mediator but to find an electrode material on which the reduced enzyme GOD/FADH ${ }_{2}$ can be directly oxidized:- 
Chromatogr. 6, 1727-43.

Roston, D. A. and Kissinger, P. T. (1982). Series dual electrode detector for liquid chromatography/electrochemistry. Anai. Chem. 54, 429-34.

Shu, F. R, and Wilson, G. S. (1976). Rotating ring-disk electrode for surface catalysis studies. Anal. Chem. 48, 1679-1686.

Sittampalam, G. and Wilson, G.S. (1983). Surface modified electrochemical detector for liquid chromatography. Anal. Chem. 55, 1608-10.

Stamford, J.A., Kruk, Z. L., Millar, J. and Wightman, R. M. (1984). Striatal dopamine uptake in the rat: In-vivo analysis by fast cyclic voltammetry. Neurosci. Lett. 51, 133-8.

Stutts, K. J., Kovach, P.M., Kuhr, W. G. and Wightman, R. M. (1983). Enhanced electrochemical reversibility at heat-treated glassy carbon electrodes. Anal. Chem. $55,1632-4$.

— and Wightman, R.M. (1983). Electroanalysis of ascorbate oxidation with electrosynthesized surface-bound mediators. Anal. Chem. 55, 1576-9.

Swartz, D. B. and Wilson, G. S. (1971). Small-volume coulometric redoxostat. Anal. Biochem. 40, 392-400.

Thévenot, D. R., Sternberg, R., Coulet, P. R., Laurent, J. and Gautheron, D. C. (1979), Enzyme collagen membrane for electrochemical determination of glucose. Anal. Chem. 51, 96-100.

Wang, J. and Freiha, B.A. (1984). Extractive preconcentration of organic compounds at carbon paste electrode. Anal. Chem. 56, 849-52.

Hutchins, L. D. (1985). Thin-layer electrochemical detector with a glassy carbon electrode coated with a base-hydrolyzed cellulosic film. Anal. Chem, 57, 1536-41.

Weber, S. G. (1983). The dependence of current on flow rate in thin-layer electrochemical detectors used in liquid chromatography. J. Electroanal. Chem. 145, 1-7.

Weisshaar, D. E. and Kuwana, T. (1984). Electrodeposition of metal micro-particles in a polymer film on a glassy carbon electrode. J. Electroanal. Chem. 163, 395-9.

Wightman, R. M., Deakin, M.R., Kovach, P.M., Kuhr, W. G, and Stutts, K.J. (1984). Methods to improve electrochemical reversibility at carbon electrodes. $J$. Electrochem. Soc. 131, 1578-83. 
Horvath, C. and Engasser, J. M. (1974). External and internal diffusion in heterogeneous enzyme systems. Biotechnol. Bioeng. 16, 909-23.

Howell, J. O. and Wightman, R. M. (1984). Ultrafast voltammetry and voltammetry in highly resistive solutions with microvoltammetric electrodes. Anal. Chèm. 56, 524-9.

Ianniello, R. M., Lindsay, T. J. and Yacynych, A. M. (1982). Differential pulse voltammetric study of direct electron transfer at glucose oxidase chemically modified graphite electrodes. Anal. Chem. 54, 1098-1101.

Ikariyama, Y., Kunoh, H. and Aizawa, M. (1985). Sensitive bioaffinity sensor with metastable molecular complex receptor and enzyme amplifier. Anal. chem. 57, 496-500.

Ito, M. and Kuwana, T, (1971). Spectroelectrochemical study of indirect reduction of triphosphopyridine nucleotide. J. Electroanal. Chem. 32, 415-25.

Kao, W.H. and Kuwana, T. (1984), Electrocatalysis of electrodeposited spherical Pt microparticles dispersed in a polymeric film electrode. J. Am. Chem. Soc, 106, 473-6.

Krull, 1. S., Bratin, K., Shoup, R. E., Kissinger, P. T. and Blank, C. C. (1983). LCEC for trace analysis: recent advances in instrumentation, methods and applications. Am. Lab. 15, 57-65.

Kovach, P. M., Caudill, W. L., Peters, D. G. and Wightman, R. M. (1985). Faradaic electrochemistry at microcylinder, band and tubular band electrodes. $J$. Electroanal. Chem. 185, 285-95.

Kulys, J, J., Samaline, A.S. and Svirmickas, G. J.S. (1980). Electron exchange between the enzyme active center and organic metal. FEBS Lett. 114, 7-10.

Lane, R.F. and Hubbard, A. T. (1976). Differential double pulse voltammetry at chemically modified platinum electrodes for in vitro determination of catecholamines. Anal. Chem. 48, 1287-93.

Levich, V. G. (1962), Physiocochemical hydrodynamics, Prentice-Hall, Englewood Cliffs. N. J. USA.

Leypoldt, J. K. and Gough, D. A. (1984). Model of a two-substrate enzyme electrode for glucose. Anal. Chem. 56, 2896-2904.

Lin, P. Y. T., Bulawa, M. C., Wong, P., Lin, L., Scott, J. and Blank, C. L. (1984). The determination of catecholamines, indoleamines, metabolites, and related activities using three micron liquid chromatography columns. J. Liq. Chrom, 7, 509-38.

Nagy, G., Gerhardt, G. A., Oke, A. F., Rice, M. E., Adams, R. N. and Moore, R. B., III, Szentirmay, M.N. and Martin, C. R. (1985). Ion exchange and transport of neurotransmitters in nafion films on conventional and microelectrode surfaces. J. Electroanal. Chem. 188, 85-94.

Niki, K., Yagi, T., Inokuchi, H. and Kimura, K. (1979). Electrochemical behaviour of cytochrome $c_{3}$ of Desulfovibrio vulgaris, Strain Miyazaki, on the mercury electrode. J. Am. Chem. Soc. 101, 3335-40.

— Kobayashi, Y. and Matsuda, H. (1984). Determination of macroscopic standard potentials of a molecule with a reversible $n$-consecutive one electron transfer process, J. Electroanat. Chem. 178, 333-41.

Polta, J. A. and Johnson, D. C. (1983). The direct electrochemical detection of amino acids at a platinum electrode in an alkaline chromatographic effluent. J. Liq. 
electrochemical flow-through cells of the confined wall-jet type. J. Electroanal. Chem. 182, 295-313.

de Alwis, W. U. and Wilson, G.S. (1985). Rapid sub-picomole electrochemical enzyme immunoassay for immunoglobulin G. Anal. Chem. 57, 2754-56.

Doyle, M. J., Halsall, H. B. and Heineman, W, R. (1984). Enzyme linked immunosorbent assay with electrochemical detection for $\alpha_{1}$-acid glycoprotein. Anal, Chem. $56,2355-60$.

Dryhurst, G. (1977). Electrochemistry of biological molecules. Academic Press. New York.

- Kadish, K. M., Scheller, F, and Renneberg, R. (1982). Biological electrochemistry Vol. I. Academic Press, New York.

Durliat, H. and Comtat, M. (1984). Amperometric enzyme electrode for the determination of glucose based on thin-layer spectroelectrochemistry of glucose oxidase. Anal. Chem. 56, 148-52.

Eddowes, M. J, and Hill, H. A. O. (1979). Electrochemistry of horse heart cytochrome c. J. Am. Chem. Soc. 101, 4461-4464.

Albery, W.J., Hill, H.A. O, and Hillman, A. R. (1981). Mechanism of the reduction and oxidation reaction of cytochrome $c$ at a modified gold electrode. $J$. Am. Chem. Soc. 103, 3904-3910.

Eggers, H. M., Halsall, H. B. and Heineman, W. R. (1982). Enzyme immunoassay with flow amperometric detection of NADH, Clin. Chem. 28, 1848-51.

Gerhardt, G. A., Oke, A. F., Nagy, G., Moghaddem, B. and Adams, R. N. (1984). Nafion-coated electrodes with high selectivity for CNS electrochemistry. Brain Res. 290, 390-95.

Goldstein, L. (1976). Kinetic behaviour of immobilized enzyme systems. Meth. Enzymol. 44, 397-450.

Gorton, L., Torstensson, A., Jaegfeldt, H. and Johansson, G. (1984). Electrocatalytic oxidation of reduced nicotinamide coenzymes by graphite electrodes modified with an adsorbed phenoxazinium salt, meldola blue. J. Electroanal. Chem. 161, 103-20.

Gough, D. A. and Leypoldt, J. K. (1979). Membrane-covered, rotated disk electrode. Anal. Chem. 51, 439-444.

- $(1980 a)$. Rotated, membrane covered oxygen electrode. Anal. Chem. 52, 1126-30.

- (1980b). Transient studies of glucose, oxygen and hydroquinone at a membranecovered rotated disk electrode, $J$. Electrochem. Soc. 127, 1279-86.

Gunasingham, H. (1984). Large volume wall-jet cells as electrochemical detectors for high performance liquid chromatography. Anal. Chim. Acta 139, 193-47.

- and Fleet, B. (1983). Wall-jet electrode in continuous monitoring voltammetry. Anal. Chem. 55, 1409-14.

- Tay, B. T. and Ang, K. P. (1985). The electrolytic efficiency of amperometric detection on normal phase HPLC. Anal, Chim. Acta 176, 143-50.

Heineman, W. R. and Halsall, H. B. (1985). Strategies of electrochemical immunoassay. Anal. Chem. 57, 1321-31a.

Hinnen, C., Parsons, R. and Niki, K. (1983). Electrochemical and spectroreflectance studies of adsorbed horse heart cytochrome $c$ and cytochrome $c_{3}$ (D. vulgaris, Miyazaki strain) at a gold electrode, J, Electroanal. Chem. 147, 329-337. 
thus making it generally more widely applicable than potentiometric detection. The challenge lies in efficient coupling of biospecific reactions to the electrode response. Such sensors must operate in extremely heterogeneous environments and, in the case of implantable sensors, at $37^{\circ} \mathrm{C}$.

\section{References}

Adams, R.N. (1969). Electrochemistry at solid electrodes. Marcel Dekker, New York.

- (1976). Probing brain chemistry with electroanalytical techniques. Anal. Chem. 48, 1126A-1138A.

Albery, W. J. (1985). The current distribution on a wall-jet electrode. J. Electroanat. Chem. 191, 1-13.

- and Bartlett, P.N. (1985). Amperometric enzyme electrodes Part I. Theory. $J$. Electroanal. Chem. 194, 211-22.

- and Craston, D. H. (1985). Amperometric enzyme electrodes Part II. Conducting salts as electrode materials for the oxidation of glucose oxidase. $J$. Electroanal. Chem. 194, 223-35.

- Svanberg, L. R. and Wood., P. (1984). The estimation and identification of proteins by ring-disc titration Part IL. Application to liquid Chromatography. J. Electroanal. Chem. 162, 45-53.

Andrieux, C.P., Dumas-Bouchiat, J.M, and Saveant, J.M. (1984). Kinetics of electrochemical reactions mediated by redox polymer films. J. Electroanal. Chem. $169,9-21$.

Armstrong, F. A., Hill, H. A. O. and Walton, N. J. (1982), Direct electrochemical reduction of ferredoxin promoted by $\mathrm{Mg}^{2+}$. FEBS Letl. 145, 241-4.

Oliver, B. N. and Whitford, D. (1985). Direct electrochemistry of the photosynthetic blue copper protein plastocyanin. Electrostatic promotion of rapid charge transfer at an edge-oriental pyrolytic graphite electrode. J. Am. Chem. Soc, 107, 1473-6.

Bard, A. J. and Faulkner, L. R. (1980). Electrochemical Methods. Wiley, New York.

Blankespoor, R. L. and Miller, L. L. (1984). Electrochemical oxidation of NADH kinetic control by product inhibition and surface coating. J. Electroanal. Chem. 171, 231-41.

Boitieux, J. L., Thomas, D. and Desmet, G. (1984), Oxygen electrode-based enzyme immunoassay for the Amperometric determination of hepatitis B surface antigen, Anal. Chim. Acta 163, 309-13.

Brezina, M. and Zuman, P. (1958). Polarography in medicine, biochemistry and pharmacy. Interscience, New York.

Carr, P. W. and Bowers, L. D. (1980). Immobilized enzymes in analytical and clinical chemistry. Wiley, New York.

Clark, L. C. and Lyons, C. (1962). Electrode systems for continuous monitoring in cardiovascular surgery. Ann. N. Y. Acad. Sci. 102, 29-45.

Dalhuijsen, A. J., van der Meer, Th. H., Hoogendoorn, C. J. and van Bennekom, W. P. (1985). Hydrodynamic properties and mass transfer characteristics of 
Alwis and Wilson 1985). This assay can be performed with a precision and accuracy of $\pm 3 \%$ in less than 30 minutes.

Sensitivity of the enzyme immunoassay would be significantly enhanced if the immunosorbent were located as close to the amperometric sensor as possible. Unfortunately most immunological reactions have such large equilibrium constants that it is difficult to displace the antibody-antigen complex once formed. Ikariyama and co-workers (1985) have developed a clever system for displacement of the enzyme label from a membranecovered electrode so that the high affinity reaction occurs in solution and not at the sensor surface. Immunosensor surfaces yielding rapid and reproducible response are still rare.

Many biosensors are operated at a fixed potential. This provides the significant advantage of instrumental simplicity. However, there will always be a background current which can become significant at low analyte concentrations. Establishment of the background correction and in vivo calibration of biosensors are two challenging problems in need of reliable solutions. Fluctuation in these parameters can be due to 'poisoning' of the electrode by components in the medium. Sensitivity and response time are both adversely affected. If the fluctuating baseline is due to variations in concentrations of endogenous electroactive interference, then a dual (differential) electrode system may be employed. This approach has been used with the glucose electrode where one electrode is coated with a glucose oxidase membrane, the other electrode membrane contains no enzyme. Electroactive impurities are presumed to diffuse through both membranes in the same fashion (Thévenot et al. 1979). In cases where the electrode becomes fouled by matrix contaminants or by the product of an electrode reaction, a multipulse potential step protocol has been employed (Lane and Hubbard 1976; Polta and Johnson 1983). This can serve the function of preconditioning the electrode including removal of accumulated films as well as establishing a baseline in a potential region where no electrolysis occurs. Various forms of pulse polarography have also proven useful. Cyclic or linear sweep voltammetry is particularly useful in two kinds of applications. Many of the neuroactive substances are oxidized at approximately the same potential thus making it difficult to distinguish them. The complete cyclic voltammogram reflects the chemistry of the electrolysis products which are different. This information serves as a qualitative fingerprint (Stamford et al. 1984) as well as a means for quantitating overlapping electrochemistry. It has been recently shown (Wang and Freiha 1984) that organic molecules of biological interest can be concentrated on a treated electrode surface. Using a linear scan the deposited analyte is stripped from the surface yielding a well-defined peak.

Amperometry provides a promising approach to the development of both in vivo and in vitro biosensors. Wide dynamic range $\left(10^{4}\right.$ to $\left.10^{5}\right)$ is possible 
When inserted into living tissues as probes, such an electrode will sample the fluid in the 'pool' immediately surrounding the electrode.

Another extremely important electrode configuration is that of the flowthrough amperometric detector. The most commonly employed device consists of a thin-layer cell containing a planar electrode of Pt, Au, or glassy carbon. The counter electrode and reference electrode are located downstream. The cell resistance in this case can be quite high but again the currents are in the nano-ampere range so that IR drops are usually insignificant. This configuration has been very successfully and widely employed as a detector in liquid chromatography (LCEC) (Krull et al. 1983). Picomole detection limits have been reported (Lin et al. 1984). There have been a number of fundamental inyestigations of the behaviour of this type of electrode with a view to optimization of response (Gunasingham and Fleet 1983; Weber, 1983). This configuration has been used primarily for in vitro sampling often accompanied by sample clean-up and a chromatographic separation. Thévenot and co-workers, however, have demonstrated the use of such an enzyme electrode in conjunction with an extracorporeal shunt for real-time monitoring of blood glucose concentrations (see Chapter 22). The flowthrough detector is normally operated at a constant DC potential although scanning is possible in some situations. The thin-layer detector is a kind of flow reactor and one can use combinations of electrodes at different potentials to selectively convert or monitor species initially present in the sample or generated electrochemically within the detector (Roston and Kissinger 1982). The detector response is flow-rate dependent so it is important that this is carefully controlled, particularly at low detection limits. Pulsation of the solvent pump creates periodic variations in the signal. Under typical flow conditions $10-20 \%$ of the total electroactive material passing through the detector will be electrolysed. This usually produces a sharp peak whose height or area can be used as the basis for quantitation.

The sensitivity and selectivity of amperometric detection has made it well suited for immunoassays. This subject has been recently reviewed by Heineman and Halsall (1985). It is not generally feasible to detect directly antibodies electrochemically and thus it has been necessary to label the antibody or antigen (hapten) with an electroactive species. More commonly the activity of an enzyme, measured by determining the concentration of an electroactive product released in solution, forms the basis for an enzyme immunoassay. Because every mole of enzyme can produce in a reasonable time at least $10^{3}-10^{4}$ moles of product, an amplification results. Subpicomole detection is accordingly feasible. Assays for hepatitis B surface antigen (Boitieux et al. 1984), $\alpha_{\mathrm{I}}$ acid glycoprotein (Doyle et al. 1984) and phenytoin (Eggers et al. 1982) have been reported. An in situ flow-through immuno-reactor formed the basis for the femtomole detection of IgG (de 
why the response of an amperometric electrode will remain constant for an extended period and then suddenly drop. As has been pointed out (Horvath and Engasser 1974), the response of the sensor will be independent of enzyme activity as long as the activity is high enough. However, the enzyme decays gradually and eventually reaches the point where the response becomes kinetically controlled. At this point, the sensor response no longer remains constant. For more detailed discussion of the theory of enzyme electrode response and the behaviour of immobilized enzymes, the reader is referred to published work (Leypoldt and Gough 1984; Albery and Bartlett 1985; Goldstein 1976; Carr and Bowers 1980). An ideal situation would be one in which a thin membrane is employed, having the properties of favouring oxygen transport over glucose so that oxygen remains in excess within the reaction layer. The development of membranes with such special properties will undoubtedly profoundly influence the development of biosensors of all types.

\subsection{Electrodes and electrode geometry}

Although classical electrochemical measurements of analytes in biological media were made using the dropping mercury electrode (Brezina and Zuman 1958), solid electrodes constructed of $\mathrm{Pt}, \mathrm{Au}$, and various forms of carbon have been the sensors of choice in reeent years. A major limitation of solid electrodes has been the preparation of reproducible surfaces. Electrode pretreatment procedures involving polishing, heat treatment, and cycling of the electrode between several different potentials have helped both reproducibility and response. Most 'bare' electrodes, however, do not give reproducible responses after extended (several hours) exposure to proteinaceous solutions. Oxygen is by far the most common analyte monitored with an amperometric biosensor, the Clark electrode is used for this purpose (Clark and Lyons 1962). The pioneering work of Adams $(1969,1976)$ has led to the development of in vivo monitoring techniques for catecholamines and other important neuroactive substances. Electrodes designed for monitoring transient neurotransmitter response in the caudate nucleus of a rat brain must not only give rapid response but must be miniaturized so that spatial resolution is possible. Wightman and co-workers (Kovach et al. 1985; Howell and Wightman 1984) have developed a range of micro-electrode probes constructed of carbon fibers and Pt or Au wire. Electrodes with diameters of less than $0.5 \mu \mathrm{m}$ have been constructed. With such a small electrode area, measured currents are typically in the nano-ampere range. Because the characteristic electrode area to diffusion layer thickness ratio is small, the voltammetric response differs significantly from that of larger electrodes. The resulting small currents make possible the convenient use of these electrodes for cyclic voltammetry at very high scan rates $\left(10^{3}\right.$ to $\left.10^{4} \mathrm{~V} / \mathrm{s}\right)$. 
subject of several other chapters in this monograph (Davis, Chapter 14; Cardosi and Turner, Chapter 15; Aston, Chapter 16; Bennetto et al., Chapter 17).

\subsection{Theory of amperometric enzyme electrode response}

In the optimization of electrode performance it is important to understand the factors which affect stability, dynamic range, and response time. An important consideration is the kinetic behavior of the immobilized enzyme. During the mid-1970s there was a great deal of discussion concerning the effects of immobilization on the intrinsic properties of the enzyme. If the catalytic activity of the enzyme is high, it is quite possible that the overall rate of the reaction is limited by mass transfer to the catalytic surface or layer. The parameter which describes this situation is referred to in the chemical engineering literature as the Damkoehler Number

$$
D_{\mathrm{a}}=\frac{V_{\max }}{\left[\frac{D}{\delta}\right] K_{\mathrm{M}}}
$$

where $V_{\max }$ is the maximum rate of the homogeneous enzymatic reaction and $K_{\mathrm{M}}$ is the Michaelis constant (assuming that the enzyme obeys MichaelisMenten kinetics). For $D_{a} \leqslant 0.1$ the reaction will be catalysis-controlled while for $D_{\mathrm{a}} \geqslant 10$ the reaction will exhibit mass-transfer control. We demonstrated (Shu and Wilson 1976) that the properties of the rotating-disc electrode could be used to distinguish kinetic and mass-transfer effects without having to resort to variation in enzyme loading. At a rotation speed of $1600 \mathrm{rpm}$ essentially linear behaviour is observed for a Lineweaver-Burk plot suggesting that kinetic control defines the response with $D_{\mathrm{a}} \approx 0,01 . K_{\mathrm{M}}$ values similar to those for the soluble enzyme were obtained under conditions of constant (air saturated) oxygen levels. Mass-transfer limitations cause the $K_{\mathrm{M}}$ values to increase, however increased oxygen concentrations result in increased $K_{\mathrm{M}}$ values for glucose. This is a consequence of the sequence given by reactions 11.9 and 11.10 . The interplay between the enzyme kinetics and the fluxes of oxygen and glucose must be taken into account. Recent theoretical treatments have dealt with the importance of oxygen as a co-substrate (Leypoldt and Gough 1984; Albery and Bartlett 1985).

If an enzyme electrode were actually operated under kinetically controlled conditions, the current-concentration relationship would be non-linear and a useful range of less than one order of magnitude would be the result. However, as noted above, such sensors are operated with a membrane between the enzyme layer and the solution. This provides a barrier and a response proportional to the diffusional flux which is not limited by enzyme kinetics unless the activity of the enzyme becomes too low. This is the reason 
close to the electrode surface as a consequence of reaction 11.8 it does not have to diffuse very far to again undergo electron transfer. Therefore a significant enhancement of the current can be observed for only a small amount of $B_{O}$ present if the chemical reaction is rapid. The observed current may be related to the concentration of $\mathrm{B}_{\mathrm{o}}$ present, and this approach has been widely employed in electro-analytical methods. The reactions of electrogenerated $M_{R}$ are not very specific so care must be taken to exclude other potential oxidants which can compete with $\mathrm{B}_{\mathrm{O}}$. One might imagine immobilizing the mediator on the electrode or confining it in a layer near the surface.

In the development of biosensors, particularly those involving enzyme catalysed redox reactions, it would be useful to use the electrode as a 'cofactor'. The well-studied glucose oxidase system serves as an example to illustrate this point.

$$
\begin{aligned}
& \beta \text {-D-Glucose }+E_{O} \rightarrow \text { Gluconic Acid }+E_{R} \\
& E_{R}+C_{O} \rightarrow E_{O}+C_{R}
\end{aligned}
$$

$E_{O}$ and $C_{O}$ are the oxidized forms and $E_{R}$ and $C_{R}$ the reduced forms of the enzyme and cofactor respectively. In homogeneous solution, $\mathrm{C}_{\mathrm{O}}$ is ordinarily oxygen, $C_{R}$ is hydrogen peroxide. When the enzyme is immobilized to form a sensor, the objective, of course, is to obtain an amperometric response which is proportional to glucose concentration. In order for this condition to be fulfilled, reaction 11.9 must be the rate-determining step. It has been demonstrated* that fluctuation in the ambient $C_{O}$ (oxygen) level can significantly affect sensor response for both in vivo and in vitro measurements. If $\mathrm{E}_{\mathrm{R}}$ could be oxidized rapidly and directly at the electrode, then oxygen variations would not be a problem. While there is some evidence that glucose oxidase can be oxidized at an electrode (Ianniello et al. 1982; Durliat and Comtat 1984) the rates in general appear to be neither sufficiently rapid nor reproducible to be the basis for a practical device. Kulys and co-workers (1980) first reported on the use of a conducting salt formed from the $\mathrm{N}$ methylphenazinium (NMP ${ }^{*}$ ) cation and the tetracyanoquinodimethane anion (TCNQ ${ }^{-}$) as an electrode material for facilitating electron transfer of glucose oxidase. Recently Albery et al. (1985) have extended this work to include conducting salts of tetrathiafulvalene and quinoline with TCNQ. They report heterogeneous electron-transfer rates of greater than $10^{-2} \mathrm{~cm} \mathrm{~s}^{-1}$ and electrodes which are stable for at least a month. It is argued that electron transfer is direct rather than mediated as previously suggested (Kulys et al. 1980). These findings are encouraging evidence that rapid direct electron transfer involving glucose oxidase is feasible. It is also possible to mediate the oxidation of $E_{R}$ by electrogenerating $C_{0}$ in a sequence analogous to reactions 11.9 and 11.10 . The use of mediators for this purpose is the

•D. R. Thévenot (1985). Unpublished results. 
may be buried so that it cannot come in adequate contact with the electrode surface; and third, as noted above, the smaller diffusion coefficient of a macromolecule will lead to smaller currents. Strategies for direct amperometric detection of proteins must specifically address the first two problems. When the polyelectrolyte protein encounters the high field $\left(10^{4}-10^{6} \mathrm{~V} / \mathrm{cm}\right)$ at the electrode solution interface, there is good reason to believe that the molecular structure will be significantly altered. This may result in partial or complete denaturation of the molecule. Thus reduction either of the protein or electrode surface charge should lower the energy of this interaction. Using this reasoning Armstrong et al. (1982) added $\mathrm{Mg}^{2+}$ to a solution of Clostridium pasteurianum ferredoxin generating a reversible wave. Apparently ion-pair formation between the $\mathrm{Mg}^{2}$ and the negatively charged protein lowers the overall effective molecular charge. Similar results have been observed for plastocyanin (Armstrong et al. 1985). In 1979 the Oxford group (Eddowes and Hill 1979) demonstrated that an electrode could be modified by adsorption of $4,4^{\prime}$ bipyridyl. Nearly reversible behaviour for cytochrome $c$ was observed on such an electrode and subsequent studies showed that adsorption of the protein on the electrode prior to electron transfer was a requirement (Eddowes et al. 1981). The adsorption equilibrium is rapid and reversible and this is apparently the function of the bipyridyl electrode modifier. The bipyridyl is not electroactive at the potential of protein reduction. The third example is cytochrome $c_{3}$ (Desulfovibrio vulgaris) (Niki et al. 1979; Hinnen et al. 1983; Niki et al. 1984). This four-haem protein is irreversibly adsorbed on a bare electrode thus modifying it for further rapid and reversible electron transfer. The number of examples of rapid and direct electron transfer involving proteins is not great and therefore until suitable stable modified electrodes are available, the use of mediators appears to be the most promising approach.

Some years ago it was demonstrated that electrogenerated small molecular reactants could be used to couple biological redox couples to an electrode (Swartz and Wilson 1971; Ito and Kuwana 1971). The mediator serves to facilitate a biological electron transfer which is favorable thermodynamically but not kinetically. This is accomplished according to the scheme

$$
\begin{array}{ll}
\mathrm{M}_{\mathrm{O}}+\mathrm{e}^{-} \rightleftharpoons \mathrm{M}_{\mathrm{R}} & \text { Electrochemical } \\
\mathrm{B}_{\mathrm{O}}+\mathrm{e}^{-} \rightarrow \mathrm{B}_{\mathrm{R}} & \begin{array}{l}
\text { Electrochemical } \\
\text { (very slow reaction) }
\end{array} \\
\mathrm{M}_{\mathrm{R}}+\mathrm{B}_{\mathrm{O}} \rightarrow \mathrm{M}_{\mathrm{O}}+\mathrm{B}_{\mathrm{R}} & \text { Chemical }
\end{array}
$$

where $M_{O}$ and $B_{O}$ are the oxidized forms and $M_{R}$ and $B_{R}$ are the reduced forms of the mediator and biological molecule respectively. The electrochemical process will occur at the characteristic potential of the mediator. This potential is such that reaction 11.7 would also occur were it not for unfavourable heterogeneous electron-transfer kinetics. Because $\mathrm{M}_{0}$ is regenerated 
(Gerhardt et al. 1984; Nagy et al. 1985) have been shown to be effective in the selective retardation of anionic species. This is an important finding because it greatly facilitates the detection of hydrogen peroxide and positively charged neuroactive substances in the presence of large amounts of endogenous ascorbate or urate.

\subsection{Heterogeneous electron transfer}

In the last decade significant progress has been made in the utilization of amperometric techniques to enhance understanding of biologically relevant molecules. These include such small molecules as quinones, catecholamines, purines, flavins, thiols, and disulphides; and proteins such as cytochromes, ferredoxins, and flavoproteins. All of these molecules are involved in biological redox reactions. The interested reader is referred to several monographs (Dryhurst 1977; Dryhurst et al. 1982) for details. In aqueous solution $\mathrm{pH} 7$, there will be an applied potential 'window' from about +1.0 to $-0.6 \mathrm{~V}$ vs. the normal hydrogen electrode (NHE) and if analytes are electroactive within this range, they may possibly be monitored. The major problems are electrode fouling by proteins and filming of the electrode by the product or products of the electron transfer reaction. Because of its importance as a substrate or cofactor in many enzymatic reactions of analytical interest, the oxidation of reduced nicotinamide adenine dinucleotide $(\mathrm{NADH})$ has attracted considerable interest. The oxidation of this compound occurs only with difficulty and the product adsorbs on the electrode (Blankespoor and Miller 1984). For this and other systems, numerous attempts have been made to modify the electrode by heat treatment (Stutts et al. 1983; Wightman et al. 1984) by imbedding particulate metals in the surface (Kao and Kuwana 1984; Weisshaar and Kuwana 1984) or by adsorption or attachment of a chemical modifier to the surface (Stutts and Wightman 1983). It appears that the introduction of oxygen functionalities onto the surface facilitates electron transfer. Gorton et al. (1984) have reported that adsorption of meldola blue (7-dimethylamino-1, 2-benzophenoxazine) on graphite yields mediated electron transfer through the formation of a charge transfer complex. Lowered oxidation potentials are observed. Although results have been encouraging for the NADH system as well as others, the modified electrodes have not generally exhibited sufficient stability to be of practical utility. This area remains, nonetheless, an important one.

The case of electroactive proteins is somewhat more obscure. Under ordinary conditions, the vast majority of proteins exhibit no electroactivity even when electron transfer centres are known to be present in the molecule. There are perhaps three possible explanations for these observations. First, the protein may be irreversibly adsorbed on the electrode thus preventing further electron transfer from occurring. Second, the electroactive centre 
which is permselective to species of interest. The membrane serves to isolate the electrode from the biological fluid and to contain, in a thin Iayer, reagents such as enzymes essential to the detection system. Proteins from the biological medium which adsorb on the electrode and affect its response can be excluded. The membrane has two other important and not widely appreciated functions. First, it is possible for species to partition across the membrane/solution interface causing either a diminution or enhancement of response. This effect may be observed in addition to charge or molecular sieving effects. Second, the presence of a relatively thick membrane (50-1000 $\mu \mathrm{m})$ creates a substantial diffusional barrier. If too thick, a slow response (5-10 $\mathrm{min}$ ) can result. An advantage, however, is that the response of the sensor is unaffected by the motion (stirring) of the analyte solution because the external diffusion term of eqn 11.1 is much larger than the internal.

There is currently considerable interest in the properties of membranes which may be used to cover an electrochemical sensor. Traditionally the rate of transport of species across a membrane has been measured by placing it between two stirred-solution compartments containing the component of interest at two different concentrations. From the resulting flux between the two compartments, the diffusivity may be calculated if the membrane thickness is known. Stirring conditions are often not well defined. Recently the properties of a membrane-covered rotating-disc electrode (Gough and Leypoldt $1979,1980 a, b$ ) have been used to measure membrane transport. By rotating the electrode, the ratio of the external to internal diffusion (permeability) rates can be varied. The steady-state flux across the membrane is measured by electrolysis of the diffusing species as it encounters an electrode in contact with the inner side of the membrane. The resulting current, $i_{\mathrm{d}}$, is given by the relationship

$$
i_{\mathrm{d}}=i_{\mathrm{L}} \quad\left[\frac{1}{1+P_{\mathrm{s}} / P_{\mathrm{M}}}\right]
$$

where $i_{\mathrm{L}}$ is the current in the absence of the membrane. The permeability in the membrane phase $P_{\mathrm{M}}=\alpha D_{\mathrm{M}} / \delta_{\mathrm{M}}$ and the permeability in the solution phase $P_{5}=D / \delta$ define the response. The ratio of the solution to membrane permeabilities $\left(P_{s} / P_{\mathrm{M}}\right)$ is called the Biot number. The mass transfer behaviour of the system is defined by the diffusion coefficients in the membrane and solution respectively, by the membrane thickness, and by the partition coefficient $\alpha=[s]_{\text {mem }} /[s]_{\text {soln }}$ of the electroactive species (s) between the membrane and solution. The rotating-disc method in this form is limited to electroactive species, but in many cases these are the components of interest in biosensors. Negatively charged membrane-coated electrodes prepared by dip coating with a polymer solution such as cellulose acetate (Sittampalam and Wilson 1983; Wang and Hutchins 1985) or Nafion ${ }^{\mathrm{TM}}$ 
increasing the rotational speed of the electrode causes increased mass transfer and a thinner diffusion layer. The resulting steady-state current is given by the relationship

$$
i_{\mathrm{L}}=0.62 n F A D_{0}^{2 / 3} w^{1 / 2} \nu^{-1 / 6} C_{0}^{\mathrm{b}}
$$

where $D$ is the diffusion coefficient, $F$ is the faraday constant ( $96480 \mathrm{C}$ $\left.\mathrm{mol}^{-1}\right), A$ is the electrode area, $w$ is the rotation speed in $\mathrm{s}^{-1}, \nu$ is the kinematic viscosity in $\mathrm{cm} \mathrm{sec}^{-1}$, and $C_{0}{ }^{\mathrm{b}}$ is the bulk concentration. Equation 11.3 can be rewritten as

$$
\frac{1}{i_{\mathrm{T}}}=\frac{1}{i_{\mathrm{N}}}+\frac{1}{i_{\mathrm{L}}}
$$

The observed current is $i_{\mathrm{T}}$ which is partitioned between $i_{\mathrm{L}}$, a mass-transferdependent current which varies with $w$, and $i_{N}$ which is rotation-speed independent. A plot of $1 / i_{T} v s . w^{-1 / 2}$ will yield an intercept of $1 / i_{N}$. Thus the rotating dise facilitates a priori separations of $i_{N}$ and $i_{L}$. It remains to determine the physical meaning of $i_{\mathrm{N}}$. This approach has been used extensively for the characterization of electroactive polymer films (Andrieux et al. 1984).

The wall-jet electrode configuration has recently gained increasing attention and several presentations of theory have recently appeared (Dalhuijsen et al. 1985; Albery 1985). This hydrodynamic configuration offers the potential advantages of improved sensitivity due to increased masstransfer efficiency and decreased sensitivity to the characteristics of the mobile phase in a flowing stream (Gunasingham 1984). The technique has been applied in HPLC (Gunasingham et al. 1985) and for bromine titration of proteins (Albery et al, 1984).

In practical applications stirring the solution increases mass transfer thus making the diffusion layer thinner and leading to higher sensitivity. This is advantageous as long as mass transfer can be reproducibly controlled. If simple diffusion is perturbed by coupled kinetic or other processes, then both the time and magnitude of the electrode response can be significantly affected. This point will be discussed further in connection with kinetic processes. Since the diffusion coefficient, $D$, appears in the expression for the flux, it follows that the resulting current from an electroactive biological molecule will depend on its size. The diffusion coefficient for a 'large' molecule of, for example, $150 \mathrm{kD}$, is generally no less than a factor of 100 smaller than that of a typical 'small' molecule or ion. In many electrochemical experiments the current has a fractional power dependence on $D$. Thus if all other factors are equal, the difference in current between a 'large' and a 'small' molecule should not be more than a factor of 10-20. Low observed currents for 'large' molecules have been attributed to diffusional effects when, in fact, they are often controlled by some other kinetic step. 
be emphasized, however, that the terms in the above equation are not completely independent of each other.

\subsection{Diffusion/mass transfer}

Although the indicating electrode may assume a variety of different geometries, a planar configuration is illustrated schematically in Fig. 11.1. Under these conditions, a potential is applied such that species $O$ is reduced at the electrode surface at a diffusion-controlled rate. The sample concentration profile shown in Fig. 11.1 can be time dependent. Its shape will be governed both by the nature of the controlled potential perturbation of the electrode and by mass transfer through diffusion and convection. Fick's First Law,

$$
-J_{0}(x, t)=D_{0} \frac{\partial C_{0}(x, t)}{\partial x},
$$

states that the flux $J$ at the electrode surface to which the current is proportional is determined by the slope of the concentration gradient for species $O$ at that point. This is a crucial property as it may ultimately be possible to relate this flux to solution analyte concentration.

A classical work (Levich 1962) describes the flow of solution past surfaces and the fluxes generated through diffusion and convection. Probably the best defined and certainly the most useful configuration from the fundamental point of view is the rotating-disc electrode. The simple expedient of

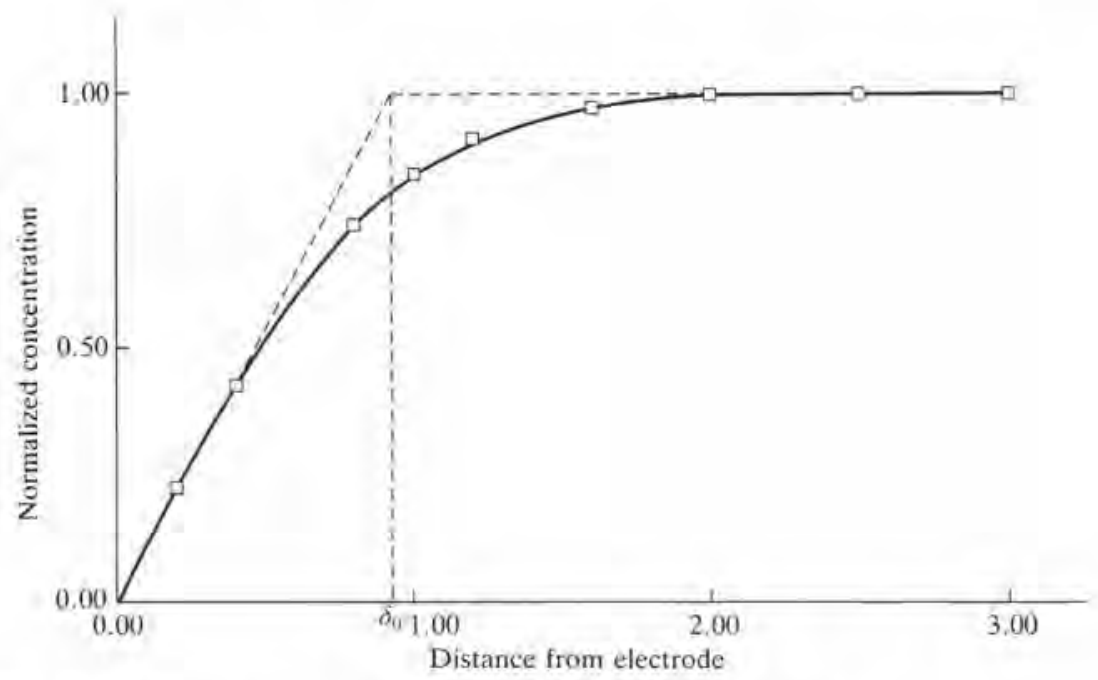

Fig, 11.1 Concentration profile for a potentiostatic experiment. 


\title{
Fundamentals of amperometric sensors
}

\author{
GEORGE S. WILSON
}

\subsection{Introduction}

Amperometric detection has found wide application to measurements in biological media. Under favourable conditions concentrations of $10^{-8}$ to $10^{-9} \mathrm{M}$ can be detected and a dynamic range of three to four orders of magnitude can be readily achieved. In the context of biosensors, it is appropriate to examine some of the fundamental features of amperometry as they influence detector response.

The application of a potential between a reference and indicating electrode can give rise to a current which, in turn, may be related to the concentration of an electroactive analyte in solution. The measured current can be directly related to the rate of the electrochemical reaction occurring at the indicating (sensing) electrode. It is important, however, to identify and control the conditions which define the rate-determining step of the overall electrolytic process. The rate of the heterogeneous electron transfer process $\left(i_{\mathrm{CT}}\right)$ occurring directly at an electrode can be controlled by variation of the applied potential according to the Butler-Vollmer equation (Bard and Faulkner 1980). Thus in many systems it is possible to choose a potential such that the current is not limited by heterogeneous electron transfer even if this process is electrochemically irreversible. If this condition is met, then the relevant ratedetermining step may be controlled by diffusion/mass transfer, adsorption, or chemical kinetic processes respectively. The overall sensor current, $i_{\tau}$, may be described by the generalized equation (11.1) below.

$$
\frac{1}{i_{\top}}=\frac{1}{i_{\mathrm{ID}}}+\frac{1}{i_{\mathrm{ED}}}+\frac{1}{i_{\mathrm{CT}}}+\frac{1}{i_{\mathrm{AD}}}+\frac{1}{i_{K}}+\ldots
$$

There are two diffusional terms, $i_{\mathrm{ID}}$ and $i_{\mathrm{ED}}$, which define the rates of internal and external diffusion respectively. The latter relates to diffusion in bulk solution up to the electrode or a membrane/solution interface. Internal diffusion involves the movement of relevant species within a membrane or reaction layer. The overall response may be controlled by charge transfer $\left(i_{\mathrm{CT}}\right)$ or by the adsorption of reactants on a membrane or electrode surface $\left(i_{\mathrm{AD}}\right)$. The movement of analyte species from the solution to the sensor may be coupled to a chemical reaction which proceeds at a finite rate $\left(i_{K}\right)$. It should 



\section{Bioelectrochemistry \\ (b) Amperometric sensors}


Wingard Jr., L. B., Cantin, L. A. and Castner, J. F. (1983). Effect of enzyme-matrix composition on potentiometric response to glucose using glucose oxidase immobilized on platinum. Biochim. Biophys. Acta 748, $21-7$.

Wingard Jr., L. B., Castner, J.F., Yao, S. J., Wolfson Jr., S. K., Drash, A. L. and Liu, C. C. (1984). Immobilized glucose oxidase in the potentiometric detection of glucose. Appl. Biochem. Biotechnol. 9, 95-104. 
use of the electrode. Therefore, additional research is needed to establish methodology for more thorough characterization of platinum-surface redox chemistry or for simple recalibration of the electrodes if potential drifting is a factor. Alternatively, a known redox couple could be attached to a potentiometrically inert electrode surface.

It probably will require considerable research before a potentiometric redox electrode can be incorporated into a viable sensor for in vivo use to regulate an insulin delivery system. The simplicity of design and of components also make a potentiometric redox electrode an attractive system for (1) monitoring of effluent systems or (2) monitoring and feedback control of certain fermentation reactors. However, in all of these systems where a significant quantity of substrate is reacted (i.e. glucose), a problem can develop if the supply of the second substrate (i.e. oxygen) is too low or if a mechanism for the regeneration of oxidized cofactor is not present.

\section{Acknowledgement}

This work was supported by a grant from the National Institutes of Health.

\section{References}

Adams, R.N. (1969). Electrochemistry at solid electrodes, pp. 206-8. Marcel Dekker, New York.

Bard, A. J. and Faulkner, L. R. (1980). Electrochemical Methods, pp. 62-72, Wiley, New York.

Castner, J. F. and Wingard Jr., L. B. (1984). Alterations in potentiometric response of glucose oxidase platinum electrodes resulting from electrochemical or thermal pretreatments of a metal surface. Anal. Chem. 56, 2891-6.

Chen, A. K., Starzmann, J. A. and Liu, C. C. (1982). Potentiometric quantitation of glycerol using immobilized glycerol dehydrogenase. Biotechnol. Bioeng. 24, 971-5.

Evans, J.F. and Kuwana, T. (1979). Introduction of functional groups onto carbon electrodes via treatment with radio-frequency plasmas. Anal. Chem. 51, $358-65$.

Hoare, J. P. (1974). In Encyclopedia of electrochemistry of the elements (ed. A. J. Bard), pp. 210-38. Marcel Dekker, New York.

Irving, H.M.N.H. (1976). Recommendations for nomenclature of ion-selective electrodes, Pure Appl. Chem. 48, 127-32.

Joseph, J.P. (1984). A miniature enzyme electrode sensitive to urea. Mikrochim. Acta 2, 473-79.

Plambeck, J.A. (1982). Electroanalytical chemistry: basic principles and applications, pp. 168-77. Wiley, New York.

Proctor, A., Castner, J. F., Wingard Jr., L. B, and Hercules, D. M. (1985). Electron spectroscopic (ESCA) studies of platinum surfaces used for enzyme electrodes. Anal, Chem. 57, 1644-9. 
In aqueous solution, platinum oxide formation is thought to begin with an oxidation in which $\mathrm{OH}$ groups attach reversibly to the surface layer of platinum atoms. At roughly monolayer coverage, the outer layers of platinum atoms undergo an irreversible rearrangement to allow $\mathrm{OH}$ groups to enter into the platinum lattice (Hoare 1974). Exactly which one-electron reactions are present is not known, but could involve the reduction of some of the platinum moieties through an intermediate +1 oxidation state. The influence of $\mathrm{pH}$ on the measured potentials was not tested directly since a change in the solution $\mathrm{pH}$ would have a major influence on the activity of the enzymes. However, varying the $\mathrm{pH}$ between 5.4 and 8.4 has no effect on the potential of a bare platinum electrode (i.e. no enzyme present). Obviously, a clear explanation of the source of the potential with platinum must await a better understanding of platinum-oxygen-water-hydrogen peroxide mechanistic chemistry.

In the case of carbon (Fig, 10.5), the positive slope of about $30 \mathrm{mV} /$ decade is indicative of a two-electron oxidation reaction taking place on the electrode surface. Porous graphite electrodes contain aldehyde as well as hydroquinone functional groups (Evans and Kuwana 1979) that can undergo twoelectron oxidations to carboxylic acid and quinone groups, respectively. However, a more detailed explanation of the source of the potentiometric response must await additional experimental work.

The magnitude of the potentiometric response is influenced by the type of pretreatment given to the platinum electrode surface. This is demonstrated by pretreating platinum electrodes as follows: (1) heating in gas flame, (2) electrochemical oxidation, (3) electrochemical reduction, (4) electrochemical neutralization, and (5) electrochemical deposition of platinic chloride. The neutral, oxidation, and flamed pretreated electrodes exhibit a significant difference in the slopes of the potentiometric response to glucose between the bare platinum and the enzyme-matrix-coated platinum electrodes (Castner and Wingard 1984). The flamed and electrochemically pretreated bare platinum electrodes were studied further using ESCA (Proctor $e t$ $a l ., 1985)$. The thermally treated platinum contains less carbonaceous surface contamination than the electrochemically pretreated platinum; and surface oxidation is greater when done electrochemically than when done by flaming. Silicon contamination of the platinum surface, due to diffusion from within the bulk metal, is observed when the platinum is pretreated by flaming.

A present limitation with the glucose oxidase-platinum electrode concerns the reproducibility of the surface potentials. Any given platinum-glucose oxidase electrode produces a linear potentiometric response when plotted against the logarithm of the glucose concentration. However, upon repeated use, there sometimes is evidence of hysteresis when the direction of change in the glucose concentration is reversed. In addition the magnitude of the baremetal potentiometric response is difficult to maintain constant with repeated 


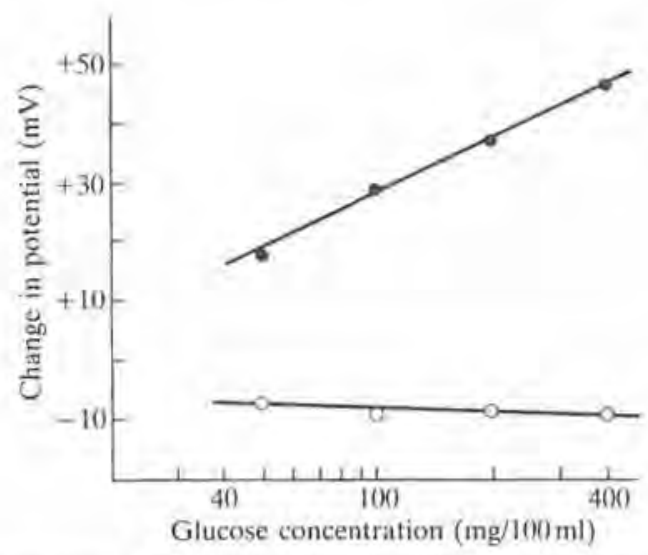

Fig. 10.5 Potentiometric response of glucose oxidase-porous graphite electrode to oxygenated glucose solution at $\mathrm{pH} 7.4$ in $0.1 \mathrm{M}$ sodium phosphate buffer, Symbols: 0 measurements made with bare graphite electrode; measurements made with glucose oxidase matrix coated graphite electrode.

placed on porous graphite instead of platinum (Fig. 10.5). However, in this case a positive potential versus log [glucose concentration] slope is obtained, as compared to a negative slope with the platinum-based electrode (Wingard et al., 1984). Possible mechanisms for these two cases are discussed next.

The source of the potentiometric response appears to involve the oxidation or reduction of electrode surface functional groups by hydrogen peroxide. This compound is generated as a product of the glucose-oxidase-catalysed oxidation of glucose. Therefore, the redox couple being measured is attached to the electrode surface instead of being free in solution as depicted in Fig. 10.3. The postulated mechanisms are summarized here, with the reader referred elsewhere for a more detailed discussion (Castner and Wingard 1984; Wingard et al. 1984). In the case of platinum (Fig. 10.4), the potential becomes more negative with increasing glucose concentration. This suggests a net reduction reaction is occurring on the platinum surface. The slope in Fig. 10.4 is about $-40 \mathrm{mV} /$ decade of glucose concentration; although as discussed later this slope is dependent upon the method of pretreatment of the platinum surface (Castner and Wingard 1984). A slope of -40 $\mathrm{mV} /$ decade is suggestive of greater than one-electron but less than twoelectron transfer, and probably results from several reactions occurring in parallel or in series. The following reactions can occur with a net positive $E^{0}$; although other one-electron transformations very likely are present:

$$
\begin{array}{ll}
\mathrm{H}_{2} \mathrm{O}_{2}=\mathrm{O}_{2}+2 \mathrm{H}^{*}+2 \mathrm{e}^{-} & E^{0}=-0.68 \mathrm{~V} \\
\mathrm{Pt}(\mathrm{OH})_{2}+2 \mathrm{H}^{+}+2 \mathrm{e}^{-}=\mathrm{Pt}+2 \mathrm{H}_{2} \mathrm{O} & E^{0}=0.98 \mathrm{~V}
\end{array}
$$




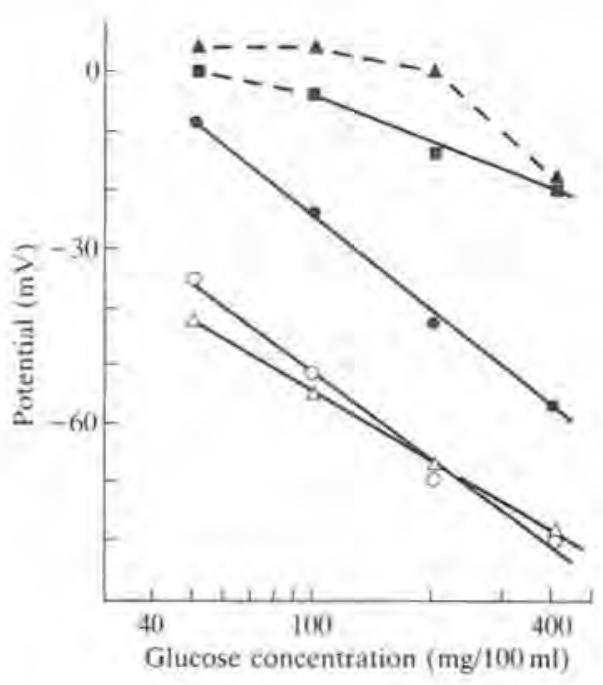

Fig. 10.4 Potentiometric response of glucose oxidase-platinum electrode to oxygenated glucose solution at $\mathrm{pH} 7.4$ in $0.1 \mathrm{M}$ sodium phosphate buffer. Potentials are the measured values minus the potential with plain buffer (no glucose). Potentials are with respect to $\mathrm{Ag} / \mathrm{AgCl}(1 \mathrm{M} \mathrm{KC} \ell)$. Lines fitted by linear least squares. Symbols: $\Delta$ and $O$ measurements made with enzyme-platinum out of mould; $\Delta(0.24 \mathrm{~cm})$, $(0.16 \mathrm{~cm}), \quad(0.05 \mathrm{~cm})$ measurements made with enzyme-platinum in mould (numbers in parentheses indicate thickness of enzyme matrix).

expected for $8 \mu \mathrm{g}$ of glucose oxidase. The low activity is attributed to diffusional constraints imposed by the cross-linked matrix. Therefore, a proportionally greater glucose oxidase activity would be expected with the thinner cross-linked enzyme-albumin matrices.

Direct potentiometric measurements are carried out by immersing the glucose oxidase electrode and an $\mathrm{Ag} / \mathrm{AgCl}$ reference electrode in an oxygensaturated solution of glucose, with the $\mathrm{pH}$ controlled at $6.0 \pm 0.1$ to $7.5 \pm 0.1$ using $0.1 \mathrm{M}$ sodium phosphate buffer. The potentials can be measured using a suitable potentiometer, such as a Keithley $610 \mathrm{C}$. Typical results are shown in Fig. 10.4 for a series of different thicknesses of enzyme matrix on one side of the platinum disc. With the enzyme-platinum disc removed from the mould, the enzyme matrix layer is very thin $(\mathrm{ca} .0 .02 \mathrm{~cm})$. Whereas with the enzyme-platinum in the mould, the layer of enzyme matrix is $0.05-0.24 \mathrm{~cm}$ thick. It is evident from Fig. 10.4 that a Nernstian relationship is observed for glucose concentrations of $50-400 \mathrm{mg} / 100 \mathrm{ml}$. This is of interest for clinical glucose determination since normal blood glucose levels occur in the range $90-120 \mathrm{mg} / 100 \mathrm{ml}$.

Similar linear results are observed when the glucose oxidase matrix is 
biocatalytic surface or (2) construction of a blocked interface. Examples of biocatalytic surfaces on redox electrodes include work with glucose oxidase (Castner and Wingard 1984; Wingard et al. 1983), glycerol dehydrogenase (Chen et al. 1982), and urease (Joseph 1984). The blocked interface system can utilize an antibody-antigen complex to mediate the potentiometric response due to changes in a given analyte concentration. These referenced systems have been characterized experimentally; but none have been brought into commercial practice as yet.

Of the above examples, the glucose oxidase redox electrode system probably is the one that has been most well characterized (Castner and Wingard 1984; Wingard et al. 1983; Proctor et al, 1985; Wingard et al. 1984). This enzyme catalyses the reaction of $\beta$-D-glucose plus $\mathrm{O}_{2}$ to produce glucono-lactone plus hydrogen peroxide. As mentioned in the introduction, biocatalytic enzyme redox electrodes involve the immobilization of an oxidoreductase enzyme on the electrode surface with the primary analyte in solution. Alternative schemes can involve (1) immobilization of an enzyme cofactor, such as a porphyrin or flavin, on the electrode surface with reliance on apoenzymes in the sample to catalyse the oxidation or reduction of the immobilized redox centre or (2) immobilization of both the enzyme and a mediator molecule on the electrode surface. In our glucose oxidase work, the enzyme is immobilized on a noble metal or a carbon electrode. The redox potential of these electrodes is thought to be dependent on the glucose, oxygen, and hydrogen peroxide in the solution and on functional groups on the platinum or carbon surface. The procedures and results for the glucose oxidase redox electrode work are summarized below.

Glucose oxidase, alone or mixed with catalase, has been immobilized on platinum, porous graphite, and gold and has been shown to produce a direct potentiometric response in the presence of glucose solution at $\mathrm{pH} 7.4$. For example, a mixture of catalase, lyophilized glucose oxidase from $A$. niger, bovine serum albumin, and glutaraldehyde is mixed in sodium phosphate buffer at pH 7.4. The mixture is poured into a mould that contains a disc of $0.05 \mathrm{~mm}$ thick platinum foil. The thickness of the cross-linked enzymealbumin matrix can be controlled, for example from $0.05 \mathrm{~cm}$ to $0.32 \mathrm{~cm}$, by the use of spacers in the mould. Glutaraldehyde cross-linking of the protein takes place over a $2 \mathrm{hr}$ period at room temperature. The cross-linked matrices are next washed with buffer for 1-2 days to remove loosely attached enzyme prior to testing for enzymatic activity or for potentiometric evaluation (Wingard et al. 1983).

A $0.32 \mathrm{~cm}$ thick cross-linked enzyme-albumin matrix contains about $8 \mu \mathrm{g}$ of glucose oxidase (about 0.8 units of activity). The apparent activity of the immobilized enzyme can be determined quite conveniently by the $o$-dianisidine/peroxidase colorimetric procedure, using glucose and oxygen as the substrates. The apparent activity is only 0.0044 units or less than $1 \%$ of that 

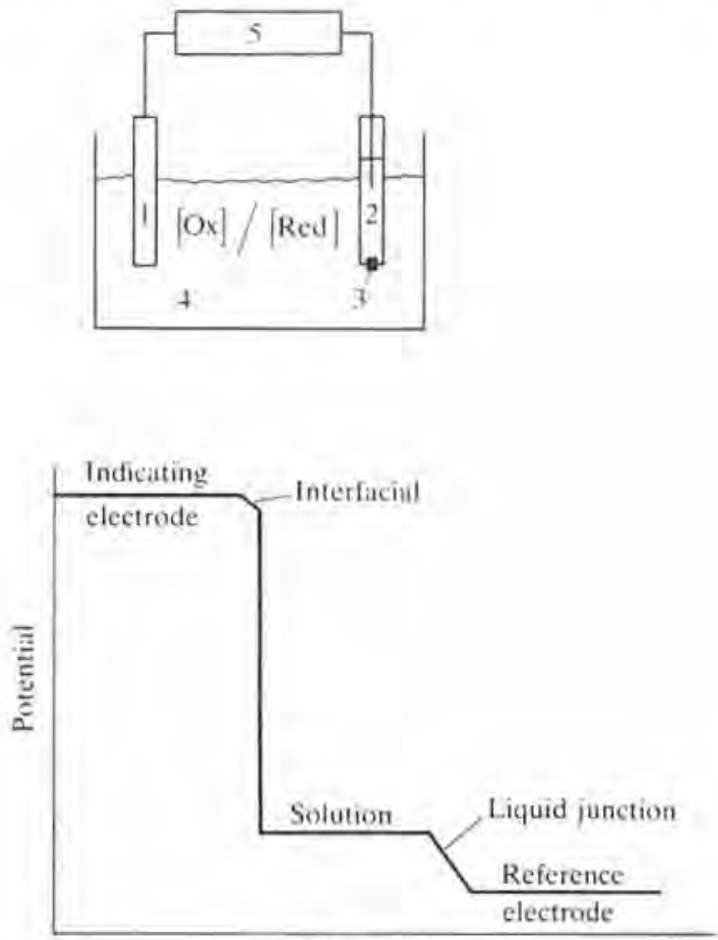

Distance

Fig. 10.3 Source of potential differences arising with a redox electrode. The components are as follows: 1 , indicating electrode; 2 , reference electrode with internal electrolyte solution; 3, porous plug (liquid junction); 4, redox material in solution (ratio of oxidized to reduced form establishes a potential); 5 , potentiometer.

systems. However, with redox systems there is the added constraint of a lack of knowledge about the relationship between electrode surface chemistry and the observed potentials. On the other hand, redox electrodes can have faster potentiometric response times due to the absence of mass transfer across interfaces. In addition, the base redox electrode exhibits lower electrical resistance than the typical ISE electrode, therefore requiring less expensive instrumentation, i.e. lower impedance devices. Redox systems are less selective and thus have a broader range of applications.

\subsection{Examples of biosensors based on redox electrodes}

Conceptually, two approaches can be taken for the construction of potentiometric biosensors based on redox electrodes. They are (1) construction of a 
$\mathrm{pH}$ glass, lanthanum fluoride crystals, and ionophore-doped PVC films. Redox electrodes typically, but not exclusively, are noble metal probes that exhibit Nernstian behaviour for changes in the concentration ratio of oxidized and reduced species in the sample solution. The selection of $\mathrm{Pt}, \mathrm{Au}$, or Pd for use as electrodes is based on the premise that these metals are good electrical conductors, while at the same time they are not chemically reactive. A corollary to the above statement is that the measured potentials for any redox couple should be independent of the electrode material. In practice this argument does not hold universally. Evidence of this is given by the warning of R. A. Adams about the use of noble metal electrodes (Adams 1969): 'One of the undisputed points with regard to platinum electrode methodology (most of the following discussion also applies to gold and noble metal electrodes in general) is that reproducible results are almost always obtained provided the general pretreatment of the electrode is duplicated each time.'

It is a common practice in reporting electrochemical measurements obtained via redox electrodes to provide the reader with procedural information about the condition of the indicating electrode. This includes (1) the method for cleaning the electrode surface, (2) the purity of the bulk material, and (3) the molecular morphology of the surface (i.e. poly vs. single crystalline material). An example showing how the type of platinum surface pretreatment influences the potentiometric response for a series of glucose oxidase redox electrodes is given in a later section. This pretreatment influence may be caused by the presence of electrochemically active functional groups on the surface of the noble metal or other electrode materials, e.g. glassy carbon. The presence of these moieties can impact upon the precision and the accuracy of the respective redox measurement.

The analytical integrity of redox electrodes also is influenced by the selection of reference electrode. However, this situation is not unique to redox probes but is shared by ISE probes as well. An in-depth treatment of this topic is beyond the scope of this section. The contribution played by the reference electrode is summarized schematically in Fig. 10.3. As shown in the figure, the sample solution is separated by a liquid junction from the reference electrode internal electrolyte. At this junction (usually through a fritted glass plug), a concentration gradient of ionic species can develop; and correspondingly, a potential difference can be generated. The magnitude of the potential will be transitory and dependent upon diffusional processes of the charged species. Control of the electrode junction potentials can be achieved experimentally in one of two ways. First, all of the ionic species on both sides of the junction can be of the same charge/mobility ratio; or second, the reference electrode can be calibrated with a standard sample solution. The theory of electrode junction potentials is covered in detail elsewhere (Bard and Faulkner 1980; Plambeck 1982 and references therein). In summary, biosensors based on redox electrodes are constrained in the same manner as ISE 
more general application. A comparison of these two potentiometric systems is presented in order to identify clearly the principles which distinguish the two approaches.

Both ISE and redox-electrode-based biosensors share a commonality of:

1) Analyte selectivity is dependent upon the biological activity associated with the material directly coupled to the indicating electrode.

2) The analytical signal, in this case the Emf, is measured under null current flow conditions, thus requiring high impedance instrumentation.

3) The potential at the indicating electrode is measured with respect to a stable reference electrode. This is shown schematically in Fig. 10.1.

The ISE and redox types of potentiometric biosensors are distinguished by the electrochemical reactions taking place at the respective sensing electrodes. ISEs operationally are permselective membrane sensors which track ion-exchange events at the membrane/solution interfaces (see Fig. 10.2) (Irving 1976). Classical examples of permselective membranes are
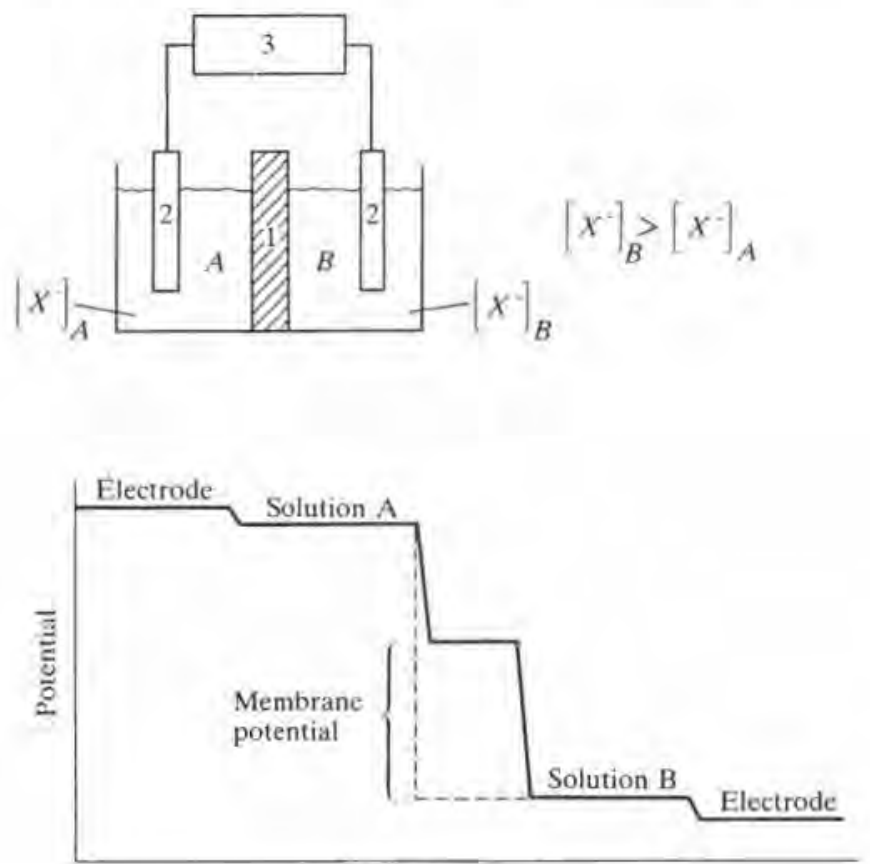

Distance

Fig. 10.2 Source of potential differences arising with an ion-selective electrode. The components are as follows: 1 , ion-exchange membrane with fixed negative charges; 2 , platinum electrodes; 3 , potentiometer. Interfacial junction potentials are shown by the potential differences between the electrodes and the adjacent solution. 


\title{
Potentiometric biosensors based on redox electrodes
}

\author{
LEMUEL B. WINGARD JR. and JAMES CASTNER
}

\subsection{Introduction}

The subject of potentiometric biosensors has been approached by many as an extension to the study of ion-selective electrodes (ISE). The basis for this perception is twofold. First, a majority of the high impedance electrochemical biosensors described in the literature contain an ISE; and second, the purpose for developing these modified electrodes has been professed by many to be simply a desire to extend the analytical utility of the base probe. This utilitarian view has sharply focused the direction of research in the field.

The theory, types, and performance characteristics of biosensors based on ISE technology have been reviewed in the preceding chapter. In this chapter, we will extend the discussion by considering another design: namely, bioprobes based on redox electrodes. Such a system has less specificity and thus

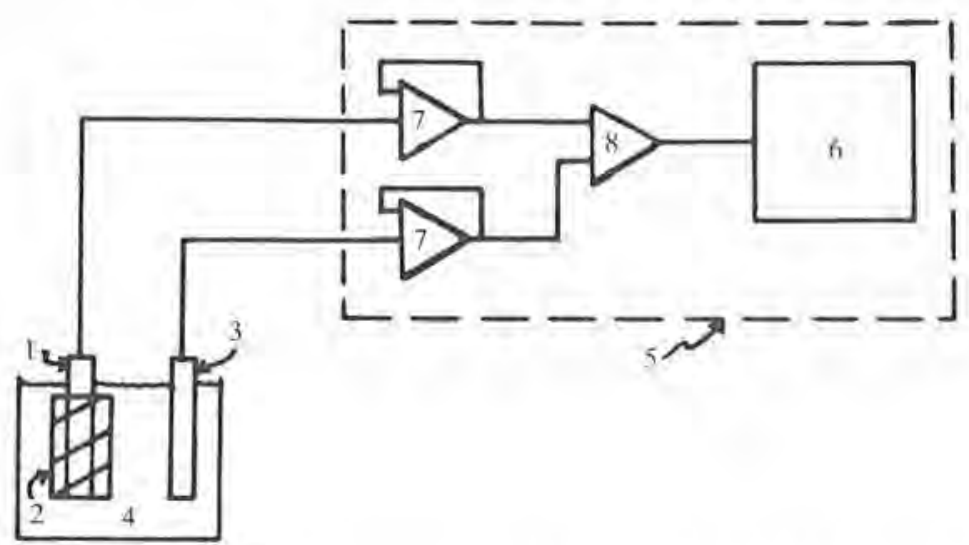

Fig. 10.1 General circuitry for measurement of potential at non-ISE-type indicating electrode. The components are as follows: 1 , indicating electrode; 2 , biological material on indicating electrode; 3 , reference electrode; 4 , electrolyte containing material to be measured; 5 , high impedance potentiometer; 6 , display unit; 7, operational amplifier current follower; and 8, comparator circuit. 
Durst). National Bureau of Standards, Washington D.C., p. 215 ,

Nagy, G., von Storp, H. and Guilbault, G. (1973). Enzyme electrode for glucose based on an iodide membrane sensor. Anal. Chim. Acta 66, 443-55.

Nilsson, H., Akerlund, A. and Mosbach, K. (1973). Determination of glucose, urea and penicillin using enzyme $\mathrm{pH}$ electrodes. Biochim. Biophys. Acta 320, 529-34.

Pick, J., Toth, K., Pungor, E., Vasak, M. and Simon, W. (1973). Potassium-selective silicone rubber membrane electrode based on a neutral carrier. Anal. Chim. Acta 64, 477-80.

Pioda, L., Wachter, M., Dohner, R. and Simon, W. (1967). Helv. Chim. Acta 50, 1373.

Pungor, E. (1967). Theory and application of anion selective electrodes. Anal. Chem. $39,(13), 28 \mathrm{~A}$.

Rechnitz, G. A. (1967). Ion selective electrodes: a review. Chem. Eng. News June 12, 146.

Stephanova, O. K., Shuitz, M.M., Materova, E. A. and Nicolsky, B.P. (1963). Vestn. Leningrad, Univ. 4, 93.

Thompson, H. and Rechnitz, G. (1974). Ion electrode based enzymic analysis of creatinine. Anal. Chem. 46, 246-9.

Tietz, N.W. (1970). Fundamentals of clinical chemistry Saunders, Philadelphia, p. 637.

Tran-Minh, C. and Brown, G. (1975). Construction and study of electrodes using cross-linked enzymes. Anal. Chem. 47, 1359.

Tsuchida, T, and Yoda, K. (1983). Multi-enzyme membrane electrodes for determination of creatinine and creatinine in serum. Clin. Chem. 29, 51-5.

Updike, S. J. and Hicks, G. P. (1971). The enzyme electrode. Nature 214, 986-8.

White, C. and Guilbault, G. (1978). Lysine specific enzyme electrode for determination of lysine in grains and food, Anal, Chem. 50, 1481-5.

Winters, 1. (1981). Acid base physiology in medicine. The London Co., Cleveland, Ohio USA. 
selectivity for potassium over sodium. Science 167, 987-8.

Fung, K. W., Kuan, S. S., Sung, H. Y, and Guilbault, G. (1979). Methionine selective enzyme electrode. Anal. Chem. 51, 2319-24.

Garrels, R. M. (1967). Ion sensitive electrodes and individual ion activity coefficients. In Glass electrodes for hydrogen and other cations (ed. G. Eisenman). Marcel Dekker, New York, pp. 344-61.

Griffiths, G. H., Moody, G. J. and Thomas, J. D. R. (1972). Optimum composition of polyvinyl chloride matrix membranes used for selective calcium-sensitive electrodes. Analyst (London) 97, 420-7.

Guilbault, G. (1984). Handbook of immobilized enzymes. Marcel Dekker, New York.

- and Hrabankova, E. (1970). L-amino acid electrode, Anal. Letters 3, 53-7.

- and Mascini, M. (1977). Urease coupled ammonia electrode for urea determination in blood serum. Anal. Chem. 49, 795-8.

- and Montalvo, J. (1969), J, Am. Chem, Soc, 91, 2164.

- (1970). J. Am. Chem. Soc. 92, 2533.

- and Coulet, P. R. (1983). Creatinine-selective enzyme electrodes, Anal. Chim. Acta 152, 223-8.

- and Nagy, G. (1973a). Improved urea electrode. Anal. Chem. 45, 417-19.

(1973b). Enzyme electrodes for the determination of L-phenylalanine. Anal. Lett. 6, 301-12.

- and Shu, F. (1971), Electrode for the determination of glutamine, Anal. Chim. Acta 56, 333-40.

- (1972). Enzyme electrodes based on the use of a carbon dioxide sensor. Anal. Chem. 44, 2161-6.

- Chen, S. and Kuan, S. (1980). A creatinine specific enzyme electrode. Anal. Letters 13, 1607-24.

- Kuan, S. and Nagy, G., (1973). Improved electrode for the assay of urea in blood. Anal. Chim. Acta 67, 195-201.

Haake (1975). Brinkman Instruments, Cantiague Rd., Westbury, New York, U.S.A. Haber, F. and Klemensiewicz, Z. (1909). Z. Phys. Chem. 67, 385.

Hattner, R.S., Johnson, J. W., Bernstein, D. S., Wachman, A. and Brackman, J, (1970). Clin. Chim. Acta 28, 67.

Joseph, J. P. (1985). An enzyme microsensor for urea based on an ammonia gas electrode. Anal. Chim. Acta 169, 249-56.

Karrenman, G. and Eisenman, G. (1962). Bull. Math. Biophys. 24, 413.

Khuri, R. N. (1969). In Ion-selective electrodes, Special Publication 314 (ed, R. A. Durst). National Bureau of Standards, Washington D.C, p. 287.

Li., T. K. and Piechoki, J. T. (1971). Clin. Chem. 17, 411.

Mascini, M., Fortunati, S., Moscone, D. and Palleschi, G. (1985), Ammonia abatement in an enzymic flow system for the determination of creatinine in blood sera and urine. Anal. Chim. Acta 171, 175-84.

Moody, G. J. and Thomas, J. D. R. (1972). Development and publication of work with ion-sensitive electrodes. Talanta 19, 623-39.

- Oke, R. B. and Thomas, J. D. R. (1970). Calcium-sensitive electrode based on a liquid ion exchange in a polyvinyl chloride matrix. Analyst (London) 95, 910-18. Moore, E. W. (1969). In Ion-selective electrodes, Special Publication 314 (ed. R. A. 
9.6.1.5 Penicillin Another very important electrode described has been the penicillin electrode, now widely used to monitor for the penicillin content of fermentation broths. The electrode is based on use of a $\mathrm{pH}$ probe, coated with immobilized enzyme penicillinase.

$$
\text { Penicillin } \stackrel{\text { Penicillinase }}{\longrightarrow} \text { Penicilloic acid }
$$

The response time is very fast $(<30 \mathrm{~s}$ ) with a slope of $52 \mathrm{mV} /$ decade over the range $5 \times 10^{-2}-10^{-4} \mathrm{M}$ (Nilsson et al. 1973).

\subsection{Commercial availability of enzyme probes}

Immobilized enzymes, together with ISE sensors, are used in several instruments available commercially. Owens-Illinois (Kimble) has designed a urea instrument using immobilized urease and an ammonia electrode probe. Patent rights to this system have been purchased by Technicon, who markets the instrument in Europe.

Self-contained electrode probes are based on ISE's available from only Universal Sensors (P. O. Box 736, New Orleans, La. 70148 USA), which offers probes for urea, creatinine, amino acids, and others based on glutaraldehyde immobilization.

\section{References}

Alexander, P. W. and Joseph J. P. (1981), Anal. Chim. Acta 131, 103.

Ammann, D., Pretsch, E. and Simon, W. (1972). Calcium ion-selective electrode based on a neutral carrier. Anal. Lett. 5, 843-50.

Anfalt, T., Granelli, A and Jagner, D. (1973). Urea electrode based on the ammonia probe. Anal, Lett, 6, 969-75.

Annino, J. S. (1967). Determination of sodium in urine by specific ion electrode. Clin. Chem. 13, 227-32.

Budd, A. L. and Jones, R. H. (1963). The Analyzer 4, 5.

Clark, L. and Lyons, C. (1962). Electrode systems for continuous monitoring in cardiovascular surgery. Ann. N. Y. Acad. Sci. 102, 29-45.

Cremer, M. (1906), Z. Biol. 47, 562.

Davies, J. E. W., Moody, G. J. and Thomas, J.D. R. (1972). Nitrate ion selective electrodes based on polyvinyl chloride matrix membranes. Analyst (London) 97, $87-94$.

Eisenman, G. (ed.) (1967), Glass electrodes for hydrogen and other cations. Marcel Dekker, New York.

- (1969). In Ion-selective electrodes, Special Publication 314 (ed. R. A. Durst). National Bureau of Standards, Washington D.C.

Eyal, E. and Rechnitz, G. A. (1971). Mechanistic studies on the valinomycin-based potassium electrode. Anal. Chem. 43, 1090-3.

Frant, M.S. and Ross, J.W. (1970). Potassium ion specific electrode with high 
A totally specific enzyme electrode for the assay of L-lysine was described by White and Guilbault (1978). No response was observed with any D- or L-amino acid, except for L-lysine. The electrode can be used for the assay of amino acid in complex matrices, without the necessity for extensive separations and extensive instrumentation (e.g. amino acid analyser). The electrodes are quite stable, with a linear range of L-lysine of $5 \times 10^{-5}$ to $0.1 \mathrm{~mol} / 1$. The only limitation is the long response time (5-10 $\mathrm{min})$.

Fung et al. (1979) have proposed a totally specific electrode for L-methionine, prepared by immobilizing L-methionine $\gamma$-lyase (EC 4.4.1.11) onto an ammonia-specific electrode. The $\alpha, \gamma$ elimination of L-methionine proceeds with formation of $\alpha$-ketobutyrate, methane thiol, and ammonia.

Only methionine reacts with purified enzyme, with a linear range of $10^{-2}-$ $10^{-5} \mathrm{~mol} / \mathrm{l}$ observed.

A potentiometric L-tyrosine electrode for the direct determination of L-tyrosine in biological fluids was described by Havas and Guilbault. The sensor element of the probe is a carbon-dioxide-gas membrane electrode covered with a layer of immobilized apo L-tyrosine decarboxylase. A linear range of $2 \times 10^{-3}$ to $4 \times 10^{-5} \mathrm{~mol} / \mathrm{l}$ is observed, with no interference from any other $\mathrm{L}$-amino acids or from D-tyrosine.

\subsubsection{Glucose and sugar electrodes As mentioned previously, glucose} assay is of extreme importance, used as a diagnostic monitor for diabetes.

Nagy et al. (1973) described a self-contained electrode for glucose based on an iodide membrane sensor:

$$
\begin{array}{lll}
\text { Glucose }+\mathrm{O}_{2} & \stackrel{\text { Glucose oxidase }}{\longrightarrow} & \text { Gluconic acid }+\mathrm{H}_{2} \mathrm{O}_{2} \\
\mathrm{H}_{2} \mathrm{O}_{2}+2 \mathrm{I}^{-}+2 \mathrm{H}^{+} \stackrel{\text { Peroxidase }}{\longrightarrow} & 2 \mathrm{H}_{2} \mathrm{O}+\mathrm{I}_{2}
\end{array}
$$

The highly sensitive iodide sensor monitors the decrease in the iodide activity at the electrode surface. The assay of glucose was performed both in a stream and at a stationary electrode. Pretreatment of the blood sample was required to remove interfering reducing agents, such as ascorbic acid, tyrosine, and uric acid.

Nilsson et al. (1973) described the use of conventional $\mathrm{pH}$ glass electrodes for the preparation of enzyme-pH electrodes by either entrapping the enzymes within polyacrylamide gels around the glass electrode, or as a liquid layer trapped within a cellophane membrane. In an assay of glucose, based on a measurement of the gluconic acid produced, the $\mathrm{pH}$ response was almost linear from $10^{-4}$ to $10^{-3} \mathrm{~mol} / 1$ with a $\mathrm{pH}$ change of about 0.85 per decade. Electrodes of this type were also constructed for urea and penicillin. The ionic strength and $\mathrm{pH}$ were controlled using a weak $(1 \mathrm{mmol} / \mathrm{l})$ phosphate buffer, $\mathrm{pH} 6.9$, and $0.1 \mathrm{~mol} / 1$ sodium sulphate. 
$\mathrm{CA} / \mathrm{CI} / \mathrm{SO}$ membrane, and one for creatine using a $\mathrm{Cl} / \mathrm{SO}$ membrane. The response time of the electrodes was $20 \mathrm{~s}$ in the rate mode, with a detection limit of $1 \mathrm{mg} /$.

More than 500 assays could be performed with one electrode, and no loss of activity was observed after nine months of storage at $4{ }^{\circ} \mathrm{C}$.

Mascini et al. (1985) proposed an enzyme reactor system, consisting of soluble NADPH and $\alpha$-ketoglutarate, with glutamate dehydrogenase immobilized onto nylon tubes, for the removal of $98 \%$ of the ammonia present in human blood and urine samples in $50 \mathrm{~s}$. This abatement of ammonia permits the use of an ammonia probe, coupled with immobilized creatininase (Carlo Erba enzyme coil, the Clinibond), for the assay of creatinine at low levels in blood and urine. Only $200 \mu \mathrm{l}$ of sample are required, and the entire process was carried out in a single-flow stream.

9.6.1.3 Amino acids Enzyme electrodes have been widely used for the assay of amino acids in clinical analysis since several amino acids (tyrosine, phenylalanine, tryptophan, methionine) are important diagnostic health indicators. The first of such analytical probes was described by Guilbault and Hrabankova (1970), who placed an immobilized layer of L-amino acid oxidase from snake venom over a monovalent cation electrode, which senses the ammonium ion formed in the enzyme catalysed oxidation of the amino acid. Gauilbault and Nagy (1973b) developed two different types of sensors for L-phenylalanine in blood: (a) an L-amino acid oxidase/peroxidase reaction layer in polyacrylamide, placed over an iodide-selective electrode. This probe senses the decrease in the activity of iodide at the electrode surface, according to reactions 9.6 and 9.7:

$$
\begin{array}{ll}
\text { L-Phenylalanine } & \stackrel{\text { Oxidase }}{\longrightarrow} \mathrm{H}_{2} \mathrm{O}_{2} \\
\mathrm{H}_{2} \mathrm{O}_{2}+2 \mathrm{H}^{+}+2 \mathrm{I}^{-} \stackrel{\text { Peroxidase }}{\longrightarrow} \mathrm{I}_{2}+2 \mathrm{H}_{2} \mathrm{O}
\end{array}
$$

the second electrode (b) used a silicone-rubber-based non-actin-type ammonium ion electrode covered with L-amino oxidase in polyacrylamide gel. This electrode has a longer linear range and is more selective than electrode (a).

Guilbault and Shu (1972) evaluated the used of a base carbon dioxide sensor coated with tyrosine decarboxylase for assay of L-tyrosine in body fluids. A linear range of $2.5 \times 10^{-4}$ to $10^{-2} \mathrm{~mol} / \mathrm{I}$ was observed, with a slightly faster response time than that recorded for a similarly based urea electrode.

Guilbault and Shu (1971) described an enzyme electrode for glutamine, prepared by entrapping glutaminase on a nylon net held between a layer of cellophane and a cation electrode. The electrode responds to glutamine over the concentration range $0.1-10^{-4} \mathrm{~mol} / 1$, with a response of only $1-2 \mathrm{~min}$. 
The response time of the electrode to urea was about 7-10 minutes, and a linear range of $5 \times 10^{-5}$ to $10^{-2} \mathrm{~mol} / 1$ was obtained with a slope of $0.8 \mathrm{pH}$ units per decade.

Still another possibility for a urea electrode is the use of a urease-covered carbon dioxide sensor, to measure the second product of the urea-urease reaction, $\mathrm{HCO}_{3}^{-}$. Guilbault and Shu (1972) showed that $\mathrm{Na}^{+}$and $\mathrm{K}^{*}$ had no influence on this electrode and the linear range was $10^{-4}$ to $10^{-2} \mathrm{~mol} / 1$.

A coated-wire urea electrode was described by Alexander and Joseph (1981), in which a pH-sensing antimony metal wire was coated with a layer of urease. Response times of 1-2 minutes were obtained, with a linear range of $5 \times 10^{-4}$ to $10^{-2} \mathrm{~mol} / \mathrm{l}$ urea and a slope of $44 \mathrm{mV} /$ decade. Later Joseph (1985) described a urea electrode based on a gas membrane, ammonia electrode, constructed of antimony metal. This microsensor responds from $10^{-4}$ to $10^{-2} \mathrm{~mol} / 1$ of urea in $30-45 \mathrm{~s}$. The ammonia sensor was reported to have a faster base-line recovery than the commercial gas membrane electrodes, thus a distinct advantage.

Guilbault and Mascini (1977) described a highly specific and reproducible enzyme electrode for urea, using the enzyme urease chemically bound and attached to a new, improved Teflon membrane, which is an integral part of the ammonia-gas membrane electrode. From 200-1000 assays could be performed on one electrode, with a coefficient of variance of $2.5 \%$, over the concentration range $5 \times 10^{-5}$ to $10^{-2} \mathrm{~mol} / \mathrm{l}$; at least 20 assays $/ \mathrm{hr}$ can be performed.

Enzyme electrodes for urea, using glutaraldehyde-attached urease and either a carbon dioxide or a cation-selective glass electrode sensor, were described by Tran-Minh and Brown (1975). A range of $10^{-5}-10^{-1} \mathrm{~mol} / \mathrm{I}$ was obtained using the ammonia glass electrode as the base sensor.

\subsubsection{Creatinine (a diagnostic indicator of kidney function) Thompson} and Rechnitz (1974) have described the use of unpurified creatininase with an ammonium probe for a creatinine electrode. Guilbault et al, (1980) used a similar system, albeit with purified creatininase immobilized onto alkylamine glass beads, packed into a stirrer, and an ammonia electrode as sensor. The residual ammonia level of blood serum was lowered using several removal techniques.

An improved direct-reading, specific electrode for creatinine was disclosed by Guilbault and Coulet (1983), using a highly specific creatininase from Carlo Erba. Linearity from 1-100 mg\% was obtained. An enzyme electrode system for creatinine proposed by Tsuchida and Yoda (1983) utilized three enzymes: creatinine amidohydrolase (CA), creatine amidohydrolase $(\mathrm{CI})$ and sarcosine oxidase (SO), co-immobilized onto the porous side of a cellulose acetate membrane. Two multi-enzyme electrodes were constructed using a hydrogen-peroxide sensing electrode, one for creatinine plus creatine using a 
The range of most enzyme electrodes is $10^{-2}$ to $10^{-4} \mathrm{M}$, with some extending up to $10^{-1} \mathrm{M}$ (depending on the solubility of the substrate in the aqueous solution) and some extending down to $10^{-5} \mathrm{M}$, or lower, depending on the detection limit of the base sensor.

Deterioration of the enzyme electrode can be seen by three changes in the response characteristics: (1) with age the upper limit will decrease, from say $10^{-1}$ to $10^{-2} \mathrm{M},(2)$ the slope of the calibration curve of potential vs. Iog [concentration], originally $60 \mathrm{mV} /$ decade, Nernstian, will drop to 50,40 perhaps $30 \mathrm{mV} /$ decade, or lower, and (3) the response time of the electrode, originally $30 \mathrm{~s}-4 \mathrm{~min}$, (approximately the same as that of the base sensor), will become longer as the enzyme ages.

In construction of an enzyme electrode, it is important that a highly purified enzyme be used (at least 10 units $/ \mathrm{mg}$ ) so that only a small amount of enzyme need to be used in the construction of the electrode. This will ensure a fast response time, approaching that of the base probe. As can be seen in Table 9.1, at least $10 \mathrm{U}(1 \mathrm{mg})$ of enzyme is generally used.

The stability of the electrode depends on the type of entrapment, chemically attached enzyme electrodes being the most stable (500-1000 runs/electrode), and a storage stability of about 6-14 months.

\subsection{Examples of enzyme electrodes based on ISEs}

\subsubsection{Some commonly used enzyme electrodes}

9.6.1.1 Urea (diagnostic indication for kidney function) The first urea electrode was prepared by Guilbault and Montalvo (1969), by immobilizing urease in a polyacrylamide matrix on nylon or dacron nets. These nets were then placed onto a Beckman cation selective electrode (which responds to ammonium ion). In later publications Guilbault and Montalvo (1970) described an improved electrode, covered with a cellophane membrane, that could be used for 21 days with no loss of activity.

In attempts to improve the selectivity of the urea determination, Guilbault and Nagy (1973a) used a silicone rubber based nonactin ammonium-ion selective electrode as the base sensor, together with immobilized urease in a polyacrylic gel. Guilbault et al. (1973) used a three electrode system, which allowed dilution to a constant interference level. Four months electrode stability resulted.

Anfalt et al. (1973) polymerized urease directly onto the surface of an Orion ammonia-gas membrane by means of glutaraldehyde. Sufficient ammonia was produced in the enzyme reaction layer, even at $\mathrm{pH}$ values as low as $7-8$, to allow direct assay of urea in the presence of large amounts of $\mathrm{Na}^{+}$and $\mathrm{K}^{+}$. A response time of $2-4$ minutes was observed.

A urea electrode, using physically entrapped urease and a glass electrode to measure the $\mathrm{pH}$ change in the solution, was described by Nilsson et al. (1973). 
Table 9.2 Continued

\begin{tabular}{|c|c|c|c|c|c|c|c|}
\hline Type & Enzyme & Sensor & Immobilization $^{\alpha}$ & Stability & $\begin{array}{l}\text { Response } \\
\text { time }\end{array}$ & $\begin{array}{l}\text { Amount of } \\
\text { enzyme (U) }\end{array}$ & Range $(\mathrm{mol} / \mathrm{l})^{b}$ \\
\hline $\begin{array}{l}\text { 4. D-Amino acids } \\
\text { (general })^{e}\end{array}$ & $\begin{array}{l}\text { D-AA oxidase } \\
\text { (EC 1.4.3.3) }\end{array}$ & Cation & Physical & 1 month & $1 \mathrm{~min}$ & 50 & $10^{-2}-5 \times 10^{-5}$ \\
\hline \multirow[t]{2}{*}{ 5. Penicillin } & \multirow{2}{*}{$\begin{array}{l}\text { Penicillinase } \\
\text { (EC 3.5.2.6) }\end{array}$} & \multirow[t]{2}{*}{$\mathrm{pH}$} & Physical & $1-2$ weeks & $0.5-2 \mathrm{~min}$ & 400 & $10^{-2}-10^{-4}$ \\
\hline & & & Soluble & 3 weeks & $2 \min$ & 1000 & $10^{-2}-10^{-4}$ \\
\hline 6. Amygdalin & $\begin{array}{l}\beta \text {-Glucosidase } \\
(\text { EC 3.2.1.21) }\end{array}$ & $\mathrm{CN}^{-}$ & Physical & 3 days $^{f}$ & $10-20 \mathrm{~min}$ & 100 & $10^{-2}-10^{-5}$ \\
\hline 7. Nitrate & $\begin{array}{c}\text { Nitrate reductase/ } \\
\text { nitrite reductase } \\
\text { (EC 1.9.6.1/ } \\
1.6 .6 .4)\end{array}$ & $\mathrm{NH}_{4}^{+}$ & Soluble & 1 day & $2-3 \mathrm{~min}$ & 10 & $10^{-2}-10^{-4}$ \\
\hline 8. Nitrite & $\begin{array}{l}\text { Nitrate reductase } \\
\text { (EC 1.6.6.4) }\end{array}$ & $\operatorname{Gas}\left(\mathrm{NH}_{3}\right)$ & Chemical & $\begin{array}{l}3-4 \\
\text { months }\end{array}$ & $2-3 \mathrm{~min}$ & 10 & $5 \times 10^{-2}-10^{-4}$ \\
\hline
\end{tabular}

a 'Physical' refers to polyacrylamide gel entrapment in all cases; 'chemical' is attachment chemically to glutaraldehyde with albumin, to polyacrylic acid, or to acrylamide, followed by physical entrapment.

${ }^{b}$ Analytically useful range, either linear or with reasonable change if curvature is observed.

${ }^{c}$ Preparation lacks stability as evidenced by constant decrease in signal each day.

${ }_{d}$ Electrode responds to L-cysteine, L-leucine, L-tyrosine, L-trytophan, L-phenylalanine, and L-methionine.

'Electrode responds to D-phenylalanine, D-alanine, D-valine, D-methionine, D-leucine, D-norleucine, and D-isoleucine.

$f$ Time required for signal to return to base line before re-use. 
Table 9.2 Typical electrodes and their characteristics

\begin{tabular}{|c|c|c|c|c|c|c|c|}
\hline Type & Enzyme & Sensor & Immobilization $^{\sigma}$ & Stability & $\begin{array}{l}\text { Response } \\
\text { time }\end{array}$ & $\begin{array}{l}\text { Amount of } \\
\text { enzyme }(U)\end{array}$ & Range $(\mathrm{mol} / \mathrm{l})^{b}$ \\
\hline \multirow[t]{7}{*}{ 1. Urea } & \multirow{7}{*}{$\begin{array}{l}\text { Urease } \\
\text { (EC 3.5.1.5) }\end{array}$} & Cation & Physical & 3 weeks & $30 \mathrm{~s}-1 \mathrm{~min}$ & 25 & $10^{-2}-5 \times 10^{-5}$ \\
\hline & & Cation & Physical & 2 weeks & $1-2 \min$ & 75 & $10^{-2}-10^{-4}$ \\
\hline & & Cation & Chemical & $>4$ months & $1-2 \min$ & 10 & $10^{-2}-10^{-4}$ \\
\hline & & $\mathrm{pH}$ & Physical & 3 weeks & $5-10 \mathrm{~min}$ & 100 & $\begin{array}{l}5 \times 10^{-3}-5 \times \\
10^{-5}\end{array}$ \\
\hline & & $\operatorname{Gas}\left(\mathrm{NH}_{3}\right)$ & Chemical & 4 months & $2-4 \mathrm{~min}$ & 10 & $\begin{array}{l}5 \times 10^{-2}-5 \times \\
10^{-5}\end{array}$ \\
\hline & & $\mathrm{Gas}\left(\mathrm{NH}_{3}\right)$ & Chemical & 20 days & $1-4 \min$ & 0.5 & $10^{-2}-10^{-4}$ \\
\hline & & $\operatorname{Gas}\left(\mathrm{CO}_{2}\right)$ & Physical & 3 weeks & $1-2 \min$ & 25 & $10^{-2}-10^{-4}$ \\
\hline \multirow[t]{2}{*}{ 2. Glucose } & \multirow{2}{*}{ Glucose oxidase } & $\mathrm{pH}$ & Soluble & 1 week & $5-10 \mathrm{~min}$ & 100 & $10^{-1}-10^{-3}$ \\
\hline & & $1^{-}$ & Chemical & $>1$ month & $2-8 \mathrm{~min}$ & 10 & $10^{-3}-10^{-4}$ \\
\hline \multirow{3}{*}{$\begin{array}{l}\text { 3. L-Amino acids } \\
\text { (general) }^{d}\end{array}$} & \multirow{3}{*}{$\begin{array}{l}\text { L-AA oxidase } \\
\quad(\text { EC 1.4.3.2) }\end{array}$} & Cation & Physical & 2 weeks & $1-2 \mathrm{~min}$ & 10 & $10^{-2}-10^{-4}$ \\
\hline & & $\mathrm{NH}_{4}$ & Chemical & $>1$ month & $1-3 \min$ & 10 & $10^{-2}-10^{4}$ \\
\hline & & 1 & Chemical & $>1$ month & $1-3 \mathrm{~min}$ & 10 & $10^{-3}-10^{-4}$ \\
\hline L-Tyrosine & $\begin{array}{l}\text { L-Tyrosine } \\
\text { decarboxylase } \\
\text { (EC 1.1.25) }\end{array}$ & $\operatorname{Gas}\left(\mathrm{CO}_{2}\right)$ & Physical & 3 weeks & $1-2 \mathrm{~min}$ & 25 & $10^{-1}-10^{-4}$ \\
\hline L-Glutamine & $\begin{array}{l}\text { Glutaminase } \\
\text { (EC 3.5.1.2) }\end{array}$ & Cation & Soluble & 2 days & $1 \mathrm{~min}$ & 50 & $10^{-1}-10^{-A}$ \\
\hline L-Glutamic acid & $\begin{array}{l}\text { Glutamate } \\
\text { dehydrogenase } \\
\text { (EC 1.4.1.3) }\end{array}$ & Cation & Soluble & 2 days $^{c}$ & $I \min$ & 50 & $10^{-1}-10^{-4}$ \\
\hline L-Asparagine & $\begin{array}{l}\text { Asparaginase } \\
\text { (EC 3.5.1.1) }\end{array}$ & Cation & Physical & 1 month & $1 \mathrm{~min}$ & 50 & $10^{-2}-5 \times 10^{-5}$ \\
\hline
\end{tabular}


into the enzyme and permit loss of entrapped air. Store the electrode in buffer (optimum for the enzyme system) in a refrigerator between use.

\subsubsection{Apparatus}

The enzyme electrode, once constructed, is used like any other ion-selective electrode. The potentiometric probes, e.g. urea, amino acids, penicillin, are plugged directly into a digital voltmeter (e.g. Orion, Corning, Sargent, Amel, etc.). The $\mathrm{mV}$ readings for each concentration tested are then plotted $v s$. concentration in a linear-log plot.

A reference electrode, generally a calomel electrode, is used together with the enzyme electrode. Alternatively, the reference electrode can be combined as an integral part of the enzyme electrode, as is the case with the ammonia, carbon dioxide, or oxygen electrode base sensors used in the urea, amino acid, glucose, or alcohol probes.

Finally, the electrode must be kept in a solution with constant stirring rate, since it has been shown that a change in stirring rate will change the potential of the electrode measured.

\subsection{Operational properties of electrodes}

Table 9.2 gives a listing of some enzyme electrodes that have been prepared for analysis of common substrates together with the enzyme used, the sensor, the immobilization method, the stability of the probe, the response time of the electrode, the units (U) of enzyme used to make the electrode, and the range of concentrations determinable. (See Guilbault (1984) for a complete listing of electrodes available.)

In several cases, many different base sensors could be used. For example, for urea, one could use either a cation electrode, which measures the $\mathrm{NH}_{4}^{+}$ion formed in the urease-catalysed hydrolysis of urea:

$$
\text { Urea } \stackrel{\text { Urease }}{\longrightarrow} \mathrm{NH}_{4}^{-}+\mathrm{HCO}_{3}^{-}
$$

or an $\mathrm{NH}_{3}$ or $\mathrm{CO}_{2}$ electrode to measure either the $\mathrm{NH}_{3}$ (formed by adding - $\mathrm{OH}$ to $\mathrm{NH}_{4}^{+} \longrightarrow \mathrm{NH}_{3}$ ) or the $\mathrm{CO}_{2}$ (formed by adding $\mathrm{H}^{+}$to the $\mathrm{HCO}_{3}^{-}$ $\longrightarrow \mathrm{CO}_{2}$ ). By far the best probe is the $\mathrm{NH}_{3}$ electrode, because of its high specifity and low limit of detection $\left(10^{-6} \mathrm{M}\right.$ compared to $5 \times 10^{-5} \mathrm{M}$ for the $\mathrm{CO}_{2}$ electrode). The disadvantage of use of this electrode is slow response time (2-4 $\mathrm{min}$ ) and long recovery time to return to the original base line (5-10 min). Guilbault and Mascini (1977), for example, showed that by chemically attaching urease to a polypropylene membrane, which is an integral part of the $\mathrm{NH}_{3}$ gas membrane electrode, that 200-1000 assays can be performed on one electrode with a C.V of $2.5 \%$ over the range of $5 \times 10^{-5}$ to $10^{-2} \mathrm{M}$. At least 20 assays $/ \mathrm{hr}$ can be made with excellent correlation with the results obtained by the spectrophotometric diacetyl procedure. 


\subsection{Preparation of a typical electrode}

Take the base sensor (chosen from Table 9.1) and turn it upside down (Fig. 9.1b). Cover the sensor with a piece of pig intestine membrane (Universal Sensors, New Orleans, USA). Wet the membrane with $30 \mu \mathrm{l}$ of $25 \%$ albumin solution, then add 10 units of enzyme, dissolving it in the albumin solution. Add $5 \mu \mathrm{l}$ of $25 \%$ glutaraldehyde to complete the immobilization and leave to dry. Then cover with a piece of dialysis membrane (cellophane, 20-25 $\mu$ m thick, Will Scientific, Arthur Thomas, Sigma, etc.) about twice the diameter of the size of the electrode sensor. Place a rubber O-ring, with a diameter that fits the electrode body snugly, around the cellophane membrane (Fig. 9.1b), and gently push the O-ring onto the electrode body, so that enzyme forms a nice uniform layer on top of the electrode surface. Place the electrode in buffer solution overnight to allow penetration of buffer
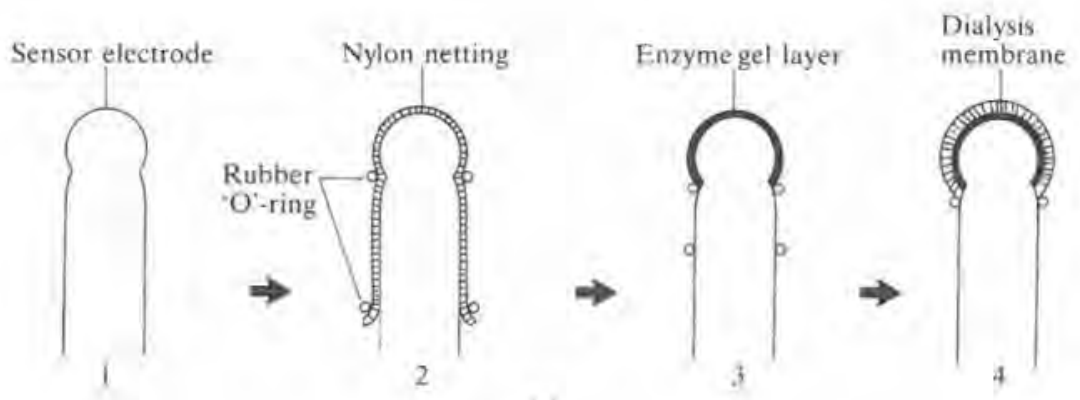

(a)

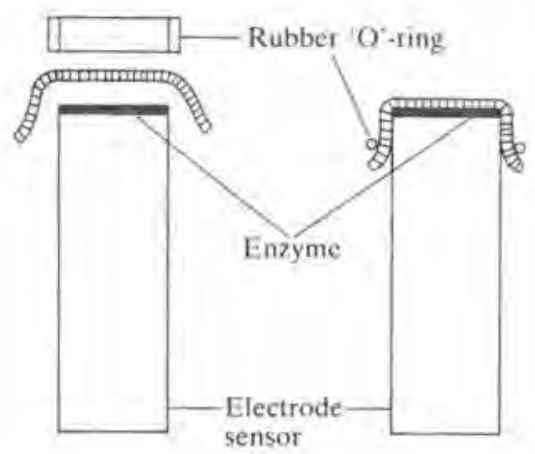

(b)

Fig. 9.1 Preparation of enzyme electrode probes

(a) using physically entrapped enzymes and

(b) using chemically attached enzymes. 
Table 9.1 Possible ISE sensors useful in construction of enzyme electrode

\begin{tabular}{ll}
\hline Potentiometric sensors & Useful for \\
\hline $\mathrm{NH}_{3}$ & $\begin{array}{l}\text { Urea, amino acids, glutamine, glutamic acid, } \\
\text { nitrate, nitrite, creatinine, lyase, and deaminase } \\
\text { enzymes. }\end{array}$ \\
& $\begin{array}{l}\text { Urea, amino acids, decarboxylative enzyme systems. } \\
\mathrm{CO}_{2}\end{array}$ \\
$\mathrm{pH}$ & $\begin{array}{l}\text { Penicillin, RNA, DNA, glucose, enzyme reactions } \\
\text { giving pH change. }\end{array}$ \\
& Glucose, amino acids, cholesterol, alcohols. \\
$\mathrm{I-}$ & Amygdalin. \\
\hline
\end{tabular}

produced is monitored) enzyme electrode was described by Guilbault and Montalvo for urea in 1969. Since this, over one hundred different electrodes have appeared in the literature; a summary of these can be found in a recent book (Guilbault 1984).

In enzyme electrodes, the enzyme is usually immobilized, thus reducing the amount of material required to perform a routine analysis, and eliminating the need for frequent assay of the enzyme preparation in order to obtain reproducible results. Furthermore, the stability of the enzyme is often improved when it is incorporated in a suitable gel matrix. An electrode for the determination of urea prepared by covering an ammonium ion selective electrode with chemically bound urease has been used for over 300 days, for example (Guilbault 1984).

Of the two methods used to immobilize an enzyme: (a) the chemical modification of the molecules by the introduction of insolubilizing groups and (b) the physical entrapment of the enzyme in an inert matrix, such as starch or polyacrylamide (Chapter 6), the technique of chemical immobilization is the best to make electrode probes.

An enzyme electrode operates via a five-step process: (i) the substrate must be transported to the surface of the electrode, (ii) the substrate must diffuse through the membrane to the active site, (iii) reaction occurs at the active site, (iv) product formed in the enzymatic reaction is transported through the membrane to the surface of the electrode, and (v) product is measured at the electrode surface. The first step, transport of the substrate, is most critically dependent on the stirring rate of the solution, so that rapid stirring will bring the substrate very rapidly to the electrode surface. If the membrane is kept very thin, using highly active enzyme, then steps 2 and 4 are eliminated or minimized; since step 3 is very fast, the response of an enzyme electrode should theoretically approach the response time of the base sensor. Many researchers have shown with experimental data that one can approach this behaviour by using a thin membrane and rapid stirring. 
sensitive to $\mathrm{NH}_{4}^{*}$ with linear near-Nernstian response of $51 \mathrm{mV}$ per decade change over the concentration range $10^{-1}$ to $10^{-4} \mathrm{M} \mathrm{NH}_{4}^{+}$. This electrode responds poorly to $\mathrm{Li}^{+}$ion activity and the selectivity for $\mathrm{NH}_{4}^{+}$over $\mathrm{K}^{+}$and $\mathrm{Na}^{+}$was reported to be superior to glass electrodes sensitive to ammonium ions. The nonactin electrode has been used in an enzyme system and will be discussed in the second part of this review. Valinomycin-based potassium ion-selective electrodes have been studied (Eyal and Rechnitz 1971). The antibiotic may be used in a suitable organic solvent or an inert matrix (Pick et al. 1973) and electrodes prepared in this manner have been used to measure the potassium content of serum (Frant and Ross 1970). Greater concentrations of sodium can be tolerated with the antibiotic electrode than with the sodium glass electrode. For a more detailed account of the neutral carrier ionselective electrodes, the chapter by Eisenman (1969) in the N.B.S. proceedings is recommended.

The final electrodes to be considered are the gas-sensing type prepared by placing a gas-permeable membrane over a housing which contains a $\mathrm{pH}$ electrode and internal filling solution. Electrodes of this type are available for ammonia, carbon dioxide, and hydrogen sulphide. The $\mathrm{CO}_{2}$ electrodes have found most use in the determination of blood $P_{\mathrm{CO}_{z}}$ (Haake 1975; Winters 1981.) Ammonia in natural waters and solids has been determined directly using an ammonia electrode and the measurement of the ammonia or ammonium content of a wide variety of samples is feasible since only gases which diffuse through the membrane would interfere with the operation of the device.

\subsection{Enzyme electrodes}

The classic potentiometric enzyme electrode is a combination of an ionselective electrode-base sensor with an immobilized (insolubilized) enzyme, which provides a highly selective and sensitive method for the determination of a given substrate. Some of the ion-selective electrodes useful in construction of enzyme electrodes are presented in Table 9.1. Advantages of such potentiometric sensors are simplicity of instrumentation (only a $\mathrm{pH}$ meter is needed, not a polarographic system as required for amperometric based probes), low cost, and easy availability of a large number of good, reliable base ISEs.

Clark and Lyons (1962) first introduced the concept of the 'soluble' enzyme electrode (Chapter 1), but the first working electrode was reported by Updike and Hicks (1971) using glucose oxidase immobilized in a gel over a polarographic oxygen electrode to measure the concentration of glucose in biological solutions and tissues. These are both voltammetric or amperometric probes, i.e. the current produced upon application of a constant applied voltage is measured. The first potentiometric (no applied voltage, the voltage 
Ammann et al. (1972). This electrode is reported to have superior selectivity for calcium over sodium and magnesium.

The family of electrodes prepared from silver sulphide alone or mixed with halogen salts of silver are well characterized (Pungor 1967; Rechnitz 1967), The precipitates are used either in the form of a pellet or mixed in an inert supporting matrix such as silicon rubber. When silver sulphide is used alone, the electrode responds to sulphide and silver ions over the concentration range $10^{\circ}$ to $10^{-7} \mathrm{M}$. The sulphide content of natural waters and low levels of silver have been determined with this electrode.

When a particular silver halide is mixed with silver sulphide to form a sensor membrane, the membrane behaves as though it was composed of the halide salt alone. Electrodes for iodide, bromide, and chloride have been prepared in this manner. The iodide electrode also responds to cyanide. An electrode for thiocyanate can be prepared by mixing silver thiocyanate with silver to form a sensor membrane. The selectivity coefficients of the halide and pseudo-halide electrodes can be estimated by:

$$
k_{i j}=\frac{\text { Solubility product of } \mathrm{Ag}_{i}}{\text { Solubility product of } \mathrm{Ag}_{j}} .
$$

Liquid ion-exchange electrodes which are sensitive to nitrate, perchlorate, fluoroborate, and chloride generally have a useful working range of $10^{-1}$ to $10^{-5} \mathrm{M}$. The liquid ion-exchange electrode for chloride is not as seriously affected by the presence of sulphide and the halogens as the solid-state chloride electrode. Therefore, it can be used for many assays in which the interference caused by sulphide or the halogens is not negligible. The perchlorate and fluoroborate electrodes have limited applications in analysis. However, the nitrate electrode has been used to directly determine nitrate in many types of samples.

The glass electrodes for determination of monovalent and divalent cations have been used in a variety of clinical and environmental studies. Garrels (1967) used a potassium/sodium glass electrode for measuring these cations in sea water. Parts-per-billion quantities of sodium in water were measured by Budd and Jones (1963) and Annino (1967) used a sodium ion-selective electrode to measure the sodium content of urine. There are many other applications of glass electrodes in the areas of environmental and clinical chemistry. The text edited by Eisenman (1967) and the chapter of the N.B.S. publication written by Khuri (1969) are recommended to those who are interested in these areas (see also Chapter 20).

Antibiotics and similar compounds have been used successfully to prepare cation-selective electrodes. The calcium ion-selective electrode developed by Ammann et al. (1972) has been mentioned earlier. Pioda et al. (1967) have studied the properties of the antibiotic nonactin for use as a sensor membrane. An electrode prepared from this material in an inert matrix is 
or masked. It is often necessary to use a buffer solution to control ionic strength, $\mathrm{pH}$, and to prevent changes in the activity of the ion being measured by oxidation, reduction, or complexation.

There have been a large number of reports of applications and progress in the design and manufacture of ion-selective electrodes in the literature. An important development has been the use of PVC in the preparation of ionselective electrodes. Electrodes manufactured with PVC are much lower in cost, they have essentially the same response characteristics and usually can be used for longer periods of time than previous electrode assemblies. Moody et al. (1970) constructed a calcium-selective electrode using a liquid ion exchanger incorporated in a PVC matrix. The optimum concentration of calcium exchanger used in preparation was described by Griffiths et al. (1972). The electrode constructed in this manner gave a near-Nerstian response ( $30 \mathrm{mV}$ per $\mathrm{pC}$ a unit) over the range $2.6 \times 10^{-2}$ to $6.0 \times 10^{-5} \mathrm{M}$ in $\mathrm{CaCl}_{2}$ solution. Davies et al. (1972) prepared nitrate ion-selective electrodes by incorporating commercially available liquid ion-exchangers in PVC. These electrodes overcome the problem of leakage that is associated with other liquid ion-exchange assemblies.

Pick et al. (1973) have used valinomycin in a variety of neutral carriers to prepare ion-selective electrodes for potassium. The response time of this type of electrode is usually less than $3 \mathrm{~s}$ and the useful range of the electrodes is $10^{-1}$ to $10^{-5} \mathrm{M}$. This electrode prepared from this material is exceptional in that there is very little or no drift in potential over a three-day period. As the working characteristics of electrodes are improved and new electrodes are introduced many new applications of these devices can be expected.

The use of ion-selective electrodes for the determination of the calcium content of biological materials has been investigated vigorously since calcium is one of the most important electrolytes in human physiology (Moore 1969). The electrode most commonly used in these studies has been a liquid ionexchanger of the calcium salt of didecyl phosphoric acid in didecyl-phenyl phosphonate. Such an electrode has a working range of $10^{\circ}$ to $10^{-5} \mathrm{M}$ in calcium. The electrode responds to ionized calcium and if the total calcium content of a sample is to be measured, calcium must be freed from ligands or chelates prior to measurement.

The normal values of calcium in serum range from 8.5 to $10.5 \mathrm{mg} / 100 \mathrm{ml}$ in elders to 9.0 to $11.0 \mathrm{mg} / 100 \mathrm{ml}$ in children (Tietz 1970). Of this, 30 to $55 \%$ is present as protein-bound calcium, 5 to $10 \%$ is present in the form of complexes and chelates and the remainder is ionized calcium (Moore 1969). Studies of apparent ionized serum calcium have been carried out by Hattner et al. (1970) and by $\mathrm{Li}$ and Piechoki (1971). In the case of serum measurements, standards are prepared using solutions with sodium content (150 mM) that approximates the sodium concentration of serum samples. A new calcium-selective electrode based on a neutral carrier has been developed by 
The value of $a_{i}$ is taken at the point where serious deviation from Nernstian response is noted (Moody and Thomas 1972).

At present, ion-selective electrodes can be divided into several categories according to the composition of their sensor membranes.

1. Glass electrodes are ion-selective electrodes in which the sensing membrane is a very thin membrane of glass usually in the shape of a bulb. The composition of the glass determines the selectivity of the membrane. Glass electrodes are available which are sensitive to $\mathrm{H}^{*}$ ( $\mathrm{pH}$ electrodes) and to cations in the order $\mathrm{Ag}^{+}>\mathrm{H}^{+}>\mathrm{K}^{+}>\mathrm{NH}_{4}^{+}>\mathrm{Na}^{+}>\mathrm{Li}^{+}$, $\mathrm{Ca}^{++}, \mathrm{Mg}^{++}$over a concentration range $10^{-1}$ to $10^{-5} \mathrm{M}$.

2. Solid-state electrodes are ion-selective electrodes in which the sensor is a thin layer of a single or mixed crystal or precipitate which is an ion conductor. Two classes of these electrodes are distinguished: homogeneous and heterogenous. Homogeneous electrodes refer to those electrodes in which the membrane is a pellet prepared from a precipitate, mixture of precipitates, or a single crystal. In the heterogeneous electrodes a precipitate or mixture or precipitates is dispersed in an inert supporting matrix such as silicone rubber or poly (vinyl chloride) (PVC).

3. Liquid ion-exchange electrodes are prepared by dissolving an organic ion exchanger in an appropriate solvent. The solution is held in an inert matrix. Ion exchangers at present used in the preparation of these electrodes may be a ligand association complex such as those formed by the transition metals with derivatives of 1,10 -phenanthroline, quarternary ammonium salts, organic-phosphate complexes, and antibiotics. In some cases the exchanger and solvent are entrapped in an inert polymer matrix such as PVC or poly (methyl methacrylate) and coated on a platinum wire or graphite rod.

4. There are special electrodes which employ a coating over the membrane of an ion-selective electrode. The coating may be a gas-permeable membrane in which case electrodes sensitive to $\mathrm{CO}_{2}$ or $\mathrm{NH}_{3}$ are the result. The gas diffuses through the membrane and alters the $\mathrm{pH}$ of an internal filling solution. The $\mathrm{pH}$ change is measured with a glass electrode and is proportional to the concentration of gas which enters the membrane. Another coating, which has been used successfully, contains an enzyme which converts a substrate to an ion which is detected by an ionselective electrode.

These electrodes will be discussed in some detail in the second part of this chapter.

When using an ion-selective electrode to make potentiometric measurements of the activity of a given ion in solution, it is important to remember that the device is affected by the activity of the ion. Therefore, species which may complex the ion of interest and lower its activity must either be removed 
activity. The response of the glass electrode was commonly believed to be a result of migration of hydrogen ions through the thin glass membrane. The studies carried out by Karrenman and Eisenman (1962) and the work of Stephanova et al. (1963) provided the insight necessary for the development of new ion-selective electrodes. At present, ion-selective electrodes (ISEs) for $\mathrm{Na}^{+}, \mathrm{K}^{+}, \mathrm{Mg}^{+}, \mathrm{Ca}^{+}, \mathrm{Cd}^{+}{ }^{*}, \mathrm{Cu}^{+}{ }^{+}, \mathrm{Ag}^{+}, \mathrm{NH}_{4}^{+}, \mathrm{S}^{-}, \mathrm{I}^{-}, \mathrm{Br}^{-}, \mathrm{Cl}^{-}, \mathrm{CN}^{-}$, $\mathrm{SCN}^{-}, \mathrm{F}^{-}, \mathrm{NO}_{3}^{-}, \mathrm{ClO}_{4}^{-}, \mathrm{BF}^{-}$, as well as $\mathrm{H}^{-}$, are available. The electrodes are available from many manufacturers and as newer methods of preparation of ISEs have been developed, several kits for the preparation of different electrodes using a common body or housing have been introduced.

An ion-selective electrode may be defined as a device that develops an electrical potential proportional to the logarithm of the activity of an ion in solution. The term 'specific' is sometimes used to describe an electrode. This term indicates that the electrode responds to only one particular ion. Since no electrode is truly specific for one ion, the term ion-selective is recommended as more appropriate.

The response of an ion-selective electrode to an ion, $i$, of activity $a_{i}$ and charge $z$, is given by the Nickolski equation:

$$
E=\text { constant }+\frac{2.303 R T}{z F} \log \left[a_{i}+k_{i j}\left(a_{j}\right)^{z / y}\right]
$$

in which $E$ is the measured potential, $R$ is the gas constant and is equal to 8.314 joules deg ${ }^{-1}, T$ is the absolute temperature in Kelvins, $F$ is the Faraday constant equal to 96487 coulombs equiv $^{-1}, k_{i j}$ is the selectivity coefficient, and $j$ is any interfering ion of charge $y$ and activity $a_{j}$. The sign of the second term on the right-hand side of eqn $(9,1)$ is positive for cations and negative for anions.

The selectivity coefficient is a numerical description of the preferential response of an ion-selective electrode to the major ion, $i$, in the presence of the interfering ion $j$. The lower the numerical value of $k_{i j}$ for a particular ion-selective electrode the greater concentration of $f$ can be tolerated before causing errors in the measurement. Values of $k_{j}$ can be calculated from:

$$
\pm \frac{E_{2}-E_{1}}{2.303 R T / z F}=\log k_{i j}+\left(\frac{z}{y}-1\right) \log a_{i}
$$

in which $E_{1}$ and $E_{2}$ are the measurements of separated solutions of the principle ion and interfering ion, respectively, at the same activity. Or, more realistically, $k_{i j}$ may be obtained by making measurements of the potential of an ion-selective electrode in solutions of constant interferent activity, $a_{j}$, and changing primary ion activity, $a_{i}$. Then $k_{i j}$ may be determined by

$$
a_{i}=k_{i j}\left(a_{j}\right)^{z / y} \text {. }
$$




\title{
9 \\ Ion-selective electrodes and biosensors based on ISEs
}

\author{
S. S. KUAN and G. G. GUILBAULT
}

\subsection{Introduction}

$\mathrm{pH}$ is one of the most commonly made measurements in the chemical laboratory. The glass electrode, selective for hydrogen ions, a reference electrode, and a pH meter combine to form an extremely useful analytical tool. The advantages of this measuring system, are speed, sensitivity, cost, reliability, and the sample is not destroyed or consumed in the process. The same advantages apply to other ion-selective electrodes which have become available in the past few years. At this time, ion-selective electrodes which are sensitive to particular cations and anions can be purchased or constructed at moderate cost. The analytically useful range of these sensors is generally from $10^{-1} \mathrm{M}$ to $10^{-5} \mathrm{M}$ although there are many sensors which are useful at even lower concentrations. Since the response of ion-selective electrodes is logarithmic, the precision of measurements is constant over their dynamic range.

Ion-selective electrodes are finding many applications in biological studies. Particularly useful applications are in the field of clinical chemistry where a large number of samples, and the need for a rapid method of analysis, rule out many slower, more involved methods.

Enzyme electrodes represent the most recent advance in analytical chemistry. These devices combine the selectivity and sensitivity of enzymatic methods of analysis with the speed and simplicity of ion-selective electrode measurements. The result is a device that can be used to determine the concentration of a given compound in solution quickly and a method that requires a minimum of sample preparation. Enzyme electrodes for the determination of glucose, urea, L-amino acids, penicillin, and other substances of clinical importance have been developed.

\subsection{Ion-selective electrodes}

The hydrogen-selective or $\mathrm{pH}$ electrode, the best known ion-selective electrode, traces its discovery to Cremer (1906) and Haber and Klemensiewicz (1909) who found that certain glasses respond to hydrogen ion 
arise when the electrode kinetics are slow and there is no product inhibition. The rate constant $k^{\prime}$ occurs in the same bracket as $L k_{\text {cat }}$ since in either case the rate-limiting step involves turnover of the enzyme. Thirdly, the other two terms involving $k^{\prime}$ are also cases where the electrode kinetics are rate limiting. In the first bracket most of the enzyme is present as either $\mathrm{ES}$ or $\mathrm{E}^{\prime} \mathrm{P}$, while in the second bracket most of the enzyme is present as $\mathrm{E}$ and therefore requires $\mathrm{S}$ to be converted to $\mathrm{E}^{\prime}$. These terms are larger the larger the concentration of $\mathrm{P}$ behind the membrane, whether this is because of the external concentration $\left(p_{\infty}\right)$ or because of slow transport of the generated $\mathrm{P}$ across the membrane $\left(j / k_{\mathrm{p}}^{\prime}\right)$. This product inhibition arises because in going from E, ES, or E'P to $\mathrm{E}^{\prime}$ and thence to the rate-limiting transition state on the electrode, $\mathrm{P}$ has to be released. This does not apply if $\mathrm{E}^{\prime}$ is the dominant enzyme species when one obtains the simple $k^{1-i}$ term.

Finally we have the last term on the right of eqn 12.8 . This term will dominate if the transport of $\mathrm{S}$ through the membrane is rate limiting. Under these condition $j$ does not depend on the enzyme concentration; the kinetics of both the enzyme and the electrode are fast enough to consume $S$ as soon as it passes through the membrane.

\subsection{Second-generation electrodes}

We now extend the treatment to second-generation electrodes. For these electrodes the enzyme $E^{\prime}$ is regenerated by reaction with a mediator $M$ rather than by direct reaction on the electrode. In some cases the mediator is immobilized on the electrode surface. For this case the rate constant $k^{\prime}$ in the reaction scheme above simply describes the heterogeneous reaction of the enzyme with the immobilized mediator. Hence the same treatment applies.

Another possibility is that the mediator is present throughout the electrolyte layer. Now the $k^{\prime}$ reaction in the scheme must be replaced by a homogeneous reaction of $\mathrm{E}^{\prime}$ with $\mathrm{M}$ :

$$
\mathrm{E}^{\prime}+\mathrm{M} \stackrel{k_{4}}{\longrightarrow} \mathrm{E}+\mathrm{M}^{\prime}
$$

In most cases the concentration of $\mathrm{M}$ will be sufficiently large for its concentration to remain uniform. Then we can replace $k^{\prime}$ throughout eqn 12.8 by the equivalent rate constant describing enzyme regeneration, $L k_{4} m$ :

$$
\begin{aligned}
\frac{e_{\mathrm{\Sigma}}}{j}= & \left\{1-\frac{j}{k_{\mathrm{S}}^{\prime} s_{\infty}}\right\}\left\{\frac{1}{L k_{\mathrm{cat}}}+\frac{1}{L k_{4} m}+\frac{K_{3}^{-1}\left(1+K_{2}^{-1}\right)}{L k_{4} m}\left[p_{\infty}+\frac{j}{k_{\mathrm{p}}^{\prime}}\right]\right\} \\
& +\frac{1}{s_{\infty}}\left\{\frac{K_{\mathrm{M}}}{L k_{\mathrm{cat}}}+\frac{K_{1}^{-1} K_{2}^{-1} K_{3}^{-1}}{L k_{4} m}\left[p_{\infty}+\frac{j}{k_{\mathrm{p}}^{\prime}}\right]\right\}+\frac{e_{\Sigma}}{k_{\mathrm{S}}^{\prime} s_{\infty}}
\end{aligned}
$$

The discussion of the significance of the different terms is similar to that following eqn 12.8 . 
In extending the treatment to second-generation electrodes, we should perhaps have included the back reaction $k_{-4}$ and the electrochemical regeneration of the mediator;

$$
\mathrm{E}^{\prime}+\mathrm{M} \underset{k_{-4}}{\stackrel{k_{4}}{\rightleftarrows}} \mathrm{E}+\mathrm{M}^{\prime}
$$

Electrode

$$
\mathbf{M}^{\prime} \stackrel{k^{\prime}}{\longrightarrow} \mathrm{M}
$$

However considering the fate of $\mathrm{M}^{\prime}$, for the $k^{\prime}$ transition state to be higher in free energy than transition state 4 we require

$$
k^{\prime}<L k_{-4} e \text {. }
$$

Since $k^{\prime}$ describes an electrochemical reaction of a small mediator molecule, the reaction can be driven by the electrode potential, and we can assume that $k^{\prime}>0.1 \mathrm{~cm} \mathrm{~s}^{-1}$. With typical values for $L$ of $10^{-2} \mathrm{~cm}$ and for $e$ of $10^{-4} \mathrm{~mol}$ $\mathrm{dm}^{-3}$ we find that the inequality in eqn 12.12 can only be satisfied if $k_{-4}$ is greater than $10^{5} \mathrm{dm}^{3} \mathrm{~mol}^{-1} \mathrm{~s}^{-1}$. Values of $k_{-4}$ are seldom documented but can be calculated from the known values of $k_{4}$ and the difference between the standard reduction potentials of the enzyme and mediator respectively. It is found that even if the rate of the forward reaction is diffusion controlled $\left(\sim 10^{9} \mathrm{dm}^{3} \mathrm{~mol}^{-1} \mathrm{~s}^{-1}\right)$ a difference in the $E^{\circ}$ values of greater than $250 \mathrm{mV}$ will lead to values of $k_{-4}$ of less than $10^{5} \mathrm{dm}^{-3} \mathrm{~mol}^{-1} \mathrm{~s}^{-1}$. In practice the values of $k_{4}$ are less than this and generally mediators are chosen with sufficient difference in $E^{\circ}$ so as to allow the reaction to go to completion. Hence we are unlikely to find examples where we have to have the more complicated reaction scheme. This is just as well since the algebra for the more complicated scheme is formidable.

\subsection{NADH electrodes}

Over 250 enzymes use the ubiquitous cofactor $\mathrm{NAD}^{+} / \mathrm{NADH}$. The conducting organic salts are excellent electrodes for the efficient oxidation of NADH (Kulys 1981; Albery and Bartlett 1984). This finding allows us to develop a family of second-generation electrodes in which the enzyme turnover is followed by the regenerative oxidation of NADH to NAD ${ }^{+}$(Chapter 15). The reaction scheme for a substrate $\mathrm{SH}_{2}$ is:

$$
\begin{aligned}
& \mathrm{SH}_{2}+\mathrm{NAD} \cdot \stackrel{\text { Enzyme }}{\longrightarrow} \mathrm{S}+\mathrm{NADH}+\mathrm{H}^{*} \\
& \mathrm{NMP}^{+} \mathrm{TCNQ}^{-} \\
& \mathrm{NADH} \stackrel{\text { Electrode }}{\longrightarrow} \mathrm{NAD}^{+}+\mathrm{H}^{+}+2 \mathrm{e}
\end{aligned}
$$


As regards the detailed enzyme kinetics we assume the following model for a one substrate one product enzyme, which converts substrate $S$ to product $P$, using the NAD ${ }^{*} / \mathrm{NADH}_{\text {cofactor. We assume that there is sufficient NAD }}$. present so that the concentration of free enzyme, E, is much smaller than the concentration of enzyme bound to NAD ${ }^{-}, \mathrm{ENAD}^{+}$. We also assume that the kinetics of the binding of the enzyme to NAD is sufficiently rapid so that equilibrium is established between $\mathrm{E}$ and ENAD * with a binding constant of $K_{0}$ :

$$
\begin{aligned}
& \mathrm{S}+\mathrm{E} . \mathrm{NAD} \cdot \frac{k_{\mathrm{t}}}{\underset{k_{-1}}{\rightleftarrows}} \mathrm{S} \text {.E.NAD }+\frac{k_{2}}{\underset{k_{-2}}{\rightleftarrows}} \mathrm{P} . \mathrm{E} \cdot \mathrm{NADH} \underset{k_{-3}}{\stackrel{k_{3}}{\rightleftarrows}} \mathrm{E}+\mathrm{NADH}+\mathrm{P}+\mathrm{H}^{+} \\
& \mathrm{E}+\mathrm{NAD}+\rightleftarrows \text { E.NAD }{ }^{+} K_{0}
\end{aligned}
$$

Electrode

$$
\mathrm{NADH} \stackrel{k^{\prime}}{\longrightarrow} \mathrm{NAD}^{+}+\mathrm{H}^{+}+2 \mathrm{e}
$$

For this scheme we have

$$
K_{\mathrm{TD}}=K_{0} K_{1} K_{2} K_{3}
$$

where $K_{\mathrm{TD}}$ describes the overall equilibrium between $\mathrm{S}+\mathrm{NAD}$ and $\mathrm{P}+\mathrm{NADH}+\mathrm{H}^{+}$.

We then follow a similar argument to that used above, to obtain the following expression for $j$ :

$$
\begin{aligned}
\frac{e_{\Sigma}}{j}= & \frac{1}{L k_{\mathrm{cat}}}\left[1-\frac{j}{k_{\mathrm{S}}^{\prime} s_{\infty}}\right]+\frac{K_{\mathrm{M}}}{L k_{\mathrm{cat}} s_{\infty}}+\frac{j e_{\Sigma}}{k_{\mathrm{p}}^{\prime} k^{\prime} K_{\mathrm{TD}} S_{\infty}\left[\mathrm{NAD}^{+}\right]}+ \\
& \frac{j^{2}}{L k_{\mathrm{p}}^{\prime} k^{\prime} K_{\mathrm{TD}} S_{\infty}\left[\mathrm{NAD}^{+}\right]}\left[\frac{1}{k_{-1}}+\frac{K_{2}}{k_{-1}}+\frac{1}{k_{-2}}\right]+\frac{e_{\mathrm{\Sigma}}}{k_{\mathrm{S}}^{\prime} s_{\infty}}
\end{aligned}
$$

To simplify the expression, we have assumed that the external product concentration, $p_{\infty}$, is zero. Comparing this expression with eqn 12.8, we find that the enzyme kinetic terms in $k_{\text {cat }}$ and $K_{\mathrm{M}} / k_{\text {cat }}$ and the substrate transport term in $k_{\mathrm{s}}^{\prime}$ are the same. This is not surprising since the reaction schemes are identical up to the point where the product is released. For the third-generation scheme the $k^{\prime}$ step on the electrode is one of the sequence of transition states that the enzyme has to cross in each cycle. Hence the $k^{\prime}$ terms in eqn 12.8 behave like an additional transition state in the saturated and unsaturated parts of the expression. For the NADH electrode, the enzyme E on its release is regenerated by the rapid binding of NAD ${ }^{+}$. However the electrochemical turnover of the NADH can be rate limiting. If the third term in eqn 12.13 is dominant then the enzyme concentration, $e_{\Sigma}$, cancels out and the flux, $j$, does not depend on $L$, the thickness of the electrolyte layer. Remembering that the 
product concentration, $p$, is given by $j / k_{\mathrm{p}}^{\prime}$, we find that the flux is given simply by:

$$
j \approx k^{\prime}[\mathrm{NADH}]_{\mathrm{eq}}
$$

where $[\mathrm{NADH}]_{\mathrm{eq}}$ is the equilibrium concentration of NADH for the particular substrate, product, and $\mathrm{NAD}^{+}$conentrations. Under these circumstances the enzyme system is a rapid pre-equilibrium and the ratelimiting process is the electrochemical turnover of the NADH.

What about the remaining term, the fourth term in eqn 12.13? At first sight these terms are rather puzzling. They have a mixture of enzyme and electrochemical kinetics and each term seems to depend on a backward-going rate constant! In fact these terms really describe the cycling of the enzyme. In the third-generation scheme we assumed that the electrochemical $k^{\prime}$ step was irreversible, so each cycle of the enzyme was cleanly separated by the $k^{\prime}$ step. In the present scheme the enzyme does not participate in an irreversible step; the NADH is destroyed on the electrode. So now we must not assume that each enzyme cycle starts and ends with E.NAD *. Like a stage army we must join up the cycles to form a continuous sequence and then look for the biggest barrier. Remembering that, as before, $p=j / k_{\mathrm{p}}^{\prime}$ and that $[\mathrm{NADH}]=j / k^{\prime}$, we can interpret the fourth term in eqn 12.13 as shown in the free-energy profiles in Fig. 12.3. The backward rate constants arise, because it is more economical to express the free-energy difference as the quotient of the full equilibrium constant, $K_{\mathrm{TD}}$, and the backward rate constant rather than the more complicated expression for the forward rate constant. The electrochemical rate constant arises because NADH is released on going to the rate-limiting transition state, and the concentration of NADH will depend on the electrode kinetics. It is interesting that eqn $\mathbf{1 2 . 1 3}$ shows that, depending on which term is dominant, one may find that $j$ varies with $[\mathrm{S}],[\mathrm{S}]^{1 / 2}$ or even $[\mathrm{S}]^{1 / 3}$.

In deriving expressions to describe the kinetics of enzyme electrodes, we emphasize the importance first of expressing the result in the reciprocal form, and secondly of interpreting the expression in terms of free-energy diagrams. We have used both approaches to good advantage in our work on homogeneous enzyme kinetics (Albery and Knowles 1976, 1987).

\subsection{No product inhibition}

Equations $12.8,12.11$, and 12.13 are complicated equations in $j$; in our view little insight can be obtained by attempting to solve cubic and quartic equations. It is however unlikely that for any real system all the terms in a particular equation will be significant. The important application of our analysis is the identification of the rate-limiting process. When we take the cases where there is no product inhibition, we find that eqns $12.8,12.11$, and 


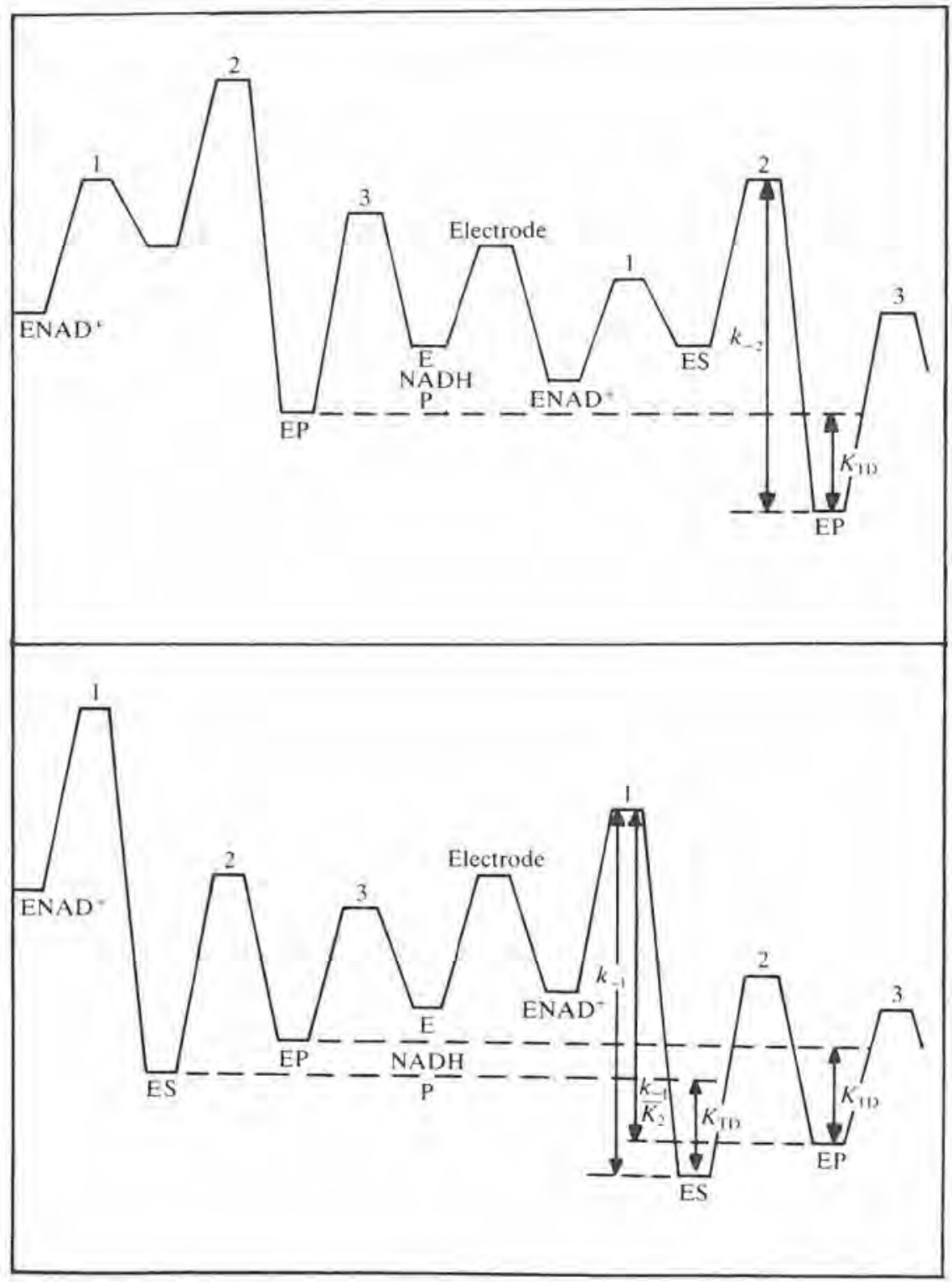

Fig. 12.3 Schematic free energy profile illustrating the fourth term in eqn 12.3. In the top case the $k_{-2}$ term is dominant and the response is controlled by a combination of the equilibrium constant between EP and ES and the rate constant $k_{2}$. In the lower case the dominant term is $k_{-1}$, and the response is controlled by the equilibrium between $\mathrm{ES}$ and $\mathrm{ENAD}^{+}$and the rate constant $k_{1}$. In the case where $k_{1} / K_{2}$ is dominant the response is controlled by a combination of the equilibrium between $\mathrm{EP}$ and ENAD ${ }^{*}$ and the rate constant $k_{1}$. 
12.13 all have the same form, so that our treatment will apply to a wide range of enzyme electrodes.

We will work with eqn 12.8 . We start by rearranging it into a form which is similar to a Hanes plot (Hanes 1932) for the analysis of Michaelis-Menten kinetics:

$$
\frac{s_{\infty}}{j}=\frac{1}{k_{\mathrm{ME}}^{\prime}}\left[1+\frac{s_{\infty}}{K_{\mathrm{ME}}}\left\{1-\frac{j}{k_{S}^{\prime} s_{\infty}}\right\}\right] .
$$

In this equation we have introduced the effective electrochemical rate constant for the enzyme electrode at low substrate concentrations, $k_{\mathrm{ME}}^{\prime}$ where

$$
1 / k_{\mathrm{ME}}^{\prime}=K_{\mathrm{M}} /\left(e_{\mathrm{\Sigma}} L k_{\mathrm{cat}}\right)+1 / k_{\mathrm{S}}^{\prime} \text {. }
$$

We have introduced a similar parameter in our treatment of modified electrodes (Albery and Hillman 1981, 1984) and indeed the $K_{\mathrm{M}}$ term corresponds to the layer case of that treatment. Depending on which is the slower, the effective electrochemical rate constant will be determined by either the enzyme kinetics, the $K_{\mathrm{M}} / k_{\text {cat }}$ term, or by the transport of the substrate through the membrane, the $k_{\mathrm{s}}^{\prime}$ term. Again note the reciprocal form.

Secondly we have introduced into eqn 12.14 the equivalent of the Michaelis constant for the enzyme electrode, $K_{\mathrm{ME}}$ where

$$
K_{\mathrm{ME}}=\frac{K_{\mathrm{M}}\left(L k_{\mathrm{cat}}\right)^{-1}+e_{\Sigma}\left(k_{\mathrm{S}}^{\prime}\right)^{-1}}{\left(L k_{\mathrm{cat}}\right)^{-1}+\left(k^{\prime}\right)^{-1}} .
$$

The significance of $K_{\mathrm{ME}}$ is similar to that of the Michaelis constant in homogenous enzyme kinetics. For concentrations smaller than $K_{\mathrm{ME}}$ the system is unsaturated, the current is proportional to the concentration of substrate and is governed by the rate constant $k_{\mathrm{ME}}$. For concentrations greater than $K_{\mathrm{ME}}$ the system becomes saturated and the flux reaches a maximum value. This flux can be characterized by the equivalent of $k_{\text {cat }}$ :

$$
\left(k_{\text {cat }, \mathrm{E}}^{\prime}\right)^{-1}=\left(L k_{\text {cat }}\right)^{-1}+\left(k^{\prime}\right)^{-1}=e_{\mathbb{S}} / j_{\text {max }} \text {. }
$$

Because $k_{\text {cat, E }}^{\prime}$ describes a flux per unit area, it has the usual dimensions (cm $\mathrm{s}^{-1}$ ) of an electrochemical rate constant. Again the reciprocal form shows that depending on which is the smaller the observed $k_{\text {cat, E }}^{\prime}$ will be either determined by the saturated enzyme kinetics, $k_{\mathrm{cai}}$, or by the electrochemical regeneration, $k^{\prime}$.

From eqns 12.15 to 12.17 we find,

$$
k_{\mathrm{cat}, \mathrm{E}}^{\prime} / K_{\mathrm{ME}}=k_{\mathrm{ME}}^{\prime} / e_{\mathrm{Y}} \text {. }
$$

For an enzyme electrode under unsaturated conditions, this equation relates the kinetic description used by enzyme kineticists $\left(k_{\mathrm{cat}} / K_{\mathrm{M}}\right)$ to the electrochemical rate contant $\left(k^{\prime}\right)$ used by electrochemists. 
The first stage of the analysis is to find $k_{\mathrm{ME}}^{\prime}$ by plotting $s_{\infty} / j$ against $s_{\infty}$. Equation 12.14 shows that this may be a curve but the limiting value as $s_{\infty} \rightarrow 0$ gives $\left[s_{\infty} / j\right]_{0}=\left(k_{\mathrm{ME}}^{\prime}\right)^{-1}$. Next for values of $s_{\infty} / j$ significantly greater than $\left[s_{\infty} / j\right]_{0}$ we calculate values of $\rho$ where

$$
\rho=\left[j / s_{\infty}\right] /\left[j / s_{\infty}\right]_{0} \leqslant 1 .
$$

Substitution in eqn 12.14 gives

$$
y=\frac{\rho^{-1}-1}{s_{\infty}}=\frac{1}{K_{\mathrm{ME}}}\left[1-\frac{\rho k_{\mathrm{ME}}^{\prime}}{k_{\mathrm{S}}^{\prime}}\right] .
$$

Equation 12.20 predicts that plots of $y$ against $\rho$ should be straight lines. From the intercept on the $y$ axis we can find the Michaelis constant for the electrode, $K_{\mathrm{ME}}$. From the intercept $\rho_{0}$ on the $x$ axis we can determine the relative importance of enzyme kinetics and transport kinetics in the observed rate constant $k_{\mathrm{ME}}^{\prime}$. From eqns 12.15 and 12.20 we find that:

$$
k_{\mathrm{S}}^{\prime}=\rho_{0} k_{\mathrm{ME}}^{\prime}
$$

and

$$
k_{\text {cat }} e_{\mathrm{S}} L / K_{\mathrm{M}}=\rho_{0} k_{\mathrm{ME}}^{\prime} /\left(\rho_{0}-1\right) .
$$

If the value of $\rho_{0}$, the intercept on the $x$ axis, is unity, then the transport of S across the membrane is clearly rate limiting. If on the other hand, a horizontal line is found, corresponding to $\rho_{0}=\infty$, then the unsaturated enzyme kinetics are rate limiting. Hence the $y / \rho$ plot is a valuable diagnostic plot. Examples of its application are given below.

Figure 12.4 shows typical $j$ versus $s_{\infty}$ curves, Hanes plots, and plots of eqn 12.20 for different values of $k_{\mathrm{ME}}^{\prime} / k_{\mathrm{S}}^{\prime}$. It is interesting that for the case where transport across the membrane is clearly rate limiting, we obtain a sharp dogleg plot of flux against concentration of substrate. This arises because under these conditions neither of the two rate-limiting processes, transport or enzyme turnover under saturated conditions, depends on the internal substrate concentration, $s_{0}$; hence the flux is simply limited by the slower of the two processes. In this section we have considered the cases where there is no product inhibition. Elsewhere (Albery and Bartlett 1985) we have considered the effects of product inhibition and have discussed the different types of diagnostic plot that one obtains for these cases.

\subsection{Sensitivity versus concentration range}

When the kinetic parameters have been determined, and the rate-limiting step identified then one is in a much better position to optimize the design of an enzyme electrode. In particular an important design feature is the permeability or otherwise of the membrane to the substrate. There are two 


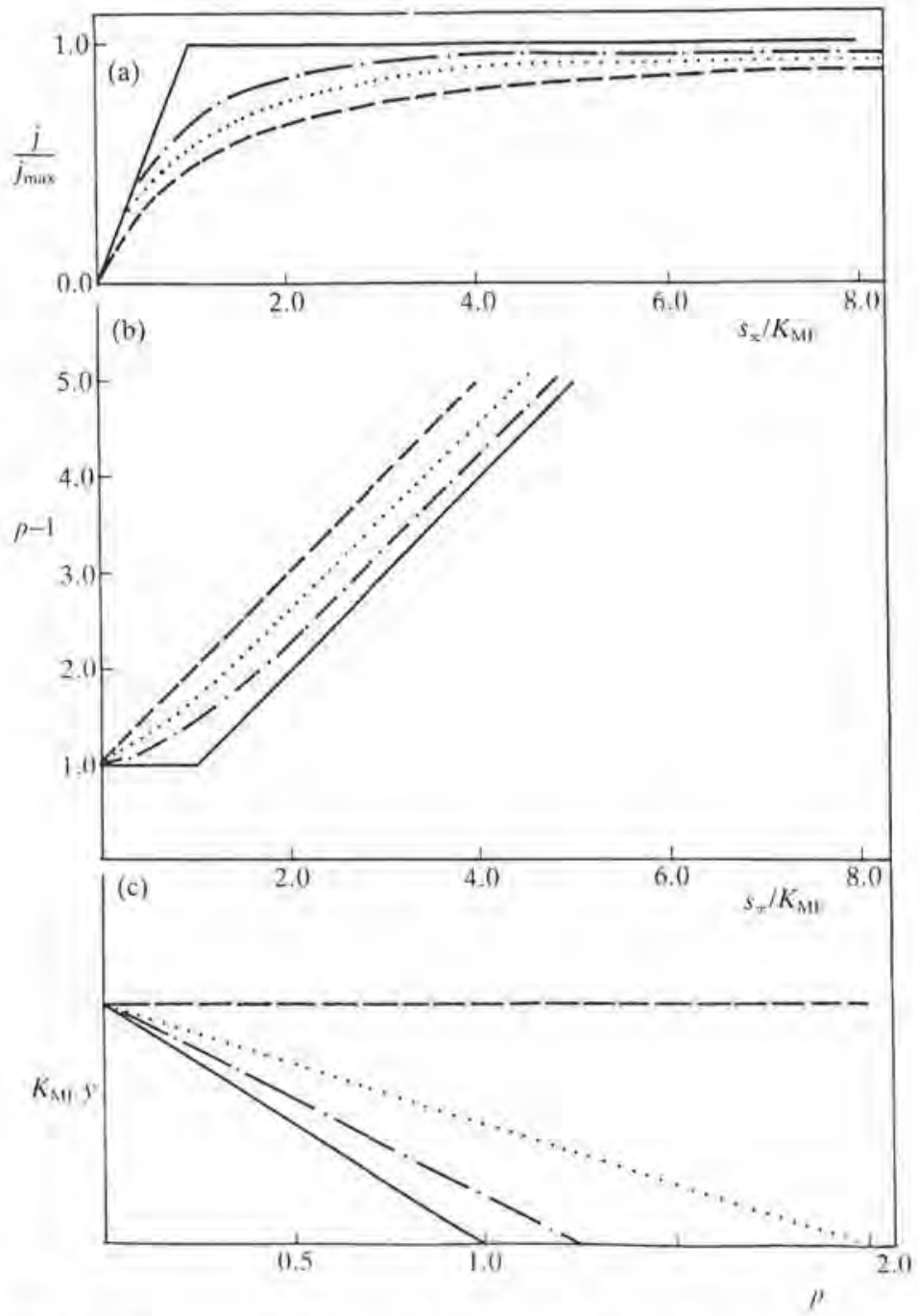

Fig. 12.4 (a) Shows typical plots of flux against substrate concentration for different values of $k_{\mathrm{ME}}^{\prime} / k_{\mathrm{SS}}^{\prime}$ for the case where there is no product inhibition. For these curves, (b) and (c) show the corresponding Hanes plots and plots of eqn 12.20 respectively. The values of $k^{\prime}{ }_{\mathrm{ME}} / k_{\mathrm{S}}^{\prime}$ are as follows:- 1.00 ; $-\bullet-0.80$; $0.5 ;-\cdots$ 0.00 .

advantages to making the transport of the substrate through the membrane rate limiting. First, the response of the electrode then depends on the transport characteristics of the substrate in the membrane. The response does not depend on the enzyme kinetics nor on the electrochemical kinetics. Since both 


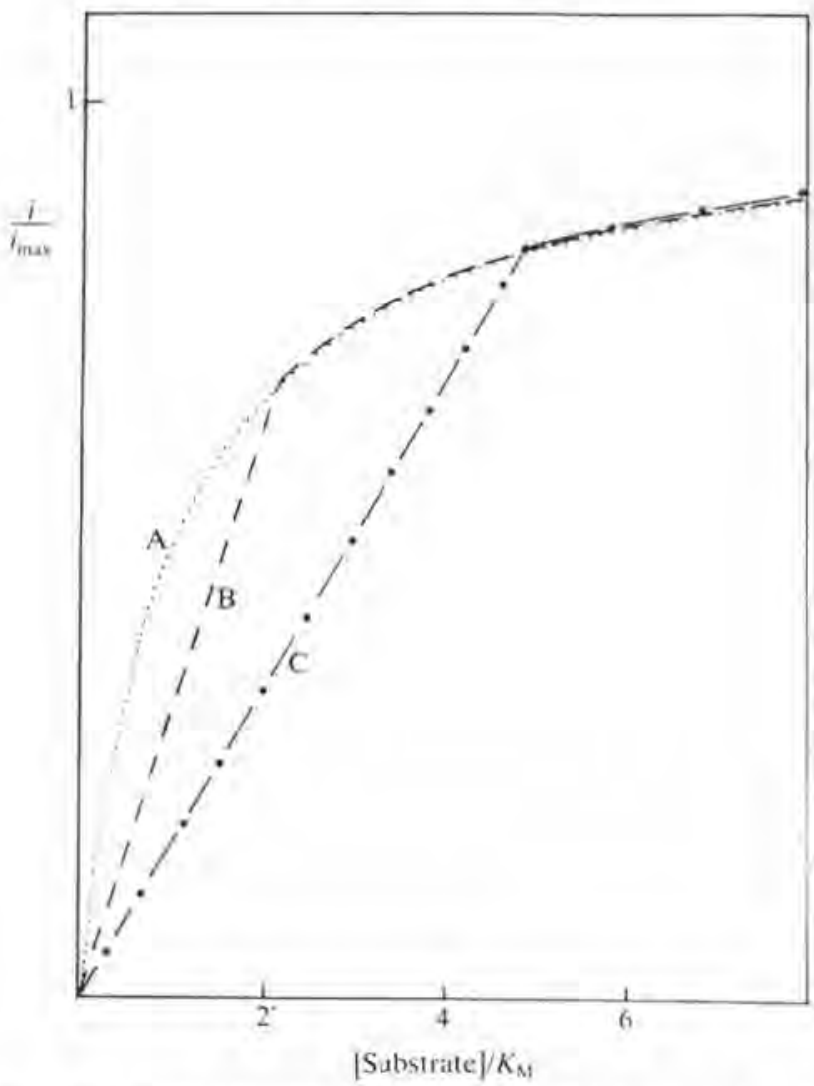

Fig. 12.5 The effect that varying the permeability of the membrane has on the theoretical current response of an enzyme electrode to increasing concentration of substrate. In $A$ the enzyme kinetics are rate limiting, while $B$ and $C$ show the effect of using less and less permeable membranes.

can be unreliable, this is good. Secondly, the range of concentration over which the enzyme responds is extended. If enzyme kinetics are rate limiting then the enzyme saturates when the concentration of the substrate is somewhat larger than $K_{\mathrm{M}}$. However if transport kinetics are rate limiting, a linear response of current to concentration may be obtained for substrate concentration levels that are much in excess of $K_{\mathrm{M}}$.

This point is illustrated in Fig. 12.5. The different schematic curves show the effect of putting on less and less permeable membranes. In A the membrane is very permeable and enzyme kinetics are rate limiting. In B and C the transport kinetics have become successively slower and at low concentrations the transport kinetics are rate limiting. The dog-leg response curves give successively higher values of the electrode Michaelis constant $K_{\mathrm{ME}}$. Of course 
the thicker membranes mean that there is less current at low concentrations. So if sensitivity is all important then it is best to use as permeable a membrane as possible. But if one has plenty of current then it is sensible to use a membrane that makes the transport of the substrate rate limiting. So far in all of our work we have used membranes prepared from dialysis tubing. Optimization through the proper design of the membrane will become increasingly important.

\subsection{Conducting organic salt electrodes}

We have investigated a number of different conducting organic salts for use as enzyme electrodes (Albery et al. 1985). The donors and acceptors are given in Table 12.1. Many of these materials were first prepared by Melby and coworkers (Melby 1965; Melby et al. 1962) and their electrochemistry has been investigated by Jaeger and Bard $(1979,1980)$. Nearly all the salts we have made show electrochemical activity with glucose oxidase. This finding some-

Table 12.1 Conducting organic salts as enzyme electrodes

Donors<smiles>C1=CSC(=C2SC=CS2)S1</smiles>

T7F<smiles>C[n+]1c2ccccc2nc2ccccc21</smiles>

$\mathrm{NMP}^{*}$

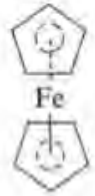

$\mathrm{Fec}^{*}$<smiles>Clc1ccccn1</smiles>

$\mathrm{Cu}(\mathrm{DPA})$<smiles></smiles>

$\mathrm{Q}^{+}$
$\left(\mathrm{CH}_{3} \mathrm{CH}_{2}\right)_{3} \mathrm{~N}^{+} \mathrm{H}$

TEA*

Acceptors<smiles>N#CC(C#N)=c1ccc(=C(C#N)C#N)cc1</smiles>

TCNQ<smiles>N#CC(C#N)=C(c1cccc([N+](=O)[O-])c1)c1cc([N+](=O)[O-])cc([N+](=O)[O-])c1</smiles>

DTF 


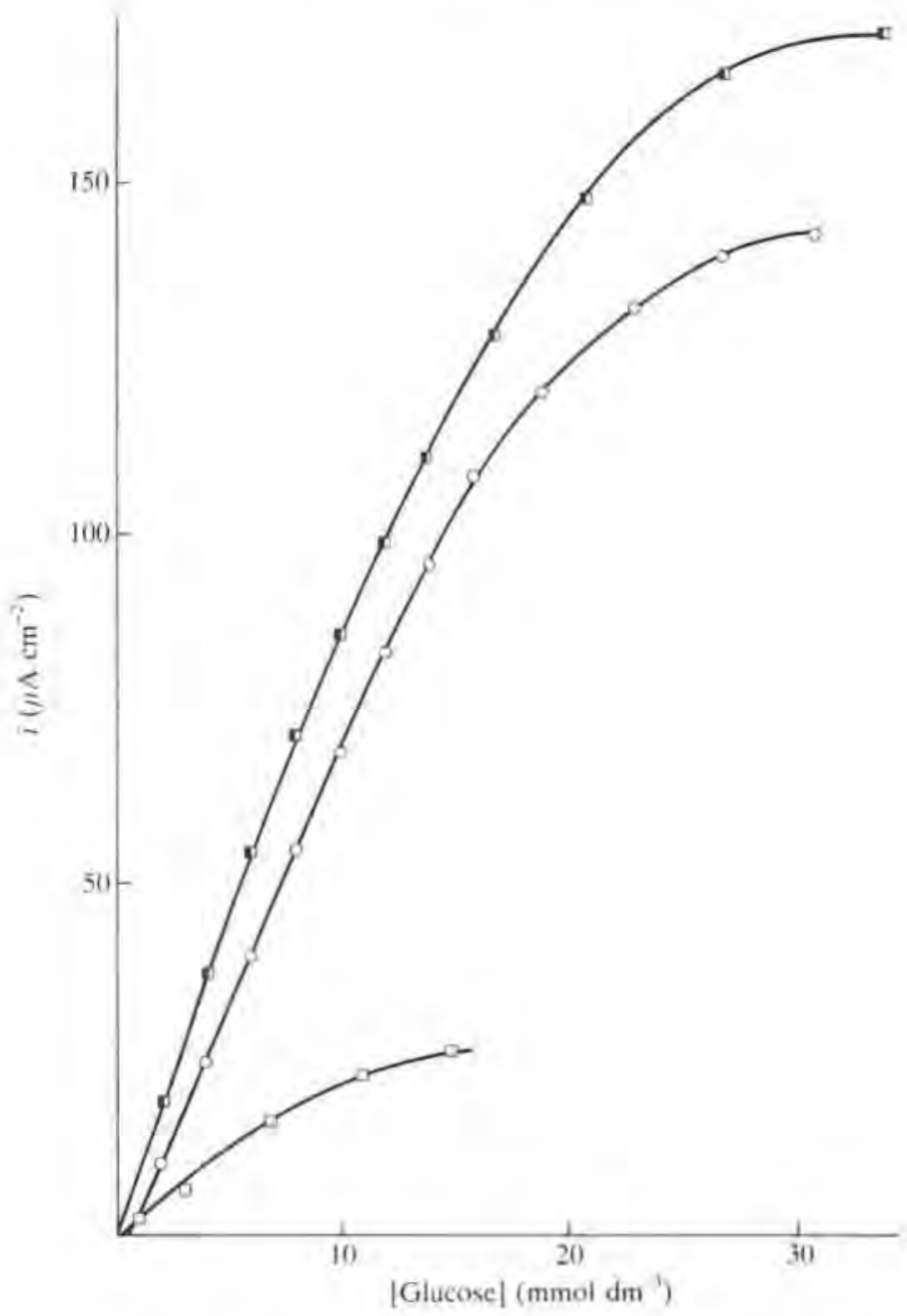

Fig. 12.6 Typical results for currents from membrane electrodes with increasing glucose concentration. The three electrode materials were TTF ' TCNQ (D), $\mathrm{NMP}^{*} \mathrm{TCNQ}^{-}(\mathrm{O}), \mathrm{Q}^{+}(\mathrm{TCNQ})_{2}^{-}(\square)$.

what surprised us, since we had thought that there would have to be rather specific interactions between the surface and the enzyme to obtain efficient electron transfer. In fact it turns out that these materials are good electrocatalysts for a number of different flavoproteins. The reason for the ubiquity of this behaviour will be discussed below.

Our preliminary work showed that the three most suitable materials with respect to background currents, and voltage range were the TCNQ salts of 


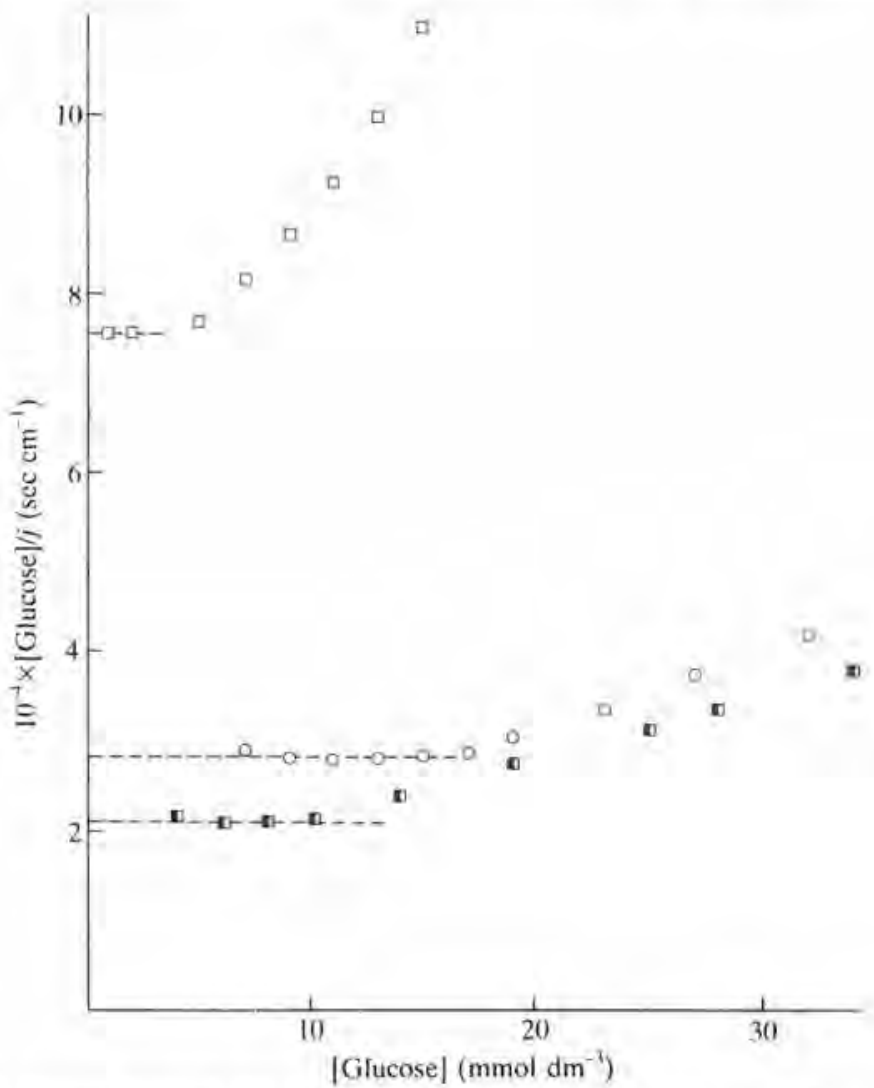

Fig. 12.7 Hanes plots of the data in Fig. 12.6. The three electrode materials were $\mathrm{TTF}^{+} \mathrm{TCNQ}^{-}$(四), $\mathrm{NMP}^{+} \mathrm{TCNQ}^{-}(\mathrm{O}), \mathrm{Q}^{+}(\mathrm{TCNQ})_{2}^{-}(\square)$.

$\mathrm{TTF}^{+}, \mathrm{NMP}^{*}$, and $\mathrm{Q}^{*}$. Membrane electrodes were therefore made of these three materials. The current from these electrodes was measured as the glucose concentration in the external solution was increased. Typical results are shown in Fig. 12.6. The data in this figure are analysed by the procedure presented above.

The first stage of the analysis is to make a Hanes plot [see eqn 12.14] of $n F A$ [Glucose] $/ i$ against [Glucose]. To compensate for the different areas of the electrode, we carry out the analysis in terms of the current densities $(i / A)$. These plots are shown in Fig. 12.7. The intercepts at zero concentration is the reciprocal of the electrochemical rate constant, $k_{\mathrm{ME}}^{\prime}$, defined in eqn 12.15 . Values of $k_{\mathrm{ME}}^{\prime}$ are collected in Table 12.2.

Next the parameter $\rho$ (eqn 12.19) is calculated where

$$
\rho=i / n F A k_{\text {ME }}^{\prime}[\text { Glucose] }
$$


Table 12.2 Results for membrane electrodes

\begin{tabular}{|c|c|c|c|}
\hline $\begin{array}{l}\text { Electrode } \\
\text { material }\end{array}$ & $\frac{k_{\mathrm{ME}}^{\prime d}}{\left(\mathrm{~cm} \mathrm{~s}^{-1}\right)}$ & $\frac{K_{\mathrm{ME}}^{b}}{\left(\mathrm{mmol} \mathrm{dm}^{-3}\right)}$ & $\frac{k_{\text {cat. }}^{\prime} \mathrm{E}^{e}}{\left(\mathrm{~cm} \mathrm{~s}^{-1}\right)}$ \\
\hline $\mathrm{TTF}^{*} \mathrm{TCNQ}^{-}$ & $4.7 \times 10^{-5}$ & 20 & $9 \times 10^{-2}$ \\
\hline NMP $\cdot$ TCNQ - & $3.0 \times 10^{-5}$ & 22 & $8 \times 10^{-2}$ \\
\hline $\mathrm{Q}^{+} \mathrm{TCNQ}^{-}$ & $1.3 \times 10^{-5}$ & II & $1.4 \times 10^{-2}$ \\
\hline
\end{tabular}

${ }^{a}$ Calculated from eqn 12.14.

${ }^{b}$ Calculated from eqn 12.20 .

c Calculated from eqn 12.18 .

and from eqn $12.20 y$ is plotted against $\rho$. Plots for the three electrodes are shown in Fig. 12.8. In each case good straight lines are obtained, showing the success of the analysis. The fact that in each case $\rho_{0}$, the intercept on the $x$ axis, is equal to unity shows, as discussed above (eqns 12.21 and 12.22), that at low substrate concentrations the rate-limiting step is the diffusion of glucose through the membrane. The subsequent enzyme and electrode steps are so fast that they are not rate limiting. This is the most desirable condition for a reliable sensor, since the enzyme and electrochemical kinetics do not affect the response of the sensor. As long as this condition is maintained any decay in the enzyme or electrode activity has no effect.

The results in Fig, 12.6 show that glucose concentrations can be determined in the range $50 \mu \mathrm{mol} \mathrm{dm}{ }^{-3}$ to $10 \mathrm{mmol} \mathrm{dm}^{-3}$. The fact that the transport of the glucose through the membrane is rate limiting explains why the values of $k_{\mathrm{ME}}^{\prime}$ in Table 12.2 are all so similar and do not depend on the electrode material. The value of $L_{\mathrm{M}}$, the thickness of the membrane, is $0.3 \mathrm{~mm}$. Substitution in eqn 12.10 gives values for $K_{\mathrm{S}} D_{\mathrm{S}}$ of the order of $10^{-6}$ $\mathrm{cm}^{2} \mathrm{~s}^{-1}$. These values are in good agreement with that found by Gough and Leypoldt (1980), who used a rotating-disc electrode method to measure the transport properties of a similar membrane.

Returning to Fig. 12.8, from the intercepts and eqn 12.20 we can calculate values of $k_{\text {ME }}$. Results from all our experiments are collected in Table 12.2. We can now explore the rate-limiting process when the enzyme electrode is saturated, and its behaviour is determined by $k_{\text {cat }_{,}^{\prime}}^{\prime}$ defined in eqn 12,17. Values of $k_{\text {eat, }}^{\prime}$ are collected in Table 12.2.

The two terms of eqn 12.17 describe saturated enzyme and electrochemical kinetics. The ratio of these two terms can be found from a knowledge of $K_{\mathrm{ME}}$, $K_{\mathrm{M}}$, and the ratio $k_{\mathrm{S}}^{\prime} / k_{\mathrm{ME}}^{\prime}$ which is determined from plots of eqn 12.20 . From eqns 12.15 and 12.16 we obtain:

$$
\frac{k_{\mathrm{cal}} L}{k^{\prime}}=\frac{K_{\mathrm{M}}}{K_{\mathrm{ME}}\left(1-k_{\mathrm{ME}}^{\prime} / k_{\mathrm{S}}^{\prime}\right)}-1 .
$$




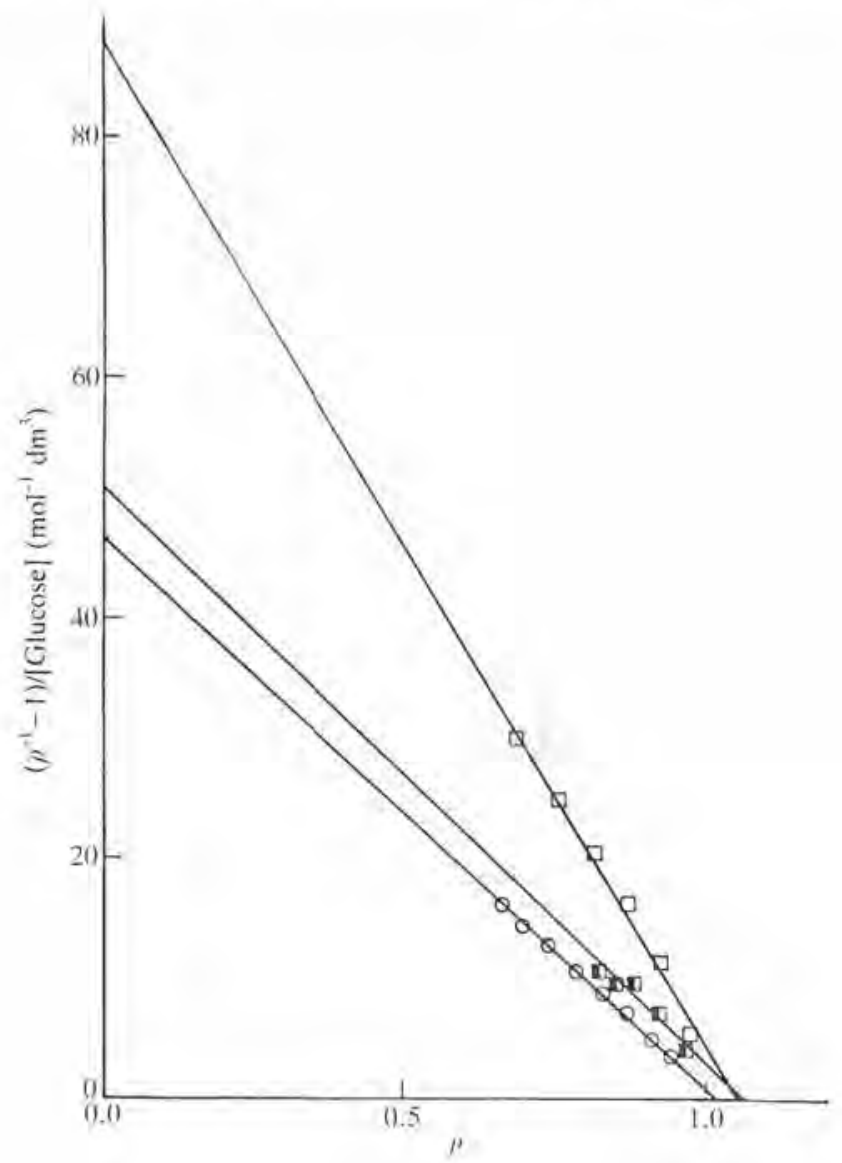

Fig. 12.8 Plots of eqn 12.20 from the data in Fig. 12.7. The three electrode materials were TTF * TCNQ ${ }^{-}(\mathbf{\square}), \mathrm{NMP}^{+} \mathrm{TCNQ}^{-}(\mathrm{O}), \mathrm{Q}^{+}(\mathrm{TCNQ})_{2}^{-}(\square)$.

The literature value for $K_{\mathrm{M}}$ is $7 \mathrm{mmol} \mathrm{dm}^{-3}$ (Cardosi 1984). The values of $K_{\mathrm{ME}}$ in Table 12.2 are about $5 \mathrm{mmol} \mathrm{dm}^{-3}$. Within experimental error we have found that for all three materials $k_{\mathrm{ME}}^{\prime}$ is equal to $k_{\mathrm{S}}^{\prime}$. We can conclude therefore that the bracket in the denominator of eqn 12.15 is smaller than 0.1 . We then find that

$$
k_{\text {cat }} L / k^{\prime}>10 \text {. }
$$

Hence we can conclude that the rate-limiting process in the saturated region is the electrode kinetics and not the enzyme kinetics. The electrochemical rate constants, $k^{\prime}$, for the three materials are given by the values of $k_{\mathrm{cat}, \mathrm{E}}^{\prime}$ in Table 12.2. With a value for $k_{\text {cat }}$ of $800 \mathrm{~s}^{-1}$ (Wiebel and Bright 1971) and with a value for $L$ of several tens of microns, we find that, in accord with our 
analysis, the inequality in eqn 12.16 is indeed satisfied. It is very satisfactory that the three materials in Table 12.2 are indeed excellent electrocatalysts for the direct oxidation of glucose oxidase with electrochemical rate constants, $k^{\prime}$, which are all greater than $10^{-2} \mathrm{~cm} \mathrm{~s}^{-1}$.

\subsection{Electrochemical mechanism}

It should be admitted that there has been some controversy as to whether the enzyme is oxidized by direct electron transfer on the electrode surface or whether there is a mediated electron transfer; in the latter case dissolved $\mathrm{TTF}^{*}$ and or TCNQ ${ }^{-}$reacts with the enzyme in the electrolyte and then these species are reoxidized on the electrode. Cenas and Kulys (1981) have claimed that the reaction of glucose oxidase on NMP ' $\mathrm{TCNQ}^{-}$takes place by the mediated mechanism. However, as argued elsewhere in greater detail (Albery et al. 1985), we do not find their arguments convincing. Using a ring-disc electrode we have tried to measure the amount of dissolved NMP ${ }^{+}$or TCNQ - in the vicinity of the electrode. Thanks be to God we failed. The concentrations must be significantly less than $1 \mu \mathrm{mol} \mathrm{dm}-3$. Coupling these concentrations with the homogeneous second-order rate constants of the order of $10^{4} \mathrm{dm}^{3} \mathrm{~mol}^{-1} \mathrm{~s}^{-1}$, measured by Cenas and Kulys, we find that in no way could the homogeneous mediated mechanism give the large values of $k_{\text {cat, E }}^{\prime}$ reported in Table 12.2. The salts are just too insoluble and the homogeneous kinetics too sluggish for the mediated transfer to be an efficient mechanism.

It is interesting to speculate as to why the conducting organic salts are such good electrocatalysts for flavoproteins. We have found that many of these enzymes are strongly adsorbed on to the electrode surface. So much so that one can make a sensor without a membrane. All one has to do is to dip the electrode into a solution of the enzyme, wash off the excess, and the electrode is ready. The adsorbed layer is so strongly held that it is not lost from the surface, even when there is no enzyme in the adjacent solution. The reason for this strong attraction may arise from the fact that the conducting salt consists of alternate stacks of positive and negative ions. A patchwork of positive and negative charges on the surface could be expected to interact strongly with a corresponding patchwork on the enzyme surface. Others prefer to invoke the hydrophobic interaction between the enzyme and the aromatic surface of the electrode. We have shown (Albery et al, 1981) the importance of attractive interactions for the catalysis of biological electron transfer. We certainly have such interactions between the flavoprotein enzymes and the conducting salts.

\subsection{Electrode stability}

Furthermore the stability of these electrode is excellent. A membrane electrode was run continuously for 28 days. The results are shown in 


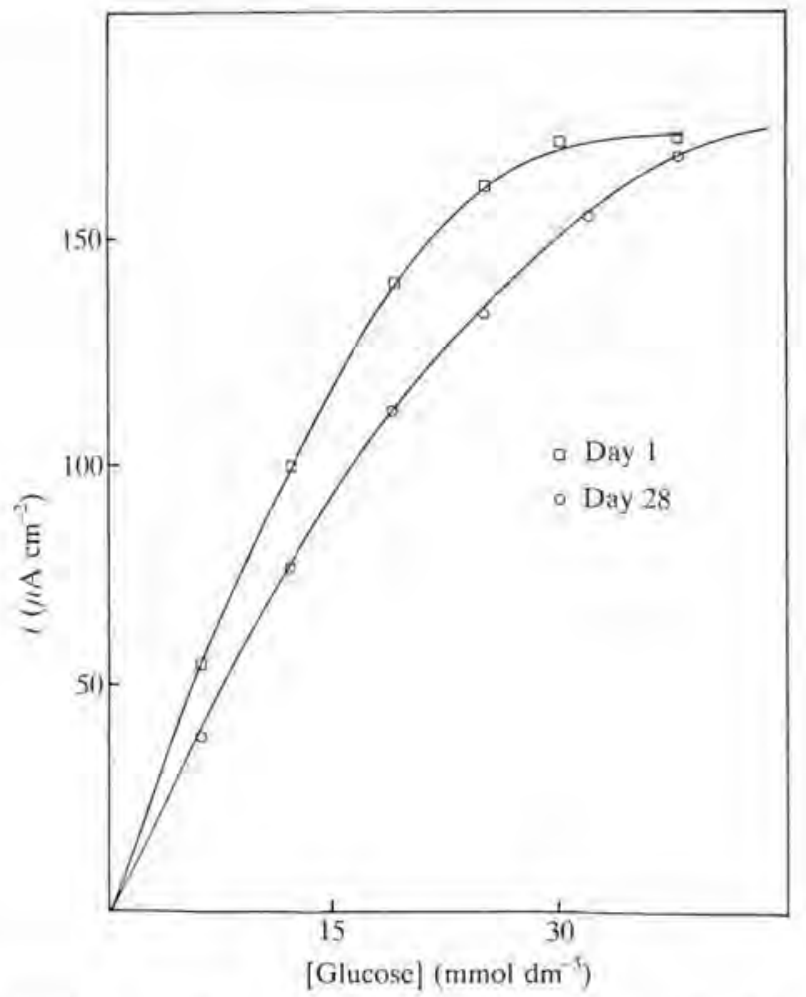

Fig. 12.9 Current response of a glucose enzyme electrode on the day of its fabrication, and the same electrode after 28 days of continuous use.

Fig. 12.9. At the end of the month the sensor had lost only $20 \%$ of its original activity. Kinetic analysis, like that presented above, showed that transport of the substrate through the membrane was still the rate-limiting step, so that the slight loss of activity must be caused by deterioration of the membrane. Again the importance of knowing that in this case the response is membrane limited is stressed.

\subsection{Other enzymes}

So far we have concentrated on the glucose oxidase system, but other enzymes will react on these electrodes. Of the different materials we have investigated (Albery et al. 1985), the salt $\mathrm{TTF}^{+}{ }^{+} \mathrm{TCNQ}^{-}$, had the lowest background current and is therefore our material of choice. We can now report results for the use of this electrode material with four other enzyme systems containing the flavin prosthetic group, FAD. In each case the reduced enzyme can be directly oxidized on the electrode. Details of the enzymes and 
Table 12.3 Enzyme substrate systems

\begin{tabular}{lllll}
\hline Enzyme & $\begin{array}{l}\text { Xanthine } \\
\text { oxidase } \\
\text { (EC 1.2.3.2) }\end{array}$ & $\begin{array}{l}\text { D-Amino acid } \\
\text { oxidase } \\
\text { (EC 1.4.3.3) }\end{array}$ & $\begin{array}{l}\text { L-Amino acid } \\
\text { oxidase } \\
\text { (EC 1.4.3.2) }\end{array}$ & $\begin{array}{l}\text { Choline } \\
\text { oxidase } \\
\text { (EC 1.1.3.17) }\end{array}$ \\
$\begin{array}{c}\text { Activity } \\
(\mu / \mathrm{mg})\end{array}$ & 1.25 & 14 & 0.44 & 50 \\
$\begin{array}{l}\text { Substrate } \\
\text { pH }\end{array}$ & Xanthine & D-Alanine & Phenylalanine & $\begin{array}{l}\text { Betaine } \\
\text { aldehyde }\end{array}$ \\
$\begin{array}{l}\text { Buffer } \\
(0.1 \mathrm{M})\end{array}$ & Phosphate & Tris & 6.5 & 7.4 \\
\hline
\end{tabular}

substrates are given in Table 12.3. For each enzyme a membrane electrode responded to increasing concentration of its substrate. Figure 12.10 shows typical results for all four systems. These curves are analysed according to the treatment presented above. Figures 12.11-12.13 show the $y / \rho$ plots of eqn 12.20 for xanthine oxidase and $\mathrm{D}$ and $\mathrm{L}$ amino acid oxidase respectively. In all three cases straight lines are obtained which pass through +1 on the $x$ axis. This behaviour shows that transport of the substrate across the membrane is rate limiting. Hence we can conclude that, like glucose oxidase, these three enzyme/TTF' TCNQ electrode combinations have sufficiently fast kinetics to handle all of each of their substrates which diffuse through the membrane.

Values of $k_{\mathrm{S}}^{\prime}$ are reported in Table 12.4. It can be seen that for these three enzymes the values are very similar and close to that observed for glucose oxidase. This is not surprising. For dialysis membrane we expect $K_{\mathrm{S}}$ to be

Table 12.4

\begin{tabular}{llll}
\hline & {$\left[k_{\mathrm{s}}\right] / \mathrm{cm} \mathrm{s}^{-1}$} & $\begin{array}{l}{\left[K_{\mathrm{ME}}\right] / \mathrm{mmol}} \\
\mathrm{dm}^{-3}\end{array}$ & {$[k]\left(\mathrm{cm} \mathrm{s}^{-1}\right)$} \\
\hline $\begin{array}{c}\text { Xanthine } \\
\text { oxidase }\end{array}$ & $1.1 \times 10^{-4}$ & 0.2 & $3.7 \times 10^{-4}$ \\
$\begin{array}{c}\text { D-Amino acid } \\
\text { oxidase }\end{array}$ & $2.6 \times 10^{-5}$ & 1.1 & $2.4 \times 10^{-4}$ \\
$\begin{array}{c}\text { L-Amino acid } \\
\text { oxidase }\end{array}$ & $2.8 \times 10^{-5}$ & 2.6 & $4.6 \times 10^{-2}$ \\
$\begin{array}{c}\text { Choline } \\
\text { oxidase }\end{array}$ & $2.8 \times 10^{-4}$ & 1.8 & $3.0 \times 10^{-3}$ \\
\hline
\end{tabular}




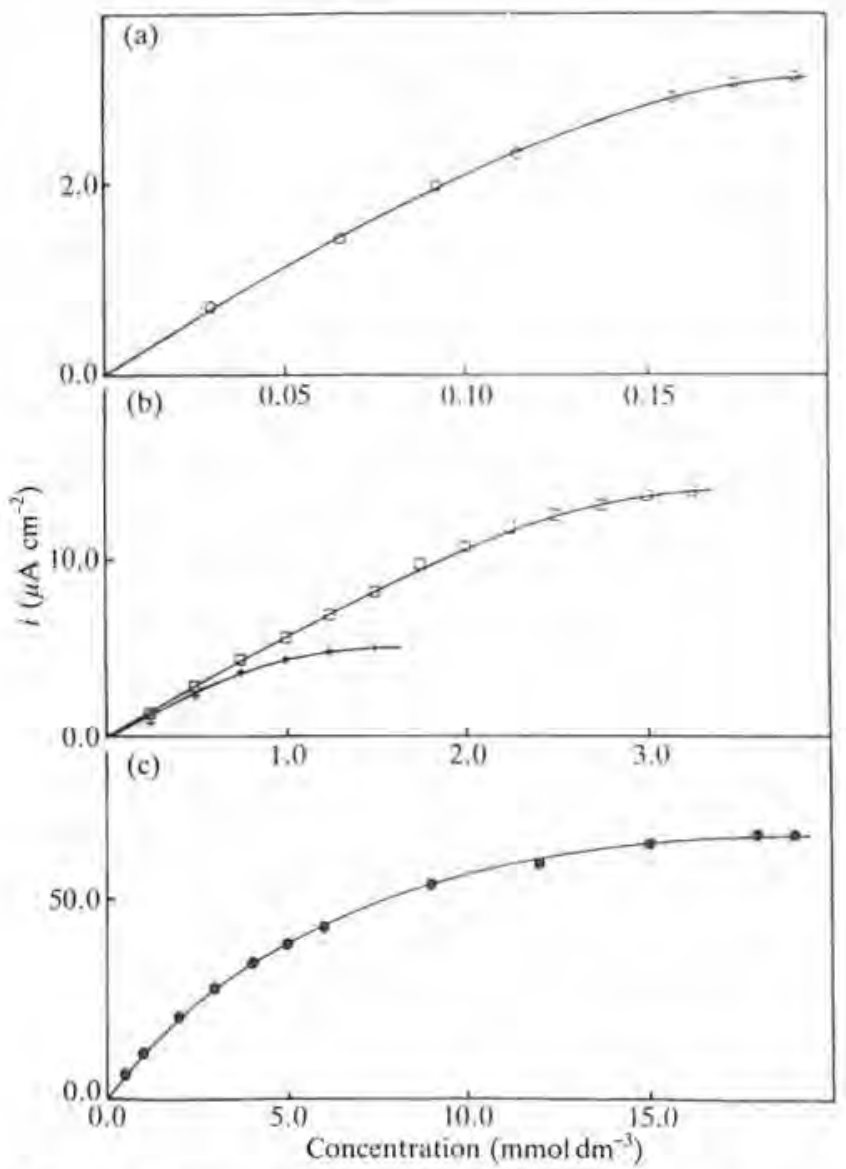

Fig, 12.10 Typical results for current with increasing substrate concentration using (a) xanthine oxidase $[O]$, (b) D-amino acid oxidase (+), L-amino acid oxidase ( $\square$ ), and (c) choline oxidase [@] enzyme electrodes. The electrode material in each case was $\mathrm{TTF}^{+} \mathrm{TCNQ}^{-}$.

close to unity and hence the different values of $k_{\mathrm{S}}^{\prime}$ for the different substrates reflect the differences in the diffusion coefficients. For similarly sized substrates these are unlikely to be large.

From the intercepts on the $y$ axis of the $y$ against $\rho$ plots in Figs 12.11-12.13, we can use eqn 12.20 to find $K_{\mathrm{ME}}$. As in our previous work on glucose oxidase, we interpret these results as being caused by the interplay of substrate transport and the electrode kinetics of the enzyme, where

$$
K_{\mathrm{ME}}=k^{\prime} e_{\Sigma} / k_{\mathrm{ME}}^{\prime}
$$




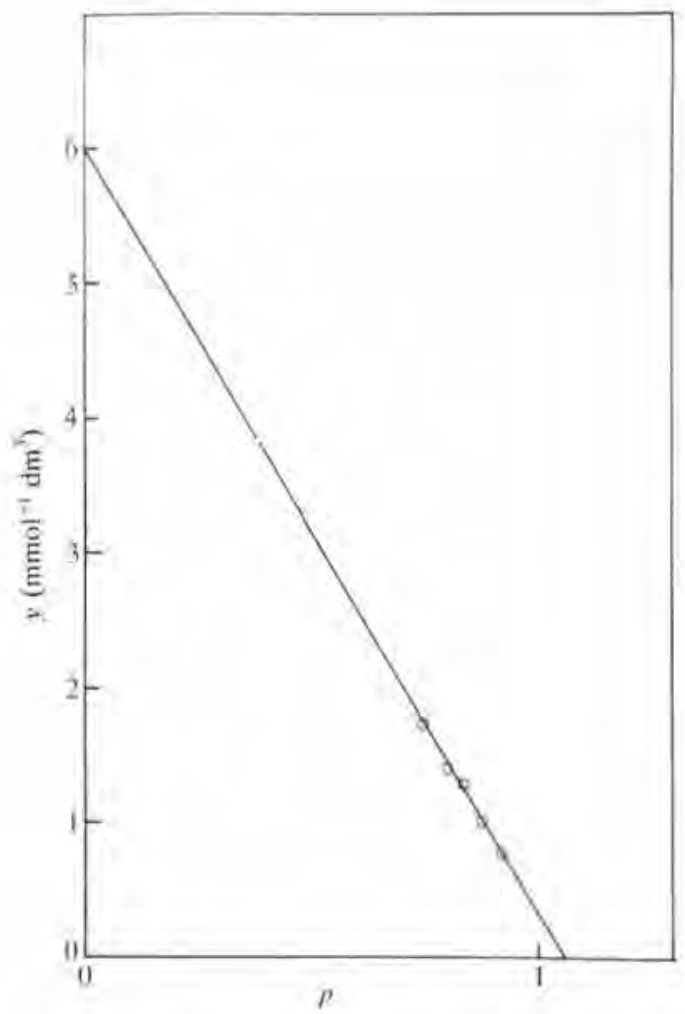

Fig. 12.11 Plots of eqn 12.20 for the xanthine oxidase enzyme electrode using the data in Fig. 12.10.

and $k^{\prime}$ describes the electrode kinetics of the enzyme. Values of the electrochemical rate constant $k^{\prime}$ are reported in Table 12.4. It can be seen that these electrochemical rate constants are all greater than $10^{-4} \mathrm{~cm} \mathrm{~s}^{-1}$. These large values show once again the advantage of using organic conductors as electrode materials for enzymes.

Turning to choline oxidase, the system is somewhat complicated because the product of the enzymatic oxidation of choline is betaine aldehyde, which is itself a substrate for the enzyme. In order to simplify the analysis, we have used betaine aldehyde as the substrate for the enzyme electrode. The $y / \rho$ plot is shown in Fig. 12.14. It can be seen in this case that the straight line passes through approximately +2 on the $x$ axis as opposed to +1 in the previous cases. A value of +2 for $\rho_{0}$ in eqns 12.20 and 12.21 shows that in this case there is an equal contribution from the enzyme and transport terms to the observed rate constant $k_{\mathrm{ME}}^{\prime}$. Again the value of $k_{\mathrm{S}}^{\prime}$, calculated from eqn 12.21 and reported in Table 12.4, is similar to the values for the other 


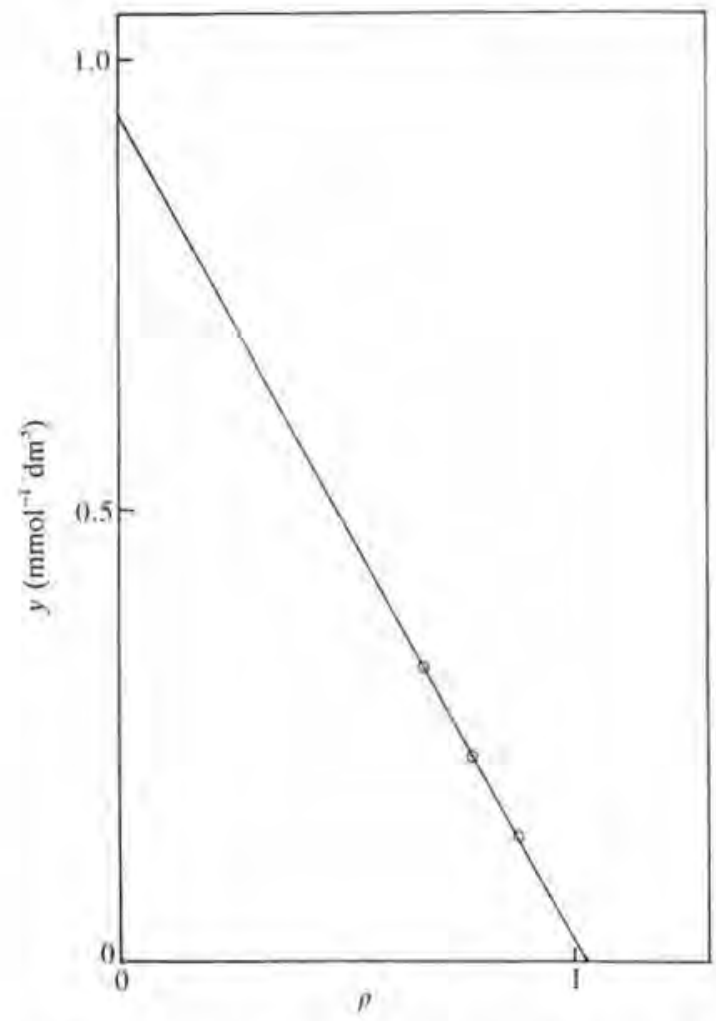

Fig. 12.12 Plots of eqn 12.20 for the D-amino acid oxidase enzyme electrode using the data in Fig. 12.10.

substrates. A value of the electrochemical rate constant, $k$, can be found by the same procedure as discussed above and is reported in Table 12.4. Again it is satisfactory that the value is as large as $10^{-3} \mathrm{~cm} \mathrm{~s}^{-1}$.

\subsection{NADH electrodes}

We now turn to a different strategy in which we use the same type of electrode material together with enzymes that use the ubiquitous cofactor $\mathrm{NAD}^{*} / \mathrm{NADH}$. We have found that the electrochemical rate constant for the oxidation of $\mathrm{NADH}$ at an electrode made of the conducting organic salt $\mathrm{NMP}^{+}$TCNQ - was as large as $10^{-2} \mathrm{~cm} \mathrm{~s}^{-1}$ (Albery and Bartlett 1984). Using this material we have developed an enzyme electrode, which uses yeast ethanol dehydrogenase (EC 1.1.1.1.) to oxidize a number of different alcohols such as ethanol, butan-1-ol, and propan-2-ol. The reaction scheme has been discussed above. 


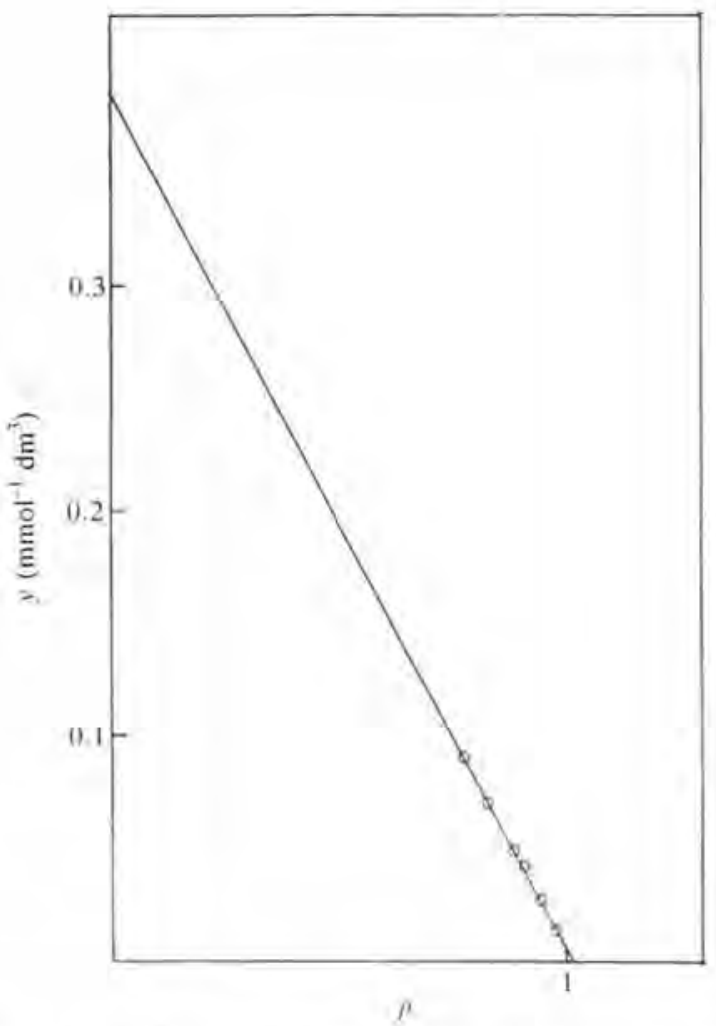

Fig. 12.13 Plots of eqn 12.20 for the L-amino acid oxidase enzyme electrode using the data in Fig. 12.10.

Typical results for the increase in current with increasing ethanol concentration are shown in Fig. 12.15. Application of the usual analysis gives the $y / \rho$ plot shown in Fig. 12.16. In this case a horizontal straight line is obtained. This corresponds to $\rho_{0}$ equal to infinity and from eqns 12.21 and 12.22 we find in this case that the enzyme kinetics are rate limiting. The fact that a straight line is obtained also allows us to conclude that the more complicated $j$ and $j^{2}$ terms on the right-hand side of eqn 12.13 are not significant; eqn 12.13 has reduced to the simple form. Indeed we find that there is no drop in current when the product acetaldehyde is added to the external solution. This means that the saturated behaviour in this case is not governed by the electrochemical kinetics, but by the saturated enzyme kinetics. In keeping with this conclusion the value of $K_{\mathrm{ME}}$ of $7.7 \mathrm{mmol} \mathrm{dm}{ }^{-3}$ measured from the intercept on the $y$ axis of the data in Fig. 12.16 is in good agreement with literature values for $K_{\mathrm{M}}$ of $13 \mathrm{mmol} \mathrm{dm}^{-3}$ (Barman 1969) and $13.1 \mathrm{mmol}$ $\mathrm{dm}^{-3}$ (Mazid and Laidler 1982). When enzyme kinetics determines both the 


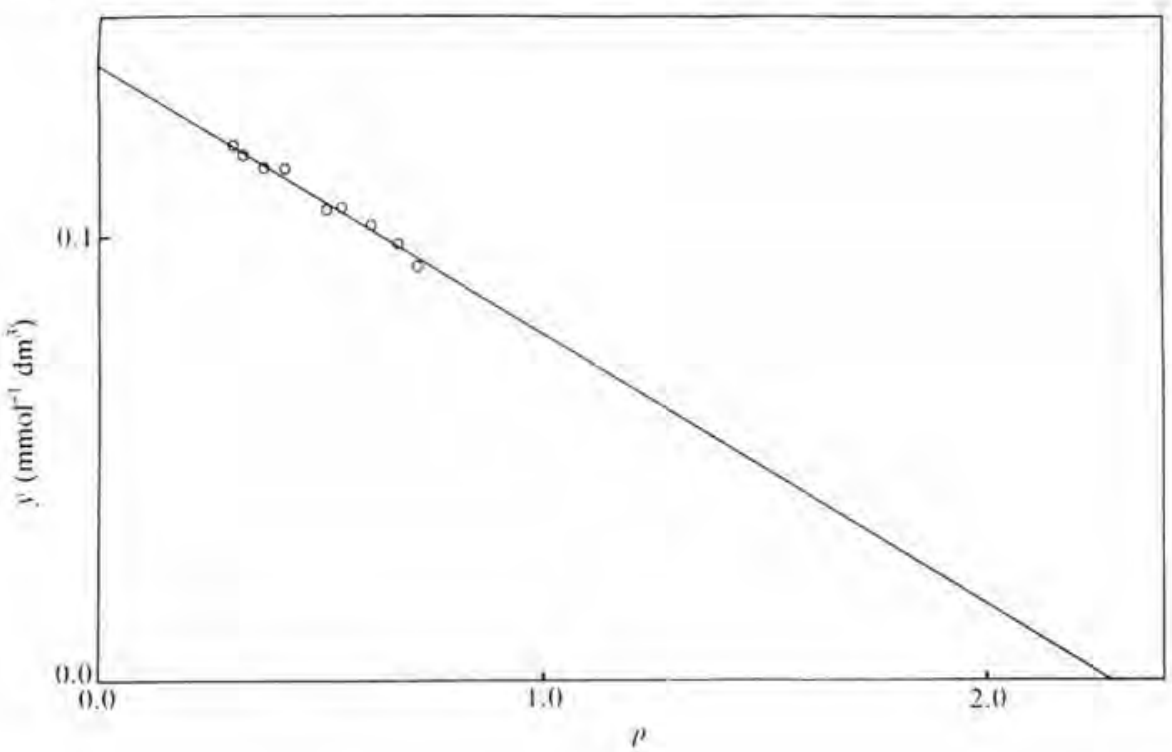

Fig. 12.14 Plots of eqn 12.20 for the choline oxidase enzyme electrode using the data in Fig. 12.10.

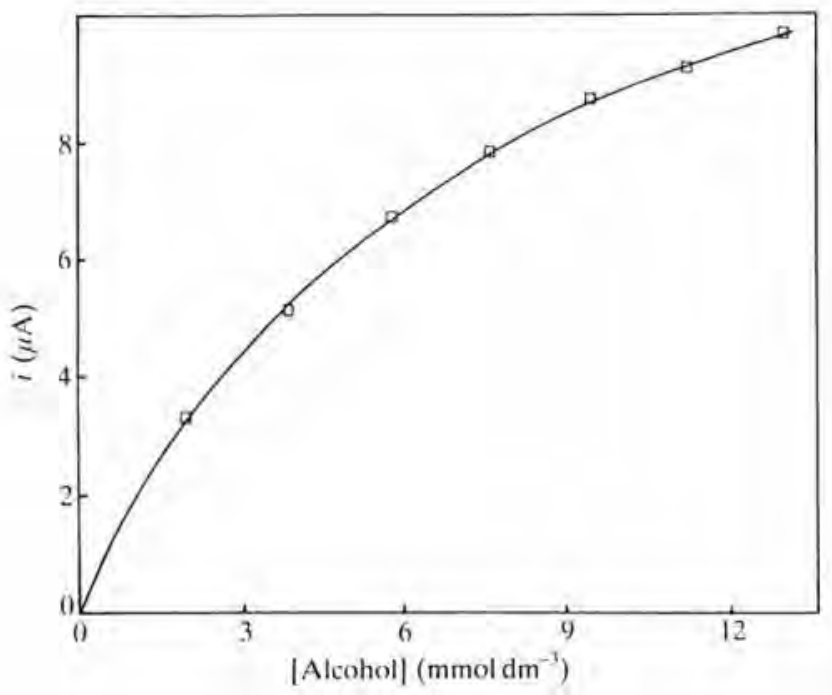

Fig. 12.15 Current response of an alcohol enzyme electrode with increasing concentrations of ethanol. 


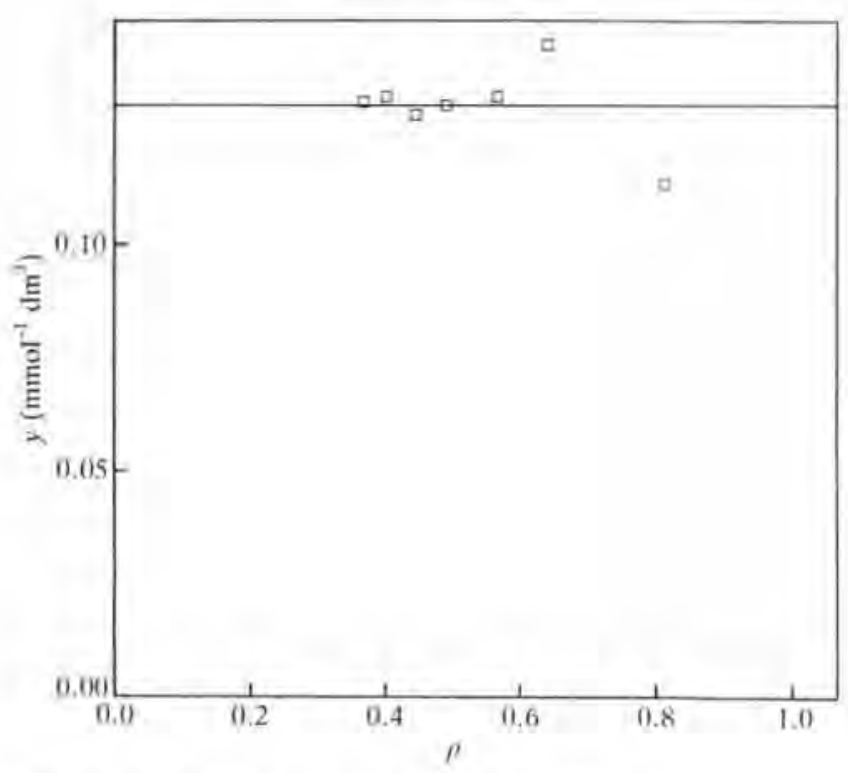

Fig. 12.16 Plots of eqn 12.20 from the data in Fig, 12,15.

unsaturated and saturated behaviour then $K_{\mathrm{ME}}=K_{\mathrm{M}}$. Hence the behaviour of this enzyme electrode where enzyme kinetics are always rate limiting may be contrasted with that of the glucose oxidase electrode where enzyme kinetics are never rate limiting.

The advantage of having a good electrode for the oxidation of NADH is that by efficiently scavenging the NADH the electrode reduces its concentration so that one does not get the dreaded back reaction, product inhibition and the $j$ and $j^{2}$ terms in eqn 12.13. We estimate that at our current densities the ambient concentration of NADH is as low as $10 \mu \mathrm{moI} \mathrm{dm}^{-3}$. The electrode helps to drive the reaction forward by destroying the NADH. A large value of $k^{\prime}$ can compensate for a small value of $K_{\mathrm{TD}}$ in the denominators of the product-inhibition terms in eqn 12.13 .

\subsection{Summary}

We have seen how different types of behaviour can be elucidated with the $y / \rho$ plot of eqn 12.20. This plot allows us to determine $K_{\mathrm{ME}}$ and $\rho_{0}$. The determination of these two parameters together with a knowledge of $K_{\mathrm{M}}$ for the enzyme will in many cases be sufficient to determine the rate-limiting step of the device. Figure 12.17 shows the behaviour of the transport, enzyme, and electrochemical kinetics as a function of substrate concentration. Inspection of the figure allows us to delineate the different cases summarized in Table 


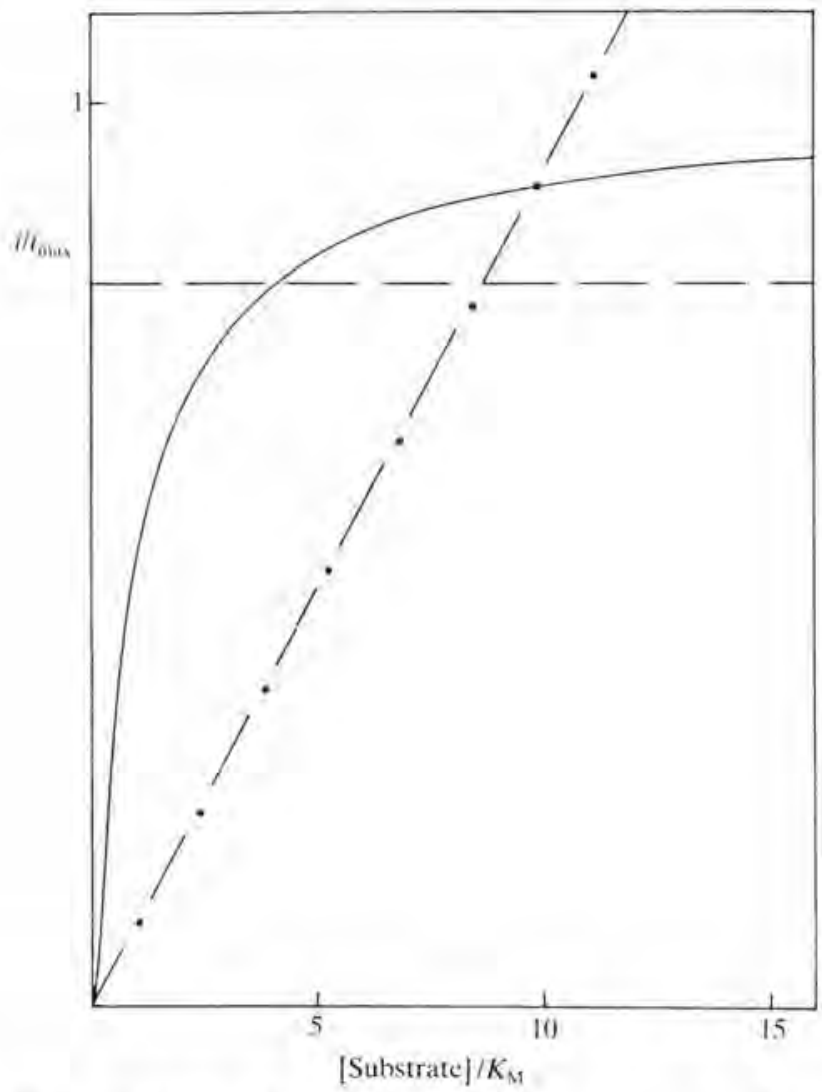

Fig. 12.17 Theoretical plots of current against concentration of substrate when the response of the device is controlled by the rate of substrate diffusion through the membrane $(-\bullet-\bullet)$, the enzyme kinetics (- $\longrightarrow$, and the electrode kinetics $(-\ldots)$, respectively.

12.5. In developing enzyme electrodes this type of analysis is crucial. For instance there is no point in worrying about the enzyme or the electrode if the problem lies with the membrane.

\section{Acknowledgements}

We are most grateful to our colleagues Drs P. N. Bartlett and A. E. G. Cass, M. Bycroft, B. J. Driscoll, and K. W. Sim whose work has been reported in this article. We thank the SERC, BP, the University of London, and Genetics International for financial support. This is a contribution from the Imperial College Sensors Group. 
Table 12.5 Summary of mechanistic deductions from $K_{\mathrm{ME}}$ and $\rho_{0}$

\begin{tabular}{llll}
\hline & $K_{\mathrm{ME}}<K_{\mathrm{M}}$ & $K_{\mathrm{ME}}=K_{\mathrm{M}}$ & $K_{\mathrm{ME}}>K_{\mathrm{M}}$ \\
\hline \multirow{2}{*}{$\rho_{0}=1$} & Transport & Transport & Transport \\
& Electrochemical & Electrochemical & $? ? ?$ \\
$\rho_{0}=\infty$ & Enzyme & Enzyme & Enzyme \\
& Electrochemical & Enzyme & Something piscine \\
\hline
\end{tabular}

\section{References}

Albery, W. J. (1975). Electrode kinetics, p. 58. Oxford University Press, Oxford.

- and Bartlett, P.N. (1984). An organic conductor electrode for the oxidation of NADH. J, Chem. Soc, Chem. Commun, 234-6.

- (1985). Amperometric enzyme electrodes, Part I. Theory. J. Electroanal. Chem. 194, $211-22$.

— and Hillman, A. R. (1981). Electrode kinetics. Ann. Rep. Prog. Chem. Sect C, 377-437.

- (1984). Transport and kinetics in modified electrodes. J. Electroanal. Chem. 170, 27-49.

- and Knowles, J. R. (1976). Evolution of enzyme function and the development of catalytic efficiency. Biochemistry 15, 5631-40.

— and Knowles, J. R. (1987). Energetics of enzyme catalysis. Isotopic experiments, enzyme conversion and oversaturation. J. Theoret. Biol. 124, 137-71.

- Bartlett, P.N. and Craston, D.H. (1985). Amperometric enzyme electrodes, Part II. Conducting organic salts as electrode materials for the oxidation of glucose oxidase, J. Electroanal. Chem. 194, 223-35.

- Eddowes, M. J., Hill, H. A. O, and Hillman, A. R. (1981). Mechanism of the reduction and oxidation reaction of cytochrome $c$ at a modified gold electrode. $J$. Am. Chem. Soc. 103, 3904-10.

Barman, T. E. (1969). Enzyme handbook Vol. 1, p. 23. Springer Verlag, New York. Cardosi, M. (1984). Ph.D thesis, Department of Biochemistry, Imperial College.

Cass, A. E. G., Davis, G., Francis, G. D., Hill, H. A. O., Aston, W. J., Higgins, 1. J., Plotkin, E. V., Scott, L. D. L. and Turner, A. P. F. (1984a). Ferrocene-mediated enzyme electrode for amperometric determination of glucose. Anal, Chem. 56, $667-71$.

- Hill, H. A. O., Higgins, I. J., Plotkin, E. V., Turner, A. P. F. and Aston, W. J. $(1984 b)$. Amperometric enzyme electrode for glucose determination. In Charge and field effects in biosystems (ed. M. J. Allen and P. N. R. Underwood), p. 475-82. Abacus Press, Tunbridge.

Cenas, N. K. and Kulys, J. J. (1981). Biocatalytic oxidation of glucose on the conductive charge transfer complexes. J. Electroanal. Chem. 128, 103-13.

Clark, L. C. and Lyons, C. (1962). Electrode systems for continuous monotoring in cardiovascular surgery, Ann. N.Y. Acad. Sci. 102, 29.

Gough, D. A. and Leypoldt, J. K. (1980). Transient studies of glucose, oxygen, and hydroquinone at a membrane covered rotating disc electrode. J. Electrochem. Soc. $127,1278-87$. 
Guilbault, G. G. and Lubrano, G. J. (1973). An enzyme electrode for the amperometric determination of glucose. Anal. Chim. Acta 64, 436.

Hanes, C.S. (1932). CLXVII. Studies on plant amylases. I. The effect of starch concentration upon the velocity of hydrolysis by the amylase of germinated barley. Biochem. J. 26, 1406.

Jaeger, C. D. and Bard, A.J. (1979). Electrochemical behaviour of tetrathiafulvalene-tetracyanoquinodimethane electrodes in aqueous media. J. Am. Chem. Soc. 101, 1690-9.

- (1980). Electrochemical behavior of donor-tetracyanoquinodimethane electrodes in aqueous media. J. Am. Chem. Soc, 102, 5435-42.

Kulys, J. J. (1981). Development of new analytical systems based on biocatalysers. Enzyme Microb. Technol. 3, 342-352.

- Samalius, A.S. and Svirmickas, G. J.S. (1980). Electron exchange between the enzyme active centre and organic metal. FEBS Lett, 114, 7-10.

Mazid, M. A. and Laidler, K. J. (1982). pH dependence of free and immobilized yeast alcohol dehydrogenase kinetics. Can. J. Biochem. 60, 100-7.

Melby, L. R. (1965). Substituted quinodimethans. VIII. Salts derived from the 7,7,8,8-tetracyanoquinodimethan anion-radical and benzologues of quaternary pyrazinium cations. Can, J. Chem. 43, 1448-53.

Harder, R. J., Hertler, W. R., Mahler, W., Benson, R. E. and Mochel, W. E. (1962). Substituted quinodimethans. II. Anion-radical derivatives and complexes of 7,7,8,8-tetracyanoquinodimethan. J. Am. Chem. Soc. 84, 3374.

Wiebel, M.K. and Bright, H.J. (1971). The glucose oxidase mechanism, interpretation of $\mathrm{pH}$ dependence. J. Biol. Chem. 246, 2734-44. 


\title{
The use of electrochemical methods in the study of modified electrodes
}

\author{
P. N. BARTLETT
}

\subsection{Introduction}

Over the past twelve years the field of modified electrodes has developed to such an extent that it is now possible, by the application of established synthetic methods, to begin to design the electrode/electrolyte interface. This ability to be able to control the molecular structure of the electrode surface is an important advance for the design of biosensors since it now becomes feasible to tailor the electrode to the requirements of the particular biological redox system. This is a particularly interesting approach to the development of amperometric biosensors since conventional 'clean' metal electrodes are generally very poor voltammetric electrodes for the direct oxidation or reduction of redox enzymes or co-enzymes. In order to proceed down this path it is necessary to know something of the properties of the modified electrode and its interaction with the substrate. In this chapter we will discuss the various techniques available for the study of these processes. Firstly, however, we begin with a brief review of the various strategies for the modification of electrodes and the theoretical models of their properties.

A variety of approaches to the modification of electrodes have been developed and investigated since the early work of Lane and Hubbard (1973 $a, b)$ on the adsorption of unsaturated monomers at electrodes. Figure 13.1 summarizes these methods. The various techniques have recently been comprehensively reviewed (Albery and Hillman 1981; Faulkner 1984; Murray 1984) and so we shall restrict ourselves to a short survey of the methods available. The early work of Lane and Hubbard was based upon the adsorption of species at the electrode surface. This type of modification is frequently a reversible process and consequently a suitable concentration of the free species in solution is important in order to maintain the coverage of the electrode surface, furthermore this method usually only yields monolayer or sub-monolayer coverages. Subsequently methods for the direct covalent attachment of redox mediators to the electrode surface were developed by Murray's group (Moses et al. 1975), Kuwana's group (Lin et al. 1977) and others. These methods rely on a direct chemical linking of the redox group to the electrode surface using silanization of the electrode to produce M-O-Si- 


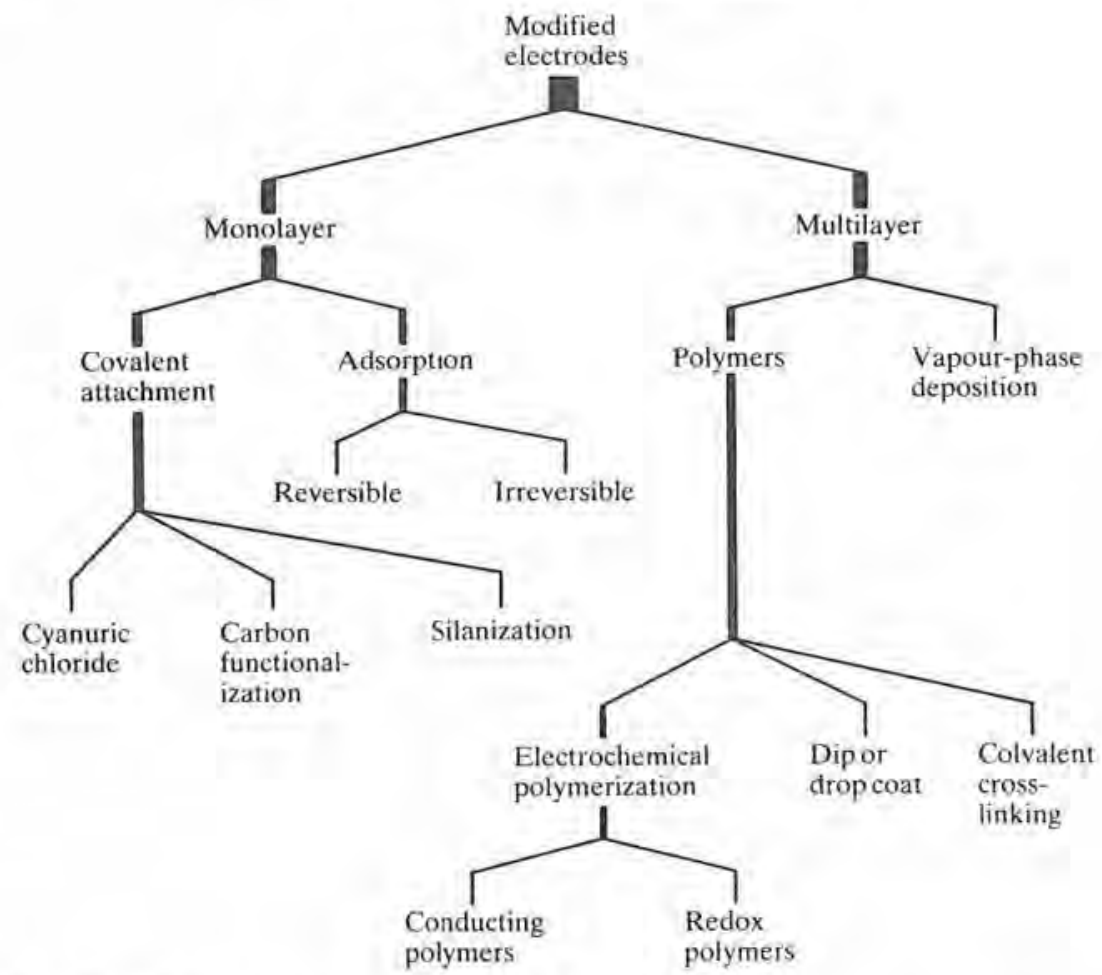

Fig. 13.1 The various types of modified electrode.

linkages, the use of cyanuric chloride to couple the redox group to the electrode, or in the case of carbon electrodes direct reaction with acidic and carbonyl functionalities on the electrode surface. These methods are most frequently employed to produce monolayer coverages, although it is possible by judicious control of the conditions to adapt these approaches to the preparation of multilayer modified electrodes (see for example Bolts and Wrighton 1979).

Multilayer coverage of electrode surfaces is most frequently achieved by the use of polymeric modification of the electrode and this is an area which is rapidly expanding. The available methods include the electrochemical polymerization of suitable monomers to yield adherent redox polymer films, the drop or dip coating of polymers onto electrodes, or the gas-phase plasma polymerization of monomers onto the electrode surface. Of these the dip or drop coating method, in which a sample of the polymer is dissolved in a suitable solvent for application to the electrode (somewhat like 'painting' the film on) has the advantage that the bulk physical properties of the polymer may be characterized before it is applied. On the other hand the electrochemical polymerization approach is very attractive because of the ease of formation and control over film thickness provided by the use of the electro- 
chemistry. Further details of the scope of these approaches can be found in the reviews of Albery and Hillman (1981) and Murray (1984).

Given that this variety of methods for the chemical modification of electrodes exists and is continually increasing it is now possible to begin to design suitable electrode surfaces for particular electrochemical reactions. As an example, let us consider the problem of the electrochemical oxidation of $\beta$-nicotinamide adenine dinucleotide $(\mathrm{NADH})$ to enzymatically active NAD ${ }^{*}$. There is considerable interest in this reaction because of the large number (over 250) of dehydrogenase enzymes which use this coenzyme or the closely related $\beta$-nicotinamide adenine dinucleotide phosphate (NADP / NADPH). These dehydrogenases catalyses reactions of the following general type:

$$
\mathrm{SH}_{2}+\mathrm{NAD}^{+} \longrightarrow \mathrm{S}+\mathrm{NADH}+\mathrm{H}^{+}
$$

where $\mathrm{SH}_{2}$ is the substrate and $\mathrm{S}$ is the product.

If the NADH formed in these reactions can be efficiently, electrochemically re-oxidized, then this can form the basis of an amperometric enzyme electrode specific to the particular substrate of the dehydrogenase chosen (Chapter 15). The great attraction of this approach is the diversity of sensors which are possible simply by changing the dehydrogenase used. Unfortunately clean (unmodified) metal or carbon electrodes are unsuitable for the oxidation of NADH. As shown by the work of Elving et al. (1976) the oxidation of $\mathrm{NADH}$ at clean electrodes only proceeds at high (about 1 volt) overpotentials. Under these conditions the oxidation proceeds through radical intermediates. Clean metal electrodes are thus unsuitable for two reasons. First, the regeneration of $\mathrm{NAD}^{+}$does not occur cleanly; dimers and other products are also formed. Second, the high overpotential required leads to problems of interferences from other species present in the sample.

In order to overcome these problems a variety of modified electrodes have been investigated. At the modified electrode the re-oxidation of NADH occurs through reaction with the immobilized mediator, rather than directly at the electrode surface. Some of the different approaches in the literature are shown in Table 13.1, These include both the use of a monolayer modified electrodes based on the adsorption of orthoquinones (Tse and Kuwana 1978; Jaegfeldt et al, 1981), and the use of multilayer modified electrodes based on polymeric orthoquinones (Degrand and Miller 1980). In addition, electrochemically deposited $\mathrm{N}$-methylphenazine has been studied by Torstensson and Gorton (1981). In all these cases some degree of success was achieved in the catalysis of the oxidation of NADH. The overpotential was considerably reduced at the modified electrode, thus alleviating the problem of interference. However, in all cases the modified electrodes proved to be too unstable for prolonged use, either because of side reactions of the mediator, or because of loss of mediator from the electrode surface. For these reasons these systems were not suitable for use in amperometric sensors. More 
Table 13.1 Modified electrodes for the oxidation of NADH

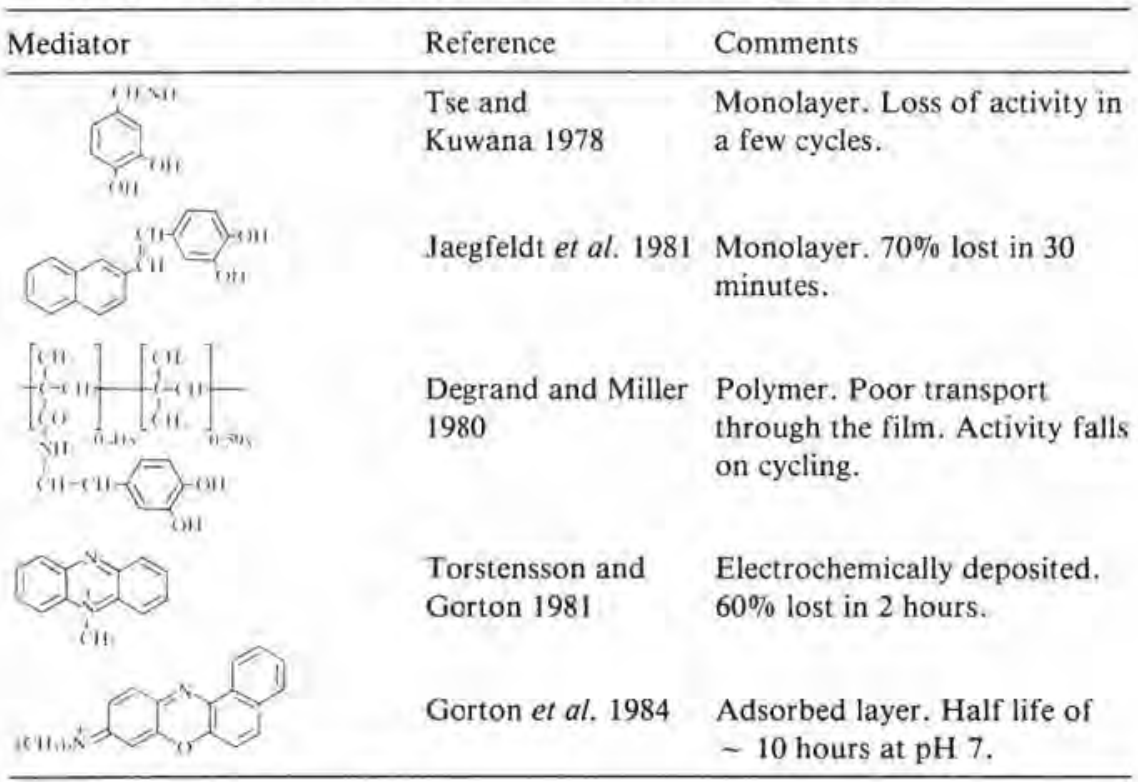

recently Gorton et al. (1985) have reported results for the oxidation of $\mathrm{NADH}$ at a graphite electrode modified by adsorption of 1,2-benzophenoxazine-7-one. They have carried out a detailed study of the kinetics of the reaction using the rotating disc electrode. They find no significant loss of activity over a period of 7 hours in the $\mathrm{pH}$ range $1.1-7.0$ and a $17 \%$ decrease over the same period at $\mathrm{pH} 8.0$. The problem of stability has been overcome by Albery and Bartlett (1984) who have shown that the one-dimensional conducting salt formed from $\mathrm{N}$-methyl phenazine with tetracyanoquinodimethane can be used as an electrode for the reaction (Chapter 12). This material does not suffer from the problems of stability encountered with other approaches.

\subsection{The kinetics of modified electrodes}

The rational design of a modified electrode for a specific application requires an understanding of three areas. Firstly, it is necessary to know something of the mechanism and kinetics of the corresponding homogeneous redox reaction. Secondly, it is necessary to know something of the electrochemistry of the mediator. Thirdly, it is necessary to have some appreciation of the electrochemical behaviour of modified electrodes in general. It is only in this way that it is possible to predict with any confidence the likely effects of 
immobilization on the kinetics and thermodynamics of the mediator reactions.

Along with the development of various methods for the modification of electrodes there has been a concomitant interest in various techniques for their characterization. These have included both electrochemical and spectroscopic studies. In this chapter we shall concentrate on the various electrochemical characterization techniques using both steady state and transient measurements at the modified electrode. The various in situ spectroscopic techniques for the study of electrode surfaces have recently been reviewed (Robinson 1984). In order to place these measurements in perspective we begin by considering the transport and kinetics in modified electrodes.

Detailed mathematical treatments of reactions at chemically modified electrodes have been published by Andrieux et al. (1982) and by Albery and Hillman (1981 and 1984). In these models the authors consider the general case of a multilayer modified electrode and they identify a number of possible rate-limiting steps. Monolayer modified electrodes are a special case of this general model. The two treatments are almost identical and their conclusions are in good agreement. For our purposes we will adopt the notation of Albery and Hillman (1984). Figure 13.2 shows the general model for the layer modified electrode. In the model the mediator couple A/B is assumed to be present, immobilized uniformly throughout a layer of thickness $L$ at the electrode surface. The mediator reacts with the substrate $Y$, present in the bulk solution, to give a product $Z$. The reaction at the modified electrode is then

$$
\mathrm{B}+\mathrm{Y} \longrightarrow \mathrm{A}+\mathrm{Z}
$$

The mediator is then oxidized (or reduced) by the electrode to regenerate B ready to react with another molecule of substrate from the solution. As we shall see a number of kinetic processes can be identified in this general scheme. It is the relative magnitudes of the characteristic rate constants for these processes which determine the behaviour of the modified electrode.

Figure 13.2 shows each of these rate processes. The diffusion of electrons through the layer, assumed to occur by electron hopping between redox centres, is characterized by a diffusion coefficient $D_{\mathrm{e}}$. (See Daum et al. 1980; Oyama and Anson 1980a.) For reaction to occur within the layer, the substrate must penetrate the layer and diffuse within it. The behaviour of the substrate within the layer is characterized by a partition coefficient $K$ and a diffusion coefficient $D_{y}$. Albery and Hillman then distinguish three possible types of reaction of the substrate and characterize each with its own rate constant. Firstly, the reaction can occur with the mediator on the outside of the layer with a rate constant $k^{\prime \prime}$. Secondly, the reaction can occur, with a rate constant $k$, with the mediator within the layer. Finally the reaction can occur directly, at the electrode surface with a rate constant $k_{\mathrm{E}}^{\prime}$. The distinction between the 
The use of electrochemical methods

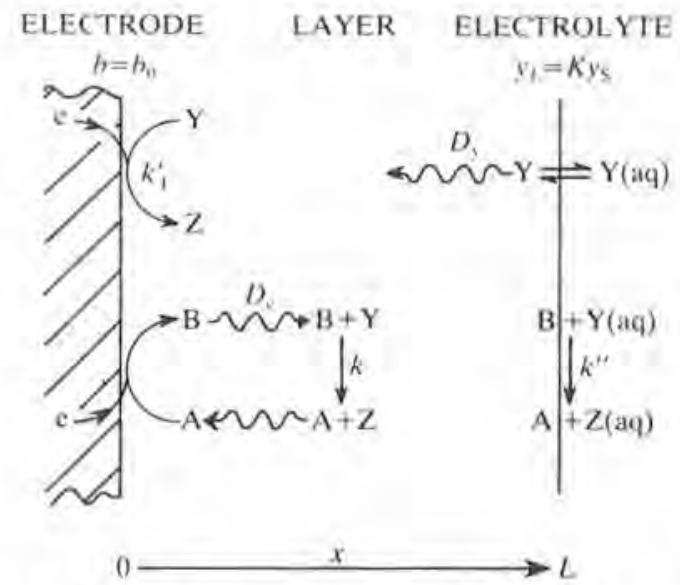

Fig. 13.2 General model for a modified electrode showing the notation. Four processes are shown, electrode surface reaction $\left(k_{\mathrm{E}}^{\prime}\right)$, partition of substrate into the film $(K)$, mediated reaction in the film $(k)$, and surface-mediated reaction $\left(k^{\prime \prime}\right)$. (After Albery and Hillman (1984) with permission.)

first two cases arises because of the differences in the solvation environment at the surface and within the layer. In these models the electron transfer reaction between the mediator $\mathrm{A} / \mathrm{B}$ and the electrode is assumed to be fast. The surface concentration of the mediator species B $\left(b_{0}\right)$ is then fixed by the electrode potential. This assumption has been discussed by Anson (1980) in terms of Marcus theory.

Two distinct transport processes within the layer can be identified; electron transport and substrate transport. The electrons enter from the electrode side (on the left in Fig. 13.2), converting A to B, and are transported through the layer by hopping. The substrate, Y, partitions into the layer from the solution (on the right in Fig. 13.2) and diffuses through the layer. The mediated conversion of substrate to product, reaction (13.1), occurs where the two species, diffusing from opposite sides of the layer, meet. The region where this occurs is called the reaction zone. The location of the reaction zone and its thickness are determined by the relative rates of transport of the two species in the layer and the rate of the mediator reaction (13.1). Thus, for example, if the diffusion of electrons in the layer is much faster than the diffusion of substrate, then the reaction is likely to occur close to the solution/layer interface. If, on the other hand, the substrate diffuses much more rapidly through the layer than the electrons, then the reaction is likely to occur close to the electrode/layer interface.

In the full treatment of the problem we can identify a total of ten possible cases associated with six possible locations for the reaction zone. Figure 13.3 shows these six locations for the reaction zone. In the figure the electrode is 


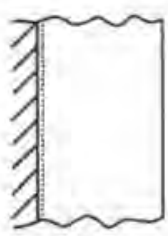

$E t_{y}$

$E k^{\prime} E$

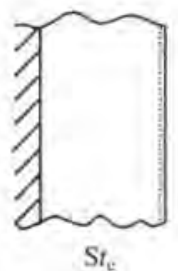

$S k "$

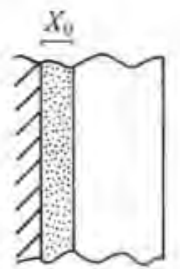

$\mathrm{LEt}_{\mathrm{y}}$

LEK

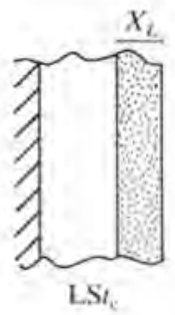

LSk

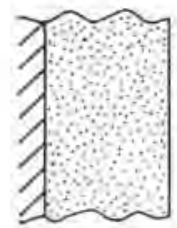

Lk

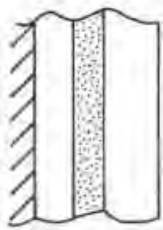

LRZ

Fig. 13.3 The location of the reaction in the ten possible cases together with the notation used to distinguish them. The electrode is on the left in each case and the location of the reaction is shown by the dotted region.

on the left in each case and the reaction zone is represented by the dotted region. The figure also gives the notation used by Albery and Hillman for each of the ten cases. Their notation uses capital letters to describe the location of the reaction zone followed by a lower case letter to distinguish the various rate-limiting processes. Table 13.2 gives the key to this notation. Thus, as an example, $\mathrm{LSt}_{\mathrm{e}}$ indicates a layer surface reaction zone determined by transport of electrons through the layer. For further details the reader is referred to the original papers.

Returning to Fig. 13.3 we can see that if we make a modified electrode for which the transport of substrate through the layer far outstrips the transport of electrons, then the reaction occurs close to the electrode (cases $\mathrm{E} t_{y}, \mathrm{E} k_{\mathrm{E}}^{\prime}$, $\mathrm{LE} t_{\mathrm{y}}$ and $\mathrm{LE} k$ ). Whether the reaction occurs at the electrode (cases $\mathrm{E} t_{\mathrm{y}}$ and $\mathrm{E} k_{\mathrm{E}}^{\prime}$ ) or in a zone of thickness $X_{0}$ close to the electrode (cases LE $t_{y}$ and LEk ) depends upon the relative magnitudes of the rate constants $k^{\prime \prime}$ and $k_{\mathrm{E}}^{\prime}$. On the other hand, if we arrange for the transport of electrons through the layer to be faster than the transport of substrate, then the reaction occurs at the outside of the layer (cases $\mathrm{St}_{\epsilon}, \mathrm{S} k^{\prime \prime}, \mathrm{LS} t_{e}$ and $\mathrm{LS} k$ ). Whether the reaction only occurs right at the surface (case $\mathrm{S} t_{\mathrm{e}}$ or $\mathrm{S} k^{\prime \prime}$ ) or occurs in a region of thickness $X_{\mathrm{L}}$ at the surface (case LSt $t_{\mathrm{e}}$ or LSk) depends upon the relative values of the rate constants $k$ and $k^{\prime \prime}$. Finally, we may find that the two transport terms are in balance. Under these circumstances we find the last two cases, $L k$ and 
Table 13.2 Case notation for modified electrodes

\begin{tabular}{ll}
\hline Symbol & Meaning \\
\hline Location of the reaction zone: \\
L & Layer \\
LS & Layer surface \\
LE & Layer electrode \\
$\mathrm{S}$ & Surface \\
$\mathrm{E}$ & Electrode \\
LRZ & Layer reaction zone \\
Rate limiting & kinetics: \\
$t_{y}$ & Transport of substrate \\
$t_{e}$ & Transport of electrons \\
$k^{\prime \prime}$ & Surface reaction \\
$k$ & Layer reaction \\
$k_{\mathrm{E}}^{\prime}$ & Electrode reaction \\
\hline
\end{tabular}

LRZ. Now the reaction occurs within the bulk of the layer. If the reaction is slow it will occur throughout the whole layer (case $\mathrm{L} k$ ). If the reaction is fast (a large $k$ ) the reaction will be confined to a narrow zone where the two reagents meet (case LRZ).

Expressions for the currents appropriate to the various cases can be found in the original papers of Albery and Hillman and of Andrieux et al.

These treatments are important for the design of biosensors because they enable us to distinguish a number of distinct strategies for the catalysis of any given reaction. These strategies have been discussed by Albery and Hillman (1984). They conclude that there are two different approaches based upon the two cases $\mathrm{S} k$ " and LS $k$. For the interfacial reaction case (Sk"), reasonably efficient catalysis by the modified electrode requires a value of $k_{2}$ (the second order homogeneous rate constant for the mediator reaction) of $k_{2}>10^{4} \mathrm{dm}^{3} \mathrm{~mol}^{-1} \mathrm{~s}^{-1}$. Under these circumstances the reaction occurs at the layer/solution interface and so the thickness of the layer is unimportant. Indeed the thicker the layer the more likely it is that transport of electrons through the layer will be a problem and so for this case it is logical to use a monolayer electrode. For the layer reaction case (LSk), on the other hand, the thickness of the layer is important. Ideally in this case the layer should be the same thickness as the reaction layer, $X_{\mathrm{L}}$. For this case we require a value of $k_{2}>10 \mathrm{dm}^{3} \mathrm{~mol}^{-1} \mathrm{~s}^{-1}$. It is not surprising that $k_{2}$ is less, under these circumstances, than for the surface case above since now many more catalytic centres throughout the layer are participating in the reaction. However to achieve this advantage it is important to ensure that the diffusion coefficient for the substrate within the layer is large; $D_{y} \sim 10^{-6} \mathrm{~cm}^{2} \mathrm{~s}^{-1}$. This in turn 
implies a fairly open, porous structure for the layer. For the catalysis of bioelectrochemical redox reactions, particularly those of large molecules such as redox enzymes, this is an important constraint on the layer electrode strategy.

It is interesting to note that in all the studies to date the rate of the homogeneous reaction between mediator and substrate has been a good guide to the rate of the heterogeneous reaction on the electrode or within the layer. This is a significant finding since it means that a logical strategy can be developed for the design of new modified electrodes based on studies of suitable homogeneous mediators. An example of this approach is the work of Kitani et al. (1981) on mediators for NADH oxidation.

The mediation of the electrochemistry of solution redox species is not the only application of modified electrodes. There is also interest in their use in the controlled release of drugs, the construction of ion gates, and the design of microelectronic devices (Thackeray et al. 1985).

In the applications of modified electrodes to the controlled release of drugs the objective is to make an electrode which has bound to it the drug, or other species, of interest. The method employed to bind the drug should be such that its release can be controlled electrochemically. In practice both electrostatic entrapment and covalent bonding have been used. Such a system, potentially, then has the ability to deliver controlled doses of the drug to localized regions on demand. With such a system the rate of delivery, the intervals between doses, and the amounts delivered are all under electrochemical control. Miller and co-workers have carried out a number of studies on polymer-modified electrodes for controlled release (Lau and Miller 1983; Lau et al. 1983; Zinger and Miller 1984). In their early work they concentrated on modified electrodes for the release of the neurotransmitter dopamine. To do this they prepared a polymer consisting of a backbone on to which they attached dopamine units using cathodically cleavable covalent bonds. The polymer consisted of a polystyrene backbone with pendant isonicotinate groups. The dopamine was attached to these pendant groups by amide linkages (Lau and Miller 1983). When the polymer was dip coated onto a glassy carbon electrode it proved possible to release dopamine into solution by application of a potential of $-1.2 \mathrm{~V}$ (vs. SCE). Glutamate and $\gamma$-aminobutyric acid (GABA) can also be released in this way (Lau et al. 1983).

Once again an understanding of the transport and kinetics of modified electrodes is important in their design. In their work Miller et al. found that the rate of charge propagation through the films $\left(D_{\mathrm{e}}\right)$ was too slow to allow delivery of the neurotransmitters at the rates desired, and that this limited the amount of neurotransmitter that could be released. This problem of slow charge propagation through polymeric layers on electrodes is quite common with non-conducting polymer backbones; especially when two-electron redox mediator groups are used. One way to overcome the problem is to make the polymer backbone conducting. There has been much work on 
conducting polymers produced by the electropolymerization of heterocyclic aromatic monomers (see, for example, Bidan et al. 1984; Bull et al. 1983; Diaz and Kanazawa 1979). Although a lot of this work has been carried out in non-aqueous solvents, conducting polymer films can be grown from aqueous solutions. Zinger and Miller (1984) have applied conducting polymers to the problem of controlled release. For this they used a polypyrrole film into which they ion-exchanged glutamate so that it was electrostatically bound in the film. Then by making the film cathodic, so that they reduced the polypyrrole, they were able to expel the glutamate counter ions, Using a conducting polymer they avoided the charge transport problem and were thus able to deliver larger amounts of neurotransmitter, and to deliver repetitive pulses.

Conducting polymers have also been used to make electrochemically controlled ion gates (Burgmayer and Murray 1982, 1984). The principle of the ion gate is very similar to that employed in the controlled release of glutamate. In its insulating (reduced) form polypyrrole is uncharged and is impermeable to both anions and cations. Upon oxidation the polymer becomes conducting and in this form is positively charged. The charged polymer is permselective; in other words anions can now penetrate the film much more readily than cations. Burgmayer $e t a l$. have used this property to make an ion gate for chloride ion. They coated polypyrrole onto a gold gauze electrode to form a coherent, continuous film. In the neutral, insulating form this film was impermeable to ions. On oxidation of the film to the charged form, it becomes permeable to chloride ions. Thus they were able to construct a membrane which could be electrochemically switched between chlorideion-permeable and impermeable states.

As we have seen modified electrodes are beginning to find application in a variety of biochemical areas. In order to exploit these applications it is necessary to apply the criteria discussed above to their design. It is also important to be able to characterize the various rate processes and the behaviour of the modified electrode. We now turn to the various electrochemical techniques available for this characterization.

\subsection{Stationary electrode techniques}

Stationary electrode techniques have been extensively applied in the study of modified electrodes because of the comparative simplicity of the apparatus required. In particular the techniques of cyclic voltammetry and the more sophisticated pulse and $\mathrm{AC}$ modulation voltammetries can be used to provide information on the coverage of the electrode and the kinetics of the electrode reaction. In addition single step potential perturbations have proved useful in the study of the kinetics of charge transport processes within the layer. 


\subsubsection{Cyclic voltammetry}

In conventional cyclic voltammetry a triangular potential waveform is applied to the electrode and the corresponding current is recorded. This technique has been widely applied in the studies of the electrochemistry of solution species (see Heinze 1984) and in the study of electrochemical reactions with subsequent chemical reaction steps (see Chapter 14 of this book). The technique has also been widely applied in the study of modified electrodes. Figure 13.4a shows a typical cyclic voltammogram of a ruthenium-trisbipyridyl-modified electrode as a function of sweep rate. Results of this type are frequently used to estimate the surface coverage of immobilized electroactive species based on the integration of the anodic and cathodic currents observed at the modified electrode in an indifferent electrolyte, where the only Faradaic reaction is the oxidation or reduction of the immobilized redox group. In order to do this it is necessary to estimate the contribution from the double layer charging current, and this is generally carried out 'by eye' since it is not possible to measure this independently. The observed double layer charging current at the clean electrode under the same

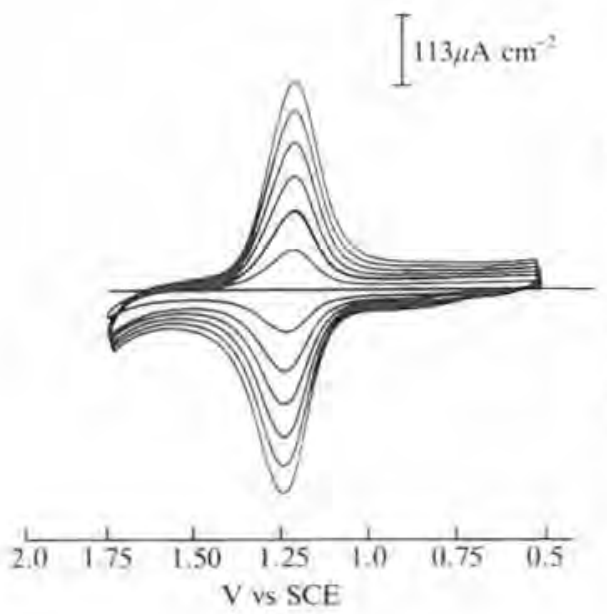

(a)

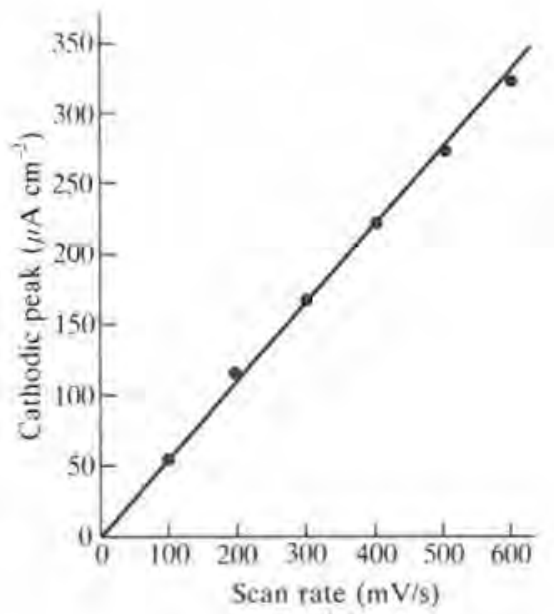

(b)

Fig. 13.4 (a) Cyclic voltammograms of a platinum electrode silanized with $\mathrm{Ru}(\text { bipy })_{3}^{2+}$ (electroactive coverage $\sim 21$ monolayers) in $0.1 \mathrm{~mol} \mathrm{dm}^{-3}$ TBAP in acetonitrile.

(b) The variation of peak height with sweep rate.

(Ghosh and Spiro 1981, reprinted by permission of the publisher, The Electrochemical Society, Inc.). 
conditions can sometimes be used as a guide but this approach should be treated with caution due to the changes in the structure of the double layer likely to be produced by the modification procedure. The integrated charge under the cyclic voltammetric peak will only give an accurate estimate of the total coverage if the whole coat, as opposed to just a few inner layers, undergoes reaction on the timescale of the measurement. Fortunately it is relatively simple to check this since if the process is kinetically controlled, with only part of the coat undergoing the redox reaction, the total charge measured is a function of the sweep rate; the slower the sweep rate the longer the time for the reaction and hence the greater the proportion of the coat that reacts. This is also reflected in the variation of the peak height with sweep rate. When diffusional transport within the film is rate limiting the peak height varies with the square root of the sweep rate $\left(\nu^{\frac{1}{2}}\right)$ and is given by precisely the same expression as that for semi-infinite linear diffusion of homogeneous solution species (Daum et al. 1980).

$$
i_{\mathrm{p}}=0.4463(n F)^{\frac{3}{2}} A D_{\mathrm{e}}^{\frac{1}{2}} \nu^{\frac{1}{2}} b_{0} /(R T)^{\frac{1}{2}}
$$

where $i_{\mathrm{p}}$ is the peak height, $n$ the number of electrons transferred, $A$ the area of the electrode, $F$ the Faraday constant, $D_{c}$ the diffusion coefficient for charge transport in the film, and $b_{0}$ is the concentration of redox species in the film. When there is no diffusional limitation the peak height varies directly with the sweep rate (see Fig. 13.4b). Thus the variation of peak height with sweep rate is a simple diagnostic for the behaviour of the modified electrode. In certain circumstances a changeover from $\nu$ to $\nu^{\frac{1}{2}}$ dependence can be observed with increasing sweep rate (see for example Oyama and Anson 1980a). This changeover is determined by the interplay between sweep rate and the kinetics of charge transport through the film. Figure 13.5 summarizes the type of behaviour found and shows schematic concentration profiles for the redox film. In an elegant set of experiments on a polyvinyl ferrocene modified electrode Daum et al. (1980) have shown that the changeover can also be brought about by reducing the rate of charge transport by cooling the electrode from 20 to $-70^{\circ} \mathrm{C}$.

The peak potentials and peak shapes in cyclic voltammetry also contain information on the kinetics of charge transport. For rapid kinetics, when the whole of the layer remains in equilibrium with the electrode the peak separation, $\Delta E$, is expected to be zero (Bard and Faulkner 1980 p. 521). In practice this is seldom observed even for monolayer modified electrodes. In general, for multilayer electrodes, the peak separation increases with increasing sweep rate whilst the mid-peak potential remains essentially constant. The wave-shapes for multilayer modified electrodes have not yet been fully analysed and exhibit a diversity of forms. This is because the processes are more complex for the multilayer electrodes and such effects as changes in solvent swelling, transport of charge compensating counterions, 

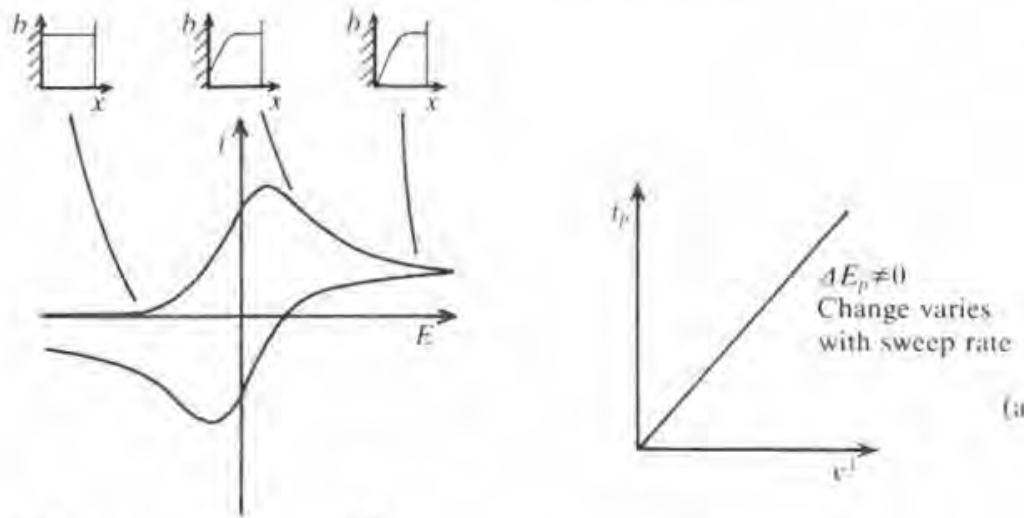

(a)
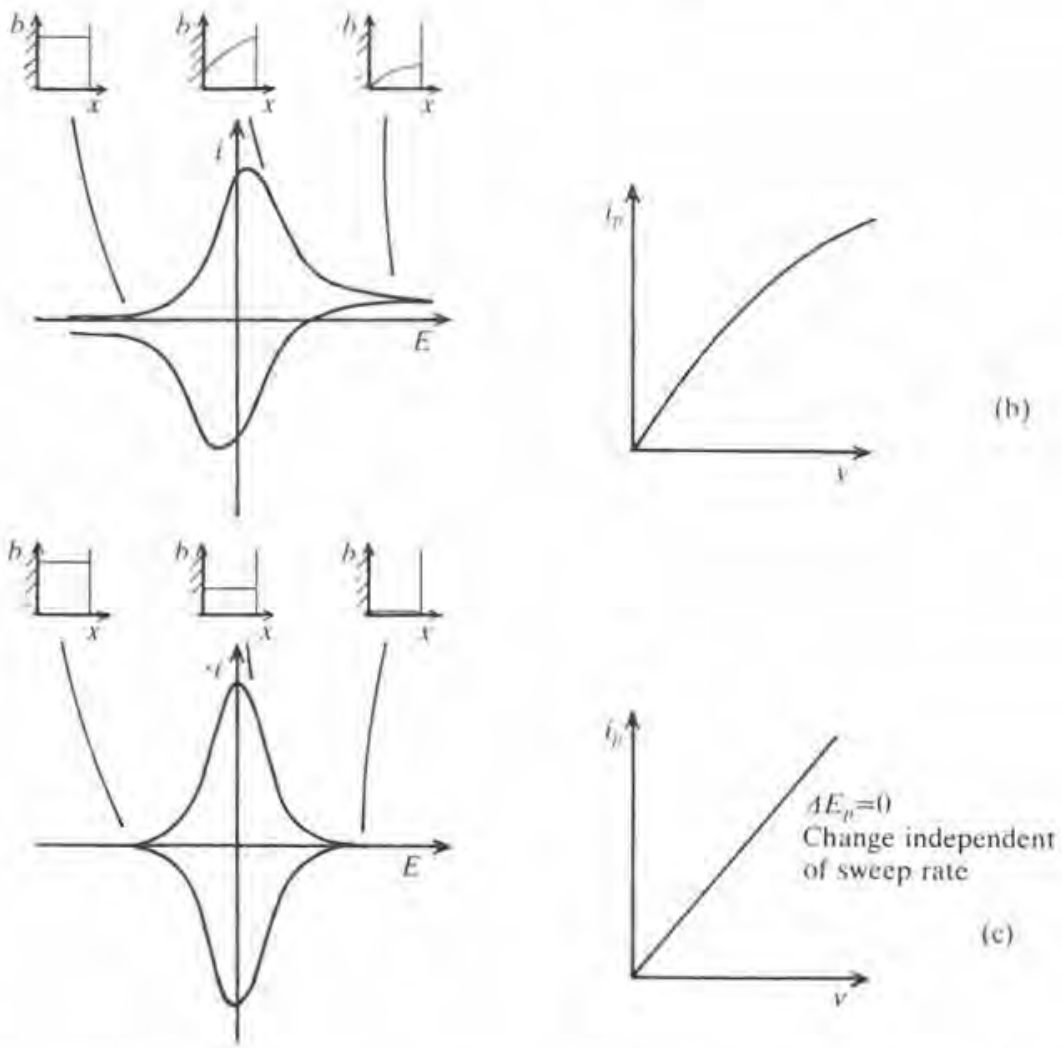

Fig. 13.5 Cyclic voltammetric behaviour of modified electrodes showing the types of behaviour found ranging from semi-infinite linear diffusion (a), through the mixed case (b), to surface reaction (c), and the appropriate dependence of peak height on sweep rate in each case. Schematic concentration profiles are shown for the films. 
and interactions between the redox groups themselves all lead to nonidealities in behaviour. Brown and Anson (1977) have proposed a model for these interactions based on activity coefficients. Further details of the mathematical treatment of waveshapes for modified electrodes may be found in the work of Aoki et al. (1983) and in a series of papers by Laviron and co-workers (Laviron 1979, 1980, 1981; Laviron and Roullier 1980; Laviron et al. 1980).

\subsubsection{Pulse polarography}

The resolution of cyclic voltammetry as a technique for the study of modified electrodes is essentially limited by the contribution of double layer charging to the observed current. This can be an especially significant problem for the study of low surface coverages encountered when using immobilized enzymes or for sub-monolayer coverage of redox species. One way to overcome this problem is to use a pulse polarographic technique and in particular differential pulse polarography. These techniques have been successfully applied to the determination of metal ions in solution at low concentrations for a number of years (Osteryoung 1981; Bard and Faulkner 1980, p. 190) and in the study of the electrochemistry of bioredox species in solution (see for example Bianco and Haladjian 1982), Pulse polarographic techniques are especially well suited to use with microprocessor-controlled electrochemical instrumentation because of the relative ease with which the required waveforms can be generated and the corresponding currents acquired and analysed. For this reason they may be expected to become increasingly popular over the next few years.

Figure 13.6 shows the principle of the technique; a series of potential pulses of amplitude $\Delta E$ (usually between 5 and $50 \mathrm{mV}$ ) are superimposed on a slow potential sweep (ca. $1 \mathrm{mV} \mathrm{s}^{-1}$ ). The pulse is repeated after a time $\tau_{\mathrm{D}}$ and is of duration $\tau_{\mathrm{D}}-\tau\left(\tau_{\mathrm{D}}\right.$ is often referred to as the 'drop time', a reference back to the original implementation of the technique at the dropping mercury electrode). The current is measured over a fixed time interval, $\delta$, just before and again towards the end of the pulse as shown in the figure. The differential pulse polarogram then consists of a plot of the difference between these two current measurements, $\Delta i$, as a function of the base potential $E_{1}$. It is more convenient in the case of digital instrumentation to use the wave-form shown in Fig. 13.6(b), rather than to use a slow potential sweep as the baseline, and indeed this is the wave-form which is most conveniently used in the theoretical treatment (Birke 1978). Brown and Anson (1977) have compared cyclic voltammetry and differential pulse polarography for the study of monolayer modified electrodes. Figure 13.7 shows some of their data for ruthenium pentamine covalently attached to a pyrolytic graphite electrode. It is clear from the figure that the technique is suitable for the study of low surface coverages.

For a monolayer modified electrode the Faradaic reaction of the surface- 

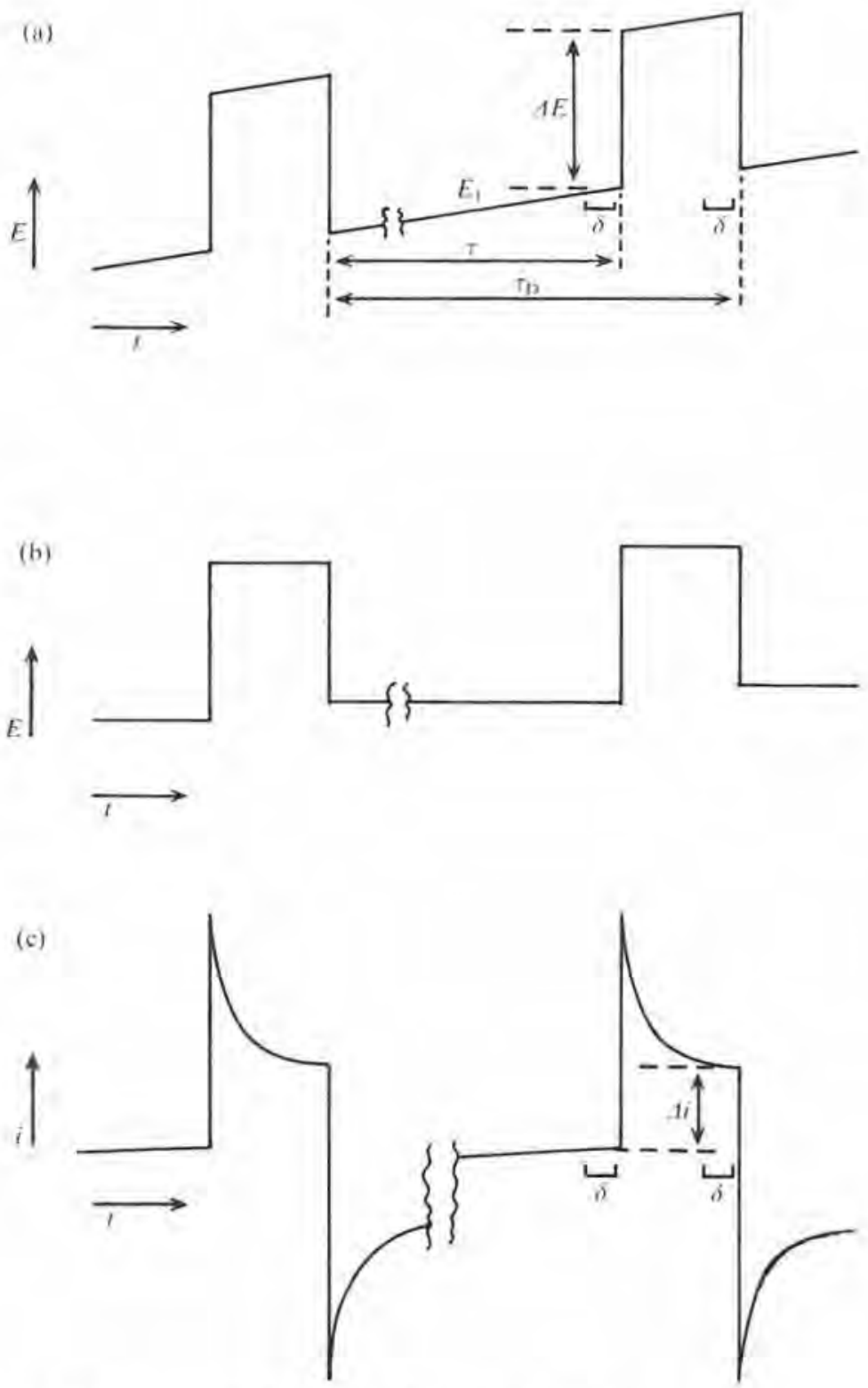

Fig. 13.6 The principle of differential pulse polarography.

(a) The potential waveform showing the notation.

(b) The corresponding waveform used with digital instrumentation.

(c) The resulting current response. 


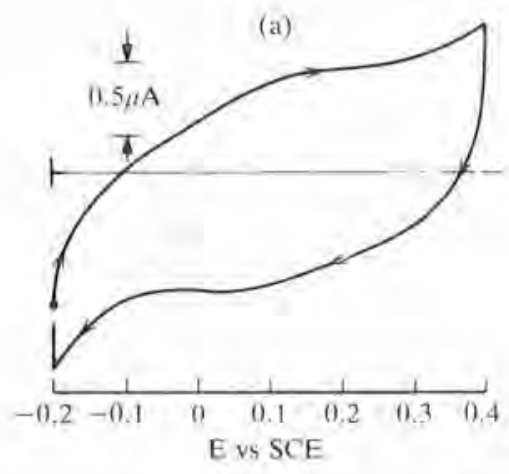

(h)
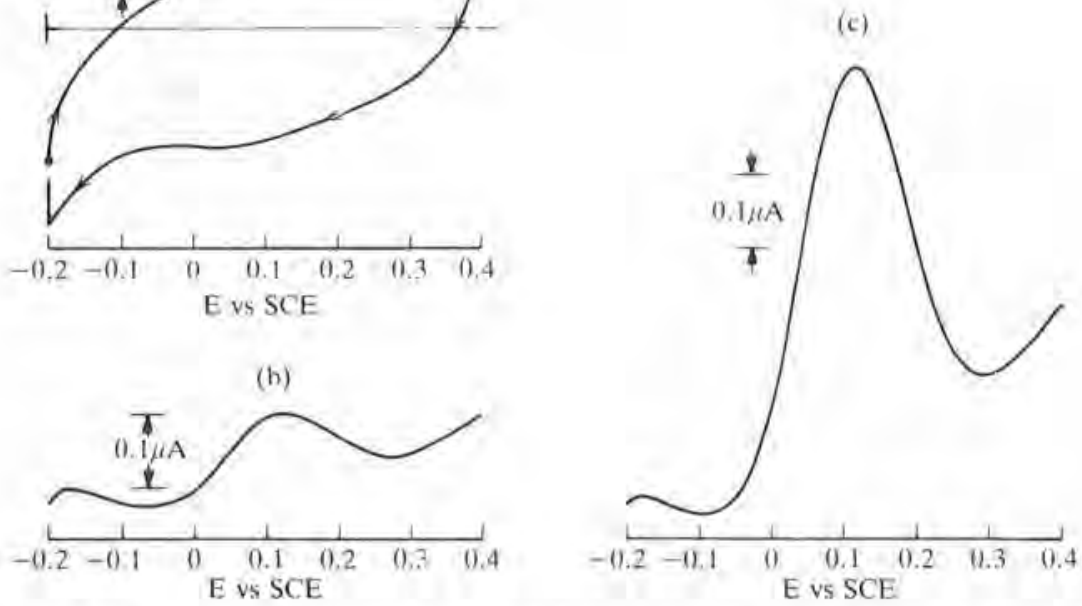

Fig. 13.7 Cyclic and differential pulse voltammograms for an edge-on pyrolytic graphite electrode with approximately $4.2 \times 10^{-11}$ moles of ruthenium (II) pentamine covalently bound to the surface. Supporting electrolyte, $1 \mathrm{~mol} \mathrm{dm}^{-3} \mathrm{CF}_{3} \mathrm{COOH}$.

(a) Cyclic voltammogram at $50 \mathrm{mV} \mathrm{s}^{-1}$.

(b) Differential pulse polarogram with $R_{\mathrm{u}} \sim 100 \Omega$.

(c) As in (b) but with $R_{\mathrm{u}}=1000 \Omega$.

(Brown and Anson 1977. Reprinted with permission from Analytical Chemistry. Copyright 1977 American Chemical Society).

bound species can be treated as a pseudo-capacitor in parallel with the double layer capacitance and Brown and Anson (1977) have presented a theoretical treatment based on this model and assuming that the surface-bound redox couple exhibits a Nernstian response. In other words they assume that the kinetics of electron transfer are rapid and that the concentrations of oxidized and reduced species bound at the electrode surface are therefore related to the electrode potential by the Nernst equation. Care should be exercised in applying this model to other modified electrodes, and in particular multilayer modified electrodes, where this assumption may be invalid due to diffusional limitations within the coating. In their model the charging of the double layer and the surface-bound redox species occurs through the uncompensated solution resistance as shown in Fig. 13.8. The current flowing in the circuit after each pulse is then given by:

$$
i=\frac{\Delta E}{R_{\mathrm{u}}} \exp -\left[\frac{t}{R_{\mathrm{u}}\left(C_{\mathrm{dl}}+C_{\mathrm{E}}\right)}\right]
$$




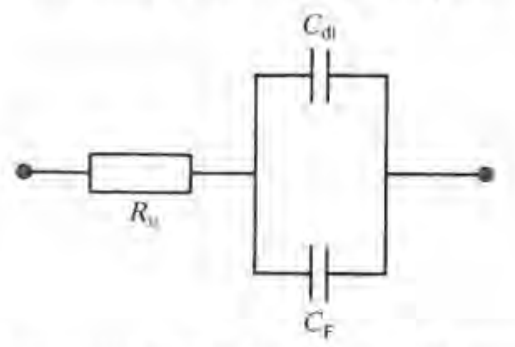

Fig. 13.8 The simple equivalent circuit for irreversibly attached reactant used by Brown and Anson (1977).

where $R_{\mathrm{u}}$ is the uncompensated solution resistance, $C_{\mathrm{dl}}$ the double layer capacitance, $C_{\mathrm{F}}$ the Faradaic pseudo-capacitance, and $t$ the time from the start of the pulse. By the clever trick of adding an additional uncompensated resistance in the measuring circuit Brown and Anson were able to enhance the sensitivity of the technique by increasing the $R C$ time of the circuit and slowing down the charging of the capacitance.

A full description of the wave-shape of the differential pulse polarogram calls for explicit knowledge of the double-layer capacitance and its variation with potential. This is an inaccurate and problematical process because of the uncertain origin of the background currents and the possible changes brought about by the surface modification process itself. The technique is, however, appealing for the detection of low coverages and the peak potentials can provide information on the standard potential of the attached redox couple. Care should be exercised in the choice of experimental parameters such as scan rate since erroneous peak potentials can result. It is therefore prudent to check the constancy of peak potentials with variations in the experimental parameters before concluding that these are in fact the same as the standard potentials for the attached redox species.

Differential pulse polarography has been used to look at enzymes immobilized at electrode surfaces where, again as a result of low coverages, cyclic voltammetry is poorly suited. Ianniello et $a l$. $(1982 a, b)$ have used the technique to study the electrochemistry of flavoproteins covalently attached by the cyanuric chloride method to graphite electrodes. Using this method they have observed shifts in the peak potentials for the oxidation/reduction of the flavin prosthetic group on attachment of glucose oxidase, xanthine oxidase, and D-and L-amino acid oxidase to electrodes and the effects of removal and subsequent replacement of the flavin moiety.

The related technique of normal pulse polarography has been used to study polymer modified electrodes (Oyama et al. 1983, 1984). In this technique a series of pulses is applied to the electrode, each of increasing amplitude but always from the same base voltage at which negligible reaction occurs (Bard and Faulkner 1980, p. 186). The wave-form used is shown in Fig. 13.9. The 


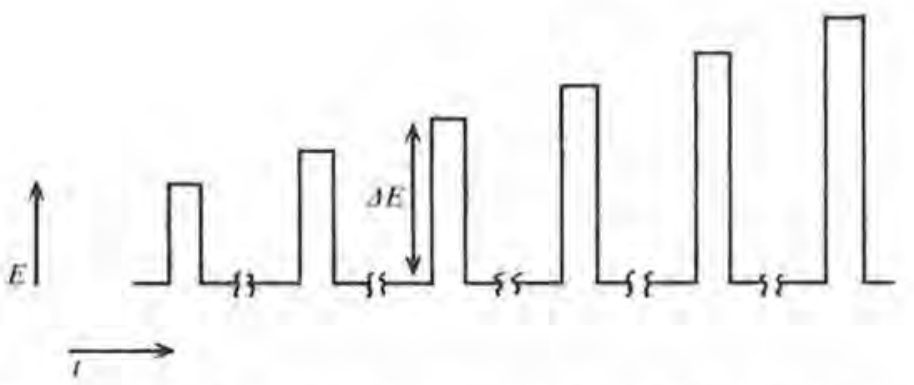

Fig. 13.9 The potential wave-form used in normal pulse polarography.

normal pulse polarogram then consists of a plot of the current measured towards the end of the pulse as a function of the pulse voltage.

\subsubsection{AC voltammetry}

As a technique for the study of modified electrodes at low coverages AC voltammetry offers similar advantages over conventional cyclic voltammetry as the differential pulse technique. In AC voltammetry a small amplitude sinusoidal modulation is imposed on top of the slow potential scan applied to the electrode. Using a lock-in amplifier the resulting AC-modulated component of the current is measured and plotted as a function of the applied mean potential. The technique has been applied by Senda and co-workers to the study of the electrochemistry of redox proteins adsorbed at the surface of mercury electrodes (Ikeda et al. 1979, 1981; Kakutani et al. 1980, 1981). The theory for the AC voltammetric response appropriate to this type of modified electrode has been presented by Kakutani and Senda (1979).

\subsubsection{Potential step chrono-amperometry}

All of the stationary electrode techniques described above have relied upon the use of repetitive potential wave-forms of some type. Single potential step experiments at modified electrodes are also frequently used to probe the kinetics of charge transport through polymeric layers of bound redox species. In this type of experiment a single potential step from an initial potential, $E_{\mathrm{i}}$ to a final potential, $E_{f}$, is applied to a stationary electrode in background electrolyte and the associated current response is recorded as a function of time. Figure 13.10 shows a typical transient for a thionine-coated electrode in $0.05 \mathrm{~mol} \mathrm{dm}^{-3}$ sulphuric acid. The effect of the potential step is to change the redox state of the coat so that the current is made up of a capacitative contribution at short times and the Faradaic reaction of the coat at longer time. The response is then a bounded diffusion problem and is essentially identical to the thin layer cell case. Using Laplace transforms to solve Fick's second law of diffusion as applied to a bounded film of 


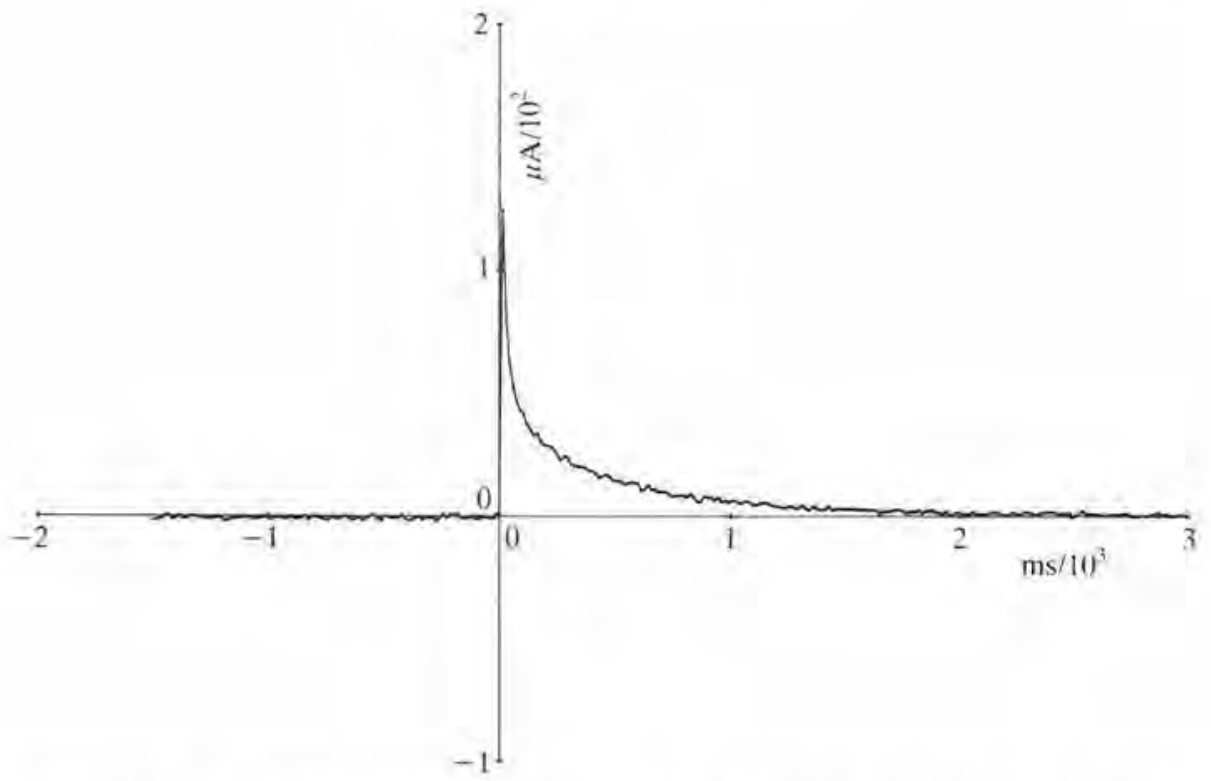

Fig. 13.10. Typical transient for a potential step at a thionine-coated electrode in $0.05 \mathrm{~mol} \mathrm{dm}^{-3} \mathrm{H}_{2} \mathrm{SO}_{4}$ recorded using a microprocessor-controlled potentiostat. The potential step is from -211 to $-191 \mathrm{mV} v s$. SCE and is averaged over eight repeat transients.

thickness $L$ gives the following expression for the transient current (Oglesby et al, 1965).

$$
i(t)=\left(\frac{D_{\mathrm{c}}}{\pi t}\right)^{\frac{1}{2}} \frac{\Delta Q}{L}\left\{1+2 \sum_{m=1}^{\infty}(-1)^{m} \exp \left(\frac{-m^{2} L^{2}}{D_{\mathrm{e}} t}\right)\right\}
$$

where $\Delta Q$ is the change in charge in the coat, $L$ is the thickness of the coat, and $D_{\mathrm{e}}$ the diffusion coefficient for charge within the coat. Equation 13.4 is a modified form of the Cottrell equation (Bard and Faulkner 1980, p. 143). The current transient is most readily analysed by plotting $i$ against $t^{-\frac{3}{2}}$ as shown in Fig. 13.11. At short times $\left(t \ll D / L^{2}\right)$, when the concentration polarization within the coating has not reached the outside of the coat, eqn 13.4 reduces to the corresponding Cottrell equation for the unbounded (semi-infinite linear diffusion) case

$$
i(t)=\left(\frac{D_{\mathrm{e}}}{\pi t}\right)^{\frac{1}{2}} \frac{\Delta Q}{L} .
$$

At longer times when $t \sim D / L^{2}$ the concentration polarization in the coat reaches the surface and the diffusion limited current falls below that given by eqn 13.5. This can be seen in the plot of $i$ against $t^{-\frac{1}{2}}$ by the deviation from the 


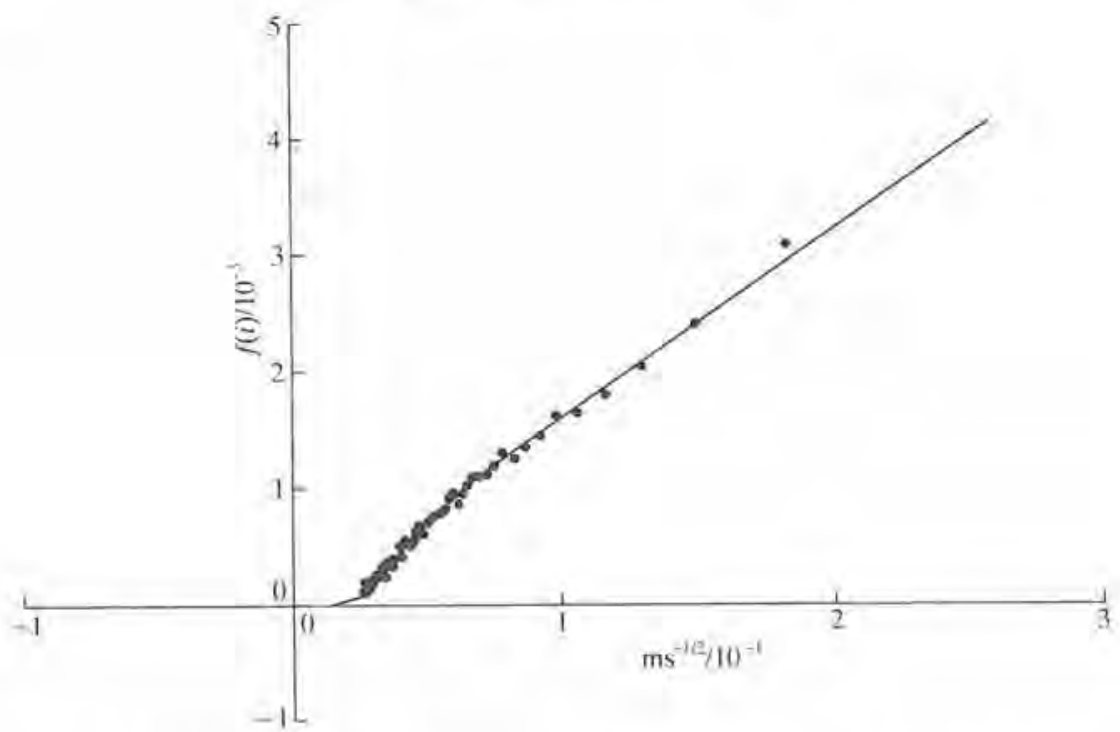

Fig. 13.11 Analysis of the thionine potential step transient according to the Cottrell equation. The line is calculated from eqn 13.4 with $D / L^{2}=0.813 \mathrm{~s}^{-1}$ and $\Delta Q=2.63$ $\times 10^{-5}$ C. $f(i)=i / \Delta Q$.

straight line through the origin as the exponential terms in eqn 13.4 become significant. The slope of the line through the origin and the shott time data gives a value of $D_{e}$ for the reaction from eqn 13.5 corresponding to the effective diffusion coefficient for charge through the coat providing $\Delta Q$ and $L$ are known. $\Delta Q$ is usually obtained by integration of the whole transient whilst $L$ can be estimated from the coverage measured by cyclic voltammetry or some other means.

Deviations from the straight line behaviour predicted by eqn 13.5 at very short times are sometimes observed due to the double layer charging current (Peerce and Bard 1980). A number of workers (see for example Murray 1984) advocate the use of large $(>500 \mathrm{mV}$ ) steps right through the redox peak in the cyclic voltammogram for the coating. This practice should be approached with caution due to the gross changes in morphology and solvation that may be induced by such large changes in the redox state of the coat and also due to the problems of double layer charging this introduces. In the experience of the present author it is much better to use a number of much smaller (10-20 mV) steps backwards and forwards about the region of redox activity of the coating and to compare these for consistency in the values of $D_{e}$ obtained. The most significant source of error in the evaluation of $D_{e}$ by this method is frequently to be found in the value of $\Delta Q$ used in eqn 13.4. For this reason it is always wise to fit the full equation to the experimental data rather 
than simply to rely on the initial slopes.

A number of groups have investigated the mechanism of charge transport through polymer films and the nature of the rate-limiting process. In certain cases $D_{\mathrm{e}}$ has been found to be dependent upon the nature of the counter ion. Thus varying the size of the counter ion has been shown to have a marked effect on the values of $D_{\mathrm{c}}$ obtained for the thionine-coated electrode (Albery et al. 1982) with values ranging from $9.1 \times 10^{-13} \mathrm{~cm}^{2} \mathrm{~s}^{-1}$ for sulphate ion to $0.9 \times 10^{-13} \mathrm{~cm}^{2} \mathrm{~s}^{-1}$ for tosylate ion. The relationship between the observed diffusion coefficient and the various rate-limiting processes has been discussed by Murray (1984).

The potential step technique measures the transient response of the electrode to a potential perturbation. A variety of other methods have been used in combination with the potential step to study charge transport and kinetic processes in modified electrodes. Most notable amongst these is the use of spectroscopic detection to monitor the time course of the change in the redox state of the coat. This approach has the advantage of avoiding the problems of the background contributions encountered with current measurements. The most commonly used method is to observe the change in the visible region of the spectrum using an optically transparent tin oxide electrode as the substrate onto which to coat the modified electrode. The spectroscopic measurement follows the integrated change in the redox state of the coating, and so the integrated form of the modified Cottrell equation is appropriate (Albery et al. 1982)

$$
\begin{aligned}
\theta= & \frac{(\mathrm{OD})_{t}-(\mathrm{OD})_{0}}{(\mathrm{OD})_{\infty}-(\mathrm{OD})_{0}} \\
= & 2\left(\frac{D_{e} t}{L^{2} \pi}\right)^{\frac{1}{2}}+2 \sum_{m=1}^{\infty}(-1)^{m}\left[2\left(\frac{D_{c} t}{L^{2} \pi}\right)^{\frac{1}{2}} \exp \left(\frac{-m^{2} L^{2}}{D_{c} t}\right)\right. \\
& \left.-2 m \operatorname{erfc}\left(\frac{m L}{D_{\mathrm{e}}^{\frac{1}{2}} t^{\frac{1}{2}}}\right)\right]
\end{aligned}
$$

where (OD) is the optical density and the subscripts $0, t$, and $\infty$ denote the initial value, the value at time $t$, and the final infinity value respectively. $L$ is the film thickness, and $D_{\mathrm{e}}$ the effective diffusion coefficient.

Figure 13.12 shows a typical plot of experimental data for the optical transient at a thionine-coated electrode. In situ electron spin resonance has also been used to study the changes in polymeric coatings in response to a potential step (Albery et al. 1984). In this experiment the authors were able to follow the concentration of bound radicals in a polynitrostyrenecoated electrode as a function of time and to analyse the results using eqn 13.6. 


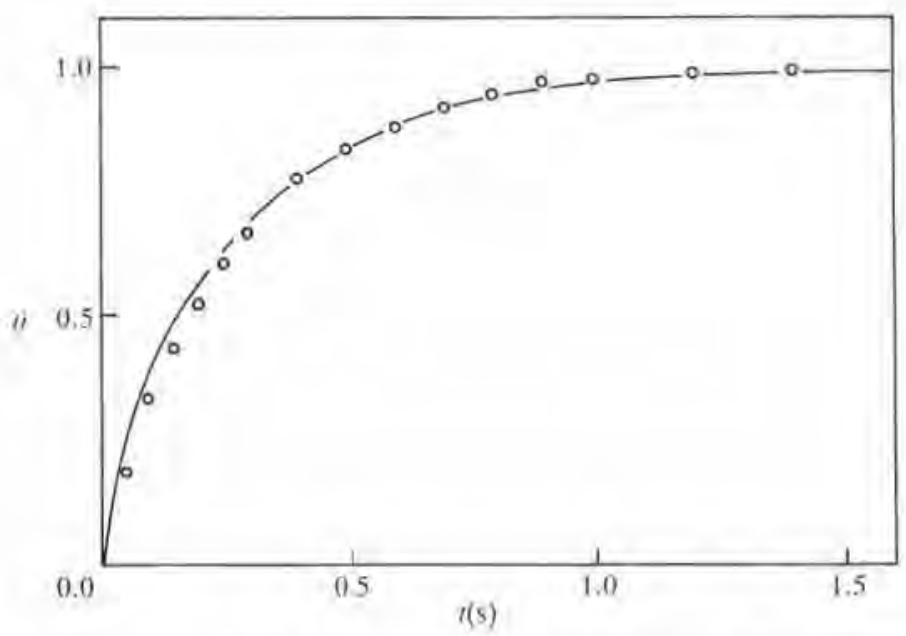

Fig. 13.12 Optical transient at a thionine-coated electrode. The solid line was calculated from eqn 13.6. (From Albery et al, 1982 with permission.)

\subsection{Forced-convection techniques}

The methods described above have all concentrated on the properties of the modified electrode itself and the kinetic processes occurring within the coat. In order to probe the equally important and interesting question of the kinetics and mechanism of the mediated reaction between the bound redox species and the substrate present in solution it is necessary to be able to calculate the surface concentration of substrate. Forced-convection electrodes, and in particular the rotating disc and ring-disc electrode geometries, are ideally suited to this type of study. This is because the controlled hydrodynamics of the rotating electrode provides reproducible, calculable, and readily experimentally controlled transport of substrate to, and product from, the electrode surface.

\subsubsection{The rotating-disc electrode}

The hydrodynamics of the rotating disc were first solved by von Karman (1921) and Cochran (1934). The rotating electrode acts as a pump drawing fresh solution up from the bulk of the solution towards the electrode surface, then spinning it around and flinging it out sideways. This flow pattern is shown in Fig. 13.13. The action of the electrode establishes a stationary boundary layer, called the diffusion layer, at the electrode surface which rotates with the electrode. Outside this stationary layer the solution is well stirred. The thickness of the diffusion layer is given by

$$
X_{\mathrm{D}}=0.643 D^{\frac{1}{3}} \nu^{\frac{1}{6}} W^{-\frac{1}{2}}
$$


(a)
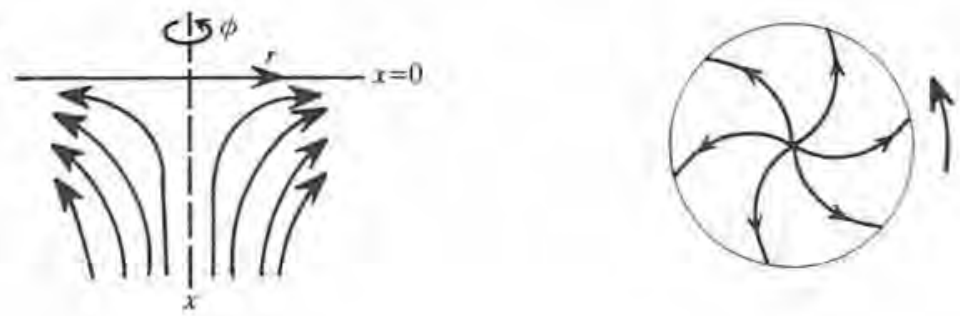

(b)

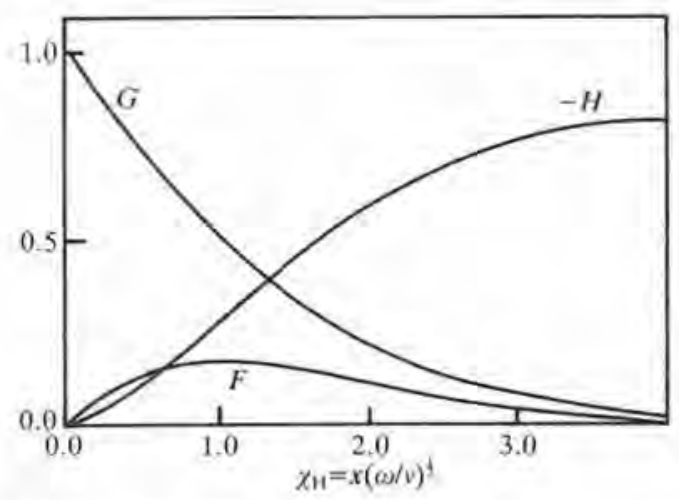

Fig. 13.13 Fluid flow at a rotating-disc electrode.

(a) Schematic representation of the streamlines.

(b) The three velocity components of the flow as a function of distance from the electrode surface: $v_{\phi}=r \omega G\left(\chi_{\mathrm{H}}\right) ; v_{r}=r \omega F\left(\chi_{\mathrm{H}}\right) ; v_{x}=(\omega / v)^{\frac{1}{2}} H\left(\chi_{\mathrm{H}}\right)$ (where $\omega$ is the rotation speed in $\mathrm{rad} \mathrm{s}^{-1}$ ).

where $D$ is the diffusion coefficient, $\nu$ is the kinematic viscosity (the viscosity divided by the density), and $W$ is the rotation speed of the electrode in $\mathrm{Hz}$. Equation 13.7 has two important features. Firstly, the thickness of the diffusion layer is dependent on the rotation speed and so can be varied experimentally. Secondly, the thickness is independent of the radial coordinate so that the stationary layer is uniform in thickness over the whole surface of the electrode; the electrode is said to be uniformly accessible and the current density will thus be uniform over the surface of the disc.

Figure 13.14 shows the concentration profiles for species $\mathrm{Y}$ reacting at a rotating-disc electrode to produce a product $Z$. At distances greater than $X_{\mathrm{D}}$ the solution is well stirred and there is no concentration polarization of $\mathrm{Y}$ or $Z$. Transport in this region is predominantly by convection. At distances closer to the electrode than $X_{\mathrm{D}}$ the solution is stationary and transport is 


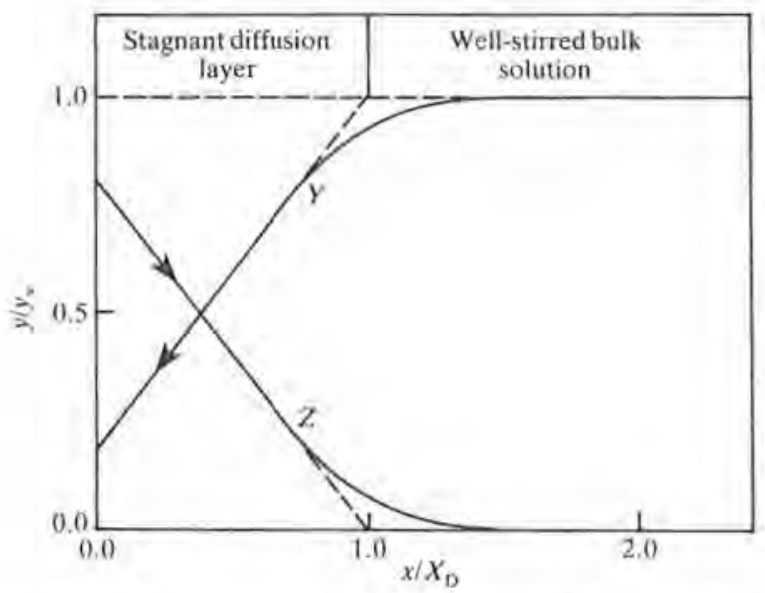

Fig. 13.14 Variation of concentration with distance at a rotating disc electrode.

purely by diffusion. The flux of species reacting at the electrode surface, $j$, is simply given by Fick's first law

$$
j=\frac{D}{X_{\mathrm{D}}}\left(y_{\infty}-y_{0}\right)=\frac{i}{n A F}
$$

where $y_{\infty}$ is the bulk concentration, $y_{0}$ the concentration at the electrode surface, $n$ is the number of electrons transferred, $A$ is the electrode area and $F$ is the Faraday constant $\left(96480 \mathrm{C} \mathrm{mol}^{-1}\right)$. Since $X_{\mathrm{D}}$ is readily calculable from eqn 13.7 and the current and bulk concentration are generally known, eqn 13.8 can be used to find the surface concentration of reacting species. This is a significant point for the study of modified electrodes since it is precisely this concentration which is important in the investigation of the kinetics of mediated reactions.

When the electrode potential is poised so that the surface concentration of substrate is reduced to zero $\left(y_{0}=0\right)$ the current becomes limited by mass transport and we obtain, from eqn 13.7 and 13.8, the Levich equation for the limiting current,

$$
i_{\mathrm{L}}=1.554 n A F D^{\frac{2}{3}} \nu^{-\frac{1}{6}} y_{\infty} W^{\frac{1}{2}} .
$$

Under these conditions the current is directly proportional to the square root of the rotation speed; the faster the electrode is rotated the thinner the stagnant layer and thus the more efficient the transport of substrate to the electrode surface. It is worth considering the possible effects of surface roughness on the hydrodynamics of the rotating-disc electrode since the polymeric coatings applied to the electrode seldom exhibit perfectly smooth surfaces. Bruckenstein et al. (1985) have investigated the effects of surface 
roughness, produced by polishing electrodes with abrasives of different sizes, on the current response of a rotating-disc electrode. They find that there is no effect on polishing with abrasives of $14 \mu \mathrm{m}$ or less. Bearing in mind that for typical values in aqueous solution the stagnant layer, $X_{\mathrm{D}}$, is of the order of $10^{-3} \mathrm{~cm}$ thick this is not surprising. As a rule of thumb, surface roughness is not likely to be important provided it is less than $-X_{\mathrm{D}}$.

Simple rotating-disc measurements are ideally suited to the investigation of mediated reactions at modified electrodes because of the calculable rate of mass transport of the substrate, and a number of authors have made use of this technique (Anson et al. 1983a, b; Albery et al. 1980; Oyama and Anson $1980 \mathrm{~b}$; Rocklin and Murray 1981; Haas and Zumbrunnen 1981). The Levich limiting current given by eqn 13.9 is seldom observed for modified electrodes since there is often another rate-limiting step other than transport of substrate to the electrode surface. Under these circumstances it is most convenient to extrapolate out the mass transport contribution and thus to obtain a value for $k_{\mathrm{ME}}^{+}$the effective heterogenous rate constant for reaction at the electrode. The value of $k_{\mathrm{ME}}^{\prime}$ can then be analysed in terms of the theoretical model for the reactions at modified electrode put forward by Albery and Hillman (1981 and 1984) and by Andrieux et al. (1982) and discussed earlier in this chapter.

In order to treat the reaction at a modified electrode we can regard the supply of substrate, $\mathrm{Y}$, from the bulk of solution as introducing an additional step in the reaction mechanism, characterized by a mass transport rate constant $k_{\mathrm{D}}^{\prime}$, where

$$
k_{\mathrm{D}}^{\prime}=D / X_{\mathrm{D}}=1.554 D^{\frac{2}{3}} \nu^{-\frac{1}{6}} W^{\frac{1}{2}}
$$

We can then write the scheme for the reaction as

$$
\mathrm{Y}_{\infty} \underset{k_{\mathrm{D}}^{\prime}}{\stackrel{k_{\mathrm{D}}^{\prime}}{\rightleftharpoons}} \mathrm{Y}_{0} \stackrel{k_{\mathrm{ME}}^{\prime}}{\longrightarrow} \mathrm{Z}
$$

where $Y_{\infty}$ is the substrate in the bulk of solution and $Y_{0}$ is the substrate at the electrode surface. In doing this we are following an analysis of the type first proposed by Koutecky and Levich (1958) for reactions at rotating-disc electrodes. Analysis of the scheme gives the following expression for the flux, $j$,

$$
\frac{1}{j}=\frac{1}{k_{\mathrm{D}}^{\prime} y_{\infty}}+\frac{1}{k_{\mathrm{ME}}^{\prime} y_{\infty}}
$$

where $y_{\infty}$ is the bulk concentration of substrate. Substituting in eqn 12 for $k_{\mathrm{D}}^{\prime}$ and using the fact that $j=i / n A F$ we obtain the Koutecky Levich equation

$$
\frac{1}{i}=\frac{1}{1.554 n F A D^{\frac{2}{3}} \nu^{-\frac{1}{6}} W^{\frac{1}{2}} y_{\infty}}+\frac{1}{n F A k_{\mathrm{ME}}^{\prime} y_{\infty}} .
$$


Thus a plot of $i^{-1}$ against $W^{-\frac{1}{3}}$ should yield a straight line whose slope is the reciprocal of the Levich slope (eqn 13.9), and whose intercept gives the value of $k_{\mathrm{ME}}^{\prime}$. This procedure allows the mass transport component to be extrapolated out of the data by going to infinite rotation speed, where the surface concentration equals the bulk concentration. Albery and Hillman (1981) have published a flowchart, based on this type of analysis, for the diagnosis of the mechanism for a reaction at a modified electrode. They also discuss those cases in which the Koutecky Levich plot is non-linear because $k_{\text {ME }}^{\prime}$ itself depends upon $y_{0}$. This analysis has been applied to the thioninecoated electrode (Albery et al, 1985b).

The Koutecky Levich analysis is appropriate in most cases in which there is some rate-limiting process in addition to mass transport at the rotating-disc electrode. As an example of the use of this type of analysis, let us consider the oxidation of $\mathrm{NADH}$ at a rotating-disc electrode made from $\mathrm{N}$-methylphenazinium tetracyanoquinodimethanide (NMP.TCNQ). Figure 13.15 shows the currents obtained plotted against the square root of the rotation speed (Levich plots) for four different concentrations. As can be seen, with increasing rotation speed the current eventually reaches a plateau, the value
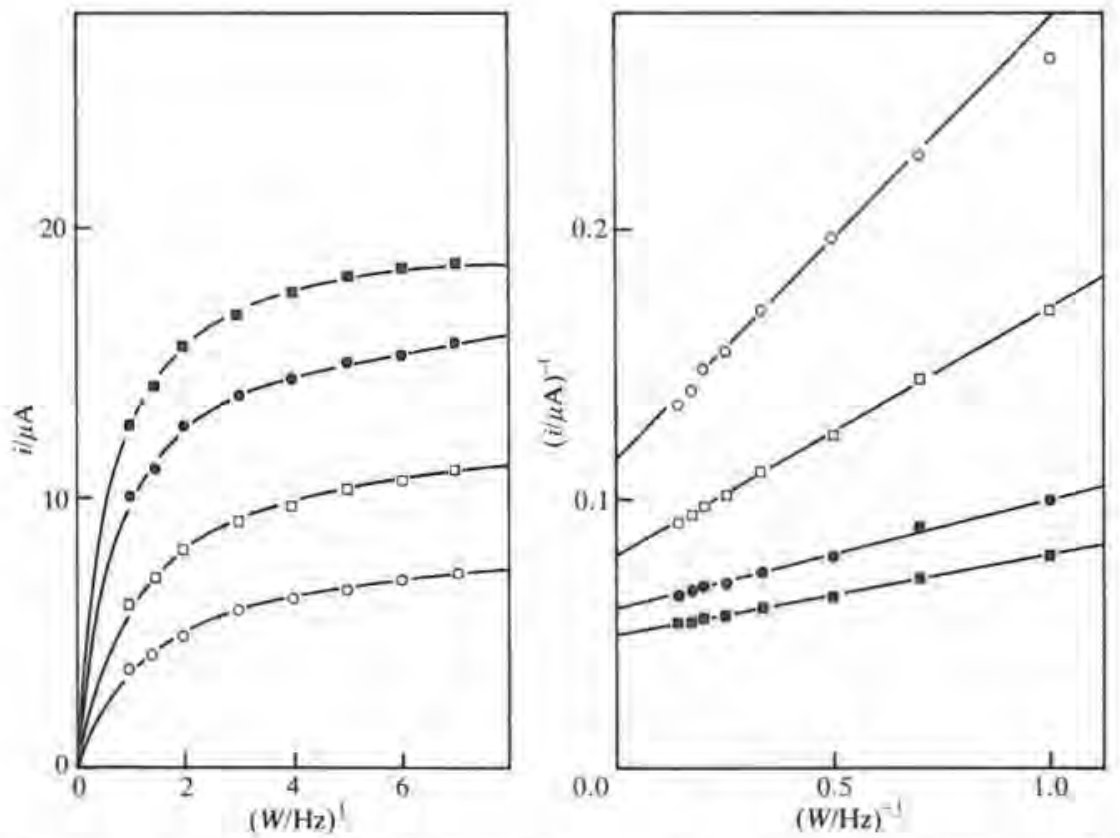

Fig. 13.15 Levich and Koutecky Levich plots for the reaction of NADH at an NMP.TCNQ rotating disc electrode for various concentrations of NADH: $0,0.38$ $\mathrm{mmol} \mathrm{dm}^{-3} ; \square, 0.25 \mathrm{mmol} \mathrm{dm}^{-3} ; \bullet, 0.13 \mathrm{mmol} \mathrm{dm}^{-3}$; 目, $0.063 \mathrm{mmol} \mathrm{dm}^{-3}$. The electrolyte was $0.5 \mathrm{~mol} \mathrm{dm}^{-3} \mathrm{KCl}, 0.1 \mathrm{~mol} \mathrm{dm}^{-3}$ Tris pH 7.0. 
of which depends on the concentration. This behaviour is indicative of some additional rate-limiting step at the electrode. Figure 13.15 also shows the corresponding Koutecky Levich plots. Now the slopes and intercepts of the straight lines obtained vary with the concentration of NADH. As expected the slopes are in agreement with the predicted slopes from the Levich equation (eqn 13,9). From the intercepts of the Koutecky Levich plots we can determine how the rate of the surface reaction varies with the concentration of NADH at the electrode surface. Using this technique Albery and Bartlett (1984) have shown that the oxidation of NADH at the NMP.TCNQ electrode proceeds through the initial adsorption of NADH at the electrode followed by oxidation to NAD*. A similar study has recently been published by Gorton et al. (1985) for the oxidation of NADH at a modified electrode.

The rotating-disc electrode has been used by Albery et al. (1981) to study the mechanism of the oxidation/reduction of horse heart cytochrome $c$ at a gold electrode modified by adsorption of $4-4^{\prime}$-bipyridyl. This reaction is similar to the NADH case described above in that electron transfer occurs to and from the adsorbed cytochrome $c$ (Chapter 15). The overall reaction involves a number of steps. These are mass transport of the protein to the electrode surface, adsorption at a suitable vacant site, electron transfer, desorption of the product, and finally mass transport of the product into the bulk of the solution. The rotating-disc electrode is well suited to the study of reactions of this type because it allows the mass transport steps to be readily controlled. Albery et al, have presented a full analysis of the rotation speed dependence for this mechanism. They find that for the cytochrome $c$ case the rate constants for the adsorption and desorption steps are identical within experimental error. Under these circumstances their full expression simplifies to a form of the Koutecky Levich equation. From an analysis of the rotation speed variation of the current they obtain values for the rate constants for the adsorption and desorption steps at the modified electrode.

Rotating-disc electrodes have also been used to study the transport of species through membranes (Gough and Leypoldt 1979, 1980a, $b$; Freese and Smart 1982). In these studies the membrane is attached to the front face of the rotating-disc electrode so that the species must diffuse through the membrane before reacting at the electrode surface. This situation is identical to the electrode reaction cases for polymer-modified electrodes discussed above (cases $E t_{y}$ and $E k_{E}^{\prime}$ of Fig. 13.3). Gough and Leypoldt (1979) have presented an analysis of the mass transport at such a membrane-covered electrode. Their resulting expression is identical to the Koutecky Levich equation. This is not surprising since the membrane simply acts as an additional, rotationspeed independent, barrier to transport to the electrode (under these conditions $k_{\mathrm{ME}}^{\prime}=K D_{y} / L$ ). This technique has been used to measure the diffusion coefficients of oxygen and hydroquinone in membranes (Gough and Leypoldt 1980b). Obviously it is restricted to electroactive species. 


\subsubsection{The rotating ring-disc electrode}

The addition of a concentric ring electrode to the rotating disc considerably extends the scope of the technique. The ring electrode is situated downstream from the disc and can thus be used to detect and quantify the products of the disc reaction. The ratio of the current on the ring electrode, $i_{R}$, to the current on the disc electrode, $i_{\mathrm{D}}$, for the detection of a stable product of the disc reaction is known as the collection efficiency, $N_{0}$. Thus if the reactions at the two electrodes are:

at the disc: $\mathrm{A}+n \mathrm{e} \longrightarrow \mathrm{B}$

at the ring: $\mathrm{B} \longrightarrow \mathrm{A}+n \mathrm{e}$

then $N_{0}=-i_{\mathrm{R}} / i_{\mathrm{D}}$

where (Albery and Hitchman 1971)

$$
\begin{aligned}
N_{0}= & 1-F(\alpha / \beta)+\beta^{\frac{2}{3}}\{1-F(\alpha)]-(1+\alpha+\beta)^{\frac{2}{7}} \\
& {[1-F[(\alpha / \beta)(1+\alpha+\beta)]], } \\
\mathrm{F}(\theta)= & \frac{3^{\frac{1}{2}}}{4 \pi} \ln \left\{\frac{\left(1+\theta^{\frac{1}{3}}\right)^{3}}{1+\theta}\right\}+\frac{3}{2 \pi} \cdot \arctan \left(\frac{2 \theta^{\frac{1}{3}}-1}{3^{\frac{1}{2}}}\right)+\frac{1}{4}, \\
\alpha= & \left(r_{2} / r_{1}\right)^{3}-1, \\
\beta= & \left(r_{3} / r_{1}\right)^{3}-\left(r_{2} / r_{1}\right)^{3},
\end{aligned}
$$
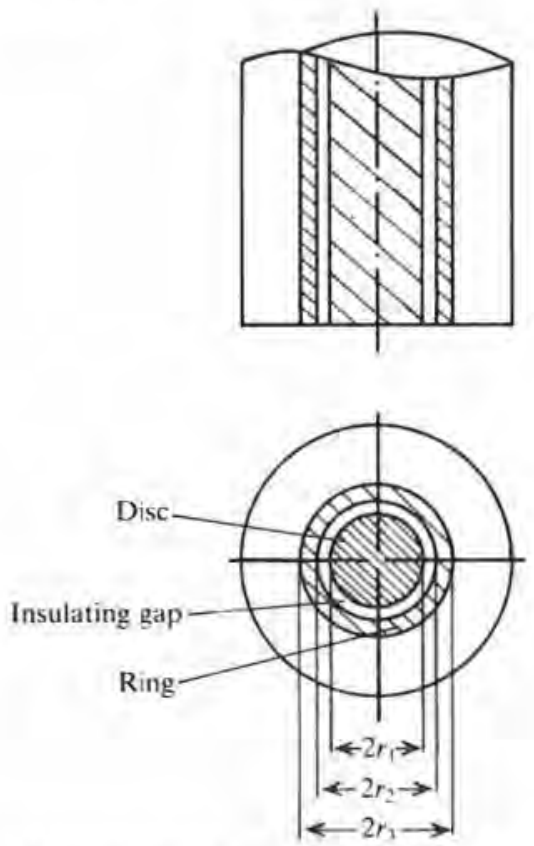

Fig. 13.16 A ring-disc electrode showing the three radii. 
and the radii $r_{1}, r_{2}$, and $r_{3}$ are shown in Fig. 13.16. It is significant that the collection efficiency, $N_{0}$, depends only upon the three radii and is independent of the rotation speed. Values of $N_{0}$ for common values of the radius ratios have been tabulated by Albery and Hitchman (1971).

The ring may also be used in the shielding mode. In this case the two electrode reactions are

at both ring and disc: $\mathrm{A}+n \mathrm{e} \longrightarrow \mathrm{B}$

Under these circumstances the ring current is reduced from its limiting value when $i_{\mathrm{D}}=0$ by a calculable fraction of the disc current (Albery and Hitchman 1971).

$$
i_{\mathrm{R}, \mathrm{L}}=i_{\mathrm{R}, \mathrm{L}}^{0}-N_{0} i_{\mathrm{D}}
$$

where $i_{R, L}$ is the observed limiting ring current and $i_{R, ~}{ }_{L}^{0}$ is the value when $i_{\mathrm{D}}=0$. These relations are easier to understand when the full polarograms are considered, Fig, 13,17. The effect of switching on the disc current from zero to $i_{\mathrm{D}}$ is to shift the ring polarogram (which is assumed to be for a reversible case in the figure) by an amount $N_{0} i_{\mathrm{D}}$.

The great advantage of the rotating ring-disc electrode is that the ring electrode can be used to work back from the observed ring current, through the known collection efficiency, to the flux on the disc electrode. This has been used to particular advantage in the study of the electrochemical polymerization of thionine (Albery et al. 1980) where only a fraction of the total disc current goes into the polymerization reaction. In this experiment the ring electrode was used to detect the amount of thionine consumed at the disc

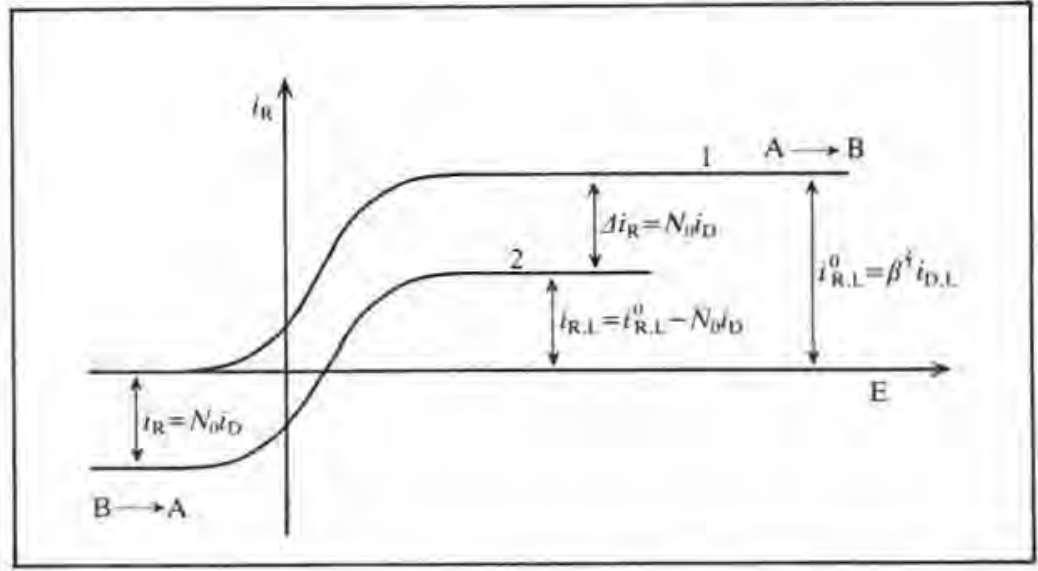

Fig. 13.17 Ring polarograms for a ting-disc electrode. Curve 1 shows the polarogram for $i_{\mathrm{D}}=0$. Curve 2 shows the case for $i_{\mathrm{D}} \neq 0$ when the reaction at the disc is $\mathrm{A}+n \mathrm{e} \rightarrow \mathrm{B}$. 
during polymerization. The measurements using the ring-disc electrode were shown to be in good agreement with the coverage calculated from cyclic voltammetry. Behret et al. (1981) have applied the technique to the study of oxygen reduction at electrodes modified by polymeric phthalocyanine coatings. In their experiments the ring electrode is used to determine the proportion of $\mathrm{H}_{2} \mathrm{O}_{2}$ produced in the oxygen reduction.

The technique has also been successfully applied to the study of immobilized enzyme systems. Kamin and Wilson (1980) have used a rotating ringdisc electrode consisting of a platinum ring electrode and a platinum, graphitic oxide or carbon-paste disc onto which glucose oxidase had been immobilized. With this type of electrode they were able to use the ring electrode to measure the fluxes of $\mathrm{H}_{2} \mathrm{O}_{2}$ produced at the disc by the reaction of glucose and oxygen with the immobilized enzyme. The system has the added advantage that the mass transport of reactants to the disc surface is also controlled and reproducible. In this way Kamin and Wilson were able to investigate the effect of immobilization on the enzyme kinetics.

In addition to its use in the steady state the ring-disc electrode can also be used in combination with transient or sinusoidal perturbation techniques for the study of modified electrodes. In the transient experiments the ring electrode is used to detect the transient in the flux of a product of the disc reaction produced by an imposed change in the disc current or potential. Albery et al. (1982) have presented a general method for working back from the observed transient ring response to the generating disc flux. This enables the components of the observed disc transient to be distinguished in those cases in which two or more processes contribute to the observed disc transient. This deconvolution procedure is trivial when the timescale of the observed ring transient is long compared to the time taken for species to cross the gap from the disc to the ring; this time is of the order of $X_{D}^{2} / D$. Under these conditions the steady-state relationship is a good approximation and

$$
i_{\mathrm{D}}(t) \simeq-i_{\mathrm{R}}(t) N_{0}^{-1} \text {. }
$$

When however the timescale of the transient is comparable with the characteristic time for crossing the gap it is necessary to use a more complex deconvolution procedure based on a set of trial functions and requiring computer fitting.

The use of sinusoidal modulation at the rotating-disc electrode can be used to study adsorption processes. In this method a low amplitude sinusoidal modulated is superimposed on the galvanostatted disc current and the corresponding in and out of phase components of the resulting modulated ring current are recorded as a function of frequency. The complex collection efficiency, $N_{\omega}$, is then given by

$$
N_{\omega}=-\tilde{i_{\mathrm{R}}} / \tilde{i_{\mathrm{D}}}=X+\mathrm{i} Y
$$


where $\tilde{i}_{\mathrm{R}}$ is the modulated component of the ring current and $\tilde{i}_{\mathrm{D}}$ is the modulated component of the disc current. $N_{\mathrm{\omega}}$ is made up of three contributions, a transport term $N_{\mathrm{tr}}$, a capacitative collection term $N_{\mathrm{C}}$, and the Faradaic term $N_{\mathrm{F}}$. Thus

$$
N_{\omega}=\left(N_{\mathrm{tr}} N_{\mathrm{F}}\right)+N_{\mathrm{C}} \text {. }
$$

In order to extract the Faradaic term it is necessary to correct the data for the effects of $N_{\mathrm{C}}$ and $N_{\mathrm{tr}}$. This is readily achieved since $N_{\mathrm{tr}}$ can be calculated; the theory for $N_{t r}$ has been verified using the ferri/ferrocyanide system at platinum/platinum ring-disc electrode (Albery et al. 1971, 1978). $N_{c}$ can be estimated by extrapolation of data obtained at high frequency where this term dominates. The Faradaic complex collection efficiency, $N_{F}$, can thus be extracted and compared with model predictions. One of the advantages of this technique is that the potential of the ring electrode can be chosen to monitor either the reactant or the product. This means that in adsorption studies the two can be measured independently. This approach has been applied to the study of thionine adsorption on platinum (Albery and Hillman 1979), the adsorption of methyl viologen on platinum (Albery et al. 1985a), and the study of the reaction of cytochrome $c$ at a modified gold electrode (Albery et al. 1981). The reaction of cytochrome $c$ is greatly facilitated at a gold electrode modified by the adsorption of $4,4^{\prime}$-bipyridyl over that at the clean electrode. Using the AC ring-disc technique in combination with rotating-disc studies the authors were able to study the adsorption of the cytochrome $c$ to the modified electrode and to calculate the overall free energy profile for the reaction.

\subsection{Conclusions}

In this chapter we have examined some of the potential applications of modified electrodes in the field of biosensors and in the wider biochemical and biomedical field. This is an area which shows tremendous promise and one that can be expected to grow as people explore the interactions between biological systems and electrodes. In order to make progress in the design and control of these interactions we must be able to design the surfaces of our electrodes. In turn this requires us to model the behaviour of our modified electrodes and to characterized their electrochemistry. I hope this chapter has shown some of the ways which we have available to do this and I hope it will encourage others to try their hand in this interesting area of chemistry.

\section{References}

Albery, W. J, and Bartlett, P, N. (1984), An organic conductor electrode for the oxidation of NADH. J. Chem. Soc. Chem. Commun. 234-6.

- and Hillman, A. R. (1979). Ring-disc electrodes. Part 19. Adsorption studies at 
low frequency A.C. J. Chem. Soc., Faraday Trans. 1, 75, 1623-34.

- (1981). Modified electrodes. Ann. Rep. Prog. Chem., Sect. C, 377-437.

-(1984). Transport and kinetics in modified electrodes. J. Electroanal. Chem. 170, 27-49.

- and Hitchman, M. L. (1971). Ring-disc electrodes. Clarendon Press, Oxford.

- Bartlett, P.N. and McMahon, A. J. (1985a). Transport and kinetics at micro-

heterogeneous electrodes. Part 5, the methyl viologen platinum system. J. Electroanal. Chem. 182, 7-23.

Boutelle, M. G. and Hillman, A. R. (1985b). The mechanism of Faradaic reactions at the thionine coated electrode. J. Electroanal. Chem. 182, 99-111.

- Compton, R. G, and Hillman, A. R. (1978). Ring-disc electrodes. Part 18. Collection efficiency at high frequency A.C. J. Chem. Soc., Faraday Trans. 1, 74, 1007-19.

— and Jones, C.C. (1984). A novel electrode for electrochemical ESR and its application to modified electrodes. J. Amer. Chem. Soc. 106, 469-73.

- Drury, J.S. and Hutchinson, A.P. (1971). Ring disc electrodes. Part 15. Alternating current measurements. Trans. Faraday Soc. 67, 2414-18.

Boutelle, M. G., Colby, P. J. and Hillman, A. R. (1982). The kinetics of electron transfer in the thionine coated electrode. J. Electroanal. Chem. 133, 135-45.

- Eddowes, M. J., Hill, H. A. O. and Hillman, A. R. (1981). Mechanism of the reduction and oxidation reaction of cytochrome $c$ at a modified gold electrode. J. Amer. Chem. Soc. 103, 3904-10.

— Foulds. A. W., Hall, K. J. and Hillman, A. R. (1980). Thionine coated electrode for photogalvanic cells. J. Electrochem. Soc. 127, 654-61.

Andrieux, C.P., Dumas-Bouchiat, J.M. and Saveant, J. M. (1982). Catalysis of electrochemical reactions at redox polymer electrodes. Kinetic model for stationary voltammetric techniques. J. Electroanal. Chem. 131, 1-35.

Anson, F. C. (1980). Kinetic behaviour expected from outer sphere redox catalysts confined within polymeric films on electrode surfaces. J. Phys, Chem. 84, 3336-8.

- Oshaka, T. and Saveant, J.-M. (1983a). Diffusional pathways for multiplycharged ions incorporated in polyelectrolyte coatings on graphite electrodes. Cobalt oxalate in coatings of protonated polylysine. J. Phys. Chem. 87, 640-647.

(1983b). Kinetics of electron transfer cross-reactions within redox polymers. Coatings of a protonated polylysine copolymer with incorporated electroactive anions. J. Amer. Chem. Soc. 105, 4883-90.

Aoki, K., Tokuda, K. and Matsuda, H. (1983). Theory of linear sweep voltammetry with finite diffusion space. J. Electroanal. Chem. 146, 417-29.

Bard, A. J. and Faulkner, L. R. (1980). Electrochemical methods. Wiley, New York.

Behret, H., Binder, H., Sandstede, G. and Scherer, G. G. (1981). On the mechanism of electrocatalytic oxygen reduction at metal chelates. Part III: metal phthalocyanines. J. Electroanal. Chem, 117, 29-42.

Bianco, P. and Haladjian, T. (1982). Electrochemical investigation of cytochrome $c_{3}$ from Desulfovibrio desulfurilars Norway at solid electrodes. $J$. Electroanal. Chem. 137, 367-76.

Bidan, G., Deronzier, A. and Moutet, J.-C. (1984). Electrochemical coating of an electrode by a poly(pyrrole) film containing viologen. J. C. S. Chem. Commun. 1185-6.

Birke, R. L. (1978). Current-potential-time relationships in differential pulse polarography: theory of reversible, quasi-reversible, and irreversible electrode processes. Anal. Chem. 50, 1489-96. 
Bolts, J.M. and Wrighton, M.S. (1979). Chemically derivatised n-type semiconducting gallium arsenide photoelectrodes. Thermodynamically uphill oxidation of surface-attached ferrocene centres. J. Amer. Chem. Soc. 101, 6179-84.

Brown, A. P. and Anson, F.C. (1977). Cyclic and differential pulse voltammetric behaviour of reactants confined to the electrode surface. Anal. Chem. 49, 1589-95.

Bruckenstein, S., Sharkey, J.W. and Yip, J.Y. (1985). Effect of polishing with different size abrasives on the current response at a rotating disc electrode. Anal. Chem. 51, 368-71.

Bull, R. A., Fan, F.-R. and Bard, A. J. (1983). Polymer films on electrodes. 13. Incorporation of catalysts into electrochemically conductive polymers - iron phthalocyanine in polypyrrole. J. Electrochem. Soc. 130, 1636-8.

Burgmayer, P. and Murray, R. W. (1982). An ion gate membrane: electrochemical control of ion permeability through a membrane with an embedded electrode. J. Amer, Chem. Soc, 104, 6139-40.

- (1984), Ion gate electrodes. Polypyrrole as a switchable ion conductor membrane. J. Phys. Chem. 88, 2515-21.

Cochran, W. G. (1934), The flow due to a rotating disc, Proc. Camb. Phil. Soc. Math. Phys. Sci. 30, 365-75.

Daum, P., Lehnard, J. R., Rolison, D. R. and Murray, R. W. (1980). Diffusion charge transport through ultrathin films of radiofrequency plasma polymerised vinylferrocene at low temperature. J. Amer. Chem. Soc, 102, 4649-53.

Degrand, C, and Miller, L.L. (1980). An electrode modified with polymerbound dopamine which catalyses NADH oxidation. J. Amer. Chem. Soc. 102, 5728-32.

Diaz, A. F. and Kanazawa, K. K. (1979). Electrochemical polymerisation of pyrrole. J. C. S. Chem. Commun, 635-6.

Elving, P. J., Schmakel, C. O, and Santhanam, K. S. V. (1976). Nicotinamide-NAD sequence: redox processes and related behaviour: behaviour and properties of intermediates and final products. CRC Crit, Rev. Anal. Chem, 6, 1-67.

Faulkner, L. R. (1984). Chemical microstructures on electrodes. Chem. and Eng. News, Feb. 27th, 28-45.

Freese, J. W. and Smart, R. B. (1982). Rotating voltammetric membrane electrode. Anal. Chem. 54, 836-7.

Ghosh, P. K. and Spiro, T. G. (1981). Electroactive coatings of tris(bipy)- and tris(o-phen) ruthenium (II) attached to electrodes via hydrosilylation and electropolymerisation of vinyl derivatives. J, Electrochem. Soc. 128, 1281-7.

Gorton, L., Torstensson, A. Jaegfeldt, H. and Johansson, G. (1984). Electrocatalytic oxidation of reduced nicotinamide coenzymes by graphite electrodes modified with an adsorbed phenoxazinium salt, Meldola blue. J. Electroanal. Chem. 161, 103-20,

- Johansson, G. and Totstensson, A. (1985). J. Electroanat. Chem, 196, 81-92.

Gough, D. A. and Leypoldt, J. K. (1979). Membrane covered rotated disc electrode. Anal. Chem. 51, 439-44.

- $(1980 a)$. Transient studies of glucose oxygen and hydroquinone at a membrane covered rotated disc electrode. J. Electrochem. Soc, 127, 1278-86.

- (1980b). Rotated membrane-coated oxygen electrode. Anal. Chem. 52, 1126-30.

Haas, O. and Zumbrunnen, H.-R. (1981). Electrochemical properties of hydroxyphenazine coated electrodes. Helv, Chim. Acta 64, 854-63. 
Heinze, J. (1984), Cyclic voltammetry - electrochemical spectroscopy. Angew. Chem., Int. Edn. Engl. 23, 831-918.

Ianniello, R. M., Lindsay, T.J. and Yacynych, A. M. (1982a). Direct electron transfer in immobilised flavoenzyme chemically modified graphite electrodes. Anal. Chim. Acta 141, 23-32.

$(1982 b)$. Differential pulse voltammetric study of direct electron transfer in glucose oxidase chemically modified graphite electrodes. Anal. Chem. 54, 1098-101.

Ikeda, T., Ando, S. and Senda, M. (1981). Electrochemical oxidation-reduction properties of covalently bound FAD of cholesterol oxidase adsorbed on a mercury electrode surface. Bull. Chem. Soc. Jpn. 54, 2189-93.

Toriyama, K. and Senda, M. (1979). Electrochemical behaviour of ferredoxins adsorbed on mercury electrode surface. Cyclic D.C. and A.C voltammetric studies with HMDE. Bull. Chem. Soc. Jpn. 52, 1937-43.

Jaegfeldt, H., Torstensson, A., Gorton, L. and Johansson, G. (1981). Catalytic oxidation of reduced nicotinamide adenine dinucleotide by graphite electrodes modified with adsorbed aromatics containing catechol functionalities. Anal. Chem, 53. 1979-82.

Kakutani, T. and Senda, M. (1979). Theory of A.C. polarisation and A.C. polarography and voltammetry of surface redox reaction. Bull. Chem. Soc. Jpn. 52, $3236-41$.

- Kano, K., Ando, S. and Senda, M. (1981). Electrochemical oxidation and reduction of FMN adsorbed on a mercury electrode surface. Bull. Chem. Soc. Jpn. 884-90.

- Toriyama, K., Ikeda, T. and Senda, M. (1980). Electrochemical oxidation and reduction of ferredoxin adsorbed on a mercury electrode surface. Phase-selective A.C. polarography at DME. Bull. Chem. Soc. Jpn. 53, 947-50.

Kamin, R.A. and Wilson, G. S. (1980). Rotating ring-disc enzyme electrode for biocatalysis kinetic studies and characterisation of the immobilised enzyme layer. Anal. Chem. 52, 1198-205.

Kitani, A., So, Y,-H, and Miller, L. L. (1981). An electrochemical study of the kinetics of NADH being oxidised by diimines derived from diaminobenzenes and diaminopyrimidines. J. Amer. Chem. Soc. 103, 7636-41.

Koutecky, J. and Levich, V. G. (1958). The use of the rotating disc electrode in the study of electrochemical kinetics and electrolytic processes. Zh. Fiz. Khim. 32, $1565-75$.

Lane, R.F. and Hubbard, A.T. (1973a). Electrochemistry of chemisorbed molecules. I. Reactants connected to electrodes through olefinic substituents. J. Phys. Chem. 77, 1401-10.

- $(1973 b)$. Electrochemistry of chemisorbed molecules. II, The influence of charged chemisorbed molecules on the electrode reactions of platinum complexes. J. Phys. Chem. 77, 1411-21.

Lau, A. N, and Miller, L. L. (1983). Electrochemical behaviour of a dopamine polymer. Dopamine release as a primary analog of a synapse. J. Amer. Chem. Soc. $105,5271-7$.

— and Zinger, B. (1983). Release of neurotransmitters glutamate and $\gamma$-aminobutyric acid from an electrode. Catalysis of slow redox propagation through a 
polymer film. J. Amer. Chem. Soc. 105, 5278-84.

Laviron, E. (1979). Use of linear potential sweep voltammetery and of A.C. voltammetery for the study of the surface electrochemical reaction of strongly adsorbed systems and of redox modified electrodes. J. Electroanal. Chem, 100, 263-70.

- (1980). A multilayer model for the study of space distributed redox modified electrodes. Part 1. Description and discussion of the model. J. Electroanal. Chem. 112, 1-9.

(1981). A multilayer model for the study of space distributed redox modified electrodes. Part III: influence of interactions between the electroactive centres in the first layer on the linear potential sweep voltammograms, J. Electroanal. Chem. $122,37-44$.

- and Roullier, L. (1980). General expression of the linear potential sweep voltammogram for a surface redox reaction with interactions between adsorbed molecules - applications to modified electrodes. J. Electroanal. Chem. 115, 65-74.

— and Degrand, C. (1980). A multilayer model for the study of space distributed redox modified electrodes. Part II. Theory and application of linear potential sweep voltammetry for a simple reaction. J. Electroanal. Chem. 112, 11-23.

Lin, A. W. C., Yeh, P., Yacynych, A. M. and Kuwana, T. (1977). Cyanuric chloride as a general linking agent for attachment of redox groups to pyrolytic graphite and metal oxide electrodes. J. Electroanal. Chem. 84, 411-19.

Moses, P. R., Weir, L. and Murray, R. W. (1975). Chemically modified tin oxide electrodes. Anal. Chem. 47, 1882-6.

Murray, R.W. (1984). Chemically modified electrodes. In Electroanalytical chemistry (ed. A. J. Bard), Vol. 13, pp. 191-38. Marcel Dekker, New York.

Oglesby, D, M., Omang, S. H. and Reilley, C. N. (1965). Thin layer electrochemical studies using controlled potential or controlled current. Anal. Chem. 37, 1312-6.

Osteryoung, J. (1981). Pulse polarography, In Water quality measurement (eds. H. B. Mark and J.S, Mattson), pp. 85-190. Marcel Dekker, New York.

Oyama, N. and Anson, F. C. (1980a). Factors affecting the electrochemical response of metal complexes at pyrolytic graphite electrodes coated with films of poly(4-vinylpyridine). J. Electrochem. Soc. 127, 640-7.

- $(1980 b)$. Catalysis of electrode processes by multiply-charged metal complexes electrostatically bound to polyelectrolyte coatings on graphite electrodes, and the use of polymer coated rotating disc electrodes in diagnosing kinetic and conduction mechanisms. Anal. Chem. 52, 1192-8.

- Oshaka, T. and Ushirogouchi, T. (1984). Charge-transfer reactions of metal complexes at electrode/film interfaces and in films. J. Phys. Chem. 88, 5274-80.

- Kaneko, M., Sato, K. and Matsuda, H. (1983). Electrode kinetics of $\mathrm{Fe}(\mathrm{CN})_{6}^{3-14-}$ and $\mathrm{Fe}(\mathrm{CN})_{5}^{2-/ 3-}$ complexes confined to polymer films on graphite surfaces. J. Amer. Chem. Soc. 105, 6003-8.

Peerce, P, J, and Bard, A. J. (1980). Polymer films on electrodes, Part II: film structure and mechanism of electron transfer with electrodeposited poly(vinylferrocene). J. Electroanal. Chem. 112, 97-115.

Robinson, J. (1984). Spectroelectrochemistry. In Specialist periodical reports, electrochemistry, Vol. 9, pp. I01-61. Royal Society of Chemistry, London.

Rocklin, R. D. and Murray, R. W. (1981), Kinetics of electrocatalysis of dibromoalkyl reductions using electrodes with covalently immobilised metallo-tetraphenyl- 
porphyrins. J. Phys. Chem. 85, 2104-12.

Thackeray, J.W., White, H.S. and Wrighton, M.S. (1985). Poly(3-methylthiophene)-coated electrodes: optical and electrical properties as a function of redox potential and amplification of electrical and chemical signals using poly(3-methylthiophene)-based microelectronic transistors. J. Phys. Chem. 89, 5133-49.

Torstensson, A. and Gorton, L. (1981). Catalytic oxidation of NADH by surfacemodified graphite electrodes. J. Electroanal. Chem. 130, 199-207.

Tse, D.C.S. and Kuwana, T. (1978). Electrocatalysis of dihydronicotinamide adenosine diphosphate with quinones and modified quinone electrodes. Anal. Chem. 50, 1315-18.

von Karman, T. (1921). Ober laminare und turbulente Reibung. Z. Angew. Math. Mech. 1, 233-52.

Zinger, B, and Miller, L. L. (1984). Timed release of chemicals from polypyrrole films, J. Amer, Chem. Soc. 106, 6861-3. 


\title{
14 \\ Cyclic voltammetry studies of enzymatic reactions for developing mediated biosensors
}

\author{
GRAHAM DAVIS
}

\subsection{Introduction}

Many amperometric biosensors use an oxidase to catalyse a substrate oxidation reaction (Carr and Bowers 1980). To date, little progress has been made towards inducing the resulting reduced enzyme-cofactor complex to undergo rapid direct electrochemical re-oxidation, consequently electron acceptors have been used to shuttle electrons from the catalytic site to the electrode. Conventionally, oxygen has been chosen since it is the natural electron acceptor for many of these enzymes and is usually available in the analyte (Chapters 1 and 18).

More recent research has attempted to replace oxygen by using nonphysiological electron acceptors (or mediators) immobilized on the electrode surface or within the enzyme layer (Chapters 15 and 16). This approach has been encouraged by studies on coating electrodes with redox species using methods including adsorption, polymer coating, and covalent attachment (Bard 1983; Murray 1980; Bartlett, this volume). Adaptation to amperometric biosensor construction may offer some advantages; for example, by using a mediator with a low redox potential a lower operating potential than that required for hydrogen peroxide detection is possible (Cass et al. 1984). This can reduce interference from electro-active species generally encountered in biological samples. Increased operational stability may also be achieved by having a fixed concentration of electron acceptor retained within the enzyme layer, thus obviating a problem of oxygen-dependent biosensors where variation in the oxygen tension can alter the response characteristics. Amperometric biosensors incorporating immobilized mediators therefore provide an interesting alternative to peroxide-detecting systems (Romette and Boitieux 1984; Shichiri et al. 1982, 1984).

To help select a suitable mediator for an amperometric biosensor the electrochemical technique of direct current cyclic voltammetry is useful and enables many important properties of the mediator to be determined. Generally, it is desirable to use a low-potential mediator with a high electrochemical rate constant, the latter is important to ensure that the response of the biosensor is not limited by electrode kinetics. Both parameters can be 
determined by single-scan cyclic voltammetry. Stability of a mediator as a function of $\mathrm{pH}$, temperature, oxygen tension, enzyme inhibitors, and interferents can also be assessed from changes in the shape of voltammograms with time. Most importantly, the technique can provide qualitative and quantitative information about electrochemically coupled enzymatic reactions, upon which mediated amperometric biosensors are based.

\subsection{Direct current cyclic voltammetry}

Direct current cyclic voltammetry is based upon the maintenance of the potential of a working electrode with respect to a reference electrode by making a current pass between the working and counter electrode (Bard and Faulkner 1980). This requires a potentiostat with a triangular waveform generator and an $X-Y$ recorder on which the current-potential curves are recorded. Experiments are usually performed with a cell containing a 2-4 mm diameter working electrode (made of gold, platinum, or carbon), a platinum-gauze counter electrode, and a saturated calomel electrode (SCE) as reference (Davis et al. 1983). As the rate of enzymatic reactions are temperature dependent, experiments are best performed under thermostatic control.

Cyclic voltammetry consists of sweeping the potential of a stationary working electrode at a constant rate in an unstirred solution, between two set limits, with the current being recorded as a function of potential, Fig. 14.1. A single scan can be performed or the electrode can be cycled continuously.

The measured current has two components, a non-Faradaic component resulting from redistribution of charged and polar species at the electrode surface and a Faradaic component resulting from exchange of electrons between the electrode and species in solution. When the rate of electron transfer (at sufficiently oxidizing or reducing potentials) is fast, the Faradaic current is controlled by the rate of diffusion to the electrode. Hence, for the reversible reduction of the redox species $\mathrm{O}$,

$$
\mathrm{O}+n \mathrm{e}^{-} \rightleftharpoons \mathrm{R}
$$

the surface concentration of the two redox forms will change in accordance with the Nernst equation,

$$
[\mathrm{O}] /[\mathrm{R}]=\exp \left[n F / R T\left(E-E^{\circ}\right)\right]
$$

and the Faradaic current will depend on the concentration gradient of $\mathrm{O}$ at the electrode surface.

$$
i_{\mathrm{f}}=n F A D_{0}(\mathrm{~d}[\mathrm{O}] / \mathrm{d} x)_{x=0} .
$$

A cyclic voltammogram is recorded by holding the working electrode at a positive potential and then sweeping towards and beyond the $E^{0}$ of a redox 


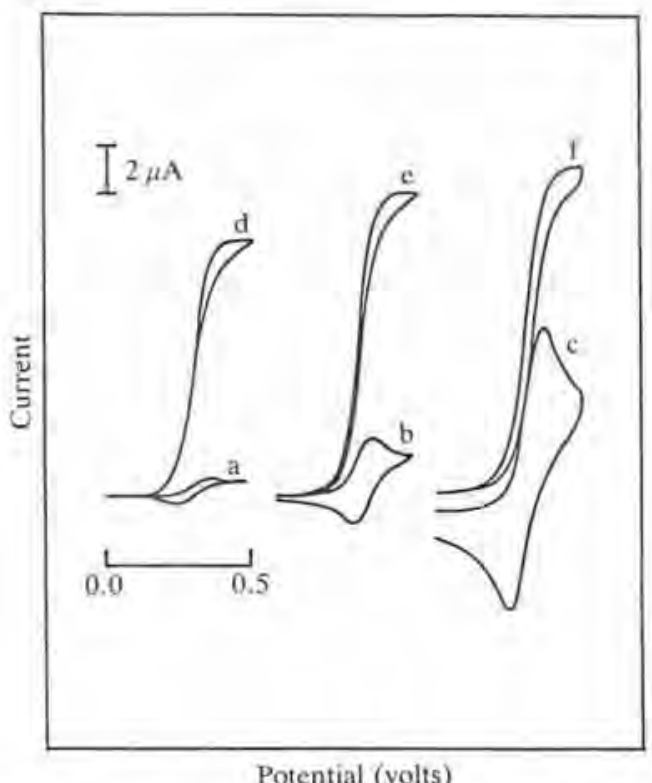

Fig. 14.1 (a-c) DC Cyclic voltammograms at a $4 \mathrm{~mm}$-diameter gold working electrode of ferrocene monocarboxylic acid $(200 \mu \mathrm{M})$ in $50 \mathrm{~mm}$ phosphate perchlorate electrolyte $\mathrm{pH} 7.0$, versus $\mathrm{SCE}$ at sweep rates of 1,10 , and $100 \mathrm{mV} \mathrm{s}^{-1}$, respectively. (d-f) With the addition of $11 \mu \mathrm{M}$ glucose oxidase and $50 \mathrm{~mm}$ glucose.

species. As reduction occurs, the concentration of $\mathrm{O}$ is depleted in the electrolyte close to the electrode, consequently the current is not maintained but peaks and then decays. When the direction of the potential scan is reversed, a peak resulting from the re-oxidation of $\mathrm{R}$ is observed. A redox couple that follows eqn. 14.2, (termed Nernstian or reversible) is illustrated in Fig. 14.1 (a-c). This shows a series of voltammograms of the ferroceneferricinium ion redox couple of ferrocene monocarboxylic acid, eqn 14.4, recorded at different potential sweep rates, $v$.
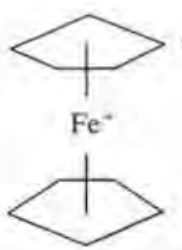

\section{$\mathrm{COOH}$}

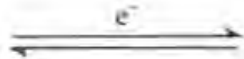

For a reversible reaction of this type, the maximum cathodic current is given by

$$
i_{\mathrm{p}}=0.4463 n F A\left(D_{0} n F \nu / R T\right)^{1 / 2} C_{0}
$$


and occurs at a potential $28.5 / n \mathrm{mV}$ cathodic of $E^{0}$ at $298 \mathrm{~K}$, independent of the potential scan rate.

Experimentally, $E^{0}$ can be estimated from the mid-point potential, $E_{1 / 2}$, of the maximum anodic and cathodic currents. If the mediator is chemically unstable and is converted to a form with different redox properties, the magnitude of the current in the initial voltammogram will decrease with time.

\subsection{Electrochemically coupled enzymatic reactions}

For a rapid reaction between the reduced enzyme-cofactor complex, Z, of an oxidoreductase and an electron acceptor both the stoichiometry and the relative redox potentials must be favourable to electron transfer (Kuwana et al. 1977). In addition, the active site of the enzyme must be 'accessible' to the acceptor molecule.

The reaction can be simplified to the following form

$$
\mathrm{Z}+\mathrm{O} \stackrel{k}{\longrightarrow} \mathrm{R} ; \mathrm{R}-\mathrm{ne}^{-} \rightleftharpoons \mathrm{O}
$$

in which component $Z$, serves to convert $O$ back to $R$ at potentials where $O$ is generated at the electrode. Qualitatively, the effect on voltammograms is to increase the anodic current and decrease the cathodic current. The theory developed by Nicholson and Shain (1964), shows that two limiting cases are possible. When the pseudo-first-order rate constant $k_{\mathrm{f}}$ is small, voltammograms will approximate those of a simple reversible electron-transfer reaction, whereas if $k_{\mathrm{f}}$ is large the current will be directly proportional to $k_{\mathrm{f}}^{1 / 2}$ and independent of the potential scan rate:

$$
i=n F A\left(D_{0} k_{f}\right)^{1 / 2} C_{0} / 1+\exp \left[n F / R T\left(E-E_{1 / 2}\right)\right] .
$$

In the latter case a limiting current rather than a peak is observed. An intermediate example is illustrated by Fig. 14.1 (d-f), which shows yoltammograms of a redox mediator in the presence of the enzyme glucose oxidase and its substrate. To confirm that a catalytically coupled reaction of the type shown in eqn 14.6, is occurring, it is useful to plot the current function, eqn 14.5 , versus the potential sweep rate. This allows the effect of sweep rate on the diffusional process to be separated from its effect on the kinetics. Fig. 14.2, curve A shows that a reversible reaction gives a horizontal straight line, as $i_{\mathrm{p}} / v^{1 / 2}$ is constant. However, for a catalytic reaction the current function only approaches curve A when the potential scan rate is so fast that the reaction does not proceed significantly before the experiment is complete, curve B. In practice, this correlation can be obtained simply by plotting $i_{\mathrm{p}} / v^{1 / 2}$, which approximates the current function, versus $\log v$. This qualitative aspect of the technique is the most important for a biosensor application, 


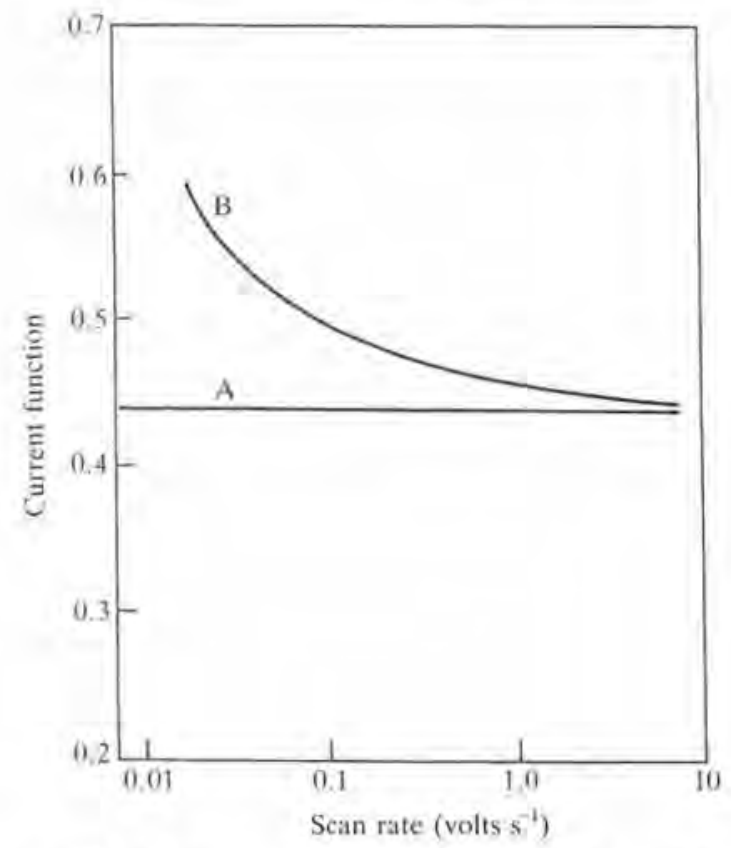

Fig. 14.2 Variation of the peak current function with voltage sweep rate: for a diffusion-controlled electrochemical reaction (curve A) and for a catalytically coupled reaction of the type shown in eqn 14.6 (curve B).

as it is usually sufficient to indicate the possibility of detecting an analyte based upon the catalytic reaction under investigation.

To derive kinetic information for the reaction, the mediator must have reversible electrochemistry and the enzyme must be saturated with substrate, $[\mathrm{S}] \gg K_{\mathrm{M}}$ (Davis et al. 1983). One of the methods is to use a working curve, Fig. 14.3, in which the ratio of the kinetic to diffusion-controlled current, $i_{\mathrm{k}} / i_{\mathrm{d}}$, measured from voltammograms like those shown in Fig. 14.1, is plotted as a function of the kinetic parameter $\left(k_{\mathrm{f}} / a\right)^{1 / 2}$, where $a=n F v / R T$ (Nicholson and Shain 1964). By measuring $i_{\mathrm{k}} / i_{\mathrm{d}}$ at different sweep rates, a set of values of $k_{\mathrm{f}} / a$ can be obtained at a fixed concentration of the enzyme. The effect of sweep rate can then be eliminate by plotting $k_{\mathrm{f}} / a$ versus $1 / v$. Under pseudo-first-order conditions, this bisects the origin with a gradient $k_{\mathrm{f}} R T / n F$, from which the scan-rate independent pseudo-first-order rate constant is calculated. The second-order rate constant, $k_{\mathrm{s}}=k_{\mathrm{r}} /[\mathrm{Z}]$, for the homogeneous reaction between the oxidized mediator and the reduced cofactor-enzyme complex, eqn 14.6 , is estimated by repeating the experiment at different enzyme concentrations.

It is important to note that this analysis assumes the diffusion coefficients of $\mathrm{O}$ and $\mathrm{Z}$ are equal. This is clearly not true for reactions involving small 


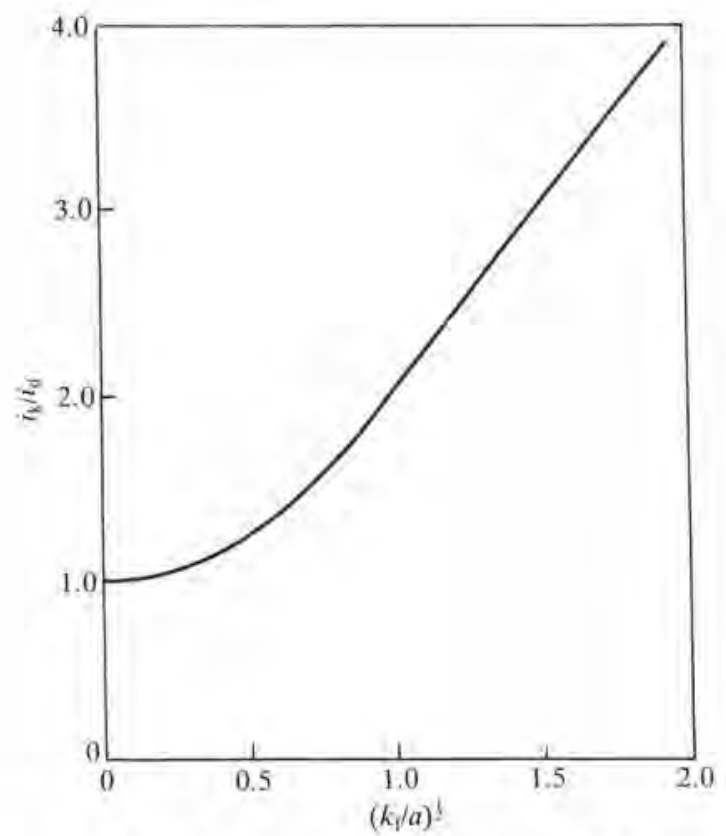

Fig. 14.3 Theoretical plot of the ratio of the kinetic to diffusion-controlled peak current, $i_{\mathrm{k}} / i_{\mathrm{d}}$, versus the kinetic parameter $\left(k_{\mathrm{f}} / a\right)^{1 / 2}$.

molecule mediators and proteins. Ryan and Wilson (1975) have shown that under these conditions, if the reaction is very fast $>10^{6} 1 \mathrm{~mol}^{-1} \mathrm{~s}^{-1}$, this can lead to an underestimation of the rate constant. Nevertheless, the method remains a useful alternative to stopped-flow kinetic techniques for studying these reaction (Weibel et al. 1969; Morton et al. 1970).

A wide range of oxidoreductases have been studied using cyclic voltammetry with the ferricinium ion of ferrocene monocarboxylic acid, eqn 14.4, as the mediator, Table 14.1. Apart from the oxygen-specific enzymes cholesterol oxidase, oxalate oxidase, and choline oxidase for which no catalytic reaction was observed (Davis 1984), a rate constant for the other oxidoreductases was estimated (Aston et al. 1984b; Cass et al. 1984, 1985a, b; Dicks et al. 1986; D'Costa et al. 1986).

The rates obtained for non-oxygen specific flavoenzymes reacting with ferrocene cover the same range $10^{4}-10^{6} 1 \mathrm{~mol}^{-1} \mathrm{~s}^{-1}$, as those for oxygen (Bright and Porter 1975; Gibson and Hastings 1962). The first authors concluded that catalytic activity of these enzymes is associated with substrate oxidation rather than subsequent re-oxidation of the flavin moiety. Comparing these data, it appears that there is no inherent disadvantage in using a non-physiological acceptor with non-oxygen specific flavoenzymes. However, for a 
Table 14.1 Electrochemically determined rate constants for the reaction of the reduced form of various enzymes with the ferricinium ion of ferrocene monocarboxylic acid at $\mathrm{pH} 7.0$ and $298 \mathrm{~K}$

\begin{tabular}{lll}
\hline Enzyme & Substrate & $k_{\mathrm{s}} \times 10^{-5} 1 \mathrm{~mol}^{-1} \mathrm{~s}^{-1}$ \\
\hline Glucose dehydrogenase & Glucose & 93.0 \\
Flavocytochrome $b_{2}$ & Lactate & 67.0 \\
Galactose oxidase & Galactose & $8.5^{a}$ \\
Xanthine oxidase & Xanthine & 4.0 \\
CO oxidoreductase & Carbon monoxide & 4.0 \\
Glutathione reductase & NADPH & 2.0 \\
Glucose oxidase & Glucose & 2.0 \\
Glycollate oxidase & Glycollate & $1.2^{b}$ \\
Alcohol dehydrogenase & Methanol & $0.6^{c}$ \\
L-Amino acid oxidase & Leucine & $0.4^{d}$ \\
Pyruvate oxidase & Pyruvate & 0.2 \\
Lipoamide dehydrogenase & NADH & 0.2 \\
Sarcosine oxidase & Sarcosine & 0.1 \\
Cholesterol oxidase & Cholesterol & - \\
Oxalate oxidase & Oxalate & $-{ }^{e}$ \\
Choline oxidase & Choline & - \\
\hline
\end{tabular}

${ }^{a} \mathrm{pH} 9.0 ;{ }^{b} \mathrm{pH} 8.3 ;{ }^{c} \mathrm{pH} 10.5 ;{ }^{d} \mathrm{pH} 7.8$ and $310 \mathrm{~K} ;{ }^{e} \mathrm{pH} 3.0$

practical biosensor based on this type of reaction, it would be important to minimize cross reactivity with oxygen.

To date, the best-studied enzyme is glucose oxidase for which rate constants were determined as a function of temperature and $\mathrm{pH}$ (Cass et al. 1984) with a range of ferrocene derivatives, Table 14.2. Whilst the reaction is thermodynamically favourable for all derivatives it is interesting to note that

Table 14.2 Electrochemically determined rate constants for the reaction of reduced glucose oxidase with the ferricinium ion of a range of ferrocene derivatives at $\mathrm{pH} 7.0$ and $298 \mathrm{~K}$

\begin{tabular}{lll}
\hline Ferrocene derivative & $E_{1 / 2} \mathrm{mV} v s . \mathrm{SCE}$ & $k_{\mathrm{s}} \times 10^{-5} 1 \mathrm{~mol}^{-1} \mathrm{~s}^{-1}$ \\
\hline 1,1'-Dimethyl & 100 & 0.8 \\
Ferrocene & 165 & 0.3 \\
Vinyl & 250 & 0.3 \\
Monocarboxylic acid & 275 & 2.0 \\
1,1'-Dicarboxylic acid & 285 & 0.3 \\
Methyl trimethylamino & 400 & 5.3 \\
Polyvinyl & 450 & - \\
\hline
\end{tabular}


a polyvinylferrocene-coated electrode, prepared according to the method of Merz and Bard (1978), did not give an observable catalytic reaction with glucose oxidase (Davis 1984). This may result from an inability of polymerbound ferrocene, unlike the monomer, to approach the active site of the enzyme and facilitate rapid electron transfer.

In addition to studying reactions between oxidoreductases and nonphysiologically related redox species, cyclic voltammetry has been used to investigate protein-protein redox reactions in vitro. The reversible electrochemistry of the redox protein, horse heart cytochrome $c$ at a bipyridylmodified gold electrode (Eddowes et al. 1981) enabled Hill and Walton (1982) to show that cytochrome oxidase can be supplied with electrons for the reduction of oxygen to water. The rate of reaction is enhanced by the presence of a second mediating redox protein, either azurin or cytochrome $c_{551}$, Table 14.3. Here, data are in good agreement with stopped-flow analysis (Morton et al, 1970). The reaction of cytochrome $c$ with carbon monoxide oxidoreductase (Aston et al. 1984b) and the lactate dehydrogenase flavocytochrome $b_{2}$ (Cass et al. 1985a) has also been studied, Table 14.3.

Cyclic voltammetry has been applied qualitatively to the study of coupled reactions involving more than one enzyme. For example, oxidation of lactate by the NAD-linked lactate dehydrogenase can be coupled electrochemically via the lipoamide dehydrogenase-ferrocene system, Table 14.1, and isocitrate can be detected via the NADP-linked isocitrate dehydrogenase coupled to the glutathione reductase-ferrocene system, Table 14.1 (Cass et al. 1985b).

\subsection{Amperometric biosensors}

All of the electrochemically coupled enzymatic reactions discussed previously form the basis for the development of mediated amperometric biosensors. In addition, work on the electron transfer protein, cytochrome $c$, demonstrates that mediators need not be limited to synthetic redox compounds.

In practical terms, Cass et al. (1984) have demonstrated the value of directcurrent cyclic voltammetry in choosing a suitable mediator for incorporation

Table 14.3 Electrochemically determined rate constants for the reaction with horse heart cytochrome $c$ at $\mathrm{pH} 7.0$ and $293 \mathrm{~K}$

\begin{tabular}{lc}
\hline Biological redox species & $k_{\mathrm{s}} \times 10^{-5} 1 \mathrm{~mol}^{-1} \mathrm{~s}^{-1}$ \\
\hline Azurin & 0.1 \\
Cytochrome $c_{551}$ & 0.2 \\
CO oxidoreductase & 0.3 \\
Flavocytochrome $b_{2}$ & 50.0 \\
\hline
\end{tabular}


into an amperometric biosensor. From the data presented in Table 14.2, 1,1 -dimethylferrocene was selected for a glucose sensor operating in whole blood. In this configuration, a sufficient excess of ferrocene was incorporated to minimize cross reactivity of glucose oxidase with oxygen. For this application it was important that the mediator had a low solubility in aqueous solution thus confining it to the porous carbon-base sensor and a low redox potential to facilitate an operating potential at which uric acid and other electroactive components of whole blood do not cause interference. Ferrocenes are particularly adaptable in this respect as their solubility and redox potential can be controlled by attaching different substituent groups to the cyclopentadienyl rings (Kuwana et al. 1977; Deeming 1982).

In addition to assaying blood samples from diabetic subjects, the glucose biosensor could be used to measure the rate of a solution reaction, for example a coupled assay for creatine kinase activity was demonstrated (Green et al. 1984). Other mediated amperometric biosensors which use ferrocene have been developed for alcohols (Aston et al. 1984a), carbon monoxide (Aston et al. 1984b) glycollate, L-amino acids, and galactose (Dicks et al, 1986) based upon the reactions shown in Table 14.1.

\section{References}

Aston, W. J., Ashby, R. E., Scott, L. D. L. and Turner, A.P. F. (1984a). Enzyme based methanol sensor. In Change and field effects in biosystems (eds. M. J. Allen and P.N.R. Usherwood), pp. 491-8. Abacus Press, Tunbridge.

- Bell, J., Colby, J., Davis, G., Higgins, I. J., Hill, H, A. O. and Turner, A. P. F. $(1984 b)$, CO: Acceptor oxidoreductase from Pseudomonas thermocarboxydovorans strain $\mathrm{C} 2$ and its use in a carbon monoxide sensor. Anal. Chim. Acta $163,161-74$.

Bard, A. J. (1983). Chemical modification of electrodes. J. Chem. Ed, 60, 302-4.

- and Faulkner, L. R. (1980). Electrochemical methods: fundamentals and applications. John Wiley, New York.

Bright, W, H. and Porter, D. J. T. (1975). Flavoprotein oxidases. In The enzymes (ed. P. D. Boyer), Vol. 12B, pp. 421-505. Academic Press, New York.

Carr, P. W. and Bowers, L. D. (1980). Immobilized enzymes in analytical and clinical chemistry. John Wiley, New York.

Cass, A.E.G., Davis, G., Green, M.J. and Hill, H.A.O. (1985b) Ferrocene monocarboxylic acid as an electron acceptor for oxidoreductases. J. Electroanal. Chem. 190, 117-27.

Hill, H. A. O. and Nancarrow, D. J. (1985a). Reaction of flavocytochrome $b_{2}$ with cytochrome $c$ and ferrocene monocarboxylic acid: Comparative kinetics by cyclic voltammetry and chronoamperometry. Biochim. Biophys, Acta 828, 51-7.

Francis, G., Hill, H. A. O., Higgins, 1. J., Aston, W. J., Plotkin, E. V., Scott, L. D. L, and Turner, A.P.F. (1984). Ferrocene mediated enzyme electrode for amperometric determination of glucose, Anal. Chem. 56, 667-71. 
Davis, G. (1984). Studies in applied bioelectrochemistry. D. Phil Thesis, University of Oxford.

—, Aston, W. J., Higgins, I. J., Hill, H. A. O. and Turner, A.P. F. (1983). Bioelectrochemical fuel cell and sensor based on a quinoprotein, alcohol dehydrogenase. Enzyme Microb. Tech, 5, 383-8.

D'Costa, E. J., Higgins, I. J. and Turner, A.P.F. (1986). Quinoprotein glucose dehydrogenase and its application in an amperometric glucose sensor. Biosensors 2, 71-87.

Deeming, A. J. (1982). Mononuclear iron compounds with hydrocarbon ligands. In Comprehensive organometallic chemistry (eds. G. Wilkinson, F, G. A. Stone and E. W. Abel), Vol. 4, pp. 377-512. Pergamon, Oxford.

Dicks, J., Aston, W. J., Davis, G. and Turner, A.P.F. (1986) Mediated amperometric biosensors for D-galactose, glycolate, and L-amino acids based on a ferrocene-modified carbon electrode. Anal. Chim. Acta 182, 103-12.

Eddowes, M. J., Albery, W. J., Hill, H, A, O, and Hillman, A. R, (1981). Mechanism of the reduction and oxidation reaction of cytochromes at a modified gold electrode. J. Am. Chem. Soc, 103, 3904-10.

Gibson, Q.H. and Hastings, J.W. (1962). The oxidation of reduced flavin mononucleotide by molecular oxygen. Biochem. J. 83, 368-77.

Green, M. J., Davis, G. and Hill, H. A. O. (1984). Creatine kinase assay using an enzyme electrode. J. Biomed. Eng. 6, 176-7.

Hill, H.A.O. and Walton, N. J. (1982). Investigation of some intermolecular electron transfer reactions of cytochrome $c$ by electrochemical methods. $J . A m$. Chem. Soc. 104, 6515-19.

Kuwana, T., Szentrimay, R, and Yeh, R, (1977). Evaluation of mediator titrants for indirect coulometric titrations of biocomponents. Am. Chem. Soc. Symp. Ser. 38, 143-58.

Merz, A. and Bard, A. J. (1978). A stable surface modified platinum electrode prepared by coating with electroactive polymer. J. Am. Chem. Soc. 100, 3222-3.

Morton, R. A., Overnell, J. and Harbury, H. A. (1970). Electron transfer between cytochromes $c$ from horse and pseudomonas. J. Biol. Chem. 245, 4653-7.

Murray, R. W. (1980). Chemically modified electrodes. Acc. Chem. Res. 13, 135-41.

Nicholson, R.S. and Shain, I. (1964). Theory of stationary electrode polarography. Anal. Chem. 36, 706-23.

Romette, J.L. and Boitieux, J.L. (1984). Computerised enzyme electrodes. J. Biomed. Eng. 6, 171-4.

Ryan, M. D. and Wilson, G.S. (1975). Some considerations in spectroelectrochemical evaluation of homogeneous electron transfer involving biological molecules. Anal. Chem. 47, 885-90.

Shichiri, M., Kawamori, R., Hakia, N., Yamasaki, Y. and Abe, H. (1982). Wearabletype artificial endocrine pancreas with needle-type glucose sensor, Lancet 2, 1129-31.

- (1984). Closed loop glycemic control with a wearable artificial endocrine pancreas. Diabetes 33, 1200-2.

Weibel, M. K., Duke, R. F., Page, D.S., Bulgrin, V.G. and Luthy, J. (1969). The glucose oxidase mechanism: enzyme activation by substrate. J. Am. Chem. Soc. 91, 3904-9. 


\title{
15 \\ The realization of electron transfer from biological molecules to electrodes
}

\author{
M.F. CARDOSI and A.P.F. TURNER
}

\subsection{Introduction}

Over the past decade there has been tremendous interest in the development of cheap, reliable biosensors for both clinical and industrial applications, One way of achieving this goal is to combine a biological catalyst with an electrochemical sensor, yielding a device which is both specific and easy to use. Furthermore, sensors based around electrochemical probes offer the most direct route for converting a chemical concentration into an electrical signal and can be readily interfaced to monitoring and control circuitry (Turner 1985).

In its simplest form an enzyme electrode consists of a thin layer of enzyme(s) held in close proximity to the active surface of a transducer, a suitable reference electrode and a circuit for measuring either the potential difference generated between the two electrodes (potentiometric) or the current that flows between them (amperometric)*. Usually the electrode is covered by a membrane which serves to protect against fouling and/or to introduce some desirable partitioning at the interface. To carry out a measurement the enzyme electrode is simply immersed into a solution containing the analyte of interest and the steady-state current or potential is read. The relationship is linear for an amperometric electrode and logarithmic for a potentiometric one.

In the potentiometric enzyme electrode the sensing head acts like a battery generating a potential difference which is measured relative to the reference under conditions of zero current flow. This has the advantage that there is no net consumption of material and hence mass transport is unimportant. Such sensors, however, suffer from two major disadvantages. Accurate information about the concentration of analyte in the solution will only be obtained if there is a local thermodynamic equilibrium at the electrode interface. This requires the electrode kinetics to be rapid with a standard electrochemical rate constant greater than $10^{-2} \mathrm{~cm} \mathrm{~s}^{-1}$ (Albery et al. 1985). This seriously

-The effect of the enzyme-catalysed reaction may be to generate an electro-active product, which can be detected amperometrically, or to alter the concentration of a particular ionic species, e.g. $\mathrm{H}^{*}$, whereby potentiometry becomes the basis for detection. 
limits the number of systems that can be used as indicator electrodes. The second disadvantage arises from the exponential dependence of the analyte concentration $(c)$ on the electrode potential $(E)$, i.e.

$$
\ln (c)=\text { constant }+n E F / R T
$$

where $n$ is the valency of the ion, $F$ is the faraday unit, $R$ is the gas constant, and $T$ is the absolute temperature. Small errors in $E$ can give quite substantial errors in $c$. For example for $n=1$ an error in $E$ of $10 \mathrm{mV}$ leads to a $19 \%$ error in the value of $c$ (Albery et al. 1985). Because of these disadvantages, biosensors based on amperometric indicator electrodes are considered to be more practical although in most cases care must be taken to control the hydrodynamics at the electrode surface.

Enzymes involved in the oxidation and reduction of biological molecules (oxidoreductases) either contain a redox centre such as iron, copper, flavin, quinone at their active site or perform their biological role in conjunction with a redox-active cofactor such as $\mathrm{NAD}(\mathrm{P})^{*}$. The difficulty in obtaining direct electrochemistry between an enzyme's redox centre and a naked electrode, together with the absence of an effective electrocatalytic surface for the efficient recycling of reduced cofactor, led to the first enzyme electrodes indirectly exploiting electrochemistry to monitor enzyme activity. The classic example is the glucose sensor proposed by Clark and Lyons (1962) and described by Updike and Hicks (1967) based on the enzyme glucose oxidase and a polarographic oxygen electrode (Chapter 1). Glucose oxidase is an FAD-containing enzyme (Fig. 15.1) which catalyses the oxidation of glucose to gluconic acid:

$$
\text { Glucose }+\mathrm{O}_{2}+\mathrm{H}_{2} \mathrm{O}=\text { Gluconic acid }+\mathrm{H}_{2} \mathrm{O}_{2}
$$

During the catalytic cycle the flavin prosthetic group is first reduced by glucose and then re-oxidized by molecular oxygen. The amount of glucose present in the solution is determined by following either the rate of oxygen consumption or the rate at which hydrogen peroxide is produced. Although functional, there are a number of disadvantages to such a system. The current will depend not only upon the glucose concentration but also on the partial oxygen tension $\left(\mathrm{pO}_{2}\right)$ of the solution. Secondly, the potentials required to reduce oxygen or oxidize hydrogen peroxide are sufficiently extreme so as to introduce the possibility of interference. Finally, the oxidation of hydrogen peroxide to oxygen is proton dependent, i.e.,

$$
\mathrm{H}_{2} \mathrm{O}_{2}=2 \mathrm{H}^{+}+\mathrm{O}_{2}+2 \mathrm{e}^{-}
$$

The current would thus vary quite considerably with the $\mathrm{pH}$ of the solution. Clearly, reliable application of this probe is only possible if both the $\mathrm{pH}$ and $\mathrm{pO}_{2}$ of the solution can be carefully controlled, a situation not often encountered even in the laboratory. If these problems could be avoided then 


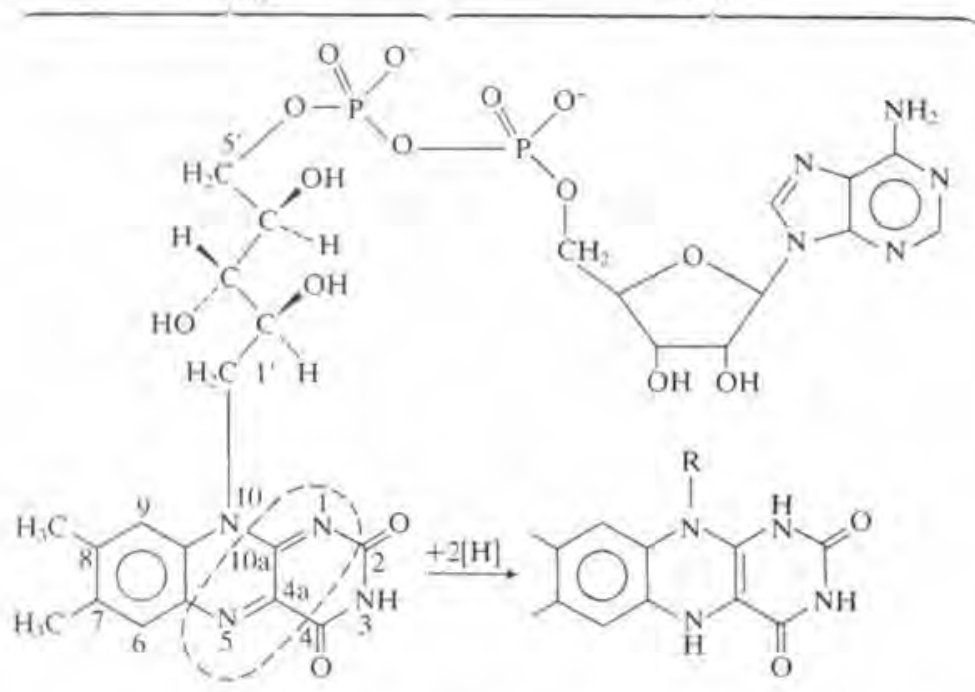

Fig. 15.1 The flavin co-enzyme. Dotted lines indicate the region that is altered following reduction.

it would be possible to develop enzyme electrodes which would gain wider acceptance. It is the development of such electrodes which forms the subject of this chapter.

\subsection{Mediators and chemically modified electrodes}

Although dioxygen is the physiological electron acceptor for oxidases such as glucose oxidase it can be replaced in the majority of cases by an electron transfer mediator. In this context, a mediator is a low-molecular-weight redox couple which shuttles electrons from the redox centre of the enzyme to the surface of the indicator electrode. During the catalytic cycle the mediator first reacts with the reduced enzyme and then diffuses to the electrode surface where it undergoes rapid charge transfer. This can be illustrated with reference to glucose oxidase:

in the solution:

$$
\begin{aligned}
\text { Glucose }+\mathrm{FAD}+\mathrm{H}_{2} \mathrm{O} & =\text { Gluconic acid }+\mathrm{FADH}_{2} \\
\mathrm{FADH}_{2}+\mathrm{M}_{\text {ox }} & =\mathrm{FAD}+\mathrm{M}_{\text {rod }}+2 \mathrm{H}^{+}
\end{aligned}
$$

at the electrode:

$$
\mathrm{M}_{\mathrm{red}}=\mathrm{M}_{\mathrm{ox}}
$$


The rate at which the reduced mediator $\left(\mathrm{M}_{\mathrm{red}}\right)$ is produced is measured amperometrically by oxidation at the electrode.

The use of a mediator introduces a number of distinct advantages. Provided the reduced mediator is unreactive with oxygen, it makes the measurement virtually independent of $\mathrm{pO}_{2}$. Secondly, the working potential of the enzyme electrode is now determined by the formal potential $\left(E^{0}\right)$ of the mediator couple. This can be particularly advantageous if the mediator has a low $E^{0}$ since it lessens the chance of interference. Finally, if the oxidation of reduced mediator does not involve protons it can make the enzyme electrode relatively $\mathrm{pH}$ insensitive (obviously the device cannot be used at extremes of $\mathrm{pH}$ where denaturation of the enzyme takes place).

The use of mediators in conjunction with oxidoreductases is by no means a recent innovation. Molecules such as quinones, organic ions, and inorganic ions such as ferricyanide and redox dyes have all been used with some degree of success (Bright and Porter 1975). A practical mediator, however, should fulfil the following criteria.

i) It should react rapidly with the reduced enzyme.

ii) It should exhibit reversible heterogeneous kinetics.

iii) The overpotential for the regeneration of the oxidized mediator should be low and $\mathrm{pH}$ independent.

iv) It should be stable in both oxidized and reduced forms.

v) The reduced mediator should not react with $\mathrm{O}_{2}$.

vi) For many applications it should be non-toxic.

Although the use of mediators does offer distinct advantages one is faced with the unnecessary complication of having to add it to the sample. A more practical configuration would be to have the mediator firmly anchored to the surface of the electrode in such a way that it was still electrochemically active and able to react with the reduced enzyme. To date, one of the most successful classes of mediator compounds that has been used in this way has been that based on ferrocene ( $\eta^{5}$-bis-cyclopentadienyl iron)

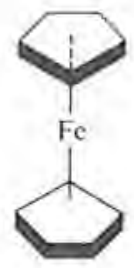

and its derivatives. Ferrocene is a transition metal $\pi$-arene complex which consists of an iron atom sandwiched between two cyclopentadienyl rings. It has a well-behaved electrochemical redox couple $\left(E^{0} /(\mathrm{mV})\right.$ versus SCE $=$ 165) with variation in physical and chemical properties available through 
substitution in either of the two rings systems (Pickett 1984). The first successful enzyme electrode based on ferrocene contained an insoluble derivative of the mediator and glucose oxidase (Cass et al. 1984). In simple terms I,I'-dimethyl ferrocene was incorporated into a graphite electrode upon which the enzyme glucose oxidase was chemically immobilized (Aston, Chapter 16). In this configuration, electrochemically generated ferricinium ions act as oxidants for reduced glucose oxidase. Once the ferricinium ion has been reduced it is re-oxidized at the electrode surface by polarizing the electrode and allowing current to flow. The sequence of reactions occurring at this electrode can be summarized as:

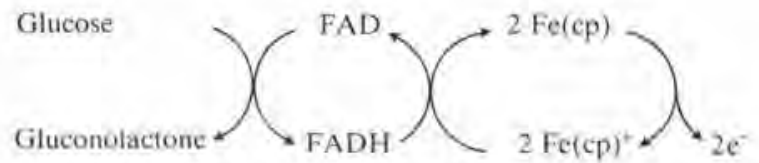

The advantages of this biosensor configuration are as follows.

i) The low formal potential needed to generate the ferricinium species ( $220 \mathrm{mV}$ versus $\mathrm{Ag} / \mathrm{AgCl}$ ) tends to minimize interference.

ii) Reduced ferrocene does not react with oxygen, making the sensor oxygen-insensitive.

iii) The electron-transfer reaction between the ferricinium ion and the reduced enzyme is fast resulting in rapid response times.

iv) Because of the low solubility of the ferrocene derivative the mediator is essentially confined at the electrode surface thus allowing the probe to be used without prior addition of mediator to the sample.

v) Because the enzyme is itself immobilized to the surface of the transducer the sensor can be used more than once.

The glucose electrode described by Cass et al. (1984) exhibited an excellent linear response for glucose (up to $30 \mathrm{~mm}$ ) beyond the physiologically relevant range whilst retaining rapid response times $(60-90 \mathrm{~s}$ to $95 \%$ steady-state current). These response characteristics were achieved by the use of a 'spongy' carbon-foil electrode which produced a reasonably defined hydrodynamic restriction to the diffusion of substrate without the need to resort to additional membranes. The electrodes showed essentially no difference in response when the analysis was performed under aerobic or anaerobic conditions and provided that an anion exclusion membrane was placed over the electrode they were relatively free from interference from metabolites, such as ascorbate, commonly found in blood plasma.

Since the original communication a variety of oxidoreductases have been used in conjunction with the ferrocene-modified electrode (Table 15.1) and 
Table 15.1 Enzymes which have been coupled to ferrocene-modified carbon in enzyme electrodes

\begin{tabular}{ll}
\hline Substrate & Enzyme \\
\hline L-Amino acids & L-Amino acid oxidase \\
Carbon monoxide & Carbon monoxide oxidoreductase \\
Glucose & Glucose oxidase or PQQ GDH \\
Glycolic acid & Glycolate oxidase \\
Lactate & Lactate oxidase or LDH \\
NADH & Glutathione reductase or Diaphorase \\
Pyruvate & Pyruvate oxidase \\
\hline
\end{tabular}

See also Table 14.1

this method of constructing enzyme electrodes is likely to be generally applicable (Turner 1986). NAD(P)'-independent dehydrogenases such as the quinoproteins offer particular advantages in this configuration (Turner et al. 1986). Whilst fericinium ions act as highly efficient electron transfer agents between reduced oxidases and electrodes under anaerobic conditions, oxidases retain a natural affinity for oxygen. The degree of oxygen interference is largely determined by the relative concentrations of oxygen and ferricinium ions at the enzyme's electron transfer site, since the rate of reaction with both reagents is similar (Davis 1985). Consequently, if the assay solutions are saturated with pure oxygen the amperometric response from typical oxidase electrodes decreases by about $30 \%$ (Cass et al. 1985; Dicks et al. 1986). Ferrocene-modified electrodes incorporating NAD(P)--independent dehydrogenases circumvent this problem completely and show no detectable decrease in current even in oxygen-saturated solutions (Aston et al. 1984; Turner et al. 1984; D'Costa et al. 1986). Although it is preferable to avoid the introduction of unstable, expensive, and soluble components such as $\mathrm{NAD}(\mathrm{P})^{+}$(Turner 1985), cofactor-dependent dehydrogenases (see below) may also be coupled to ferrocene-modified electrodes by the inclusion of a second enzyme. Both lipoamide dehydrogenase (diaphorase) and glutathione reductase may be catalytically coupled to an electrode using ferricinium ions (Cass et al. 1985) facilitating the following detection scheme:

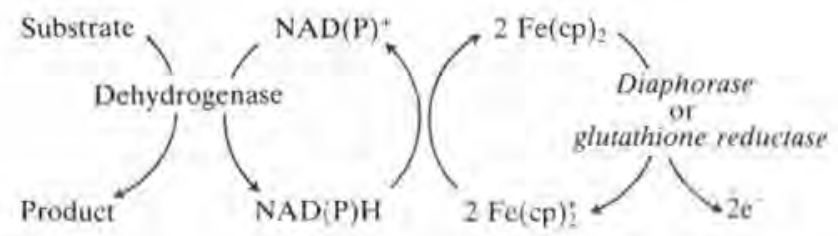


An interesting extension of the glucose oxidase/ferrocene electrode has been to couple it to other analytes using enzymes that compete with glucose oxidase for its substrate. In the presence of ATP and hexokinase, for example, glucose is diverted to glucose-6-phosphate (G-6-P). The glucose electrode, therefore, can be used to detect ATP (Davis 1984):

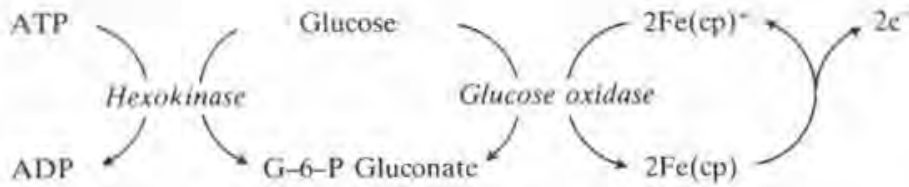

Through the detection of ATP, NAD , and NADP ' a greater range of analytes become accessible, including clinically relevant enzymes. Addition of creatine phosphate to the ATP assay above, for example, allows the detection of the enzyme creatine kinase, which is an important marker in the diagnosis of myocardial infarction.

These examples have concentrated on the ferrocene-based glucose sensor, but a range of other configurations employing enzyme modulation are possible providing novel enzyme electrodes (Scheller et al. 1985) and immunosensors (Green, Chapter 4).

Modified electrodes for the regeneration of oxidized enzyme have also been based on redox polymers containing $p$-and $o$-quinone groups adsorded onto the surface of electrodes, Fig. 15.2 (Cenas et al, 1983, 1984). These electrodes have been shown to be efficient oxidants for reduced glucose oxidase, L-lactate oxidase, and xanthine oxidase. Cenas et al. $(1983,1984)$
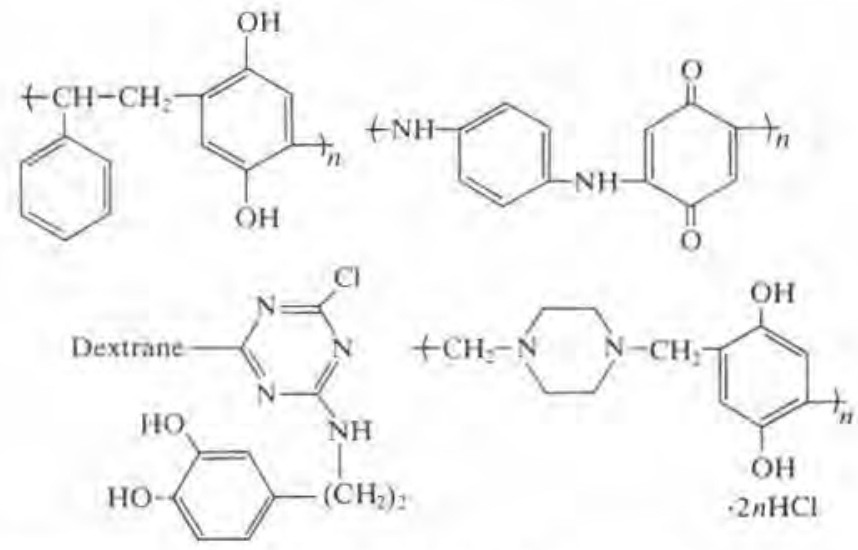

Fig. 15.2 Types of polymers based on $o$ and p-quinones which have been used to modify carbon and platinum electrodes. 
found that these enzymes could be re-oxidised in the range 0.05 to $0.5 \mathrm{~V}$ (versus $\mathrm{Ag} / \mathrm{AgCl}$ ) at $\mathrm{pH} 7$. The oxidation of these enzymes was found to occur at the oxidation potential of the polymer modifier suggesting that these acted in a mediatory way. A major drawback of these redox polymer electrodes, however, was that they lost their electrocatalytic activity after a relatively short period of time, typically 5 days (Cenas et al. 1984). More recently, Jonsson and Gorton (1985) have described an amperometric glucose sensor based on glucose oxidase immobilized onto graphite which had been previously treated with $N$-methyl-phenazinium (NMP). The authors found that the response of the electrode to glucose was strictly linear over the range 0.5 to $150 \mu \mathrm{M}$ and the sensor was usable up to about $2 \mathrm{~mm}$. The immobilized glucose oxidase was found to be stable for several months but the mediator had to be renewed on a daily basis.

\subsection{Enzyme electrodes based on cofactor regeneration}

Another group of enzymes which can be used in biosensor design are the nicotinamide adenine dinucleotide (NAD* and NADP*)-dependent dehydrogenases. These enzymes differ from the aforementioned oxidases in that they do not contain an active-site redox centre per se but rather carry out

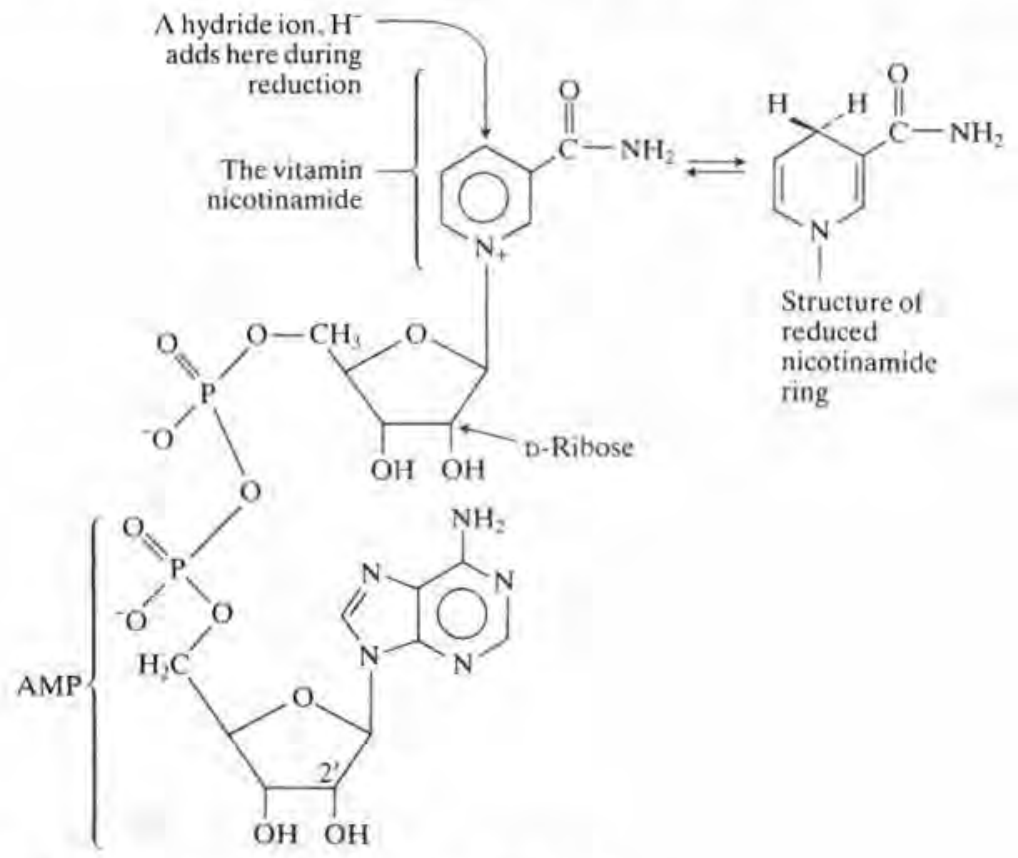

Fig. 15.3 The hydrogen-carrying co-enzyme NAD. 
their catalytic function with the help of a nicotinamide dinucleotide cofactor, Fig. 15,3.

The types of reaction normally associated with the nicotinamide dinucleotide cofactors and hydrogen transfer reactions are of the type:

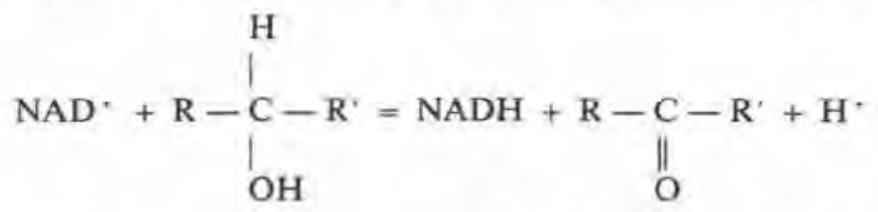

In this reaction one hydrogen atom of the substrate is directly transferred to $\mathrm{NAD}^{+}$and the other appears in the solvent. Both electrons lost by the substrate are transferred to the nicotinamide ring. In an amperometric enzyme electrode based on this type of dehydrogenase the enzymatic activity is measured by recycling reduced $\mathrm{NAD}(\mathrm{P})+$ at a suitable electrode:

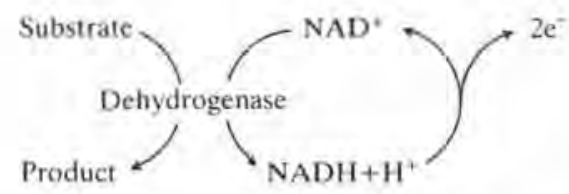

The essential element in making a successful biosensor of this type is to provide a suitable electrocatalytic surface which can re-oxidize reduced coenzyme both efficiently and in the correct biological form, i.e., in a form that will be recognized by the enzyme. Although it is possible to regenerate NAD* from NADH at naked electrodes under ideal conditions $(0.1 \mathrm{M} \mathrm{NADH}$, $\mathrm{pH} 7$ ) this approach does have its disadvantages. A large overvoltage is needed, i.e., $1.1 \mathrm{~V}$ (versus SCE) for the regeneration of NAD * at platinum and prolonged usage results in fouling of the electrode surface due to the accumulation of high-molecular-weight oxidation products. The need to overcome these difficulties has led to the development of suitable modified surfaces based on species such as catechols (Jaegfeldt et al. 1981), hydroquinones (Tse and Kuwana 1977), and redox dyes (Huck et al. 1984; Gorton et al. 1984). The rationale behind the preparation of a chemically modified electrode for NADH oxidation is that if a redox couple in solution can oxidize NADH and can in some way be retained at the surface of the electrode, then the resulting modified electrode should be able to carry out the electrocatalytic regeneration of NAD +. Preparation of suitable modified electrodes may simply be achieved by passive adsorption of the redox couple to the surface of the electrode, or can involve a synthetic route where the redox couple is covalently bound to the electrode via a bifunctional reagent such as a substituted silane or cyanuric chloride. An excellent review on the topic of chemically modified electrodes is presented by Murray (1984) and the 
kinetics of such electrodes is discussed in detail by Bartlett (Chapter 13). The oxidation of NADH at a modified electrode procedes by a mediatory route catalysed by the immobilized redox $(\mathrm{O} / \mathrm{R})$ couple:

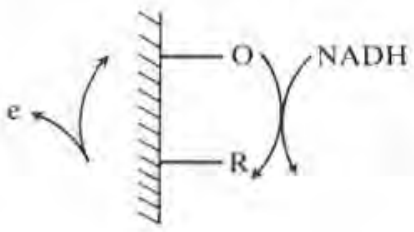

where $\mathrm{O}$ and $\mathrm{R}$ are the oxidized and reduced forms of the surface mediator. If the rate of the mediation reaction is fast and the rate of reduction or oxidation of the immobilized couple faster, the substrate (NADH) becomes oxidized at a potential near that of the $\mathrm{O} / \mathrm{R}$ formal potential $E^{0}$. This has the effect of greatly lowering the overpotential of the electrochemical reaction, thus reducing interference. Furthermore, the oxidation of NADH is carried out with higher efficiency (in terms of recycling biologically useful NAD+) and without deleterious effects on the electrode.

Although offering a significant advantage, modified electrodes do suffer from a lack of long-term operational stability, Instability usually manifests itself as the desorption of mediator from the electrode surface resulting in decreased electrocatalytic activity. This is particularly the case where the mediator is adsorbed to the electrode surface. Although mediators which are covalently attached to electrode surfaces do exhibit better stability this must be offset by the complicated and often time-consuming chemistry involved.

More recently, a novel electrode based on highly conducting organic metals has proved a most useful system for the regeneration of NAD + from NADH (Kulys 1981; Albery and Bartlett 1984; Chapter 12). These electrodes are based on stable charge-transfer complexes formed by the partial transfer of an electron from a donor such as 7,7,8,8,-tetracyano-p-quinodimethane (TCNQ) to an acceptor such as tetrathia-fulvalene (TTF) or N-methylphenazinium (NMP):
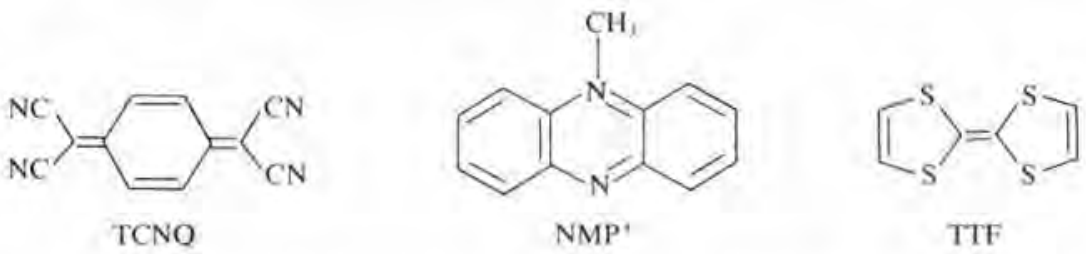

These donor acceptor complexes are metallic at room temperature with a conductivity of $500(\mathrm{ohms} \mathrm{cm})^{-1}$ for the TTF+TCNQ - complex and 200 
(ohms cm) ${ }^{-1}$ for the NMP+TCNQ - complex (Bryce and Murphy 1984). The chemistry and physics of organic metals has been well documented (Engler 1976) as have their electrochemical properties (Jaeger and Bard 1980). Kulys (1981) observed the oxidation of NADH at NMP - TCNQ electrodes at $-0.2 \mathrm{~V}$ (versus $\mathrm{Ag} / \mathrm{AgCl}$ ). When the electrode was used in conjunction with alcohol dehydrogenase the current was found to increase in the presence of ethanol (Kulys and Razumas 1983). The following experimental parameters were observed: $k_{\mathrm{M(app)}}, 15.4 \mathrm{mM} ; i_{\max }, 1.3 \mu \mathrm{A} \mathrm{cm}^{-2}$. The same electrode could be used to detect acetaldehyde in the presence of NADH with a $k_{\text {M(app) }}$ of $54 \mu \mathrm{M}$. The oxidation of NADH at the NMP + TCNQ electrode is thought to take place via a mediatory route (Kulys 1981). The reaction is believed to involve electrogenerated NMP - ions since the NMA - TCNQ - complex does not exhibit any electrocatalytic oxidation of NADH.

A problem that still has to be solved before a dehydrogenase can be incorporated into a practical device is the need to add exogenous cofactor to the solution. This requirement clearly limits any direct or on-line application these sensors may have. A possible way of overcoming this limitation may be the use of permselective membranes to localize the $\mathrm{NAD}(\mathrm{P})$ ' at the electrode surface. Retention could also be achieved by chemical immobilization or by an exclusion principle in which the effective weight of the cofactor is increased by covalent attachment to a high-molecular-weight species such as dextran (Davies and Mosbach 1974; Chapter 6),

\subsection{Amperometric sensors based on redox proteins}

The essence of this type of coupled amperometric enzyme electrode is that the reduced oxidoreductase does not participate in a redox reaction directly at an electrode surface but rather charge transfer occurs via an attendant redox protein such as cytochrome $c$. In effect, this configuration is analogous to the mediated system described above except that in this example the mediator is not a simple ion but a complex protein molecule. In the coupled electrode the oxidoreductase provides the specificity for the sensor and the redox protein the means of shuttling electrons from the active site of the enzyme to the surface of the electrode. Cytochrome $c$ has proved a useful model in this type of sensor design because not only does it have well-defined electrochemistry, but it is also found in nature as the natural electron acceptor in many enzyme complexes. (For a review on the structure and function of cytochrome $c$ the reader is referred to Salemme 1977.)

Studies on the electrochemistry of cytochrome $c$ were initiated in the early 1970s by Betso and coworkers (Betso et al., 1972) who were able to show that the iron centre of the protein was reducible at both mercury and platinum electrodes, albeit at a slow rate. Although charge transfer had been observed, the slow kinetics made this an impractical approach for biosensor design. The 
first major breakthrough in obtaining an analytical device based on cytochrome $c$ electrochemistry came when Eddowes and Hill (1977) described a simple treatment of gold which allowed quasi-reversible (i.e. fast) charge-transfer kinetics. They observed that the addition of $4,4^{\prime}$-bipyridyl (bipy),

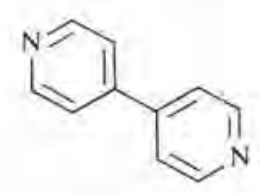

to a buffered electrolyte solution enhanced the rate of electron transfer between the haem site of cytochrome $c$ and the gold surface. It is important to appreciate that although $4,4^{\prime}$-bipyridyl greatly enhanced charge transfer from the haem centre to the gold electrode it is itself electro-inactive in the potential region of interest and thus does not merely act as a charge-transfer mediator. The mechanism for the enhanced heterogeneous charge transfer is believed to depend upon the adsorption of bypyridyl to the electrode surface and the rapid on-off binding of the cytochrome at the modified electrode-solution interface. This ensures that the protein is held sufficiently close to the electrode surface and in the correct orientation to allow the electron transfer step to take place efficiently (Albery et al. 1981; Bartlett, Chapter 13). Fast adsorption/desorption kinetics are considered important otherwise blocking of the modified interface would occur.

The interaction of cytochrome $c$ with the gold/bipyridyl electrode has been suggested to occur through specific lysine residues, on the surface of the protein, hydrogen bonding to one of the nitrogen atoms of the promoter. The reasoning for this suggestion comes from the similarity between the electrochemical system and the protein-protein electron exchange properties of cytochrome $c$. Poly-lysine, for example, has long been known to inhibit the reaction between cytochrome $c$ and its biological redox partner cytochrome $c$ oxidase. When poly-lysine is added to an electrochemical cell containing cytochrome $c$ and the bipyridyl-modified gold electrode the heterogeneous charge transfer is similarly inhibited. In an analogous fashion modification of the surface side-chains in cytochrome $c$ is equally effective in inhibiting both the electrochemical charge-transfer step and the reaction with cytochrome $c$ oxidase (Cass et al. 1984; Pickett 1984; Higgins and Hill, 1985).

Other activating molecules besides $4,4^{\prime}$-bipyridyl have been examined and shown to promote electron transfer from the haem centre of cytochrome $c$ to the gold electrode (Eddowes and Hill 1982). The pyridines (III) and (IV), for example, allow a well-developed cyclic voltammetric response of the protein 
to be observed at a gold electrode (Taniguchi et al. 1982). Walton and coworkers (Allen et al. 1984) have surveyed some fifty-five bifunctional organic molecules as possible promoters and concluded that bifunctional molecules containing both a surface-active group (a Lewis base with acceptor properties using $\mathrm{N}, \mathrm{S}$, or $\mathrm{P}$ ) and a weakly basic or anionic functional group appropriately orientated with respect to one another were suitable as promoters. More recently, gold electrodes modified by the chemisorption of pyridine aldehyde thiosemicarbozones (V) have extended this work to promote electrode reactions not only with cytochrome $c$ but also with proteins such as plastocyanin, which have a negatively charged binding domain (Hill et al. 1981).

The cytochrome $c$ gold/bipyridyl system has been incorporated in enzymebased sensors for L-lactate (Cass et al. 1985), carbon monoxide (Turner et al. 1984), and hydrogen peroxide (Higgins and Hill 1985). Horse heart cytochrome $c$ is reduced by an enzyme-catalysed reaction:

(i)

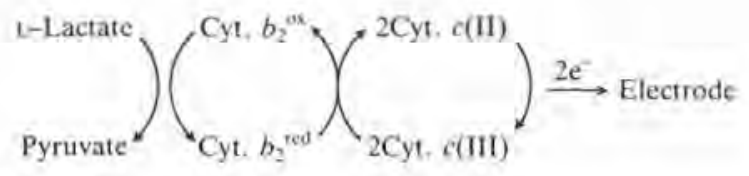

(ii)
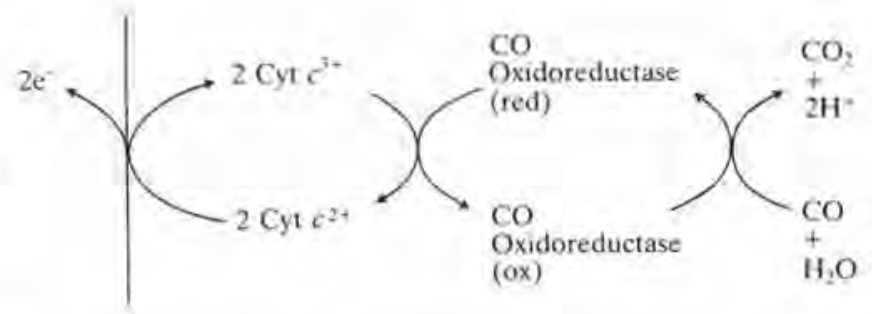

Reaction (i) is catalysed by yeast flavo-cytochrome $c$ and reaction (ii) by a bacterial carbon monoxide oxidoreductase (Turner et al. 1984). The reaction of cytochrome $c$ at the 4,4'-bipyridyl electrode has also been coupled via a terminal oxidase to the reduction of dioxygen to water, thus providing the basis for an oxygen sensor, Fig. 15.4 (Hill et al. 1981).

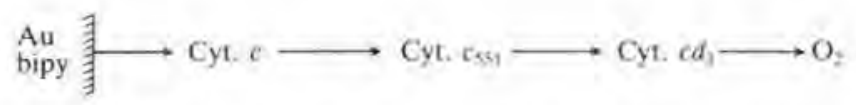

Fig. 15.4 Reaction scheme for a biosensor based on cytochrome $c$ for the detection of dioxygen. 
Cytochrome $c_{551}$, the 'blue' copper protein Azurin, was necessary because the rate of electron transfer between horse heart cytochrome $c$ and Pseudomonas

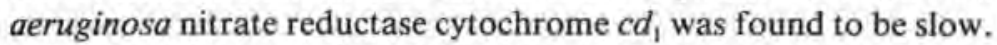

The cytochrome $c /$ gold/bipyridyl electrode has been used to facilitate charge transfer to and from intact systems as well as isolated enzymes. For example, when rat liver mitochondria and protoplasts prepared from Paracoccus denitrificans were used with the above electrode, electrons could be fed into these systems from the gold electrode. In addition, electrons could be extracted from added reducing agents such as NADH via cytochrome $c$ (Higgins and Hill 1985). Although no practical biosensor has been developed using a coupled intact biological system in this way, a variety of possibilities exist (Higgins et al. 1980; Higgins and Hill 1979; Turner et al. 1983). Clearly, any system capable of either oxidizing or reducing cytochrome $c$ may be coupled to an electrode in this way providing a route to monitoring chemicals, enzymes, cell components, or intact micro-organisms.

\subsection{Conducting organic metal electrodes coupled to oxidases}

Another interesting application of the charge-transfer-type electrodes mentioned above has been to couple them directly to oxidases. Kulys and coworkers, for example, have reported efficient coupling of the redox centres of cytochrome $b_{2}$ (L-lactate ferricytochrome $c$ oxidoreductase) (Kulys and Svirmickas, 1979), glucose oxidase (Kulys et al. 1980), horse-radish peroxidase (Kulys et al. 1980) and xanthine peroxidase (Kulys and Razumas 1983) to NMP - TCNQ + or NMA - (methyl acridinium) TCNQ + electrodes. The construction of these electrodes was relatively simple; either powdered enzyme was mixed with a finely divided preparation of the charge-transfer complex and pressed into a disc $\left(10^{5}\right.$ pounds in $\left.{ }^{-2}\right)$ or an aliquot of enzyme solution was entrapped at the electrode surface behind a membrane. Enzyme electrodes containing cytochrome $b_{2}$ adsorbed on NMP+TCNQ + electrodes oxidized L-lactate in the region of -0.2 to $0.5 \mathrm{~V}$ (versus SCE). The $k_{\mathrm{M}(\mathrm{app})}$ of the electrode was approximately $2 \mathrm{mM}$ with an $i_{\max }$ of $25-37 \mu \mathrm{A} \mathrm{cm}^{-2}$. The electrode response was the same in the presence and absence of oxygen. The maximum current was reached at $\mathrm{pH} 6.6$ whereas the native enzyme exhibits maximum activity at $\mathrm{pH} 7.2$. The electrodes were found to retain $45-50 \%$ of their initial sensitivity after three to nine days at room temperature.

Electrodes based on glucose oxidase adsorbed on NMA $+\mathrm{TCNQ}^{-}$or $\mathrm{NMP}+\mathrm{TCNQ}$ - electrodes responded to glucose only at potentials higher than $0.1 \mathrm{~V}$ relative to $\mathrm{Ag} / \mathrm{AgCl}$; i.e. the peak oxidation potential of NMP + and NMA+, The electrodes showed a good linear range and remarkable stability retaining their activity for more than 100 days (Kulys et al. 1980).

Electroreduction of hydrogen peroxide on NMA-TCNQ+ electrodes containing adsorbed peroxidase starts at 0.3 to $0.35 \mathrm{~V}$. Studies using rotating- 
disc techniques have shown that the process is limited by the catalytic activity of the enzyme. The $k_{\mathrm{M}(\mathrm{app})}$ of the system is $80 \mu \mathrm{M}$ and $i_{\max }=0.27 \mathrm{~mA} \mathrm{~cm}^{-2}$. The heterogeneous rate constant has been calculated to be $8.5 \times 10^{-4} \mathrm{~cm} \mathrm{~s}^{-1}$. These electrodes have also been used in conjunction with glucose oxidase to construct bienzyme glucose-sensitive electrodes (Kulys et al. 1981). This configuration exploited the bioelectrocatalytic reduction of hydrogen peroxide produced by the action of glucose oxidase.

More recently, Albery and co-workers (1985) have also investigated the use of organic metal electrodes as suitable charge-transfer surfaces for redox enzymes (Chapter 12). They have extended this work by looking at the charge-transfer properties of glucose oxidase at a variety of different conducting organic electrodes. A common feature they found with all the electrodes was low background currents in the absence of glucose. All materials gave increased currents on the addition of glucose however the best materials were found to be the $\mathrm{TCNQ}^{-}$salts of $\mathrm{NMP}^{-},\left(\mathrm{TTF}^{+}\right)$, and quinolinium $\left(\mathrm{Q}^{+}\right)$

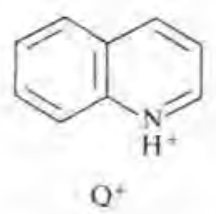

in terms of giving the lowest background currents and widest ranges of operating voltages. Albery et al. (1985) have also reported the system to be stable. After a period of 28 days continuous operation, the response of the glucose electrode was found to have decreased by only $20 \%$.

An important point which still has to be resolved is the exact nature of the electrode mechanism, i.e., whether or not the enzyme is oxidized by direct electron transfer to the electrode or whether it is a mediated process. In the latter case TTF *, NMP *, and/or TCNQ- react with the enzyme and are then oxidized at the electrode. Kulys and his school (Kulys and Svirmickas 1980) claim that in the case of cytochrome $c$ and peroxidase-containing electrodes the exchange of electrons between the redox centre and the electrode is direct. They cite as their evidence the lack of current dependence of these electrodes on the potential and data from kinetic studies using rotating disc (Kulys and Samalius 1982). In the case of the flavin-containing oxidases, glucose oxidase, and xanthine oxidase, Kulys claims a mechanism mediated by NMP - This conclusion is supported by the fact that substrate oxidation only proceeds at potentials corresponding to the mediator redox conversion potentials. They suggest that mediators are formed in the layer near the electrode surface during the slight dissolution of the organic metal. In this context it is interesting to note that Kulys and Cenas (1983) have shown that, in solution, $\mathrm{TCNQ}^{\circ}$ is an extremely efficient oxidant of reduced glucose 
oxidase $\left(5_{\mathrm{ox}} \times 10^{-4}\left(\mathrm{M}^{-1} \mathrm{~s}^{-1}\right)=150\right)$. We have since shown that graphite electrodes modified with TCNQ act as efficient electrocatalytic surfaces for the oxidation of reduced glucose oxidase and can operate at very modest potentials $\left(\mathrm{O} \mathrm{mV}\right.$ vs $\mathrm{Ag} / \mathrm{AgCl}$ ). We have also shown that $\mathrm{TTF}^{*}$ is an efficient mediator for reduced glucose oxidase, both in homogenous solution and when adsorbed on to an electrode surface (Turner et al. 1987). Albery et al. (1985) disagree with this final postulate and argue that charge transfer occurs via the direct route for glucose oxidase. They cite rotating-ring-disc data as their main evidence in that they have not been able to detect the products of electrode dissolution on the down-stream ring electrode. (Chapter 12).

\subsection{Conclusion}

The search for new electron-transfer agents and explanations of their mechanism of action will doubtless continue apace, driven by the commercial significance of this type of biosensor configuration. ${ }^{*}$ New products based on this general technology are imminent and it seems probable that the area will be advanced rapidly. The key to success lies in finding simple and stable configurations capable of mass production at low cost. The hope is that this will be achieved without sacrificing the accuracy and sensitivity associated with conventional laboratory analyses.

\section{Acknowledgement}

A. P.F. Turner is a Senior Fellow of the British Diabetic Association.

\section{References}

Albery, W. J. and Bartlett, P.N. (1984). An organic conductor electrode for the oxidation of NADH. J. Chem. Soc. Chem. Commun. 234-6.

- Craston, D. H. and Hagett, B. G. D. (1985). The development of novel biosensors. In The World Biotech Report 1985, Vol. 1, 359-82. Online Publications, Pinner, U.K.

— Eddowes, M. J., Hill, H. A. O. and Hillman, A. R. (1981) Mechanism of the reduction and oxidation of cytochrome $c$ at a modified gold electrode. J. Amer. Chem. Soc. 103, 3904-10.

Allen, P.M., Hill, H.A.O. and Walton, N.J. (1984). Surface modifiers for the promotion for the promotion of direct electrochemistry of cytochrome $c, J$. Electroanal. Chem. 178, 69-86.

Aston, W. J., Ashby, R. E., Higgins, I. J., Scott, L.D. L. and Turner, A. P. F. (1984). Enzyme based methanol sensor. In Charge and field effects in bio-

*Whilst glucose sensors have been used as the ubiquitous example, the importance of finding a scheme which is applicable to a wide range of enzymatic reactions must be stressed, since this will facilitate the development of multianalyte sensors. 
systems (eds. M.J. Allen and P.N.R. Usherwood), 491-8. Abacus Press, Turnbridge Wells.

Betso, S. R., Klepper, M. H, and Anderson, L. B. (1972). The electrochemical behaviour of cytochrome c. J. Amer. Chem. Soc. 94, 8197-204.

Bright, H. J, and Porter, D. J. T. (1975). In The enzymes (ed. H. Boyer), Vol. 12, 421-86. Academic Press, New York.

Bryce, M. R. and Murphy, L. C. (1984). Organic metals. Nature 309, 119-26.

Cass, A. E. G. (1984). Protein electrochemistry, Life Chem. Reporis. 2, 321-362.

- Davis, G., Hill, H.A.O. and Nancarrow, D. J. (1985). The reaction of flavocytochrone $b_{2}$ with cytochrome $c$ and ferricinium carboxylate. Comparative kinetics by cyclic voltammetry and chronoamperometry. Biochim. Biophys. Acta. 828, 51-7.

-Francis, D. G., Hill, H. A. O., Aston, W. J., Higgins, I. J., Plotkin, E. V., Scott, L. D. L. and Turner, A.P.F. (1984). Ferrocene-mediated enzyme electrode for amperometric determination of glucose. Anal. Chem. 56, 667-71.

Cenas, N. K., Kanapieniene, J.J. and Kulys, J, J. (1984). NADH oxidation by quinone electron acceptors. Biochem. Biophys. Acta. 767, 108-12.

- Pocius, A.K. and Kulys. J.J. (1983). Electron exchange between flavin and heme containing enzymes and electrodes modified by redox polymers. Bioelectrochem. Bioenerg. 11, 61-73.

- and Kulys, J. J. (1984). Bioelectrocatalytic conversion of substances on polymer modified electrodes. Bioelectrochem. Bioenerg. 12, 583-91.

Clark, L. C. and Lyons, C. (1962). Electrode system for continuous monitoring in cardiovascular surgery. Ann. N. Y. Acad. Sci. 102, 29-33.

Davies, P. and Mosbach, K. (1974). The application of immobilized NAD - in an enzyme electrode and in model enzyme reactors. Biochim. Biophys. Acta, 370, 329-338.

Davis, G. (1985). Electrochemical techniques for the development of amperometric biosensors. Biosensors. 1, 161-78.

- (1984). Studies in applied bioelectrochemistry. Ph. D. Thesis, University of Oxford.

D'Costa, E. J., Higgins, 1. J. and Turner, A.P. F. (1986). Quinoprotein glucose dehydrogenase and its application in an amperometric glucose sensor. Biosensors 2, 71-89.

Dicks, J. M., Aston, W. J., Davis, G. and Turner, A. P. F. (1986). Mediated amperometric biosensors for D-galactose, glycolate and L-amino acids based on a ferrocene-modified carbon paste electrode. Anal. Chim. Acta. 182, 103-112.

Eddowes, M. J. and Hill, H. A. O. (1977). A novel method for the investigation of the electrochemistry of metalloproteins: cytochrome c. J. Chem. Soc. Chem. Commun. 71.

(1982). Binding as a prerequisite for rapid electron transfer reactions of metalloproteins. Am. Chem. Soc. Adv. Chem. Ser. 201, 173-8.

Engler, E. M. (1976), Organic metals. Chemtech. 4, 274-9.

Gorton, L., Torstensson, A., Jaegfeldt, H. and Johansson, G. (1984). Electrcatalytic oxidation of reduced nicotinamide coenzymes by graphite electrodes modified with an adsorbed phenoxazinium salt, Meldola Blue, J. Electroanal. Chem. 161, 103-20. 
Higgins, I. J. and Hill, H. A. O. (1979). Microbial generation and interconversion of energy sources. In Microbial Technology. (eds. A. T. Bull, D. C. Ellwood and C. Ratledge) Society of General Microbiology Symposium No. 19, pp 359-76.

- (1985). Bioelectrochemistry, Essays in Biochemistry 21, 119-45.

Higgins, I. J., Best, D. J. and Hammond, R. C. (1980). New findings in methaneutilising bacteria highlight their importance in the biosphere and their commercial potential. Nature 286, 561-4.

Hill, H. A. O,, Walton, N. J, and Higgins, I. J. (1981). Electrochemical reduction of dioxygen using a terminal oxidase. FEBS Lett. 126, 282-4.

Huck, H., Schelter-Graf, A., Danzer, J., Kirch, P. and Schmidt, H. (1984). Bioelectrochemical detection systems for substrates of dehydrogenases. Analyst 109 , 147.

Jaeger, C, D, and Bard, A. J. (1978). Electrochemical behaviour of tetrathiafulvalene tetracyanoquinodimethane electrodes in aqueous media. J. Amer. Chem. Soc. 101, 1690-99.

(1980). Electrochemical behaviour of donor-tetracyanoquinodimethane electrodes in aqueous media. J. Amer. Chem. Soc, 102, 5435-42.

Jaegfeldt. H., Torstensson, A. B. C., Gorton, L. G. O. and Johansson, G. (1981). Catalytic oxidation of reduced nicotinamide adenine dinucleotide by graphite electrodes modified with adsorbed aromatics containing catechol functionalities. Anal. Chem. 53, 1979-82.

Jonsson, G. and Gorton, L. (1985). An amperometric glucose sensor made by modification of a graphite electrode surface with immobilized glucose oxidase and adsorbed mediator. Biosensors. 1, 355-68.

Kulys, J. J. (1981), Development of new analytical systems based on biocatalysers. Enzyme Microb. Technol. 3, 342-52.

— and Cenas, N. K. (1983). Oxidation of glucose oxidase from Penicillium vitale by one- and two-electron acceptors. Biochim. Biophys. Acta. 744, 57-63.

- and Razamas, V.J. (1983). Biocatalysis in electrochemistry of organic compounds. Mokslas, Vilinius. (In Russian.)

_ and Samalius, A. S. (1982). Acceleration of electrode processes by biocatalysts. Lietuvos. TSR. Mokslu. Akademijas, Darabi. B ser, 2(129), 3-9.

- and Svirmickas, G.J.S. (1979). Bioelectrocatalysis. Electron transfer from the active centre of cytochrome $b_{2}$ to organic metals. Dokl. Akad. Nauk. SSSR, 245, 137-40.

(1980). Reagentless lactate sensor based on cytochrome $b_{2}$, Anal. Chim. Acta, $117,115-20$.

- Pesliakiene, M. V. and Samalius, A.S. (1981). The development of bienzyme glucose electrodes. Bioelectrochem. Bioenerg. 8, 81-8.

Samalius, A.S. and Svirmickas, G. J.S. (1980). Electron exchange between the enzyme active centre and organic metal. FEBS Lett. 114, 7-15.

Murray, R.W. (1984). Chemically modified electrodes. In Electroanalytical chemistry, (ed. A. J. Bard) Vol. 13, pp. 191-387, Marcel Dekker, New York.

Pickett (1984). Electrochemistry of metal complexes. In Electrochemistry (ed. D. Pletcher), Vol. 9, pp. 162-221, Roy, Soc. Chem, London.

Salemme, R. (1977). Structure and function of cytochrome c. Ann. Rev. Biochem. 46, 229-39.

Scheller, F. W., Rennberg, F.S. R., Müller, H., Janchen, M. and Weise, H. (1985). Biosensors: trends and commercialization. Biosensors. 1, 135-61. 
Taniguchi, I., Toyosawa, K., Tamaguchi, M. and Yasukouchi, K. (1982). Voltammetric response of horse heart cytochrom $c$ at a gold electrode in the presence of sulphur bridged bipyridines. J. Elecroanal. Chem. 140, 187-93.

Tse, D. C. S, and Kuwana, T. (1977). Oxidation of NADH at a modified electrode. Anal. Chem. 49, 1589-95.

Turner, A.P. F. (1985) Biosensors for process monitoring and control. In The World Biotech Report 1985, Vol. 1, Online Publications, Pinner, UK, pp. 181-92.

- (1988). Amperometric biosensors based on mediator-motified electrodes. In Methods in enzymology: immobilized enzymes and cells (ed. K. Mosbach). Academic Press, New York.

D'Costa, E. J. and Higgins, I. J. (1986). The use of glucose dehydrogenase and other quinoproteins in analytical systems. In Enzyme Engineering. Plenum, New York. (In press.)

- Ramsay, G. and Higgins, I. J. (1983). Applications of electron transfer between biological systems and electrodes. In Industrial and Medical Applications of Bioelectrochemistry. Biochem. Soc. Trans. 11, 445-448.

- Hendry, S. P. and Cardosi, M. F. (1987). Tetrathioful valene: new mediator for amperotemic biosensors. In Biosensors, Instrumentation and Processing. Online, London, pp. 125-37.

Aston, W. J., Davis, G., Higgins, I. J., Hill, H. A. O. and Colby, J. (1984). Enzyme based carbon monoxide sensors. Microbial Gas Metabolism. (Eds. R. K. Poole and D.S. Dow). Academic Press, New York, pp. 161-70.

Updike, S. J, and Hicks, G. P. (1967) The enzyme electrode. Nature 214, 986-8. 


\title{
The construction of mediated amperometric biosensors
}

\author{
W.J. ASTON
}

The ideal method for the determination of substances whether in the field of medical or industrial analysis requires the procedure to be technically simple, rapid, inexpensive, sensitive, precise, and accurate, using stable reagents which are non-hazardous. Biological catalysts such as enzymes possess many of these properties being capable of catalysing specific reactions in mixed samples under mild conditions. It is these properties coupled with their increased availability which has resulted in their incorporation into diagnostic tests replacing many of the earlier non-enzymic assays which involved chemical oxidation-reduction techniques or condensation methods (Dietzler and Smith 1980). These chemical methods were subject to positive error resulting from the reaction with substances that normally occur in the blood and, in the case of the condensation reactions, used hazardous reagents such as hot, highly concentrated sulphuric acid. A frequently assayed metabolite, glucose, may be assayed by a variety of methods (Passey et al. 1977), the National Reference Method for the determination for this substrate for instance is a spectrophotometric assay incorporating two enzymic reactions (Neese et al. 1976). In the presense of glucose the enzymes, hexokinase (EC 2.7.1.1) and glucose-6-phosphate dehydrogenase (EC 1.1.1.49) result in the production of $\mathrm{NADH}$, the resulting increase in absorbance being measured spectrophotometrically at $340 \mathrm{~nm}$. A commonlyencountered technique utilizes the enzyme glucose oxidase, the product of the reaction may be determined spectrophotometrically by incorporation of peroxidase (EC 1.11.1.7) and linking the subsequent oxidation of hydrogen peroxide to a chromogen such as $o$-dianisidine (Guidotti et al. 1961). The use of chromogens has resulted in new diagnostic products referred to as 'dry chemistry' systems in which all the reagents and auxiliary substances necessary for the reaction are embedded in a paper or plastic matrix in their dry state, thus removing the need to prepare reagent solutions (Walter 1983; Sherwood et al. 1983). Although other techniques, such as calorimetric (Mosbach and Danielsson 1981) and opto-electronic methods (Lowe et al. 1983) have been proposed, the most practical alternative is the use of electrochemical detection systems. It is the construction of these amperometric devices and their possible development which is the subject of this chapter. 
The majority of electrochemical devices function by measuring the enzymic consumption or production of a naturally occurring electrochemically active species. Glucose, for instance, may be determined by measuring the production of hydrogen peroxide or consumption of oxygen by the action of glucose oxidase (Chapter 1). Indeed a diverse range of biosensors utilizing electrochemical techniques have been described (Carr and Bowers 1980; Aston and Turner 1984). Few devices, however, have been successfully developed commercially (Thai and Yeo 1983; Chapter 18). An alternative is to link biological redox reactions to an electrode, via a mediator, and measure the flow of electrons amperometrically (Chapter 15). Enzyme electrodes have many advantages as analytical devices enabling particular substrates to be assayed with the minimum of pretreatment. Since these devices are generally non-destructive; multiple determinations of samples may be performed. Although the response may be affected by chemical and physical factors, it is rapid, usually within 30-600 seconds.

\subsection{Bio-fuel cells}

Biological fuel cells are devices in which the reactions at one or both electrodes are catalysed biologically at ambient temperatures and pressures. The diversity of microbial metabolism allows a large range of fuels to be utilized including many industrial wastes by incorporating either whole organisms (Bennetto 1984; Chapter 17) or enzyme preparations (Plotkin et al, 1981, Higgins et al, 1984). Bio-fuel cells have generally been classified into one of two types depending on the mode of interaction of the biological catalyst with the electrode (Shaw 1963): in type A cells the fuel, such as hydrogen, is generated biologically in a separate chamber to that in which its subsequent electrochemical oxidation occurs. Type B bio-fuel cells require the direct interaction of the biological catalyst with the electrode to provide a continuous source of electrons, a process often facilitated by the use of a mediator. The mediator should be capable of rapid reversible electron transfer and have a redox potential close to that of the biological catalyst which it is transferring electrons from the electrode. The mediator should be non-toxic, not a substrate for the catalyst, and must be stable for prolonged periods of time during both storage and operation. It is this latter type of cell, utilizing a mediator, which will be described in this section.

Bio-fuel cells usually consist of two inert electrodes, such as gold, platinum, or carbon, maintained in a buffered solution. Prior to use the electrodes are cleaned by sonication in buffer or by cyclic voltammetry in sulphuric acid $(500 \mathrm{mM})$ in the potential range $-0.26 \mathrm{~V}$ to $+1.3 \mathrm{~V}$ (versus the standard calomel reference electrode, SCE) until distinct hydrogen/oxygen, oxidation-reduction peaks are observed (Sawyer and Roberts 1974). The electrodes are separated by an ion-exchange membrane 


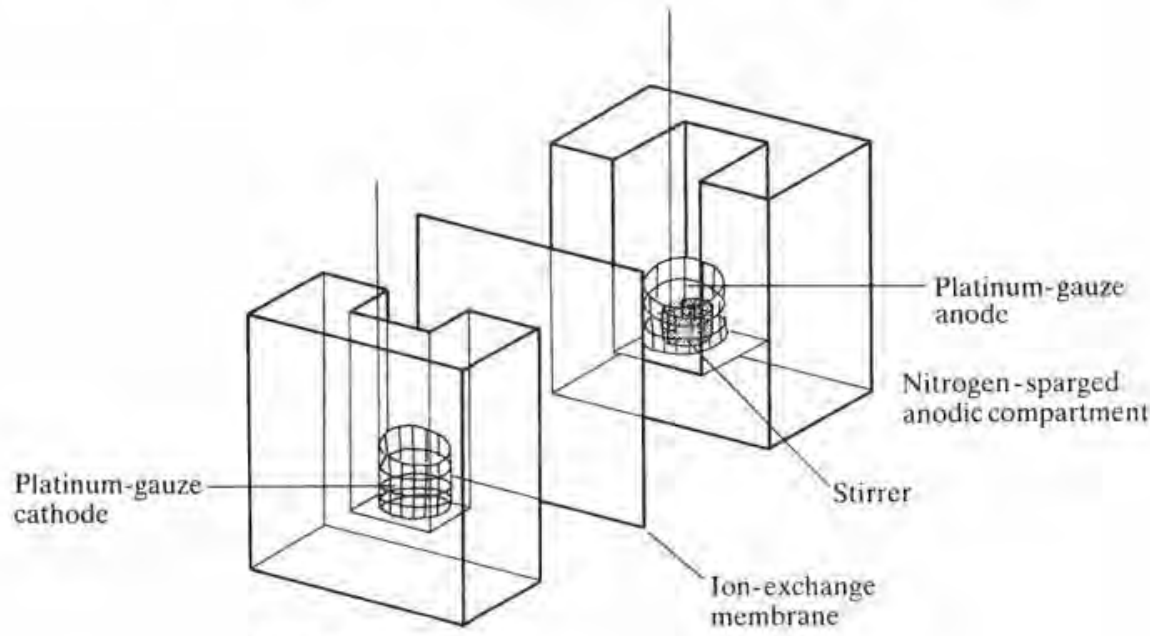

Air-or oxygensparged cathodic compartment

Fig. 16.1 Exploded view of bio-fuel cell. The perspex cell consisted of an anode and cathode $(35 \times 16 \times 15 \mathrm{~mm}$ and $35 \times 15 \times 11 \mathrm{~mm})$ separated by an ion-exchange membrane. Set up as described in the text (see Table 16.1).

and sparged with air/oxygen (cathode) and nitrogen (anode) (Fig. 16.1). The membrane separates the reactions occurring in the two compartments whilst allowing the exchange of protons. A range of suitable membranes are available commercially (BDH Ltd., Poole, Dorset, UK; Nafion, DuPont (UK) Ltd., Hemel Hempstead, Herts, UK). Higher charge-transfer efficiencies are attained if the nitrogen gas is passed through a gas purification unit (Nilox,

Table 16.1 Components of a glucose-oxidase-based bio-fuel cell, mediated by TMPD

Anodic compartment

Acetate buffer (50 mM, pH 4.5)

Sodium chloride (150 mM)

Mediator (TMPD) (8.0 mM)

Glucose oxidase $(8.0 \mathrm{mg})$

Total volume $4.2 \mathrm{ml}$

Pt electrode 50 mesh

$1.6 \times 4.8 \mathrm{~cm}$

Sparged with oxygen-free nitrogen and magnetically stirred
Cathodic compartment

Acetate buffer (50 mM, pH 4.5)

Sodium chloride (150 mM)

Total volume $3.5 \mathrm{mI}$

Pt electrode 50 mesh

$1.6 \times 4.8 \mathrm{~cm}$

Sparged with air 


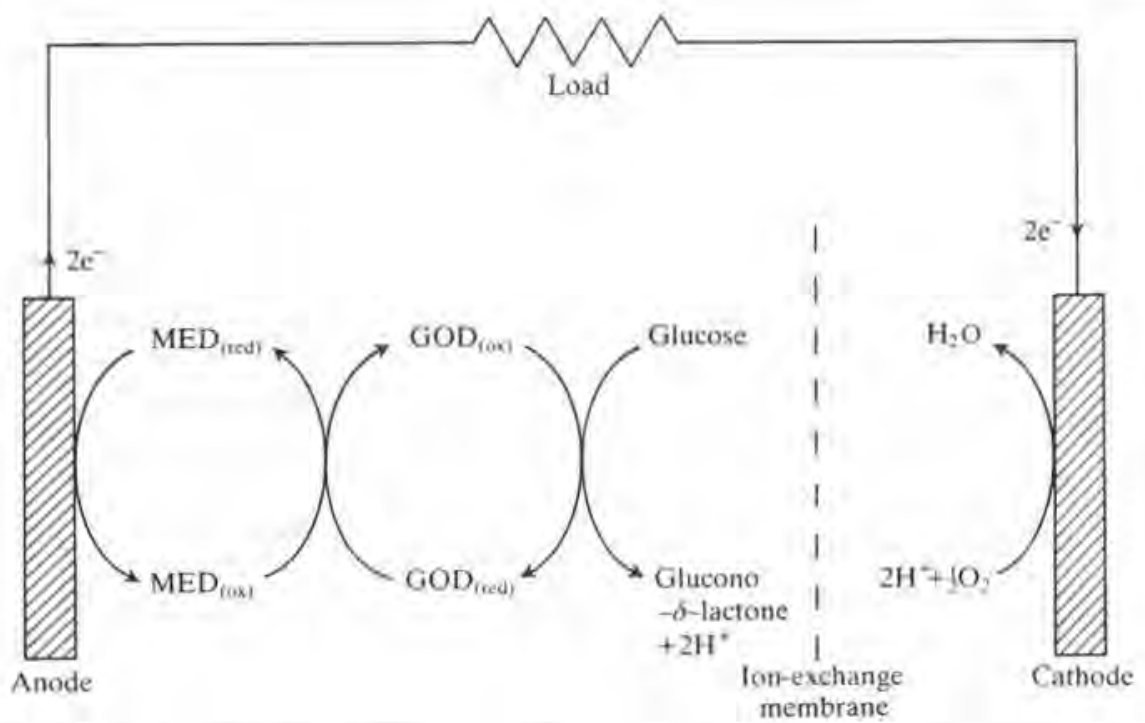

Fig. 16.2 Schematic diagram of a glucose-oxidase-based bio-fuel cell where $\mathrm{MED}_{(\mathrm{ox} / \mathrm{red})}$ are the oxidized and reduced forms of the mediator. $\mathrm{GOD}_{(\mathrm{ox} / \mathrm{red})}$ are the oxidized and reduced forms of the prosthetic group of the enzyme.

Jencons Scientific Ltd, Leighton Buzzard, Bedfordshire, UK) and water prior to use, thereby reducing oxygen interference and loss of solution by evaporation respectively. The addition of glucose to a bio-fuel cell containing glucose oxidase and a soluble mediator, e.g. $N, N, N^{\prime}, N^{\prime}$-tetramethyl- $p$ -phenylenediamine (TMPD) (Table 16.1) maintained at $20^{\circ} \mathrm{C}$ results in the flow of electrons from the enzyme to the anode, via the mediator. The electrons flow around the external circuit to the cathode, where under ideal conditions in the presence of protons and oxygen, water is produced (Fig, 16.2). The resulting current, measured as a voltage across a known resistance, is proportional to the addition of a limiting component until saturation is achieved. Glucose at concentrations as low as $0.1 \mathrm{~mm}$ may be determined rapidly (within five seconds) by measuring steady-state currents (Fig. 16.3). Lower concentrations may be determined by measuring the charge passed, determined by integration of the areas under the current/time profiles.

As a sensor the bio-fuel cell described above suffers limitations due to the presence of an auto-oxidizable mediator although this may be reduced by the incorporation of an insoluble mediator (Higgins et al. 1984), the requirement for an oxygen cathode, and primarily to the need for a membrane. Whilst the diffusion properties of ion-exchange membranes are affected by the $\mathrm{pH}$ of 


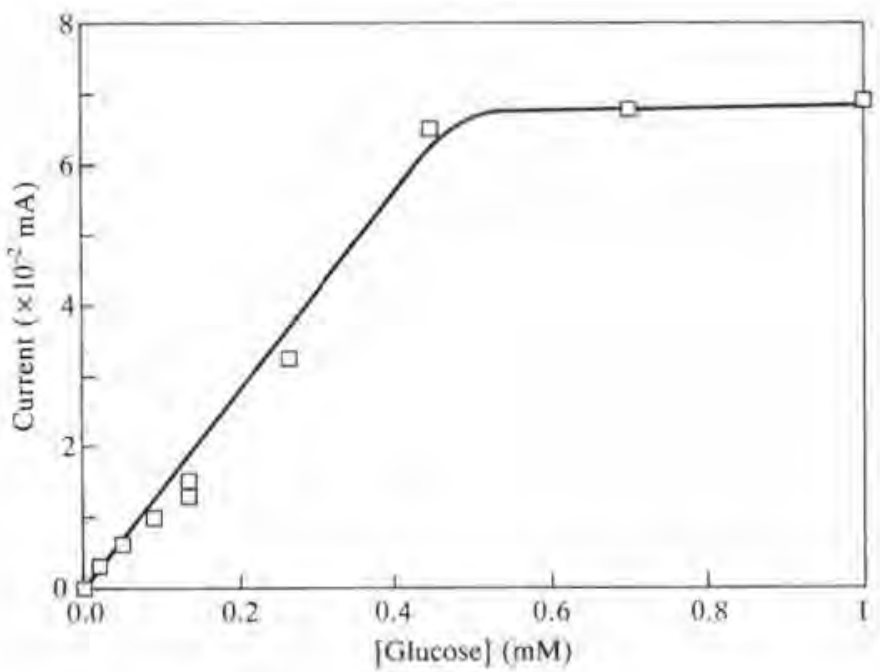

Fig. 16.3 Steady-state current response of a glucose-oxidase-based bio-fuel cell, measured as a voltage across a resistance.

the buffer, the majority retain enzymes whilst allowing the passage of lowmolecular-weight components such as gases, mediators, and substrates (Turner et al. 1980). The cell is affected by changes in the rate of agitation of the anodic compartment, stirring being required to enhance the rate of electron-transfer from the enzyme/mediator to the electrode. The diffusion of components across the membrane results in a reduction in electrontransfer efficiency due to competitive sidereactions occurring. Due to these limitations the device is unsuitable as a sensor in the majority of situations where the quantitative determination of a substrate is required.

\subsection{Poised potential configurations}

The requirement for an air cathode and semi-permeable membrane may be eliminated by the use of a conventional or computer-controlled potentiostat. These devices maintain the potential of the working electrode in a cell with respect to a reference electrode, such as a calomel electrode,

Oxygen interference may be reduced by the use of an oxygen-independent enzyme such as methanol dehydrogenase. The enzyme is capable of oxidizing a range of primary alcohols to the corresponding aldehydes and acids. Incorporation of methanol dehydrogenase into a fixed potential cell in the presence of a soluble mediator, phenazine ethosulphate, enables methanol to be determined in solution at much lower concentrations than is possible using conventional gas-liquid chromatography (Aston et al. 1984). The device 
(Fig. 16.4) consisted of a jacketed reaction vessel $(5.0 \mathrm{ml})$ (Quickfit, Gallenkamp and $\mathrm{Co}$. Ltd., London, UK) maintained at $30^{\circ} \mathrm{C}$, containing borate buffer $(3.0 \mathrm{ml}, 250 \mathrm{mM}, \mathrm{pH} 9.0)$, ammonium chloride $(50 \mathrm{~mm})$, phenazine ethosulphate $(1.0 \mathrm{~mm})$ and crude extract of Methylosinus trichosporium OB3b (3.0 mg protein) prepared from organisms grown on methane as the sole source of carbon and energy (Scott et al. 1981). The mixture was continuously stirred using a magnetic stirrer and purged with nitrogen as described above. The platinum working electrode was cleaned by

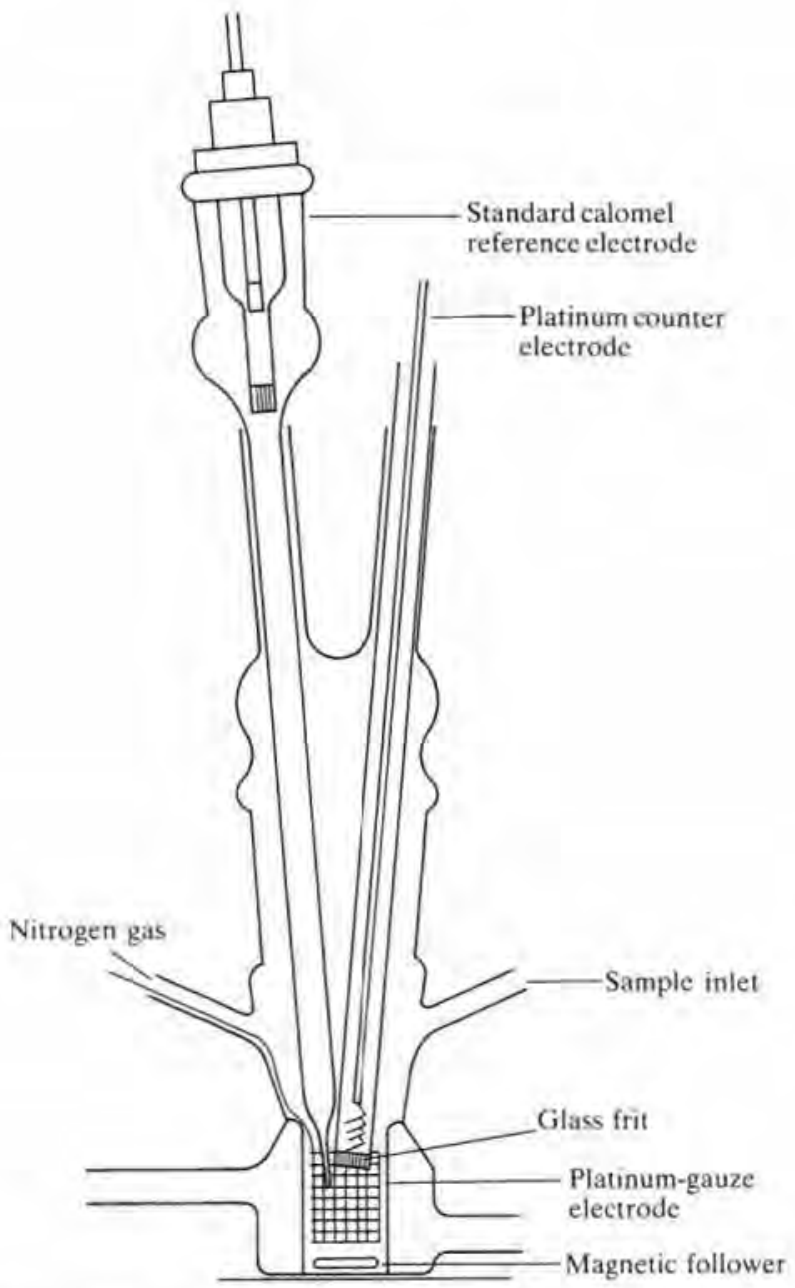

Fig. 16.4 Schematic diagram of a fixed-potential enzyme-based sensor (containing soluble components). (See text for details.) 
cyclic voltammetry, immersed in the reaction mixture, and maintained at $+100 \mathrm{mV}$ (versus SCE) using a potentiostat (H.B. Thompson and Associates, Newcastle Upon Tyne, UK). A platinum counter-electrode was used; isolated from the reaction mixture by means of a glass frit. It was possible to determine formaldehyde and methanol at concentrations as low as $0.02 \mu \mathrm{M}$ by measuring the charge passed upon the addition of aliquots of sample using a CDP4 computing integrator (Pye Unicam Ltd., Cambridge, UK).

This configuration has many advantages over the previously described biofuel cell as an electrochemical sensor. The primary advantage being that it does not utilize a membrane or require sparging with oxygen. However, as in the case of the bio-fuel cell, it is dependent upon the rate of stirring and needs to be maintained anaerobic in the presence of an auto-oxidizable mediator. An alternative is to use a non-auto-oxidizable mediator such as ferrocene, capable of eliciting a high charge-transfer efficiency irrespective of the oxygen tension. Ferrocene and its derivatives are a group of organometallic compounds capable of single-electron transfer between a range of oxidoreductase enzymes such as glucose oxidase, pyruvate oxidase, xanthane oxidase, oxalate oxidase, sarcosine oxidase, lipoamide dehydrogenase, glutathione reductase (Cass et al. 1985), and quinoproteins (Duine and Frank 1981) such as methanol dehydrogenase (Aston et al. 1984) glucose dehydrogenase (D'Costa et al. 1984) and lactate dehydrogenase (Preneta et al. 1984). The incorporation of dimethyl-trimethylferrocene methiodate (1.0 $\mathrm{mm})$, a soluble derivative of ferrocene, in the methanol sensor described above, enabled methanol $(3.0 \mu \mathrm{M})$ to be determined irrespective of varying oxygen tension. This was demonstrated by sparging the cell with either oxygen or nitrogen and measuring the resulting peak current and charge passed. Whilst the device did not require highly trained personal or expensive equipment it did require stirring, and was volume and temperature dependent.

\subsection{Probe configurations}

The incorporation of insoluble mediators into carbon pastes has enabled probe devices to be constructed. The paste, comprising graphite $(2.5 \mathrm{~g})$, liquid paraffin $(1.5 \mathrm{ml})$ and the mediator $(250 \mathrm{mg})$ is thoroughly mixed and placed in a recess in the electrode (Fig. 16.5). The electrode consists of a disc of platinum set into the end $(2.0 \mathrm{~mm})$ of a length of glass tubing $(0.6 \mathrm{~cm}$ diameter) using a non-conductive epoxy resin (Ciba-Geigy Ltd., Cambridge, $\mathrm{UK}$ ). Contact to the external circiut is made via a single-stranded wire bonded to the disc using a silver-loaded epoxy resin (Adams 1969). Prior to use, the platinum disc is cleaned using an aluminium oxide slurry $(0.2 \mu \mathrm{m})$. The enzyme is retained at the surface of the paste electrode using a membrane held 


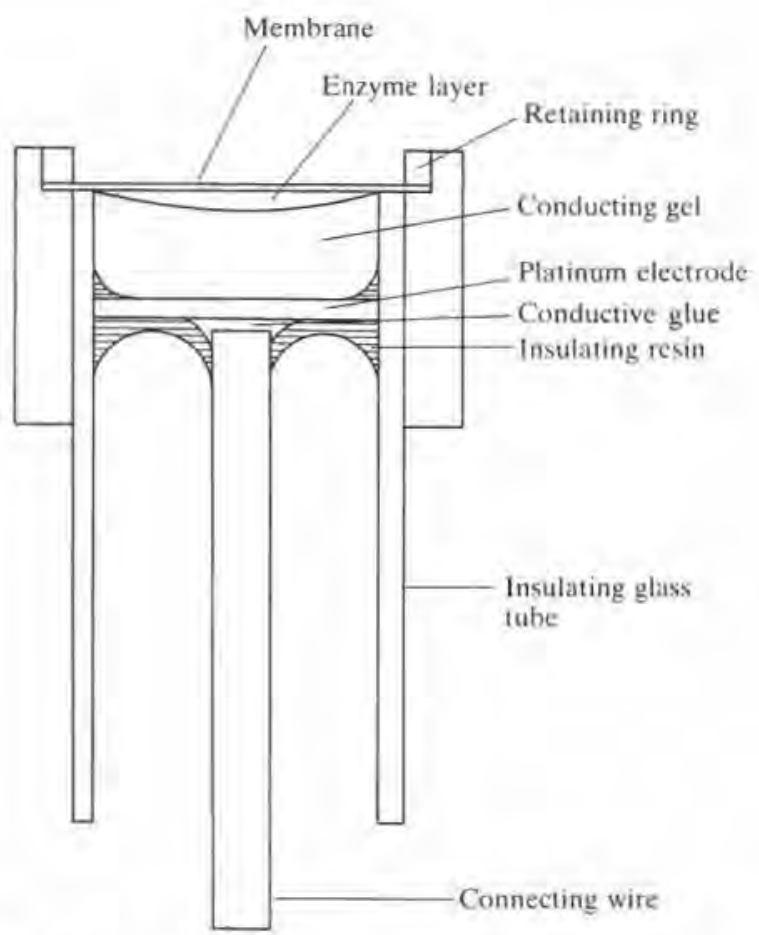

Fig. 16.5 A membrane-retained graphite-paste enzyme-based sensor. The electrode consisted of a platinum disc $(6.0 \mathrm{~mm}$ diameter) set into a pasteur pipette using conductive and non-conductive resins as described in the text.

in position by an ' $\mathrm{O}$ ' ring. Whilst this method of retension is suitable for flexible membranes, inflexible membranes such as ion-exchange membranes require mounting in a holder (Fig. 16.5). The choice of membrane, of which a variety are available commercially (e.g. dialysis, Nuclepore), affects the response time, interference characteristics, and the linear range of the biosensor. Using dialysis membrane, a glucose electrode exhibits a linear response up to $15 \mathrm{~mm}$ (Fig. 16.6). As in the case of the previously described configurations the response is rapid with the steady-state response being attained within a minute. Generally, the thinner the layer of paste, the more reproducible the electrode response. Poised at $+150 \mathrm{mV}$ (versus $\mathrm{SCE}$ ) a glucose oxidase/1,1'-dimethylferrocene electrode with a paste $1.0 \mathrm{~mm}$ thick measured over ten replicates produced a background current of $1.2 \mathrm{~mA} \pm 0.5 \%$ in the absense of glucose, whilst an electrode with a paste $3.0 \mathrm{~mm}$ thick produced a current of $1.4 \mathrm{~mA} \pm 2.0 \%$. In the presence of glucose $(5.0 \mathrm{~mm})$ the current responses with pastes of 1.0 and $3.0 \mathrm{~mm}$ thick were $1.4 \mathrm{~mA} \pm 0.64 \%$ and $1.6 \mathrm{~mA} \pm 2.5 \%$, respectively. A more reproducible surface is achieved by 


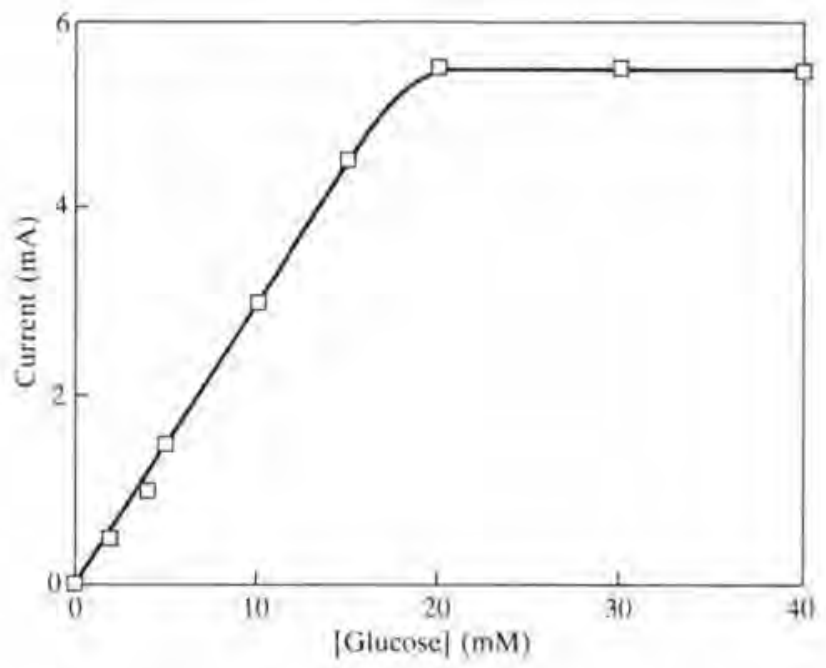

Fig. 16.6 Calibration curve for a membrane-retained glucose-oxidase-based biosensor.

using a glass sphere to form a recess in the carbon paste into which the components may be placed (Fig. 16.5). By incorporating carbon monoxide oxidoreductase in the probe and placing a silver/silver chloride reference around the membrane holder in the presence of buffer has enabled carbon monoxide gas to be detemined (Turner et al. 1984).

The covalent immobilization of glucose oxidase in the presence of 1,1'-dimethylferrocene has enabled convenient glucose analyses to be performed (Cass et al. 1984). A graphite foil (Union Carbide, Ohio, USA) was adopted as the supporting electrode since it possesses a high surface area for immobilization of the components. Discs of carbon $(6.0 \mathrm{~mm}$ diameter $)$ are connected to lengths of wire using a silver-loaded epoxy resin (Johnson Matthey Chemicals, Royston, UK) and allowed to dry. The electrodes are mounted onto the end of a length of glass tubing $(6.0 \mathrm{~mm}$ diameter) using a non-conducting epoxy resin (Ciba-Geigy Ltd, Cambridge, UK); care being taken to ensure that the resin does not seal the edges of the carbon, through which the enzyme gains access. Sealing these edges causes up to $75 \%$ of the current to be lost due to enzyme immobilization predominantly occurring on the electrode surface. Once dry, the electrode is inverted and insulated using a mixture of epoxy resin $(1.8 \mathrm{~g}$, grade 814$)$ and catalyst, triethylenetetramine $(0.23 \mathrm{~g})$ (Polysciences Ltd., Moulton Park, Northampton, UK). Drying may be performed overnight at room temperture or more rapidly by heating in an air oven at $60^{\circ} \mathrm{C}$ for 1.5 hours. Once fabricated, the resistance between the 
connecting wire and the electrode surface is measured and any electrodes exhibiting resistances greater than $3.0 \mathrm{ohms}$ are discarded. Electrodes with high or variable resistances do not elicit linear responses and are not reproducible. The electrodes are doped with the mediator, 1, ' -dimethylferrocene ( $25 \mu \mathrm{l}, 100 \mathrm{~mm}$ ) (Strem Chemicals, Newburyport, USA) dissolved in a suitable solvent, such as toluene, and allowed to air dry. Immobilization of the enzyme is done initially by placing the graphite electrode in acetate buffer (100 mM, pH 4.5) containing 1-cyclohexy1-3(2-morpholinoethyl)carbodiimide metho- $p$-toluenesulformate $(100 \mathrm{mM})$ for 80 minutes at room temperature. The electrodes are subsequently washed with distilled water and placed in glucose oxidase $\left(50 \mathrm{mg} \mathrm{ml}^{-1}\right.$ protein) in acetate buffer $(100 \mathrm{mM}$, $\mathrm{pH} 4.5$ ) overnight or for an hour using carbonate buffer (100 mM, pH 9.5). After immobilization the electrodes are washed and stored frozen at $-20^{\circ} \mathrm{C}$ in phosphate buffer (100 mM, pH 7.4).

Poised at $+150 \mathrm{mV}$ (versus SCE) and maintained at $30^{\circ} \mathrm{C}$, the electrodes exhibited linear responses up to $30 \mathrm{~mm}$ glucose within $30 \mathrm{~s}$ (Cass et al. 1984). At low glucose concentrations $(8.0 \mathrm{mM})$ they were unaffected by changes in the $\mathrm{pH}$ range 7.0-9.0 although an effect was observed at higher concentrations and the response increased with increasing temperature at a rate of $4.0 \%{ }^{\circ} \mathrm{C}^{-1}$ up to $45^{\circ} \mathrm{C}$, above which inactivation occured. The presence of oxygen caused a reduction in the currents produced since it was the natural electron acceptor for the enzyme. The apparent $K_{\mathrm{M}}$ for glucose by glucose oxidase immobilized by this method was $24 \mathrm{~mm}$. This was unaffected by placing a polycarbonate membrane over the probe although a dialysis membrane altered the apparent $K_{\mathrm{M}}$ to $74 \mathrm{~mm}$ changing the electrode response from a kinetically controlled configuration to a diffusion-controlled system, a mechanism which can be used to tailor the range over which linearity is desired.

The electrode has advantages over the previously described systems in that it enables rapid, multiple determinations to be performed on samples. It is simple, easily calibrated and when operated at glucose concentrations normally encountered in the body does not demonstrate the $\mathrm{pH}$-dependence characteristic of the soluble enzyme. It therefore exhibits many of the properties suitable for clinical analysis and is readily applied to in vitro analysis. The application of micromanipulation techniques to minaturized electrodes (Silver 1976; Chapter 21) should facilitate the evolution of in vivo devices.

\subsection{Strip devices}

The low currents produced by these configurations enabled the electronics to be simplified by coupling the reference and auxiliary electrodes, i.e. using a single counter electrode such as the silver/silver chloride reference electrode. 
The adoption of the two-electrode configuration allows both the working and counter electrodes to be mounted in close proximity on a suitable base material thereby reducing the volume of sample required (Fig. 16.7). The silver/silver chloride reference electrodes made from silver foil (BDH Chemical Ltd., Poole, Dorset, UK) were polished using an aluminium oxide slurry $(0,2 \mu \mathrm{m})$ (BDH Chemicals Ltd., Poole, Dorset, UK). When used in association with the previously described poised potential devices the foil $(40 \times 5.0 \times 0.13 \mathrm{~mm})$ is connected to the external circuit by means of a soldered wire insulated using a non-conductive epoxy resin (Giba-Geigy, Duxford, Cambridge, UK). The electrode is immersed in a solution of hydrochloric acid $(1.0 \mathrm{M})$ at a potential of $+400 \mathrm{mV}$ (versus SCE) for $30 \mathrm{~s}$ and rinsed in distilled water prior to use. When incorporated onto a strip device one surface is polished with an aluminium oxide slurry, cut into squares $(3.0 \times 3.0 \mathrm{~mm})$, and bonded to the base using a conductive silver resin (Johnson Matthey Chemicals Ltd. UK).

Squares of papyex $(3.0 \times 3.0 \mathrm{~mm})$ were bonded onto the base electrode using colloidal graphite, after firmly pressing into position, any excess carbon was removed. Prior to immobilization of the enzyme, as described previously, it is necessary to insulate the reference electrode using a layer of silicone rubber to eliminate inactivation of the enzyme by silver ions. In the

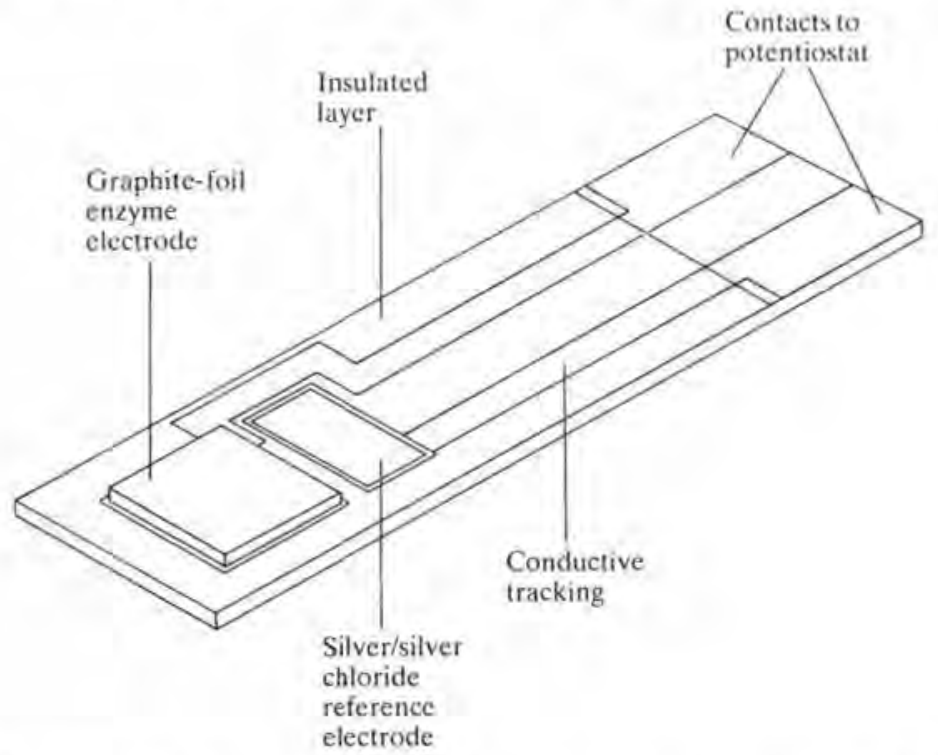

Fig. 16.7 Schematic diagram of a glucose-oxidase-based enzyme electrode. Both the working and silver/silver chloride electrodes were mounted horizontally on a ceramic base. (See text for details.) 
case of glucose oxidase, immobilization is performed as described previously with the silicone rubber protector being removed prior to use.

\subsection{Manufacturing considerations}

Amperometric enzyme electrodes have been shown to operate under laboratory conditions, although they may be considered to be in their infancy and problems may arise when applied to practical situations. These stem from the requirement to control the chemical and physical conditions which influence the catalytic reactions occurring within the electrode. The use of a non-auto-oxidizable mediator reduces the effect of oxygen interference with a further reduction achieved by the use of an oxygen-independent enzyme, e.g. quinoproteins. Temperature and $\mathrm{pH}$ dependence may be reduced by the incorporation of an excess of enzyme.

This configuration although overcoming many of the problems encountered with those described previously, is not amenable to mass production. In order to produce enzyme electrodes economically and satisfy commercial requirements several criteria must be fulfilled with the components being available in sufficient quantity and quality and fabricated under stringent manufacturing protocols (Sharp 1983).

If the strip device is developed it is necessary to be able to mount both electrodes in close proximity on the same base and connect these to the external circuit. A variety of possible base materials are available ranging from ceramics to plastics and even cardboard. Once a suitable base material is obtained it is necessary to deposit a low resistance conducting bridge between the edge connector and electrodes. A variety of techniques are available depending on the temperature the base material is capable of withstanding (Bunshah et al. 1982). Whilst ceramics are capable of withstanding temperatures in the region of $150^{\circ} \mathrm{C}$, plastics deform at these elevated temperatures. Two methods which allow contacts to be deposited at low temperatures include electroless goldplating (Feldstein 1974) and vacuum deposition techniques (Smith 1976). By exposing areas of the base, the desired electrode and connector may be deposited with the connecting tracking subsequenty insulated. Porous carbon electrodes may be manufactued by a continuous technique involving sequential exposure of the material to reagents contained in tanks. Costs may be reduced if immobilization of the enzyme is not incorporated in the procedure and adsorption is sufficient. In the case of the immobilization of glucose oxidase to porous carbon, omitting 1-cyclohexyl-3(2-morpholinoethyl)-carbodiimide metho- $p$ toluenesulformate from the immobilization procedure causes only a $25 \%$ reduction of the initial current indicating that adsorption of the enzyme may be sufficient for these electrodes to function.

Reference electrodes may be preformed as described previously, cut, and 
bonded to the base, The working electrodes may be coated with a membrane thereby changing the response time and the linearity of the electrode. Whilst commercial membranes are available which may be bonded to the base it is possible to spray-coat membranes, such as cellulose acetate; the properties of the membrane being dependent on the particular solvent, or solvents, used. The effect of membranes and electrode configurations on the electrode linearity can be seen from the results presented. In the case of the fuel cell and the first poised potential device described in which a membrane is not used, the response is linear up to $0.45 \mathrm{~mm}$ (Fig. 16.3). By variation in the configuration or the use of a membrane, the linearity can be extended to cover the range up to $20 \mathrm{mM}$ (Fig. 16.6).

Once completed, enzyme electrodes must be stable during storage prior to use. The majority of enzymes may be stored frozen in buffer, as above, or refrigerated at $4{ }^{\circ} \mathrm{C}$ (Yellow Springs Instruments, Ohio, USA; Thai and Yeo 1983). A more practical approach is to store the electrodes dry. This may be achieved by air drying or freeze drying and storing the electrodes desiccated in the manner adopted by manufacturers of diagnostic strip devices (Ames Company Ltd., Bucks, UK).

\subsection{Conclusion}

Our knowledge of electrochemical biosensors has advanced rapidly over the last few years. This has been due to the interaction of biochemical, electrochemical, and electronic disciplines coupled with corresponding financial stimulation. The commercial development of enzyme electrodes in diagnostic analyses has already been demonstrated, enabling metabolites such as glucose concentrations to be routinely determined in clinical laboratories. Self monitoring usually involves patients performing a visual comparison with a colour chart or more accurately using a reflectance meter (Chiasson et al. 1984). The amperometric systems described in this chapter have particular advantages over presently available methods including the ability to quantitatively determine the concentration of a specific metabolite, irrespective of the oxygen tension, without pretreating the sample or washing prior to the determination. Electrodes ideally should exhibit stability both during storage and during operation, with their reproducibility of response falling within predetermined constraints. In addition, they must not be subject to interferences from other metabolites. The ability of these electrodes to perform well in turbid solutions such as blood may result in their application in other areas such as environmental and industrial analysis.

\section{Acknowledgements}

The author would like to thank his colleagues at Genetics International Inc. in particular Dr D. Scott. 


\section{References}

Adams, R. M. (1969), Electrochemistry at solid electrodes (ed. A. J. Bard). Marcel Dekker, New York.

Aston, W. J, and Turner, A.P.F. (1984). Biosensors and biofuel cells. In Biotechnology and genetic engineering reviews, (ed. G. E. Russel), Vol. 1 pp. 89-120. Intercept, Newcastle upon Tyne.

- Ashby, R. E. A., Scott, L. D., Higgins, I. J, and Turner, A. P. F. (1984). Enzyme based methanol sensor. In Change and field effects in biosystems (eds. M. J. Allen and P.N. R. Usherwood), pp. 491-8. Abacus Press, Tunbridge Wells.

Bennetto, H. P. (1984). Microbial fuel cells. Life Chemistry Reports 2, 363-453.

Birch, K., Hilderbrandt, P., Marshall, M. O. and Sestoff, L. (1981). Self monitoring of blood glucose without a meter. Diabeles Care 4, 414-16.

Bunshah, R. F., Blocher, J. M., Bomfield, T. D., Fish, J. C., Ghate, P. B., Jacobson, B. E., Mattox, D. M., McGurie, G. E., Schwartz, M., Thornson, J. A. and Tucker, R.C. (1982). Deposition technologies for films and coatings, development and applications. Nayes Publications, New Jersey.

Carr, P. W. and Bowers, L. D. (1980). Immobilised enzymes. Analytical clinical chemistry. John Wiley, New York.

Cass, A. E. G., Davis, G., Green, M. J. and Hill, H. A. O. (1985). Ferricinium as an electron acceptor for oxido-reductases. Journal of Electroanalytical Chemistry. $190117-9$.

-Francis, G. D., Hill, H. A. O., Aston, W. J., Higgins, I. J., Plotkin, E. V., Scott, L. D. L. and Turner, A.P. F. (1984). Ferrocene-mediated enzyme electrode for the amperometric determination of glucose. Analytical Chemistry 56, 667-71.

Chiasson, J. L., Morrisaet, R. and Hamet, P. (1984). Precision and costs of techniques for self-monitoring of serum glucose levels. Canadian Medical Research Association Journal 130, 38-43.

Davis, G., Hill, H. A. O., Aston, W. J., Turner, A. P.F. and Higgins, I. J. (1983). Bioelectrochemical fuel cell and sensor based on a quinoprotein. Enzyme and Microbial Technology 5, 383-8.

D'Costa, E. J., Duine, J. A., Dokter, P., Turner, A. P. F. and Higgins, I. J. (1984). Kinetics of a microbial quinoprotein glucose dehydrogenase. Society for General Microbiology Quarterly 11, M11.

Dietzler, D. N. and Smith, C.H. (1980). Carbohydrates. In Gradwolls clinical laboratory methods and diagnosis (eds. A. C. Sonnenwirth and L. Jareft), Vol. 1, pp. 210-49. C. D. Mosby, St. Louis.

Duine, J. A. and Frank, J. (1981). Quinoproteins: a novel class of dehydrogenases. Trends in Biochemical Science 6, 278-80,

Feldstein, N, (1984). Electroless plating in the electronics industry. Plating 141-53.

Guidotti, G., Colombo, J.P. and Foa, P.P. (1961). Enzymic determination of glucose. Analytical Chemistry 33, 151-2.

Higgins, I. J., Aston, W. J., Best, D. J., Turner, A. P. F., Jezequel, S. G. and Hill, H.A.O. (1984). Applied aspects of methylotrophy: Biochemical applications, purification of methanol dehydrogenase, mechanism of methane monooxygenase. In Microbial growth on CI compounds (eds. R. L. Crawford and R. S. Hanson). American Society for Microbiology, Washington. 
Lowe, C., Goldfinch, M. J. and Lias, R.T. (1983). Some novel biomedical biosensors. In Biotech 83 , pp, 665-78. Proceedings of the International Conference on the Commercial Applications and Implications of Biotechnology. Online Publications, Northwood, London.

Mosbach, K. and Danielsson, B. (1981). Enzyme thermister devices. Analytical Chemistry 53, 83-94.

Neese, J. W., Duncan, P., Bayse, D., Robinson, M., Cooper, T. and Stewart, C. (1976). Development and evaluation of a Hexakinase/glucose-6-phosphate dehydrogenase procedure for use as a National Reference Method. U.S. Department of Health, Education and Welfare. US. Public Health Service Center for Disease control, Atlanta, GA. HEW Publication Number (CDC) 77-8330, 147pp.

Passey, R. B., Gillum, R. L., Fuller, J.B., Urry, F.M. and Giles, M. L. (1977). Evaluation and comparison of 10 glucose methods and the reference method recommonded in the proposed product class standard (1974). Clinical Chemistry 23, 131-9.

Plotkin, E. V., Higgins, I. J. and Hill, H. A. O. (1981). Methanol dehydrogenase bioelectrochemical cell and alcohol detector. Biotechnology Letters 3, 187-92.

Preneta, A. P., Turner, A. P.F. and Higgins, I. J. (1984). Enzyme-based lactate sensor. Society for General Microbiology Quarterly 11, M11.

Sawyer, D. and Roberts, J. (1974). Experimental electrochemistry for chemists, pp. 67-79. Wiley, New York.

Scott, D. Brannan, J. and Higgins, I. J. (1981). The effect of growth conditions on Intracytoplasmic membranes and methane mono-oxygenase activities in Methylosinus trichosporium OB3b. Journal of General Microbiology 125, 63-72.

Sharp, J. R. (1983). Guide to good pharmaceutical manufacturing practice. HMSO publications. Grovenor Press, Portsmouth.

Shaw, M. (1963). Biochemical fuel cells. In Proceedings of the 17th Annual Power Sources Conference. 1, 53-6.

Sherwood, M. J., Warchal, M.E. and Chen, S,-T. (1983). A new reagent strip (Visidex) for determination of glucose in whole blood. Clinical Chemistry 29. 438-46.

Silver, I. A. (1976). An ultramicro glucose electrode. In Ion and electrode in biology and medicine (eds. M. Kessler, L.C. Clark, D. Lubbers, I. A. Silver and W. Simon), pp, 189-92. Urban and Schwarzenberg, Munich, Berlin.

Smith, H. R. (1976). Vacuum deposition techniques, methods of aluminium evaporation. Metal Finishing Sept., 42-47.

Thai, A. C. and Yeo, P.P. B. (1983). Stable blood glucose test strips and reflectance meters. Singapore Medical Journal 24, 45-7.

Turner, A. P.F., Aston, W. J., Higgins, I. J., Davis, G. and Hill, H. A. O. (1980). Applied aspects of bioelectrochemistry: Fuel cells, sensors and bioorganic synthesis. Biotechnology bioengineering Symposium 12, 401-12.

_ Bell, J. M., Colby, J., Davis, G. and Hill, H. A. O. (1984). Carbon monoxide: Acceptor oxidoreductase from Psuedomonas thermocarboxydvorans strain C2 and its use in a carbon monoxide sensor. Analytica Chimica Acta 163, 161-74.

Walter, B. (1983). Dry reagent chemistry in clinical chemistry. Analytical Chemistry $55,499 a-514 a$. 


\title{
Redox-mediated electrochemistry of whole micro-organisms: from fuel cells to biosensors
}

\author{
H. PETER BENNETTO, JONATHAN BOX, GERARD \\ M. DELANEY, JEREMY R. MASON, SIBEL D. ROLLER, \\ JOHN L. STIRLING, and CHRISTOPHER F. THURSTON
}

\subsection{Introduction}

\subsection{I 'Direct' and 'indirect' whole-cell sensors}

Until recently the use of intact microbes in biosensors has been restricted to an 'indirect' mode wherein the biocatalyst functions in conjunction with attractively simple, familiar sensing elements such as the $\mathrm{pH}$ electrode (substrate-induced generation of acidic or basic products) or the conventional oxygen electrode (substrate-dependent respiration). The general utility of these devices may be judged from the account given by Karube in chapter 2 of this volume and from recent reviews (Aston and Turner 1984; Guilbault 1984; Mărgineanu et al, 1985). In particular, attention has been drawn to their potential for use in clinical analysis of biological fluids, for monitoring fermentation systems, in toxological studies, and for estimation of antibiotics (Kobos 1983; Simpson and Kobos 1984; Corcoran and Rechnitz 1985; Findl et al. 1985).

In the present article we discuss a different approach, based largely on recent studies of microbial fuel cells, in which micro-organisms give a 'direct' electrical signal. The biochemical fuel cell containing cells or cell components has long attracted attention as a source of 'alternative energy' from biological fuels (Aston and Turner 1984; Bennetto 1984), and lately as a possible route to synthesis of compounds of potential commercial interest (van Dijk et al. 1985). Various types of bio-fuel cell were intensively studied in the 1960s and 1970s, with motivation from NASA-funded research programmes aimed at the development of ancillary power sources. However, most of these devices relied on the principle of electrochemical oxidation of a secondary metabolic product such as formate or hydrogen for production of power, and were relatively inefficient. Nevertheless the production of hydrogen by Clostridium butyricum was ingeniously used in the first fuel cell-type microbial sensors for measurement of BOD (biological oxygen demand) in wastewaters (Karube et al. 1977) and for the estimation of formic acid (Matsunaga et al. 1980).

Recently a renewed interest in microbial fuel cells and sensors has resulted 
from the discovery that 'redox' coupling reagents can be used to link microbial respiratory processes to an electrode directly and effectively. The source of power in a 'direct' microbial fuel cell is the well-known reducing power of the micro-organisms stemming from 'redox'-active substances produced in the initial or intermediate stages of catabolism. Thus electrons from intracellular electron-rich substances can be diverted from the normal respiratorychain pathways by appropriate coupling reactions, and thence delivered via the anode to an external circuit (Bennetto et al. 1980, 1983). With some design modification it is possible to adapt the fuel cell for sensor use, since the electron flow resulting from the electrochemical oxidative process is readily available for measurement by amperometric or other methods, and under suitable conditions the signal becomes substrate-dependent (Turner et al. 1982). The essential difference between the 'direct' and 'indirect' modes of operation is that in the indirect method signals are generated by the Nernstian response of an electrode to concentrations of stable metabolic products, or by means of the polarographic response to oxygen, whereas in the direct method a true bioelectrochemical connection is established between substrate and electrode via the electroactive products of biocatalysis.

In the following account we outline the bioelectrochemical background, and attempt to draw up guidelines for the construction of mediated wholecell sensors. Illustrative preliminary results on glucose and alcohol sensors are also presented.

\subsubsection{Electron transduction from enzymes and whole cells}

There are intrinsic differences between the mechanism of signal-generation from whole cells and that of mediated enzyme electrodes, which is discussed elsewhere in this volume by Cardosi and Turner (Chapter 15) and by Albery and Craston (Chapter 12). In the latter case electrons are taken from reduced enzymes at a donor site (or a limited number of sites) provided that there is relatively free access for the mediator. Access of a mediator to sources of reducing power within an organism is restricted, however, by the cell walls and membrane, and these same sources may be varied in number and location (enzymes, pyridine nucleotides, quinone intermediates, cytochromes). In principle it is possible for a mediator to interact with a particular intracellular electron donor, but since complex interactive redox states are present it is useful for many purposes to view the electrons as residing in a 'reducing pool' within the cytoplasm. Some of the factors to be considered in these systems also relate to sensor applications of protein electrochemistry and electron transduction to and from cytochrome centres, which have been recently reviewed (Turner et al. 1982; Cass 1984). 


\subsection{Whole cells as catalysts in biosensors}

Since most of the enzymes employed for use in sensors have been isolated from micro-organisms, it is logical that the organisms themselves should be regarded as potential biocatalysts, even if their manipulation appears to present more complex problems (Aston and Turner 1984). Microbes can be judiciously selected for particular sensor applications from an enormous range at our disposal (aerobes, anaerobes, chemolithotrophs, photosynthetic organisms, etc.), bearing in mind their widely differing respiratory physiology and biochemistry. There are no well-defined guidelines for selection, apart from what is known about the utility of the enzymes and with due regard to the lessons from many previous studies of indirect microbial sensors. The directions of future work with micro-organisms may be influenced, however, by a number of distinct advantages and disadvantages which they offer.

(a) The versatility of micro-organisms makes them potentially suitable as catalysts for a very wide range of substrates, and thus potential analytes, that can be utilized. In principle this covers almost every type of naturallyoccurring carbon compound (Gunsalus and Schuster 1961).

(b) The cost of production is very moderate for many organisms, whereas the isolation of an enzyme from its source can be expensive.

(c) Where oxidation in the cells involves several degradative stages to provide reducing intermediates, whole cells might provide a bigger electrochemical signal than a single enzyme. For example, in one type of glucose sensor the oxidation of glucose to gluconolactone using glucose oxidase yields two electrons per molecule of substrate (e.g. see Cass et al. 1984):

$$
\mathrm{C}_{6} \mathrm{H}_{12} \mathrm{O}_{6}=\text { Gluconolactone }+2 \mathrm{H}^{+}+2 \mathrm{e}^{-}
$$

In contrast, the complete oxidation of glucose in a whole-cell may be represented by the equation

$$
\mathrm{C}_{6} \mathrm{H}_{12} \mathrm{O}_{6}+6 \mathrm{H}_{2} \mathrm{O}=6 \mathrm{CO}_{2}+24 \mathrm{H}^{+}+24 \mathrm{e}^{-}
$$

A substantial proportion of this large electron yield may be realized in practice (Delaney et al. 1984). It should be noted, however, that microbial catalysts are bulkier than enzymes, and estimates of the relative activity of whole cells and enzymes in bio-fuel cells suggest that a given weight of whole cells is about as effective as the weight of the relevant enzyme(s) it contains.

(d) Some potentially useful enzymes are unstable, or may need a hydrophobic environment or sophisticated methods of immobilization in order to function. In whole micro-organisms, stability and activity is conferred by the natural medium of the cell in a way that is difficult to mimic, and the cells themselves can be immobilized by simpler procedures (Guilbault 1984; Karube and Suzuki 1983). 
(e) Enzymes might be better shielded within micro-organisms from interfering (signal-generating) substances and inhibitory solutes such as heavymetal compounds, which would be present in many test samples.

(f) The use of enzymes in bioelectrochemical electron transfer frequently demands the presence of a co-enzyme. Addition of exogenous cofactors is unnecessary, however, in the case of whole organisms, because these substances are regenerated within the cells.

(g) A number of micro-organisms are genetically well-characterized, and methods of strain-selection for increasing particular enzyme proteins are well developed. The judicious use of mutants could provide an additional range of activity, selectivity, and specificity.

(h) Both the direct and indirect modes of signal-transduction from whole cells offer scope for non-genetic biotechnological manipulations. In particular, the direct method promises a versatility for transduction of electrons from various intermediate stages of the oxidative pathway, possibly with some selectivity provided by differences in the response to different mediators (see section 17.3). In the indirect method, production of a response-producing active product by a multi-step oxidation process is more likely to be subject to kinetic inhibition, whereas in the direct method, electron transduction usually by-passes much of the respiratory chain.

The advantages of using micro-organisms for sensor applications must, however, be balanced against the known disadvantages, and the following points are particularly relevant.

(a) The great flexibility and versatility of micro-organisms might also limit the selectivity and sensitivity of microbial sensors. The organisms may oxidize substrates other than the ones(s) we wish to sense, which could be a particular problem in biological fluids containing high concentrations of active substances such as glucose. Also cells deprived of one substrate may 'switch' to an alternative metabolic path.

(b) There may be problems of biological stability attached to the storage and retention of activity of organisms over long periods. Surprisingly little is known of the long-term stabilities of either enzymes or whole cells.

(c) In reductive reactions of organisms, the 'tapping' of respiratory activity with the aid of mediators may be subject to interference from atmospheric oxygen.

(d) Difficulties are associated with the need to prevent loss of soluble mediators by immobilization or other methods of retention.

Work in progress suggests the feasibility of constructing sensors containing both Gram-positive and Gram-negative organisms, as well as strict. aerobes and facultative anaerobes (see Table 17.1). Several of the advantages and disadvantages outlined above are borne out by our recent experimental 
Table 17.1 Whole-organism sensors based on the bio-fuel cell principle: organisms used or under investigation

\begin{tabular}{|c|c|c|}
\hline Organism & Substrate & Reference $^{a}$ \\
\hline Clostridium butyricum & $\mathrm{BOD}^{b, c}$ & Karube et al, 1977 \\
\hline Clostridium butyricum & Formic acid ${ }^{e}$ & Matsunaga et al. 1980 \\
\hline E. coli & Glucose & Hanazato and Shiono 1983 \\
\hline E. coli ML308 & Lactose & Roller et at. 1983 \\
\hline Proteus vulgaris & Glucose, sucrose & \\
\hline Anabaena variabilis & $\mathrm{CO}_{2}, \mathrm{~h} p$ & Bennetto et al. 1984 \\
\hline Methylomonas methylovora & Ethanol, methanol & Box and Mason 1986 \\
\hline Alcaligenes eutrophus & Succinate, pyruvate & \\
\hline Pseudomonas putida & Succinate & \\
\hline Erwinia carotovora & Sucrose & \\
\hline Nocardia salmonicolor & Acetate & \\
\hline Hansenula anomala & D, L-Lactate & Scheller et al. 1985 \\
\hline Lactobacillus fermenti & Vitamin $B_{1}$ & Scheller et al. 1985 \\
\hline
\end{tabular}

${ }^{2}$ This work, and unpublished results, unless otherwise stated.

${ }^{\mathrm{B}} \mathrm{BOD}=$ biological oxygen demand.

"Indirect' fuel-cell sensor depending on microbial production of hydrogen (no mediator).

findings, some of which are detailed in Section 17.4, while possible methods of improvement are discussed in Section 17.5.

\subsection{Generation of electricity by micro-organisms}

\subsubsection{Microbial fuel cell studies}

Studies of biochemical fuel cells have demonstrated that electrontransduction from micro-organisms is remarkably efficient if a suitable 'redox' mediator is included in the analyte (Bennetto et al. 1980, 1983). Progress in the development of direct bio-fuel cells has been reviewed elsewhere (Wingard et al. 1982; Aston and Turner 1984; Bennetto 1984). The aim of many of these studies has been to identify factors which generally govern the generation of electricity from microbes, in particular the crucial rôle of redox mediators in promoting transfer of electrons across the cell wall and membrane (Roller et al. 1984; Delaney et al. 1984), but the potential for bioanode sensor applications was also established (Turner et al. 1982). We also note that the rate of decolourization of redox dyes such as resazurin has long been used to measure bacterial activity in foods and milk (e.g. Proctor and Greenlie 1939); it is but a short step to couple this type of reaction to an electrode or an optical sensor. 


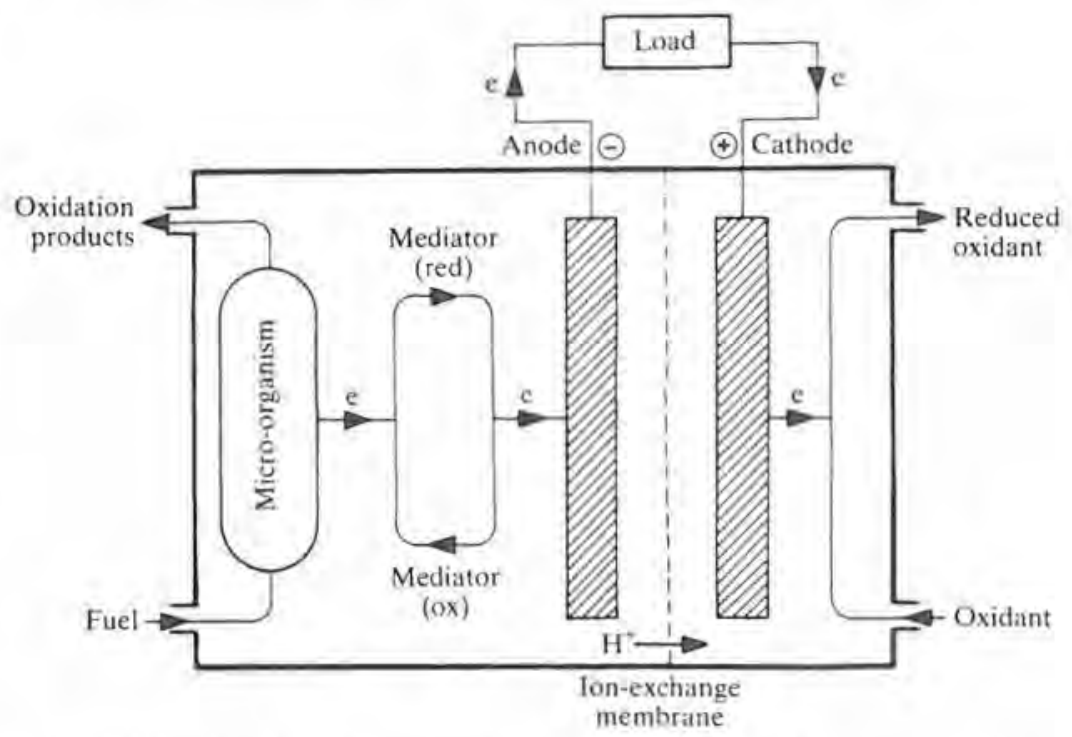

Fig. 17.1 Microbial fuel cell; schematic representation. See Delaney et al. (1984) and Bennetto (1984) for details.

The mode of operation of the microbial fuel cell is shown in Fig. 17.1 (Roller et al. 1984). The typical discharge behaviour observed, and the effect produced by further addition of substrate, is shown in Fig. 17.2 for the thionine-mediated glucose $P$. vulgaris cell. The amount of electricity drawn from the cell, given by the area under the graph, is proportional to the quantity of substrate added. A recent detailed analysis of this system has established the fate of glucose in the anode compartment, and confirms that the coulombic yield from oxidation of glucose is about $50 \%$ (Thurston et al. 1985). These results were obtained with $30 \mathrm{mg}$ freely suspended bacteria, which easily generate a signal of milliamp or hundreds of $\mathrm{mV}$ proportions. Figure 17.2 also illustrates the rapid regeneration of power on a subsequent addition of substrate. For sensing purposes, however, such a device is rather bulky ( $15 \mathrm{~cm}^{3}$ anode compartment) and the response rather slow (several minutes), so that substantial changes of size and configuration are required to convert it to a convenient sensor format. These developments are considered in Section 17.5.

\subsubsection{Mediator-organism interactions}

Fuel cell studies have revealed much information which has a direct bearing on the development of whole-cell sensors. Mediator reduction rates affect 


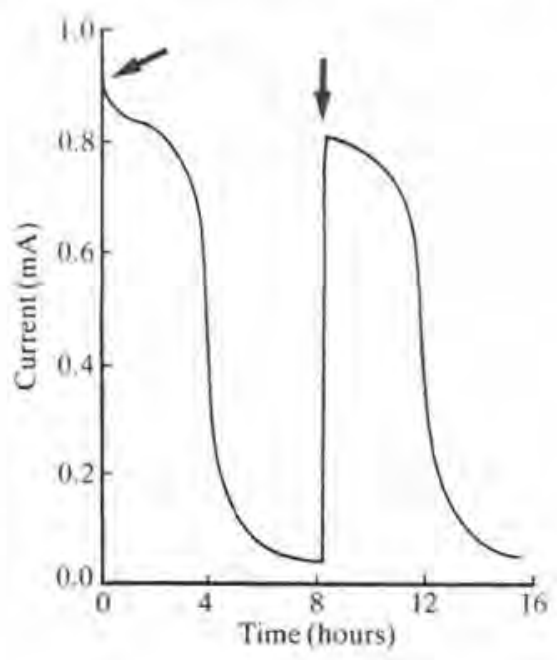

Fig. 17.2 Electricity generation from a glucose/ $P$. vulgaris microbial fuel cell. The analyte contained $0.1 \mathrm{M}$ phosphate buffer, $\mathrm{pH} 7.0 ; 1 \mathrm{mM}$ thionine; $30 \mathrm{mg}$ (dry wt.) organism; $\left(30^{\circ} \mathrm{C}\right)$. Discharge of the cell was through $560 \mathrm{ohm}$ load. Arrows mark additions of $10 \mu \mathrm{mol}$ glucose.

the efficiency of signal-transduction, and the considerable variation for particular organisms and mediators is illustrated by a selection of data in Table 17.2 taken from recent work (Stirling et al. 1983; Roller et al. 1984). Such differences in reactivity, whatever the cause, might be used to differentiate between types of organism, and could be applied to differential sensing and cell-counting. The rates of reduction generally show a dependence on concentrations of organisms, mediator (up to certain limits), and substrate (depending on load conditions). For a given organism and mediator they are

Table 17.2 Rates of reduction of mediators by micro-organisms, $\mu \mathrm{mol} \mathrm{g}^{-1} \mathrm{~s}^{-1}$ (substrate, glucose; $30^{\circ} \mathrm{C}$ )

\begin{tabular}{llllll}
\hline Organism & $Q_{\mathrm{O}_{2}}$ & $\mathrm{TH}$ & $\mathrm{MB}$ & $\mathrm{BCB}$ & $\mathrm{BV}$ \\
\hline E. coli $\mathrm{B} / \mathrm{r}$ & 1.76 & 3.65 & 4.16 & 0.99 & 2.23 \\
$P$. vulgaris & 0.69 & 7.10 & 1.70 & 1.03 & 6.11 \\
$P$. aeruginosa & 1,03 & 1.54 & - & 0.63 & 0.31 \\
\hline
\end{tabular}

$\mathrm{TH}$, thionine; $\mathrm{MB}$, methylene blue; $\mathrm{BCB}$, brilliant cresyl blue; $\mathrm{BV}$, benzyl viologen; $Q_{\mathrm{O}_{2}}$ is the respiratory quotient, or rate of reduction of dioxygen. (It should be noted that the reductions involve $4,2,2,2$, and 1 electrons for $\mathrm{O}_{2}, \mathrm{TH}, \mathrm{MB}, \mathrm{BCB}$, and $\mathrm{BV}$ respectively.) 
quite reproducible and show only small variations (up to $20 \%$ ) depending on growth rates.

The question of what type of mediator molecule or molecular property gives fast electron transfer in whole-cell systems is one which cannot be easily answered. There is no simple dependence on mediator charge, but lipophilicity may play an important rôle in aiding penetration of lipid membranes (Bennetto et al. 1981). The various factors involved have been extensively reviewed elsewhere (Bennetto 1984). The redox levels which are accessible appear to vary for different organisms and mediators, but it is not easy to identify specific reducing centres. Attempts at pinpointing the reducing source may not be meaningful, since the species immediately interacting with the mediator might not be the key source of electrons, and the concentrations and redox states of both the cellular intermediates and the mediator will be interdependent. Some insights into the mechanistic aspects might be gained, however, from kinetic differences in the reduction of mediators. An example is provided by thionine and 2-hydroxy-1,4-naphthoquinone (HNQ), which can both be used to good effect as mediators with whole cells (Bennetto et al. 1985). Thionine reacts particularly rapidly with free NADH in buffer solution (and orders of magnitude faster than many of its derivatives); in contrast, HNQ is not reduced at all by free NADH. This suggests that NADH alone cannot reduce HNQ in the cell, and that electrons must therefore come from a different intermediate. Interestingly, HNQ is reduced by NADH in the presence of membrane particles (Delaney et al. 1986).

A drawback of many mediator substances for sensor use is their poor longterm stability, usually more serious for reduced forms. It should also be noted that many of the requirements of mediators for use in bio-fuel cells differ from those for use in sensors, where some compromises are necessary. Soluble mediators present a problem of mediator loss, whereas insoluble ones may give rise to diffusion-limited currents. The effects of solubilizing groups are complex: positively charged groups will encourage migration of reduced mediator to the anode but tend to promote undesirable absorption there (as in the case of thionine), while negative charges inhibit penetration of negatively charged cell walls and charge transfer to a negative electrode, though this is not evident in the case of the HNQ anion. Attention has been focused recently on the use of ferrocene and its derivatives as mediators, mainly because the solubility and electrochemical properties of these compounds can be varied by substituents as required. Insoluble ferrocenes have been exploited in the construction of a glucose enzyme sensor (Cass et al. 1984), but some uncertainty remains relating to the mechanism of mediation. Thus importance is attached to the diffusion of oxidized ferrocene cation away from the electrode, but it is not clear how the very insoluble neutral forms move electrons to the electrode following reduction of the cation by the enzyme. 


\subsubsection{Electrochemical considerations}

17.3.3.1 Mode of operation of microbial sensors Direct sensors will probably be operated to greater advantage in the amperometric, rather than the potentiometric mode, and will depend on the rapid establishment of poised steady-state electrode potentials and currents through the action of a mediator. Following the addition of substrate, generation of electrons by the organism leads to an increase in the concentration of reduced mediator (and hence the redox ratio), which in turn gives a potential shift and drives a current through an external load. With an appropriate choice of load resistance and component concentrations, the amperometric response is measured under steady-state conditions, and the depolarizing action of the organism becomes substrate-dependent. The limits of sensitivity, the accuracy, and the response time of the sensor will depend upon the amount of current obtainable from a given amount of organism and substrate. Apart from the biological considerations, this current will depend on the efficiency of electron-transfer reactions at each end of the electron-transduction process: (a) electron transfer from the electron source in the micro-organism to a mediator, and (b) transfer of electrons from the mediator to the base electrode. Both of these are affected by electrochemical activation or mass transport limitations, and could lead to high polarization effects and poor performance.

17.3.3.2 Polarization effects In considering first the reducing action [process (a)], an essential requirement for the potential to be established rapidly is fast penetration of the mediator both into and out of the cell surface, as in the case of the microbial fuel cell. If rapid penetration is not achieved, either of these steps may become rate determining, and the sensor will respond only sluggishly. (In unfavourable cases, where the cell exterior is less permeable, some improvement might result from the use of more sophisticated mediating systems, as discussed in Section 17.5.) Since the permeation rates are concentration-dependent, there are advantages in poising the potential of the system artificially by potentiostatic means (as illustrated in the example below), so that the concentrations of either the reduced or oxidized mediator do not become too small, and the redox ratio does not approach extreme values. Also, when current is drawn, it should not be so great as to remove excessive amounts of mediator from the reaction area, which would amount to an undesirable effect of concentration polarization. Let us suppose, by way of illustration, that a sensor is designed which uses $1 \mathrm{mg}$ (dry wt.) organism, and the equivalent of $0.1 \mathrm{ml}$ of $1.0 \mathrm{mM}$ mediator solution (a one-electron redox reagent). It therefore contains $10^{-7} \mathrm{~mol}$ mediator, and, using the Faraday constant $F=10^{5} \mathrm{C} \mathrm{mol}^{-1}$, would require $10^{-2}$ coulombs of electricity for complete reduction of the mediator. Experiment shows that 
many active organisms are capable of sustaining mediator-coupled currents of up to $100 \mu \mathrm{A} \mathrm{mg}{ }^{-1}$, equivalent to $10^{-4} \mathrm{C} \mathrm{s}^{-1}$, and would thus produce a relatively small perturbation in mediator concentration. Such a system would (for glucose) remove substrate from the test solution at rates of the order of $10 \mathrm{nmol} \mathrm{s}^{-1}$, so that the test would be virtually non-destructive over an experimental period of, say, 0.5-5.0 min.

Considerations of permeability into the lipid membranes of cells indicate that the ingress or escape of a mediator should equally not be limiting at mediator concentrations of around millimolar (Bennetto 1984). In practice, rapid reduction of the mediator lends itself to a fast-responding sensing device in favourable cases. Thus, response times of $0.5-5 \mathrm{~min}$ have been observed in fuel cells, and these can be much reduced in designs having a mediator which is localized at or near the electrode surface. The rate-determining step for the response of such electrodes may then be the uptake of substrate by the micro-organisms, which is often a very rapid process and should not present serious problems. It is interesting to note that uptake of glucose by $P$. vulgaris in a fuel cell is more rapid when the cell is under load (Thurston et al. 1985).

To assess the importance of the electron-transfer step [process (b), above] it is instructive to use the previous example and express the current in terms of the electrochemical rate equation

$$
i=F k c A
$$

where $c$ is the concentration of reduced mediator delivering charge to the electrode, $A$ is the area of the working electrode (conveniently taken as $1 \mathrm{~cm}^{2}$ ), and $k$ is the specific electrochemical rate constant. Cyclic voltammetry measurements show that several effective mediators have $k$ values of around $10^{-2}-10^{-3} \mathrm{~cm} \mathrm{~s}^{-1}$. Using these figures, the equation above predicts that they would be capable of supporting $0.1-1.0 \mathrm{~mA}$ at $1.0 \mathrm{mM}$ concentration, and polarization of the electrode should therefore be negligible.

By comparison, many indirect sensors depending on oxygen tension or product concentration are poorly poised and provide sluggish electrode kinetics. This, in part, arises from diffusional limitations; the dependence on oxygen consumption is a particular weakness of many systems containing dense populations of aerobic organisms, in which oxygen tension may become vanishingly small and oxygen transfer may be very slow (Clarke et al. 1985). A distinction should therefore be made between whole-cell sensing methods employing the oxygen electrode, which is essentially a polarographic device, and redox-mediated amperometry, which is not.

17.3.3.3 Electrode potentials of mediators For microbial fuel cells, mediators of low redox potential have been favoured in order to maximize the obtainable voltage, but this is not essential for sensors, provided that an 
adequate signal is produced. Likely mediators are those of higher $E^{0}$ which are generally more stable and less prone to re-oxidation by molecular oxygen. Interference from oxygen might be less serious, however, where the kinetics of mediator-organism and mediator-electrode interactions are favourable for rapid electron transduction.

17.3.3.4 Electrode materials Presently available information favours the use of carbon electrodes for satisfactory interfacing of the biocatalyst. These have a range of useful properties (Wang 1981; Besenhard and Fritz 1983) and surface modification by controlled oxidation can provide groups which are ideal for constructing binding links to whole organisms (see section 17.5).

\subsection{Experimental whole-cell biosensors}

The use of electrical outputs from 'direct' bio-fuel cells operated in a sensor mode is illustrated below with results for glucose and ethanol. The two substrate-dependent parameters considered were

(i) total coulombic output of cells under constant load,

(ii) rate of current (or potential) rise.

Peak current, potentiometric, potentiostatic, impedance, and capacitative responses are all also worthy of investigation. Figure 17.3 shows the

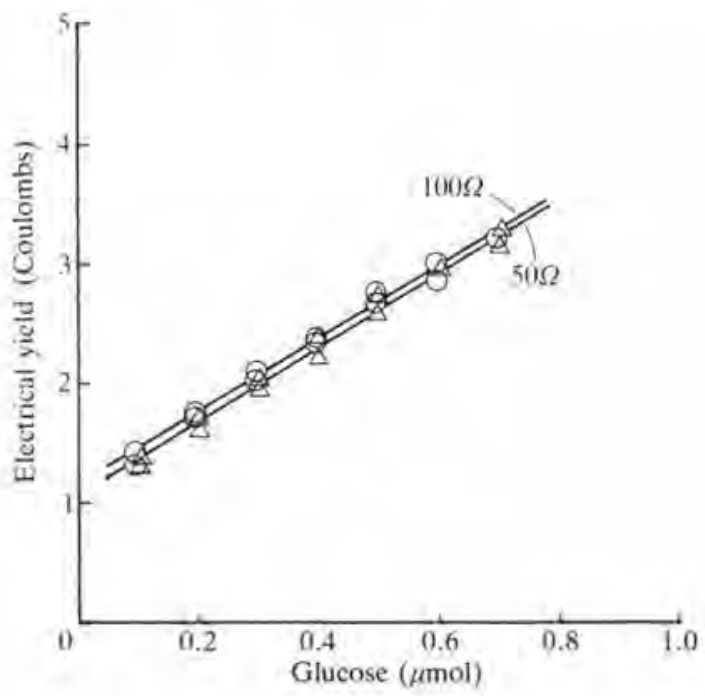

Fig. 17.3 Correlation of electrical output with glucose for a $P$. vulgaris fuel-cell sensor. $60 \mathrm{mg}$ (dry wt.) organism. Resistive loads were $100 \mathrm{ohm}, O$; and $50 \mathrm{ohm}, \Delta$ $\left(30^{\circ} \mathrm{C}\right)$. 


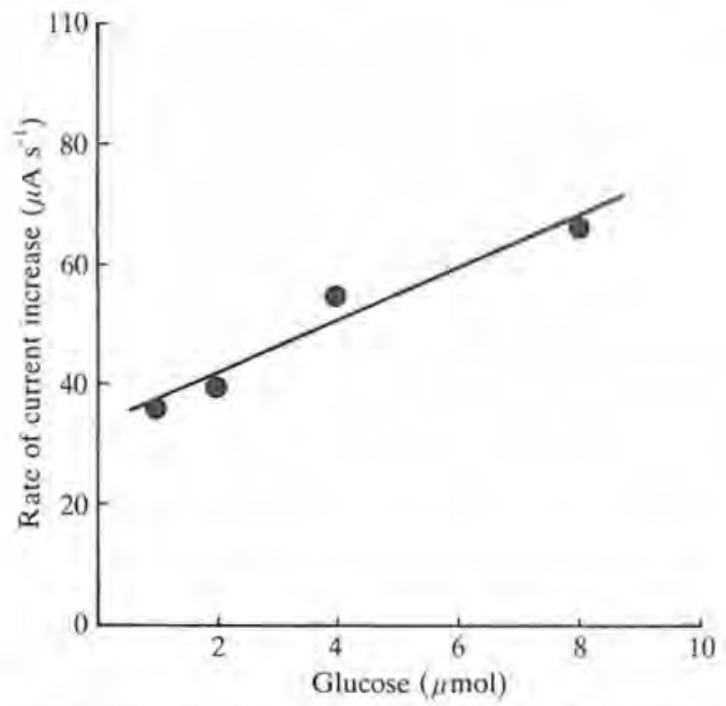

Fig. 17.4 Rate of current increase $v s$. glucose for a $P$. vulgaris sensor fuel cell. $8 \mathrm{~mm}$ 2-hydroxyl-1, 4-naphthoquinone; $50 \mathrm{mg}$ (dry wt.) organism, $\left(30^{\circ} \mathrm{C}\right)$. Resistive load was varied authomatically to maintain constant 0.53 volts.

correlation of coulombic output with glucose concentration for $P$. vulgaris sensor cells similar to those described above (Fig. 17.2). Figure 17.4 shows the concentration dependence of current-time response for a similar cell using HNQ as mediator, and a constant anode potential maintained with an active load device.

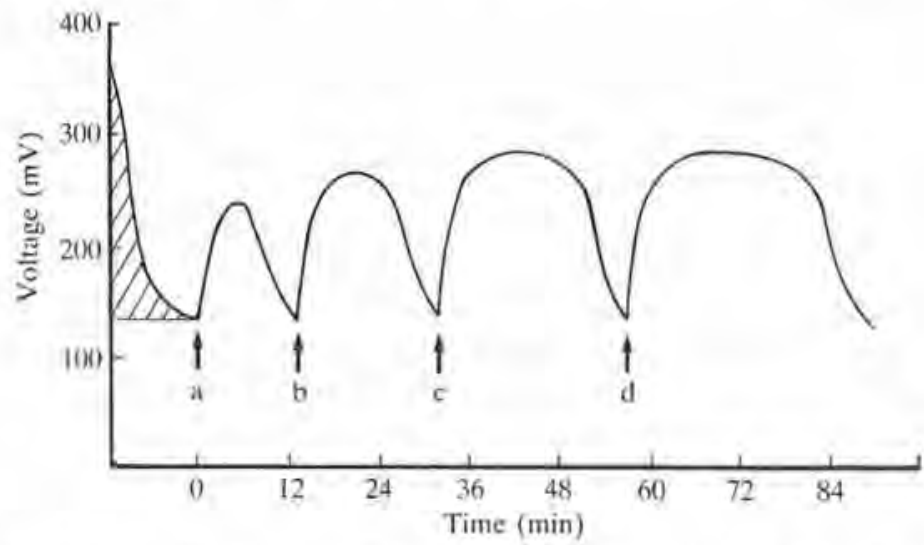

Fig. 17.5 Current-time behaviour of Methylomonas methylovora fuel cell. $1 \mathrm{~mm}$ thionine; $60 \mathrm{mg}$ (dry wt.) organism; $560 \mathrm{ohm}$. Additions of $0.5,1.0,1.5,2.0 \mu \mathrm{mol}$ ethanol were made at a, b, c, d. 


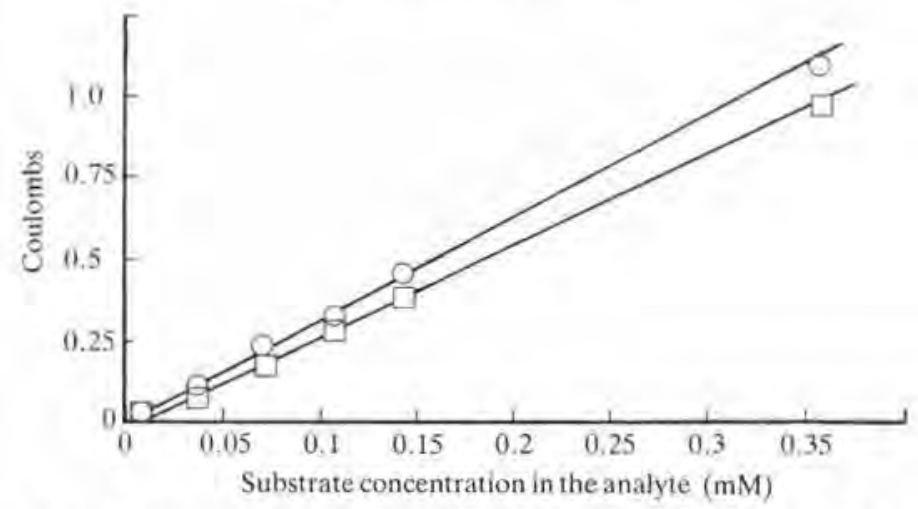

Fig. 17.6 Coulombic output of ethanol/methanol fuel-cell sensor as a function of substrate concentration in analyte. Conditions as for Fig. 17.5. $\square$, ethanol; $\bigcirc$, methanol.

The current-time plots for a Methylomonas methylovora fuel cell from successive additions of ethanol are shown in Fig. 17.5. In this experiment the endogenous capacity of the system was first exhausted (shaded portion). The system responds equally well to ethanol or methanol, the carbon source on which the organisms were grown. The correlation of coulombic output with quantity of ethanol or methanol added is illustrated in Fig. 17.6. The increase of cell voltage (decrease in bio-anode potential) following additions of ethanol was also followed as a function of time over a $30 \mathrm{~s}$ period, and Fig. 17.7 shows the relationship between these rates and the substrate concentration. This system clearly obeys Michaelis-Menten kinetics over the ethanol range $0.05-1.5 \mathrm{mmol}$ (analyte concentration $3.5-105 \mu \mathrm{M}$ ), and thus functions as a very sensitive and reasonably fast sensor. It is interesting that the active enzyme in this methylotroph is an NAD-linked dehydrogenase (cf. Section 17.2.1 (f)) and can be used selectively, since it will not oxidize carbohydrates ( $c f$. section 17.2.2 (a)). On the debit side, however, it is rather unstable ( $c f .17 .2 .2(\mathrm{~b}))$.

\subsection{Future developments}

\subsubsection{General considerations for design of microbial sensors}

Modifications are under way in many laboratories which will transform the 'fuel cell' sensor into the form of a probe. Reduction in the volume of a fuelcell-type sensor from $15 \mathrm{~cm}^{3}$ to $0.5 \mathrm{~cm}^{3}$ reduces the 'dead' time (for mixing and equilibration at porous electrodes) from several minutes to less than $30 \mathrm{~s}$, and the logical development is to localize organisms within a small volume adjacent to the electrode. 


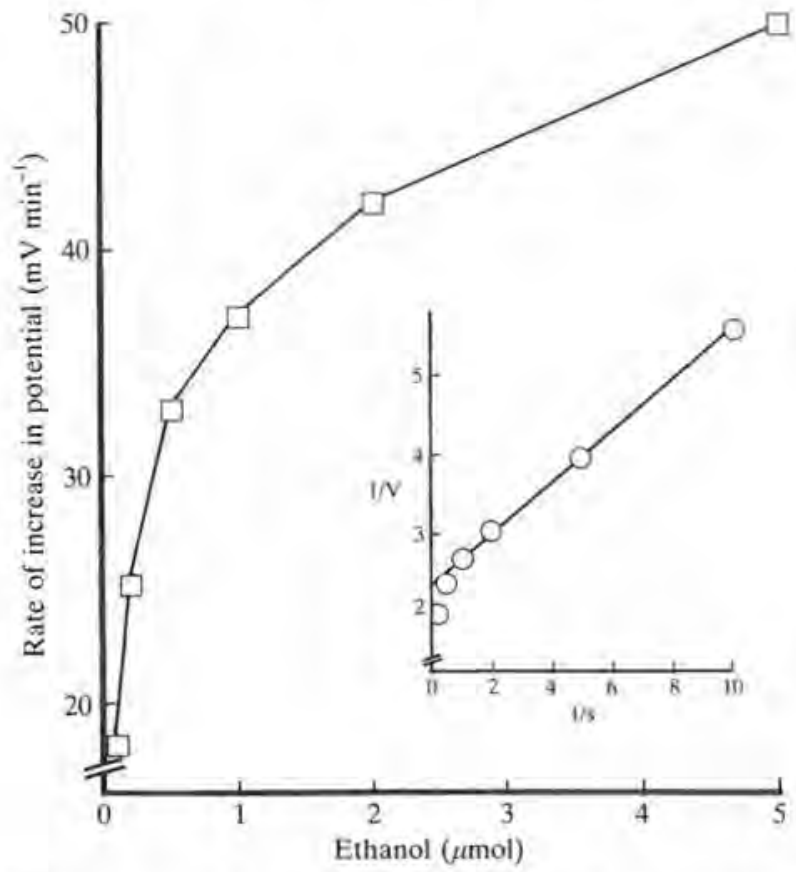

Fig. 17.7 Kinetic response of ethanol sensor. Rates were measured over a $30 \mathrm{~s} \mathrm{period}$ after addition of substrate to the cell. Inset shows the data as a Lineweaver-Burk plot; $S$ is ethanol concentration $(\mu \mathrm{mol}) ; V$ is rate of potential increase $\times 100 ; K_{\mathrm{S}}=18 \mu \mathrm{M}$.

A schematic representation of a multi-layer sensor in the form of a conventional electrode-probe is shown in Fig. 17.8. As with other sensors, a choice of design may be made between the durable and 'throwaway' types in which the working part of the probe is a disposable electrode disc or something similar. The latter possibility is attractive in view of the simplicity of construction and inexpensive bulk manufacture promised by electrodes formed with a carbon film coating on a paper or plastic base (popularly known as 'credit card' technology). A miniaturized version used in conjunction with a retractable probe would have advantages for clinical use; it is possible to extract samples (e.g. blood) and carry out an ex vivo test, so eliminating any possibility of contamination by sensor components (the main toxicity risks being presented by the organism and mediator). A needle-type glucose probe is described by Shichiri et al. in Chapter 23 of this volume.

\subsubsection{Design and construction of bio-active layers}

The simple representation given in Fig. 17.8 is clearly only a rough guide to the possibilities for sensor design. Various configurations for enzyme sensors 


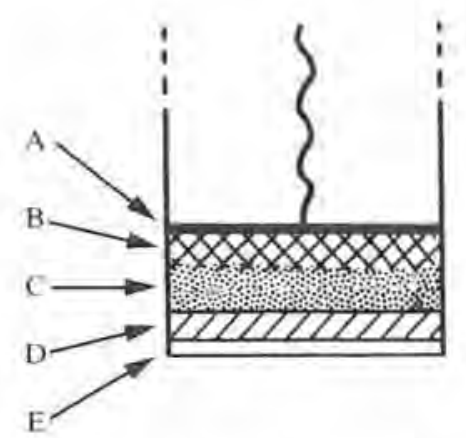

Fig. 17.8 Proposed construction of microbial sensor. A, carbon backing plate; B, porous carbon matrix; $\mathrm{C}$, bio-active layer; $\mathrm{D}$, filtering layer; $\mathrm{E}$, protective membrane.

have been outlined previously, notably by Scheller and co-workers (Scheller et al. 1984), who focus attention on the characteristics of the bio-active layer which forms the essential working part of the biosensor. One of the main goals in the development of prototype microbial sensors is to understand what happens in such an active layer containing biocatalyst, mediator(s), and other essential components; its composition and structure can then be modified in the light of test experiments. Various strategies for constructing the active layer are discussed below.

\subsubsection{Immobilization of micro-organisms}

Many techniques for immobilization of organisms have been developed (Chibata and Wingard 1983). Of these, the following are best suited to retain organisms on or adjacent to the electrodes:

17.5.3.1 Adsorption In the absence of a binding agent, micro-organisms bind strongly to absorbent surfaces, including carbons, but to a widely varying degree (Ward and Berkeley 1980). This simple approach suffers from the disadvantages that the biomass loading is difficult to control, and desorption effects may result from changes in $\mathrm{pH}$, ionic strength, etc.

17.5.3.2 Physical entrapment Physical entrapment of organisms within a porous electrode may be achieved using a gel matrix such as alginate, polyacrylamide, carrageenan, or a photo-cross-linked polymer which can be specifically tailored to modify its hydrophobicity (Fukui and Tanaka 1982). Mild preparation conditions allow the biomass loading to be carefully controlled. Although penetration times for a test solution containing substrate should be of the order of a few seconds, mass-transfer limitations may arise. These same properties could be used to advantage, however, since 
judicious selection of physical characteristics might facilitate the specific exclusion of contaminating substances in the analyte, or the regulation of mediator mobility and redox properties.

17.5.3.3 Covalent attachment Covalent attachment methods used for enzymes can be adapted for whole cells (Wiseman 1985), A high density of cells may be conveniently and firmly held at the surface, while diffusion problems are avoided. The more successful methods employ a two-stage process in which surface groups such as carboxylate are activated, e.g. using a carbodiimide coupling reagent, and the resulting surface polymer is reacted with the organisms. High biomass loading may be achieved, and biological stability can be enhanced considerably. Recent results show that satisfactory sensor signals (microamps) can be obtained using a monolayer bound to the electrode having $1 \mathrm{mg}$ (dry wt.) of micro-organism per $100 \mathrm{~cm}^{2}$ (real area) attached by this method.

\subsubsection{Redox mediating systems}

Previous work with mediated fuel cells (outlined above) and enzyme electrodes has provided insight on the various questions relating to redox mediators. There is a particular need to identify or synthesize suitable mediators for incorporation into systems which can couple the biocatalyst to the electrode while not allowing the active redox component to be leached out during measurement, though loss of mediators and other components may be tolerable in 'throwaway' designs. In durable probes, where the signal may be dependent on mediator concentration and loss of mediator could lead to poor reproducibility, the concentration could be maintained by a controlled delivery from a reservoir of micro-encapsulated reagent (Williams 1984; Kost and Langer 1984). Alternatively, mediators or derivatized mediators could be found which would be too large to escape from the network of a substratepermeable gel or some other structure which itself could be part of the immobilizing support for the micro-organisms. Ionic mediators might also be held by an oppositely charged polymer or co-polymer component, appropriately located or distributed. Several other possible approaches currently being explored are considered below.

17.5.4.1 'Anchored' mediators In recent years there have been significant advances in synthesis of 'speciality' polymers (Ise and Tabushi 1983) and there are numerous ways in which organic or inorganic mediators could be 'anchored' on polymer supports (Sheats et al. 1984), or attached to the cell wall. For example, an arrangement such as that illustrated in Fig. 17.9 can be envisaged in which a redox-active pendant mediator is suspended by a flexible molecular chain from an anchoring site $(\mathrm{P})$ on a linear chain polymer of open structure. In this 'swinging arm' arrangement the oxidized moiety $\left(\mathrm{M}_{\mathrm{ox}}\right)$ gains access to the intracellular source of electrons by penetrating the outer cell wall 


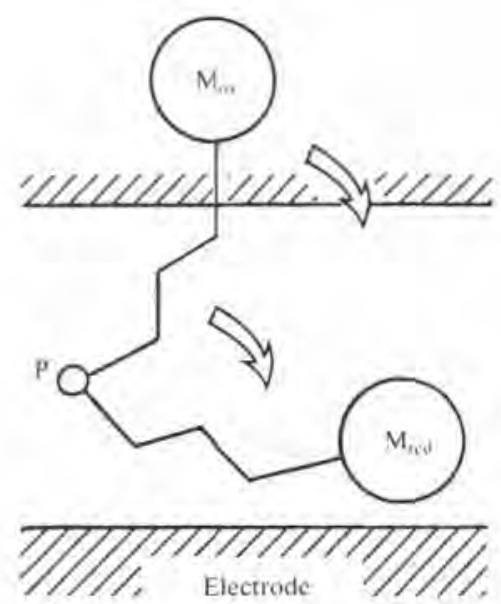

Fig. 17.9 Design of biocatalyst layers containing micro-organisms: the polymeranchored pendant mediator.

(upper part of figure) and on reduction the reduced form $\left(\mathrm{M}_{\mathrm{red}}\right)$ diffuses to the electrode and is re-oxidized.

The outer cell walls of micro-organisms are extremely varied in structure and composition, and the feasibility of the mechanism proposed above will therefore depend on the type of organism under study: generally 'Gram negative' organisms are more complex than 'Gram positive'. Access to reducing areas will be easy where the organism has exoenzymes but progressively more difficult for periplasmic enzymes, cytoplasmic enzymes, and mitochondria in eukaryotic cells. But many bacteria, for example, have walls of $5-20 \mathrm{~nm}$ thickness consisting principally of peptidoglycan, a chain-like disaccharide heteropolymer glycan with peptide substituents (Ward and Berkeley 1980; Inoue 1980). A pendant chain of $10-15-\mathrm{CH}_{2}-$ groups would in principle enable electrons to be ferried across a 5-10 nm gap by this mechanism, sufficient to mediate charge transfer from many organisms even with a "thinly spread' mediator. (A single mediator moiety in every $20 \mathrm{~nm}^{2}$, or 1 per $100 \mathrm{~nm}^{3}$ for a $5 \mathrm{~nm}$ gap, gives a mediator density of 0.01 molecules $\mathrm{nm}^{-3}$, i.e. a local concentration $=0.01 \mathrm{~mol} \mathrm{dm}^{-3}$ ).

17.5.4.2 'Functionalized' organisms Much is known about the electron transfer which constitutes the 'internal circuitry' of biochemical pathways (Losada et al. 1983; Dreyer 1984), but various ways of making connections to external circuits by manipulation and 'functionalization' of organisms await exploration. Mechanisms for mediation other than via mobile mediators can be envisaged, such as depicted in Fig. 17.10, which involves direct 


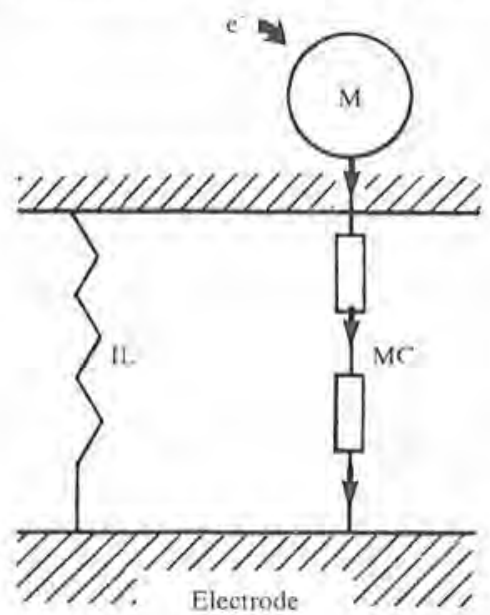

Fig. 17.10 Transduction from micro-organism via molecular conductor. $M$, receptor-mediator; IL, immobilizing link; MC, molecular conductor.

transduction through a molecular conductor. Here a mediator centre accepts electrons from the reducing pool inside the organism and channels them to the electrode along a conducting organic pathway (Aviram 1983; Munn 1984; Bryce and Murphy 1984). The molecular conductor (MC) should of course be 'bio-compatible' with the outer cell structure. There could be advantages in the development of an immobilizing/conducting matrix made of conducting polymers such as doped poly( $N$-vinylcarbazole $)$ which have been proposed for battery construction (Kakuta et al. 1985).

In more complex organisms the interfacing process presents many difficulties, particularly where the reducing centres are located in mitochondria, but 'functionalization' of the micro-organism by implanting appropriately modified redox reagents could allow electrons to 'hop' to accessible outer parts. Such electron-bridging systems are a real possibility in view of recent evidence on the long-distance tunnelling of electrons in functionalized proteins (Williams and Concar 1986). Properties required for the acceptor centre might be achieved by the tailoring of a functionalizing reagent such as a transition metal complex to a desired redox potential whilst matching it to the intracellular enzyme and its predominantly hydrophobic environment, and then partitioning it into the organism. Organic solvent components, mild detergents, and other reagents designed for facilitated transport of solutes across membranes might be employed for introducing and positioning 'foreign' components within organisms, which can tolerate such treatments for limited periods (Felix 1982; Leive 1968). 
of a mediator in either the biocatalyst layer or an adjacent layer introduces diffusion requirements which might affect electrochemical behaviour. Transfer of oxidized and reduced forms of mediators (free or bound) should not be so slow as to affect seriously the response time or the steady-state amperometric currents through concentration polarization. The limiting current density, $i_{\mathrm{d}}$, for a mobile species of diffusion constant, $D$, at a concentration, $c$, in a diffusion layer of thickness, $\delta$, is approximated by the expression

$$
i_{d}=\frac{D F c}{\delta} .
$$

The value of $i_{\mathrm{d}}$ for the pendant mediator scheme described above (calculated using a conservatively estimated value of $D$ of $10^{-7} \mathrm{~cm}^{2} \mathrm{~s}^{-1}$, and with $c=$ $0.01 \mathrm{~mol} \mathrm{dm}^{-3}, \hat{\delta}=5 \mathrm{~nm}$ ), is $0.2 \mathrm{~A} \mathrm{~cm}^{-2}$, which suggests that mediator diffusion need not present a serious limitation on sensor signals. Experimental checks on such restrictions imposed by the matrix can be assessed by comparisons of electrochemical responses (cyclic voltammetry) of immobilized forms with those from freely suspended components (organisms and mediators). Appropriate interfacing of the active layers through modified electrodes (Murray 1980, 1984; Albery and Hillman 1981) may be achieved using carbon electrodes, making use of surface carboxylate, quinone, and sulphur-containing groups.

\subsubsection{Selectivity, specificity, and interference}

Many ways of tailoring the sensor to avoid interferences and improve selectivity present themselves, and we here outline just some of the areas for investigation:

17.5.5.1 Manipulation of organisms By comparison with a single enzyme, a micro-organism is a multi-function biocatalyst which imposes multiparameter restrictions, but affords a wide range of opportunities for manipulation. Although use of an organism which utilizes both ascorbate and glucose as substrates, say, may give rise to signal interference when assaying one in the presence of the other, the uptake or catabolism of the interferent could be repressed in a number of ways. For example, the $K$ values afford differing selectivity over different concentration ranges, and could be modified according to the design of the bioactive layer or by biological manipulation. Another approach to increased specificity lies in the use of mutants: strains which do not metabolize glucose, for instance, could be used to detect a second substrate in a glucose-rich medium.

The use of micro-organisms in a secondary capacity as filters for substances, e.g. glucose and oxygen, which affect the primary electrochemical or bio-electrochemical sensing reactions, can in principle extend the 
range of usefulness of biosensors and other sensors. Such a 'scavenging layer' might be usefully placed between the active layer and the test solution (see Fig. 17.8).

17.5.5.2 Differential measurements In common with general analytical practice, interference problems for measurements in biological and industrial fluids can be minimized by judicious use of 'blanks'. For example, measurements could be carried out in a differential cell in which two cells are placed 'back to back'. The sensing part consists of two thin bio-anodes placed on one probe module, an arrangement which has advantages for calibration using a null method and gives additional compensation for temperature, $\mathrm{pH}$, and ionic-strength variations. Compensation for the effect of interfering substrates can also be devised by a 'blank' sensor having no analyte biocatalyst but which contains a biocatalyst for the interferant, matched appropriately to blank out its amperometric effect in the sensor proper.

\subsubsection{Other modifications of the bioactive layer For a probe which} depends on reductive biological action for its signal, the sensitivity and time of response may be affected by the oxygen tension. Although some sensors might function even in the presence of competitive oxidation reactions, particularly with very active organisms which rapidly establish their own anaerobic environment, it may be convenient to use oxygen-insensitive mediators, such as ferrocenes (Cass et al. 1984). These problems might also avoided by the use of a scavenging layer rendered inactive or partly inactive to the analyte, or by the use of 'electrochemical modulation' (Scheller et al. 1985). In this approach the oxygen tension can be controlled by use of an internal electrode grid. Such methods have also been used to increase the selectivity of a glucose enzyme electrode by decreasing the flux of ascorbate to the biocatalytic area.

In the preliminary work described in section 17.4 , the substrate was diluted in the measuring cell from a small aliquot of added test solution. The dilution step could be avoided by use of a microbial probe, but the micro-organisms may become substrate-saturated in concentrated solutions, which might confer a long-lasting reducing activity and so limit the substrate-sensitivity. This problem (which would be less serious for a single measurement) might be solved by limiting the amount of substrate penetrating through to the active layer. Use could be made of a scavenging layer or diffusion-limiting $\mathrm{gel} /$ polymer membrane between the solution and the biocatalyst ( $c f$. section 17.5.3.1). The extent of substrate utilization would also be dependent on the particular mediator used, as well as its concentration and location, and could accordingly be de-sensitized by tailored design. 


\subsection{Future prospects}

In this article we have attempted to describe an emerging biosensor technology which promises faster response times and sensitivity as a result of the mechanism of signal transduction, i.e. electrical 'connection' to the early stages of microbial catabolism. This avoids the requirement of the indirect method for the organism to reach a steady state of product generation in response to the analyte concentration. It is clear, however, that 'direct' microbial biosensors share many of the advantages and disadvantages already revealed for those of the 'indirect' type: many of the applications will be the same ( $c f$. section 17.2), and much work remains to be done in further exploration of both methods.

At the time of writing there are reservations about the acceptibility of whole-cell sensors for many applications, for example clinical use, fermentation control, and the food industries. Though such reservations do not affect the impetus of research work in some countries, notably Japan, it is worth examining the possible reasons for this scepticism. If we accept that the biosensors could be made economically, perhaps along the lines suggested here and elsewhere in this volume, the two main requirements are that they should work, and that they should be safe. At present it may be unrealistic to expect the implementation of test procedures which would, for example, satisfy the (rather demanding) standards of the best industrial analytical chemistry laboratories, but results in the literature and work carried out in industrial laboratories suggest that some whole-cell sensors do not behave very reproducibly, and have not been adequately or objectively tested. Improvements can be expected to follow, however, from a closer examination of the fundamental mechanistic aspects, together with the use of new materials and designs. The risk of contamination of test materials by whole-cell biocatalysts, and the fears it engenders, present a serious problem which will only be overcome after rigorous demonstrations of safe application, which will be all the more convincing if the performance of biosensors can generally be improved. Biotechnological innovations are becoming rapidly assimilated and accepted, however, and some of the prejudice directed against the use of 'bugs' will perhaps soon be swallowed and digested, together with helpings of mycelial protein now available in supermarket pies!

\section{Acknowledgements}

We thank Johnson Matthey for the loan of electrode materials and (in part) Cambridge Life Sciences plc. for support (for SDR). 


\section{References}

Albery, W. J. and Hillman, R.A. (1981). Modified electrodes. Ann. Rep. Prog. Chem., C, 78, 377-437.

Aston, W. J. and Turner, A. P. F. (1984). Biosensors and biofuel cells, In Biotech. Genet. Eng. Rev, (ed. G. Russell), Vol. 1, pp. 89-120. Intercept, Newcastle-uponTyne.

Aviram, A, (1983). Molecular components for electronic device function - an overview, Proc. First World Conf. Commercial Applications and Implications of Biotechnology (Biotech '83), pp. 695-704. Online Publications, London.

Bennetto, H. P. (1984). Microbial fuel cells. In Life chemistry reports (eds. A. M. Michelson and J. V. Bannister), Vol. 2, no. 4, pp. 363-453. Harwood Academic, London.

- Stirling, J. L. and Tanaka, K. (1985). Reduction of 'redox' mediators by NADH and electron transduction in bioelectrochemical systems, Chem. and Ind. (Lond.), 695-7.

- Tanaka, K, and Matsuda, K. (1984). Bio-fuel cell containing algae. In Charge and field effects in biosystems (eds. M.J. Allen and P.N.R. Usherwood), pp, 515-522. Abacus Press, Tunbridge Wells.

- Dew, M. E., Stirling, J. L. and Tanaka, K. (1981). Rates of reduction of phenothiazine 'redox' dyes by E. coli. Chem. and Ind. (Lond.) 776-8.

- Stirling, J. L., Tanaka, K. and Vega, C. A. (1980). Microbial fuel cells. Soc, Gen. Microbiol. Quarterly, 8, 37.

- (1983). Anodic reactions in microbial fuel cells. Biotechnol. Bioeng. 25, 559-68.

Besenhard, J.O. and Fritz, H.P. (1983). The electrochemistry of carbon blacks. Angew. Chemie 22, 950-975.

Bryce, M. R. and Murphy, L. C. (1984). Organic Metals. Nature 309, 119-126.

Cass, A. E. G. (1984). Protein electrochemistry: current studies and potential applications. In Life Chemistry Reports (eds. A.M. Michelson and J. V. Bannister), Vol. 2, no. 4, pp. 321-362. Harwood Academic, London.

Cass, A. E. G., Davis, G., Francis, G. D., Hill, H. A. O., Aston, W. J., Higgins, I. J., Plotkin, E. V., Scott, L. D. L. and Turner, A.P. F. (1984). Ferrocene-mediated enzyme electrode for amperometric determination of glucose. Anal. Chem. 56, $667-71$.

Chibata, I. and Wingard, L. B. Jr. (1983). Applied biochemistry and bioengineering; Vol. 4: Immobilized cells. Academic Press, London.

Clarke, D. J., Calder, M. R., Carr, R.J. G., Blake-Coleman, B. C,, Moody, S. C. and Collinge, T.A. (1985). The development and application of biosensing devices for bioreactor monitoring and control. Biosensors 1, 213-320.

Corcoran, C.A. and Rechnitz, G.A. (1985). Cell-based biosensors. Trends in Biotechnol. 3, 92-6.

Delaney, G. M., Bennetto, H. P., Mason, J. R., Roller, S. D., Stirling, J. L. and Thurston, C. F. (1984). Electron transfer coupling in microbial fuel cells. 2. Performance of fuel cells containing selected micro-organism-mediator-substrate combinations. J. Chem. Tech. Biotechnol. 34B, 13-27.

- Bennetto, H. P., Mason, J. R., Roller, S. D., Stirling, J. L, and Thurston, C. F. (1986). Electron transduction from enzymes and bacteria. Anal. Proc, 23, 143-4.

Dreyer, J, L. (1984). Electron transfer in biological systems: an overview, Experientia 40, 653-776. 
Felix, H. (1982). Permeabilized cells. Anal. Biochem. 120, 211-234.

Findl, E., Strope, E. R. and Conti, J. C. (1985). Electrochemical techniques in the biological sciences. In Comprehensive treatise of electrochemistry, Vol. 10, Bioelectrochemistry, (eds. S. Srinivasan, Y. A. Chizmadzhev, J. O.' M. Bockris, B. E. Conway and E. Yeager), pp. 491-529. Plenum Press, New York.

Fukui, S, and Tanaka, A. (1982). Immobilized microbial cells. Ann. Rev. Microbiol. 36, 145-72.

Guilbault, G. G. (1984). Analytical uses of immobilized enzymes. Chapter 3v, pp. 211-26. Marcel Dekker, New York.

Gunsalus, I. G, and Schuster, C. W. (1961). Metabolism. In The bacteria (eds. I. Gunsalus and R. Y. Stanier), Vol. 2. Academic Press, New York.

Hanazato, Y. and Shiono, S. (1983). Bioelectrode using two hydrogen ion sensitive field effect transistors and a platinum wire pseudo reference electrode. In Chemical sensors (eds. T. Seiyama, K. Fueki, J, Shiokawa and S. Suzuki), Analytical Chem. Symp. Series, Vol. 17, pp. 513-8. Kodansha/Elsevier, Tokyo.

Inoue, M. (1980). Bacterial outer membranes: Biogenesis and functions. Academic Press, London.

Ise, N. and Tabushi, 1. (1983), Introduction to speciality polymers. Cambridge University Press, London.

Kakuta, T., Shirota, Y, and Mikawa, H. (1985). A rechargeable battery using electrochemically doped poly ( $N$-vinylcarbazole). J. Chem. Soc. Chem. Commun. 553-5.

Karube, I. and Suzuki, S. (1983). Application of biosensor to fermentation processes. Ann. Rep. Ferment. Processes 6, 203-236.

- (1984). Amperometric and potentiometric determinations with immobilised enzymes and micro-organisms. Ion-selective Elec. Rev, 6, 15-58.

-Matsunaga, T. and Suzuki, S. (1977). A new microbial electrode for BOD estimation. J. Solid Phase Biochem. 2, 97-104.

Kobos, R.K. (1983). Microbe-based electrochemical sensing systems. Trends in Analyt. Chem. 2, 154-7.

Kost, J. and Langer, R. (1984). Controlled release of bioactive agents. Trends in Biotechnol. 2, 47-51.

Leive, L. (1968). Studies on the permeability change produced in coliform bacteria by ethylenediaminetetracetate. J. Biol. Chem. 243, 2373-2380.

Losada, M., Hervas, M., De La Rosa M. A. and De La Rosa, F. F. (1983). Energy transduction in bioelectrochemical systems. Bioelectrochem. Bioenerg. 11, $193-230$.

Mărgineanu, D.-G., Vais, H. and Ardelean, I. (1985). Bioselective electrodes with immobilized bacteria. J. Biolechnol. 3, 1-9.

Matsunaga, T., Karube, I. and Suzuki, S. (1980). A specific microbial sensor for formic acid. European J. Appl. Microbiol. Biotech. 10, 235-43.

Munn, R. W. (1984). Molecular electronics. Chem. in Britain, 20, 518-24.

Murray, R. W. (1980). Chemically modified electrodes. Accounts Chem. Res. 13, $135-41$.

- (1984). Chemically modified electrodes. In Electroanalytical chemistry (ed. A. J. Bard), Vol. 13. pp. 191-368. Marcel Dekker, New York and Basel.

Proctor, B. E. and Greenlie, D. G. (1939). Reduction-oxidation potential indicators in quality control of foods. 1. Correlation of resazurin reduction rates and bacterial plate counts. Food Res. 4, 41-9. 
Roller, S. D., Bennetto, H. P., Delaney, G.M., Mason, J, R., Stirling, J. L. and Thurston, C.F. (1984). Electron transfer coupling in microbial fuel cells. 1. Comparison of redox-mediator reduction rates and respiratory rates of bacteria. J. Chem. Tech. Biotechnol. 34B, 3-12.

- and White Jr., D. R. (1983). A bio-fuel cell for utilisation of lactose wastes. Proc. First World Conf. Commercial Applications and Implications of Biotechnology (Biotech '83), pp. 655-663. Online Publications, London.

Scheller, F. W., Strnad, G., Renneberg, R, and Kirstein, D. (1984). Potentialities of protein electrochemistry in analytics. In Charge and field effects in biosystems (eds. M. J. Allen and P. N. R. Usherwood), pp. 483-90. Abacus Press, Tunbridge Wells. - Schubert, F., Renneberg, R., Muller, H.-G., Janchen, M. and Weise, H. (1985). Biosensors: trends and commercialisation. Biosensors 1, 135-160.

Sheats, J. E., Pittman, C. U. Jr. and Carraher, C. E. Jr. (1984), Organometallic polymers. Chem. in Britain 20, 709-15.

Simpson, D. L. and Kobos, R, K. (1984), Ammonia gas sensor for microbial assay of tetracycline, gentamycin, streptomycin and neomycin. Anat. Chim. Acta 164, 273-7.

Stirling, J. L., Bennetto, H. P., Delaney, G. M., Mason, J. R., Roller, S. D., Tanaka, K. and Thurston, C.F. (1983). Microbial fuel cells. Biochemical Society Transactions 11, 45I-3.

Thurston, C. F., Bennetto, H. P., Delaney, G. M., Mason, J. R., Roller, S. D. and Stirling, J, L, (1985), Glucose metabolism in a microbial fuel cell. Stoichiometry of product formation in a thionine-mediated Proteus vulgaris fuel cell and its relation to coulombic yields, J. Gen. Microbiol. 131, 1393-1401.

Turner, A. P. F., Aston, W. J., Higgins, I. J., Davis, G. and Hill, H. A. O. (1982). Applied aspects of bioelectrochemistry; fuel cells, sensors and bioorganic synthesis. Biotech. Bioeng. Symp. No. 12, 401-12.

van Dijk, C., Laane, C. and Veeger, C. (1985). Biochemical fuel cells and amperometric sensors. Recl. Trav. Chim. Pays-Bas 104, 245-52.

Wang, J. (1981). Reticulated vitreous carbon - a new versatile electrode material. Electrochim. Acta 26, 1721-6.

Ward J. B. and Berkeley, R. C. W. (1980), The microbial cell surface and adhesion, In Microbial adhesion to surfaces (eds. R. C. W. Berkeley, J. M. Lynch, J. Melling, P. R. Rutter, and B. Vincent), pp. 47-66. Soc. Chem. Ind./Ellis Horwood, Chichester.

Williams, A. (1984). The controlled release of bioactive agents. Chem. in Britain 20, $221-4$.

Williams, R. J. P. and Concar, D. (1986). Long-range electron transfer. Nature 322. 213-4.

Wingard, L. B., Jr., Shaw, C. H. and Castner, J. F. (1982). Bioelectrochemical fuel cells. Enzyme Microb. Technol. 4, 137-142.

Wiseman, A. (1985), Handbook of Enzyme Biotechnology. (2nd edn), Ellis Horwood, Chichester. 


\title{
Application of enzyme-based amperometric biosensors to the analysis of 'real' samples
}

\author{
FRIEDER W, SCHELLER, DOROTHEA PFEIFFER, \\ FLORIAN SCHUBERT, REINHARD RENNEBERG, \\ and DIETER KIRSTEIN
}

\subsection{Introduction}

Amperometric biosensors represent the highest-developed branch of biospecific electrodes as it is reflected by the number of publications, patents, and commercialized analysers. They combine the advantages of faradaic electrode processes, e.g. high sensitivity, linear concentration dependence, selectivity by changing the electrode potential, and independence of sample buffer capacity with the high substrate specificity of enzymes or higher integrated biocatalytic systems like organelles, micro-organisms, or tissue slices. Based on the principle of detecting a concentration gradient of the electrodeactive product, amperometric sensors are only able to detect the formation or consumption of reaction partners, and are not suited to indicate changes of the electron density resulting from the sole complex formation process without chemical conversion. Furthermore, amperometric sensors are generally restricted to two-substrate reactions catalysed by oxidoreductases, since redox reactions are based on the transfer of electrons between two substances. Therefore the concentrations of both the substrate and the cofactor influence the reaction rate. This stimulated the development of principles which eliminate the limitations of the cofactor concentration, e.g., the oxygen-regenerating auxiliary electrode, mediator chemically modified indicator electrodes, or the exploitation of the direct electron transfer between the protein prosthetic group and the redox electrode resulting in a reagentless measuring regime. In this way, in addition to the enzyme the cofactor is also eliminated as a reagent in analyses using amperometric biosensors. In addition to the drastic reduction of reagent costs the most important advantage of applying amperometric biosensors is the considerable simplification of the measuring devices: They represent the spatial unity of dialyser, enzyme reactor, and electrochemical detector (Fig, 18.1). This is the predominant feature of analysers based on amperometric biosensors.

Among biosensors, oxidase-catalysed reactions dominate. This fact is related to the simple handling of electrochemical $\mathrm{O}_{2}$ and $\mathrm{H}_{2} \mathrm{O}_{2}$ detection. 


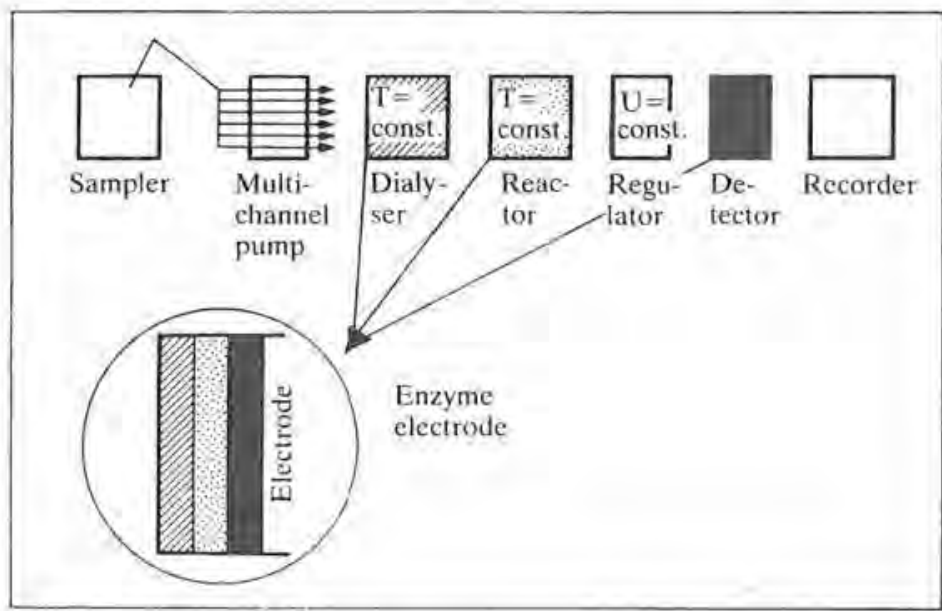

Fig. 18.1 The unit of dialyser, enzyme reactor, and electrochemical sensor in enzyme electrodes.

When no useful product is obtained in the analyte molecule conversion, a readily measurable substance can be formed in a succeeding enzyme reaction. These sequence-type reactions are generally used with esters, oligosaccharides, and amides. Other types of coupled reaction are based on recycling the molecule of interest in order to produce a multiple of the product, or to eliminate disturbing substances.

In patents and publications, amperometric biosensors have been described for the determination of about 80 different substances including substrates, cofactors, prosthetic groups, enzyme activities, antibodies, inhibitors, and activators. Linear concentration ranges of these sensors usually extend over two to four decades with a limit of detection at 1-100 micromolar concentration. Analysers based on amperometric biosensors for the determination of eleven different substances have been commercialized.

The aim of this contribution is to illustrate the potentials and limitations of the routine application of amperometric biosensors in clinical diagnostics, fermentation control, food production, and pollution control.

\subsection{Application of amperometric biosensors}

\subsubsection{Low-molecular weight soluble substances}

The majority of analytically important substances belong to this group. Typical representatives are monosaccharides, oligosaccharides, alcohols, organic acids, and amino acids. Also uric acid and creatinine, being soluble in the micromolar range, shall be discussed in this chapter. The electro- 
enzymatic measurement of these substances is to some extent used in the analytical routine. Furthermore, inorganic ions acting as activators or inhibitors and prosthetic groups, e.g. FAD, have been determined on laboratory scale using amperometric biosensors.

18.2.1.1 Determination of glucose Exact and rapid determination of glucose is essential not only in analytical clinical laboratories but also for online supervising of diabetic patients. In microbiological and food industries the glucose sensor is important for process control, and application should be expanded to di-and polysaccharides and amylase determinations. In order to meet these requirements, about 50 groups from various countries deal with the development and optimization of glucose sensors and analysers.

18.2.1.2 Blood glucose The determination of blood glucose levels is an indispensable test for the exact diagnosis and therapy of diabetes mellitus as well as for many kinds of disorders. Whereas the normal blood glucose level is about $5 \mathrm{mmol} / 1$, the pathological value may increase up to $50 \mathrm{mmol} / 1$. In the case of urine glucose the normal value is about $1 \mathrm{mmol} / \mathrm{l}$ but with most glucose assays the interferences cause serious problems.

Approximately 5 per cent of the adult population of industrialized countries has diabetes. Analytical chemistry has played and is continuing to play a major role in the conquest of diabetes mellitus. Innumerable methods have been developed but the specificity of enzyme reactions and the sensitivity of electrochemical techniques resulted in the popularization of glucose sensors. The scheme of glucose-oxidase (GOD, EC 1.1.3.4) catalysed glucose oxidation reveals two main possibilities for glucose measurement.

$$
\text { D-Glucose }+\mathrm{O}_{2} \underset{\text { oxidase }}{\stackrel{\text { Glucose }}{\longrightarrow}} \mathrm{H}_{2} \mathrm{O}_{2}+\text { D-Gluconolactone }
$$

One can register the consumption of oxygen either by cathodic reduction or by the production of hydrogen peroxide by anodic oxidation.

18.2.1.3 Interferences Various reducing substances present in biological samples, for instance ascorbic acid, uric acid, glutathione, etc., may considerably influence the oxidation of $\mathrm{H}_{2} \mathrm{O}_{2}$. To eliminate this disadvantage, four fundamental approaches are possible.

i) Lobel and Rishpon (1981) eliminated a part of the interference by using a negatively charged dialysis membrane, which rejected up to $0.0852 \mathrm{mmol} / \mathrm{I}$ of ascorbic acid and $0.464 \mathrm{mmol} / 1$ of uric acid. Higher concentration of these substances and also glutathione and bilirubin influence the glucose signal.

ii) Thévenot et al. (1982 and Chapter 22) included a compensating 
electrode with a non-enzymatic collagen membrane and registered the difference between the two sensor currents.

iii) $\mathrm{A} \mathrm{H}_{2} \mathrm{O}_{2}$-selective asymmetrical cellulose-acetate membrane excluding most of the potentially interfering substances in front of the electrode is used by Yellow Springs Instruments Corp., USA (Newman 1976) and by the Japanese company Fuji Electric (Tsuchida and Yoda 1981). But this composed membrane costs more.

iv) Without a permselective membrane exact and fast glucose measurements are possible by the derivative method in connection with an additional diffusion resistance behind the enzyme layer (Scheller and Pfeiffer 1978).

In the case of blood glucose detection by means of electrochemical oxygen detection deoxyhemoglobin binds oxygen in the measuring solution. Therefore the measured values do not agree with the true ones.

18.2.1.4 Sensitivity Contrary to $\mathrm{H}_{2} \mathrm{O}_{2}$-assay which starts from a very low background current and permits a sensitive detector (detection limit of $10^{-6} \mathrm{~mol} / \mathrm{l}$ glucose), in the case of $\mathrm{O}_{2}$-reduction a difference from the basic oxygen current is registered. Therefore the sensitivity is usually lower by two to three orders of magnitude (Thévenot 1982). The linear measuring region of glucose sensors extends over four decades. Obviously it is limited by the oxygen diffusion into the reaction layer. By applying an additional external diffusion barier in front of the glucose oxidase membrane (Scheller and Pfeiffer 1978) the linear measuring range can be shifted to much higher concentrations.

18.2.1.5 Stability The stability of glucose in blood samples is an important problem in clinical diagnostics. With intact erythrocytes the concentration of blood glucose is decreased up to $20 \%$ within two hours, also in the presence of $24 \mathrm{mmol} / \mathrm{I} \mathrm{NaF}$, a glycolysis inhibitor. By diluting the blood sample with a hypotonic buffer leading to blood haemolysis, glycolysis is completely eliminated and the glucose concentration is stable over 24 hours (A.B.2 1985).

18.2.1.6 Glucose analysers The effort of studying the sensor problems resulted in the development of various glucose analysers by relevant companies. A comparison of analytically interesting parameters is represented in Table 18.1. The first amperometric enzyme electrode-based glucose measuring device was developed by Yellow Springs Instruments Corp. in 1979 (YSI-23 A) (see Chapter 1). The correlations between values obtained by the usual hexokinase-glucose 6-phosphate-dehydrogenase method and those by the YSI-23 A are satisfactory for plasma and serum. Results with whole blood are not presented (Chua and Tan 1978). The research kit of the Hungarian company Radelkis (Havas et al. 1980) and the Glucoroder-E from 
Table 18.1 Glucose analysers based on amperometric enzyme electrodes

\begin{tabular}{|c|c|c|c|c|c|c|}
\hline Company & $\begin{array}{l}\text { Enzyme } \\
+ \text { added } \\
\text { reactant }\end{array}$ & $\begin{array}{l}\text { Measuring } \\
\text { range } \\
(\mathrm{mmol} / 1)\end{array}$ & $\begin{array}{l}\text { Sample } \\
(\mu 1)\end{array}$ & $\begin{array}{l}\text { Measuring } \\
\text { frequency } \\
\text { (sample/hr) }\end{array}$ & $\begin{array}{l}\text { Serial } \\
\text { precision } \\
(\%)\end{array}$ & Stability \\
\hline \multicolumn{7}{|l|}{ Manual analysers } \\
\hline Yellow Springs Instruments & glucose & $1.0-45.0$ & 25 & 40 & 2.0 & 300 samples \\
\hline $\begin{array}{l}\text { Corp. (USA): } \\
\text { Model } 23 \mathrm{~A}\end{array}$ & $\begin{array}{l}\text { oxidase } \\
\text { (GOD) }\end{array}$ & & & & & \\
\hline $\begin{array}{l}\text { ZWG, } \\
\text { Acad. of Sciences (GDR): } \\
\text { Glukometer GKM } 01\end{array}$ & GOD & $0.5-50.0$ & $20-25$ & $60-90$ & 1.5 & 1000 samples \\
\hline $\begin{array}{l}\text { Radelkis (Hungary): } \\
\text { OP-G1-7113-S }\end{array}$ & GOD & $1.7-2,0$ & 100 & 40 & $5.0-10.0$ & 250 days \\
\hline $\begin{array}{l}\text { Inst. Biochem, Vilnius } \\
\text { (USSR): Enzalyst-G }\end{array}$ & GOD & $0.5-30.0$ & 50 & 60 & 5.0 & n.c. \\
\hline Ferment (USSR): Aplama & GOD & $2.5-30.0$ & n.c. & 20 & 3.0 & n.c. \\
\hline $\begin{array}{l}\text { Fuji Electric (Japan): } \\
\text { Gluco } 20 \mathrm{~A}\end{array}$ & GOD & $0-27.0$ & 20 & $80-90$ & 1.7 & 500 samples \\
\hline Seres (France): Enzymat & GOD & $1.0-22.0$ & 200 & 60 & n.c. & 500 samples \\
\hline $\begin{array}{l}\text { Solea-Tacussel (France): } \\
\text { Glucose electrode }\end{array}$ & GOD & $0.0001-1.0$ & n.c. & n.c. & 2.0 & 1000 samples \\
\hline $\begin{array}{l}\text { Hoffmann-La Roche \& Co. } \\
\text { (Switzerland): } \\
\text { Glucose Analyzer } 5410\end{array}$ & $\begin{array}{l}\mathrm{GOD}+ \\
{\left[\mathrm{Fe}(\mathrm{CN})_{6}\right]^{-3}}\end{array}$ & $2.5-27.5$ & 100 & $t_{r}=60 \mathrm{~s}$ & 1.5 & 8 weeks \\
\hline $\begin{array}{l}\text { Inst. Techn. Chem., } \\
\text { Acad. Sciences GDR }\end{array}$ & $\begin{array}{l}\mathrm{GOD}+ \\
p \text {-quinone }\end{array}$ & $0-55.5$ & 800 & 15 & 3.0 & 8 weeks \\
\hline
\end{tabular}


Table 18.1 cont.

\begin{tabular}{|c|c|c|c|c|c|c|}
\hline Company & $\begin{array}{l}\text { Enzyme } \\
+ \text { added } \\
\text { reactant }\end{array}$ & $\begin{array}{l}\text { Measuring } \\
\text { range } \\
(\mathrm{mmol} / \mathrm{l})\end{array}$ & $\begin{array}{l}\text { Sample } \\
(\mu 1)\end{array}$ & $\begin{array}{l}\text { Measuring } \\
\text { frequency } \\
\text { (sample/hr) }\end{array}$ & $\begin{array}{l}\text { Serial } \\
\text { precision } \\
(\%)\end{array}$ & Stability \\
\hline $\begin{array}{l}\text { Analytical Instruments } \\
\text { (Japan): Glucoroder-E }\end{array}$ & GOD & $0-55.5$ & $20-40$ & $120-150$ & 2.0 & n.c. \\
\hline \multicolumn{7}{|l|}{$\begin{array}{l}\text { Automatic flow } \\
\text { analysers }\end{array}$} \\
\hline $\begin{array}{l}\text { Daiichi (Japan): } \\
\text { Auto \& Stat GA-1110 }\end{array}$ & GOD & $1.0-40.0$ & $100-250$ & n.c. & 1.0 & n.c. \\
\hline $\begin{array}{l}\text { MLW (GDR): } \\
\text { ME Glucose } 6\end{array}$ & GOD & $1.0-44.0$ & 20 & $80-120$ & 1.2 & 1000 samples \\
\hline $\begin{array}{l}\text { Charles Univ. Prague } \\
\text { (Czechoslovakia) } \\
\text { Dept. Biochem, }\end{array}$ & GOD & $0.0006-5.0$ & n.c. & 60 & 3.5 & $30 d$ \\
\hline On-line devices & & & $\begin{array}{l}\text { lag time } \\
\text { (min) }\end{array}$ & & & \\
\hline $\begin{array}{l}\text { Life Science Instr., } \\
\text { Div. Miles Lab. (USA): } \\
\text { Biostator GC IIS }\end{array}$ & GOD & up to 27.5 & 2 & & 5 & $50 \mathrm{~h}$ \\
\hline $\begin{array}{l}\text { Centr. Inst. Diabetes, } \\
\text { Karlsburg (GDR) }\end{array}$ & GOD & up to 40.0 & $15-20$ & & n.c. & n.e. \\
\hline $\begin{array}{l}\text { Osaka Univ. Med. } \\
\text { School } \\
\text { Dept. Med. (Japan) }\end{array}$ & GOD & $2,85-22,0$ & n.c. & & n.c. & $3 d$ \\
\hline
\end{tabular}

n.c., not communicated

$t_{r}$, response time 
Analytical Instruments (Japan) are based on the oxygen consumption mode. Therefore these devices are not appropriate for blood glucose measurements.

No data about correlations between usual methods and precision are published by Seres (France), Solea-Tacussel (France), and the Biochemical Institute of Vilnius (USSR). Similarly, only a little information has been published about enzyme electrodes available from Universal Sensors (USA) (Guilbault 1984; Chapter 9).

One of the most significant problems of blood glucose measurement is demonstrated by the Japanese companies Fuji Electric Co. and Daiichi. In the Gluco $20 \mathrm{~A}$ (Fuji) the sensor works with $20 \mu \mathrm{l}$ of undiluted whole blood and good correlation between Gluco $20 \mathrm{~A}$ and the hexokinase method for serum is obtained. However, comparative studies show that the glucose values of whole blood are always $13 \%$ smaller than those of serum (Niwa et al. 1981). Similar results with whole blood are obtained with the Auto and Stat GA-1110 (Daiichi, Japan). The following correlation to an enzymatic method is published: $y=0.793 x+0.471 \mathrm{mmol} / 1$. The problem was investigated by the author's group. Using the Glukometer GKM 01 from ZWG Berlin (GDR), undiluted, EDTA-stabilized blood and 1:10-diluted samples in isotonic dextrane phosphate buffer were compared. The correlation curve obtained is shown in Fig. 18.2. At direct injection of undiluted blood the

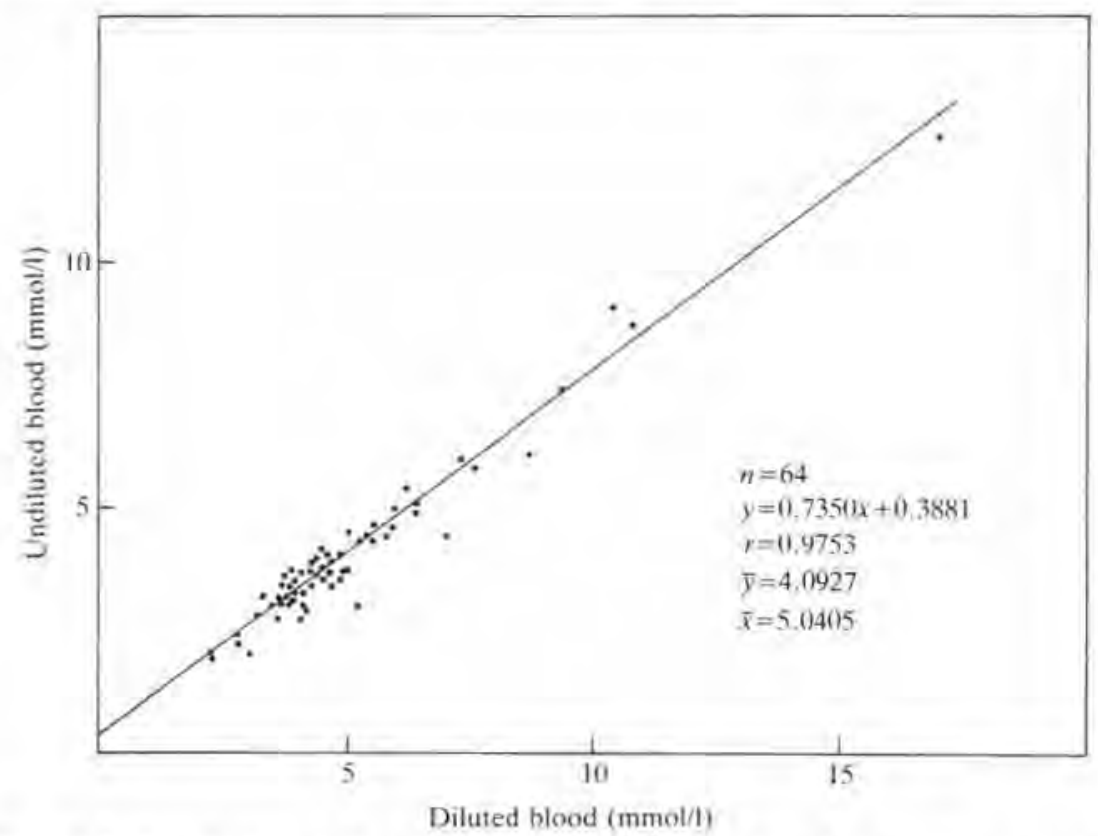

Fig. 18.2 Correlation of glucose concentration of 1:10-diluted and undiluted whole blood samples by glucose oxidase electrode. 
values are $18.8 \%$ lower than those obtained with 1:10-prediluted samples. This difference reflects in the case of direct injection of undiluted whole blood an incomplete indication of the glucose present in erythrocytes. That is why the glucose determined by this mode does not represent the real value. Using 1:10-diluted blood or serum and the derivative hydrogen peroxide method the results obtained with the Glukometer GKM 01 agree well with the glucose oxidase-peroxidase method. With the same GOD-sensor the values of blood glucose assay carried out by the ME Glucose 6 (Müller et al. 1985) agree excellently with the highly specific glucose dehydrogenase method:

$$
\begin{aligned}
& y=(1.003 \pm 0.006) x-(0.015 \pm 0.045) \mathrm{mmol} / 1, \\
& r=0.996, \\
& n=196 .
\end{aligned}
$$

Supervising of diabetic patients demands a glucose-controlled insulin infusion system. Great efforts are being made to overcome difficulties like stability of enzyme membrane and wide linear concentration range. Fogt et al. (1978) published the first feedback control system, the Biostator ${ }^{60}$. Using diluted blood, the enzyme electrode is stable for up to 50 hours, the linearity extends up to $27.5 \mathrm{mmol} / \mathrm{l}$. Implantable sensors are being developed by Abel et al. (1984) and Shichiri et al. (1984; Chapter 23) for glucose monitoring using needle-type electrodes. Both make use of the smaller glucose concentrations of the interstitium as compared with venous blood. Shichiri's investigations resulted in a wearable artificial endocrine pancreas (400 g) consisting of the needle-type sensor, minicomputer, and two syringe driving systems.

A ferrocene-modified glucose oxidase sensor was used for the prototype of a personal glucose monitor for diabetes in easily portable form by the Cranfield/Oxford groups (Cass et al. 1984). The signal does not depend on oxygen and for undiluted whole blood a good agreement with standard methods for plasma is obtained (see also Chapters 15 and 16).

18.2.1.7 Fermentation control In fermentation control, monitoring of various substrates and products of biochemical reactions is a key problem (Enfors 1982). The varying concentration of oxygen in fermentation broth and the need for oxygen in the glucose-oxidase-catalysed reaction are the main difficulties in this field.

For discrete measurements of fermentation samples, Mor and Guarnaccia (1977) performed a differential measurement between a glucose sensor and an auxiliary electrode using hexacyanoferrate (III) as electron acceptor. The increase of the concentration of the reduced hydrogen acceptor, $p$-quinone, with additional inert gas was used by Asperger and Krabisch (1985).

Romette et al. (1979) developed a glucose electrode with an enzyme 
membrane possessing a high oxygen solubility. The membrane is loaded with air prior to the analysis. This reservoir supplies the GOD reaction with sufficient oxygen but would not be applicable to in situ analysis in fermentation processes.

The most interesting development in this field is an oxygen-stabilized glucose electrode based on electrolytic generation of oxygen (Enfors 1982). Thus, variation of sample-dissolved oxygen does not disturb the signal output (see also Chapter 19).

\subsubsection{Galactose and lactose Galactose provides an alternative carbo-} hydrate source and improves homeostatic regulation of glucose in the premature infant. Because of its potentially toxic effects, sensitive methods for monitoring its concentration are needed. The normal range of serum galactose is below $0.24 \mathrm{mmol} / 1$. The disaccharide lactose is present uniquely in milk. The average concentration in human milk is $0.3-0.6 \mathrm{~mol} / \mathrm{t}$ and in cows milk $0.25-0.28 \mathrm{~mol} / 1$. There has been considerable interest in the development of methods for the determination of lactose, as the lactose content in foodstuffs is indicative of the amount of skimmed milk powder that has been added.

Both galactose and lactose have been determined using galactose oxidase (EC 1.1.3.9) immobilized on a hydrogen-peroxide-selective cellulose acetate membrane in front of the electrode (Taylor et al. 1977). The linear range of the measurement was at least 0 to $30 \mathrm{mmol} / \mathrm{l}$ of galactose or lactose with a membrane working life of typically 10 days. The repeatibility was shown to be $2 \%$. The only physiologically important interference found was dihydroxyacetone.

Determination of lactose using an immobilized enzyme electrode is included as a special kit in the YSI Industrial Analyzer Model 27 and the automatic device Enzymat from Seres (France). In addition Yellow Springs describes in the specification sheet the determination of fructose on the basis of immobilized galactose oxidase, a reaction unknown in literature up to now (Johnson et al. 1982).

Lactose sensors have been also developed by co-immobilizing $\beta$ galactosidase (EC 3.2.1.23) and glucose oxidase (Cordonnier et al. 1975; Bertrand et al, 1981).

18.2.1.9 Sucrose Sucrose is the most important representative of disaccharides contained in different foods and drinks as a sweetener and as a nutrient in fermentation broths. It is produced from sugar cane or sugar beets having a typical content of sucrose between 15 and $25 \%$. Sucrose determination is required in sugar production processes, quality control of foods, and fermentation control.

Up to now all enzyme electrodes for sucrose are based on invertase (EC 
3.2.26) -catalysed sucrose hydrolysis and subsequent glucose oxidation producing the electrode-active species. (Bertrand et al. 1981, Kulys et al. 1979. Macholán and Konečna 1983). Since GOD converts only the $\beta$-conformer of glucose, the co-immobilization of mutarotase (EC 5.1.3.3) which accelerates the conversion of the originally produced $\alpha$-glucose into the $\beta$-form results in an almost tenfold increased sensitivity, however, in parallel the linear measuring range is reduced by the same factor (Scheller and Karsten 1983; Cordonnier et al. 1975). This effect is based on the increased rate of mutarotation by the enzyme as compared with the slow spontaneous reaction.

For sucrose determination with the YSI analyser the procedure is the same as that for glucose, except that an invertase-mutarotase-GOD membrane has to be installed. The sucrose reading takes about 60 seconds to reach steady state, Raffinose and melibiose give readings of two per cent and eight per cent, respectively. The linear range extends up to $90 \mathrm{mmol} / 1$, the membrane life time is typically 10 days. Since the final enzyme reaction is the same as that used in glucose determination, sucrose determination is subject to interference by the glucose content of the sample. For such samples YSI suggests determining the sum of glucose and sucrose and additionally the glucose content by using the 'sucrose membrane' and the simple 'glucose membrane', respectively. This procedure appears to be not very convenient, since the membranes have to be exchanged during the measurement. It is more reasonable to carry out the inversion of sucrose outside the measuring cell using soluble invertase and to measure the glucose content before and after splitting of sucrose of soluble invertase.

The problem of glucose interference in sucrose determination can be solved by converting the glucose by GOD and catalase to non-disturbing products. For this purpose the indicating enzyme layer is covered with an anti-interference layer containing GOD and catalase (EC 1.11.1.6). $\beta$-DGlucose permeating into the anti-interference layer is completely eliminated. Using this anti-interference layer, determination of sucrose (Scheller and Renneberg 1983) is unaffected by endogenous glucose if the glucose concentration in the measuring cell does not exceed $2 \mathrm{mmol} / \mathrm{l}$. In this manner sucrose concentrations were determined directly in the juice of sugar beets or in samples of instant cocoa using the Glukometer (Scheller and Renneberg 1983). The same principle was extended to the elimination of other interfering substances, e.g. lactate.

18.2.1.10 Lactate Many critically sick patients develop acidosis as a result of respiratory, haemodynamic, or metabolic abnormalities. Elevated plasma lactate levels commonly result from metabolic disturbances producing acidosis particularly with associated vascular collapse. Determination of blood L-lactate is important to distinguish lactic acidosis from other causes 


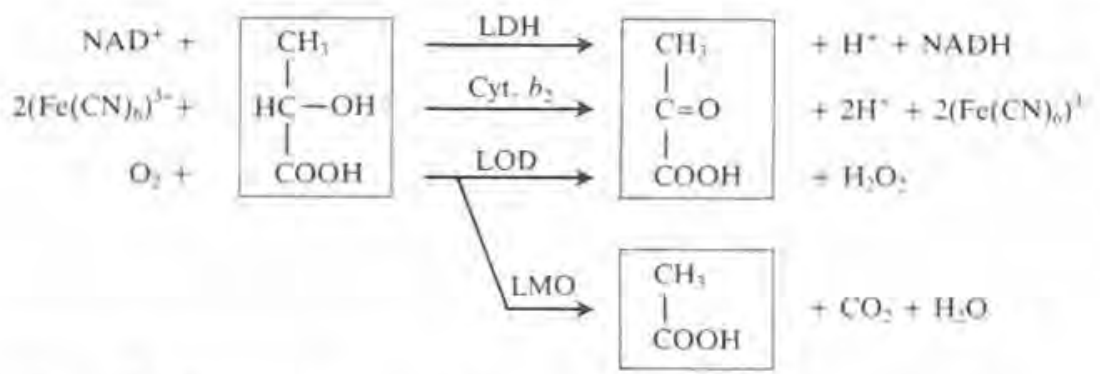

Scheme 18.1 Enzyme reactions for lactate determination.

and in the following treatment. A particular field where rapid and accurate blood lactate determination is desired is exercise control in sports medicine. Furthermore, lactate measurement in cerebrospinal fluid is of help in the differentiation between viral and purulent meningitis and in the detection of cerebral oxygen deficiency. Reference lactate values in blood and liquor are below $2.7 \mathrm{mmol} / \mathrm{I}$ and $1.2-2.1 \mathrm{mmol} / 1$, respectively. Lactate measurement in serum does not reflect the true blood concentration, since the increase of lactate strongly depends on the time between blood withdrawal and separation of corpuscular constituents.

Four different enzymes are suited for L-lactate determination with amperometric biosensors: Lactate dehydrogenase (LDH, EC 1.1.1.27), cytochrome $b_{2}$ (EC 1.1.2.3), lactate oxidase (LOD, EC 1.1.3.2), and lactate monooxygenase (LMO, EC 1.13.12.4). Their catalytic reactions are shown in Scheme 18.1. The LDH reaction can be coupled to redox electrodes by anodic oxidation of NADH, either directly (Yao and Musha 1979; Blaedel and Engstrom 1980; Laval et al. 1984; Cenas et al. 1984) or via electron mediators such as phenazine methosulphate (Malinauskas and Kulys 1978) or flavine mononucleotide (Suzuki et al. 1975). These sensors provided insight into the problems of electrochemical cofactor regeneration and mediated electron transfer but are not suited for routine application, presumably because of the electrode fouling by NADH or mediator oxidation products.

In biosensors based on $\mathrm{LMO}$, a decarboxylating enzyme which is often designated as 'lactate oxidase', the immobilized enzyme is fixed to Clarktype oxygen electrodes (Schindler and von Gülich 1981; Mascini et al. 1984). With such a sensor, Mascini et al. (1984) obtained a linear concentration dependence of up to $0.25 \mathrm{mmol} / 1$ in the measuring cell and a correlation coefficient of $r=0.995(y=1.094 x-0.128 \mathrm{mmol} / \mathrm{l})$ for measurement in reconstituted human sera. However, as with all $\mathrm{O}_{2}$-electrode-based sensors, problems may arise from differences in the oxygen content of buffer and sample. 
The reaction of the pyridine-nucleotide-independent lactate dehydrogenase, cytochrome $b_{2}$, is coupled to amperometric electrodes by the enzymes ability to transfer electrons from lactate to several mediators including thionine, dichlorophenol indophenol, and potassium ferricyanide, of which the latter reacts with highest rate (Kulys and Razumas 1983). Also the anodic oxidation of organic metal complexes co-immobilized with the enzyme and functioning as mediators has been employed (Kulys and Švirmickas 1980; Chapter 15). As an approach to in vivo lactate determination the natural electron acceptor, cytochrome $c$, has been co-immobilized with cytochrome $b_{2}$ and its reduced form then measured electrochemically (Durliat and Comtat 1980). These studies did not exceed the laboratory scale.

Lactate determination with LOD is appropriately carried out with the immobilized enzyme combined with a hydrogen peroxide probe.

Self-contained enzyme-electrode-based L-lactate analysers are marketed by La Roche, Switzerland, OMRON Tateisi Electronics Co., Japan, and Yellow Springs Instruments Corp., USA. The Roche Lactate Analyzer 640, which was introduced in 1976, uses a cytochrome $b_{2}$ sensor, the enzyme solution being simply entrapped in a reaction chamber in front of a platinum probe polarized to $+0.28 \mathrm{~V}$ for ferrocyanide oxidation. The device permits analysis of $20-30$ blood samples of $100 \mu \mathrm{l}$ per hour with lactate concentrations between 1 and $12 \mathrm{mmol} / 1$, with a precision of $5 \%$. Results are obtained within 2-3 min after blood withdrawal from the patients. The stability of one sensor preparation containing about $2 \mathrm{U}$ of enzyme is one month. Correlation with an optical LDH-method yields $r=0.998(y=$ $1.094 x-0.215 \mathrm{mmol} / \mathrm{l})$.

LOD electrodes are applied in the OMRON HER 100 and YSI Model 23L lactate analysers, both introduced in 1983. The OMRON instrument allows up to $8.9 \mathrm{mmol} / \mathrm{l}$ of blood lactate to be measured with a response time of $80 \mathrm{~s}$ and a precision $<5 \%$. The sample amount required is $100 \mu \mathrm{l}$. The temperature dependence of the enzyme reaction is compensated by a built-in thermistor in close proximity to the $\mathrm{H}_{2} \mathrm{O}_{2}$-indicating probe. Stability of the LOD sensor is 13 days and a correlation coefficient of $r=0.998$ is obtained with control serum (Tsuchida et al. 1985). Lactate in cell cultures can also be measured with the device. The YSI Model $23 \mathrm{~L}$ is applicable to lactate determination in whole blood as well as plasma and liquor cerebrospinalis. The respective correlation coefficients with the photometric Boehringer method are $0.997,0.997$, and 0.9996 . Linearity is obtained up to $15 \mathrm{mmol} / \mathrm{l}$ and only $25 \mu \mathrm{l}$ sample are needed. 42 samples per hour can be assayed.

A sensor for determination of the medically unimportant D-isomer of lactate is part of the Enzymat analyser offered by Seres, France. It is based on D-lactate-oxidase-containing cell-free extract coupled to a dissolved $\mathrm{O}_{2}$-probe. The measurable concentration range is 0.5 to $20 \mathrm{mmol} / 1$.

An L-lactate sensor with immobilized LOD was successfully used by 
Mascini et al. (1985) for in vivo studies with an endocrine artificial pancreas, the Biostator ${ }^{\text {. }}$. The sensor was inserted downstream from the glucose sensor and permitted to measure the body response of a diabetic patient to exercise, glucose infusion, and insulin infusion. This method should be of help in the design of infusion algorithms for extreme cases such as surgery of diabetics.

An innovative principle for L-lactate measurement is being introduced in the Glukometer analyser. With a lactate pyruvate recycling system consisting of LDH and cytochrome $b_{2}$ the sensitivity to lactate is amplified by at least one order of magnitude (Schubert et al. 1985a). With this sensor, lactate can be assayed using as little as $1 \mu \mathrm{l}$ blood. Use of an anti-interference membrane containing LMO to oxidize endogenous blood lactate to non-disturbing acetate and $\mathrm{CO}_{2}$ makes the sensor also applicable to fast determination of alanine aminotransferase activity (Schubert et al. 1984b).

18.2.1.11 Urea The urea concentration in blood, which is usually expressed by blood urea nitrogen (BUN) concentration is an important parameter in clinical chemistry, because it is a good index of the kidney function. The normal value is $3.6-8.9 \mathrm{mmol} / 1$.

Methods of amperometric urea determination have been developed much later than potentiometric and conductometric procedures. The first amperometric urea electrode developed by Suzuki's group in Japan consisted of a combination of a urease membrane with nitrifying bacteria which metabolize the formed ammonia and consume oxygen (Chapter 2). This oxygen consumption was measured with a Clark-type electrode (Okada et al. 1982). The described electrode contained five membranes and therefore a relatively high response time of $2 \mathrm{~min}$ for rate assays or $7 \mathrm{~min}$ for steady-state measurements is obtained. The absence of buffer interference, a correlation coefficient with optical methods of 0.97 , an operational stability of 10 days, and a linear signal-concentration relationship between 2 and $200 \mathrm{mmol} / \mathrm{l}$ are quite sufficient. The large measuring volume of $50 \mathrm{ml}$ at high final concentrations and a serial variation coefficient of $5 \%$ at $150 \mathrm{mmol} / 1$ characterize this method as applicable for urine analysis only.

Another possibility of amperometric urea determination has been developed on the basis of $\mathrm{pH}$-dependent hydrazine oxidation by Kirstein et al. (1985a). The advantages of this method are the linear calibration curve contrary to the logarithmic response of potentiometric sensors (Fig. 18.3), the excellent reproducibility $(\mathrm{CV}=1 \%$ ), a sample throughput of 40 per hour using rate assays (Kirstein et al. 1985b), and a linear signal-concentration curve between 0.025 and $2 \mathrm{mmol} / 1(1-80 \mathrm{mmol} / 1$ sample concentration with $50 \mu \mathrm{l}$ sample volume). The sensitivity is $75 \mathrm{nA} \mathrm{mmol}-1$, whereas 4.4 is obtained with the hybrid urea sensor described above. However this $\mathrm{pH}$ sensitive method suffers from interferences of compounds which determine 


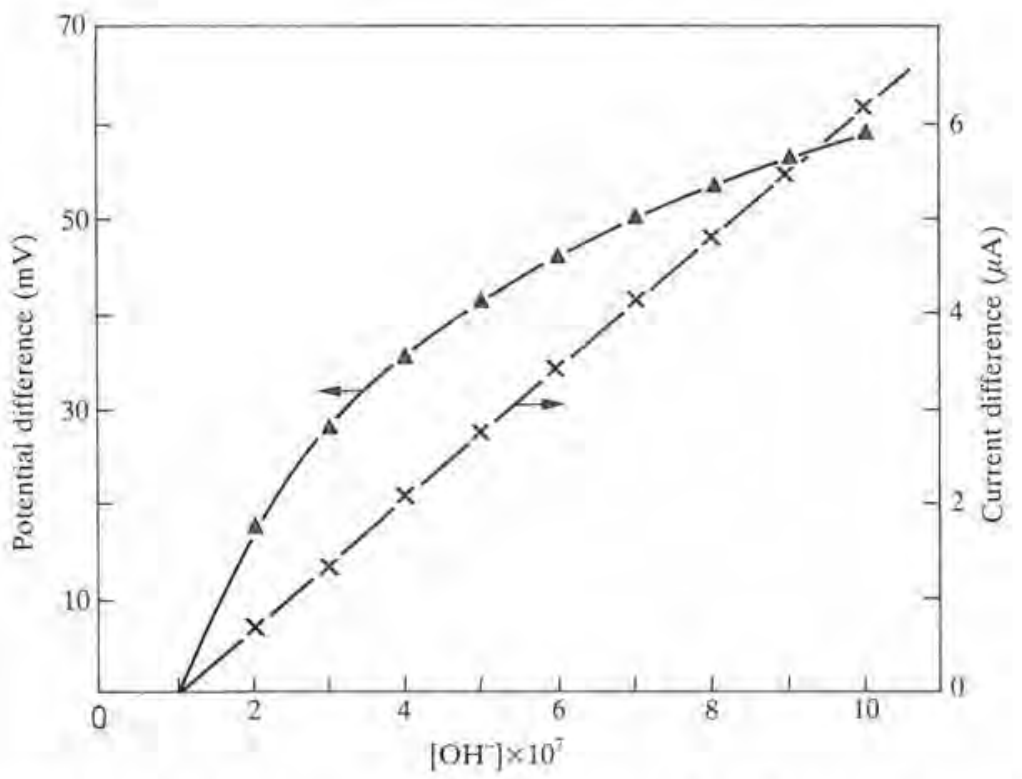

Fig. 18.3 Response characteristics of the amperometric and a potentiometric pH sensor.

$\mathrm{pH}$ and buffer capacity of biological fluids, such as proteins or hydrogen carbonate.

18.2.1.12 Creatinine and creatine Determination of creatinine and creatine in biological fluids is of significant value for diagnosis of renal, muscular, and thyroid function. Normal concentrations are at about $100 \mu \mathrm{mol} / 1$.

Two types of amperometric multi-enzyme sequence electrodes have been developed by Tsuchida and Yoda (1983) for creatinine and creatine determination. Hydrogen-peroxide-selective asymmetric cellulose acetate membranes bear co-immobilized creatinine amidohydrolase (CA, EC 3.5.2.10), creatine amidinohydrolase (CI, EC 3.5.3.3), and sarcosine oxidase (SO, EC 1.5.3.1), or only CI and SO, respectively. Hydrogen peroxide is the final product determined amperometrically. Both electrodes respond linearly up to $760 \mu \mathrm{mol} / 1$ of the substrates. The response time is $20 \mathrm{~s}$ (rate assay) and the detection limit $7.6 \mu \mathrm{mol} / 1$. The required sample volume is $25 \mu \mathrm{l}$, the sensitivity $11 \mathrm{nA} \mathrm{mmol}^{-1}$. The $\mathrm{CV}$ (within-day) is between 1.3 and $11.7 \%$ for creatinine and between 4.8 and $7.6 \%$ for creatine. The correlations with the optical Jaffé method are $y=1.078 x-23.0 \mu \mathrm{mol} / \mathrm{l} ; r=0.985$ for creatinine and $y=1.101 x-19.0 \mu \mathrm{mol} / 1 ; r=0.962$ for creatine in serum. Within 11 days there is less than $20 \%$ loss of activity. 
18.2.1.13 Uric acid The assay of uric acid for diagnosis and treatment of haematology disorders has been recognized. The normal range is $140-420 \mu \mathrm{mol} / \mathrm{l}$.

Uric acid is oxidized in presence of uricase (urate oxidase, EC 1.7.3.3) by molecular oxygen according to:

$$
\text { Uric acid }+\mathrm{O}_{2} \longrightarrow \text { Allantoin }+\mathrm{CO}_{2}+\mathrm{H}_{2} \mathrm{O}_{2}
$$

The first amperometric method for the quantitative determination of uric acid in biological fluids was published by Nanjo and Guilbault (1974). These authors measured the disappearance of dissolved oxygen because they could not separate the signals of $\mathrm{H}_{2} \mathrm{O}_{2}$ and the unreacted uric acid. In subsequent studies by other authors uric acid electrodes have been developed on the basis of $\mathrm{H}_{2} \mathrm{O}_{2}$ and/or uric acid electro-oxidation. A survey of the results is given in Table 18.2. Different modes of operation (rate or steady-state measurements) for oxygen and $\mathrm{H}_{2} \mathrm{O}_{2}$-sensitive electrodes, respectively, and a direct electrochemical uric acid oxidation were compared by Jănchen et al. (1983) (Fig, 18.4). The elimination of interferences by other electrode-active constituents of biological samples (e.g. ascorbic acid) was achieved by Kulys et al. (1983) when horse-radish peroxidase served as catalyst for the reaction between $\mathrm{H}_{2} \mathrm{O}_{2}$ and hexacyanoferrate (II) followed by reduction of the $\mathrm{Fe}^{\mathrm{III} \text {. }}$ complex at $\mathrm{O} \mathrm{V}$ vs. $\mathrm{Ag} / \mathrm{AgCl}$ on glassy carbon. Yoshino and Osawa (1980) coupled uricase at a $\mathrm{H}_{2} \mathrm{O}_{2}$ permselective membrane which is applied in the commercial uric acid analyser UA 300 A (Fuji Electric, Japan). Similar results were obtained with the GKM 02 variant of the Glukometer (Jänchen et al. 1983),

18.2.1.14 Ethanol Ethanol is the most common toxic substance involved in legal cases. Drunk driving and acute ethanol intoxication require fast and reliable determination of ethanol in blood. Measurement of the substance is also important in food and beverages and in fermentation processes.

So far, amperometric biosensors employing alcohol dehydrogenase (Malinauskas and Kulys 1978; Blaedel and Engstrom 1980) have not been successfully applied to ethanol analysis in 'real' samples. In contrast, alcohol oxidase (EC 1.1.3.13) from various microbial sources appears well suited for use in alcohol sensors. The enzyme catalyses the oxidation of lower primary alcohols according to

$$
\mathrm{R}-\mathrm{CH}_{2} \mathrm{OH}+\mathrm{O}_{2} \longrightarrow \mathrm{R}-\mathrm{CHO}+\mathrm{H}_{2} \mathrm{O}_{2}
$$

Guilbault et al. (1983) measured ethanol added to blood samples with an $\mathrm{O}_{2}$-electrode-based enzyme sensor employing a commercial alcohol oxidase from Candida boidinii. Deviation of the results from those obtained by gas chromatography was only $2.5 \%$. An alcohol-oxidase sensor was also applied in a flow-injection system for ethanol determination in beer (Schelter-Graf 
Table 18.2 Uricase electrodes

\begin{tabular}{|c|c|c|c|c|c|c|c|c|}
\hline \multirow{2}{*}{$\begin{array}{l}\text { Measured } \\
\text { species }\end{array}$} & \multirow{2}{*}{$\begin{array}{l}\text { Sample } \\
\text { volume } \\
(\mu \mathrm{l})\end{array}$} & \multirow{2}{*}{$\begin{array}{l}\text { Linear } \\
\text { up to } \\
(\mathrm{mmol} / \mathrm{l})\end{array}$} & \multirow{2}{*}{$\begin{array}{l}\text { Operational } \\
\text { stability }\end{array}$} & \multirow{2}{*}{$\begin{array}{l}\text { Precision } \\
(\%)\end{array}$} & \multicolumn{3}{|c|}{$\begin{array}{l}\text { Correlation with optical } \\
\text { methods } \\
y=a x+b ; r\end{array}$} & \multirow[b]{2}{*}{ References } \\
\hline & & & & & $a$ & $b(\mathrm{mmol} / \mathrm{l})$ & $r$ & \\
\hline $\mathrm{O}_{2}$ & 500 & 0.5 & $\begin{array}{l}100 \text { days, } 70 \% \\
\text { residual } \\
\text { activity }\end{array}$ & $\begin{array}{l}9 \\
4\end{array}$ & $\begin{array}{l}0.96 \\
0.97\end{array}$ & $\begin{array}{l}0.049 \\
0.357\end{array}$ & $\begin{array}{l}1.02 \\
1.0\end{array}$ & $\begin{array}{l}\text { Nanjo and Guilbault } \\
\text { (1974) }\end{array}$ \\
\hline $\begin{array}{l}{\left[\mathrm{Fe}(\mathrm{CN})_{6}\right]^{3-}} \\
\quad \text { (via } \mathrm{HRP} \text { ) }\end{array}$ & 10 & 0.035 & $\begin{array}{l}40 \text { days, } 50 \% \\
\text { residual } \\
\text { activity }\end{array}$ & - & - & - & - & Kulys el al. (1983) \\
\hline $\mathrm{O}_{2}$ & 100 & 1.2 & 7 days & $3.2-4.8$ & & & & Jänchen et al. (1983) \\
\hline $\mathrm{H}_{2} \mathrm{O}_{2}$ & 100 & 1.2 & & $1.8-2.0$ & 0.943 & 0.0198 & 0.9948 & \\
\hline $\mathrm{H}_{2} \mathrm{O}_{2}$ & 20 & 0.6 & 500 samples & $0.5-2.7$ & 1.10 & $2.44 \times 10^{-3}$ & 0.974 & Yoshino and Osawa (1980) \\
\hline $\mathrm{H}_{2} \mathrm{O}_{2}$ & 25 & 3.0 & $\begin{array}{l}17 \text { days } \\
\quad(1000 \text { samples })\end{array}$ & $0.6-2.2$ & 0.977 & $3 \times 10^{-3}$ & 0.985 & Tsuchida and Yoda (1983) \\
\hline
\end{tabular}




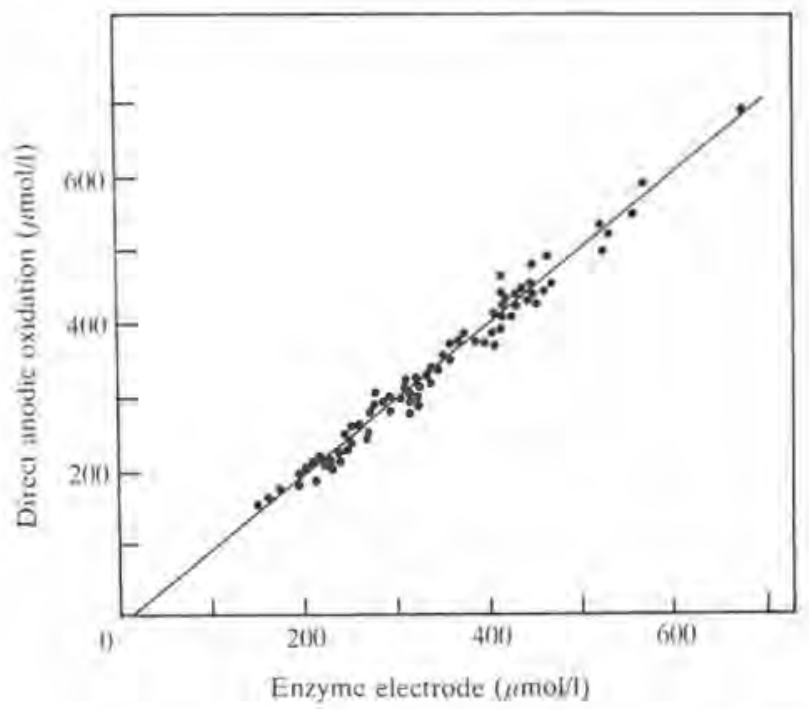

Fig. 18.4 Correlation of uric acid concentrations determined by direct anodic oxidation (ordinate) and by a uricase enzyme electrode (abscissa); $y=1.018 x+12.5$ $\mu \mathrm{mol} / \mathrm{l} ; r=0.9921$.

et al. 1983). Linearity of the method was up to $30 \mathrm{mmol} / \mathrm{l}$, the sensor half-life 6.5 days, and the measuring frequency 60 per hour. Accurate measurement of beer ethanol with the system was possible.

Using alcohol oxidase from Hansenula polymorpha together with catalase, Verduyn et al. (1984) developed a sensor for direct, continuous ethanol measurement in fermentation processes. The dissolved oxygen tension in the fermenter was kept between 95 and $100 \%$ by vigorous stirring and aeration. In the fermentation broth the electrode was stable only for 3-5 days as compared with several weeks when used in buffer. Another drawback of the method is the narrow range of substrate concentration for which a linear response is obtained (up to $1 \mathrm{mmol} / \mathrm{l}$ ). Therefore ethanol production can only be followed for a short period of fermentation time. Nevertheless, the sensor enables a rapid estimation of the fermentative capacity of aerobic yeast cultures to be made.

Alcohol analysis is possible with the YSI Model 27 industrial analyser. In this device the alcohol oxidase membrane is sandwiched between a polycarbonate and a cellulose acetate membrane. It has a lifetime of seven days. The instrument permits ethanol to be determined in beverages (Mason 1983) in concentrations up to $94 \mathrm{mmol} / 1$ with good accuracy. Precision is below $2 \%$. The method is subject to severe interference by methanol, but this substance is scarcely present in ethanol samples of interest. 
18.2.1.15 Glutamate, lysine A large number of amperometric biosensors for amino acids using either non-specific or highly selective amino acid oxidases have been studied. Of these only the sensors for glutamate and for L-lysine are likely to be applicable for routine analytical purposes. In the lysine electrode, highly selective L-lysine $\alpha$-oxidase (EC 1.4.3.-) from Trichoderma viride immobilized in a gelatin matrix and fixed to an $\mathrm{O}_{2}$-electrode is used (Romette et al. 1983). The properties of the carrier minimize the influence of sample oxygen content. The linear measuring range of the sensor is small, but approximation of the calibration curve and use of a microcomputer provide easy access to values between 0.2 and $3 \mathrm{mmol} / \mathrm{l}$. Enzyme inactivation during the course of the reaction is kept minimal by kinetic measurement, so that 3000 assays can be performed with one lysine oxidase membrane. The lysine sensor is employed in a flow system and its application in fermentation control is possible. The sensor is used in the Enzymat analyser offered by Seres, France.

An L-glutamate sensor using L-glutamate oxidase (EC 1.4.3.11), a newly isolated enzyme, was developed by Yamauchi et al. (1984) (see also Section 18.2.4.2). Glutamate in soy sauce is determined with good accuracy (correlation with L-glutamate decarboxylase method, $r=0.978$ ).

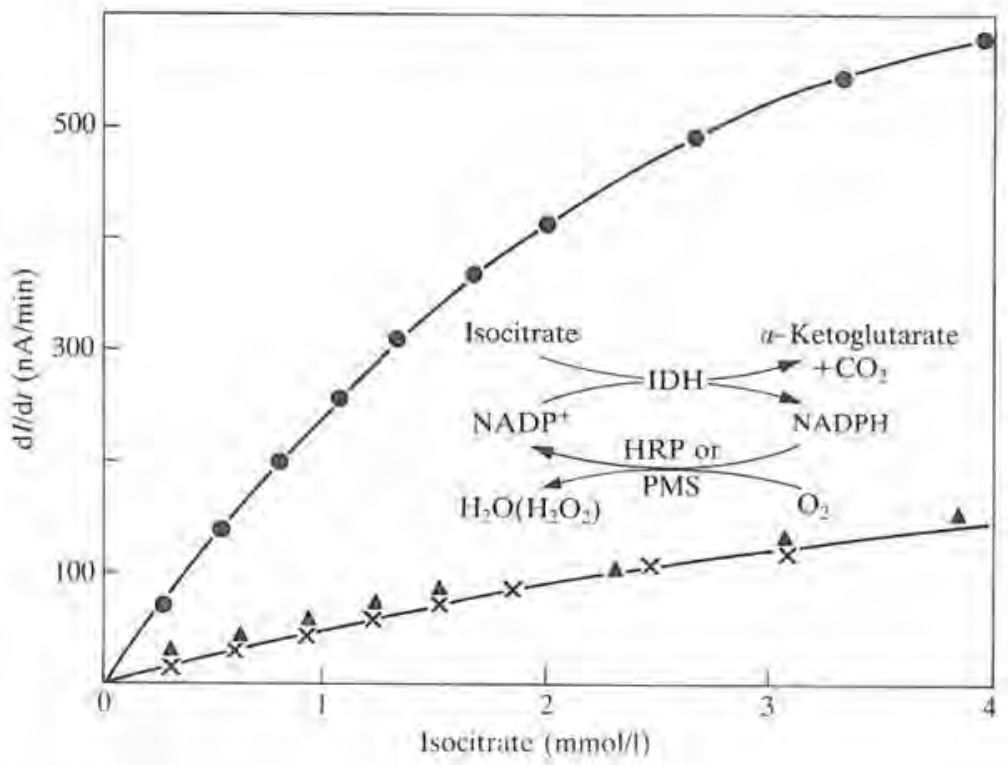

Fig. 18.5 Scheme and calibration curves of amperometric isocitrate determination with isocitrate dehydrogenase (IDH)-membrane electrodes. Cofactor recycling by: $x$, soluble PMS; $\boldsymbol{\Delta}$, co-immobilized PMS;

, co-immobilized HRP (and soluble co-catalysts). 
18.2.1.16 Isocitrate Isocitrate is a by-product of microbial citric acid production. High contents in fermentation broths can reduce the yield of crystalline citric acid. Its content depends on the strain of micro-organisms as well as on process conditions and therefore an analytical observation of the production can be advantageous.

Isocitrate is oxidatively decarboxylated by isocitrate dehydrogenase (IDH, EC 1.1.1.42), which is an NADP+-dependent enzyme (Fig. 18.5). It was determined amperometrically with unsatisfactory results by Nakamura et al. (1980), who utilized mediators immobilized together with the enzyme on the electrode surface. Better results are obtained when the oxygen consumption during catalytic NADPH reoxidation was measured by a Clark electrode. For this purpose, IDH was co-immobilized with horse-radish peroxidase (HRP, EC 1.11.1.17). The bi-enzyme electrode was used for isocitrate determination in pure isocitrate solutions by Schubert et al. (1985b). Application in fermentation solutions suffered from interferences by other constituents of the sample. Therefore HRP was replaced by phenazine methosulphate (PMS) in soluble form by Kirstein et al. (1984) or immobilized together with IDH (see Fig. 18.5). In all cases the cofactor was included in the background solution thus permeating the enzyme layer before isocitrate was injected.

18.2.1.17 Phosphate Phosphate determination in blood and urine is important in diagnosis of renal failure, Vitamin D deficiency, and hypervitaminosis, bone diseases, diabetic ketoacidosis, and other disturbances. Phosphorous is also of interest in agricultural chemistry and environmental protection, since it occurs in fertilizers and detergents.

Already in 1975, Guilbault and Nanjo proposed to use the combination of the phosphate-inhibitable enzyme, alkaline phosphatase, and GOD in a biosensor for phosphate ion. Using a similar approach, an acid phosphatase (EC 3.1.3.2)-containing potato tissue slice was immobilized on a layer of GOD in front of an oxygen electrode to assemble a phosphate sensor which is readily applicable in the Glukometer instrument (Schubert et al. 1984a). Glucose 6-phosphate present in the background buffer is hydrolysed by acid phosphatase, the glucose thus formed giving a stable base signal of the sensor. Addition of the phosphatase inhibitor, inorganic phosphate, causes a signal corresponding to the diminished glucose liberation. With this plant tissue hybrid sensor phosphate is measured in concentrations between 1 and $30 \mathrm{mmol} / \mathrm{l}$ using a $100 \mu \mathrm{l}$ sample. The stability is 300 assays or three weeks, precision below $2 \%$, and 15 samples per hour can be analysed. Of 14 substances tested, only tetraborate and molybdate, and to a lower extent borate and nitrate, interfere. Phosphate in urine and fertilizer samples is measured with good accuracy. For determination of blood phosphorous, however, where the normal concentrations are $0.5-1.8 \mathrm{mmol} / 1$, the sensor is not sensitive enough. 


\subsubsection{Low molecular weight, highly surface-active substances}

Organic substances possessing both a hydrophobic and a hydrophilic part tend to form micelles. This peculiarity is generally found with different lipids and cholesterol esters. In order to overcome this problem, detergents are added to the sample to get a molecular disperse solution. However, the presence of detergents changes the solubility of oxygen thus interfering with the oxygen consumption measurement. These detergents may be accumulated at the membranes of the electrode resulting in alteration of the masstransport rates.

Together with plasma phospholipids and triglycerides, cholesterol is transported through the bloodstream bound to specific proteins. These lipoproteins can be visualized as a sphere with an outer solubilizing coating of protein and phospholipid and an inner hydrophobic, neutral core of triglyceride and cholesterol. Neutral lipids (triglycerides), phospholipids, and cholesterol esters are split by specific hydrolases to form a substrate for the respective oxidase. Owing to the problems described above, the hydrolytic reaction catalysed by soluble enzyme is separated from the concentration measurement which is performed with an amperometric oxidase electrode.

18.2.2.1 Cholesterol Today everyone worries about cholesterol, because a relationship was found between the concentration of cholesterol in plasma and the amount of cardiovascular diseases, i.e., the number of heart attacks increases with the value of cholesterol (Levy 1981). A plasma cholesterol of 3.1-6.7 mmol/1 would be the average or 'normal' for a man or woman in middle age where about $70 \%$ of the total cholesterol is esterified by fatty acids.

Enzyme electrodes for the determination of free cholesterol have been developed by Satoh et al. (1977), Bertrand et al. (1979), and Mascini et al. (1983) using cholesterol oxidase (COD, EC 1.1.3.6) immobilized at the surface of collagen membranes or at a nylon net. The concentration of free cholesterol was determined with an accuracy of about 5-25\%. After incubating the serum in triton $\mathrm{X} 100$-or deoxycholate-containing background solutions with cholesterol ester hydrolase (CEH, EC 3.1.1.13), total cholesterol concentration is also susceptible. The same reaction sequence is used in the lipid analyser ICA-LG 400 from Toyo Jozo, Japan. $30 \mu \mathrm{l}$ of the serum sample are pretreated with $7.5 \mu \mathrm{l} \mathrm{CEH}$ solution for $11 \mathrm{~min}$ at $37^{\circ} \mathrm{C}$ to give the free cholesterol. Its concentration is measured using COD immobilized in front of an oxygen electrode. The fast response of only $15 \mathrm{~s}$ is based on the rate method of oxygen consumption and the measuring value is corrected by the signal of an enzyme-free oxygen sensor. Cholesterol concentration of 40 samples per hour is measured together with the simultaneous determination of triglycerides and phospholid concentration (see below). Ascorbic acid and 


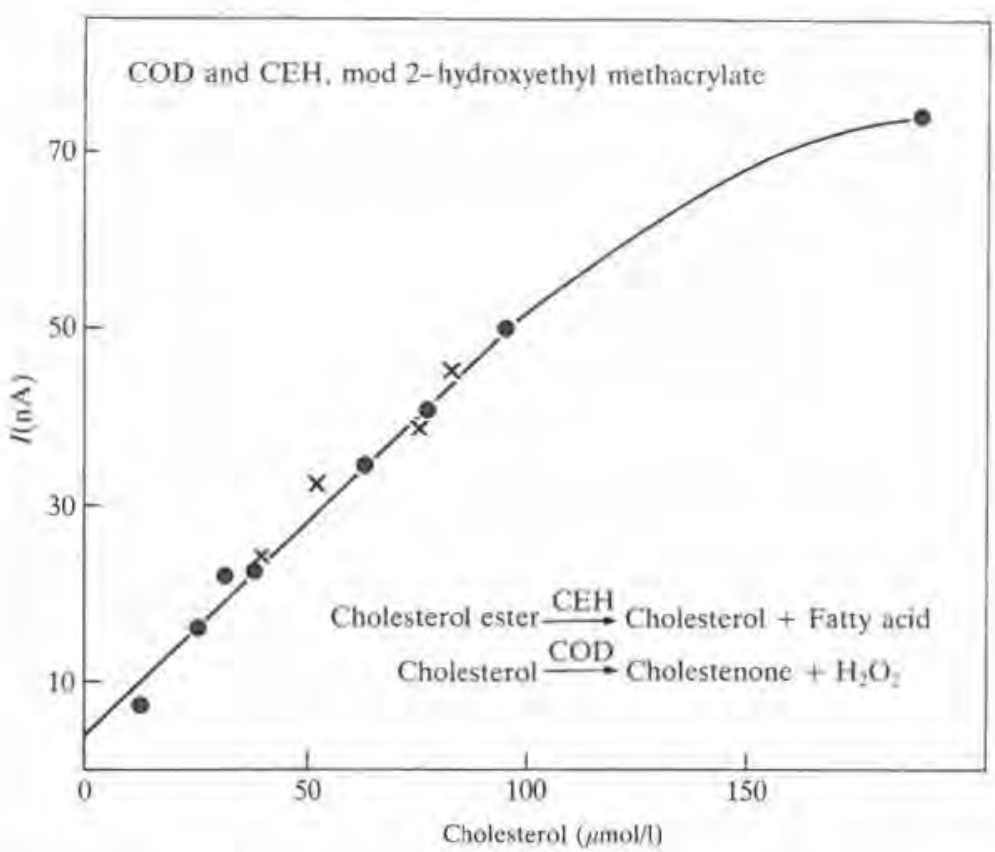

Fig. 18.6 Calibration graph for the determination of total cholesterol in aqueous cholesterol standards $(\bullet)$ and serum solutions $(x)$ obtained with the CEH/COD enzyme-sequence electrode.

bilirubin do not interfere. A correlation equation to a non-specified reference method of

$$
y=1.03 x-0.15 \mathrm{mmol} / 1 ; r=0.985(n=50)
$$

for total cholesterol is given.

An enzyme electrode with direct spatial contact of COD and CEH immobilized at the surface of spheron particles has been described by Wollenberger et al. (1983). Both free cholesterol and cholesterol esters were measured with the same sensitivity (Fig. 18.6) giving evidence for complete ester hydrolysis. Five minutes after addition of $50 \mu \mathrm{l}$ of serum sample the total cholesterol content is indicated in the $\mathrm{H}_{2} \mathrm{O}_{2}$ steady-state mode. However the two-enzyme sensor is stable only for one day.

18.2.2.2 Triglycerides Serum triglyceride analysis is a crucial test in the diagnosis and classification of hyperlipidaemia. Hyperlipidaemia, primarily known as a coronary risk factor, is also associated with many other disorders. The normal concentration of serum triglycerides is $0.35-1.7 \mathrm{mmol} / 1$.

In triglyceride determination the first step of the reaction sequence is 
catalysed by microbial lipoprotein lipase (EC 3.1.1.3) where glycerol and fatty acids are formed. The following reactions couple the glycerol conversion to the formation of an easily measurable substance. Soluble or immobilized glycerol dehydrogenase (EC 1.1.1.6) coupled with the diaphorasecatalysed conversion of NADH has been used in the amperometric determination of triglycerides (Kelly and Christian 1984; Winartasaputra et al. 1982). On the other hand, glycerol kinase (EC 2.7.1.30) and glycerophosphate oxidase (EC 1.1.3.-) are used in the lipid analyser ICA-LG 400 (Toyo Jozo, Japan) where the rate of oxygen consumption is evaluated in the same manner as described for cholesterol. Forty serum samples per hour can be analysed. A possible application of glycerol oxidase should result in a considerable simplification of the reaction system.

18.2.2.3 Phospholipids The normal concentration range of phospholipids in plasma extends from 2.5 to $3.0 \mathrm{mmol} / 1$. The main constituent is phosphatidyl choline. Enzymatic determination of phospholipids involves the combination of phospholipase D (EC 3.1.4.4) and choline oxidase (EC 1.1.99.1). Pre-incubation of the serum sample with soluble phospholipase D is combined with measurement of the free choline by an amperometric oxidase electrode in the ICA-LGA 400 of Toyo Jozo.

The hydrogen peroxide formed in an enzyme reactor has been measured electrochemically using the enzymes immobilized together on a hydrophobic agarose gel (Karube et al. 1979). The response time of the enzyme reactor flow device is $2 \mathrm{~min}$ and the linear range between 0 and $4 \mathrm{mmol} / \mathrm{l}$.

\subsubsection{High-molecular-weight soluble substances}

There are two ways to determine enzymatically inactive, water-soluble highmolecular-weight substances with amperometric biosensors:

(i) They have to be split into units small enough to penetrate the membranes covering the immobilized enzyme layer where they are converted to electrode-active products.

(ii) They can be detected by immunological reactions if the respective antibodies or antigens are available.

Potentiometric biosensors have been proposed which are based on indication of the changes of charge density resulting from the specific complex formation of a bioaffin and the high-molecular analyte.

18.2.3.I Polysaccharides The most abundant carbohydrate is cellulose, a polymer of glucose connected by $1,4-\beta$-glycosidic bonds. Its world-wide use is estimated at 800 million tons per year. Another homopolymer made up of glucose is starch where glucose units are connected in the main chain by $1,4-\alpha$-glycosidic bonds. The new biotechnological product pullulan is a linear 
polysaccharide containing maltotriose units connected by $1,6-\alpha-$ glycosidic bonds,

Prior to analysis, polysaccharides are acid-hydrolysed to glucose or they are split by specific enzymes, e.g. cellulases, amylases, or pullulanases.

18.2.3.2 Starch The YSI Industrial Analyzer Model 27 equipped with a glucose oxidase membrane is adaptable by pretreatment procedures to starch determination. It uses two approaches to starch measurement:

(i) For internal starch hydrolysis a constant amount of glucoamylase is injected into the measuring solution and after equilibration the starch sample is added. Sixty seconds later, a reading of the glucose produced by the starch hydrolysis is displayed.

(ii) In external hydrolysis procedures for starch hydrolysis glucoamylase alone or together with $\alpha$-amylase (EC 3.2.1.1) is used, and the glucose content in the hydrolysed starch sample is determined.

Corn, rice, wheat, and potato starch, but also starch in cornmeal, breakfast cereals, and pancake mixes have been determined. The glucose measured ranges from 0 to $25 \mathrm{mmol} / 1$ with a reproducibility of $\pm 2 \%$.

A more elegant and economical approach is to co-immobilize glucose oxidase with glucoamylase for measurement of saccharides able to penetrate the protective dialysis membrane in front of the two-enzyme layer. Using the Glukometer GKM 01 with a glucoamylase-glucose oxidase enzyme electrode (Renneberg et al. 1983b) starch is directly determined within $0.5-1 \mathrm{~min}$ with a linear range of up to $0.3 \%$ (final concentration) if a constant amount of $\alpha$ amylase (1 $\mathrm{U}$ ) is added to the measuring solution (Fig. 18.7). The problem of interference by endogenous glucose was solved by covering the glucoamylase-glucose oxidase electrode with an anti-interference enzyme later containing glucose oxidase and catalase (Fig. 18.7) (see also Section 18.2.1.9).

Pullulan concentrations can be measured using the Glukometer GKM 01 equipped with a glucoamylase-glucose oxidase membrane on a modified oxygen electrode (Renneberg et al. 1985). After addition of a constant amount of soluble pullulanase $(2 \mathrm{U} / \mathrm{ml})$ to the measuring solution, pullulan was determined up to $0.1 \%$ (final concentration). The Glukometer was also used to determine soluble cellulose by adding a constant amount of cellulase (EC 3.2.1.4) into the measuring solution and following the glucose produced hydrolytically (Pfeiffer et al. 1980),

18.2.3.3 Antigens and antibodies Up to now, no commercial biosensor system has been described for antigens and antibodies. The recently developed enzyme immunosensors measure the activity of marker-enzymes bound to a defined amount of antigen. The enzyme-marked antigen and the 


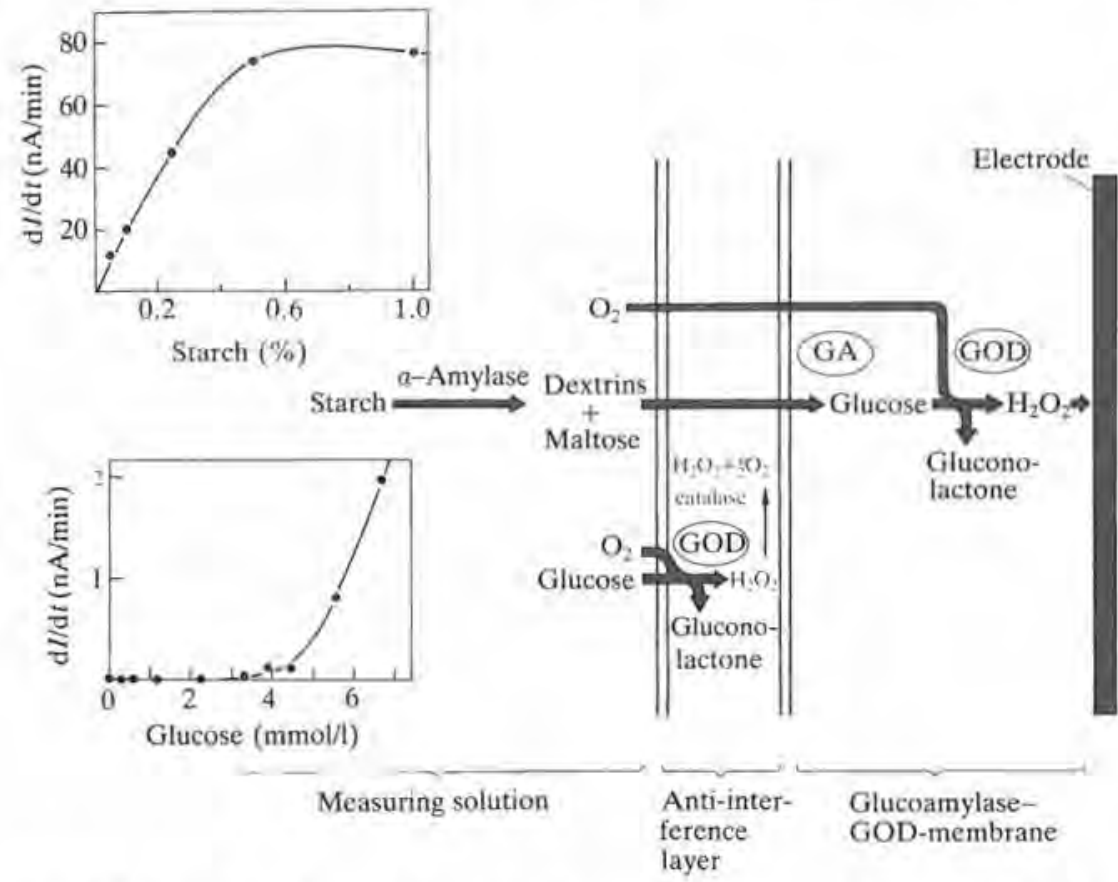

Fig. 18.7 Principle of starch determination using a glucose-eliminating multi-layer sensor.

unlabelled antigen to be determined compete for the binding sites of antibodies on the membrane covering the electrode. The unknown concentration of unlabelled antigens is inversely related to the activity of the markerenzymes measured by the electrode. However, at present the sensitivity is low as compared with established enzyme immunoassays; fast measurements are impossible because the measuring cell is occupied for the whole incubation time (up to several hours) to permit formation of the antigen-antibody complex.

At present, the development of both potentiometric and amperometric electrode-based enzyme immunoassays seems to be the only practical way to avoid these disadvantages. The antigen-antibody complex formation is carried out independently of the sensor system. When the incubation period is finished, the substrate of the marker enzyme is added and the rate of substrate conversion is followed by the sensor. With the Glukometer, 1.6 to $16 \mathrm{ng}$ of factor-VIII-related antigen could be measured with a glucose sensor in human plasma using alkaline phosphatase as marker-enzyme and glucose 6-phosphate as substrate (Renneberg et al. 1983a). 
Amperometric detection of superoxide anion formed on interaction of neutrophils with IgG adsorbed at the electrode surface seems to open up new possibilities to more direct immunoelectrodes (Green et al. 1984; Chapter 4).

\subsubsection{Enzyme activities}

In clinical diagnostics the determination of enzyme activities in body fluids is a rapidly developing field. Elevated enzyme activities found in blood, serum, plasma, or urine are due to leakage from damaged tissues and cells. For fermentation control the measurement of the activity of industrial enzymes, e.g. proteases or amylases, is of great importance. Sensors for enzyme activity determination are mainly based on the measurement of the initial rate of substrate conversion by the enzyme of interest, which is added to the measuring solution containing a saturating concentration of the substrate. The high concentration of substrate is required to obtain zero-order reaction kinetics, where the substrate conversion rate depends only on the enzyme activity. To date commercial self-contained enzyme sensors are available only for $\alpha$-amylase (Fuji Electric, Japan).

\subsubsection{Lactate dehydrogenase Lactate dehydrogenase (LDH) is a} tetramer allowing the formation of five isoenzymes of LDH differing in subunit structure and electrophoretic mobility. In mammalian cells LDH is only located in the cytoplasm. Thus, after cellular damage (liver and heart diseases), LDH is readily released from the cytoplasm. Total LDH activity in serum is of clinical importance in differentiating disorders, such as acute myocardial infarction, congestive heart disease, pernicious anaemia, and hepatitis. The normal ranges for $\mathrm{LDH}$ in serum of adult males and females are 63-155 and 62-131 U/l.

With a pyruvate-oxidase (EC 1.2.3.3)-based sensor the LDH activity of human sera was determined in the range 25-135 U/1 (Minoura et al. 1982) using the maximum current decrease. The sensor of Mizutani et al. (1983), using immobilized lactate oxidase, permits the sequential determination of both L-lactate and $\mathrm{LDH}$. The minimum activity of $\mathrm{LDH}$ which could be determined was $1 \mathrm{U} / 1$. The relative standard deviation is $2.6 \%$ in ten successive measurements. The total measuring time for $\mathrm{LDH}$ activity is $5 \mathrm{~min}$. The sensor is stable for two weeks with ten measurements per day. Serum samples with LDH activities ranging from 138 to $414 \mathrm{U} / \mathrm{I}$ can be determined with a correlation coefficient of 0.995 between the sensor and a conventional method.

No self-contained LDH-analysers have been described up to now. However, the L-lactate analysers of La Roche, OMRON, Yellow Springs Instruments Corp., and the Glukometer could well be adapted to LDH activity measurements (see also Section 18.2.1.10). Problems could arise from 
the relatively small amounts of lactate formed in the LDH reaction demanding a pre-incubation step, and from the lactate present in serum.

18.2.4.2 Amylases Amylases catalyse the hydrolysis of oligosaccharides and polysaccharides (starch and glycogen). $\alpha$-Amylase yields primarily maltose and some other oligosaccharides (4-12 glucose units). Determination of $\alpha$-amylase in blood and urine is of decisive importance in the diagnosis of acute pancreatitis. The normal activity of $\alpha$-amylase in serum extends from 60 to $150 \mathrm{U} / 1$.

The activity of $\alpha$-amylase in serum has been determined by different biosensor systems. The commercial analyser of Fuji Electric (Osawa et al. 1981) uses a GOD sensor measuring first the endogenous glucose concentration of the sample and, after addition of maltopentaose and a constant amount of $\alpha$ glucosidase (maltase, EC 3.2.1.20), the $\alpha$-amylase activity of the sample. However, this method is expensive due to high consumption of the soluble enzyme and the special substrate and gives wrong results if the endogenous glucose concentration is high. A more efficient approach seems to be the use of bi-enzyme sensors with co-immobilized GOD and glucoamylase (Pfeiffer et al, 1980) or $\alpha$-glucosidase (Yoda and Tsuchida 1983). In both cases maltose and oligosaccharides formed in the $\alpha$-amylase-catalysed starch hydrolysis diffuse into the bi-enzyme membrane where they are converted by glucoamylase or $\alpha$-glucosidase to glucose which is indicated by the GOD reaction. $\alpha$-Amylase activity in human serum is determinable with the $\alpha$-glucosidase-GOD membrane mounted on an electrode of the Yellow Springs Instruments Ǵlucose Analyzer $23 \mathrm{~A}$. Thirty seconds after injection of $25 \mu \mathrm{l}$ of human serum into a phosphate buffer containing $0.1 \%$ soluble $\mathrm{starch}$, the current increase is recorded for $30 \mathrm{~s}$. Total assay time is $100 \mathrm{~s}$. Within-day precision with three different sera $(n=20)$ is between 4.4 and $7.3 \%$. Repeated assays of control human sera over 17 days showed a between-day coefficient of variation of $5 \%$. After 1000 assays in over 17 days $70 \%$ of the initial activity was still present.

$\alpha$-amylase activities of bacterial origin have been measured with the Glukometer GKM 01 and the bi-enzyme sensor (glucoamylase and GOD). The calibration curve is linear up to $4.0 \mathrm{U} \alpha$-amylase in the measuring solution (Renneberg et al. 1983b). With the same bi-enzyme sensor pullulanase activity was also determined (Renneberg et al. 1985). A linear range of the maximal slope of the current-time curve is obtained with a constant amount of $0.1 \%$ pullulan up to a pullulanase activity of $0.7 \mathrm{U} / \mathrm{ml}$. The limit of detection is $0.05 \mathrm{U} / \mathrm{ml}$.

18.2.4.3 Transaminases The importance of determination of alanine and aspartate aminotransferase (ALT, EC 2.6.1.2 and AST, EC 2.6.1.1, formerly: GPT and GOT) activities ranges not far below that of glucose 
measurement. Increased serum activities of these enzymes indicate myocardial, hepatic, and jaundice diseases, all of which are increasingly common in industrialized countries. Normal activities are 5-24 U/I for ALT and 5-20 U/l for AST. They can increase 100-1000 fold, especially in acute hepatitis and alcoholism. Therefore a large detection range is desirable for biosensors measuring these activities.

According to the reactions catalysed by ALT (eqn. 18.4) and AST (eqn. 18.5)

L-alanine $+\alpha$-Ketoglutarate $\rightarrow$ L-Glutamate + Pyruvate

$\mathrm{L}$-Aspartate $+\alpha$-Ketoglutarate $\rightarrow$ L-Glutamate + Oxaloacetate

biosensing of the products, glutamate, pyruvate, or oxaloacetate, is possible. Kihara et al, (1984) applied a bi-enzyme electrode comprising poly (vinyl chloride)-adsorbed oxaloacetate decarboxylase (EC 4.1.1.3) and pyruvate oxidase (EC 1.2.3.3) for the sequential measurement of both transaminases. The base sensor was a hydrogen-peroxide electrode. First, AST activity is determined by adding the sample to aspartate- and $\alpha$-ketoglutaratecontaining measuring solution. The slope of the current-time curve reflects AST activity. Then a substrate solution containing alanine is added and a further increase of the slope observed. The difference between the two slopes is linearly related to ALT activity. The measuring range is up to $1500 \mathrm{U} / \mathrm{I}$ with either enzyme. Assay of both activities is completed within less than $4 \mathrm{~min}$. The correlation coefficients between the sensor procedure and spectrophotometric methods as calculated for 25 serum samples are 0.99 for ALT as well as AST determination.

Another, equally promising approach is to use L-glutamate oxidase. This newly isolated flavoprotein selectively catalyses the oxidative deamination of the amino acid, yielding $\mathrm{NH}_{3}, \alpha$-ketoglutarate, and $\mathrm{H}_{2} \mathrm{O}_{2}$. Coupling of immobilized glutamate oxidase to an $\mathrm{O}_{2}$ or $\mathrm{H}_{2} \mathrm{O}_{2}$ probe results in a glutamate sensor also useful for ALT and AST measurement (Yamauchi et al. 1984). However, the method requires pre-incubation of the sample with the respective substrate mixtures for $30 \mathrm{~min}$. Linearity between anodic $\mathrm{H}_{2} \mathrm{O}_{2}$ oxidation current and ALT and AST activity, respectively, is at least up to $200 \mathrm{U} / \mathrm{l}$.

Fast ALT measurement is possible with the cytochrome $b_{2} / \mathrm{LDH}$ sensor described in the Section 18.2,1,10 (Schubert et al. 1984b).

\subsection{Conclusions}

Amperometric biosensors for substrate determination are a reliable and highly specific tool. Highest economical benefit is effected if they are applied in automatic flow devices like the ADM 300 of VEB MLW, the Enzymat of Seres, and the announced flow-injection analyser of Control Equipment Co., Princeton. Whilst in commercial analysers the highest sample throughput is 
120 per hour, in a flow-injection analysis apparatus 300 glucose samples per hour can be measured (Olsson et al. 1986). Substrate recycling results in a considerable increase in sensitivity allowing high sample dilution or reduction of sample volume to the order of less than $1 \mu \mathrm{l}$. This principle opens up a new avenue to measurements in the picomolar range - the range of hormones and antigens. Further progress will be achieved by combining biocatalysts with microelectronic elements which transduce and amplify signals.

\section{References}

Abel, P., Müller, A. and Fischer, U. (1984). Experience with an implantable glucose sensor as a prerequisite of an artificial beta cell, Biomed. Biochim. Acta 43,577-84 .

A. B. 2-GDR (1985). Arzneimittelbuch der DDR. Akademie-Verlag, Berlin.

Asperger, L. and Krabisch, Ch. (1986), Uberprüfung amperometrischer Me $\beta$ prinzipien zur Glucosebestimmung mit Enzymelektroden. Acta Biotechnol. In press.

Bertrand, C., Coulet, P. R. and Gautheron, D. C. (1979). Enzyme electrode with collagen-immobilized cholesterol oxidase for the microdetermination of free cholesterol, Anal. Lett. 12, 1477-88.

- (1981). Multipurpose electrode with different enzyme systems bound to collagen films. Anal. Chim. Acta 126, 23-34.

Blaedel, W.J. and Engstrom, R.C. (1980). Reagentless enzyme electrodes for ethanol, lactate, and malate. Anal. Chem. 52, 1691-7.

Cass, A. E. G., Davis, G. . Francis, G. D., Hill, H. A. O., Aston, W. J., Higgins, I. J., Plotkin, E. V., Scott, L. D. L. and Turner, A.P. F. (1984). Ferrocene-mediated enzyme electrode for amperometric determination of glucose. Anal. Chem. 56, 667-71.

Čenas, N., Rozgaite, J. and Kulys, J. (1984), Lactate, pyruvate, ethanol, and glucose-6-phosphate determination by enzyme electrode. Biotechnol. Bioengn. 26. 551-3.

Chua, K, S. and Tan, I. K. (1978). Plasma glucose measurement with the Yellow Springs Glucose Analyzer. Clin. Chem. 24/1, 150-2.

Cordonnier, M., Lawny, F., Chapot, D. and Thomas, D. (1975). Magnetic enzyme membranes as active elements of electrochemical sensors. Lactose, saccharose, maltose bienzyme electrodes. FEBS Lett. 59/2, 263-7.

Durliat, H. and Comtat, M. (1980). Reagentless amperometric lactate electrode. Anal. Chem. 52, 2109-12.

Enfors, S.-O. (1982). A glucose electrode for fermentation control. Appl. Biochem. Biotechnol. 7, 113-9.

Fogt, E. J., Dodd, L. M., Jenning, E. M. and Clemens, A. H. (1978). Development and evaluation of a glucose analyzer for a glucose controlled insulin infusion system (Biostators). Clin. Chem. 24, 1366-76.

Green, M. J., Hill, H. A. O., Tew, D. G. and Wolton, N. J. (1984). An opsonised electrode. FEBS Lett. 170, 69-72.

Guilbault, G. G. (1984). Analytical uses of immobilized enzymes, p. 350. Marcel Dekker, New York.

— and Nanjo, M. (1975). A phosphate-selective electrode based on immobilized 
alkaline phosphatase and glucose oxidase. Anal, Chim. Acta 78, 69-80.

- Danielsson, B., Mandenius, C. F. and Mosbach, K. (1983). Enzyme electrode and thermistor probes for determination of alcohols with alcohol oxidase. Anal. Chem. 55, 1582-5.

Havas, J., Porjesz, E., Nagy, G. and Pungor, M. (1980). Glucose selective sensor. Determination of glucose content of blood and urine. Hung. Sci. Instruments 49 , 53-9.

Jänchen, M., Walzel, G., Neef, B., Wolf, B., Scheller, F, Kühn, M., Pfeiffer, D., Sojka, W. and Jaross, W, (1983). Harnsäurebestimmung in verdünntem Serum mit enzymelektrochemischem und enzymlosem Sensor. Biomed. Biochim. Acta 9, 1055-65.

Johnson, J.M., Halsall, H. B. and Heineman, W. R. (1982). Galactose oxidase enzyme electrode with internal solution potential control, Anal. Chem. 54, 1394-9.

Karube, I., Hara, K., Satoh, I and Suzuki, S. (1979). Amperometric determination of phosphatidyl choline in serum with use of immobilized phospholipase D and choline oxidase. Anal. Chim, Acta 106, 243-50.

Kelly, T.A. and Christian, G.D. (1984). Amperometric determination of glycerol and triglycerides using an oxygen electrode. Analyst 109, 453-6.

Kihara, K., Yasukawa, E. and Hirose, S. (1984). Sequential determination of glutamate-oxalacetate transaminase and glutamate-pyruvate transaminase activities in serum using an immobilized bienzyme-poly (vinyl chloride) membrane electrode. Anal, Chem, 56, 1876-80.

Kirstein, D., Schubert, F., Scheller, F., Abraham, M. and Boross, L. (1984). Amperometrische Isocitrat-bestimmung in Fermentationslosungen der mikrobiologischen Citronensäureproduktion. Abstracts of the 16th Annual Meeting of the Biochemical Society of the GDR, p. 37.

- Kirstein, L. and Scheller, F. (1985a). Enzyme electrode for urea with amperometric indication: part $\mathrm{I}$ - basic principle, Biosensors 1, 117-30.

- Scheller, F., Olsson, B. and Johansson, G. (1985b). Enzyme electrode for urea with amperometric indication: part II - electrode with diffusional limitation. Anal, Chim. Acta 171, 345-50.

Kulys, J. J., Ralys, E. V. and Penkova, R. S. (1979). Automatic analyzer of sucrose using immobilized enzymes. Prikl. Biokhim. Mikrobiol. 15/2, 282-90.

- and Razumas, V.J. (1983), Biocatalysis in electrochemistry of organic compounds (Russian), p. 61. Mokslas, Vilnius.

- and Švirmickas, G.-J. S. (1980). Reagentless lactate sensor based on cytochrome $b_{2}$. Anal, Chim. Acta 117, 115-20.

- Laurinavičius, V.S. A., Pesliakiene, M. V. and Gurevičiene, V.V. (1983). The determination of glucose, hypoxanthine and uric acid with use of bi-enzyme amperometric electrodes, Anal. Chim. Acta 148, 13-18.

Laval, J.-M., Bourdillon, Ch. and Moiroux, J. (1984). Enzymatic electroanalysis: electrochemical regeneration of NAD * with immobilized lactate dehydrogenase modified electrodes. J. Am. Chem. Soc. 106, 4701-06.

Levy, R. (1981). Cholesterol, lipoproteins, apoproteins, and heart disease: Present status and future prospects. Clin. Chem, 27, 653-62.

Lobel, E. and Rishpon, J. (1981). Enzyme electrode for determination of glucose. Anal. Chem, 53, 51-3. 
Macholán, L. and Konečna, H. (1983). A biospecific membrane sensor for the determination of sucrose. Coll. Czech. Chem. Commun. 48, 798-804.

Malinauskas, A and Kulys, J. (1978). Alcohol and glutamate sensors based on oxidoreductases with regeneration of nicotinamide adenine dinucleotide. Anal. Chim. Acta 98, 31-7.

Mascini, M., Moscone, D. and Palleschi, G. (1984). A lactate electrode with lactate oxidase immobilized on nylon net for blood serum samples in flow systems. Anal. Chim. Acta 157, 45-51.

- Tomassetti, T. and Iannello, M. (1983). Determination of free and total cholesterol in human bile samples using an enzyme electrode. Clin. Chim. Acta 132, 7-15.

- Fortunati, S., Moscone, D., Palleschi, G., Massi-Renedetti, M. and Fabietti, P. (1985). A L-lactate sensor with immobilized enzyme for use in in-vivo studies with an endocrine artificial pancreas. Clin. Chem. 31, 451-3.

Mason, M. (1983). Determination of glucose, sucrose, lactose and ethanol in foods and beverages, using immobilized enzyme electrodes. J. Assoc, Off. Anal. Chem. $66,981-4$.

Minoura, N., Yamada, S., Karube, I., Kubo, I. and Suzuki, S. (1982). Determination of lactate dehydrogenase in serum by using a pyruvate sensor. Anal. Chim. Acta 135, 355-7.

Mizutani, F., Sasaki, K. and Shimura, Y. (1983). Sequential determination of L-lactate and lactate dehydrogenase with immobilized enzyme electrode. Anal. Chem. 55, 35-8.

Mor, J.-R. and Guarnaccia, R. (1977). Assay of glucose using an electrochemical enzymatic sensor. Anal. Biochem. 79, 319-28.

Müller, E., Kühnel, S., Trommler, Ch. and Günther, R. (1985). Automatisierte elektrochemische Bestimmung mit Durchflu $\beta$-meßzellen. Labortechnik, 18, 8-9.

Nakamura, K., Nankai, S. and Iijime, T. (1980). Bioelectrochemical sensor using immobilized enzyme electrodes, National Tech. Rep. 26, 497-506.

Nanjo, M. and Guilbault, G. G. (1974). Enzyme electrode sensing $\mathrm{O}_{2}$ for uric acid in serum and urine. Anal. Chem. 46, 1769-72.

Newman, D.P. (1976). Membrane for enzyme electrodes. US-Patent 3979274. Int.Cl. G $01 \mathrm{~N} \mathrm{27/46.}$

Niwa, H., Itoh, K., Nagata, A, and Osawa, H. (1981). Studies on the rapid determination of glucose level in blood using the enzyme electrode, the 'glucometer'. Tokaj. J. Exp. Clin. Med. 6/4, 403-14.

Okada, T., Karube, I. and Suzuki, S. (1982). Hybrid urea sensor using nitrifying bacteria. Eur. J. Appl. Microbiol. Biotechnol. 14, 149-54.

Olsson, B., Lundbäck, H., Johansson, G., Scheller, F. and Nentwig, J. (1986), Theory and application of diffusion-limited amperometric enzyme electrode detection in flow injection analysis of glucose. Anal. Chem. 58, 1046-52.

Osawa, H., Akiyama, S. and Hamada, T. (1981). Determination of uric acid, glucose and amylace in whole blood using enzyme electrode. Proc. 1st Int. Sensor Symp. 163-8.

Pfeiffer, D., Scheller, F., Jänchen, M. and Bertermann, K. (1980). Glucose oxidase bienzyme electrodes for ATP, NAD*, starch and disaccharides. Biochimie 62, 587-93. 
Renneberg, R., Schößler, W. and Scheller, F. (1983a). Amperometric enzyme sensorbased immunoassay for factor VIII related antigen. Anal. Lett. 16 (B I6), 1279-89, - Kaiser, G., Scheller, F. and Tsujisaka, Y. (1985). Enzyme sensor for pullulan and pullulanase activity. Biotechnol. Lett. 11, 809-12.

- Scheller, F., Riedel, K., Litschko, E. and Richter, M. (1983b). Development of anti-interference enzyme layer for $\alpha$-amylase measurement of glucose containing samples. Anal. Lett. 16 (B 12), 877-90.

Romette, J. L., Froment, B, and Thomas, D. (1979). Glucose-oxidase electrode. Measurements of glucose in samples exhibiting high variability in oxygen content. Clin. Chim. Acta. 95, 249-53.

— Yang, J.S., Kusakabe, H. and Thomas, D. (1983). Enzyme electrode for specific determination of L-lysine. Biotechnol. Bioengn, 25, 2557-66.

Satoh, 1., Karube, 1. and Suzuki, S. (1977). Enzyme electrode for free cholesterol. Biotechnol. Bioeng. 19, 1095-100.

Scheller, F. and Karsten, Ch. (1983). A combination of invertase reactor and glucose oxidase electrode for the successive determination of glucose and sucrose. Anal. Chim. Acta 155, 29-36.

and Pfeiffer, D. (1978). Enzymelektroden. Z. Chem. 18, 50-57.

and Renneberg, R. (1983). Glucose-eliminating enzyme electrode for direct sucrose determination in glucose-containing samples. Anal. Chim. Acta 152, 265-9.

Schelter-Graf, A., Huck, H. and Schmidt, H.-L. (1983). Rasche und genaue Bestimmung von Ethanol mittels einer Oxidase-Elektrode in einem Strömungs-in jektionssystem. Z. Lebensm. Unters, Forsch. 177, 356-8.

Schindler, J.G. and von Gülich, M. (1981). L-Lactat-Durchflußelektrode mit immobilisierter Lactat-Oxidase. Fresenitus Z. Anal, Chem, 308, 434-6.

Schubert, F., Kirstein, D., Schröder, K. L. and Scheller, F. W. (1985a). Enzyme electrodes with substrate and coenzyme amplification. Anal. Chim. Acta, 169, 391-6.

- Renneberg, R., Scheller, F. W. and Kirstein, L. (1984a). Plant tissue hybrid electrode for determination of phosphate and fluoride. Anal. Chem. 56, 1677-82. - Kirstein, D., Abraham, M., Scheller, F. and Boross, L. (1985b). Horseradish peroxidase based bienzyme electrode for isocitrate. Acta Biotechnol. 5, 275-8.

- Scheller, F., Kirstein, D., Schröder, K. L. and Chojnacki, A. (1984b). Verfahren zur elektrochemischen Bestimmung von Lactat, Pyruvat und der Activităt von Alaninaminotransferase. DD-Patent 222 896, Int. Cl. C 12 Q1/26.

Shichiri, M., Kawamori, R., Hakui, N., Asakawa, N., Yamasaki, Y, and Abe, H. (1984). The development of wearable-type artificial endocrine pancreas and its usefulness in glycaemic control of human diabetes mellitus. Biomed. Biochim. Acta 43, 561-8.

Suzuki, S., Takahashi, F., Satoh, I. and Sonobe, N. (1975). Ethanol and lactic acid sensors using electrodes coated with dehydrogenase-collagen membranes. Bull. Chem. Soc. Japan 48, 3246-9.

Taylor, P. J., Kmetec, E. and Johnson, J. M. (1977). Design, construction, and applications of a galactose selective electrode. Anal. Chem. 49, 789-94.

Thévenot, D. R. (1982). Problems in adapting a glucose oxidase electrochemical sensor into an implantable glucose-sensing device. Diabeles Care 5/3, 184-9. 
Sternberg, R. and Coulet, P. (1982). A glucose electrode using high-stability glucose-oxidase collagen membranes. Diabetes Care 5/3, 203-6.

Tsuchida, T, and Yoda K. (1981), Immobilization of D-glucose oxidase onto a hydrogen peroxide permselective membrane and application for an enzyme electrode. Enzyme Microb. Technol, 3 326-30.

- (1983). Multi-enzyme membrane electrodes for determination of creatinine and creatine in serum. Clin. Chem. 29, 51-6.

- Takasugi, H., Yoka, K., Takizawa, K. and Kobayashi, S. (1985). Application of $1-(+)$ - lactate electrode for clinical analysis and monitoring of ússue culture medium. Biotechnol. Bioengn. 27, 837-41.

Verduyn, C., Zomerdijk, T.P. L., van Dijken, J.P. and Scheffers, W. A. (1984). Continuous measurement of ethanol production by aerobic yeast suspensions with an enzyme electrode. Appl. Microbiol. Biotechnol. 19, 181-5.

Winartasaputra, H., Kuan, S. S. and Guilbault, G. G. (1982). Amperometric enzymic determination of triglycerides in serum. Anal. Chem. 54, 1987-90.

Wollenberger, U., Kühn. M., Scheller, F., Deppmeyer, V.and Jänchen, M. (I983). Amperometric enzyme sequence electrodes for cholesterol. Bioelectrochem. Bioenerg. 11, 307-17.

Yamauchi, H., Kusakabe, H., Midorikawa, Y., Fujishima, T. and Kuninaka, A. (1984). Enzyme electrode for determination of L-glutamate. Abstr. 3rd Eur. Congr, Biotechnol., pp. J-705-10, Verlag Chemie, Weinheim.

Yao, T. and Musha, S. (1979). Electrochemical enzymatic determination of ethanol and L-lactic acid with carbon paste electrode modified chemically with NADH. Anal. Chim. Acta 110, 203-9.

Yoda, K. and Tsuchida, T. (1983). Bi-enzyme electrode for determination of $\alpha$-amylase activity in serum. Proc, 2nd Int. Sensor Symp. Fukuoka, 648-53.

Yoshino, F. and Osawa, H. (1980). Rapid measurements of glucose and uric acid in whole blood using the enzyme electrodes. Clin. Chem. 26, 1060. 


\title{
Compensated enzyme-electrodes for in situ process control
}

\author{
SVEN-OLOF ENFORS
}

\subsection{Introduction}

A great number of the enzyme electrodes described in the literature are based on oxygen-utilizing enzymes. These electrodes must be furnished with enough oxygen to permit the reaction to run without oxygen limitation unless other electron carriers are utilized. Enzyme electrodes are mostly designed to operate according to the dynamic mode, which means that the initial rate of signal change after the exposure of the electrode to the sample is used as the measure of concentration of the analyte. In this mode of operation oxygen limitation can be avoided through oxygenation of the sample prior to its injection into the measuring chamber of the electrode. A further improvement which permits injection of samples without oxygen has been presented by Romette et al. (1979). They developed an enzyme membrane with high oxygen solubility and showed that it is possible to saturate the membrane with oxygen prior to the exposure of the electrode to oxygen-free samples.

However, the dynamic mode of operation complicates the utilization of enzyme electrodes for process control. This mode offers the very important advantage of easy re-calibration during the measurements but an automatic sampling procedure is required if it is to be used for process control.

A possibility to avoid the sampling problem in fermentation control with enzyme electrodes would be to design electrodes for in situ operation in the bioreactor. However, this mode of operation introduces several problems that must be solved: (i) sterilizability of the probe, (ii) continuous operation with a steady-state signal representing the analyte concentration, (iii) operation without sample treatment like dilution or addition of oxygen or other cosubstrates of the reaction, and (iv) re-calibration during the operation.

This chapter describes two principles that contribute to the solution of the problems of continuous operation of glucose electrodes in situ in a bioreactor: the oxygen-stabilized glucose electrode and the externally buffered glucose electrode. In both systems the micro-environment of the enzyme is controlled by the operator to compensate for unfavourable conditions for the enzyme. 


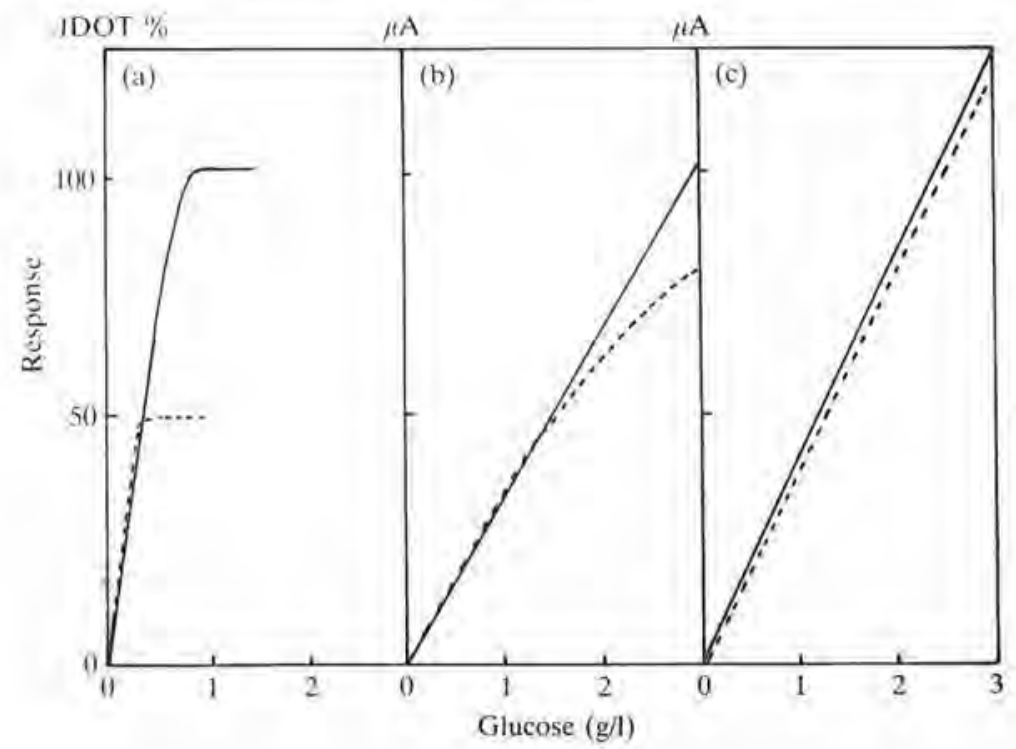

Fig. 19.1 Steady-state responses of glucose electrodes to increasing concentrations of glucose in phosphate buffer at pH. (a) An oxygen-diffusion-dependent electrode. (b) An oxygen-stabilized electrode working with an internal oxygen tension corresponding to $50 \%$ of the air saturation value. (c) An oxygen stabilized electrode working at an internal oxygen tension of $100 \%$ of the air saturation value. Solid lines: Oxygen tension of sample is $100 \%$ of air saturation. Dotted lines: Oxygen tension of sample is $50 \%$ of air saturation.

\subsection{The oxygen-stabilized glucose electrode}

The net enzyme reaction of a glucose-oxidase-based glucose electrode with catalase is:

$$
\beta \text {-D-Glucose }+1 / 2 \mathrm{O}_{2} \longrightarrow \text { Gluconate }+\mathrm{H}^{*}
$$

The oxygen solubility in water is about $0.25 \mathrm{~mm}$ and the $K_{\mathrm{M}}$ value of glucose oxidase with respect to oxygen is unusually high - about $0.5 \mathrm{~mm}$ (Linek et al. 1980). In order to respond linearly to increasing concentration of glucose the reaction rate of the electrode must be controlled by the rate of diffusion of glucose into the enzyme layer. However, the high $K_{\mathrm{M}}$ value in relation to the low oxygen solubility makes the response non-linear at glucose concentrations above about $1 \mathrm{~g} / \mathrm{l}$, (Fig. 19.1a). Furthermore, the oxygen concentration in a fermenter is normally much lower and may approach zero which turns the enzymatic reaction from glucose limitation to oxygen limitation at a point that cannot be controlled. Thus, oxygen-diffusion-dependent glucose electrodes are not suitable for in situ operation in a fermenter. 
The principle of oxygen compensation of the enzymatic reaction was developed to solve this problem (Enfors 1981). It is depicted in Fig. 19.2. The enzymes glucose oxidase (EC 1.1.3.4) from Aspergillus niger, and catalase (EC 1.11.1.6) from beef liver are immobilized by cross-linking in bovine serum albumine with glutaraldehyde on a platinum screen. This screen is then attached close to the oxygen-sensitive tip of an oxygen electrode. If the enzyme is contaminated with micro-organisms during the assembling of the electrode it must be disinfected, e.g. by dipping in a $2.5 \%$ glutaraldehyde solution. Finally, the sterile unit is inserted into the autoclaved electrode housing fitted with a cellulose acetate membrane at its end. The enzymescreen must be tightly pressed against the cellulose acetate membrane to reduce the response time. The platinum electrode is connected as the anode in the electrolysis circuit shown in Fig. 19.2. When glucose diffuses into the enzyme layer the enzymatic reaction consumes oxygen at a rate that is proportional to the rate of glucose transport which, at steady state, is proportional to the sample concentration of glucose.

The signal from the oxygen electrode is compared with a constant reference signal and the enzyme reaction causes oxygen depletion and a deviation between these potentials. The electronic circuit generates a current through the anode on which the enzyme is immobilized and oxygen is formed by electrolysis of water at the platinum surface. The oxygen diffuses into the enzyme layer and supplies the reaction. The electrolysis current is controlled by the difference between the two potentials in such a way that the oxygen

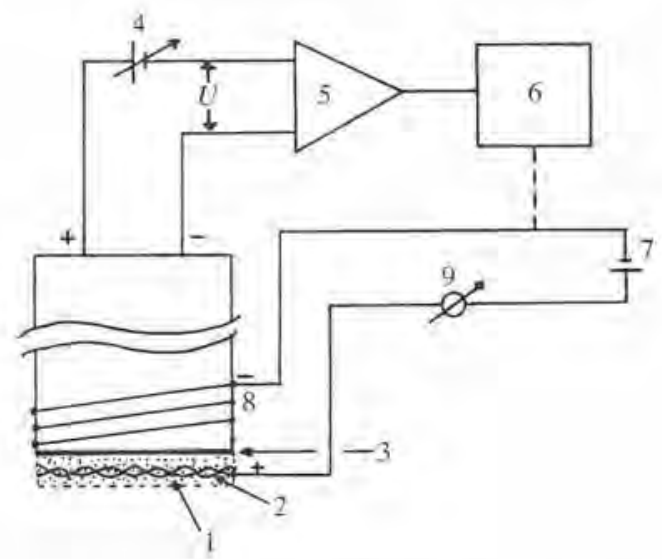

Fig. 19.2 Design principle of the oxygen-stabilized glucose electrode. 1, Immobilized enzymes. 2, Platinum net. 3, Teflon membrane of oxygen electrode. 4, Reference voltage. 5, Differential amplifier, 6, PID-controller that controls the current through the electrolysis circuit to keep the differential voltage $(U)$ zero. 7 , Voltage source of electrolysis circuit. 8, Platinum coil around the electrode. 9, Microammeter. 
Table 19.1 Summary of enzymatic and electrochemical reactions of the oxygen-stabilized glucose electrode

\begin{tabular}{|c|c|c|c|}
\hline \multicolumn{2}{|c|}{$\mathrm{C}_{6} \mathrm{H}_{12} \mathrm{O}_{6}+\mathrm{O}_{2}+\mathrm{H}_{2} \mathrm{O}$} & \multirow{2}{*}{$\underset{\text { Catalase }}{\stackrel{\text { Glucose oxidase }}{\longrightarrow}}$} & $\mathrm{C}_{6} \mathrm{H}_{12} \mathrm{O}_{7}+\mathrm{H}_{2} \mathrm{O}_{2}$ \\
\hline & $\mathrm{H}_{2} \mathrm{O}_{2}$ & & $1 / 2 \mathrm{O}_{2}+\mathrm{H}_{2} \mathrm{O}$ \\
\hline Anode: & $\mathrm{H}_{2} \mathrm{O}$ & $\longrightarrow$ & $1 / 2 \mathrm{O}_{2}+2 \mathrm{H}^{+}+2 \mathrm{e}^{-}$ \\
\hline Cathode: & $2 \mathrm{H}^{+}+2 \mathrm{e}^{-}$ & $\longrightarrow$ & $\mathrm{H}_{2}$ \\
\hline Net reactio & $\mathrm{H}_{12} \mathrm{O}_{6}+\mathrm{H}_{2} \mathrm{O}$ & $\longrightarrow$ & $\mathrm{C}_{6} \mathrm{H}_{12} \mathrm{O}_{7}+\mathrm{H}_{2}$ \\
\hline
\end{tabular}

production rate increases until the deviation is reduced to zero. Thus, the oxygen tension is maintained constant in the enzyme layer independent of the glucose concentration but the higher the reaction rate the higher is the electrolysis current. A platinum wire around the electrode body is connected as cathode of the electrolysis circuit.

The enzymatic and electrochemical reactions in this oxygen-stabilized electrode are summarized in Table 19.1; each oxidized molecule of glucose corresponds to two electrons through the electrolysis circuit. Thus, if there is no exchange of oxygen between the enzyme electrode and the sample medium the electrolysis current should be a measure of the glucose concentration. The linear relationship between electrolysis current and glucose concentration is shown in Fig. 19.1b, and c.

One way to minimize the exchange of oxygen would be to use an external oxygen sensor as a reference signal for the electrolysis control. This is possible, but during fermentation process control the external oxygen concentration can be very low and then the response range is considerably reduced because the oxygen tension in the enzyme will be low (compare $b$ and c in Fig. 19.1). By applying high reference voltages the oxygen tension in the electrode can be forced to values exceeding the air saturation value and this is a way to increase the linear response range.

However, when the oxygen tension in the electrode is higher than in the sample an error will occur that is caused by loss of electrolytically produced oxygen through diffusion to the sample medium. A compensation method for this has been developed (Enfors and Cleland 1983) and the correct glucose concentration is given by:

$$
S=\left(I-k_{\mathrm{D}}\left(\mathrm{DOT}_{\mathrm{i}}-\mathrm{DOT}_{\mathrm{o}}\right)\right) / k_{\mathrm{G}}
$$

where $S=$ glucose concentration $(\mathrm{g} / \mathrm{l}), I=$ electrolysis current $(\mu \mathrm{A})$, $k_{\mathrm{D}}=$ mass transfer coefficient $(\mu \mathrm{A} / \%$ air saturation $), \mathrm{DOT}_{\mathrm{i}}$ and $\mathrm{DOT}_{\mathrm{o}}$ 


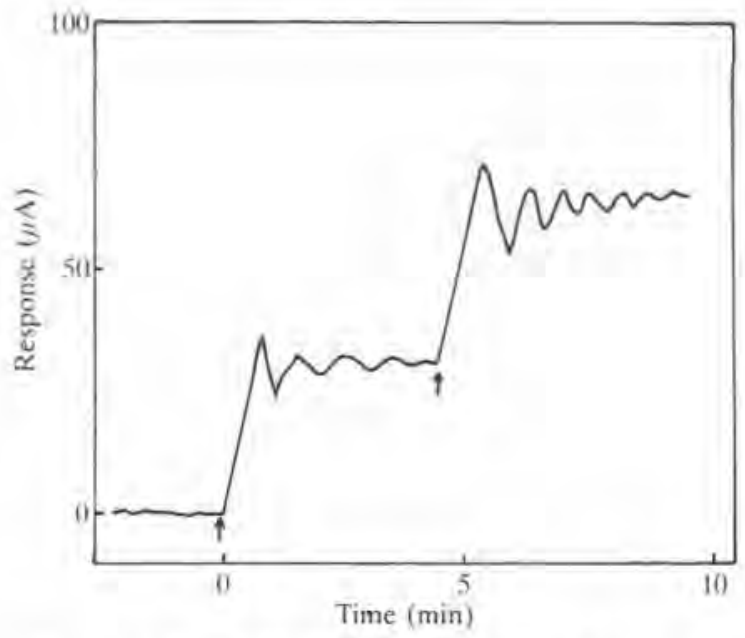

Fig. 19.3 Time-response curve of the oxygen-stabilized glucose electrode. At the arrows additions of glucose were made to increase the concentration by about $1 \mathrm{~g} / \mathrm{I}$ in a phosphate buffer at $\mathrm{pH} 7.0$.

$=$ internal and external dissolved oxygen tension, respectively $(\%$ air saturation), and $k_{\mathrm{G}}=$ electrode sensitivity when $\mathrm{DOT}_{\mathrm{i}}=\mathrm{DOT}_{\mathrm{o}},\left(\mu \mathrm{A} / \mathrm{I} \mathrm{g}^{-1}\right)$.

The response-time curve of this electrode is demonstrated in Fig. 19.3. It shows that the time constant $\left(t_{95}\right)$ of the enzymatic reaction is about two minutes. However, the internal DOT control system has not yet been optimized and the time constant of the total electrode output is about $5-10 \mathrm{~min}$.

The author uses this electrode for control of glucose concentration in E. coli $\mathrm{K} 12$ processes which are suffering from catabolite repression, which means that a considerable amount of glucose is converted to inhibitory products of anaerobic energy metabolism unless the glucose concentration is kept below $1 \mathrm{~g} / 1$. The first experiments to control the glucose concentration exhibited pronounced drift of the electrode sensitivity (Cleland and Enfors 1983). A somewhat surprising increase of the electrode sensitivity during the first hours of the process caused the fermenter glucose concentration to stabilize at about $0.5 \mathrm{~g} / \mathrm{I}$ when the controller was set at $1 \mathrm{~g} / 1$. This increasing sensitivity of the electrode is never observed as long as measurements are performed in buffer solutions.

The reason for the increased sensitivity during the first hours of operation in the bioreactor can be explained. During the assembling of the electrode the enzyme was infected with bacteria (mainly staphylococci) from the hands. As long as the electrode was used in buffer these bacteria did not grow and were too few to influence the electrode. As soon as the electrode was inserted in the fermenter with growth medium the cells increased in number so that they 
could compete with the glucose oxidase in the consumption of the glucose that diffused into the electrode. This should not influence the electrode performance if the stoichiometry of the two reactions were identical. However, the stoichiometry of the glucose-oxidase-catalysed reaction gives $1 / 2$ oxygen per glucose while the stoichiometry of the bacteria-catalyzed reaction is

$$
\mathrm{C}_{6} \mathrm{H}_{12} \mathrm{O}_{6}+6 \mathrm{O}_{2} \longrightarrow 6 \mathrm{CO}_{2}+6 \mathrm{H}_{2} \mathrm{O}
$$

for pure respiration and about half that oxygen consumption if glucose is used for growth. Thus, the pure respiration-based electrode gives 12 times higher sensitivity than the glucose-oxidase-based electrode. The resulting performance of the infected electrode is intermediary depending on the relative extent of the different reactions occurring in the electrode.

\subsection{Principle of the externally buffered enzyme electrode}

A major limitation of enzyme electrodes that are used in situ is that the sample cannot be treated prior to analysis by, for example dilution, change of $\mathrm{pH}$, or addition of co-substrates of the enzymatic reaction. To solve these problems and also to prevent possible accumulation of inhibitory products in the enzyme the principle of the externally buffered enzyme electrode was introduced and applied to glucose analysis (Cleland and Enfors 1984). It was soon found that the oxygen for the enzymatic reaction could be supplied from the buffer flow but that the response of such an electrode was nonlinear. This electrode also worked best when equipped with the oxygenstabilization system described above when applied to glucose analysis.

The design of an externally buffered glucose electrode with electrolytic oxygen production is shown in Fig. 19.4. The enzyme with the electrolysis system is identical with that described above. However, the enzyme disc is placed in a chamber created by placing it between two 15 mesh nylon nets which are pressed tightly between the oxygen electrode surface and a cellulose acetate membrane. This chamber is furnished with inlet and outlet channels for the buffer composed of $1 \mathrm{~mm}$ inner diameter stainless steel syringes connected to drain or a buffer feeding pump through Tygon-tubing. The buffer is kept above room temperature to avoid air bubble clogging.

The glucose diffuses through the cellulose acetate membrane and is mixed in the turbulent buffer flow in the enzyme chamber. Thus, this electrode operates under much lower glucose concentration than the former one at corresponding sample concentrations. This has the effect of extending the linear response range. Depending on the buffer flow-rate, linear responses obtained are from $0-10 \mathrm{~g} / 1$ at low flow rates $(0.042 \mathrm{ml} / \mathrm{min})$ to $0-100 \mathrm{~g} / 1$ at $0.45 \mathrm{ml} / \mathrm{min}$. Other properties of the externally buffered glucose electrode are that it can operate in samples with extreme $\mathrm{pH}$ values since the buffer flow protects the enzyme. It may for instance be used at $\mathrm{pH} 2.0$. At a buffer flow 


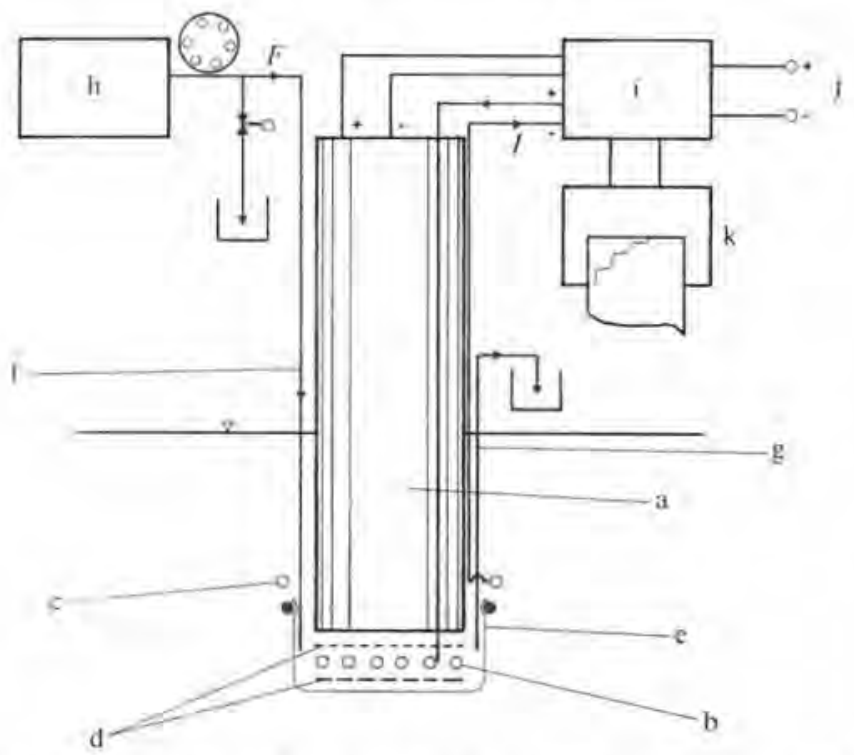

Fig. 19.4 Main parts of the externally buffered enzyme electrode: a, oxygen electrode; $b$, Pt gauze with immobilized enzymes; c, Pt coil (cathode); d, nylon nets; e, dialysis membrane; $f$, in-going buffer stream; $g$, buffer effluent; $h$, buffer reservoir; i, PID controller, j, reference potential, k, recorder; $I$, electrolysis current; $F$, buffer flow. (From Anal. Chem. (1984) 56, 1880, by permission of the American Chemical Society).

rate of $0.15 \mathrm{ml} / 1$ the internal glucose concentration was about $8 \%$ of the external concentration. Of this glucose about $3.75 \%$ was converted to gluconic acid.

\subsection{Compensated enzyme-electrodes for process control}

The two principles of compensation described above solve some problems in fermentation control. The oxygen-stabilized electrode without the external buffering is sensitive enough to be used for control of glucose feeding, in processes which are catabolite repressed. The oxygen-stabilization system makes analysis possible and independent of the oxygen concentration in the reactor.

The externally buffered glucose electrode of fers the possibility of monitoring the glucose consumption in processes where the initial concentration is high: $100 \mathrm{~g} / \mathrm{l}$ is a common initial concentration in processes where there is no inhibitory effect of the sugar. Here the diluting effect of the buffer adjusts the glucose concentration to values that are suitable for the measuring 


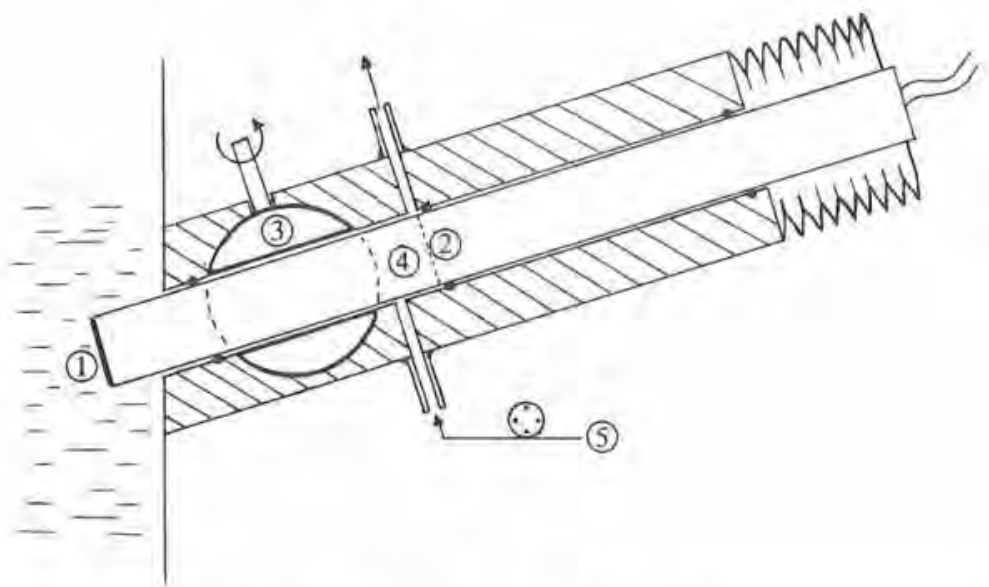

Fig. 19.5 Calibration housing for an in situ sensor. The electrode (1) is inserted through an open ball valve (3) into the reactor. For calibration it is withdrawn to a position (2) where the valve can be closed and a calibration solution (5) is pumped through the calibration chamber (4). After calibration the valve is opened and the electrode is re-inserted for continued operation.

system. Furthermore, the protecting effect of the buffer on the enzyme makes analysis possible in environments that are detrimental to the enzyme.

The main difficulty preventing a breakthrough in the application of enzyme electrodes to process control is the poor stability. Declining response might be caused by rapid inactivation of the enzyme. However, since the reaction is diffusion-rate controlled rather than kinetically controlled it also suffers from fouling of the membrane. Such fouling can be expected irrespective of whether the analyte is diffusing directly into an in situ electrode, into a dialyser, or forced through a filter for further transport to an external sensor. In each case the mass transport over the membrane must be subject to calibration if measurements are to be performed over long periods.

This calibration problem complicates all applications of biosensors for online process control. However, calibration may be performed in a way that is rather simple in the case of the in situ sensor. One principle for calibration that is applied in the author's laboratory is described in Fig. 19.5. Basically, the probe is inserted through a ball valve and it is intermittently partly withdrawn for calibration as described in the figure.

Much still remains to be improved if biosensors are to be used extensively in the harsh environment within a fermenter. However, the possibility of compensating for environmental factors and calibrating the sensor when it is placed in the reactor make the in situ application of biosensors for bioprocess control very promising. 


\section{References}

Cleland N. and Enfors S.-O. (1983). Control of glucose-fed batch cultivations of E. coli by means of an oxygen stabilized enzyme electrode. Eur. J. Appl. Microbiol. Biotechnol. 18, 141-7.

(1984). Externally buffered enzyme electrode for determination of glucose. Anal. Chem. 56, 1880-4.

Enfors, S.-O. (1981). Oxygen-stabilized enzyme electrode for glucose analysis in fermentation broths. Enzyme Microb. Technol. 3, 29-32.

- and Cleland N. (1983). Calibration of oxygen- and pH-based enzyme electrodes for fermentation control. In Chemical sensors (ed. T. Seiyama), pp. 672-676. Elsevier, Amsterdam.

Linek, V., Benes, P., Sinkula, J., Holecek, O. and Maly, V. (1980). Oxidation of D-glucose in the presence of glucose oxidase and catalase. Biotechnol, Bioeng. 22, 2515-27.

Romette, J. L., Froment, B. and Thomas D. (1979). Glucose oxidase electrode. Measurements of glucose in samples exhibiting high variability in oxygen content. Clin. Chim. Acta 95, 249-53. 


\title{
In vivo chemical sensors and biosensors in clinical medicine
}

\author{
DENZIL J. CLAREMONT and JOHN C. PICKUP
}

\subsection{Introduction}

For the purpose of this chapter we have defined an in vivo biosensor as a small, probe-type device that is either inserted into or attached to the body for continuously measuring (without added reagent) the concentration of a substance of pathological or therapeutic importance.

Many sensors have been described as being potentially implantable and although they often represent considerable scientific achievements, sometimes little thought has been given to the clinical justification for continuously monitoring the concentration of a particular analyte. Any in vivo biosensor (unless it were totally non-invasive) presents a risk to the patient or volunteer and it is therefore important to define the criteria to be used when selecting an analyte for continuous monitoring. The prime requisite, we think, is that it should be a substance which changes in concentration so rapidly (e.g. blood glucose or arterial oxygen tension, $\mathrm{P}_{\mathrm{a}} \mathrm{O}_{2}$ ) that conventional in vitro analysis would not be adequate to follow the trend on a minute-to-minute basis. Secondly, the change in concentration should have important physiological or clinical implications. The overall aim in developing in vivo biosensors in medicine is to improve patient management and, based on the above considerations, the main analytes that warrant continuous monitoring at present are blood gases, $\mathrm{pH}$, glucose, and potassium.

In the future, it is possible that 'closed-loop' systems will be developed where the blood levels of various drugs are monitored with a biosensor and feedback control of drug delivery rates from an infusion pump is accomplished so as to maintain blood levels within a narrow, therapeutic range. There is already considerable interest in the advantages of controlled 'openloop' infusion of several drugs (Pickup 1984; Prescott and Nimmo 1985).

Implantable biosensors may also be useful for intermittent analyte measurement under certain eircumstances. In theory, miniature sensors provide (a) access to highly-localized areas within the body, (b) the ability to make measurements within small volumes of body fluids (e.g. interstitial fluid), without consumption or withdrawal of fluid, and (c) rapidly available results. One may speculate that these advantages could be of use, for 
example, for detecting analytes, at operation, within a tissue or a blood vessel draining the tissue, allowing localization of a tumour, ensuring adequacy of its excision, or even establishing its biochemical nature.

In the following discussion we have attempted to review the main clinical applications for various in vivo biosensors. The methodology for most of these devices is described elsewhere in this volume or can be found in the original references.

\subsection{Blood gases}

In the healthy human, the partial pressure of oxygen and carbon dioxide in arterial blood is kept within tightly controlled limits $\left(\mathrm{P}_{\mathrm{a}} \mathrm{O}_{2} 12.6-13.3 \mathrm{kPa}\right.$; $\mathrm{P}_{\mathrm{a}} \mathrm{CO}_{2} 4.5-6.1 \mathrm{kPa}$ ). However, in a variety of disorders and disease states affecting primarily the cardiovascular or respiratory system or metabolic regulation (see Table 20.1) changes in $\mathrm{P}_{3} \mathrm{O}_{2}$ and $\mathrm{P}_{3} \mathrm{CO}_{2}$ can, if corrective measures are not implemented, produce serious and sometimes fatal clinical consequences.

Table 20.1 Disorders associated with blood-gas abnormalities
Infant respiratory distress syndrome
Adult respiratory distress syndrome
Chronic obstructive lung disease
Cardiac failure
Congenital heart defects
Cardiac surgery

There are three main categories of patients in whom continuous blood gas monitoring is useful: premature neonates, patients in acute cardiovascular or respiratory failure (particularly those requiring mechanical ventilation), and those undergoing open-heart surgery.

\subsubsection{Blood-gas monitoring in premature neonates}

The need to monitor cardiovascular and respiratory function in premature neonates has been the prime driving force in the development of in vivo oxygen sensors. In many premature infants, the lungs are underdeveloped and do not produce surfactant, and it is necessary, therefore, to ventilate them mechanically. Hypoxia, on the one hand, can cause permanent brain damage, but administration of high concentrations of oxygen can produce retrolental fibroplasia and result in blindness. Thus it is essential to strike a balance which does not compromise life or result in unacceptable morbidity. Parker et al. (1971) developed a Clark-type poloragraphic oxygen electrode for insertion into the umbilical artery of neonates. It consisted of a silver 


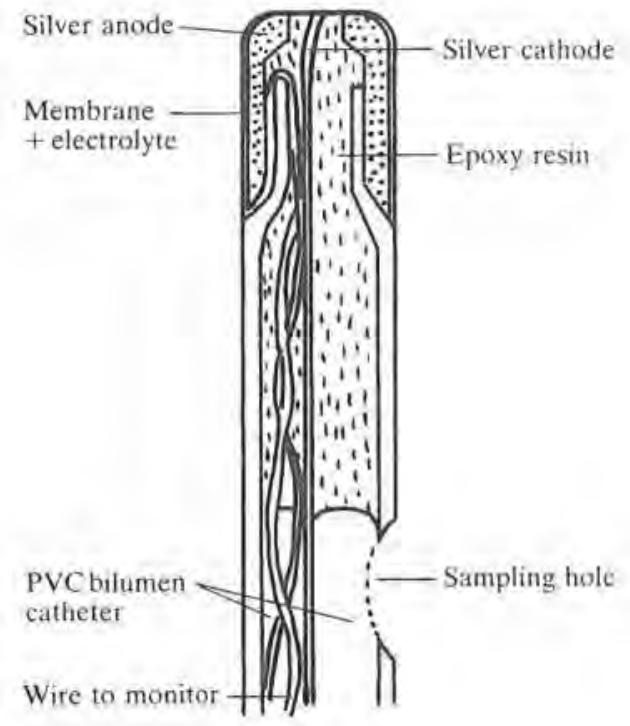

Fig. 20.1 The catheter-tip $\mathrm{PO}_{2}$ electrode.

anode and cathode separated by a dry potassium chloride electrolyte (Fig. 20.1). The electrode was poised at $750 \mathrm{mV}$ and the current generated was found to be proportional to the partial pressure of oxygen in the blood. This type of sensor has also been evaluated clinically by Conway et al. (1976) and Pollitzer et al. (1980).

Intravascular monitoring is not without limitations. Probe failure due to thrombotic deposition on the sensing element can be a problem and, as with any indwelling vascular device, it can provide a source of emboli and a route for infection. Relatively non-invasive sensing alternatives have, therefore, been the subject of much investigation.

Gerlach demonstrated as long ago as 1851 that oxygen diffuses through the skin. The first reported measurements of transcutaneous oxygen tension $\left(\mathrm{TcPO}_{2}\right)$ were by Baumberger and Goodfriend (1951): they used a dropping mercury electrode to determine polarographically the $\mathrm{PO}_{2}$ of a layer of liquid on the skin. Later, Rooth et al. (1957) reported a similar experiment using the $\mathrm{PO}_{2}$ electrode developed by Clark one year previously and found that, after a period of equilibration, a similar $\mathrm{PO}_{2}$ to that of arterial values was obtained, provided the skin was warmed so as to produce a hyperaemia. Huch et al. (1969) also induced a hyperaemia (in the scalp of neonates) by the local application of a nicotinic acid derivative. A catheter platinum $\mathrm{PO}_{2}$ electrode was attached to the skin and electrode readings showed a definite relationship to arterial values. The experience gained with this type of electrode prompted the development of a more practical flat, button- 


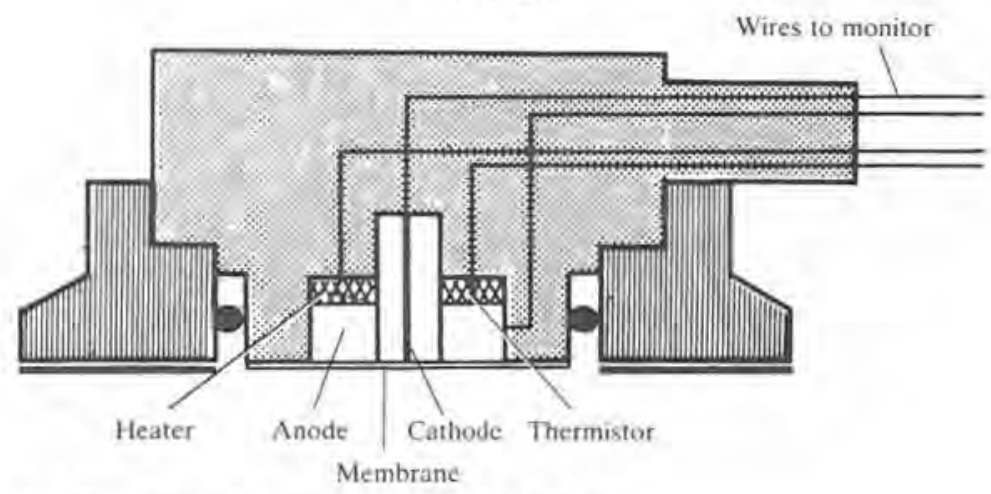

Fig. 20.2 Diagram of a transcutaneous $\mathrm{PO}_{2}$ electrode.

shaped design of sensor (Fig. 20.2). The electrode used by Eberhardt et al. (1973) consisted of a large gold cathode which was directly heated so as to produce local hyperaemia. This pioneering research work has led to the development of a number of commercially available systems (e.g. Draeger Medical Ltd, Orange Medical Instruments Ltd, Roche).

Since it is relatively non-invasive, continuous transcutaneous monitoring of $\mathrm{PO}_{2}$ has aroused considerable interest in neonatal medicine. In recent years, the proceedings of two international meetings on this topic have been published (Huch et al. 1979; Huch and Huch 1983) and contain numerous reports on the development and reliability of $\mathrm{TcPO}_{2}$ electrodes as well as information on various physiological and clinical studies. The system has undoubtedly contributed towards improving the management of severely ill neonates, resulting in a decrease in both mortality and morbidity. Yamanouchi et al. (1983), for example, used a Hellinge Oximonitor (SM361) to measure transcutaenons oxygen partial pressure in 810 low birth-weight infants who required mechanical ventilation or oxygen administration. Although their study was not a controlled one, they conclude that because the upper limit of $\mathrm{TcPO}_{2}$ was maintained between $50-80$ torr $(6.6-10.6 \mathrm{kPa})$ retinopathy occurred in only 20 infants (none of them were blind). This, they believe, to be the lowest incidence published to date.

The success and clinical utility of $\mathrm{TcPO}_{2}$ monitoring has prompted the development of similar systems for monitoring $\mathrm{CO}_{2}\left(\mathrm{TcPCO}_{2}\right)$. In spite of the large number of publications of this topic, however (see Huch and Huch 1983), the clinical value of $\mathrm{TcPCO}_{2}$ monitoring has yet to be established.

\subsubsection{Blood-gas monitoring in patients with respiratory insufficiency}

At present, there is some controversy as to which measurement (arterial oxygen partial pressure, $\mathrm{P}_{\mathrm{a}} \mathrm{O}_{2}$; mixed venous oxygen partial pressure $\mathrm{P}_{\mathrm{v}} \mathrm{O}_{2}$, mixed venous oxygen saturation, $\mathrm{S}_{\mathrm{v}} \mathrm{O}_{2}$ ) is the best index of tissue oxygenation in patients suffering from hypoxaemia due either to respiratory disease or 
secondary to cardiac failure (Downs 1983). (As this is a physiological issue, it will not be discussed any further in this chapter, but the methods used to monitor these parameters will be described). The $\mathrm{P}_{\mathrm{a}} \mathrm{O}_{2}$ in respiratory disease can be monitored directly using intravascular $\mathrm{PO}_{2}$ electrodes as described in the section on neonatal monitoring. Armstrong et al. (1978) flow-guided intravascular $\mathrm{PO}_{2}$ electrodes into the pulmonary artery of 25 patients in acute respiratory failure who required intermittent positive pressure ventilation. They found that if the $\mathrm{P}_{\mathrm{v}} \mathrm{O}_{2}$ fell below $5.3 \mathrm{kPa}$ this was indicative of respiratory or cardiac deterioration which was not always obvious by clinical observation. Moxham and Armstrong (1981) placed the same type of electrodes in the right atrium of 26 patients with myocardial infarction. When breathing air, 11 of the patients had a right atrial oxygen partial pressure, $\mathrm{RAP}_{\mathrm{v}} \mathrm{O}_{2}$, of less that $4.53 \mathrm{kPa}$. In this group there were eight deaths. Fifteen patients had a $\mathrm{RAP}_{\mathrm{v}} \mathrm{O}_{2}>4.53 \mathrm{kPa}$ and in this group there were no deaths. Jamieson et al. (1982) have reported the use of a fibre-optic oximeter to monitor $\mathrm{S}_{\mathrm{v}} \mathrm{O}_{2}$ and have shown it to be a reliable index of tissue perfusion and oxygenation.

The catheter used in this system was of $7.5 \mathrm{~F}$ diameter, containing two plastic fibre-optic light guides. The optical module contained three lightemitting diodes providing light of three wavelengths in the range $600-100 \mu \mathrm{m}$. Each wavelength of light was pulsed in sequence at $244 \mathrm{~Hz}$ through a single optical fibre to illuminate the blood flowing past the catheter tip. The light reflected by the blood was transmitted along the second optical fibre to a photodetector. These light signals were converted into electrical signals and then processed to display the $\mathrm{S}_{\mathrm{v}} \mathrm{O}_{2}$ over the previous five seconds. This average was updated every second. Kandel and Aberman (1983) argue that in patients in cardiopulmonary failure $\mathrm{S}_{\mathrm{v}} \mathrm{O}_{2}$ is a better indicator of cardiopulmonary function than $\mathrm{P}_{\mathrm{v}} \mathrm{O}_{2}$. This is because $\mathrm{S}_{\mathrm{v}} \mathrm{O}_{2}$ is determined only by the components of the oxygen transport system (cardiac output, haemoglobin, arterial oxygen saturation and oxygen consumption) where as $\mathrm{P}_{\mathrm{v}} \mathrm{O}_{2}$ can change solely because of a shift in the oxygen-haemoglobin dissociation curve, without disturbances in oxygen transport.

Hutchison et al. (1981) compared $\mathrm{TcPO}_{2}$, measured with a heated electrode, with the $\mathrm{PO}_{2}$ of arterial samples from three groups of patients, measured on an in vitro analyser. The first group consisted of 20 patients with chronic respiratory disease and the correlation between the two methods was $r=0.93$. The second group comprised of eight hypothermic patients studied immediately after cardiopulmonary bypass surgery; there was no correlation between the methods even after supplementary heating was applied to the skin. Presumably this was due to the poor peripheral circulation. The final group was 14 patients in an intensive care unit receiving various concentrations of inspired oxygen; the correlation was 0.90 .

Fatt and Deutsch (1983) investigated a further non-invasive technique 


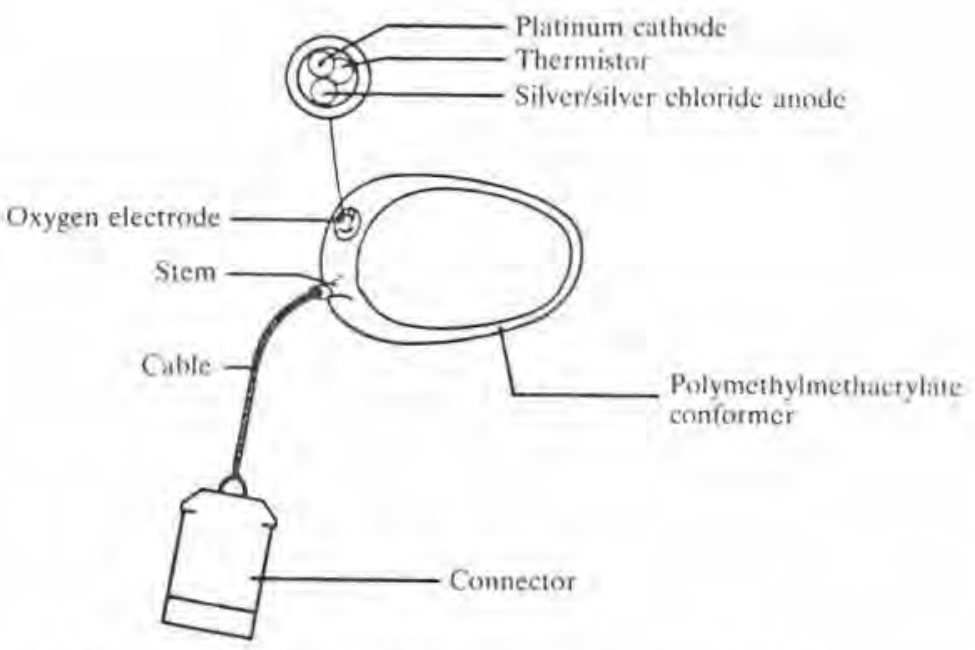

Fig. 20.3 Diagram of Orange Medical Instruments conjunctival $\mathrm{PO}_{2}$ sensor,

when they showed that a polarographic oxygen electrode placed in contact with the palpebral conjunctiva measures values of oxygen tension related to that in the underlying capillary bed. Furthermore, a mathematical analysis based on equations describing the diffusion of oxygen in an oxygen-consuming tissue indicated that the measured $\mathrm{PO}_{2}$ would only be about 5-10 torr below that in the capillary bed. Isenberg and Shoemaker (1983) used a transconjunctival oxygen minitor to continuously measure the $\mathrm{PO}_{2}$ of the palpebral conjunctiva in 19 patients undergoing general anaesthesia for surgery or in an intensive care unit. Arterial $\mathrm{PO}_{2}$ was also determined on blood samples withdrawn from the radial artery. The sensor was a miniature Clark-type electrode (Orange Medical Instruments). The electrode is mounted on an oval conformer (Fig. 20.3) that fits into the superior and inferior conjunctival fornicies. An integral solid-state thermistor measures conjunctival temperature. Figure 20.4 shows the relationship they found between mean transconjunctival $\mathrm{PO}_{2}$ and radial artery $\mathrm{PO}_{2}$.

\subsubsection{Blood-gas monitoring during and after cardiac surgery}

Cardiac surgery for coronary artery bypass, the replacement of defective heart valves, and the correction of congenital heart defects is commonplace today. To provide a dry and motionless operative field, it is necessary to virtually stop the flow of blood through the heart and lungs, and this can be achieved by the technique known as cardio-pulmonary bypass (CPB). The function of the heart and lungs are taken over by an extracorporeal machine which essentially comprises of a pump and a gas-exchange unit. During CPB, 


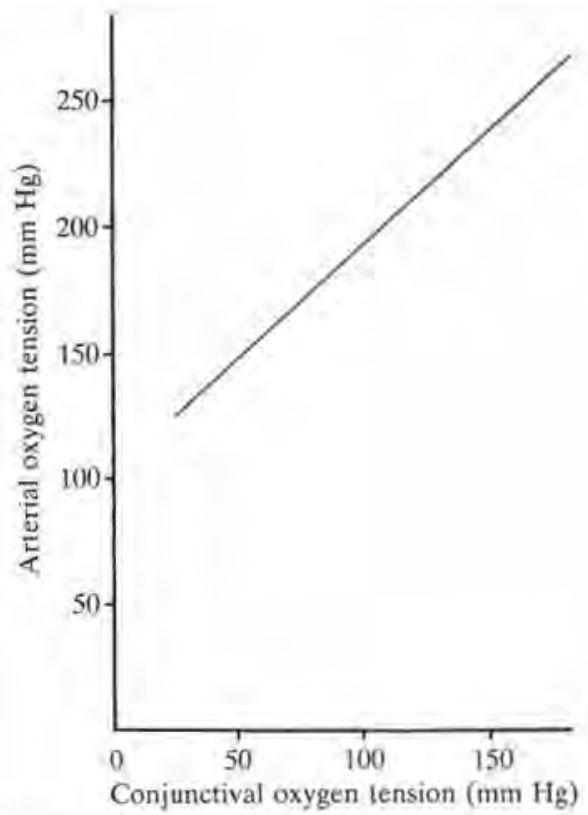

Fig. 20.4 The relationship between the mean oxygen tensions of the conjunctiva and the radial artery in the studied subjects. (Redrawn after Isenberg and Shoemaker 1983.)

when gas exchange is under the control of the perfusionist, a knowledge of arterial blood gas tensions is essential to ensure that the performance of the oxygenator in the extracorporeal circuit is optimal. Until recently, this could only be establised by intermittent, but frequent, collection of samples of arterial blood from the patient or from an appropriate point in the extracorporeal circuit and subsequent measurement of the $\mathrm{P}_{\mathrm{a}} \mathrm{O}_{2}, \mathrm{P}_{\mathrm{a}} \mathrm{CO}_{2}$, and $\mathrm{pH}$ on a blood-gas analyser. CPB is a dynamic procedure (Walpoth et al. 1981; Claremont and Pagdin 1985), with rapid changes in both $\mathrm{PO}_{2}$ and temperature, and the inevitable delay between sampling and obtaining results causes inaccuracies with in vitro measurement. Consequently, adjustments to the gas-exchange unit are made on retrospective data and may be physiologically inappropriate. Futhermore, under conditions of hypothermia it is necessary to correct in vitro blood-gas measurement made at $37^{\circ} \mathrm{C}$ to the patients blood temperature using one of several nomograms (Kelman and Nunn 1966; Severinghaus 1966; Thomas 1972) which may themselves contain inaccuracies (Andstritch et al. 1981).

A number of devices have been developed specifically for monitoring the oxygen status of blood in heart-lung machines. Although, strictly speaking, these are ex vivo instruments since they are attached to the heart-lung 
machine rather than directly to the patient, they are of interest for a discussion of in vivo sensors. The Oxytrode (Critikon Ltd, Tampa, Florida, USA) consists of a precalibrated, sterile, disposable Clark-type electrode which is in direct contact with the blood, and once inserted into the heart-lung machine a calibration check is only possible by adjusting the sensor reading to correspond to the value of a sample measured on a blood gas analyser.

In an attempt to overcome these problems Parker et al. (1983) developed a system in which the electrode is separated from the blood by a sterile, gaspermeable membrane and a contact fluid. This instrument, the In-Line Oxygen Monitoring Systems, ILOMS, (Orange Medical Instruments, High Wycombe, Bucks, England) is shown schematically in Fig. 20.5. Essentially, it consists of a sterile disposable 'T-piece' connector with a gas window. This is placed in the arterial return line of the heart-lung machine. A re-usable Clark-type electrode with an integral thermistor is inserted into the 'T-piece' and secured in place with a bayonet cap. The electrode is calibrated in air, and, if necessary, calibration checks can be made during bypass without
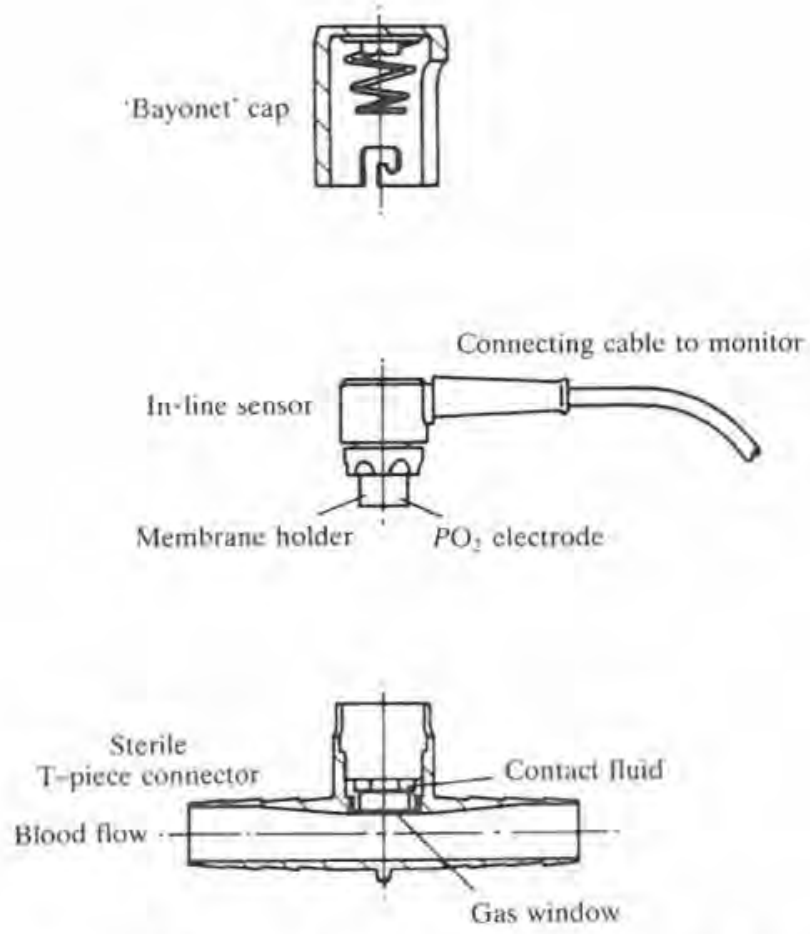

Fig. 20.5 Diagram of ILOMS (In-Line Oxygen Monitoring System) sensor/connector assembly. (From Claremont et al. 1984.) 
compromising the sterility of the blood. Moreover, since calibration is without reference to another instrument (except a barometer for determining atmospheric pressure) it is absolute and, theoretically, error-free. This instrument has been evaluated in vitro (Claremont et al. 1984). There was no significant difference between the ILOMS reading and the calculated $\mathrm{PO}_{2}$ of blood in a circuit. There was, however, a significant difference between the ILOMS readings and the value of samples measured on a conventional blood gas analyser. The instrument has also been evaluated clinically in 46 patients (Claremont and Pagdin 1985). When the sensor was placed in the arterial return line of the heart-lung machine, rapid and large changes in $\mathrm{PO}_{2}$ were seen. However, when placed in the venous side of the machine, readings from the monitor were almost constant, in spite of the changes in pump flow-rate and blood temperature. This suggests that mixed venous $\mathrm{PO}_{2}$ is an insensitive index of tissue perfusion.

Jamieson et al. (1982) used a fibre-optic catheter oximeter (Oximeter Shaw TM, Catheter Oximeter System, Oximetrix, Inc., Mountain View, California, USA) to monitor continuously the mixed venous oxygen saturation $\left(\mathrm{S}_{\mathrm{v}} \mathrm{O}_{2}\right)$ during and after cardiac surgery. An $\mathrm{S}_{4} \mathrm{O}_{2}$ of $>65 \%$ was taken as indicative of a normal cardiac output, but a fall in $\mathrm{S}_{\mathrm{\gamma}} \mathrm{O}_{2}$ of $10 \%$ was seen before changes in blood pressure or heart rate, due to a decrease in cardiac output.

\subsection{Potassium monitoring}

Potassium is an electrolyte of paramount physiological and pathological importance. It is the main cation within cells and plays an essential role in maintaining the membrane potential of electrically excitable cells such as cardiac muscle and nerve tissue. Normally, the plasma concentration of $\mathrm{K}^{*}$ is kept within narrow limits $(3,8-5.5 \mathrm{mmol} / \mathrm{l})$, but this delicate balance can be disturbed in a number of disorders including renal, adrenal and gastrointestinal disease, drug therapy (e.g. diuretics), and diabetes melltius. Changes in $\mathrm{K}$ * can have profound effects on the rhythm of the heart; hyperkalaemia, the most serious potassium abnormality, causes bradycardia, ventricular fibrilation, and, if severe, cardiac arrest. Indeed, the patients in whom continuous $\mathrm{K}$ - monitoring is most likely to be of clinical value, are those suffering from cardiac disorders (changes in $\mathrm{K}+$ due to renal disease or diabetes are unlikely to be so rapid that conventional in vitro analysis would not suffice).

A number of workers have developed catheter-tip K* sensors either based on a conventional potentiometric ion-selective electrode using the antibiotic valinomycin as the $\mathrm{K} \cdot$ ionophore (Treasure and Band 1977; Webb et al. 1983) or solid-state devices such as ion-selective field-effect transistors (ISFETs) coated with an ion-selective membrane (McKinlay et al. 1980).

One of the most interesting and useful applications of an in vivo $\mathrm{K}^{+}$sensor is that reported by Webb et al (1983). In two patients undergoing percu- 
taneous transluminal coronary angioplasty, with a Grunzig balloon catheter for coronary artery stenosis (a newly developed technique for dilating narrowed blood vessels without recourse to major surgery), a $\mathrm{K}$ * electrode was placed in the coronary sinus (this receives blood that has circulated through the heart muscle). Three consecutive ballon inflations were performed with a duration of 20 seconds at 80 -second intervals. During angioplasty occlusion the patients did not experience any chest pain nor were there any significant changes in the surface electrocardiogram. No change in coronary sinus $\mathrm{K}^{+}$was seen during balloon inflation but 4.5 seconds after deflation, there was a transient rise in coronary sinus $\mathrm{K}^{+}$of $0.3 \mathrm{mmol} / \mathrm{I}$ above a base line level of $4.0 \mathrm{mmol} / 1$. This was intepreted as the washout of $\mathrm{K}+$ from myocardial cells following a few seconds of ischaemia. If coronary sinus $\mathrm{K}^{+}$is an early indicator of myocardial ischaemia, indwelling $\mathrm{K}^{+}$ electrodes may prove to be a useful adjunct in the management of patients following acute myocardial infarction, or coronary artery surgery.

\subsection{Hydrogen ion concentration}

Continuous in vivo recording of $\mathrm{pH}$ has not yet become an established or routine practice but several investigations point to clinically useful applications, especially for intravascular, tissue, and gastric $\mathrm{pH}$ measurement.

Catheter-tip pH electrodes for insertion into vessels may be constructed using a variety of ion-selective membranes, such as those incorporating the $\mathrm{H}^{+}$ionophore $p$-octadecyloxy $m$-chlorophenylhydrazine-mesoxalonitrite, OCPH (Coon et al. 1976; LeBlanc et al. 1976; Cobbe and Poole-Wilson 1979). Such electrodes are less fragile than conventional glass $\mathrm{pH}$ sensors.

Rithalia et al. (1979) have investigated continuous subcutaneous tissue $\mathrm{pH}$ monitoring in 22 adults, 14 during and after open heart surgery. They used a miniature glass $\mathrm{pH}$ probe, and found a highly significant correlation with arterial blood $\mathrm{pH}$ measurements made by intermittent sampling. However, as expected, the relationship was poor in those patients with impaired tissue perfusion.

Twenty-four hour oesophageal $\mathrm{pH}$ monitoring uses an indwelling $\mathrm{pH}$ electrode, placed in the lower oesophagus, to detect gastro-oesophageal reflux (Johnson and DeMeester 1974). To avoid the problems of continuous intubation, and restriction of acticity by electrical contacts, portable, radiotelemetry systems have recently been developed and evaluated (Falor et al. 1981; Branicki et al. 1982).

\subsection{Glucose}

Diabetic patients have a relative or absolute lack of insulin which causes the blood glucose concentration to exceed the normal narrow limits (about 
$3.5-5 \mathrm{mmol} / \mathrm{l}$ in the fasting state). About $20 \%$ of diabetics, who mostly contract the disease under the age of about 30 years, have suffered a complete or near-complete destruction of the insulin-secreting cells (islets of Langerhans) in the pancreas; this type of diabetes is called insulin-dependent or type I diabetes and these patients need insulin replacement to live. Insulin is usually given by subcutaneous injection and although preserving life and largely preventing the symptoms of acute hyperglycaemia, injections cannot maintain non-diabetic blood glucose levels ('control'). The values sometimes slip too low (hypoglycaemia), causing unpleasant symptoms and dangerous impairment of consciousness; and often are too high, causing, it is strongly suspected, serious long-term tissue complications in the eyes, nerves, kidneys, and blood vessels.

There has, therefore, been an intense drive in the last few years to improve diabetic control. One important approach has been the controlled infusion of insulin from 'open-loop' portable pumps (Pickup et al. 1978; Pickup and Rothwell 1984), a strategy aiming at mimicking non-diabetic insulin secretion profiles. Near-normoglycaemia can be obtained with these devices for periods of at least several years but control can be lost in a variety of circumstances such as after strenuous exercise and during intercurrent illness and menstruation. A logical development of these systems is 'closed-loop' feedback-control of the insulin infusion rate via an implanted glucose sensor. Ultimately, this type of artificial endrocrine pancreas may become totally implantable but progress in this direction is at a very early stage and immense technological, biological, and ethical problems must be met and solved for it to become a reality.

In the interim, implantable glucose sensors which are not connected to pumps would still have the considerable advantages of providing a hypoglycaemia alarm, of warning against impending hyperglycaemia and

Table 20.2 Characteristics of the ideal in vivo glucose sensor

1. Site: non-invasive or subcutaneous

2. Size: $<25 \mathrm{G}$ needle

3. Biocompatible: minimal tissue reaction

4. Linearity: $0-20 \mathrm{~mm}$

5. Resolution: $<1 \mathrm{~mm}$ (for a hypoglycaemia monitor)

6. Specificity: not affected by metabolites

7. Drift: $<10 \%$ per day

8. In vitro response time: $95 \%<2-3$ minutes

9. Calibration: factory or easy single point home calibration

10. Storage: in stable and convenient form

11. Construction: amenable to mass production

12. Cost: cheap 
ketoacidosis, and generally supplying continuous information of blood glucose control to enable the patient to correct and adjust insulin therapy himself.

At this point it is appropriate, then, to consider the characteristics (Table 20.2) of an ideal in vivo glucose electrode.

20.5.0.1 Site Although the concentration of glucose in the blood is the parameter of main clinical interest, the risk of serious infection and the formation of thrombi on the sensing element preclude the blood stream as a site for long-term monitoring. A non-invasive technique such as transcutaneous monitoring would be the method of choice. At present there is little information on the permeability of the skin to glucose, but Clark (1979) has shown in the anaesthetized cat that if glucose oxidase is injected under the skin and a transcutaneous $\mathrm{PO}_{2}$ electrode is placed over the injection site, the $\mathrm{PO}_{2}$ reading of the electrode changes in response to an intravenous injection of glucose. Furthermore, he suggests that it may be possible to retain the enzyme in a silastic membrane which could be implanted on a long-term basis.

The aqueous humor of the eye has also been proposed as a site for monitoring glucose. Using an optical rotation technique, March et al. (1982) reported a good correlation between the glucose concentration of samples of rabbit aqueous humour and blood glucose but problems of bulky, inconvenient instrumentation and interfering substances would seem to limit the method for in vivo human use.

From a practical point of view, the subcutaneous tissue seems to be the most suitable site; it has been used as an implantation site for continuous insulin infusion for several years without serious infection or untoward local tissue reaction (Pickup et al. 1980). Moreover, it is an injection site that is well tolerated by most diabetic patients. However, there is little independent information at present on the concentration of glucose in subcutaneous tissue and its relationship to blood glucose levels. Wolfson et al. (1982) implanted modified Guyton capsules, dialysis sacs, and Milipore membrane devices in the subcutaneous extracellular space, pericardium, pleura, and peritoneum of baboons and/or rabbits for periods of up to six months. The glucose concentration of the fluid in these devices ranged between 50 and $115 \mathrm{mg} / \mathrm{dl}$ $(2.8-6.4 \mathrm{mmol} / \mathrm{l})$ but this technique did not reveal any information on the dynamics of blood tissue exchange. See below for sensor information on this topic.

20.5.0.2 Size This is an important consideration from at least three aspects. Firstly, patient acceptability of a large sensor is obviously low. Secondly, large sensors inevitably cause more tissue damage during insertion than smaller sensors and, apart from causing haemorrhage, may alter the 
tissue/blood glucose relationship through changes in local blood flow and vascular permeability. Thirdly, fine needle-type sensors seem to cause less tissue reaction than larger, flat configurations (Woodward 1982).

20.5.0.3 Biocompatibility A variety of factors determine the biocompatibility of an implantable device, but the nature of the material is probably most important. Although volumes have been written on the biocompatibility of polymers and plastics, most of it is not relevant to biosensors. Let us consider the materials that would be used in constructing a glucose sensor. In all probability, the sensor will be a needle and will be made of metal; platinum and stainless steel both have good biocompatibility and mechanical properties.

Most glucose sensors are coated with a membrane such as polyurethane or cellulose acetate (e.g. Shichiri et al. 1982; Chapter 23; Pickup and Claremont 1985 ) and it is essentially this which creates the interface between the body and the sensor proper and determines the biocompatibility. Membranes also have the function of trapping at the electrode sensor components such as enzymes, mediators, and cofactors, controlling the access of glucose and potentially interfering substances to the sensor, imposing a diffusional barrier which extends the linear range without sample dilution and determining sensor kinetics in general (Pickup 1985).

20.5.0.4 Linearity Since diabetics commonly display blood glucose values of $2-30 \mathrm{mmol} / 1$ the sensor should be linear or of predictable response within approximately this range.

20.5.0.5 Resolution The level of resolution should be about $1 \mathrm{~mm}$ or less if the device is to be effective as a hypoglycaemia indicator but in the higher ranges (about $15 \mathrm{mmol} / 1$, say) an accuracy of $\pm 20 \%$ may suffice.

20.5.0.6 Specificity The sensor should be specific for glucose. The output of the instrument should not be affected by drugs or by the many other metabolites which are disordered in diabetes, other than glucose.

20.5 0.7 Drift The sensor should be stable and not drift by more than about $10 \%$ a day.

20.5.0.8 In vitro response time At present, there is little information on the relative timing of changes of blood and tissue glucose concentration. From our studies in pigs (see below), it seems that there is reasonably close temporal relationship between blood glucose and subcutaneous tissue glucose levels. An in vitro $95 \%$ response time of $<2$ minutes should be suitable in these circumstances. 
20.5.0.9 Calibration Ideally, the sensor should be so stable and reliable that factory calibration would be possible. Failing this, an easy single-point home calibration would have to suffice.

20.5.0.10 Construction The design of the electrode should be such that it can be manufactured reproducibly in large numbers, easily sterilized, and produced at low cost.

The numerous reports on possible sensing strategies have been reviewed recently (e.g. Pickup 1985; Turner and Pickup 1985) and are discussed elsewhere in this volume. Most glucose sensors consist of amperometric or potentiometric enzyme electrodes, electrocatalytic sensors without enzymes, and opto-electronic systems such as the bioaffinity probe of Mansouri and Schulz (1984), see Chapter 32. Here, we shall confine our review to the reports of in vivo testing of some of the glucose sensors in humans and/or animals.

Soeldner et al. (1973) were amongst the first to evaluate in vitro and in vivo an electrocatalytic glucose sensor in a fuel-cell configuration. Disc-shaped sensors $2 \times 0.2 \mathrm{~cm}$ were implanted in the subcutaneous tissue of Rhesus monkeys and rabbits. During acute studies, sensor current followed blood glucose after a meal or administration of intravenous glucose with a latency of about $0-15 \mathrm{~min}$. There was apparently no conversion of the sensor responses to glucose concentrations and although sensors were implanted for several weeks at a time, little information on drift and biocompatibility were reported. Lewandowski et al. (1982) used an electrocatalytic glucose sensor in a blood flow-through chamber inserted in an arteriovenous shunt in dogs. A decrease in total anodically directed current in the range -0.4 to $-0.8 \mathrm{~V}$ was closely related to blood glucose concentrations during short-term experiments.

A glucose-oxidase enzyme electrode based on the detection of oxygen consumption was constructed and tested in dogs by Bessman et al. (1981). The sensor consisted of two oxygen electrodes covered by polypropylene membrane within a circular ( $15 \mathrm{~mm}$ diameter) plastic housing; the enzyme was immobilized over one electrode and current decreases compared with the control electrode. The relationship between the difference current and glucose concentration was non-linear over the range $0-20 \mathrm{mmol} / \mathrm{I}$ and responses substantially decreased by a lowered oxygen tension. Electrodes implanted in the subcutaneous tissue of dogs recorded glucose levels which were approximately half those of the blood. An implantable closed-loop system was also constructed consisting of the sensor and a reciprocative insulin pump, but this failed to maintain euglycaemia in the diabetic dogs. The authors considered that this was because the sensor underestimated the true tissue glucose levels because of the lower tissue $\mathrm{PO}_{2}$, in spite of the differential sensor operating mode. 
Kondo et al. (1982) also used as differential glucose sensor based on the measurement of oxygen consumption at a Clark-type oxygen electrode. The sensor was tested in dogs where an external arterio-venous shunt was created between the carotid artery and the jugular vein and the electrodes placed in the shunt. Four of eleven experiments failed because of thrombosis or electrical problems, but in other studies there was a good correlation between sensor output and blood glucose concentrations.

An amperometric sensor based on immobilized glucose oxidase and detecting hydrogen peroxide production was tested in vivo by Clark and Duggan (1982; Chapter I). A variety of provisional short-term studies in subcutaneous tissue and blood were performed but few results therefrom presented. Shichiri and his colleagues (1982, 1983; chapter 23) have developed a similar needle-type hydrogen peroxide detector and have performed extensive testing in animals and man. There was a significant relationship between subcutaneous glucose and blood glucose levels in dogs although after acute intravenous glucose loads the subcutaneous increases were delayed by $5-15 \mathrm{~min}$ and were about $65 \%$ lower than blood peaks. The sensitivity of the subcutaneously implanted sensor decreased to $94 \%$ at $24 \mathrm{~h}$, $90 \%$ at $48 \mathrm{~h}$, and $57 \%$ at $72 \mathrm{~h}$, compared with the initial output. Current outputs were not significantly affected by a drop in tissue oxygen tension from about 38 to $25 \mathrm{~mm} \mathrm{Hg}$. The device was also incorporated in a wearable closed-loop system and tested in three pancreatectomized dogs. Good control was obtained for 7 days by renewing the sensor every fourth day.

Shichiri et al. (1984) have also tried their needle-type sensor and wearable 'artificial pancreas' in human diabetics and succeeded in obtaining nearnormoglycaemia over several days. The glucose concentrations were reported as being about $25 \%$ lower in subcutaneous tissue compared to blood but followed almost immediately an increase in blood glucose (Shichiri and Kawamori 1983).

Another amperomeric glucose oxidase/hydrogen peroxide detector was recently tested in dogs by Abel et al. (1984) using either an ex vivo, extracorporeal blood flow-through system or subcutaneous implantation. The tissue glucose concentration was found to be $30-50 \%$ of the blood concentration.

We have recently begun (Pickup and Claremont 1985) in vivo testing of an amperometric sensor using the ferrocene-mediated electron transfer principle originally decribed by Cass ef al. (1984) (see also Chapters 15 and 16). We constructed miniaturized sensors based on $1 \mathrm{~mm}$ wide strips of graphite foil, impregnated with 1,1-dimethylferrocene and immobilized glucose oxidase, and covered with a polyurethane membrane. In vitro, the sensors were linear to at least $20 \mathrm{mmol} / 1$ and were virtually insensitive to the changes in oxygen tension likely to be found in vivo. When implanted in the subcutaneous tissue of non-diabetic pigs, sensor glucose concentra- 


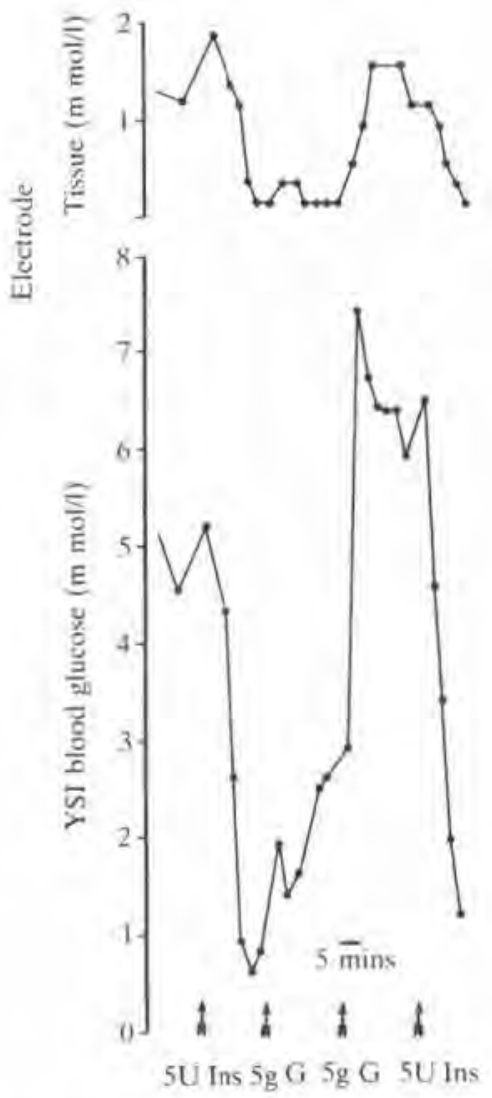

Fig. 20.6 Results from an experiment when a ferrocene mediated amperometric glucose electrode was implanted into the subcutaneous adipose tissue of an anaesthetized pig. Blood glucose concentration was manipulated by intravenous injections of insulin (Ins) or glucose (G).

tions were generally some $20 \%$ of those recorded in the blood by intermittent sampling and conventional laboratory assay. However, acute changes in blood glucose concentration caused by intravenous injection of insulin or glucose solution were mirrored by electrode responses, with little detectable latency (Fig. 20.6).

The fibre-optic bioaffinity glucose sensor based on concanavalin $\mathrm{A}$ as a binding agent (Mansouri and Schulz 1984; Chapter 32) has been evaluated in dogs in a blood flow-through chamber. Sensor responses corresponded reasonably well to blood glucose levels estimated conventionally but gave lower values after about two hours, the authors attributing this to a fall in blood temperature and $\mathrm{pH}$ over this time. 
In conclusion, we can say that in vivo testing of glucose sensors is still at an early stage. Several sensor configurations perform adequately in the shortterm but there is very little study of sensor drift and biocompatibility over periods of weeks. The subcutaneous tissue is a feasible site for sensor implantation; generally, the glucose levels here are lower, but significantly correlated with blood concentrations. Problems such as how to sterilize the sensor, how to calibrate in vivo, will the device be patient-acceptable, and can it ever be reliable enough to incorporate into a closed-loop system for routine use, are all largely unaddressed.

\subsection{Concluding remarks}

In this chapter we have attempted to review what we consider to be the most significant clinical applications of in vivo biosensors. The first clinical use of an in vivo biosensor was reported by Clark and co-workers some 25 years ago (Clark and Bargeron 1959) when they used an intravascular platinum cathode to detect a left-to-right cardiac shunt. Over the last two decades, blood-gas monitoring has progressed from relatively crude (bio-incompatible, unreliable) intravascular electrodes to sophisticated, non-invasive devices. Continuous blood-gas monitoring has undoubtedly made a significant contribution to the management of patients with severe cardiopulmonary disorders.

Potassium monitoring is still in its infancy, and it will probably only have a limited clinical application.

Although the development of in vitro glucose electrodes began at about the same time as blood-gas electrodes, progress has been much slower. At present, there are no commercially available in vivo glucose sensors. However, recent work will, hopefully, rectify this shortcoming.

One can tentatively speculate that in the future in vivo sensors for drugs (e.g. cytotoxics) may be useful in trying to establish the optimal dose (i.e. a concentration which produces the desired therapeutic effect with minimal unwanted side effects) for an individual patient.

Biosensors and biotechnology are currently in vogue and are a source of immense scientific interest. However, when developing in vivo biosensors, careful thought should be given to the clinical value of continuously monitoring a particular analyte (see Chapter 36 ).

\section{References}

Abel, P., Miller A. and Fischer U. (1984). Experience with an implantable glucose sensor as a prerequisite of an artificial beta cell. Biomed. Biochim. Acta 5, 577-84. Andstritch, R. F., Muravchick, S. and Gold, M. I. (1981). Temperature correction of blood gas parameters. Anaesthesiology 55, 311-16. 
Armstrong, R, F., Secker-Walker, J., St. Andrew, D., Cobbe, S., Cohen, S. L, and Lincoln, J. C. R. (1978). Continuous monitoring of mixed venous oxygen tension in cardio-respiratory disorders. Lancet 1, 632-4.

Baumberger, J.P. and Goodfriend, R. B. (1951). Determination of arterial oxygen tension in man by equilibration through intact skin. Fed. Proc. 10, 10-11.

Bessman, S. P., Thomas, L. J, Kojima, H., Sayler. D. F. and Layne, E. C. (1981). The implantation of a closed-loop artificial beta cell in dogs. Trans. Am. Soc. Art. Int, Org. 27, 7-17.

Branicki F. J., Evans, D. F., Ogline, A.C., Atkinson, M. and Hardcastle J.D. (1982). Ambulatory monitoring of oesophageal $\mathrm{pH}$ in reflux oesophagitis using a portable radiotelemetry system. Gut $23,992-8$.

Cass, A. E. G., Davis, G., Francis. G. D., Hill, H. A. O., Aston, W. J., Higgins, I. J., Plotkin, E. V., Scott, L. D. L. and Turner, A. P. F. (1984). Ferrocene-mediated enzyme electrode for amperometric determination of glucose. Anal, Chem. 56, 667-71.

Claremont, D. J. and Pagdin, T. M. (1985). Evaluation of a new re-usable electrode for continuous monitoring of blood $\mathrm{PO}_{2}$ during open heart surgery. J. Med. Eng. Tech. 9, (4), 174-9.

Walton, N. (1984). Continuous monitoring of blood $\mathrm{PO}_{2}$ in extracorporeal systems. Anaesthesia 39 362-9.

Clark, L. C. Jr (1979). Continuous measurement of circulating glucose using the transcutaneous $\mathrm{PO}_{2}$ electrode. In: Continuous transcutaneous blood gas monitoring. Birth Defects. Original Article Series (eds, A. Huch, R. Huch, and J, F, Lucey) XV, no. 4, pp. 39-42. Alan R Liss Inc., New York.

- and Bargeron L. M. (1959). Left-to-right shunt detection by an intravascular electrode with hydrogen as an indicator. Science, 130, 709-710.

— and Duggan C. A. (1982). Implanted electroenzymatic glucose sensors. Diabetes Care 5. 174-80.

Cobbe S. M. and Poole-Wilson P.A. (1979). Continuous measurement of $\mathrm{pH}$ in central arteries and veins. Lancet 2, 444-5.

Conway, M., Durbin, G. M., Ingram, D., McIntosh, N., Parker, D., Reynolds, E. R. and Soutter, L. P. (1976). Continuous monitoring of arterial oxygen tension using a catheter-tip polarographic electrode in infants. Paediatrics 57, 244-50.

Coon, R. L., Lai, N. C. J. and Kampine, J. P. (1976). Evaluation of a dual-function $\mathrm{pH}$ and $\mathrm{PCO}_{2}$ in vivo sensor, J. Appl. Physiol. 40, 625-9.

Downs, J.B. (1983). Monitoring oxygen delivery in acute respiratory failure. Respiratory Care 28 (5), 608-13.

Eberhardt, P., Hammacher, K, and Minat, W. (1973). Methode zur kutanen Messung des asuerstoff-druckes. Biomed. Techn. (Stuttg), 6, 216-21.

Falor, W. H., Change, B., White, H. A., Kraus, J. M., Taylor, B, Hansel, J. R., and Kraus, F.C. (1981). Twenty-four hour oesophageal pH monitoring by telemetry. Am. J. Surg. 142, 514-16.

Fatt, I. and Deutsch, T.A. (1983). The relation of conjunctival $\mathrm{PO}_{2}$ to capillary bed $\mathrm{PO}_{2}$. Critical Care Med. $11(6), 445-8$.

Huch, R, and Huch, A. (eds) (1983). Continuous transcutaneous blood gas monitoring, Reproductive medicine. Vol, 5. Marcel Dekker Inc., New York and Basel. 
Huch, A., Huch, R. and Lubbers, D. W. (1969). Quantitative polarographische sauerstoffdruckmessung auf der kopfhaut des neurgebornen, Arch. Gynaekol. 207, 443-52.

- and Lucey, J.R. (eds) (1979). Continuous transcutanenous blood gas monitoring. Birth defects. Original Article Series XV, no. 4. Alan R. Liss, New York.

Hutchison, D. C.S., Rocca. G. and Honeybourne, D. (1981). Estimation of arterial oxygen tension in adult subjects using a transcutaneous electrode. Thorax 36, 473-7.

Isenberg, S. J. and Shoemaker, W.C. (1983). The transconjunctival oxygen monitor. Am. J. Opthalmol. 95, 803-6.

Jamieson, W. R. E.. Turnbull, K. W., Larriea, A. J., Dodds, W. A., Allison, J. C. and Tyers, G, F, O, (1982). Continuous monitoring of mixed venous saturation in cardiac surgery, Canad. J. Surg. 25 (5), 538-43.

Johnson, L. F, and DeMeester, T. R. (1974). Twently-four hour pH monitoring of the distal esophagus. A quantitative measure of gastroesophageal reflux. $A m . J$. Gastroenterol. 62, 325-32.

Kandel, G. and Aberman, A. (1983). Mixed venous oxygen saturation: its role in the assessment of the critically ill patient. Arch. Intern. Med. 143, 1400-2.

Kelman, G. R. and Nunn, J.F. (1966). Nomograms for correction of blood $\mathrm{PO}_{2}$, $\mathrm{PCO}_{2} \mathrm{pH}$ and base excess for time and temperature, J. Appl. Physiol. 21, 1484-90.

Kondo, T., Ito, K., Ohkura, K., Ito, K. and Ikeda, S. (1982). A miniature glucose sensor, implantable in the blood stream. Diabeles Care 5, 218-21.

LeBlanc, D. H., Brown, J. F., Klebe, J. F., Niedrach, L. W., Shusartzuk G. M. J. and Stoddard, W. H. (1976). Polymer membrane sensors for continuous intravascular monitoring of blood pH. J, Appl, Physiol, 40, 644-7.

Lewandowski, J.J. Szczepanska-Sadowski, E., Krzymien, J. and Nalecz, M. (1982). Amperometric glucose sensor: short-term in vivo test. Diabetes Care 5, 238-44.

Mansouri, S. and Schulz, J. S. (1984). A miniature optical glucose sensor based on affinity binding. Biotechnology 2, 885-90.

March, W. F., Rabinovitch, B, Adams R. L., Wise, J. R, and Melton, M. (1982). Ocular glucose sensor. Trans, Am. Soc, Artif. Intern. Organs XXVIII, 232-5.

McKinley, B. A., Saffle, J., Jordan, W. S., Janata, J., Moss, S. D, and Westernskow, D. R. (1980). In vivo continuous monitoring of $\mathrm{K}^{+}$in animals using ISFET probes. Medical Instrumentation 14, 93-7.

Moxham, J. and Armstrong, R.F. (1981). Continuous monitoring of right atrial oxygen tension in patients with myocardial infarction. Intensive Care Med. 7, 157-64.

Parker, D., Delpy, D. T. and Halsall, D.N. (1983). A new approach to in-line gas monitoring. Development of an oxygen sensor. Med. Biol. Eng. Comp. 21. 134-7.

- Key, A. and Davies, R.S. (1971). Catheter-tip transducer for continuous in vivo measurement of oxygen tension. Lancet 1, 952.

Pickup, J, C. (1984), Clinical applications of infusion systems. J. Med. Eng. Technol. 8, 101-7.

(1985). Biosensors: a clinical perspective. Lancet 2, 817-20.

Pickup J.C. and Claremont D. J. (1985). A potentially implantable glucose sensor with direct electron transfer, Diab. Res. Clin, Prac, Suppl. 1, 447. 
and Rothwell, D. (1984). Technology and the diabetic patient. Med. Biol. Eng. Comput. 22, 385-400.

- Keen, H., Parsons, J.A. and Alberti, K.G. M. M. (1978), Continuous subcutaneous insulin infusion: an approach to achieving normoglycaemia. Brit. Med. J. 1, 204-7.

- Viberti, G.C., White, M.C., Kohner, E.M., Parsons, J.A. and Alberti, K. G. M. M. (1980). Continuous subcutaneous insulin infusion in the treatment of diabetes mellitus. Diabetes Care 3, 290-300.

Pollitzer, M., Soutter, L. P., Reynolds, E. R. and Whitehead, M. (1980). Continuous monitoring of arterial oxygen tension in infants: four years of experience with an intravascular oxygen electrode. Paediatrics 66 (1), 31-6.

Prescott, L.F. and Nimmo, W.S. (eds) (1985). Rate Control in Drug Therapy. Churchill Livingstone, Edinburgh.

Rithalia, S. V.S., Herbert, P. and Tinker, J. (1979). Continuous monitoring of tissue $\mathrm{pH}$, Brit. Med. I. 1, 1460.

Rooth, G., Sjostedt, S. and Caligora, F. (1957). Bloodless determination of oxygen tension by polarography. Science Tools 4, 37-45.

Severinghaus, J. W. (1966). Blood gas calculation. J. Appl. Physiol. 21. 1108-16.

Shichiri, M. and Kawamori, R. (1983). Feasibility of needle-type glucose sensor and the wearable artificial endocrine pancreas. In Diabetes treatment with implantable insulin infusion systems (eds. K. Irsigler, H. Kritz, and R. Lovett.), pp. 224-30. Urban and Schwarzenberg, Munich.

- Hakui, N., Yamasaki, Y. and Abe, H. (1984). Closed-loop glycemic control with a wearable artificial endocrine pancreas. Diabetes 33, 1200-2.

Yamasaki, Y., Haukui, N. and Abe, H. (1982). Wearable-type artificial pancreas with needle-type glucose sensor. Lancet 2, 1129-31.

- Goriya, Y., Yamasaki, Y., Abmura, M., Kaui, N, and Abe, H. (1983). Glycaemic control in pancreatectomised dogs with a wearable artificial pancreas. Diabetologia 24, 179-84.

Soeldner, J.S., Change, K. W., Aisenberg, S. and Hiebert, J. M. (1973). Progress towards an implantable glucose sensor and an artificial beta cell. In Temporal aspects of therapeutics (eds. J. Urquhart and F.E. Yates), pp. 181-207. Plenum, New York.

Thomas, L. J. (1972). Algorithms for selected blood acid-base blood gas calculations. J. Appl. Physiol. 33, 154-8.

Treasure, T. and Band D. M. (1977). A catheter-tip potassium selective electrode. J. Med. Eng. Tech. 1, 271 .

Turner, A.P. F. and Pickup, J.C. (1985). Diabetes mellitus: biosensors for research and management, Biosensors 1, 85-115.

Walpoth, B., Dernierre, D., Eglott, L. and Turina, M. (1981). Continuous oxygen partial pressure monitoring in cardiac surgery. Proc. Eur. Soc. Art. Org. 8, 301-8.

Webb, S. C., Rickards, A.F. and Poole-Wilson, P.A. (1983), Coronary artery potassium concentration recorded during coronary angioplasty. Br. Heart. J. 50, 146-8.

Wolfson, S. K., Tokarsky, J. F. and Krupper, M. A. (1982), Glucose concentration at possible sensor sites. Diabetes Care 5, 162-5. 
Woodward, S. C. (1982). How fibroblasts and giant cells encapsulate implants: considerations in design of glucose sensors. Diabetes Care 5, 278-81.

Yamanouchi, 1., Igarashi, 1. and Ouchi, E. (1983). Successful prevention of retinopathy of prematurity via transcutaneous oxygen partial pressure monitoring In Continuous transcutaneous blood gas monitoring. Reproductive medicine (eds R. Huch and A. Huch), Vol. 5, pp. 333-40. Marcel Dekker, New York and Basel. 


\title{
Thin-film micro-electrodes for in vivo electrochemical analysis
}

\author{
O. PROHASKA*
}

\subsection{Summary}

Multiple parameter monitoring is required in order to analyse complex biomedical processes within living tissue. Most often the probes have to be extremely small and the sensors closely spaced. Methods developed in thinfilm physics and solid-state electronics provide the possibility to realize miniaturized multiple sensor arrays which have been successfully used in brain research. Besides the specific design problems, caused by the fabrication constraints, the miniaturized thin-film metal electrodes display all the disadvantages of metal wire electrodes. A new chamber-type electrode design will be discussed which seems to be able to overcome most of these problems, enabling, in addition, the construction of miniaturized electrochemical cells.

\subsection{Introduction}

Investigations of miniaturized chemical sensors are motivated by economic as well as methodological reasons. Improvement in monitoring techniques can be accomplished in part by arranging 'on-chip' sensors close to the integrated electronic circuit and by keeping the sensors extremely small in order not to damage or destroy the sample. In brain research, for example, multiple electrode arrangements are required in order to elucidate the spatial neuronal interconnections (Petsche et al. 1984; Krueger 1983) and multiple parameter recordings have to be performed in order to explain normal as well as pathological neuronal activities (Caspers et al. 1980; Elger et al. 1981). Conventional glue techniques allow the arrangement of only a limited number of glass or metal micro-electrodes in order to form a multiple sensor. Techniques which were developed in modern thin-film physics and solid-state electronics, and which enable the fabrication of precisely defined structures in the micrometre dimension range, might be very helpful in designing new instruments

-The above described work was performed at the Institut fuer Allgemeine Elektrotechnik und Elektronik at the University of Vienna, Austria together with H. Dragaun, P. Goiser, A. Jachimowicz, F. Kohl, W. Morais, F, Olcaytug, K. Pirker, P. Pfundner, R. Schallauer, and G. Urban. 
for medical research, surgery, and intensive-care patient monitoring.

This chapter will be concerned with the fabrication and design problems, the limitations, and new aspects of miniaturized electrical and electrochemical thin-film sensors for in vivo research studies. The design of mechanically and electrically stable miniaturized $\mathrm{Ag} / \mathrm{AgCl}$ reference electrodes, as well as the construction of new miniaturized chamber-type sensors, will be described.

\subsection{Miniaturized thin-film multiple electrode probes}

Thin-film and solid-state techniques (Maissel and Glang 1970), mainly developed in order to improve the quality, density, and mass production of integrated circuits, enable the fabrication of precisely arranged electrode arrays (Prohaska et al. 1981). These are placed on small, needle-shaped substrates, which can be inserted in tissue. An example of such a multiple electrode probe is shown in Fig. 21.1. The probe was designed in order to measure the electrical activity of brain tissue, especially within the cortex of rabbits. Eight electrodes with recording site areas of $2500 \mu \mathrm{m}^{2}$ are placed in one row $300 \mu \mathrm{m}$ apart from one another; a ninth electrode is located $1 \mathrm{~mm}$ beneath the eighth in order to simultaneously monitor the hippocampus activities underneath the cortical structure (Petsche et al. 1984).

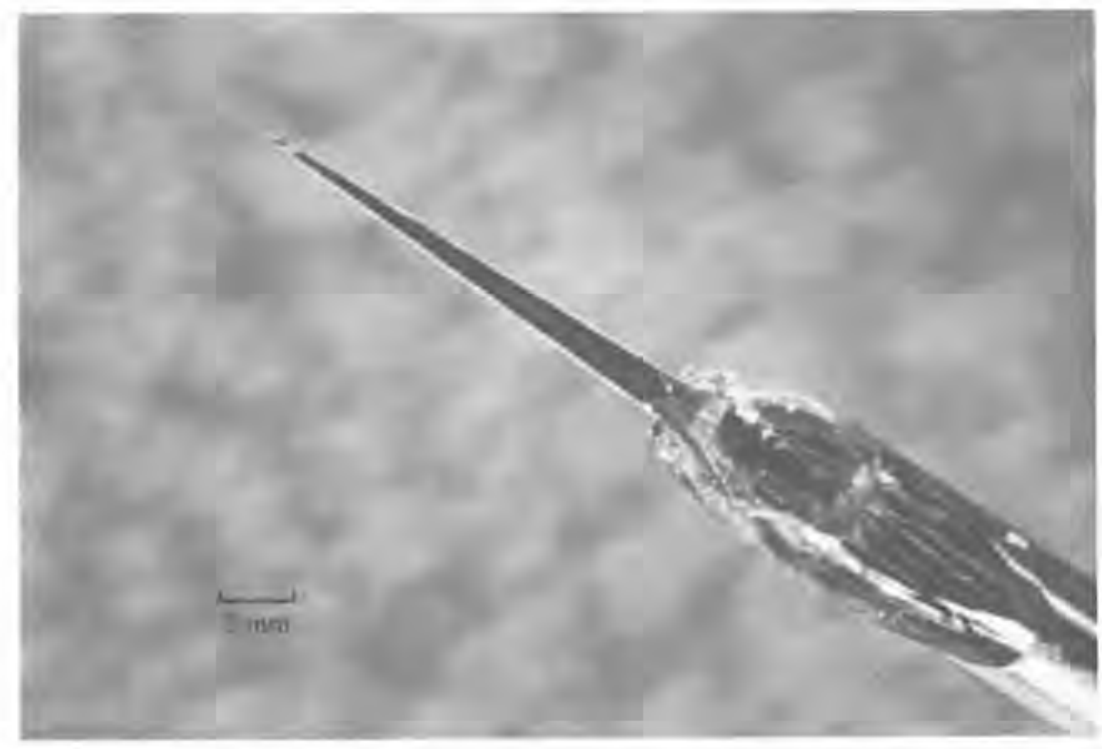

Fig. 21.1 Thin-film multiple electrode probe designed in order to register the electrical activities of the brain in animals. It contains nine recording sites, $2500 \mu \mathrm{m}^{2}$ in size, placed in one row on a needle-shaped $0.1 \mathrm{~mm}$-thick glass carrier. 


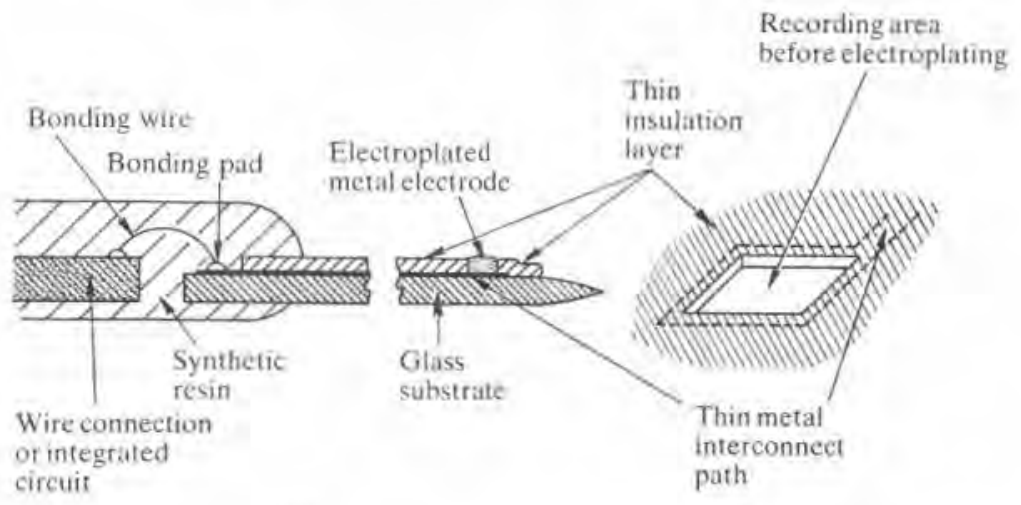

(a)

(b)

Fig. 21.2 (a) Cross section of a thin-film multiple electrode probe and (b) enlarged view of a thin-film electrode.

\subsubsection{Probe fabrication}

The schematic drawings in Fig. 21.2 show a cross-section (a) of the probe and a thin-film electrode (b). The photolithographic production steps provide a high degree of flexibility in the electrode and electrode arrangement design and allow a match of an adequate electrode pattern with the anatomical tissue structure.

First, a layout is drawn of the metal and insulator structure and photographically reduced up to several hundred times, defining the desired electrode size and arrangement on a so-called mask. Figure 21.3 shows an example of a metal (a) and an insulation layer mask (b) of a 16-fold electrode probe. The electrodes are arranged in one row $100 \mu \mathrm{m}$ apart with recording sites of $225 \mu \mathrm{m}^{2}$.

Second, a $0.1 \mu \mathrm{m}$ thick $\mathrm{Cr}$-Au-Cr triple layer ( $\mathrm{Cr}$ serves as an adhesion layer) is evaporated onto the surface of a carefully cleaned $100 \mu \mathrm{m}$ thick glass substrate. The metal layer is then completely covered by a layer of photoresist which changes its solubility in a solvent after having been exposed to ultraviolet light. After the first UV exposure through the metal mask and proper solvent treatment ('development'), the photoresist only covers the parts of the thin metal film which form the electrode areas, the thin metal interconnect paths, and the bonding pads. The uncovered metal film is etched off in selective $\mathrm{Cr}$ and Au etchants (Vossen and Kern 1978). Afterwards a 2-3 $\mu \mathrm{m}$ thick $\mathrm{Si}_{3} \mathrm{~N}_{4}$ insulation layer is produced by plasma-enhanced chemical vapour deposition methods (Vossen and Kern 1978), covering the metal structure as well as the glass substrate. After a second photolithographic step using the insulation layer mask, the photoresist covers the insulation layer with the exception of the recording site and bonding pad areas. Plasma etching 


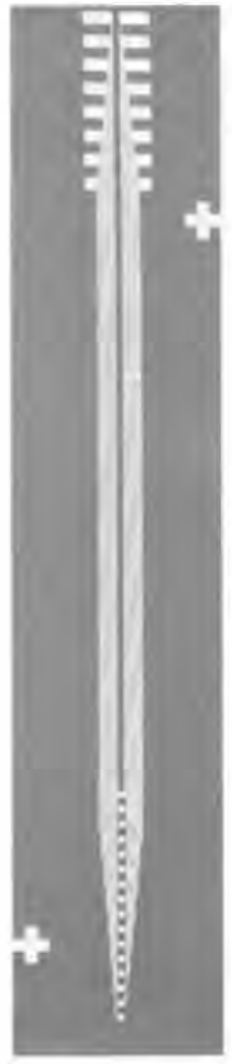

(a)

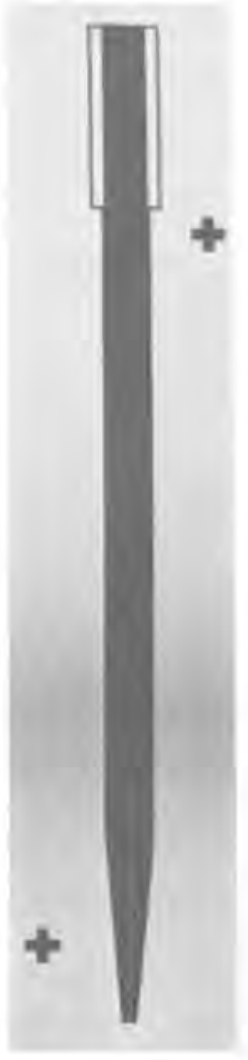

(b)

Fig. 21.3 Metal (a) and insulation layer (b) mask structure of a 16-fold electrode probe; any required two-dimensional electrode array can be produced using the described photolithographic fabrication procedures.

techniques allow the fabrication of precisely defined electrode areas, even in the $\mu \mathrm{m}$ size range. Thereafter, the glass substrate is cut in a needle-like shape by a diamond scriber under microscopic control. By means of a modified glass micro-electrode fabrication procedure, a fine probe tip is created which, in turn, allows smooth insertion of the probe into the brain tissue. The thin metal structure can be bonded to insulated copper leads or directly to integrated electronic circuits. The bonding areas have to be electrically as well as mechanically protected and are embedded in synthetic resin and silicone rubber. The upper $\mathrm{Cr}$ layer is removed at the recording sites so that the thin Au layer can be covered electrolytically by various metal layers such as gold, platinum, or silver (which can be converted to $\mathrm{AgCl}$ to provide a reference 
electrode in order to create a smooth probe surface as well as to serve various application purposes, which will be discussed below.

Other substrate, insulation, and metal layer materials are also used (Shamma-Donaghue et al. 1982; May et al. 1979; Wise and Angell 1975; Kuperstein and Whitington 1981; Edell 1982) for multiple electrode probes and a wide range of applications is opened up by the integration of electronic circuits directly behind the electrode structure. The possibility of adding the integrated circuit directly onto the same silicon substrate is also being investigated (Wise and Najafi 1984; Takahashi and Matsuo 1984).

\subsubsection{Electrical probe characteristics}

The electrodes form an electrode-electrolyte system with the tissue. The electrical properties of that system determine the signal transfer, the cross talk between two thin-metal interconnect paths, and the signal coupling across the insulation layer. In order to assure disturbance-free recordings, the electrical characteristics of that system have to be analysed, since it is dependent on the signal frequency, the electrode size, the electrode material, and the electrolyte,

The electrode-electrolyte interface can be represented by an equivalent electronic circuit, taking into account the charge-transfer processes at the electrode-electrolyte double layer as well as the diffusion, crystallization, and reaction processes at the electrode surface (Vetter 1962).

In recording the electrical activity of the brain tissue, the potential changes between the thin-film electrodes and a large reference electrode are measured. The current flow during the measurements has to be minimized in order to avoid tissue irritation. This means that crystallization and reaction processes at the electrode surfaces can be excluded and the equivalent circuit, representing the electrode-electrolyte interface, can be reduced to a

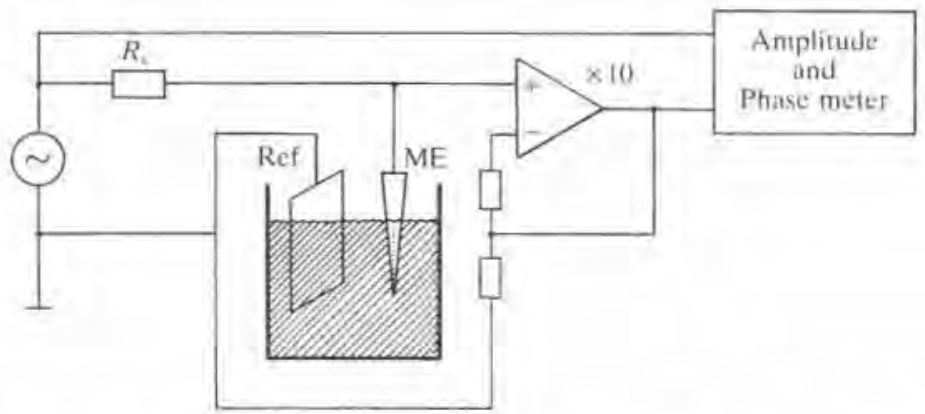

Fig. 21.4 Electrode-electrolyte impedance measurement set-up; amplitude and phase measurements allow the calculation of the capacitive and ohmic part of the impedance. 
resistance-capacitance $(R-C)$ combination. $R$ and $C$ can be determined by current-voltage measurements. In order to be able to compare the results with larger-sized electrode impedances (Geddes 1972) the $R-C$ series equivalent circuit will be used here as well.

Figure 21.4 shows the measurement set-up which was used in order to determined the electrode-electrolyte impedance $Z$ and its ohmic and capacitive components. $R$ and $C$ were calculated according to the voltage divider rule from the measured relation between the applied voltage and the voltage drop at the electrode impedance as well as their offset in phase. Our experiments showed, in accordance with theory (Vetter 1962) and other recordings (Geddes 1972), that the electrode-electrolyte impedance becomes a function of the current density as soon as a critical current density value is exceeded during the impedance measurements; we therefore kept the current density below $1 \mu \mathrm{A} / \mathrm{mm}^{2}$ throughout the electrode-electrolyte interface investigations.

Figure 21.5 shows the strong frequency dependence of $Z, R$, and $C$ of thinfilm gold electrodes with electrode areas of $0.25 \mathrm{~mm}^{2}$ (subscript 1), $0.01 \mathrm{~mm}^{2}$ (subscript 2), and $2500 \mu \mathrm{m}^{2}$ (subscript 3). The recordings also show that the electrode impedances are, in accordance with the theory, inversely proportional to the electrode area. The capacitive part of the impedance is almost frequency independent, indicating that the double-layer capacitance determines the capacitive part of the metal electrode impedance; the ohmic part,

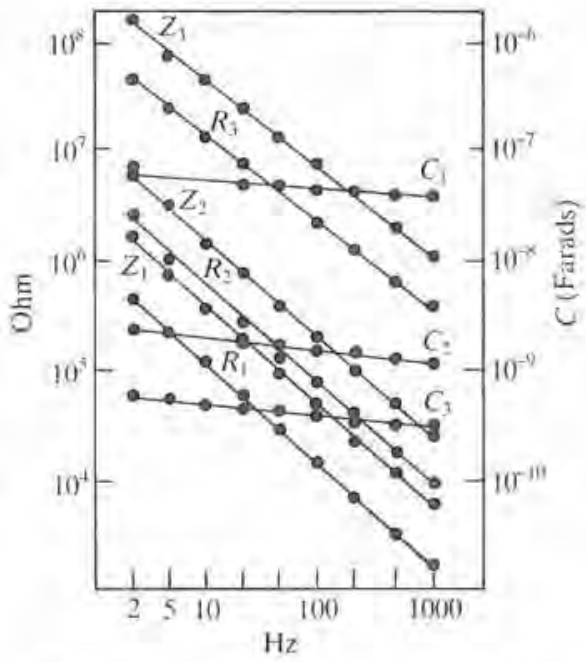

Fig. 21.5 Frequency dependence of $Z, R$, and $C$ of thin-film gold electrodes; subscript 1, electrode area of $0.25 \mathrm{~mm}^{2}$; subscript 2, electrode area of $0.01 \mathrm{~mm}^{2}$, subscript 3 electrode area of $2500 \mu \mathrm{m}^{2}$. 


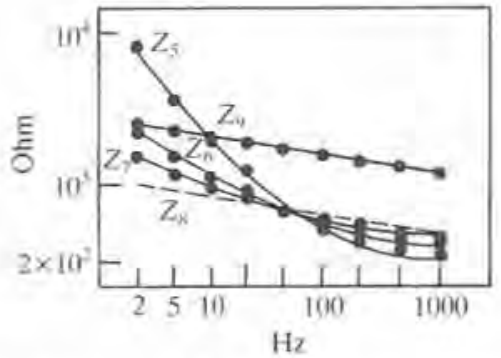

Fig. 21.6 Frequency dependence of $Z, R$, and $C$ of a mechanically and electrically stable thin-film Ag/ $\mathrm{AgCl}$ electrode, $2500 \mu \mathrm{m}^{2}$ in geometrical size (the actual electrode surface area is much larger).

however, is strongly frequency dependent and therefore mainly determined by the diffusion resistance.

High quality $\mathrm{Ag} / \mathrm{AgCl}$ electrodes show a very different behaviour; their fabrication procedure is discussed below. In Fig. $21.6 \mathrm{Z}$ and $R$ of an $\mathrm{Ag} / \mathrm{AgCl}$ electrode, $2500 \mu \mathrm{m}^{2}$ in geometrical size, are almost frequency independent, whereas the capacitive part of the impedance is negligible. Furthermore, the impedance value of $\mathrm{Ag} / \mathrm{AgCl}$ electrodes is more than 100 times smaller than that of Au electrodes of the same geometrical size for frequencies below $10 \mathrm{~Hz}$,

The different electrode characteristics make these two types of electrodes preferable for various applications. Single neuronal activities will be better studied with miniaturized gold electrodes, whereas potential changes in the frequency range below $100 \mathrm{~Hz}$ will be recordable with less perturbances as long as $\mathrm{Ag} / \mathrm{AgCl}$ electrodes are used.

The fabrication of mechanically stable $\mathrm{Ag} / \mathrm{AgCl}$ electrodes is a precondition for their electrical stability. This is particularly important since $\mathrm{Ag} / \mathrm{AgCl}$ electrodes can also be used as miniaturized reference electrodes. Therefore, appropriate fabrication steps shall be briefly outlined. The thin-film gold electrode areas are first electrolytically covered by a $1 \mu \mathrm{m}$ thick silver layer which is electrolytically converted to $\mathrm{AgCl}$ in a $1 \% \mathrm{NaCl}$ solution. The amplitude and the frequency dependence of $\mathrm{Ag} / \mathrm{AgCl}$ electrode impedances depend strongly on the amount of charge which was used for the chloridizing process. Figure 21.7 shows the impedance changes of $\mathrm{Ag} / \mathrm{AgCl}$ electrodes which were produced, using a current density of $0.03 \mathrm{~mA} / \mathrm{mm}^{2}$ for $10 \mathrm{~s}$ (subscript 5), $50 \mathrm{~s}$ (subscript 6), $100 \mathrm{~s}$ (subscript 7), and $180 \mathrm{~s}$ (subscript 9). These impedance changes support the assumption that the $\mathrm{Ag} / \mathrm{AgCl}$ growth starts at energetically preferred points on the silver surface (Jaenicke et al. 1955); a strong frequency dependence below $100 \mathrm{~Hz}$ is caused by the remaining silver surface. With on-going electrodeposition time a continuous $\mathrm{Ag} / \mathrm{AgCl}$ 


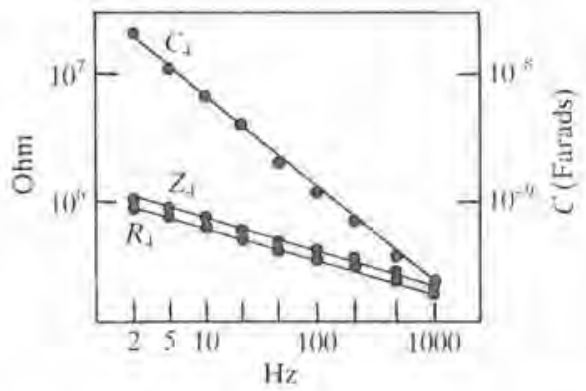

Fig. 21.7 Frequency dependence changes of the electrode-electrolyte impedance of a silver electrode which was chlorided for $10 \mathrm{~s}(5), 50 \mathrm{~s}(6), 100 \mathrm{~s}(7)$, and $180 \mathrm{~s}$ (9) with a current density of $0.03 \mathrm{~mA} / \mathrm{mm}^{2}$.

layer will be formed with minimum impedance values $\left(Z_{8}\right)$. Further electrolysis results in an increase of the $\mathrm{Ag} / \mathrm{AgCl}$ layer thickness, increasing the impedance of the $\mathrm{Ag} / \mathrm{AgCl}$ layer due to the fact that silver chloride has a resistivity of only $10^{5} \mathrm{Ohm} / \mathrm{cm}^{2}$ (Jaenicke et al, 1955).

The formation of mechanically stable $\mathrm{Ag} / \mathrm{AgCl}$ layers depends on the current density used during the process: SEM photos of $\mathrm{Ag} / \mathrm{AgCl}$ layers which were produced using current densities of $0.15 \mathrm{~mA} / \mathrm{mm}^{2}$ for $2 \mathrm{~s}$ (Fig, 21.8a), $25 \mathrm{~s}$ (Fig. 21.8b) and $35 \mathrm{~s}$ (Fig. 21.8c) show the large surface structure difference compared with the mechanically very stable $\mathrm{Ag} / \mathrm{AgCl}$ layer which was formed with a current density of $0.03 \mathrm{~mA} / \mathrm{mm}^{2}$ for $200 \mathrm{~s}$ (Fig. 21.8d). The latter fabrication procedure was successfully used for the production of all our miniaturized $\mathrm{Ag} / \mathrm{AgCl}$ electrodes.

\subsubsection{Sources of signal disturbances and application limits}

An adequate probe design is necessary in order to obtain disturbance-free signals from the thin-film multiple electrodes. Significant signal transfer distortions can be caused by the Johnson noise (Johnson 1928) of the electrode-electrolyte impedance, by the shunt capacitance along the thin-film metal interconnecting paths across the insulation layer, and by the cross talk between evaporated conducting paths. The electrode size and material determines the dimensions of the thin metal interconnecting paths and the thickness of the insulation layer (Prohaska et al. 1986). It should be emphasized at this point, that the available thin-film and solid-state equipment enables the fabrication in principle of even sub-micrometer structures and may enable a broader field of applications for micro-miniaturized electrodes.

In covering the thin-film electrodes with a reference electrolyte and an ionselective membrane (Burgess et al. 1982), thin-film ion-selective electrodes can be fabricated. Two and three electrode arrangements might be able to serve as voltammetric recording systems with the advantage of the better 


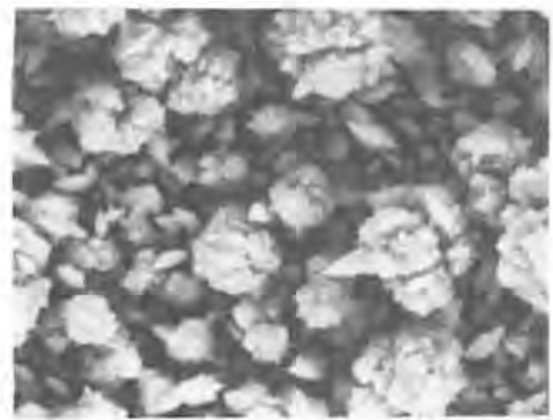

a

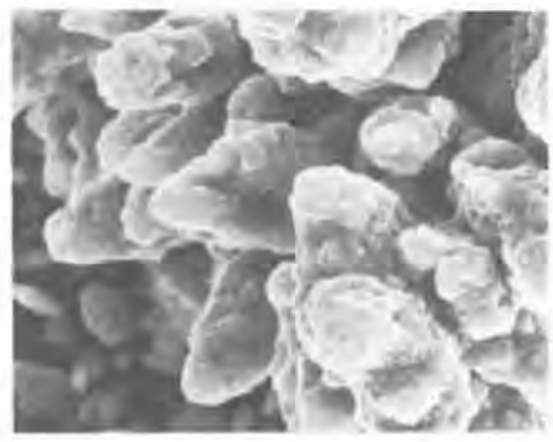

c

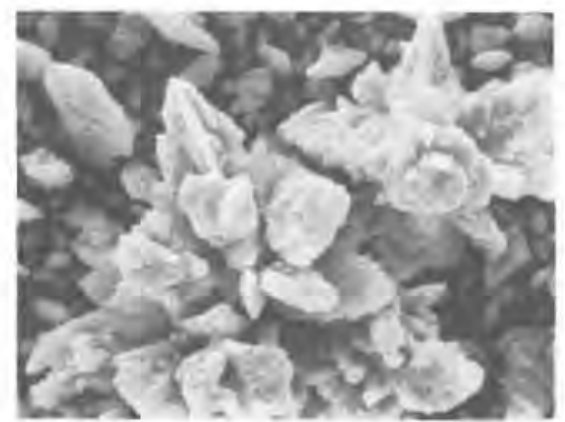

b

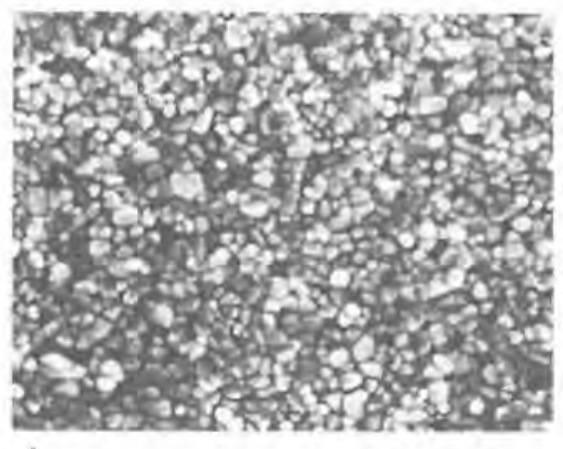

d

$2 \mu \mathrm{m}$

Fig. 21.8 Changes of the $\mathrm{Ag} / \mathrm{AgCl}$ surface, depending on the electrolysis time: (a) $2 \mathrm{~s}$; (b) $25 \mathrm{~s}$; (c) $35 \mathrm{~s}$. The current density was $0.15 \mathrm{~mA} / \mathrm{mm}^{2}$ respectively. (d) $\mathrm{Ag} / \mathrm{AgCl}$ electrode surface after a chloriding procedure with $0.03 \mathrm{~mA} / \mathrm{mm}^{2}$ for $200 \mathrm{~s}$.

recording qualities which are observed with micro-working electrodes (Caudill et al. 1982).

The main application limitations of thin-film electrodes are set by the quality of the electrode and insulation material and by the way potentiometric and voltammetric chemical sensors have to be designed. One problem is that the $\mathrm{Ag} / \mathrm{AgCl}$ electrodes are $\mathrm{Cl}$ - ion concentration dependent, a fact which can cause severe signal distortions. Another problem is that the reference electrode for voltammetric recordings in the case of a two electrode system should be larger by far then the working electrode; in the case of a three electrode system, the minimum size of the $\mathrm{Ag} / \mathrm{AgCl}$ reference electrode is determined by the leakage current of the amplifier system since this current can be large enough to modulate the $\mathrm{AgCl}$ deposit on the $\mathrm{Ag} / \mathrm{AgCl}$ electrode and thereby change the electrode potential. Furthermore, in vivo voltammetric studies become impossible as soon as the tissue becomes irritated 


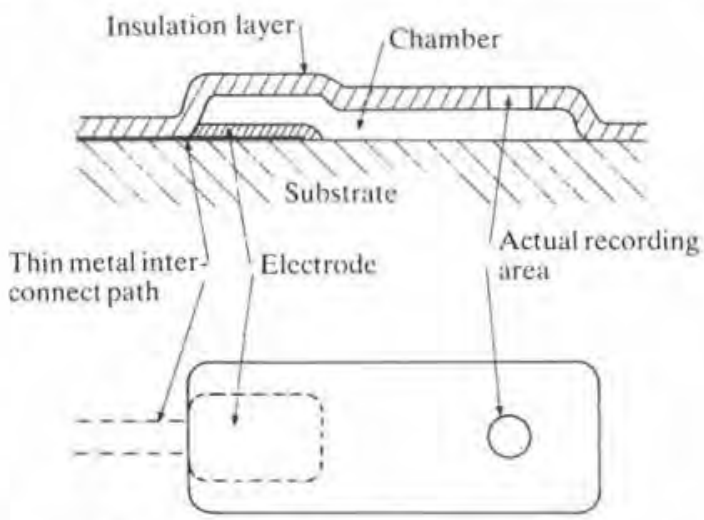

Fig. 21.9 Cross section and top view of a chamber-type electrode.

by the voltammetric current. A first step in order to overcome these problems was made by developing new miniaturized chamber-type electrodes.

\subsection{Chamber-type electrodes}

Figure 21.9 shows a cross section and a top view of a chamber-type electrode. In contrast to the flat thin insulation layer in Fig. 21.2 which covers the thin metal interconnecting paths of the thin-film electrode arrangement, the insulation layer is now bent upward and forms a chamber which covers the electrode. The contact between the electrode and the sample is effected through a hole in the chamber which defines the actual recording site size. The chamber is filled with an electrolyte. The SEM photo in Fig. 21.10 shows

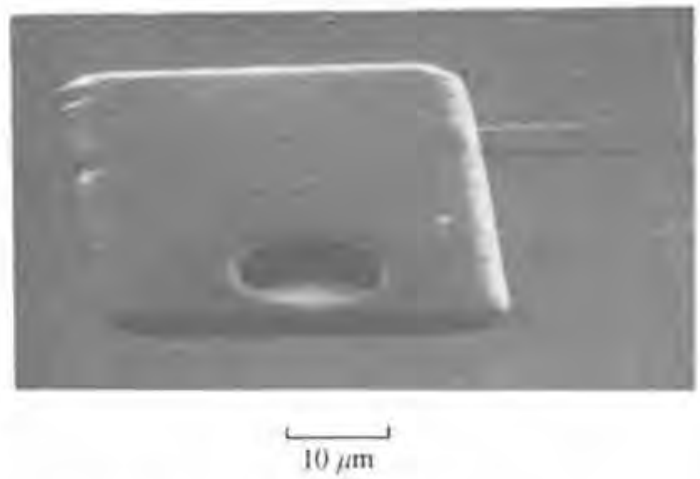

Fig. 21.10 SEM photo of a chamber-type electrode. 
a chamber-type electrode formed by a $3 \mu \mathrm{m}$ thick $\mathrm{Si}_{3} \mathrm{~N}_{4}$ insulation layer which is produced by plasma-enhanced chemical vapour deposition. (Vossen and Kern 1978; Olcaytug et al. 1980). The holes have, in this case, a diameter of $15 \mu \mathrm{m}$. The chamber is about $3 \mu \mathrm{m}$ high, $45 \mu \mathrm{m}$ wide and $80 \mu \mathrm{m}$ long. The measured impedance-frequency relationship indicates that the resistance of the electrolyte bridge in that chamber is below the $\mathrm{M} \Omega$ range. Furthermore, the $\mathrm{Ag} / \mathrm{AgCl}$ electrodes, covered by these chambers, become insensitive to rapid fluctuations in chloride ion concentration. The response time for $\mathrm{Cl}^{-}$ concentration changes can be increased up to $8 \mathrm{~s}$ if the distance between the $\mathrm{Ag} / \mathrm{AgCl}$ electrode and the hole is $30 \mu \mathrm{m}$ and up to $150 \mathrm{~s}$ if that distance is $80 \mu \mathrm{m}$. These results let us hope that a chamber design can be found which drastically improves miniaturized reference electrode qualities.

The main advantages of the chamber construction are:

a) The direct contact between tissue and electrode material can be avoided.

b) The recording area, being independent of the size of the electrodes, is defined by the size of the hole.

c) Since larger electrode areas can be used, signal disturbances can be kept minimal.

d) Miniaturized electrochemical cells can be designed, forming potentiometric and voltammetric sensors.

In filling the chamber with an ion-selective membrane, these types of sensors can be used as ion-selective electrodes. An enzyme electrode can be designed by supplying the chamber with an enzyme layer. The chamber not only protects the selective membranes and layers from being mechanically affected by the sample or the measuring process, but it also allows only a small portion of the selective membrane to be exposed to the sample, thus increasing the lifetime of the miniaturized sensor. In the event that the chamber is covering an electrode set, voltammetric recording techniques can be used advantageously to analyse the sample composition; the chamber construction is especially valuable in the case of diffusion-controlled processes, as it protects the electrode and provides it with a stable environment.

By using a $25 \mu \mathrm{m}^{2}$ gold electrode placed directly beneath the hole and a $1600 \mu \mathrm{m}^{2} \mathrm{Ag} / \mathrm{AgCl}$ reference electrode, an oxygen sensor was designed for amperometric measurements in the brain of animals. The response time is in the range of $0.3 \mathrm{~s}$, which agrees well with calculated values. The main advantage of this arrangement is that the current flow takes place only within the chamber and does not affect the sample under test, which is especially important in the case of nerve tissue. 


\subsection{Concluding remarks}

Thin-film and solid-state techniques seem to offer a large variety of possibilities in designing miniaturized electrochemical sensors. Although intensive studies will still be necessary in order to reach the quality and stability of macroscopic sensors, advantageous features of miniaturized thinfilm electrodes have already been demonstrated (Davis et al. 1986). A major advantage might also be that multiple parameter measurements could be possible; closely spaced chamber-type sensors, forming in themselves closed units, do not interfere with each other and physical parameters may be recordable at the same time using high resolution thin-film temperature sensors (Urban et al. 1982) and pressure sensors (Guckel et al. 1984; Lee and Wise 1982; Ko et al. 1982) integrated onto the same substrate.

\section{Acknowledgement}

The research was sponsored by the Austrian Fonds zur Foerderung der wissenschaftlichen Forschung, project no. S22/09 as well as by the Austrian Ludwig Boltzman Society. We want to thank Professors W. Fallmann, F. Paschke, H. Petsche, and R. Vollmer for the stimulating discussions and their support.

\section{References}

Burgess, B., Burleigh, P. and Diamond, H. (1982). Thin film solid state electrochemical sensors. Proc, Biosensors, 48-50. Los Angeles, 1982.

Caspers, H., Speckmann, E. J. and Lehmenkuehler, A. (1980). Electrogenesis of cortical DC potentials, In Motivation, motor and sensory processes of the brain. (eds. H. H. Kornhuber and L. Duecke) Progr. Brain Res. 54, 3-15.

Caudill, W. L., Howell, J. O. and Wightman, R. M. (1982). Flow rate independent amperometric cell. Anal. Chem. 54, 2532-5.

Davis, G.,Prohaska, O. and Olcaytug, F. (1986). Enzyme coupled reactions at microvoltammetric electrodes, Communication for Anal. Chem. In preparation.

Edell, D.J. (1982). A biocompatible, multi-channel neuroelectric interface, Proc. 35 th $A C E M B$, p. 6.

Elger, C.E., Speckmann, E. J., Prohaska, O. and Caspers, H. (1981), Pattern of intracortical potential distribution during focal interictal epileptiform discharges (FIED) and its relation to spinal field potentials in the rat. Electroenceph. Clin. Neurophysiol. 51, 393-402.

Geddes, L. A. (1972). Electrodes and the measurement of bioelectric everts, WileyInterscience, New York.

Guckel, H. and Burns, D. W. (1984). Planar processed polysilicon sealed cavities for pressure transducer arrays, Technical Digest IEEE IEDM, p. 223.

Jaenicke, W., Tischer, R.P. and Gerischer, H. (1955). Die anodische Bildung von Silberchlorid-Deckschichten und Umlagerungserscheinungen nach ihrer 
kathodischen Reduktion zu Silber, Z. Elektrochem. Angew. Physik. Chem. 59, 448-55.

Johnson, J. B. (1928). Therminal agitation of electricity in conductors. Phys. Rev. 32, 97-109.

Ko, W. H., Bao, M. and Hong, Y. (1982). A high-sensitivity integrated-circuit capacitive pressure transducer, IEEE Trans. Electron Devices 29, 48-56.

Krueger, J. (1983). Simultaneous individual recordings from many cerebral neurons: techniques and results. Rev. Physiol, Biochem. Pharmacol. 98, 177-233.

Kuperstein, M. and Whitington, D. A. (1981). A practical 24 channel microelectrode for neural recording in vivo. IEEE Trans, BME 28, 288-293.

Lee, Y.S. and Wise, K. D. (1982). A batch-fabricated silicon capacitive pressure transducer with low temperature sensitivity IEEE Trans. Electron Devices 29, $42-48$.

Maissel, L. and Glang, R. (1970). Handbook of thin-film technology. McGraw-Hill, New York.

May, G. A., Shamma, S. A. and White, R. L. (1979). A tantalum-on-sapphire microelectrode array, IEEE Trans. Electron Devices 26, 1932-39.

Olcaytug, F., Riedling, K. and Fallmann, W. (1980). A low temperature process for the reactive formation of $\mathrm{Si}_{3} \mathrm{~N}_{4}$ layers on InSb. Thin Solid Films, 67, 321-4.

Petsche, H., Pockberger, H. and Rappelsberger, P. (1984). On the search for the sources of the electroencephalogram. Neuroscience, 11, 1-29.

Prohaska, O., Olcaytug, F., Pfundner, P. and Dragaun, H. G. (1986), Thin-film multiple electrode probes: possibilities and limitations. IEEE Trans, Biomed. Eng. 33, 223-9.

- Womastek, K. and Petsche, H. (1981). A multielectrode for intracortical recordings produced by thin-film technology, Electroenceph. Clin. Neurophysiol. 42, 421-2.

Shamma-Donoghue, S. A., May, G. A., Cotter, N. E. and White, R. L. (1982). Thinfilm multiple arrays for a cochlear prostheses, IEEE Trans, Electron Devices 29. 136-144.

Takahashi, K, and Matsuo, T, (1984). Integration of multi-microelectrode and interface circuits by silicon planar and three-dimensional fabrication technology, Sensors and Actuators 5, 89-99.

Urban, G., Kohl, F., Olcaytug, F., Vollmer R. and Prohaska, O. (1982). Duennschichttemperaturfuehler fuer Mehrfachmessungen im Kortex, Wiss. Berichte, Jahrestagung Oesterr. Ges. BMT, 273-6.

Vetter, K. J. (1962). Electrochemical kinetics, Academic Press, New York.

Vossen, J.L. and Kern, W. (1978). Thin-film processes. Acad. Press, New York.

Wise, K. D. and Najafi, K. (1984). A micromachined integrated sensor with on-chip self-test capabilities, Proc. IEEE Solid-State Sensor Conference, 12-16.

- and Angell, J.B. (1975). A low-capacitance multielectrode probe for neurophysiology, IEEE Trans. Biomed, Eng. 22, 212-19. 


\title{
The design and development of in vivo glucose sensors for an artificial endocrine pancreas
}

\author{
GILBERTO D. VELHO, GERARD REACH, and \\ DANIEL R. THÉVENOT
}

\subsection{Introduction}

Insulin, a polypeptide hormone produced by the beta-cells of the pancreas, is essential in many metabolic pathways for carbohydrates, proteins, and fats; in the absence of normal insulin secretion, body fuel homeostasis is deranged. Diabetes mellitus is characterized by a relative or an absolute insulin deficiency manifested by loss of control of the circulating blood glucose levels, and by other metabolic abnormalities.

Diabetes is a common disease in affluent societies, affecting from one to three per cent of the population, and often five to ten per cent of those over 40 years of age (Hamman 1983). Where systematic surveys have been performed in the developing nations, rates of one to two per cent of the total population prevail (Bennett 1983). Thus, diabetes is a major world-wide health problem with a great social and economic impact due largely to its later complications. Albisser and Spencer (1982) referring to the Report of the National Commission on Diabetes to the Congress of the United States (1976) suggest that, in that country, diabetics are 25 times more prone to blindness than non-diabetics, 17 times more prone to kidney disease, 5 times more prone to gangrene, twice as prone to heart disease, and have a life expectancy of approximately one-third less than the general population.

Diabetes mellitus is a heterogeneous disease and only a minority of patients, representing however 3 per 1000 of the general population, are so severely insulinopenic as to require insulin therapy. Since its introduction in the early twenties up to the last years of the seventies, insulin therapy was possible only through discontinuous insulin administration, by one, two, or occasionally, several daily insulin injections.

The search for better methods for treating insulin-dependent diabetes and its complications has led to the development of new devices for insulin therapy in the last decade. Infusion systems for continuous insulin delivery (insulin pump), including a reservoir, a pump, and a power supply packed into a portable single unit, have been made available to clinicians and diabetic patients. Efforts to develop a portable self-regulated system, associating an 


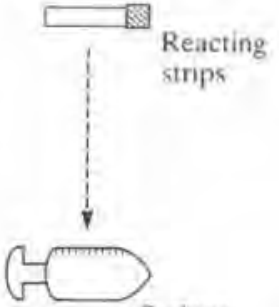

Syringe

a. Conventional therapy

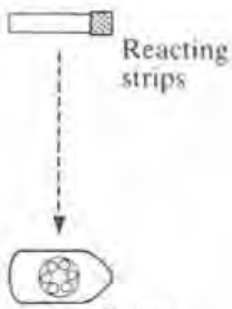

Pump

b. Open-loop system

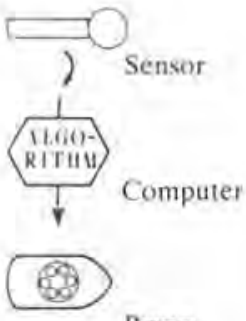

Pump

c. Closed-loop system

Fig. 22.1 Scheme of three possible methods of insulin therapy.

(a) Intensive conventional therapy: multiple insulin injections dependent on manual glucose measurement with reacting strips.

(b) Open-loop system: continuous preprogrammed insulin infusion dependent on manual glucose measurement with reacting strips.

(c) Closed-loop system: continuous self-regulated insulin infusion. The glucose level, continuously measured by the sensor, is translated by the computer in a variable rate of insulin delivery.

implantable glucose sensor with the insulin delivery device, are in progress in several laboratories. This system is referred to as a closed-loop system, in contrast to the former, non self-regulated system, known as the open-loop system (Fig. 22.1). The control of insulin delivery by open-loop and closedloop systems, as compared to the physiological regulation of insulin secretion, is shown in Fig. 22.2.

This chapter will review the advantages of the closed-loop system of insulin therapy, the requirements for an implantable glucose sensor, and the state of present development and applications of glucose sensors.

\subsection{Are closed-loop insulin infusion systems really necessary?}

The evidence of a relationship between the microvascular complications of diabetes mellitus and hyperglycaemia (Tchobroutsky 1978) led to the intensification of insulin therapy, either by multiple daily insulin injections or continuous insulin infusion, in the hope that it would improve metabolic control and therefore prevent the occurrence of these late complications. Rizza et al. (1980) comparing the control of blood sugar by an artificial endocrine pancreas (closed-loop system), continuous subcutaneous insulin infusion (open-loop system), and intensified conventional insulin therapy, in insulindependent diabetes, found no significant differences among the three regimens and suggested that all three methods have the potential to achieve a similar near-normalization of glycaemia. 


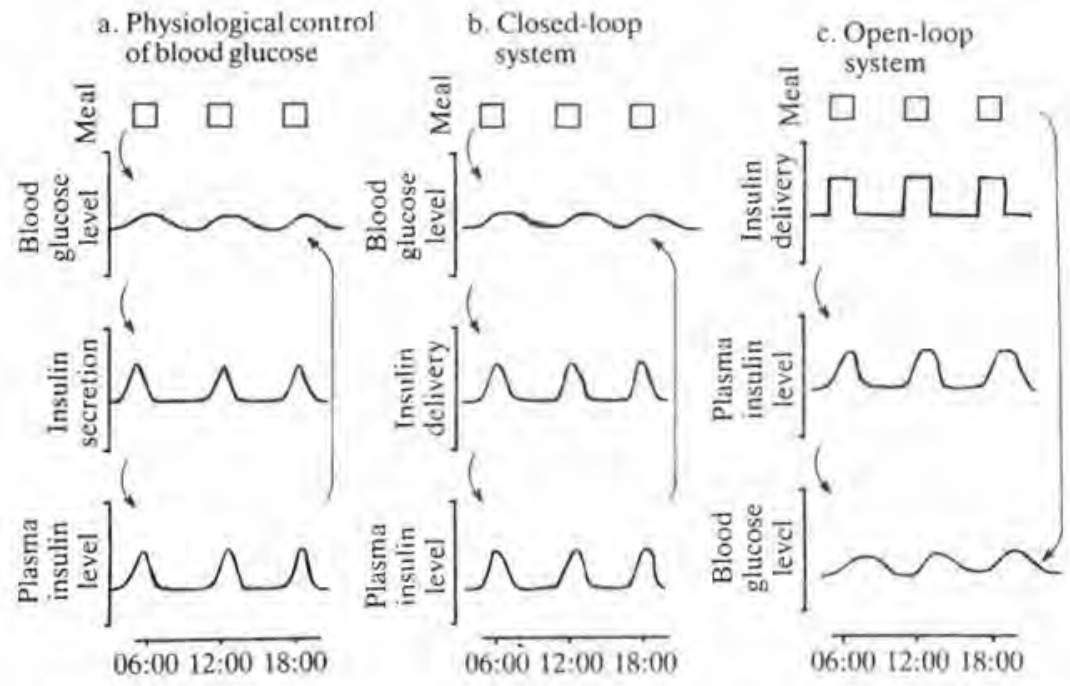

Fig. 22.2 Physiological regulation of blood glucose by the endocrine pancreas (a), control of insulin delivery in closed-loop (b) and open-loop (c) systems. In the openloop system, insulin delivery is programmed to normalize the blood glucose but it is not regulated by the glucose level.

The main advantages and disadvantages presented by these three methods of insulin therapy are summarized in Table 22.1. Intensive conventional insulin therapy is inexpensive, calls for no special equipment and is immediately available to every patient. However, multiple daily injections of insulin are necessary to achieve a near normal glycaemic control.

Open-loop devices do not have this limitation. Nevertheless, they are expensive, must be carried by the patient, and like any mechanical devices, are subject to malfunction. The problem of insulin aggregation, with loss of biological activity and obstruction of the internal passages of the device has been described (Lougheed et al. 1980). Both conventional insulin therapy and open-loop therapy need frequent glucose measurements by the patient to maintain adequate glycaemic control since diet and exercise demand an adaptation of insulin doses. Both therapy methods present, however, further advantages concerning the timing of mealtime insulin bolus and the route of insulin delivery. The mealtime insulin bolus is not controlled by nutrient absorption in the gut, as in normal individuals and in closed-loop therapy (Fig. 22.2), and thus, must be controlled by the patient. As the postprandial blood glucose and insulin levels are affected by the interval between insulin administration and meal ingestion, this interval, if appropriately chosen, may contribute to the normalization of glycaemia and insulin 
Table 22.1 Main advantages and disadvantages of different methods of insulin therapy

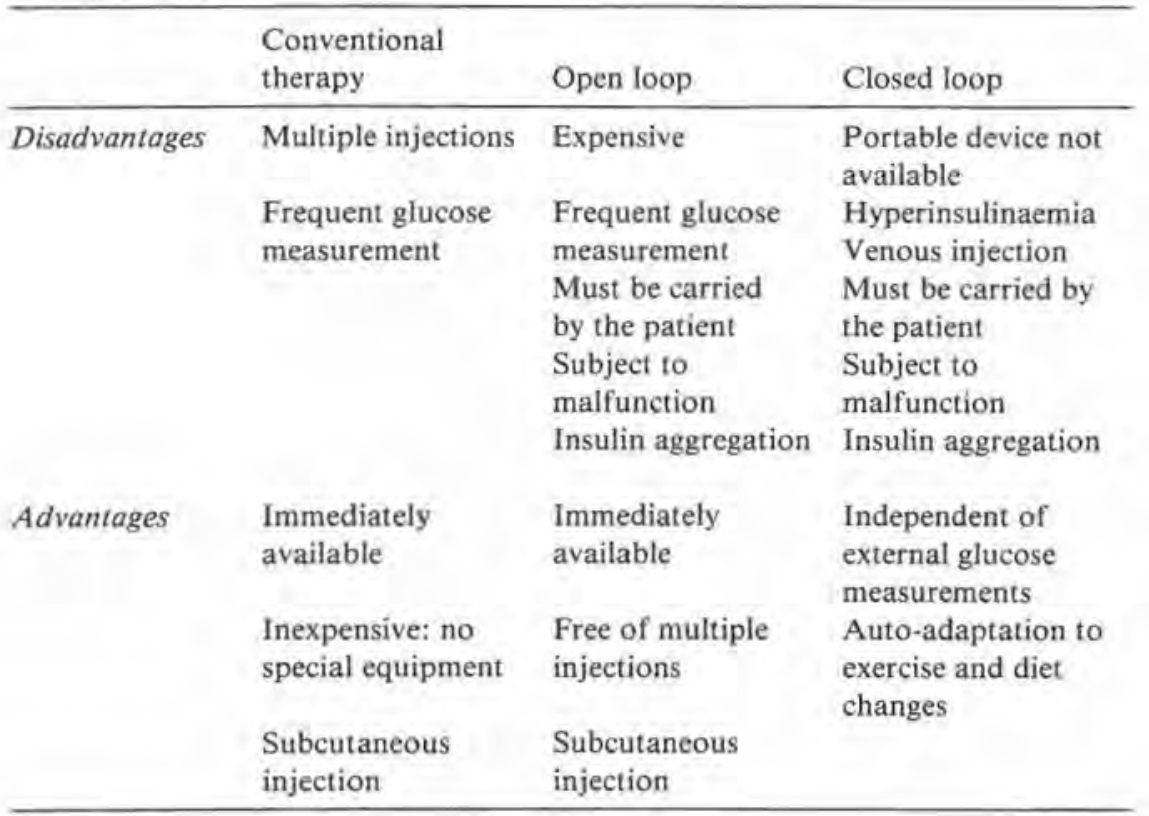

profile. Dimitriadis and Gerich (1983) compared the effects of 30-min subcutaneous insulin infusions started $60 \mathrm{~min}, 30 \mathrm{~min}$, and immediately before meal ingestion on postprandial plasma glucose and insulin profiles in subjects with insulin-dependent diabetes mellitus. They found that administration of insulin $60 \mathrm{~min}$ before meal ingestion provided plasma glucose and insulin profiles closest to normal and permitted less insulin to be used. This anticipation of 60 minutes may be necessary for two complementary reasons: first, part of this time may be required to build up a physiological hepatic insulinization from insulin delivered subcutaneously. Second, insulin secretion in non-diabetic subjects is not controlled only by blood glucose rise: the response of insulin-secreting cells is anticipated under the influence of different nerves and of gastroenteric hormones. Thus, if insulin doses and the timing of injection or bolus infusion are carefully chosen, near normal glucose control can be obtained by intensive conventional therapy and open-loop systems through the subcutaneous route of insulin delivery. In that way the complications associated with long-term vascular access for intravenous insulin infusion can be avoided.

The main advantage of a closed-loop insulin delivery device is its independence of external glucose measurements and its ability to cope with the 
variations of insulin requirement brought about by exercise and diet. Nevertheless, the glycaemic normalization achieved by these devices is frequently associated with peripheral hyperinsulinaemia (Horwitz et al, 1980). Hyperinsulinaemia is a common feature of any insulin administration through a peripheral route, and is mainly due to the absence of the portal-peripheric insulin gradient. Furthermore hyperinsulinaemia might also be the consequence of the time lag in insulin administration in response to the glucose challenge. Thus, this hyperinsulinaemia can be avoided by the combination of the feedback-controlled insulin administration with a pre-programmed preprandial insulin infusion (Calabrese et al. 1982). Therefore, the possibility of a 'manual' or 'semi-automatic' mode should be considered in the design of closed-loop systems.

Currently, closed-loop systems present several disadvantages. They are cumbersome bedside devices that need continuous blood withdrawal from the subject in order to ensure the automatic glucose analysis. A small portable device is not yet commercially available. Closed-loop systems, portable or not, need long-term venous access for insulin delivery. Insulin absorption by a subcutaneous or peritoneal route is not fast enough to enable an efficient feedback control of the infusion rate. Finally, closed-loop systems, being automatic devices, should be extremely reliable, both mechanically and in the reading of the glucose sensor, otherwise their main advantage, i.e. less demanding glucose control by the patient, would be lost. Pump-induced insulin aggregation seems to be an additional problem to be solved (Brennan et al. 1985).

\subsection{Why is a portable closed-loop insulin infusion device not yet available?}

Such a device consists essentially of a glucose sensor, a pump and a computer that translates the information provided by the sensor into a variable rate of insulin infusion. The pump and the computer components of the device are commercially available. By contrast, implantable glucose sensors that prove to be reliable are still to be developed.

The great majority of the glucose sensors developed so far operate through the oxidation of $\beta$-D-glucose by dissolved oxygen in the presence of $\beta$-D-glucose oxidase (GOD EC 1.1.3.4.), according to the following reaction:

$$
\text { Glucose }+\mathrm{O}_{2} \stackrel{\text { Glucose oxidase }}{\longrightarrow} \text { Gluconic acid }+\mathrm{H}_{2} \mathrm{O}_{2}
$$

They consist of electrochemical detectors (electrodes) associated in different ways with the enzyme support. The chemical reaction may be monitored via three of its constituents, i.e., oxygen depletion, gluconic acid, or hydrogen peroxide formation. 
Table 22.2 Main requirements for a glucose sensor for an artificial beta-cell

High specificity for glucose

Linearity of response from 1 to $15 \mathrm{mmol} / 1$ of glucose

Response time less than 10 minutes

Response independent of hydrodynamies and oxygen variations in tissues

Stability of glucose oxidase membrane at $37{ }^{\circ} \mathrm{C}$ in tissues

Biocompatibility

Prolonged lifetime (at least several days)

Miniaturization of the sensor head

The requirements to be fulfilled by a portable or implantable glucose oxidase type of sensor for use in a closed-loop device (Thévenot 1982) are described below and summarized in Table 22.2. General requirements, also valid for other types of glucose sensors, are marked with an asterisk.

1) High specificity for glucose*. In the case of glucose oxidase sensors this includes high enzymatic specificity and high electrochemical specificity of associated detectors. The first condition is always valid since glucose oxidase catalyses the oxidation of only very few species besides glucose, and at a much lower rate (Barman 1969). On the contrary, the second condition is often non-valid and depends mainly upon the type of electrochemical detector used (see Section 22.4).

2) Linearity of in vivo response from 1 to $15 \mathrm{mmol} / 1$ (Fig. 22.3b)*; this rather limited linear range is justified by the recent findings of Harrison et al. (1985) who described the properties of isolated human islets of Langerhans (Fig. 22.3a). The threshold concentration of glucose required for stimulation of insulin release was between 2 and $4 \mathrm{mmol} / 1$, insulin secretory response to glucose stimulation had half-maximal values at a glucose concentration of approximately $5 \mathrm{mmol} / \mathrm{l}$ and a plateau at $10 \mathrm{mmol} / \mathrm{l}$. Under in vivo conditions the calibration curve of a glucose oxidase sensor may not always be linear over this range (example: Fig. 22.4, curve A). In fact, the tissue or blood glucose level, especially in diabetics, may be higher than the apparent Michaelis constant $\left(K_{\mathrm{M}}\right)$ of glucose oxidase solutions for glucose in air-saturated solutions, i.e. 4 to $10 \mathrm{mmol} / 1$ (Apotheker A., Thévenot D. R., and Wilson G.S., unpublished data). However, it is possible to get a calibration curve linear over a much higher concentration range, i.e. up to $20-30 \mathrm{mmol} / 1$, if the glucose flux is reduced by membranes of low permeability to glucose. This may be achieved by an external membrane covering the enzymatic membrane (Fig. 22.4, curve C) or by the enzymatic membrane itself (Fig. 22.4 curve B).

3) Response time less than $10 \mathrm{~min} *$; Sorensen et al. (1982) using a theoretical physiological pharmacokinetic model of glucose homeostasis 

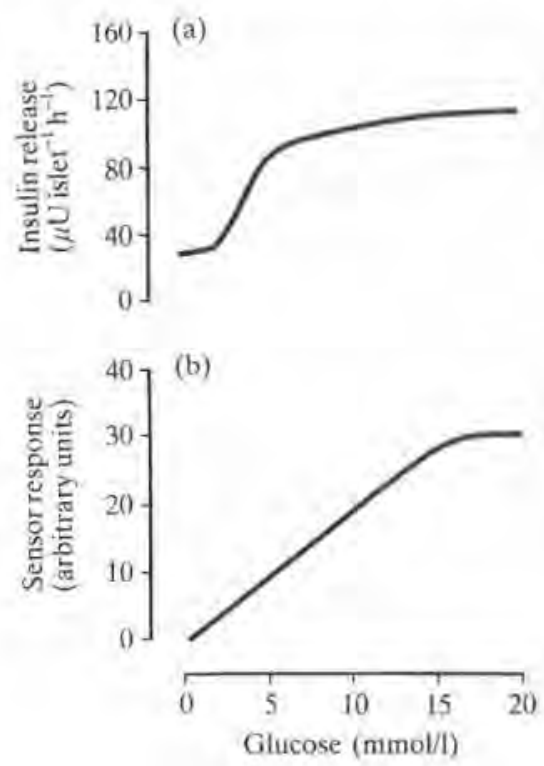

Fig. 22.3 (a) Insulin secretion by isolated islets in respone to glucose (after Harrison et al. 1985), Note the sigmoidal relationship and the plateau observed at values higher than $10 \mathrm{mmol} / \mathrm{L}$. (b) Linearity of an implantable glucose sensor for the artificial endocrine pancreas. Due to the response of natural beta cells, the linearity of the sensor response may be limited to $15 \mathrm{mmol} / \mathrm{l}$ of glucose.

showed that increases in sensor delay resulted in progressive loss in glucose regulation, exacerbation of hyperinsulinaemia, and increased insulin requirements.

4) Independence of sensor response to fluid hydrodynamics in vessels or tissues*.

5)Independence of sensor response to oxygen level variations in the sensor surroundings and oxygen consumption by the sensor itself. Oxidation of glucose by dissolved oxygen is an irreversible process with a steady state that may be controlled either by the enzymatic oxidation reaction with high temperature dependence $\left(6-10 \% /{ }^{\circ} \mathrm{C}\right)$ or by substrate diffusion with low temperature dependence $\left(2-4 \% /{ }^{\circ} \mathrm{C}\right.$ ) (Racine and Mindt 1971 ; Kamin and Wilson 1980). Under such heterogeneous kinetics, the glucose electrode consumes what it is supposed to monitor. This is a characteristic common to Clark's oxygen sensor (see Section 22.4.1.). Whatever the electrochemical detector associated with the glucose oxidase membrane, the stability of its readings is affected by external diffusion (i.e. fluid flowrate near the membrane), internal diffusion (i.e. permeability to substrates), as well as by oxygen concentration level in or near the mem- 


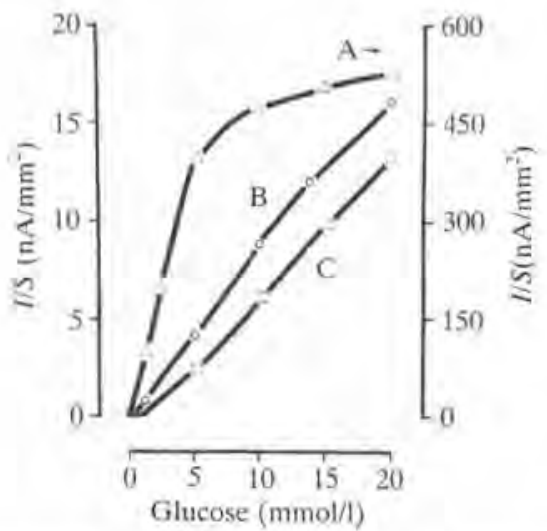

Fig. 22.4 Different types of calibration curves for a glucose sensor using different glucose oxidase membranes (after Sternberg R., Tallagrand T., and Thévenot D. R., unpublished data). (a) GOD collagen membrane (right-side $Y$ axis). (b) GOD cellulose acetate membrane (left-side $Y$ axis). (c) GOD collagen membrane covered with a pinholed Teflon membrane (left-side $Y$ axis). Note that the use of an additional non-enzymatic membrane or of a cellulose acetate membrane both extends the linear range of the system and impairs its sensitivity.

branes. In an ideal situation these factors should be kept constant. In the case of implantable glucose electrodes these ideal conditions are difficult to obtain. Clark's oxygen detectors that have a cathode diameter approximately equal to the membrane thickness (10-20 microns), are less dependent on hydrodynamics, due to the hemispheric diffusion pattern (in contrast with the linear pattern) obtained in this particular situation (Bard and Faulkner 1980; Wightman 1981). Accessibility of substrates and adequate oxygen level at enzymatic sites can be indirectly controlled by using an additional external membrane more permeable to oxygen than to glucose and/or by using enzymatic layers with a high partition coefficient for oxygen.

6) Long-term mechanical, chemical, and enzymatic stability of glucose oxidase and its support at $37^{\circ} \mathrm{C}$, in whole blood, lymph, or tissue.

7) No leaking of glucose oxidase into fluids and tissues surrounding the sensor; being a foreign enzyme, its recognition by the immune system would provoke an immune reaction.

8) Biocompatibility of all implanted parts of the sensor; absence of implant encapsulation by fibroblasts and giant cells*, Woodward (1982) suggested that the optimal configuration for a subcutaneously implantable sensor is in the form of a wire or filament. Such a structure, if measuring less than about $2 \mathrm{~mm}$ in diameter, would evoke a minimal tissue response. 
9) The scaling down of the sensor should not modify the geometrical, physical, and enzymatic characteristics which control its analytical properties.

10) The system should require minimal calibration and zero adjustment*.

11) Finally, the sensor should have a prolonged lifetime, it should be easily replaceable if necessary and not be expensive if it has to be replaced*. In the case of sensors to be partially inserted in the subcutaneous tissue, in a needle-like fashion, a lifetime of several days, if not weeks, could be accepted. Obviously, a totally implantable device would require a much longer lifetime.

In the remaining sections of this chapter an overview is presented of the significant results obtained in the development of glucose sensors and their application in closed-loop insulin infusion devices.

\subsection{Glucose oxidase electrochemical sensors for the artificial endocrine pancreas: types of detectors.}

\subsubsection{Oxygen detectors}

Clark and Lyons (1962) described the first specific glucose electrode (Chapter 1), The enzyme was retained on a polymer membrane and an amperometric oxygen electrode estimated the decrease of oxygen as the reaction proceeded. The Clark-type oxygen electrodes are almost insensitive to all types of interfering substances, but they are obviously very sensitive to variations in partial pressure of oxygen within the fluid in contact with the electrode. Thus, misreadings due to physiological or pathological fluctuations of oxygen partial pressure are to be expected under in vivo conditions. This problem may be surmounted by the addition of a second electrode, not associated with a glucose oxidase membrane, forming a differential system (Updike and Hicks 1967).

Improvements in this system by Bessmann and Schultz (1973) led to a prototype implantable sensor using two galvanic oxygen electrodes as detector. Oxygen had access to the electrodes through a polypropylene membrane, the external side of which was fastened to a matrix of nylon cloth. Glucose oxidase was covalently bound to the matrix, in the working electrode, by glutaraldehyde. The whole was contained in a plastic disc of $2 \mathrm{~cm}$ diameter by $0.25 \mathrm{~cm}$ depth. The sensor had a useful in vivo lifetime of four days but a less than optimal sensitivity to glucose, due in part to the low oxygen partial pressure in subcutaneous tissues (Bessman et al, 1977).

An additional problem with this type of sensor (Fig. 22.5a) is the competition for oxygen between the glucose oxidase membrane (flux $v_{2}$ ) and the oxygen detector itself (flux $v_{1}$ ); if the cathode is not small enough, the latter flux may interfere with the apparent glucose oxidase activity. 


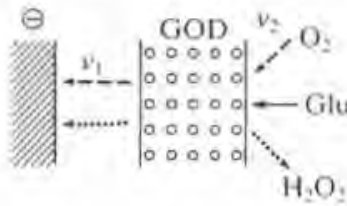

$E=-0,4 \mathrm{~V} / \mathrm{AgCl}$

a. Oxygen cathodic detector

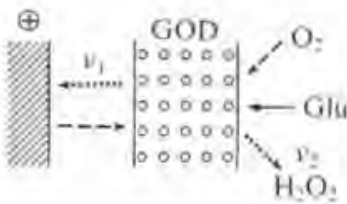

$E=+0,6 \mathrm{~V} / \mathrm{AgCl}$

b. Hydrogen peroxide anodic detector

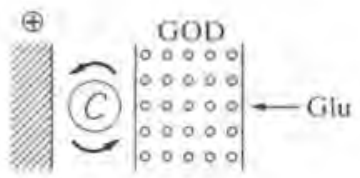

$E=+1)] 6 \mathrm{~V} / \mathrm{AgCl}$

c. Cofactor detector

Fig. 22.5 Types of detectors used in glucose oxidase electrochemical sensors for artificial endocrine pancreas: (a) oxygen cathodic; (b) hydrogen peroxide anodic; and (c) cofactor detector. See text for explanation.

\subsection{2 $\mathrm{pH}$ detectors}

Glucose sensors based on the detection of gluconic acid via a pH electrode have been developed. Nevertheless they present poor sensitivity, selectivity, and linearity of calibration curves (Nilson et al. 1973) and thus cannot be implanted in the highly buffered body fluids.

\subsubsection{Hydrogen peroxide amperometric detectors}

Amperometric detection of enzymatically generated hydrogen peroxide is probably the most-developed type of glucose sensor (Guilbaut and Lubrano 1973; Scheller et al. 1977; Thévenot et al. 1978) (Fig. 22.5b). Clemens et al. (1977) adapted one of such sensors for use in a bedside-type artificial pancreas. Similar sensors have been adapted for the same purpose by several groups (see Section 22.7). Over the last ten years, improvements have been made in the sensor design, the binding of the enzyme to its support, and the functional characteristics of the electrodes.

This type of detector is very sensitive to glucose; its lowest detection limit may reach $10 \mathrm{nmol} / \mathrm{l}$ (Thévenot et al. 1978). Hydrogen peroxide amperometric detection is also very sensitive to naturally occurring electron donors, such as ascorbate, urate, and tyrosine. Methods have been developed to increase the selectivity of the glucose electrode towards such interfering substances. Either the response is compensated by a non-enzymatic detector (Thévenot et al. 1978) or the platinum anode is covered by selectively impermeable membranes (cellulose acetate, for instance) with pores that will exclude ascorbate and most other potential interfering substances (Yellow Springs Instrument Co. 1975).

The independence on oxygen concentration of hydrogen peroxide detection is an advantage in sensor design. Nevertheless the local oxygen level 
necessary for the enzymatic reaction to occur must be taken into account: in that way, membrane partition and the diffusion coefficient for oxygen play an important role in glucose response patterns, Oxygen is regenerated during the electrochemical oxidation of hydrogen peroxide on the platinum surface according to the reaction:

$$
\mathrm{H}_{2} \mathrm{O}_{2} \longrightarrow \mathrm{O}_{2}+2 \mathrm{H}^{+}+2 \mathrm{e}^{-}
$$

Optimization of the collection efficiency of the detector (Fig. 22.5b) i.e., the ratio between the part of the enzymatically generated hydrogen-peroxideflux oxidized on the platinum $\left(v_{1}\right)$ and the total flux $\left(v_{1}+v_{2}\right), v_{2}$ being the part of the flux diffusing towards the bulk solution, would result in a greater availability of oxygen in the enzymatic layer and in a greater independence of oxygen diffusion from the bathing fluids, once the reaction had started (Coulet et al, 1980).

Finally, hydrogen peroxide anodic oxidation is not always diffusion controlled and its rate may limit the signal from the sensor. This rate may depend on electrode conditioning (Dubois 1984).

\subsubsection{Hydrogen peroxide potentiometric detection}

Potentiometric measurement of glucose concentration is the principle of a sensor developed by Schiller, Wingard, and Liu (1982; Chapter 10). Glucose oxidase is immobilized directly on the platinum surface of the working electrode by methods including entrapment in polyacrylamide gel, crosslinking in an albumin matrix with glutaraldehyde, and coupling to platinum through gamma-aminopropyltriethoxy silane (Wingard et al, 1979).

In contrast to amperometric detections in which an external potential is applied between the electrodes, and in which oxygen or hydrogen peroxide local concentrations are directly monitored through the generated current, potentiometric detection measures a pseudo-equilibrium potential inside the system. The electrochemcial reaction responsible for this potential appears to result from the interactions between the enzymatically generated hydrogen peroxide and the platinum surface (Wingard et al. 1982). The electrode cleaning procedure is always critical to the functioning of the system. Linearity of response, as the logarithm of glucose concentration, was achieved in in vitro studies over the range of about 0.6 to $22 \mathrm{mmol} / 1$. Theoretical advantages of this system for in vivo utilization, due to the low potential generated, include minimal electrochemical interference and the possibility of micro-miniaturization of the electrode.

\subsubsection{Cofactor detectors}

The concept of cofactor detectors is based on the ability of cofactors to act as temporary acceptors of the protons and electrons released during the oxidation of substrates by oxidation-reduction enzymes (Chapter 15). The general 
idea is to have a solid-state-type electrode in which a naturally-occurring or an artificial cofactor is an integral part of the electron-conducting support and the enzyme is immobilized with the cofactor. The electrode, as a whole, behaves as a cofactor, i.e. an electron acceptor or donor for an enzymatic reaction (Fig. 22.5c).

The coupling of riboflavin to solid carbon, forming a solid-state pathway for easy electron transfer, has been described (Wingard 1982). Subsequent developments (Wingard 1983a) included the conversion of immobilized riboflavin to FAD and the appearence of enzymatic activity on the addition of the apoenzyme of glucose oxidase. Later, Cass et al. (1984) used entrapped ferrocene derivatives, as ferricinium ions, which may be electrochemically oxidized and react with reduced glucose oxidase. If such reagents are present in sufficient excess, then the supply of oxygen to the catalytic layer would have little effect on the enzymatic rate (see Chapters 15 and 16). Recently, Ikeda et al. (1985) described a glucose sensor using benzoquinone as a cofactor. Glucose oxidase was immobilized on the surface of a $p$ benzoquinone-carbon paste electrode by coating the enzyme-loaded surface with a nitrocellulose film. Properties of the sensor include the electrocatalytic oxidation of glucose with a linear range up to $15 \mathrm{mmol} / 1$, the response time of about 20 seconds and the insensitivity to variations of oxygen tension in sample solutions.

\subsection{Designs of in vivo glucose oxidase sensors}

The latest developments towards an implantable glucose sensor have favoured three types of sensor design: the plane-geometry type, the vesselshaped, and the needle type. Plane-geometry sensors consist basically of a plane surface support containing the metal working electrode and the reference and counter electrodes coated by various combinations of enzymatic and non-enzymatic, hydrophilic and hydrophobic membranes. The membranes provide a support for the enzyme, an environment for the chemical reaction, and a diffusion barrier assuring the optimal concentrations of glucose and oxygen in this environment. Fischer and Abel (1982) described a plane-geometry sensor mounted into a flow chamber. It consisted of a platinum anode for the measurement of hydrogen peroxide, a silver/silver chloride reference and counter electrode, glucose oxidase immobilized onto sepharose and held by hydrophilic cellulose acetate membranes, and an hydrophobic perforated Teflon membrane in front of the anode. In vivo tests using normal and diabetic dogs showed reasonable correlation between the sensor output and the plasma glucose reference values with a response time between 90 and 120 seconds. The linear range for in vitro calibration was up to $40 \mathrm{mmol} / \mathrm{l}$ of glucose.

An original approach was described by Kondo et al. (1981): the sensor is a 
vessel-shaped device through which the blood flows. Oxygen-type electrodes and membranes are disposed around its wall. The sensor is introduced into the circulatory system in a fashion similar to an external arterio-venous shunt for haemodialysis. The linear range is up to $16 \mathrm{mmol} / \mathrm{l}$ of glucose and the response time is about 10 minutes.

The needle-type sensors are usually micro-electrodes having a platinum core (anode) isolated from an external silver/silver chloride cathode reference and counter electrode. The electrode is coated with glucose oxidase immobilized in a solution of a matrix material (cellulose diacetate, for instance) in a volatile solvent (acetone, for instance). Shichiri et al. (1982; Chapter 23) have described a subcutaneously implantable needle-type sensor having an in vivo response time of 2 to $5 \mathrm{~min}$ and a linear response of up to $27 \mathrm{mmol} / 1$ of glucose (see Section 22.7).

\subsection{Glucose sensors: possible alternative approaches}

Glucose sensors based on non-enzymatic approaches have been known for many years. Although they purport to avoid the difficulties associated with heterogeneous enzyme kinetics none of these systems is presently sufficiently developed to permit in vivo implantation.

The characteristics of direct electrochemical sensors, consisting of platinum electrodes not associated with glucose oxidase have been studied (Soeldner et al. 1973; Gebhardt et al. 1978; Richter et al. 1982). The signal is generated by direct glucose oxidation at the anodic surface of a platinum electrode, in response to alternate anodic and cathodic potentials. Their specificity to glucose, in biological fluids, is still less than optimal, due to the interference of endogenous oxidizable substances such as amino acids, urea, ascorbic acid, and of exogenous substances such as alcohol and several drugs. The selection of adequate working potentials and the use of an external selective membrane brings real improvement to the system specificity. An additional problem with this type of detector is the poisoning of the platinum surface by adsorption of gluconic acid and amino acids, which leads to the gradual deactivation of the anode catalyst and inhibition of further oxidation. The deactivation can be offset with regeneration of the working electrode by repeated surface oxidation by electrochemical pulsing. Nevertheless, oxidized radicals are generated and desorbed from the electrode surface together with products of electrode degradation. The present status of the electrocatalytic glucose sensor does not favour its use as an implantable device.

The competition of glucose and fluorescein-labelled polydextran for the binding sites of the protein concanavalin A, immobilized on the inside surface of a hollow dialysis fibre, is the principle of a sensor developed by Schultz et al. (1982; Chapter 32). This affinity sensor is completed by an 
optical fibre inserted in the lumen of the dialysis fibre that allows the measuring of the unbound labelled dextran. This approach presents an advantage, compared with glucose oxidase sensors: the response is determined by the competitive equilibrium between glucose and the signal producing ligand. Thus, kinetics of enzyme reactions and electrode fouling do not affect the magnitude of the sensor response. Optimal specificity and sensitivity could be obtained by the selection of appropriate binding protein and competitive ligand; specific antibodies could be used, for instance. The sensor still suffers from limited stability and relatively long response times when employed as an in vivo sensor.

The concept of non-invasive glucose monitoring of the aqueous humour of the eye, by the measurement of the degree of optical rotation produced by the local concentration of glucose, has been advanced by March et al. (1979). The requirement of heavy optical equipment is an important drawback in terms of its development into a portable device.

Several endogenous enzymes that use glucose as the primary substrate might be utilized in an enzymatic glucose sensor. They include glucose dehydrogenase, glucokinase, glucose-6-phosphatase and glucose isomerase (Wingard 1983b). In the case of glucose dehydrogenase, NAD - NADH concentrations could be monitored using a miniature fibre-optic spectrometer. At the present time this system is still a theoretical speculation.

The last part of this chapter will deal will the glucose sensor as a part of a closed-loop insulin infusion system. The main characteristics of some implantable sensors are described in Table 22.3.

Table 22.3 Main characteristics of some implantable glucose sensors

\begin{tabular}{|c|c|c|c|c|}
\hline & \multicolumn{4}{|c|}{ Authors } \\
\hline & Bessman & Fischer & Kondo & Shichiri \\
\hline Type of detector & Galvanic cell & $\begin{array}{l}\mathrm{Pt} \text { anode/ } \\
\mathrm{H}_{2} \mathrm{O}_{2}\end{array}$ & $\mathrm{O}_{2}$ (Clark) & $\mathrm{Pt}$ anode $/ \mathrm{H}_{2} \mathrm{O}_{2}$ \\
\hline $\begin{array}{l}\text { Enzymatic } \\
\text { membrane material }\end{array}$ & Nylon & Sepharose & Nylon & $\begin{array}{l}\text { Cellulose } \\
\text { acetate }\end{array}$ \\
\hline $\begin{array}{l}\text { Immobilization } \\
\text { procedure }\end{array}$ & $\begin{array}{l}\text { Covalently } \\
\text { bound by } \\
\text { glutaraldehyde }\end{array}$ & $\begin{array}{l}\text { Covalently } \\
\text { bound by } \\
\text { cyanogen } \\
\text { bromide }\end{array}$ & $\begin{array}{l}\text { Covalently } \\
\text { bound by } \\
\text { glutaraldehyde }\end{array}$ & $\begin{array}{l}\text { Covalently } \\
\text { bound by } \\
\text { glutaraldehyde }\end{array}$ \\
\hline $\begin{array}{l}\text { Non-enzymatic ( } 1 \text { ) } \\
\text { membrane material }\end{array}$ & Polypropylene & $\begin{array}{l}\text { Celluilose } \\
\text { acetate }\end{array}$ & Polypropylene & Polyurethane \\
\hline $\begin{array}{l}\text { Non-enzymatic ( } 2 \text { ) } \\
\text { membrane material }\end{array}$ & & $\begin{array}{l}\text { Perforated } \\
\text { Teflon }\end{array}$ & $\begin{array}{l}\text { Perforated } \\
\text { Teflon }\end{array}$ & $\begin{array}{l}\text { Polyvinyl- } \\
\text { alcohol }\end{array}$ \\
\hline Sensor geometry & $\begin{array}{l}\text { Plane } \\
\text { geometry }\end{array}$ & $\begin{array}{l}\text { Plane } \\
\text { geometry }\end{array}$ & $\begin{array}{l}\text { Vessel- } \\
\text { shaped }\end{array}$ & $\begin{array}{l}\text { Needle- } \\
\text { shaped }\end{array}$ \\
\hline
\end{tabular}




\subsection{The artificial beta cell}

The earliest external electromechanical device used as a closed-loop insulin infusion system was described by Kadish (1964). Whenever the blood glucose exceeded $1.5 \mathrm{~g} / 1(8.33 \mathrm{mmol} / \mathrm{l})$ or fell under $0.5 \mathrm{~g} / \mathrm{l}(2.77 \mathrm{mmol} / \mathrm{l})$, insulin or glucagon, respectively, were infused. However this on-off system was not able to normalize the glycaemia. A resurgence of interest in the seventies for this bedside instrument, which became known as the artificial beta-cell, led to the refining of the feed-back controlled systems commanding the insulin delivery. Kadish's device was improved by Albisser et al. (1974) who subjected the control of insulin delivery to a computer-calculated predicted value based on the minute-to-minute variations of blood sugar. Clemens et al. (1977) constructed the first of these devices to be commercially available. It was named Biostator Glucose-Controlled Insulin Infusion System (60 kg, $42 \times 46 \times 46 \mathrm{~cm})$. A number of similar artificial beta cells, using extracorporeal glucose sensors, have been since then fabricated and evaluated by several groups, including Mirouze et al. (1977), Slama et al. (1977), Kraegen et al. (1979), Goriya et al. (1979) and Fischer et al (1980).

Bessman et al. (1977) reported the implantation into a diabetic dog of a small artificial beta cell consisting of an oxygen-detector glucose sensor, electronics, a micro-pump, and a power supply. The sensor was similar to the one previously described in Section 22.4 .1. The pump was a piezoelectric device separated from the insulin reservoir by a solenoid valve. Insulin was delivered into the peritoneal cavity when appropriately phased pulses were applied to the pump and valve. However, in this experiment, as well as in the observations on seven additional dogs (Bessman et al. 1981), the amount of insulin administered to the dogs was clearly insufficient, due to the inadequate response of the glucose sensor to the glucose levels.

A remarkable achievement in terms of miniaturization was reported by Shichiri et al, (1982) who developed a wearable closed-loop device ( $400 \mathrm{~g}$, $12 \times 15 \times 6 \mathrm{~cm})$ associated with an implantable needle-type sensor. Short term glycaemic control was achieved in diabetic patients connected to the instrument (Shichiri et al. 1984). These results are presented in Chapter 23 of this book.

\subsection{Conclusion}

Our understanding of the physiological, physicochemical, and electrochemical mechanisms underlying the basic requirements for an in vivo glucose sensor has expanded in recent years. The fruits of this understanding, in terms of technology, are beginning to be available. However, several questions remain unanswered and several answers are still not translated into practice. 
Concerning the sensor functioning under conditions of in vivo implantation, the optimal arrangement of the glucose oxidase support and the protective membranes has still to be found, allowing long-term enzymatic stability and adequate glucose and oxygen local concentrations with minimal tissue reaction. A better understanding of the operational properties of such sensors, both in vitro and in vivo, would allow their design and performance to be optimized.

Other approaches than the glucose oxidase sensor may prove to be worthwhile. The affinity type of sensor could be a promising alternative. Implantable sensors usually require a membrane barrier between the sensing element and the biological fluid. It is clear that the failure of such membranes to maintain reproducible analyte transport characteristics is a major cause of biosensor malfunction.

Finally, the expectations aroused by the development of a reliable sensor for long-term use in a portable closed-loop insulin infusion system justify the efforts being made in on-going studies. More easily attainable good glycaemic control in diabetic subjects could, hopefully, prove to be a major step in the prevention of the late complications of diabetes.

\section{Acknowledgements}

The support of the Caisse Nationale de l'Assurance Maladie des Travailleurs salariés (France Grant CNAMIS-INSERM 85. 3, 54. 8. E, of National Institute of Health (U.S.) Grant AM 30718, and of Association des Jeunes Diabétiques (Paris, France) are gratefully acknowledged. Furthermore, Dr Gilberto Velho was supported by a grant from C.N.Pq.

\section{References}

Albisser, A. M. and Spencer, W. J. (1982). Electronics and the diabetic. IEEE Trans Biomed Eng. 29, 239-48.

- Leibel, B. S., Ewart, G., Davidovac, Z,, Botz, C. K, and Zingg, W. (1974). An artificial endocrine pancreas. Diabetes 23, 389-96.

Bard, A. J, and Faulkner, L. R. (1980). Mass transfer by migration and diffusion. In Electrochemical methods, Fundamentals and applications (eds, A, J, Bard and L. R. Faulkner), pp. 119-35. Wiley, New York.

Barman, T.E. (1969). Glueose oxidase. In Enzyme handbook (ed. T. E. Barman), Vol. 1, pp. 112-113. Springer-Verlag, Berlin.

Bennett, P. H. (1983). Diabetes in developing countries and unusual populations. In Diabetes in epidemiological perspective (eds. J.I. Mann, K. Pyorala and A. Teuscher), pp. 43-57. Churchill Livingstone, Edinburgh.

Bessman, S. P. and Schultz, R. D. (1973). Prototype glucose-oxygen sensor for the artificial pancreas. Trans. Am. Soc. Artif. Intern. Organs, 19, 361-4. 
Hellyer, J.M., Layne, B. C., Takada, G., Thomas, L. J. Jr. and Sayler, D. (1977). The total implantation of an artificial $\beta$-cell in a dog: Progress report. Diabetes, Excerpta Medica-International Congress Series 413, 496-501,

- Thomas, L. J., Kojima, H., Sayler, D. F. and Layne E. C. (1981). The implantation of a closed loop artificial beta cell in dogs. Trans. Am. Soc. Artif. Intern. Organs, 27, 7-17.

Brennan, J. R., Gebhart, S. S. P. and Blackard, W. G. (1985), Pump-induced insulin aggregation: a problem with the Biostator. Diabetes 34, 353-9.

Calabrese, G., Bueti, A., Zega, G., Giombolini, A., Bellomo, G., Antonella, M. A., Massi-Benedetti, M. and Brunetti, P. (1982). Improvement of artificial endocrine pancreas (Biostator; GCIIS) performance combining feedback controled insulin administration with a pre-programmed insulin infusion. Horm. Metabol. Res. 14, 505-7.

Cass, A. E. G., Davis, G., Francis, G. D., Hill, H. A. O., Aston, W. J., Higgins, I. J., Plotkin, E. V., Scott, L. D. L. and Turner, A. P. F. (1984). Ferrocene-mediated enzyme electrode for amperometric determination of glucose. Anal. Chem. 56, $667-71$.

Clark, L. C. Jr. and Lyons, C. (1962). Electrode systems for continuous monitoring in cardiovascular surgery. Ann. N,Y, Acad. Sci. 102, 29-46.

Clemens, A.H., Chang, P.H. and Myers, R.W, (1977). The development of Biostator, a giucose controlled insulin infusion system (GCIIS). Horm. Metab. Res. suppl. 7: 23-33.

Coulet, P. R., Sternberg R. and Thévenot, D.R. (1980). Electrochemical study of reactions at interfaces of glucose oxidase collagen membranes. Biochim. Biophys. Acta 612, 317-27.

Dimitriadis, G. D. and Gerich, J. E. (1983). Importance of timing of preprandial subcutaneous insulin administration in the management of diabetes mellitus. Diabetes Care 6, 374-7.

Dubois, C. (1984). Caractérisation électrochimique des membranes utilisées dans les électrodes à enzymes, D.E.A. de Cinétique Chimique Appliquée. Université Pierre et Marie Curie, Paris.

Fischer, U. and Abel, P. (1982). A membrane combination for immplantable glucose sensors. Measurements in undiluted biological fluids. Trans. Am. Soc, Artif. Intern. Organs 28, 245-8.

- Jutzi, E., Bombor, H., Freyse, E. J., Salzsieder, E., Albrecht, G., Besch, W. and Bruns, W. (1980). Assessment of an algorithm for the artificial $\beta$-cell using the normal insulin-glucose relationship in diabetic dogs and men. Diabetologia 18, 97-107.

Gebhardt, U., Luft, G., Richter, G. J. and Von Sturm F. (1978). Development of an implantable electrocatalytic glucose sensor. Bioelectrochemistry and Bioenergetics 5, 607-24.

Goriya, Y., Kawamori, R., Shichiri, M. and Abe, H. (1979). The development of an artificial beta cell system and its validation in depancreatized dogs: the physiological restoration of blood glucose homeostasis. Med. Prog. Technol. 6, 99-108.

Guilbault, G. G. and Lubrano, G. J. (1973). An enzyme electrode for the amperometric determination of glucose. Anal. Chim. Acta. 64, 439-45. 
Hamman, R. F. (1983). Diabetes in affluent societies. In Diabetes in epidemiological perspective (eds. J.I. Mann, K. Pyorala and A. Teuscher), pp. 7-42. Churchill Livingstone, Edinburgh.

Harrison, D. E., Christie, M. R. and Gray, D. W. R. (1985), Properties of isolated human islets of Langerhans: insulin secretion, glucose oxidation and protein phosphorylation. Diabetologia 28, 99-103.

Horwitz, D. L., Zeidler, A., Gonen, B, and Jaspan, J. B. (1980). Hyperinsulinism complicating control of diabetes mellitus by an artificial beta cell. Diabetes Care 3, 274-7.

Ikeda, T., Hamada, H., Miki, K. and Senda, M. (1985). Glucose oxidase immobilized benzoquinone - carbon paste electrode as a glucose sensor. Agric. Biol. Chem. 49, 541-3.

Kadish, A. (1964). Automation control of blood sugar. A servomechanism for glucose monitoring and control. Am. J. Med. Electron, 3, 82-6.

Kamin, R. and Wilson, G.S. (1980). Rotating ring-disk enzyme electrode for biocatalysis kinetic studies and characterization of the immobilized enzyme layer. Anal. Chem. 52, 1198-205.

Kondo, T., Kojima, H., Ohkura, K., Ikeda, S. and Ito, K. (1981). Trial of new vessel access type glucose sensor for implantable artificial pancreas in vivo. Trans. Am. Soc, Artif. Intern. Organs, 27, 250-3.

Kraegen, E. W., Whiteside, R., Bell, D., Chia, Y.O. and Lazarus L. (1979). Development of a closed-loop artificial pancreas. Horm. Metab. Res. suppl. 8, $38-42$.

Lougheed, W. D., Woulfe-Flanagan, H., Clement, J. R. and Albisser, A. M. (1980). Insulin aggregation in artificial delivery systems. Diabetologia 19, 1-9.

March, W., Engerman, R. and Rabinovitch, B. (1979). Optical monitor of glucose. Trans. Am. Soc. Artif. Intern. Organs. 25, 28-31.

Mirouze J., Selarn J. L., Pham, T.C. and Cavadore, D. (1977). Evaluation of exogenous insulin homeostasis by the artificial pancreas in insulin dependent diabetes. Diabetologia 13, 273-8.

Nilson, H., Akerlind, A. C, and Mosbach, K. (1973). Determination of glucose, urea and penicillin using enzyme-pH electrodes Biochim. Biophys. Acta, 320, 529-34.

Racine, P. and Mindt, W. (1971). On the role of substrate diffusion in enzyme electrodes. Experientia suppl. 18, 524-34.

Report of the National Commission on Diabetes to the Congress of the United States (1976), U.S, Dep. Health, Educ., Welfare, Public Health Service, Nat, Inst. of Health, DHEW Publication No. (NIH) 76, 1021-8.

Richter, G. J., Luft, G. and Gebhardt, U. (1982). Development and present status of an electrocatalytic glucose sensor. Diabetes Care 5, 224-8.

Rizza, R.A., Gerich, J.E., Haymond, M. W., Westland, R. E., Hall, L. D., Clemens, A.H., and Service, F. J. (1980). Control of blood sugar in insulin dependent diabetes: comparison of an artificial endocrine pancreas, continuous subcutaneous insulin infusion, and intensified conventional insulin therapy. N. Engl. J. Med, 303, 1313-8.

Scheller, F., Janchen, M., Pfeiffer, D., Seyer, I, and Muller, K. (1977). Enzymelektrode zum Nachweis von Glucose. Z. Med. Labor. Diagn. 18, $312-16$. 
Schiller, J. G., Wingard, L. B. Jr. and Liu, C. C. (I982). Potentiometric detection of hydrogen peroxide and apparatus therefore. U.S. Patent 4,340,448.

Schultz, J.S., Mansouri, S. and Goldstein, I. J. (1982). Affinity sensors: a new technique for developing implantable sensors for glucose and other metabolites. Diabetes Care 5, 245-53.

Shichiri, M., Yamasaki, Y., Kawamori, R., Hakui, N. and Abe, H. (1982). Wearable artificial endocrine pancreas with needle-type glucose sensor, Lancet 2, 1129-31.

- Kawamori, R., Hakui, N., Yamasaki, Y. and Abe, H. (1984). Closed-loop glycaemic control with a wearable artificial endocrine pancreas. Variation in daily insulin requirements to glycaemic responses. Diabetes 33, 1200-2.

Slama, G., Klein, J. C., Tardieu, M. C. and Tchobroutsky, G. (1977). Normalisation de la glycémie par pancréas artificial non miniaturisé, Application pendant 24 heures chez 7 diabétiques insulino-dépendants. Nouv. Presse Med. 6, 2309-15.

Soeldner, J.S., Chang, K. W., Aisenberg, S. and Hiebert, I. M. (1973). Progress towards an implantable glucose sensor and an artificial beta cell. In Temporal aspects of therapeutics (eds. J. Urquhart and F. E. Yates), pp. 181-207. Plenum Press, New York-London.

Sorensen, J. T., Colton, C. K., Hillman, R.S, and Soeldner, J. S. (1982). Use of a physiologic pharmacokinetic model of glucose homeostasis for assessment of performance requirements for improved insulin therapies. Diabetes Care $\mathbf{5}$, 148-57.

Tchobroutsky, G. (1978). Relation of diabetic control to development of microvascular complications. Diabetologia 15, 143-52.

Thévenot, D. R. (1982). Problems in adapting a glucose oxidase electrochemical sensor into an implantable glucose-sensing device. Diabetes Care 5, 184-9.

- Coulet, P. R., Sternberg, R. and Gautheron, D. C. (1978). A highly sensitive glucose electrode using glucose oxidase collagen film. Bioelectrochem. Bioenerg. 5, 548-53.

Updike, S. J. and Hicks, G. P. (1967). The enzyme electrode. Nature 214, 986-8.

Wightman, R. M. (1981). Microvoltametric electrodes. Anal. Chem. 53, 1125-34 A.

Wingard, L. B. Jr. (1982). Possibility for an immobilized flavin fuel cell electrode for glucose measurement. Diabeles Care 5, 222-3.

- (1983a). Prospects for electrochemical devices and processes based on biotechnology. In Biotech 83 pp. 613-24. Online Publications Ltd. Northwood, UK.

(1983b). Immobilized enzyme electrodes for glucose determination for the artificial pancreas. Federation Proc. 42, 288-291.

— Liu, C. C., Wolfson, S. K., Yao, S. J. and Drash, A. L. (1982). Potentiometric measurement of glucose concentration with an immobilized glucose oxidase/catalase electrode. Diabetes Care 5, 199-202.

_ Schiller, J. G., Wolfson, S. K., Liu, C. C., Drash, A. L.and Yao, S. J. (1979). Immobilized enzyme electrodes for the potentiometric measurement of glucose concentration: immobilization techniques and materials. J. Biomed. Mater. Res. 13, 921-35.

Woodward, S, C. (1982). How fibroblasts and giant cells encapsulate implants: - considerations in design of glucose sensors. Diabetes Care 5, 278-81.

Yellow Spring Instruments Co. (1975). Instruction manual Y.S.I. model 23 A. 


\title{
23
}

\section{Needle-type glucose sensor and its clinical applications}

\author{
MOTOAKI SHICHIRI, RYUZO KAWAMORI, and \\ YOSHIMITSU YAMASAKI
}

\subsection{Introduction}

Several types of glucose sensors have been proposed, however, only a few have been applied to in vivo clinical use. Chang et al. (1972) proposed a discshaped electrochemical (non-enzymatic) glucose sensor. They reported that on the 117th day of implantation in the subcutaneous tissue of a Rhesus monkey the glucose electrode produced a signal which correlated significantly with corresponding blood sugar levels following intravenous glucose administration (Soeldner et al. 1976). However, since some other electrochemically active species influence the sensor output, this sensor has not yet been applied to in vivo monitoring of human subjects.

In contrast, glucose sensors using glucose oxidase (Updike and Hicks 1967; Guilbault and Lubrano 1973) have been used for in vitro and in vivo monitoring because of their specificity to glucose and precision in glucose determination. Bessman et al. first reported an implantable glucose sensor consisting of two galvanic oxygen electrodes, which was incorporated into an implantable closed-loop artificial beta cell (Bessman et al. 1981). They reported that all of these units had functioned fairly well but none had brought the animal under complete control, partially because the glucose sensors were insensitive to the tissue glucose concentration, giving about half of the expected level. There have been no reports on human monitoring by this type of glucose sensor.

The authors have developed a needle-type glucose sensor which retained in vitro and in vivo characteristics suitable for tissue glucose monitoring (Shichiri et al. 1982, 1983). By applying the glucose sensor as a glucose monitoring device, a wearable artificial endocrine pancreas system enabled closedloop glycaemic regulation in diabetic patients for more than six days (Shichiri et al. 1984).

\subsection{The principle of glucose measurement by an intracorporeal glucose sensor}

In the presence of glucose and oxygen, the glucose oxidase used in enzymatic glucose sensors catalyses the oxidation of glucose and produces gluconic acid 
and hydrogen peroxide. Because the physiological concentration of oxygen in blood or tissue fluid is much lower (Bartlett and Tenney 1963) than the $K_{\mathrm{M}}$ values of the enzyme (Gibson et al. 1964), not only glucose concentration but also oxygen tension may regulate the rate of glucose oxidation. Therefore, when a glucose sensor is implanted, output of the sensor might be nonlinearly proportional to glucose concentration (Bessman et al. 1981). In order to solve this problem, a membrane which is more permeable to oxygen than to glucose is useful (Ikeda et al. 1980; Yamasaki 1984) because it limits delivery of glucose to the enzyme layer of the sensor. Thus the output of the sensor with such a membrane shows linearity over a wide range of glucose concentrations and insensitivity to fluctuation of oxygen tension.

Concerning the host response to a sensor, the size and surface configurations of the intracorporeal device are also important. Woodward (1982)

Table 23.1 Enzymatic glucose sensors used in in vivo monitoring

\begin{tabular}{|c|c|c|c|}
\hline & California* & Nagoya** & Osaka*** \\
\hline Shape & Disc & $\begin{array}{l}\text { Venous } \\
\text { access-type }\end{array}$ & Needle-type \\
\hline Size & $\begin{array}{l}\text { Diameter } 20 \mathrm{~mm} \\
\text { Depth } 2 \mathrm{~mm}\end{array}$ & - & $\begin{array}{l}\text { Diameter } 0.4-0.8 \mathrm{~mm} \\
\text { Length } 20 \mathrm{~mm}\end{array}$ \\
\hline $\begin{array}{l}\text { Semi-permeable } \\
\text { membrane }\end{array}$ & - & Polypropylene & Polyurethane \\
\hline $\begin{array}{l}\text { Enzyme-bound } \\
\text { membrane }\end{array}$ & Nylon & $\begin{array}{l}\text { Millipore } \\
\text { nylon filter }\end{array}$ & $\begin{array}{l}\text { Cellulose } \\
\text { diacetate }\end{array}$ \\
\hline $\begin{array}{l}\text { Gas-permeable } \\
\text { membrane }\end{array}$ & Polypropylene & Teflon & - \\
\hline Determinant & Oxygen & Oxygen & Hydrogen peroxide \\
\hline Anode & $\mathrm{Pb}$ & $\mathrm{Ag}$ & $\mathrm{Pt}$ \\
\hline Cathode & $\mathrm{Ag}$ & $\mathrm{Pt}$ & $\mathrm{Ag}$ \\
\hline Electrolyte & $\mathrm{KOH}$ & $\mathrm{NaCl}$ & Body fluid \\
\hline $\begin{array}{l}\text { Response to glucose } \\
\text { concentration }(\mathrm{mmol} / \mathrm{l})\end{array}$ & $0-8.3$ & $0-38.5$ & $0-27.5$ \\
\hline Implanted site & $\mathrm{se}$ tissue & Blood vessel & $\begin{array}{l}\text { Blood vessel, } \\
\text { sc tissue }\end{array}$ \\
\hline In vivo monitoring & Rabbit, dog & Dog, human & Dog, human \\
\hline Control experiment & $\begin{array}{l}\text { Stz-diabetic } \\
\text { dog }\end{array}$ & Px dog & $\begin{array}{l}\text { Px dog, } \\
\text { human diabetics }\end{array}$ \\
\hline
\end{tabular}

Abbreviations; stz (streptozotocine), px (pancreatectomized), sc (subcutaneous)

* Layne et al. 1976; Bessman et al. 1981

** Ikeda et al. 1980

*** Shichiri et al. 1982; Shichiri et al. 1983; Shichiri et al, 1984 
suggested that if the sensor could be fabricated in the form of a wire or filament measuring less than about $2 \mathrm{~mm}$ in diameter, a minimal host response would be evoked. Therefore, a miniature needle shape is one of the ideal designs for an indwelling glucose sensor as opposed to a disc shape.

The structure and membrane design of the intracorporeal glucose sensors reported are listed in Table 23.1 along with their in vitro characteristics.

\subsection{Preparation of a needle-type glucose sensor}

A hydrogen peroxide electrode is prepared according to the method described by Hagihara et al (1981) modified as follows. The tip of a platinum wire (diameter $0.2 \mathrm{~mm}$, length $4 \mathrm{~cm}$ ) is melted in an oxygen natural gas flame to form a small bulb (diameter $0.3-0.7 \mathrm{~mm}$ ), then it is sealed into a soft glass capillary by melting also in an oxygen natural gas flame. Then the tip of the electrode is polished with fine sandpaper (No. 2000) until the platinum surface (anode) is uncovered. The platinum-glass anode is inserted into a silver-plated stainless-steel tube (inner diameter $0.4 \mathrm{~mm}$, length $2 \mathrm{~cm}$ ) as the cathode of the electrode and fixed tightly by heating in an oxygen gas flame.

The electrode tip is dipped into $1 \%$ cellulose diacetate solution (Eastman Kodak Co., USA) dissolved in $50 \%$ acetone- $50 \%$ ethanol solution for $5 \mathrm{~s}$, and then exposed to acetone vapour for $5 \mathrm{~min}$. These procedures are repeated twice. The tip is then dipped into $2.5 \%$ cellulose diacetate solution dissolved in $50 \%$ acetone $-50 \%$ ethanol solution for $30 \mathrm{~s}$. Then, $0.2 \mu \mathrm{l}$ of glucose oxidase solution, in which $50 \mathrm{mg}$ of glucose oxidase (from Aspergillus niger, type II, $17300 \mathrm{U} / \mathrm{g}$, Sigma Chemical, Co., USA) is dissolved in $1 \mathrm{ml}$ of distilled water, is dropped onto the electrode tip, the dipped end being kept upwards. For the immobilization of glucose oxidase, $0.1 \mu \mathrm{l}$ of $2 \%$ glutaraldehyde solution (Wako Pure Chemical Industries, Ltd., Japan) is dropped onto the electrode tip. The electrode is kept in air for $2 \mathrm{hr}$ at $25^{\circ} \mathrm{C}$ and then is exposed to acetone vapour for $5 \mathrm{~min}$ at $25{ }^{\circ} \mathrm{C}$. The tip is dipped into $2 \%$ polyurethane (Japan Erastran Co., Japan) dissolved in tetrahydrofuran (Wako Pure Chemical Industries, Ltd., Japan) for $2 \mathrm{~s}$ followed by drying in air. Then, the tip is dipped into $15 \%$ polyurethane in $50 \%$ tetrahydrofuran-50\% dimethylformamide (Wako Pure Chemical Industries Ltd., Japan) for another $10 \mathrm{~s}$. Finally the needle-type glucose sensor (Fig. 23.1) thus prepared is stored in the refrigerator until it is used.

\subsection{In vitro characteristics of the glucose sensor}

\subsubsection{Procedure for determining in vitro characteristics}

A needle-type glucose sensor polarized at a voltage of $+0.6 \mathrm{~V}$ is connected to the current-voltage converting amplifier (POG-200A, Unique Medical Co., 


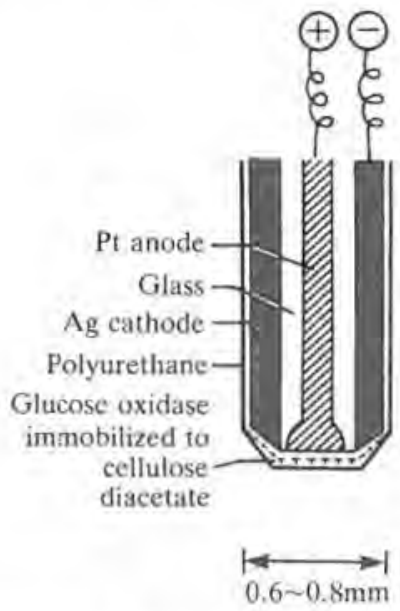

Fig. 23.1 Structure of a needle-type glucose sensor.

Ltd., Japan), which amplifies current of $1 \mathrm{nA}$ to a voltage of $100 \mathrm{mV}$. A pen recorder (VP6621A, Matsushita Communication Industrial Co., Ltd., Japan) is connected to record sensor outputs.

The in vitro characteristics of the sensor are tested in $0.9 \% \mathrm{NaCl}$ solution containing 7\% bovine albumin (Fraction V, Miles, USA) with varying glucose concentrations in a temperature-, flow-rate-, and oxygen-tensioncontrollable chamber. The output current of the sensor is calibrated initially after a stabilization period of at least $10 \mathrm{~min}$.

\subsubsection{Drift and noise range of measurement}

The drift of the base line and noise range of the sensor are expressed as a percentage change of the sensor output in response to $5.5 \mathrm{mmol} / 1$ glucose solution. The base-line drift was $0.8 \pm 0.3 \%$ per $24 \mathrm{hr}$ and the noise range was $0.3 \pm 0.4 \%$. The residual current against glucose free saline solution was 1.3 $\pm 0.6 \%$ (Table 23.2).

\subsubsection{Dose response against glucose}

The dose response pattern and rapidity of the sensor output in response to the alteration in glucose concentration were measured by infusing solutions with $0-27.5 \mathrm{mmol} / 1$ glucose at $37^{\circ} \mathrm{C}$. The sensor output responded well to the changes in glucose concentrations. The rapidity in response shown by $T_{90 \%}$ was $16.2 \pm 6.2 \mathrm{~s}$. Linear response was obtained in the range $0-27.5 \mathrm{mmol} / 1$.

\subsubsection{Effect of temperature and oxygen tension}

The temperature coefficient measured by changing the temperature of the solution from $33{ }^{\circ} \mathrm{C}$ to $42{ }^{\circ} \mathrm{C}$ was $2.3 \pm 1.0 \% / 1{ }^{\circ} \mathrm{C}$. The current 
Table 23.2 Typical characteristics of a needle-type glucose sensor in vitro

\begin{tabular}{lc}
\hline Test & Performance \\
\hline Residual current $(\%)$ & $1.3 \pm 0.6$ \\
Baseline drift $(\% / 24 \mathrm{hr})$ & $0.8 \pm 1.3$ \\
Noise range $(\%)$ & $0.3 \pm 0.4$ \\
Output generated to 5.5 mmol/4 glucose $(\mathrm{nA})$ & $1.2 \pm 0.4$ \\
Range of glucose concentrations producing & $0-27.5$ \\
a linear dose-response pattern $($ mmol/1) & $16.2 \pm 6.2$ \\
T $_{90 \%}$ response time $(\mathrm{s})$ & $2.3 \pm 1.0$ \\
Temperature coefficient $\left(\% / 1^{\circ} \mathrm{C}\right)$ & \\
\hline
\end{tabular}

Results are shown as mean \pm SD for 15 sensors. The performance is expressed as percentage change of the output at $5.5 \mathrm{mmol} / 1$ glucose.

dependency of oxygen tension was checked by admitting varying oxygen/nitrogen gas mixtures to the solution and by monitoring the oxygen tension $(15-150 \mathrm{~mm} \mathrm{Hg})$ with an oxygen sensor. The output current in response to $5.5 \mathrm{mmol} / \mathrm{l}$ of glucose concentration increased only by $0.1 \%$ per $1 \mathrm{~mm} \mathrm{Hg}$.

\subsubsection{Life expectancy}

The life expectancy of the glucose sensor was examined in the chamber by continuous recirculation of a solution containing glucose of $5.5 \mathrm{mmol} / \mathrm{A}$ at $37^{\circ} \mathrm{C}$. Each sensor was equilibrated in this solution for $2 \mathrm{hr}$ and output currents were continuously recorded for 7 days without calibration. During continuous monitoring in vitro, the output current gradually decreased to $76.2 \pm 6.9 \%$ of the initial value at 7 days after the initiation of the monitoring.

\subsection{In vivo characteristics of the glucose sensor}

\subsection{Procedure for determining in vivo characteristics}

For in vivo monitoring, a needle-type glucose sensor is connected to a current-voltage converting amplifier device which was constructed by using a CMOS operational amplifier (ICU 7613, Intersil Inc., USA). A polarizing voltage in the glucose sensor is supplied by a lithium battery built in the device. The pen recorder is connected to the amplifier to monitor sensor outputs.

Each sensor's output is calibrated with a standard glucose solution in which $11 \mathrm{mmol}$ of glucose is dissolved in $100 \mathrm{ml}$ of sterilized $0.9 \% \mathrm{NaCl}$ solution maintained at $37^{\circ} \mathrm{C}$. Then, a glucose sensor is inserted by means of an 
indwelling needle (gauge no. 18) into the jugular vein or subcutaneous tissue of healthy and diabetic dogs, or into subcutaneous tissue of the forearm in healthy and diabetic volunteers. The sensor output is compared with blood glucose concentrations simultaneously measured by a bedside-type artificial endocrine pancreas system (Shichiri et al. 1979; Kawamori et al. 1980).

\subsubsection{Noise range of in vivo measurement}

The noise range of in vivo monitoring with the sensor inserted into subcutaneous tissue in generally anesthetized and unanesthetized normal dogs was $1.3 \pm 0.5 \%(n=5)$, and $3.1 \pm 0.8 \%(n=5)$, respectively. Strenuous muscular exercise in dogs produced noise in the range up to $13.4 \%$ of output.

\subsubsection{Response of the sensor to blood glucose}

The outputs of the sensor when kept in the jugular vein of dogs $(Y)$ was related to the results of intravenous glucose monitoring by the bedside-type of artificial endocrine pancreas system $(X)(Y=0.98 X+2, r=0.998$, $n=92$ ). A significant relationship also existed between the glucose concentrations obtained by the needle-type glucose sensors in subcutaneous tissue $(Y)$ and the blood glucose concentrations $(X)$ determined by the bedside-type monitoring system in dogs $(Y=0.85 X+3, r=0.956, n=144)$. In human volunteers, a high correlation $(Y=0.79 X+17, r=0.96, n=115)$ was also observed (Fig. 23.2).

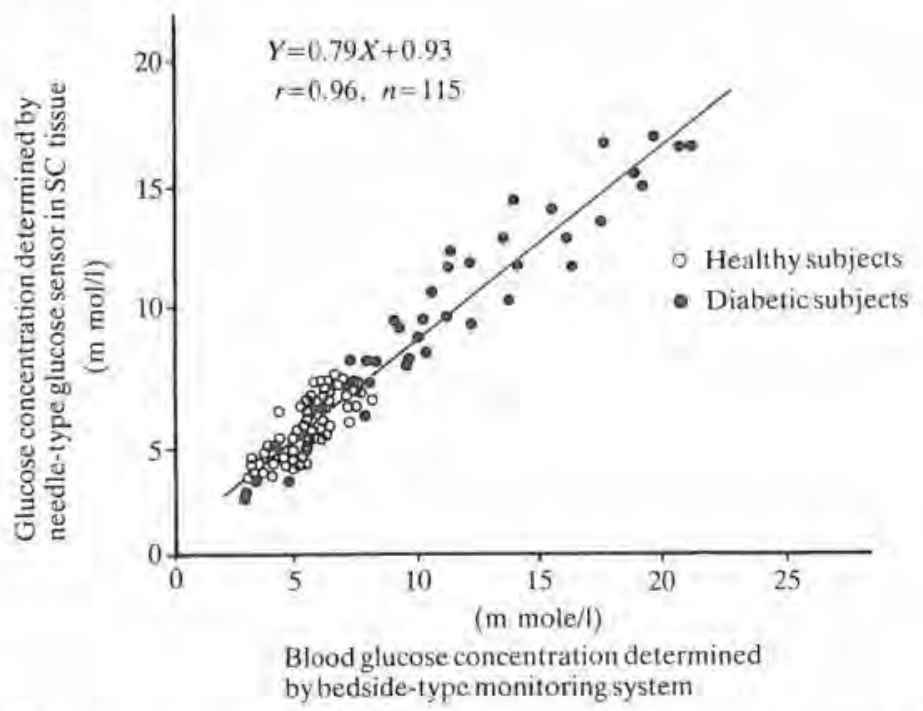

Fig. 23,2 The relationship between glucose concentration determined by a needletype glucose sensor inserted into subcutaneous tissue and blood glucose concentration determined by a bedside-type artificial endocrine pancreas in normal and diabetic volunteers. 


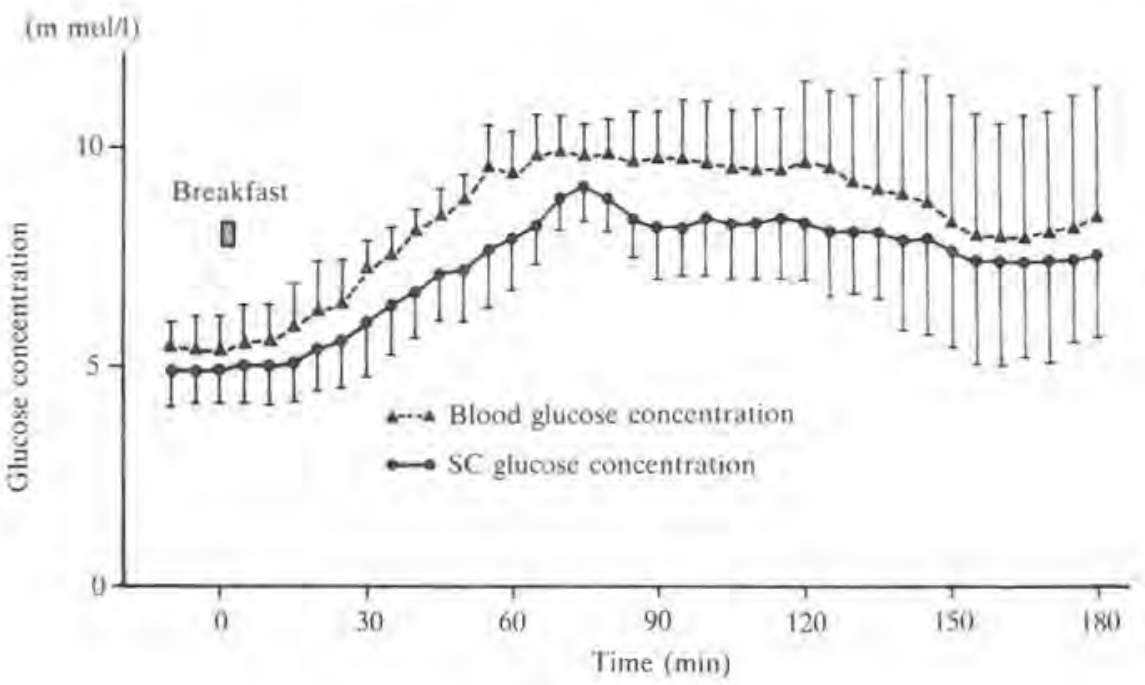

Fig. 23.3 Postprandial subcutaneous glucose concentration determined by a needletype glucose sensor and blood glucose concentration determined by a bedside-type artificial endocrine pancreas in human diabetics $(n=5)$. Data are shown as mean \pm SD.

\subsubsection{Response of the sensor to the change in blood glucose}

In order to check the sensor response to a change in blood glucose concentration, both subcutaneous glucose concentration and plasma glucose concentration were monitored in normal volunteers to whom glucose was intravenously delivered at a dose of $0.55 \mathrm{mmol} \mathrm{kg-1} \mathrm{min}^{-1}$ for $30 \mathrm{~min}$. The subcutaneous glucose concentration started to rise 5-10 min after the rise in the plasma glucose concentration. Also, the subcutaneous glucose concentration showed a peak 5 min later (Fig. 23.3).

\subsubsection{In vivo effect of oxygen tension}

In normal dogs, a reference oxygen electrode was also inserted into the subcutaneous tissue $2-3 \mathrm{~cm}$ away from the glucose sensor to monitor background oxygen tension in the subcutaneous tissue. After monitoring the base line for more than $30 \mathrm{~min}$, the dogs inhaled $100 \%$ nitrogen gas or $95 \%$ oxygen plus $5 \%$ carbon dioxide gas. The reference oxygen electrode showed fluctuations in subcutaneous tissue oxygen tension in the range of $26-50 \mathrm{~mm} \mathrm{Hg}$. However, the glucose sensor showed stable output regardless of oxygen tension changes and the output was consistent with monitored blood glucose concentrations (Fig. 23,4). 


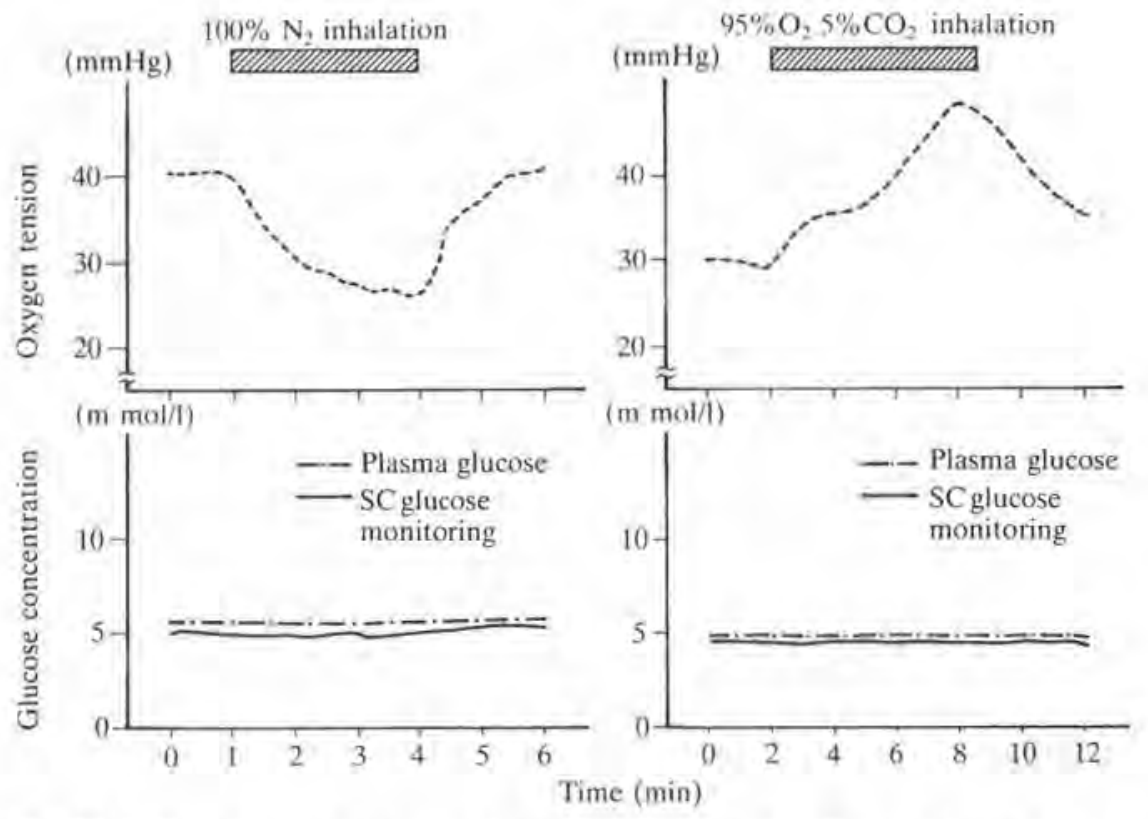

Fig. 23.4 Tissue glucose concentrations monitored by a needle-type glucose sensor. The dog inhaled $100 \% \mathrm{~N}_{2}$ gas for 3 min (left panel) or $95 \% \mathrm{O}_{2}$ plus $5 \% \mathrm{CO}_{2}$ gas for 6 min (right panel). Plasma glucose concentrations were measured by discrete samplings. Tissue oxygen tension was monitored by a needle-type oxygen electrode.

\subsubsection{Life expectancy on an in vivo basis}

Changes in in vitro characteristics during continuous monitoring cannot be determined on the sensor implanted in subcutaneous tissue. Therefore, to estimate the in vivo sensor characteristics and the possible tissue reaction against sensor implantation, both 'relative' output current and 'relative' response time of the sensor are determined as follows: 'relative' output of the sensor kept in subcutaneous tissue for three days is calculated by comparing the sensor's output with simultaneously monitored blood glucose concentration. 'Relative' response time of the sensor is determined as the time lag between the rise in blood glucose and the rise in sensor's output after meal intake. After three days, the 'relative' output decreased to $73.5 \%$ of the initial level and the 'relative' response time increased from $5.1 \mathrm{~min}$ to $13.5 \mathrm{~min}$. On the contrary, in vitro characteristics of the sensor determined after removal showed at $23 \%$ reduction in output and a $14 \mathrm{~s}$ delay in response (Table 23.3). Thus, reduction in in vitro characteristics cannot completely account for the reduction in the performance of the implanted sensor. Reduction in perfusion flow rate of interstitial fluid during the sensor's 
Table 23.3 'Relative' output current and 'relative' response time of sensors inserted into subcutaneous tissue during continuous monitoring

In vitro characteristics

Before application $\quad 3$ days after application

Residual current

(nA)

$1,0 \pm 0.4$

$1.4 \pm 1.2$

Output current generated to

$2.2 \pm 0.5$

$1.7 \pm 0.1$

$5.5 \mathrm{mmol} / \mathrm{Iglucose}$

(nA)

$\mathrm{T}_{90 \% \mathrm{O}}$

(s)

$29 \pm 6$

$43 \pm 6$

In vivo characteristics

\begin{tabular}{|c|c|c|}
\hline 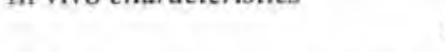 & Just after application & 3 days after application \\
\hline 'Relative' output current* $(\%)$ & 100 & $74 \pm 3$ \\
\hline 'Relative' response time ${ }^{* *}(\mathrm{~min})$ & $5.1 \pm 2.2$ & $13.5 \pm 1.5$ \\
\hline
\end{tabular}

Results are shown as mean $\pm \mathrm{SD}(n=5)$.

Sensors used in in vivo monitoring had a different rot number from that of the sensors examined in in vitro basis (Table 23.2).

- 'Relative' output current of the sensor kept in subcutaneous tissue for 3 days was calculated by comparing the sensor's output with blood glucose concentration.

** 'Relative' response time was determined as the time lag between the rise in blood glucose and the rise in sensor's output after meal intake.

implantation might result partially in the decrease in performance of implanted sensor.

\subsubsection{Scanning electron-microscope examination of the sensor's surface}

Scanning electron-microscope examinations were carried out on glucose sensors kept in subcutaneous tissue of normal dogs for 3, 7, and 14 days. Figure 23.5 shows one example of the scanning electron-microscope examinations of the membrane of the sensor. After a three-day continuous use in the subcutaneous tissue, a slight fixation of protein was observed and small pits were noted on the surface of the membrane. After seven and fourteen days of continuous use, the membrane was heavily coated with protein but the small pits on the surface were not observed. However, in these situations, fixation of fibroblasts and giant cells was not demonstrated on the surface.

Histologic changes in subcutaneous tissue around the sensor insertion area were examined in normal dogs. After a three-day application, migration of leukocytes and slight fibrin deposition was recognized in the insertion area.

\subsection{In vivo monitoring}

\subsubsection{Telemetry glucose monitoring system}

Because of the in vivo characteristics of the sensor, such as close linearity of 
sensor output to blood glucose concentration, responsiveness to glycaemic change, and long-lived stability of output, a needle-type glucose sensor is quite useful for in vivo monitoring. For this purpose, a telemetry glucose monitoring system has been constructed using a needle-type glucose sensor.

The system consists of a glucose sensor-transmitter and a receiver. The transmitter converts a current signal generated by a sensor to a very high frequency (VHF) audio signal. The small enclosure is packed with a current-voltage converting amplifier (ICU 7613, Intersil, Inc., USA), a voltage-frequency converter, and a lithium battery, is $4 \times 6 \times 2 \mathrm{~cm}$ and weighs $50 \mathrm{~g}$. The receiver demodulates the audio-frequency signal received to a voltage, and the glucose concentration calculated from the voltage is continuously displayed on the LED display. Hyperglycaemia or hypoglycaemia beyond the pre-fixed threshold sets off an alarm. This device composed of a VHF oscillator and batteries is $10 \times 12 \times 5 \mathrm{~cm}$. The receiver can detect the sensor signals from a distance of $20 \mathrm{~m}$ from the transmitter.

\subsubsection{Procedure for in vivo monitoring using a telemetry monitoring system}

After the calibration using sterilized saline solution with and without glucose ( $5.5 \mathrm{mmol} / \mathrm{l})$, a glucose sensor was inserted into subcutaneous tissue of the

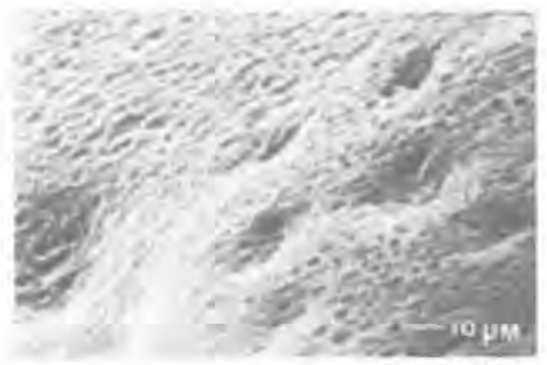

Before

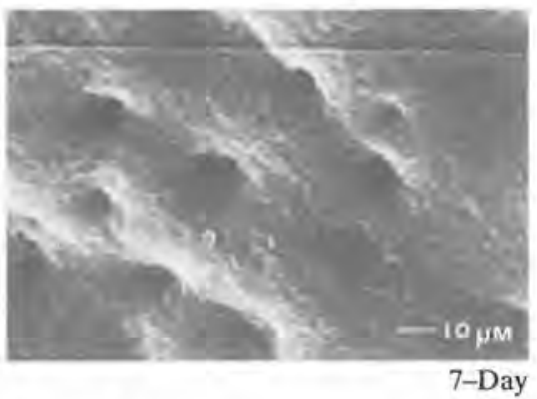

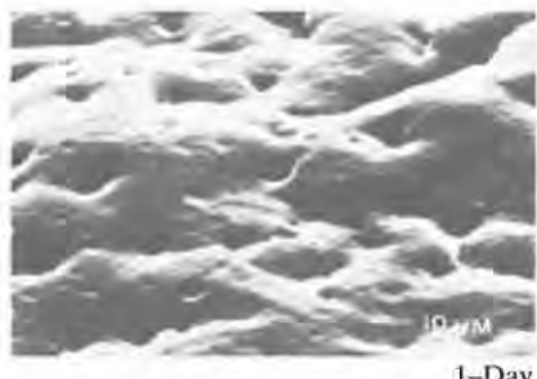

1-Day

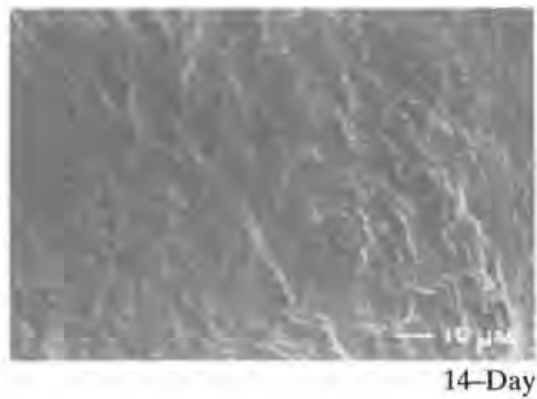

Fig. 23.5 Scanning electron-microscope examinations of glucose sensors kept in subcutaneous tissue of normal dogs before or after 3,7, and 14 days of implantation. 


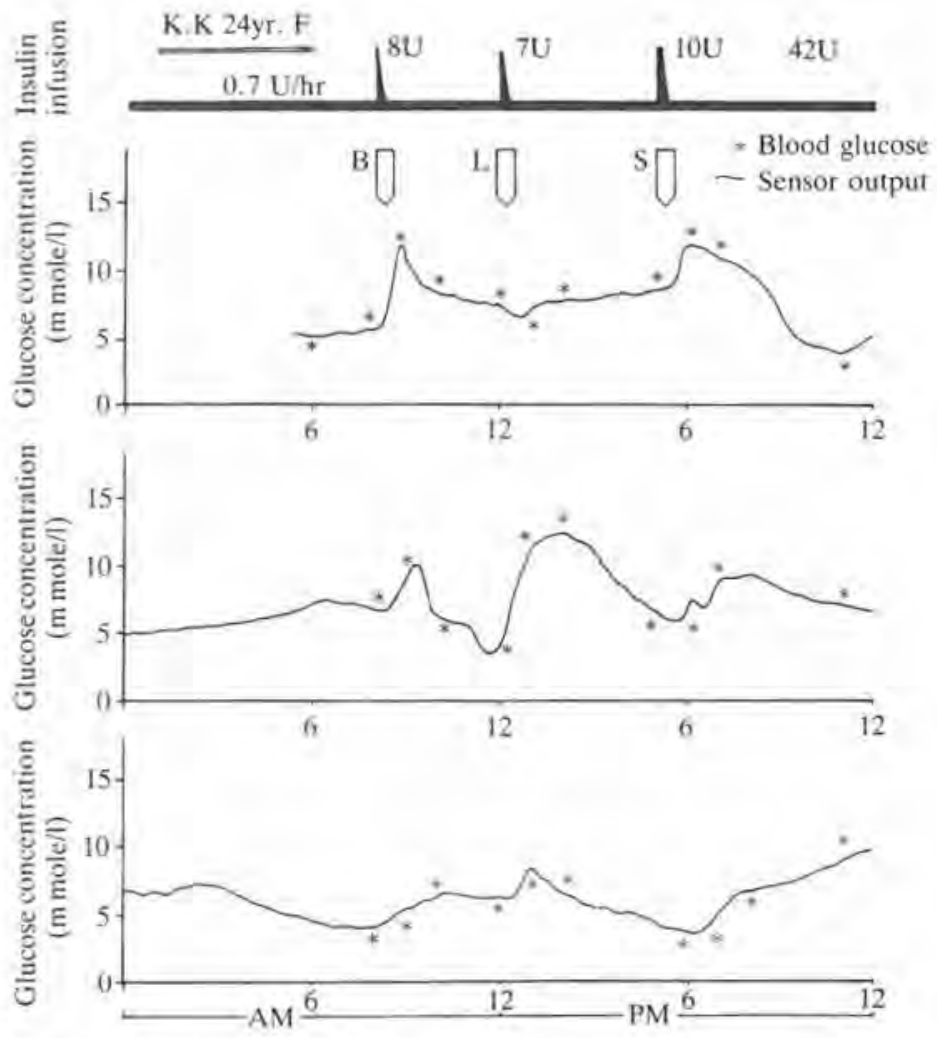

Fig. 23.6 Three days' continuous glycaemic monitoring using a telemetry glucose monitoring system in an insulin-dependent diabetic patient treated by continuous subcutaneous insulin infusion. B, L, and S denote breakfast, lunch, and supper, respectively. Asterisks indicate blood glucose concentrations determined by discrete samplings. The pattern of subcutaneous insulin infusion by the open-loop system is also depicted.

forearm of diabetic subjects by means of an indwelling needle (gauge no. 18). The sensor was fixed in situ with an adhesive bandage. In some patients, the sensor was replaced with a new one after three days' continuous monitoring. The transmitter was fixed to the forearm or was anchored to a waist belt.

\subsubsection{In vivo monitoring}

Figure 23.6 shows one representative case of three days' continuous record of an insulin-dependent diabetic treated with continuous subcutaneous insulin infusion. The continuous monitoring of glucose concentration disclosed a day-by-day variation of glycaemia in diabetics. The subcutaneous glucose 


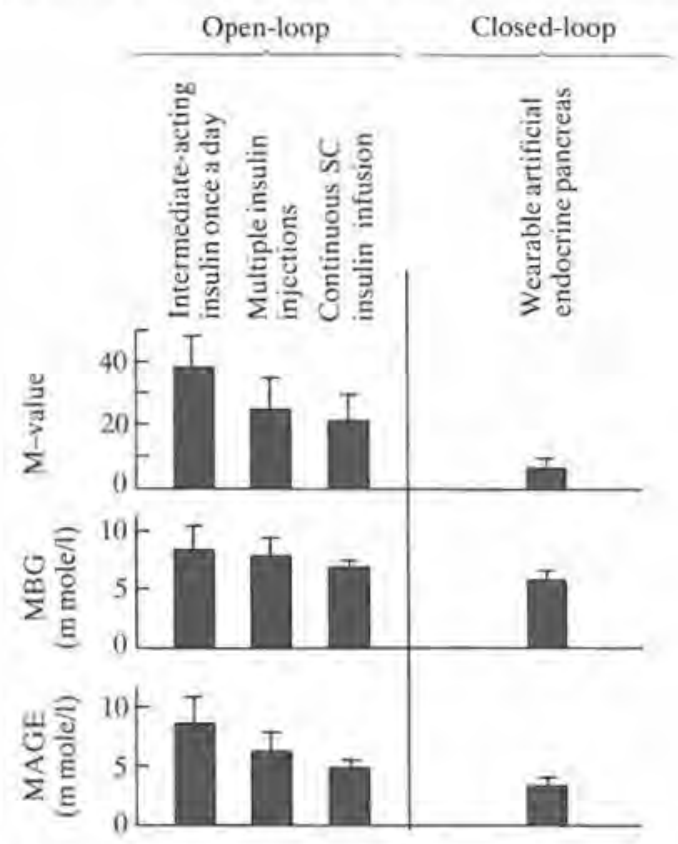

Fig. 23.7 Blood glucose regulatory indices in five insulin-dependent diabetic subjects controlled with intermediate-acting insulin injection once a day, multiple insulin injections, continuous subcutaneous insulin infusion, and wearable artificial endocrine pancreas.

concentrations determined by the sensor were consistent with the plasma glucose concentration. Results of daily glycaemic excursion demonstrated by the telemetry glucose monitoring system in diabetics treated with several insulin regimens are shown in Fig, 23.7

\subsection{Application of a closed-loop glycaemic control system}

\subsubsection{Wearable artificial endocrine pancreas}

A needle-type glucose sensor provides sensor characteristics suitable for application to a closed-loop control system and allows wearability at the same time. Thus, the author developed a wearable artificial endocrine pancreas, which consists of a needle-type glucose sensor, a microcomputer system, two syringe delivery units for insulin and glucagon infusions, and lithium batteries. The total system was packed into a small unit $(12 \times 15 \times$ $6 \mathrm{~cm}$ ) weighing $400 \mathrm{~g}$. 


\subsubsection{Computer algorithm for closed-loop insulin and glucagon infusion}

Intravenous insulin and glucagon infusion algorithms in this system are the same as those of a bedside type (Shichiri et al. 1979; Kawamori et al. 1980).

Insulin infusion rate, $\operatorname{IIR}(t)\left(\mathrm{mU} \mathrm{kg}^{-1} \mathrm{~min}^{-1}\right)$ is expressed as follows:

$$
\operatorname{IIR}(t)=K_{\mathrm{p}} \cdot \mathrm{BG}(t)+K_{\mathrm{d}} \cdot \Delta \mathrm{BG}(t)+K_{\mathrm{c}}
$$

where, $\mathrm{BG}(t)$ and $\Delta \mathrm{BG}(t)$ are blood glucose concentration $(\mathrm{mmol} / \mathrm{l})$ and its rate of change ( $\left.\mathrm{mmol}^{-1} \min ^{-1}\right)$ at time $t$, respectively, and $K_{\mathrm{p}}$, and $K_{\mathrm{d}}$ are coefficients for proportional and derivative action, respectively, and $K_{c}$ is a constant for basal insulin supplementation. By selecting proper parameters, $\left(K_{\mathrm{p}}=0.51, K_{\mathrm{d}}=4.89, K_{\mathrm{c}}=-2.02\right)$ this algorithm was proven to establish perfect glycaemic control with physiological insulinaemia.

Glucagon infusion rate, $\operatorname{GIR}(t)\left(\mathrm{ng} \mathrm{kg} \mathrm{min}^{-1}\right)$ is expressed as follows;

$$
\operatorname{GIR}(t)=G_{p} \cdot\left(\mathrm{BG}_{p}-\mathrm{BG}(t-\tau)\right)+\mathrm{G}_{\mathrm{d}} \cdot(-\Delta \mathrm{BG}(t-\tau))+G_{c}
$$

where, $\mathrm{BG}_{\mathrm{p}}$ is the projected value of blood glucose concentration set as $4.4 \mathrm{mmol} / \mathrm{l}$ and $\tau$ is the delay time for initiation of glucagon infusion, $G_{\mathrm{p}}$ and $G_{\mathrm{d}}$ are coefficients for proportional and derivative actions, respectively, and $G_{\mathrm{c}}$ is a constant for basal glucagon supplementation.

\subsubsection{Noise reduction}

The current generated by the glucose sensor is so small that noise can interfere with the output. Thus, hardware and software noise filters are built into the system. Because the sensor's signal is a direct current, low and high pass filters are effective in eliminating noises of low and high frequency waves and are used as a hardware noise filter. As a software noise filter, the computer algorithm has several program steps for noise reduction as follows: The computer calculates an average of ten samples of output current obtained every ten microseconds then the computer rejects a new data point when it shows a greater deviation from the previous I-min of data than a pre-fixed threshold.

\subsubsection{Procedure for closed-loop glycaemic control with a wearable artificial endocrine pancreas}

Glycaemic control in insulin-dependent diabetic patients with the wearable artificial endocrine pancreas was attempted. The parameters of insulin and glucagon infusion algorithms were; $K_{\mathrm{p}}=0.51, K_{\mathrm{d}}=4.89, K_{\mathrm{c}}=-2.02$, $G_{\mathrm{p}}=3.6, G_{\mathrm{d}}=7.2, G_{\mathrm{c}}=0.4$, and $\tau=10$. The sensor was replaced with a new one after three days' use. Glycaemic control was compared in each patient with that obtained by intensified multiple insulin injection regimens and continuous subcutaneous insulin infusion therapy. 


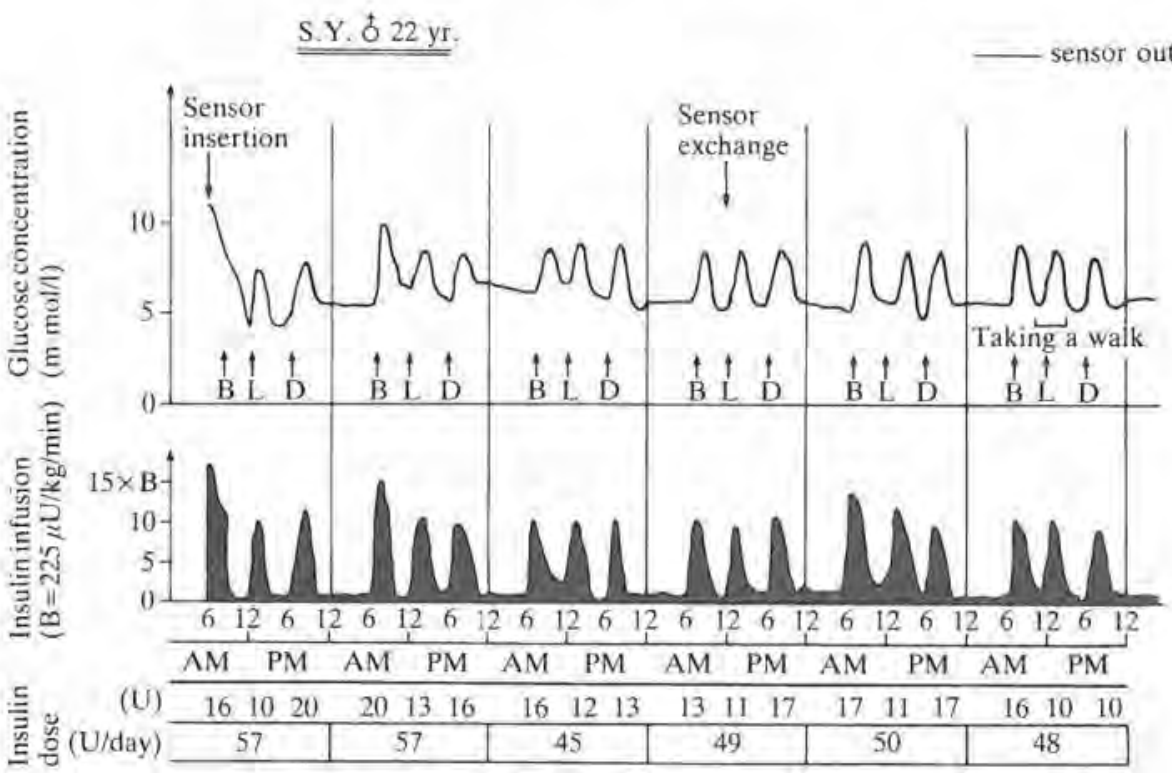

Fig. 23.8 A 6-day continuous glycaemic control in an insulin-dependent diabetic patient with a wearable artificial endocrine pancreas. The sensor was replaced on the fourth day. The patterns of insulin infusion and cumulative insulin requirement doses are also depicted. B, L, and S denote breakfast, lunch, and supper, respectively.

\subsubsection{Closed-loop glycaemic control in human diabetics}

The typical glycaemic control for six days in an insulin-dependent diabetic patient is depicted in Fig. 23.8. In all patients studied, physiological glycaemic regulations were established. As shown in Fig. 23.7, indices of daily glycaemic excursions such as MBG (mean blood glucose), $M$-value (Schlichtkrull et al. 1965), and MAGE (Service et al. 1970) were improved significantly in diabetics controlled by the wearable artificial endocrine pancreas, compared with the patients treated with the conventional insulin therapy, multiple insulin injections therapy, and continuous subcutaneous insulin infusion therapy.

\subsection{Conclusion}

The successful glycaemic control in human diabetics with the artificial pancreas (Albisser et al. 1974; Pfeiffer et al. 1974; Kawamori et al. 1978) underlines the importance of continuous glycaemic monitoring to strict glycaemic control. However, the major obstacle to extending the term of 
glycaemic control in human diabetics is the development of an implantable glucose sensor with high precision in tissue glucose determination.

A needle-type glucose sensor, which is a miniature hydrogen peroxide electrode covered by a membrane with biological activity, is easy to implant and replace. The sensor has the in vitro and in vivo characteristics suitable for continuous tissue-glucose monitoring.

A telemetry system using a needle-type glucose sensor is capable of monitoring glucose concentration in ambulatory diabetics. In addition, a wearable artificial endocrine pancreas, which incorporates a needle-type glucose sensor, has been devised and regulated glycaemia physiologically in human diabetics for more than six days.

Further improvements in sensor design, especially in membrane biocompatibility, might reduce the host reactions to the sensor implanted in tissue and thus extend its biological life.

\section{References}

Albisser, A. M., Leibel, B. S., Ewart, T. G., Davidovac, Z., Botz, C. K., Zingg, W., Schipper, H. and Gander, R. (1974). Clinical control of diabetes by the artificial pancreas. Diabetes 23, 397-404.

Bartlett, D. Jr. and Tenney, S. M. (1963). Tissue gas tensions in experimental anemia. J. Appl. Physiol. 18, 734-8.

Bessman, S. P., Thomas, L. J., Kojima, H., Sayler, D. F. and Layne, E. C. (1981). The implantation of a closed-loop artifical beta cell in dogs. Trans. Am. Soc. Artif. Intern. Organs 27, 7-18.

Chang, K. W., Aisenberg, S. and Soeldner, J.S. (1972). In vitro tests of an implantable glucose sensor. Proc, of 25th Ann. Conf. on Eng. in Med. and Biol. pp. 58 .

Gibson, Q. H., Swoboda, B. E. P. and Massey, V. (1964). Kinetics and mechanism of action of glucose oxidase. J. Biol. Chem 239, 3927-34.

Gough, D. A., Anderson, F, L., Giner, J., Colton, C. K. and Soeldner, J. S. (1981). Effect of coreactants on electrochemical glucose oxidation. Anal. Chem. 50, 941-4,

Guilbault, G. G. and Lubrano, A. (1973). An enzyme electrode for the amperometric determination of glucose. Anal. Chim. Acta 64, 439-55.

Hagihara, B., Ishibashi, F., Sato, N., Minami, T., Okada, Y. and Sugimoto, T. (1981). Intravascular oxygen monitoring with a polarographic oxygen cathode. J. Biomed. Eng. 3, 9-16.

Ikeda, S., Aoyama. N., Ito, K., Ohkura, K., Yamamoto, T., Ichihashi, H. and Kondo, T. (1980). Artificial pancreas - study of the new vessel access type glucose sensor. Jpn. J. Artif. Organ 9, 182-92.

Kawamori, R., Shichiri, M., Goriya, Y., Yamasaki, Y., Shigeta, Y, and Abe, H. (1978). Importance of insulin secretion based on the rate of change in blood glucose concentration in glucose tolerance, assessed by the artificial beta cell. Acta Endocrinol. 87, 339-51. 
Kawamori, R., Shichiri, M., Kikuchi, M., Yamasaki, Y. and Abe, H. (1980). Perfect normalization of excessive glucagon responses to intravenous arginine in human diabetes mellitus with the artificial beta cell. Diabetes 29, 762-5.

Layne, E. C., Schultz, R. D., Thomas, L. J., Slama, G., Sayler, D. F. and Bessman, S. P. (1976). Continuous extracorporeal monitoring of animal blood using the glucose electrde. Diabetes 25, 81-9.

Pfeiffer, E. F., Thum, Ch. and Clemens, A. H. (1974). The artificial beta-cell - a continuous control of blood sugar by external regulation of insulin infusion (glucose controlled insulin infusion system). Horm. Metab. Res. 6, 339-42.

Schlichtkrull, J., Munk, O., Jersild, M. (1965). The M-value, an index of blood-sugar control in diabetes. Acta Med. Scand. 177, 95-102.

Service, F. J., Molnar, G. D, Rosevear, J. W., Ackerman, E., Gatewood, L. C., Taylor, W.F. (1970). Mean amplitude of glycemic excursions, a measure of diabetic instability. Diabefes 19, 644-755.

Shichiri, M., Kawamori, R. and Abe, H. (1979). Normalization of paradoxic secretion of glucagon in diabetics who were controlled by the artificial beta cell. Diabetes 28, 272-5.

- Hakui, N., Yamasaki, Y, and Abe, H. (1984). Closed-loop glycemic control with a wearable artificial endocrine pancreas - Validations in daily insulin requirements to glycemic response. Diabetes 33, 1200-1202.

Yamasaki, Y., Hakui, N. and Abe, H. (1982). Wearable-type artificial endocrine pancreas with needle-type glucose sensor. Lancet 2, 1129-31.

- Goriya, Y., Yamasaki, Y., Hakui, N., Asakawa, N. and Abe, H. (1983). Glycaemic control in pancreatectomized dogs with a wearable artificial endocrine pancreas. Diabetologia 24, 179-84.

Soeldner, J.S., Chang, K. W., Aisenberg, S., Hiebert, J. M. and Egdahl, R. H. (1976). Diabetes mellitus a bioengineering approach - An implantable glucose sensor. In Diabetes mellitus, Forgarty International Center Series on Preventive Medicine (ed. S.S. Fajan), Vol. 4, pp, 267-77. Dept. of Health, Education and Welfare Public Health Service, National Institutes of Health.

Updike, S. J, and Hicks, G. P. (1967). The enzyme electrode, a miniature chemical transducer using immobilized enzyme activity. Nature 214, 986-8.

Woodward, S. C. (1982). How fibroblasts and giant cells encapsulate implants: Considerations in design of glucose sensors. Diabetes Care 5, 278-81.

Yamasaki, Y. (1984). The development of a needle-type glucose sensor for wearable artificial endocrine pancreas. Med, J. Osaka Univ. 35, 25-34. 

Bioelectrochemistry
(c) Analysis of electrical impedance 



\title{
24
}

\section{The principles and potential of electrical admittance spectroscopy: an introduction}

\author{
DOUGLAS B. KELL
}

\subsection{Introduction and overview}

In many electrochemical techniques, one applies a (clamped) DC potential to the working electrode and measures the resultant current flowing in a circuit completed by a counter electrode (e.g. Bard and Faulkner 1980; Bond 1980; Kissinger and Heineman 1984). Even in pulse voltammetric techniques, the measuring system is designed such that the potential difference between the working and reference electrodes, and the current ultimately measured, is constant for a greater or lesser period. However, the last 20 years or so have witnessed the increasing exploitation of sinusoidal exciting voltages in the study of electrode processes in aqueous media (e.g. Breyer and Bauer 1963; Schwan 1966; Smith 1966; Sluyters-Rehbach and Sluyters 1970; Macdonald 1977; Archer and Armstrong 1980; Bard and Faulkner 1980; Bond 1980; Gabrielli 1980; Buck 1982; Macdonald and McKubre 1982) an approach which possesses two advantages in particular: (1) the sinusoid offers convenient technical and mathematical features in such systems, together with an excellent signal: noise ratio predicated upon the use of a 'steady-state' analysis (e.g. Creason et al. 1973; Gabrielli and Keddam 1974; Diamond and Machen 1983; Marshall 1983), and (2) the frequency, as well as the voltage, of the exciting wave-form may be altered, so that we may consider or use the technique as a form of spectroscopy.

To put the foregoing in another way, we may raise the idea, with which we are all familiar, that the frequency-dependent absorption of ultra-violet, visible, and infra-red light may be used in the analysis of biological (and other) materials. Yet light is only a form of electromagnetic radiation, albeit of a rather high frequency $\left(10^{14} \mathrm{~Hz}\right.$ or so), and there is thus no reason why the frequency-dependent absorption of electrical energy of lower frequencies might not similarly be exploited in bio-analytical devices. In such cases, at least below $30 \mathrm{MHz}$ or so, one requires electrodes to act as an interface between the exciting electrical field and the sample, so that, as in the 'pure' electrochemical case above, one may study the frequency-dependent, passive electrical properties of the system consisting of the electrodes plus the biological sample; in other words, one may study the frequency-dependent 
impedance or admittance of the system.

In the following, therefore, 1 shall (1) outline in very elementary terms what is meant by the concepts of electrical impedance and admittance, (2) discuss the application of such measurements in (predominantly nonfaradaic) electroanalysis, and (3) introduce the cognate concept of the dielectric spectroscopy of biological substances. These considerations will pave the way for (4) a discussion of the use of AC techniques, including frequency response analysis (FRA), in biosensor applications sensu lato. Because of the relative magnitudes of the topic and the space available, I will make no attempt to be comprehensive; my aim will be predominantly to provide, for the general reader, an introduction to a field which I believe has been widely neglected by biologists and biophysicists (despite its many spectacular successes), yet which underlies a great many present and future biosensor applications.

\subsection{Electrical impedance and admittance}

Let us consider a sinusoidally modulated voltage, of the form $V=V_{\mathrm{m}} \sin \omega t$, where $\omega$ is the frequency in radians $s^{-1}(\omega=2 \pi f$, where $f$ is the frequency in $\mathrm{Hz}$ ), $V_{\mathrm{m}}$ is the maximum (peak-to-peak) voltage, and $V$ the voltage at any given instant. If this voltage appears across the terminals of a passive circuit, device, or 'system', which may consist of pure electrical components or of a biological or chemical sample separating a pair of electrodes, the current flowing in the circuit (after any transients have died down) may be related to the voltage both by its magnitude and its phase, and is of the form $i=i_{\mathrm{m}} \sin$ $(\omega t+\theta)$. Thus (Fig. 24.Ia), although the frequency and sinusoidal nature of the wave-form are unchanged by interaction with the system, the characteristics of the system are reflected in the ratio $V_{\mathrm{m}} / i_{\mathrm{m}}$ and by the value of $\theta$.

Now, systems may exhibit resistive, capacitive, and inductive properties, properties which (by definition) may be distinguished from each other by their effects upon a sinusoidal voltage. Thus, for a pure resistor ( $R$ Ohms), the current due to our exciting waveform $\left(V_{\mathrm{m}} \sin \omega t\right)$ is given by:

$$
i=\left(V_{\mathrm{m}} / R\right) \sin \omega t .
$$

For a pure capacitor ( $C$ farads):

$$
i=\omega C V_{\mathrm{m}} \sin \left(\omega t+\frac{\pi}{2}\right)
$$

whilst for a pure (self-) inductance of $L$ henries:

$$
i=\left(V_{\mathrm{m}} / \omega L\right) \sin \left(\omega t-\frac{\pi}{2}\right) .
$$

Thus, for a pure resistor, there is no phase difference between $V$ and $i$. In 
3
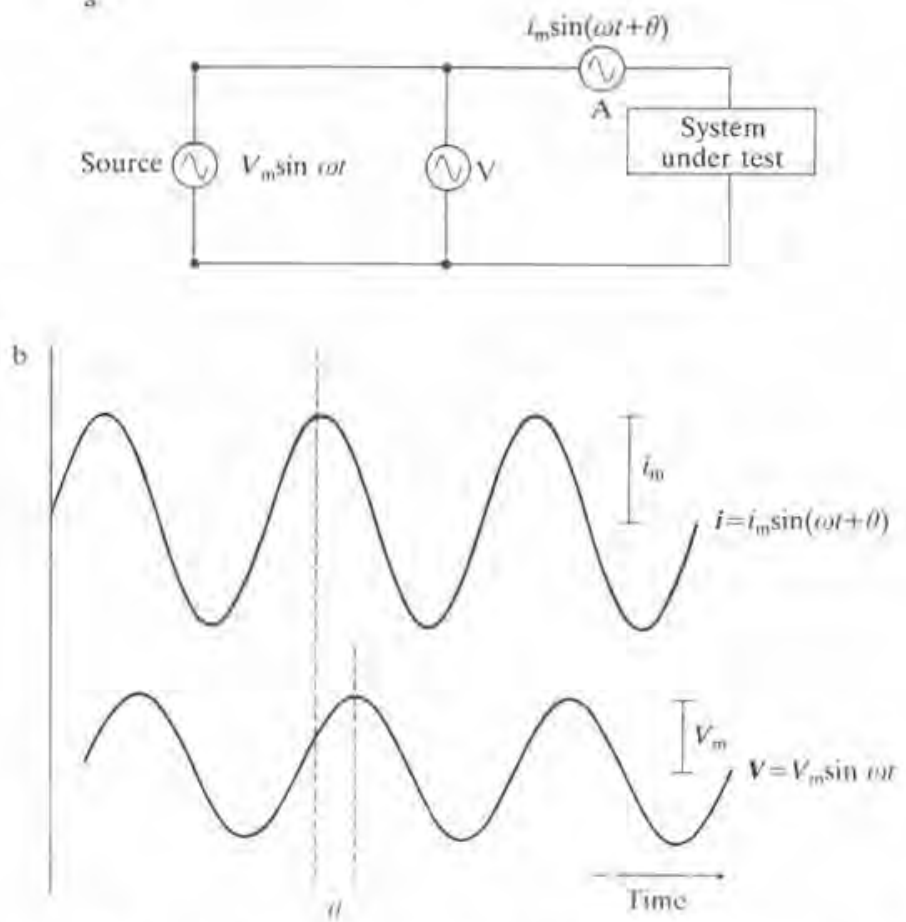

Fig. 24.1 (a), The impedimetric experiment, in which a small-amplitude perturbation, in the form of a sinusoidal voltage, is applied to the system of interest. The sinusoidal voltage across the system may be measured using a (high-impedance) AC vector voltmeter, $\mathrm{V}$, whilst the sinusoidal current flowing in the circuit may be measured by means of an $A C$ vector ammeter $A$. In practice $(a, b)$, it is found that the phase of the current differs from that of the voltage by an amount $\theta$; in the case shown it leads the voltage.

contrast, for a pure capacitor, the current leads the voltage by $\pi / 2$ radians $\left(90^{\circ}\right)$ whilst for a pure inductor the current lags the voltage by the same amount. Now, except in active biological systems such as nerve axons (e.g. Cole 1972; Jack et al. 1975; De Felice 1981), and in certain electrochemical systems, particularly those involving corrosion and electro-deposition (Gabrielli 1980), inductances are negligible, and we shall for the most part ignore them. We may therefore imagine intuitively (and correctly) that for a 'real' system, which possesses both resistive and capacitive properties (i.e. behaves as a leaky capacitor), $\theta$ takes a value between 0 and $\pi / 2$, as illustrated in Fig. 24.1b.

We may then define a vector quantity $Z$, the impedance, with modulus 


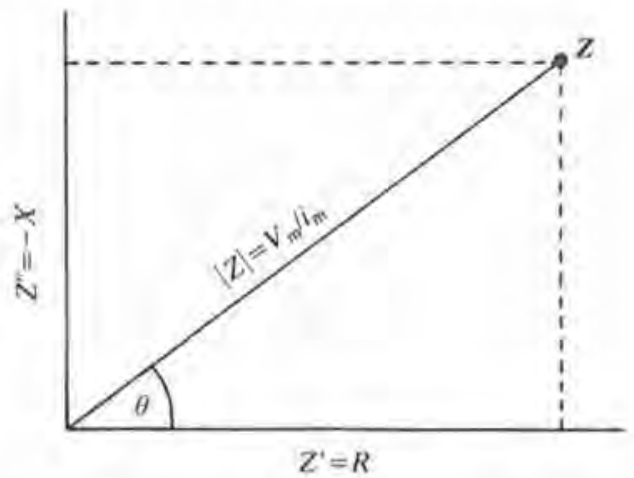

Fig, 24.2 Impedance as a complex quantity. There is a mathematical function, known as Euler's identity, which states that $A \mathrm{e}^{=\sqrt{\theta}}=A \cos \theta \pm A \mathrm{j} \sin \theta$, where $\mathrm{j}=$ $\sqrt{-1}$. Thus, any complex quantity may be split up into its real and imaginary part. The figure shows the manner in which this is done for the impedance function $Z=R+\mathrm{j} X$. Simple geometrical considerations indicate (i) that $Z^{2}=R^{2}+X^{2}$, and (ii) that $R=|Z| \cos \theta$ and $X=-|Z| \sin \theta$. Thus $R$ and $X$ may be obtained from measurements of $|Z|$ and $\theta$, and are known respectively as the 'in phase' and $\left(90^{\circ}\right)$ 'out-of-phase' components.

(magnitude) $|Z|$ and argument ('direction') $\theta$, in a form analogous to that of a complex number $a+\mathrm{j} b$ (where $\mathrm{j}=\sqrt{-1}$ ) as in Fig. 24,2, where the modulus $|Z|$ of the impedance is equal to the ratio $V_{\mathrm{m}} / i_{\mathrm{m}}$. Thus, the impedance has both real and imaginary parts, and is defined as $Z=R+\mathrm{j} X$, where the reactance $X=-1 / \omega C$, and the system is treated as though it consisted of a resistance and capacitance in series.

We may also treat the system as consisting of an equivalent conductor ( $G$ siemens $\left.=1 / R^{\prime} S\right)$ and capacitor $\left(C^{\prime}\right)$ in parallel. In this case, we define an admittance $Y$, as a vector with modulus $|Y|=i_{m} / V_{m}=1 /|Z|$ and argument $\theta$, such that $Y=1 / Z=\mathrm{G}+\mathrm{j} B$, where $B$, the susceptance, $=\omega C^{\prime}$.

As succinctly stated by Falk and Fatt (1968), the distinction between the two sets of treatments is as follows: in the impedance representation, we take the impedance to represent the dependence of the voltage on the current, the terminals of the system under study (in an arrangement such as that of Fig. 24.1a) being considered as being connected to a current source of infinite resistance (i.e. open circuited), In contrast, in the admittance representation we take the admittance to represent the dependence of the current on the voltage, the terminals being considered as being connected to a voltage source of zero resistance (short-circuited).

Since the above distinctions are only distinctions in the way we treat the sample, it is obvious that we can move from the impedance to the admittance 


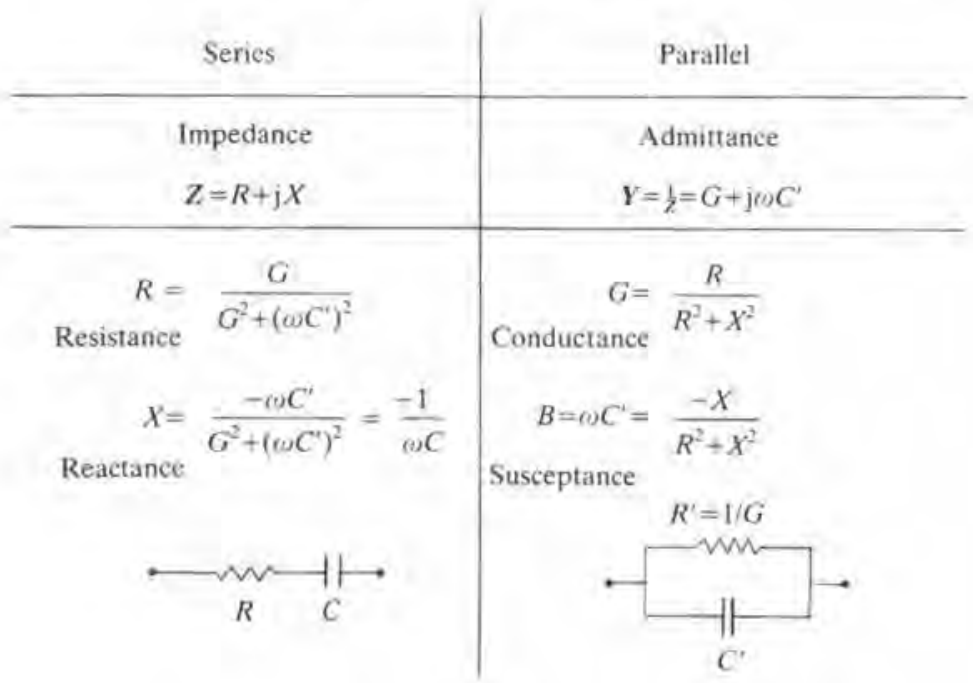

Fig. 24.3 The relationships between impedance and admittance, and their real and imaginary components. For discussion, see text.

domain, and vice versa, by the choice of appropriate values of $R, C, G$, and $C^{\prime}$. For convenience, we give the relevant equations in Fig. 24.3. In other words, regardless of the actual complexity of (the equivalent electrical circuit of) the system between the terminals of the measuring instrument, when we make measurements at a given frequency, we merely treat the system as though it consists of a single resistance (conductance) in series or in parallel with a single capacitance. For real circuits, then, the impedance $Z(\omega)$ or admittance $Y(\omega)$, and their component real and imaginary parts, are frequency-dependent quantities, the frequency-dependence of which may be used to describe the actual equivalent electrical circuits. It should be noted that, by definition, the impedance and admittance are independent of the voltage across, and current flowing in, the system under study, and this 'linear property' should be taken into account when use is made of these representations.

In general, the most convenient means by which we can extract the magnitudes and topological relationship of the components constituting the equivalent circuit is by means of complex plane diagrams, a topic to which we now turn.

\subsection{Impedance diagrams}

If we make measurements of the frequency-dependent impedance of an electrical circuit consisting of a $2.671 \mathrm{k} \Omega$ resistor in parallel with a $220.4 \mathrm{pF}$ 
432 The principles and potential of electrical admittance spectroscopy

a

Model impedance network

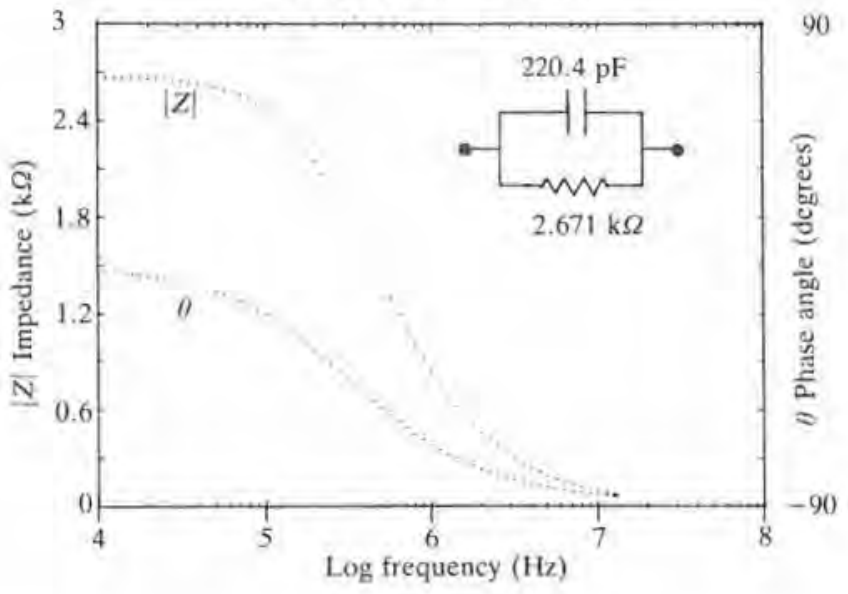

b

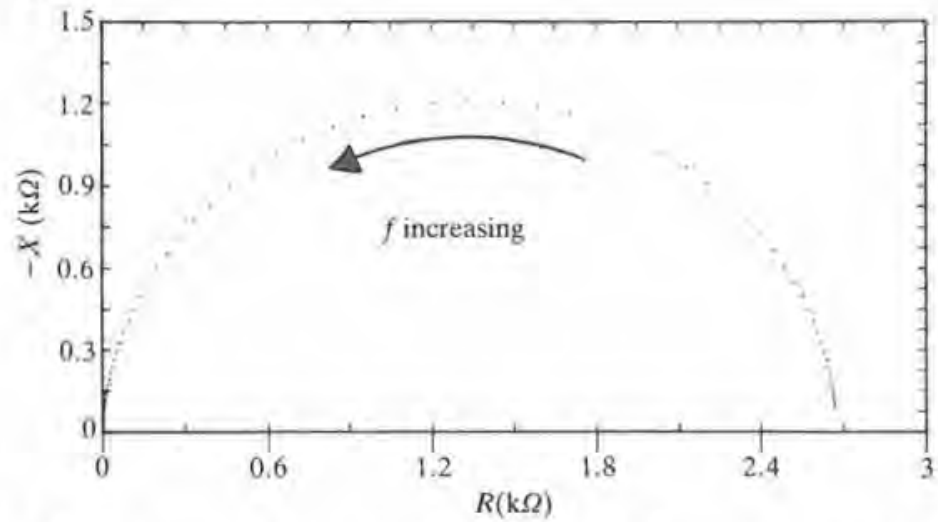

Fig. 24.4 The frequency-dependent impedance of a model electrical circuit. Measurements were made, and the data plotted, using the frequency-domain impedimetric system described by Harris and Kell (1983). (a) The impedance modulus and the phase angle as a function of the frequency. Note the existence of two plateau regions in frequency ranges that are respectively low and high relative to that of $f_{\mathrm{c}}$. (b) A reactance/resistance plot, showing that the circuit has a single time constant. For further discussion, see text.

capacitor, the behaviour shown in Fig. 24.4 is obtained. (Remember that although the circuit is actually a parallel network, we treat it, in the impedance representation, as though components were connected in series.) Thus (Fig. 24.4a), as the measuring frequency is increased, we find (i) that the phase angle ( $\theta$, as defined in Fig. 24.1), decreases from approximately $0^{\circ}$ 
(purely resistive behaviour) to approximately $-90^{\circ}$ (purely capacitive or reactive behaviour), and (ii) the modulus of the impedance $|\boldsymbol{Z}|$ decreases from roughly $2.67 \mathrm{k} \Omega$ to roughly zero. The frequency at which the transition is half completed, the so-called critical or characteristic frequency $f_{\mathrm{c}}$, may be seen, by inspection of Fig. 24.4a, to occur at approximately $300 \mathrm{kHz}$. Since the product of a resistance and a capacitance has the dimensions of time (seconds), and is equal by definition to the relaxation time $\tau$ ('time constant') for such a circuit, and since $\tau=1 / 2 \pi f_{\mathrm{c}}$, we may also calculate $\tau\left(5.89 \times 10^{-7} \mathrm{~s}\right)$ and $f_{c}(270 \mathrm{kHz})$ simply from the values of the resistor and capacitor in the circuit.

Now, as shown in Fig. 24.2, we can calculate the real $(R)$ and imaginary $(X)$ parts of the impedance from the measured values of $|Z|$ and $\theta$, and (since these change with frequency) plot the negative reactance against the resistance with frequency as the parameter. This is done in Fig. 24.4b, where it may be observed that the resultant plot takes the form of a semicircle, whose centre would lie on the abscissa and which has a maximal value of $-X$ which occurs ( $c f$. Figs. 24.4a, 24.4b) at the characteristic frequency; further, had measurements been made over a wider frequency range, it is evident (or at least plausible) that the semicircle would have extrapolated to values of 0 and $2.67 \mathrm{k} \Omega$. Thus, as discussed in many introductory textbooks of electrical circuit analysis (e.g. Bleaney and Bleaney 1976; Duffin 1980; Bobrow 1981; Brown et al. 1982; Harter and Lin 1982), these impedance diagrams reflect, and may be used to obtain, the values of the elements of an equivalent electrical circuit.

Using the equations given in Fig. 24.3, one may also derive from Fig. 24.4a the equivalent values of $G$ and $B$ pertinent to a representation in the admittance domain. In this case, a plot of $B$ versus $G$ (an admittance diagram) would also give a semicircle, with its centre on the abscissa and the maximum value of $B$ when the exciting frequency $=f_{c}$. The production of such a plot is left as an exercise for the reader. As we shall also see when we come to consider complex conductivity and permittivity, although the information contained in each plot is the same, the relative weightings of the data can serve to enhance different frequency regions (Macdonald et al. 1982).

\subsection{Impedance diagrams in electrochemical systems}

For a variety of historical and other reasons, the impedance $(R / X)$ representation has dominated the electrochemical literature, although J.R. Macdonald and his colleagues (e.g. Macdonald 1980; Macdonald et al. 1982) have stressed the utility of the three-dimensional perspective $R / X / \log f$ plot. Now, the general aim in studies of purely electrochemical and, in many cases, of solid-state (as opposed to biological) impedances is to gain information 


\section{The principles and potential of electrical admittance spectroscopy}

about the mechanisms of electrode processes, i.e. of processes occurring at the electrode/electrolyte interface. Thus, since such processes are obviously dependent upon the 'mean' potential of the working electrode, one should arrange to poise this potential at a known value, either by including both pairs of a redox couple of known $E_{0}^{\prime}$ in the medium (faradaic impedance) or electronically. In the latter case in particular, it is usual to use a threeelectrode system (Bard and Faulkner 1980; Bond 1980; Gabrielli 1980). In such two- or three-electrode measurements, of course, one should either use identical electrodes or make the impedance of the working electrode very much greater than that of the counter electrode.

The interpretation of electrochemical impedances is a vast, detailed, and complex field, and for the present purposes I shall merely give the simplest possible description of the salient ideas. These are: (1) that the electrical double layer (e.g. Mohilner 1966; Bockris and Reddy 1970; Sparnaay 1972; Martynov and Salem 1983) at the electrode/solution interface possesses, due to its molecular thickness, a significant capacitance (of some $\mu \mathrm{F}$ per $\mathrm{cm}^{2}$ actual electrode area, under typical conditions) which must be charged up before any faradaic current can flow; (ii) that the rate of the subsequent reaction may be limited by a charge-transfer step, by diffusion of electroactive reactant to the reaction layer, or by both, in which latter case one finds the superposition of a straight line and a semicircle in the $R / X$ plot; (iii) that the residual resistance at very high frequencies represents the resistance of the bulk solution between the electrodes; (iv) the diffusional impedance is often

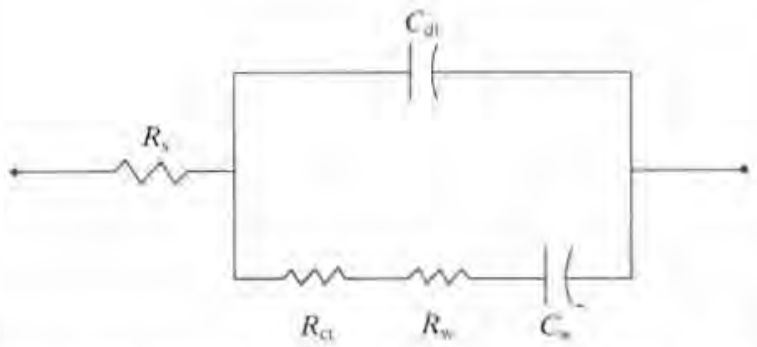

Fig. 24.5 Very general equivalent circuit for an electrochemical cell. The doublelayer capacitance $C_{\mathrm{dl}}$ is in parallel with a resistance representing the charge-transfer (faradaic) step, since geometrically they occur in (essentially) the same place. This structure is in series with a 'Warburg' impedance $Z_{w}$, comprised of resistive and capacitive parts, equivalent in essence to the 'diffusion zone'. Finally, the whole arrangement is in series with the (' $i R$ drop') bulk electrolyte solution resistance $R_{s}$. Obviously the actual magnitude of these components determines the exact frequency response of the system. The symbols used for the capacitors are to indicate the presence of some heterogeneity in the structures which they represent. 
referred to as the Warburg impedance $Z_{\mathrm{w}}$, and represented as a resistor and capacitor in series. The equivalent electrical circuit describing this behaviour, and which is usually ascribed to Randles (1947), is given in Fig. 24.5; it may be noted that we are here beginning to equate our electrical circuit components with mechanistic explanations of electrode behaviour.
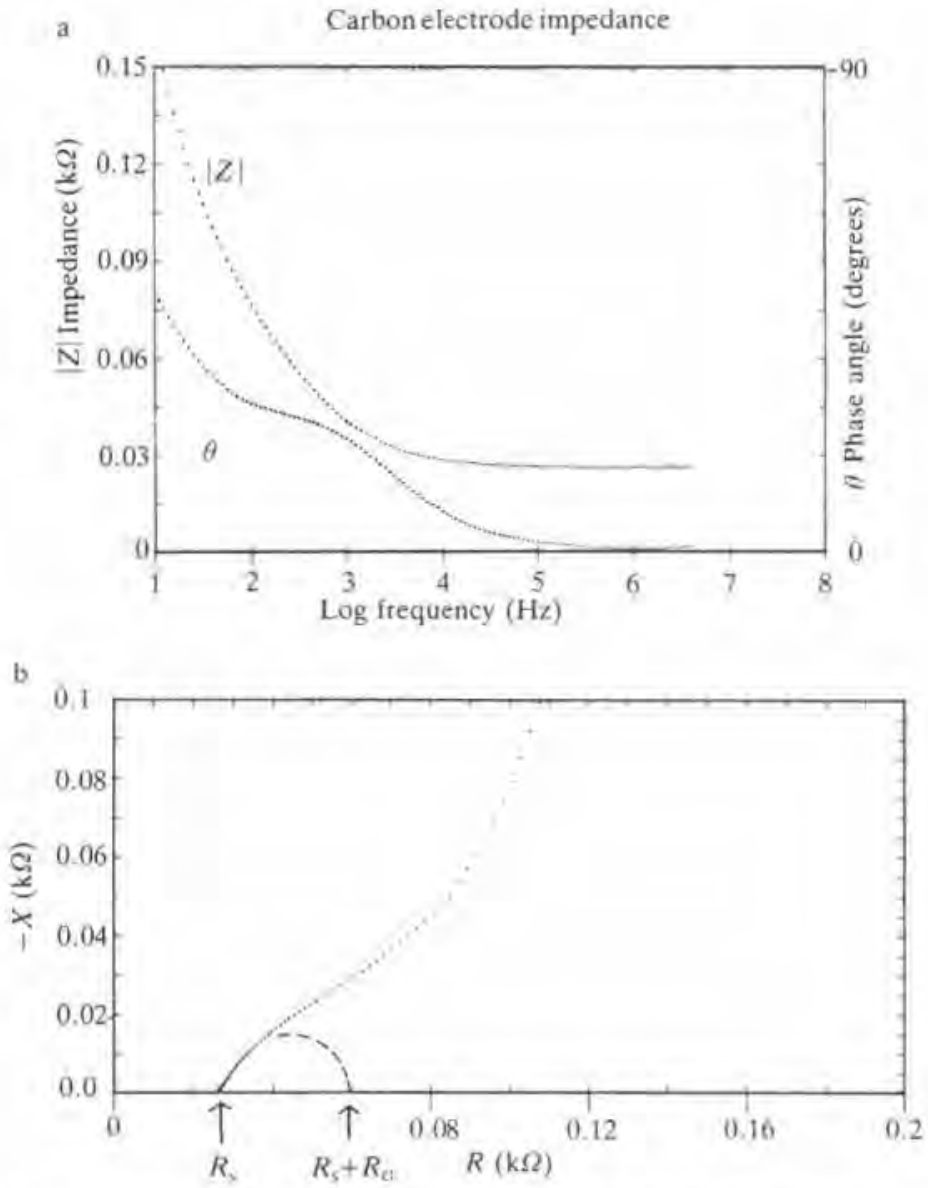

Fig. 24.6 The frequency-dependent impedance of a pair of graphite electrodes immersed in $100 \mathrm{mM} \mathrm{KCl}$. The modulating voltage was $50 \mathrm{mV}$ and measurements were performed using the apparatus described by Harris and Kell (1983). (a) Impedance modulus and phase angle versus logarithmic frequency. (b) Impedance diagram, showing how one may derive the values of $R_{5}$ and $R_{\mathrm{ct}}$ from the semicircular portion of such a plot (Hung et al. 1979). In a classical Warburg-type system, the low-frequency (right hand) part of the impedance locus should make an angle of $45^{\circ}$ with the abscissa. The characteristic frequency of the semicircular part of the plot may be used to obtain the values of $C_{\mathrm{dl}}$ from the relation $C_{\mathrm{dl}}=1 / 2 \pi f_{\mathrm{c}} R_{\mathrm{cl}}$. 
Now, it should be stressed that much more complicated behaviour than the above may be observed in practice. Nevertheless, Fig. 24.6 shows the experimentally obtained impedance diagram of a pair of cylindrical graphite electrodes ( $c a .4 \mathrm{~mm}$ radius, $20 \mathrm{~mm}$ length, surface roughness unknown, separation $10 \mathrm{~mm}$ ) immersed in $100 \mathrm{mM} \mathrm{KCl}$, a diagram which, it may be observed, corresponds fairly accurately to the behaviour described above (and see Besenhard and Fritz 1983). The following points may be made with respect to this figure: (1) the semicircular locus is by no means perfect, and is poorly separated from the straight line portion, and it is not realistic to fit it such that its centre lies on the abscissa - this may be ascribed to heterogeneity in the structures underlying $C_{\mathrm{dl}}$ and $R_{\mathrm{c}} ;(2)$ the frequency dependence of the impedance extends over an enormous range - at least seven orders of magnitude in the present case; (3) there is no frequency dependence (in this range) of the impedance of the material between the electrodes (which is simply an ionic solution) - all the observed frequency dependence is caused by electrochemical behaviour at the electrodes.

Now, it is obvious that the measured resistance and reactance of our electrochemical cell is a function of the electrode size and geometry; electrodes of larger area and closer separation will, all else being equal, appear to have a lower impedance. Since, in many cases, it is the intensive properties of the system which are of interest, we must needs take account of this; to do so we will make use of the admittance representation (Fig. 24.7), and introduce the notions of permittivity and conductivity.

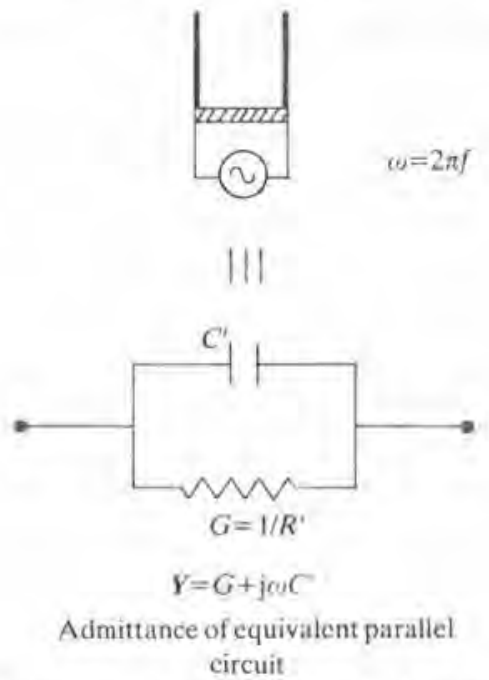

Fig. 24.7 At any given frequency, the passive electrical properties of a system may be completely described by the admittance $G+\mathrm{j} \omega C$ of the equivalent parallel circuit. 


\subsection{Permittivity, conductivity and dielectric dispersion}

For a specimen held between two parallel electrodes of area $A$ separated by a distance $d$, the intrinsic passive electrical properties are completely specified by the conductivity $\sigma^{\prime}$ and the permittivity $\epsilon^{\prime}$, which are related to the measured conductance $G$ and capacitance $C^{\prime}$ by the equations:

$$
\begin{aligned}
& G=\sigma^{\prime}(A / d) \\
& C^{\prime}=\epsilon^{\prime} \epsilon_{\mathrm{r}}(A / d)
\end{aligned}
$$

From eqn 24,4 , we find that to obtain the conductivity, we multiply the measured conductance by $d / A$, a factor which has the dimensions of length ${ }^{-1}$ (e.g. $\mathrm{cm}^{-1}$ ) and is known as the cell constant. In eqn 24.5, $\epsilon_{\mathrm{r}}$ (sometimes called $\epsilon_{0}$ in the literature) is the capacitance of a cell of unit dimensions containing a vacuum, equal to $8.854 \times 10^{-14} \mathrm{~F} / \mathrm{cm}$, so that any matter existing between the electrodes will have the effect of raising the capacitance by a factor $\epsilon^{\prime}$, a factor which was formerly called the dielectric constant, but (since it is not constant) is more properly referred to as the permittivity. The permittivity of water at $25^{\circ} \mathrm{C}$ is approximately 78.4 , so that, as may be calculated from eqns 24.4 and 24.5 , a cell of cell constant $1 \mathrm{~cm}^{-1}$ containing water at this temperature will have a capacitance of $6.94 \mathrm{pF}$. The presence of ionic electrolytes has only a rather modest effect upon the permittivity of aqueous solutions, such that the permittivity of $1 \mathrm{M} \mathrm{NaCl}$ at $25^{\circ} \mathrm{C}$ is approximately 61.6 (Davies 1965).

Now, for many purposes, it is useful to make use of the complex permittivity $\epsilon^{*}=\epsilon^{\prime}-\mathbf{j} \epsilon^{\prime \prime}$, which, as with impedance and admittance, has both real and imaginary parts, and the imaginary part of which, the dielectric loss $\epsilon^{\prime \prime}$, is related to the conductivity by the equation

$$
\epsilon^{\prime \prime}=\frac{\sigma^{\prime}-\sigma_{L}^{\prime}}{2 \pi f \epsilon_{\mathrm{r}}}
$$

where $\sigma_{\mathrm{L}}^{\prime}$ represents any DC or 'low frequency' contribution to the conductivity.

In a given frequency range, the dielectric properties of any material between the electrodes may not be constant (i.e. the material exhibits dielectric dispersion), and, as with the impedance of the model circuit in Fig. 24.4, may change between two 'plateau' values $\epsilon_{\mathrm{L}}^{\prime}$ and $\epsilon_{\infty}^{\prime}$, according to the equation

$$
\epsilon^{*}=\epsilon_{\infty}^{\prime}+\frac{\epsilon_{L}^{\prime}-\epsilon_{\infty}^{\prime}}{1+j \omega \tau}
$$

where, as before, $\tau\left(=\frac{1}{2} \pi f_{\mathrm{e}}\right)$ is the relaxation time. Equation 24.7 separates into

$$
E^{\prime}=\epsilon_{\infty}^{\prime}+\frac{\epsilon_{L}^{\prime}-\epsilon_{\infty}^{\prime}}{1+(\omega \tau)^{2}},
$$




$$
\epsilon^{\prime \prime}=\frac{\left(\epsilon_{i}^{\prime}-\epsilon_{\infty}^{\prime}\right) \omega \tau}{1+(\omega \tau)^{2}},
$$

and a plot of $\epsilon$ " versus $\epsilon$ 'gives a circle whose centre is located on the $\epsilon$ axis. However, in practice it is often observed that semicirlces result whose centre lies below the abscissa, and it was shown by Cole and Cole (1941) that this behaviour may be described by an equation of the form

$$
\epsilon^{*}=\epsilon_{\infty}^{\prime}+\frac{\epsilon_{L}^{\prime}-\epsilon_{\infty}^{\prime}}{1+(j \omega \tau)^{1-\alpha}}
$$

such that a line between the centre of the circle and the points at which the $\epsilon^{\prime \prime} / \epsilon^{\prime}$ locus crosses the abscissa makes an angle $\alpha \pi / 2$ radians with the abscissa. Although the Cole-Cole representation is entirely empirical (it is generally taken to represent some kind of distribution of relaxation times), it is now commonplace to express data in the form of a Cole-Cole plot, such that the dispersion is characterized by the 'dielectric increment' $\Delta \epsilon^{\prime}=\epsilon_{\mathrm{L}}^{\prime}-\epsilon_{\infty}^{\prime}$ and by the Cole-Cole $\alpha$. Many other dielectric relaxation time distributions have been suggested (reviewed by Boyd 1980 and see Marshall and Roe 1978), but they have not achieved widespread usage in biological systems, and are not discussed further here.

Complementarily, one may make use of the 'complex conductivity' plot of $\sigma^{n}$ versus $\sigma^{\prime}$, where

$$
\sigma^{\prime \prime}=2 \pi f \epsilon_{i}\left(\epsilon^{\prime}-\epsilon_{\infty}^{\prime}\right) \text {. }
$$

As discussed above, the two representations have the effect of weighting the appearance of the data differently; I will illustrate this by using (in Fig. 24.8) the data (Fig. 24.6) from the carbon electrode impedance spectrum.

As Fig. 24.8a shows, the apparent permittivity of the system at low frequencies reaches truly enormous values $\left(2 \times 10^{8}\right.$ at $\left.10 \mathrm{~Hz}\right)$, the measured capacitance at this frequency being approximately $70 \mu \mathrm{F}$, an effect which forms the basis of the 'electrolytic' type of capacitor used in electrical and electronic circuits. Of course, the permittivity of the electrolyte between the electrodes is only about 78 , and, if we use this value for the 'high frequency'

Fig. 24.8 Admittance properties of carbon electrodes. Data were obtained as described in the legend to Fig. 24.4. (a) Conductivity and permittivity, as obtained from the measured capacitance and conductance by means of the cell constant. (b) Admittance (complex conductivity) plot, using $\epsilon_{\infty}^{\prime}=78.4$. The fit of the two semicircles is empirical, there being no satisfactory way (in the absence of additional knowledge) of separating overlapping dispersions. Extrapolation gives $\sigma_{\mathrm{t}}$, whence $\Delta \sigma^{\prime}$ for each dispersion may be obtained. (c) Complex permittivity (Cole-Cole) plot, using the value of $\sigma_{L}$ obtained in $b$, and illustrating the estimation of the Cole-Cole $\alpha$ and the extrapolation (to low frequencies) by which one may obtain $\epsilon_{\mathrm{L}}^{\mathrm{i}}$. It may be 

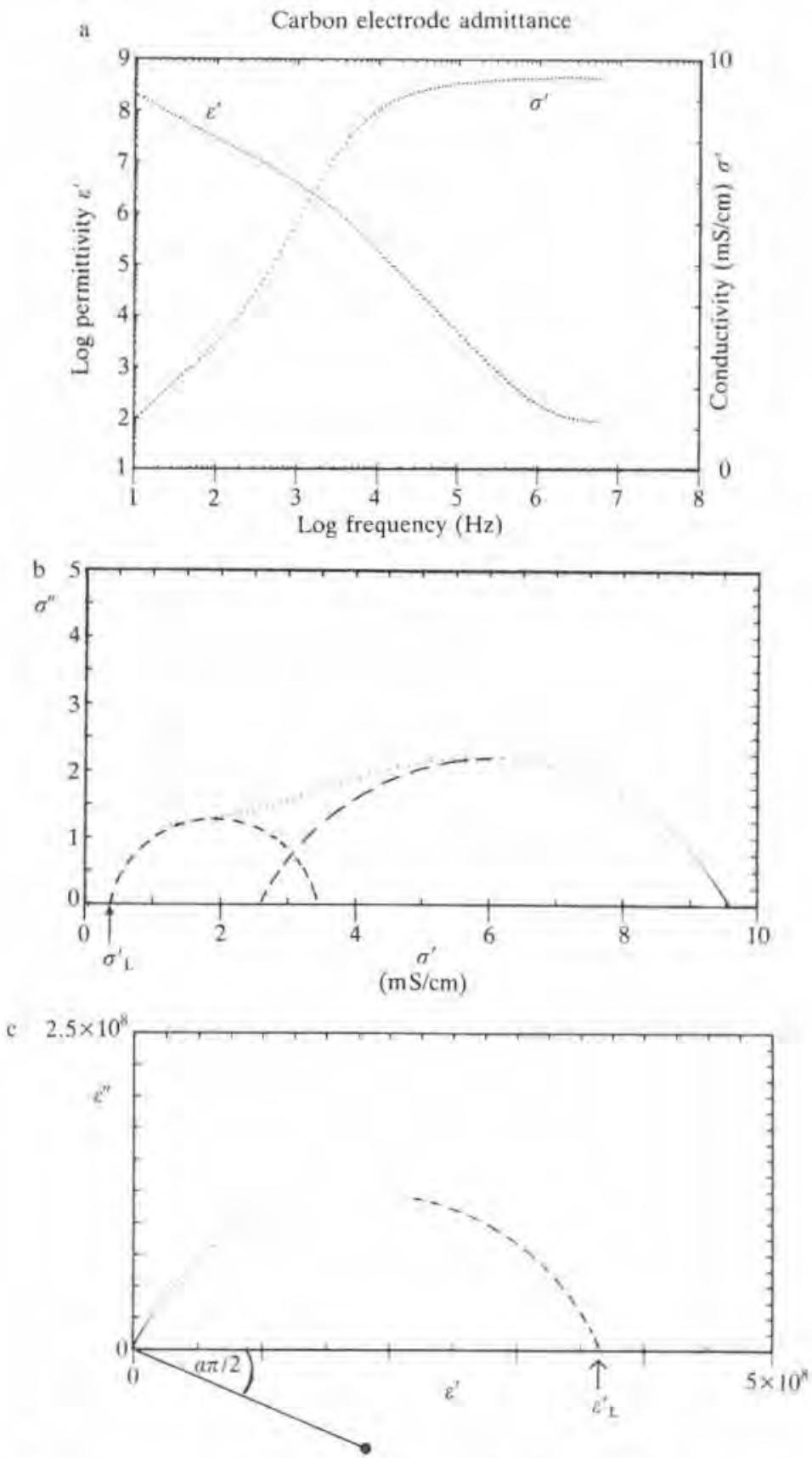

noted that two dispersions are not discernible in this diagram, illustrating how the admittance and complex permittivity plots weight the data differently. Note that, for a given dispersion in which the Cole-Cole $\alpha$ is not too large, $\tau$ is given by $\Delta \epsilon^{\prime} \epsilon_{\mathrm{r}} / \Delta \sigma^{\prime}$. 
permittivity, we obtain the admittance plot shown in Fig. 24.8b; as in the impedance diagram (Fig. 24.4), two separate processes may be discerned, extrapolation of the latter to low frequencies giving the value of $\sigma_{\mathrm{L}}$ $(0.4 \mathrm{mS} / \mathrm{cm})$ to be used in constructing the Cole-Cole plot in Fig. 24.8c. As stressed by Macdonald inter alia, the Cole-Cole plot is not really suitable for use in describing electrochemical impedances since permittivity and conductivity are intrinsic properties of materials which may be held between the electrodes, and this should be borne in mind. However, the representation in Fig. 24.8 serves to illustrate the means by which we treat data of this type, and it is hoped that this elementary exposition will assist the novice or tyro who may wish to delve further into these matters. For completeness, it should be mentioned that some literature, particularly that concerned with electrical insulators, specifies the so-called 'dissipation factor', $D=\tan \delta=\epsilon^{\prime \prime} / \epsilon$ ? For materials lacking DC conductivity, $D=\mathrm{G} / \omega C=1 / Q$, where $Q$ is the socalled quality factor or $Q$-factor.

We are now more or less in a position to consider some of the mechanistic bases for the frequency-dependent electrical behaviour of systems held between electrodes and which consist not only of ionic solutions but of biological materials. However, the dielectric (passive electrical) properties of biological and chemical (Stock 1984) substances have attracted study for a great many years (e.g, Osterhout 1922), both from a scientific and an analytical standpoint. Thus, for instance, Stewart (1899a) noted that the lowfrequency conductivity of blood plasma exceeded that of the whole blood from which it had been derived by an amount that was a monotonic function of the haematocrit, and derived an equation wherewith to estimate the latter by means of conductivity measurements. Since this time, a vast and increasing literature on biological impedances has accumulated, an amount far too great adequately to be reviewed herein, and what I shall therefore do is: (i) draw attention to the many excellent books, review articles, and monographs on the subject of the dielectric spectroscopy of biological substances, (ii) outline the salient observable and mechanistic features of the dielectric dispersions that have been described in biological systems, and the relationships between the dielectric increment and the effective molecular dipole moments underlying the dispersions, and (iii) describe some of the analytical methods and devices that have been used or proposed, and which have as their basis the measurement of conductivity, permittivity, or their vector sum. I shall then outline some of the technical and methodological aspects which should be borne in mind when one considers making measurements of biological impedances, and draw attention to the distinctions one may make between measurements in the time and frequency domain. This will lead us to an outline of the role of time series analysis in biosensing generally. Finally, I shall seek to bring together the ideas and facts described above in suggesting some novel approaches to the design and exploitation of biosensors. 


\subsection{Dielectric spectroscopy of biological susbtances}

Of the many books available on the dielectric behaviour of condensed matter, those of most biological relevance, and which are especially recommended, are by Daniel (1967), Cole (1972), Hasted (1973), Grant et al. (1978), Schanne and Ceretti (1978) and Pethig (1979). Schwan, the doyen of biological impedance determinations, has written several excellent reviews (e.g. Schwan 1957, 1963, 1977, 1981 a, b, 1983a, b; Schwan and Foster 1980; Stoy et al. 1982), and overviews of these matters may also be found in the review articles of Salter (1979), Pilla (1980; Pilla et al. 1983), Zimmermann (1982) and Pethig (1984). The latter gives an extensive discussion of measurements on proteins, which are also discussed in the reviews by Oncley (1943), Takashima (1969; Takashima and Minakata 1975), Grant and South (1972; Grant 1982, 1983), Petersen and Cone (1975), Wada (1976), Hasted et al. (1983), Kell and Hitchens (1983) and Kell and Westerhoff (1985). Our own work (Harris and Kell 1983; Kell 1983; Harris et al. 1984; Harris and Kell 1985a; Kell and Harris $1985 a, b$ ) has concentrated on microbial membranes, the latter two articles containing a fair amount of review material on this topic. Work with natural (Pauly and Packer 1960; Pauly et al. 1960; Falk and Fatt 1968; Irimajiri et al. 1979; Asami et al. 1980a, 1984) and pure phospholipid membrane vesicles (Schwan et al. 1970; Redwood et al. 1972; Asami and Irimajiri 1984; Pottel et al. 1984) and planar membranes (Hanai et al, 1964, 1965; Tien 1974; Fettiplace et al. 1975; Haydon et al. 1977; and Laver et al. 1984) may also be cited, whilst an entrée to the microbial literature may also be gained from the papers of Pauly (1962), Asami et al. (1976, 1980b), Clarke et al. (1984, 1985), Blake-Coleman et al. (1984), and Harris and Kell (1985b). Almost all charged polyelectrolytes exhibit enormous permittivities at low frequencies (e.g. Dukhin and Shilov 1974; O'Brien 1982), whilst those displayed by DNA are discussed at some length in the articles of Vreugdenhil et al, (1979) and Sorriso and Surowiec (1982). Most of the papers cited in this section concern work at frequencies below $100 \mathrm{MHz}$ or so; the higher frequency work, with which we have not had experience to date, is discussed by Foster and Schepps (1981), Foster et al. 1982, Illinger (1981), Stuchly et al. (1981), Clegg et al. (1982), Kraszewski et al. (1982), Gabriel et al. (1983), Magin and Burdette (1983) and Clegg et al. (1984), This extensive citation list indicates very clearly the great breadth and depth of literature on biological impedance determinations. What take-home messages may one distil from this work?

In general, it has become usual to point out that biological cells and tissues exhibit three broad and more-or-less separable dielectric dispersions, centred respectively in the audio-, radio- and UHF-frequency regions and referred to as the $\alpha$-, $\beta$ - and $\gamma$-dispersions, Subsidiary $\delta$ - and $\beta_{1}$-dispersions, located between the $\beta$-and $\gamma$-dispersions, may also be noted, especially in protein 
solutions (Essex et al. 1977; Grant 1982), whilst a low-frequency $\mu$-dispersion was described by Kell (1983), Harris et al. (1984), and Harris and Kell $(1985 a)$. The major mechanisms thought to underlie these dispersions are as follows: relaxation of the ion cloud tangential to charged membrane surfaces ( $\alpha$-dispersion); Maxwell-Wagner-type relaxation at the interface between the poorly conducting cell membranes and their adjacent aqueous solutions ( $\beta$-dispersion); rotation of small charged and/or dipolar molecules ( $\gamma$-dispersion); relaxation of tissue-bound water ( $\delta$-dispersion); protein rotation ( $\beta_{1}$-dispersion) and diffusional movements of membrane-associated components ( $\mu$-dispersion). Where applicable, the superposition principle states that each of these mechanisms is independent and additive, and we would stress that any potential charge or dipole mobility will lead to the existence of a dielectric dispersion. In this sense, dipole rotations are electrically indistinguishable from any other motions such as the hopping of charges between different sites (Jonscher 1975; Lewis 1977; Ngai et al. 1979), and it is therefore obvious that a plethora of molecular mechanisms can in fact underlie the relatively broad dielectric dispersions observed in practice.

As discussed above, we can describe or characterize a dielectric dispersion by its dielectric increment, its 'mean' relaxation time and by the extent of distribution of the relaxation times as embodied in the Cole-Cole $\alpha$. Now, the dielectric increment may be said to constitute the outward and visible sign of a molecular property, the dipole moment (or, for hopping of charges, etc, the effective dipole moment), $\mu$. Dipole moments are traditionally measured in debyes (D), where 1D $=3.33 \times 10^{-30} \mathrm{C} \mathrm{m}$; in other words, since the unit electrical charge $=1.6 \times 10^{-19} \mathrm{C}$, a pair of charges of opposite sign separated by $10^{-10} \mathrm{~m}(1 \AA)$ have a dipole moment of $4.8 \mathrm{D}$. It is the molecular dipole moment that serves to tell us what fraction of the dipoles are actually responding at a given field strength, according to the Langevin function (Fig. 24.9)

$$
L(x)=\operatorname{coth}(x)-1 / x
$$

where $x=\mu E_{1} / k T, E_{1}$ is the local electrical field, $k$ is Boltzmann's constant, and $T$ is the absolute temperature. Since, in complex biological systems especially, we are likely to know only the macroscopic field (i.e. the peak potential difference between the electrodes divided by the distance between them) rather than the local field, it is appropriate to use the former and to add an empirical constant. For the rotation of aqueous globular proteins with a permanent dipole moment, we use the factor $H=5.8$ (obtained from a comparison between theory and experiment for the amino acid glycine) (Oncley 1943), and we have:

$$
\mu=\sqrt{\left(9000 k T \Delta \epsilon^{\prime} / 4 \pi N H C\right)}
$$

where $N$ is Avogadro's number and $C$ is the molar protein concentration. 


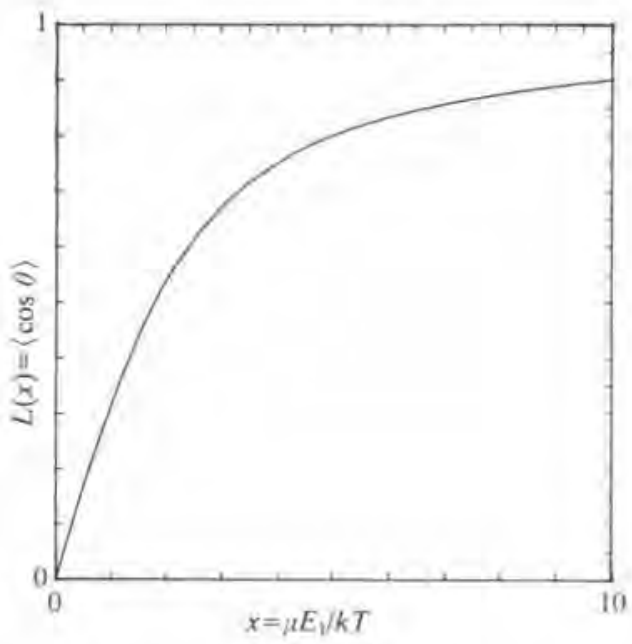

Fig. 24.9 The Langevin function. This relates the average angle between the field (at a low frequency, relative to that of $f$ ) and the (effective) dipole of interest $\langle\cos \theta\rangle$ to the field strength and effective molecular dipole moment $\mu \mathrm{E}_{1} / k T$, where $E_{1}$ is the local field, $k$ is Boltzmann's constant and $T$ the absolute temperature. For $x\left(=\mu E_{1} / k T\right)<$ 1, the Langevin function reduces to $\langle\cos \theta\rangle=\mu E_{1} / 3 k T$, and the dielectric increment is independent of the field strength (i.e. we are in the linear region), the number of particles actually moving in response to the field being proportional to $E_{1}$.

The magnitudes of the dipole moments of protein solutions observed in practice are equivalent roughly to $1-15$ relative permittivity units $(\mathrm{g} / 100 \mathrm{ml})$, corresponding to roughly 5-20 D per kilodalton (e.g. Gerber et al. 1972; Schwan 1981a). One may therefore calculate that, in a typical dielectric experiment in which the field strength is most unlikely to exceed $0.5 \mathrm{~V} / \mathrm{cm}$, and is likely to be as little as $1 / 10$ of this, the Langevin function has a very small value, such that the number of proteins actually rotating is in fact an extremely small fraction of the total. We shall have cause to return to this point later.

To summarize the discussions in this section as they relate to our overall considerations, we may make the following remarks: (1) there is an enormous literature indicating that all types of cells, tissues, and biomolecules possess dielectric properties different from those of simple ionic solution; (2) especially since dielectric spectroscopy is a non-invasive technique, one may exploit it to assay for the former in the presence of the latter; (3) because of the strong frequency-dependence of dielectric properties, one may assay for different substances or features by choosing different frequencies; (4) in such cases, a consideration of where the field lines go is likely to prove informative; (5) because of the relative insensitivity of the 
technique, and the breadth of the spectra obtained (which reflect relaxation rather than resonance), it is likely to be most useful in 'bulk' measurements when practised conventionally. From a bioanalytical standpoint, one must also add that, especially at low frequencies, one is likely also to be measuring the electrode properties, in addition to those of the material between the electrodes, although this does not of itself impair the potential analytical utility of the method. 1 would also mention that a recent and otherwise excellent book Biological Spectroscopy (Campbell and Dwek 1984) did not even mention the concept of dielectric spectroscopy, a rather clear indication that indeed the method is ripe for exploitation.

In this vein, therefore, I turn to a discussion of some of the articles which have sought to use the principles described herein in analytical devices.

\subsection{Some bioanalytical uses of conductimetry and impedimetry}

Obviously this is a vast topic as well, and I shall therefore aim for some selectivity in choosing the examples with which I shall draw attention to the use of these methods. One particular use, which is attracting increasing attention (see Firstenberg-Eden and Eden 1984; Harris and Kell 1985b), is in the exploitation of impedimetry in assessing the numbers of micro-organisms present in sparse populations, since changes in the electrical properties of microbial culture media have been known to be associated with microbial growth since the last century (Stewart 1899b). Conductimetry (e.g. Richards et al. 1978; Mackey and Derrick 1984), impedimetry (e.g. Cady 1978) and capacitimetry (Firstenberg-Eden and Zindulis 1984) have all been used (Firstenberg-Eden and Eden 1984); in the latter case especially, the microorganism-dependent changes are due to effects at the electrodes (Hause et al. 1981), since any micro-organism-dependent changes in the bulk permittivity would here be neglible.

Since the electrical conductivity (at frequencies below that of the Maxwell-Wagner type $\beta$-dispersion) of a suspension is lower than that of the fluid in which it is suspended, one may thus detect the presence of suspended matter directly by its effects upon an electrical field. Such measurements have been made both in bulk suspension (see for example irimajiri et al. 1975; Harris and Kell 1983; Lovitt et al. 1986) and in hydrodynamically focused flowing streams in devices based upon the principle of the Coulter Counter ${ }^{\mathrm{TM}}$ (e.g. Kubitschek 1969; Dow et al. 1979). Clarke and his colleagues (Blake-Coleman et al. 1984; Clarke et al. 1984, 1985) have also successfully applied impedimetry to the direct assessment of microbial biomass, and our own studies and those of others (op. cit.) have indeed shown that the dielectric properties of cells of a given radius, scale monotonically with the volume fraction of the suspended phase.

As regards the possibilities of distinguishing or identifying cells by their 
frequency-dependent dielectric properties, it is certainly true that both the size and surface charge (density), inter alia, differ for different bacteria. For instance, Gram-positive and Gram-negative bacteria have entirely different $\alpha$-dispersions (e.g. Harris and Kell 1985a). However, size and surface charge depend critically on both the $\mathrm{pH}$ and physiological status (e.g. growth rate) of micro-organisms, and simple dielectric spectra are unlikely to contain enough information, in the absence of other tests, to be diagnostic. Similarly, in non-axenic cell suspensions, the dielectric properties of the largest cells will tend to dominate those of the suspension, so that deconvolution, already difficult, would probably be impossible in all but the most favourable cases. However, 1 see no reason in principle why the Coulter Counter ${ }^{\mathrm{TM}}$ method should not be extended to exploit measurements of the frequency-dependent electrical properties of individual cells. In particular (and see later), the magnitude of the electrical fields used would allow one to make use of the non-linear electrical properties of cells, properties which may be expected to be far more cell-specific than simple linear behaviour might lead one to suppose. Thus, although I do not see that the dielectric spectroscopy of microbial cell suspensions is likely to be diagnostic of the specific microorganism (measurement of colonies might be more productive), the use of more advanced techniques does hold out some promise for the characterization of unknown cells. However, since published dielectric spectra of microbial cells do not cover more than ten species (of unknown physiological status) to date, much more work is required before one may make an adequate assessment of the many exciting possibilities in this area.

Other techniques exploiting the bulk permittivity, conductivity, or impedance of cells and tissues, and which have enjoyed a reasonably widespread use, include impedance plethysmography (e.g. Nyboer 1970; Wheeler and Penney 1982; Brown 1983; Anderson 1984) and pneumography (Pacela 1966; Henderson and Webster 1978), whilst measurements of the dielectric properties of excised tissue have been used in the testing of freshness (Faure et al, 1972; To et al. 1974; Kent 1975; Kent and Jason 1975), and quality (Pfutzner and Fialik 1982) of foods. As regards tissue measurements, it may also be mentioned that there are significant local decreases of skin impedance in the area of the meridian points recognized as significant in the science of acupuncture (e.g. Becker and Marino 1982; Jakoubek and Rohlicek 1982), estimations of which, it may well be argued, really constitute biosensing sensu stricto.

Obviously, measurements of the conductance of homogeneous solutions are widely used in environmental monitoring, and are the method of choice in estimating the salinity of the marine environment (see for example Brown 1968; Ben-Yaakov 1981; Wilson 1981). Similarly, resistivity methods have also enjoyed use in geophysical prospecting (Keller and Frischknecht 1966), although the physical and mechanistic interpretation of the data is by no 
means free of difficulty (Hasted 1973; Phillips 1984). It may also be mentioned that the time resolution of solution conductivity measurements may be made extremely good by using microwave frequencies (de Haas and Warman 1982). Schügerl (1984) gives a useful discussion of an elegant conductimetric method for monitoring bubble size and velocity distribution in microbial fermenters (and see later), whilst the utility of impedimetry in the monitoring of chromatographic eluents is discussed, for instance, by Alder et al. (1984).

As regards conductimetry in biosensors generally, Lowe $(1984,1985)$ and Ballot et al, (1984) have recently stressed that a great many of the reactions exploited in potentiometric and amperometric enzyme electrodes, for instance the urea-dependent $\mathrm{pH}$ and $\mathrm{pI}$ change in urease-containing electrodes, might be equally or better assessed conductimetrically. Similarly, Arwin and colleagues (1982) have made use of enzyme reaction-dependent changes in the double-layer capacitance of symmetrical metal electrodes as a measure of enzyme or substrate activities. Workers tend to make such measurements at a single frequency, and it goes without saying that yet more selective and informative sensors might be based upon multiple-frequency methods.

Finally, we may mention the use of conductimetry in improving the response time (Powley et al. 1980) and selectivity (Powley and Nieman 1983) of ion-selective electrodes that are normally used in a potentiometric mode. As one would expect from the properties of electrode impedances described above, there is an optimal time (frequency) window for these measurements, in this case a delay of $0.1 \mathrm{~ms}$ between the stimulus and the measurement of the response being used.

Naturally one could give many, many more examples of the above type. However, what I wish to convey is that by choosing appropriate frequencies and/or analyte matrices, a great many determinants may be monitored in real time and non-invasively by the use of impedimetry in various embodiments, and that the predominant response may be due to the behaviour of the electrode, of the bulk solution, or of the interfacial region. This concept brings us to a brief discussion of some technical aspects of this type of measurement.

\subsection{The realization of impedimetric systems}

I have not thus far laid much stress upon the technical and instrumental considerations underlying impedimetry, since, as far as the typical user is concerned, the methods to be employed follow directly from the underlying principles. Many reviews discuss the measurement of chemical impedances (e.g. Shedlovsky 1949; Blake 1950; Reilley 1954; Loveland 1963; Thomas and Pertel 1963; Pungor 1965; Bennett and Calderwood 1971; Holler and Enke 


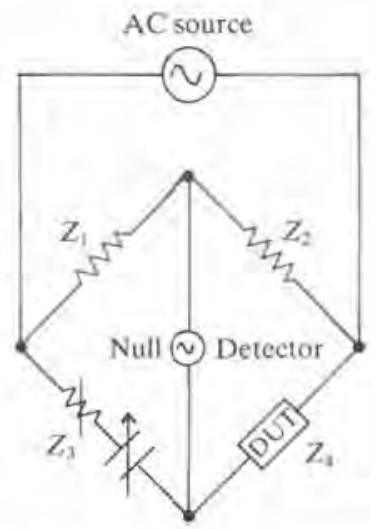

Fig. 24.10 The principle of a two-terminal impedance bridge. The device under test (DUT) forms one arm of the bridge $\left(Z_{4}\right)$, which is in many ways similar to the familiar (DC) Wheatstone bridge, excet that the voltage source is a sinusoidal oscillator of variable frequency, the null detector is $\mathrm{AC}$ sensitive and the adjustable arm of the bridge $\left(Z_{3}\right)$ contains both resistive and capacitive components. When the bridge is balanced (i.e. no current flows in the part of the circuit containing the null detector), and if $Z_{1}=Z_{2}$, then $Z_{3}=Z_{4}$, since, generally, $Z_{1} Z_{4}=Z_{2} Z_{3}$.

1984; and see electrochemical references above) and biological impedances (Schwan 1963; Hasted 1973; Grant et al. 1978; Pethig 1979; Marmarelis and Marmarelis 1978; de Felice 1981) in the range up to $30 \mathrm{MHz}$ or so. I use this frequency criterion because it is roughly here that the wavelength of electromagnetic radiation approaches the dimensions of the measuring system, such that at frequencies greater than this, the lumped circuit description implicit in the above ceases solely to be applicable, and one should also consider a field description based on the Maxwell equations (see for example Bleaney and Bleaney 1976; Lorrain and Corson 1979; Cheng 1981). Similarly, electrode impedances are now negligible. An entrée to the recent literature on these very high frequency methodologies may be gained from the articles by Dawkins et al. 1979, Burdette et al. 1980, Stuchly and Stuchly 1980, Athey et al. 1982; Foster et al. 1982, and Steel et al. 1984; we do not here discuss these matters further.

In the frequency range below $10^{7}$ to $10^{8} \mathrm{~Hz}$ or so, bridge methods (Fig. 24.10) remain the most widely used and are appropriate. Traditionally, manually balanced bridges were used, but modern instruments are computer controlled and auto-balancing. Frequency response analysers provide, albeit at some loss in precision, an extremely convenient means of obtaining dielectric spectra (Morse 1974; Gabrielli 1980). The system illustrated schematically in Fig. 24.11, which is that used by the present author, measures $V_{m}, i_{m}$, and $\theta$ (see Fig. 24.1) by means of a vector voltmeter and ammeter, 


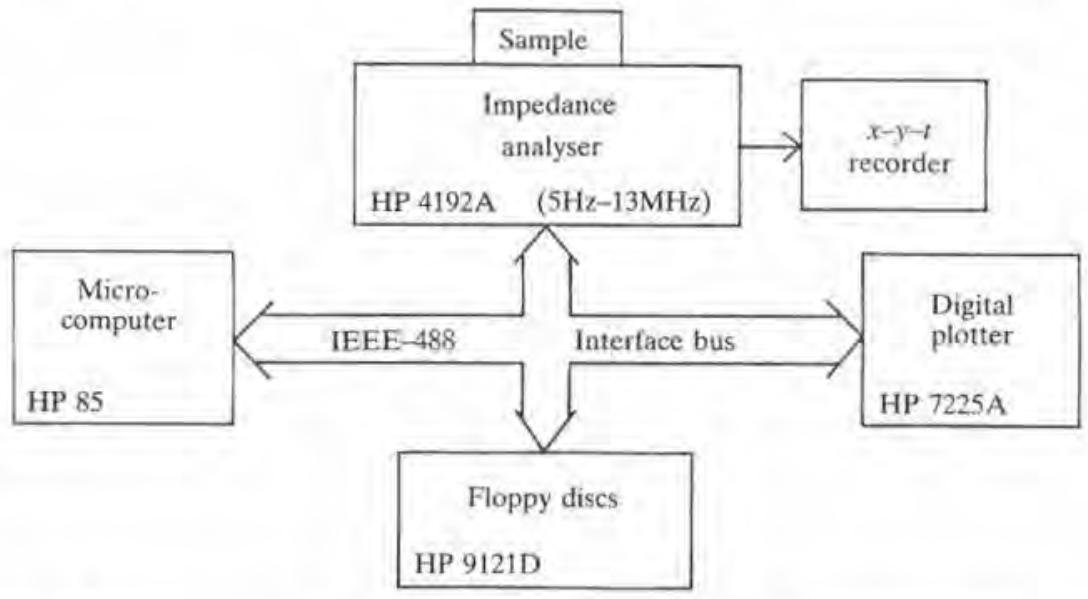

Fig. 24.11 A computer-controlled, frequency-domain dielectric spectrometer, based upon commercially available components and usable in the range $5 \mathrm{~Hz}-13 \mathrm{MHz}$. The microcomputer drives the impedance analyser, stores the data obtained both in RAM and on disc, and permits the data to be plotted in a variety of forms (see Figs 24.4. 24.6, 24.8, and Harris and Kell 1983).

whence all required information may be calculated and displayed. Its implementation of the IEEE-488 standard interface makes it extremely convenient in use, and logarithmic scans may be made at a rate of $6 \mathrm{~s}$ (and 20 measurement frequencies) per decade. In this type of system the sinusoidal frequencies are applied one at a time, and these methods are thus called frequency-domain methods.

In systems of the above type, two-terminal measurements are the more common. However, this means that one is always measuring the impedance of the sample plus the electrodes, and, particularly at low frequencies and high conductance, the latter, which may be of no scientific or analytical interest, can dominate the measurements. In such cases, four-electrode techniques are used (Fig. 24.12), by which electrode polarization problems are in principle avoided (see for example Schwan 1963, 1966, 1968; Schwan and Ferris 1968; Nakamura et al. 1981), although a careful consideration of the exact location of the electrical field lines is necessary (Schwan 1955; Schwan and Ferris 1968). In such cases, the cell constant is determined by the positioning of the voltage electrodes (Tamamushi and Takahashi 1974). The minimization of electrode polarization generally, by using $\mathrm{Pt}$ black electrodes (e.g. Schwan 1963), and the preparation of such electrodes using electrolysis in Kohlrausch solution (Geddes 1972), are discussed elsewhere.

In recent years, time-domain methods have become popular. In this type of approach, one applies a step voltage to the sample and follow, depending 


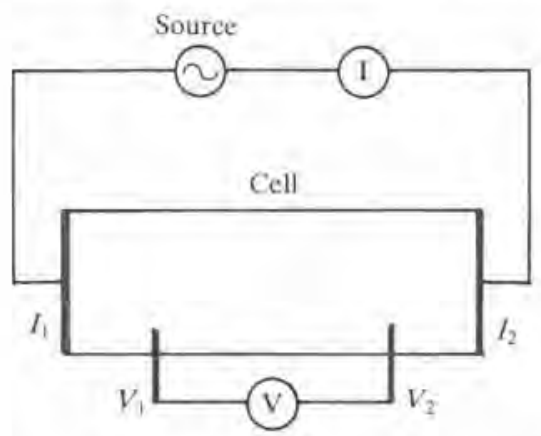

Fig. 24.12 The principle of the four-electrode technique for measuring bulk, lowfrequency impedances with minimal interference from the impedance of electrode/electrolyte interfaces. Current from the AC source is measured with an ammeter and flows through the system via two current electrodes $\left(I_{1}\right.$ and $\left.I_{2}\right)$. The voltage drop across the relevant part of the system is measured using two voltage 'pick-up' electrodes ( $V_{1}$ and $\left.V_{2}\right)$, connected to a voltmeter of high input impedance, such that negligible current flows through them and thus no electrode polarization impedance is measured (see Schwan and Ferris 1968; Ferris 1974).

upon the frequency range, the time-dependent (dis)charging current flow, or the wave behaviour, of the equivalent $R C$ circuit. Deconvolution of such data, usually by use of the fast Fourier transform (see later), gives the equivalent frequency-dependent dielectric properties. Such methods are of value at both high frequencies (see for example Cole 1975; Dawkins et al. 1979; Stuchly and Stuchly 1980; Burdette et al. 1980; Boned and Peyrelasse 1982; Steel et al. 1984) and low frequencies (e.g. Singh et al. 1979; Eden et al. 1980; Hart 1982; Schmukler and Pilla 1982; Mopsik 1984).

This concept, of the equivalence of the time- and frequency-domain behaviour of a system, leads us finally and naturally to the idea that we might broadly use an input wave-form of any shape in order to assess the passive electrical properties of a system, and this is in fact to a good approximation true. We will therefore include an introductory section on modern methods of signal analysis.

To summarize this section, we would stress again (i) that care must always be taken to be sure of the extent to which electrode polarization is contributing to the measured biological impedances, and (ii) that one should properly be aware of the pathways taken by the field lines between the electrodes.

\subsection{Spectral analysis as an integral element of biosensing}

The means most commonly used, in the general case, to analyse the frequency dependence of the response of a system to an input wave-form (e,g. Jenkins 
and Watts 1968; Priestley 1981) are the same as those used in the proper characterization of any time-dependent signal or 'time series' (e.g. Bendat and Piersol 1971; Box and Jenkins 1976; Chatfield 1984). In particular, they exploit transform techniques such as the Fourier transform (e.g. Champeney 1973; Bloomfield 1976; Bracewell 1978; Marshall 1978, 1982, 1983) and, whilst yet more advanced approaches and treatments may be mentioned (e.g. Childers 1978; Kay and Marple 1981; Chen 1982a,b; Fu 1982; Ahmed and Natarajan 1983; Geckinli and Yavuz 1983), we shall confine our short discussion to the more standard approaches that may be applied to linear, stationary, or periodic (quasi-)ergodic systems.

Any periodic signal $x(t)$ (of period $T$ ) may be represented by a Fourier series, which may be written thus:

$$
x(t)=\sum_{-\infty}^{+\infty} C_{n} \mathrm{e}^{\mathrm{j} 2 \pi j t}
$$

where

$$
C_{n}=\frac{1}{T} \int_{-T / 2}^{T / 2} x(t) \mathrm{e}^{-j 2 \pi f t} \cdot \mathrm{d} t
$$

and where $\mathrm{j}=\sqrt{-1}$ and the 'fundamental frequency' $f=1 / T$.

The Fourier series may also be written

$$
\begin{aligned}
S_{x}(f)= & F[x(t)]=X_{0}+X_{1}(\cos 2 \pi f t+\mathrm{j} \sin 2 \pi f t) \\
& +X_{2}(\cos 4 \pi f t+\mathrm{j} \sin 4 \pi f t)+\ldots \ldots \\
& +X_{n}(\cos 2 n \pi f t+\mathrm{j} \sin 2 n \pi f t)
\end{aligned}
$$

For non-periodic data, a continuous spectral representation must be obtained from a Fourier integral, given by

$$
X(f)=\int_{-\infty}^{+\infty} x(t) \mathrm{e}^{-\mathrm{j} 2 \pi f} \cdot \mathrm{d} t .
$$

These equalities thus relate signals in the time domain to those in the frequency domain, and show that any signal may be represented as a sum of sinusoids of defined frequency, amplitude, and phase.

If we take an apparently 'random' signal, such as that in the top half of Fig. 24.13a, we may wish to characterize it further, and to decide, for instance, to what extent if any it may differ materially from that of another, apparently equally random, signal such as that in the top half of Fig. 24.13b. A convenient means by which this may be accomplished is by determining the autocorrelation function $R_{x}(\tau)$, which measures the degree to which a signal correlates with a displaced replica of itself: 

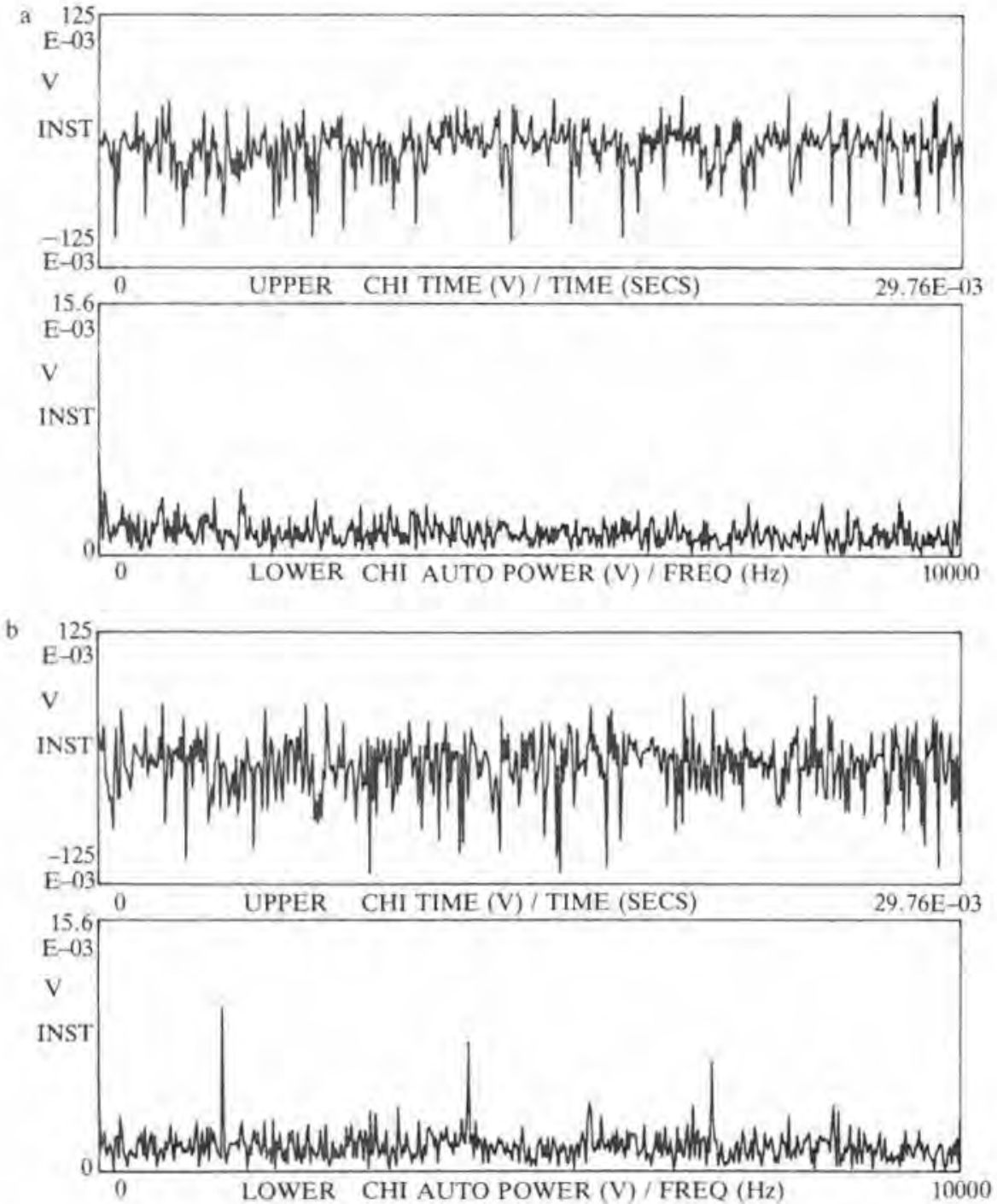

Fig. 24.13 Two 'random' signals and their autopower spectra. The upper half of each figure contains the time history of the signal, whilst the lower half is the autopower spectrum (see text) of the data. It is clear that, whilst the degree of 'randomness' in the original data is apparently similar in the two cases, the autopower spectra reveal that the signal in b has a substantial component centred at a frequency of about $1.4 \mathrm{kHz}$. In fact, the signals are constituted in each case by the output of a 'white noise' generator, that in $b$ being mixed with the output of a sinusoidal oscillator operating at $1420 \mathrm{~Hz}$. The data were analysed using a Solartron 1200 Signal Processor and plotted using a Hewlett-Packard 7470 digital plotter. 


$$
R_{x}(\tau)=\lim _{T \rightarrow \infty} \frac{1}{T} \int_{0}^{T} x(t) \cdot x(t+\tau) \cdot \mathrm{d} t
$$

At zero time displacement $(\tau=0)$, the value of $R_{x}(\tau)$ equals the mean square value of the signal $x(t)$. A 'purely' random signal ('white noise') has an autocorrelation function that is independent of the value of $t$. Such functions have found use in the on-line estimation of the time constant of electrodes (Turner and Howell 1984).

The autocorrelation function is the inverse Fourier transform of the autopower spectrum $G_{x}(f)$, i.e. $R_{x}(\tau)=F^{-1}\left[G_{x}(f)\right]$, and describes the general frequency composition of a time series in terms of the spectral density of its mean square value:

$$
G_{x}=\lim _{\Delta t \rightarrow 0} \frac{1}{(\Delta f)}\left[\lim _{T \rightarrow \infty} \frac{1}{T} \int_{0}^{T} x^{2}(x, t, \Delta f) \cdot \mathrm{d} t\right] .
$$

(The autocorrelation function and autopower spectra thus ignore phase relations). Figure 24.13 illustrates the utility of the autopower spectrum in 'picking out' a periodic signal from a noisy set of data; whilst one would be hard pressed to perceive any analytical use for data as noisy as those in the time domain representations, the autopower spectra clearly show that signal $\mathrm{b}$ indeed contains a significant component with a frequency of $1.4 \mathrm{kHz}$ or so (plus harmonics), and in this area of the spectrum the signal:noise is quite acceptable for analytical usage.

The above analyses have considered single signals alone. We may also define a cross-correlation function $R_{x y}(\tau)$ between signals $x(t)$ and $y(t)$, such that

$$
R_{x y}(\tau)=\lim _{T \rightarrow \infty} \frac{1}{T} \int_{0}^{T} x(t) \cdot y(t+\tau) \cdot \mathrm{d} t
$$

This function tells us the extent to which one signal correlates with another, and is the inverse Fourier transform of the so-called cross-power spectrum $G_{x y}(f)$, i.e.

$$
R_{x y}(\tau)=F^{-1}\left[G_{x y}(f)\right] .
$$

Finally, we may use these concepts to define the transfer function of a system, $H(f)$, which serves to define the input/output relationship of a generalized transmission system. Thus, if in a test system such as that of Fig. 24.1a, the input signal $x(t)$ has an autopower spectrum $G_{x}(f)$, and the output signal $y(t)$ is so modified by the system that the cross-power spectrum is $G_{x y}(f)$, then 


$$
H(f)=G_{x y}(f) / G_{x}(f) .
$$

In principle, therefore, any input signal might therefore be used to obtain the transfer function, and hence the impedance, since in this case the transfer function may also be defined (notation as in Figs. 24.1 and 24.2) as

$$
Z(f)=|Z(f)| \mathrm{e}^{\mathrm{j} \theta} \text {. }
$$

In practice, certain wave-forms are favoured, for reasons connected with the measuring time (Creason et al. 1973; Gabrielli et al. 1982); similarly, accuracy is improved by stressing frequency components related to the relaxation times of the system under study (one might here imagine the exploitation of an iterative system (see also Kell and Harris 1985a)). Nevertheless, despite the need to average, the 'pseudo-random' input remains popular in neurophysiological (e.g. Marmarelis and Marmarelis 1978; De Felice 1981; Fernández et al. 1984) and dielectric (Nakamura et al, 1981) work, and is that exploited in Fig. 24.13.

We may therefore state that this type of analysis is already extremely important and useful, and will become increasingly cheap, widespread, and significant as digital electronic technology advances. (All the spectral functions are implemented in a hard-wired form and in real time in the system used to construct Fig. 24.13). Although I have included a discussion of these matters because they naturally complement the concept of admittance spectroscopy, I would stress that spectral analysis in general, i.e. what is often referred to as 'pattern recognition', should be considered as an integral design goal by all workers actively developing biosensing devices. Although these methods have been used for many years in photometric systems (e.g. Berne and Pecora 1976), and Fourier techniques are widely used in NMR and IR spectroscopy, ete. (e.g. Marshall 1983; Campbell and Dwek 1984), I do not as yet perceive their exploitation in biosensing systems on the wide scale that their potency merits. Therefore, and although the applicability of these techniques to fermentation technology constitutes our own main present direction, I will end by describing two possible general uses of fluctuation or spectral analysis.

\subsection{Conductimetric correlation functions in the assessment of two- phase flows in bioreactors}

Many systems, such as laboratory and industrial bioreactors and fermenters, exhibit highly complex and multiphase fluid dynamics (e.g. Bryant 1977). Leaving aside, for the present, particulate matter and biological cells, such systems may broadly be modelled as consisting of a heterogeneous suspension of non-conducting gas bubbles in an aqueous ionic solution. When stated thus, it is evident that conductivity (or impedimetry generally) can 
provide a convenient approach to the measurement of the passage of gas bubbles, on a similar principle to that employed in the Coulter Counter ${ }^{\mathrm{TM}}$ (see Harris and Kell 1985b). In particular, the use of more than one probe in a bioreactor allows the estimation of the cross-correlation and/or coherence functions of the conductivity fluctuations between probes, a direct indication not only of bubble size and dynamics but of bubble velocity (Bucholz and Schügerl 1979a,b; Schügerl 1984; Sekoguchi et al. 1984). Spectral analysis of pressure fluctuations has also been used to gain otherwise-unobtainable, and real-time, information on the mixing dynamics in two-phase bioreactors (Gerson 1980).

Extending such ideas, we may state (accurately) that the 'problem of scaleup' (e.g. Lilly 1983) is largely ascribable to the fact that conventional measuring practice considers only the mean, and not the (rapid) fluctuations about the mean, of signals derived from probes. It should be obvious that the proper characterization of the 'state' of a culture, by means of environmental measurements, thus requires the full characterization of the time-dependent behaviour of such measurements, including their fluctuations. It is our view that this area in particular represents one of the most fruitful in which future progress may be expected.

Now, whilst the type of signal analysis discussed in this section relies upon the assessment of signals generated by macroscopic probes in microbial fermenters, we wish finally and speculatively to discuss a potentially novel approach to biosensing sensu stricto, based upon the measurement of nonlinear electrical transfer functions in relatively microscopic proteinaceous systems.

\subsection{Use of the multi-dimensional dielectric spectrum of intramolecular protein motions in biosensing devices}

The overwhelming majority of biosensing devices proposed or realized to date rely upon the juxtaposition of an enzyme (or protein) and either a potentiometric or an amperometric electrode. What I wish to discuss here is the possibility of exploiting the specific, non-faradaic and non-linear electrical behaviour of proteins that are bound (or adjacent) to electrodes.

It is now becoming widely recognized that the atoms of even protein crystals, let alone aqueous solutions of globular proteins, exhibit many and complex fluctuations about their mean or average positions, even when at thermodynamic equilibrium (above $0 \mathrm{~K}$ ) (reviews: Welch et al, 1982; Somogyi et al. 1984; Welch 1986; and references therein). Such intramolecular fluctuations are not wholly independent from each other (Kell and Hitchens 1983). Further, since proteins contain numerous charged and dipolar species, it is to be expected that the intramolecular mobilities of such groups will be (i) protein specific and (ii) changed upon substrate (ligand) 
binding, enzymatic activity, or energy transduction (Welch and Kell 1985), so that a non-invasive dielectric spectroscopic assessment of protein dynamics might form the basis of an entire family of novel biosensing devices (since this principle would apply to any protein-ligand(protein) interaction). However, since the (linear) dielectric dispersions exhibited by proteins are rather broad (reflecting, presumably, the numerous underlying processes contributing to the macroscopic observables), the problem reduces to that of signal handling, i.e. to deconvoluting the dielectric spectra. To approach this, we propose (i) to exploit the non-linear dielectric properties of proteins (or indeed any other macromolecule) and (ii) to exploit two- (or multi-) dimensional analysis of the electrical transfer functions of protein-ligand systems. We shall also need to consider the appropriate frequency range for maximizing the protein specificity of the signal.

Now, as discussed above, the fraction of charges or dipoles actually moving in response to an electrical field of the appropriate frequency is given by the Langevin function (see Fig. 24.9), so that, in calculating the fields necessary to drive at least say $80 \%$ of a given type of dipole to its extreme position we require that $\mu E_{1} / k T$ exceeds 5 , a value significantly outside the linear domain (see Fig. 24.9). Since many of the effective intramolecular dipole moments in which our interest lies probably do not exceed say 5 charge-A (24 D), and since for $\mu E_{1} / k T=5$ we require (at $298 \mathrm{~K}$ ) a field of $6.159 \times 10^{9} \mathrm{~V} \mathrm{~m}^{-1} \mathrm{D}^{-1}$ (Kell and Harris 1985a), the type of field we are likely to require is of the order of $2.5 \times 10^{8} \mathrm{~V} / \mathrm{m}$. Thus, to keep the voltages small, or at least realistic, we must use electrodes separated by as small a distance as possible, a suitable design being that of intercalated or comb electrodes (e.g. on a silicon substrate) (Fig. 24.14), as used for instance in the Eumetric ${ }^{\text {TM }}$ system (Micromet Instruments Inc, Cambridge, MA 02139, USA) for lowfrequency permittivity measurements.

Now, because of the protein-specific intramolecular connections of the different charged and dipolar groups, the imposition of a field (such that $\mu E_{1} / k T$ is greater than say 5 ) at one frequency will measurably affect the dielectric properties measured at another frequency. Thus, by measuring the frequency-dependent dielectric properties as a function of the frequency of a high electric field, we may seek to deconvulate the intramolecular electrical properties, in much the same spirit as NMR spectroscopists measure the socalled J-and NOE-connectivities or cross-relaxation pathways of NMRactive nuclei by two-dimensional techniques (e.g. Kumar et al. 1980; Jardetsky and Roberts 1981; Winter and Kimmich 1982; Wuthrich 1982; Campbell and Dwek 1984; Markley et al. 1984). In other words, one would excite (with a high field intensity $\left(E_{1}\right)$ ) at one frequency $\left(f_{1}\right)$ and interrogate (with a field $\left.E_{11}\right)$ at other frequencies $\left(f_{2}\right)$, either simultaneously $(t=0)$ or subsequently $(t>0)$, with $f_{1}$ and $f_{2}$ and/or $t$ and perhaps also $E_{1}$ and $E_{11}$ being varied throughout. What sort of frequencies should we consider? 


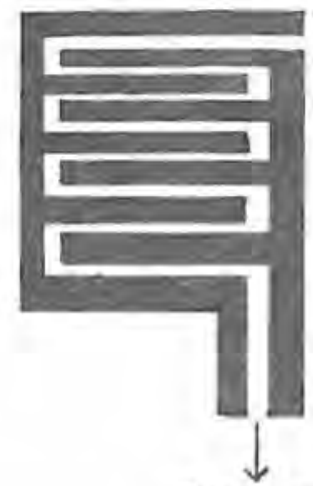

To generator and analyser

Fig. 24.14 The principle of using comb electrodes to give a high field for a reasonably low voltage, whilst covering a reasonable surface area (and thereby lowering the impedance). The alternating comb electrodes (seen here from above) are closely spaced (say $1 \mu \mathrm{m}$ or less), and attached to the signal-generating and signal-analysing circuitry. The protein or biological component of interest is placed over the surface of the device, either by covalent attachment or otherwise, and the ligand-dependent change in the multi-dimensional dielectric spectrum assessed. Available variables for obtaining the multi-dimensional matrix include (see text) $f_{1}, f_{2}, E_{1}, E_{\mathrm{II}}$, and $t$.

We might expect that many of the most interesting intramolecular relaxations would lie at frequencies in the more technically difficult range above $1 \mathrm{MHz}$ or so, not least because simple protein rotation is likely to dominate the measured spectra below this frequency. However, increasing the local solvent viscosity, e.g. with phospholipids, or chemical cross-linking of electrode-associated enzyme molecules, would serve to lower the appropriate frequency range. Notwithstanding, at the lower frequencies a significant contribution from double layer and faradaic electrode processes would be observed, and whilst this does not affect the pattern-recognition approach per se, it seems likely that the biospecific signal/noise ratio of such a device will be greater the greater the contribution from the protein dynamics.

The exact features of such multi-dimensional dielectric spectra which are likely to prove of most bioanalytical value can not easily be defined at the present time. However, it is easy to predict that a difference spectrum of protein-plus-ligand minus protein alone is likely to give the best type of definition of the ligand-selective signal analysis required, whether the biological responses in vivo are a function of the occupancy or the rate of occupancy of the proteinaceous receptor in question. Similarly, whilst I do not in any way underestimate the technical difficulties involved, one should state that if proteins recognize (bind to) ligands and each other by means of 
such frequency-dependent electrical processes, there is no fundamental reason why we should not do so as well.

The possibility of placing such a device, of the type alluded to herein, on an electrophoretic gel and therewith identifying a protein or nucleic acid in a band or a spot, seems sufficient justification alone to cause one further to explore the development of such a principle.

\section{Acknowledgement}

1 thank the Science and Engineering Research Council for financial support, Christine Harris and Professor Gareth Morris for useful discussions, Anthony Pugh for photographic assistance, and Sian Evans for typing the manuscript.

\section{References}

Adey, W. R. (1981). Tissue interactions with nonionising electromagnetic fields. Physiol. Rev. 61, 435-514.

Ahmed, N. and Natarajan, T. (1983). Discrete-time signals and systems. Reston Publishing, Reston, Virginia, USA.

Alder, J. F., Fielden, P. R. and Clark, A. J. (1984). Simultaneous conductivity and permittivity detector with a single cell for liquid chromatography. Anal. Chem.56, 985-8.

Anderson, F. A. Jr. (1984). Impedance plethysmography in the diagnosis of arterial and venous disease. Ann. Biomed. Eng. 12, 79-102.

Archer, W. I. and Armstrong, R. D. (1980). The application of A.C. impedance methods to solid electrolytes. In Electrochemistry (ed. H. R. Thirsk), Vol. 7, pp. 157-202. Specialist Periodical Reports, The Chemical Society, London.

Arwin, H., Lundström, I. and Palmqvist, A. (1982). Electrode adsorption method for determination of enzymatic activity. Med. Biol. Eng. Comput. 20, 362-74.

Asami, K. and Irimajiri, A. (1984). Dielectric dispersion of a single spherical bilayer membrane in suspension. Biochim. Biophys. Acta 769, 370-6.

- Hanai, T. and Koizumi, N. (1976). Dielectric properties of yeast cells. J, Membr. Biol, 28, 169-180.

— and Koizumi, N. (1980a). Dielectric approach to suspensions of ellipsoidal particles covered with a shell, in particular reference to biological cells. Jap. $J$. Appl. Phys, 19, 359-65.

- and Koizumi, N, (1980b). Dielectric analysis of Escherichia coli in the light of the theory of interfacial polarisation. Biophys. J. 31, 215-28.

- Irimajiri, A., Hanai, T. Shiraishi, N. and Utsumi, K. (1984). Dielectric analysis of mitochondria isolated from rat liver. I. Swollen mitoplasts as simulated by a single-shell model. Biochim. Biophys. Acta 77i, 559-69.

Athey, T. W., Stuchly, M. A. and Stuchly, S. S. (1982). Dielectric properties of biological substances at radio frequencies. Part 1. Measurement method. IEEE Trans. Microwave Theory Tech. MTT-30, 82-6. 
Ballot, C. Saizonou-Manika, B., Mealet, C., Favre-Bonvin, G. and Wallach, J. M. (1984). Conductimetric measurements of enzyme activities, Anal. Chïm. Acta 163, 305-8.

Bard, A, J. and Faulkner, L. R. (1980). Electrochemical methods. Wiley, Chichester.

Becker, R. O. and Marino, A. A. (1982). Electromagnetism and life. State University of New York Press, Albany.

Bendat, J.S. and Piersol, A. G. (1971). Random data: analysis and measurement procedures. Wiley-Interscience, New York.

Bennett, R. G. and Calderwood, J.H. (1971). Experimental techniques in dielectric studies. In Complex permittivity (ed. B.K.P. Scaife), pp. 112-70. English University Press, London.

Ben-Yaakov, S. (1981), Electrochemical instrumentation. In Marine electrochemistry (eds M. Whitfield and D, Jagner), pp. 99-122. Wiley, Chichester.

Berne, B. J, and Pecora, R. (1976). Dynamic light scattering, Wiley-Interscience, New York.

Besenhard, J.O. and Fritz, H.P. (1983). The electrochemistry of black carbons. Angew. Chem. Int. Ed. 22, 950-75.

Blake, G. G. (1950). Conductimetric analysis at radio-frequency. Chapman and Hall, London.

Blake-Coleman, B. C., Calder, M. R., Carr, R. J. G., Moody, S. C. and Clarke, D. J. (1984). Direct monitoring of reactor biomass in fermentation control. Trends Anal. Chem. 3, 229-35.

Bleaney, B. I, and Bleaney, B. (1976), Electricity and magnetism, (3rd edn), Oxford University Press, Oxford.

Bloomfield, P. (1976). Fourier analysis of time series. An introduction. Wiley, New York.

Bobrow, L.S. (1981). Elementary linear circuit analysis. Holt, Rinehart and Winston, New York.

Bockris, J. O’M. and Reddy, A.K. N. (1970). Modern electrochemistry, Vols. I and 2. Plenum Press, New York.

Bond, A. M. (1980). Modern polarographic methods in analytical chemistry. Marcel Dekker, New York.

Boned, C. and Peyrelasse, J. (1982). Automatic measurement of complex permittivity (from $2 \mathrm{MHz}$ to $8 \mathrm{GHz}$ ) using time-domain spectroscopy, J. Phys, E. Sci. Instr. 15, 534-8.

Box, G. E.P. and Jenkins, G.M. (1976). Time series analysis: forecasting and control. Revised Edition. Holden-Day, Oakland, California.

Boyd, R. H. (1980). Dielectric constant and loss. In Methods of experimental physics (ed. R. A. Fava), Vol, 16C, pp. 379-421. Academic Press, New York.

Bracewell, R.N. (1978). The Fourier transform and its applications (2nd edn). McGraw-Hill Kogakusha, Tokyo.

Breyer, B. and Bauer, H. (1963). Alternating current polarography and tensammetry. Wiley-Interscience, New York.

Brown, B. H. (1983). Tissue impedance methods. In Imaging with non-ionizing radiations (ed. D. F. Jackson), pp. 85-110. Surrey University Press, Guildford.

Brown, N. L. (1968). An in situ salinometer for use in the deep ocean. In Marine sciences instrumentation (ed. F. Alt), Vol. 4, pp. 563-77. 
Brown, P. B., France, G, N. and Moraff, H. (1982), Electronics for the modern scientist. Elsevier, Amsterdam.

Bryant, J. (1977). The characterization of mixing in fermenters. Adv. Biochem. Eng. $5,101-23$.

Bucholz, R. and Schügerl, K. (1979a). Bubble column reactors. 1. Methods for measuring the bubble size. Eur. J. Appl. Microbiol. Technol. 6, 301-13.

- $(1979 b)$. Methods for measuring the bubble size in bubble column bioreactors. II. Eur. J. Appl. Microbiol. Biotechnol. 6, 315-23.

Buck, R.P. (1982). The impedance method applied to the investigation of ionselective electrodes. Ion-Selective Electrode Rev. 4, 3-74.

Burdette, E. C., Cain, F. L. and Seals, J. (1980). In vivo probe measurement technique at VHF through microwave frequencies. IEEE Trans. Microwave Theory Tech. MTT-28, 414-27.

Cady, P. (1978). Progress in impedance measurements in microbiology. In Mechanizing microbiology (eds. A. N. Sharpe and D.S. Clark), pp. 199-239. Charles C. Thomas, Springfield, Illinois.

Campbell, L.D. and Dwek, R, A. (1984). Biological spectroscopy. BenjaminCummings, London.

Cartensen, E. L. and Marquis, R. E. (1975). Dielectric and electrochemical properties of bacterial cells. In Spores VI (eds. P. Gerhardt, R, N. Costilow and H. L. Sadoff), pp. 563-71. American Society for Microbiology, Washington, D.C.

Champeney, D.C. (1973). Fourier transforms and their physical applications. Academic Press, New York.

Chat field, C. (1984). The analysis of time series: an introduction (3rd edn). Chapman and Hall, London.

Chen, C. H. (1982a) (ed.). Digital waveform processing and recognition. CRC Press, Boca Rabon, Florida.

- $(1982 b)$ (ed.). Nonlinear maximum entropy spectral analysis methods for signal recognition. Research Studies Press, Chichester.

Cheng, D. K. (1983). Field and wave electromagnetics. Addison-Wesley, London. Childers, D. G. (1978) (ed.). Modern spectrum analysis. IEEE Press, New York.

Clarke, D. J., Blake-Coleman, B. C., Calder, M. R., Carr, R. J. G. and Moody, S. C. (1984). Sensors for bioreactor monitoring and control - a perspective. $J$. Biotechnol. 1, 135-58.

- Calder, M. R., Carr, R. J. G., Blake-Coleman, B. C. and Moody, S. C. (1985). The development and application of biosensing devices for bioreactor monitoring and control. Biosensors J. 1, 213-320.

Clegg, J.S., McClean, V. E. R., Szwarnowski, S. and Sheppard, R. J. (1984). Microwave dielectric measurements $(0.8-70 \mathrm{GHz})$ on Artemia cysts at variable water content. Phys. Med. Biol. 29, 1409-19.

- Szwarnowski, S., McClean, V. E. R., Sheppard, R. J. and Grant, E. H. (1982). Interrelationships between water and cell metabolism in Artemia cysts. X. Microwave dielectric studies. Biochim. Biophys. Acta 721, 458-68.

Cole, K. S. (1972). Membranes, ions and impulses. University of California Press.

Cole, R. H. (1975). Evaluation of dielectric behaviour by time domain spectroscopy.

1. Dielectric response by real time analysis. J. Phys. Chem. 79, 1459-69.

Cole, K.S. and Cole, R.H. (1941). Dispersion and absorption in dielectrics. 
1. Alternating current characteristics. J. Chem. Phys, 9, 341-51.

Creason, S. C., Hayes, J. W. and Smith, D. E. (1973). Fourier transform faradaic admittance measurements. III. Comparison of measurement efficiency for various test signal waveforms. J. Electroanal. Chem. Interfacial Electrochem 47, 9-46.

Daniel, V. V. (1967). Dielectric relaxation. Academic Press, London.

Davies, M. (1965), Some electrical and optical aspects of molecular behaviour, pp. 96-7, Pergamon Press, Oxford.

Dawkins, A. W. J., Sheppard, R. J. and Grant, E. H. (1979). An outline computerbased system for performing time domain spectroscopy. 1. Main features of the basic system. J. Phys. E. Sci. Instrum. 12, 1091-9.

de Felice, L. J. (1981). Introduction to membrane noise. Plenum Press, New York. de Haas, M. P. and Warman, J. M. (1982). Photon-induced molecular charge separation studied by nanosecond time-resolved microwave conductivity. Chem. Phys. $73,35-53$.

Diamond, J, M, and Machen, T, E. (1983). Impedance analysis in epithelia and the problem of gastric acid secretion. J. Membr. Biol. 72, 17-41.

Dow, C.S., France, A. D., Khan, M.S. and Johnson, T. (1979). Particle size distribution analysis for the rapid detection of microbial infection of urine. $J$. Clin. Pathol. 32, 386-90.

Duffin, W. J. (1980). Electricity and magnetism, (3rd edn.). McGraw-Hill, London.

Dukhin, S. S. and Shilov, V. N. (1974). Dielectric phenomena and the double layer in disease systems and polyelectrolytes. Wiley, Chichester.

Eden, J., Gascoyne, P.R, C, and Pethig, R, (1980). Dielectric and electrical properties of hydrated bovine serum albumin. JCS Faraday I, 76, 426-34.

Essex, C.G., Symonds, M.S., Sheppard, R.J., Grant, E. H., Lamotte, R., Soetewey, F., Rosseneu, M. Y. and Peeters, H. (1977). Five-component dielectric dispersion in bovine serum albumin solution. Phys. Med. Biol. 22, 1160-7.

Falk, G. and Fatt, P. (1968). Passive electrical properties of rod outer segments. $J$. Physiol. 198, 627-46.

Faure, N., Flachat, C., Jenin, P., Lenoir, J. Roullet, C. and Thomasset, A. (1972). Contribution a l'etude de la tendrete et de la maturation des viandes par la methode de la conductibilite electrique en basse et haute frequence. Rev. Med. Vet. 123, $1517-27$.

Fernández, J. M., Neher, E. and Gomperts, B. D. (1984). Capacitance measurements reveal stepwise fusion events in degranulating mast cells. Nature 312, 453-5.

Ferris, C. D. (1974). Introduction to bioelectrodes. Plenum Press, New York.

Fettiplace, R, , Gordon, L. G. M., Hladky, S. B., Requena, J., Zingsheim, H. P. and Haydon, D. A. (1975). Techniques in the formation and examination of 'black' lipid bilayer membranes. In Methods of membrane biology (ed. E. D. Korn), Vol. 4, pp. 1-75. Plenum Press, New York.

Firstenberg-Eden, R. and Eden, G. (1984). Impedance microbiology. Research Studies Press, Letchworth.

— and Zindulis, J. (1984). Electrochemical changes in media due to microbial growth. J. Microbiol. Methods. 2, 103-15.

Foster, K. R. and Schepps, J. L. (1981). Dielectric properties of tumor and normal tissues at radio through microwave frequencies. J. Microwave Power 16, 107-19.

— and Epstein, B. R. (1982). Microwave dielectric studies on proteins, tissues and 
heterogenous suspensions. Bioelectromagnetics 3, 29-43,

Fu, K.S. (1982) (ed.). Applications of pattern recognition. CRC Press, Boca Raton, Florida.

Gabriel, C., Sheppard, R. J. and Grant, E. H. (1983), Dielectric properties of ocular tissue at $37^{\circ} \mathrm{C}$. Phys. Med. Biol. 28, 43-49.

Gabrielli, C. (1980). Identification of electrochemical processes by frequency response analysis. Solartron Electronic Group, Farnborough.

— and Keddam, M. (1974). Progrès récent dans la mesure des impédances electrochemiques en régime sinusoidal. Electrochim. Acta 19, 355-62.

- and Takenouti, H. (1963). The use of A.C. techniques in the study of corrosion and passivity. In Corrosion: aqueous processes and passive films. Treatise on materials science and technology (ed. J, C, Scully), Vol. 23, pp. 395-451. Academic Press, New York.

- Huet, F., Keddam, M. and Lizee, J.F. (1982). Measurement-time versus accuracy trade-off analysed for electrochemical impedance measurements by means of sine, white noise and step signals. J. Electroanal. Chem. 138, 201-8.

Geckinli, N. C. and Yavuz, D. (1983). Discrete Fourier transformation and its applications to power spectra estimation. Elsevier, Amsterdam.

Geddes, L. A. (1972). Electrodes and the measurement of bioelectric events. WileyInterscience, New York.

Gerber, B. R., Routledge, L. M. and Takashima, S. (1972). Self-assembly of bacterial flagellar protein: Dielectric behaviour of monomers and polymers. J. Mol. Biol. 71, 317-37.

Gerson, D.F. (1980). The pressure fluctuation spectrum as a measure of mixing and emulsification in a biochemical reactor. Eur. J. Appl. Microbiol. Biotechnol. 10, 59-72.

Grant, E. H. (1982). The dielectric method of investigating bound water in biological material: an appraisal of the technique. Bioelectromagnetics 3, 17-24.

(1983). Molecular interpretation of the dielectric behaviour of biological materials. In Biological effects of dosimetry of nonionizing radiation (eds. M. Gandolfo, S. M. Michaelson, and A. Rindi), pp. 179-94. Plenum Press, New York,

— and South, G. P. (1972). Dielectric relaxation of proteins in aqueous solutions. Adv, Mol, Rel. Proc, 3, 355-77.

- Sheppard, R. J. and South, G.P. (1978). Dielectric behaviour of biological molecules in solution. Oxford University Press, London.

Hanai, T., Haydon, D. A. and Taylor, J. (1964). An investigation by electrical methods of lecithin-in-hydrocarbon films in aqueous solutions, Proc, R, Soc, Ser. A, 281, 377-91.

- (1965). Polar group orientation and the electrical properties of lecithin biomolecular leaflets. J. Theoret. Biol. 9, 278-96.

Harris, C.M. and Kell, D. B. (1983). The radio-frequency dielectric properties of yeast cells measured with a rapid, automated, frequency-domain dielectric spectrometer. Bioelectrochem. Bioenerg. 11, 15-28.

- (1985a). On the dielectrically observable consequences of the diffusional motions of lipids and proteins in membranes. 2. Experiments with microbial cells, protoplasts and membrane vesicles. Eur. Biophys. J., 13, 11-24.

- (1985b). The estimation of microbial biomass. Biosensors J. 1, 17-84. 
Hitchens, G. D. and Kell, D. B. (1984). Dielectric spectroscopy of microbial membrane systems. In Charge and field effects in biosystems (eds. M. J. Allen and P.N. R. Usberwood), pp. 179-85. Abacus Press, Tunbridge Wells, UK.

Hart, F. X. (1982). The use of time domain dielectric spectroscopy to characterize the progress of wound repair. J. Bioelectricity 1, 313-28.

Harter, J. H. and Lin, P.Y. (1982). Essentials of electric circuits. Reston Publishing Company, Reston, Virginia USA.

Hasted, J. B. (1973). Aqueous dielectrics. Chapman and Hall, London,

- Husain, S, K., Ko, A. Y,, Rosen, D, Nicol, E. and Birch, J. R. (1983). Excitations of proteins by electric fields. In Coherent excitations in biological systems (eds. H. Frohlich and F. Kremer), pp. 71-83. Springer-Verlag, Heidelberg.

Hause, L. L., Komorowski, R. A. and Gayon, F. (1981). Electrode and electrolyte impedance in the detection of bacterial growth. IEEE Trans, Biomed. Eng. BME-28, 403-10.

Haydon, D. A., Hendry, B. M., Levinson, S. R. and Requena, J. (1977). Anaesthesia by the $n$-alkanes. A comparative study of nerve impulse blockage and the properties of black lipid bilayer membranes. Biochim. Biophys. Acta 470, 17-34.

Henderson, R.P. and Webster, J. G. (1978). An impedance camera for spatially specific measurements of the thorax. IEEE Trans. Biomed. Eng. BME-25, 250-54.

Holler, F, J. and Enke, C, G. (1984). Conductivity and conductimetry. In Laboratory techniques in electroanalytical chemistry (eds. P. T. Kissinger and W. R. Heineman), pp. 235-66. Marcel Dekker, New York.

Hung, B. N., Beard, R. B., Brownstein, M., Dubin, S. E., Niazy, N and Miller, A. J. (1979). Correlation of linear A.C. polarization impedance studies with tissue ingrowth for porous stimulating electrodes. In Electrical properties of bone and cartilage (eds. C. T. Brighton, J. Black and S. R. Pollack), pp, 249-66. Grune and Stratton, New York.

Illinger, K. H. (1981). Electromagnetic-field interaction with biological systems in the microwave and far-infrared region. Physical basis. ACS Symp. Ser. 157, 1-46.

Irimajiri, A, Hanai, T. and Inouye, S. (1975). Evaluation of a conductometric method to determine the volume fraction of the suspensions of biomembranebounded particles. Experientia 31, 1373-74.

- (1979). A dielectric theory of 'multi-stratified shell' model with its application to a lymphoma cell. J. Theoret. Biol. 78, 251-69.

Jack, J. J. B., Noble, D. and Tsien, R. W. (1975). Electric current flow in excitable cells. Clarendon Press, Oxford.

Jakoubek, B. and Rohlicek, V. (1982). Changes of electrodermal properties in the 'acupuncture points' in men and rats. Physiol. Bohem. 31, 143-149.

Jardetzky, O. and Roberts, G. C. K. (1981). Protein dynamics. In NMR in molecular biology, pp. 448-92, Academic Press, New York.

Jenkins, G. M. and Watts, D.G. (1968). Spectral analysis and its applications. Holden-Day, Oakland, California.

Jonscher, A. K. (1975). Physical basis of dielectric loss. Nature 253, 717-19.

Kay, S. M. and Marple, S. L. (1981). Spectrum analysis - a modern perspective. Proc. IEEE 69, 1380-1419.

Kell, D. B, (1983). Dielectric properties of bacterial chromatophores. Bioelectrochem. Bioenerg. 11, 405-15. 
- and Harris, C.M. (1985a). On the dielectrically observable consequences of the diffusional motions of lipids and proteins in membranes. 1. Theory and overview, Eur. Biophys. J., 12, 181-197.

(1985b). Dielectric spectroscopy and membrane organisation. J. Bioelectricity, 4. 317-48.

- and Hitchens, G. D. (1983). Coherent properties of the membranous systems of electron transport phosphorylation. In Coherent excitations in biological systems (eds. H. Fróhlich and F. Kremer), pp. 178-98. Springer-Verlag, Heidelberg.

- and Westerhoff, H.V. (1985). Catalytic facilitation and membrane bioenergetics. In Organised multienzyme systems: catalytic properties (ed. G. R. Welch), pp. 63-139. Academic Press, New York.

Keller, G. V. and Frischknecht, F. C. (1966). Electrical methods in geophysical prospecting. Pergamon Press, Oxford.

Kent, M. (1975). Time domain measurements of the dielectric properties of frozen fish. J. Microwave Power 10, 37-48.

- and Jason, A.C. (1975). Dielectric properties of food in relation to interactions between water and the substrate. In Water relations of foods (ed. R. B. Duckworth), pp. 211-231. Academic Press, London.

Kissinger, P. T. and Heineman, W. R. (1984) (eds.), Laboratory techniques in electroanalytical chemistry. Marcel Dekker, New York.

Kraszewski, A., Stuchly, M. A., Stuchly, S. S. and Smith, A. M. (1982). In vivo and in vitro dielectric properties of animal tissues at radio frequencies. Bioeletromagnelics $3,421-32$.

Kubitschek, H.E. (1969). Counting and sizing microorganisms with the Coulter counter. In Methods in microbiology (eds. J. R. Norris and D. W. Ribbons), Vol. 1, pp. 593-610. Academic Press, London.

Kumar, A., Wagner, G., Ernst, R, R. and Wuthrich, K. (1980). Studies of J-connectivities and selective ${ }^{1} \mathrm{H}-{ }^{-} \mathrm{H}$ Overhauser effects in $\mathrm{H}_{2} \mathrm{O}$ solutions of biological macromolecules by two-dimensional NMR experiments. Biochem. Biophys. Res. Comm. 96, 1156-63.

Laver, D. R., Smith, J. R. and Coster, H. G. L. (1984). The thickness of the hydrophobic and polar regions of glycerol monooleate bilayers determined from the frequency dependence of bilayer capacitance. Biochim. Biophys. Acta 772, 1-9.

Lewis, T. J. (1977). The dielectric behaviour of non-crystalline solids. Diel. Rel. Mol. Proc. 3, 186-218.

Lilly, M. D. (1983). Problems in process scale-up. In Bioactive microbial products 2; Development and production (eds. L. J. Nisbet and D. J. Winstanley), pp. 79-89. Academic Press, London.

Lorrain, P. and Corson, D. R. (1979). Electromagnetism. W. H. Freeman, San Francisco.

Loveland, J. W. (1963). Conductometry and oscillometry. In Treatise on analytical chemistry (eds. 1. M. Kolthoff and P. J. Elving), Vol. 4, pp. 2569-629.

Lovitt, R. W., Walter, R.P., Morris, J. G, and Kell, D. B. (1986). Conductimetric assessment of the biomass content of immobilised (gel-entrapped) microorganisms. Appl. Microbiol. Biotechnol. 23, 168-73.

Lowe, C, R, (1984). Biosensors. Trends Biotechnol. 2, 59-65.

- (1985). An introduction to the concepts and technology of biosensors. Biosensors 1, 3-16. 


\section{The principles and potentiat of electrical admittance spectroscopy}

Macdonald. D. D. (1977). Transient techiques in electrochemistry. Plenum Press, New York.

- and McKubre, M. C. H. (1982). Impedance measurements in electrochemical systems. In Modern aspects of electrochemistry (eds, J. O'M. Bockris and B. E. Conway), Vol. 4, pp. 61-150. Plenum Press, New York.

Macdonald, J. R, (1980), Interface effects in the electrical response of non-metallic conducting solids and liquids, IEEE. Trans, Electr. Insul. EI-15, 65-82.

- Schoonman, J. and Lehnen, A. P. (1982). The applicability and power of complex non-linear least squares for the analysis of impedance and admittance data. J. Electroanal. Chem. 131, 77-95.

Mackey, B.M. and Derrick, C.M. (1984). Conductance measurements of the lag phase of injured Salmonella typhimurium. J. Appl. Bact. 57, 299-308.

Magin, R. L. and Burdette, E.C. (1983). Measurement of electrical properties of tissue at microwave frequencies: a new approach and treatment of abnormalities. In Non-invasive measurements (ed. P. Rolfe), Vol. 2, pp. 353-376. Academic Press, London.

Markley, J, L., Westler, W. M., Tze-Ming Chan, Kojiro, C. L. and Ulrich, E. L. (1984). Two-dimensional NMR approaches to the study of protein structure and function. Fed. Proc. 43, 2648-56.

Marmarelis, P.Z. and Marmarelis, V.Z. (1978). Analysis of physiological systems. The white-noise approach. Plenum Press, New York.

Marshall, A.G. (1978). Biophysical chemistry; principles, techniques and applications. Wiley, New York.

- (1982) (ed.). Fourier, Hadamard and Hilbert transforms in chemistry. Plenum Press, New York.

- (1983). Transform techniques in chemistry. In Physical methods in modern chemical analysis (ed. T. Kuwana), Vol. 3, pp. 57-135. Academic Press, New York.

- and Roe, D. C. (1978). Dispersion versus absorption: spectral line shape analysis for radiofrequency and microwave spectroscopy. Anal. Chem. 50, 756-763.

Martynov, G. A. and Salem, R, R. (1983). Electrical double layer at a metal-dilute electrolyte solution interface. Springer-Verlag, Berlin.

Mohilner, D. M. (1966). The electrical double layer. Part 1. Elements of double layer theory. In Electroanalytical chemistry (ed. A. J. Bard), Vol. 1, pp. 241-409. Edward Arnold, London.

Mopsik, F. (1984). Precision time-domain dielectric spectrometer. Rev. Sci. Instr. 55, 79-87.

Morse, C. T. (1974). A computer controlled apparatus for measuring AC properties of materials over the frequency rane $10^{-5}$ to $10^{5} \mathrm{~Hz}$. J. Phys. E. Sci. Instr. 7, 657-62.

Nakamura, H., Hushimi, Y, and Wada, A. (1981). Time domain measurement of dielectric spectra of aqueous polyelectrolyte solutions at low frequencies. J. Appl. Phys. 52, 3053-61.

Ngai, K. L., Jonscher, A. K. and White, C. T. (1979). On the origin of the universal dielectric response in condensed matter. Nature 277, 185-9.

Nyboer, J. (1970). Electrical impedance plethysmography, (2nd edn.). Charles C. Thomas, Springfield, Illinois. 
O'Brien, R.W. (1982). The response of a colloidal suspension to an alternating electrical field. Adv. Colloid. Interf. Sci. 16, 281-320.

Oncley, J.L. (1943). The electric moments and the relaxation times of proteins as measured from their influence upon the dielectric constants of solutions. In Proteins, amino acids and peptides (eds. E. J. Cohn and J. T. Edsall), pp. 543-568. Reinhold, New York.

Osterhout, W. J. V. (1922). Injury, recovery and death, in relation to conductivity and permeability. J. B. Lippincott, Philadephia and London.

Pacela, A. F. (1966). Impedance pneumography - a survey of instrumentation techniques. Med. Biol. Eng. 4, 1-15.

Pauly, H. (1962). Electrical properties of the cytoplasmic membrane and the cytoplasm of bacteria and of protoplasts. IRE Trans. Biomed. Electron 9, 93-95.

- and Packer, L. (1960), The relationship of internal conductance and membrane capacity to mitochondrial volume, J. Biophys. Biochem. Cytol. 7, 603-12.

- and Schwan, H.P. (1960). Electrical properties of mitochondrial membranes. $J$. Biophys. Biochem. Cytol. 7, 589-601.

Petersen, D.C. and Cone, R.A. (1975). The electric dipole moment of thodopsin solubilised in Triton X-100. Biophys. J. 15, 1181-1200.

Pethig, R. (1979). Dielectric and Electronic Properties of Biological Materials. John Wiley, Chichester.

(1984). Dielectric properties of biological materials: biophysical and medical applications. IEEE Trans. Electr. Insul. EI-19, 453-74.

Pfutzner, H. and Fialik, E, (1982). A new electrophysical method for rapid detection of exudative porcine muscle. $\mathrm{Zbl}$. Vet. Med. A. 29, 637-45.

Phillips, W.J. (1984). Resonance effects in complex resistivity data and their significance in mineral exploration. Trans, Inst. Min. Metall. (Sect. B. Appl. Earth Sci.) 93. BI-11.

Pilla, A.A. (1980). Electrochemical information transfer at cell surfaces and junctions; applications to the study and manipulation of cell regulation. In Bioelectrochemistry (eds. H. Keyser and F. Gutmann), pp. 353-396. Plenum Press, New York.

- Sechaud, P. and McLeod, B. R. (1983). Electrochemical and electrical aspects of low-frequency electromagnetic current induction in biological systems. J. Biol. Phys. 11, 51-58.

Pottel, R., Gopel, K.-D., Henze, R., Kaatze, U. and Uhlendorf, V. (1984). The dielectric permittivity spectrum of aqueous colloidal phospholipid solutions between $1 \mathrm{kHz}$ and $60 \mathrm{GHz}$. Biophys. Chem. 19, 233-44.

Powley, C. R. and Nieman, T. A. (1983). Bipolar pulse conductometric monitoring of ion-selective electrodes. Part 4. Interferences from electroactive species in measurements with the calcium electrode. Anal. Chim. Acta 155, 1-9.

- Geiger, R. F. Jr, and Nieman, T. A. (1980). Bipolar pulse conductance measurements with a calcium ion-selective electrode. Anal. Chem. 52, 705-9.

Priestley, M. B. (1981). Spectral analysis and time series, 2 vols. Academic Press, New York.

Pungor, E. (1965). Conductometry and oscillometry. Pergamon Press, Oxford.

Randles, J. E. B. (1947). Kinetics of rapid electrode reactions. Disc. Faraday Soc. 1, 11-19. 
Redwood, W.R., Takashima, S., Schwan, H.P. and Thompson, T. L. (1972). Dielectric studies on homogenous phosphatidylcholine vesicles. Biochim. Biophys. Acta. 255, 557-66.

Reilley, C. N. (1954). High-frequency methods. In New instrumental methods in electrochemistry (ed. P. Delahay), pp. 319-345. Interscience, New York.

Richards, J. C. S., Jason, A. C., Hobbs, G., Gibson, D. M. and Christie, R. H. (1978) Electronic measurement of bacterial growth. J. Phys. E. Sci. Instrum. 11, 560-8.

Salter, D.C. (1979). Quantifying skin disease and healing in vivo using electrical impedance measurements. In Non-invasive physiological measurements (ed. P. Rolfe), Vol. 1, pp. 2I-64. Academic Press, London.

Schanne, O. F. and Ceretti, E. R.P. (1978). Impedance measurements in biological cells. John Wiley, Chichester.

Schmukler, R. and Pilla, A. A. (1982). A transient impedance approach to nonfaradaic electrochemical kinetics at living cell membranes. J. Electrochem. Soc. 129, 526-8.

Schügerl, K. (1984). On-line process analysis and control in biotechnology. Trends. Anal. Chem. 3, 239-45.

Schwan, H.P. (1955). Electrical properties of body tissues and impedance plethysmography. IRE Trans. Biomed. Eng. 3, 32-46.

- (1957). Electrical properties of tissue and cell suspensions. In Advances in biological and medical physics (eds. J. H. Lawrence and C. A. Tobias), Vol. 5, pp. 147-209. Academic Press, New York.

- (1963). Determination of biological impedances. In Physical techniques in biological research (ed. W. L. Nastuk), Vol. VIB, pp. 323-407. Academic Press, New York.

- (1966). Alternating current electrode polarisation. Biophysik 3, 181-201.

(1968). Electrode polarisation impedance and measurements in biological materials. Ann. N.Y. Acad. Sci. 148, 191-209.

- (1977). Field interactions with biological matter. Ann. N.Y. Acad. Sci. 303, 198-213.

- (1981a). Dielectric properties of biological tissue and biophysical mechanisms of electromagnetic field interactions, ACS Symp. Ser, 157, 109-131.

- $(1981 b)$. Electrical properties of cells: Principles, some recent results, and some unresolved problems. In The biophysical approach to excitable systems (eds. W. J. Adelmann Jr. and D. E. Goldman), pp. 3-24. Plenum Press, New York.

(1983a). Dielectric properties of biological tissue and Cells at RF- and MWfrequencies. In Biological effects and dosimetry and non-ionizing radiation (eds. M. Gandolfo, S. M. Michaelson and A. Rindi), pp. 195-211. Plenum Press, New York.

- (1983b). Dielectric properties of biological tissues and cells at ELF-frequencies.

In Biological effects and dosimetry of non-ionizing radiation (eds. M. Gandolfo,

S. M. Michaelson, and A. Rindi), pp. 549-59. Plenum Press, New York.

— and Ferris, C. D. (1968). Four-electrode null techniques for impedance measurement with high resolution. Rev. Sci. Instr. 39, 481-5.

- and Foster, K. R. (1980). RF-field interactions with biological systems: electrical properties and biophysical mechanisms. Proc. IEEE 68, 104-13.

— Takashima, S., Miyamoto, V. K, and Stoekenius, W, (1970), Electrical 
properties of phospholipid vesicles. Biophys, $J$, 10, 1102-19.

Sekoguchi, K., Takeishi, M., Hironaga, K. and Nishiura, T. (1984). Velocity measurement with electrical double-sensing devices in two-phase flow. In Measuring techniques in gas-liquid two-phase flows (eds. J. M. Delahaye and G. Cognet), pp. 455-477. Springer-Verlag, Heidelberg.

Shedlovsky, T. (1949). Conductometry. In Physical methods of organic chemistry (ed. A. Weissberger), Part 2, pp, 1651-83. Interscience, New York.

Singh, B., Smith, C. W. and Hughes, R. (1979). In vivo dielectric spectrometer. Med. Biol. Eng. Comput. 17, 45-60.

Sluyters-Rehbach, M. and Sluyters, J. H. (1970). Sine wave methods for the study of electrode processes. In Electroanalytical chemistry (ed. A. J. Bard), Vol. 4, pp. 1-128, Marcel Dekker, New York.

Smith, D. E. (1966). A.C. polarography and related techniques; theory and practice. In Electroanalytical chemistry (ed. A. J. Bard), Vol. 1, pp. 1-155. Edward Arnold, London.

Somogyi, B., Welch, G, R. and Damjanovich, S. (1984). The dynamic basis of energy transduction in enzymes. Biochim. Biophys. Acta 768, 81-112.

Sorriso, S. and Surowiec, A. (1982). Molecular dynamics investigations of DNA by dielectric relaxation measurements. Adv. Mol. Rel. Interaction Proc. 22, 259-79. Sparnaay, M. J. (1972). The electrical double layer. Pergamon Press, Oxford.

Steel, M., Sheppard, R, J. and Grant, E. H. (1984). A precision method for measuring the complex permittivity of solid tissue in the frequency domain between 2 and $18 \mathrm{GHz}$. J. Phys. E. Sci. Instrum. 17, 29-34.

Stewart, G.N. (1899a). The relative volume or weight of corpuscles and plasma in blood. J. Physiol. 24, 356-73.

- $(1899 b)$. The changes produced by the growth of bacteria in the molecular concentration and electrical conductivity of culture media. J. Exp. Med. 4, 235-43.

Stock, J. T. (1984). Two centuries of quantitative electrolytic conductivity. Anal. Chem. 56, 561A-570A.

Stoy, R. D., Foster, K. R. and Schwan, H. P. (1982). Dielectric properties of mammalian tissue from 0.1 to $100 \mathrm{MHz}$ : a summary of recent data. Phys. Med. Biol, 27, 501-13.

Stuchly, M.A. and Stuchly, S.S. (1980). Coaxial line methods for measuring dielectric properties of biological substances at radio and microwave frequencies a review, IEEE Trans. Instrum. Meas IM-29, 176-93.

Athey, T. W., Stuchly, S. S., Samaras, G. M. and Taylor, G. (1981). Dielectric properties of animal tissues in vivo at frequencies $10 \mathrm{MHz}-1 \mathrm{GHz}$. Bioelectromagnetics 2, 93-103.

Takashima, S. (1969). Dielectric properties of proteins. 1. Dielectric relaxation. In Physical principles and techniques of protein chemistry, Part A (ed. S. J. Leach), pp. 291-333. Academic, New York.

- and Minakata, A. (1975). Dielectric behaviour of biological macromolecules. In Digest of dielectric literature 37, pp, 602-53. National Research Council, Washington D.C.

Tamamushi, R. and Takahashi, K. (1974). Instrumental study of electolytic conductance using four-electrode cells. J. Electroanal. Chem, 50, 277-84.

Thomas, B. W. and Pertel, R. (1963). Measurement of capacity: analytical uses of the 
dielectric constant. In Treatise on analytical chemistry (eds. 1. M. Kolthoff and P. J. Ewing), Vol. 4, pp. 2631-2672. Interscience, New York.

Tien, H. T. (1974). Bilayer lipid membranes (BLM). Theory and practice. Marcel Dekker, New York.

To, E. C., Mudgett, R. E., Wang, D. I. C., Goldbluth, S. A. and Decareau, R. V. (1974). Dielectric properties of food materials. J. Microwave Power 9, 303-15.

Turner, G. and Howell, J.A. (1984). On-line estimation of the time constant of oxygen electrodes by time series analyses. Biotechnol. Lett. 6, 215-20.

Vreugdenhil, Th., van der Touw, F. and Mandel, M. (1979). Electric permittivity and dielectric dispersion of low molecular weight DNA of low ionic strength. Biophys. Chem. 10, 67-80.

Wada, A. (1976). The $\alpha$-helix as an electric macrodipole. Adv. Biophys, 9, 1-63.

Welch, G. R. (1986) (ed.). The fluctuating enzyme. Wiley, New York.

- and Kell, D. B. (1985). Not just catalysts: the bioenergetics of molecular machines. In The fluctuating enzyme (ed, G. R. Welch), pp. 451-92. Wiley, New York.

- Somogyi, B. and Damjanovich, S. (1982). The role of protein fluctuations in enzyme action: a review. Progr. Biophys. Mol. Biol. 39, 109-46.

Wheeler, H. B. and Penney, C. (1982). Impedance plethysmography: theoretical and experimental basis. In Non-invasive diagnostic techniques in vascular disease (ed. E. F. Bernstein), pp. 104-116. C. V. Mosby, St Louis.

Wilson, T.R.S. (1981), Conductometry. In Marine electrochemistry (eds, M. Whitfield and D. Jagner), pp. 145-185. Wiley, Chichester.

Winter, F. and Kimmich, R. (1982). NMR field-cycling relaxation spectroscopy of bovine serum albumin, muscie tissue. Micrococcus luteus and yeast. ${ }^{14} \mathrm{~N}^{1} \mathrm{H}-$ quadrupole dips. Biochim. Biophys. Acta 719, 292-8.

Wuthrich, K. (1982). Nuclear magnetic resonance studies of internal mobility in globular proteins. Biochem. Soc. Symp. 46, 17-37.

Zimmerman, U. (1982). Electric field-mediated fusion and related electrical phenomena. Biochim. Biophys, Acta. 694, 227-77. 


\section{Bioelectrochemistry \\ (d) Silicon-based sensors}





\title{
25
}

\section{Micro-biosensors based on silicon fabrication technology}

\author{
ISAO KARUBE
}

\subsection{Introduction}

Methods for the selective determination of organic compounds in biological fluids, such as blood, are very important in clinical analysis. Most analyses of organic compounds can be performed by spectrophotometric methods based on specific enzyme-catalysed reactions. However, these methods often require a long reaction time and complicated procedures. On the other hand, electrochemical sensors employing immobilized biocatalysts have definite advantages. Namely, an enzyme sensor possesses excellent selectivity for biological substrates and can directly determine single compounds in a complicated mixture without need for a prior separation step (Karube and Suzuki 1984, 1985). Miniaturization of the enzyme sensors is a prerequisite for medical application. This has been achieved using semiconductor fabrication technology combined with enzyme immobilization techniques to produce highly selective miniature sensors.

In this chapter, ion-sensitive field-effect transistors (ISFET) and microelectrodes, prepared by silicon fabrication technology, are employed as micro-biosensor transducers. Micro-biosensors for urea, ATP (adenosine triphosphate), glucose, and glutamate constructed from micro-transducers and immobilized-enzyme thin membranes are detailed and their characteristics discussed.

\subsection{FET-based sensors}

\subsubsection{Micro-urea sensor}

The ISFET was first reported by Bergveld in 1970. Matsuo and Wise (1974) improved the ISFET properties by utilizing silicon nitride $\left(\mathrm{Si}_{2} \mathrm{~N}_{4}\right)$ as the gate insulator, reporting its use as a pH sensor. In 1980, Caras and Janata demonstrated that an immobilized penicillinase layer over the gate insulator of the ISFET could be used as a penicillin sensor (see Chapter 26). We have also reported an enzyme-FET sensor (Miyahara et al. 1983).

The assay of urea in blood and urine is a very important diagnostic test to evaluate kidney function and condition. Conventional assay methods for 
urea are based on spectrophotometry, but involve complicated and delicate procedures. Therefore, the development of an inexpensive and miniaturized sensor that is highly selective and sensitive, yet easy to use, is extremely desirable. Realization of these goals can be achieved using the ISFET transducer.

Fabrication of the ISFET uses basically the same procedures employed for the metal-insulator-semiconductor FET (MISFET), as reported by Matsuo and Esashi (1981). The structure of the ISFET is shown in Fig. 25.1.

The gate insulator of the ISFET is composed of two layers; the lower is thermally grown silicon dioxide $\left(\mathrm{SiO}_{2}\right)$, the upper being silicon nitride $\left(\mathrm{Si}_{3} \mathrm{~N}_{4}\right)$, which is sensitive to $\mathrm{H}^{*}$ ions and also has a barrier effect on ion penetration. The thickness of the $\mathrm{SiO}_{2}$ and $\mathrm{Si}_{3} \mathrm{~N}_{4}$ layers are approximately $0.1 \mu \mathrm{m}$. The sensor system consists of two ISFETs; one ISFET is covered with a crosslinked polyvinylbutyral membrane containing amino groups, onto which urease was immobilized through a Schiff base linkage (urea-sensitive ISFET, ENFET), and the other ISFET is only covered with a cross-linked polyvinylbutyral resin membrane (only pH-sensitive ISFET, REFFET).

The polyvinylbutyral membrane was spread onto the gate insulator of the ISFETs by a dropping method. Approximately $0.1 \mathrm{~g}$ of polyvinylbutyral resin and $1 \mathrm{ml}$ of 1,8-diamino-4-aminomethyloctane were dissolved in $10 \mathrm{ml}$ of dichloromethane. This polymer solution was dropped onto the gate insulator of the two ISFETs and then immersed in a $5 \%$ glutaraldehyde solution at room temperature for approximately one day to advance the cross-linking reaction. Urease was immobilized on the ENFET by immersing it in a

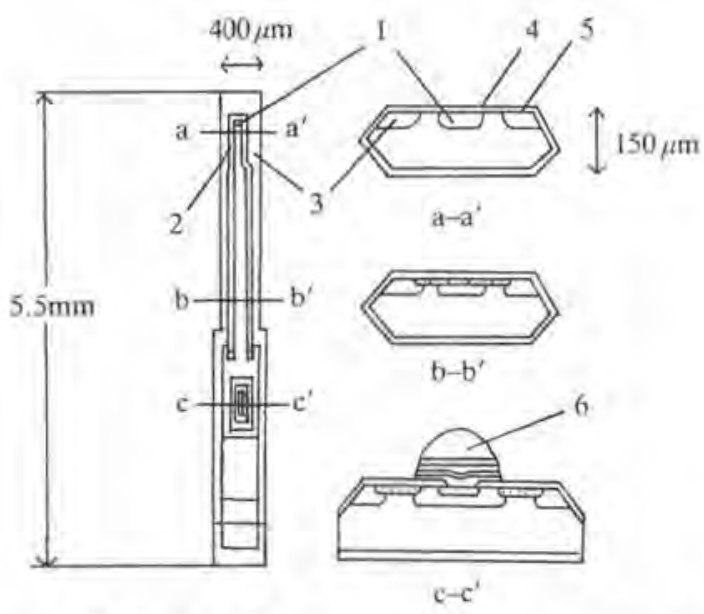

Fig. 25.1 Structure of ISFET. 1, Drain; 2, gate; 3, source; $4, \mathrm{Si}_{3} \mathrm{~N}_{4} ; 5, \mathrm{SiO}_{2}$; 6 , solder. 


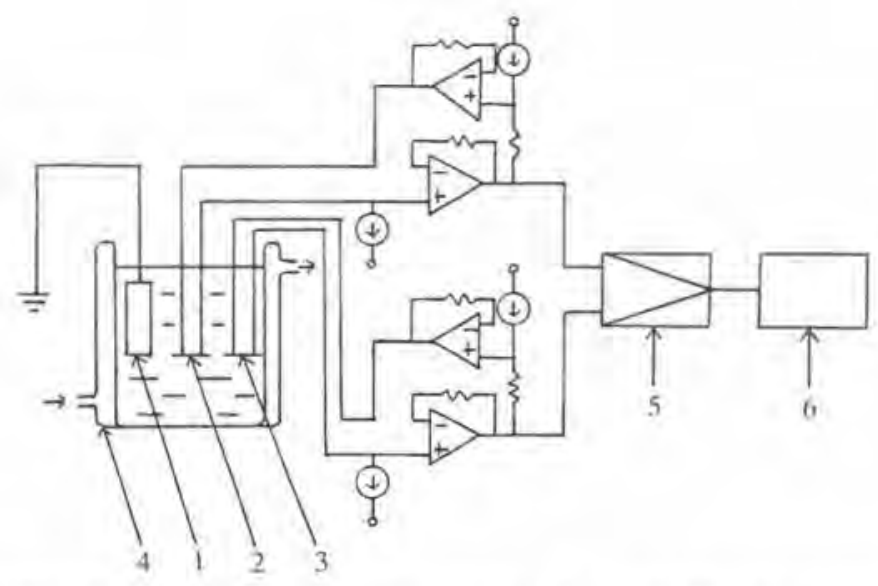

Fig. 25.2 Circuit diagram of measuring system. $1, \mathrm{Ag} / \mathrm{AgCl}$ reference electrode; 2, ENFET; 3, REFFET; 4, cell; 5 , differential amp,; 6 , recorder.

$5 \mathrm{mg} \mathrm{ml}^{-1}$ urease solution at $4{ }^{\circ} \mathrm{C}$ for approximately one day.

Measurements of urea concentration were performed in a differential mode, by comparing the difference in gate output voltage of the urea-sensing gate and the reference gate. A schematic diagram of the circuit is shown in Fig. 25.2. An Ag/ $\mathrm{AgCl}$ reference electrode was placed directly in solution with the ENFET and the REFFET, and a gate voltage applied between the $\mathrm{Ag} / \mathrm{AgCl}$ reference electrode and the source of the ENFET and the REFFET. A change in solution $\mathrm{pH}$ affects the gate insulator surface potential, with a concomitant proportional change in the gate output voltage. $100 \mu \mathrm{l}$ aliquots of urea were injected into a solution of $5 \mathrm{~mm}$ Tris- $\mathrm{HCl}$ buffer at $37^{\circ} \mathrm{C} \pm 1{ }^{\circ} \mathrm{C}$, and the differential gate output voltage change recorded for 10-20 $\mathrm{min}$.

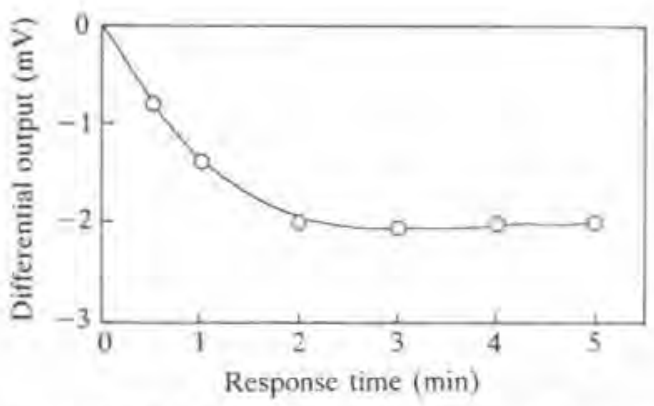

Fig. 25.3 Response curve to $1.7 \mathrm{~mm}$ urea. Experiments were performed at $37^{\circ} \mathrm{C}, \mathrm{pH}$ 7.0. 
Figure 25.3 shows a typical urea response curve of the sensor system. The differential gate output voltage reached a steady state approximately two minutes after injection of urea.

The initial rate of change of the differential gate output voltage after injection was plotted against the logarithm of the urea concentration. Figure 25.4 shows a calibration curve of the urea sensor system. A linear relationship was obtained between the initial rate of voltage change and the logarithm of urea concentration over the range 1.3 to $16.7 \mathrm{~mm}$ urea. An examination of the selectivity of the urea sensor system showed that it did not respond to $6.3 \mathrm{~mm}$ glucose, $10 \mathrm{mM}$ creatinine, and $3.6 \mu \mathrm{M}$ albumin.

The stability of the urea sensor system was also examined. The ENFET was stored at $4{ }^{\circ} \mathrm{C}$ between measurements, and exhibited a response to $16.7 \mathrm{mM}$ urea for at least two weeks.

\subsubsection{Micro-ATP sensor}

The determination of ATP (adenosine triphosphate) is important in fermentation processes and for clinical analysis. Conventional methods of ATP assay are based on spectrophotometric and bioluminescence measurements. These methods, however, require complicated and delicate procedures and a simpler and more inexpensive assay is desirable.

$\mathrm{H}^{+}$-ATPase (EC 3.6.1.3) in biological membranes catalyses production or hydrolysis of ATP. Furthermore, the enzyme has many functions, such as proton transport, which could be utilized for a bio-molecular device. Several

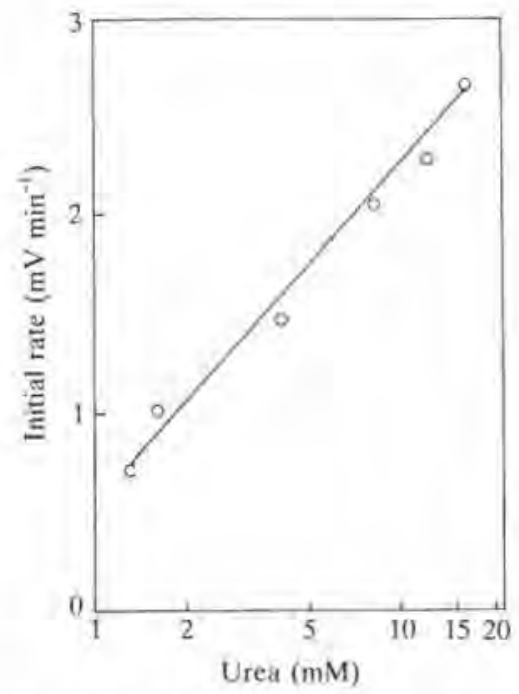

Fig. 25.4 Urea calibration curve. Experimental conditions were the same as in Fig. 25.3. 


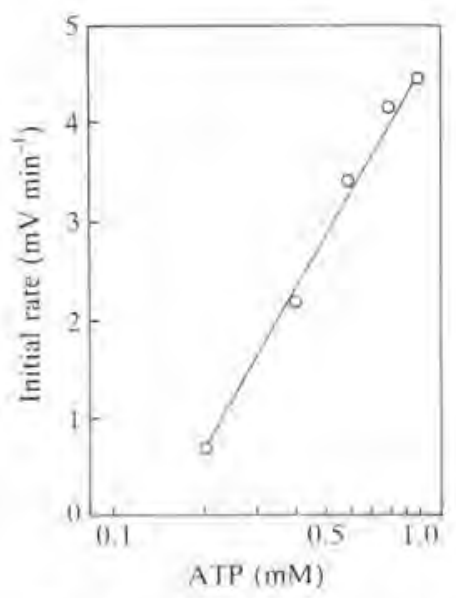

Fig. 25.5 ATP calibration curve. Experiments were performed at $40^{\circ} \mathrm{C}, \mathrm{pH} 7.0$.

studies on the properties and function of the enzyme in biological membranes have been reported by Kagawa and co-workers (Yoshida et al. 1977; Kagawa 1984). $\mathrm{H}^{*}$-ATPase was prepared from a thermophilic bacterium PS 3 and is classified as thermophilic $\mathrm{F}_{1}\left(\mathrm{TF}_{1}\right) \mathrm{ATP}$. The procedures employed in constructing the ATP sensor and measurements of gate voltage were identical to those of the urea sensor. $50 \mathrm{~mm}$ Tris-maleate buffer was used at $40^{\circ} \mathrm{C} \pm$ $1{ }^{\circ} \mathrm{C}$. The differential gate output voltage reached steady state approximately 4-5 minutes after injection of ATP.

The initial rate of change of the differential gate output voltage after injection of ATP was plotted against the logarithm of the ATP concentration. Figure 25.5 shows a calibration curve of the ATP sensor system. A linear relationship was obtained between the initial rate of the voltage change and the logarithm of ATP concentration over the range 0.2 to $1.0 \mathrm{mM}$ ATP.

Slight responses were obtained when $1 \mathrm{~mm}$ glucose, urea, and creatinine were applied to the system. The response of the system to $1 \mathrm{mM}$ ATP was retained for 18 days.

\subsection{Micro-electrode based sensors}

\subsubsection{Glucose sensor based on a micro-hydrogen peroxide electrode}

The determination of glucose in blood samples is important in clinical fields, and the development of bioelectrochemical devices would be of considerable help in routine laboratory work.

The development of miniaturized and implantable enzyme sensors employing micro-transducers is required in the medical field. Therefore, a 

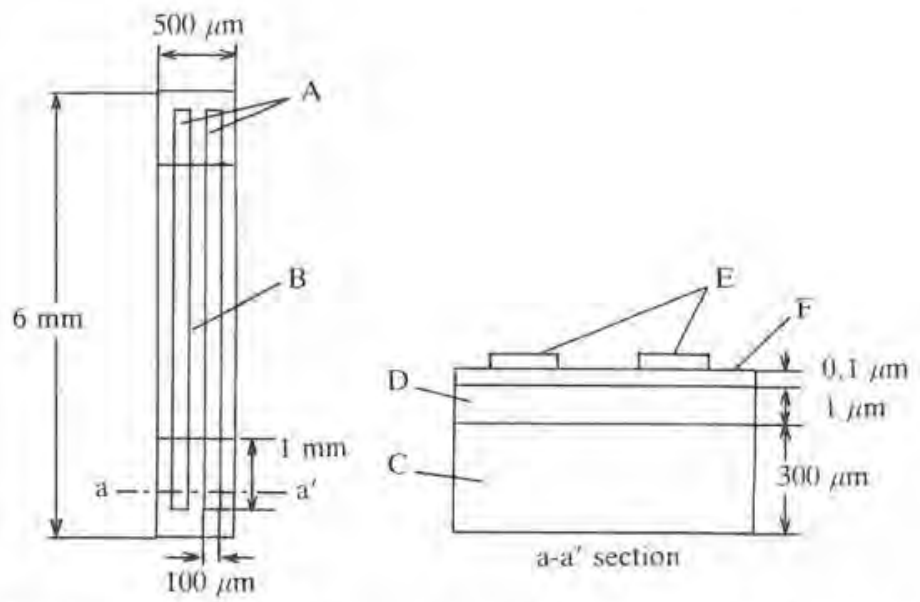

Fig. 25.6 Schematic diagram of a micro-electrode $\mathrm{A}, \mathrm{E}, \mathrm{Au} ; \mathrm{B}, \mathrm{Ta}_{2} \mathrm{O}_{5} ; \mathrm{C}, \mathrm{Si} ; \mathrm{D}, \mathrm{SiO}_{2}$; $\mathrm{F}, \mathrm{Si}_{3} \mathrm{~N}_{4}$.

micro-hydrogen peroxide $\left(\mathrm{H}_{2} \mathrm{O}_{2}\right)$ sensor has been developed utilizing the currently available integrated circuit technology. The structure of the micro- $\mathrm{H}_{2} \mathrm{O}_{2}$ sensor is shown in Fig. 25.6. Micro-Au electrodes were created on the silicon nitride surface using the vapour deposition method and partially insulated by coating with $\mathrm{Ta}_{2} \mathrm{O}_{5}$. The $\mathrm{H}_{2} \mathrm{O}_{2}$ electrode was placed in a sample solution containing $\mathrm{H}_{2} \mathrm{O}_{2}$ and the over-potential fixed at $1.1 \mathrm{~V}$. The output current of the sensor immediately increased and reached a steadystate value within one minute. A linear relationship was observed between the $\mathrm{H}_{2} \mathrm{O}_{2}$ concentration and the steady-state current in the range $1 \mu \mathrm{M}$ to $1 \mathrm{mM}$ $\mathrm{H}_{2} \mathrm{O}_{2}$. This electrode was then employed as the transducer in a micro-glucose sensor. The procedure for glucose oxidase (GOD) immobilization onto the micro-electrode is as follows. Approximately $100 \mu \mathrm{l}$ of $\gamma$-aminopropyltriethoxysilane was vapourized at $80^{\circ} \mathrm{C}, 0.5$ torr for $30 \mathrm{~min}$ onto the electrode surface, followed by $100 \mu \mathrm{l}$ of $50 \%$ glutaraldehyde vapourized under the same conditions. The modified micro-electrodes were then immersed in GOD solution containing BSA and glutaraldehyde, the GOD becoming chemically bound to the surface of the micro-electrode by a Schiff linkage. Figure 25.7 shows a typical response curve for the micro-glucose sensor. The output current increased after injection of a sample solution, steady state being reached within $5 \mathrm{~min}$. Figure 25.8 shows a calibration curve for the micro-glucose sensor.

A linear relationship was observed between the current increase (the difference between the initial and steady-state currents) and glucose concentration in the range 0.1 to $10 \mathrm{mg} \mathrm{dl}^{-1}$ glucose. Examination of the selectivity of 


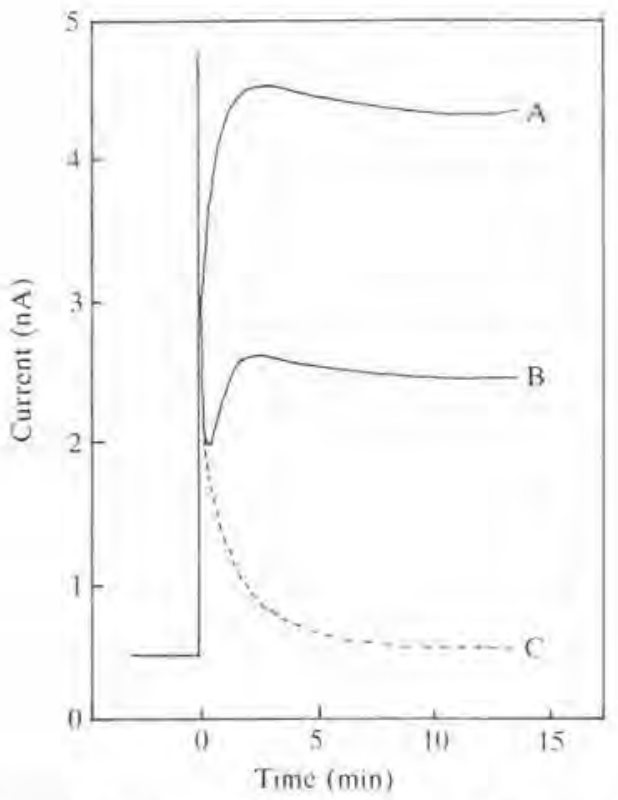

Fig. 25.7 Glucose response curves. Experiments were performed at $37{ }^{\circ} \mathrm{C}, \mathrm{pH} 7.0$. A, $10 \mathrm{mg} \mathrm{dl}^{-1}$ glucose; B, $5 \mathrm{mg} \mathrm{dl}^{-1}$ glucose; C, no glucose.

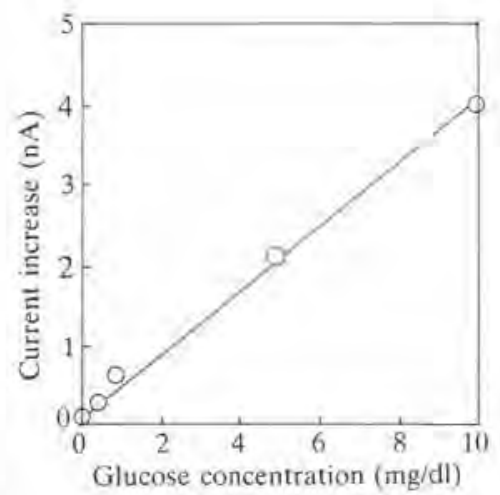

Fig. 25.8 Glucose calibration curve. Experiments were performed at $37^{\circ} \mathrm{C}, \mathrm{pH} 7.0$.

the glucose micro-sensor indicated no response to other compounds such as galactose, mannose, fructose, and maltose. Therefore, the selectivity of this sensor for glucose is highly satisfactory.

Figure 25.9 shows the effect of temperature on the current increase of the sensor system. The optimum temperature for the sensor was $55^{\circ} \mathrm{C}$. 


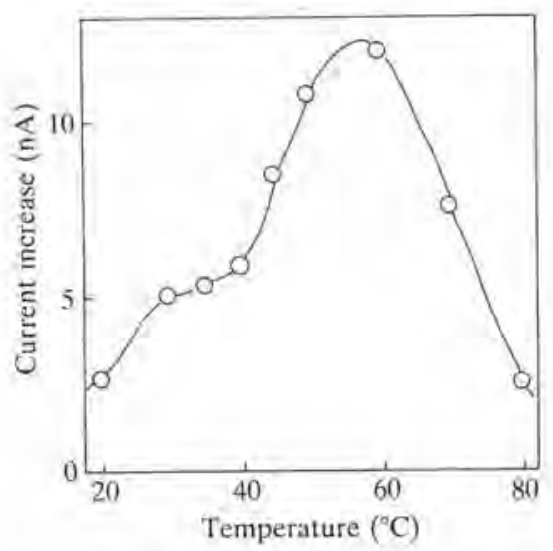

Fig. 25.9 Effect of temperature.

However, because the enzyme gradually denatures at $55^{\circ} \mathrm{C}$, the stability of the sensor at this temperature is poor. Therefore, all other experiments were performed at $37{ }^{\circ} \mathrm{C}$. Continuous operation of the sensor in $10 \mathrm{mg} \mathrm{dl}^{-1}$ glucose produced a constant current output for more than 15 days and 150 assays. Therefore, this micro-glucose sensor possesses both selectivity and good stability, its potential use as a micro-glucose sensor being very good.

\subsubsection{Micro- $\mathrm{O}_{2}$ electrode based glutamate sensor}

The determination of L-glutamic acid (L-Glu) is very important in the food industry, because large amounts of L-Glu are produced by fermentation to be used as a food seasoning. Various glutamate sensors, consisting of immobilized enzyme and an electrochemical device, have been developed for the fermentation and food industries. Glutamate oxidase catalyses the oxidation of glutamate, oxygen being consumed by the reaction. Therefore, an oxygen sensor can be employed as the transducer for a glutamate sensor. A microoxygen sensor was developed by modifiying the micro- $\mathrm{H}_{2} \mathrm{O}_{2}$ electrode,

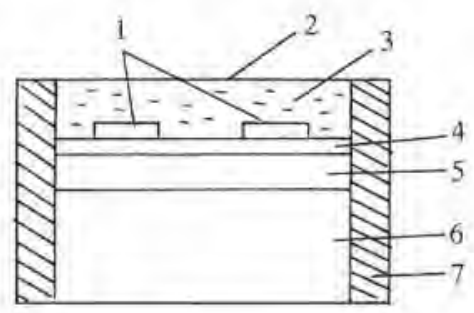

Fig. 25.10 Schematic diagram of an oxygen electrode. 1, Au; 2, Teflon membrane; 3, $0.1 \mathrm{M} \mathrm{KOH} ; 4, \mathrm{Si}_{3} \mathrm{~N}_{4} ; 5, \mathrm{SiO}_{2} ; 6, \mathrm{Si} ; 7$, silicon rubber. 
prepared as previously described.

Figure 25.10 shows the structure of the micro-oxygen sensor. It consists of a gas-permeable Teflon membrane, two micro-Au electrodes and $0.1 \mathrm{M} \mathrm{KOH}$ electrolyte solution. The characteristics of the micro-oxygen electrode were examined by cyclic voltammetry at various concentrations of dissolved oxygen (oxygen and nitrogen mixture was sparged through the sample solution). A peak current was observed due to reduction of oxygen, when a voltage of approximately $1.1 \mathrm{~V}$ was applied to the Au electrodes. A linear relationship was observed between the oxygen concentration and the peak current obtained from the cyclic voltammograms (Fig. 25.11). These results indicate that the micro-oxygen electrode can be used for oxygen concentration determination. Therefore, the micro-oxygen electrode was employed as the transducer in a micro-glutamate sensor.

Glutamate oxidase was immobilized on a cellulose triacetate membrane containing glutaraldehyde and triamine (1,8-diamino-4-aminomethyloctane). The glutamate oxidase membrane was placed on the Teflon membrane of the micro-oxygen sensor and covered with a nylon net. Application of a glutamate sample solution to the sensor system produced a rapid drop in the current output to a steady-state value, resulting from glutamate oxidation.

Figure 25.12 shows the relationship between current decrease and the glutamate concentration. When the current decrease at $5 \mathrm{~min}$ was used as the measure of activity, a linear relationship was observed between the current decrease and the glutamate concentration in the range $5-50 \mathrm{mM}$. The effect of temperature on the peak current decrease of the sensor was examined. The optimum temperature for the sensor was approximately $40^{\circ} \mathrm{C}$, but gradual denaturation of the enzyme reduced the stability of the sensor. Therefore, all other experiments were performed at $30^{\circ} \mathrm{C}$.

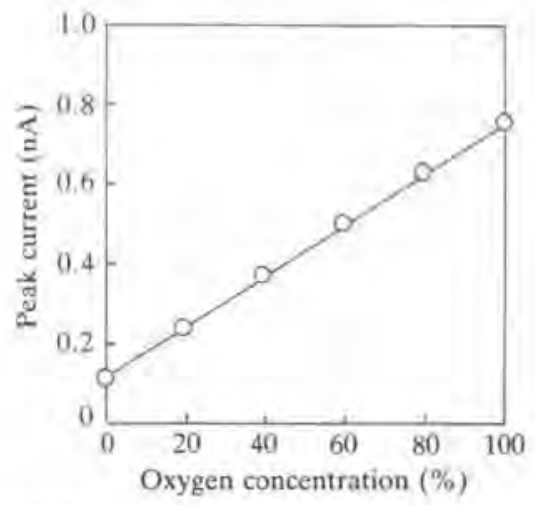

Fig. 25.11 Calibration curve of the oxygen electrode. Potential range: $-1.25 \mathrm{~V}$ $1 \mathrm{~V}$; scan rate: $100 \mathrm{mV}^{-1}$. 


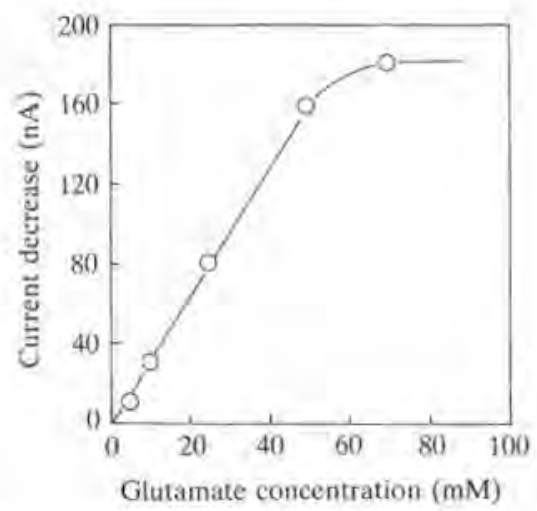

Fig. 25.12 Calibration curve of the micro-glutamate sensor. Experiments were performed at $40^{\circ} \mathrm{C}, \mathrm{pH} 7.5$.

The selectivity of the sensor for glutamate was found to be satisfactory and hence its application to fermentation process control and food analysis is very promising.

\section{References}

Bergveld, P. (1970). Development of an ion-sensitive solid-state device for neurophysiological measurements. IEEE Trans, on BME. BME-17, 70-1.

Caras, S. and Janata, J. (1980). Field effect transistor sensitive to penicillin. Anal. Chem. 52, 1935-7.

Kagawa, Y. (1984). A new model of proton motive ATP synthesis: acid-base cluster hypothesis. J. Biochem. 95, 295-98.

Karube, I. and Suzuki, S. (1984). Amperometric and potentiometric determinations with immobilized enzymes and micro-organisms. Ion-Selective Electrode Review 6, $15-58$.

- (1985). Immobilized enzymes for clinical analysis. In Enzymes and immobilized cells in biotechnology (ed. A. 1. Laskin), pp, 209-226. Benjamin/Cumming Publishing, London.

Matsuo, T. and Esashi, M. (1981). Methods of ISFET fabrication, Sensors and Actuators 1, 77-96.

Matsuo, T, and Wise, K. D. (1974). An integrated field-effect electrode for biopotential. IEEE Trans, on BME. BME-21, 485-7.

Miyahara, Y., Matsu, F., Moriizumi, T., Matsuoka, H., Karube, I. and Suzuki, S. (1983). Micro enzyme sensors using semiconductor and enzyme-immobilization techniques. In Proceedings of the international meeting on chemical sensors, Kodansha, Tokyo, pp. 501-6. Elsevier, New York.

Yoshida, M., Sone, N., Hirata, H. and Kagawa, Y. (1977). Reconstitution of adenosine triphosphatase of thermophilic bacterium from purified individual subunits. J. Biol. Chem. 252, 3480-5. 


\section{6 \\ Chemically sensitive field-effect transistors}

GARYF. BLACKBURN

\subsection{Introduction}

The chemically sensitive field-effect transistor (CHEMFET) was born out of the integration of two well developed technologies: solid-state integrated circuits and ion-selective electrodes (ISE). Bergveld (1970) demonstrated the first CHEMFET which used a silicon dioxide layer to impart sensitivity to hydrogen ions upon an insulated-gate field-effect transistor (IGFET). Since that time, considerable development has taken place, especially in the area of ion-selective field-effect transistors (ISFET). The ion-selective membranes which had previously been developed for the ISE technology could be directly applied to the fabrication of ISFETs and thus their development was straightforward. The development of CHEMFET sensors sensitive to other chemicals has also seen considerable research effort although not to the same extent as the ISFET.

The purpose of this text is not to exhaustively review the literature of the field. Rather, the purpose is to present an overview of the current areas of active research. For reviews of the literature pertaining to the CHEMFET, the reader is referred to Zemel (1975) or Janata and Huber (1980).

\subsection{Theory of FET chemical sensors}

To understand the operation of the chemically sensitive field-effect transistor, it is necessary to first understand the physics of the insulatedgate field-effect transistor from which the CHEMFET is fashioned. First, the electronic properties of semiconductor materials will be described in a qualitative way followed by a description of the metal-insulatorsemiconductor (MIS) structure which is the precursor of the IGFET. Once the operation of the IGFET is understood, the theory can easily be extended to describe the operation of the CHEMFET. A rigorous description of semiconductor physics is beyond the scope of this book and is not necessary to understand the operation of CHEMFETs. The interested reader should consult either Muller and Kamins (1977) or Sze (1981) for more detailed descriptions of the physics of semiconductor devices. 
It is important to note that the metal-oxide-semiconductor field-effect transistor (MOSFET), the metal-insulator-semiconductor field-effect transistor (MISFET), and the metal-nitride-oxide-semiconductor field-effect transistor (MNOSFET) are each just special cases of the IGFET.

\subsubsection{Semiconductor physics}

To understand the physics of semiconductor materials at a level required for the comprehension of the operation of the IGFET and CHEMFET, a description of the energy-band diagrams of semiconductor materials is required. Figure 26.I represents the energy band diagram for silicon. Increasing energy for electrons is drawn upward while the abscissa represents distance in the silicon. The cross-hatched areas represent near continua of allowed energy levels for electrons in the silicon. The upper band is called the conduction band with its lowest energy level denoted by $E_{\mathrm{c}}$. The lower band represents the valence band of silicon and its highest energy is usually denoted by $E_{\mathrm{v}}$. All energy levels are referenced to the 'vacuum level', $E_{0}$, which is defined as the energy of an electron if it were just free from the influence of the given material. The energy region between $E_{\mathrm{c}}$ and $E_{\mathrm{v}}$ represents the 'forbidden' band-gap where no allowed energy levels exist for electrons. The difference in energy between $E_{\mathrm{c}}$ and $E_{\mathrm{v}}$ is called the band-gap energy, $E_{\mathrm{g}}$, and is equal to $1.1 \mathrm{eV}$ for silicon. At a temperature of absolute zero ( $0 \mathrm{~K})$, all allowed energy levels in the valence band are filled with electrons and all levels in the conduction band are empty. Even at room temperature $(300 \mathrm{~K})$, the average kinetic energy of the electrons is only $0.04 \mathrm{eV}$ which is less than $4 \%$ of the band-gap energy. Therefore, the number of electrons with sufficient energy to jump to the conduction band remains extremely small.

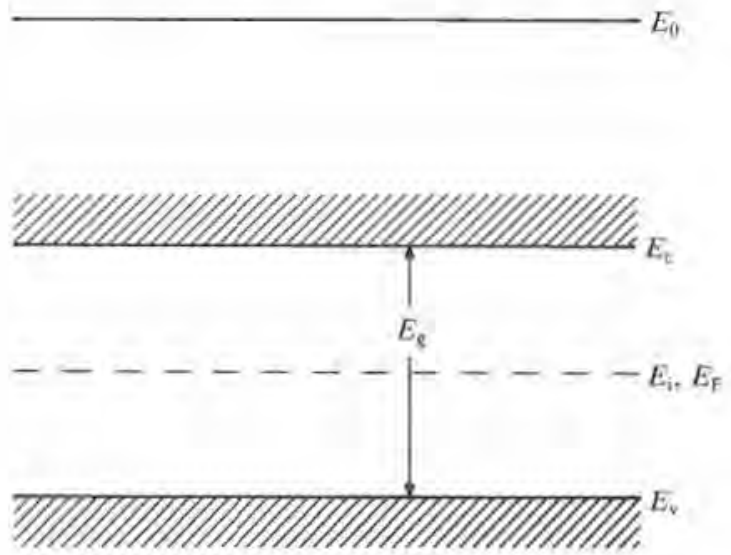

Fig. 26.1 Energy-band diagram for undoped silicon. 
For electronic conduction to occur, electrons must move in the semiconductor in response to any electric field. If the electrons move, then they must increase their kinetic energy which requires that they move to a higher, empty energy level. In undoped (intrinsic) silicon, then, conduction is insignificant because all of the valence energy levels are filled so there are no vacant energy levels for electrons to move to if they attain any additional kinetic energy. The electrons in the conduction band see a continuum of vacant energy levels to move to but because their number is extremely small, conduction in intrinsic silicon is insignificant at room temperature,

To impart conductivity on silicon, impurity atoms can be added to the crystal. Silicon is made an $n$-type semiconductor by doping the crystal with atoms from group V of the periodic table, e.g. phosphorous or arsenic. These atoms have one more valence electron than silicon and thus donate electrons to the crystal when included in the crystal lattice. Referring to the energyband diagram in Fig. 26.2a, the impurity atoms have an energy level, $E_{\mathrm{d}}$, which is within the band-gap and near the conduction band. At room temperature, most of the electrons at this donor energy level possess sufficient energy to jump to the conduction band. The increased concentration of electrons contributes to the electrical conductivity in the silicon. Silicon is made a $p$-type semiconductor by incorporationg impurity atoms from group III of the periodic table, e.g. boron or aluminium, into the silicon crystal. These atoms have one less valence atom than silicon and create

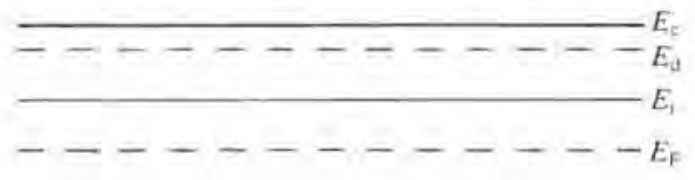

(a)

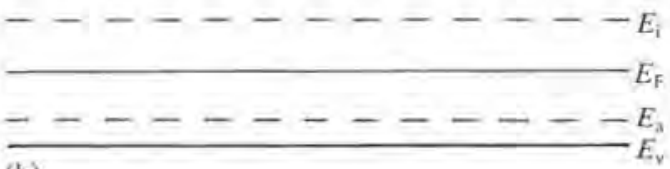

(b)

Fig. 26.2 Energy-band diagram for doped silicon. (a) n-type silicon, (b) $p$-type silicon. 
immobile 'traps' which capture free electrons. Referring to the energy-band diagram in Fig. 26.2b, the impurity atoms have an energy level, $E_{\mathrm{a}}$, which is near the valence band of the silicon. Most of the electrons in the valence band have sufficient energy to jump to these acceptor levels and thus nearly fully populate them. Each valence electron which populates one of the acceptor levels leaves behind a 'hole' or a vacant allowed energy level in the valence band, into which the remaining electrons may move to attain kinetic energy in response to an electric field, contributing to conductivity in the $p$-type silicon.

The Fermi level, $E_{\mathrm{P}}$, of a semiconductor is defined as the energy at which the probability of occupation by an electron equals one half. In intrinsic silicon (Fig. 26.1) the Fermi level is at the midpoint between the conduction and valence bands and is called the intrinsic level, $E_{\mathrm{i}}$. In n-type silicon (Fig. 26.2a) the Fermi level is closer to the conduction band because the number of electrons above $E_{\mathrm{i}}$ is larger than in intrinsic silicon. For $p$-type silicon (Fig. 26.2b) the effect is reversed. $E_{\mathrm{F}}$ is below $E_{\mathrm{i}}$ and closer to the valence band. The concentration of added impurity atoms (dopant atoms) affects the position of $E_{\mathrm{F}}$ within the band gap. Increasing the concentration of dopant atoms moves $E_{\mathrm{F}}$ further from the intrinsic level for either $n$-type or p-type silicon.

With this level of understanding of this energy-band description of semiconductor materials, a discussion of the operation of semiconductor devices (e.g., the IGFET and CHEMFET) can be presented.

\subsubsection{Metal-insulator-semiconductor structure}

The physics of the IGFET are best introduced by first examining the metalinsulator-semiconductor (MIS) structure which consists of a metal electrode and a semiconductor material separated by a thin (e.g. $100 \mathrm{~nm})$ insulating material such as silicon dioxide $\left(\mathrm{SiO}_{2}\right)$. This insulator is assumed to be ideal, i.e. no current can pass through the insulator. To simplify the analysis we will assume that the structure has the following 'ideal' characteristics. (1) The work function of the electrons in the metal, $\Phi_{\mathrm{m}}$, and the semiconductor, $\Phi_{5}$, are equal; (2) there is no net charge anywhere in the insulator; (3) there are no mobile charged species in the insulator; and (4) there are no surface states at the interface between the semiconductor and the insulator. The analysis of the MIS structure will involve the determination of the charge and potential distribution as a function of the potential applied between the metal and semiconductor. Once the analysis of this 'ideal' MIS structure is complete, the non-ideal characteristics can be accommodated rather easily.

The energy band diagram for the ideal MIS structure with zero applied voltage is shown in Fig. 26.3. The semiconductor is assumed to be silicon doped with acceptor impurities, i.e. it is $p$-type silicon. The energy barrier to electron transport through the insulator is denoted $q \phi_{\mathrm{B}}$. As is shown, at equilibrium the Fermi levels for the metal and silicon are equal and the 


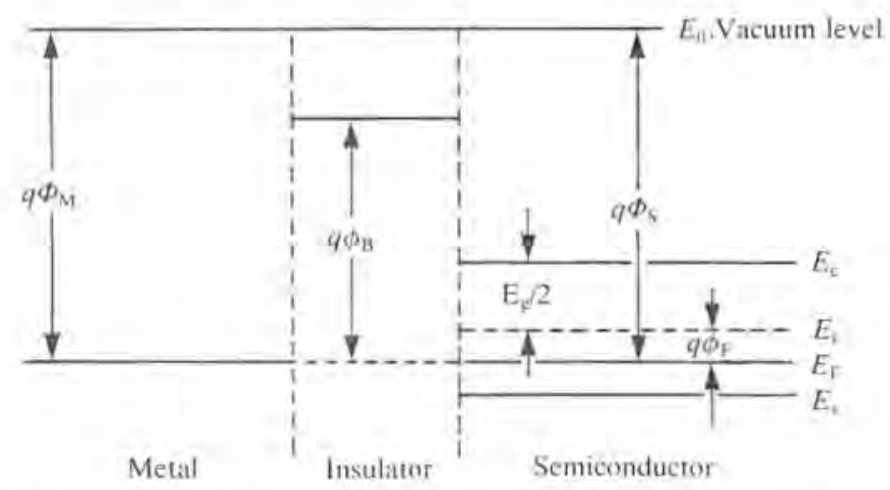

Fig. 26.3 Energy-band diagram for the ideal metal-insulator-semiconductor structure with no applied voltage.

potential and charge distributions are everywhere constant. When a potential is applied to the metal while the silicon is held at ground potential, however, the system is forced from equilibrium and the Fermi levels of the two materials separate by an amount equal to the applied potential. The system then behaves as a charged capacitor; the metal and semiconductor form the two charged plates of the capacitor.

If a negative potential is applied to the metal and the silicon is held at ground potential, then the field which is created will attract positivelycharged holes from the silicon to the silicon-insulator interface and, likewise, will attract electrons from the metal to the metal-insulator interface. Figure 26.4a illustrates the energy band diagram for this condition which is known as 'accumulation'. The $(+)$ symbols near the valence band at the siliconinsulator interface represent the accumulated holes. Note that since the convention is to depict higher energy for electrons in an upward direction, the Fermi level for the metal is drawn higher than that of the silicon even though the metal is at lower energy. It would be wise at this point to consider the shape of the energy bands in the diagram. The negative applied potential effectively increases the hole concentration at the silicon-insulator interface which means that the Fermi level must move closer to the valence band at the interface. Because the charge carriers are at thermal equilibrium in the silicon (otherwise, a current would flow to bring the charge carriers to equilibrium) the Fermi level in the silicon must be flat; therefore, the valence band must curve towards the Fermi level. Since the separation between the valence band and the conduction band, $E_{z}$, is a constant for the material, the conduction band must also bend upwards near the interface. Likewise, because the position of the intrinsic Fermi level is midway between the conduction and valence bands, it must also bend upwards at the interface. In the metal, 

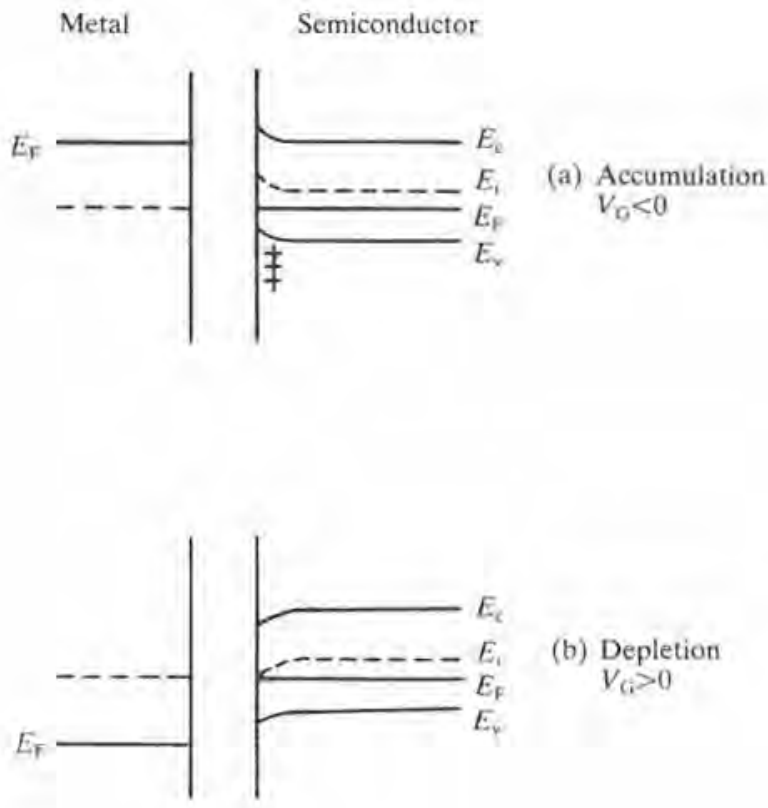

(b) Depletion $V_{\mathrm{G}}>0$

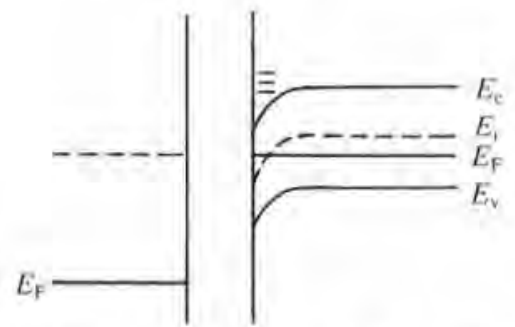

(c) Inversion $V_{G}>>0$

Fig. 26.4 Energy-band diagrams for the MIS structure in (a) accumulation, (b) depletion, and (c) inversion.

because the concentration of electrons and the density of vacant energy levels is so large, the Fermi level is constant up to the interface.

If a small positive potential is applied to the metal relative to the semiconductor then the field drives the positive mobile charge carriers (i.e., the holes) away from the silicon-insulator interface resulting in a condition known as 'depletion'. Since the hole concentration in the interfacial region of the silicon is decreased, the Fermi level must move further away from the 
valence band. Thus, the energy bands bend downwards at the interface as shown in Fig. 26.4b. This depletion region which is created will have a net negative charge density since the negatively charged dopant atoms are immobile and cannot move in the electric field.

If the magnitude of the positive potential applied to the metal is increased, then the energy bands bend down further at the interface. At some applied potential, the intrinsic level, $E_{1}$, will bend below the Fermi level, $E_{\mathrm{F}}$, as is depicted in Fig, 26.4c. At the point where the Fermi level is equal to the intrinsic level, the hole and electron concentrations will be equal. When the Fermi level bends below the intrinsic level, the electron concentration near the interface exceeds the hole concentration and the silicon in this region inverts from $p$-type to $n$-type. This condition is commonly referred to as the 'inversion' condition and the thin layer of $n$-type silicon is called the 'inversion layer'. The (-) symbols in Fig. 26.4c represent the electrons in the inversion layer.

The potential which must be applied to bring about inversion (i.e. to bend the intrinsic level to the level of the Fermi level) is shown in Fig. 26.3 as $\phi_{F}$. For the analysis of the IGFET below, a more useful definition is that of 'strong inversion' which occurs when the band movement at the interface is equal to $2 q \phi_{\mathrm{F}}$. Since the electron density at the interface depends exponentially on the quantity $\left(E_{\mathrm{i}}-E_{\mathrm{F}}\right)$, the density of electrons in the inversion layer increases extremely rapidly beyond the point of strong inversion. As a result, the bands bend further only slightly when a potential larger than that required for strong inversion is applied. The potential which must be applied between the metal and silicon to bring about strong inversion is normally referred to as the threshold voltage, $V_{\mathrm{T}}$, given by

$$
V_{\mathrm{r}}=-\frac{Q_{\mathrm{B}}}{C_{0}}+2 \phi_{\mathrm{F}}
$$

where $Q_{13}$ is the charge per unit area in the surface space-charge region and $C_{0}$ is the capacitance per unit area of the insulator. The first term on the right represents the portion of the applied voltage which is dropped across the insulator while the second term represents that portion which is dropped in the surface of the silicon.

Equation 26.1 applies only to the ideal MIS structure to which we have confined our discussion up to this point. Non-ideal effects generally result in band bending when zero voltage is applied between the metal and insulator. These effects are taken into account individually by altering the definition of the threshold voltage. Thus, if the effect of one of the non-ideal effects is to cause a natural upward bending of the bands at zero applied voltage, then a larger $V_{\mathrm{T}}$ must be applied in order to bend the bands down to the strong inversion condition. Each of the non-ideal effects will be dealt with in this way below. 
If the work function difference between the metal and semiconductor $\Phi_{\mathrm{ms}}$, is not equal to zero, then at zero applied voltage, the metal and semiconductor will not be at thermal equilibrium unless electrons move from the material with the smaller work function to the material with the larger work function. This would result in a bending of the energy bands at zero applied voltage, unlike the ideal system. To bring the bands back to the 'flat-band' condition, a potential would have to be applied to counter the difference in work functions of the two materials. The threshold voltage is therefore increased by the amount $\Phi_{\text {ms }}$

$$
V_{T}=\Phi_{\mathrm{ms}}-\frac{Q_{\mathrm{B}}}{C_{0}}+2 \phi_{\mathrm{F}} .
$$

A non-zero charge in the insulator is handled similarly. The charge in the insulator will induce an image charge in both the metal and the silicon and will again result in band bending even with zero applied voltage. The threshold voltage can again be corrected as follows (Muller and Kamins 1977):

$$
V_{\mathrm{T}}=\Phi_{\mathrm{ms}}-\frac{1}{C_{0}} \int_{0}^{d} \frac{x}{d} \rho(x) \mathrm{d} x-\frac{Q_{\mathrm{B}}}{C_{0}}+2 \phi_{\mathrm{F}}
$$

where $x$ is the distance from the metal-insulator interface, $\rho(x)$ is the charge density as a function of $x$, and $d$ is the thickness of the insulator.

In addition to the charge dispersed in the insulator, there is also a layer of charge in the insulator next to the interface, essentially at $x=d$. This charge is generally treated separately from the disperse charge in the insulator and is usually designated $Q_{\mathrm{ss}}$ (charge per unit area). Considering this surface charge, the threshold voltage is given by

$$
V_{\mathrm{T}}=\Phi_{\mathrm{ms}}-\frac{1}{C_{0}} \int_{0}^{d} \frac{x}{d} \rho(x) \mathrm{d} x-\frac{Q_{\mathrm{ss}}}{C_{0}}-\frac{Q_{\mathrm{B}}}{C_{0}}+2 \phi_{\mathrm{F}}
$$

The final non-ideality which must be considered is the deviation of the ideal band structure at the silicon-insulator interface where the periodic lattice structure of the silicon crystal is interrupted. This interruption of the lattice results in a nearly continuous distribution of allowed energy levels, called 'surface states', within the forbidden band-gap at the interface. The analysis of the influence of these surface states is complex and is treated elsewhere (Sze 1981). Fortunately, the annealing techniques which are currently employed in the fabrication of the MIS structure effectively reduce the number of surface states to a level where their effect is negligible, at least for the silicon/silicon dioxide system. Therefore, we will not further consider the existence of surface states.

The three terms in the threshold voltage equation (eqn 26.4) which deal 
with the non-idealities of the system are conveniently grouped into a single term called the 'flat-band' voltage, $V_{\mathrm{FB}}$. This is the voltage which must be applied to the metal in order to bring the energy bands to a flat condition (i.e. to the ideal condition). Therefore,

$$
V_{\mathrm{T}}=V_{\mathrm{FB}}+2 \phi_{\mathrm{F}}-\frac{Q_{\mathrm{B}}}{C_{0}}
$$

where

$$
V_{\mathrm{FB}}=\Phi_{\mathrm{ms}}-\frac{Q_{\mathrm{ss}}}{C_{0}}-\frac{1}{C_{0}} \int_{0}^{d} \frac{x}{d} \rho(x) \mathrm{d} x .
$$

Equations 26.5 and 26.6 describe the potential which must be applied to the MIS structure in order to bring about strong inversion. These equations are also essential in describing the physics of the IGFET which, in essence, is simply an MIS structure with provisions for measuring the conductivity of the surface inversion layer.

\subsubsection{Insulated-gate field-effect transistor}

The structure of the IGFET shown in Fig. 26.5 is very similar to that of the MIS capacitor described in the preceding section. The gate region of the transistor consists of the p-type silicon substrate (1); the insulator, usually $\mathrm{SiO}_{2}(2)$; and the gate metal (3); thus forming the familiar MIS structure. The structure is complicated by the addition of two $n$-type silicon areas known as the source (4) and drain (5). Metal contacts to the source and drain (6) permit

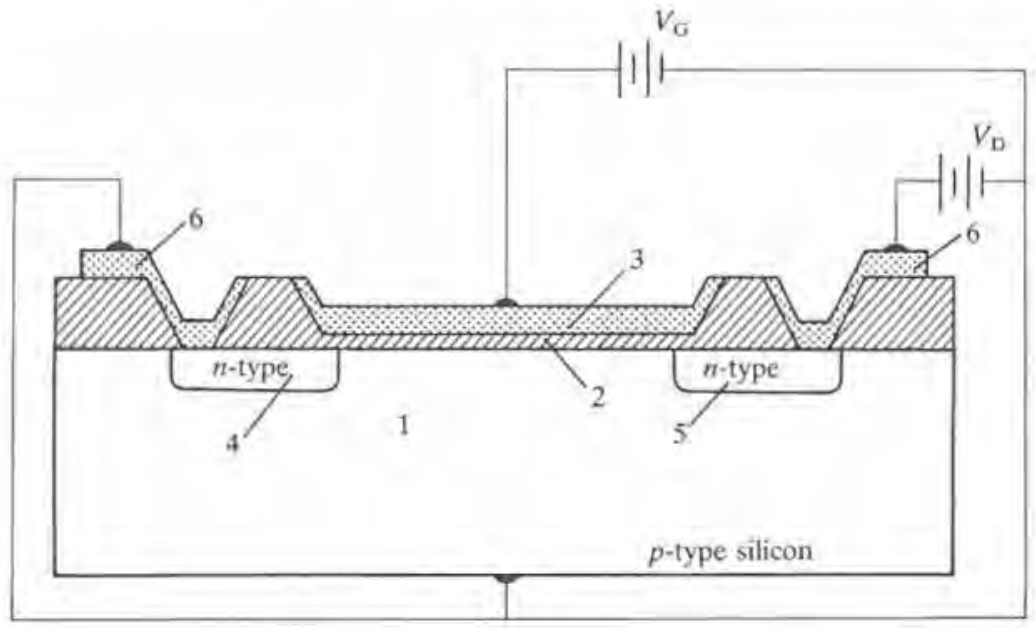

Fig. 26.5 Diagram of the IGFET. (1) p-type silicon substrate, (2) insulator, (3) gate metal, (4) n-type source, (5) n-type drain, (6) metal contacts to source and drain. 
electrical contact. The source and drain allow the conductivity of the inversion layer at the surface of the $p$-type substrate to be measured. A voltage, $V_{G}$, is applied to the gate metal and a voltage, $V_{D}$, is appilied to the drain. The substrate and the source are normally tied to ground potential. In operation, the current that flows from the drain to the source, $I_{\mathrm{D}}$, is measured as a function of $V_{\mathrm{G}}$ and $V_{\mathrm{D}}$.

The mechanism for operation of the IGFET can be understood rather easily, at least in a qualitative manner. With a small positive voltage $V_{\mathrm{D}}$ applied to the drain and a voltage $V_{\mathrm{G}}$ of magnitude less than the threshold voltage $\left(V_{\mathrm{G}}<V_{\mathrm{T}}\right)$ applied to the gate metal, the surface of the silicon is either depleted or accumulated (i.e. it is not inverted and remains $p$-type silicon). Current cannot flow from the drain to the source under these conditions because the drain ( $n$-type silicon) is biased positive with respect to the stibstrate ( $p$-type silicon) resulting in a reverse-biased $p-n$ junction which essentially blocks the current. When $V_{\mathrm{G}}$ is raised above the threshold voltage, however, a surface inversion layer forms and the surface of the silicon substrate becomes $n$-type silicon. Current can now pass from the drain to the source through the $n$-type inversion layer without crossing the reverse-biased $p-n$ junction. Above the threshold voltage, the magnitude of the gate voltage modulates the number of electrons in the inversion layer and thus alters the effective conductance of the inversion layer. The resultant control of the drain current, $I_{\mathrm{D}}$, by the gate voltage forms the basis of the transistor action of the IGFET.

Analysis of the physics of the IGFET involves the derivation of the drain current as a function of the applied voltages, $V_{\mathrm{G}}$ and $V_{\mathrm{D}}$, and the geometry of the device. The derivation will follow the 'charge-control analysis' (Muller and Kamins 1977) which makes several simplifying assumptions but which results in a set of equations which agree well with experimental results and are easily understood.

Figure 26.6a is a schematic diagram of the IGFET showing the depletion layer (often called the space-charge region) and the inversion layer (often called the channel). The applied gate voltage, $V_{\mathrm{G}}$, is greater than the threshold voltage (i.e. the device is in strong inversion) and $V_{\mathrm{D}}$ is assumed to be very small so that the potential in the channel does not vary strongly with position between the source and drain. In this case, the current which flows in the channel can easily be related to the charge in the channel, $Q_{\mathrm{n}}$, and the transit time for the electron movement across the channel, $T_{\mathrm{tr}}$ by the equation

$$
I_{\mathrm{D}}=-Q_{\mathrm{n}} / T_{\mathrm{tr}} .
$$

The transit time is simply the length of the channel, $L$, divided by the electron drift velocity, $v_{\mathrm{d}}$, which is related to the field, $V_{\mathrm{D}} / L$, and the electron mobility in the channel, $\mu_{\mathrm{n}}$, by the equation

$$
v_{\mathrm{d}}=\mu_{\mathrm{n}} V_{\mathrm{D}} / L \text {, }
$$




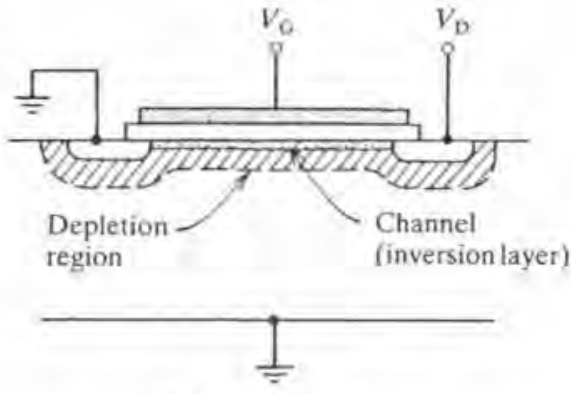

(a) $V_{G}>V_{T}, V_{D} \approx 0$
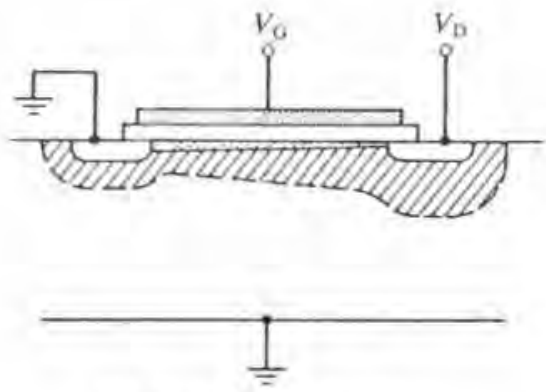

(b) $V_{\mathrm{G}}>V_{\mathrm{T}}, V_{\mathrm{D}}<V_{G}-V_{\mathrm{T}}$

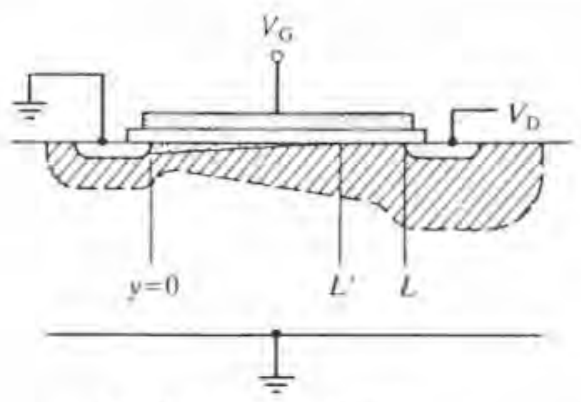

(c) $\left.V_{\mathrm{D}}>V_{\mathrm{G}}-V_{T}\right), V_{\mathrm{G}}>V_{T}$

Fig. 26.6 IGFET cross sections showing the effects of various bias conditions on the inversion layer and depletion layer. (a) The drain voltage is very small, (b) The drain voltage is large enough to cause significant variation of the thickness of the inversion and depletion layers. (c) The drain voltage exceeds the saturation value and the effective channel length is reduced from $L$ to $L^{\prime}$ referenced to $y=0$ at the source (after Muller and Kamins 1977).

The transit time is therefore

$$
T_{\mathrm{tr}}=L^{2} / \mu_{\mathrm{n}} V_{\mathrm{D}} \text {. }
$$

The charge in the channel, $Q_{\mathrm{n}}$, is simply the capacitance of the insulator, $C_{0}$, multiplied by $V_{\mathrm{G}}-V_{\mathrm{T}}$ (i.e. the portion of the gate voltage which created the channel). Since the insulator capacitance is expressed as a capacitance per unit area, we must multiply by the area of the gate, $W L$ :

$$
Q_{\mathrm{n}}=-C_{0}\left(V_{\mathrm{G}}-V_{\mathrm{T}}\right) W L
$$

where $W$ is the width of the gate, and the threshold voltage, $V_{\mathrm{T}}$, is given by eqn 26.5 . 
Substituting eqns 26.9 and 26.10 into eqn 26.7 , the drain current is given by

$$
I_{\mathrm{D}}=\frac{\mu_{\mathrm{n}} W C_{0}}{L}\left(V_{\mathrm{G}}-V_{\mathrm{T}}\right) V_{\mathrm{D}}
$$

If we now consider the case with an applied drain voltage which is not negligible compared to the gate voltage, then the charge distribution in the channel and space-charge regions are altered as shown schematically in Fig. 26.6b. The electron density in the channel near the drain is now much smaller because the effective channel bias near the drain is reduced. Similarly, the thickness of the depletion region near the drain is increased because of the applied drain voltage. A first-order analysis of the charge in the channel accounting for the applied drain voltage involves approximating the average potential between the gate and channel as $\left\{V_{\mathrm{G}}-\left(V_{\mathrm{D}} / 2\right)\right\}$. This leads to a channel charge, $Q_{n}$, different from that calculated in eqn 26.10:

$$
Q_{\mathrm{n}}=-C_{0}\left[V_{\mathrm{G}}-V_{\mathrm{T}}-\left(V_{\mathrm{D}} / 2\right)\right\} W L .
$$

The drain current therefore becomes

$$
I_{\mathrm{D}}=\frac{\mu_{\mathrm{n}} W C_{0}}{L}\left(V_{\mathrm{G}}-V_{\mathrm{T}}-\frac{V_{\mathrm{D}}}{2}\right) V_{\mathrm{D}}, V_{\mathrm{D}}<V_{\text {Dsat }}
$$

Equation 26.13 is only applicable for drain voltages less than $V_{\mathrm{G}}-V_{\mathrm{T}}$ (i.e. for the 'unsaturated' region of operation). When $V_{\mathrm{D}}>V_{\mathrm{G}}-V_{\mathrm{T}}$ (i.e. the 'saturated' region of operation), the voltage applied between the gate and the channel near the drain is actually less than or equal to zero. As a consequence the channel disappears near the drain when $V_{\mathrm{D}}>V_{\mathrm{G}}-V_{\mathrm{T}}$ as shown in Fig. 26.6c. When the IGFET is biased in the saturated region, the electrons in the channel see no energy barrier to their flow across this depletion region; rather, the region has a high electric field which accelerates the electrons to their limiting drift velocities. Consequently, the drain current is not affected by changes in the drain voltage when $V_{\mathrm{D}}>V_{\mathrm{G}}-V_{\mathrm{T}}$. The drain current equation for the saturated region of operation is therefore obtained by substituting the drain saturation voltage, $V_{\text {Dsat }}=V_{\mathrm{G}}-V_{\mathrm{T}}$, into eqn 26.13:

$$
I_{\mathrm{D}}=\frac{\mu_{\mathrm{n}} W C_{0}}{2 L}\left(V_{\mathrm{G}}-V_{\mathrm{T}}\right)^{2}, \quad V_{\mathrm{D}}>V_{\text {Dsat }} .
$$

Equations 26.13 and 26.14 give rise to the set of curves shown in Fig. 26.7. While the equations are not quantitatively correct, they do contain the correct qualitative features, i.e., the saturated and unsaturated regions, the constant current above $V_{\text {Dsat }}$, and the dependence of $V_{\text {Dsat }}$ on $V_{\mathrm{G}}$ and $V_{\mathrm{T}}$.

Figure 26.8 shows the dependence of the drain current on the gate voltage, $V_{G}$, as predicted by eqn 26.13 and 26.14 . In the saturated region (the curved region at low currents) the current is proportional to the square of the gate voltage while in the unsaturated region the current depends linearly upon the 


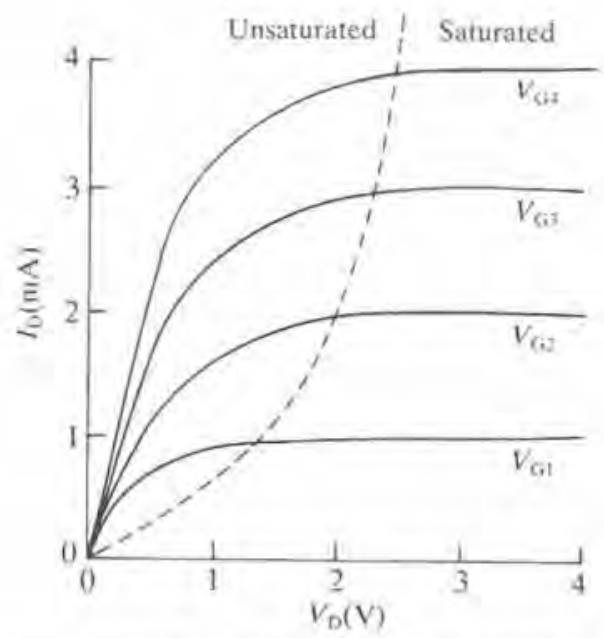

Fig, 26.7 Drain current versus drain voltage curves at several gate voltages showing the saturated and unsaturated regions of operation.

gate voltage. At higher gate voltages, however, the measured drain currents typically begin to saturate, deviating from the predicted linear curves. This deviation from predicted values arises from two separate effects. First, at large applied gate voltages, the field in the inversion layer perpendicular to the direction of current flow causes a decrease in the effective electron

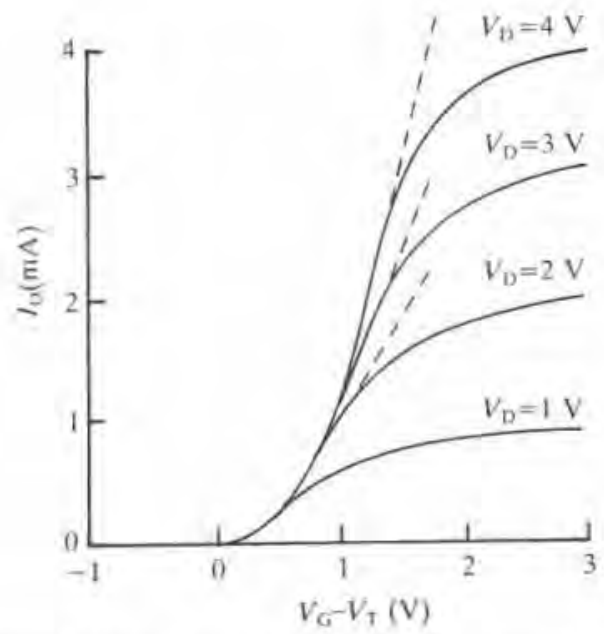

Fig. 26.8 Drain current versus gate voltage curves at several drain voltages. The dashed lines represent the theoretical linear relationship and the solid lines represent empirical curves. 
mobility, $\mu_{n}$, as the probability of the electron interacting with the surface increases. Second, in the typical geometrical layout of an IGFET on the silicon substrate, the bonding pads for the application of the control voltages are typically placed at the edges of the chip whereas the transistors are placed on the interior surface area of the chip. Thin metal strips or heavily doped silicon 'runners' usually connect the transistors to the bonding pads. Because these connections cannot be made resistance free, they contribute a resistance in series with the drain-source circuit of the IGFET. At high drain currents the resistive voltage drop caused by this series resistance, $R_{\mathrm{S}}$, becomes appreciable and the actual voltage between the drain and source becomes $V_{\mathrm{D}}-I_{\mathrm{D}} R_{\mathrm{S}}$ rather than the applied voltage $V_{\mathrm{D}}$. The series resistance, therefore, causes the drain current to be lower than expected at high current levels.

\subsubsection{The chemically sensitive field-effect transistor}

The IGFET described above is transformed into a CHEMFET, conceptually at least, by replacing the metal gate with a chemically sensitive membrane. In the case of CHEMFETs sensitive to species in aqueous solution, electrical potentials are applied to the gate region through a reference electrode in the bathing solution as shown in Fig. 26.9. From a comparison of Figs 26.9 and 26.5 (CHEMFET and IGFET) it is apparent that the only difference between the electrical circuits is the replacement of the metal gate of the IGFET by the

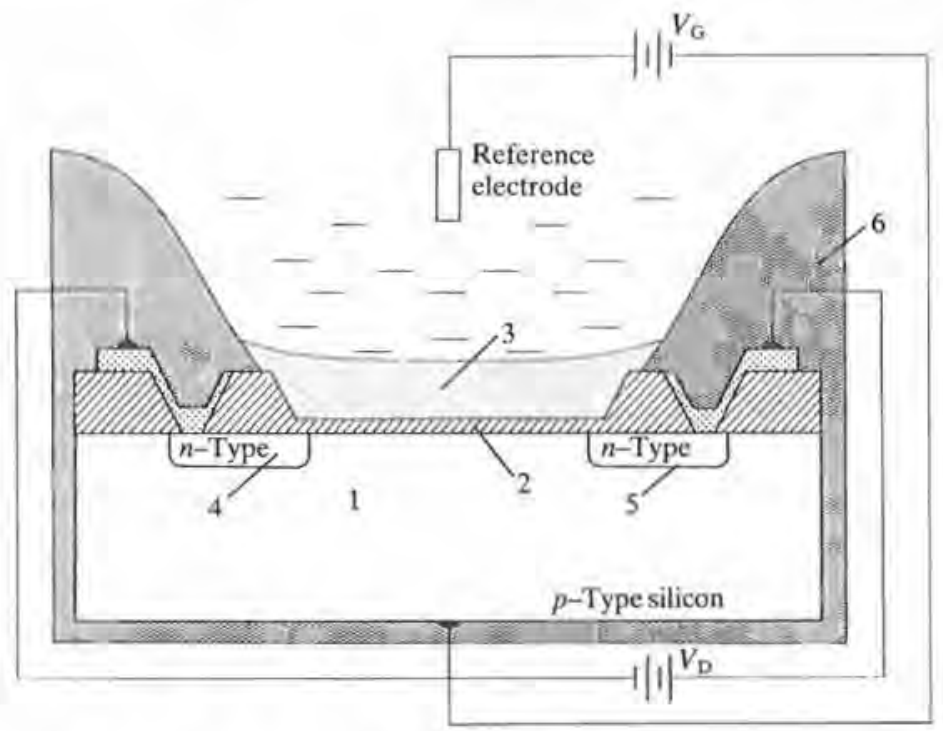

Fig. 26.9 Diagram of the CHEMFET. (1) Silicon substrate, (2) insulator, (3) chemically sensitive membrane, (4) source, (5) drain, and (6) insulating encapsulant. 
series combination of the reference electrode, solution, and chemically sensitive membrane.

The equations describing the operation of the CHEMFET are derived from the analogous equations for the IGFET by taking into account the potential differences between the new elements in the circuit. In this section, a set of general equations will be developed which describe the current-voltage characteristics for the device in terms of the potential differences at the newly created interfaces but which do not describe how these potential differences depend on the chemical properties of the bathing electrolyte. Rather, the mechanism for the chemical sensitivity of each type of CHEMFET will be described independently and specific equations will be developed in the corresponding sections below.

Referring to eqns 26.13 and $26.14, V_{\mathrm{G}}$ is the voltage applied to the gate metal and, therefore, is the potential at the surface of the insulator next to the gate metal. Analogous equations can be written for the CHEMFET by replacing $V_{\mathrm{G}}$ in these equations with the new potential at the surface of the insulator. This potential will simply be the voltage applied to the metal wire of the reference electrode, $V_{G}$, plus the sum of the potentials at the reference electrode-solution interface, $E_{\text {rei }}$, and the solution-membrane interface, $\phi_{\text {sol-mem. }}$. The general equations for the CHEMFET in solution are, therefore,

$I_{\mathrm{D}}=\frac{\mu_{\mathrm{n}} W C_{0}}{L}\left(V_{\mathrm{G}}-V_{\mathrm{T}}^{*}-E_{\text {rè }}-\phi_{\text {sol-mem }}-\frac{V_{\mathrm{D}}}{2}\right) V_{\mathrm{D}} \quad V_{\mathrm{D}}<V_{\text {Dsal }}$

and

$$
I_{\mathrm{D}}=\frac{\mu_{\mathrm{n}} W C_{0}}{2 L}\left(V_{\mathrm{G}}-V_{\mathrm{T}}^{*}-E_{\text {ref }}-\Phi_{\text {sol-mem }}\right)^{2} \quad V_{\mathrm{D}}>V_{\text {Dsal }} .
$$

As is evident, the threshold voltage is redefined as $V_{\mathrm{T}}{ }^{*}$ because the original definition (eqns 26.5 and 26.6 ) included the term $\Phi_{m s}$, the work function difference between the metal and semiconductor. For the CHEMFET in solution, the work function difference should be divided into individual terms:

$$
\Phi_{\mathrm{ms}}=\Phi_{\mathrm{m}-\mathrm{sol}}+\Phi_{\text {sol-mem }}+\Phi_{\text {mem-s }}
$$

where $\Phi_{\mathrm{m}-\mathrm{s} \mid}, \Phi_{\text {sol-mem }}$ and $\Phi_{\mathrm{met}-\mathrm{s}}$ are the work function differences between the metal and solution, solution and membrane, and membrane and semiconductor, respectively. The terms $E_{\text {tef }}$ and $\phi_{\text {sol-mem }}$ already account for the work function differences, $\Phi_{\mathrm{m}-\mathrm{sol}}$ and $\Phi_{\text {sol-mem }}$ respectively so the definition of the threshold voltage must be redefined as

$$
V_{\mathrm{T}}^{*}=V_{\mathrm{T}}-\Phi_{\text {m-sol }}-\Phi_{\text {sol-mem }}=\Phi_{\text {mem-s }}-\frac{Q_{\mathrm{s5}}}{C_{0}}-\frac{Q_{\mathrm{B}}}{C_{0}}+2 \phi_{\mathrm{F}}
$$

where the charge in the insulator is assumed to be negligible. 
Equations 26.15-26.18, therefore describe the current-voltage characteristics for the CHEMFET without defining the mechanism for the generation of the potential difference at the solution-membrane interface, $\phi_{\text {sol-mem }}$. The important point is that the magnitude of the drain current is dependent upon this potential difference. The CHEMFET, therefore, acts as an extremely high impedance transducer to detect changes in this potential difference. By using membranes that develop potentials which are dependent upon the solution concentration of a particular species, the FET transducer is rendered chemically sensitive. Following sections will describe various schemes which create a field-effect transistor sensitive to a variety of different chemicals.

\subsection{Sensor fabrication}

The process for the fabrication of a CHEMFET sensor can be divided into two parts. The first part of the process includes the fabrication of the devices in the silicon wafer (usually a 50 or $75 \mathrm{~mm}$ diameter wafer). In this first phase, each process step affects every chip simultaneously (typically several hundred to thousands of chips) and is thus very cost and labour efficient. The second part of the sensor fabrication process include those steps which are taken after the wafer is diced into individual chips and includes mounting the chip on a support, making electrical connections, encapsulating the sensor, and, usually, depositing a chemically sensitive membrane. Since each sensor is treated individually, the effort per sensor can be significant.

\subsubsection{Wafer micro-fabrication}

Each laboratory which fabricates CHEMFET devices uses different processing sequences and procedures and thus a description of the complete process is both impractical and beyond the scope of this text. For a detailed description of semiconductor processing, the reader is referred to texts specifically devoted to the topic such as Colclaser (1980).

For an $n$-channel CHEMFET, one starts with a silicon wafer which is doped with boron, making it $p$-type. The $n$-type source and drain are then formed by masking the wafer with the proper pattern by photolithographic techniques and introducing phosphorous atoms into the surface of the silicon by either ion implantation or chemical diffusion. The silicon-dioxide gate insulator (typically $50-100 \mathrm{~nm}$ thick) is formed by the thermal oxidation of the silicon surface at $1000-1200{ }^{\circ} \mathrm{C}$ in an oxygen atmosphere. Silicon nitride, which is often used as a second insulator on top of the silicon dioxide to provide better resistance to hydration, is typically grown by chemical vapour deposition at $600-800{ }^{\circ} \mathrm{C}$ in a nitrogen atmosphere containing silane $\left(\mathrm{SiH}_{4}\right)$ and ammonia $\left(\mathrm{NH}_{3}\right)$, again, $50-100 \mathrm{~nm}$ thick. Electrical contacts are made to the source, drain, and substrate by etching holes through the insulator and 


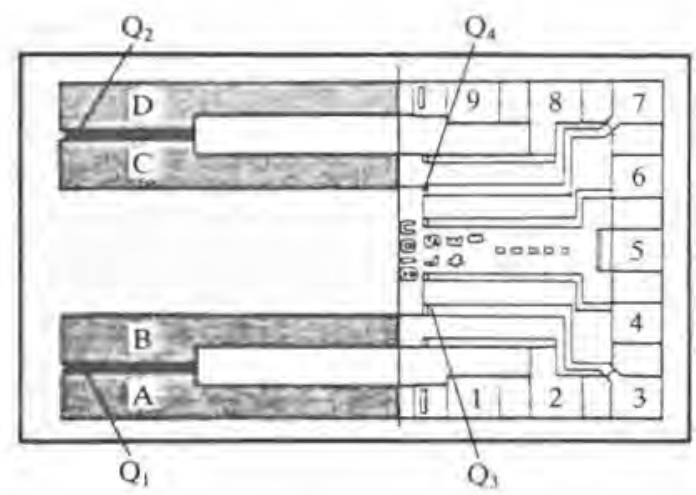

Fig. 26.10 Diagram of a typical CHEMFET chip. See text for description (after Janata and Huber 1979).

evaporating metal (generally aluminium) runners which connect these regions to bonding pads at the periphery of the chip. Upon the completion of the micro-fabrication process, the wafer is scribed with a diamond tip stylus and is then broken into individual chips.

The geometrical layout of the chips also varies among the laboratories investigating CHEMFETs. Figure 26.10 is a drawing of a chip which has been used extensively at the University of Utah (Janata and Huber 1979) and is typical of most. The chip has dimensions of $1.28 \times 2.16 \mathrm{~mm}$ and contains four FET devices; $Q_{1}$ and $Q_{3}$ are CHEMFETs and $Q_{2}$ and $Q_{4}$ are conventional IGFETs. The dimensions of the gates are all $20 \times 400 \mu \mathrm{m}$. A, B, C, and D are the drain and source regions for the two CHEMFETs. The numbered regions 1-9 are metal bonding pads to which electrical contacts are made to the external control circuitry. The two CHEMFET devices are placed at one end of the chip while the bonding pads are placed at the opposite end to facilitate coverage of the bonding pads, connecting wires, and edges of the chip with encapsulant while leaving the gates of the CHEMFETs uncovered.

\subsubsection{Sensor packaging}

Once again, each laboratory has its own techniques for packaging (supporting, wiring, and encapsulating) the CHEMFET sensors. Figure 26.11 represents the configuration used by this author for most of his research (Blackburn 1983). The support for the chip (1) is a length of $3 \mathrm{~mm}$ outer diameter glass tubing. Insulated copper wires (2) are threaded through the glass tubing, bent at right angles at one end and then anchored in place with epoxy (3). After curing, the surface of the epoxy is ground off and filed smooth to give a flat surface with the ends of the copper wires exposed to 


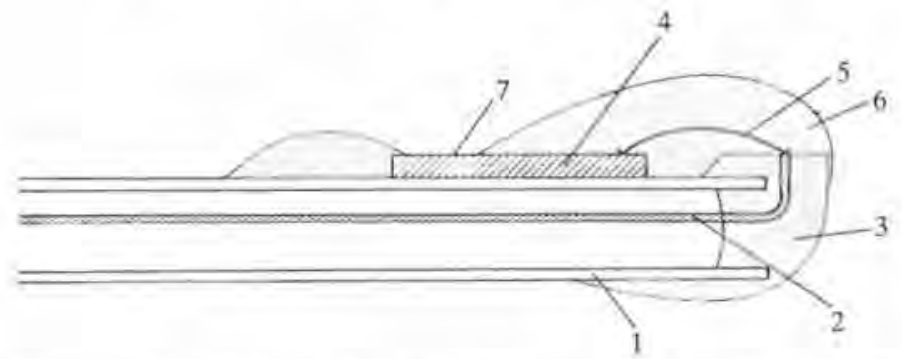

Fig. 26.11 Diagram of a typical CHEMFET sensor. (1) Glass tubing, (2) copper wires, (3) epoxy encapsulant, (4) CHEMFET chip, (5) wirebond connections, (6) epoxy encapsulant, and (7) exposed gate areas of CHEMFET chip.

serve as bonding pads for the connections to the chip. The CHEMFET chip (4) is then mounted on the outer surface of the glass tubing with cyanoacrylate adhesive. Electrical connections (5) between the chip and copper wires are made with $25 \mu \mathrm{m}$ diameter aluminium wire using an ultrasonic wirebonder. The devices are then encapsulated with epoxy (6), covering the wirebond wires, the copper wires, and all of the chip except the gate areas (7). The epoxy encapsulant serves to insulate the chip from the solution and must remain insulating for long periods of immersion in aqueous solutions. After curing the epoxy, chemically sensitive membranes are generally applied as described in the appropriate sections below. Most of the steps are performed under a $10-40 \times$ stereomicroscope to aid in visualizing the small areas of the sensor.

Ho et al. (1983) have described a technique for packaging the CHEMFET sensor which can potentially be automated. As discussed above, automation of the encapsulation process would significantly reduce the cost of and concomitantly enhance the commercial viability of CHEMFET sensors. Figure 26.12 depicts the packaging technique. The chip is mounted on the substrate (polyimide tape) and a second strip of polyimide tape containing a copper beam lead pattern is aligned and placed over the chip. The beam leads are then bonded to the chip's bonding pads either by ultrasonic or thermocompression bonding. A third strip of polyimide tape containing windows is then aligned and bonded over the surface of the chip, which leaves the entire chip encapsulated except for the chemically sensitive gates of the FET. To date, the process is still performed manually under a microscope, but the process could potentially be automated.

\subsection{Control and measurement circuitry}

Two different operating modes are typically used in making measurements with CHEMFETs. In the first, the gate voltage is held constant and the drain 


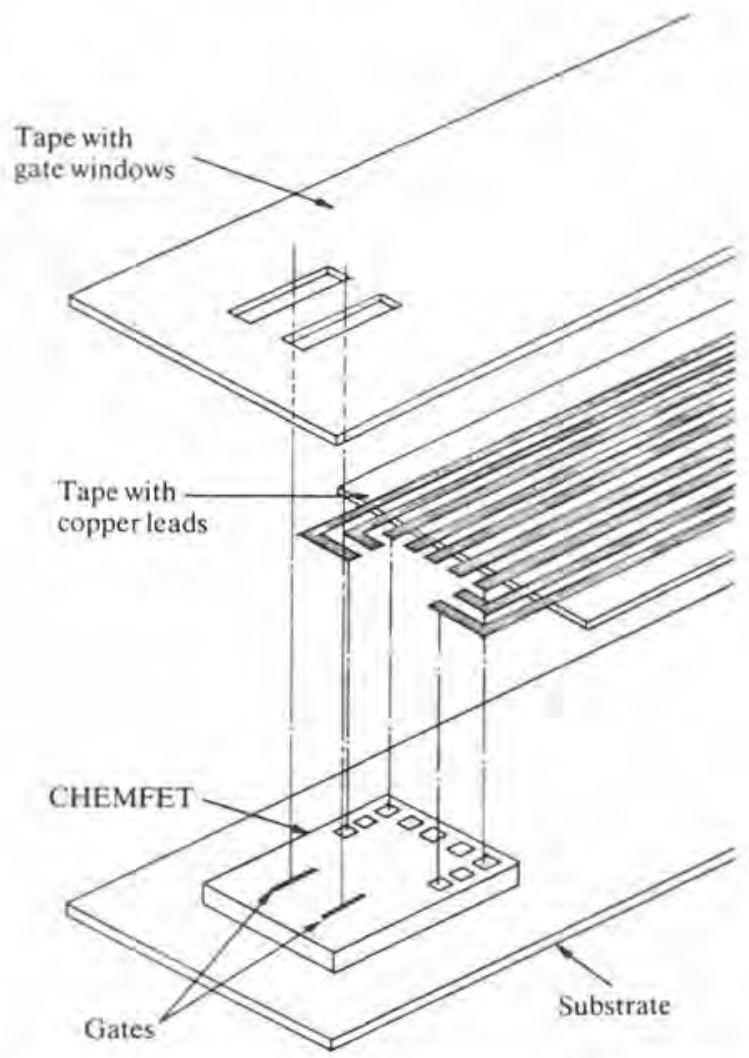

Fig. 26.12 Diagram depicting an encapsulation scheme with potential for automation utilizing polyimide tape as the encapsulant (after Ho et al. 1983).

current is monitored. In the second method, the gate voltage is changed as necessary by a feedback circuit to maintain a constant drain current. The analog control and measurement circuitry described below represents probably the least complex circuitry which could be employed. Although more elaborate circuits have been designed and tested and microprocessor control is straightforward, the circuits shown here provide an adequate and simple means for the operation of CHEMFET sensors.

\subsubsection{Constant gate voltage operation}

A simple circuit for this mode of operation is shown in Fig. 26.13 (Blackburn 1983). Constant voltages, $V_{\mathrm{D}}$ and $V_{\mathrm{G}}$, are applied to the drain and reference electrode, respectively. The substrate and the source are shorted together on the clip and the lead is fed into an op amp which serves as a current-to-voltage converter. The output voltage of the op amp is given by the product $-R_{1} I_{\mathrm{D}}$. 


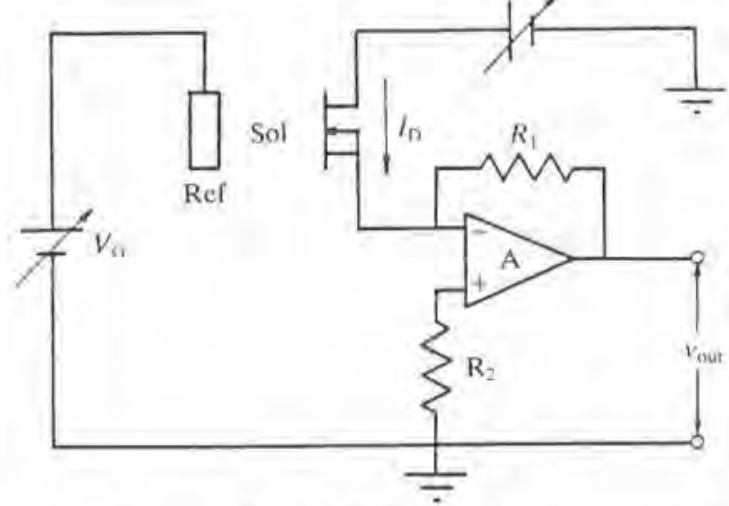

Fig. 26.13 Schematic diagram of circuit for measuring $I_{\mathrm{D}}$ at constant gate voltage. $A$, operational amplifier; $R_{1}=1 \mathrm{k} \Omega ; R_{2}=470 \Omega$.

Changes in the potential at the membrane-solution interface are monitored as changes in the drain current. The drain current is not a linear function of the gate voltage over its entire range, however. Thus, the relationship between the two variables must be known for every operating point to calculate the interfacial potential change from this change in drain current. The advantage of this mode of operation is that because the gate voltage is constant, several devices can be monitored simultarieously in the same solution with one reference electrode serving to bias all.

\subsubsection{Constant drain current operation}

A simple circuit for this mode of operation is shown in Fig. 26, 14 (Blackburn 1983). As before, the voltage applied to the drain is constant and the drain current is measured by op amp $A_{1}$. The output of the op amp, $V_{1}$, is fed into a voltage divider comprised of $R_{2}$ and $R_{3}$. The voltage at the other end of the divider is controlled by $V_{\text {set }}$. Since $R_{2}$ and $R_{3}$ are equal values, the voltage between them will be the average of $V_{1}$ and $V_{\text {set. }}$. This voltage is measured by op amp $A_{2}$. The output of this op amp is fed to the reference electrode and thus affects the drain current of the CHEMFET. The negative input of op amp $\mathrm{A}_{2}$ is tied to ground through $R_{4}$. In operation, since the two inputs must be at the same voltage, the voltage on both inputs must be zero which requires that the output of $\mathrm{A}_{1}$ be $-V_{\text {set }}$. Since the output of op amp $\mathrm{A}_{1}$ is $-R_{1} I_{\mathrm{D}}, I_{\mathrm{D}}$ must be equal to $V_{\text {set }} / R_{1}$. This requirement is met by the feedback op amp $\mathrm{A}_{2}$ which adjusts its output, $V_{\mathrm{G}}$, to control the drain current. Thus, op amp $\mathrm{A}_{2}$ holds the drain current constant $\left(I_{\mathrm{D}}=V_{\text {set }} / R_{\mathrm{f}}\right)$ by changing the voltage applied to the reference electrode. If the potential of the membrane-solution interface changes, then the feedback circuit compensates an equal and 


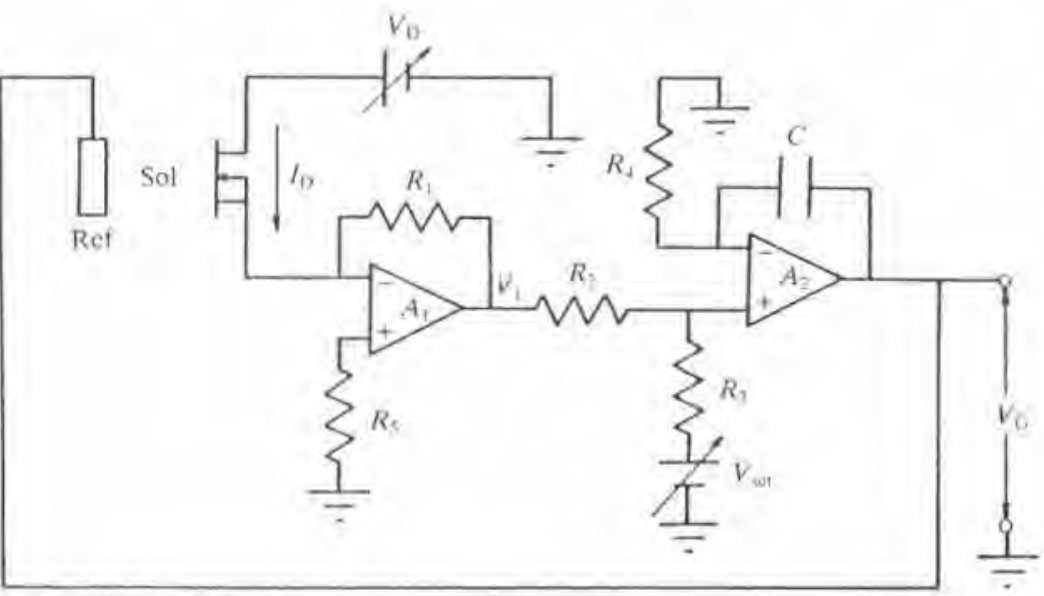

Fig. 26.14 Schematic diagram of the circuit for measuring changes in $V_{\mathrm{G}}$ at constant drain current. $\mathrm{A}_{1}, \mathrm{~A}_{2}$; operational amplifiers; $R_{1}=1 \mathrm{k} \Omega ; R_{2}=R_{3}=100 \mathrm{k} \Omega$; $R_{4}=20 \mathrm{k} \Omega ; R_{5}=470 \Omega ; C=10 \mathrm{pF}$,

opposite amount to maintain a constant drain current. In this way, the changes in the interfacial potential can be monitored directly.

This mode of operation has the disadvantage that only one sensor can be monitored at a time because the gate voltage for each must be independently controlled and the solution can only contain a single reference electrode. It is usually the operational mode of choice, however, because it provides a direct indication of the change in the interfacial potential.

\subsection{Ion-selective field-effect transistor}

The ion-selective field-effect transistor represents the marriage of the technologies of the ion-selective electrode and solid-state electronics. One of the inherent problems in the use of conventional ISEs is that the output signal from the sensor is typically rather noisy as a consequence of the high electrical impedance of the ion-selective membrane; the wires connecting the high impedance electrode to the amplifier (typically a high input impedance $\mathrm{pH}$ meter) serve as 'antennae', responding to any change in the local electromagnetic field. To reduce the electrical noise problem, the wires are generally fabricated from shielded cable and their length is kept to a minimum. Even with these precautions, it is often necessary to attempt to limit the sources of interference (e.g. it is usually necessary for the operator to stand extremely still or to step away from the electrode and electronics to achieve a stable reading). The ISFET represents this solution (i.e. using shortened cables) taken to the extreme that the cable length is reduced to zero, i.e. the ion- 
selective membrane is placed directly on the gate of the high impedance amplifier's input transistor. To facilitate measurement, the transistor is separated from the amplifier and placed on an electrode-like support which can be placed in the sample solution. The low impedance output of the FET is connected to the electronics through wires of any convenient length without the need for shielding; the ISFET makes an in situ impedance transformation and therefore is not susceptible to interference from changes in local electromagnetic fields.

\subsection{Theory}

The ion sensitivity of the ISFET arises from the use of ion-selective membranes as the chemically sensitive layer over the gate of the transistor. The membranes are identical to those developed for use with ion-selective electrodes as described by Freiser $(1978,1980)$ and Koryta $(1975)$. The reader is referred to these sources for a thorough description of the mechanisms and thermodynamics of ion-selective membranes.

The ion-selectivity and sensitivity of a 'good' ion-selective membrane results from a very small energy barrier for the transport of one particular ion across the solution-membrane interface while a rather large energy barrier is maintained for the transport of all other ions. An interface with these properties is known in electrochemistry as 'non-polarized.' When the solution concentration of the ion of interest changes, those ions experiencing only a small energy barrier tend to diffuse down the newly changed concentration gradient across the interface. In contrast, all other ions see a large energy barrier and are unable to cross the interface. As an example, if the ionselective membrane is selective for potassium ions and if the concentration of potassium chloride is increased in solution, then potassium ions will begin to flow from the solution into the membrane; the chloride ions, however, are unable to diffuse into the membrane because of the high energy barrier. As a result, a charge imbalance is created across the interface as ions of one charge move across the interface and their counter ions are excluded. This separation of charge creates a change in the potential across the interface which acts to force the ions in a direction opposite to that of the concentration gradient. The potential change thus slows and eventually stops the net flow of ions across the interface. The change in concentration of the particular ion therefore results in an interfacial potential change, $\Delta \phi_{\text {sol-mem, }}$ which can be measured by the ISFET. An analysis of the thermodynamics of the system reveals the relationship between the potential change and the concentration of the ion.

If ion $i$ can freely cross the interface between the solution and membrane, then at equilibrium the electrochemical potentials, $\tilde{\mu}_{\text {ifol }}$ and $\tilde{\mu}_{i_{\mathrm{mem}}}$, for the ion must be equal in the two phases:

$$
\tilde{\mu}_{i_{\text {sol }}}=\tilde{\mu}_{i_{\text {mein }}}
$$


the electrochemical potential for species $i$ is defined as

$$
\tilde{\mu}_{i}=\mu_{i}+z_{i} F \phi_{i}
$$

where $z_{i}$ is the charge of species $i, F$ is the Faraday constant, and $\phi_{i}$ is the bulk potential in the phase in which the ions exist. $\mu_{i}$ is the chemical potential of species $i$, defined as

$$
\mu_{i}=R T \ln \left(a_{i}\right)+\mu_{i}^{0}
$$

where $R$ is the gas constant, $T$ is the temperature (Kelvin), and $\mu_{i}^{0}$ is the standard chemical potential for species $i . a_{i}$ is the activity of species $i$ (approximately equal to the concentration for dilute solutions). Equations 26.20 and 26.21 can be written for species $i$ in both the membrane and solution phases. Substitution of these definitions into eqn 26.19 yields the relationship between the activity of the ion and the potential at the interface, the Nernst equation:

$$
E=\phi_{\text {sol-mem }}=E^{0}-\frac{R t}{z F} \ln \left(a_{i}\right)
$$

where $a_{i}$ is now assumed to represent the activity of the ion in solution. The activity of the ion in the membrane is usually assumed to be large and constant and is therefore included in the term $E^{0}$. Equation 26.22 can now be substituted into eqns 26.15 and 26.16 to yield the ion-selective response of the ISFET to the change of activity of ion $i$

$$
I_{\mathrm{D}}=\frac{\mu_{\mathrm{n}} W C_{0}}{L}\left\{V_{\mathrm{G}}-V_{\mathrm{T}}{ }^{*}-E_{\text {ref }}-E^{0}+\frac{R T}{z F} \ln \left(a_{i}\right)-\frac{V_{\mathrm{D}}}{2}\right\} V_{\mathrm{D}}
$$

and

$$
I_{\mathrm{D}}=\frac{\mu_{\mathrm{n}} W C_{0}}{2 L}\left\{V_{\mathrm{G}}-V_{\mathrm{T}}^{*}-E_{\mathrm{ref}}-E^{0}+\frac{R T}{z F} \ln \left(a_{t}\right)\right\}^{2}, V_{\mathrm{D}}>V_{\text {Dsat }} .
$$

\subsubsection{Ion-selective membranes for the ISFET}

As stated above, the membranes which are used with ISFETs are usually identical to those used with ISEs. These two types of sensors simply represent two different schemes for the measurement of changes in the potential at the solution-membrane interface. The membranes and therefore the potential generating mechanisms are identical. Each of the three types of ion-selective membranes which have been employed in ISFETs will be discussed below.

26.5.2.1 Solid-state membranes The first reported ISFET by Bergveld (1970) used a solid-state $\mathrm{pH}$ membrane of silicon dioxide $\left(\mathrm{SiO}_{2}\right)$. Shortly afterwards, Matsuo et al. (1971) described an ISFET which utilized a solid- 
state membrane of silicon nitride $\left(\mathrm{Si}_{3} \mathrm{~N}_{4}\right)$. Since these early reports, $\mathrm{SiO}_{2}$ has largely been abandoned as a $\mathrm{pH}$ membrane for ISFETs because it rapidly hydrates in aqueous solutions and loses its insulating properties which are essential for the operation of the CHEMFET. Solid-state $\mathrm{pH}$ membranes of $\mathrm{Al}_{2} \mathrm{O}_{3}$ (Abe et al, 1979), $\mathrm{ZrO}_{2}$, and $\mathrm{Ta}_{2} \mathrm{O}_{5}$ (Akiyama et al. 1982) have also been investigated.

Solid-state membranes are particularly attractive as ion-selective membranes for ISFETs because they can be deposited using common integrated circuit fabrication techniques. In addition, the membranes are deposited on the entire wafer of sensor chips simultaneously before it is broken up into individual chips. The effort and cost per sensor are thus reduced considerably.

Of the above mentioned inorganic materials, $\mathrm{Al}_{2} \mathrm{O}_{3}$ and $\mathrm{Ta}_{2} \mathrm{O}_{5}$ demonstrate the most desirable characterisics, giving $\mathrm{pH}$ sensitivities of $52-58 \mathrm{mV} / \mathrm{pH}$, a $95 \%$ response time of, at most, a few seconds, nearly negligible drift, and very little hysteresis (Abe et al. 1979; Akiyama et al. 1982).

The development of solid-state membranes for species other than the hydrogen ion have had only limited success. Many materials which are used routinely in ISEs cannot be deposited by conventional integrated circuit fabrication techniques and thus have not been exploited. Nevertheless, Buck and Hackleman (1977) developed a silver bromide solid-state membrane sensitive to bromide ions and Esashi and Matsuo (1978) developed sodium ion-selective solid-state membranes of aluminosilicate or borosilicate glass. These membranes both exhibit a sodium response of $55 \mathrm{mV} / \mathrm{pNa}$ in the range of $\mathrm{pNa} 0-3$ and a measurable response down to $\mathrm{pNa} 5$.

26.5.2.2 Polymer membranes The polymer membranes which are employed in the fabrication of ISFETs are identical to those used in ISEs. In general, any polymeric membrane which has been developed for ISEs can be used directly with ISFETs. Because of this significant overlap, no attempt will be made to repeat the description of all types of ion-selective membranes in this section.

The polymer membranes are normaily applied to the gate region of the ISFET by solvent casting from a volatile solvent. Multiple castings to attain membranes thicker than approximately $50 \mu \mathrm{m}$ are usually necessary to achieve pinhole-free membranes. If pinholes are present in the membranes they act as electrical shunts through the membrane when filled with electrolyte, shorting out the electrochemical potential and rendering the ISFET useless. The most widely studied ion-selective polymer membranes coupled with ISFETs are those sensitive to potassium and calcium ions. The potassium-selective membrane contains the ionophore valinomycin in a PVC polymer matrix which is highly plasticized with dioctyladipate according to the formulation of Band et al. (1978). The calcium-selective membrane 


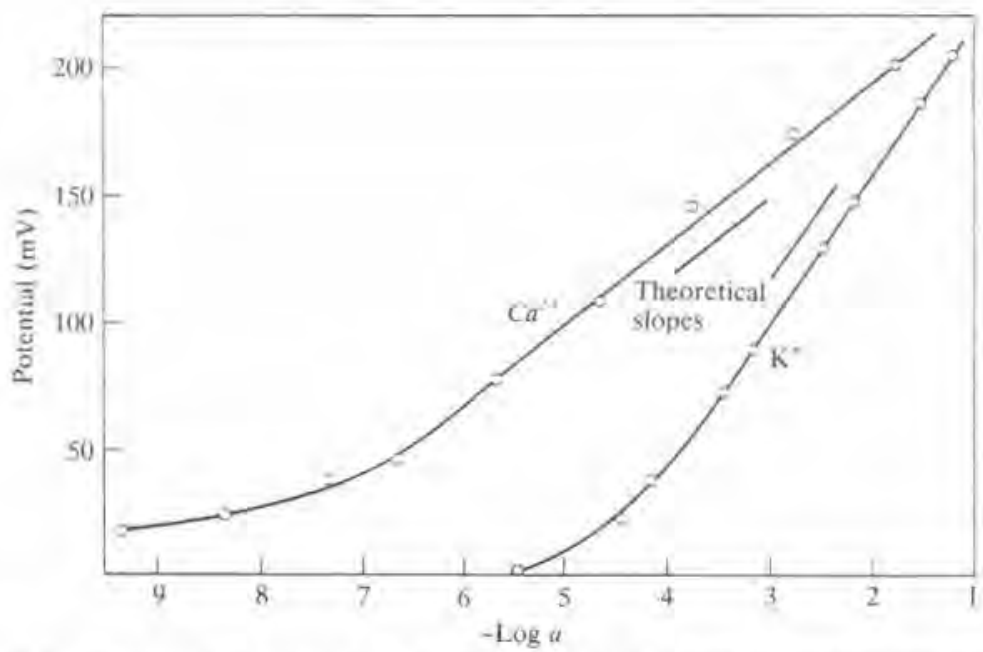

Fig. 26.15 Response of calcium and potassium ISFET with polymeric ion-selective membranes (after McBride et al. 1978).

utilizes tHODPP [ $p$-(1,1,3,3-tetramethylbutylphenyl) phosphoric acid] as the ionophore in a similar plasticized PVC matrix according to Griffiths et al, (1972). The membranes are cast from a solvent mixture of $1: 1(\mathrm{v} / \mathrm{v})$ tetrahydrofuran and cyclohexanone. Figure 26.15 shows the typical response for ISFETs using these membranes according to McBride et al. (1978).

26.5.2.3 Heterogeneous membranes Heterogeneous membranes were pioneered by Pungor (1967) and are of special interest for ISFETs because their use circumvents the difficulties encountered in the fabrication of many types of solid-state membranes. In general, the membranes consist of a semiconducting electrode material, usually an inorganic salt of low solubility in the form of a finely divided powder, immobilized in a polymer matrix. Shiramizu et al. (1979) reported the development of heterogeneous membrane ISFETs sensitive to chloride, iodide, and cyanide ions using silver salts in the elastomeric polymer, polyfluorinated phosphazine (PNF). Chloride-sensitive membranes were formulated from silver chloride powder in PNF at a weight ratio of $3: 1$. An improved membrane was formulated from a 4:1 mixture of silver chloride and silver sulphide. A heterogeneous membrane sensitive to both iodide and cyanide was formulated from a 3:1 mixture of $75 \%$ silver iodide and $25 \%$ silver sulphide in PNF. The response of these various ISFETs is shown in Fig. 26.16, where the $\mathrm{Cl}^{-}$membrane contains both silver chloride and silver sulphide while the $\mathrm{Cl}^{-}{ }_{\mathrm{b}}$ membrane contains only silver chloride. 


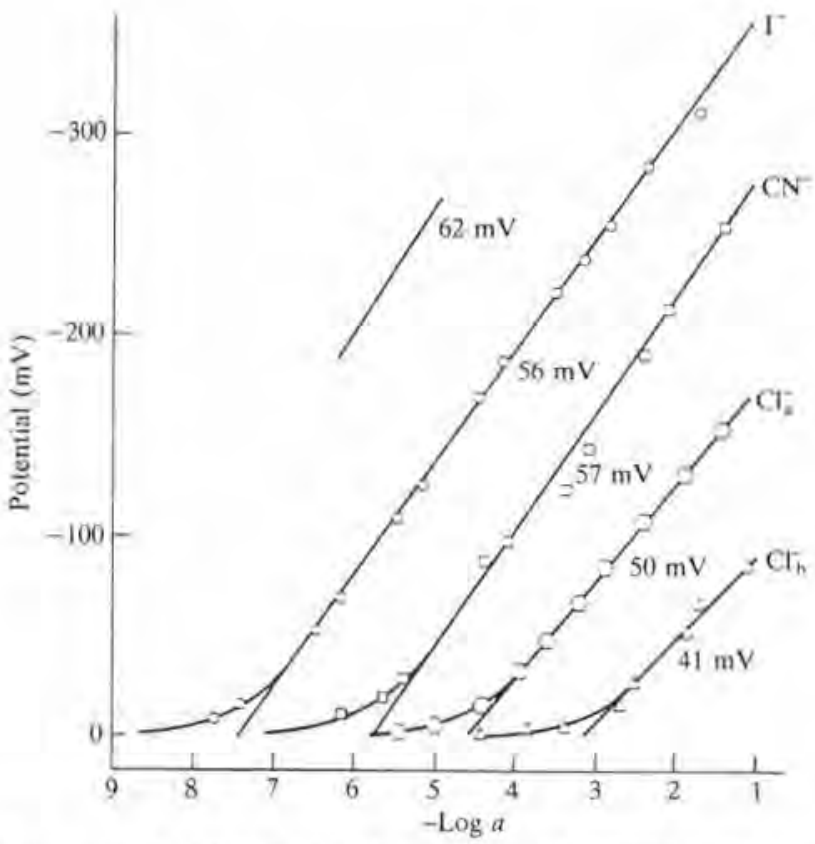

Fig. 26.16 Response of chloride, iodide, and cyanide ISFETs with heterogeneous membranes (after Janata and Huber 1980).

\subsubsection{Time response}

One of the primary advantages of the ISFET is that it makes an in situ impedance transformation, eliminating the electrical lead between the membrane and the amplifier. The parasitic capacitance of the leads is thus eliminated. As a consequence, the $R C$ time constant for the response of the ISFET should be much smaller than that of conventional ISEs. McBride et al. (1978) reported the measurement of electrical and chemical time responses for $\mathrm{Si}_{3} \mathrm{~N}_{4}$ solid-state $\mathrm{pH}$ membranes and for calcium-sensitive polymer membranes (see Fig. 26.17). The electrical time responses were measured by applying a square-wave voltage to the reference electrode and measuring the response of the drain current. The chemical time responses were measured by injecting a stream of solution across the membrane from a syringe containing a higher concentration of the ion. It was found that the polymer membrane exhibited a longer response time to potential changes due to the dynamic limitations of the membrane over the gate. The longer response time to changes in concentration as compared to changes in potential indicates that the response is limited by a diffusion layer at the membrane-solution interface. Thus, a shorter response time for ISFETs as compared to ISEs should not be expected. 
Bare $\mathrm{Si}_{3} \mathrm{~N}_{4}$

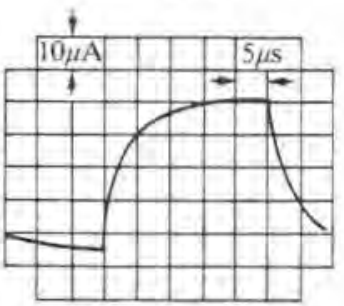

$\mathrm{Ca}^{2+}$ membrane

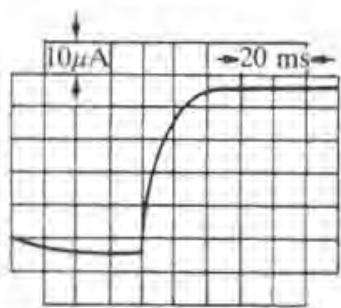

(a) Electrical response
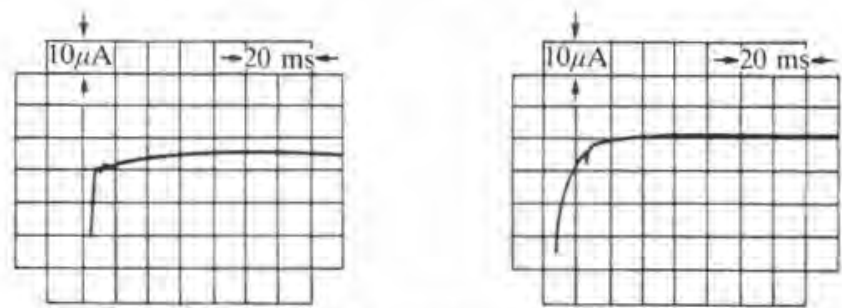

(b) Chemical response

Fig. 26.17 Time response of silicon nitride $\mathrm{pH}$-sensitive and calcium-sensitive membrane ISFET's (after McBride et al. 1978).

\subsubsection{Suspended mesh ISFET}

One of the inherent problems in the use of ISFETs with polymer ion-selective membranes is that the adhesion of the membrane to the surface of the sensor is generally very poor. In the conventional ISE, the membrane is mechanically clamped to the end of a tube so that adhesion is not a problem. In the ISFET, however, the membrane is simply cast over the gate area of the sensor and must rely on physical or chemical adhesion to the surface. This problem has been circumvented in several laboratories by applying the membrane over a large area of the sensor or by using a PVC anchoring ring embedded in the encapsulant around the perimeter of the gate area (McKinley et al. 1980). Others have approached the problem by changing the chemical composition of the membrane at the expense of the electrochemical performance.

The poor adhesion results in a gradual detachment of the membrane from the surrounding encapsulation and transistor surface and the development of electrolytic shunts around the membrane. The measurement of the electrochemical potential at the membrane-solution interface is thus erroneous and unpredictable. Any mechanical stress on the membrane, such as would be encountered in a high electrolyte flow situation or during in vivo experiments 


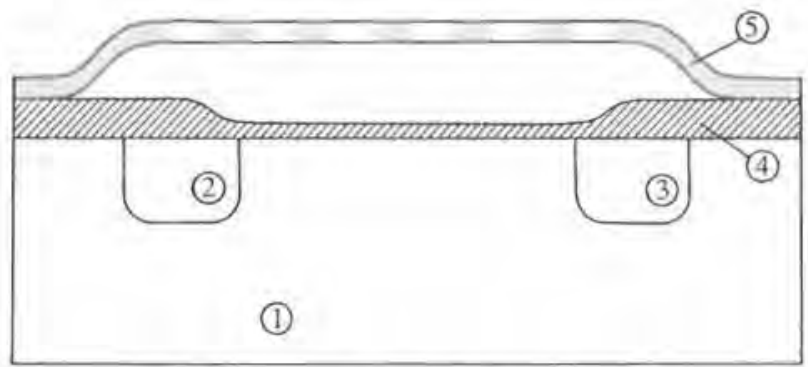

Fig. 26.18 Diagram of the suspended mesh ISFET chip before encapsulation and membrane deposition. (1) Silicon substrate, (2) source, (3) drain, (4) insulator, and (5) polymer suspended mesh (after Blackburn and Janata 1982).

when the device is inserted into tissue, usually results in complete detachment of the membrane from the device.

As a solution to the problem, Blackburn and Janata (1982) developed the suspended mesh (SM) ISFET. The device incorporates a three-dimensional structure on the surface of the FET over the sensing gates which acts to anchor the membrane. As shown schematically in Fig. 26.18, the suspended mesh consists of a polymer film suspended above the gate. The polymer film contains an array of holes through the film over the gate area of the FET. When a polymer ion-selective membrane is solvent cast over the gate area, the membrane flows under the mesh as well as over it, filling the air gap under the mesh. When the solvent evaporates, the suspended mesh becomes an integral part of the membrane, anchoring it to the surface as shown in Fig. 26.19.

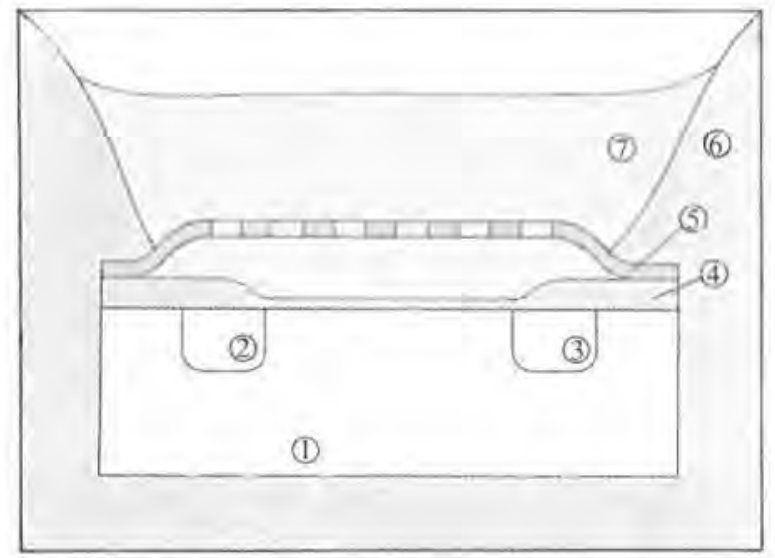

Fig. 26.19 Diagram of the suspended mesh ISFET chip after encapsulation and membrane deposition. (1) Silicon substrate, (2) source, (3) drain, (4) insulator, (5) polymer suspended mesh, (6) encapsulant, and (7) ion-selective membrane (after Blackburn and Janata 1982). 
The suspended mesh is fabricated by forming the polymer film (polyimide and photoresist) over a rectangle of thin $(1 \mu \mathrm{m})$ aluminium. The array of holes (10 $\mu \mathrm{m}$ square) is defined photolithographically and etched by conventional integrated circuit fabrication techniques. The aluminium is then etched from underneath the polymer mesh through the holes, leaving the mesh suspended approximately $1 \mu \mathrm{m}$ above the gate insulator.

Electrical and chemical testing of the SM ISFET demonstrated that the mesh had the desired effect. The electrical characteristics of the sensor were not affected by the modification. The chemical response for two sets of potassium-selective ISFETs, one set with no means for improving the membrane adhesion and the other set with the SM modification, was monitored for several weeks. After 24 hours in solution, the first set of ISFETs all began to show the first signs of poor membrane adhesion; the potential exhibited a substantial drift after step changes in the potassium concentration. The SM ISFETs, however, all demonstrated drift-free operation for over 60 days before they failed for other reasons. Figure 26.20

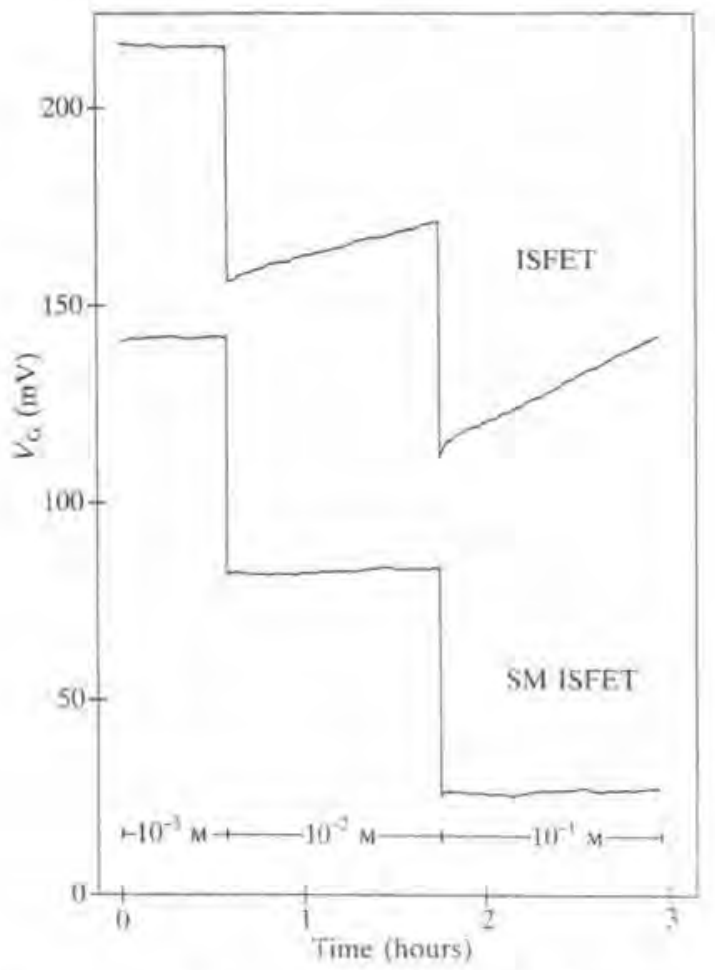

Fig. 26.20 Typical potassium standardization curves for the normal ISFET and the suspended mesh ISFET after seven days in solution (after Blackburn and Janata 1982). 
shows typical response curves for a device from each set after seven days in solution.

\subsection{Enzyme-based CHEMFET}

Enzyme electrodes as described in this text (Chapter 1 and p. 133ff) and reviewed recently by Guilbault (1982) have generated considerable interest. They represent one of the few methods currently available for the electrochemical detection of biological molecules. The sensors generally incorporate a permeable membrane which entraps an enzyme in a thin layer over the surface of, for example, an ISE. The reaction of the enzyme with its substrate either generates products or consumes reactants whose concentrations can be monitored by the underlying ISE. The use of an ISFET as a substitute for the ISE to monitor the chemical concentrations has several distinct advantages. The enzyme-based field-effect transistor (ENFET) displays the usual advantages of the ISFET over its counterpart, the ISE. In addition, the small size and well-defined geometry of the ENFET requires only minute quantities of enzyme which has important implications when expensive enzymes are used. The configuration described by Caras et al. (1985a) also has the advantages that the thickness of the enzyme-loaded membrane can be easily controlled and the adhesion of the membrane to the surface of the ENFET is very good, eliminating the need for any type of retaining membrane as is usually necessary with conventional enzyme electrodes. A further advantage of the ENFET is that it normally has multiple transistors on the same chip, allowing a second transistor to function as a reference which responds to all electrical, chemical, and physical stimuli except the enzyme-substrate reaction. The mathematical difference between the signals from the two FETs, therefore, contains only the desired chemical information with substantially reduced extraneous signals.

Although the literature in the area of enzyme electrodes is extensive, there have been only a few reports of ENFETs. Danielsson et al. (1979) first reported a urea-sensitive ENFET based on an ammonia-gas-sensitive FET. Subsequently, Caras and Janata $(1980,1985)$ have developed a penicillinsensitive ENFET, Hanazoto and Shiono (1983) and Caras et al. (1985a) have developed a glucose-sensitive ENFET, and Miyahara et al. (1983) have developed ENFETs sensitive to urea and acetylcholine (Ach).

\subsubsection{Theory}

The enzyme-sensitive FET is fabricated from an ISFET by applying a thin layer of gel which contains the enzyme over the ion-selective membrane. Figure 26.21 is a diagram of a typical sensor. The mechanism and theory of the electrochemical response are identical to that described by Turner (Chapter 15) for the enzyme electrode and by Caras et al. (1985b) for the 


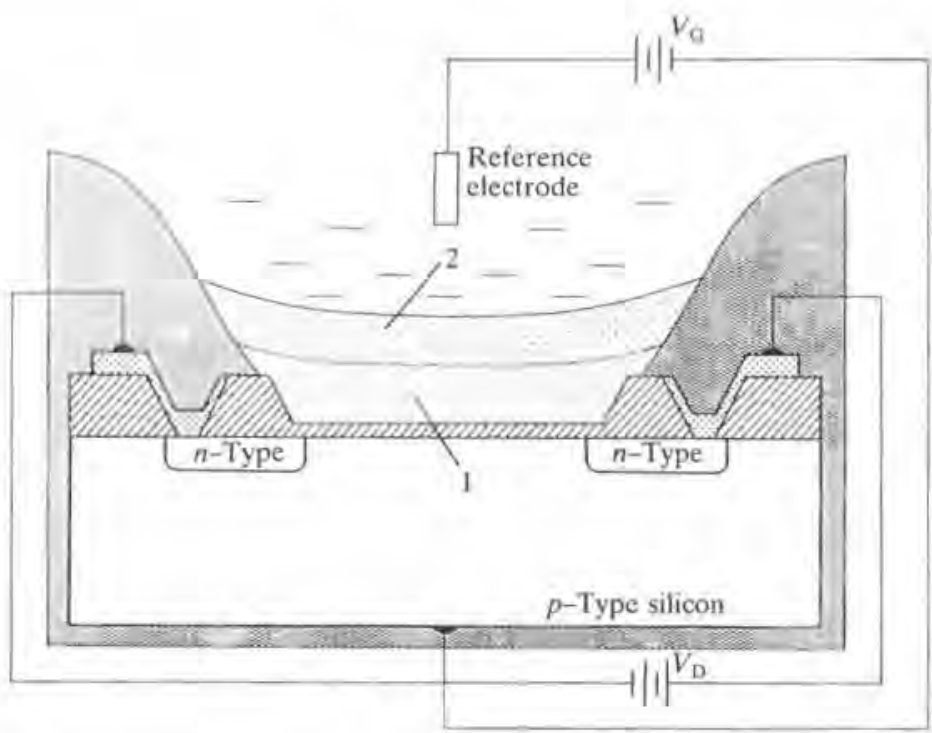

Fig. 26.21 Diagram of a typical ENFET sensor with (1) ion-selective membrane and (2) immobilized-enzyme gel layer.

ENFET. Therefore, only a qualitative presentation is offered here.

Referring to Fig. 26.21, the enzyme is immobilized in the gel layer over the ion-selective membrane. When the substrate for the enzyme is added to the solution it diffuses into the gel layer down the concentration gradient where the enzyme catalyses its chemical modification. Either the consumed reactant or generated product is monitored by the underlying ISFET. In the case where the created products are monitored, the newly formed species in the gel layer diffuse in all directions, both toward the ion-selective membrane and out of the gel layer into the solution. Those molecules which diffuse toward the ion-selective membrane cause an increase in concentration at the surface of that membrane. A steady-state condition develops in which the concentration of the species at the interface between the gel layer and the ion-selective membrane remains constant. Since the reaction rate depends upon the substrate concentration, this steady-state concentration is determined by the solution concentration of the substrate. In the case where a reactant in the solution and gel layer is consumed by the substrate reaction, then the concentration of the monitored reagent is lowered at the membrane-gel interface, again reaching a steady-state condition where this concentration is constant. The relation between the response of the ISFET and the solution concentration of the substrate is complicated and extremely difficult to solve mathematically, depending upon the rate constants for the reactions involved, the diffusion constants of each chemical, immobilized enzyme 
concentration, product-inhibition of the enzymatic reaction, etc. Caras et al. $(1985 b)$ have developed a model which considers most of these parameters, including the effect of a mobile buffer in the gel layer. The solution to the resulting equations is in close agreement with experimental results for ENFETs sensitive to penicillin and glucose.

\subsubsection{Practice}

With the exception of the urea-sensitive ENFET developed by Daniellsson et al. (1979) which is based on an ammonia-gas-sensitive FET, all ENFETs to date use $\mathrm{pH}$-sensitive ISFETs in which the hydrogen ions are produced or consumed by an enzymatic reaction according to the equations
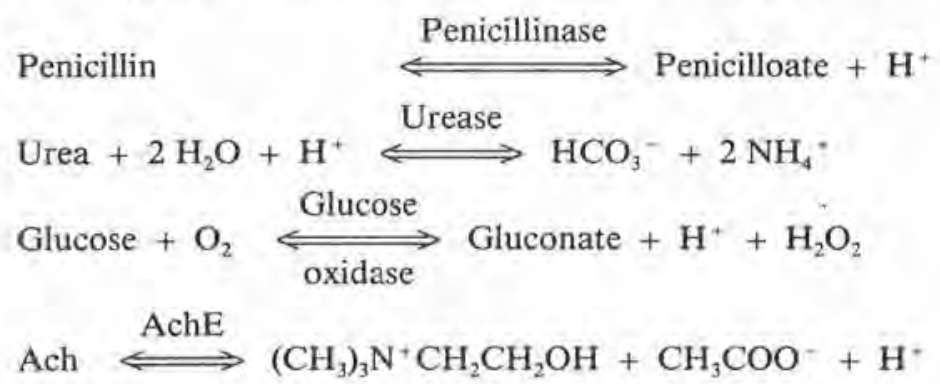

where AchE is acetylcholinesterase.

These enzyme systems were initially chosen because the $\mathrm{pH}$-sensitive ISFET does not require a polymer ion-selective membrane; the $\mathrm{pH}$-sensitive silicon nitride insulator is used directly. The enzyme-gel membranes are typically formed by immobilizing the enzyme in a matrix of cross-linked albumin, poly(acrylamide), or triacetylcellulose.

In practice, a dual-gate ISFET chip is normally employed so that one of the FETs can be used as a reference for the ENFET. This reference ISFET is coated with the gel membrane minus the enzyme and thus retains the same $\mathrm{pH}$ and temperature sensitivity as the ENFET. Because the ENFET and reference FET respond nearly identically to fluctuations in the solution potential, a stable reference electrode is not required; a wire contact to the solution is usually adequate. Thus, if the difference between the two drain currents is monitored, the signal is insensitive to changes in the solution $\mathrm{pH}$, temperature, or electrical fluctuations (noise); only changes in $\mathrm{pH}$ within the gel membrane of the ENFET caused by the enzymatic reaction are detected.

Figure 26.22 illustrates a typical time profile of the response of a penicillinsensitive ENFET to a step change in the substrate concentration (Caras and Janata 1980). The time response of the sensors is primarily a function of the thickness of the gel membrane since the substrate and detected species must diffuse through the membrane, For 50-100 $\mu$ m thick membranes in the sited reference, $63 \%$ responses typically required $10-25$ seconds. Other 


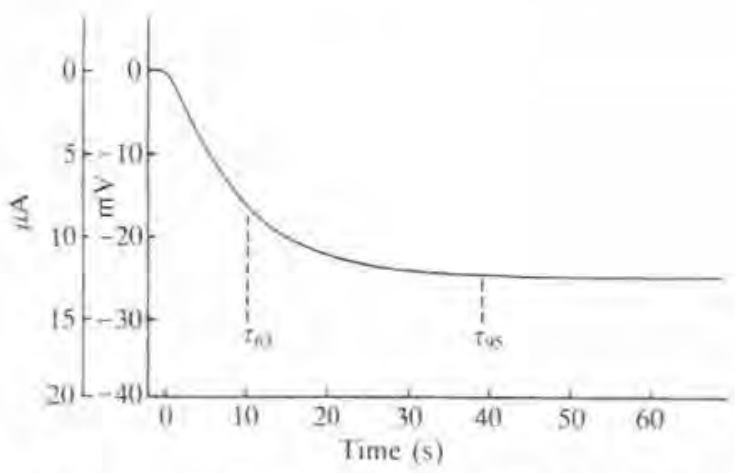

Fig. 26.22 Typical differential time response curve of a penicillin ENFET to a step change in penicillin concentration from 0 to $10 \mathrm{mM}$ in $0.02 \mathrm{M}$ phosphate buffer, $\mathrm{pH}$ 7.2; $\mathrm{T}=25^{\circ} \mathrm{C}$ (after Caras and Janata 1980).

researchers report slightly longer time constants but do not control the thickness of the gel membrane as accurately.

Figure 26.23 shows the response of the same penicillin sensitive ENFET (Caras and Janata 1980) as a function of substrate concentration. The shape of the response curve is typical for all reported ENFETs. Each type of ENFET generally demonstrates a measureable response in the concentration range of from approximately $1 \times 10^{-4}$ to $1 \times 10^{-2} \mathrm{M}$. The lifetime of the sensors is typically limited by the stability of the enzyme and thus is highly dependent on which enzyme is used and the storage conditions of the sensor. Caras and Janata (1980) and Miyahara et al. (1983) reported lifetimes for penicillin, urea, and acetylcholine ENFETs to be in the range of one to two months when the sensors were refrigerated between experiments.

Although to date all of the ENFETs which use ion-selective FETs as the sensor have employed the $\mathrm{pH}$-sensitive ISFET, there is no reason that the concept cannot be extended to other ion-selective systems as well. The sensors are simply more complicated to fabricate if a polymeric ion-selective membrane must be deposited before the gel membrane is applied. Any enzyme electrode which utilizes a macro-ISE as the sensor could realistically be adapted to utilize an ISFET in order to attain the advantages mentioned previously.

\subsection{Immunochemically sensitive field-effect transistors}

The size of the molecules involved in an immunochemical reaction (10 000 to 500000 daltons) places special requirements on the electrochemical properties of the sensing membrane for any immunoelectrode. In an ordinary ion-selective electrode, the selectivity arises from the ability of only one type 


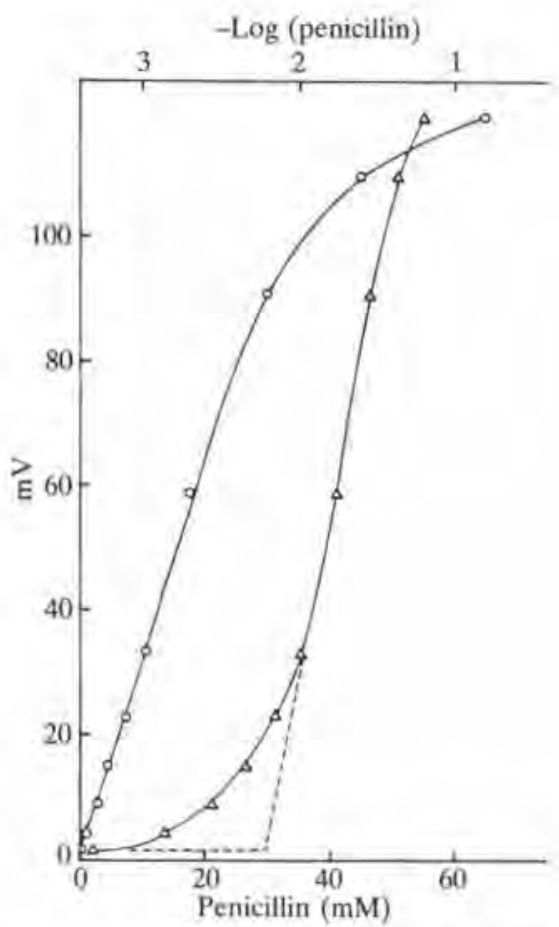

Fig. 26.23 Differential response of the penicillin ENFET. Circles are plotted according to the linear scale and triangles according to the logarithmic scale. $T=37^{\circ} \mathrm{C}, 0.02 \mathrm{M}$ phosphate buffer, $\mathrm{pH} 7.2$ (after Caras and Janata 1980).

of ion to permeate from the solution into the membrane with a high exchange-current density. This selectivity is created by the incorporation of ionophores in the membrane which specifically bind one type of ion. It is difficult to imagine that a membrane could be designed so that it would allow specific immunochemical species to permeate easily from the solution into the membrane with a high current density while excluding the permeation of all other molecules. In other words, it is unlikely that the selectivity mechanism of ISEs could be applied in the design of an immunoelectrode. A different mechanism is required which allows the detection of the immunochemical electrochemically without requiring the movement of the immunochemical into the membrane. While it is hoped that the FET can satisfy this requirement, to date all attempts have been unsuccessful.

\subsubsection{Theory}

A field-effect transistor is basically a charge measuring device, i.e., any change in the excess interfacial charge at the outer insulator surface will be 


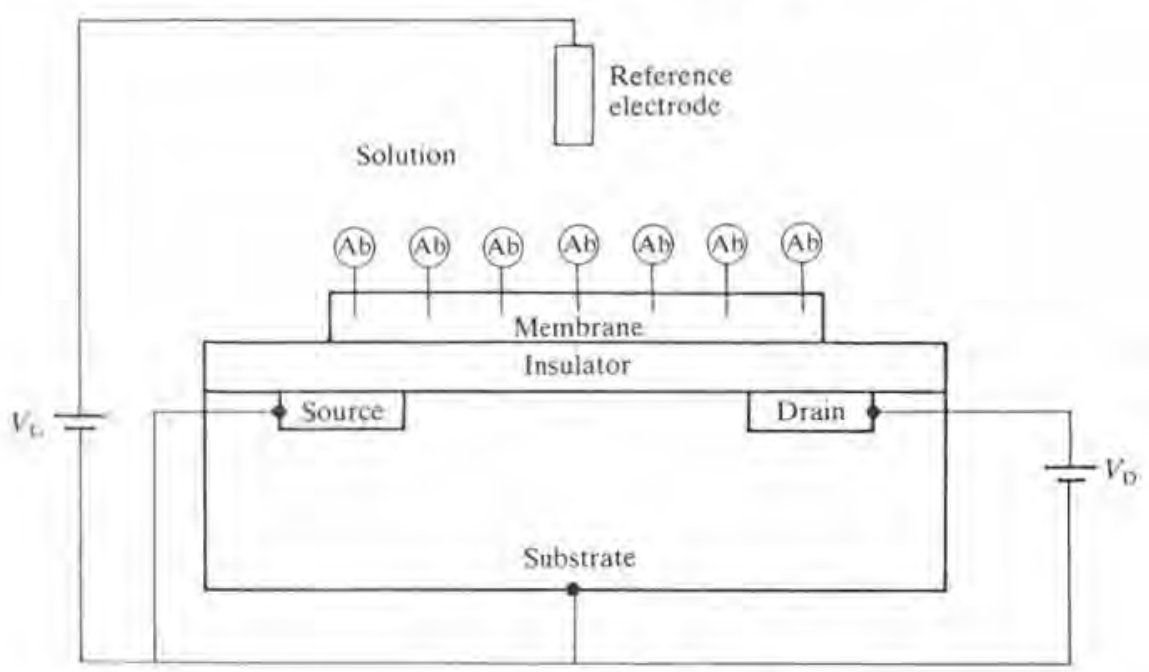

Fig. 26.24 Schematic representation of the IMFET. Ab represents antibodies immobilized at the solution-membrane interface (after Janata et al. 1984).

mirrored by an equal and opposite charge change in the inversion layer of the FET. If the solution-membrane interface of the CHEMFET is ideally polarized, i.e. if charge cannot cross the interface, then the CHEMFET can measure the adsorption of charged species at the interface as will be shown below. Since antibodies, antigens, and proteins are generally electrically charged molecules, then the polarized CHEMFET could be used to monitor their non-specific adsorption at the solution-membrane interface. To render the polarized CHEMFET selective for a given antigen and thus create the so-called immunochemically sensitive FET (IMFET), the specific antibody for that antigen could be immobilized on the surface of the CHEMFET as shown schematically in Fig. 26.24. The adsorption of this antigen would then be specifically enhanced over other molecules in the solution and the signal measured by the CHEMFET would be mostly due to the adsorption of that particular antigen.

This scheme for the measurement of the adsorption of charged species is feasible only if charge cannot cross the interface, which thus behaves as a perfect capacitor. The capacitance of a polarized interface is described by electrical double-layer theory (Bockris and Reddy 1970) and is usually modelled as a series combination of two capacitors, $C_{\mathrm{G}}$ and $C_{\mathrm{H}}$, where $C_{\mathrm{G}}$ is the capacitance of the diffuse Gouy-Chapman part of the double layer and $C_{\mathrm{H}}$ is the capacitance of the Helmholtz part of the double layer. The total capacitance, $C_{\mathrm{d} 1}$, is therefore

$$
1 / C_{\mathrm{dl}}=1 / C_{\mathrm{G}}+1 / C_{\mathrm{H}} \text {. }
$$




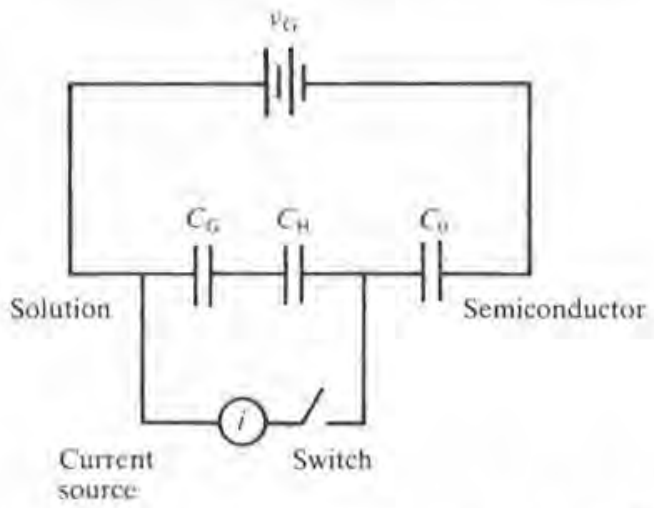

Fig. 26.25 Electrical model for the measurement of charge adsorption with the CHEMFET (after Janata and Blackburn 1984).

The electrical circuit through the gate of a CHEMFET with an ideally polarized interface can be modelled, therefore, as a series combination of $C_{\mathrm{a}}$, $C_{\mathrm{H}}$, and $C_{0}$ as drawn in Fig. 26.25 where $C_{0}$ is the capacitance of the insulator. To the left of the Helmholtz capacitor is the bulk of the solution and to the right of the insulator capacitor is the bulk of the semiconductor. A voltage, $V_{\mathrm{G}}$, is applied through a reference electrode between the solution and the semiconductor. The process of adsorption of charged molecules can be modelled as the transfer of a quantity of charge from the solution to the surface of the transistor as would occur if the switch were closed for a short time period allowing the current source to transfer the charge. As adsorption occurs, the charge on each plate of the capacitors will change to accommodate the new charge balance. The charge change on capacitor $C_{0}$ is the quantity of interest since it represents the charge in the inversion layer of the FET, $Q_{i}$, and will affect the drain current of the transistor. If a quantity of charge, $Q_{\text {ads }}$, is transferred by the adsorption of charged molecules, then the charge change on $C_{0}, Q_{1}$, can be represented by

$$
Q_{1}=Q_{\text {ads }}\left\{C_{0} /\left(C_{0}+C_{\text {di }}\right)\right\} \text {. }
$$

Thus, only a fraction of the adsorbed charge will be mirrored in the transistor. When adsorption occurs, since electroneutrality must be observed in the system, an equal quantity of the opposite charge must either enter the inversion layer of the FET or enter the double layer from solution. Equation 26.26 predicts that part of the image charge will come from the solution as ions entering the double layer with the adsorbing species. This fraction of charge which is mirrored in the inversion layer of the FET will be defined as $\beta$. According to the model being considered, $\beta$ is defined as

$$
\beta=Q_{\mathrm{i}} / Q_{\mathrm{ads}}=C_{0} /\left(C_{0}+C_{\mathrm{di}}\right) .
$$


In the case of a typical CHEMFET, $C_{0}$ is approximately $0.03 \mu \mathrm{F} / \mathrm{cm}^{2}$. The double-layer capacitance, $C_{\mathrm{dl}}$, is approximately $10 \mu \mathrm{F} / \mathrm{cm}^{2}$ for electrolyte concentrations of $0.1 \mathrm{M}$. Thus, according to this model, only $0.3 \%$ of the charge on the adsorbing molecules will be mirrored in the inversion layer of the FET. In more dilute solutions, however, where $C_{\mathrm{d}}$ will be smaller, the fraction will be larger. In the case of the adsorption of large molecules such as immunochemicals, the adsorbed charge will not be confined to the interface but will be distributed through some distance ( $c a .1-10 \mathrm{~nm}$ ) away from the surface. The fraction of charge mirrored in the inversion layer of the FET will thus be even smaller; a reasonable estimate of $\beta$ is probably on the order of $10^{-4}$.

Referring to eqns 26.15 and 26.16 which describe the drain current of the CHEMFET as a function of the potential at the solution-membrane interface, it is clear that a relationship between the adsorbed charge and interfacial potential, $\phi_{\text {sol-mem }}$, is necessary to describe the chemical response of the IMFET. This potential is simply the charge change induced in the inversion layer divided by the insulator capacitance:

$$
\phi_{\text {sol-mem }}=Q_{\mathrm{i}} / C_{0}=\beta Q_{\text {ads }} / C_{0} .
$$

Substitution of this expression into eqns 26.15 and 26.16 yields the response equations for the polarized CHEMFET.

If the binding of antigen to antibody at the interface is described as

$$
\mathrm{Ab}+\mathrm{Ag} \Longleftrightarrow \mathrm{AbAg}
$$

where $\mathrm{Ab}$ is the antibody, $\mathrm{Ag}$ is the antigen, and $\mathrm{AbAg}$ is the complex. The reaction is characterized by the equilibrium constant $K$,

$$
K=[\mathrm{AbAg}] /[\mathrm{Ab}][\mathrm{Ag}] .
$$

The total charge change at the interface due to the binding, $Q_{i}$, can be shown to be

$$
Q_{1}=\beta Q_{\text {ads }}=\beta z F \frac{K[\mathrm{Ag}][\mathrm{S}]}{1+[\mathrm{Ag}]}
$$

where $z$ is the ionic charge of the antigen and [S] is the surface concentration of binding sites (the surface concentration of immobilized antibodies before binding). Substitution of this expression into eqn 26.28 yields

$$
\phi_{\text {fol-mem }}=\frac{\beta z F K[\mathrm{Ag}][\mathrm{S}]}{C_{0}(1+[\mathrm{Ag}])}
$$

From this expression, the limit and range of detection for the IMFET can be predicted. If one assumes that the equilibrium constant will be in the usual range of $10^{5}-10^{9}$ (Eisen 1974), that $\beta=10^{-4}$, that the antibodies are immobilized with a surface concentration of one molecule per $10 \mathrm{~nm}^{2}$, that 
the charge on the antigen is five electronic charges, and that the minimum signal measureable is $10 \mathrm{mV}$, the limit of detection would be in the range of $10^{-7}-10^{-11} \mathrm{M}$. The antigen concentration which gives $90 \%$ surface coverage can similarly be calculated to be in the range of $10^{-4}-10^{-8} \mathrm{M}$. The response range for the IMFET is thus estimated to be three orders of magnitude. If a mixture of antibodies were immobilized having a range of equilibrium constants, the response range for the sensor could be extended.

Similar equations can be derived for the case where the antigen is immobilized at the interface rather than the antibody. In this case, the IMFET would detect the concentration of specific antibodies.

Reversibility of the antigen-antibody binding reaction would determine the reversibility of the sensor. If the binding is not reversible, then the sensor could only measure immunochemical concentrations which are higher than those previously measured. This requirement would obviously limit the usefulness of the sensor for many applications such as real-time in vivo monitoring of immunochemical concentrations. For in vitro applications, however, the immunochemicals might be dissociated by some chemical treatment such as flushing with a high ionic strength solution, which would make the sensor re-useable.

Up to this point, the solution-membrane interface has been assumed to be ideal, i.e., that it behaves as a perfect capacitor with no leakage current. In reality, no interface is ideal and must be modelled as a capacitor in parallel with a resistor where the capacitor is $C_{\mathrm{dl}}$ and the resistance is the chargetransfer resistance, $R_{\mathrm{ct}}$. The consequence of this non-ideality for the IMFET is that any interfacial charge change due to the adsorption of charged species will not remain distributed amongst the model capacitors as presented above. Rather, any charge separation will leak across the solution-membrane interface, and decay in an exponential manner with a time constant equal to $R_{\mathrm{ct}} C_{\mathrm{d} 1}$. For a typical electrolyte interface where $C_{\mathrm{dl}}=10 \mu \mathrm{F} \mathrm{cm}^{-2}, R_{\mathrm{ct}}$ must be at least $10^{7} \Omega \mathrm{cm}^{2}$ to attain a time constant of $100 \mathrm{~s}$. Charge-transfer resistances of this magnitude are rarely observed unless extreme measures are taken to eliminate any mechanism for charge transfer across the interface. The concept upon which the IMFET is based can be verified, therefore, only if a membrane material can be found which exhibits a charge transfer resistance greater than $10^{7} \Omega \mathrm{cm}^{2}$.

In principle, then, the CHEMFET with an ideally polarized interface is capable of measuring the concentrations of immunochemicals in solution, having a very low limit of detection and a broad response range. The specificity would only be limited by the specificity of the immunochemicals used in the device, a specificity which is second to none.

\subsubsection{Practice}

In practice, three classes of membranes have been employed in the investi- 
gation of the polarized CHEMFET (Janata and Blackburn 1984; Blackburn 1983): (1) thin conductive metals, e.g. gold and platinum; (2) thick conducting hydrophobic polymers, e.g. polyvinylchloride (PVC) and polystyrene; and (3) thin insulating membranes, e.g. Langmuir-Blodgett films of cadmium stearate. The conclusions drawn from the investigation of each of these types of membrane will be presented below.

Certain metals are routinely used in electrochemical experiments that require a polarized interface, particularly gold, platinum, and mercury. For most electroanalytical techniques, e.g. cyclic voltammetry or polarography, an electrode is considered polarized if the background current in the absence of electroactive species is small compared to the current when the electroactive species of interest is added. This requirement is much less strict than that for the polarized CHEMFET. Because the metals are electronic conductors, small concentrations of any electroactive molecule in the solution can provide a mechanism for electrons to cross the interface. To attain the charge-transfer resistance necessary for the measurement of interfacial charge changes with the CHEMFET, extreme care must be taken to eliminate all electroactive species from the solution. Following such precautions, Cohen and Janata (1983) demonstrated the adsorption of iodide ion on gold-gate CHEMFETs. In solutions containing small concentrations of immunochemicals or proteins which are inherently 'contaminated' by many electroactive species, however, the charge-transfer resistance was shown to be too low to allow the measurement of the adsorption of charged molecules (Blackburn 1983). Because these contaminants are ordinarily present in any solution containing immunochemicals, and because they decrease the charge-transfer resistance of any metal electrode, the conclusion can be drawn that metal films are not suitable as membranes for the implementation of the IMFET.

Thick polymer membranes have been employed in several reports of immunochemical electrodes. For example, Aizawa et al. (1977) reported an immunoelectrode sensitive to the syphilis antibody with a membrane containing PVC, cholesterol, cardiolipin, and phosphatidylcholine. In the absence of an ionophore in the polymer that promotes the ion flux across the interface, it was hoped that the membrane-solution interface would be ideally polarized but the bulk would demonstrate some conductivity to allow electrical measurements.

It should be pointed out that a thick $(100 \mu \mathrm{m})$ membrane fabricated from a polymer which is normally thought of as insulating may act as a conducting membrane when used over the gate of a CHEMFET. Since the input resistance of the transistor is approximately $10^{15} \Omega$, any membrane with a resistance approximately an order of magnitude smaller than this will appear conducting in the gate circuit.

Collins and Janata (1982) demonstrated that the membrane used by 
Aizawa et al. (1977) responds to changes in the concentration of many small inorganic ions present in the solution and that DC electric current could pass through the membrane. The solution-membrane interface was therefore not polarized, by definition. Similar results have been obtained for pure polymer membranes of PVC, polystyrene, and polystyrene-polybutadiene block copolymer (Blackburn 1983). The charge-transfer resistance for such membranes was shown to be between that of good ion-selective membranes and polarized electrodes. The potential at the interface is a mixed potential in which the ionic fluxes of more than one species control the potential. It is believed that the observed response to immunochemicals can be attributed to the coupling of the protein adsorption to the ion-exchange process to change the mixed potential (Collins and Janata 1982). Because the protein adsorption can be stimulated by immobilized immunochemicals, the false conclusion can be drawn that the response is due to the immunochemical reaction. Actually, the response is a secondary phenomenon; the primary response is to numerous inorganic ions. Even a minute change in the concentration of any of these ions will change the mixed potential at the interface. Thus, the very purpose of this research, the design of a highly specific immunochemical sensor, was defeated because the sensor is highly nonspecific. To date, no polymer investigated remains ideally polarized when placed in aqueous solution.

Langmuir-Blodgett films (Gaines 1965) of cadmium stearate have been investigated by Blackburn (1983) as a possible method for creating the ideally polarized interface. Langmuir-Blodgett films are formed one molecular monolayer at a time by depositing the monolayer onto a substrate from the air-water interface. The molecules are oriented by the air-water interface so that the hydrophilic end groups are situated in the water at the surface and the hydrophobic hydrocarbon chains of the molecule extend above the surface into the air, oriented away from the water at nearly a right angle. The oriented molecules are physically compressed together before deposition so that the hydrocarbon chains form a closely packed array. When deposited on a substrate, each successive monolayer is usually oriented $180^{\circ}$ relative to the previous one, giving a head-to-tail, tail-to-head configuration. Langmuir-Blodgett films have promise as a means for creating the ideally polarized interface because of this tightly packed layer of oriented hydrocarbon chains. Each successive two monolayers of the Langmuir-Blodgett film create a layer of tightly packed hydrocarbon chains approximately $4.5 \mathrm{~nm}$ thick. The energy barrier for either water molecules or ions to cross such a region should be extremely large; thus, the interface should be nearly ideally polarized if the structure of the film is perfect.

Blackburn (1983) reported that Langmuir-Blodgett films of cadmium stearate on aluminum electrodes (11 monolayers, $27.5 \mathrm{~nm}$ thick) had the desired effect of increasing the charge-transfer resistance of the interface, but 


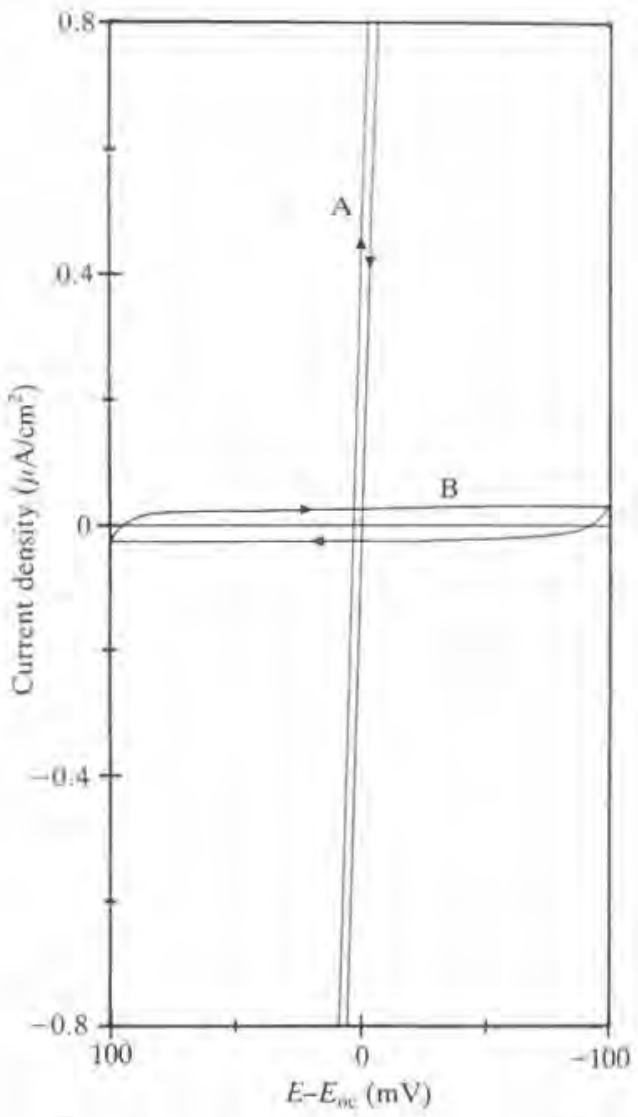

Fig. 26.26 Results of cyclic voltammetry experiments for (A) bare aluminum electrode and $(\mathrm{B})$ aluminum electrode covered with 11 monolayers of cadmium stearate in $0.1 \mathrm{M} \mathrm{NaCl} . E_{\mathrm{oc}}$ represents open-circuit voltage versus $\mathrm{Ag} / \mathrm{AgCl}$ reference clectrode.

not to the extent necessary to create an ideally polarized interface (see Fig. 26.26). The current which could be induced to flow across the interface was attenuated by approximately three orders of magnitude but the measured slope of the current-voltage curve indicated that the resistance (a reasonable estimate for the charge-transfer resistance) was only $2 \times 10^{3} \Omega \mathrm{cm}^{2}$, much lower than the desired $10^{7} \Omega \mathrm{cm}^{2}$. The conclusion was drawn that the films probably contain defects or grain boundaries that interrupt the perfect closepacked array of hydrocarbon chains, permitting the passage of charged species. This study was only a preliminary investigation of the films' properties in aqueous solution, however, and a larger effort must be undertaken to optimize the system. 
It can be concluded that the failure to date to design an immunochemically sensitive potentiometric sensor (electrode or FET) is a consequence of the failure to find the ideally polarized interface. Numerous reports of immunochemical potentiometric sensors should be regarded as experimental artifacts that can be explained on the basis of electrode kinetics, i.e., changes in the mixed potential at non-ideally polarized interfaces. Janata and Blackburn (1984) state that, in their opinion, while there is still hope for a potentiometric immunochemical sensor, it is highly unlikely that an interface with a chargetransfer resistance as high as $10^{7} \Omega \mathrm{cm}^{2}$ will be found. It is this fact that causes the creation of such a sensor to be somewhat improbable.

\subsection{Gas sensitive field-effect transistor}

\subsubsection{Hydrogen-sensitive palladium gate IGFET}

The first FET sensor sensitive to gaseous species was reported by Lundstrom et al. (1975). The device was of the conventional IGFET configuration, i.e., with a metal gate deposited between the drain and source of the transistor over the insulator. The design of the IGFET was novel in that the gate metal consisted of a catalytic metal, palladium. Particular transition metals such as palladium and platinum are unique for two reasons: first, they catalyse the decomposition of hydrogen molecules, $\mathrm{H}_{2}$, to hydrogen atoms at the metal-gas interface where the atoms then adsorb and, second, the hydrogen atoms are soluble in palladium and platinum permitting them to diffuse from the metal-gas interface into the bulk of the gate metal as depicted in Fig. 26.27. Lundstrom (1981) and Lundstrom and Soderberg (1981/82) have shown that some of the hydrogen atoms which dissolve in the gate metal

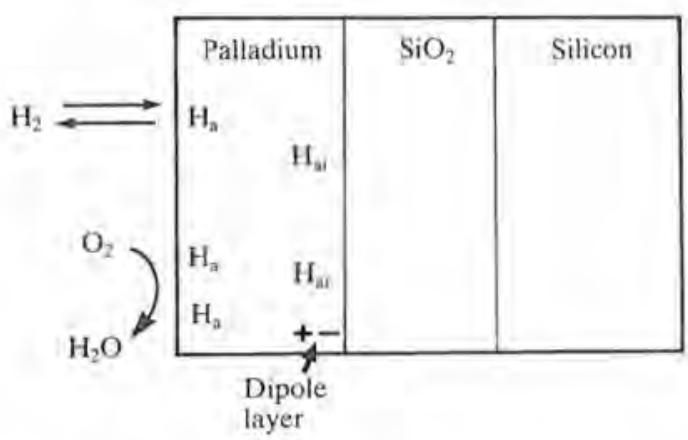

Fig. 26.27 Schematic representation for the mechanism of hydrogen and oxygen sensitivity of palladium gate IGFET. $H_{\mathrm{a}}$ represents adsorbed hydrogen atoms, $H_{\mathrm{b}}$ represents hydrogen atoms dissolved in the bulk of the palladium, and $H_{\mathrm{ai}}$ represents hydrogen atoms adsorbed at the metal-insulator interface, contributing to the dipole layer. 
spontaneously adsorb at the metal-insulator interface. The adsorbed hydrogen atoms' induced dipole moment contribute to a change in the work function of the metal gate. Referring to eqn 26.13 and 26.14 , the drain current of the IGFET is sensitive to the difference in work functions between the gate metal and semiconductor. The change in the work function of the platinum or palladium gate metal therefore induces a change in the drain current of the transistor; changes of the drain current, therefore, are directly related to the concentration of hydrogen molecules in the gas ambient around the gate area of the transistor.

In the presence of oxygen (or other 'oxidizing' gases) chemical reactions can occur with the adsorbed hydrogen atoms, depleting the surface of hydrogen atoms as shown in Fig. 26.27. Since the hydrogen atoms adsorbed at the metal-insulator interface are in equilibrium with the atoms adsorbed at the metal-gas interface, the introduction of oxygen into the system decreases the magnitude of the dipole layer potential. In this manner the hydrogen sensitive IGFET can be used as an oxygen sensor if the ambient hydrogen pressure is held constant (Lundstrom 1981).

Figure 26.28 shows a typical response for a Pd-MOS transistor in air (Lundstrom et al. 1977) in air. The temperature of the transistor was maintained at $120^{\circ} \mathrm{C}$, a condition which is necessary to attain reasonable response time for the sensors. The minimum detectable limit for hydrogen as reported

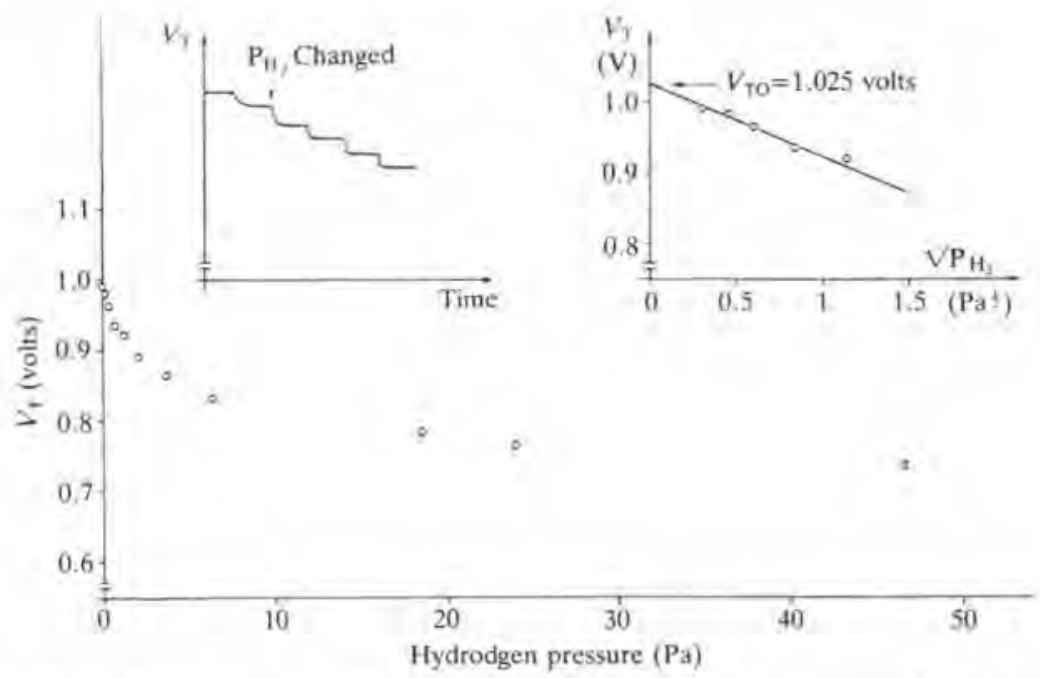

Fig. 26.28 Response of a palladium gate IGFET which was exposed to different concentrations of hydrogen in air. $V_{\text {TO }}$ represents is the threshold voltage with the $\mathrm{Pd}$ film completely discharged of hydrogen. The temperature of the transistor was maintained at $120^{\circ} \mathrm{C}$ (after Lundstrom et al. 1977). 
by Lundstrom (1981) is approximately $3 \times 10^{-5} \mathrm{~Pa}$ in an inert atmosphere and $5 \times 10^{-4} \mathrm{~Pa}$ in air. The higher value in air is caused by the decrease in adsorbed hydrogen as a consequence of reactivity with atmospheric oxygen.

Palladium gate IGFETs have also been demonstrated to be sensitive to other molecules containing hydrogen such as ammonia and hydrogen sulphide (Lundstrom 1981) as well as to methane and butane (Poteat and Lalevic 1982). It is believed that the metal catalyses the decomposition of these molecules to hydrogen atoms and other species. For each of these gases, the detection scheme involves the formation of a dipole layer of hydrogen atoms at the metal-insulator interface, which is identical to the scheme for hydrogen gas sensitivity. The selectivity of the sensors for hydrogencontaining gases is a result of the high solubility of hydrogen in palladium and the low solubility of all other molecules.

Several researchers have reported palladium gate IGFETs which are sensitive to carbon monoxide gas. In order to attain sensitivity to gases other than hydrogenous species it is necessary to create a gate metal structure with 'holes' so that the metal-insulator interface is accessible to the gas molecules; for non-porous metals, the only mechanism to reach the interface requires solubility in and diffusion through the metal. Lundstrom et al. (1981/82) described a porous palladium gate IGFET and Krey et al. (1982/83) have described a gate structure with lithographically defined holes through the palladium which allow the gas molecules access to the metal-insulator interface. Figure 26.29 shows a cross section through the Pd-gate hole structure IGFET sensor. The CO sensitivity of the sensor is approximately $75 \mathrm{mV}$ at 0.1 torr with a maximum signal of about $150 \mathrm{mV}$. The sensor is also sensitive to hydrogen, methane, butane, and other hydrogen-containing gases as expected. Krey et al. (1982/83) have shown, however, that the hydrogen sensitivity can be reduced by at least an order of magnitude by covering the

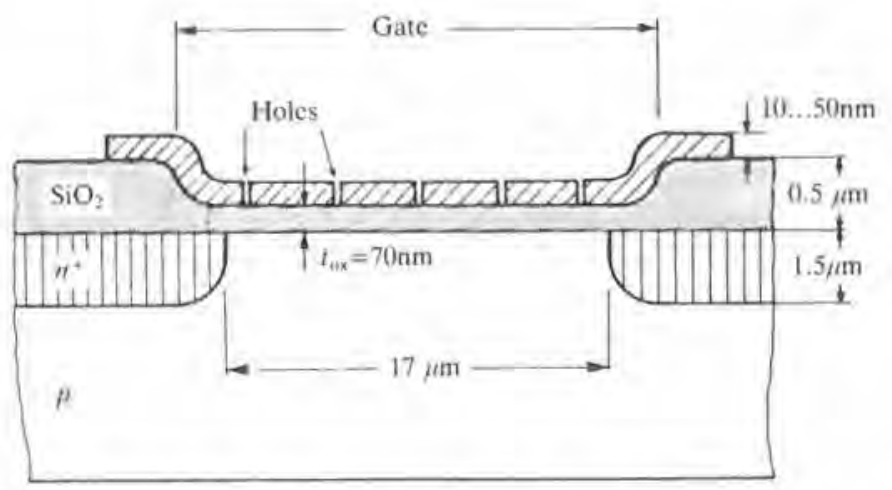

Fig. 26.29 Cross section of a palladium gate IGFET with photolithographically defined holes (after Krey et al. 1982/83). 
palladium with a $20 \mathrm{~nm}$ thick film of aluminum. The modification also reduces the $\mathrm{CO}$ sensitivity, but only by a factor of two. The aluminum apparently inhibits the diffusion of the hydrogen into the palladium.

\subsubsection{Suspended gate GASFET}

The transition-metal gate FET sensors discussed above are selective for hydrogen-containing molecules because only hydrogen is appreciably soluble in the gate metal. Unfortunately, such selectivity for other gases using different gate metals has not been demonstrated. To generate a sensitivity and selectivity to other gases, a gate structure is required which allows the gases access to the metal-insulator interface and which could potentially be made selective by surface modification of these interfaces.

Stenberg and Dahlenback (1983) described an IGFET structure in which a portion of the insulator under the poly-silicon gate is etched away, creating an air gap between the gate and the silicon over a small portion of the gate.

Blackburn et al. (1983) described a 'suspended-gate' GASFET with a platinum gate suspended over the entire gate region of the transistor as shown in Fig. 26.30. An array of holes in the platinum gate allows the ambient gas access to the air gap between the metal and insulator. The structure is similar to that of the suspended mesh ISFET described in section 26.5.4., above.

The gap between the metal and insulator can be viewed as an additional insulator with a permittivity close to 1 . When gaseous molecules with a dipole moment diffuse into the gap, the permittivity of the air gap is changed imperceptibly. However, when the molecules adsorb either on the metal surface or the insulator surface with some preferred orientation a dipole potential is created, contributing to the surface potential $\chi$ :

$$
x=\frac{1}{\epsilon_{0}} \Sigma N_{i} \mu_{i}
$$

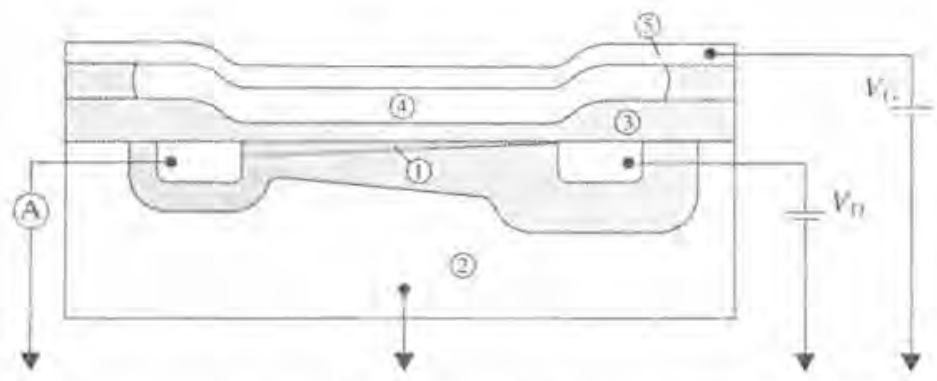

Fig. 26.30 Cross section through the suspended gate GASFET. (1) Inversion layer, (2) silicon substrate, (3) insulator, (4) air gap, and (5) suspended platinum gate (after Blackburn et al. 1983). 
where $N_{i}$ is the density of adsorbed molecules $i, \mu_{i}$ is the vertical component of their dipole moment (not to be confused with the chemical potential having the same symbol), and $\epsilon_{0}$ is the permittivity of free space. This potential can be considered as a voltage source in series with the applied gate voltage, $V_{\mathrm{G}}$. A change in the density of adsorbed dipoles will, therefore, give rise to a change in the overall electric field and alter the drain current. Using the constant current mode of operation, this change in surface potential can be monitored directly.

The suspended metal mesh is formed on a silicon wafer upon which an array of FETs without any gate metallization has already been fabricated. The suspended mesh is formed by fabricating the array of holes in a platinum film over a layer of aluminum. The aluminum is then etched from underneath the platinum through the array of holes, leaving the mesh suspended over the gate of the FET. The initial aluminum layer is typically $100 \mathrm{~nm}$ thick so the resultant air gap is approximately the same dimension. The holes are $5 \mu \mathrm{m}$ in diameter and spaced $10 \mu \mathrm{m}$ apart. A detailed description of the fabrication process is given by Blackburn et al. (1983).

The chemical response has been preliminarily tested under flowing conditions by injecting $5 \mu \mathrm{l}$ of tested substance into a $5 \mathrm{ml}$ evaporation chamber from which it was flushed out in exponential fashion past the GASFET. The response curves are shown in Fig. 26.31. The lack of response to pentane and

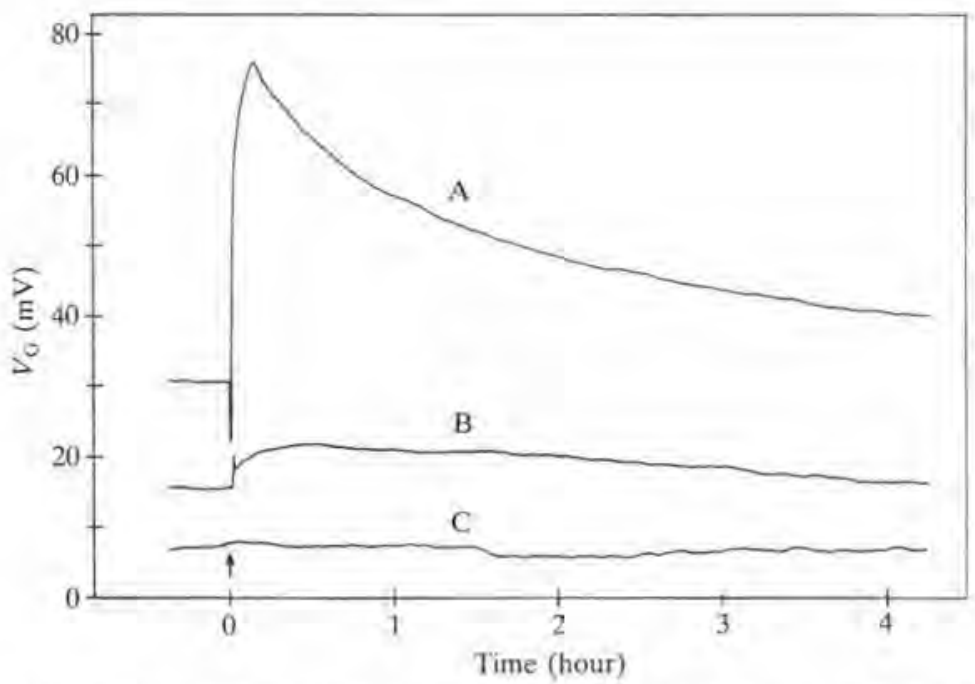

Fig. 26.31 Response of the suspended gate GASFET to addition of $5 \mu \mathrm{l}$ of (A) methanol, (B) methylene chloride, and (C) n-heptane to a flowing stream of nitrogen (after Blackburn et al. 1983). 
other non-polar molecules confirms the concept of the change in surface potential. The initial, fast response signal followed by the strong, slow response which was obtained upon addition of methanol or methylene chloride could be due to different kinetics of adsorption and/or orientation of these molecules on the platinum and/or insulator (silicon nitride) and is the subject of present studies. The long time required for the signal to return to its preinjection value results from the slow desorption from the walls of the gas delivery system; subsequent experimentation with the sensor attached at the exit of a gas chromatograph column have given much faster response times.

The suspended gate GASFET is an important development because the sensor's selectivity is not limited to only one species as it is for the palladium gate GASFETs. Indeed, in its present state, the suspended gate sensor is sensitive to most polar molecules. Chemical modification of the surfaces of the gate structure is being investigated and will hopefully impart some selectivity to this new class of gas sensors.

\subsection{Conclusion}

This chapter has presented the theory and implementation of several types of chemically sensitive field-effect transistors. The purpose was not to provide an exhaustive review of the literature, but rather to provide an overview of the different areas of interest of investigators in the field.

The ion-selective FET has seen the greatest development effort of the various types of CHEMFET sensors. This is due, in part, to the availability of ion-selective membranes which have seen considerable scientific development for application in ISEs. The ISFET should be viewed as a complement to the ISE which may have distinct advantages in particular applications. The in situ impedance transformation eliminates the need for cumbersome shielded cables while maintaining a desirable low noise level in the measured signals. This advantage, combined with the small size of the sensor itself makes the ISFET ideal for applications such as in vivo monitoring of electrolytes where the small size of both the sensor and cable is essential. The solidstate construction of the sensors (in particular, the elimination of the internal filling solution of ISEs) makes the ISFET small, lightweight, and rugged. Because the size of each FET on the surface of the chip can be very small, the potential exists for the development of sensors which monitor many different chemicals simultaneously. This development awaits a membrane deposition method which allows many small membranes to be reliably deposited close to one another on a single chip. Because the sensors are fabricated on a semiconductor substrate, additional signal processing circuitry can easily be added to the chip thereby providing such functions as multiplexing and analog-to-digital signal conversion. Finally, because the chips are fabricated thousands at a time in silicon wafers, the cost of production can potentially be very small. This reduction in cost and effort, however, awaits the 
development of techniques for automatic encapsulation and membrane deposition.

The chemically sensitive field-effect transistor is also an exciting development in that it makes possible the detection of many chemicals using mechanisms which are previously either difficult or impossible to demonstrate. In particular, the polarized CHEMFET opens up the possibility of measuring changes in interfacial charge which can be induced by specific surface interactions of immunochemicals. Unfortunately, the requirement for a nearly ideal polarized interface may not allow the development of this class of sensors. The development of gas sensors using FET devices represents another area where new sensing mechanisms are being developed which could not have been conveniently explored previously.

Although the field of chemically sensitive field-effect transistors is still in its infancy and considerable work has yet to be accomplished, this class of sensors shows considerable promise for creating chemical sensors which have advantages over their existing counterparts. In addition, the CHEMFET provides new mechanisms to measure conveniently many chemicals which were not previously possible.

\section{References}

Abe, H., Esashi, M, and Matsuo, T. (1979). ISFET's using inorganic gate thin films. IEEE Trans. Electron Devices ED-26, 1939-44.

Aizawa, M., Kato, S. and Suzuki, S. (1977). Immunoresponsive membrane I. Membrane potential change associated with an immunochemical reaction between membrane-bound antigen and free antibody. J. Membrane Sci. 2, 125-32.

Akiyama, T., Ujihira, Y., Okabe, Y., Sugano, T. and Niki, E. (1982), Ion-sensitive field-effect transistors with inorganic gate oxide for $\mathrm{pH}$ sensing. IEEE Trans. Electron Devices ED-29, 1936-41.

Band, D.M., Kratochvil, J., Poole Wilson, P.A. and Treasure, T. (1978), Relationship between activity and concentration of plasma potassium. Analyst $103,246-51$.

Bergveld, P. (1970). Development of an ion-selective solid-state device for neurophysiological measurements. IEEE Trans. Biomed. Eng. BME-17, 70-1.

Blackburn, G.F. (1983). Molecular adsorption measurement with chemically sensitive field effect transistors. Ph.D. Dissertation, University of Utah, USA.

- and Janata, J. (1982). The suspended mesh ion selective field effect transistor. $J$. Electrochem. Soc, 129, 2580-4.

- Levy, M. L. and Janata, J. (1983). Field-effect transistor sensitive to dipolar molecules. Appl. Phys. Lett. 43, 700-1.

Bockris, J. O'M, and Reddy, A.K. N. (1970). Modern electrochemistry, Vol. 2. Plenum Press, New York.

Buck, R. P. and Hackleman, D. E. (1977). Field effect potentiometric sensors, Anal. Chem. 49, 2315-21.

Caras, S. D. and Janata, J. (1980). Field effect transistor sensitive to penicillin. Anal. 


\section{Chem. 52 1935-7.}

(1985). pH based enzyme potentiometric sensors. Part 3. Penicillin sensitive field effect transistor. Anal. Chem. 57, 1924-5.

- Petelenz, D. and Janata, J. (1985a). pH based enzyme potentiometric sensors. Part 2. Glucose sensitive field effect transistor. Anal. Chem. 57. 1920-3.

_ Janata, J., Saupe, D. and Schmitt, K. (1985b). pH based enzyme potentiometric sensors. Part 1. Theory, Anal. Chem. 57, 1917-20.

Cohen, R. M. and Janata, J. (1983). Measurement of excess charge at polarized electrodes with field effect transistors, Part 1. Direction determination of the Esin-Markov coefficient. J. Electroanat. Chem, 151, 33-9.

Colclaser, R. A. (1980). Microelectronics: processing and device design. John Wiley, New York.

Collins, S. and Janata, J. (1982). A critical evaluation of the mechanism of potential response of antigen polymer membranes to the corresponding antiserum. Anal. Chim. Acta. 136, 93-99.

Danielsson, B., Lundstrom, I., Winquist, F, and Mosbach, K. (1979). On a new enzyme transducer combination: the enzyme transistor. A nal, Letl, B. 12, 1189-99.

Eisen, H.N. (1974). Immunology, an introduction to molecular and cellular principles of the immune response. Harper and Row, New York.

Esashi, M. and Matsuo, T. (1978). Integrated micro-multi-ion sensor using field effect of semiconductor. IEEE Trans. Biomed. Eng, BME-25, 184-92.

Freiser, H. (ed.) (1978). Ion-selective electrodes in analytical chemistry, Vol. 1. Plenum Press, New York.

Freiser, H. (ed.) (1980). Ion-selective electrodes in analytical chemistry, Vol. 2. Plenum Press, New York.

Gaines, G. L. (1965). Insoluble monolayers at liquid-gas interfaces. Interscience Publishers, New York.

Griffiths, G. H., Moody, G. J. and Thomas, J. D. R. (1972). An investigation of the optimum composition of poly(vinyl chloride) matrix membranes used for selective calcium-sensitive electrodes. Analyst 97, 420-7.

Guilbault, G. G. (1982). Immobilized enzymes as analytical reagents. Appl. Biochem. Biotechnol. 7, 85-98.

Hanazoto, Y, and Shiono, S. (1983). Bioelectrode using two hydrogen sensitive field effect transistors and a platinum wire pseudo reference electrode. In Proceedings of the international meeting on chemical sensors, Fukuoka, Japan, September 19-22, 1983, 513-518. Elsevier, New York.

Ho, N. J., Kratochvil, J., Blackburn, G.F. and Janata, J. (1983). Encapsulation of polymeric membrane-based ion-selective field effect transistors. Sensors and Actuators 4, 413-21.

Janata, J. and Blackburn, G. F. (1984). Immunochemical potentiometric sensors. Annals of the New York Academy of Sciences 428, 286-92.

and Huber, R. J. (1979). Ion sensitive field effect transistors, Ion-Sel. Electrode Rev. 1, 31-78.

(1980). Chemically sensitive field effect transistors. In Ion-selective electrodes in analytical chemistry (ed. H. Freiser), pp. 107-74. Plenum Press, New York.

Koryta, J. (1975). Ion-selective electrodes. Cambridge University Press.

Krey, D., Dobos, K. and Zimmer, G. (1982/83). An integrated CO-sensitive MOS 
transistor. Sensors and Acfuators 3, 169-77.

Lundstrom, I. (1981). Hydrogen sensitive MOS-structure Part 1: Principles and applications. Sensors and Actuators 1, 403-26.

- and Soderberg, D. (1981/82). Hydrogen sensitive MOS-structures Part 2: Characterization. Sensors and Actuators 2, 105-38.

- Shivaraman, M.S. and Svensson, C. (1977). Chemical reactions on palladium surfaces studied with Pd-MOS structures. Surface Science 64, 497-519.

- and Lundqvist, L. (1975). A hydrogen sensitive MOS field-effect transistor. Appl, Phys. Lett. 26, 55-7.

Matsuo, T., Esashi, M. and Iinuma, K. (1971). Biomedical active electrode utilizing field-effect of solid state device (1). Digests of Joint Meeting of Tohoku Sections of I.E.E.J., October 1971.

McBride, P. T., Janata, J., Comte, P.A., Moss, S. D. and Johnson, C. C. (1978). Ion-selective field-effect transistors with polymeric membranes, Anal, Chim. Acta. 101, 239-45.

McKinley, B. A., Saffle, J., Jordan, W. S., Janata, J., Moss, S. D. and Westenskow, D. R. (1980). In vivo continuous monitoring of $\mathrm{K}^{\prime}$ in animals using ISFETs. Med. Instrum. 14, 93-7.

Miyahara, Y., Matsu, F. and Moriizumi, T. (1983). Micro-enzyme sensors using semiconductor and enzyme-immobilization techniques. In Proceedings of the international meeting on chemical sensors, Fukuoka, Japan, September 19-22, 1983, 513-518. Elsevier, New York.

Muller, R.S. and Kamins, T. I. (1977). Device electronics for integrated circuits. John Wiley, New York.

Poteat, T. L, and Lalevic, B. (1982). Transition metal-gate MOS gaseous detectors. IEEE Trans. Electron Devices ED-29, 123-9.

Pungor, E., Toth. K. and Havas, J. (1966). Theory and application of heterogenous rubber membrane electrodes in the determination of some ions. Mikrochim. Acta 1966, 689-98.

Shiramizu, B. T., Janata, J. and Moss, S. D. (1979), Ion-selective field effect transistors with heterogeneous membranes. Anal. Chim. Acta 108, 161-7.

Stenberg, M. and Dahlenback, B. I. (1983). Surface-accessible FET for gas sensing. Sensors and Actuators 4, 273-81.

Sze, S.E. (1981). Physics of semiconductor devices (2nd edn). John Wiley, New York.

Zemel, J. N, (1975). Ion-sensitive field effect transistors and related devices. Anal. Chem. 47, 255A-66A. 


\title{
Biosensors based on semiconductor gas sensors
}

\author{
BENGT DANIELSSON and FREDRIK WINQUIST
}

\subsection{Introduction}

In this chapter we will describe some biological applications of semiconductor gas sensors based on PdMOS-components developed by Lundström et al. (1975). These are primarily hydrogen-sensitive fieldeffect transistors fabricated by metal oxide semiconductor technique (PdMOSFET) although they could display a certain sensitivity towards small hydrogen-containing molecules, such as ammonia and hydrogen sulphide. Indeed, in our first study in this field, Danielsson et al. (1979) demonstrated the feasibility of using an ammonia-sensitive PdMOSFET as a transducer in an 'enzyme transistor' utilizing the enzymes urease and creatinine iminohydrolase. After this preliminary study we started investigations of the use of hydrogen-sensitive PdMOS components for monitoring hydrogenproducing systems including the enzyme hydrogenase (EC 1.12.1.2) (Danielsson et al. 1983). Following the discovery of techniques for reproducible enhancement of the ammonia sensitivity of PdMOS-devices by Winquist et al. (1983), attention was again directed towards the combination of this group of sensors with biological systems. Recent studies show that such ammonia sensors can be used in highly specific and sensitive biosensors (Winquist et al. 1984a, $b ; 1985$ ).

Biotechnology today is receiving tremendous political, economical, scientific, and general world-wide interest as one of the most promising fields for future development. Biosensors, in particular, have been recognized as a concept bearing much promise for the future, both as a biotechnology product and especially as useful sensors for measurements in biotechnology, in complex media, fermentation broths and in vivo. For such measurements the sensor must be rugged, have good operational stability, and withstand clogging, fouling, and interfering compounds. In many cases, it must also have high sensitivity and small dimensions. In this context, we have had very good experience with our biosensors based on gas sensors, in which the detector is separated from the sample solution by a gas-permeable membrane with only the biological component of the biosensor directly exposed to the sample. We are generally using small columns containing an immobilized 
preparation of the biocatalyst, which ensures high operational stability. In most cases, a large excess of catalytic activity can be employed resulting in virtually unchanged performance of the reactor despite variations in sample composition, buffer capacity, $\mathrm{pH}$, ionic strength, colour, turbidity, temperature, denaturing, and even, to some extent, to inhibitor concentration.

This is in contrast to biosensors based on ISFETs (ion-sensitive field-effect transistors) which are operated in the sample solution and consequently, will have their response affected by $\mathrm{pH}$, buffer capacity, and the concentration of interfering ionic species similar to the measured compound.

Semiconductors, in general, have the advantage of cheap fabrication by common semiconductor technology, resulting in small size and the possibility to develop multifunctional devices as well as the direct integration with electronics for signal processing - 'smart sensors'. These features are especially attractive for in vivo sensors as they would allow for miniaturized biosensors with several enzyme patches on a single chip, either for different analyses or with several parallel channels for increased reliability. A fourfunction CHEMFET for simultaneous measurement of potassium, sodium, calcium, and hydrogen ions has been described by Sibbald et al. (1984). A further advantage of the semiconductor sensors is their low-impedance connection to associated electronics, which makes the signal leads less sensitive to electrical disturbances.

An interesting aspect of using a gas detector to follow a biochemical reaction that occurs in solution is that the measured compound will be present in a relatively higher concentration in the gas phase than in the liquid phase since the detector can be placed in a comparatively small gas volume. This is reflected by the fact that a sensitivity for $\mathrm{NH}_{4}{ }^{+}$in aqueous solution of $0.1 \mu \mathrm{M}$ can easily be obtained with a sensor having only $1 \mathrm{ppm} \mathrm{NH}_{3}$ (g)-sensitivity. Similar results are obtained with the hydrogen sensors.

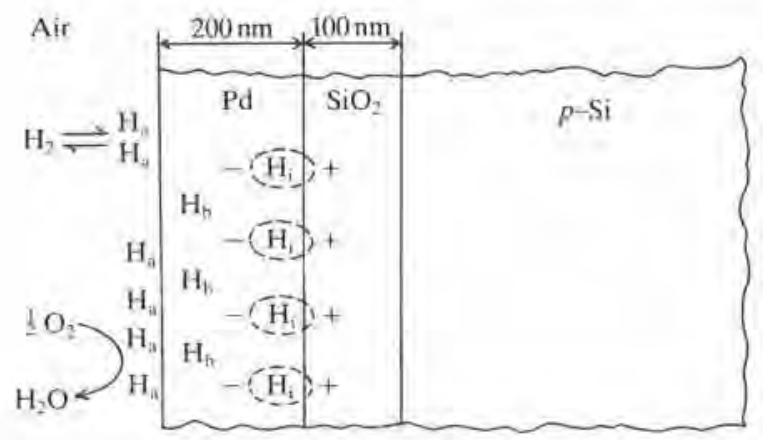

Fig. 27.1 Schematics of the chemical reactions occurring at a palladium interface. 


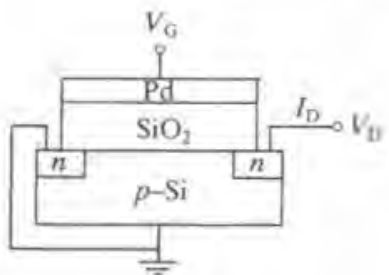

Pd MOS-FEI

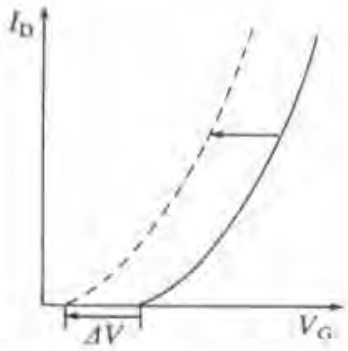

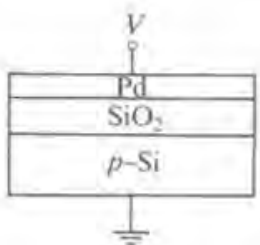

Pd MOS-capacitor

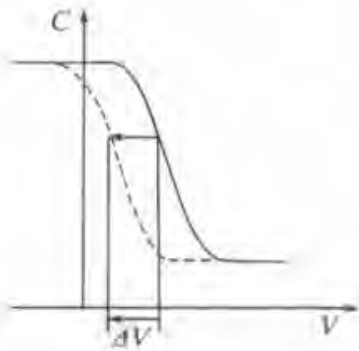

Fig. 27.2 Left: Schematic illustration of a PdMOS field effect transistor and the corresponding $I_{D^{-}} V_{\mathrm{G}}$ characteristics. Right: Schematic illustration of a PdMOScapacitor and the corresponding $C(V)$-curve. Exposure to hydrogen will shift the characteristics to the left as indicated by the dotted lines.

In the following we will describe theory, fabrication, and measurement techniques involving $\mathrm{H}_{2-}$ and $\mathrm{NH}_{3}$-sensing semiconductor structures. Application examples include the use of hydrogenase as a hydrogen-producing system, recycling systems, and various ammonia-producing enzymes, such as urease, creatininase, and amino acid deaminases.

\subsection{Physical background}

Hydrogen-detecting PdMOS-structures are made by evaporating a nonporous, $100-200 \mathrm{~nm}$ thick Pd-film on a $p$-silicon chip with a thermally grown $100 \mathrm{~nm}$ thick oxide layer (Fig. 27.1). The sensors can be fabricated as capacitors with an aluminium film as back contact, or as field-effect transistors (Fig. 27.2). In the latter case, a temperature-controlled heating circuitry is included on the same chip. (Fig. 27.3). The capacitors, which are easier to make and therefore are the component of choice for exploring studies, e.g. of film composition, are placed on a small temperature-controlled metal plate when used. The size of the components is of the order of $1 \mathrm{~mm}^{2}$.

The reactions constituting the hydrogen sensitivity of PdMOS-structures 


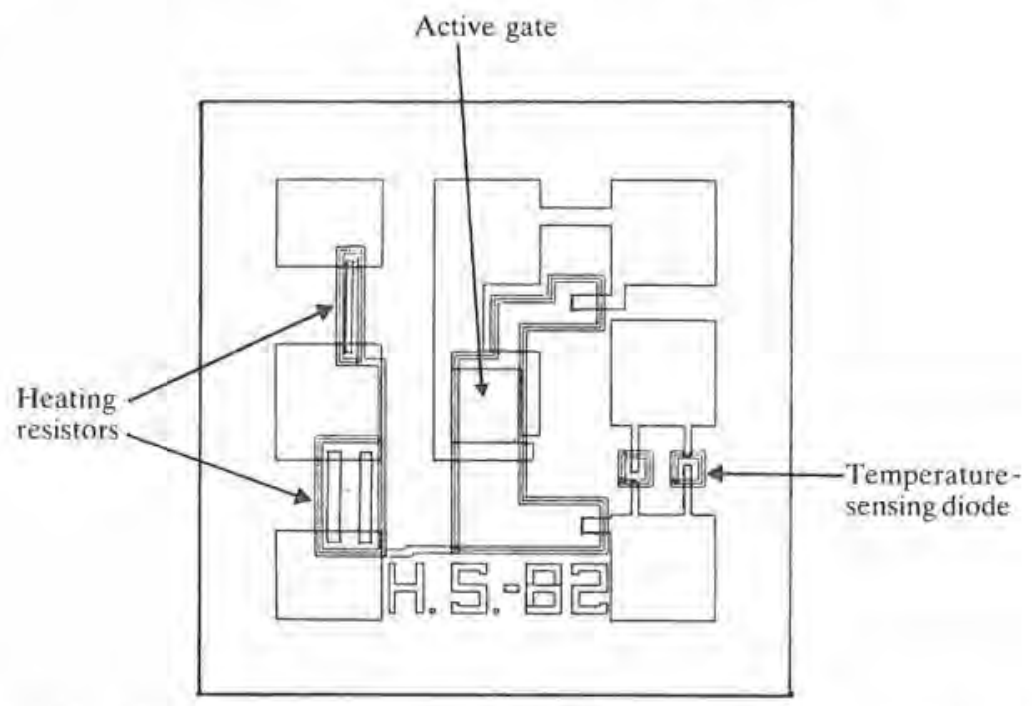

Fig. 27.3 A $2.5 \times 2.5 \mathrm{~mm}^{2}$ chip containing a PdMOSFET with integrated temperature control. The chip can be mounted in a TO-18 package.

occur on the surface and inside of the Pd layer. As indicated in Fig. 27.1, hydrogen molecules in the surrounding gas are dissociated on the surface and some of the adsorbed hydrogen atoms diffuse into the Pd layer where they become polarized in the electrical field over the component. The dipole layer thus formed will cause a voltage drop in the electric field, which in turn will shift the $I_{\mathrm{D}}-V_{\mathrm{G}}$ characteristics of a transistor or the $C(V)$-curve of a capacitor along the voltage axis (Fig. 27.2). The voltage drop is related to the ambient hydrogen pressure following the equation:

$$
\Delta V=C_{1} \times\left(P_{\mathrm{H}_{2}}\right)^{0.5} \text { for } P_{\mathrm{H}_{2}} \leqslant 50 \mathrm{ppm}
$$

where $C_{1}$ is a constant. $C_{1}$ depends on the properties of the Pd layer, film thickness, size of the active area, etc. A typical value of $C_{1}$ is $27 \mathrm{mV} / \mathrm{ppm}$. Hydrogen atoms leave the $\mathrm{Pd}$ layer on recombination with hydrogen molecules or on combination with oxygen (if present) to water. Consequently, the sensitivity will be considerably higher in the absence of oxygen, $\sim 0.01 \mathrm{ppm}$, than in the presence of oxygen, $\sim 1 \mathrm{ppm}$. The recovery time will also be much shorter in the presence of oxygen, although it can be reduced by increasing the working temperature of the sensor to $100-150^{\circ} \mathrm{C}$, which is the normal working temperature of a PdMOSFET. This temperature also prevents water molecules from sticking to the sensor surface. The response time is about $1 \mathrm{~min}$ at low concentrations of hydrogen. PdMOS devices were thoroughly described by Lundström (1981). 


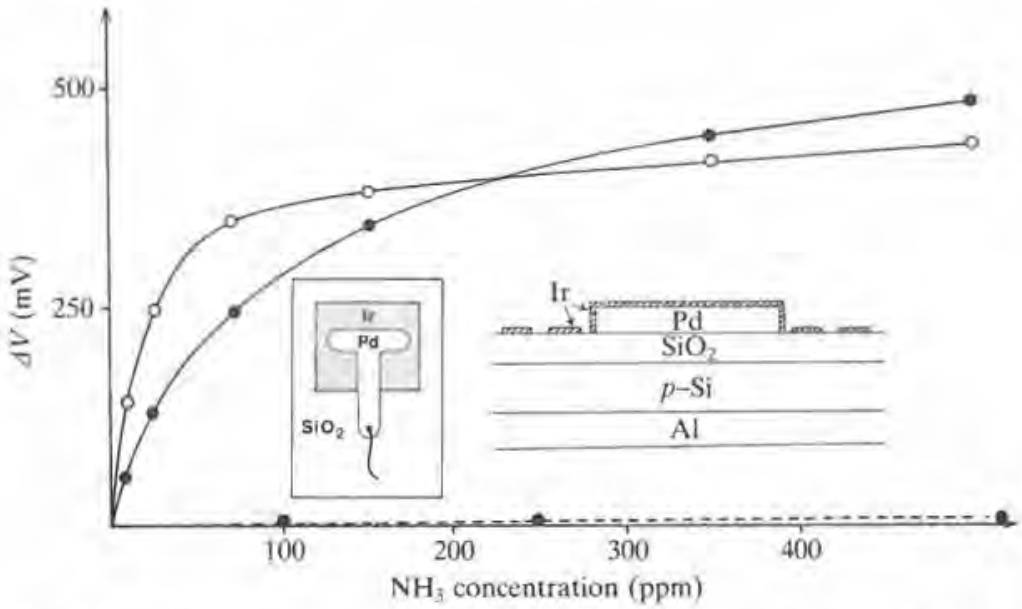

Fig. 27.4 Steady-state response for different modifications of a PdMOS-structure in comparison with that of an unmodified device. ( $-\rightarrow$ Ir-modified device: $(-0-)$ Pt-modified device; (---) unmodified device. The inserts show a schematic drawing and the cross-section of a PdMOS-capacitor modified with a thin Ir-film.

\subsubsection{Increased ammonia gas sensitivity}

Normal PdMOS devices have only a low $\mathrm{NH}_{3}$-sensitivity. As shown by Winquist and co-workers (1983), the $\mathrm{NH}_{3}$-sensitivity can be considerably enhanced by incorporating a second submonolayer of a suitable catalytic metal such as Ir or Pt (Fig. 27.4). Ir has usually been chosen in the following studies since it gives a high $\mathrm{NH}_{3}$-sensitivity at the same time as the $\mathrm{H}_{2}$-sensitivity is comparatively low. Hitherto, devices in the form of capacitors have been used with an Ir layer with a nominal thickness of $3 \mathrm{~nm}$ resistively evaporated through a metal mask over a T-shaped Pd film as shown in the insert of Fig. 27.4.

$\mathrm{NH}_{3}$-sensors will now also be available as MOSFETs with integrated temperature control. In contrast to the $\mathrm{H}_{2}$-sensors, our $\mathrm{NH}_{3}$-sensors are usually operated at room temperature or at a slightly higher temperature, $35^{\circ} \mathrm{C}$, to prevent water condensation. This means that in a biosensor, the biochemical component can be placed very close to the sensing area. The maximum $\mathrm{NH}_{3}$-sensitivity is $1 \mathrm{ppm}$. At low $\mathrm{NH}_{3}$ concentrations ( $\leqslant 50 \mathrm{ppm}$ ) the response follows the equation

$$
\Delta V=C_{2} \times\left(P_{\mathrm{NH}_{3}}\right)^{a}
$$

where $C_{2}$ and $a$ are constants (typical values are $C_{2}=24 \mathrm{mV} / \mathrm{ppm}$ and $a=0.55)$. Furthermore, Winquist et al. $(1984 b)$ have shown that if an IrMOS is exposed to $\mathrm{NH}_{3}$ for only a short time, $\Delta t$, at low concentration 
( $\leqslant 50 \mathrm{ppm}$ ) then the maximum voltage shift for an ammonia pulse is

$\Delta V=k \times P_{\mathrm{NH}}, \Delta t$, where $k$ is a constant.

A similar linear relation applies to $\mathrm{H}_{2}$-sensitive PdMOS devices as well. Normally $\Delta t \leqslant 60 \mathrm{~s}$, which is short enough to achieve linearity, but a sufficiently long time for reliable measurements.

\subsection{Experimental}

For gaseous samples the experimental arrangement can be quite simple. It has turned out to be advantageous to work with rather short sample pulses as this gives linear response for low concentrations (eqn 27.3) and makes the determination less affected by any base-line drift. The sample can be introduced via a (timer-controlled) miniature solenoid valve that is normally open to the air but can open for the sample for a short period, e.g. 10 s. At continuous monitoring this can be automatically repeated by the timer, e.g. every $10 \mathrm{~min}$. The samples are conveniently drawn through the valve to the sensor with a peristaltic pump $(\sim 1 \mathrm{ml} / \mathrm{min})$. Due to the relatively high sensitivity a dilution of the sample usually has to be made. Immediately after the pump the sample stream is therefore mixed with a stream of air or nitrogen controlled by a needle-valve or a flow-controller to give a suitable dilution factor. The combined gas stream is then led through a small flow cell in which the gas sensor is mounted. The dilution gas also efficiently flushes the cell between samples.

With aqueous samples the compound to be measured must be transferred to the gas phase in a controlled fashion in relation to its concentration. This can be done with the equipment shown in Fig. 27.5, in which the separation is accomplished with a porous, gas-permeable Teflon membrane (Fluoropore, Millipore, USA) with a pore diameter of 0.2 to $5 \mu \mathrm{m}$. A membrane area of 1-3 $\mathrm{cm}^{2}$ suffices for the flow rates normally employed, i.e. a fluid stream of

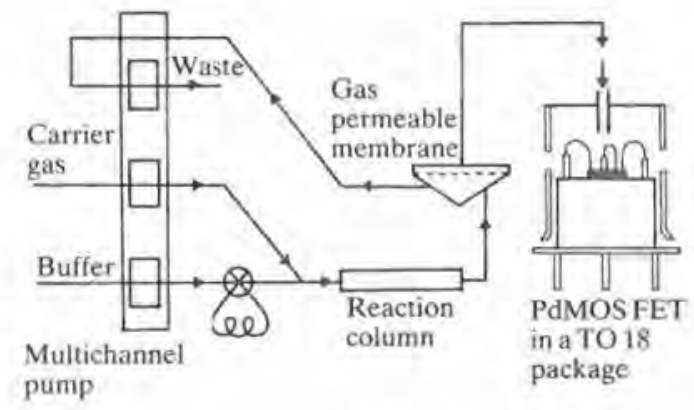

Fig. 27.5 Flow system for the determination of hydrogen in solutions. 


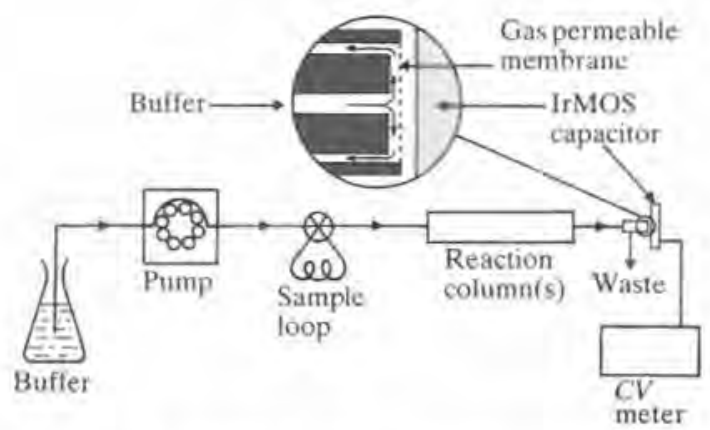

Fig. 27.6 Experimental set-up for the flow-injection analysis of ammonia with an IrMOS-capacitor.

$0.5-1 \mathrm{ml} / \mathrm{min}$ mixed with $2.5 \mathrm{ml} / \mathrm{min}$ of air or nitrogen. From the other side of the membrane a gas stream of $1 \mathrm{ml} / \mathrm{min}$ leads to the detector cell. Samples are introduced via a sample injection valve with a $0.5 \mathrm{ml}$ loop. It is convenient to use a multichannel peristaltic pump (for instance a Minipuls H8, Gilson, France) for administering the different flow streams. The pump can also be used for any additions or dilutions required. At high concentrations, requiring more extensive dilutions, this set-up could be combined with the apparatus described above.

For work with $\mathrm{NH}_{3}$-sensitive IrMOS capacitors a somewhat different approach can be used since the capacitors are mounted in such a way that the sensing area is very close to the gas-permeable membrane. Over such short distances gas transport by diffusion is satisfactory, obviating the need for a carrier gas, (See Fig. 27.6.) A peristaltic pump is used to pump buffer and sample at a flow rate of $0.4 \mathrm{ml} / \mathrm{min}$ past the membrane (porous Teflon with $5 \mu \mathrm{m}$ pore size, diameter $4 \mathrm{~mm}$ ) which is placed $0.2 \mathrm{~mm}$ above the sensor. In order to increase the pressure over the membrane a tubing $0.5 \mathrm{~m} \times 0.2 \mathrm{~mm}$ internal diameter is attached to the outlet of the flow-cell. Samples are introduced through an injection valve with a $0.2 \mathrm{ml}$ loop. If necessary, it is simple to raise the $\mathrm{pH}$ of the effluent of the enzyme column or of the $\mathrm{NH}_{4}{ }^{+}$solution by mixing with an alkaline solution in order to increase the proportion of $\mathrm{NH}_{3}$. However, remarkably high sensitivity is obtained even at rather low pH. Figure 27.7 compares the response obtained with $\mathrm{NH}_{4}{ }^{*}$ samples at pH 7.7 and 12.7. It should be noted that ammonia-producing enzymic reactions normally have rather high $\mathrm{pH}$-optima $(\mathrm{pH}>8)$.

An alternative, enzyme electrode-like design was recently developed by Winquist and co-workers (1984a). Here, the enzyme probe consists of an enzyme layer placed directly on the gas-permeable membrane, which in turn is placed in close proximity to the IrMOS capacitor (see below).

PdMOSFETs and equipment for hydrogen determination are available 


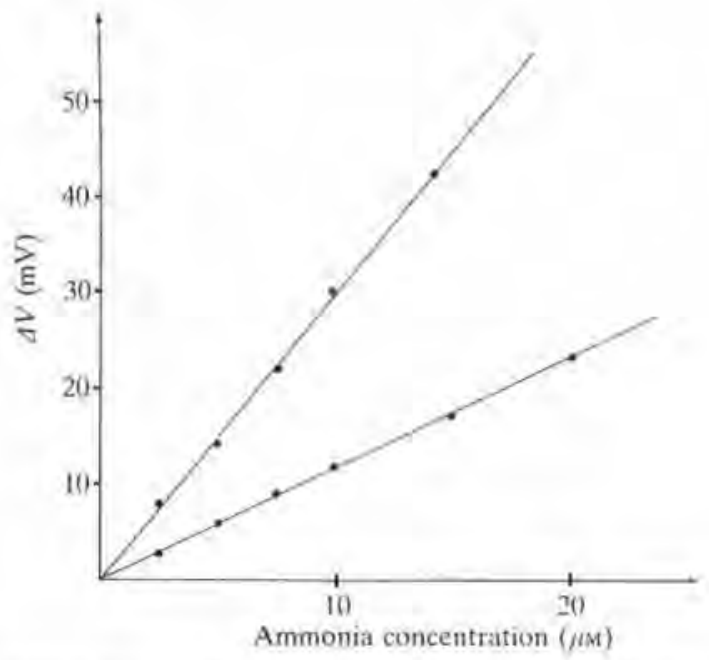

Fig. 27.7 Calibration graphs obtained from $0.2 \mathrm{ml}$ ammonia- $\mathrm{N}$ standards at $\mathrm{pH} 7.7$ and 12.7, respectively, with the flow-injection system for ammonia- $\mathrm{N}$ determination.

from Sensistor AB, Linköping, Sweden. IrMOSFETs will soon be available for $\mathrm{NH}_{3}$-determinations with the same equipment. The experiments described here involving $\mathrm{NH}_{3}$ were carried out with a capacitance meter and temperature controller assembled at the Applied Physics Laboratory at the University of Linköping.

\subsubsection{Preparation of immobilized hydrogenase}

Hydrogenase (EC 1.12.1.2) is a key enzyme for dehydrogenase assays utilizing a PdMOSFET, which becomes evident upon examination of the reaction catalysed by hydrogenase $(\mathrm{HDH})$ :

$$
\mathrm{NAD}(\mathrm{P})^{+}+\mathrm{H}_{2} \stackrel{\mathrm{HDH}}{\rightleftarrows} \mathrm{NAD}(\mathrm{P}) \mathrm{H}+\mathrm{H}^{+}
$$

We have used an $\mathrm{HDH}$ isolated from Alcaligenes eutrophus $\mathrm{H} 16$ and immobilized on controlled pore glass (CPG) following procedures described by Winquist et al. (1986b).

\subsection{Results}

\subsection{Hydrogen gas determination}

The hydrogen gas sensor produced by the Sensistor Co. is primarily intended for use as a hydrogen leak detector. As an example, hydrogen can be used as a cheap, non-poisonous test gas for localization of damage to underground 
electricity or telecommunication cables. Hydrogen is introduced at one end of the cable and any leakage can easily be localized as hydrogen readily leaks through the soil.

Many important routes lead to hydrogen evolution or consumption and hydrogen is produced by many micro-organisms under anaerobic conditions. Furthermore, various hydrogenase systems are attracting considerable interest since they are involved in microbial or photosynthetic hydrogen production as a future energy source of great potential. We have previously demonstrated that a PdMOSFET in the set-up shown in Fig. 27.5 can be conveniently used for monitoring microbial hydrogen production (Winquist et al. 1982). In this study we particularly studied Clostridium acetobutylicum immobilized in alginate beads, with the effluent after dilution fed to the apparatus shown in Fig. 27.5.

Cleland et al. (1984) used a PdMOS sensor in a study of the hydrogen evolution from an $E$. coli cultivation. Hydrogen is produced only under anaerobic conditions and this was clearly demonstrated as hydrogen evolution sharply increased when the oxygen level fell to zero. The hydrogen production rapidly decreased when oxygen was admitted as a consequence of the inhibitory effect of oxygen on the hydrogenase system. It was suggested that the PdMOS sensor could be used to detect inhomogeneity of mixing, e.g. in scale-up studies of bioreactors, because any 'partial anaerobiosis' would lead to hydrogen evolution that would readily be detectable with the highly sensitive hydrogen detector.

The susceptibility of Enterobacteriaceae to ampicillin was investigated by Hörnsten et al. (1985) using a hydrogen-sensitive PdMOSFET. A large number of isolates of enterobacteria from urinary tract infections were cultured in sealed tubes and the amount of hydrogen produced was measured by injecting a $2 \mathrm{ml}$ gas sample into a measuring cell housing the PdMOSsensor. Molecular hydrogen is an end product of mixed acid fermentation by Enterobacteriaceae, and therefore the degree of inhibition of hydrogen evolution is a measure of ampicillin susceptibility. In all but a few cases it could be established within five hours whether the cells were viable or not in the presence of ampicillin. The results agreed well with common, routine disc-diffusion results. In conclusion, assay of hydrogen production promises to become a useful, quick, and simple technique for establishing antibiotic susceptibility.

In a recent study, Hörnsten et al. (1986a) investigated the ampicillin susceptibility of an $E$. coli strain causing urinary tract infection using a PdMOSFET sensor for determining hydrogen production (Hornsten et al. $1986 b$ ) in comparison with three other parameters: heat production (as measured with use of an enzyme thermistor unit (Danielsson 1986; see also Chapter 29)), intracellular ATP-level, and acid/base production. Hydrogen evolution, heat production, and acid/base production were measured on-line 


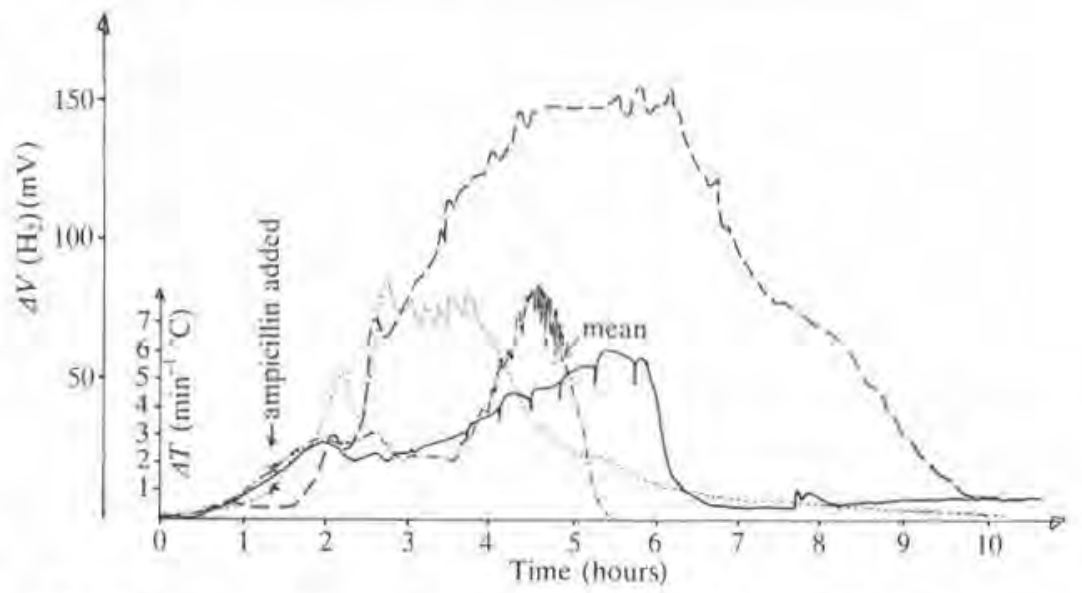

Fig. 27.8 Hydrogen and heat production by $E$. coli under anaerobic conditions with and without ampicillin added at minimal inhibitory concentration $(\mathrm{MIC}=4 \mathrm{mg} / \mathrm{l}$ ): -.-.-, thermogram with and _-, without ampicillin; $\cdots \cdot \cdot$, hydrogen concentration with and -., without ampicillin.

from fermentations in various media of 2-2.51 of E. coli suspensions with different additions of ampicillin. Hydrogen was sampled by a peristaltic pump through a timer-operated solenoid valve and mixed with an air-stream for proper dilution as described above (Section 27.3). All four methods were found to give valuable information. The use of two of the methods in parallel evidently facilitates correct conclusions. Hydrogen monitoring during fermentations gives rapid indication of antibiotic susceptibility and requires only rather simple techniques and equipment. Figure 27.8 compares two different runs with and without ampicillin added.

27.4.1.1 Hydrogenase Small reaction columns containing about $1 \mathrm{ml}$ of HDH immobilized on CPG were inserted in the analytical system shown in Fig. 27.5 for quantification of $\mathrm{NAD}^{-}$and NADH. Samples of NADH

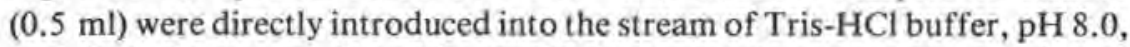
and the hydrogen produced by the $\mathrm{HDH}$-catalysed reaction was monitored with a PdMOSFET. The calibration curve was linear up to $0.5 \mathrm{mM} \mathrm{NADH}$ $\left(C_{\mathrm{NADH}}(\mu \mathrm{M})=0.1 \times \Delta V(\mathrm{mV})\right)$ with a detectability of $0.03 \mathrm{mM}$. At least ten samples per hour could be analysed (Danielsson et al. 1983).

The same system could be used for determination of $\mathrm{NAD}^{+}$based on the reversed $\mathrm{HDH}$ reaction in which $\mathrm{H}_{2}$ is consumed. In this case the working buffer was supplemented with $100 \mathrm{ppm}$ of $\mathrm{H}_{2}$ and $0.1 \mathrm{mM} \mathrm{NADH}$. The calibration curve was linear over the range $0.05-0.6 \mathrm{~mm}$. Furthermore, we have studied combinations of various dehydrogenases and $\mathrm{HDH}$ to extend 
the applicability of hydrogen sensors. For instance, ethanol could be quantified using the following reaction sequence:

$$
\mathrm{C}_{2} \mathrm{H}_{5} \mathrm{OH}+\mathrm{NAD}^{+} \underset{-\mathrm{CH}_{3} \mathrm{CHO}}{\stackrel{\mathrm{ADH}}{\longrightarrow}} \mathrm{NADH}+\mathrm{H}^{\cdot} \stackrel{\mathrm{HDH}}{\longrightarrow} \mathrm{NAD}^{*}+\mathrm{H}_{2}(27.5)
$$

An interesting approach to the analysis of volatile dehydrogenase substrates directly in gaseous samples is possible through the use of co-immobilized dehydrogenase/HDH in a 'moist' column saturated with buffer and coenzyme. The sample is introduced into a carrier gas stream that transports the hydrogen produced in the reaction column to the PdMOS-detector. Encouraging results have been obtained in preliminary tests with alcohol and aldehyde vapours.

As immobilized HDH is comparatively stable, it of fers an attractive way of coenzyme regeneration, since the reaction solution is not polluted by any other substrates/products than $\mathrm{H}_{2} / \mathrm{H}^{+}$. We have demonstrated the feasibility of such a system using co-immobilized alanine dehydrogenase/HDH for the formation of alanine from pyruvate accompanied by continuous regeneration of NADH (Danielsson et al. 1982):

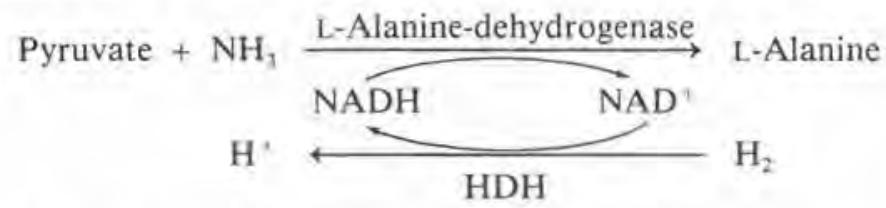

It can be anticipated that this type of coenzyme regenerative system could find use for signal amplification in combination with PdMOS-sensors in a similar way as described by Scheller and co-workers (1985) using substrate amplifying systems together with an enzyme thermistor. This should make very sensitive determinations possible.

\subsubsection{Measurements involving $\mathrm{NH}_{3}$}

Ammonium is a weak acid with $\mathrm{p} K_{\mathrm{a}}=9.25$, which means that ammonium ions are in equilibrium with ammonia in the $\mathrm{pH}$ range $6-12$. In measurements of ammonia in aqueous solutions with the flow system based on the IrMOScapacitance the fraction of $\mathrm{NH}_{3}$ is measured. Consequently, the $\mathrm{pH}$ of the solution is an important parameter that must be carefully controlled. If the $\mathrm{pH}$ is raised, formation of $\mathrm{NH}_{3}(\mathrm{~g})$ will be favoured and the sensitivity will be higher. Maximum sensitivity is obtained for $\mathrm{pH}$ values above 11. For determination of ammonia in inorganic samples, such as rain and river water, we have worked at $\mathrm{pH} 12.5$. With biological samples the $\mathrm{pH}$ could not be raised so far, because $\mathrm{NH}_{3}$ could be released from labile nitrogen compounds under strong alkaline conditions. For blood samples the $\mathrm{pH}$ is not allowed to exceed 8.5. 


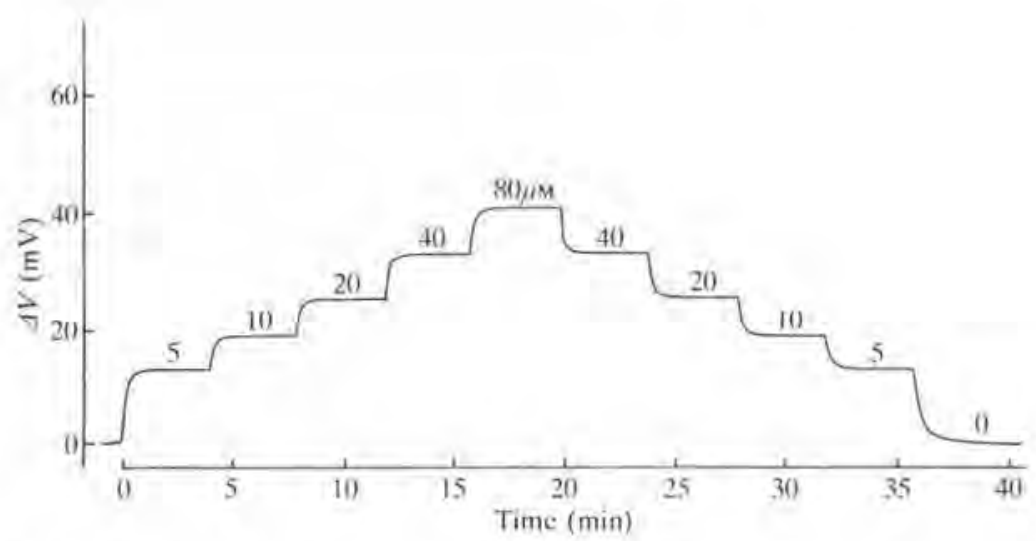

Fig. 27.9 Recording from a continuous monitoring of the ammonium concentration in buffer, $\mathrm{pH} 7.7$. The ammonium concentration was changed stepwise every $4 \mathrm{~min}$.

The concentration of ammonia- $\mathrm{N}$ (total amount of ammonia and ammonium ions) in aqueous solutions can be determined with the flowinjection system depicted in Fig. 27.6 with the reaction column omitted. The linear calibration graphs obtained with $0.2 \mathrm{ml}$ samples at $\mathrm{pH} 7.7$ and 12.7 respectively, are displayed in Fig. 27.7. The lower limit of detection (at $S / N$ $=3$ ) was $0.4 \mu \mathrm{M}$ at $\mathrm{pH} 7.7$ and $0.2 \mu \mathrm{M}$ at $\mathrm{pH} 12.7$ and the graphs were linear up to $50 \mu \mathrm{M}$. The high sensitivity makes it possible to perform determination of ammonia- $\mathrm{N}$ in only $20 \mu \mathrm{l}$ samples of blood or blood serum, even after tenfold dilution. The expected concentration range is $10-70 \mu \mathrm{M}$. This method would be useful for paediatric samples due to the small sample volume needed and it should be noted that the technique works equally well with whole blood since the colour or turbidity of the sample will not interfere.

To give an idea of the performance of the sensor a continuous and a pulsewise recording of ammonia- $\mathrm{N}$ in buffer $\mathrm{pH} 7.7$ is given in Fig. 27.9. The concentration was changed stepwise every $4 \mathrm{~min}$.

A disadvantage of the present IrPdMOS sensor is that some low-molecular-weight amines as well as $\mathrm{H}_{2}$ interfere with the $\mathrm{NH}_{3}$ determination. Table 27.1 lists the response obtained with some amines in comparison with ammonia at $\mathrm{pH} 7.7$. It was found that the response for amines was even higher for gaseous samples (Winquist et al. 1984b). This indicates that the phase separation step and the membrane properties are important factors to consider in any efforts to reduce the interferences. It may also be possible to change the catalytic properties of the sensor surface. Another possibility is to use scavenger (enzyme) columns or to make differential measurements. In the applications studied to date, however, we have not found that interferences would be a serious problem in practice. 
Table 27.1 Response of an IrMOS-capacitor for various amines $(50 \mu \mathrm{M})$ at $\mathrm{pH} 7.7$ in relation to the response for $50 \mu \mathrm{M}$ ammonia- $\mathrm{N}$

\begin{tabular}{lc}
\hline Compound & $\begin{array}{c}\text { Relative sensitivity } \\
\%\end{array}$ \\
\hline Ammonia-N & 100 \\
Methylamine & 10 \\
Ethylamine & 5 \\
Butylamine & 0 \\
Diethylamine & 2 \\
Ethanolamine & 2 \\
Ethylenediamine & 1 \\
\hline
\end{tabular}

27.4.2.1 Urea determinations Urea was determined by the flow system shown in Fig. 27.6 using a $40 \times 2 \mathrm{~mm}$ Eupergit $C$ (polyacrylic beads from Röhm-Pharma GmbH, Darmstadt, West Germany) column with 40 IU of urease. Urease produces two molecules of $\mathrm{NH}_{3}$ per urea molecule:

$$
\left(\mathrm{NH}_{2}\right)_{2} \mathrm{CO}+\mathrm{H}_{2} \mathrm{O} \stackrel{\text { Urease }}{\Longleftarrow} \mathrm{CO}_{2}+2 \mathrm{NH}_{3}
$$

Due to the large amount of enzyme on the column, 100\% conversion and unchanged performance was achieved for at least one month (Winquist et al. 1984a) despite unfavourable $\mathrm{pH}$ of the working buffer. The assay was

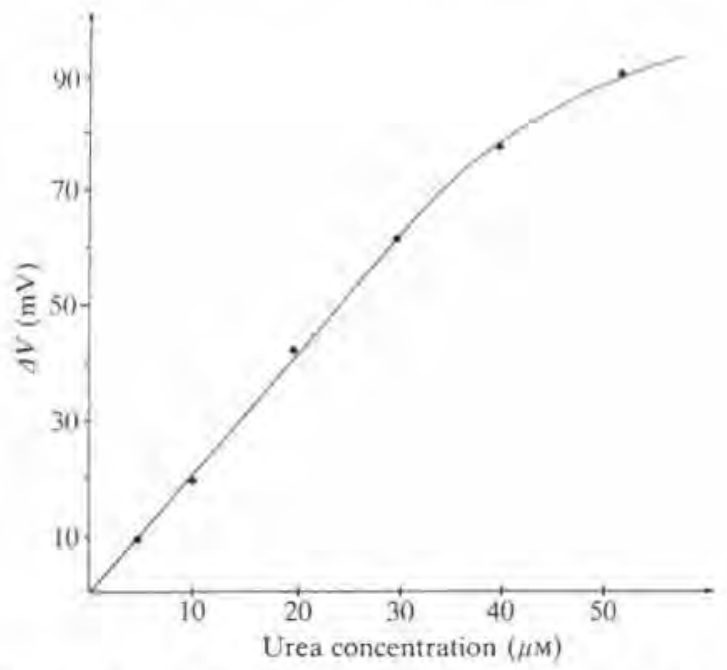

Fig. 27.10 Calibration curve for $0.2 \mathrm{ml}$ urea standards at $\mathrm{pH} 8.1$ using a reaction column containing $40 \mathrm{IU}$ of urease. 
made in $0.05 \mathrm{M}$ Tris- $\mathrm{HCl}, \mathrm{pH} 8.1$, while the $\mathrm{pH}$ optimum for urease activity is 7.0. The calibration curve for $0.2 \mathrm{ml}$ samples was linear up to $40 \mu \mathrm{M}$ with a lower limit of detection of $0.2 \mu \mathrm{M}$ (Fig. 27.10). Twenty 500-fold diluted serum samples could be assayed per hour with a precision of about $2 \%$. The sampling rate evidently is too low for large collections of samples and should be increased. One possibility would be to use several parallel sensors (and many enzyme columns) in an automatically switching arrangement. The present flow system is, however, excellent for a limited number of samples and is a suitable back-up system due to its high operational stability and short start-up time.

27.4.2.2 Determination of L-asparagine Several enzymes are available which more or less selectively deaminate various amino acids. An example is L-asparaginase (EC 3.5.1.1):

$$
\text { L-Asparagine }+\mathrm{H}_{2} \mathrm{O} \stackrel{\text { L-Asparaginase }}{\longrightarrow} \text { L-Aspartate }+\mathrm{NH}_{4}{ }^{*}
$$

For assays of samples that normally contain ammonia, such as blood and serum samples, an ammonia-scavenging column of L-glutamatedehydrogenase can be used to remove the ammonia prior to the analysis of other ammonia-producing compounds:

$\mathrm{NH}_{4}{ }^{+}+\mathrm{NADH}+\alpha$-Oxoglutarate $\stackrel{\begin{array}{c}\text { L-Glutamate- } \\ \text { dehydrogenase }\end{array}}{\longrightarrow} \mathrm{NAD}^{+}+$L-Glutamate

The use of a scavenger column can be illustrated by an assay proposed for the determination of L-asparagine in blood (serum) (Winquist et al. 1986b). Twenty units of L-glutamate-dehydrogenase and five units of L-asparaginase, respectively, were bound to Eupergit $C$ and packed in two consecutive Teflon columns $(32 \times 2 \mathrm{~mm})$ connected to the flow system shown in Fig. 27.6. The working buffer was $0.05 \mathrm{M}$ Tris- $\mathrm{HCl}, \mathrm{pH} 8.2$, containing $1 \mathrm{mM} \mathrm{NADH}, 0.5 \mathrm{~mm} \alpha$-oxoglutaric acid, and $3 \mathrm{mM} \mathrm{NaN}_{3}$. Under the conditions at room temperature the L-glutamate-dehydrogenase column was capable of completely removing up to $0.5 \mathrm{~mm}$ ammonia- $\mathrm{N}$ in $0.2 \mathrm{mI}$ samples at a flow rate of $0.4 \mathrm{ml} / \mathrm{min}$, which gives good margins for diluted blood samples. The calibration graph for L-asparagine was linear up to $40 \mu \mathrm{M}$; the voltage shift was $0.8 \mathrm{mV} / \mu \mathrm{M}$. After every 20 samples, columns and tubings were washed for $5 \mathrm{~min}$ with $0.1 \mathrm{M} \mathrm{K}$-phosphate, $\mathrm{pH} 7.0$, containing $0.8 \mathrm{M} \mathrm{NaCl}$.

27.4.2.3 Creatinine determinations A method for determination of creatinine in serum and urine was recently developed by Winquist et al. 
(1986a). Ammonia, released by creatinine iminohydrolase (EC 3.5.4.21) immobilized on oxirane acrylic beads (Eupergit C),

$$
\text { Creatinine }+\mathrm{H}_{2} \mathrm{O} \stackrel{\text { Creatininase }}{\longrightarrow} \mathrm{N} \text {-Methylhydantoin }+\mathrm{NH}_{3}
$$

was measured with an IrMOS-capacitor. The creatininase column was preceded by a L-glutamate-dehydrogenase column for removal of endogeneous ammonia- $\mathrm{N}$. The working buffer was $0.05 \mathrm{M}$ Tris- $\mathrm{HCl}, \mathrm{pH} 8.5$ and the procedure was the same as for determination of L-asparagine. With $6 \mathrm{IU}$ of creatininase (obtained from Aalto Bio Reagents Ltd, Dublin, Ireland) in a $0.1 \mathrm{ml}$ column, the sensor response was linear up to $30 \mu \mathrm{M}$ for $85 \mu \mathrm{l}$ samples with a detection limit of $0.2 \mu \mathrm{M}$. Very satisfactory results were obtained in comparison with conventional methods; 25 -fold diluted serum and 1000 -fold diluted urine samples were analysed with a precision of about $3 \%$ without any serious interferences. Thus, the sample consumption was very low, which makes this method well suited for paediatric samples, although the relatively low sample throughput ( 15 samples/h) is a hindrance for larger series of samples to be processed.

\subsection{Concluding remarks}

The flow system equipped with an enzyme reactor (Fig. 27.6) has been used for a number of enzyme-substrate combinations. If the activity of the reactor is large enough for complete substrate conversion, a given amount of substrate will give the same amount of ammonia irrespective of enzyme-substrate combination. This is shown in Table 27.2 where the

Table 27.2 Responses obtained with an IrMOS-capacitor for different enzyme-substrate combinations. Buffer; $0.05 \mathrm{M}$ Tris- $\mathrm{HCl}, \mathrm{pH}$ 8.5. Substrate concentration: $10 \mu \mathrm{M}$ during $30 \mathrm{~s}$

\begin{tabular}{|c|c|c|c|}
\hline Substrate & Enzyme & Product & $\begin{array}{l}\text { Voltage } \\
\text { shift (mV) }\end{array}$ \\
\hline Urea & Urease & $\mathrm{CO}_{2}+2 \mathrm{NH}_{3}$ & 16 \\
\hline L-Asparagine & Asparaginase & Aspartate $+\mathrm{NH}_{3}$ & 8 \\
\hline L-Aspartate & Aspartase & Fumarate $+\mathrm{NH}_{3}$ & 8 \\
\hline L-Glutamate & $\begin{array}{l}\text { Glutamate } \\
\text { dehydrogenase }\end{array}$ & $\begin{array}{l}\alpha \text {-oxoglutarate + NADH } \\
+\mathrm{NH}_{3}\end{array}$ & 8 \\
\hline Adenosine & Adenosine deaminase & Inosine $+\mathrm{NH}_{3}$ & 8 \\
\hline Creatinine & $\begin{array}{l}\text { Creatinine imino- } \\
\text { hydrolase }\end{array}$ & $\mathrm{N}$-Methylhydantoin $+\mathrm{NH}_{3}$ & 8 \\
\hline $\mathrm{NH}_{3}$ & - & $\mathrm{NH}_{3}$ & 8 \\
\hline
\end{tabular}


responses for various combinations are compared under identical conditions. Urea gives twice the voltage shift when compared with the other substrates because it produces two ammonia molecules. Detection ranges for some $\mathrm{NH}_{3}$-producing systems under the operating conditions described in the previous section are summarized in Table 27.3.

Table 27.3 Operating ranges for $\mathrm{NH}_{3}$ and some enzyme-substrate systems with a flow-injection system according to Fig. 27.6. Sample volume: $0,2 \mathrm{ml}$; Flow rate: $0.4 \mathrm{ml} / \mathrm{min}$

\begin{tabular}{llcc}
\hline Substrate & Enzyme & $\mathrm{pH}_{\exp }$ & Linear range $(\mu \mathrm{M})^{*}$ \\
\hline $\mathrm{NH}_{3}$ & - & 7.7 & $0.4-50$ \\
$\mathrm{NH}_{3}$ & - & 12.7 & $<0.2-50$ \\
L-Asparagine & Asparaginase & 8.2 & $0.2-40$ \\
Creatinine & Creatinine & & \\
& $\quad$ imminohydrolase & 8.5 & $0.2-30$ \\
Urea & Urease & 8.1 & $0.2-40$ \\
\hline
\end{tabular}

* The first number corresponds to the lower limit of detection $(S / N=3)$.

In the introduction we discussed advantages and disadvantages of the present sensor design that must be operated in the gas phase. In many applications the measuring procedure would be greatly simplified if the transducer could be placed directly in the sample solution. This would be of special value in probes intended for in vivo monitoring. Work is in progress at the Linköping laboratory to modify the PdMOS-structures for operation in electrolyte solutions. These experiments reveal that the approach is possible, but no attempts have been made as yet to combine these sensors with enzymes. Slow responses to changes in the hydrogen concentration is presently a problem. In particular, the recovery time is much longer in electrolytes than in gases.

The membrane used for phase separation in the biosensor designs described in this chapter can, however, be placed very close to the sensor surface. Thus we have recently prepared a probe-like construction with relatively small dimensions which rather easily could be further reduced, if desired (Winquist et al. 1985). Figure 27.11 shows the design and the responses for urea and ammonia of a urease probe based on an IrMOScapacitor. The sensor is embedded with a polyester resin in a small tube leaving only the porous Teflon membrane $\left(3 \mathrm{~mm}^{2}\right)$ exposed to the solution. About $200 \mathrm{IU}$ of urease were applied onto the membrane and cross-linked with glutaraldehyde. This bio-probe has a rather high sensitivity, although its response is slower than that of the $\mathrm{NH}_{3}$-detecting flow system. The sensitivity for urea is higher than for $\mathrm{NH}_{4}{ }^{+}$, since each urea molecule produces two ammonia molecules. By using one probe with active urease and one with 


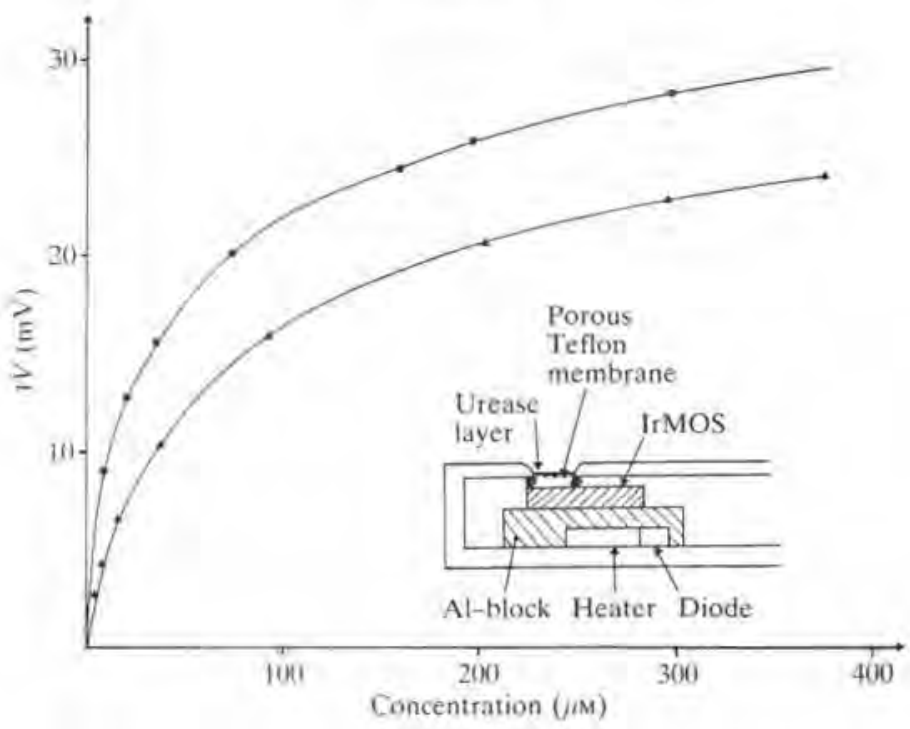

Fig. 27.11 Steady-state response for urea ( $(\bullet)$ and $\mathrm{NH}_{4}^{+}(\mathbf{\Delta})$ of a urease probe. The insert shows a cross-section of the probe.

inactivated enzyme it was possible to determine urea concentrations in samples containing endogenous ammonia. Future developments could further reduce the dimensions of the probes and also combine several sensor elements for incorporation of a reference or for the simultaneous measurement of several components.

The $\mathrm{H}_{2} \mathrm{~S}$-sensitivity of PdMOS-sensors was earlier demonstrated by Shivaraman (1976). It is of the same order as that of $\mathrm{NH}_{3}$ and $\mathrm{H}_{2}$. No attempts have been made to date to exploit this property, but there should be many interesting biological applications, both with pure enzyme systems for detection of sulphur-containing metabolites and with micro-organisms.

There is no doubt that future developments along the lines described here will bring semiconductor sensors closer to usable in vivo sensors, multicomponent sensors, 'smart sensors', and maybe also towards the biochip concept.

\section{References}

Cleland, N., Hörnsten, G., Elwing, H., Enfors, S.-O. and Lundström, I. (1984). Measurement of hydrogen evolution by oxygen-limited $E$. coli by means of a hydrogen sensitive Pd-MOS sensor. Appl. Microbiol. Biotechnol. 20, 268-70.

Danielsson, B. (1986). Enzyme thermistor devices. Methods in Enzymol. In press.

- Lundström, 1., Mosbach, K. and Stiblert, L. (1979). On a new enzyme transducer combination: The enzyme transistor. Anal. Lett, 12, 1189-99.

- Winquist, F., Malpote, J,-Y, and Mosbach, K. (1982). Regeneration of NADH 
with immobilized systems of alanine dehydrogenase and hydrogen dehydrogenase. Biotechnol. Lett. 4, 673-8.

- Mosbach, K. and Lundström, 1. (1983). Bioanalytical applications of hydrogenand ammonia-sensitive palladium gate MOS devices. In Proc. int. meeting on chemical sensors. Fukuoka, Kodansha, Tokyo, pp. 507-512. Elsevier, New York.

Hörnsten, G., Elwing, H, , Kihlström, E. and Lundström, I. (1985). Determination of molecular hydrogen in investigations of the susceptibility of Enterobacteriaceae to ampicillin. J. Antimicrob. Chemother. 15, 695-700.

- Danielsson, B., Nilsson, L. and Lundström, I. (1986a), Physiological studies of Escherichia coli under ampicillin stress and anaerobic conditions. Submitted.

Hörnsten, G., Danielsson, B., Elwing, H., and Lundström, I. (1986b). Sensorized on line determinations of molecular hydrogen in Escherichia coli fermentations. Appl. Microbiol. Biotechnol. 24, 117-21.

Lundström, I. (1981). Hydrogen-sensitive MOS-structures. Part I: Principles and applications. Sensors and Actuators 1, 403-26.

- Shivaraman, S, and Svensson, C. (1975). A hydrogen sensitive Pd-gate MOStransistor. J. Appl. Phys, 46, 3876-81.

Scheller, F., Siegbahn, N., Danielsson, B. and Mosbach, K. (1985). High-sensitivity enzyme thermistor determination of L-lactate by substrate recycling. Anal. Chem. 57, 1740-3.

Shivaraman, S. (1976). Detection of hydrogen sulfide with palladium-gate MOS fieldeffect transistors. J. Appl. Phys, 47, 3592-3.

Sibbald, A., Whalley, P. D. and Covington, A. K. (1984). A miniature flow-through cell with a four-function ChemFET integrated circuit for simultaneous measurements of potassium, hydrogen, calcium and sodium ions. Anal. Chim. Acta 159, 47-62.

Winquist, F., Lundström. I. and Danielsson, B. (1986a). Determination of creatinine by an ammonia-sensitive semiconductor structure and immobilized enzymes. Anal. Chem. 58, 145-8.

- Danielsson, B., Lundström, 1. and Mosbach, K. (1982). Use of hydrogensensitive Pd-MOS materials in biochemical analysis. App. Biochem. Biotechnol. 7 , 135-9.

- $(1986 b)$. The use of hydrogen and ammonia sensitive semiconductor structures in analytical biochemistry - 'Enzyme transistors'. Methods in Enzymol. In press.

- Spetz, A., Lundström, I. and Danielsson, B. (1984a). Determination of urea with an ammonia gas-sensitive semiconductor device in combination with urease. Anal. Chim. Acta 163, 143-9.

- $(1984 b)$. Determination of ammonia in air and aqueous samples with a gassensitive semiconductor capacitor. Anal. Chim. Acta 164, 127-38.

— Armgarth, M., Nylander, C, and Lundström, I. (1983). Modified palladium metal-oxide-semiconductor structures with increased ammonia gas sensitivity. Appl. Phys. Lett. 43, 839-41.

— Lundström, I. and Danielsson, B. (1985). Biosensors based on ammonia sensitive metal-oxide-semiconductor structures. Sensors and Actuators. 8, 91-100. 
Mechanical and acoustic impedance 



\title{
28
}

\section{Principles and potential of piezo-electric transducers and acoustical techniques}

\author{
DAVID J. CLARK, BARRIE C. BLAKE-COLEMAN, and \\ MICHAEL R. CALDER
}

\subsection{Introduction}

Acoustical methods have become highly sophisticated particularly through audio engineering and developments in naval instrumentation (e.g. echo sounding and acoustic signatures of vessels). Accompanying such developments have been significant adyances in piezo-electric transducer technology, particularly at the upper ultrasonic frequency limits and in the development of piezo-electric polymers. However, exploitations of acoustical techniques in the biological sciences have remained relatively few and dispersed until recently. Most notable are advances in acoustic microscopy, surface mass detecting sensors, acoustic resonance densitometry, and acoustic impedance of inhomogeneous systems. The principles and application of such techniques are discussed.

\subsection{Piezo-electric transducers}

Piezo-electric transducers are central to most acoustic techniques. Piezoelectricity was discovered in 1880 by the Curie brothers as a phenomenon where electric dipoles (developing a potential difference) are generated in anisotropic natural crystals subjected to mechanical stress (Curie and Curie 1880). Such materials also exhibit the converse effect in suffering dimensional change under the influence of an electric field. Some piezo-electric materials are also pyro-electric, electric polarization resulting from thermal absorption by the material (Cady 1946). All materials exhibiting an anisotropic effect, such as piezo-electricity, have no centre of symmetry in their crystal structure. All such crystals belong to one of 32 point groups (crystal classes), 20 of the 32 classes exhibit piezo-electric effects and ten of these pyro-electric effects. A few naturally abundant crystals (e.g. quartz, tourmaline, and Rochelle salt) are piezo-electric (Cady 1946). Although manmade ceramic piezo-electrics are most widely applied (van Randeraat and Setterington 1974), more recent piezo-electric polymers (Kocharayan et al, 1967) are finding increasing exploitation. Since polymeric materials are not 
usually obtained as single crystals of appreciable size, piezo-electric effects in these materials are usually observed in the uniaxially oriented state. According to the state of orientation, four types of symmetry are found (Fukada 1974). Certain anisotropic biological structures (e.g. DNA, proteins) can also be considered to be piezo-electric and pyro-electric (Fukada 1968, 1974), a point which could well prove important in molecular biosensor research.

\section{2,1 Ceramics}

The existence of a polar axis in natural piezo-electric crystals gives rise to an inherent polarization, before any electric field is applied. The highly polar structure of quartz crystals under different conditions of loading is outlined in Fig. 28.1a. Man-made piezo-electric ceramics have improved piezoelectric properties and are polycrystalline in structure (e.g. barium titanate and various lead zirconate-titanates). The individual crystallite domains possess polar axes which are randomly aligned and do not have piezo-electric properties in their original state. Polarization in intense electric fields at elevated temperatures (above the Curie point) aligns the polar axes of the individual crystallite domains (Fig. 28.1b). Ceramics are highly chemically stable and mechanically rigid, and can be sintered into a wide variety of shapes and sizes (van Randeraat and Setterington 1974).

\subsubsection{Polymers}

Kocharayan et al. (1967) first identified that the high polarity of the unit cells of polytrifluoroethylene, plasticized, and rigid polyvinyl chloride yielded their high piezo-electric activities, Polyvinylidene fluoride (PVDF) was later shown to be susceptible to still higher levels of poling (Kawai 1969). PVDF is an approximate equal mixture of amorphous and crystalline polymer, the latter principally having a non-polar alpha form and a highly polar beta form, where the hydrogen and fluorine atoms are arranged to give maximum dipole moment per unit cell (Fig. 28.2a). These are randomly oriented along the polymer chain until poled (Fig. 28.2b). Commercial processes also exploit uniaxial or biaxial mechanical stretching of the extruded or cast polymer to ensure that the beta form predominates. Unlike ceramics, PVDF films are highly compliant. Apart from their chemical resistance, they are more sensitive transducers of mechanical to electrical energy than ceramics or quartz crystals.

\subsubsection{Modes of transduction}

Dipoles are largely poled normal to the major plane of the piezo-electric material. Piezo-electrics are anisotropic, their electrical, mechanical, and electromechanical properties differing for electrical or mechanical transduction along their respective axes. Convention uses numbered subscripts to identify the vector and tensor directions (Fig. 28.3). The first subscript iden- 

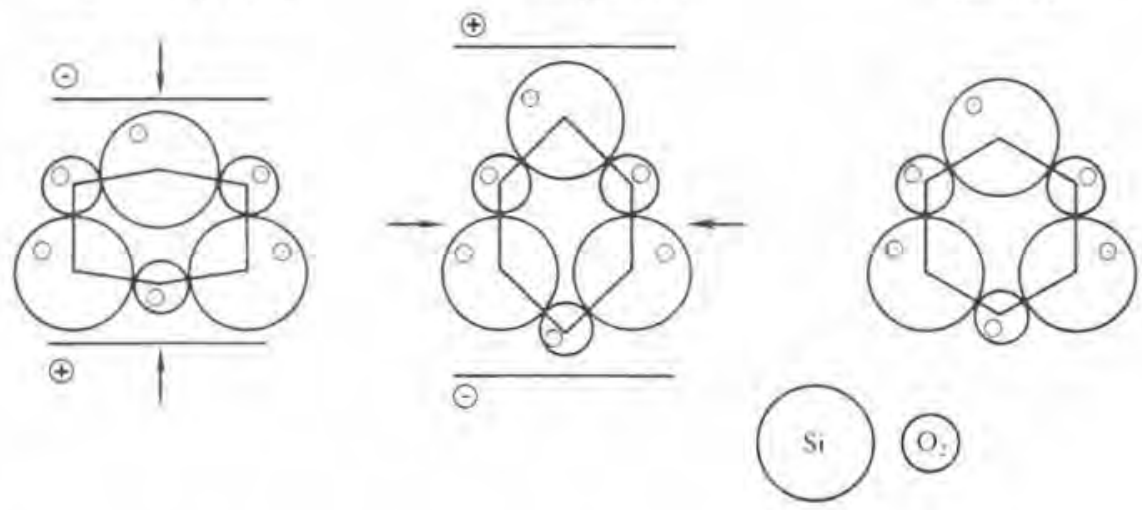

Crystal structure uf dipole

Polarized zones of dipolex
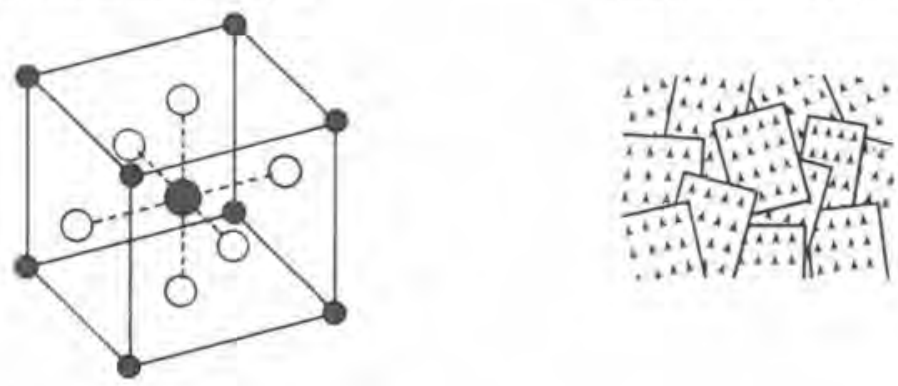

Barium
Oxygen
Oitanium

Fig. 28.1 Crystal and domain structures of piezo-electric ceramics. (a) Crystal structure of quartz showing changes in dipole moment underloading. (b) Barium titanate ceramic structure.

tifies the polarization and direction of field, the second the mechanical stress or strain axis (see Fukada 1974). Generally, polarization is along the $y$ axis (subscript 3), when force can be applied along the $x$ (subscript 1), $y$, or $z$ (subscript 2) axes. Fig. 28.3 summarizes these conventions for the piezoelectric constant $(d)$. Mechanical stress or strain $(g)$, compliance ratio $(s)$, dielectric constant $(\epsilon)$, etc. are similarly described (Fukada 1974). Thus transducer plates can be length, width, or thickness expanders (Fig. 28.3). Similarly, discs can be radial $\left(d_{31}\right)$ or thickness expanders $\left(d_{33}\right)$ and hoops or tubes, 
$a$ form

(non-polar, antiparallel dipole chains)

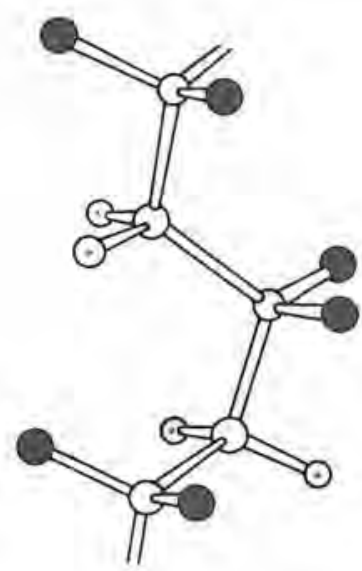

(a) $\bigcirc$ Hydrogen $\bigcirc$ Fluorine

Before polarization

(b)

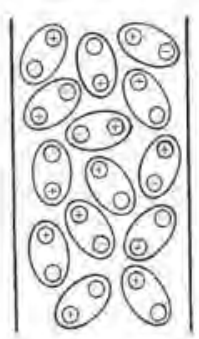

$\beta$ form

(polar, parallet dipole chains)
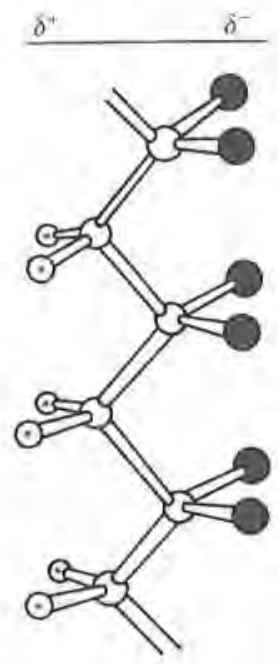

Carbon polarization

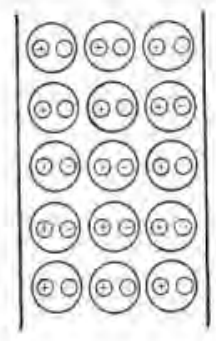

Fig. 28.2 Molecular structure and domain structure of polyvinylidene fluoride piezoelectric film. (a) Molecular structure of polyvinylidine fluoride. (b) Orientation of dipoles in polyvinylidine fluoride film.

length $\left(d_{31}\right)$ or thickness expanders $\left(d_{33}\right)$. Piezo-electric layers are often applied to non-piezo-electric materials to manufacture shear plates (e.g., $d_{15}$ to achieve $x$ and $z$ plane stress). Laminates (multimorphs) of piezo-electric material, arranged serially or in parallel, can be manufactured to allow bending. Further, manufacturing processes are capable of altering the relative magnitudes of electrical, mechanical, and electromechanical properties. 


\begin{tabular}{|c|c|c|c|c|c|}
\hline & Poled & $\begin{array}{l}\text { Field } \\
\text { axis }\end{array}$ & $\begin{array}{l}\text { Stress or } \\
\text { strain axis }\end{array}$ & $\begin{array}{l}\text { Free } \\
\text { axis }\end{array}$ & $\begin{array}{l}\text { Piezo-electric } \\
\text { constants }\end{array}$ \\
\hline $\begin{array}{l}\text { Length } \\
\text { expander }\end{array}$ & 1 & 1 & & & $d_{31}$ \\
\hline $\begin{array}{l}\text { Width } \\
\text { expander }\end{array}$ & 1 & 1 & & & $d_{0}$ \\
\hline $\begin{array}{l}\text { Thickness } \\
\text { expander }\end{array}$ & 1 & 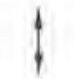 & 1 & & $d_{33}$ \\
\hline
\end{tabular}

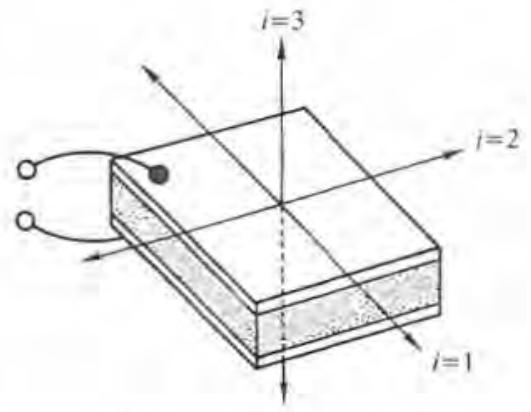

$s_{i}=\frac{\Delta x_{i}}{x_{i}}=d_{N} E$

Fig. 28.3 Anisotropy of mechanical, electrical, and electromechanical parameters in typical piezo-electric materials where the relative deformation $\left(s_{i}\right)$ of the body in the $x_{i}$ direction at a certain electrical field strength $(E)$ is related to the piezoelectric strain constant $\left(d_{3 i}\right)$ shown in the table (above).

of the piezo-electric material itself to suit particular applications. Consequently, considerable attention is required in selecting the appropriate piezoelectric for any application. Similarly, mounting piezo-electric transducers in enclosed probes requires attention to design models (e.g. Krimholtz et al. 1970). The nature of transducer backing structure and materials, together with the nature, properties, and structure of the coupling materials separating the piezo-electric material from the external medium under study, affect probe performance significantly (Tamura et al, 1975; Silk 1980; Bainton and Silk 1980; Bainton et al. 1981). 


\subsection{Discrete biesensor devices using piezo-electric transducers}

Despite the existence of a wide range of piezo-electric materials and accompanying measurement principles, virtually all reported exploitations of piezo-electric transducers in discrete sensor devices have used specially coated, oscillating quartz crystals as sensitive detectors of changes in surface mass (gravimetric sensors). This principle has been widely used in volatile and gas-phase analysis (Alder and McCallum 1983), but application to liquidphase measurements has proved problematical.

\subsubsection{Principles of electrogravimetric sensors}

Industrially grown, rather than natural, quartz crystals are used almost exclusively for electrogravimetric sensors because of their higher purity. Although transducer thickness is the principal determinant of oscillator frequency of piezo-electrics, special ' $Y$ ' cut quartz crystals oscillating in shear mode can be used to overcome harmonic and overtone interference. However, temperature coefficient and oscillator frequency also varies with the angle of rotation (Lack et al. 1934). AT $\left(+35^{\circ} 15^{\prime}\right)$ and BT $\left(-49^{\circ} 00^{\prime}\right)$ cut crystals have minimal temperature coefficients (Lack et al. 1934; Heising 1946). Other performance criteria are well studied (e.g. Heising, 1946). The majority of electrogravimetric sensors use crystals cut along the AT plane into thin $(10-15 \mathrm{~mm})$ plates or discs with the electric field being applied along the $y$ axis. These oscillate in the thickness shear mode, parallel to the major axis, and yield antimodally displacing surfaces (Heising 1946; Guilbault 1980; Alder and McCallum 1983). Crystals are placed in an oscillator circuit and the resonant frequency measured using conventional electronic techniques (Fig. 28.4). The frequency of oscillation largely depends on the combined mass of the crystal and its coatings, as described for a AT crystal microbalance (Sauerbrey 1959; Stockbridge 1966). The change in resonant frequency $(\Delta /)$ resulting from adsorption of detected analyte can be calculated (Guilbault 1980; Alder and McCallum 1983) as providing extremely high sensitivity (approx. 500 to $2500 \mathrm{~Hz} / \mu \mathrm{g}$ ) sensors with pg detection limits for commercially available quartz crystals,

$$
\Delta f=-2.3 \times 10^{6} f^{2} \frac{\Delta m}{A}
$$

where $f$ is the frequency $(\mathrm{Hz})$ of the crystal, $\Delta m(\mathrm{~g})$ the mass of adsorbed material, and $A(\mathrm{~cm})$ is the adsorbing or sensing area.

\subsubsection{Gravimetric biosensors}

King $(1964,1965)$ first reported use of piezo-electric crystals as detectors for gas chromatography. Guilbault (Hlavay and Guilbault, 1977; Guilbault 1980,1982 ) and Alder and McCallum (1983) have provided excellent reviews 


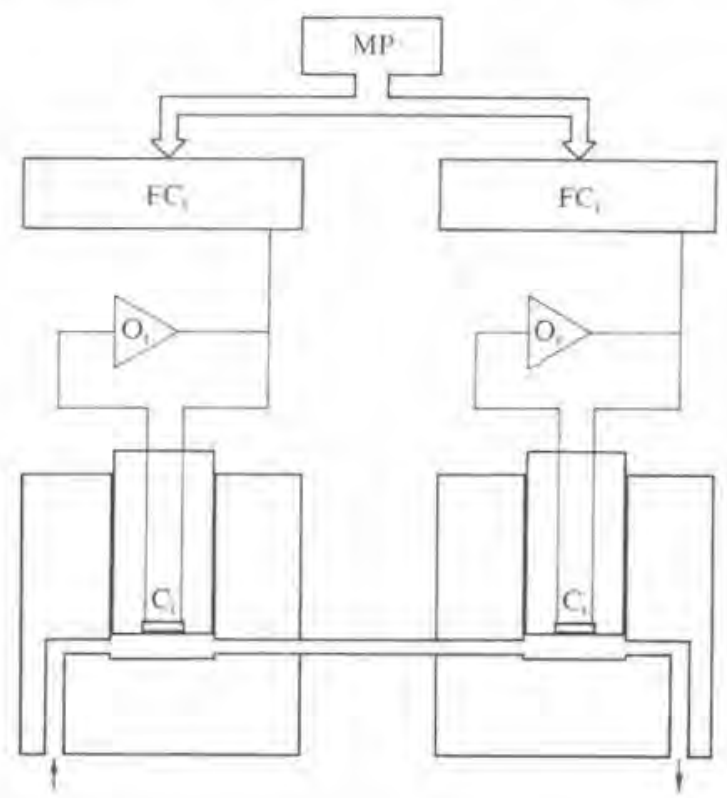

Fig. 28.4 Typical system for electrogravimetric sensor analyses incorporating reference $\left(C_{r}\right)$ and test $\left(C_{t}\right)$ erystal sensors, individually held in oscillating circuits $\left(\mathrm{O}_{t}\right.$ and $\mathrm{O}_{t}$, respectively) serviced with separate frequency counters ( $\mathrm{FC}_{\mathrm{r}}$ and $\mathrm{FC}_{\mathrm{t}}$, respectively), interfaced to a common microprocessor.

of the development and application of piezo-electric crystals in analytical chemistry by coating crystals with compounds which selectively adsorb analytes of interest. The majority of work concerns detection of gases (e.g. sulphur dioxide, carbon monoxide, hydrogen chloride) or volatile species (aromatic and aliphatic hydrocarbons) and, in so much, does not have significant biological applications. Most require relative humidity to be low and held constant. However, ammonia sensors using a variety of chemical, biochemical, and polymer coatings (Karmarker and Guilbault 1975; Karmarker et al. 1976; Hlavay and Guilbault 1978; Edmonds et al. 1982) and a dissolved carbon dioxide sensor using didodecylamine- or dioctadecylaminecoated crystals separated from the solution with a Teflon membrane (Schuman and Fogelman 1976), could well be useful in fermentation monitoring (Clarke et al. 1984, 1985). King (1970) has also reported a methane sensor, which lthough effective is perhaps less useful for monitoring bioconversion of wastes to methane than other hydrocarbon sensors (Clarke et al. 1984, 1985). Volatile organoleptic compounds are important in many biological processes (e.g. fermentation and food). It is conceivable that sufficiently selective coatings could be developed for these substances to replace 
GCMS monitoring and other spectrometric analyses. In a similar manner to ion-selective electrode analyses (Clarke et al. 1982), it has been suggested that multicomponent analysis/interference correction could be carried out to improve the practical selectivity of coated crystals (Alder and McCallum 1983).

King (1972) first suggested use of similar surface adsorption principles in biological fluids; the problems of achieving a practical device were quickly pointed out by Richardson (1972). Subsequently Downes (1979) failed to monitor the growth rate of micro-organisms through their interactions with crystals, apparently, for reasons of slow growth rates and the possibility of ultrasonic disruption of the cells. Although using a sensitive diaphragm microphone Hill (1983) analysed noise (using Fourier transform techniques) from cilia and flagellae, whose motion is rapidly sensitive to the energy state of cells.

Viscous damping of oscillation, medium temperature fluctuations, and the greater likelihood of non-specific adorptions are envisaged as the major problems of such applications. Nomura has been particularly active in applying piezo-electric crystals in fluids for a number of reasons. The change in oscillation frequency on immersing crystals in solvent solutions was expectedly found to depend principally on the density $(\rho)$ and viscosity $(\eta)$ of the solution (Nomura et al. 1981; Nomura and Okuhara 1982), according to the following empirical relationship.

$$
\Delta f=a \rho^{\frac{1}{2}}+b \eta^{\frac{1}{2}}-c
$$

where $a, b$, and $c$ are constants. Further, the resonant frequency also decreased with increasing electrolyte concentration and specific conductivity, as would be expected, largely through their proportionality with solution density. However, some curious (Alder and McCallum 1983) effects of solution concentration were noted (Nomura and Maruyama 1983), where linearity with specific conductivity was noted up to approximately $2 \mathrm{~mm}$ of metal phosphate solutions, when significant deviations from linearity due to viscosity and density were reported. At concentrations in excess of $20 \mathrm{mM}$, the solution was reported as short circuiting the quartz crystal causing dramatic changes in resonant frequency. Many compounds can be determined by their electrodeposition on electrodes. Iodide determination was achieved through its deposition on the $\mathrm{Pt}-\mathrm{Ag} / \mathrm{AgCl}$ coating of the gold electrode of a quartz crystal (Nomura and Mimatsu 1982). Many metal ions also adsorb onto surfaces from solution. Such tests were similarly arranged for determination of iron III, lead II, and aluminium III through adsorption of their phosphate salts onto a thin glass slide (to prevent electrodeposition) virtually contiguous with the crystal surface (i.e. electrode), producing relatively linear sensors in the concentration range 10 to $100 \mu \mathrm{M}$ (Nomura and Maruyama 1983). Similar principles have been exploited in attempts to 
develop an electrogravimetric immunosensor (Roederer and Baastians 1983). In this case, ST cut SAW (surface acoustic wave) crystals consisting of interdigitized (in the manner of plate condensers) nickel transducers. Areas between electrodes were etched prior to silanization and attachment of antibody (goat antihuman IgG). Significant decreases in resonant frequency were noted on immersion in buffer solutions, which were dependent on the volume presented to the devices. The responses to buffer alone to antibody-modified and non-modified (reference) crystals should be the same (Konash and Baastians 1980). The observation that they were not was apportioned to differences in non-specific binding due to the absence of protein modification on the reference sensor. However, a major problem was insufficient sensitivity and improvements in detection limits of about three orders of magnitude would be required to achieve a clinically useful device (e.g. for gravimetric immunosensors). These improvements are being sought through blocking non-specific adsorption sites, increasing the density of coverage with immobilized antibody, immobilizing inert protein on the reference crystal and using more sensitive crystals (Roederer and Baastians 1983), when significant improvements in performance appear to be possible (Baastians, unpublished). In a similar manner to those workers now considering closer and more exclusive coupling between the detecting principle (not only the detector) and sensing ligand principle (see Clarke et al. 1985 for a review), piezo-electric biosensors could be similarly improved. Further, use could well be made of the natural piezo-electric properties of biopolymers and their aggregates/structures, rather than concentrating solely on surface binding.

Damping of crystal resonance in fluid media has been overcome by the rather simple procedure of allowing adsorption to take place in solution and then air drying the sensor prior to measurement of the resonant frequency. This was demonstrated by immersing crystals in chloroform-extracted 8-quinolinate lead chelate (Nomura et al. 1982). Rather than relying on nonspecific adsorption and/or retention prior to drying of the extracted lead, the chelator (and other sensor ligands) could be immobilized onto the crystal surface in a similar manner to other types of sensors (see Clarke et al. 1985 for a review).

\subsection{Acoustic wave propagation and acoustic impedance}

There exists sophisticated analyses of the passage of acoustic waves through low viscosity and visco-elastic materials. The natural complexity and inherent dynamic behaviour of solutions, suspensions, or pellets of biological materials would be expected to present significant difficulties in achieving specific or biologically meaningful measurement from such analyses. Simultaneous multiparameter monitoring and multicomponent analyses would be expected to overcome many of these drawbacks. Despite 
the potential significance of this area, relatively few biological studies have been forthcoming, perhaps disproportionately restricting our attention to the area.

\subsubsection{Basic principles}

Most methods of acoustic and ultrasonic analysis are long established, although the significant benefits of modern signal handling instrumentation are now available. In general, acoustic waves are introduced into the signal modifying medium and either received by the same or a separate transducer. Other monitoring techniques (e.g, optical) are occasionally used.

In low viscosity liquids, shear stiffness is considered negligible in comparison to the compressional stiffness and reverberation methods can be applied at low frequencies. Quantities of interest are the complex bulk modulus $\left(K=K^{\prime}+\mathrm{j} K^{\prime \prime}\right)$ and the imaginary component of the complex shear modulus $\left(G=G^{\prime}+G^{\prime \prime}\right)$. Both shear components must be considered in visco-elastic materials, requiring further measurements (e.g. longitudinal wave amplitude measurements and shear mechanics). Although in certain cases low viscosity liquid parameters can be measured directly, in most cases, multiple measurements (introduced into some form of model) are required. The predominating loss mechanisms and thermal effects cause attenuation to vary as the square of frequency, such that losses are so small at low $\mathrm{KHz}$ range frequencies as to require use of resonance techniques and/or use of large volumes of liquid. Ceramic piezo-electrics can be used to excite single radial modes and to monitor signal decay when excitation is switched off after achievement of steady state (Mulders 1948). Since at higher frequencies, high overtone modes can be difficult to identify, the simultaneous induction of multiple modes by frequency modulation is preferred. Decay patterns can then be similarly monitored (Lawley and Reed 1955),

Acoustic interferometry is a long established technique for monitoring wave velocities and attenuation, most often configured by causing the wave to be reflected back across a variable distance, towards a single transducer (Del Grosso et al. 1954). Alternatively the reflector can be replaced with an identical piezo-electric, when the received signal will progress through maxima and minima as the distance is varied (Fry 1949). Fixed path interferometers can also be used (Carstensen 1954).

Perhaps one of the most useful techniques for determining attenuation and velocity was introduced by Pellam and Galt (1946). A piezo-electric transducer radiates a short train of waves, subsequently acting as a receiver for the reflected signal (the 'pulse echo' technique). Transit time provides velocity and loss is determined from the attenuation of the waves over various reflected distances. Wave diffraction is significant in such methods, particularly at low frequencies. Although velocities can be determined from direct 
delay measurements, phase comparison techniques are similarly useful (McSkimin 1960).

The Debeye and Sears (1932) photo-acoustic effect has allowed determination of wave velocities and attenuations. The alternate compressions and rarefactions accompanying the passage of a sound wave in fluids, effectively produce a diffraction grating for light and diffraction angles can be used to determine the sound wavelength and therefore velocity. Measurement of the intensity of the diffracted orders provides measurements of loss. Such methods are not to be confused with photo-acoustic spectroscopy, relying on the acoustic wave accompanying the generation of a thermal wave through absorption of modulated light (e.g. Cahen et al. 1980; Yip and Yeung 1983). This method has been particularly useful for infrared and visible spectroscopy of optically dense biological samples (e.g. Carpenter et al. 1983a, b).

\subsubsection{Applications}

Generally, molecular acoustics has played a relatively small role in studying homogeneous solutions of biological molecules. Measurement of ultrasonic velocities can supply information about the inter- and intramolecular interactions of macromolecules (Passynski 1938; Jacobson 1950; Stuehr and Yeager 1965). Ultrasonic absorption spectroscopy has provided a means of studying the kinetics and thermodynamics of proton transfer, since relaxation times $(<100 \mathrm{ps})$ lie within the time constants of achievable ultrasound frequencies. Such techniques require precise methods or measuring propagation velocity (Eggers and Funck 1973; Sarvazayan and Khorakov 1977) such that the compressibility and hydration of macromolecules can be analysed (Sarvazayan et al, 1979). Ionizable groups tend to be a principal influence on the absorption spectra of amino acids and corresponding $\mathrm{p} K$ values could be identified. Similar influences on the absorption spectra of protein solutions (metmyoglobin) were noted. A velocity change due to protein denaturation was also identified (see also Cho et al. 1985; Jurgens and Baumann 1985).

Acoustical analysis of inhomogeneous fluids (i.e. particles suspended in electrolyte solutions, e,g. microbial cultures) is particularly difficult. Measurement of sludge concentration of wastewaters has been achieved ultrasonically (Hayakawa and Kori 1972). Growth of yeast (and other) cultures has been monitored ultrasonically using a flexible piezo-electric membrane transducer consisting of a polyacetal resin, chlorinated polyethylene, and lead zirconate titanate (Ishimori et al. 1981). The measurement cell consisted of two piezo-electric membranes (each $2.5 \times 1.5 \mathrm{~cm}$ and $0.2 \mathrm{~mm}$ thick) separated by $2.5 \mathrm{~mm}$ of culture fluid. The oscillation frequency of the transmitting membrane was fixed at $40 \mathrm{KHz}$ such that approximately $20-100 \mathrm{mV}$ peak to peak amplitudes were generated at the receiving membrane. Although output voltage should increase with increasing medium concentration (Stuehr and Yeager 1965), little increase in amplitude (approx. $5 \mathrm{mV}$ ) 
was observed over the concentration range $10 \mathrm{~mm}$ to $500 \mathrm{~mm}$. Similarly, increase in sound velocity with temperature (Stuehr and Yeager 1965) was slight in this system over the range 25 to $40^{\circ} \mathrm{C}$. Since culture medium density often changes during growth of cultures, the response of the sensor to various glycerol concentrations (from densities of 1 to 1.10 ) was monitored. Changes in amplitude again were small. Conversely, introduction of populations of bacteria and yeast provoked significantly greater responses (amplitudes varying from $20 \mathrm{mV}$ to $50-80 \mathrm{mV}$ (approx.) for variations in numbers of 10 to 10 per $\mathrm{ml}$ ). Output was relatively linear with cell number up to 10 cells $/ \mathrm{ml}$ and appeared to provide better growth curve data than culture conductivity measurements (e.g. Cady 1978). Although the transducer could withstand a small number of steam sterilization cycles, eventual cracking of the piezomembrane proved to be a problem. The precise underlying principles of the method are unclear, except that suspension compressibility appeared to play a greater role in the measurement than sound velocity and density (Ishimori et al. 1981).

Significant advances in understanding the propagation of ultrasonic waves in inhomogeneous suspensions (of non-biological particles) has been achieved, particularly through the work of Chivers and Anson (Chivers 1980; Anson and Chivers, 1981 a, b; Chivers and Anson 1982). Table 28.1 outlines the main parameters of interest for modelling the acoustic interactions of the medium and suspended particle components of inhomogeneous systems. Although these would be expected to result in complex formulations, particularly when the dynamics of microbial cultures and attendant gas bubbles are considered, a wide range of measurements could be applied (Table 28.1) to multicomponent analysis methods. This would require extension of the

Table 28.1 Some analytically useful measurements for multicomponent analysis of the various influences on acoustic wave propagation in inhomogeneous suspensions of particles in electrolyte media

Some analytically useful parameters

Homogeneous medium

Suspended particles

Attenuation

Specific absorption

(resonance)

Propagation delay

Density

Density

Longitudinal wave absorption

Viscosity

Longitudinal wave absorption

Surface shear attenuation

Wave shape (Fourier

Bulk modulus

Elasticity analysis)

Scatter and coherency

Scatter direction

Thermal expansion Specific heat

Thermal expansion Specific heat 
above models for more biological suspensions. Despite their complexity, biologically meaningful measurements could result.

Ultrasound fields have also been used to polarize and aggregate cell populations. One important exploitation of these effects has been in electroacoustic cell fusion procedures (Vienken et al. 1985; see also Coakley 1985; Coakley et al. 1986). When cell suspensions are exposed to ultrasound fields, two forces can operate to bring cells close together. In a standing wave situation, the first force operates to move cells close to the velocity maxima in the sound field. The other operates to bring cells into contact perpendicular to the direction of the velocity amplitude in the absence of the cells. Their combined effects will move cells to preferred areas of the sound field, where the aggregation forces will be at a maximum (e.g. Vienken et al. 1985).

\subsection{Acoustic microscopy}

Although acoustic microscopy cannot be considered to be a biosensing technique, recent developments are worthwhile considering because of the capabilities likely to be offered to the biologist. Not only are resolutions comparable to the resolution of optical microscopes, but the penetration of acoustic waves through solid material is usefully exploited in resolving structural information at various depths (for reviews see Attal 1983; Wickramansinghe 1984; Wiczkowski 1984). Further, the extensive developments undertaken over the last decade have significantly improved broader understanding in a number of relevant areas.

Lemons and Quate (1974; see also Quate et al. 1979) first demonstrated scanning acoustic microscopy (SAM), as first suggested by Sokolov's (1949) realization that $\mathrm{GHz}$ acoustic waves in water had similar wavelengths to visible light.

Figure 28.5 jointly outlines the respective configurations of transmission and reflective acoustic microscopy. In the former configuration, identical sapphire lens-piezo-electric transducer (lithium niobate below $150 \mathrm{MHz}$ and zinc oxide at higher frequencies) configurations are used, whereas the reflective configuration employs a single lens-transducer configuration alternately switched between excitation and receiver modes. Exciting radio-frequency (RF) signals are applied to the transmitting lens which excites longitudinal waves in the sapphire rod. These are focused to a diffraction-limited beam waist by the subsequent lens. In the transmission configuration, the receiver lens is confocal with the transmitter, collecting and collimating the acoustic signal prior to its reconversion into an electrical signal. Resolution in such a configuration is principally defined by the beam waist. The spot diameter can approach $0.7 \lambda$, using a high quality wide aperture lens $(f / 0.7)$. It is conceivable that the theoretical resolving power of acoustic microscopy could be increased further by data analysis techniques. Walker (1983) has 


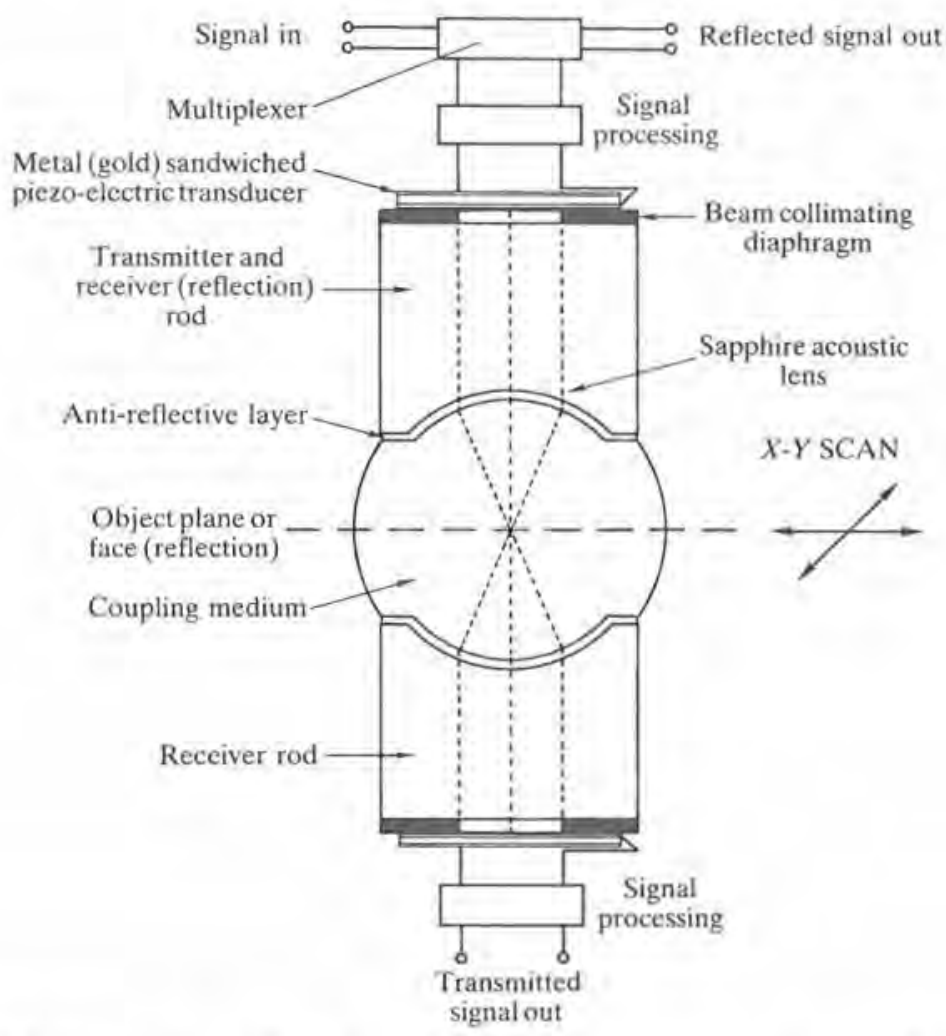

Fig. 28.5 Schematic configuration of a scanning acoustic microscope illustrating transmission and reflection modes of operation (see text for further details).

demonstrated experimentally that imaging resolutions greater than the Rayleigh criterion should be achievable using an iterative spectral extrapolation algorithm based on the Gerchberg method.

Many of the imaging modes of optical microscopy (viz. stereo imaging, dark field contrast, phase contrast, and differential phase contrast) have counterparts in acoustic microscopy (Wickramansinghe 1984). The piezoelectric transducers generate coherent waves when excited with a RF signal, which are converted back to a coherent RF signal in the receive mode. Consequently, at each pixel point of the scan, it is possible to measure phase as well as amplitude. Stereo imaging has been demonstrated by taking two images at different scan plane angles and dark field by replacing the transmitter with a plane wave transducer and introducing a zero order stop at the aperture centre of the receiver lens. Photo-acoustic spectroscopy principles have also been incorporated into acoustic microscopes. Quantitative acoustic 
measurements have also been made at each pixel point.

Biological materials have high acoustic contrast without resort to staining techniques. Living red blood cells and chick fibroblasts have been studied using scanning acoustic microscopy (see Wickramansinghe 1984). Clearly many more interesting applications await this highly developed tool.

\subsection{Acoustic resonance densitometry (ARD)}

The idea of mass determination by the mass damping of a vibrating resonant body can be attributed to Rayleigh $(1870,1875)$. Although vibrating viscometer devices were reported in the 1930s (e.g. Philipoff 1934), use of mass damping in mechanically resonating bodies as sensitive measures of mass change are due mainly to Kratky and Leopold (1968).

The tubular (see Fig. 28.6) or spherical sample chamber of ARD instruments encloses a fixed test volume. It should be elastically self-supporting and possess a low temperature coefficient (e.g. by using a silica, stainless steel, or nickel alloy construction) to minimize thermal expansion, stress, and loading. Resonance is initially excited and sustained by applying to the sample chamber a pair of solenoids connected within a closed oscillatory circuit using a fixed-gain AC amplifier. The amplifier ensures resonant displacement of the sample chamber within its linear range, preventing nonharmonic oscillations (radial and longitudinal) and ensuring the almost exclusive transverse displacements required for tubular sample chamber configurations (Fig. 28.6). When such conditions are maintained there exists

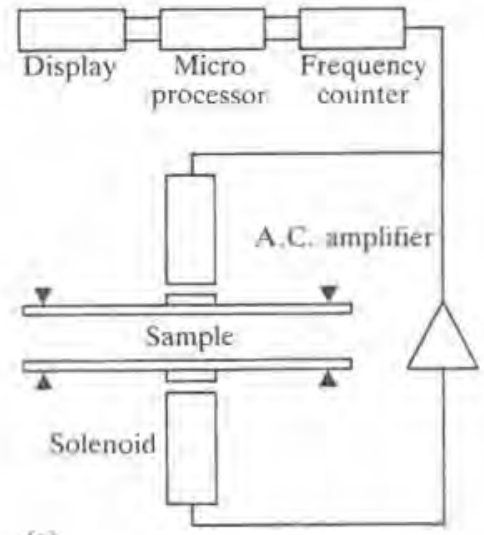

(a)

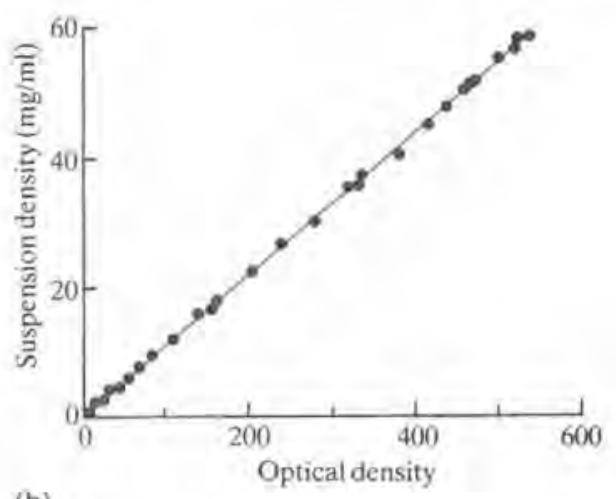

(b)

Fig. 28.6 Monitoring of microbial biomass by acoustic resonance densitometry (ARD). (a) Configuration of ARD apparatus, (b) Correlation of ARD measurement of microbial suspension density with conventional optical density measurement (see text for details). 
a direct relation between the density of the sample and the square of the oscillation frequency, which excludes parameters such as sample viscocity. A simple approach has been to hold the sample chamber at constant temperature and convert the frequency (period, $\tau$ ) to density $(\rho)$ using two calibration constants $(A$ and $B)$,

$$
\rho=\frac{1}{A}\left(\tau^{2}-B\right) \text {. }
$$

Alternatively, measured temperature corrections can be incorporated into such formulations.

It is surprising that the precise (4-6 digit) density measurements afforded by such instruments have not found biological exploitation to any significant extent. However, it has been recently demonstrated (Blake-Coleman and Clarke 1984a, b; Blake-Coleman et al. 1984, 1986), that ARD can operate as a linear monitor of microbial biomass over an extremely wide range of culture density (Fig. 28.6). Application in harvested-culture processing as well as monitoring culture growth should thereby be afforded (BlakeColeman et al. 1986). The transducer is robust and does not suffer adverse effects on steam sterilization. The natural resonance of the sample chamber tends to discourage microbial fouling and the incorporation of sonic (or steam flushing) cleaning cycles can be arranged. Since most micro-organisms have a higher density than their suspending medium, increase in their numbers provides a linear relation with density. Cultures treated by a variety of cell breakage procedures maintain the same density (Blake-Coleman et al. 1986), illustrating that true bulk mass per unit volume changes are involved, rather than some more subtle mass rearrangement within the cell structure. The medium density per se also changes throughout the course of growth of some cultures. Consequently, in such cases, medium density changes must be simultaneously monitored and these values subtracted from the measured composite culture density changes (Blake-Coleman et al. 1984, 1986). Although this can be achieved aseptically using dual-sample-chamber configurations, one of which is supplied by a tangential-flow filtration assembly, instrumental methods of achieving precise correction for dynamic changes in medium density are currently being developed (Blake-Coleman and Clarke $1984 a, b$; Clarke and Blake-Coleman 1985).

\subsection{Conclusions and future potential}

Although acoustical techniques have not found application comparable to other biosensing techniques, a number of principles and techniques are now developing which can tackle the complexities of biological measurements. However, our understanding of the acoustical properties of biological materials are only partially developed, and further developments will require 
sophisticated measurement, modelling and data analysis techniques. Considerable attention has been devoted to the development of piezo-electric sensors with chemical sensitivity. Similar attention to biochemical specificities would be expected to provide a number of piezo-electric biosensors. However, in common with other biosensing principles, close attention needs to be devoted to achieving more direct, close, and exclusive coupling between the biosensing and detector materials.

\section{References}

Alder, J. F. and McCallum, J.F. (1983). Piezo-electric crsytals for mass and chemical measurements. The Analyst 108 (1291), 1169-89.

Anson, L. W. and Chivers, R. C. (1981a). Frequency dependence of the acoustic radiation force function ( $\mathrm{Yp}$ ) for spherical targets of a wide range of materials. J. Acoust. Soc. Am. 69, 1618-22.

- $(1981 b)$. The use of absorbing polymeric materials for suspended sphere ultrasonic radiometry, Acoust. Letts. 4(4), 74-80.

Attal, J. (1983). La microscopie acoustique. La Recherche 14 (Mai), 664-667.

Bainton, K.F. and Silk, M. G. (1980). Some factors which affect the performance of ultrasonic transducers, Brit. $J$, of N.D. T, 22, 15-21.

- Hillier, M. J. and Silk, M. G. (1981). An easily constructed broad bandwidth ultrasonic probe for research purposes. J. Phys. E (Sci. Instrum.) 14, 1313-9.

Blake-Coleman, B, C. and Clarke, D, J. (1984a). Coil geometry and ARD apparatus. Brit. Pat. Appl. No. $84085 / 27$.

- (1984b). Determination of biomass using ARD. Brit. Pat. Appl, No. 84085/28. - Calder, M. R., Carr, R. J. G., Moody, S. C. and Clarke, D. J. (1984). Direct monitoring of reactor biomass in fermentation control. Trends in Anal. Chem. 3(9), 229-32.

- Clarke, D. J., Calder, M. R. and Moody, S. C. (1986). Determination of reactor biomass by acoustic resonance densitometry. Biotechnol. \& Bioeng. 28(8), 1241-7.

Cady, P. (1978). Progress in impedance measurements in microbiology. In Mechanizing microbiology (eds A. N. Sharpe, D.S. Clark and A. Balows), pp. 199, John Wiley, New York.

Cady, W. G. (1946). Piezo-electricity. McGraw Hill, New York and London.

Cahen, D. G., Garty, M. and Becker, S. (1980). Photoacoustic calorimetry of concentrated fluorescent solutions. J. Phys. Chem. 84, 3384-9.

Carpenter, R., Larue, B. and Leblane, R. M. (1983a). Photoacoustic spectroscopy of Anacystis nidulans I. Arch. Biochem. \& Biophys. 222(2), 403-10.

- (1983b). Photoacoustic spectroscopy of Anacystic nidulans II. Arch. Biochem. Biophys. 222(2), 411-5.

Carstensen, E. L. (1954). Measurement of dispersion velocity of sound in liquids. J. Acoust. Soc. Am. 26, 858-9I.

Chivers, R. C. (1980). Acoustic wave fluctuations in inhomogeneous media. J. Phys. D 13, 1947-51.

- and Anson, L.W. (1982). Choice of target and accuracy of measurement in suspended sphere ultrasonic radiometry. J. Acoust. Soc. Am. 72, 1670-95. 
Cho, K., Leung, W. P., Mok, H. Y. and Choy, C. L. (1985). Ultrasonic absorption in myoglobin and other globular proteins. Biochem, Biophys. Acta 830, 36-44.

Clarke, D. 1. and Blake-Coleman, B. C. (1985). Improvements in filters. Brit. Pat. Appl. No. 8514899 .

- Kell, D. B., Burns, A. and Morris, J. G. (1982). The role of ion-selective electrodes in fermentation control. I. S. E. Reviews 4, 75-133.

- Blake-Coleman, B. C., Calder, M. R., Carr, R. J. G. and Moody, S. C. (1984). Sensors for bioreactor monitoring and control - a perspective. J. Biotechnol. 1, 135-58.

- Calder, M.R., Carr, R.J.G., Blake-Coleman, B.C., Moody, S.C. and Collinge, T.A. (1985). The development and application of biosensor devices for bioreactor monitoring and control. Biosensors 1(3), 213-320.

Coakley, W. T. (1985). Interfacial instability and cell membranes. APSM Bulletin 5 . 16-18.

- Hewison, L. A. and Tilley, D. (1986). Interfacial instability and the agglutination of erythrocytes by polymers. Studia Biophysica. In press.

Curie, J, and Curie, P. (1880). Development, par pression, de l'electricite polarise dans les crystaux hemiedries et faces inclines. Comp. Rend. 91, 294-7.

Debeye, P. and Sears, F. W. (1932), Scattering of light by sound. Proc, Natl. Acad. Sci. (U.S. A.) 18, 410-15.

Del Grosso, V. A., Smura, J. A. and Fougere, P. F. (1954). Ultrasonic investigations in liquids. Naval Res, Lab. Reports NRL-4439.

Downes, J. (1979). Monitoring of microbial growth using piezoelectric crystals. Internal Report, Imperial College, London, UK.

Edmonds, T. E., Fraser, S. M. and West, T. S. (1982). Polyvinylpyrrolidone coated piezoelectric quartz crystals as an ammonia sensor. Poster at Roy. Soc. of Chem. Int. Conf. on 'Detection and Measurement of Hazardous Substances in the Atmosphere', City Univ., London, 20-22 December 1982.

Eggers, F. and Funck, Th. (1973), Ultrasonic measurements with millilitre liquid samples in the 0.5 to $100 \mathrm{MHz}$ range. Rev. Sci, Instrum. 44(8), 969-73.

Fry, W. J. (1949). The double crystal acoustic interferometer. J. Acoust. Soc. Am. 21, 17-21.

Fukada, E. (1968). Piezoelectricity in polymers and biological materials, Ultrasonics October 1968, 229-330.

- (1974). Piezoelectric properties of biological macromolecules. Adv. in Biophys. 6, 121-55.

Guilbault, G. G. (1980). Use of piezoelectric crystal detector in analytical chemistry. I.S.E. Reviews 2(1), 4-15.

(1982), Piezoelectric crystal detectors in analytical chemistry. Anal. Proc. 19, 68-79.

Hayakawa, N. and Kori, A. (1972), Ultrasonic probe for monitoring the concentration of wastewater sludge. Mizu Shori Gijutsu 13, 57-63.

Heising, R. A. (1946). Quartz crystals for electrical circuits. p. 24. Van Nostrad, New York.

Hill, R.J. (1983). The frequency dependent emission of low frequency sound by motile cultures of the ciliate Tetrahymena thermophilia. Biochem. Biophys, Res. Commun. 117(1), 190-5. 
Hlavay, J. and Guilbault, G. G. (1977). Application of the piezoelectric crystal detector in analytical chemistry, Anal. Chem. 49, 890-2.

- (1978). Applications of piezoelectric crystal detectors in analytical chemistry. Anal. Chem. 50, 1044-8.

Ishimori, Y., Karube, I. and Suzuki, S. (1981). Determination of microbial populations with piezoelectric membranes. Applied \& Environ. Microbiol, 42(4), 632-7.

Jacobson, B. (1950). On the adiabatic compressibility of aqueous solutions. Ark. Kemi. 2, 177-81.

Jurgens, K. D, and Baumann, R. (1985). Ultrasonic absorption studies of proteinbuffer interactions. Eur. J. Biophys. 12, 217-22.

Karmarker, K. H. and Guilbault, G. G. (1975). The determination of ammonia and nitrogen dioxide at the parts per billion level with coated piezoelectric crystal detectors. Anal. Chim. Acta 75, 111-15.

- Webber, L. M. and Guilbault, G. G. (1976). Measurement of sulfur dioxide in automobile exhausts and industrial stack gases with a coated piezoelectric crystal detector. Anal. Chim. Acta 81(2), 265-71.

Kawai, H. (1969). The piezoelectricity of poly(vinylidene fluoride). Jap. J. Appl. Phys. 8, 975-9.

King W. H. Jr. (1964). Piezoelectric sorption detector. Anal Chem. 36, 1735-9.

- (1965). Piezoelectric sorption detector. U.S. Patent 3, 164, 004 (Jan 5), 1965.

(1970). Monitoring of hydrogen, methane and hydrocarbons in the atmosphere. Environ. Sci. \& Technol. 4(12), 1136-40.

- (1972). Chemical analysis using piezoelectric crystal detectors. Bull. N. Y. Acad. Sci. 48, 459-69.

Kocharayan, N. M., Pachadzhyan, B. and Tivriktsyan, Zh. (1967). 'Induced' piezoelectric effect in some polymers. Dokl. Akad. Nauk. Ann. S. R. R. 44(3), 111-6.

Konash, P. L. and Baastians, G. J. (1980). Piezoelectric crystal detectors in liquid chromatography. Anal. Chem. 52, 1929-32.

Kratky, O. and Leopold, H. (1968). Acoustic resonance densitometry, Austrian Patent No. 2704.

Krimholtz, R., Loedom, D. and Matthaei, G. (1970). New equivalent circuits of elementary piezoelectric transducers. Electron Letts, 6, 398-402.

Lack, F. R., Willard, G. W. and Farni, Z. E. (1934). Angle of rotation of quartz crystals. Bell Syst. Tech. J. 13, 453-6.

Lawley, L. E. and Reed, R. D. C. (1955). A reverberation method for the measurement of the adsorption of ultrasonics in liquids. Acoustica 5, 316-19.

Lemons, R. A. and Quate, C. F. (1974). Integrated circuits as viewed with an acoustic microscope. Appl. Phys. Letts. 24, 163-6.

McSkimin, H. J. (1960). Performance of high frequency barium titanate transducers for generating ultrasonic waves in liquids. J. Acoust. Soc. Am. 32, 1401-5.

Mulders, L.E. (1948). Ultrasonic reverbation measurements in liquids. Appl. Scí. Res, B1, 149-52.

Nomura, T. and Maruyama, M. (1983). Effect of metal ions on a piezoelectric quartz crystal in aqueous solution and the adsorptive determination of iron III as its phosphate. Anal. Chim. Acta 147, 365-9.

- and Mimatsu, T. (1982). Electrolytic determination of traces of iodide in solution with a piezoelectric quartz crystal. Anal. Chim. Acta 143, 237-41. 
and Okuhara, M (1982). Frequency shifts of piezoelectric quartz crystals immersed in organic liquids. Anal. Chim. Acta 142, 281-5.

- Yamashita, T. and West, T.S. (1982). Determination of lead by adsorption of the extracted 8-quinolinolate on the electrodes of a piezoelectric quartz crystal. Anal. Chim. Acta 143, 247.

- Okuhara, M., Murata, K. and Hattori, O. (1981). Behaviour of a piezoelectric quartz crystal in organic solvents. Bunskei Kagaku 30(6), 417-21.

Passynski, A. (1938). Compressibility of electrolytes. Acta Physiochem U.R.S.S. 8 , 385-9.

Pellam, J. R. and Galt, J. K. (1946). Ultrasonic propagation in liquids. I Application of pulse techniques to velocity and absorption measurements at 15 megacycles. J. Chem. Phys. 14, 608-12.

Philipoff, W. (1934). Dynamische untersuchungen an kolloiden systemen. Physik Zeitschr. 35, 884-905.

Quate, C.F., Atlar, A. and Wickramansinghe, H. K. (1979). Acoustic microscopy with mechanical scanning. Proc. I, E, E. 67, 1092-6.

Rayleigh, Baron (alias Strutt, J. W.) (1870). On the theory of resonance. Philos. Trans, 161, 77-9.

- (1875). Vibrations of a liquid in a cylindrical vessel. Nature 12, 251.

Richardson, P. D. (1972). The operation of piezoelectric crystal detectors in fluids. Bull. N. Y. Acad. Med. 48, 465-9.

Roederer, J.E. and Baastians, G. J. (1983). Microgravimetric immunoassay with piezoelectric crystals. Anal. Chem. 55, 2333-6.

Sarvazayan, A.P. \& Khorakov, D.P. (1977). Molecular and cellular biophysics, pp. 93. Nakau, Moscow.

- and Hemmes, P. (1979). Ultrasonic investigation of the $\mathrm{pH}$ dependence solutesolvent interactions in aqueous solutions of amino acids and proteins. J. Phys. Chem. 83(13), 1796-9.

Sauerbrey, G. Z. (1959). Use of a quartz vibrator for weighing thin layers on a microbalance. Z. Physik. 155, 206-10.

Schuman, M.S. and Fogelman, W. W. (1976). Nature of analysis for inorganics in water, J, Water Pollut. Control Fed. 49(6), 90I-5.

Silk, M. G. (1980). The effect of constructional variations on ultrasonic probe performance. U. K. Atomic Energy Res. Est. Reps. AERE-R9, 761-5.

Sokolov, S. (1949). An acoustic microscope. Dokl. Akad. Nauk. 64, 333-6.

Stockbridge, C. D. (1966). Hydrostatic pressure on quartz crystal resonators. Vac. Microbalance Tech. 5, 193-7.

Stuehr, J, and Yeager, E. (1965). Physical acoustics (ed. W. P. Mason), Vol. 2, Part A, 211-49.

Tamura, M., Yamaguchi, T., Oyaba, T. and Yoshimi, T. (1975). Electroacoustic transducers with piezoelectric high polymer films. J. Audio. Eng. Soc. 23, 21-5,

Van Randeraat, J. and Setterington, R. E. (1974). Piezoelectric ceramics. Mullard Ltd.

Vienken, J., Zimmermann, U., Zenner, H.P., Coakley, W.T. and Gould, R. K. (1985). Electro-acoustic fusion of erythrocytes and of myeloma cells. Biochem. Biophys. Acta 820, 259-64.

Walker, J.G. (1983). Optical imaging with resolution exceeding the Rayleigh 
criterion. Optica Acta 30(4), 1197-202.

Wickramansinghe, H.K. (1984). Acoustic microscopy: present and future. I.E.E. Proc. 131, Part A, No. 4, 282-91.

Wiczkowski, J. (1984). Son et lumiere. Lab Practice October 1984, 15-20.

Yip, B. C. and Yeung, E. S. (1983). Photoacoustic spectroscopy in gases based on wavelength modulation, Anal. Chem, 55, 978-87. 

Calorimetry 



\title{
Theory and application of calorimetric sensors
}

\author{
BENGT DANIELSSON and KLAUS MOSBACH
}

\subsection{Introduction}

In recent years experience gained in the field of enzyme immobilization has led to the development of bio-analytical devices in which the 'sensing' enzymes are placed in close proximity to the actual measuring part, the transducer. The best known example of such a combination of enzyme and transducer is the enzyme electrode. Various other combinations have been described, including the enzyme thermistor and other thermal bio-analysers. These devices are based on a general detection principle, the measurement of the heat of reaction. Enzymic reactions, in particular, are accompanied by a considerable heat evolution, generally in the range of 25 to $100 \mathrm{~kJ} / \mathrm{mol}$ (Table 29.1), which makes enzyme calorimetry a highly versatile technique. The lack of specificity due to the general detection principle is adequately compensated for by the use of specific, immobilized biocatalysts, such as enzymes. Other common advantages associated with the use of an immobilized biocatalyst proximal to the transducer include repeated use of the biocatalyst, higher sensitivity, quicker response time, possibility of continuous flow operation, and probable stabilization of the biocatalyst.

Although many applications of calorimetry in biochemical analysis have been reported (Johansson et al. 1976; Martin and Marini 1977; Grime 1980), calorimetry has not gained widespread use in routine bio-analysis and might be attributed to the high cost and complexity of available instruments and tedious, time-consuming operation. Several research groups have attempted to develop simple and less expensive calorimeters for routine use with immobilized enzymes. A 'small volume calorimeter', in which the enzyme was attached to a thin aluminium foil placed on the surface of a Peltier element as a temperature sensor (Pennington 1976), was one of the first instruments developed. A drop of the sample was applied on the enzyme layer with the amount of substrate detected as a very small temperature change. The sensitivity, however, was poor and continuous-flow operation was not possible.

A most straightforward approach was used in the thermal enzyme probes (TEP) (Cooney et al. 1974; Mosbach et al. 1974; Weaver et al. 1976) in which the enzyme was directly attached to the temperature transducer, a thermistor, 
Table 29.1 Molar enthalpies of enzyme-catalysed reactions

\begin{tabular}{llll}
\hline Enzyme & Substrate & $-\Delta H(\mathrm{~kJ} / \mathrm{mol})$ & References \\
\hline Catalase & Hydrogen peroxide & 100 & Rehak and Young 1978 \\
Cholesterol oxidase & Cholesterol & 53 & Rehak and Young 1978 \\
Glucose oxidase & Glucose & 80 & Schmidt et al. 1976 \\
Hexokinase & Glucose & $28(75)^{*}$ & McGlothlin and Jordan 1975 \\
Lactate dehydrogenase & Na-pyruvate & 62 & Brown 1969 \\
NADH-dehydrogenase & NADH & 225 & Poe et al. 1967 \\
Penicillinase & Penicillin G & $67(115)^{*}$ & Grime and Tan 1979 \\
Trypsin & Benzoyl-L-arginineamide & 29 & Brown 1969 \\
Urease & Urea (phosphate buffer, pH 7.5) & 61 & Grime 1985 \\
Uricase & Urate & 49 & Rehak and Young 1978 \\
\hline
\end{tabular}

* The $\Delta H$ values in parenthesis include protonation of Tris $(-47.5 \mathrm{~kJ} / \mathrm{mol}$ (Rehak and Young 1978)). 
by either cross-linking or entrapping the enzyme in a dialysis bag enclosing the thermistor. Unfortunately, the major part of the heat evolved in the enzymic reaction was lost to the surrounding solution without being detected by the thermistor. Consequently, the sensitivity was low and even if this advantage to some extent was alleviated in later designs (Tran-Minh and Vallin 1978; Rich et al. 1979), the TEP concept was primarily intended for batch operation.

A considerably more efficient detection of the reaction heat was possible in systems employing small columns with enzyme bound to support particles, as in the 'enzyme thermistor' (Mosbach and Danielsson 1974; Mosbach et al. 1975 ) and in the 'immobilized enzyme flow-enthalpimetric analyser' (Bowers et al. 1976). The combination of a flow enthalpimeter of commercial design with a thermostatted, immobilized enzyme column has also been described (Kiba et al. 1984). In these cases the heat was transported by the liquid passed through the column to or along the temperature sensor that was mounted at the top of the column or at its outlet.

The possibility for continuous analysis is an additional advantage when using flow-through arrangements. Most of these closely related devices have rather similar performances. As established from reactions of known enthalpy (Table 29.1) or electrical calibration (Danielsson et al. 1979), as much as $80 \%$ of heat evolved in such 'semi-adiabatic' instruments can be registered as a temperature change. For a given substrate present at a concentration of $1 \mathrm{mmol} / \mathrm{l}$ and with a molar enthalpy change of $80 \mathrm{~kJ} / \mathrm{mol}$, a peak height corresponding to $10^{-2}{ }^{\circ} \mathrm{C}$ or higher will be expected, and a temperature resolution of $10^{-4}{ }^{\circ} \mathrm{C}$ is required in order to give $1 \%$ accuracy in the measurement. As previously mentioned, most enzymic reactions are accompanied by considerable heat production in the range of 25 to $100 \mathrm{~kJ} / \mathrm{mol}$; therefore, measurements of concentrations as low as $0.1 \mathrm{mmol} / 1$ should not present any problems.

\subsection{Experimental}

\subsection{Apparatus}

The enzyme thermistor developed in the authors' laboratory has previously been described (Danielsson et al. 1981a). A recent design of the apparatus is schematically shown in Fig. 29.1. Figure 29.2 shows all the instruments needed for performing enzyme thermistor analysis. Inside a temperaturecontrolled $\left(25,30\right.$, or $\left.37^{\circ} \mathrm{C}\right)$ aluminium cylinder placed in a polyurethane foam insulated casing is another aluminium cylinder separated by a thin airspace that provides a certain degree of thermal insulation. Heat is transported between the two blocks mainly by convection and by the fluid pumped from the main heat exchanger in the outer cylinder to the short, secondary heat exchanger in the inner cylinder. The columns will therefore be 


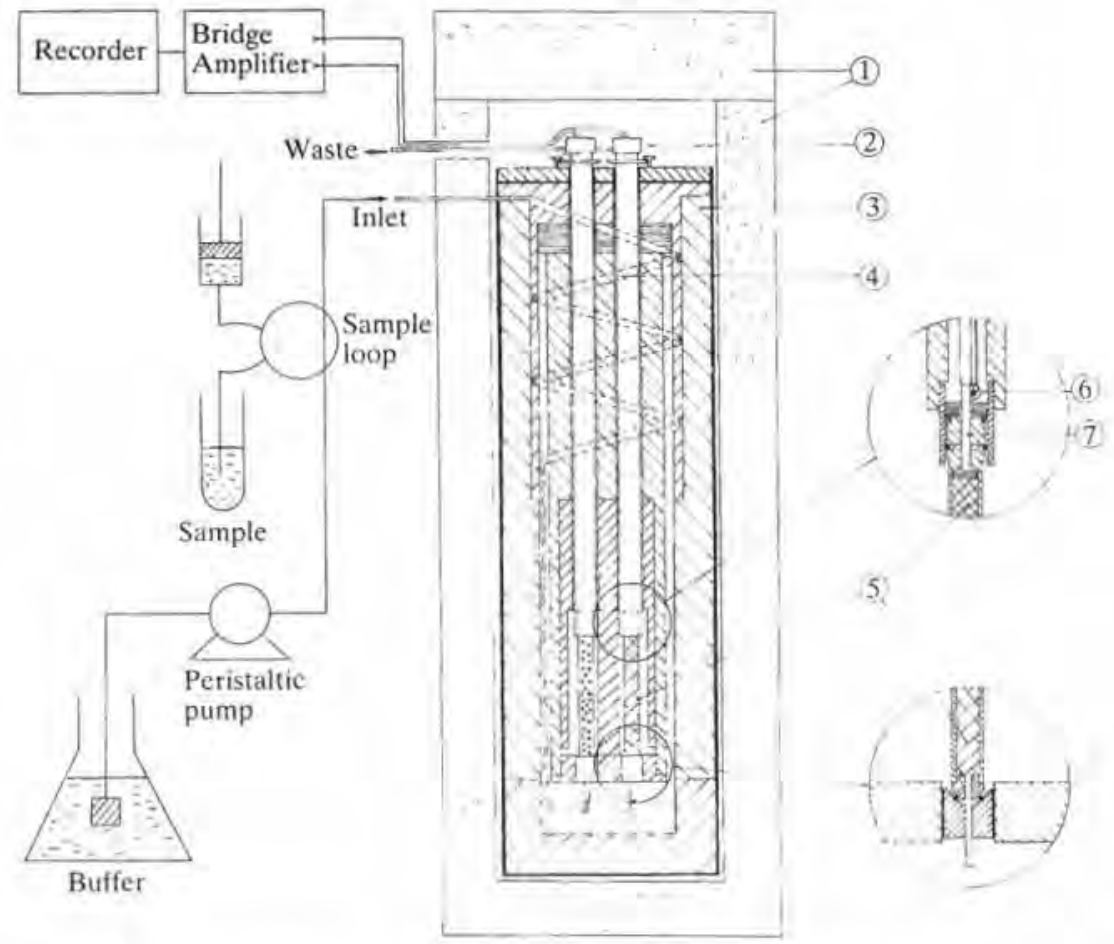

Fig. 29.1 Schematic cross-section of an enzyme thermistor with aluminium constanttemperature jacket in a typical set-up: (1) Polyurethane insulation, (2) plexiglas tube/thermistor probe for column insertion, (3) thermostatted aluminium cylinder, (4) heat exchanger, (5) enzyme column, (6) thermistor attached to a gold capillary, (7) column outlet. There are two identical column ports that can be used independently or one of the ports can be used as a reference channel (split-flow).

surrounded by an environment with a very stable temperature.

The major part of the heat produced in the enzyme column is transported out of the column by the flow stream. The temperature at the outlet of the column is measured with a small thermistor mounted on a short gold capillary tube. A dual bead isotherm thermistor (type A395, Victory Engineering Corp., Springfield, N. J., USA) with a resistance of $16 \mathrm{k} \Omega$ at $25^{\circ} \mathrm{C}$ and a temperature coefficient of $-3.9 \% /{ }^{\circ} \mathrm{C}$ is commonly used. In addition, a reference thermistor mounted in the inner block for differential measurements is also utilized which results in increased base-line stability.

Temperature registration is made by a DC-coupled Wheatstone bridge equipped with a chopper-stabilized amplifier and wire-wound precision resistors with a low temperature coefficient. At maximum sensitivity this bridge produces a $100 \mathrm{mV}$ change in the recorder signal for a temperature 


\title{
32 \\ Design of fibre-optic biosensors based on bioreceptors
}

\author{
JEROME S. SCHULTZ
}

\subsection{Introduction}

The use of optical-fibre wave guides for miniaturization of spectrophotometric methods to monitor samples of the order of 0.1 lambda in volume has taken on special significance in recent years due to the ready availability of a variety of optical fibres and opto-electronic devices for light sources and detectors (Chabay 1982). Optical fibres have been used to fabricate microcalorimeters and micro-fluorimeters (Vureck and Browman 1969). However, it is only recently that optical fibres and detector elements have been coupled to biochemical reactions for the purposes of developing miniaturized biosensors.

There have been adequate demonstrations of fibre-optic based analytical devices for physical properties such as temperature and simple analytes such as $\mathrm{pH}, \mathrm{CO}_{2}, \mathrm{O}_{2}$ (Lubbers and Opitz 1983; Peterson et al. 1980; Saari and Seitz 1982). Some recent reviews of developments in optical biosensors have been published by Seitz (1984; Chapter 30) and Peterson and Vurek (1984).

Schultz and Sims (1979) introduced an approach to measure biochemicals based on competitive binding between specific receptors and fluorescently labelled analogue-analytes. The system was miniaturized by using a single hollow dialysis fibre as a microscopic 'test tube', and by inserting a single optical fibre into this reaction chamber spectrophotometric changes can be monitored. An important characteristic of this system is that it is reagentless, i.e. since the reactions are reversible and the reagents are retained within the dialysis chamber by utilizing macromolecular derivatives, the systems is selfcontained and does not need to be regenerated.

\subsection{Optical fibres}

The basic construction of an optical fibre is shown in Fig. 32.1. It consists of a core material which has a higher refractive index than the cladding material (Lacey 1982). The result of this construction is that light that enters the fibre is totally internally reflected at the interface between the two transparent materials and is guided along the length of the fibre. Modern materials for 
Tsuji, F. I. (1978). Cypridina luciferin and luciferase. In Methods in Enzymology, Vol. 57 (ed. M. DeLuca), pp. 364-72. Academic Press, New York.

Ward, W. W. and Cormier, M. J. (1979). An Energy transfer protein in coelenterate bioluminescence. J. Biol. Chem. 254, 781-88.

Weeks, I., Campbell, A. K. and Woodhead, J. S. (1983). Two-site immunochemiluminometric assay for human alpha-fetoprotein Chin. Chem. 29, 1480-3.

Beheshti, I., McCapra, F., Campbell, A. K. and Woodhead, J.S. (1983). Acridinium esters as high specific activity labels in immunoassay. Chin. Chem. 29, 1474-9.

—, Sturgess, M. Siddle, K. Jones M. K. and Woodhead, J. S. (1984). A High sensitivity immunochemiluminometric assay for human thyrotropin. Clin. Endocrinol. 20, 489-95.

White, E. H., Miano, J. D. and Umbreit, M. (1975). On the mechanism of firefly luciferin luminescence, J. Am. Chem. Soc, 97, 198-200.

Wilson, T. (1976). Chemiluminescence in the liquid phase. Int, Sci, Rev. 9. 265-322.

Wienhausen, G. K., Kricka, L. J., Hinckley, J. E. and DeLuca, M. (1982). Properties of bacterial Luciferase/NADH-FMN oxidoreductase and firefly luciferase immobilised onto sepharose. Appl, Biochem. Biotech, 7, 463-72.

Whitehead, T. P., Thorpe, G. H. G., Carter, T. J. N., Groucutt, C. and Kricka, L. J. (1983). Nature Lond. 305, 158-9.

Wynberg, H., Meijer, E. W, and Hummelen, J. C. (1981). 1.2-Dioxetanes as chemiluminescent probes and labels. In Bioluminescence and chemiluminescence (eds. $\mathrm{M}$, DeLuca and W. D. McElroy), pp. 687-9. Academic Press, New York. 
Patel, A. (1983). The development of homogeneous chemiluminescence immunoassay, $\mathrm{Ph}, \mathrm{D}$, Thesis, the University of Wales, Cardiff, Wales, UK.

- Campbell, A. K. and McCapra, F. (1983). Chemiluminescence energy transfer: a new technique applicable to the study of ligand-ligand interactions in intact cells. Anal. Biochem. 129, 162-9.

Rauhut, M. M. (1979). Chemiluminescence. In Kirk-Othmer encyclopedia of chemical technology, Vol. 5, p. 416.

Reynolds, G. T. (1978). Applications of photosensitive devices of bioluminescence studies, Photochem. Photobiol. 27, 405.

- (1979). Localisation of free ionised calcium in cells by means of image intensification. In Detection and Measurement of Free Calcium Ions (eds. C. C. Ashley and A. K. Campbell), p. 227. Elsevier-North Holland, Amsterdam.

Richardson A. P. (1985). Novel chemiluminescence immunoassays. D. Phil. Thesis, University of Sussex, England.

Roda, A., Girotta, S., Ghini, S., Grigola, B., Carrea, G. and Bovara, R. (1984). Development of a continuous-flow analysis for serum and salivary bile acids using bacterial bioluminescent enzymes immobilised on nylon coil. In Clinical and biochemical tuminescence (eds. L. J. Kricka and T. J, N. Carter), p. 129. Dekker, New York.

Roswell, D. F. and White, E. H. (1978). Chemiluminescence of luminol and related hydrazides, In Methods in enzymology, Vol. 57 (ed. M. Deluca) p. 409. Academic Press, New York.

Salerno, R. Moneti, G., Magini, A., Tomasi, A. and Pazzagli, M. (1984). Evaluation of luminescent immunoassay methods for urinary steroids. In Analytical applications of bioluminescence and chemiluminescence (eds. L. J. Kricka, P. E. Stanley, G. H. G. Thorpe, and T. P. Whitehead), p. 179. Academic Press, London.

Schroeder, H.R., Boguslaski, R. C., Carrico, R.J. and Buckler, R. T. (1978). Monitoring specific protein binding reactions with chemiluminescence. In Methods in enzymology, Vol, 57 (ed. M. DeL uca), pp. 424-45. Academic Press, New York. Schroeder, H. R., Vogelhut, P.O., Carrico, R. J., Boguslaski, R. C. and Buckler, R.T. (1976), Competitive protein binding assay for biotin monitored by chemiluminescence. Anal. Chem. 48, 1933-7.

Schuster, G. B. (1979). Chemiluminescence of organic compounds: conversion of ground state reagents to excited state products by the CIEEL mechanism. Acct. Chem. Res, 12, 366-73.

Seliger, H. H, and McElroy, W. D. (1960). Pathways of energy transfer in bioluminescence. Radiation Res. Suppl. 2, 528-38.

Stanley, P.E. (1982). Instrumentation. In Clinical and biochemical luminescence (eds. L. J, Kricka and T. J. N. Carter), pp. 219-260. Dekker, New York.

Strehler, B.L. (1968). Bioluminescence assay: principles and practice, Methods Biochem. Anal. 16, 99-181.

Thorpe, G. H. G., Gillespie, E., Haggart, R., Kricka, L. J. and Whitehead, T. P. (1984). An immunoassay for serum thyroxine employing enhanced luminescent quantitation of horseradish peroxidase. In Analytical applications of bioluminescence and chemiluminescence. (eds. L. J. Kricka, P. E. Stanley, G. H. G. Thorpe and T. P. Whitehead), pp. 234-8. Academic Press, London.

Tseng, S. and Rauhut, M. M. (1981). Europ. Pat, Appl. No. 81100369.8. 
and NADPH specific FMN oxidoreductases from Beneckea harveyi. Biochem. 16 2932-6.

(1982). Analytical applications of bioluminescence: Marine bacterial system. In Clinical and biochemical luminescence (eds. L. J, Kricka and T. J. N. Carter), pp. 75-87. Dekker, New York.

Kohen, F., Bayer, E. A., Wilchek, M., Barnard, G., Kim, J. B., Collins, W.P., Beheshti, 1., Richardson, A. P. and McCapra, F. (1984). Development of luminescence based assays for haptens and protein hormones. In Analytical applications of bioluminescence and chemiluminescence (eds, L. J. Kricka, P. E. Stanley, G. H. G. Thorpe, and T. P. Whitehead), pp. 149-58. Academic Press, London.

Kohen, F., Pazzagli, M., Kim, J. B. and Lindner, H. R. (1979). An Assay Procedure from Plasma Progesterone Based on Antibody Enhanced Chemiluminescence, FEBS Lett. 104, 201-5.

Kricka, L. J. and Carter, T.J.N. (eds.) (1982). Clinical and biochemical luminescence. Dekker, New York.

- Stanley P. E., Thorpe, G. H. G. and Whitehead, T. P. (eds.) (1984). Analytical applications of bioluminescence and chemiluminescence. Academic Press, London.

- Wienhausen, G. K., Hinkley, J.E. and DeLuca, M. (1983). Automated bioluminescence assays for NADH, glucose 6-phosphate, primary bile acids and ATP. Anal. biochem. 129, 392-401.

Lamola, A.A. and Turro, N.J. (1969). Energy transfer and organic photochemistry. In Techniques of organic chemistry (ed. A. Weissberger), Vol. 14. Wiley, New York.

Lundin A. (1982), Analytical applications of bioluminescence: the firefly system. In Clinical and biochemical luminescence (eds. L. J. Kricka and T. J.N. Carter), pp. 43-74. Dekker, New York.

Maier, C. L. (1978). Assay of pharmacologically, immunologically and biochemically active compounds in biological fluids. U.S. Patent 4,104,029.

McCapra, F. (1973). The chemiluminescence of organic compounds. In Progress in Organic Chemistry (eds. W. Carruthers and J. K. Sutherland), Vol. 8, pp. 231-77. Butterworths, London.

- (1975). The chemistry of bioluminescence. Acct. Chem. Res. 9. 201-8.

(1982). The chemistry of bioluminescence. Proc. R. Soc. Lond. B. 215, 247-72.

- and Manning, M. (1973). Bioluminescence of coelenterates: chemiluminescent model compounds, Chem. Commun. 467-8.

Chang, Y.C. and Francois, V. (1968). The chemiluminescence of a firefly luciferin analogue. Chem. Commun. 22-3.

- Roth, M., Hysert, D. and Zaklika, K. A. (1973). Model compounds in the study of bioluminescence. In Chemiluminescence and bioluminescence (eds. M.J. Cormier, D. M. Hercules, and J. Lee), pp.313-23. Plenum Press, London.

Michelson, A. M. (1978), Purification and properties of Pholas dactylus luciferin and luciferase. In Methods in enzymology, Vol. 57 (ed. M. DeLuca), pp. 385-406. Academic Press, New York.

Mulkerrin, M. G. and Wampler, J.E. (1978). Assaying hydrogen peroxide using the earthworm system. In Methods in enzymology, Vol. 57 (ed. M. DeLuca), pp. 375-81. Academic Press, New York. 
Brolin, S. E., Berggren, P. O. and Naeser P, (1984). Application of light guides for enhancement of signal to noise ratios at low levels of luminescence detectability. In Analytical applications of bioluminescence and chemiluminescence (eds. L. J. Kricka, P. E. Stanley, G. H. G. Thorpe, and T. P. Whitehead), p. 479. Academic Press, London.

Campbell, A.K. and Patel, A. (1983). A homogeneous immunoassay for cyclic nucleotides based on chemiluinscence energy transfer. Biochem. J. 216, 185-194. Carter, T. J. N., Kricka, L. J., Bullock, D. G., Bunce, R. A. and Whitehead T. P. (1979). Optimisation of luminescence instrumentation. In Proc, International Symposium on Analytical Applications of Bioluminescence and Chemiluminescence (eds. E. Schram and P. Stanley), pp. 637-51. State Printing and Publishing, Westlake Village, CA, USA.

Charbonneau, H., Walsh, K. A., McCann, R. O., Prendergast, F. G., Cormier, M. J. and Vanaman, T.C. (1985). Amino acid sequence of the calcium-dependent photoprotein aequorin. Biochem., 24, 6762-71.

Cormier, M.J. (1978). Comparative biochemistry of animal systems. In Bioluminescence in action (ed. P. J. Herring), pp. 75-108. Academic Press, London.

- Hori, K. Karkhanis, Y. D., Anderson, J. M., Wampler, J. E., Morin, J. G. and Hastings, J. W. (1973). Evidence for similar biochemical requirements for bioluminescence among the coelenterates. J. Cell. Physiol, 81, 291-7.

DeLuca, M. (ed.) (1978). Bioluminescence and chemiluminescence. Methods in enzymology, Vol. 57, Academic Press, New York.

- (1984). Bioluminescence assays using co-immobilised enzymes. In Analytical applications of bioluminescence and chemiluminescence (eds. L. J. Kricka, P. E. Stanley, G. H. G. Thorpe, and T.P. Whitehead), pp., 111-23. Academic Press, London.

Freeman, T. M. and Seitz, W. R. (1978). Chemiluminescence fiber optic probe for hydrogen peroxide based on the luminol reaction. Anal. Chem. 50, 1242-6.

Goto, T. and Fukatsu, H. (1969). Cypridina Luminescence. Chemiluminescence in micelle solutions - a model system for cypridina bioluminescence. Tetrahedron Leiters 4299-302.

Gundermann, K.-D. (1968). Chemilumineszenz organischer verbindungen. SpringerVerlag, Berlin.

Hallett, M. B. and Campbell, A. K. (19XX). Applications of coelenterate luminescent proteins. In Clinical and biochemical luminescence (eds. L. J. Kricka and T. J. N. Carter), pp. 89-133. Dekker, New York.

Hastings, J.W. (1978). Bacterial and dinoflagellate luminescent systems. In Bioluminescence in action (ed. P. J. Herring), pp. 129-70. Academic Press. London.

_ and Nealson, K. H. (1977). Bacterial bioluminescence, A. Rev. Microbiol. 31, 549-95.

- Baldwin, T. O. and Nicoli, M. Z. (1978). Bacterial luciferase: Assay, purification and properties. In Methods in enzymology (ed. M. DeLuca), Vol. 57, pp. 135-52. Academic Press, New York.

Herring, P. J. (ed.) (1978). Bioluminescence in action. Academic Press, London. Jablonski, E. G. and DeLuca, M. (1977). Purification and properties of the NADH 
this article can be made limiting with respect to the analyte only. At present the only way in which this flexibility can be utilized is by having the lumimescent compound present in excess. Such 'buffering' by the reagent presents no problems in ordinary analytical work, but considerable ingenuity will have to be deployed to produce the same effect within the restrictions of a sensor with severely limited volume. However, with the very reasonable assumption that the analytes to be examined by this technique will themselves be at the lowest end of the concentration range (made extremely likely by the unrivalled sensitivity) this should not prevent the design of suitable devices. An interesting natural example of the longevity of a luciferin supply is provided by the firefly itself. The organism emerges from the pupa with all the luciferin required for a lifetime (about one month) of almost continuous nightly flashing! Given the very much reduced need for photons that photomultipliers have as compared with the intense firefly flash, it should be possible to estimate the length of time over which the sensor may operate. Until the attempt is made, no precise answer to the question can be given, but in the best cases this may well be not too different from the presently available lifetime of the enzymes employed in existing biosensors. Chemiluminescent compounds will function as solids or pastes, and semi-permeable membranes in conjunction with suitably altered and thus sequestered compounds can be envisaged.

Finally, the question of interference must be considered. It is difficult to discuss this in general since there are many different reactions which lead to luminescence. However there are several compounds available that can be used to overcome particular problems. For example, luminol is oxidized with the emission of light as a result of the catalytic effect of iron compounds such as haem. Its use for other purposes in the presence of whole blood is thus precluded. We have developed the use of acridinium esters for glucose measurement in whole blood with excellent results-identical to those in aqueous buffer and serum. In conclusion, no claim can be made at present that luminescent reactions are ready for incorporation into a robust sensor, but their advantages of easy detectability and extreme sensitivity make further investigations worth pursuing.

\section{References}

Anderson, J. M., Faini, G. J., Wampler, J. E. and Stimson A. (1974), Photometry and radiometry for engineers. Interscience, New York.

Ashley C. C. and Campbell A. K. (eds.) (1979). Detection and measurement of free calcium in cells. Elsevier-North Holland, Amsterdam.

Baldwin, T. O., Johnson, T. C. and Swanson, R. (1984), Recent progress in bioluminescence: Cloning of the structural genes encoding bacterial luciferase, analysis of the encoded sequences and crystallisation of the enzyme. In Flavins and Flavoproteins (eds, R. C. Bray, P. C, Engel, and S. G. Mayhew), pp. 345-58. de Gruyter,-Berlin. 
(Freeman and Seitz 1978; Seitz, this volume Chapter 30.) Peroxidase is immobilized in a clear polyacrylamide gel and used to measure the concentration of hydrogen peroxide in a buffer solution using luminol both in the gel and solution. Concentrations down to $10^{-6} \mathrm{M}$ were detectable by a photomultiplier tube at the other end of the two-foot-long fibre. It was pointed out that unlike other enzyme electrodes, there is no need to have the product of the enzymic reaction diffuse to the surface of the electrode. Thus response time is very short at about four seconds. A major problem noted was that the response was mass transfer limited. Use of a simple form of light guide has supported this idea as a convenient and workable configuration (Brolinet al. 1984). The major problems in pursuing this approach are probably those associated with enzyme immobilization. In the case of an immunosensor of course the difficulty presented by the slow rate of the ligand-antibody binding equilibrium itself looms large. Nevertheless the high sensitivity of light detection and independence from electrode phenomena make further research seem very worthwhile.

The availability of the luciferases has been considered a threat to the development of analytical devices based on bioluminescence. Recently however there have been some encouraging advances which suggest that this is no longer a problem. Bacterial luciferase (Baldwin et al. 1984) and the photoprotein aequorin (Charbonneau et al, 1985) have been cloned. There is now the prospect that the rarity of the organism will no longer inhibit attempts to devise new analyses based on their very desirable characteristics. Some problems remain to be solved before unusual luciferases are made available in quantity, but attention will increasingly turn to bioluminescence with this restraint removed.

As with all devices based on the use of enzymes, the question of stability arises. A fair amount of experience has already been gained on the use of luciferases immobilized on various supports. Firefly luciferase has not yet been found to be sufficiently stable as to prove suitable for sensor applications, but bacterial luciferase has shown a steady improvement in stability as an understanding of the best methods of immobilizing it has increased. It can now undergo several hundreds of re-use cycles and there is every hope that these enzymes will be no different from many others. Whether they will prove as durable as the relatively few stalwarts in common use remains a matter for future research.

Another problem facing chemiluminescence and bioluminescence in sensor applications is the need for reagent replenishment. If the easy detectability and high sensitivity of light emission are to be exploited, the configuration of the device must take this into account. It must be understood that the phenomenon requires the irreversible oxidation of the substrate-the luciferin in the case of bioluminescence-and the readily available small organic molecule in chemiluminescence, Many of the reactions described in 
detector. The associated electronics are well developed and basic easily constructed sensitive systems have been described (Anderson et al. 1974). Modern photon (pulse) counting instrumentation increases sensitivity by lowering noise levels and is increasingly used in commercially available luminometers. Photomultiplier tubes are most noise free when sensitive in the blue region of the spectrum, ( 350 to $450 \mathrm{~mm}$ ), and are thus suitable, if not ideal, for the great majority of chemiluminescent reactions. Only the firefly system is seriously disadvantaged since its peak emission occurs at a fairly long wavelength $(560 \mathrm{~mm})$. The very robust, cheap, and adaptable lightsensitive diodes are less sensitive than photomultiplier tubes, particularly in the blue region. Photosensitive film, especially modern enhanced sensitivity types can also be used. The merits of each of these has been discussed (Carter et al. 1979). In favourable cases, with sufficient light available, photodiodebased systems provide cheap portable devices, with a variety of commercially available ATP detectors being available for simple field-testing for bacterial infection. This and some thirty other commercial luminometers have been described (Stanley 1982). A portable prototype using photodiodes for glucose analysis has been developed in the author's laboratory.

Light measurement in the homogeneous energy transfer assay previously mentioned provides certain challenges and advantages. For example, the measurement of a ratio of wavelengths provides an intrinsic improvement in precision since some variations in signal must cancel. Two separate phototubes may be used, with an appropriate filter for each (Campbell and Patel 1983; Patel 1983). The use of a single phototube with rotating filters frequency locked to phase-sensitive amplifiers provides a more elegant and potentially less noisy detecting system (Richardson 1985).

\subsection{Possible biosensor applications}

If we were to define biosensors in their ideal form, capable of implantation, continuous monitoring, uniquely sensitive, and free from interference, then luminescence methods have a long way to go. However, it is abundantly clear that as a method of translating the presence in tiny amounts of biological compounds into an easily processed signal, they are successful.

They also possess characteristics such as sufficient sensitivity and an ability to sample without separation that strongly suggest that there is considerable potential. Existing configurations are on the whole, classical, in that a prepared sample is placed in a cell or cuvette and light emission detected by placing it in front of a photomultiplier tube. Recently however there have been developments which suggest that the manufacture of convenient devices is on the horizon.

A device which may well prove to be a model for others based on the reactions described in this chapter utilizes a $1 / 8$ inch diameter fibre optic. 


\subsubsection{Light-measuring techniques}

Chemiluminescent reactions respond to variables such as $\mathrm{pH}$ and catalysts in a number of ways. From some luminescent systems it is possible to obtain a flash with a duration of milliseconds or a prolonged lower light level lasting some hours. Bioluminescent reactions are less easily manipulated, but similar characteristics are obtainable. Thus detection can be arranged for maximum sensitivity or convenience. It is obvious that for a given number of photons maximum sensitivity is obtained by arranging light emission in the shortest possible time. This has its drawbacks because mixing errors are at a maximum in the initial phases. Enzyme turn-over (as in the peroxidase reactions) provides amplifications which result in prolonged, relatively high intensity emission since the luminescent compound is in excess and not stoicheometrically linked to the analyte. Peak sensing, integration of the light and monitoring changes in the rate of reaction are also possible. The various possibilities are shown diagrammatically in Fig. 31.1.

\subsubsection{Kinetic measurements}

All luminescent reactions are subject to variations in rate. This is a potentially powerful means of analysis since the high detectability of light emission gives opportunities for rapid sampling of small portions of a reaction sequence.

\subsubsection{Light-measuring devices}

Photomultiplier tubes are the most powerful and inost common light

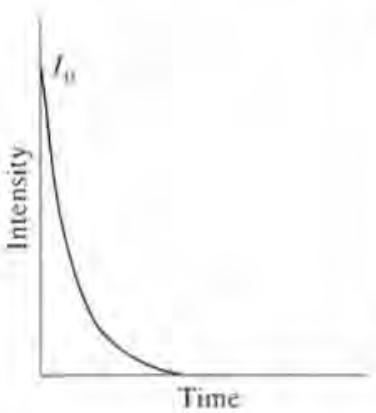

(a)

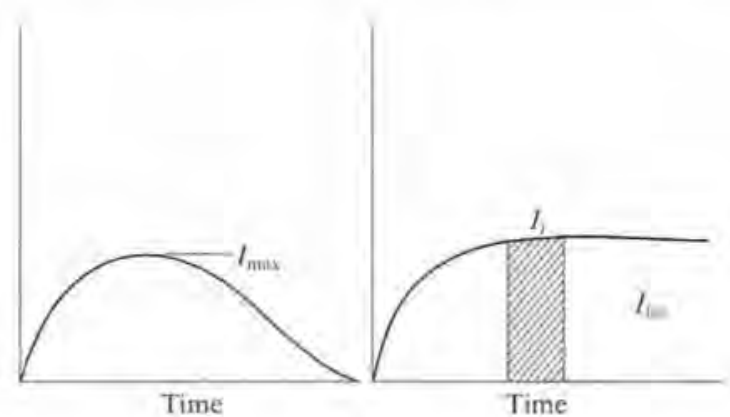

(b)

(c)

Fig. 31.1 (a) Initial intensity, $I_{\mathrm{o}}$, provides maximum sensitivity, but may give a lesser, still acceptable precision.

(b) Peak sensing devices provide automatic quantification. The peak $I_{\max }$ can be produced by variation of the reaction characteristics so as to produce optimum sensitivity consistent with reproduceability.

(c) Devices similar to those used in (b) can integrate for a variable time after a variable delay $\left(I_{t}\right)$. Alternatively the light signal can be integrated for as long as is convenient to produce the necessary sensitivity. 
luminescence possesses, whereas radioactivity does not. Luminescence from the luciferins in bioluminescence, both in vivo and in vitro is much affected with regard to wavelength and efficiency on binding to proteins or on being placed in an otherwise non-aqueous environment (Ward and Cormier 1979; McCapra and Manning 1973; Goto and Fukatsu 1969; McCapra et al. 1973).

These effects have been exploited in a model biotin-avidin system in which the light intensity increases 10 -fold on the binding of the attached luminol (Schroeder 1976). Several homogeneous steroidal assays make use of the adventitious enhancement of light from the reaction of the attached isoluminol which results when the steroid is bound to the antibody (Kohen et al. 1979; Salerno et al. 1984).

A more fundamental investigation has recently demonstrated a potentially very important technique (Patel et al. 1983; Campbell and Patel 1983). The system is based on transfer of the original electronic excitation of the chemiluminescent product to a fluorescent energy acceptor. Such a phenomenon is well known in spectroscopy and photochemistry (Lamola and Turro 1969).

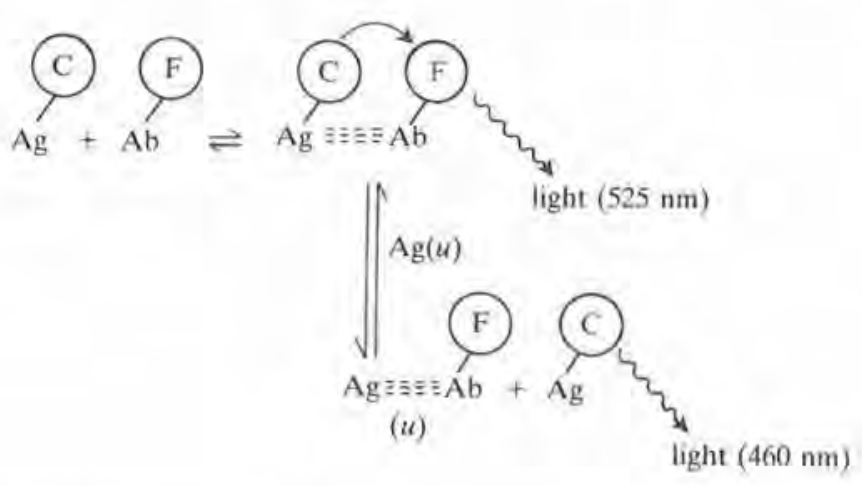

The ligand (e.g. cAMP or progesterone) is covalently attached to the chemiluminescent compound (ABEI) and the antibody made fluorescent by attachment of fluorescein. The trasfer of energy is governed by the Förster equation, requiring a close approach (ca $50 \AA$ ) of donor and acceptor (Lamola and Turro 1969). Thus the bound fraction emits green light and the unbound, the unaltered blue emission of isoluminol. Various filters can be used (the emission bands are broad) with the ratio at $460 / 525 \mathrm{~nm}$ producing the best results. In general the principle can be described thus where $\mathrm{Ag}$ (the antigen) is labelled with a chemiluminescent compound (C) and $\mathrm{Ab}$ (the antibody) is labelled with a fluorescent acceptor (E). When $\mathrm{Ag}-\mathrm{C}$ is displaced by the analyte of unknown concentration $\mathrm{Ag}(u)$, the distance between $\mathrm{C}$ and $\mathrm{F}$ is too great for energy transfer, so that bound and unbound are readily distinguished. 


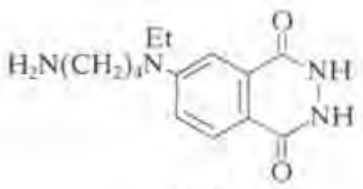

ABEI

fluorescent compounds to proteins or tyrosine (for subsequent iodination) to haptens. Isothiocyanate, $N$-hydroxysuccinimidyl ester and imido-ethers have all been used. A favourite label first applied by Schroeder et al. in 1978 is 6-[N-(4-aminobutyl)- $N$-ethyl]amino-2, 3-dihydro-1,4-phthalazine-1,4-dione (ABEI). Thyroxine was labelled by this and other hydrazides. Various steroidal assays have been similarly developed (Salerno et al. 1984; Kohen et al. 1984). Reactions such as these require injection of the reagents and give an easily quantified, but brief, emission of light. Excellent results in the quantification of protein and peptide hormones such as hCG (human chorionic gonadotrophin, Kohen et al. 1984) TSH (thyrotropin) (Weeks et al. 1984) and $\alpha$-fetoprotein (Weeks et al. 1983) have been achieved. Acridinium salts are particularly useful in this area.

As already noted the reactions which emit the detectable light are usually fast. This has advantages in sensitivity but enzyme labelling of antigen or antibody, coupled to light emission provides a much longer lived emission, and could thus be used in principle for continuous monitoring. Peroxidases in conjunction with luminol are the best developed systems of this sort. For example horse-radish peroxidase has been coupled to all the components necessary for complete thyroid-function analysis, including both proteins and haptens (Thorpe et al. 1984). Such techniques while successful are still in their infancy. Improvements, as demonstrated by the use of synergistic enhancements of sensitivity (Whitehead et al. 1983) can be reasonably expected as investigation proceeds. This approach is very similar to that of the well-known enzyme immunoassays, but has two very significant advantages of potential use in biosensor design. The light level is directly proportional to analyte concentration (there is no build up in intensity as in a colorimetric assay) and the detectability is very much higher.

\subsubsection{Homogeneous assay All of the assays outlined above are separa-} tion assays. Continuous monitoring of biologically significant compounds in a biosensor requires both a continuing signal and a direct response to changing concentrations of analyte without resort to separation of the materials being analysed. Homogeneous immunoassay methods have recently been developed, using two very different phenomena.

The essential requirement of course is to distinguish bound from unbound ligand, and the influence of the protein on ligand properties is a feature that 


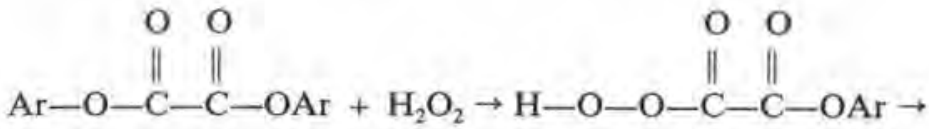

$$
\begin{aligned}
& + \text { Fluorescer } \rightarrow \text { [Fluorescer }]^{*} \mathrm{CO}_{2}{ }^{*}+\mathrm{CO}_{2} \\
& \rightarrow \text { Fluorescer* }+\mathrm{CO}_{2} \rightarrow \text { Fluorescer }+ \text { Light }
\end{aligned}
$$

Lastly, mention must be made of dioxetans such as (10) and (11). These are insoluble compounds which on mild heating give light, with no other reagent or intermediate involved (Wilson 1976).<smiles>CC(C)(C)COC(C)(C)C</smiles>

10

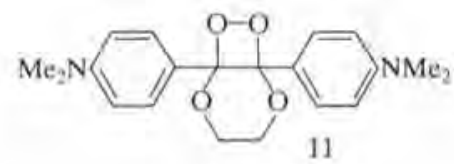

Simple dioxetans such as (10) give almost exclusively triplet states, but others such as (I1) give high yields of singlet states.

\subsubsection{Chemiluminescent immunoassays}

Use of the bioluminescent systems in bio-analysis has the advantages of an inbuilt specificity since the luciferases and associated enzymes are naturally specific. However by labelling either antibody or antigen (occasionally both) with a chemiluminescent compound, all the advantages of enzyme- and radio-immunoassays (RIA) are obtained with none of the disadvantages. Sensitivity can be higher than for RIA (Weeks et al. 1984), stability of the label is excellent, measurement is both flexible and easy, and the instrumentation is simple and relatively cheap (Kricka et al. 1984; Kricka and Carter 1982).

31.3.2.1 Types of assay In spite of the relatively short time in which chemiluminescent immunoassays have been studied, several different sorts of assay have been developed. The simplest is the direct replacement of ${ }^{125} \mathrm{I}$ by the luminescent compound. Many such applications have been reported, using cyclic hydrazides such as isoluminol (Schroeder et al. 1978), luminol itself (Maier 1978), acridinium phenyl esters (Weeks et al. 1983), dioxetans (Wynberg et al. 1981) to label haptens or small peptides.

The methods of labelling are very similar to those developed for attaching 
analysis. It is not possible to do other than outline the reaction scheme in this context.

In aqueous solution catalysts and hydrogen peroxide are both essential for strong light emission, although base and oxygen alone are sufficient in dipolar aprotic solvents such as dimethylsulphoxide.

\subsubsection{Mechanisms of chemiluminescence}

Only a very brief account is necessary here, although it is an interesting and important area, an understanding of which can be expected to improve the application of the phenomenon. Concentration on the best-understood reactions will give a useful basis, even if it does beg many questions. Compounds may react with oxygen, usually in the form of a carbanion or electron-rich species to produce a peroxide. A closely related peroxide can also be produced if the structure of the compound invites attack by hydrogen peroxide, which is a very powerful nucleophile in aqueous solution. The purpose of the metal catalysis in the luminol reaction is to produce an intermediate derived from luminol which will react with $\mathrm{H}_{2} \mathrm{O}_{2}$ or its oxidation product $\mathrm{O}_{2}{ }^{-}$, superoxide ion. The acridinium salts and acridans can be used to demonstrate both routes.
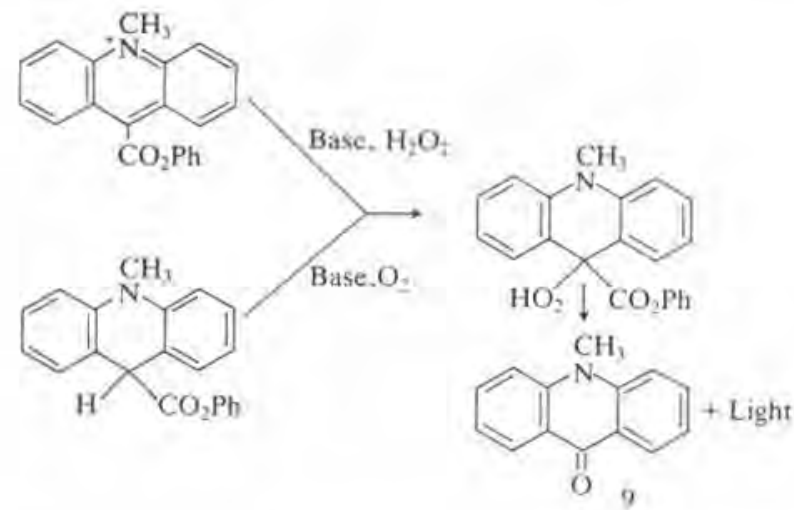

In this case the fluorescent product (9) is directly derived from the reactant. In the very flexible active oxalate series, the reactant gives an intermediate which is not fluorescent, but can react with a large variety of added fluorescers, resulting in very high light yields. Incidentally, this reaction demonstrates what is believed to be the nature of the actual excitation step involving electron transfer (McCapra 1973; Schuster 1979). The group $\mathrm{Ar}$ must be strongly electronegative, the most common being dinitrophenyl and trichlorophenyl although many others are possible. Some of these are particularly suitable for use in aqueous systems (Tseng and Rauhut 1981). 


$$
\begin{aligned}
& \mathrm{R} \stackrel{\phi_{\mathrm{C}} \phi_{\mathrm{E}}}{\longrightarrow} \mathrm{P}^{*}, \quad \mathrm{P}^{*} \stackrel{\phi_{\mathrm{E}}}{\longrightarrow} \mathrm{P}+h \nu, \\
& \phi_{\mathrm{Cl}}=\phi_{\mathrm{C}} \times \phi_{\mathrm{F}} \times \phi_{\mathrm{E}} .
\end{aligned}
$$

A high chemical yield $\phi_{\mathrm{C}}$ of excited product $\mathrm{P}^{*}$ is obviously required, and the fluorescence yield $\phi_{\mathrm{F}}$ should be as near unity as possible. The third term $\phi_{\mathrm{E}}$ denotes the proportion of molecules entering the excited state, and this depends intimately on the reaction mechanism. The in vitro firefly reaction is the most efficient known with $\phi_{\mathrm{CL}}=0.88$ (Seliger and McElroy 1960) but the simple chemical reaction of a derivative of firefly luciferin can almost match this with $\phi_{C L}=0.33$ (White et al. 1975). Other quantum yields range from 1 $\times 10^{-8}$ for Grignard compounds up to 0.25 for the reaction of active oxalates with suitable fluorescers (Rauhut 1979).

A variety of solvents can be used for the reactions, with aqueous conditions being the least suitable for high quantum yields. Exceptions to this observation are the reactions of the cyclic hydrazides such as luminol, acridinium salts and to a certain extent, special active oxalate esters.

For maximum detectability, the electronically excited molecule formed in the reaction should be in the singlet state. This is because the alternative triplet state is much longer lived, and subject to quenching by other molecules present, particularly oxygen. The light observed is thus a chemically produced fluorescence.

The best-known chemiluminescent compound is undoubtedly luminol (7). Much is known about its properties and those of a large number of analogues (Roswell and White 1978; Gundermann 1968). The fact that there is no agreed mechanism for the actual light-emitting step for this most studied of molecules has not hindered extensive application. It is not the most efficient hydrazide known, partly because the excited product, the diphthalate ion (8), is not particularly fluorescent. Under the best conditions the chemiluminescence quantum yield is only about $1 \%$.

The mechanism as already indicated is not well understood. This is particularly true of the catalysed reaction which has been of greatest use in

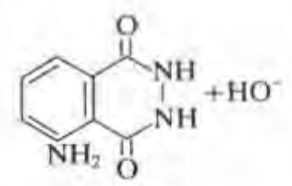

7

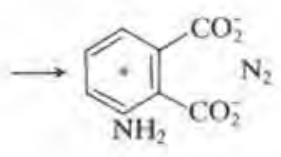

$\rightarrow \underbrace{\mathrm{CO}_{2}^{-}}_{\mathrm{NH}_{2}}{ }_{8}^{\text {Light }}$ 
much more active but exceedingly scarce, peroxidase system is that isolated from the clam Pholas dactylus (Michelson 1978).

\subsection{Chemiluminescence}

Although the fundamental mechanisms underlying light emission from biological and chemical reactions must be the same, the two fields developed independently until about 15 years ago. Fully convincing detailed mechanisms are available only for the firefly, Cypridina, and coelenterate systems, but very many more organic compounds react by well-understood mechanisms.

Chemiluminescence in solution is almost invariably an oxidative reaction. Molecular oxygen and hydrogen peroxide are most often involved. A very wide range of chemical structure can be found (McCapra 1973; Gundermann 1968) and a few chemiluminescent compounds are shown below to illustrate the diversity.<smiles>Nc1cccc2c(=O)[nH][nH]c(=O)c12</smiles><smiles>Cc1nc2ccccc2c(C(c2ccccc2)c2c3ccccc3nc3ccccc23)c1[N+](=O)[O-]</smiles><smiles>c1ccc(-c2nc(-c3ccccc3)c(-c3ccccc3)[nH]2)cc1</smiles><smiles>CC(C)=Nc1c2ccccc2cc2ccccc12</smiles><smiles>CC1N=C2C=CC=CN2C1=O</smiles>

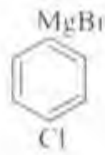<smiles>CN1c2ccccc2C(C(=O)O)c2ccccc21</smiles><smiles>CC12CC3CC4C3(C)C41OO2</smiles><smiles>C1CCC(PC2CCCCC2)CC1</smiles>

The efficiency of light emission is measured in terms of the number of photons produced per molecule reacting, stated in terms of a quantum yield $\phi$. This in turn depends on three factors, 
<smiles>CCC(C)c1nc2c(CCCNC(=N)N)[nH]c(-c3c[nH]c4ccccc34)cn-2c1=O</smiles>

2<smiles>O=c1c(Cc2ccc(O)cc2)nc2c(Cc3ccccc3)[nH]c(-c3ccc(O)cc3)cn1-2</smiles>

The chemical mechanism proposed for these luciferins is closely related to that of several efficient, purely chemical, systems, representing a notable success of interpretative organic chemistry (McCapra 1975).

Other luciferins for which no mechanism exists for light emission are those of the fresh water limpet Latia neritoides (4), the earthworm Diplocardia longa (5) and the dinoflagellate Pyrocystis lumula (6).

The luciferase from Diplocardia longa is a copper protein, and has peroxidase activity. Its value in assays for $\mathrm{H}_{2} \mathrm{O}_{2}$ and some oxidases has been assessed (Mulkerrin and Wampler 1978). As will be seen later, peroxidase reactions make excellent chemiluminescent analyses and assays. Another,<smiles>CC1=C(CC/C=C/OC=O)C(C)(C)CCC1</smiles>

4<smiles>CC(C)CC(=O)NCCC=O</smiles>

5<smiles>[R]OC(=O)CCC1=C(C)C(=O)N=C1C1CC(=O)c2c([nH]c(/C=c3\[nH]/c(=C/C4NC(=O)C(C)=C4C)c(C)c3C)c2C)N1</smiles> 
of the metabolites is not great at present and the example is more illustrative of the value to analysis of continued research into bioluminescence.

$$
\begin{aligned}
& \text { Luciferyl sulphate }+ \text { PAP } \stackrel{\text { Sulphokinase }}{\longrightarrow} \text { Luciferin }+ \text { PAPS } \\
& \text { Luciferin }+\mathrm{O}_{2} \stackrel{\text { Luciferase }}{\longrightarrow} \text { Oxyluciferin }+\mathrm{CO}_{2}+\text { Light }
\end{aligned}
$$

The central role of $\mathrm{Ca}^{2+}$ in cellular processes makes it an attractive target for quantitation (Ashley and Campbell 1979). It is even possible to localize the source of $\mathrm{Ca}^{2}$ - within large cells since image intensification devices (Reynolds 1978, 1979) allow microscopic visualization with a resolution of about $2 \mu \mathrm{M}$. The technique depends on the fact that certain coelenterates (principally Acquorea and Obelia) store the luciferin as a peroxide (1) bound to the luciferase, making a single entity called a photoprotein. Isolation of this photoprotein is achieved in the presence of a high concentration of EDTA to inactivate endogenous calcium ion. Cytoplasmic $\mathrm{Ca}^{2}$ can be detected at about the $10^{-7} \mathrm{M}$ level on micro-injection of the photoprotein (called aequorin).

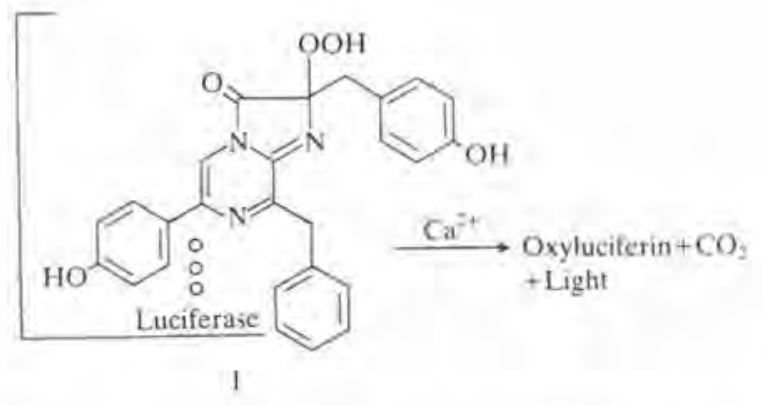

Serum levels of $\mathrm{Ca}^{2+}$ are too high for direct examination by this technique, causing saturation (Campbell and Patel 1983). Again this specialized use of luminescence serves more as an indicator of future possibilities than as a practical biosensor, since the technique is among the more difficult to master. Nevertheless it is not unreasonable to suggest that the chemist may eventually mimic the triggering event which gives rise to the easily detected light signal. A variety of synthetic compounds whose light emission is catalysed by an ion or other ligand can be envisaged suggesting an important research goal.

The ostracod crustacean Cypridina hilgendorfii generates light by the same chemical mechanism as the firefly in spite of the very different structure of the luciferin (2). A related luciferin (3) first characterized from the sea pansy Renilla reniformis has subsequently been found in a remarkably wide variety of organisms, including fish, shrimp, squid, and very many coelenterates (e.g. the jellyfish Aequorea aequorea) (Cormier et al. 1973). 
$\mathrm{FMNH}_{2}+$ Luciferase $+\mathrm{O}_{2} \rightarrow \mathrm{FMNH}(\mathrm{OOH}) \cdot$ luciferase

$\mathrm{FMNH}(\mathrm{OOH}) \cdot$ luciferase $+\mathrm{RCHO} \rightarrow \mathrm{FMN}+\mathrm{R} \cdot \mathrm{CO}_{2} \mathrm{H}+$ Luciferase $+\mathrm{H}_{2} \mathrm{O}+$ Light

\subsubsection{Applications of bacterial bioluminescence}

Mention has already been made of the use of the firefly system in ATP analysis, and there is little doubt that it is the method of choice for this ubiquitous substance. Immobilization of the enzyme has been achieved. A more versatile immobilized enzyme, and one that is very accessible, is the bacterial luciferase. The number of assays of enzymes which can be coupled to either the production or consumption of $N A D H$ and $N A D(P) H$ is very large. Many of these have been listed (Jablonski and DeLuca 1982). Some examples and the assay limit are NADH $(0.1 \mathrm{fmol})$, glucose $(0.5-6 \mathrm{pmol})$, malate $(20-250 \mathrm{pmol})$, testosterone $(0.2-5 \mathrm{nmol})$, TNT $(30 \mathrm{fmol})$. The analysis of TNT for example was achieved by inducing a TNT reductase which used NADH as cofactor. This technique could obviously be extended to other reduceable compounds for which enzymes may be induced.

Detailed descriptions of the methods used for the immobilization of the reductase and luciferase have been given (Jablonski and DeLuca 1977). Glass rods were coated by cementing arylamine glass beads using epoxy adhesive, and the enzyme coupled to the beads by diazotization.

The active rod can be used over 100 times without loss of activity although the specific activity of the enzymes may be reduced by about a thousand-fold. Light detection is carried out by placing the rod in the buffer-analyte solution in front of a phototube. Considerable retention of substrates, particularly $\mathrm{NADH}$ at high concentrations, necessitates extensive washing which can reduce activity still further.

Improvements to this system are being continuously made and cyanogenbromide-activated Sepharose (DeLuca 1984) and nylon-6 (Roda et al. 1984) are effective carriers. Some 25 different enzymes have been immobilized with excellent retention of enzyme activity in both the 'analysing' enzyme and the light-emitting components. NAD(P)H can be measured at the $6 \mathrm{fmol}$ level using a flow system.

\subsubsection{Other bioluminescent systems}

The only other prominent uses of the many bioluminescent organisms concern the jellyfish Aequorea, the hydroid Obelia, and the sea pansy Renilla.

Renilla luciferin is stored in the organism as an enol sulphate (Cormier 1978 ) and is released by a sulphokinase. The reaction sequence shown below shows how 3', 5'-phosphoadenosine phosphate (PAP) and the product of the transfer $3^{\prime}$-phosphoadenosine-5'-phosphosulphate (PAPS) can be coupled to the light reaction and analysed at the 10 to $100 \mathrm{pmol}$ level. The significance 


\subsubsection{Firefly luciferase in ATP analysis}

Since ATP occupies such a central place in the biochemistry of all living systems, it is not surprising to find that there is a very large number (over 1000 papers) of applications. There is little point in giving a description of these since the most significant references have been collected, and the methods described (DeLuca 1978; Kricka et al. 1984). They include biomass determinations, detection of bacterial infection (not species specific), antibiotic assay, and any enzymic reaction either producing or utilising ATP.

Although it is possible to produce the materials for these assays as required (DeLuca 1978), the need for good, consistent quality reagents has made the use of commercially available reagents (e.g. from Sigma or LKB-Wallac, Turku, Finland) very attractive.

This historically important technique is however not well suited to biosensor applications in view of its complexity unless ATP is the specific target. High sensitivity can be more readily achieved by the much more flexible chemiluminescent systems described later. Nevertheless the coimmobilization of luciferase and other analytically useful enzymes is being explored with some success (DeLuca 1984). The clinically important enzyme creatine kinase (in heart disease) can be measured at the 1 femtomole level, and the successful addition of automated flow systems suggests that convenient sensing devices are possible (Wienhausen et al. 1982; Kricka et al. 1983).

\subsubsection{Bacterial luminescence}

Luminous bacteria (e.g. Photobacterium phosphoreum, Vibrio harveyi) are found in almost all marine environments, as saprophytes, free-living organisms, and symbionts. Many fish use the light of colonies of such bacteria for purposes such as mating, shoaling, and attracting prey. They are easily cultured and a large amount is known about their biochemistry (Hastings 1978; Hastings and Nealson 1977). One very attractive feature is the large amount of easily purified luciferase (up to $5 \%$ of the cellular contents) that can be obtained. There is no luciferin in the same sense as in the firefly. The light-emitting species seems to be a complex of luciferase, reduced flavin (FMNH), and a long chain fatty aldehyde. Although the chemical mechanism of light emission is not fully established, the outline shown is accepted. The bacterial luciferase sold commercially contains the necessary NAD(P)H:FMN oxidoreductase in varying amounts but contamination by other enzymes severely limits its usefulness. Isolation of both enzymes from a cultured cell paste in sufficient purity for analytical purposes is relatively easy. (Hastings et al. 1978; Jablonski and DeLuca 1977)

$$
\mathrm{NAD}(\mathrm{P}) \mathrm{H}+\mathrm{FMN} \underset{\text { reductase }}{\stackrel{\text { Oxido }}{\longrightarrow}} \mathrm{FMNH}_{2}+\mathrm{NAD}(\mathrm{P})
$$


the extraction and purification have been published (DeLuca 1978; Lundin 1982). The luciferase is a moderately stable enzyme, readily obtainable at various levels of purification. Potent inhibitors of the enzyme such as dehydroluciferin (found as an impurity in commercial preparations), pyrophosphate, and the products of the reaction, oxyluciferin and adenosine monophosphate, must be removed for maximum sensitivity. The avoidance of their effects on the analysis has been discussed (Lundin 1982).

The luciferase catalyses all of the reactions in the sequence shown below. For ease of understanding, the chemical events are shown separately. Indeed the chemical reactions can be performed without enzyme with a reduction in efficiency to about $25 \%$ of that of the enzymic reaction (White et al. 1975; McCapra et al. 1968). This chemiluminescent reaction does not require ATP and thus loses contact with the assay under discussion. It is analogous to chemiluminescent systems discussed later.

Luciferin + Luciferase $+\mathrm{Mg}^{2}+$ + ATP $\rightarrow$ Luciferyl adenylate L-AMP L-AMP-luciferase $+\mathrm{O}_{2} \rightarrow$ Oxyluciferin*-Luciferase

Oxyluciferin*-Luciferase $\rightarrow$ Oxyluciferin-Luciferase + Light (562 nm)

The overall efficiency, measured as the quantum yield $\phi$ (see later) is a remarkable $88 \%$ (Seliger and McElroy 1960 ).<smiles>O=C(O)C1CSC(c2nc3ccc(O)cc3s2)=N1</smiles><smiles>CCOC(=O)C1=NC(c2nc3ccc(Cl)cc3s2)C(c2nc3ccc(OC)cc3s2)=N1</smiles><smiles>COc1ccc2nc(C3=NC4(CS3)OOC4=O)sc2c1</smiles><smiles>C[N+](C)(C)Oc1ccc2nc(C3=NC(=O)CS3)sc2c1</smiles>

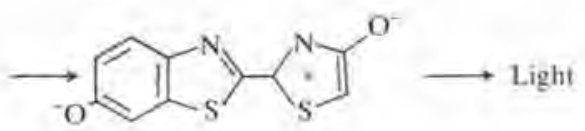


biochemistry, where known, is as interesting and almost wholly unexploited. An excellent modern survey of all aspects of bioluminescence, with particular reference to the biological aspects is available (Herring 1978).

Various biochemical sequences are used to produce light, but the simplest in terms of its components, and having an accepted reaction mechanism, is that of the little ostracod crustacean Cypridina hilgendorfii (Tsuji 1978). It provides the basic description of light emission in luminescent organisms.

$$
\begin{aligned}
& \text { Luciferin }+ \text { Luciferase } \frac{\mathrm{O}_{2}}{\mathrm{H}_{2} \mathrm{O}} \text { Oxyluciferin* } \\
& \text { Oxyluciferin } * \text { Oxyluciferin }+ \text { Light }
\end{aligned}
$$

In the simplest cases, the luciferin is a small heterocyclic organic molecule whose enzyme-catalysed oxidation leads to the formation of the product, oxyluciferin, in a singlet excited state. Radiation from this state is identical to that of fluorescence, which can be produced by irradiating the oxyluciferin in the usual way.

Several of the enzymes (luciferases) involved use common cofactors in the reaction. These cofactors are often central to metabolic processes in general, and the light-emitting reaction can thus be coupled to many reactions of biological significance. Foremost among this type of bioluminescent reaction is that found in the firefly (ATP as cofactor), Renilla (PAPS as cofactor), luminous bacteria (NADH or $\mathrm{NAD}(\mathrm{P}) \mathrm{H}$ and $\mathrm{FMN}$ as cofactors) and the jelly-fish Aequorea $\left(\mathrm{Ca}^{2+}\right)$. Other less well-understood organisms such as the rare boring clam Pholas dactylus and the earthworm Diplocardia have had brief examination as potential analytical tools. A very good review in the series Methods in enzymology of all of the above types is available (DeLuca 1978). A further volume in the series is in preparation.

It is not appropriate to discuss the detailed chemistry and biochemistry of bioluminescence, but it would be helpful to give an outline of the two mostused systems since an understanding of the principles underlies any possible application.

\subsubsection{Firefly bioluminescence}

This is the most studied and best understood of the luminous organisms. Its chemistry, biochemistry, and biology are all well reviewed (McCapra 1975, 1982; DeLuca 1978; Herring 1978). It was also the first organism which allowed the demonstration of the power of light detection in analysis (Strehler 1968).

Although the luciferin is readily synthesized, the luciferase is naturally only obtainable from the firefly itself. Several other related members of the Coleoptera also use the same light system, but these organisms are relatively rare and have not been exploited. Methods for the synthesis of luciferin and 


\title{
Potential applications of bioluminescence and chemiluminescence in biosensors
}

\author{
F. McCAPRA
}

\subsection{Introduction}

It is obvious from discussions of the concept of biosensors, especially as presented in this monograph, that there are several possible definitions. The use of biologically derived entities such as whole cells, enzymes, or immunoglobulins in primary contact with the analyte is almost universal. Problems associated with the application of these are common to all biosensor techniques and will not be discussed here. On the other hand, signal handling methods are very well developed, often only needing miniaturization or other adaptation to the specific purpose. In the middle, presenting many opportunities for invention and imagination, are the transducers, translating biochemical events into electronic effects or electrical signals. Sensitivity is at a premium here, together with an ability to operate in biological fluids without undue interference.

Recently both bioluminescent and chemiluminescent reactions have been shown to provide sensitivity second to none, especially when used in conjunction with the very best photon-counting equipment. However their use in convenient devices has been relatively unexplored. The purpose of the present article is to describe the principles of luminescent reactions, their success as rivals to established enzyme and immunoassay methods, and to provide indications of the ways in which their special properties may be exploited. Although their use in biosensors is only at a preliminary stage, there is an extensive literature on analytical uses. (DeLuca 1978; Kricka and Carter 1982; Kricka et al. 1984.)

\subsection{Bioluminescence}

The emission of light from reactions of organic molecules in solution is an interesting and by now reasonably well-understood phenomenon. Bioluminescent organisms such as fireflies, glow-worms, angler-fish, and those responsible for the phosphorescence of the sea are often familiar. However there are very many organisms, particularly marine, such as squid, shrimp, and deep sea fish which show even more dramatic effects. Their 
VoelkI, K. P., Optiz, N. and Luebbers, D. W. (1980), Continuous measurement of concentrations of alcohol using a fluorescence-photometric enzymatic method. Fres. Z. Anal. Chem. 301, 162-3.

Vurek, G. G., Feustel, P. J, and Severinghaus, J.W. (1983) A fiber optic $\mathrm{pCO}_{2}$ sensor. Annals of Biomed. Eng. 11, 499-510.

Wolfbeis, O.S. Fuerlinger, E., Kroneis, H. and Marsoner, H. (1983). Fluorimetric analysis. 1. A study of fluorescent indicators for measuring near neutral ('physiologiucal' pH-values. Fres. Z. Anal. Chem. 314, 119-24.

Offenbacher, H., Kroneis, H. and Marsoner, H. (1984). A fast responding fluorescence sensor for oxygen, Mikrochimica Acta 153-8.

Zhujun, Z. and Seitz, W, R. (1984a). A fluorescence sensor for quantifying $\mathrm{pH}$ in the range from 6.5 to 8.5 Anal. Chim. Acta 160, 47-55.

$(1984 b)$. A carbon dioxide sensor based on fluorescence. Anal. Chim. Acta $160,305-9$. 
Speiser, P. P. and Bisson, H. J. (1977). Nanoencapsulated fluorescence indicator molecules measuring $\mathrm{pH}$ and $\mathrm{pO}_{2}$ down to submicroscopial regions on the basis of the optode principle. Z. Naturforsch. 32c, 133-4.

MacDonald, B. F, and Seitz, W, R. (1982). Tetrakis N-dimethylaminoethylene is an extraordinarily sensitive reagent for oxygen. Anal. Lett. 15(A1), 57-66.

Milanovich, F. P., Hirschfield, T. B., Wang, F. T. and Klainer, S. M. (1984) Clinical measurements using fiber optics and optrodes, Proc. SPIE-Int. Soc. Opt. Engl 494, 18-24.

Opitz, N. and Luebbers, D. W. (1976). Simultaneous measurement of blood gases by means of fluorescence indicators. Pfluegers Arch. 362, R52.

- (1983). New fluorescence photometrical techniques for simultaneous and continuous measurements of ionic strength and hydrogen ion activities. Sensors and Acruators 4, 473-9.

- (1984) A correction method for ionic strength-independent fluorescence photometric pH measurement. Adv. Exp. Med. Biol. 169, 907-12.

Peterson, J. I. and Vurek, G. G. (1984). Fiber optic sensors for biomedical applications, Science 224, 123-127.

Fitzgerald, R. V. and Buckhold, D.K. (1984). A fiber-optic $\mathrm{pO}_{2}$ sensor for physiological use. Anal. Chem. 56, 62-7.

Peterson, J. I., Goldstein, S. R., Fitzgerald, R. V. and Buckhold, D. K. (1980). Fiber optic pH probe for physiological use. Anal. Chem. 52, 864-9.

Saari, L. A. and Seitz, W. R. (1982). pH sensor based on immobilized fluoresceinamine. Anal. Chem, 54, 821-3.

(1983). Immobilized morin as fluorescence sensor for determination of aluminium (I11), Anal. Chem. 55, 667-70.

Schultz, J.S. and Sims, G. (1979). Affinity sensors for individual metabolites. Biotechnol. Bioeng. Symp. 9, 65-71.

- Mansoure, S. and Goldstein, I, J. (1982). Affinity sensor: A new technique for developing implantable sensors for glucose and other metabolites. Diabetes Care 5D, 245-53.

Seitz, W. R. (1984). Chemical sensors based on fiber optics. Anal. Chem. 56, 16A.

- Saari, L.A., Zhujun, Z., Pokornicki, S., Hudson, R. D., Sieber, S. C. and Ditzler, M. A. (1985). Metal ion sensors based on immobilized fluorogenic ligands. In Advances in Luminescence Spectromietry (eds. L. J. Cline Love and D. Eastwood), pp. 63-77. ASTM Pub. no. 863, Philadelphia.

Sepaniak, M. J., Tromberg, B. J. and Eastham, J. F. (1983). Optical fiber fluoroprobes in clinical analysis. Clin. Chem. 29, 1678-82.

Sutherland, R. M., Daehne, C. and Place, J.F. (1984a). Preliminary results obtained with a no-label homogeneous, optical immunoassay for human immunoglobulin G. Anal. Lett. 17, 43-53.

- and Ringrose, A.S. (1984b). Optical detection of antibody-antigen reactions at a glass-liquid interface, Clin. Chem, 30, 1533-8.

Urbano, E., Offenbacher, H. and Wolfbeis, O.S. (I984). Optical sensor for continuous determination of halides. Anal. Chem. 56, 427-9.

Uwira, N., Opitz, N. and Luebbers, D. W, (1984). Influence of enzyme concentration and thickness of the enzyme layer on the calibration curve of the continuously measuring glucose optode, Adv. Exp. Med. Biol. 169, 913-21. 


\subsubsection{Halide sensors}

Two different types of optical sensors for halide ions have been reported. One type is based on halide quenching of fluorescence from an immobilized organic cation (Urbano et al. 1984). Because heavy atoms are better quenchers than lighter atoms, the sensitivity of the sensor follows the order $\mathrm{I}^{-}>\mathrm{Br}^{-}>\mathrm{Cl}^{-}$. The other approach is based on silver fluoresceinate as a reagent (Hirschfield et al. 1983). The effect of added halide is to pull the silver away from the fluorescein rendering it fluorescent. This sensor follows the same sensitivity order as the first sensor. However, in the case of the second sensor the sensitivity order is determined by the relative solubilities of the various silver halide salts.

\subsubsection{Other sensors}

Sensors for glucose and for antigens based on competitive binding have been referred to earlier in the chapter and are described in detail in other chapters. As sensors for $\mathrm{pH}, \mathrm{CO}_{2}$, oxygen and other analytes are developed to a higher degree of refinement, one can expect that they will be coupled to other biological reagents to make sensors for new analytes. Already, an optical oxygen sensor has been coupled with immobilized oxidase enzymes to make sensors responding to the oxidase substrates (Voelkl et al. 1980; Uwira et al. 1984).

\section{References}

Arnold, M. A. (1985). Enzyme-based fiber optic sensor. Anal. Chem. 57, 565-6.

Chen, R.F. (1968). Fluorescent pH indicators: Spectral changes of 4-methylumbelliferone. Anal. Lett 1, 423-8.

Freeman, T.M. and Seitz, W. R. (1981). Oxygen probe based on tetrakis(alklylamino)ethylene chemiluminescence. Anal. Chem, 53, 98-102,

Hirschfield, T, Deaton, T., Milanovich, F. and Klainer, S. (1983). Feasibility of using fiber optics for monitoring groundwater contaminants. Opt. Eng. 22, 527-31.

Kawahara, F. K., Fiutem, R. A., Silvus, H.S., Newman, F.M. and Frazar, J. H. (1983). Development of a novel method for monitoring oils in water. Anal. Chim. Acta 151, 315-27.

Kirkbright, G.F., Narayanaswamy, R. and Welti, N.A. (1984a), Studies with immobilised chemical reagents using a flow-cell for the development of chemically sensitive fibre-optic devices. Analyst 109, 15-17.

- (1984b). Fibre-optic $\mathrm{pH}$ probe based on the use of an immobilised colorimetric indicator. Analyst 109, 1025-8.

Kroneis, H. W. and Marsoner, H. J. (1983). A fluorescence-based sterilizable oxygen probe for use in bioreactors. Sensors and Actuators 4, 587-92.

Luebbers, D. W. and Opitz, N. (1975). The $\mathrm{pCO}_{2}-/ \mathrm{pO}_{2}$-optode. New probe for measurement of partial pressure of carbon dioxide or partial pressure of oxygen in fluids and gases. Z. Naturforsch., C: Biosci. 30c, 532-3.

- (1983). Optical fluorescence sensors for continuous measurement of chemical concentration in biological systems. Sensors and Actuators 4, 641-54. 
water was found to reduce the susceptibility of the fluorophor to quenching (Peterson et al. 1984). The reference intensity for oxygen sensors has been the source intensity at the excitation wavelength measured either directly or as backscattered by the reagent phase. An alternative approach is to add a reference fluorophor that is not susceptible to oxygen quenching and emits at a different wavelength from the oxygen-susceptible fluorophor.

For oxygen measurements based on a change in intensity rather than a shift in spectrum, any change in response due to reagent degradation or changing susceptibility to quenching can only be determined by recalibrating the sensor. (This differs from the situation in optical $\mathrm{pH}$ sensors where the ratio of acid to base is the measured parameter.) The problem of reagent degradation can be dealt with by measuring the fluorescence lifetime rather than the fluorescence intensity. The ratio of the fluorescence lifetime in the absence of quencher to the lifetime in the presence of quencher is equivalent to $I_{\mathrm{fo}} / I_{\mathrm{f}}$. The lifetime approach to oxygen measurement will be easier to implement if a fluorophor or phosphor can be found with a relatively long lifetime so that the instrumental requirements for accurate lifetime measurements are simplified.

Another approach would be to base an oxygen sensor on a reagent that changes colour while reversibly binding oxygen. For example, an oxygen sensor can be based on changes in the absorption/reflection spectrum of immobilized haemoglobin. The problem with this approach is that reagents which reversibly bind oxygen tend to be subject to slow irreversible oxidation. Fot it to be viable a reagent phase which is sufficiently stable with respect to oxidation must be developed. The attractive feature of this approach is that it creates the possibility of measuring the ratio of the amount of reagent combined with oxygen to the amount of reagent not combined with oxygen.

\subsubsection{Metal ion sensors}

Metal ion sensors have been reported based on immobilized ligands that form fluorescent complexes (Saari and Seitz 1983; Seitz et al. 1985). In general any ligand that changes optical properties, either colour or fluorescence, upon complexation can potentially be used as an indicator in the reagent phase of a metal ion sensor. Just as the response range of a pH sensor depends on the $\mathrm{p} K_{\mathrm{a}}$ of the immobilized acid-base indicator, the range of metal concentration that can be sensed with an immobilized ligand as the reagent depend on the formation constant for complex formation. One of the problems inherent in designing metal ion sensors is that complex formation often involves the displacement of one or more protons by the metal ion. When this happens, response depends on a conditional formation constant which depends on $\mathrm{pH}$. Thus $\mathrm{pH}$ control is required for metal sensing. 
$\mathrm{CO}_{2}$ sensors are actually simpler than $\mathrm{pH}$ sensors. Because the internal filling solution is isolated from the sample by a hydrophobic gas-permeable membrane, variation in the ionic strength of the sample is less of a problem. Furthermore, the indicator can be dissolved in the internal filling solution rather than having to be immobilized on a solid substrate. The major problem in developing optical $\mathrm{CO}_{2}$ sensors is to achieve a short response time since the time to reach equilibrium in the internal filling solution is added to the intrinsic response time of the $\mathrm{pH}$ sensor. The solution to this problem lies primarily in engineering the sensor to minimize the distance that $\mathrm{CO}_{2}$ has to diffuse in solution for the device to reach equilibrium.

\subsubsection{Oxygen}

Most optical oxygen sensors reported to date are based on fluorescence quenching. Because they involve an equilibrium measurement, they are inherently less sensitive to variations in temperature and flow conditions than the widely used polarographic oxygen electrode. The fluorescent reagent can be in solution separated from the sample by a hydrophobic oxygenpermeable membrane or it can be incorporated into or onto a solid phase. In general the susceptibility of the reagent to oxygen quenching depends both on the fluorophor and the medium. Linear calibration curves with an intercept of 1.00 are observed plotting $I_{\mathrm{fo}} / \mathrm{I}_{\mathrm{f}} v s$ partial pressure of oxygen where $I_{\mathrm{fo}}$ is the fluorescence intensity in the absence of oxygen and $I_{\mathrm{r}}$ is the intensity in the presence of oxygen (eqn 30.6 assuming fluorescence intensity is proportional to unquenched reagent). Because curves are linear, a one-point calibration is possible.

A variety of reagent phases have been used in optical oxygen sensors. Pyrenebutyric acid has been used both in solution (Luebbers and Optiz 1975, 1983) and immobilized on a solid substrate (Wolfbeis et al. 1984). Because pyrenebutyrate has a long fluorescence lifetime, it has time to interact with oxygen and is therefore more susceptible to quenching than most fluorophors. However, pyrenebutyric acid requires excitation in the ultraviolet $(342 \mathrm{~nm})$. Perylenedibutyrate adsorbed on a hydrophobic matrix was selected as the reagent for an optical oxygen sensor after a screening study involving 70 dyes (Peterson et al. 1984). Because perylenedibutyrate is excited at $468 \mathrm{~nm}$ and emits at $514 \mathrm{~nm}$, it can be used in a sensor with plastic optical fibre. A sterilizable oxygen sensor based on a fluorophor cast directly into a silicone membrane has been reported (Kroneis and Marsoner 1983). Response times for optical oxygen sensors can be shorter than one second.

Because the reagent phases used for optical oxygen sensors are confined by a hydrophobic membrane, they are only subject to interference from volatile components of the sample. Certain anaesthetics such as halogenated hydrocarbons interfere by quenching fluorescence (Peterson et al, 1984). When a hydrophilic substrate was used for fluorophor immobilization, adsorbed 
HPTS provides a ratio measurement like 4-methylumbelliferone but can be used with glass optical fibre and an incandescent source.

Because of the three sulphonate groups the solution $\mathrm{p} K_{\mathrm{a}}$ of HPTS has a strong dependence on ionic strength. In fact, the difference in $\mathrm{pH}$ as measured with 4-methylumbelliferone from the $\mathrm{pH}$ measured with HPTS has been proposed as an optical indicator of ionic strength (Opitz and Luebbers $1983,1984)$. HPTS has been immobilized both covalently and by ion exchange (Zhujun and Seitz 1984a). Immobilization changes and can even reverse the ionic strength dependence of the $\mathrm{p} K_{\mathrm{a}}$. If HPTS can be immobilized on a substrate that keeps the ionic strength dependence of the $\mathrm{p} K_{\mathrm{a}}$ at an acceptable level it is likely to prove to be the indicator of choice for physiological pH measurements. (It should be noted that the author has stated in a paper that the $\mathrm{pH}$ measured by HPTS immobilized on an ion-exchange membrane is independent of ionic strength (Zhujun and Seitz 1984a). This was meant only to imply that the use of an intensity ratio measurement eliminates the effect of variations in fluorescence efficiency as a function of ionic strength and not that the $\mathrm{p} K_{\mathrm{a}}$ is independent of ionic strength when HPTS is immobilized on the membrane.)

Fluorescein and its derivatives can be also be used as fluorometric $\mathrm{pH}$ indicators (Milanovitch et al, 1984; Saari and Seitz 1982). They have the advantage that they can be efficiently excited by an argon ion laser.

Optical sensors for $\mathrm{pH}$ have a working range of 1 to $2 \mathrm{pH}$ units centred around the $\mathrm{p} K_{\mathrm{a}}$ of the indicator. Although there has been considerable effort directed towards finding indicators that are suitable for the physiological $\mathrm{pH}$ range, relatively little has been done with $\mathrm{pH}$ indicators that respond outside this range. A series of immobilized colorimetric indicators that respond at a variety of $\mathrm{pHs}$ has been evaluated for reflectance-based $\mathrm{pH}$ sensing (Kirkbright et al, 1984a, b).

\section{$30.5 .2 \mathrm{pCO}_{2}$}

Optical $\mathrm{CO}_{2}$ sensors are analogous to the Severinghaus $\mathrm{CO}_{2}$ electrode. An optical $\mathrm{pH}$ sensor is placed in contact with a reservoir of bicarbonate solution and covered with a $\mathrm{CO}_{2}$-permeable membrane, usually silicone. The $\mathrm{CO}_{2}$ partial pressure determines the concentration of carbonic acid in the internal filling solution which in turn determines the $\mathrm{pH}$ of the carbonic acid/bicarbonate buffer system. The range of $\mathrm{CO}_{2}$ partial pressures sensed depends on the bicarbonate concentration and on the $\mathrm{pH}$ sensitivity of the optical $\mathrm{pH}$ sensor. Optical sensors that respond to physiological $\mathrm{pHs}$ are suitable as the internal sensing element for sensors that respond to $\mathrm{CO}_{2}$ partial pressures in the range of physiological interest. The $\mathrm{pH}$ sensors that have received extensive evaluation for physiological applications have also been used as the internal sensing element in $\mathrm{pCO}_{2}$ sensors (Vurek et al. 1983; Zhujun and Seitz 1984b; Luebbers and Opitz 1975, 1983). 
time of 0.7 minute to reach $(1-(1 / \mathrm{e}))$ or $63 \%$ of the final value. The limitation of this sensor is that it is based on a change in absorbance/reflectance rather than fluorescence.

4-Methylumbelliferone has the attractive feature that the acid and base forms fluoresce at different wavelengths making it possible to relate $\mathrm{pH}$ to the ratio of intensities for the two forms of the indicator (Chen 1968; Luebbers et al. 1977). However, because 4-methylumbelliferone is excited in the ultraviolet region of the spectrum $(318 \mathrm{~nm})$, it requires relatively expensive instrumentation, i.e. an arc lamp source and fused silica optical fibre.

The trisodium salt of 8-hydroxy-1,3,6-pyrenetrisulfonic acid (HPTS) is another fluorescent indicator that has attracted considerable attention. In a study of solution indicators, it was judged the most suitable for physiological pH measurements (Wolfbeis et al. 1983). The spectral features of HPTS are shown in Fig. 30.2. The base form of the indicator is specifically excited at $470 \mathrm{~nm}$ while the acid form is selectively excited at $405 \mathrm{~nm}$. In a buffered medium over the physiological pH range, the acid form of HPTS undergoes excited state deprotonation more rapidly than it fluoresces. As a consequence, emission from the base form of the indicator is observed even when the ground state indicator is in the acid form. The ratio of fluorescence intensities excited at $405 \mathrm{~nm}$ and $470 \mathrm{~nm}$ is the measured parameter related to $\mathrm{pH}$.

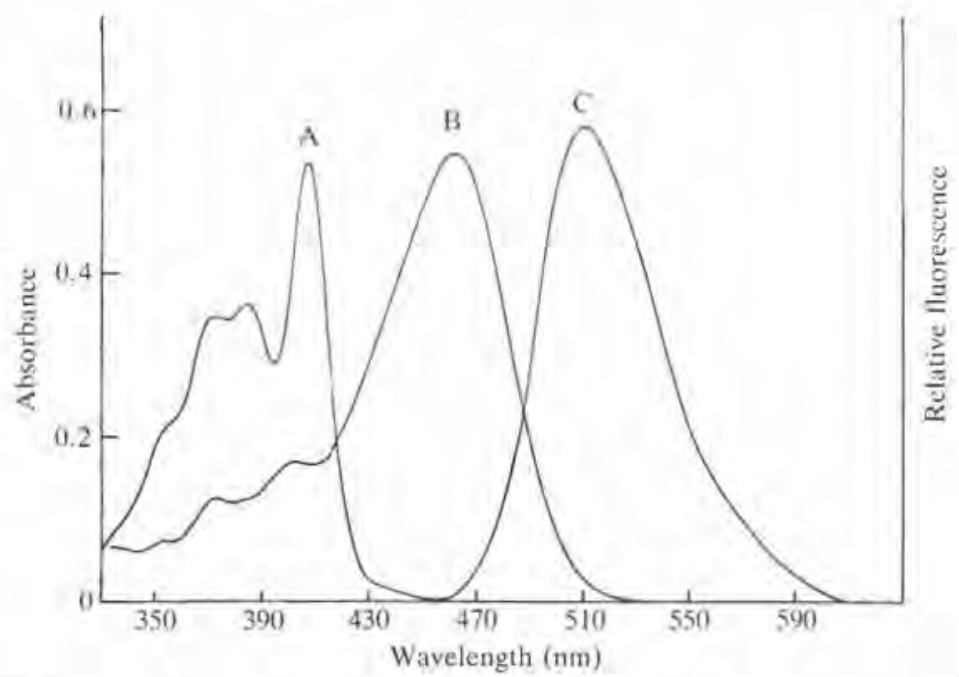

Fig. 30.2 Spectral Properties of HPTS. A is the absorption spectrum of the acid form of HPTS, B is the absorption spectrum of the base form of HPTS and C is the fluorescence emission spectrum of the base form of HPTS. At neutral pH's the fluorescence of the base is observed even when the indicator is in the ground state acid form. 
than being proportional to the concentration of product. The measured parameter is steady-state light emission as analyte diffuses into the reagent phase and reacts. Once formed the product does not contribute further to the observed signal. The rate of the light-producing process will slow down as reagent is consumed. This problem can be minimized, however, if the amount of reagent is large relative to the rate of reagent consumption. A sensitive oxygen sensor based on tetrakis-alkyaminoethylene chemiluminescence has been reported (Freeman and Seitz 1981; MacDonald and Seitz 1982).

Sensors based on chemi/bioluminescence have the attractive feature that no excitation source is required since the analytical reaction generates its own light. However, because the measured signal involves a steady state, it is inherently sensitive to parameters influencing either the supply of analyte to the sensor surface or the rate of the light producing process. The possibilities of such sensors are discussed in more detail in the chapter by McCapra (Chapter 31).

\section{0,4.5 Adsorbent}

The reagent phase can be an adsorbent of some sort that selectively concentrates an optically detectable analyte in the field of view of the optical fibre. In effect this involves the combination of a separation with direct spectroscopy. A sensor for polyaromatic hydrocarbons based on this principle has been reported (Kawahara et al. 1983). However, while there has been interest in direct in vivo measurement of optically detectable analytes (Sepaniak et al. 1983), there are as yet no examples of biological applications of this approach.

\subsection{Applications}

\section{$30.5 .1 \mathrm{pH}$}

Because optical sensors offer the prospect of improved stability relative to electrodes with respect to calibration, considerable effort has been devoted to the development of an optical $\mathrm{pH}$ sensor suitable for continuous in vivo measurement. Various indicators have been used, each with its own set of advantages and disadvantages. Phenol red changes adsorption characteristics with $\mathrm{pH}$ at wavelengths longer than $450 \mathrm{~nm}$ and thus can be used with plastic optical fibres (Peterson et al. 1980). The covalently immobilized indicator has a $\mathrm{p} K_{\mathrm{a}}$ of 7.6 compared to the solution $\mathrm{p} K_{\mathrm{a}}$ of 7.9 . The measured parameter is the ratio of intensity reflected/transmitted by the reagent phase at $558 \mathrm{~nm}$ where the base form of the indicator absorbs to the intensity at $600 \mathrm{~nm}$ where neither form of the indicator absorbs. The sensor measures $\mathrm{pH}$ to $0.01 \mathrm{pH}$ unit in the range from 7.0 to 7.4 . It is stable, with respect to calibration, for hours. It responds exponentially to changes in $\mathrm{pH}$ with a response 
The use of two wavelengths for the measurement effectively provides a convenient reference intensity.

In addition to the glucose sensor (Schultz et al. 1982) the competitive binding approach can potentially be applied to many other reactions. In addition to providing access to analytes that cannot be sensed directly, it has the attractive feature that the range of analyte concentrations that are sensed can be controlled to some degree by varying the ligand concentration.

The major limitation of the competitive binding approach is likely to be the rate of response. A strong association between ligand/analyte and reagent inherently means that the rate of dissociation is slow. Since dissociation of ligand/analyte from the reagent is necessary for the sensor to respond, this means a slow response time.

The competitive binding approach to sensing is described in more depth in Chapter 32.

\subsubsection{Catalyst}

The immobilized reagent can catalyse transformation of an analyte to a product with different optical properties. For example, a sensor has been reported based on the use of immobilized alkaline phosphatase to catalyse the hydrolysis of $p$-nitrophenylphosphate to $p$-nitrophenoxide (Arnold 1985). The measured signal is the steady-state absorbance of product under conditions where the rate of product generation is balanced by the rate of product diffusion away from the sensor. This approach can potentially be applied using other enzymes and thus lead to a whole class of new devices.

Catalyst-based sensors require careful control of conditions. Factors affecting both the rate of the catalysed reaction and the efficiency of mass transfer to and from the sensor surface will affect the steady-state signal. While the approach warrants further study, it remains to be demonstrated whether or not these sensors will prove to be practical.

\subsubsection{Chemiluminescence}

In general it is not possible to develop continuous sensing devices using a reagent phase which reacts irreversibly with analyte to form a product (unless there are provisions for removing the product and renewing the reagent). Instead, these devices would act as integrating sensors since the amount of product formed would be proportional to the amount of analyte that has contacted the sensor since it was first placed in operation. In some cases the product can be removed by exposing the immobilized reagent phase to a different medium (Sutherland et al. 1984a, b). In this case the sensor is 'rechargeable' and can be used for multiple measurements on an integrating basis.

Devices based on chemiluminescence and bioluminescence represent an exception to the above generalization. The reason for this is that chemi/bioluminescence is proportional to the rate at which product is generated rather 
immobilization matrix (e.g. by causing it to swell) thereby influencing $K_{\mathrm{e}}$. Problems of this sort are likely to be identified as optical sensors reach the point where they are evaluated for long term response characteristics in practical contexts.

The above analysis assumes that the amount of analyte combining with or released by the immobilized reagent is small relative to the amount of analyte in the sample. If this is not the case, systematic errors can arise when the concentration of analyte changes.

\subsubsection{Competitive binding}

The concept of sensors based on competitive binding was first proposed by Schultz and Sims (1979). In this type of sensor, the immobilized reagent phase includes both a selective reagent and a 'ligand' which binds to the reagent. Added analyte displaces the ligand from the reagent,

$$
\mathrm{A}+\mathrm{LR}<\longrightarrow \mathrm{L}+\mathrm{AR}
$$

where $\mathrm{L}=$ ligand. For this reation to occur, the ligand or the reagent must be in solution. Whichever of the two is in solution must be larger than the analyte so it can be immobilized by confinement behind a size-selective membrane which is permeable to analyte.

The measured optical parameter is based on the ligand-reagent interaction. The first reported competitive binding sensor was developed for glucose. In this device, the reagent, concanavalin A, is immobilized on the inside surface of a hollow fibre permeable to glucose (Schultz et al. 1982). When the end of the optical fibre is placed inside the hollow fibre, the immobilized concanavalin A is out of the optical path. As a consequence fluorophor-labelled ligand fluorescein-labelled dextran, is not excited when bound to concanavalin A. Added glucose displaces the dextran allowing it to diffuse into the optical path where fluorescence is excited. Therefore, increasing glucose levels are accompanied by increases in the observed fluorescence signal. Because the dextran is too large to pass through the hollow fibre, it remains immobilized.

In on-going work in the author's laboratory, competitive binding sensors are being developed using fluorescence energy transfer. In this case the ligand is labelled with a donor and the reagent with an acceptor (or vice versa). The excitation wavelength is set to selectively excite the donor. When ligand is bound to reagent the distance between the donor and acceptor is short so that energy transfer from the donor to the acceptor occurs. When analyte binds to reagent displacing ligand, the distance between the donor and acceptor increases so that energy transfer does not occur. This leads to an increase in donor emission and a decrease in acceptor emission. The measured parameter is the ratio of emission intensities for donor and acceptor fluorescence. 
immobilized phase are equivalent for $\mathrm{R}$ and $\mathrm{AR}$ and thus cancel.

$A R$ and $R$ vary with analyte concentration as follows:

$$
\begin{aligned}
& A R=K_{\mathrm{e}}[\mathrm{A}] C_{\mathrm{r}} /\left(1+K_{\mathrm{e}}[\mathrm{A}]\right) \\
& R=C_{\mathrm{r}} /\left(1+K_{\mathrm{e}}[\mathrm{A}]\right)
\end{aligned}
$$

where $C_{r}$ is the sum of free and combined reagent:

$$
C_{\mathrm{t}}=A R+R \text {. }
$$

$C_{\mathrm{r}}$ is necessarily fixed since there will be a constant number of moles of reagents in the immobilized phase.

If the measured optical parameter is proportional to $A R$, then response is proportional to $[\mathrm{A}]$ at low concentrations ([A] $\ll 1 / K_{\mathrm{e}}$ ) and shows saturation behaviour reaching a limiting value as $[\mathrm{A}]$ increases to values $\gg 1 / K_{e}$.

If the measured parameter is proportional to $R$, then the signal decreases with added analyte. In this case, a linear working curve is obtained based on a rearranged form of eqn 30.4 ,

$$
C_{r} / R=1+K_{\mathrm{e}}[\mathrm{A}] \text {. }
$$

The ratio of signal in the absence of added analyte to the signal in the presence of analyte is the measured parameter. This situation is commonly encountered in sensors based on fluorescence quenching. In this case $K_{\mathrm{e}}$ is not a true equilibrium constant. Instead it is a measure of the fluorescent reagent's susceptibility to quenching and depends on the rate of quenching relative to the rates of other excited state processes.

The dependence on $C_{r}$, the amount of immobilized indicator, can be eliminated if one can measure both $A R$ and $R$, since the ratio of the two is directly proportional to analyte concentration,

$$
A R / R=K_{\mathrm{e}}[\mathrm{A}] \text {. }
$$

Where feasible, this is the preferred situation. Effectively, the measurement of $R$ serves as the reference for the measurement of $A R$, since the measured ratio is independent of instrumental fluctuations and any changes in reagent phase optical properties.

Because the reagent is serving as an indicator, the response necessarily depends on $K_{\mathrm{e}^{2}}$. For a reagent to be useful for an optical sensor, it must not only have suitable optical characteristics but must also have a $K_{\mathrm{e}}$ appropriate to the range of concentrations to be measured. (For example, the range of $\mathrm{pH}$ s measured by an optical $\mathrm{pH}$ sensor depends on the $\mathrm{p} K_{\mathrm{a}}$ of the immobilized indicator.) Any uncontrolled variable that influences $K_{\mathrm{e}}$ is a potential source of error. For example, because the activity of charged reagents depends on ionic strength, variations in ionic strength can be a source of error. In addition to direct effects on $K_{\mathrm{e}}$, there can also be indirect effects. For example, variations in temperature and/or ionic strength can affect the 
in absorption by the reagent phase, then the magnitude of the inner filter effect on fluorescence will vary with fluorophor concentration and the measured intensity will no longer be proportional to concentration. For cxample, using equations reported in the literature (Zhujun and Seitz 1984a), it can be calculated that the response of a fluorescent indicator to $\mathrm{pH}$ will show substantial shifts when there are large inner filter effects.

In sensors based on colour changes, the problem of developing a theoretical relationship between concentration of absorber and measured intensity is considerably more difficult. The first problem is that there is no way to measure the reference intensity at the same wavelength in the absence of absorber. Instead the reference intensity has to be measured at a different wavelength where the incident radiation is not absorbed or through a separate optical path which bypasses the reagent phase. The second problem is that the interaction of the incident radiation with the coloured reagent will involve both reflection and absorption. While optical sensors based on colour changes have been shown to respond linearly to analyte over relatively small concentration ranges (Peterson et al. 1980), the difficulty in developing a quantitative relationship between signal and analyte concentration remains a problem. In addition to optical convenience, this is another reason why sensors based on changes in fluorescence are preferred where feasible.

\subsection{Reagent considerations}

The immobilized reagent phase of an optical sensor can interact with analyte in a variety of ways which are considered below.

\subsubsection{Indicator}

The reagent can act as an indicator, reversibly reacting with the analyte to form a product that has different optical properties from the uncombined reagent. Where feasible, this approach is attractive because the measured signal involves an equilibrium. The majority of optical sensors reported to date involve reagents acting as indicators. An example would be a $\mathrm{pH}$ sensor where the reagent can be considered to be the base form of an indicator, the analyte is hydrogen ion and the product is protonated indicator.

The interaction of an indicator reagent with analyte may be represented;

$$
\mathrm{A}+\mathrm{R}<\longrightarrow \mathrm{AR}
$$

where $\mathrm{A}=$ analyte, $\mathrm{R}=$ reagent, and $\mathrm{AR}=$ combined reagent. If the indicator reaction is $1: 1$, then the equilibrium constant, $K_{\mathrm{e}}$, will be

$$
K_{e}=A R /[\mathrm{A}] R
$$

where $R$ and $A R$ are the moles of free and combined reagent molecules in the immobilized phase, respectively. It is assumed that activity effects in the 
fibres are used to transmit light to and from an immobilized reagent phase. The reagent is immobilized on solid substrate particles represented as spheres in the figure. A tubular membrane slipped over the two fibres serves to hold the reagent in place. Analyte diffuses through the membrane to interact with the reagent. A cap at the end of the tubular membrane blocks the incident radiation from directly interacting with the sample, avoiding a potential source of interference. In addition to serving as the immobilization substrate, the solid particles scatter the incident radiation so that some of it is redirected into the second fibre which leads to a detection system. The arrangement in Fig. 30.1a has been successfully employed in sensors designed for biochemical measurements of $\mathrm{pH}$ and oxygen (Peterson et al. 1980; Peterson et al. 1984). The reagent phase is a typically few millimeters long in this type of sensor.

The arrangement in Fig. 30.1 b differs from that in Fig. 30.1a only in that a single fibre is used to transmit light both to and from the reagent phase. This arrangement is suitable for sensors based on changes in fluorescence because the fluorescence of interest is readily distinguished from scattered excitation radiation on the basis of wavelength.

In Fig. 30.1c the cladding is removed from part of the optical fibre and replaced by the reagent phase. This arrangement takes advantage of the fact that light transmitted through the optical fibre penetrates a small distance into the cladding. Changes in the refractive index and/or the absorptive properties of the reagent phase will modify the intensity of light transmitted through the fibre. Furthermore, it is possible to excite fluorescence from reagents on the surface of fibre. This optical configuration has been used for immunoassay (Sutherland et al. 1984a,b), an application which is described in more detail in Chapter 33. However it is unsuitable for many applications because a relatively long length of fibre has to be coated with reagent,

The immobilized reagent phase of an optical sensor is generally not a welldefined medium for spectroscopy. If the reagent is immobilized on a solid phase, there will be considerable scattering of the incident radiation. This is particulary true if the reagent phase consists of solid particles as shown in Fig. $30.1 \mathrm{a}$ and b. Because the immobilized reagent phase is used on a continuous basis, the effect of optical inhomogeneities on the measured signal can be accounted for by calibration. However, the calibration procedure will be simpler and more reliable if the relationship between the optical signal and the measured parameter is known. In sensors based on fluorescence, it has generally been assumed that fluorescence intensity is directly proportional to the concentration of fluorophor. While this is probably a good assumption in most cases, it should be recognized that there may be significant inner filter effects due to absorption of the excitation radiation by the immobilized reagent. If changes in fluorophor concentration are accompanied by changes 
the best approach is to incorporate the reference signal into the immobilized reagent itself. For example, in a $\mathrm{pH}$ sensor based on an indicator with acid and base forms that fluoresce at different wavelengths, the measured parameter can be the ratio of fluorescence for the two forms (Zhujun and Seitz 1984a). This type of reference compensates not only for instrumental fluctuations and variations in reagent phase optical properties but also for changes in the amount of immobilized indicator due to slow decomposition or some other process.

The type of optical fibre used influences the cost and performance of optical sensors. Plastic has the lowest cost and is the safest, but is limited to wavelengths above about $450 \mathrm{~nm}$. Glass fibres are somewhat more expensive but are suitable for measurements down to about $380 \mathrm{~nm}$. Because measurements below $380 \mathrm{~nm}$ require arc lamp sources and considerably more expensive fused silica fibre, there is a significant cost incentive to use immobilized reagent phases that change optical properties in the visible region of the spectrum. Fibre diameters have typically been in the range from 50 to 200 micrometers.

Optical sensors can be configured in several ways, some of which are illustrated in Fig. 30.1. Figure 30.1a shows a bifurcated device in which separate

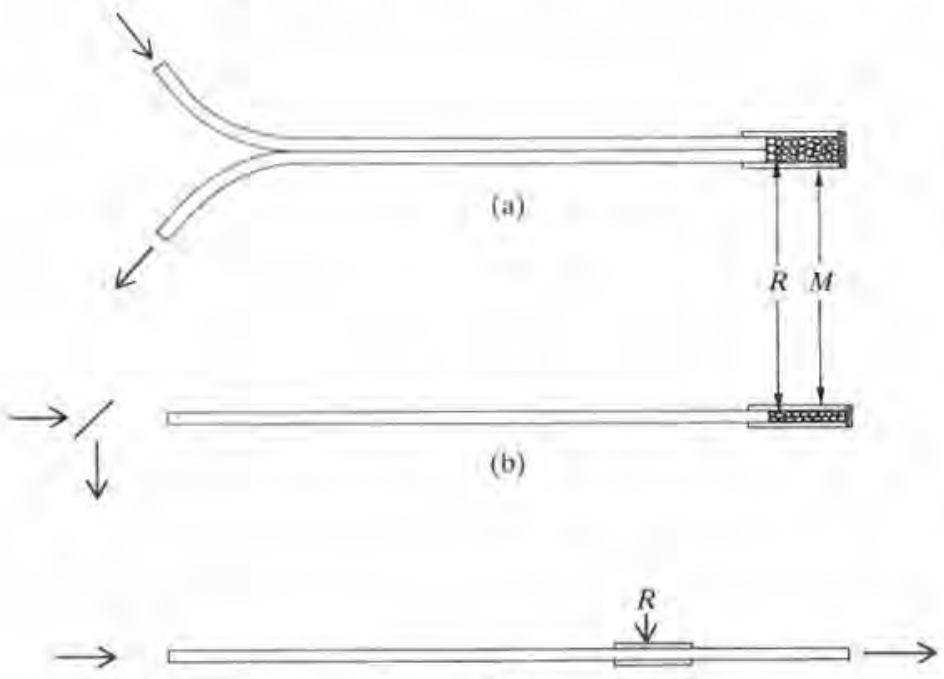

(c)

Fig. 30.1 Optical Sensor Configurations. M, membrane permeable to analyte; $\mathrm{R}$, immobilized reagent phase. The arrows represent the direction in which light is travelling. (a) Bifurcated sensor in which separate fibres carry light to and from the immobilized reagent. (b) Sensor in which the same fibre(s) carry light to and from the reagent, and a beam splitter is used to redirect emerging light to a detection system.

(c) Sensor in which the reagent phase is coated on the outside of the fibre. 
difficult at best to find reagent phases that can be used reliably for longer time periods.

5) Response times for some optical sensors may be slow because there must be mass transfer of analyte to or from the reagent phase before constant response is reached. While response times can be reduced by making the reagent phase smaller, this is accompanied by a decrease in the amount of reagent and the magnitude of the optical signal.

\subsection{Instrumental considerations}

The instrumentation required for optical sensors depends on the intended application. Fortunately, most biomedical applications do not require that the signal be transmitted for long distances through optical fibre. This means that the degree of signal attenuation in the fibre is relatively small and successful sensors can be designed using conventional continuum sources rather than requiring the greater intensity available only with lasers. This not only reduces instrumentation costs but also allows greater flexibility in choice of immobilized reagent phases since there are fewer limitations on available wavelengths. The majority of biomedical applications reported to date as well as the first commercial chemical sensor system based on fibre optics (Cardiovascular Devices Inc.) involve incandescent sources and interference filters. Measurements at more than one wavelength can be accomplished by arrangements for sequentially inserting different filters into the optical path or by splitting the beam after it returns from the immobilized reagent phase.

In some applications an argon laser has been used as a source (Milanovich et al. 1984; Schultz et al. 1982). The high intensity available from the laser makes it possible to miniaturize the sensor while maintaining acceptable intensities. It is particularly well suited for immobilized reagents based on fluorescein derivatives because the $488 \mathrm{~nm}$ laser line efficiently excites fluorescein fluorescence.

While, unlike electrodes, optical sensors do not require a separate reference sensor, the response characteristics of optical sensors are generally improved by comparing the analytical signal relative to a reference intensity. This can be accomplished in several ways. The simplest is to directly measure source intensity at the analytical wavelength to compensate for source fluctuations. A more attractive approach is to use a reference intensity which follows an optical path through the immobilized reagent since this will compensate for any changes in the optical properties of the reagent phase, (e.g., changes in scattering by the reagent phase due to variation in refractive index of the sample with time). Back-scattered excitation radiation has been used as a reference in an oxygen sensor based on fluorescence (Peterson et al. 1984). Alternatively, a fluorophor that is insensitive to analyte can be incorporated into the reagent phase to provide a reference signal. Where feasible, 
will be particularly true if the sensor involves the measurement of the ratio of intensities at two different wavelengths. For example, a highly stable $\mathrm{pH}$ sensor can be prepared if one optically measures the amounts of both the acid and base forms of an indicator and relates $\mathrm{pH}$ to the ratio of the two.

7) Optical sensors that simultaneously respond to more than one analyte can be prepared using multiple immobilized reagents with different wavelength characteristics. For example, a sensor responding simultaneously to $\mathrm{O}_{2}$ and $\mathrm{CO}_{2}$ has been reported (Opitz and Luebbers 1976).

8) Multiwavelength measurements can be used to monitor any change in the optical properties of the immobilized reagent phase which might indicate decomposition of the reagent or some other process affecting the ability of the immobilized reagent phase to respond accurately. This information could be used to decide when to replace the reagent phase.

The last three advantages of optical sensors reflect the fact that optical sensors have the potential for higher information content than electrical sensors because there is a complete spectrum of information available. In addition, in luminescence-based sensors there is further information that can be acquired by measuring luminescence lifetimes. The challenge for the chemist is to design immobilized reagent phases that exploit this potential. If appropriate reagent phases can not be developed, then the advantages of optical sensors will not be realized in practice.

In general, optical sensors have the following limitations relative to electrical sensors.

1) They are subject to background from ambient light. This can be eliminated by excluding light and/or modulation techniques.

2) Compared to some electrical sensors, optical sensors have limited dynamic ranges. For example, the $\mathrm{pH}$ electrode has a dynamic range of greater than $10^{12}$ compared to a typical range of $10^{2}$ for an optical $\mathrm{pH}$ sensor.

3) Optical sensors are 'extensive' devices. The signal depends on the amount of reagent. Thus miniaturizing the sensor causes a decrease in the magnitude of measured intensities which in turn complicates the technology of the measurement.

4) Long-term stability of immobilized reagents subjected to incident light is likely to be a problem. The stability of a particular reagent will depend on several factors involving both the intrinsic reactivity of the reagent and operating parameters of the sensor such as temperature and the intensity and duration of incident light. While it is reasonable to expect development of reagent phases that are stable for days and weeks, it will be 
nuous basis and will not consider systems where the immobilized reagent is used on a one-time basis. It will, however, include systems where immobilized reagents have been used for continuous optical measurements without fibre optics, since these reagents could easily be adapted for use with optical fibres.

Prior reviews have considered biomedical applications of all types of fibreoptic sensors (Peterson and Vurek 1984) and general characteristics of chemical sensors based on fibre optics (Seitz 1984).

\subsection{Advantages and limitations}

Because most optical sensors reported to date respond to analytes that can also be sensed electrically, electrochemical sensors have provided the frame of reference for evaluating the performance of optical sensors. A general comparison must be treated with caution, however. Since optical sensors are based on very different principles than electrical sensors, the relative merit of the two will depend on both the particular analyte being measured and the demands of a particular application. In general, optical sensors offer the following advantages relative to electrodes.

1) No 'reference electrode' is required. (However, as discussed below, it is good practice to measure the signal of interest relative to a reference intensity of some sort. This can involve the addition of a reference compound to the reagent phase.)

2) Because the signal is optical, it is not subject to electrical interference. This advantage is particularly important for sensors operated in electrically noisy environments.

3) The immobilized reagent phase does not have to be in physical contact with the optical fibre. This simplifies the development of sensors in which the immobilized reagent phase can be conveniently discarded and replaced. This is likely to be important in practice because it will be difficult to develop reagent phases with sufficient stability to be useful on an indefinite basis.

4) Optical devices are inherently safer than electrical devices when used for in vivo biomedical measurements because there is no danger of electrical shock.

5) Certain analytes, most notably oxygen, can be sensed on an equilibrium basis optically but not electrochemically. Because, once equilibrium is reached, 'equilibrium' sensors do not require a steady-state supply of analyte to the sensor surface, they are inherently less sensitive to fluctuations in temperature and flow conditions in the sample than amperometric electrochemical sensors.

6) Optical sensors can be highly stable with respect to calibration. This 


\title{
Optical sensors based on immobilized reagents
}

\author{
W. RUDOLF SEITZ
}

\section{0,1 Introduction}

This chapter will deal with devices involving an immobilized reagent phase on the end of a single optical fibre or a fibre bundle. Interaction of the component being measured (i.e. the analyte) with the immobilized reagent phase causes a change in the optical properties of the reagent phase which is measured through the optical fibre. An example would be a $\mathrm{pH}$ sensor prepared by placing an immobilized acid-base indicator on the end of an optical fibre bundle.

These sensors involve the synthesis of two ideas. One is the use of optical fibres to bring light from a spectrometer to a sample and back. The other is the use of immobilization as a means of allowing a chemical reagent to be used on a continuous rather than on a one-time basis. While both ideas have been applied individually for many years, their combination is relatively new and presents exciting possibilities that are just beginning to be realized. The use of optical fibres effectively permits scientists to 'bring the spectrometer to the sample', while the use of immobilized reagent phases makes it possible to do chemistry on the sample in situ.

D. W. Luebbers and co-workers were the first to use immobilized indicators for continuous measurements in biological fluids (Luebbers and Opitz 1975). They used the term 'optode' for their devices in analogy to 'electrode'. Subsequently, T. Hirshfeld and co-workers have introduced the term 'optrode'. Since the ' $r$ ' belongs to the root word, electrical, 'optode' is the grammatically correct term. However, because 'optode' suggests a kind of small wart-causing amphibian, the more euphonious 'optrode' may become the accepted term. In this chapter both terms will be avoided in favour of 'optical sensors'.

\subsubsection{Scope of chapter}

This chapter will review the advantages and limitations of optical sensors relative to electrodes. The instrumentation required for an optical sensor system will be briefly described. The ways in which the immobilized reagent phase can interact with analyte will be described and illustrated with specific devices. The chapter will confine itself to devices which respond on a conti- 

Photometry 

Scheller, F., Siegbahn, N., Danielsson, B, and Mosbach, K. (1985). High-sensitivity enzyme thermistor of L-lactate by substrate recycling. Anal. Chem. 57, 1740-3.

Schmidt, H.-L., Krisam, G. and Grenner, G. (1976). Microcalorimetric methods for substrate determinations in flow streams with immobilized enzymes. Biochim. Biophys. Acta 429, 283-90.

Tran-Minh, C. and Vallin, D. (1978). Enzyme-bound thermistor as an enthalpimetric sensor. Anal. Chem. 50, 1874-8.

Weaver, J.C., Cooney, C. L., Fulton, S.P., Schuler, D. and Tannenbaum, S. R. (1976). Experiments and calculation concerning a thermal enzyme probe. Biochim. Biophys. Acla 452, 285-91.

Winquist, F., Danielsson, B., Malpote, J.-Y., Persson, L. and Larsson, M.-B. (1985). Enzyme thermistor determination of oxalate with immobilized oxalate oxidase. Anal. Lett. 18, 573-88. 
glucose oxidase column. Talanta 31, 131-2.

Mandenius, C. F., Bülow, L., Danielsson, B. and Mosbach, K. (1985). Monitoring and control of enzymic sucrose hydrolysis using on-line biosensors. Appl. Microbiol, Biotechnol, 21, 135-42.

Marconi, W. (1978). Biomedical applications of enzymatic fibres. In Enzyme engineering (eds. G. B. Broun, G. Manecke, and L. B. Wingard, Ir.), Vol. 4, p. 179-86. Plenum Press, New York.

Martin, C. J, and Marini, M. A. (1977). Microcalorimetry in biochemical analysis. CRC Crit, Rev. in Anal. Chem. 8, 221-86.

Mattiasson, B, and Danielsson, B. (1982). Calorimetric analysis of sugars and sugar derivatives with aid of an enzyme thermistor. Carbohydr, Res. 102, 273-82.

— and Mosbach, K. (1976). A split-flow enzyme thermistor. Anal. Lett., 9, 867-89. Borrebaeck, C., Sanfridsson, B, and Mosbach, K. (1977). Thermometric enzyme linked immunosorbent assay: TELISA. Biochim. Biophys. Acta 483, 221-7.

- Danielsson, B., Winquist, F., Nilsson, H. and Mosbach, K. (1981). Enzyme thermistor analysis of penicillin in standard solutions and in fermentation broths. Appl. Environ. Microbiol. 41, 903-8.

- Mandenius, C.F., Axelsson, J.P., Danielsson B. and Hagander, P. (1983). Computer control of fermentation with biosensors. Ann. N. Y. Acad. Sci. 413, 193-6.

McGlothlin, C. D. and Jordan, J. (1975). Enzymatic enthalpimetry, a new approach to clinical analysis: glucose determination by hexokinase catalyzed phosphorylation. Anal. Chem. 47, 786-90.

Mosbach, K, and Danielsson, B. (1974). An enzyme thermistor. Biochim. Biophys. Acta 364, 140-5.

— and Danielsson, B. (1981). Thermal bioanalyzers in flow streams-enzyme thermistor devices, Anal. Chem. 53, 83A-94A.

- Borgerud, A. and Scott, M. (1975). Determination of heat changes in the proximity of immobilized enzymes with an enzyme thermistor and its use for the assay of metabolites, Biochim, Biophys. Acta 403, 256-65.

- Mattiasson, B., Gestrelius, S., Srere, P.A. and Danielsson, B. (1974). Theoretical and practical aspects of immobilized multi-step enzyme systems. In Enzyme engineering (eds. E.K. Pye and L. B. Wingard, Jr.), Vol, 2, p. 151. Plenum, New York.

Pennington, S. N. (1976). A small volume microcalorimeter for analytical determinations. Anal. Biochem. 72, 230-7.

Poe, M., Gutfreund, H. and Estabrook, R. W. (1967). Kinetic studies of temperature changes and oxygen uptake in a differential calorimeter: The heat of oxidation of NADH and succinate. Arch. Biochem. Biophys, 122, 204-11.

Rehak, N. N. and Young, D.S. (1978). Prospective applications of calorimetry in the clinical laboratory, Clin. Chem. 24, 1414-19.

Rich, S., Ianiello, R. M. and Jespersen, N. D. (1979). Development and application of a thermistor enzyme probe in the urea-urease system, Anal. Chem. 51, 204-6.

Satoh, I., Danielsson, B. and Mosbach, K. (1981). Triglyceride determination with use of an enzyme thermistor. Anal. Chim. Acta 131, 255-62.

- Ogawa, T. and Danielsson, B. (1986). Calorimetric phospholipid determination. Submitted. 
Canning, L.M., Sayers, C.N. and Carr, P.W. (1976). Rapid flowenthalpimetric determination of urea in serum with use of an immobilized urease reactor. Clin. Chem. 22, 1314-8.

Brown, H. D. (1969). Calorimetry of enzyme catalyzed reactions. In Biochemical microcalorimetry (ed. H. D. Brown), p. 149. Academic Press, New York.

Cooney, C. L., Weaver, J. C., Tannenbaum, S. R., Faller, D. V., Shields, A. and Jahnke, M. (1974). The thermal enzyme probe - a novel approach to chemical analysis. In enzyme engineering (eds. E. K. Pye and L. B. Wingard, Jr.), Vol. 2. p. 411-7. Plenum. New York.

Danielsson, B. and Mosbach, K. (1979). Determination of enzyme activities with the enzyme thermistor unit. FEBS Lett. 101, 47-50.

- Mattiasson, B. and Mosbach, K. (1981a), Enzyme thermistor devices and their analytical applications. Appl. Biochem. Bioeng. 3, 97-143.

- Gadd, K., Mattiasson, B, and Mosbach, K. (1977). Enzyme thermistor determination of glucose in serum using immobilized glucose oxidase. Clin. Chim. Acta 81, 163-75.

(1976). Determination of urea with an enzyme thermistor using immobilized urease. Anal. Lett. 9, 987-1001.

Mattiasson, B., Karlsson, R. and Winquist, F. (1979). Use of an enzyme thermistor in continuous measurements and enzyme reactor control. Biotechnol. Bioeng. 21, 1749-66.

- Bülow, L., Lowe, C. R., Satoh, I, and Mosbach, K. (1981b). Evaluation of the enzyme thermistor as a specific detector for chromatographic procedures. Anal. Biochem. 117, 84-93.

- Rieke, E., Mattiasson, B., Winquist, F, and Mosbach, K. (1981c). Determination by the enzyme thermistor of cellobiose formed on degradation of cellulose. Appl. Biochem. Biotechnol. 6, 207-22.

Decristoforo, G. and Danielsson, B. (1984). Flow injection analysis with enzyme thermistor detector for automated determination of $\beta$-lactams. Anal. Chem. 56, 263-8.

Fulton, S. P., Cooney, C.L. and Weaver, J.C. (1980). Thermal enzyme probe differential temperature measurements in a laminar flow-through cell. Anal. Chem. 52, 505-8.

Grime, J.K. (1980). Biochemical and clinical analysis by enthalpimetric measurements - a realistic alternative approach? Anal. Chim. Acta 118, 191-225.

(1985). Application of solution calorimetry to biochemical and clinical analyses. In Analytical solution calorimetry (ed. J. K. Grime), p. 345. Wiley-Interscience, New York.

- and Tan, B. (1979). The determination of some selected penicillins by enzymatic enthalpimetry. Anal. Chim. Acta 107, 319-26.

Guilbault, G. G., Danielsson, B., Mandenius, C.F. and Mosbach, K. (1983). A comparison of enzyme electrode and thermistor probes for assay of alcohols using alcohol oxidase. Anal. Chem. 55, 1582-5.

Johansson, А., Mattiasson, B. and Mosbach, K. (1976). Immobilized enzymes in microcalorimetry. Methods in Enzymol. 44, 659-67.

Kiba, N., Tomiyasu, T. and Furusawa, M. (1984). Flow enthalpimetric determination of glucose based on oxidation by 1.4-benzoquinone with use of immobilized 
the case of cyanide, that can be determined with a sensitivity of $10^{-5} \mathrm{M}$ using the enzyme rhodanese (Danielsson et al. 1981a). Even for pesticides and acetylcholine esterase inhibitors a direct approach can be envisaged (Danielsson et al. 1981a). Alternatively, one may utilize immobilized, intact cells, organelles, or multi-enzyme systems placed in the column in case a more general detection of poisonous material is required. With such a system a larger number of potentially toxic compounds would be detected as these affect the overall metabolism, leading to decreased heat generation.

\subsection{Conclusion}

Despite many attractive properties, surprisingly few immobilized enzyme probes have yet been applied in commercial instruments. To date only a very limited number of enzyme-electrode based systems have been introduced. The rapidly growing importance of biotechnology will lead to an increasing demand for flow stream analysers to be used in process control, fermentation monitoring, and downstream analysis. In this field immobilized enzyme probes will certainly find their applications as they allow for highly specific continuous flow analysis. The use of immobilized enzymes cuts down enzyme costs and increases the operational stability. Thermal flow analysers appear to be particularly attractive due to the possibility of analysing turbid, particulate, or coloured samples. Furthermore, they are based on the most general detection principle, the detection of the heat of reaction, which makes them directly applicable to most enzymic reactions. The heat formed by the primary enzymic reaction is usually sufficient for reliable measurements thus obviating the need for auxiliary enzymic reactions generally required in other analytical procedures (often involving expensive co-enzymes - especially disadvantageous in continuous flow analysis). The prospects are also good for applications of thermal flow analysers in medical instruments, for instance in flow analysers for metabolite monitoring. Parallel with the development of highly sophisticated, high-capacity multichannel laboratory systems there is a development of small dedicated single-channel instruments, such as creatinine and glucose analysers. Excellent flow stream analysers of this type could be based on enzyme thermistors as section 29.3 above indicated, for example in an oxalate analyser.

\section{References}

Birnbaum, S., Bülow, L., Danielsson, B., Hardy, K. and Mosbach, K. (1986). Rapid automated analysis of human proinsulin produced by Escherichia coli. Anal. Biochem. 158, 12-15.

Bowers, L. D. and Carr, P.W. (1976). Immobilized-enzyme flow-enthalpimetric analyzer: application to glucose determination by direct phosphorylation catalyzed by hexokinase. Clin. Chem. 22, 1427-33. 
relation to a calibration curve for the lower concentration range. The coefficient of correlation between analytical results obtained with the calorimetric method and those from conventional assays was 0.997 for broth samples. Penicillin determination by the enzyme thermistor in the antibiotics industry has proven superior to commonly used methods such as high performance liquid chromatography (HPLC) and colorimetric procedures. Several instruments have been installed to replace the latter techniques (Decristoforo and Danielsson 1984).

Most fermentation analyses are complex systems containing particulate matter. Methods to prevent entry of these particles must be devised in order to prevent clogging of the analytical device. This can be accomplished either by the insertion of a dialysis membrane (of the type Technicon is using in its Auto Analyzer system (Danielsson et al. 1981c) or by employing enzymes bound to the inside of nylon tubing instead of using regular columns. With the former arrangement, the important requirement for sterility in fermentation process can also be met. Since the nylon tubing has a more limited binding capacity than that of CPG, the linear range will also be limited. In addition, the sensitivity of the CPG-system is high enough to permit a higher degree of dilution, which is a third way of reducing the problem of clogging. With the enzyme thermistor unit, it is possible not only to monitor continuously a product (e.g. penicillin as it is formed in a fermentation process) as well as substrates (e.g. glucose being consumed), but also to register simultaneously the overall thermal behaviour of such a microbial process, yielding a thermogram (power-time curve) which provides valuable information (Danielsson et al. 1981a). In this case the micro-organism suspension is mixed with a substrate solution and the temperature increase after appropriate reaction time is measured. The procedure is similar to that of thermal enzyme activity determination.

\subsubsection{Environmental analysis}

Thermal analysis can also be applied to both discontinuous and continuous environmental control analysis (Danielsson et al. 1981a); in the latter case acting as a kind of toxi-guard. If detection of a specific toxic compound is required, a limiting amount of an enzyme that is inhibited by the compound can be immobilized in the enzyme column of an enzyme thermistor. Heavy metals, for instance $\mathrm{Hg}^{2}$ + in concentrations down to $0.2 \mathrm{ppb}$, could be determined due to the inhibitory action on immobilized urease (Danielsson et al. $1981 a$ ). The heavy metal concentration of a sample was determined by comparison of the temperature response to a substrate pulse (containing an excess amount of urea) before and after introduction of the sample. A special washing step completely restored the original urease activity permitting repeated analysis with the same enzyme column. In some instances there are enzymes available that act directly on the substance to be measured. Such is 
thermistor (Fig. 29.5). The heat signal registered by the thermistor unit was used via a proportional/integral-controller to regulate the flow of substrate through the reactor. Thus, it was possible to keep the product composition (e.g. glucose) in the effluent constant, despite clogging occurring in the column.

As discussed, specific, continuous-flow assays for several sugars have been devised. Different types of process control involving such sugars (Mattiasson et al. 1983; Mandenius et al. 1985) are being investigated by the authors. The experiments indicate that the enzyme thermistor is stable enough to monitor and control a process over several days without any need for change of enzyme cartridge or recalibration. It is advisable to check the base line once a day. The system is also fast enough to respond to sudden changes ( 1 to 3 minutes) and has been used with analogue controllers and digital computers. In principle, the same approach could be used to control the blood glucose level in a patient by dosing insulin in response to the signal from an enzyme thermistor which continuously measures the glucose concentration.

In another study penicillin present in fermentation broth was analysed with a unit containing immobilized penicillinase ( $\beta$-lactamase) (Mattiasson et al. 1981). With CPG as enzyme matrix, the useful linear range was found to be at least 0.01 to $100 \mathrm{mmol} / 1$. This study provides a comparison between CPGbound and nylon tubing-bound penicillinase. In Fig. 29.6 some penicillin G determinations in samples made up in fermentation broth are plotted in

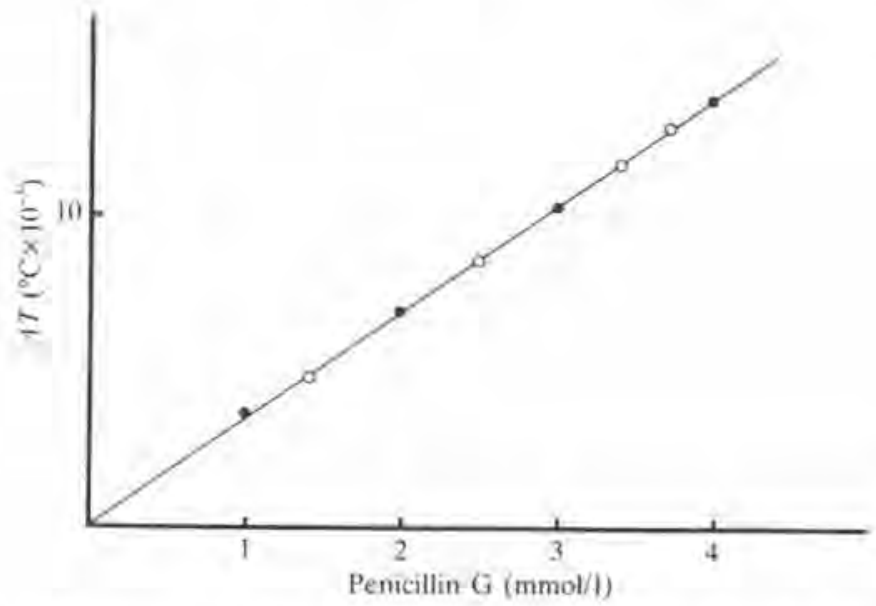

Fig. 29.6 Calibration curve for penicillin $\mathrm{G}$ in $0.3 \mathrm{M}$ sodium phosphate buffer, $\mathrm{pH}$ $7.0(\bullet)$, with samples containing known amounts of penicillin $G$ in 10 -fold diluted fermentation broth plotted in the same diagram $(O)$. The sample volume was $1 \mathrm{ml}$, flow rate $1 \mathrm{ml} /$ minute, and the enzyme thermistor column contained CPG-bound penicillinase. 
a strong ultraviolet absorbance, thereby often precluding on-line assay of a particular enzyme either by UV-monitoring or by spectrophotometric monitoring of changes in $\mathrm{NAD}(\mathrm{P}) \mathrm{H}$ concentration. Thus, calorimetric detection of the eluted enzyme activity should have definite advantages for monitoring this type of affinity chromatography.

\subsubsection{Process control and fermentation analysis}

One of the most promising application fields for thermal bio-analysers appears to be in process control and fermentation analysis. As biotechnology advances, a growing interest can be expected in direct methods for on-line determination of specific components formed or consumed in processes. With the exception of methods for determining physical variables, such as $\mathrm{pH}, \mathrm{pO}_{2}$, and $\mathrm{pCO}_{2}$, most analytical techniques currently used for process control are discontinuous, off-line procedures. In contrast, continuous online methods reduce the costs of sample handling and personnel and give more information, making them more suitable for process control. Much of the knowledge gained in this field is directly applicable to medical areas, for instance to in vivo monitoring systems. The suitability of the enzyme thermistor for continuous monitoring and control was investigated. In one model study, the authors used an enzyme thermistor to control the effluent composition of an enzyme reactor containing $\beta$-galactosidase (Danielsson et al. 1979). Lactose present in whey from cow's milk pumped through the reactor was hydrolysed to glucose and galactose and the glucose level in the effluent was measured with use of a glucose oxidase/catalase-loaded enzyme

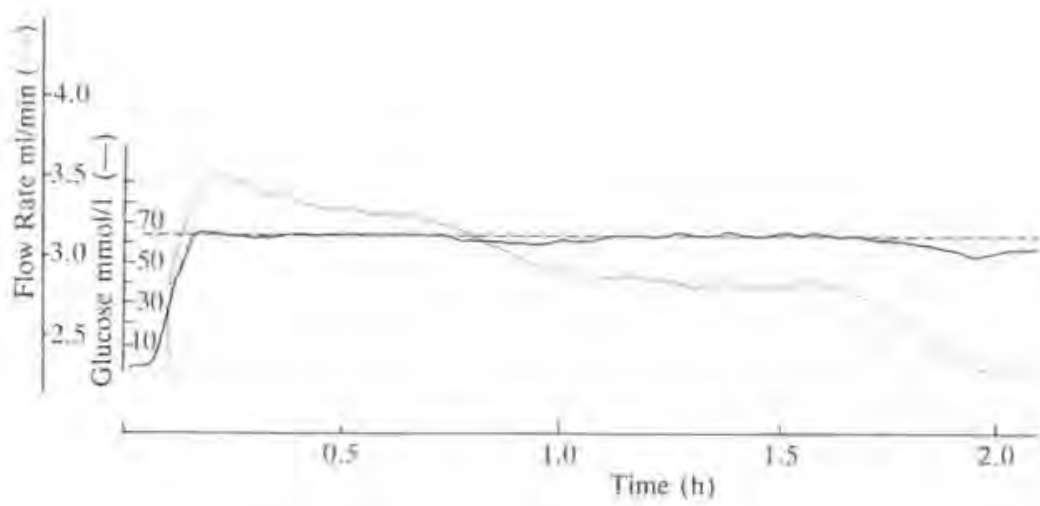

Fig. 29.5 Glucose concentration measured by a glucose oxidase/catalase thermistor $(\longrightarrow$ ) and pump speed ( $\cdots \cdot)$ upon pumping whey (150 $\mathrm{m}$ in lactose) through a plugflow reactor containing Sepharose-bound lactase. The glucose concentration was set to $63 \mathrm{M} \mathrm{(-)}$ (Danielsson et al. 1979). Reproduced with permission from the publisher John Wiley and Sons, Inc,, New York, N.Y., USA. 
the enzyme in excess are each passed through a heat exchanger prior to being mixed and rapidly passed through one of the short, inner heat exchangers to eliminate heat generated on mixing, before entering a reaction chamber. The reaction chamber (about $1 \mathrm{ml}$ volume) replaces the enzyme column normally used in the apparatus and consists of either an inactive column or a piece of Teflon tubing forming a 'reaction coil'. The temperature at the outlet of the reaction chamber is continuously measured with one of the thermistor probes as described in the experimental section (29.2). Linear correlation has been found between temperature response and enzyme activity for a variety of enzymes (Danielsson and Mosbach 1979). The sensitivity is in the order 0.01 to $0.1 \mathrm{IU} / \mathrm{ml}$ depending on the enzyme. Determination of soluble enzyme activities by calorimetry could be of interest in the clinical area, as well as for monitoring enzyme purification processes. Although the absolute sensitivity of this technique is rather low, it has the advantage of being a direct, continuous flow method that can be used on crude samples with inexpensive substrates (no need for expensive substrates to produce coloured products).

\subsubsection{Calorimetric monitoring of chromatography}

The enzyme thermistor has been successfully applied as an instrument for the specific monitoring of gel filtration, ion exchange chromatography, and affinity chromatography (Danielsson et $a l .1981 b$ ). Since the enzyme thermistor can be used for continuous monitoring of enzymic activities directly on crude samples, it provides possibilities for direct identification and localization of a specific component in a complex chromatogram, for instance, in the initial steps of an enzyme purification scheme (Fig. 29.4). Furthermore, elution in affinity chromatography is frequently accomplished with co-enzymes having

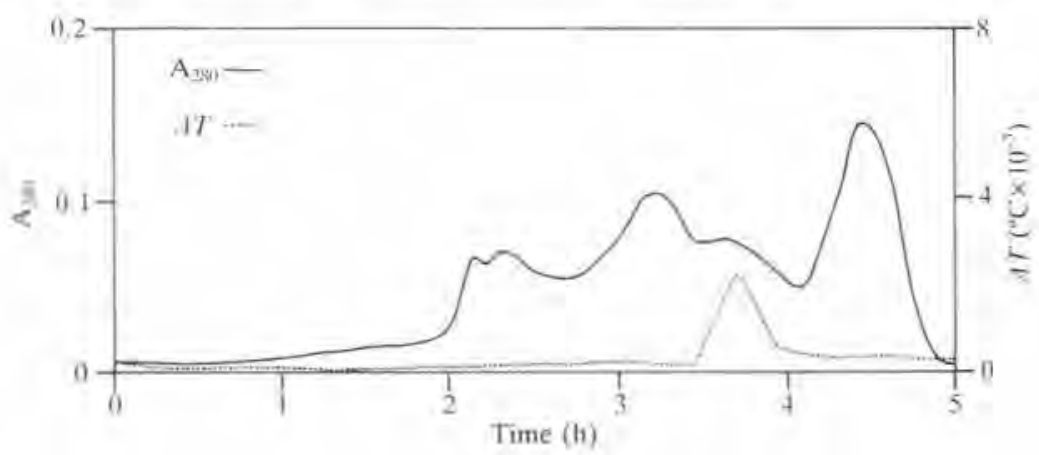

Fig. 29.4 Gel filtration of $1 \mathrm{ml}$ crude yeast extract on an Ultrogel AcA 44 column eluted with $0.2 \mathrm{M}$ Tris- $\mathrm{HCl}, 0.0133 \mathrm{M} \mathrm{MgCl}_{2}, \mathrm{pH} 7.8$, at a flow rate of $0.75 \mathrm{ml} / \mathrm{min}$. For enzyme thermistor assay of hexokinase (dotted line) the effluent was mixed with substrate solution containing $0.54 \mathrm{M}$ glucose, $0.011 \mathrm{M} \mathrm{ATP}$, flow rate $0.2 \mathrm{ml} / \mathrm{min}$ (Danielsson et al. 1981b). Reproduced with permission from the publisher Academic Press, Inc., Orlando, Florida, USA. 

a. Mattiasson and Danielsson 1982
b. Mosbach and Danielsson 1974
c. Danielsson et al. $1981 a$
d. Schmidt et al. 1976
e. Kiba et al. 1984
f. Danielsson et al. 1977
g. Marconi 1978
h. Bowers and Carr 1976
i. Danielsson et al. unpublished
j. Winquist et al. 1985
k. Satoh et al. 1981
l. Tran-Minh and Vallin 1978

m. Rich et al. 1979

n. Bowers et al. 1976

o. Fulton et al. 1980

p. Danielsson et al. 1976

q. Danielsson and Mosbach 1979

r. Danielsson et al. 1981 c

s. Mattiasson et al. 1977

t. Birnbaum et al. 1986

u. Danielsson et al, $1981 b$

v. Guilbault et al. 1983

x. Mattiasson el al. 1981

y. Decristoforo and Danielsson 1984

described above (Scheller et al. 1985), resulting in an extremely high sensitivity $(10 \mathrm{nM})$. This enzyme can, of course, also be used, preferably together with catalase, in the common type of enzyme thermistor assay, then with a performance similar to that of the glucose oxidase/catalase system.

A calorimetric assay for determining oxalic acid content in urine and in serum has also been developed. The enzyme oxalate oxidase (EC 1.2.3.4) has become available (Boehringer Mannheim). Very promising results have been obtained with this preparation and with the authors' preparations from barley seedlings. The oxalic acid concentration can be determined directly in 5- to 10-fold diluted urine in the range of 0.01 to $0.5 \mathrm{mmol} / \mathrm{l}$ (Winquist et al. 1985). This assay is also useful with food samples.

Rapid enzymic determination of ethanol is of interest in clinical chemistry as well as in biotechnology. In a recent study, enzyme electrode assays, based on a polarographic oxygen electrode, and enzyme thermistor alcohol assays were compared using the alcohol oxidase from Candida boidinii (EC 1.1.3.13) (Guilbault et al. 1983). The stability of the CPG-bound enzyme in the enzyme thermistor was drastically improved by co-immobilizing catalase. Catalase continuously removes the deleterious hydrogen peroxide formed and simultaneously recovers half of the oxygen consumed, and the reaction approximately doubles the heat formation. Both methods were found to give quite adequate sensitivities for blood ethanol determination. With the thermistor probe, as little as $0.2 \mathrm{mmol} / \mathrm{l}$ (with linearity up to $2 \mathrm{mmol} / \mathrm{l}$ ) could be assayed with a precision of $1.5 \%$. In addition to ethanol, methanol, propanol, and butanol were found to give good responses with both techniques.

\subsubsection{Enzyme activity determination}

With a minor modification of the flow system, the enzyme thermistor allows for determination of soluble enzyme activity. The sample solution containing the enzyme and an appropriate substrate solution containing a substrate to 
Table 29.2 Substances analysed with enzyme thermistors

\begin{tabular}{|c|c|c|c|}
\hline Substance & $\begin{array}{l}\text { Immobilized } \\
\text { biocatalyst }\end{array}$ & $\begin{array}{l}\text { Concentration } \\
\text { range }(\mathrm{mmol} / \mathrm{l})\end{array}$ & References \\
\hline \multicolumn{4}{|l|}{ Clinical analysis } \\
\hline Ascorbic acid & Ascorbic acid oxidase & $0.05-0.6$ & $a$ \\
\hline ATP & Apyrase & $1-8$ & $b$ \\
\hline Cholesterol & Cholesterol oxidase & $0.03-0.15$ & c \\
\hline Cholesterol esters & $\begin{array}{l}\text { Cholesterol esterase }+ \\
\text { cholesterol oxidase }\end{array}$ & $0.03-0.15$ & $c$ \\
\hline Creatinine & $\begin{array}{l}\text { Creatinine } \\
\text { iminohydrolase }\end{array}$ & $0.01-10$ & $c$ \\
\hline Glucose & $\begin{array}{l}\text { Glucose oxidase + } \\
\text { catalase }\end{array}$ & $0.002-0.8$ & $d, e, f, g$ \\
\hline Glucose & Hexokinase & $0.5-25$ & h \\
\hline Lactate & Lactate & & \\
\hline & 2-monooxygenase & $0.005-2$ & $c, i$ \\
\hline Oxalic acid & Oxalate oxidase & $0.005-0.5$ & $j$ \\
\hline Oxalic acid & Oxalate decarboxylase & $0.1-3$ & $c$ \\
\hline Triglycerides & Lipoprotein lipase & $0.1-5$ & $k$ \\
\hline Urea & Urease & $0.01-500$ & $l, m, n, o, p$ \\
\hline Uric acid & Uricase & $0.05-4$ & $c$ \\
\hline \multicolumn{4}{|c|}{ Soluble enzyme analysis } \\
\hline Urea & Urease (soluble) & $0.1-100 \mathrm{U} / \mathrm{ml}$ & $q$ \\
\hline $\mathrm{H}_{2} \mathrm{O}_{2}$ & Catalase (soluble) & $0.1-100 \mathrm{U} / \mathrm{ml}$ & $q$ \\
\hline Glucose + ATP & Hexokinase (soluble) & $0.1-2.5 \mathrm{U} / \mathrm{ml}$ & $r$ \\
\hline \multicolumn{4}{|c|}{ Immunological analysis, TELISA } \\
\hline Albumin (antigen) & $\begin{array}{l}\text { Immobilized antibodies } \\
+ \text { enzyme-linked } \\
\text { antigen }\end{array}$ & $10^{-10}-$ & $s$ \\
\hline \multirow{2}{*}{$\begin{array}{l}\text { Gentamicin (antigen) } \\
\text { Insulin (antigen) }\end{array}$} & " & $0.1-\mu \mathrm{g} / \mathrm{ml}$ & $c$ \\
\hline & $"$ & $\begin{array}{l}0.1-1.0 \mathrm{U} / \mathrm{ml} \\
0.1-50 \mu \mathrm{g} / \mathrm{ml}\end{array}$ & $t$ \\
\hline \multicolumn{4}{|c|}{ Fermentation analysis and process control } \\
\hline Cellobiose & $\begin{array}{l}\beta \text {-Glucosidase }+ \\
\text { glucose oxidase }+ \\
\text { catalase }\end{array}$ & $0.05-5$ & $u$ \\
\hline Cephalosporin & Cephalosporinase & $0.005-10$ & $c$ \\
\hline Ethanol & Alcohol oxidase & $0.01-2$ & $v$ \\
\hline Galactose & Galactose oxidase & $0.01-1$ & $a$ \\
\hline Lactose & $\begin{array}{l}\text { Lactase and glucose } \\
\text { oxidase + catalase }\end{array}$ & $0.05-10$ & $a$ \\
\hline Penicillin G & Penicillinase & $0.05-500$ & $x, y$ \\
\hline Sucrose & Invertase & $0.05-100$ & $a$ \\
\hline
\end{tabular}




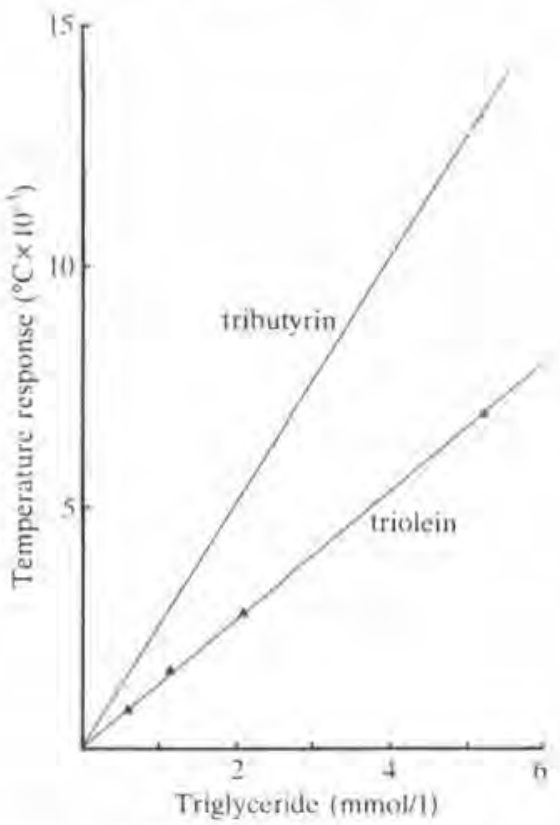

Fig. 29.3 Calibration curves for a split-flow enzyme thermistor containing lipoprotein lipase bound to CPG.

column was charged with only choline oxidase and catalase co-immobilized on CPG. The linear range was between 0.03 and $0.19 \mathrm{mM}$ and serum samples could be directly analysed after 10 -fold dilution and with good correlation with conventional methods. Co-immobilization of all three enzymes involved resulted in rather low operational stability, but the proposed technique permitted eight weeks of operation or at least 1600 analyses with each column.

The authors' current studies on applications of the enzyme thermistor in biotechnology include determination of lactate in fermentation broth. This metabolite is also of clinical interest. Using lactate 2-monooxygenase (EC $1,13.12 .4)$ bound to $\mathrm{CPG}$, lactate concentrations in aqueous standards as well as in biological samples can be determined with high sensitivity and stability from $0.005 \mathrm{mmol} / /$ up to $3 \mathrm{mmol} / 1$, with a linear response up to about 0.5 to $1 \mathrm{mmol} / \mathrm{I}$. A comparison between calorimetric and electrochemical determination of lactate showed that the enzyme thermistor gave a higher sensitivity than a polarographic oxygen electrode in a flow cell combined with a lactate oxidase column of the same type as used in the enzyme thermistor (B. Danielsson, I. Satoh, and B. Mattiasson, unpublished results).

Furthermore, an oxidase from Pediococcus pseudomonas (EC 1.1.3.2) is now available and has been used particularly in the recycling system 
samples was determined in the course of a day, both with the single-column and split-flow apparatus. Glucose concentrations determined by the enzyme thermistor agreed well with the values obtained from a conventional, spectrophotometric, enzymic technique used in routine hospital diagnosis. The operational stability of the system was at least as good as that of the urease thermistor. Similar results were obtained by Schmidt and co-workers (1976), who also used glucose oxidase co-immobilized with catalase, and in a study (Marconi 1978) where these enzymes were fibre entrapped, resulting in a broader range of linear response (up to $5 \mathrm{mmol} / \mathrm{l}$ glucose), although at the expense of sensitivity and probably also with lower operational stability.

By employing an alternative electron acceptor to oxygen, benzoquinone, the linear range of glucose oxidase can be considerably extended. Addition of $45 \mathrm{~mm}$ benzoquinone to the working buffer resulted in a linear range of 0.1 to $70 \mathrm{~mm}$ (Kiba et al. 1984).

A glucose oxidase/catalase thermistor in combination with a disaccharidesplitting enzyme can be applied for the determination of disaccharides containing glucose (e.g. cellobiose (Danielsson et al. 1981c) and lactose (Mattiasson and Danielsson 1982)). In addition, sucrose has been measured directly with the enzyme invertase (EC 3.2.1.26) in the range of 0.05 to $100 \mathrm{mmol} / 1$ (Mattiasson and Danielsson 1982). This example illustrates one of the advantages of the enzyme thermistor since this reaction can be followed directly without any coupled reaction which is not possible with other techniques, such as colorimetry. Galactose oxidase (EC 1.1.3.9) can be used for galactose assays and ascorbate oxidase (EC 1.10.3.3) can be used for vitamin $C$ assays with linearity between 0.05 and $0.6 \mathrm{mmol} / 1$ (Mattiasson and Danielsson 1982).

Triglyceride determination (Satoh et al. 1981) is a current example of enzyme calorimetry employing a single enzyme, where in most other techniques several enzymes have to be used. Lipoprotein lipase (EC 3.1.1.34) was immobilized on CPG having a pore size of $2000 \AA$. The assay was performed with a split-flow apparatus in $0.1 \mathrm{M}$ Tris buffer, $\mathrm{pH} 8.0$, containing $0.5 \%$ Triton X-100. A linear temperature response was obtained for 0.05 to $10 \mathrm{mmol} / 1$ of tributyrin and for 0.1 to $5 \mathrm{mmol} / 1$ of triolein (Fig, 29.3). The triglyceride concentration in serum samples could be determined directly after two-fold dilution (up to a concentration of $3 \mathrm{mmol} / \mathrm{l}$ ) with the Tris buffer. The results correlated well with those obtained with conventional spectrophotometric enzymic methods.

Another example of lipid assay comes from a recent study of phospholipid determination employing three consecutively acting enzymes; phospholipase D, choline oxidase, and catalase (Satoh et al. 1986). Of these enzymes soluble phospholipase D (36 I U) was added directly to $0.05 \mathrm{ml}$ sample, which was then injected into the buffer stream $(1 \mathrm{ml} / \mathrm{min}$. of $0.1 \mathrm{M}$ Tris-HC1, $\mathrm{pH} 8.0$, containing $15 \mathrm{mM} \mathrm{CaCl}_{2}$ and $0.5 \%$ Triton $\mathrm{X}-100$ ), whereas the enzyme 
peroxide, the substrate of catalase, into the flow stream. Sensitivities as low as $10^{-13} \mathrm{~mol} / \mathrm{I}$ have currently been obtained with the TELISA technique. After the determination, the immunosorbent is readily regenerated by a glycine wash at low $\mathrm{pH}$ and a complete measuring cycle will only take 10-15 minutes to perform. The advantage of this technique is that occasional samples can be rapidly and sensitively analysed in a very simple procedure. For use in biotechnology, for instance for monitoring the production of hormones and proteins by genetically engineered micro-organisms, we have automated the TELISA procedure (Birnbaum et al. 1986). The sample valve and valves for substrate and wash solutions are then operated by a programmable controller. The cycle time is 15 minutes and the samples can be taken from the process stream or from a sample changer.

\subsubsection{Applications in clinical chemistry}

Several routine methods based on immobilized urease for the determination of urea in serum have been described (Tran-Minh and Vallin 1978; Rich et al. 1979; Bowers et al. 1976; Fulton et al. 1980; Danielsson et al. 1976). We found that a remarkable wide range of linearity could be achieved; typically 0.01 to $200 \mathrm{mmol} / \mathrm{I}$ (Danielsson et al. 1976). Since serum samples were diluted 10 -fold, most samples fell within the 0,3 and $10 \mathrm{mmol} / 1 \mathrm{range}$. Consequently, there was a considerable margin for enzyme inactivation; i.e. the operational stability could be expected to be high. An enzyme column could be used for several months or for several hundreds of determinations of serum samples. The time required per sample (about two to three minutes) is acceptable for a short series of samples and the precision with a relative standard deviation of about $1 \%$ is good.

For determining glucose concentrations, either hexokinase or glucose oxidase (with catalase) have been used. Soluble hexokinase was used in a direct-injection enthalpimetric assay for glucose samples in the range of 0.5 to $50 \mathrm{mmol} / 1$ (McGlothlin and Jordan 1975). Immobilized hexokinase was used in a similar system in the range of 0.5 to $25 \mathrm{mmol} / \mathrm{I}$ (Bowers and Carr 1976). The throughput of 40 samples/hour, the accuracy, the precision, in addition to the long-term stability and reproducibility of the latter system make it very acceptable as a routine clinical instrument for determining glucose concentrations.

The use of glucose oxidase instead of hexokinase was preferred due to better enzymic stability and no cofactor requirements (Danielsson et al. 1977). A disadvantage when using glucose oxidase is, however, that linearity is obtained up to $0.45 \mathrm{mmol} / 1$ glucose or $0.7 \mathrm{mmol} / 1$ with co-immobilized glucose oxidase and catalase. This limitation can be overcome by diluting samples 50 - to 100 -fold, or by injecting small serum volumes ( 5 to $20 \mu \mathrm{l}$ ) directly into the buffer stream entering the enzyme thermistor. The precision of the determinations was high. A $0.6 \%$ relative standard deviation for 
The sensitivity of a calorimetric assay can be greatly increased by substrate recycling, for instance with an oxidase and a dehydrogenase acting on the same substrate/product pair. It was recently demonstrated that a 1000 -fold increased sensitivity for lactate or pyruvate could be obtained with use of lactate oxidase and catalase co-immobilized with lactate dehydrogenase (Scheller et al. 1985). While passing through the column, lactate is repeatedly oxidized by the lactate oxidase and the pyruvate reduced to lactate by the lactate dehydrogenase with simultaneous consumption of NADH. The total enthalpy charge will be approximately the same as for the oxidation of NADH by oxygen, which is a highly exothermic reaction (cf. Table 29.1). Lactate or pyruvate concentrations as low as $10 \mathrm{nM}$ could be determined with this technique using a $0.8 \mathrm{ml}$ column with $0.5 \mathrm{ml}$ samples at a flow rate of $1 \mathrm{ml} / \mathrm{min}$. Under similar conditions the detectability for $\mathrm{NAD}(\mathrm{H})$ could be increased up to 80 -fold by co-enzyme recycling using the co-immobilized enzyme couple lactate dehydrogenase and glucose 6-phosphate dehydrogenase.

\subsection{Applications}

Highly promising results have been obtained with enzyme thermistors and similar devices in many areas of bio-analysis including clinical analysis, process control, fermentation analysis, and environmental control. The application to chromatographic monitoring was recently described demonstrating the possibility of specific detection of a particular enzyme in a complex mixture. In addition, a flow system for immunoassay based on the enzyme thermistor has been developed. Table 29.2 summarizes a number of the many analyses that have been studied with the enzyme calorimeters presented, although some of these studies are of a rather early date showing detection ranges that probably could be considerably improved with modern equipment.

\subsubsection{Thermometric enzyme-linked immunosorbent assay (TELISA)}

The enzyme thermistor can also be applied to a growing area of immunochemical analysis: antigen/antibody determination. For this alternative procedure, the authors have suggested the name 'thermometric enzymelinked immunosorbent assay' (TELISA) (Mattiasson et al. 1977). In principle, the column of the enzyme thermistor is filled with immunosorbent, such as antibodies immobilized on Sepharose CL-4B. The antigen to be determined and an enzyme (e.g. catalase)-labelled antigen are introduced into the flow; the amount of catalase-bound antigen remaining bound to the column is a function of the content of antigen. The less antigen that is present in the sample, the more catalase-labelled antigen will be found in the column and thus evolve more heat after the subsequent introduction of hydrogen 
calculate the concentration of each sample based on peak heights or peak areas. When the sample pulse length is sufficiently increased to more than 1 to 5 minutes, the temperature response will eventually reach a constant value proportional to the concentration. Consequently, the concentration of an enzyme substrate can be continuously monitored.

\subsubsection{Amplification}

A number of 'tricks' can be applied to amplify heat formation. (Although many enzymic reactions have sufficiently high molar enthalpies, permitting determinations with devices such as the enzyme thermistor with a sensitivity as low as $10^{-5} \mathrm{~mol} / \mathrm{I}$, some reactions, such as enzymic hydrolysis of esters, produce very little heat). For example, hydrolysis of acetylcholine by choline esterase has an enthalpy change close to zero. Nevertheless, this reaction can be detected calorimetrically because the hydrolytic step produces a proton which can protonate a suitable buffer (Tris) with high protonation enthalpy (e.g. Tris, $\Delta H=-47.5 \mathrm{~kJ} / \mathrm{mol}$ (Rehak and Young 1978)) making the total process strongly exothermic. In another example, the response of an enzyme thermistor charged with immobilized trypsin was studied in Tris as well as in phosphate buffer $(\Delta H=-4.7 \mathrm{~kJ} / \mathrm{mol})$ (Mosbach and Danielsson 1974).

Sensitivity can also be increased by co-immobilizing sequentially operating enzymes. Each reaction step will contribute to the temperature signal registered and represent the sum of the reaction enthalpies. Determination of glucose, for example, with glucose oxidase (Glucose $+\mathrm{O}_{2} \stackrel{\text { Glucose oxidase }}{\longrightarrow}$ Glucono- $\delta$-lactone $+\mathrm{H}_{2} \mathrm{O}_{2}$ ) could be made about twice as sensitive by including catalase $\left(\mathrm{H}_{2} \mathrm{O}_{2} \stackrel{\text { catalase }}{\longrightarrow} 1 / 2 \mathrm{O}_{2}+\mathrm{H}_{2} \mathrm{O}\right)$ in the enzyme thermistor column. At the same time, half of the oxygen consumed in the glucose oxidase reaction is regenerated thereby increasing the upper concentration limit for linearity from about 0.45 to $0.7 \mathrm{mmol} / 1$. Co-immobilized, sequentially operating multi-step enzyme systems may offer further advantages, such as faster substrate conversion as compared with the situation where the enzymes are immobilized on separate polymer beads and better efficiency at low substrate concentrations (Danielsson et al. 1977).

When the first reaction is only weakly exothermic, the corresponding enzyme may be placed in a precolumn outside the enzyme thermistor, leaving only the subsequent enzyme(s) inside the calorimeter. This allows for an increased flexibility and improves the overall performance since more efficient enzyme columns can be used, especially in the first step. Determination of cellobiose using $\beta$-glucosidase (Danielsson et al. 1981c) and determination of lactose using $\beta$-galactosidase (Mattiasson and Danielsson 1982) were made in this manner. Because the hydrolytic steps have a very low enthalpy, the actual thermal measurements were performed on the glucose formed by hydrolysis, using a glucose oxidase/catalase enzyme thermistor. 
and Danielsson 1981). Different carrier materials can show considerable variation in their adsorption of sample constituents depending for instance on the actual surface distribution of ionic or hydrophobic groups. Since such adsorption almost certainly will give rise to non-specific heat and may even affect the enzymic reaction, the choice of enzyme support material could be rather crucial. Using CPG with pore sizes in the range of $500 \AA$ to $2000 \AA$ and with a particle size usually around 80 mesh has given good results. When using either untreated CPG or alkyl(propyl)amino-derivatized CPG obtained from different suppliers, glutaraldehyde activation of the glass and subsequent binding of the enzyme almost invariably gives good results. It should be noted that a rather large excess of enzyme (often 100 units) is generally applied to an enzyme thermistor column. This procedure ensures good operational stability and unchanged performance of the system over a long series of samples or extended periods of continuous monitoring. The column should be functional for several months.

\subsubsection{Procedure}

The instrumental arrangement for enzyme thermistor analysis permits the inclusion of an inactive reference column for compensation of non-specific heat. The split-flow arrangement (Mattiasson et al. 1976) has proven valuable in many situations involving crude samples (e.g. for determination of triglycerides (Satoh et al. 1981)), but in most cases it is possible to avoid non-specific effects by careful design of the procedure, so that variations in solvent composition (mixing and solution heats) are avoided and adsorption of macromolecules or micro-organisms is prevented.

Buffer is continuously pumped through each functioning channel at a flow rate of 0.5 to $3 \mathrm{ml} /$ minute. Samples are generally introduced as short pulses through a chromatography valve equipped with 0.1 to $1 \mathrm{ml}$ loop. Thermal steady state will not be obtained for short sample pulses, but the enzymic reaction will generate a temperature peak, the height of which is normally taken as a measure of the substrate concentration (Danielsson et al. 1981a).

The linear range of the relationship between temperature peak height and substrate concentration is usually at least $10^{-5}$ to $10^{-1} \mathrm{~mol} / 1$ when not limited by reactant concentrations (as oxidases are by the supply of oxygen) or by other factors. The maximum number of samples that can be analysed by the present system is 15 to 60 per hour with the stated sample volumes. The latter figure is obtained by decreasing the sample volume to $0.1 \mathrm{ml}$ and increasing the flow rate to 2 to $3 \mathrm{ml} /$ minute. In order to demonstrate simplified sample handling, a sample changer for an Auto Analyzer system (Technicon Instruments Corp., Tarrytown, New York, USA.) was modified and interfaced with a small desktop computer that also controls the sampling valve, This system can be left unattended, for instance, overnight. If the sample series has also included calibration standards, the computer is programmed to 


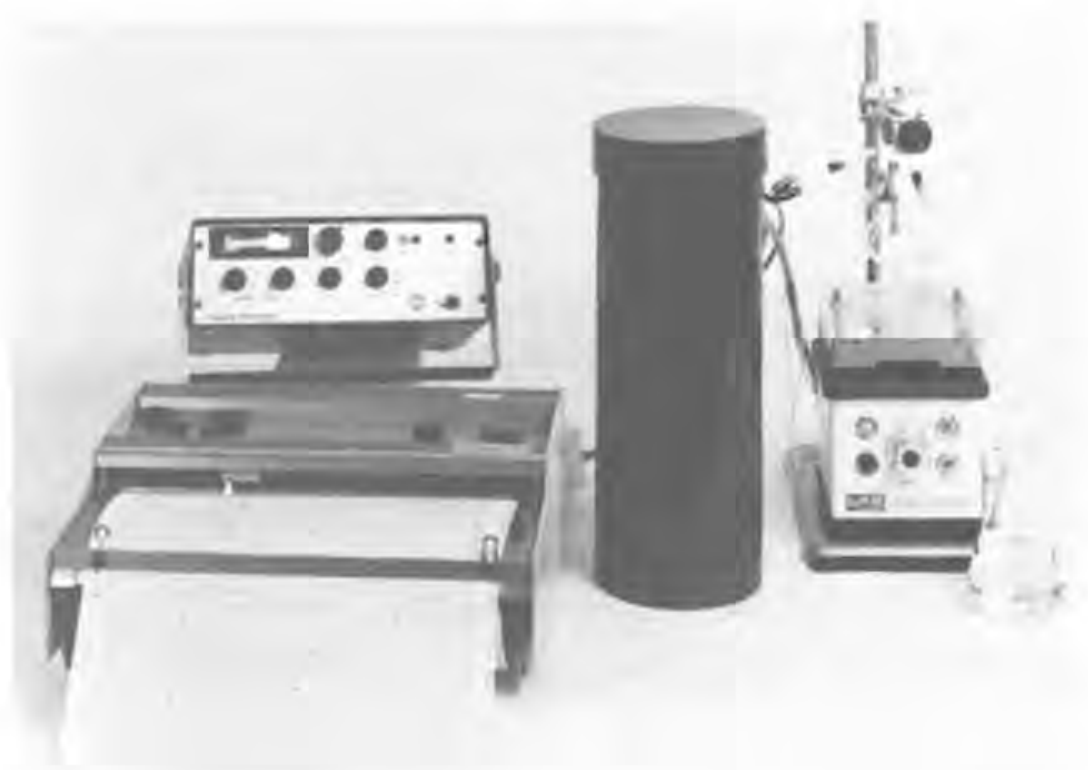

Fig. 29.2 Enzyme thermistor equipment for manual operation.

change of $0.001{ }^{\circ} \mathrm{C}$. The lowest practical useful range, mainly dictated by temperature fluctuations created by friction and turbulence in the column, is usually higher, typically $0.01{ }^{\circ} \mathrm{C}$. It should be noted that a substrate concentration of $0.5 \mathrm{mmol} / \mathrm{l}$ to $1 \mathrm{mmol} / \mathrm{I}$ in many reactions gives a temperature change of $0.01{ }^{\circ} \mathrm{C}$. Over the past five years more than 15 devices as shown in Figure 29.2 have been produced at our institute and utilized in various laboratories.

\subsubsection{Enzyme column}

Changing columns is very easy since the column is simply inserted into the end of a plastic tube (containing the outlet tubing and the temperature sensor) by which it is fitted into the apparatus. Columns of different diameters (maximum internal diameter $7 \mathrm{~mm}$ ) and bed heights (maximum $30 \mathrm{~mm}$ ) can be used. Nylon tubing wound around a special adaptor which fits into the column holder and connects the tubing to the flow system can also be used as enzyme support. (Nylon tubing is advantageous for analysis of crude samples containing particulates (Mattiasson et al. 1981) but suffers from low enzyme loading capacity.) Thus, the carrier material usually used is CPG (controlled pore glass) that offers high enzyme-coupling capacity, good mechanical, chemical, and microbial stability, as well as relatively simple coupling procedures. Other materials have also been used (Danielsson et al, 1981 $a$; Mosbach 


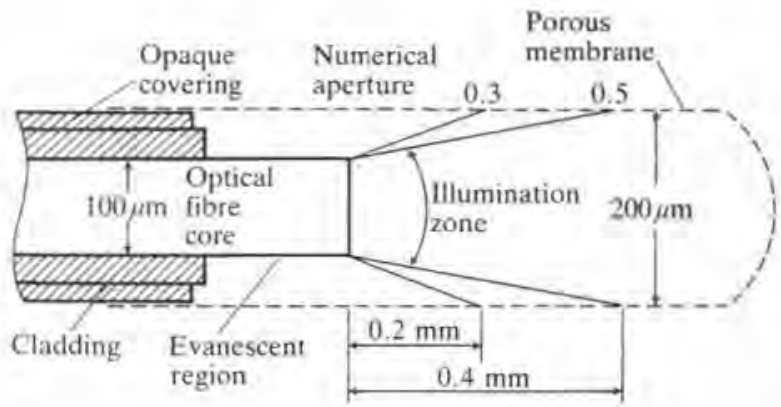

Fig. 32.1 Basic construction of an optical fibre illustrating the two mechanisms of light exchange, i.e. an illumination cone from the end of the fibre, and an evanescent wave from a decladded portion of the fibre. In usual practice only one of these modes is utilized in a given application.

optical fibres are highly purified so that the extent of attenuation due to absorption or scattering is relatively insignificant for fibre lengths of the order of several metres, which would be the case for most analytical systems of interest here.

For the purposes of using fibre optics in biosensor applications, there are two principle mechanisms by which light interacts with the external environment. Primarily, light emanates from the end of the core. If the light source is a laser, then the exit beam will be highly columnated with a diameter about equal to that of the incident laser beam. This cylindrical beam is of particular importance in the 'separation' techniques described below. If the light source is a focused beam from a typical lamp, then the light leaves the core and diverges in a cone of illumination as shown in Fig. 32.1. The dispersion of the cone of light (called the numerical aperture of the fibre) depends on the difference in refractive indices between the core material and the external fluid. Regions of illumination for typical commercially available fibres are shown in this figure. The active biochemical constituents of a biosensor are often sheltered from the external environment by one or more membrane layers that cover the end of the optical fibre. The characteristics of these membranes are selected for particular functions in different analyses.

Light intensity diminishes as the region of illumination widens with distance from the end of the cleaved fibre. Also, the fraction of light emitted by fluorescence that returns to the optical fibre and is transmitted to the detection system falls off rapidly with distance. Some idea of the geometrical considerations for detection are shown in Fig. 32.2 for an axial beam of light. The total amount of light that re-enters the fibre is the sum of all these point sources in the region of illumination, but for practical purposes, only a zone 


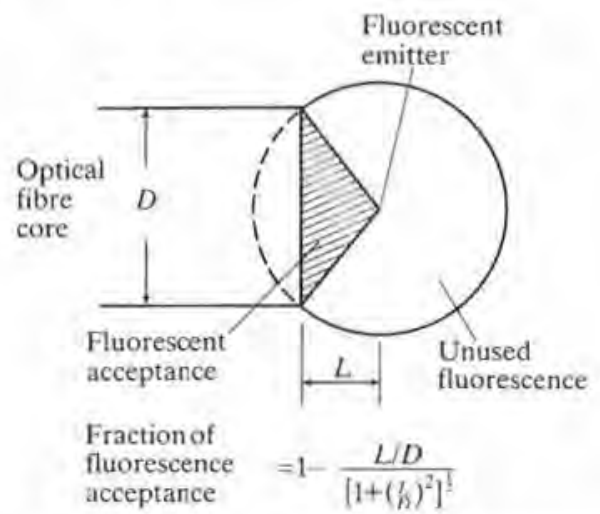

Fig. 32.2 Geometric considerations of the light-gathering power of an optical fibre. The shaded region is the cone of light from a point emitter on the axis that enters the optical fibre.

of about ten fibre diameters in length provides useful signals back to the detector. Thus as shown in Fig. 32.3. for fibres of the order of 200 microns in diameter, the effective sensor zone is about $2 \mathrm{~mm}$ in length.

Another mechanism for allowing light to penetrate the outside environment is to remove the cladding from the central core. Provided the refractive index of the core is still higher than the external fluid, the light will still be

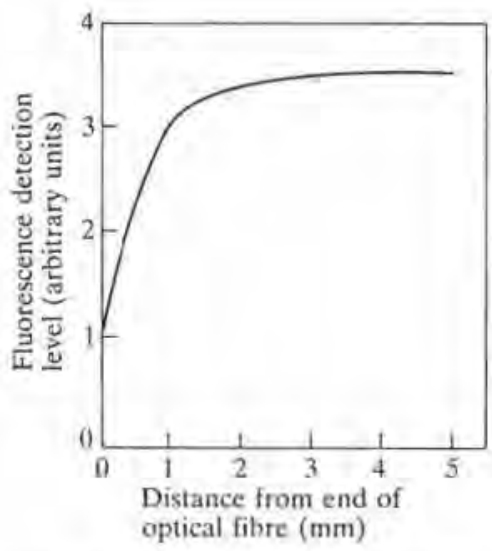

Fig. 32.3 Effect of chamber length on total amount of fluorescence detected by an optical fibre, 
transmitted down the fibre, but a small portion will escape into the external phase. This evanescent wave of light energy decreases exponentially with distance from the core surface, and for all practical purposes is only effective for distances of the order of 100 angstroms.

\subsection{Biosensors based on bioreceptors}

In this chapter, we restrict our discussion to biosensors that are based on the reversible binding between analytes and specific receptors. The most common form of this type of assay in biology are the immunoassay procedures based on specific antibodies developed for low molecular weight haptens (i.e. in this context, analytes). Although the original implementation of immunoassays utilized radioactive tracers to monitor the extent of binding, over the last few years the use of fluorescent labels has become of increasing interest because of the elimination of possible radiation hazards (Smith et al. 1981). Fortunately, most of the technology that has been developed for fluro-immunoassays can be readily adapted for biosensor development.

In addition to the availability of antibodies as receptor candidates for biosensors, there are several other classes of proteins that have the desired characteristics of reversible selective binding. For example, lectins have specific binding characteristics for different sugars (Goldstein and Hayes 1978); membrane binding proteins with specificities for different amino acids and sugars have been isolated and described (Landich and Oxender 1982); and enzymes have non-reactive binding sites for allosteric effectors (Lehninger 1970).

The technology associated with affinity chromatography can provide a wealth of information and materials for 'affinity-pairs'. Many combinations of receptors and ligands have been evaluated in the context of purification of proteins by affinity chromatography (Chaiken et al. 1984). Some of these systems could be adapted for use in a biosensor, e.g. flavin and flavinbinding protein.

The design of biosensors based on reversible binding to biological receptors will be discussed in two categories - direct and indirect methods.

\subsubsection{Direct method}

The direct method involves only the reversible reaction of the analyte and receptor species:

$$
\text { Analyte }+ \text { Receptor } \rightleftharpoons \text { Analyte:receptor }
$$

In order to use this reaction directly, there must be a spectral change in at least one of these three species. For example, if the absorption spectrum of the receptor changes when the analyte is bound, then monitoring this change 
would provide a direct measure of the extent of binding and thus a measure of analyte concentration. A classical case is the spectral change that occurs when oxygen binds to haemoglobin. Although this colour change has been used primarily to monitor the percent oxygenation of haemoglobin, the effect could be turned around for the estimation of the partial pressure of oxygen colorimetrically,

Of course since the binding of oxygen to haemoglobin is sensitive to $\mathrm{pH}$, carbon dioxide, and temperature, this reaction would not be the first choice for constructing an oxygen sensor. But, it does remind one that for a sensor one needs to evaluate the effect of environmental conditions on binding behavior.

Another, and somewhat more prevalent optical effect that has been used to measure the extent of reaction is the quenching of fluorescence when the analyte binds to the protein. Most proteins fluoresce when excited in the green due to the residues of tryptophan in the molecule. This fluorescence can be quenched when the analyte binds to the protein if the absorption spectrum of the analyte overlaps the emission spectrum of tryptophan. An example of clinical importance is the fluorescent assay of aminoglycoside antibiotics as described by Shaw et al. (1977) for gentamicin.

It should be noted that for direct systems, it is not necessary to have a membrane separating the chemical constituents of the sensor from the sample fluid if the receptor is conserved by immobilizing it to the surface of the optical probe. However it may be desirable to use a membrane anyway, to protect the receptor protein from other constituents of the sample fluid, e.g. enzymes.

\subsubsection{Indirect method}

If neither the analyte nor receptor exhibit a spectral change on binding, then one must resort to the use of analyte-analogues that have some measurable response. In these indirect methods the general chemical equation are:

$$
\begin{aligned}
& \text { Analyte }+ \text { Receptor } \rightleftharpoons \text { Analyte:receptor } \\
& \text { Analogue-analyte }+ \text { Receptor } \rightleftharpoons \text { Analogue-analyte:receptor }
\end{aligned}
$$

Where the analogue-analyte either has an inherent measurable optical characteristic, or induces some optical change on binding to the receptor. If one can monitor the extent of the second reaction then, indirectly, one can estimate the concentration of the analyte since changes in analyte concentration will alter the extent of the second reaction. Some possibilities for implementing this concept in the design of biosensors are discussed below.

32.3.2.I Spatial separation techniques As mentioned in the description of optical fibre characteristics, the emanating beam from an optical fibre is 


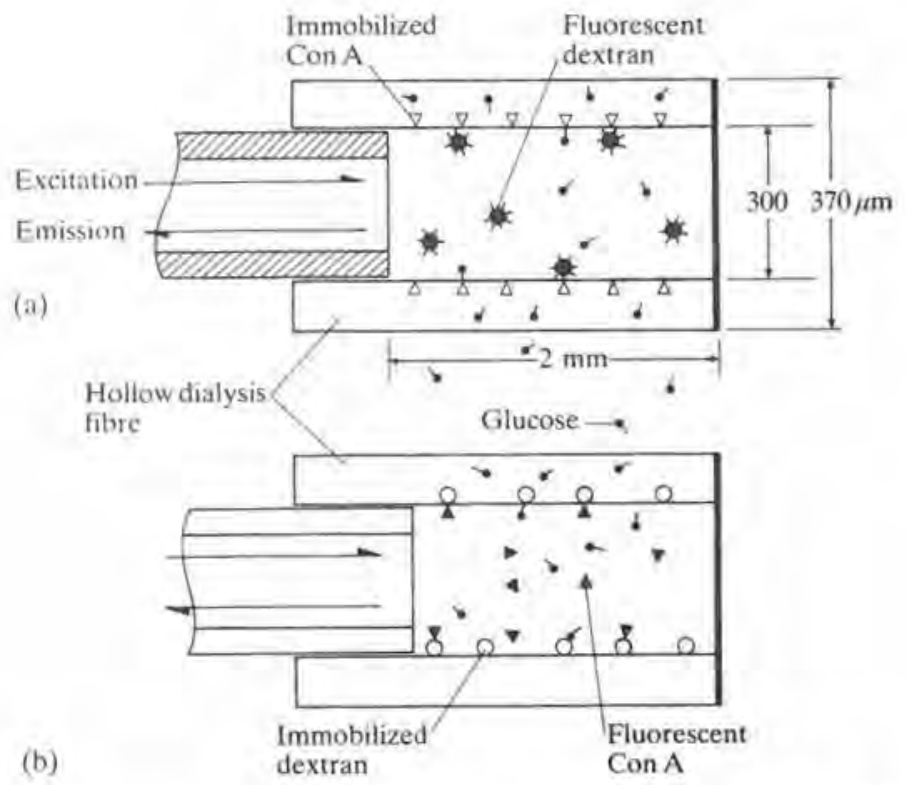

Fig. 32.4 Configurations for a glucose biosensor based on the 'separation' principle, i,e. the measurement of only the unbound fluorescently labeled macromolecule.

(a) Glucose sensor utilizing immobilized Con A as the bioreceptor and fluorescently labeled dextran as the freely mobile analogue-analyte (Schultz et al. 1982).

(b) An alternative scheme for a glucose sensor where the analog-analyte, i.e. dextran, is immobilized and the bioreceptor, i.e. Con A, is fluorescently labeled and freely mobile.

focused to some extent, depending on the illumination source and numerical aperture of the optical fibre. This property can be used to advantage to monitor independently individual constituents if one of the components of the system is immobilized out of the field of view of the optical fibre. Figure $32.4 \mathrm{a}$ shows one arrangement to accomplish this task.

Here the receptor protein is immobilized to the interior surface of a hollow dialysis fibre that defines the transducer chamber. This configuration was used successfully for a prototype glucose sensor (Schultz et al. 1982; Mansouri and Schultz 1984). Concanavalin A (con A) provided the specific receptor for sugars and high molecular weight $(70000)$, fluorescently labelled dextran (FITC-dextran) was chosen as the analogue-analyte. Con A was covalently immobilized to the cellulose hollow fibre (Sirinivasan et al. 1986), essentially out of the view of the optical fibre.

In the absence of sugar, a signal equivalent to about $20 \%$ of the maximum fluorescence was measured. Through a variety of tests (Mansouri 1983) it was 


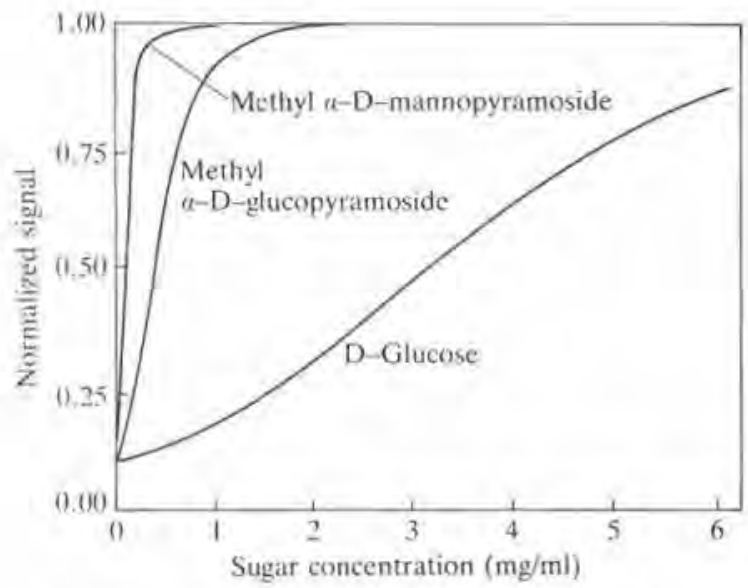

Fig. 32.5 Typical sugar calibration curves for a con A-based biosensor (Mansouri 1983).

estimated that about three quarters of this base-line signal was from free FITC-dextran and the balance from dextran bound to con A on the dialysis tube. As the sensor is placed in solutions of increasing sugar concentration, increasing amounts of fluorescent dextran are displaced from the wall into the field of view of the optical fibre. Eventually, at high sugar concentrations, all the dextran is free and no further increase in fluorescence is obtained. The saturation-type of calibration curves shown in Fig. 32.5 are typical of the response from systems involving receptor binding. Simplified mathematical models for these response curves are discussed below.

The response for three different sugars are shown in Fig. 32.5 to illustrate that the sensitivity of the method is directly related to the binding constant between the analyte and receptor. The sensor is more than ten times more sensitive to methyl mannoside than to glucose, with a mid-range response of about 100 micrograms per $\mathrm{ml}$ for the mannose derivative. These curves also show that if a solution contained a mixture of sugars, then the sensor output would provide some weighted mean level of the sugars present. Fortunately, if the sensor is used to monitor glucose in blood, there are no other sugars of consequence present in blood to compete with glucose for the binding sites. The ultimate sensitivity of this device is related to the lowest fluorescein concentration that can be measured, this was to be of the order of 100 nanograms of fluorescein per $\mathrm{ml}$.

It should be pointed out that an alternative labelling scheme with essentially equivalent characteristics as the above case would be to label con $\mathrm{A}$ with 


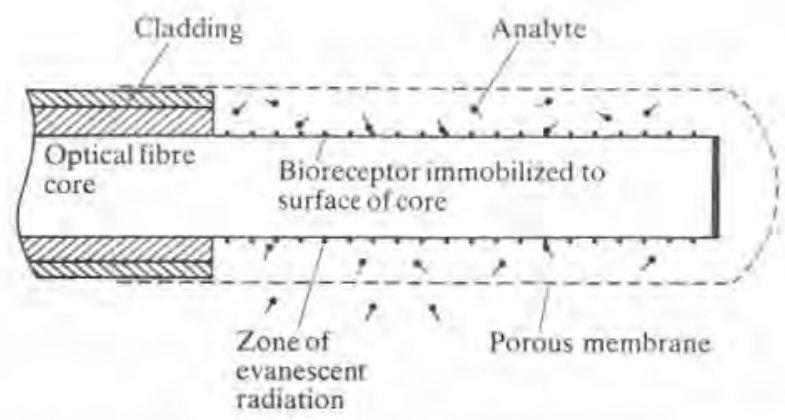

Fig. 32.6 Configuration for a biosensor based on the use of the evanescent wave for detection of the bound bioreceptor-analogue-analyte complexes.

fluorescein and immobilize the dextran (unlabelled) to the hollow dialysis fibre. This situation which is depicted in Fig. 32.4b could have some advantages if it were easier to label con A with fluorescein or if one could obtain a higher density of immobilized dextran than immobilized con A.

Instead of monitoring the free fluorescent species, the fluorescence of the immobilized fraction could be measured as well. There are a number of optical techniques for doing this, but perhaps the most elegant is the use of the evanescent wave approach introduced by Kronick and Little (1975). In this method, the receptor would be bound to the core of a decladed optical fibre as shown in Fig. 32.6. Since the extent of penetration of the evanescent wave into the fluid is only on the order of 100 angstroms, the primary volume sampled will be the absorbed layer of receptor molecules and bound fraction of analyte. Andrade et al. (1985) have demonstrated the feasibility of using the evanescent wave in this fashion.

The sensitivity of these methods may be increased by the use of timeresolved fluorimetry (Soini and Hemmila 1979). In this technique, fluorescent moieties are chosen that have relatively long lifetimes (persistence after excitation on the order of microseconds rather than nanoseconds, as is typical for fluorescein). This allows one to measure fluorescent emission after turning off the excitation beam. An advantage of this approach is that interferences from background scattering of the excitation beam and background fluorescence due to impurities (usually short-time in nature) are eliminated.

Typically rare-earth chelate fluorophores have been found to have the appropriate properties for time-resolved fluorimetry. For example, Leung and Mears (1977) has shown that a 'bifunctional' chelation agent can be used to covalently attach heavy metal chelates to albumin, allowing measurement 
down to the micromolar level. Special gated fluorimeters for this purpose have been designed (Soini and Kojola 1983).

32.3.2.2 Homogeneous techniques There are a number of other optical techniques that can be used with the indirect method and that have the additional advantage that no immobilization chemistry is required.

Fluorescent energy transfer.

Uliman (Uliman et al. 1976) introduced the concept and proved the utility of fluorescent excitement energy transfer immunoassays. The essence of the approach can be represented as follows:

$$
\mathrm{Ag}-\mathrm{F}+\mathrm{Ab}-\mathrm{R} \rightleftharpoons \mathrm{Ag}-\mathrm{F}: \mathrm{Ab}-\mathrm{R}
$$

where $\mathrm{F}=$ fluorescein, $\mathrm{R}=$ rhodamine, $\mathrm{Ag}=$ antigen, and $\mathrm{Ab}=$ antibody (receptor). Thus, light of the appropriate frequency is used to excite the fluorescence of fluorescein. When the antigen (Ag-F) is in the free form (left side of the equation) emitted light is detected by the optics. On the other hand, when the fluorescein moiety is in close proximity to rhodamine (that has an absorption spectrum that overlaps the Ag-F fluorescence spectrum) about $70 \%$ of the emitted light is quenched by direct energy transfer from fluorescein to rhodamine. Thus an optical biosensor can be constructed on this principle:

$$
\begin{aligned}
& \text { Analyte }+ \text { Receptor- } \mathrm{R} \rightleftharpoons \text { Analyte:receptor- } \mathrm{R} \\
& \text { Analogue-analyte- } \mathrm{F}+\mathrm{Receptor}-\mathrm{R} \rightleftharpoons \text { Analogue-analyte-F: receptor- } \mathrm{R}
\end{aligned}
$$

(fluorescent) (quenched)

The measurement of fluorescence quenching gives an indirect estimate of the unlabelled analyte concentration through the competitive binding mechanism.

A particular advantage of this technique is that neither the receptor nor the analogue-analyte needs to be immobilized, since the quenching occurs in solution. Furthermore, one has more complete control of the concentration of receptor and analogue-analyte in the transducer compartment and can more easily optimize the conditions for maximum assay sensitivity. Also, the choice of membrane materials for the hollow fibre would be expanded because one is not limited to materials that can be easily activated for immobilization and the strength of the hollow fibre would not be compromised by the immobilization chemistry.

At least two physical configurations are possible in this case, either a single fibre arrangement as shown in Fig. 32.7a, or a fibre bundle arrangement as 


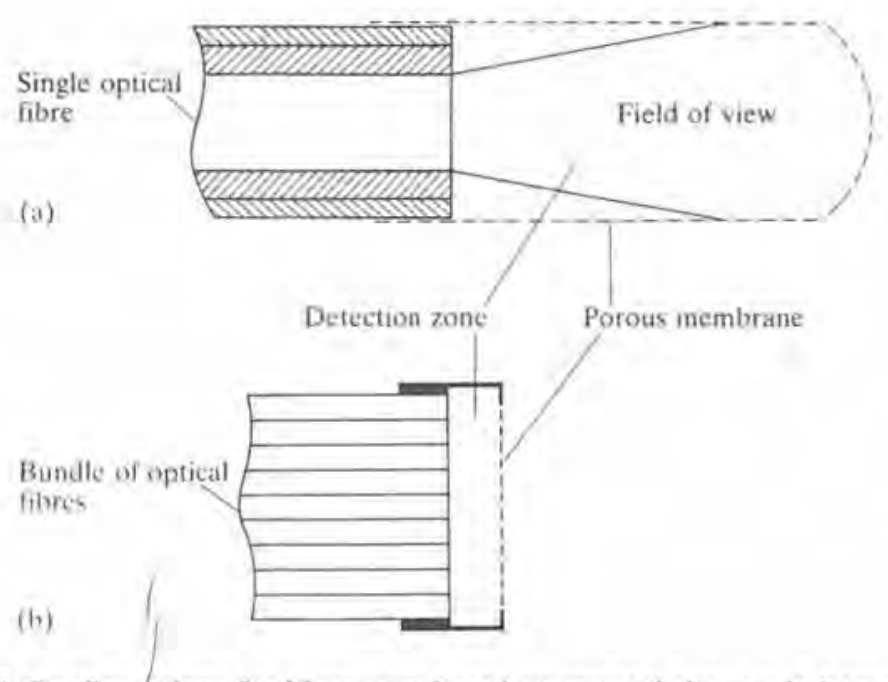

Fig. 32.7 Configurations for biosensors based on spectral changes in homogeneous reactions. (a) Single optical fibre, (b) use of a bundle of optical fibres, with a detection chamber of thickness $t$.

shown in Fig. 32.7b. The latter type has some advantages in that there is more light gathering power with multiple fibres, as a result the chamber thickness (dimension $t$ in the figure) can be made very small, which will decrease the diffusion path length and increase the response rate of the system.

Fluorescence depolarization

An alternative to energy transfer is the method of fluorescence depolarization introduced by Dandlicker (Dandlicker and Saussuve 1970). Here the principle is based on the difference in rotational diffusion rates between the free analogue-analyte and the receptor-bound analogue-analyte. When the analogue-analyte is bound to the large molecular receptor, its rotation rate is slowed so that the depolarization of emitted light is reduced. Thus, an increase in the amount of polarized fluorescence is directly related to the amount of analogue bound. In the immunoassay kits based on this principle low-molecular-weight analogues can be used because conservation of reactants is not an issue. For biosensor applications, the analogue would have to be large enough to be retained within the dialysis tube and thus a molecular weight of the order of several thousand is required. With larger analogueanalytes the sensitivity of the method will be lessened because the difference in rotational diffusion rates between the free and bound analogue will be reduced.

Normal optical fibres scramble polarized light, but recent developments in 
the use of graded index glass allow retention of polarization for short lengths of fibres. Configurations similar to that shown in Fig. 32.7 could be used with the fluorescence depolarization approach.

Turbidimetric methods

Another method for estimating the extent of reaction between macromolecular analogue-analytes and receptors is by measuring changes in the intensity of back-scattered light in sensors in the form shown in Fig. 32.7. For polymers with diameters of the order of tens of angstroms, the intensity of back-scattered light is proportional to the square of molecular weight. If, for example in a glucose sensor, dextran with a size equal to con A (molecular weight about 60000 ) is chosen as the competing analyte, then the intensity of scattered light from the complex dextran-con A will be about four times that of either component alone. Thus there will be a decrease in scattered light as reaction 32.3 proceeds in the left direction as glucose enters the sensor chamber.

\subsection{Approximate models for response characteristics}

Assuming that the reactions involved are at equilibrium, the mathematical representation of response characteristics for biosensors based on reversible binding can be obtained fairly directly for simple univalent binding systems. The practical attainment of chemical equilibrium should be considered in the light of the kinetics of other processes that are involved in the sensor response. For example, in most biosensors a membrane will be interposed between the chemical constituents and the sample fluid. Then the rate of analyte diffusion through the membrane(s) will be an important factor in the overall sensor response. For example, the time constant for diffusion of glucose through a hollow dialysis fibre with a 30 micron wall thickness is about five minutes (Schultz et al. 1982). The time constant for glucose binding to con $\mathrm{A}$ is on the order of milliseconds, and thus the reaction is always at equilibrium with respect to the diffusion dynamics. On the other hand, the kinetics of some antibody-antigen interactions is fairly slow. For example, Kranz et al. (1982) report on antifluorescyl monoclonal antibodies with dissociation half-lifes from $10^{-2}$ to $10^{3}$ seconds. For antibodies it generally appears that the higher the affinity constant the lower the dissociation rate, in some cases as long as hours. Obviously, in the latter situation an equilibrium assumption will not be valid.

\subsection{Direct method}

The chemistry of simple binding can be represented as

$$
\underset{\text { analyte }}{\mathrm{A}}+\underset{\text { receptor }}{\mathrm{R}} \rightleftharpoons \underset{\text { bound analyte }}{\mathrm{A}: \mathrm{R}}
$$




$$
\begin{aligned}
& {[\mathrm{R}]_{1}=[\mathrm{R}]+[\mathrm{A}: \mathrm{R}]} \\
& K_{\mathrm{a}}=[\mathrm{A}: \mathrm{R}] /[\mathrm{A}][\mathrm{R}] \\
& {[\mathrm{R}] /[\mathrm{R}]_{\mathrm{l}}=1 /\left[1+\left([\mathrm{A}] K_{\mathrm{a}}\right)\right]}
\end{aligned}
$$

where $[R]$ is concentration of free receptor, $[R]$, is total receptor concentration and $K_{\mathrm{a}}$ is equilibrium binding constant between analyte and receptor.

Thus if the receptor (R), normally fluoresces but is quenched when $A$ is bound, then $[R] /[R]_{t}$ is the residual fluorescence as a function of the concentration of A. As with systems that obey Langmuir isotherms, the response is one-half maximum when [A] is numerically equal to $K_{\mathrm{a}}{ }^{-1}$. Note that the sensitivity of this type of system is independent of receptor concentration, and if the detection system were able to measure $R$ over the range $0.1<$ $[\mathrm{R}] /[\mathrm{R}]_{\mathrm{t}}<0.9$, this corresponds to an analyte range of $(1 / 9) / K_{\mathrm{a}}<[\mathrm{A}]$ $<9 / K_{\mathrm{a}}$.

\subsubsection{Indirect methods}

As shown in Fig. 32.5 for our glucose sensor, the calibration curves for sensors based on equilibrium binding are generally hyperbolic in nature.

For practical reasons, i.e. attempting to obtain the maximum performance from the sensor, it is desirable to have the background output as close to zero as possible and about 50 to $80 \%$ maximal response at the operational expected concentration of the analyte for a given application. The selection criteria for receptor and analogue-analyte as related to binding constants and concentration effects can be estimated from a simplified mathematical analysis of the system.

$$
\begin{gathered}
\underset{\mathrm{A}}{\text { analyte }}+\mathrm{R} \rightleftharpoons \begin{array}{c}
\mathrm{A}: \mathrm{R} \\
\text { receptor } \\
\text { bound analyte }
\end{array} \\
\mathrm{A}^{*}+\mathrm{R} \rightleftharpoons \mathrm{A}^{*}: \mathrm{R}
\end{gathered}
$$

analogue-analyte receptor bound analogue-analyte

If it is assumed that the binding reactions are unimolecular (more complex behaviour will be discussed later), then assuming chemical equilibrium between all of the constituents, the following algebraic equations will pertain:

$$
\begin{aligned}
& K_{\mathrm{a}}=[\mathrm{A}: \mathrm{R}] /[\mathrm{A}][\mathrm{R}] \\
& K_{\mathrm{a}}^{*}=\left[\mathrm{A}^{*}: \mathrm{R}\right] /\left[\mathrm{A}^{*}\right][\mathrm{R}]
\end{aligned}
$$

where $[\mathrm{A}]$, etc., are the concentration of the various species.

Because of the construction of the sensor, the total amount of receptor and analogue ligand are conserved over time and thus the following material balance equations can be written.

$$
\begin{aligned}
& {[R]_{t}=[R]+[A: R]+\left[A^{*}: R\right]} \\
& {\left[A^{*}\right]_{t}=\left[A^{*}\right]+\left[A^{*} ; R\right]}
\end{aligned}
$$


where $[R]_{1}$ and $\left[A^{*}\right]_{t}$ are the total (or initial) concentrations of these two species within the sensor chamber.

These four equations can be solved for the ratio of unbound to total detector ligand concentration:

$$
\begin{aligned}
& \left(\frac{\left[\mathrm{A}^{*}\right]}{\left[\mathrm{A}^{*}\right]_{\mathrm{t}}}\right)^{2}+\frac{\left[\mathrm{A}^{*}\right]}{\left[\mathrm{A}^{*}\right]_{\mathrm{t}}}\left[\left(\frac{[\mathrm{R}]_{\mathrm{t}}}{\left[\mathrm{A}^{*}\right]_{\mathrm{t}}}-1\right)+\left(\frac{K_{\mathrm{a}}[\mathrm{A}]+1}{\left[\mathrm{~A}^{*}\right]_{\mathrm{l}} K_{\mathrm{a}}{ }^{*}}\right)\right]- \\
& \left.\frac{K_{\mathrm{a}}[\mathrm{A}]+1}{\left[\mathrm{~A}^{*}\right] K_{\mathrm{a}}^{*}}\right)=0 .
\end{aligned}
$$

The normalized response is given as $\left[\mathrm{A}^{*}\right] /\left[\mathrm{A}^{*}\right]_{[}$; this ratio, between 0 and 1 , of the free analogue-analyte to the total analogue-analyte concentration represents the range of output of the sensor. The sensitivity of the sensor will depend somewhat on the amount of analogue-analyte used in preparing the sensor $\left[\mathrm{A}^{*}\right]_{\text {. }}$. From practical considerations minimum concentration of the analogue ligand that can be used in the sensor depends on the capability of the optical system for measuring low levels of fluorescence and the presence of other possible interfering substances, e.g. bilirubin in blood.

From eqn 32.17 it can be seen that the normalized response is a function of two groups of parameters $[\mathrm{R}]_{\mathrm{l}} /\left[\mathrm{A}^{*}\right]_{\mathrm{l}}$ and $\left(K_{\mathrm{a}}[\mathrm{A}]+1\right) /\left(\left[\mathrm{A}^{*}\right]_{l} K_{\mathrm{a}}{ }^{*}\right)$. The latter group contains the independent variable of interest, $[\mathrm{A}]$ the concentration of

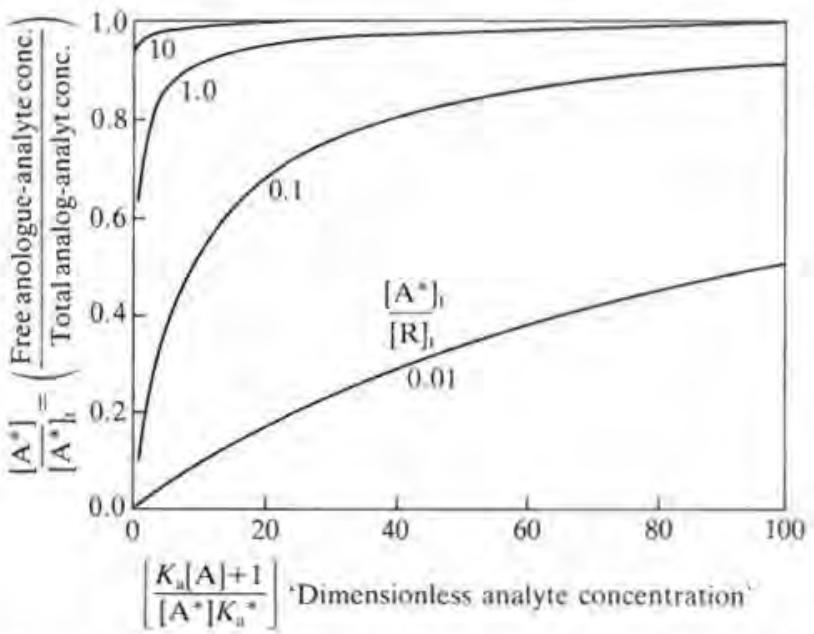

Fig. 32.8 Parametric plot of eqn 32.17 relating the expected ratio of free analogueanalyte to total analogue-analyte in the detection chamber as a function of the dimensionless group $\left.\left[K_{\mathrm{a}}[\mathrm{A}]+1\right) /\left[\mathrm{A}^{*}\right] K_{\mathrm{a}}{ }^{*}\right]$ which is directly related to the free analyte concentration. Parametric curves of $\left[A^{*}\right]_{t} /[R]_{t}$ give the effect of relative levels of analogue-analyte concentration within the detector chamber. 
analyte. Thus a dimensionless plot of $\left[\mathrm{A}^{*}\right] /\left[\mathrm{A}^{*}\right]_{1}$ vs. $\left(K_{\mathrm{a}}[\mathrm{A}]+1\right) /\left(\left[\mathrm{A}^{*}\right]_{1} K_{\mathrm{a}}{ }^{*}\right)$ provides a complete characterization of the system as shown in Fig. 32.8.

From this figure it is clear that at low values of $[R]_{t} /\left[A^{*}\right]_{t}$, the responsiveness of the sensor to changes in analyte concentration will be very slight. Physically this condition represents the situation where the analogue-analyte concentration is so high relative to the amount of available receptor sites that there is a great excess of unbound analogue ligand. Thus the additional release of analogue ligand from receptor sites by analyte does not appreciably affect the free and measurable $\left[\mathrm{A}^{*}\right]$. Thus in designing biosensors of this type it is clear that in order to obtain an analyte calibration curve over a range of concentrations of interest a reasonable rule of thumb is to maintain the ratio

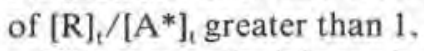

This same principle can be seen from another point of view. Operationally, it is desirable for the signal to be low at low analyte concentration, and high at the maximum analyte concentration expected for a given application. This behaviour can be met if $[R]_{t} /\left[A^{*}\right]_{t}$ is of the order of 10 . On the other hand, if $[R]_{\mathrm{l}} /\left[\mathrm{A}^{*}\right]_{\mathrm{t}}$ is much larger, (e.g. of the order of 100 ), some other problems may result, namely that the actual concentration of analogue-analyte may be too low for dependable detection by the optical system.

These considerations can be used to estimate the physical chemical constants required for a given sensor application. First, it is desirable for the output of the sensor to be low when the analyte concentration is zero. Substituting the values $[A]=0,[R]_{t} /\left[A^{*}\right]_{1}=10$, and a minimal sensor response, i.e. $\left[\mathrm{A}^{*}\right] /\left[\mathrm{A}^{*}\right]_{\mathrm{k}}=0.1$, we find from eqn 32.17 that the group $\left[\mathrm{A}^{*}\right]_{\mathrm{R}} K_{\mathrm{a}}{ }^{*}$ needs to be of the order of 1.0 .

Now in order to obtain a $50 \%$ response when the analyte concentration is in mid-range, let $\left[\mathrm{A}^{*}\right] /\left[\mathrm{A}^{*}\right]_{\mathrm{t}}=0.5$, and we find that the group $K_{\mathrm{a}}\left[\mathrm{A}^{\prime}\right]$ should be of the order of 10 (where $\left[\mathrm{A}^{\prime}\right]$ is the mid-range analyte concentration).

Using the glucose sensor as an example, the range of blood glucose in diabetics is on the order of 1 to $5 \mathrm{mg} / \mathrm{ml}$, thus $\left[\mathrm{A}^{\prime}\right]=5 \mathrm{mg} / \mathrm{ml}$ or $0.025 \mathrm{M}$. The association binding constant of glucose to con $\mathrm{A}\left(K_{\mathrm{a}}\right)$ is about $320 \mathrm{M}^{-1}$.

The maximum amount of con A that we were able to immobilize to the interior surface of the hollow dialysis fibre resulted in an effective concentration of $10^{-5} \mathrm{M}$. The affinity binding constant between FITC dextran $\left(70000 \mathrm{MW}\right.$ ) and con $\mathrm{A}$ is about $7.5 \times 10^{-4} \mathrm{M}^{-1}$ and the total concentration of FITC dextran was about $1.5 \times 10^{-6} \mathrm{M}$. The value of the $K_{\mathrm{a}}\left[\mathrm{A}^{\prime}\right]$ group was about 6 at a glucose value of $2.5 \mathrm{mg} / \mathrm{ml}$, and $[R]_{t} /\left[\mathrm{A}^{*}\right]_{t}$ was 7 . Thus for our particular glucose sensor these dimensionless groups are approximately in the range of the criteria given above.

From these considerations a rational approach to designing a biosensor can be outlined for a specific analyte.

1. Estimate the mid-range analyte concentration of interest [ $\left.\mathrm{A}^{\prime}\right]$. 
2. Select a bioreceptor with a binding constant with a numerical value on the order of $10 /\left[\mathrm{A}^{\prime}\right]$.

3. Estimate the minimum concentration of analogue-analyte ligand that can be detected by the optical system. $[\mathrm{A}]_{\mathrm{m}}$ and presume that $\left[\mathrm{A}^{*}\right]_{\mathrm{t}}$ will be on the order of $50[\mathrm{~A}]_{\mathrm{m}}$.

4. Select, synthesize, or modify competing analogue candidate compounds so that the criteria $K_{\mathrm{a}}{ }^{*}=1 /[\mathrm{A}]_{\mathrm{m}}$ is satisfied.

5. Develop methodologies to load the sensor transducer compartment with the bioreceptor so that the total concentration of sites is of the order of $100\left[\mathrm{~A}^{*}\right]_{\text {. }}$.

\subsubsection{Multivalent binding behaviour}

So far in this discussion we have limited the analysis to monovalent interactions between ligands and receptors. Actually, most antibodies are at least divalent (i.e. have two receptor sites) and many are multivalent. Concanavalin A has four receptor sites. Also, haptens (analogue-analytes) usually have multiple active groups. Thus the simple models given above are not exact for these more complex systems, and general mathematical approaches are still being developed (Perelson 1984). However, as a first approximation for estimating biosensor behaviour one can use the above formulations with pseudo-univalent binding constants. For example, as mentioned above the binding constant between glucose and con A is $320 \mathrm{M}^{-1}$ whereas for FITC-dextran, linear glucose polymer with many pendant glucose units, the effective binding constant with con $\mathrm{A}$ is about $7.5 \times 10^{4} \mathrm{M}^{-1}$ or a factor $\mathbf{2 0}$ times higher.

\subsection{Summary}

The capabilities of fibre-optic systems for miniaturizing analytical techniques based on the properties of light provides an enormous resource that can be harnessed for biosensor applications. The fast pace of developments in opto-electronic devices, i.e. solid-state lasers, integrated optoelectronic chips, new types of optical fibres, connectors, multiplexers, portends a future where not only the sensor is miniaturized but the measuring equipment as well. In addition, modern developments in molecular biology and protein engineering in particular will make it possible to design bioreceptors with the desired characteristics for specific use in biosensors. These two trends should result in a completely new generation of highly selective, miniature, portable, stable, and inexpensive biosensors that can be used in both biomedical and industrial applications.

\section{Acknowledgement}

This work was partially supported by NIH Grant RO1 AM 26858. 


\section{References}

Andrade, J. D., VanWagenen, R. A., Gregonis, D. E., Newby, K. and Lin, J.-N. (1985). Remote fibre-optic biosensors based on evanescent-excited fluoroimmunnoassay: Concept and Progress. IEEE Trans. Electron Devices ED-32. $1175-9$.

Chabay, I. (1982). Opical waveguides. Anal. Chem. 54, 1071A-80A.

Chaiken, I., Wilchech, M., Paritch, I. (eds.) (1984). Affinity chromatography and biological recognition. Academic Press, New York.

Dandlicker, W. and Saussuve, V.A. (1970). Review of fluorescence polarization in immunochemistry. Immunochem. 7, 799-805.

Goldstein, I. J. and Hayes, C. E. (1978). The lectins; carbohydrate-binding proteins of plants and animals. In Advances in carbohydrate chemistry and biochemistry. (eds. R. S. Typson and D. Horton), Vol. 35, 127-145.

Kranz, D. M., Herron, J.N. and Voss E.W., Jr, (1982). Mechanisms of ligand binding by monoclonal antifluoresyl antibodies, J. Biol. Chem., 257, 6987-95.

Kronick, N. M. and Little, W. A. (1975). J. Immunol. Methods. 8, 235-240.

Lacey, E. A. (1982). Fiber optics. Prentice Hall, Englewood, NJ, USA.

Landich, R. and Oxender, D. (1982). Periplasmic binding proteins. In Membranes and Transport (ed. Martonosi), Vol. 2, pp, 81-88. Plenum, New York.

Lehninger, A. (1970), Biochemistry. Worth, New York.

Leung, C. and Mears, C. (1977). Attachment of fluorescent metal chelated to macromolecules using 'bifunctional' chelating agents. Biochem. Biophys. Res. Comm. $75,149-55$.

Lubbers, D. W. and Opitz, N. (1983). Blood gas analysis with fluorescent dyes as an example of their usefulness as quantitative chemical sensors. In Proc. intl. mig. chemical sensors, Fukuoda, Japan. Elsevier, Amsterdam.

Mansouri, S. (1983). Optical glucose sensor based on affinity binding. Ph.D. Thesis, University of Michigan.

Mansouri, S. and Schultz, J, S. (1984). A miniature optical sensor based on affinity binding. Biotechnology 2, 385-90.

Perelson, A.S. (1984). Some mathematical models of receptor clustering by multivalent ligands. In Cell surface dynamics: concepts and models (eds. A.S. Perelson, C. DeLisi, and F. W. Wiegel), pp. 223-276. Marcel Dekker, New York.

Peterson, J.1., Goldstein, S. R., Fitzgerald, R. V. and Ruckold, D. K. (1980). Fiberoptic pH probe for physiological use. Anal. Chem. 52, 864-9.

Peterson, J. I. and Vurek, G. G. (1984). Fiber-optic sensors for biomedical applications. Science. 224, 123-7.

Saari, L. and Seitz, W. R. (1982). pH sensor based on immobilized fluorescein amine. Anal. Chem. 54, 821-3.

Schultz, J.S., Mansouri, S. and Goldstein, I. J. (1982). Affinity sensor: a new technique for developing implantable sensors for glucose and other metabolites. Diabetes Care. 5, 245-53.

Schultz, J. S. and Sims, G. (1979). Biolech. Bioeng. Symp. 9, 65-71.

Seitz, W. R. (1984). Chemical sensors based on fiber optics. Anal. Chem. 56, 16A-34A.

Sirinivasan, K. R., Mansouri, S. and Schultz, J.S. (1986). Coupling of Concanavalin 
A to cellulose hollow fibers for use in a glucose affinity sensor. Biotech. Bioeng, 28, In press.

Shaw, E. J., Watson, R. A. A., London, J., Smith, D. S. (1977). Estimation of serum gentamicin by quenching fluoroimmunoassay. J. Clin, Pathol. 30, 562-31.

Smith, D.S., Al-Hakiem, M. and Landon, J. (1981). A review of fluorimmunassay and immunofluorimetric assay. Anal. Clin. Biochem. 18, 253-274.

Soini, E. and Hemmila, I. (1979). Fluorimmunoassay: present status and key problems. Chin. Chem. 25, 353-61.

Soini, E. and Kojola, H. (1983). Time-resolved fluorometer for lanthanide chelates a new generation of nonisotopic immunoassays. Clin Chem. 29, 65-8.

Ullman, E. F., Schwarzberg, M. and Rubenstein, K. (1976). Fluorescent excitation transfer immunoassay, a general method for determination of antigens. J. Biol. Chem. 251, 4172.

Vureck, G. G. and Bowman, R.L. (1969). Fiber-optic colorimeter for submicroliter samples. Anal. Biochem. 29, 238-47. 


\title{
33
}

\section{IRS devices for optical immunoassays}

\author{
RANALD M. SUTHERLAND and CLAUS DÄHNE
}

\subsection{Introduction}

Internal reflection spectroscopy (IRS) is an established technique for monitoring reaction systems within the order of a wavelength of light at a continuous surface (Harrick 1967). IRS is based on the optical reflection characteristics between two transparent media of different refractive indices. When a light beam is totally internally reflected within the optically denser medium, an electromagnetic wave-form is generated in the optically rarer medium close to the reflecting surface. This evanescent wave is part of the internally reflected light beam and penetrates a fraction of a light wavelength into the lower refractive index medium. The evanescent wave is the 'sensing' component and can optically interact with compounds close to or at the surface. This optical interaction can be followed as a change in the intensity of light which exits from the optically denser medium. The latter is commonly called the internal reflection element (IRE) and can be designed as a single (Fig. 33.1a) or multiple (Fig. 33.1b) reflection element, Other common terms for multiple reflection elements are light-guide or waveguide. The characteristics of this type of sensor allow the continuous monitoring of reactions at the interface with minimal interference from compounds distant from the waveguide surface (of the order of a light wavelength).

Previous biological applications of IRS devices are mainly based on the interaction of proteins with or at surfaces, the latter pretreated to make them hydrophobic or hydrophilic. The two optical techniques most frequently used for measuring these interactions are attenuated total reflection (ATR) and total internal reflection fluorescence (TIRF). ATR is defined as 'reflection which occurs when an absorbing coupling mechanism acts in the process of total internal reflection to make the reflectance less than unity' (Harrick 1967). That is, when an optically absorbing film is present on the waveguide interface, light energy absorbed from the evanescent wave can be monitored as an attenuation of the internally reflected light beam. ATR spectroscopy has been extensively applied to monitoring protein interactions at surfaces (Baier and Dutton 1969; Brash and Lyman 1971) in the infra-red (IR) region. Gendreau and co-workers have combined ATR and Fourier- 


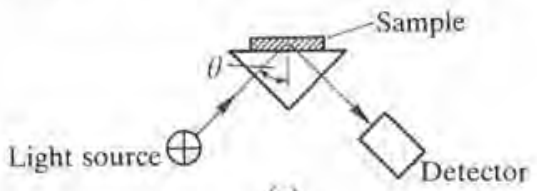

(a)

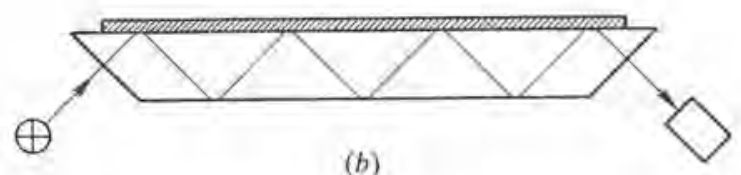

(b)

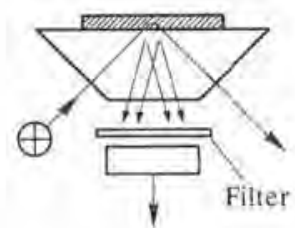

(c)

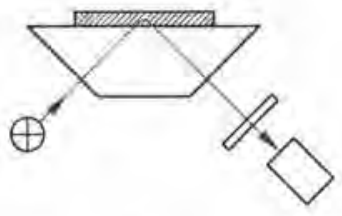

(d)

Fig. 33.1 Schematic representations of various internal reflection elements; (a) a single reflection prism; (b) a multiple-internal reflection element; (c) detection of right-angle fluorescence; (d) detection of in-line fluorescence.

transform infra-red spectroscopy to rapidly generate complete spectra of proteins adsorbed to a Germanium waveguide (Gendreau et al. 1981, 1982).

TIRF has also been applied to the study of protein interactions at surfaces using a number of fluorescence techniques. It can be considered that TIRF is a form of ATR spectroscopy, as in TIRF the absorption of evanescent photons by surface-bound molecules is the first of a two-step process where, in a second step the photons are re-emitted at a longer wavelength as fluorescence. Harrick and Loeb (1973) applied TIRF to monitoring bovine serum albumin (BSA)-dansyl chloride bound to the surface of a quartz waveguide, using TIRF. Watkins and Robertson (1977) measured fluoresceine (FITC)-bovine- $\gamma$-globulin binding to the surface of silicon rubber, and Lok et $a l .(1983 a, b)$ presented data on the binding of FITC-BSA also to silicon rubber, Van Wagenen et al. $(1980,1982)$ used the intrinsic fluorescence of protein tryptophan residues to analyse BSA bound to a quartz waveguide.

Two other techniques have been applied to TIRF measurement, Burghardt 
and Axelrod (1981) used photobleaching recovery to study tetramethylrhodamine-BSA adsorbed to a quartz slide, and Thompson et al. (1981) developed a fluorescence correlation spectroscopic method to measure the interaction of rhodamine-labelled IgG and rhodamine-labelled insulin with a BSA-coated quartz slide.

The potential advantages of using IRS devices for immunoassay lie mainly in the ability to monitor surface reactions with high sensitivity. Conventionally (e.g. Kirkham and Hunter 1971), immunoassays are a multi-step procedure involving several incubations and penultimately the separation of antibody-bound materials from non-bound components prior to measurement of the specific binding signal. This separation step is a major source of assay imprecision, is tedious, and is technically demanding. There has been considerable research into producing non-separation (i.e. homogeneous) immunoassays (e.g. fluorescence polarization (Dandliker et al. 1980), laser nephelometry (Deaton et al. 1976), homogeneous enzyme immunoassays (EMIT, Syva Corporation)) all of which have been applied in a routine clinical laboratory environment. However, there is still the requirement for a generally applicable method both for research and for routine immunoassays. IRS devices seem to offer an alternative approach as a novel form of homogeneous immunoassay with the advantage of being able to use a number of different optical detection techniques.

The key characteristic in the context of immunoassay is the ability to monitor surface reactions without major interferences from the bulk of solution. The concept lies in fixing one of the immunological binding pair to the surface of the waveguide, and monitoring its reaction with the complementary antigen (or antibody) without the need to carry out a formal separation step. This is because an 'in situ' separation occurs at the waveguide surface within the optically sensitive region of the evanescent wave thus precluding the physical separation of antibody-bound from 'free' molecules.

The following paragraphs describe the optical theory behind some of the techniques used for measuring surface reactions with an evanescent wave, the important factors of optical system design with respect to commonly used IREs, a review of available literature on IRS devices for immunoassay, and finally some comments on what the future may hold.

\subsection{Theoretical aspects}

\subsubsection{Principles of internal reflection spectroscopy}

When a light beam irradiates the interface between two transparent media (Fig. 33.2), striking from the medium of higher refractive index $\left(n_{1}>n_{2}\right)$, total internal reflection occurs (Harrick 1967) when the angle of reflection $\theta$ is larger than the critical angle $\theta_{c}$ :

$$
\theta_{\varepsilon}=\sin ^{-1}\left(n_{2} / n_{1}\right) \text {. }
$$




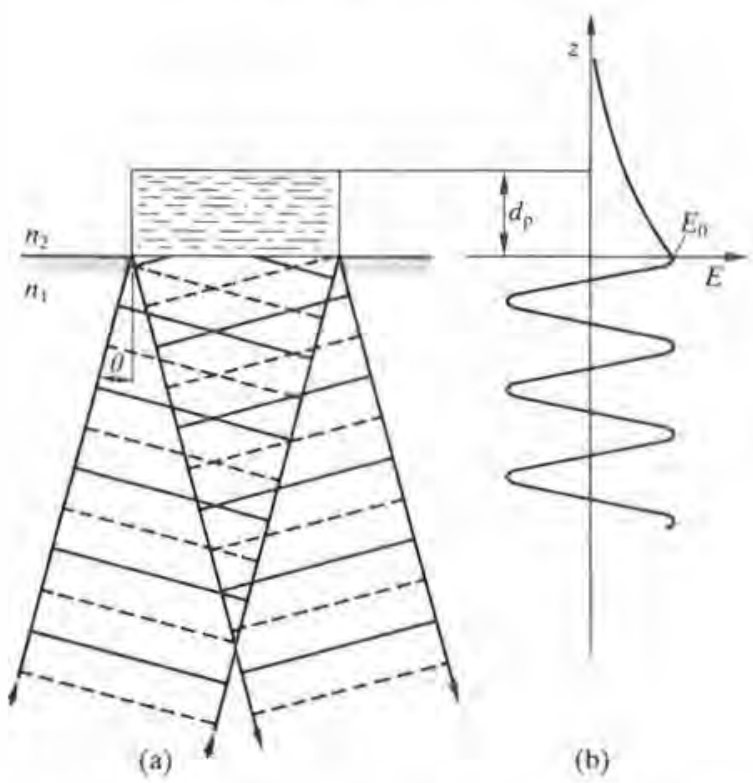

Fig. 33.2 Generation of the evanescent wave at an interface between two optical media; (a) where $n_{1}>n_{2}$ and $\theta>\theta_{c}, \theta_{c}$ is the critical angle at which refraction occurs; the evanescent wave is generated at the reflecting surface. (b) Same as (a), but representing the electric field amplitude $E$, on both sides of the reflecting surface $(Z=$ distance into the rarer medium, $d_{\mathrm{p}}=$ the characteristic penetration depth of the evanescent wave).

In this case the evanescent wave penetrates a distance $\left(d_{\mathrm{p}}\right)$, of the order of a fraction of a wavelength, beyond the reflecting surface into the rarer medium $\left(n_{2}\right)$. According to Maxwell's equations, a standing sinusoidal wave, perpendicular to the reflecting surface, is established in the denser medium (Fig. 33.1b). Although there is no net flow of energy into a non-absorbing, rarer medium, there is an evanescent field in that medium. Because of continuity conditions of the field vectors the electric field amplitude $(E)$ is largest at the surface interface $\left(E_{0}\right)$ and decays exponentially with distance $(Z)$ from the surface:

$$
E=E_{0} \cdot \exp \left(-Z / d_{\mathrm{p}}\right) .
$$

The depth of penetration $\left(d_{\mathrm{p}}\right)$, defined as the distance required for the electric field amplitude to fall to $\exp (-1)$ of its value at the surface, is given by

$$
d_{p}=\frac{\lambda / n_{1}}{2 \Pi\left[\sin ^{2} \theta-\left(n_{2} / n_{1}\right)^{2}\right]^{1 / 2}} .
$$


This quantity $d_{p}$ decreases with increasing $\theta$ and increases with closer index matching (i.e. as $n_{2} / n_{1} \rightarrow 1$ ). Also, because $d_{\mathrm{p}}$ is proportional to wavelength, it is greater at longer wavelengths.

Thus, by an appropriate choice of the refractive index $n_{1}$ of the IRE, of the incident angle, and of the wavelength, one can select a $d_{\mathrm{p}}$ to promote optical interaction mainly with compounds close to or affixed at the interface and minimally with bulk solution.

As an example, if the waveguide is made of quartz $\left(n_{1}=1.46\right)$, and the rarer medium is an aqueous sample $\left(n_{2}=1.34\right.$ ), $\theta_{c}$ is $66^{\circ}$ (eqn 33.1). If $\theta$ is selected as $70^{\circ}, \lambda$ as $500 \mathrm{~nm}$, the resultant $d_{p}$ is approximately $270 \mathrm{~nm}$ into the solution (see eqn 33.3). The estimated size of an $\mathrm{IgG}$ molecule (i.e. an antibody) is approximately $10 \mathrm{~nm}$ by $6 \mathrm{~nm}$ (Amzel and Poljak 1979). Thus a 'sandwich-type' immunological complex at the surface, consisting of three layers of $\mathrm{IgG}$ may have an average diameter of around $25 \mathrm{~nm}$. At $25 \mathrm{~nm}$ the field strength is still $91 \%$ of $E_{0}$ (see eqn 33.2). However at double or treble this distance, the field strength falls off to $83 \%$ and $76 \%$ respectively, due to the exponential decay characteristics.

The depth of penetration is one of four factors which determine the attenuation caused by absorbing films in internal reflection. The other factors are, the polarization-dependent electric field intensity at the reflecting interface, the sampling area which increases with increasing $\theta$, and matching of the refractive index of the denser medium to that of the rarer medium which in turn controls the strength of the optical coupling. The appropriate quantity which takes account of all these factors in the effective thickness, $d_{c}$. It represents the actual thickness of film that would be required to obtain the same absorption in a transmission experiment.

In order to enhance sensitivity, multiple reflection elements are often used. The number of reflections $(N)$ is a function of the length $(L)$, thickness $(T)$ of the waveguide and angle of incidence $(\theta)$.

$$
N=L / T \cdot \cot \theta
$$

The longer and thinner the waveguide, the larger is $N$ and the more frequently the evanescent wave interacts with the surface layer of antibody-antigen complexes. If for one reflection the reflectivity $(R)$ is

$$
R=1-\alpha \cdot d_{\varepsilon}
$$

where $\alpha$ is the absorption coefficient and $d_{e}$ is the effective thickness of a weakly absorbing layer, after $N$ reflections the reflection loss is

$$
R^{N}=1-N \cdot \alpha \cdot d_{\mathrm{e}}
$$

i.e. it is increased by a factor of $N$.

The evanescent wave can be used to monitor surface reactions by a number of optical techniques. Emphasis will be placed on two major forms of optical 
detection. Firstly, systems which are based on the optical absorbance or fluorescence characteristics of the compound to be measured, i.e. ATR and TIRF. Secondly, systems based on the change in the thickness/refractive index of the immunological film will be discussed. The latter includes surface plasmon resonance (SPR).

\subsubsection{Attenuated total reflection (ATR) and total internal reflection fluorescence (TIRF)}

When an absorbing material is placed in contact with the reflecting surface of an IRE, the resultant internally reflected light beam is said to be attenuated (Harrick 1967). In the case of ATR techniques, what is measured is the attenuated intensity as a function of incident wavelength, In TIRF, fluorescent materials are used, and thus the absorbed energy is partly reemitted as fluorescent light which is in turn detected.

To collect the fluorescence at a waveguide/liquid interface different signal collection techniques can be employed. Fluorescence emitted at an interface can be detected either conventionally where the detector is placed at right angles to the interface (Fig. 33.1c) or in-line with the primary light beam (Fig. 33.1d). Considering the very small solid angle of emission in in-line detection compared with the emission angle of right-angle geometry detection, the former would not seem to be very efficient. However, there is an enhancement effect, and theory predicts that for a fused silica waveguide with water as the $n_{2}$ medium, the in-line fluorescence intensity can be 50 times higher than the fluorescence emitted at right-angles to the waveguide. This effect, that fluorescence is tunnelled back into the waveguide is verified both theoretically and experimentally (e.g. Lee et al. 1979; Carniglia et al. 1972).

The following is a verbal explanation: in a first step, an incident plane wave generates an evanescent wave which excites molecules near the surface with a local distribution proportional to the evanescent electric field intensity (see eqn 33.2). After a characteristic excited-state lifetime, these molecules emit fluorescent radiation with a local distribution in the vicinity of the surface very similar to the exciting intensity distribution described by eqn 33.2 , i.e. that of an evanescent wave, but at the fluorescent wavelength. The question of what happens to the fluorescent evanescent wave can be answered by applying the principle of optical reciprocity, which states that this light is coupled back into the waveguide as a plane wave in the same way as the primary process when a plane wave generates an evanescent wave. Theory shows that the fluorescent intensity emission peaks at the critical angle of total internal reflection so that it can be internally reflected. To increase sensitivity, the IRE can be structured to collect fluorescent light from several reflections and guide it to the detector.

This is especially advantageous when an optical fibre is used as an IRE, as fluorescent light emitted at right-angles from an elongated fibre cannot be 
easily collected onto a detector. In-line detection also avoids measurement of fluorescence through the bulk of the sample solution surrounding the fibre, which otherwise can give significant interference, depending on the fluorescent dye used (Soini and Hemmilä 1979).

\subsubsection{Surface plasmon resonance (SPR)}

Surface plasmon resonance (also called surface plasmon oscillation) is a wellestablished concept that has been studied both theoretically and experimentally for a number of years. Detailed reviews of surface plasmon oscillations are given by Raether $(1977,1980)$. However, it is a new technique in the field of chemical and biological measurement.

Surface plasmons exist in the boundary of a solid (metal or semiconductor) whose electrons behave like those of a quasi-free electron gas. The plasmons represent the quanta of the oscillations of surface charges, which are produced by exterior electrical fields in the boundary. These charge oscillations are coupled with high frequency electromagnetic fields extending into space. Surface plasmons can be excited by electron beams or by light. Two types of surface plasmons can be distinguished: radiative and nonradiative. For sensing applications, non-radiative plasmons excited by light are of most interest. This plasmon is characterized by an exponential decrease of the electric field with distance from the boundary. Non-radiative surface plasmons are not produced by 'direct' illumination (i.e. using light reflected at a metal surface) as the momentum of the incoming photons is too small. The method mainly applied for sensing applications is the excitation of plasmons by evanescent waves. The optical configuration is the ATR, or prism arrangement called the Kretschmann configuration (Kretschmann and Raether 1968). Figure 33.3 illustrates the system, and the resemblance with the basic ATR approach can be seen (e.g. Fig. 33.1a) as an IRE in the form of a glass prism with refractive index $n_{1}$ is irradiated with light incident at the prism base at an angle $\theta$. The probed region below the prism base has a refrac-

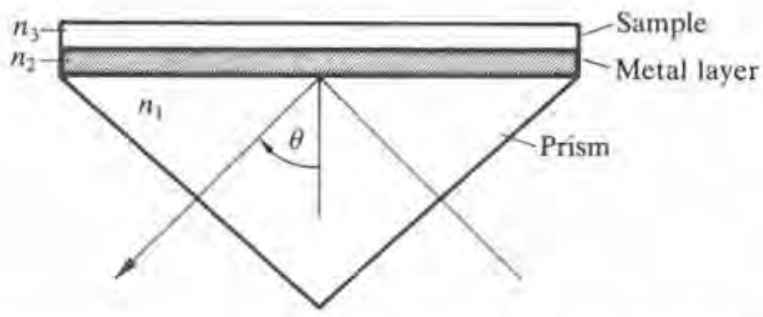

Fig. 33.3 Attenuated total reflection method of exciting non-radiative plasmons using the Kretschmann arrangement (Kretschmann and Raether 1968). $\theta$ is the incident angle of the light; $n_{1}, n_{2}$, and $n_{3}$ are the refractive indices of the glass prism, metal layer, and sample, respectively. 
tive index $n_{3}$ where $n_{1}>n_{3}$. The characteristic of SPR is the metal layer between the prism and sample. When the incident field is $p$-polarized and the angle of incidence $\theta$ is such that the photon momentum along the surface matches that of the plasmon, light can couple to the electron plasma in the metal. This is the surface plasmon resonance. It can be seen in the intensity of the totally-reflected light as a sharp drop in transmission as a function of the angle of incidence. The depth and width of this resonance minimum is determined by the characteristic absorption and the thickness of the metal film. For a given metal a thickness can be selected to give a reflected intensity of effectively zero (e.g. $c a .60 \mathrm{~nm}$ for silver). Variation of the wavelength of the incident light can displace the position and width of the minimum.

Figure 33.4 illustrates the spatial distribution of the energy density, $|\mathrm{H}|^{2}$, for three different angles of incidence and a $60 \mathrm{~nm}$ layer of silver as the metal; $\theta_{0}=50^{\circ}$, this is outside the resonance and the field energy decays exponentially inside the plasma; $\theta_{0}=45.4^{\circ}$, this is near the resonance and the field energy drops at first before reaching a higher value at the boundary; $\theta_{0}=45.2^{\circ}$, this is at resonance and the field energy is maximum at the boundary. In this case, the energy density is about eighty times higher as compared to the value without resonance. That is, there is an evanescent

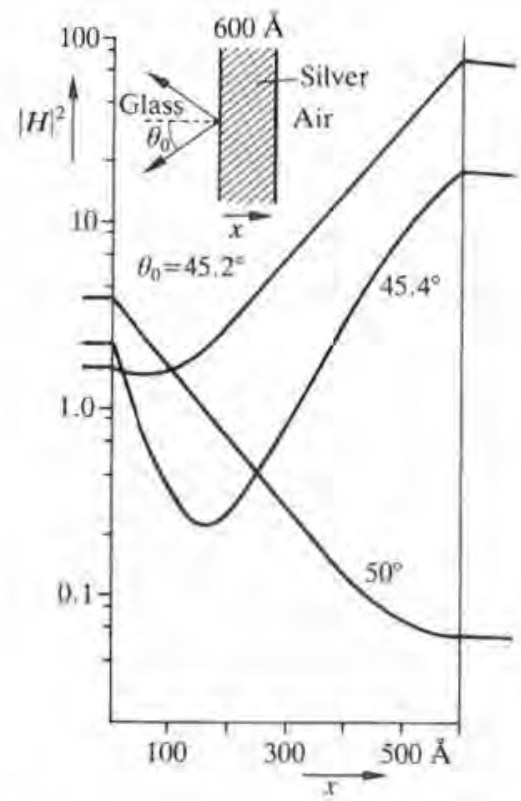

Fig. 33.4 The calculated electromagnetic density $|H(x)|^{2}$ in a $600-\AA ̊ \Omega$ silver film (left medium: glass, right medium: air) with the angle of incidence as the parameter $(\lambda=6000 \AA)$. (After Raether 1980, with permission.) 


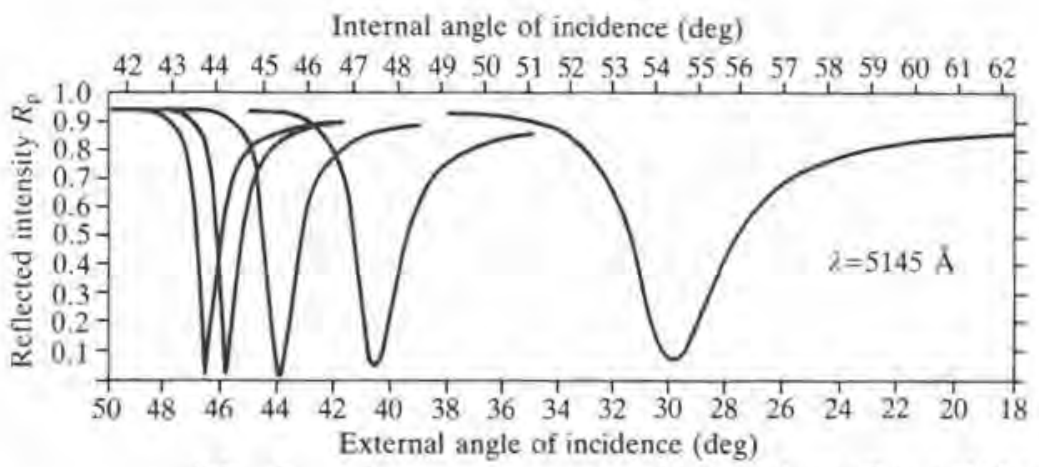

0136

12 Monolayers Cd-arachidate on silver

Fig. 33.5 Attenuated total reflectivity curves for silver films covered with different numbers of Cd-arachidate monolayers, as indicated $(\lambda=5145 \AA$ ) (after Pockrand et al. 1977, with permission).

wave outside the metal (in air in Fig. 33.4) which has the same penetration depth but is amplified eighty-fold when compared with ATR without a metal film. This is one reason for the high sensitivity of SPR. The other is that the angular position of the resonance minimum is very sensitive to variations in refractive index of the medium just outside the metal film, e.g. changing from air $(n=1.0)$ to water $(n=1.33)$ results in shifting the resonance angle from $43^{\circ}$ to $68^{\circ}$, using a silver film and a HeNe light source (Liedberg et al. 1983). Since the electric field probes the medium only a few hundred nm from the metal surface, the resonance is very sensitive to thin films at that surface. This is the effect used for sensing applications.

As an example, Fig. 33.5 shows the dependence of the resonance minimum on the number of Cd-arachidate monolayers each $26.8 \AA$ thick. It can be seen that the resonance position shifts to greater angles and its width increases with increasing numbers of layers. In terms of sensitivity, if the optical system can resolve better than $0.05^{\circ}$, coatings of a few angstroms thick can be measured.

\subsubsection{Ellipsometry}

Ellipsometry is probably one of the most commonly used techniques for measuring thin films deposited on a solid substrate. For detailed explanations of the theory, instrumentation, and applications, the reader is referred to the many review articles available (e.g. Poste and Moss 1972; Rothen 1974; Muller 1976; Azzam and Bashara 1977; Hauge 1980). Ellipsometry is mentioned in this chapter as it is one of the most sensitive techniques for measurement of thin films. Although it is based on external, not internal, 
reflection techniques, Carter et al. (1982) have suggested that ellipsometric measurements of thin biological films can be carried out using either singleor multiple-internal reflection waveguide systems. Published information on combining ellipsometry with IRS devices is very limited. It is not clear how useful this technique is, and it will not be discussed in any detail.

\subsection{Practical considerations in choosing and using IREs}

\subsubsection{IRE}

The key element to an IRS system is the IRE. The geometry of the IRE is a function of both the nature of the sample (in this instance usually a small quantity of liquid) and the IRS technique employed. It is not intended to review each of these techniques with reference to the detailed optical requirements and the reader is referred to the references in each of the preceding sections for such detail. However it is useful to pick out some of the important factors required to generate an evanescent wave at a liquid/waveguide interface.

A large variety of IREs have been developed (Harrick 1967), the simplest of which is the single reflection prism (Fig. 33.1a). The prism is generally used at a fixed angle $(\theta)$ and liquids can be held at the reflecting surface using a flow-cell arrangement (Van Wagenen et al, 1982). The single reflection prism is generally not sufficiently sensitive to allow the absorption measurements of immunoassays. However, fluorescence emission spectra can be obtained in right-angle geometry (Fig. 33.1c) (e.g. Van Wagenen et al. 1982). To obtain the high sensitivity required for ATR immunoassay, multiple internal reflection elements (e.g. plates, Fig, 33.1b) must be employed as increasing the number of reflections, $N$, enhances the contrast of a measured spectrum (see eqn 33,6). $N$ is increased by making the plate longer and/or thinner (see eqn 33.4). Because of practical considerations both the length $(L)$ and the thickness $(T)$ are limited. For example, with fast reactions the plate has to be sufficiently long to maximize sensitivity, but short enough to avoid filling delays. Also as $T$ decreases, the aperture is reduced limiting the usable light power. When $T$ is so small that light focused on the entrance aperture fills it completely, there may be problems designing a flow cell as gaskets may contact the light-guiding area. In this case the gasket is a potential source of light loss through absorption, refraction and scattering. Also the gasket may 'creep' during the test protocol. The design shown (Fig. 33.6) avoids contact of light with the gasket as light is introduced via a quartz prism which directs light away from the gasket. Variable-angle prisms were used to allow changing the angle of incidence $(\theta)$ and thus control the depth of penetration (see eqn 33.3) such that the spectral contrast can be optimized. In this case $\theta$ is varied by the linear movement or rotation of a mirror (Fig. 33.6).

A very promising multiple-reflection IRE is the optical fibre. Here light is 


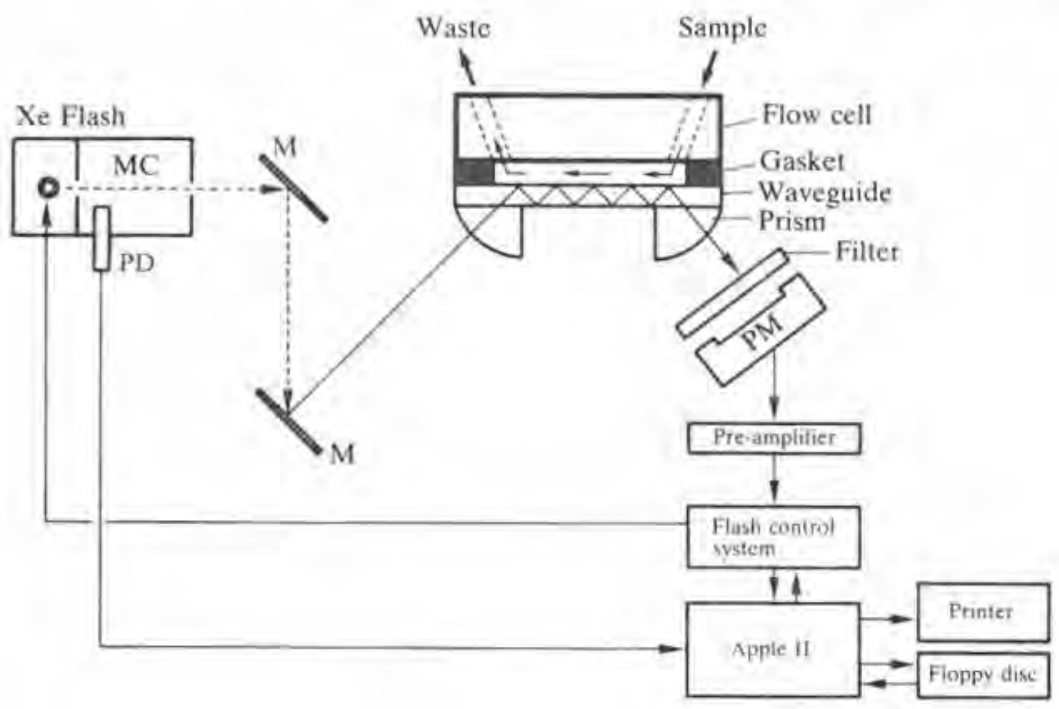

Fig. 33.6 Diagram of instrumental layout used for measuring immunoassays with a multiple-internal reflection plate. PM, photomultiplier tube; PD, photodiode; MC, monochromator; $\mathrm{M}$, mirrors.

also introduced at angles exceeding $\theta_{c}$ and propagates down the fibre by total internal reflection. Optical fibres manufactured for communication purposes are available with high optical quality. Fibres have been employed as IREs because of their small diameter and (potentially) unlimited length so that the effective number of reflections can be very large. To allow the fibre surface to interact with the sample, the protective coating and cladding have to be removed from communication fibres. This is relatively easy for plastic clad silica fibres (available core diameters from 0.2 to $1.5 \mathrm{~mm}$, e.g. Fibre Optique Industries, Pithiviers, France). Figure 33.7 shows a diagram of a fibre optic assembly ( $0.6 \mathrm{~mm}$ diameter fibre core). Within a cylindrical flow cell, the straight fibre is exposed to the sample solution along an active length of $64 \mathrm{~mm}$. The fibre ends are held in metal fittings and polished. The lenses define the angular aperture of around $70^{\circ}$.

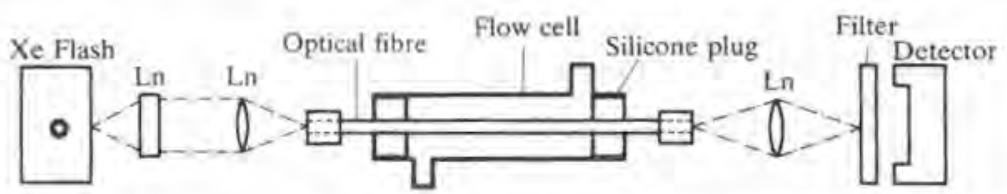

Fig. 33.7 Diagram of fibre-optic assembly with flow cell and light coupling optics. Ln are lenses. 
Other types of fibres, e.g. quartz long-distance communications fibres (core diameters $5-50 \mu \mathrm{m}$ ), are difficult to handle because of their small size. Similarly, because of the complicated optical paths and mode conversion effects introduced by bends in longer optical fibres, calculations of angles of incidence and hence effective thickness cannot easily be made. There are no data available using such fibres for immunoassay.

\subsection{2 $S P R$}

The experimental components used for SPR are similar to those of an ATR system (c.f. Fig. 33.3 and Fig. 33.1a). The IREs are prisms coated on the sensor side with a thin film of metal (e.g. $\mathrm{Au}, \mathrm{Ag}, \mathrm{Al}$, or $\mathrm{Cu}$ ).

Several methods are used for detecting the plasmon resonance. The most common is measuring the attenuated reflection as a function of incident angle at a fixed wavelength, or as a function of wavelength for a fixed incident angle. Using the former approach angular shifts of the order of $0.0005^{\circ}$ have been measured (Eagen and Weber 1979). By choosing an angle of incidence half-way down the reflectance minimum and measuring the intensity of reflected light at that constant angle, changes in the refractive index of the order of 0.00001 were detected (Liedberg et al. 1983). Another method measures the scattered light coupled out by the surface roughness of the film as a function of incident angle at a fixed wavelength. This geometry is similar to that of TIRF with right-angle detection (see Fig, 33,1d).

\subsubsection{Material requirements}

When choosing an optical material for IREs consideration is given to optical characteristics (refractive index, wavelength transmission limits, surface quality), mechanical characteristics (hardness, brittleness), and chemical characteristics (inertness, adsorption efficiency of proteins, availability of chemical groups for covalent protein attachment).

The optical and mechanical aspects are discussed in detail by Harrick (1967) who gives refractive indices and transmission limits for a number of materials for the visible, UV, IR, and far IR regions. Opposing surfaces must be flat and parallel, and the surface quality must be sufficiently high to minimize light losses due to scattering. Hardness and brittleness are also important to reduce physical handling problems both in producing an IRE and particularly in preparation of the surface,

The material most often used for IREs is quartz due to its well-characterized optical qualities and chemical properties. The transmission range of quartz is from 0.3 to $2.3 \mu \mathrm{m}$ allowing measurements to be carried out in the UV visible and near-IR. Also its hardness makes it a relatively simple material to handle. Quartz IREs are readily available as microscope slides and as the core material of plastic-clad silica fibres. 


\subsubsection{Antibody immobilization}

The immobilization of one of the immunological binding pair to the IRE surface is a key factor in the successful development of an IRS device for immunoassay. Not only does the immobilization procedure have to meet requirements of reproducibility, high protein uptake, retained immunological activity, and stability, but also the procedure should not cause chemical or physical changes to the surface which cause undesirable optical effects (e.g. light scattering). Since most references apply to quartz or glass, this discussion will be limited to these materials. Also the following paragraphs will deal with protein immobilization only. Many non-protein antigens may be immobilized to IRE surfaces but the individual chemistries are not generally applicable and will not be discussed here. However, one approach is to coat the IRE with a protein (e.g. bovine serum albumin) and then couple with non-protein antigen to this material (Kronick and Little 1975).

The two most commonly used techniques for protein immobilization to silica surfaces are physical adsorption and covalent coupling. There is a considerable quantity of information available on this subject (e.g. see reviews: Weetall, 1972, 1974, 1975; Jakoby and Wilchek 1974; Mosbach 1976) and the reader is referred to these reviews for detailed procedures as the following discussion will be limited to general principles.

Physical adsorption of proteins to silica surfaces is probably the result of weak-force interactions such as charge-charge (e.g. ionic, salt-bridge) or hydrogen-bonding (Messing 1976). However, protein-glass bonds formed during adsorption are so tight that strong acids or alkalis are required to recover the proteins. Physical adsorption as a method of preparing antibodycoated IREs, is attractive as proteins can be rapidly adsorbed onto glass surfaces in a single-step procedure. Also adsorption does not change the optical characteristics. A typical approach would be to first wash the surface clean using one or a combination of strong acids, detergents, organic solvents, and physical means such as heating to high temperatures or ion bombardment (Burghardt and Axelrod 1981; Thompson 1982). A hydrophobic surface can then be generated (e.g. by washing the IRE in dichloromethyl silane (Elwing and Stenberg 1981)) which encourages protein adsorption. The IRE is then dipped into the appropriate protein solution for several hours, washed to remove unbound materials, incubated with a nonspecific protein solution to block unfilled binding sites, washed again and stored dry at $4{ }^{\circ} \mathrm{C}$ until required.

Adsorption of proteins to surfaces is a complex and generally poorly understood reaction (Lundström 1983). As such, the reproducible preparation of antibody-coated waveguides is based on an empirical 'trial and error' approach. Also with such a technique it is difficult to predict the configuration of proteins on the surface and the effects of the surface adsorption 
event on availability of antibody-binding sites. Covalent linkage is a more attractive approach as it potentially allows control of the immobilization reaction and a certain degree of predictability.

Most work of the covalent attachment of proteins to silica has been carried out with controlled pore glass particles as the solid-phase (for a review see Lynn 1975). The most often used and probably the simplest technique is reaction with silane coupling agents. These agents are monomeric silanes which have organo-functional groups at one end and groups which will react with the inorganic surface at the other end. A typical agent is $\gamma$-aminopropyltriethoxy silane (APTS) which can be used in a three-step procedure to attach proteins to silica. In an aqueous acid environment, the ester groups on the ATPS hydrolyse to form hydroxyls which can then condense with surface silanol groups to give a silica surface with pendant amino groups (eqn 33.7).

$$
\begin{aligned}
& \text { Silica - SiOH }+\mathrm{C}_{2} \mathrm{H}_{5}-\mathrm{OSi}\left(\mathrm{OC}_{2} \mathrm{H}_{5}\right)_{2}\left(\mathrm{CH}_{2}\right)_{3} \rightarrow \\
& \text { Silica-SiOSi }\left(\mathrm{OC}_{2} \mathrm{H}_{5}\right)_{2}\left(\mathrm{CH}_{2}\right)_{3}-\mathrm{NH}_{2}
\end{aligned}
$$

In a second step, the amino groups are used to prepare an active aldehyde intermediate by reaction with glutaraldehyde at neutral $\mathrm{pH}$ (eqn 33.8):

Silica $-\mathrm{NH}_{2}+\mathrm{OCH}\left(\mathrm{CH}_{2}\right)_{2} \mathrm{CHO} \rightarrow$ Silica $-\mathrm{N}=\mathrm{CH}\left(\mathrm{CH}_{2}\right)_{2} \mathrm{CHO}$

The final step is to react the aldehyde group with a primary amine on the protein to form an imine coupling (eqn 33.9):

$$
\text { Silica }-\mathrm{CHO}+\mathrm{NH}_{2} \text {-Antibody } \rightarrow \text { Silica }-\mathrm{CH}=\mathrm{N} \text {-Antibody }
$$

This approach has been applied to antibody immobilization to planar quartz and optical fibre IREs (Sutherland et al. 1984b). However, there are a number of potential pitfalls with this chemistry as the optimum concentration of active aldehyde groups on the surface falls within a narrow useful range. A minimum number of these groups is required for a stable antibody preparation and too many groups can result in multiple site attachment of the antibody to the surface resulting in decreased immunological activity. Also, the silane layer thickness is a function of many factors including $\mathrm{pH}$, temperature, time of reaction, surface area, silane concentration and the reaction solvent (Schroder et al. 1967). Thus for reproducibility each of these factors has to be considered when optimizing the coupling conditions.

Alternative covalent systems have been used with reflectance devices (Nilsson and Mosbach 1981). Here silicon wafers are alkylated, hydroxyl groups formed on the alkylated surface and then activated with tresyl chloride. The tresylate groups can then react with amino groups on the protein. This technique was applied to the attachment of concanavalin A to silicon surfaces which were subsequently reacted with $S$. aureus cells and the reaction monitored by ellipsometry (Mandenius et al. 1984). 


\subsection{Application of IRS devices to immunoassay: review}

\subsection{ATR}

ATR has been applied to monitoring immunoassays in the IR, visible, and ultra-violet (UV) regions of the spectrum. The basic principles are those given in the above theoretical section, where sequestration of antigen (or antibody) at a waveguide/liquid interface is measured as an attenuation of the internally reflected light beam. This attenuation is wavelength specific for an absorption maximum characteristic of one of the immunological binding pair.

In the IR region, Ockman (1978) has applied ATR to monitoring the reaction between various sera albumin and their respective antisera, at a germanium/liquid interface. The waveguide surface was initially immersed in the antigen solution and the transmission spectra obtained of the absorbed protein layer. Following reaction with dilute antiserum solution, bound antibody was identified as a decrease in transmission in the Amide 1 region $\left(1650 \mathrm{~cm}^{-1}\right)$. The change in optical density was 0.032 units for specific binding (antibody-antigen reaction) while only 0.007 units for non-specific binding (in the absence of antigen).

This end-point approach, though successful, does not make full use of the potential of IRS methods which should allow continuous monitoring of the immunological reaction as it occurs. Such continuous analyses have been carried out in both the visible and UV regions.

As a model system, the reaction between haemoglobin and rabbit antihaemoglobin antisera has been monitored by ATR techniques (Sutherland, R. M., Kulhanek, E., and Dähne, C., unpublished observations). In this case, rabbit antibodies were covalently immobilized to the surface of a quartz microscope slide, then reacted with different concentrations of haemoglobin. By monitoring the attenuation of the reflected light at $410 \mathrm{~nm}$ (absorption maximum of haemoglobin) a three-phase response curve was obtained (Fig. 33.8). Initially a base-line was established in the presence of a physiological buffer system containing sheep serum (to minimize non-specific binding of haemoglobin to the surface). On introduction of the antigen solution there was an immediate fall in transmission due to light absorption by haemoglobin molecules diffusing within the penetration depth of the evanescent wave. This was followed by a rapid attenuation in transmission as the haemoglobin molecules were bound close to the surface by antibodies. Finally as the antibody-binding sites became saturated the rate of change in transmission became smaller. Also shown are the results of repeating the experiment with BSA replacing the antibody of the surface. The difference between the two curves represents specific binding of haemoglobin at the surface.

Similar results were found in the UV region, where specific antibody- 


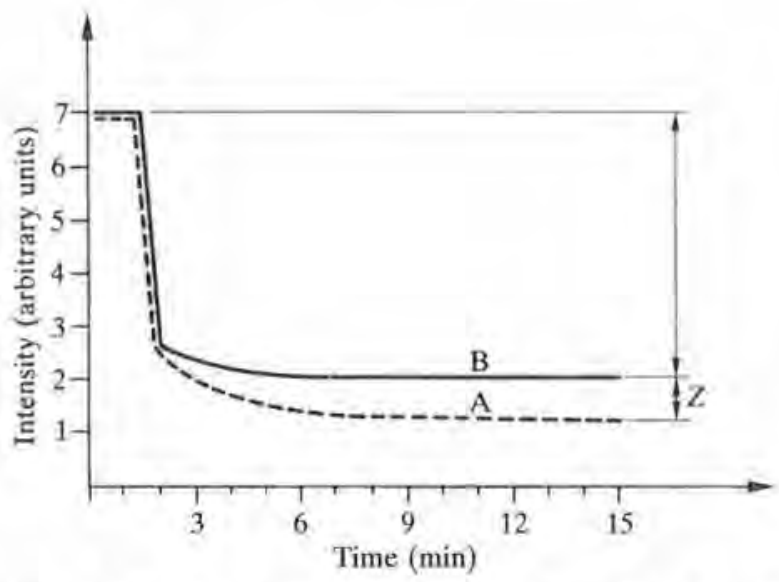

Fig. 33.8 Injection of a $10 \mathrm{mg} / \mathrm{ml}$ solution of haemoglobin into the flow cell illustrated in Fig. 33.6, with (A) rabbit anti-haemoglobin (code No. A118, Dako Immunoglobulins, Copenhagen, Denmark) attached to the surface of the multipleinternal reflection element by a covalent bond (aminopropyltriethoxy silane; see text for details) and (B) bovine serum albumin similarly immobilized to a second IRE. The reaction was monitored by the attenuation of the internally-reflected light beam at $410 \mathrm{~nm}$. The difference between the two curves $(Z)$ is taken to represent specific binding (Sutherland, R. M., Kulhanek E. and Dähne, C,; unpublished observations).

binding of the chemotherapeutic agent methotrexate, at a quartz/liquid interface was monitored by the attenuation in transmission at $310 \mathrm{~nm}$ (Sutherland et al. 1984a). Plots of the absolute change in transmission, or rate of change in transmission, gave a dose-response curve with an estimated limit of detection of $0.3 \mu \mathrm{mol} / 1$ for methotrexate.

\subsubsection{TIRF}

The original work using TIRF to measure immunological reactions was carried out by Kronick and Little $(1973,1975,1976)$. Haptens such as phenylarsonic acid or morphine were immobilized to the surface of a quartz microscope slide via a hapten-albumin conjugate. FITC-labelled antibody which bound to the immobilized hapten could be detected by exciting fluorescence at the surface using the evanescent wave. On addition of free hapten to the bulk of solution the binding rate of the FITC-antibody to the surface was reduced in a concentration-dependent fashion. Monitoring the fluorescence at right-angles (see Fig. 33.1c), a minimum detection limit of $0.2 \mu \mathrm{mol} / 1$ of morphine could be measured.

Thompson (1982) used a similar approach combined with fluorescence correlation spectroscopy. Here, dinitrophenol was immobilized to a quartz 
slide via adsorbed albumin. Both monovalent and bivalent rhodaminelabelled antibodies were reacted with the surface-bound hapten. One of the main findings associated with these experiments was the large proportion of non-specifically bound antibody (up to $60 \%$ of the signal). One conclusion by Thompson was that the high level of non-specific binding prevented continuous measurement of the kinetics of binding.

Sutherland et al. (1984b) used the reaction between human $\mathrm{IgG}$ and two antisera to investigate some of the critical factors which might overcome the non-specific binding in a TIRF system, with FITC as the Iabel. Preliminary experiments demonstrated that inclusion of an excess (200-fold relative to the antibody) of an appropriate non-immune animal serum into the buffer, reduced non-specific binding of the FITC-labelled protein to acceptable levels. The non-immune serum probably competed for and saturated, nonspecific binding sites on the waveguide surface and similarly competitively inhibited non-specific interactions between the fluorescently-labelled proteins and immobilized antibody.

With assay buffers containing the animal serum, experiments were carried out to partially optimize a two-site immunofluorometric assay for IgG. A sheep anti-IgG was covalently immobilized to the waveguide surface and reacted with standard solutions of human IgG. Following rinsing with buffer to remove unbound materials, the reaction between immunologically immobilized IgG and a second (FITC-labelled) rabbit antibody was monitored by measuring the right-angle fluorescence. The signal generated using this approach is illustrated (Fig. 33.9), with an explanation of the various signal components. The detection limit of this system was approximately $5 \mu \mathrm{g} / \mathrm{ml}$ of IgG.

All these TIRF systems are based on right-angle detection of the fluorescent signal. It is also possible to use in-line detection (Fig. 33.1d). Sutherland et al. (Sutherland et al. 1984a; Dähne et al, 1984) have used both quartz microscope slides and quartz optical fibres to monitor the two-site immunofluoremetric assay for IgG, with signal detection in the in-line mode. After a ten-minute incubation with the FITC-antibody, these authors achieved a detection limit of 3.0 and $1.5 \mu \mathrm{g} / \mathrm{ml}$ of $\mathrm{IgG}$, for the slide and fibre waveguides respectively.

\section{$33.4 .3 S P R$}

Nylander et al. (1982) first suggested using SPR as a technique to monitor immunological reactions and measured changes as small as $30 \AA$ in the thickness of an immunological layer. In later work, using the reaction between human IgG and anti-IgG as a model, the antigen was absorbed to the silvered surface of a prism to give a layer of protein up to $50 \AA$ thick (Liedberg et al. 1983). Various concentrations of anti-IgG were individually incubated with the absorbed protein and the shift in resonance angle monitored at a fixed 

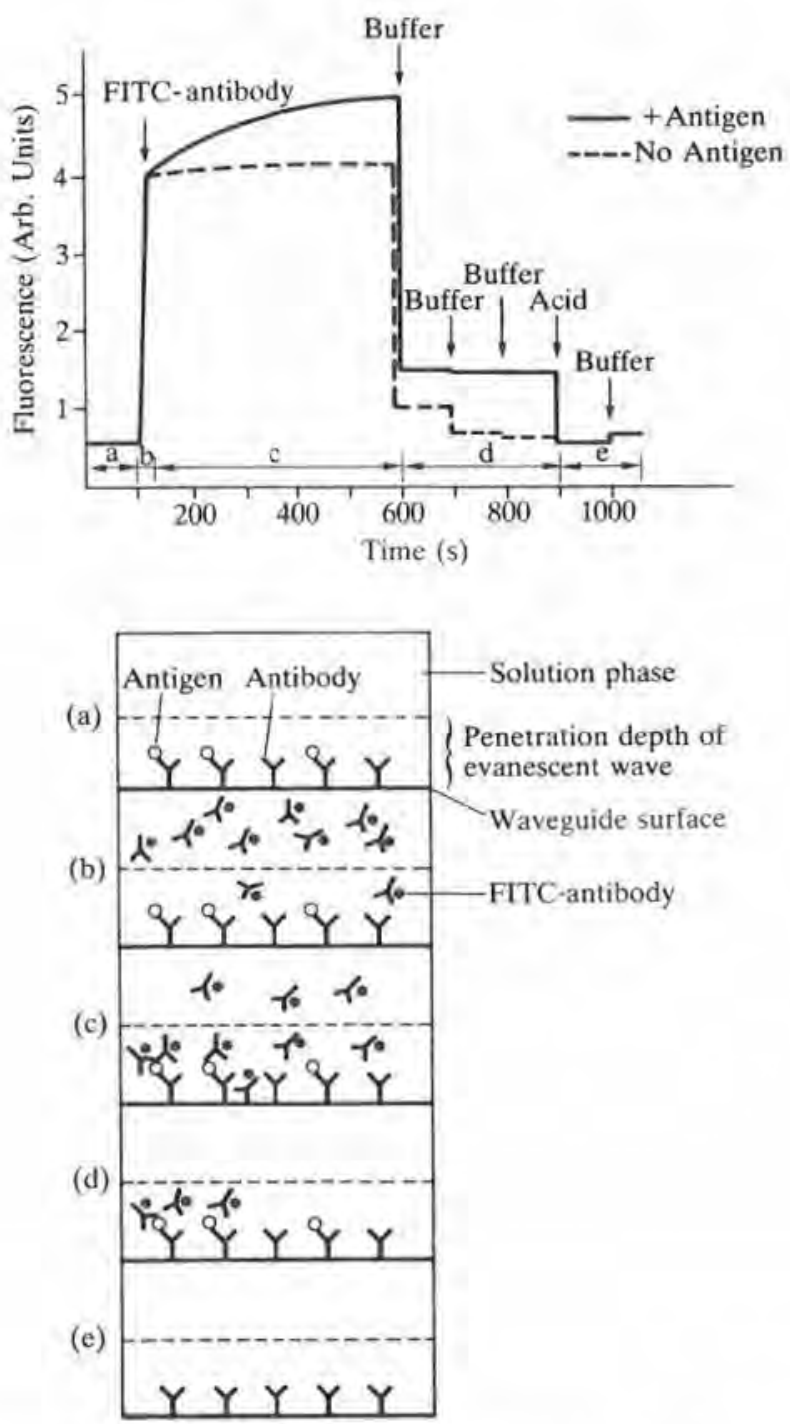

Fig. 33.9 Binding curves and a schematic representation of a two-site immunofluorimetric assay monitored by TIRF. Here the IRE surface is coated with antibody raised against Human IgG. Following reaction of the coated waveguide with a standard IgG solution ( $10 \mu \mathrm{g} / \mathrm{ml})$ a second FITC-labelled anti-IgG is allowed to react with the immobilized antigen and the reaction monitored by measuring fluorescence either at right-angles (Sutherland et al, 1984b) or with an in-line geometry (Sutherland et al. 1984a). (a) The prepared IRE; (b) signal generated by unbound FITC-anti-IgG within the $d_{\mathrm{p}}$ of the evanescent wave; (c) binding curves of FITC-anti-IgG in the 


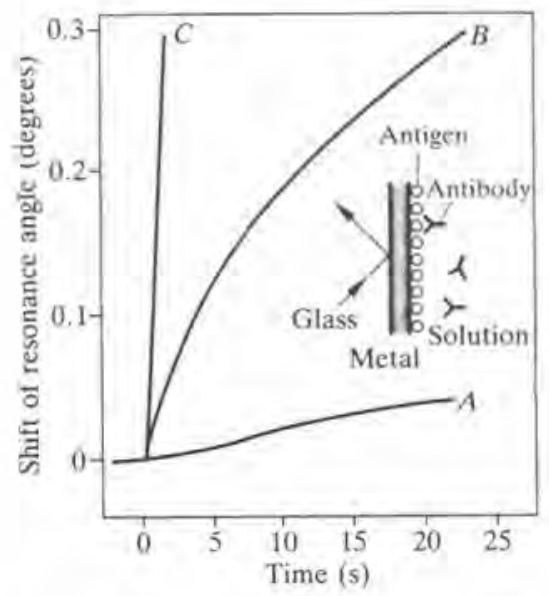

Fig, 33.10 Shift in resonance angle versus time for three different anti-IgG concentrations; $A, B$ and $C$ for 2,20 and $200 \mu \mathrm{g} / \mathrm{ml}$ of $\mathrm{IgG}$ respectively. The shift is measured as an increase of the reflected light intensity. The insert illustrates the antibody binding event. (After Liedberg et al. 1983, with permission.)

angle of incident light (Fig. 33.10). Using this approach the SPR system could differentiate between 0.2 and $2 \mu \mathrm{g} / \mathrm{ml}$ of antibody after $25 \mathrm{~s}$.

Flanagan and Pantell (1984) carried out similar experiments using human serum albumin as the antigen. The initial layer of antigen was $60 \AA$ thick. This increased to $200 \AA$ following reaction with the antibody, corresponding to up to 4 layers of $\mathrm{IgG}$. The authors suggested that there was a proportion of non-specifically adsorbed antibodies in this second layer, although cooperativity between antibodies may also be a factor.

\subsection{Discussion}

The potential for IRS devices in immunoassay is based on the ability to monitor surface reactions without major interferences from the bulk of solution components. The above examples demonstrate the basic feasibility of this concept. However considerable effort will have to be expended before these systems achieve their potential in both research and commercial environments. Two factors are essential to the successful application of IRS devices for immunoassays. Firstly, antibodies as reagents allow the specific measurement of a compound (or family of compounds) from complex

presence (solid line) and absence (dotted line) of antigen; (d) washing away unbound materials leaving the specific (solid line) and non-specifically (dotted line) bound signals; (e) disrupting the immunologically bound materials with dilute acid. 
mixtures such as found in clinical samples (e.g. serum, plasma, or whole blood). Considerable research has been carried out to adopt procedures in conventional immunoassays, which do not compromise this specificity. However, with IRS devices some new (and old) problems may occur. Each of the three systems, ATR, TIRF, and SPR, will be sensitive to non-specific optical and/or physical interactions at the interface. Both the ATR and TIRF techniques will be sensitive to substances which will optically interfere within the penetration depth of the evanescent wave (e,g. substances which absorb at the same wavelength, or quench fluorescence). All three systems, but probably SPR in particular will be sensitive to physical non-specific binding events at the interface. As SPR is essentially a measure of an effective layer thickness and refractive index, non-specific changes in either of these parameters may be difficult to differentiate from specific binding events.

A second factor is that conventional immunoassays involving a separation step (i.e. they are heterogeneous), are sensitive systems which can detect compounds down to femtomolar levels using radioactive labels. As yet the ATR, TIRF, and SPR available data show detection limits of approximately $1 \mu \mathrm{mol} / 1,10 \mathrm{nmol} / 1$, and $<5 \mathrm{nmol} / 1$. For assay systems approaching the sensitivity of conventional heterogeneous assays significant progress will have to be made.

One key element in enhancing sensitivity is the use of multiple internal reflections, as yet applied to ATR and TIRF systems. Using thinner and/or longer waveguides is rewarded by an increase in sensitivity factorially related to the number of reflections (see eqn 33.6). In this context, optical fibres seem to offer considerable advantages, as they can be manufactured very thin and, with appropriate optics, effectively all of the external surface is used as the sensor. One design for a disposable optical fibre diagnostic is available (Fig. 33.11). There may be, however, a considerable challenge in manufacturing such a device.

If adequate sensitivity can be achieved with reasonable assay times (minutes, not hours) IRS devices such as optical fibres may find application in vivo as well as in vitro. Optical fibres have already enjoyed success in the fields of surgery, optical investigative procedures (e.g. endoscopy), and in vivo analyses (e.g. blood pressure, blood oxygenation, $\mathrm{pH}$ ). This is due to their small size and physical flexibility as well as light-guiding properties. Used as in vivo IRS devices for immunoassay other characteristics may be equally important, such as being chemically inert, the possibility of construction from biocompatible materials and fabrication costs may be sufficiently low to warrant a disposable system. Two major problems will have to be addressed however. Firstly how to sterilize such a disposable without destroying the antibodies. Secondly how to use the device to continuously monitor. The latter problem is due to the affinity constant of the antibody which makes binding effectively irreversible, and thus the sensor may not 


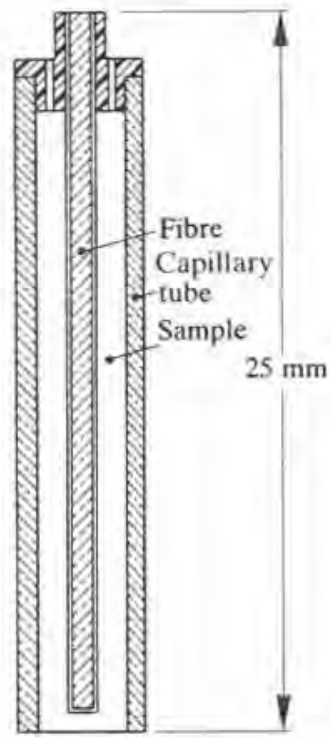

Fig. 33.11 A disposable fibre-optic IRE for TIRF immunoassays. The fibre is held within a capillary tube which allows easy sampling (the sample is introduced into the cuvette by capillary action) and the capillary tube accurately defines the sample volume. (After Hirschfeld 1984, with permission).

respond rapidly to changes (reductions) in antigen concentration.

One of the aims for an IRS device applied to optical immunoassays must be to supply easier test systems useful in clinical diagnostics. Due to the lack of a separation step such assay systems could be operated in a relatively nonskilled environment. To achieve this potential requires not only suitable IRE development but also design of a simple photometric system in which to carry out measurements. As yet such a system is not readily available.

In conclusion, IRS devices for immunoassays have great potential for both research and commercial applications as they offer a relatively flexible and simple alternative to the conventional immunoassay. As such, appropriate development could result in these devices entering the in vitro and even in vivo diagnostics market place.

\section{Acknowledgements}

We wish to acknowledge the technical assistance of Mr. A. Bregnard in the development of the fibre-optic system, Mr. A. Ringrose for his considerable efforts in designing the planar waveguide system used in our laboratories, and also to Ms. E. Kulhanek for her work on the haemoglobin system 
reported above. We also acknowledge the help of the secretarial staff of the Battelle Institute for their typing of this manuscript under a tight schedule and especially Ms. J. Boëque for proof-reading the manuseript. Some of the above work was financially supported by Prutec Ltd.

\section{References}

Amzel, L. M. and Poljak, R. J. (1979). Three-dimensional structure of immunoglobulins. Ann. Rev, Biochem, 48, 961-98.

Azzam, R. M. A. and Bashara, N. M. (1977). Ellipsometry and polarized light. North Holland Publishing Co., Amsterdam.

Baier, R. E. and Dutton, R. C. (1969). Initial events in interaction of blood with a foreign surface. I. Biomed. Mater. Res. 3, 191-206.

Brash, J.L. and Lyman, D. J. (1971). Adsorption of proteins and lipids to nonbiological surfaces. In The chemistry of biosurfaces (ed. M. L. Hair), pp. 177-232. Marcel Dekker Inc., New York.

Burghardt, T.P. and Axelrod, D. (1981). Total internal reflection/fluorescence photobleaching recovery study of serum albumin adsorption dynamics. Biophys, $J$. $33,455-68$.

Carniglia, C. K., Mandel, L. and Drexhage, H. (1972). Absorption and emission of evanescent photons. J. Opt. Soc. Amer. 62, 479-86.

Carter, T., Dähne, C, and Place, J.F. (1982). European Patent Application EP 81-801255.0.

Dähne, C., Sutherland, R. M., Place, J. F. and Ringrose, A. R. (1984). Detection of antibody-antigen reactions at a glass-liquid interface: a novel fibre-optic sensor concept. In OFS ' 84 , Proceedings of the second international conference on optical fibre sensors (eds. R. T. Kersten and R. Kist), pp. 75-9. VDE-Verlag, Berlin.

Dandliker, W. B., Hsu, M.-L. and Vanderlaan, W.P. (1980). Fluorescence polarization immuno/receptor assays. In Immunoassays, clinical laboratory techniques for the 1980's (eds. R. M. Nakamura, W. R. Dito, and E. S. Tucker), pp. 65-88. Alan R. Liss Inc., New York.

Deaton, C. D., Maxwell, K. W., Smith, R. S. and Creveling, R. L. (1976). The use of laser nephelometry in the measurement of serum proteins. Clin. Chem. 22, $1465-70$.

Eagen, C. F. and Weber, W. H. (1979). Modulated surface-plasmon resonance for adsorption studies. Phys, Rev. 19, 5068-82.

Elwing, H. and Stenberg, M. (1981). Biospecific bimolecular binding reactions $-\mathrm{a}$ new ellipsometric method for their detection, quantitation and characterization. J. Immunol. Methods. 44, 343-9.

Flanagan, M.T. and Pantell, R.M. (1984). Surface plasmon resonance and immunosensors. Electr. Lett, 20, 968-70.

Gendreau, R.M., Winters, S., Leininger, R. I., Fink, D., Hassler, C. R. and Jakobsen, R. J. (1981). Fourier-transform infrared spectroscopy of protein adsorption from whole blood: Ex-vivo dog studies. App. Spectr. 35, 355-7.

- Leininger, R. I., Winters, S. and Jacobsen, R. J. (1982). Fourier-transform infrared spectroscopy for protein-surface studies. In Biomaterials: Interfacial 
phenomena and applications (eds. S. L. Cooper and N. A. Peppas), Adv. Chem. Ser, 199, 371-95.

Harrick, N. J. (1967), Internal reflection spectroscopy. Interscience, New York. and Loeb, G. I. (1973). Multiple internal reflection spectrometry. Anal. Chem. 45, 687-91.

Hauge, P. S. (1980). Recent developments in instrumentation in ellipsometry. Surf. Sci. 96, 108-40.

Hirschfeld, T, E, (1984). US Pat. 4,447,546.

Jakoby, W.B. and Wilchek, M. (eds.) (1974), Affinity techniques. Methods in enzymology. Vol. 34, Academic Press, New York.

Kirkham, K. E. and Hunter, W. M. (1971). Radioimmunoassay methods. ChurchillLivingstone, Edinburgh.

Kretschmann, E. and Raether, H. (1968). Radiative decay of non-radiative surface plasmons excited by light. Naturforschung 123, 2135-6.

Kronick, M.N. and Little, W.A. (1973). A new fluorescent immunoassay. Bull. Amer. Phys. Soc. 18, 782.

and Little, W. A. (1975). A new immunoassay based on fluorescence excitation by internal reflection spectroscopy. J. Immunol. Methods. 8, 235-42. (1976). US Pat. No. 3,939,350.

Lee, E. H., Benner, R. E., Fenn, J. B. and Chang, R. K. (1979). Angular distribution of fluorescence from liquids and monodispersed spheres by evanescent wave excitation. App. Opt. 18, 862-70.

Liedberg, B., Nylander, C. and Lundström, 1. (1983). Surface plasmon resonance for gas detection and biosensing. Sensors and Actuators 4, 299-304.

Lok, B. K., Cheng, Y.-L. and Robertson, C. R. (1983a). Protein adsorption on crosslinked polydimethylsiloxane using total reflection fluorescence. J. Coll, Interf. Sci. 91, 104-116.

- $(1983 b)$. Total internal reflection fluorescence: A technique for examining interaction of macromolecules with solid surfaces. Ibid. 87-103.

Lundström, I. (1983). Surface physics and biological phenomena. Phys. Scrip. T4, 5-13.

Lynn, M. (1975). Inorganic support intermediates: covalent coupling of enzymes on inorganic supports. In Immobilized enzyme, antigens, antibodies and peptides (ed. H. H. Weetall), pp. 1-48, Marcel Dekker Inc., New York.

Mandenius, C. F., Welin, S., Danielsson, B., Lundström, I. and Mosbach, K. (1984). The interaction of proteins and cells with affinity ligands covalently coupled to silicon surfaces as monitored by ellipsometry. Anal. Biochem. 137, 106-14.

Messing, R. A. (1976). Adsorption and inorganic bridge formation. In Immobilized enzymes (ed. Mosbach, K.). Methods in enzymology, Vol. 64, pp. 148-169. Academic Press, New York.

Mosbach, K. (ed.) (1976). Immobilized enzymes. Methods in enzymology. Vol. 64. Academic Press, New York.

Muller, R. H. (1976). Present state of automatic ellipsometers. Surf. Sci. 56, 19-36. Nilsson, K. and Mosbach, K. (1981). Immobilization of enzymes and affinity ligands to various hydroxyl group carrying supports using highly reactive sulfonyl chlorides. Biochem. Biophys. Res. Comm. 102, 449-57.

Nylander, C., Liedberg, B. and Lund, T. (1982). Gas detection by means of surface 
plasmon resonance. Sens. Actuators 3, 79-88.

Ockman, N. (1978). The antibody-antigen interaction at an aqueous-solid interface: a study by means of polarized infrared ATR spectroscopy. Biopolymers 17, 1273-84.

Pockrand, I., Swalen, J. D., Gordon, I. and Philpott, M. R. (1977). Surface plasmon spectroscopy of organic monolayer assemblies. Surf. Sci. 74, 237-44.

Poste, G. and Moss, C. (1972). Antigen-antibody reactions in thin films. In The study of surface reactions in biological systems by ellipsometry (eds. G. Poste and C. Moss), pp. 206-231. Pergamon Press, New York.

Raether, H. (1977). Surface plasmon oscillations and their applications. In Physics of thin films, advances in research and development (eds. G. Haas and M. H. Francombe), Vol. 9, pp. 145-261. Academic Press, New York.

- (1980). Excitation of plasmons and interband transitions by electrons. Springer, tracts in modern physics, Vol. 88. Springer-Verlag, Berlin, FRG.

Rothen, A. (1974). Ellipsometric studies of thin films. In Progress in surface and membrane science (eds. J.F. Danielli and M. D. Rosenberg), pp. 81-118. Academic Press, New York.

Schroder, M. E., Lerner, I. and D'Oria, F. J. (1967). Radioisotope study of coupling agents in reinforced plastics. Med. Plast. 45, 195-7.

Soini, E. and Hemmilä, I. (1979). Fluoroimmunoassay: Present status and key problems. Clin. Chem. 25, 353-61.

Sutherland, R. M., Dähne, C., Place, J.F, and Ringrose, A. R. (1984a). Optical detectin of antibody-antigen reactions at a glass liquid interface. Clin. Chem. 30, 1533-8.

(1984b). Immunoassays at a quartz-liquid interface: theory, instrumentation and preliminary application to the fluorescent immunoassay of human immunoglobulin G. J. Immun. Methods, 74, 253-65.

Thompson, N. L. (1982). Ph.D. Thesis. University of Michigan.

- Burghardt, T.P. and Axelrod, D. (1981). Measuring surface dynamics of biomolecules by total internal reflection fluorescence with photobleaching recovery and correlation spectroscopy. Biophys, J, 33, 435-54.

Van Wagenen, R. A., Rockhold, S. and Andrade, J.D. (1982). Probing protein adsorption: total internal reflection intrinsic fluorescence. In Biomaterials. Interfacial phenomena and applications (eds. S. L. Cooper and N.A. Peppas). Adv. Chem. Ser. Vol. 199, pp. 351-370.

—Z Zdasiuk, B. J. and Andrade, J. D. (1980). Total internal reflection fluorescence studies of albumin adsorption onto quartz. Org. Coat. Plast. Chem, 42, 749-53.

Watkins, R. W, and Robertson, C. R. (1977). A total internal reflection technique for the examination of protein adsorption. J. Biomed. Mater. Res. 11, 915-38.

Weetall, H.H. (1972). Insolubilized antigens and antibodies. The chemistry of biosurfaces (ed. M. L. Hair) Vol. 2, pp. 597-631. Marcel Decker, New York.

- (1974). Preparation, characterization and application of enzymes immobilized on inorganic supports. Adv. Exp. Med. Biol. 42, 191-2.

(ed.) (1975). Immobilized enzymes, antigens, antibodies and peptides: Preparation and characterization. Marcel Decker, New York. 


\title{
34
}

\section{Laser light scattering and related techniques}

\author{
ROBERT J. G. CARR, ROBERT G. W. BROWN, JOHN \\ G. RARITY, and DAVID J. CLARKE
}

\section{4,1 Introduction}

Optical techniques have long been central to the analysis of biological systems and have been utilized in a wide variety of forms. In recent years however the development of new and improved optical measuring mechanisms has been stimulated by the advent of the laser. The laser celebrated the 25 th anniversary of its invention last year (1985). Since its appearance it has contributed to some of the most significant advances in measurement science, not only in the research laboratory environment but increasingly in the industrial world as well.

The laser is a light source having unique properties. It is an emitter of collimated monochromatic light that can range from $\mathrm{X}$-ray to microwave (Maser) through the ultra-violet, visible, and infra-red regions of the electromagnetic spectrum. The light output can be continuous or pulsed, with pulse lengths as short as a few femto-seconds (approx. $1 \times 10^{-15}$ seconds) and output powers ranging from microwatts to megawatts and more. Laser light can be highly polarized (electric field oscillation in one direction only) and is usually coherent both temporally and spatially (with field phase relationships being maintained along the beam and across it). There exists an extensive introductory literature describing the wide range of lasers that exist, their properties, and their uses (e.g. Brown 1980; Forsyth and Wilson 1980; Butler 1980; O'Shea et al. 1979).

Laser light is very different from that produced by conventional white light sources. Its properties can be individually exploited to great effect in laserbased optical measuring systems.

Concomitant with the development of the laser has been the development of optical-fibre technology (Cherin 1983), the major thrust being provided by the rapid expansion of the optically based data communications systems industry. It is the combination of laser and fibre-optic technologies that promises the development of a new generation of optical sensors that will enable the application of many of the existing optical techniques to situations previously considered inaccessible or inappropriate to optically based analysis or monitoring. 
Optical-fibre sensors can be broadly classified into two categories: intrinsic and extrinsic sensors (Culshaw 1983). Intrinsic sensors, in which propagation time (or group or phase velocity), intensity, or polarization state of the light propagating down a fibre may be modulated by an external force acting on the fibre, have been developed for a wide range of physical variables, for example, electrical, magnetic, and acoustic fields; angular and linear displacement; velocity and acceleration; temperature, pressure, stress, and strain; and radiation dosage (Dakin 1983; Moore and Ramer 1983; Giallorenzi et al, 1982; Brenci et al. 1983). Extrinsic sensors merely use the fibre as a means of transporting light to and from an analyte- or measurandsensitive element or volume that is responsible for modifying one or more properties of the light used (such as intensity, state of polarization, or wavelength).

Fibre sensors have been demonstrated for the measurement of an increasing number of analytes in biological systems in vitro (Milanovich et al. 1984) and in vivo (Vurek 1984) by modification of the fibre cladding or tip (endface) with analyte-sensitive reagents or biological molecules. The advantages afforded by such remote optical-fibre sensors, for example, miniaturization, electromagnetic immunity, ruggedness, physical flexibility, and ease of manipulation, longevity, and the ability to be multiplexed, have ensured a great deal of interest in their development (Cramp and Ried, $1982 a, b$; Carter et al. 1982). Thus, a wide variety of optical techniques lend themselves to fibre-optic technology including the monitoring of light scattered by particles.

Light scattering has long been a method of deriving information as to the size, shape, and composition of particles but new, more powerful, techniques of analysis by laser light scattering have been developed in recent years that are likely to find application in fibre-optic sensors. This chapter will discuss how laser light can be scattered by particles (macromolecules to microorganisms and above in size) and used to study particle size, shape, velocity, and other potentially useful parameters for the biotechnologist and, further, how these techniques may be configured into biosensors.

\subsection{Theory and principles of light scattering}

A laser beam possesses a number of properties that can be very stable with time and easily and specifically modified by interactions with many types of materials, the modification to be later analysed with high precision. Some of these properties were mentioned in the introduction; intensity, monochromaticity phase, polarization, etc. The specific advantages afforded by the laser concern its ability to yield these parameters to often better than $1 \%$ accuracy and yet non-intrusively (i,e. without perturbation or contamination). 
The scattering of light by small particles has been the subject of intensive study for over 100 years and is well reviewed and documented (e.g. van de Hulst 1957; Kerker 1969; Huglin 1972; Degiorgio 1983; Ford 1985). For biosensing we are primarily concerned with three types of light scattered by particles in particular size ranges and all of which are inter-related: 1) Rayleigh scattering, 2) Rayleigh-Gans-Debye scattering, and 3) Mie scattering.

\subsection{Rayleigh scattering}

Consider a particle of dielectric (non-absorbing) material illuminated by collimated plane-polarized light of wavelength much greater than its largest dimension.

The incident field induces a fluctuating dipole moment, and the particle acts as a point source radiating equally in all directions perpendicular to the dipole axis. The intensity $(I)$ scattered by the particle in this plane is inversely proportional to the fourth power of the wavelength $(\lambda)$ and directly proportional to the square of the particle volume $(V)$ :

$$
I \propto \frac{V^{2}}{\lambda^{4}} .
$$

In the plane parallel with the induced dipole (thus parallel to the incident field polarization) the scattered intensity falls to zero at 90 degrees (longitudinal waves are not supported in electromagnetic radiation). The scattering polar diagram is shown schematically in Fig. 34.1a.

\subsubsection{Rayleigh-Gans-Debye (RGD) scattering}

For particles of dimension comparable with the wavelength of incident light and refractive index close $(\Delta n \leqslant 0.05)$ to its surrounding medium, i.e. a weak scatterer, the polar diagram shows less light is scattered back towards the original laser beam (Fig. 34.1b). The forward lobe can be crudely explained by saying that in all but the forward direction destructive interference occurs between light scattered from different points within the particle. Thus

$$
I \propto \frac{V^{2}}{\lambda^{4}}, P(K)
$$

where $P(K)$ contains the interference effects caused by the particle dimension approaching that of the incident wavelength, and the angular dependence arises through the scattering vector modulus

$$
|K|=\frac{4 \pi n_{1}}{\lambda} \sin \frac{\theta}{2}
$$

where $n_{t}$ is the refractive index of the medium surrounding the particle, $\lambda$ is the wavelength of the incident beam, and $\theta$ is the angle of measurement 
(a)

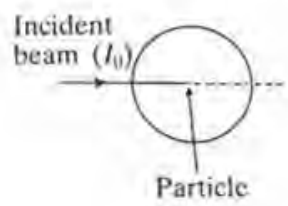

Incident

(b)

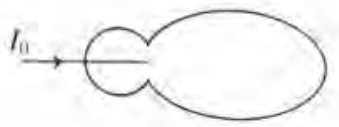

(c)

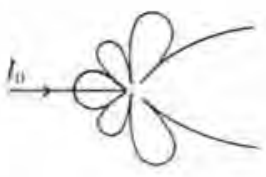

Scattered

intensity

$\left(I_{\mathrm{s}}\right)$
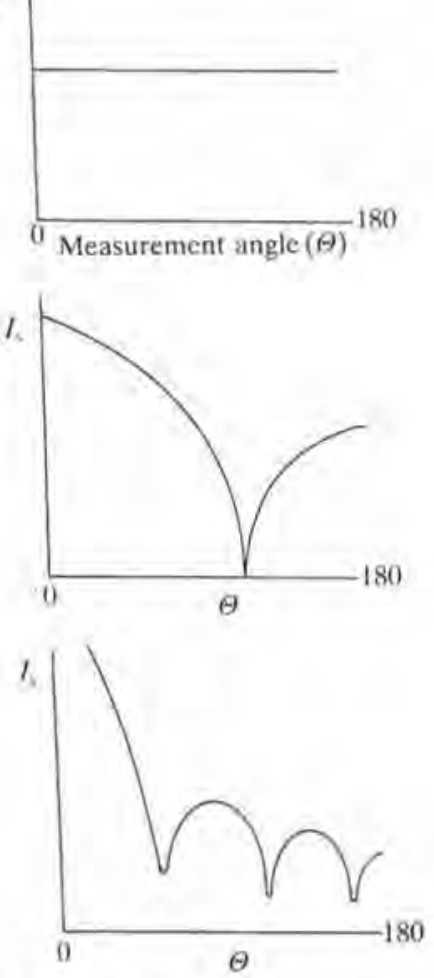

Fig. 34.1 Light scattered from (a) Rayleigh, (b) Reyleigh-Gans-Debye, and (c) Mie scattering particles. For each type of scatterer the light scattered $\left(I_{5}\right)$ in the plane perpendicular to the plane of polarization of the incident beam $\left(I_{0}\right)$ is shown both graphically (intensity as a function of measuring angle, $\theta$ ) and as a polar scattering diagram (representing the change in scattering intensity over $\pm 180^{\circ}$ circle for a symmetrical particle).

relative to the beam direction. At certain values of $\theta$, no light is scattered, thus forming a pattern of scattering lobes surrounding the particle. Many biological macromolecules and micro-organisms are RGD scatterers, close to forward scattering angles (Koch 1968).

\subsubsection{Mie scattering}

For particles of dimension $\geqslant 1$ wavelength and of arbitrary refractive index the Mie theory of light scattering is required. Again forward scattering predominates (Fig. 34.1c) with several lobes appearing in the polar diagram. The minima are more numerous but less pronounced than in the RGD 
approximation due to phase differences in the distribution of the light energy around the particles' surface.

The Mie formula for scattering intensity involves a series summation of complex functions; thus its evaluation is computationally expensive. However, there exist two simpler analytic approximations that are often applicable. The Fraunhofer approximation (Swithenbank et al, 1977) assumes opaque particles of size larger than the wavelength of light giving rise to the familiar Airy pattern. The anomalous diffraction (van de Hulst 1957) approximation assumes similar size but particle refractive index close to that of the medium.

\subsection{Light-scattering techniques}

There are a large number of techniques that utilize light scattering as a sensing or analysis method. Such methods may be conveniently divided into two areas: (1) static light scattering in which the magnitude of scattering is observed directly and (2) dynamic light scattering in which one observes the timescale of intensity fluctuations (which is not dependent on the absolute or relative magnitudes of intensity).

\subsubsection{Static light-scattering techniques}

Static light-scattering techniques include those long familiar to biochemistry, microbiology, and other biological disciplines. Thus turbidimetry, nephelometry, and angular or differential light scattering, etc. are well characterized and common methods for the routine analysis of biological samples and need not be discussed in any detail here. Whilst there remain many aspects of light scattering that are poorly defined, e.g. the problem of arbitrary shape (Drain et al. 1982; Williams 1984; Walters 1980), in general, light scattering has proved an important means of particle sizing (Barth and Sun 1985; Barth, 1984) and characterization.

Latimer (1982) has comprehensively reviewed the theoretical and practical aspects of light scattering by cells and subcellular particles and Koch (1968) and Koch and Ehrenfeld (1968) have similarly discussed extensively light scattering by bacteria and bacteria-like particles. Light-scattering principles are widely employed, for instance in the assessment of nuclear and cellular morphology using forward and perpendicular scatter in a flow cytometry system (Benson et al. 1984), in the characterization of epidermal cells using goniometric techniques (Bruls and van der Luen 1984) and laser light is used in the monitoring of erythrocyte deformation (Plasek and Marik 1982). Lasers have also been used in the rapid screening of urine for the presence of bacteriuria in a nephelometer configuration (Bayardelle and Richet 1984).

Multi- and low-angle laser scattering techniques may be used in the study of particles as small as lysozyme (Jones and Midgley 1984) or can be used as 
the basis of a technique for the characterization of microbial populations, i.e. angular or differential light scattering (Wyatt 1975a,b). More recent techniques, such as circular intensity differential scattering (CIDS; Salzman and Gregg, 1984) have evolved from established light scattering techniques, in this case circular dichroism (e.g. Kreuger 1984), and are under commercial development (Mesa Diagnostics Inc., San Diego) for application in the area of clinical microbiology. Related to the above are the electric dichroism and electric birefringence techniques (Oakley et al. 1982) used to study samples orientated in an electric field. Such techniques have been widely employed in the study of DNA by, for instance, Yamaoka and his co-workers (Yamaoka and Yueda 1982; Charney and Yamaoka 1982; Yamaoka and Matsuda 1980, 1981).

Lasers are also used in Raman and Brillouin scattering (Yariv 1975). Brillouin scattering arises from the interaction of monochromatic (laser) light with thermally driven acoustic fluctuations within the substance being studied, Brillouin scattering and its use in the analysis of biological systems has been discussed by Randall and Vaughan (1979). Raman scattering arises from the interaction of coherent laser incident light with the substance and results in frequency shifted scattered light containing information about the molecular vibrational properties of the sample. Raman spectroscopy has been used in the in vitro study of many biological molecules (Theophanides 1979; Prescott et al. 1984) and, more recently, of intact cells (Jeannesson et al. 1983).

A further technique that has been employed primarily in the size analysis of inorganic and mineral (e.g. fuel sprays, exhaust gas) particles of size greater than $1 \mu \mathrm{m}$ is diffraction light scattering usually assuming the Fraunhofer approximation. Its potential as a technique of use to the biotechnologist makes it worthy of further discussion. In diffraction light scattering it is usual to direct an expanded parallel beam of continuous laser light through an ensemble of static or moving macromolecules or particles and analyse the spatial distribution of diffracted optical energy by means of detecting the back focal plane intensity distribution (or Airy pattern) of a lens symmetrically centred in the laser beam and focused at infinity (Fig. 34.2). For a monodisperse sample the particle radius $(a)$ is

$$
a=\frac{0.61 \lambda f}{r}
$$

where $\lambda$ is the wavelength of the incident beam, $f$ is the focal length of the (Fourier) lens, and $r$ is the radius of the first zero intensity ring in the diffracted intensity distribution. For polydisperse samples, the particlesize distribution is calculated through a Bessel function transformation. Particle sizes from approx $1 \mu \mathrm{m}$ to $1 \mathrm{~mm}$ can be measured, particularly at high concentration, using this technique (Bertero and Pike 1983; Nakadate 

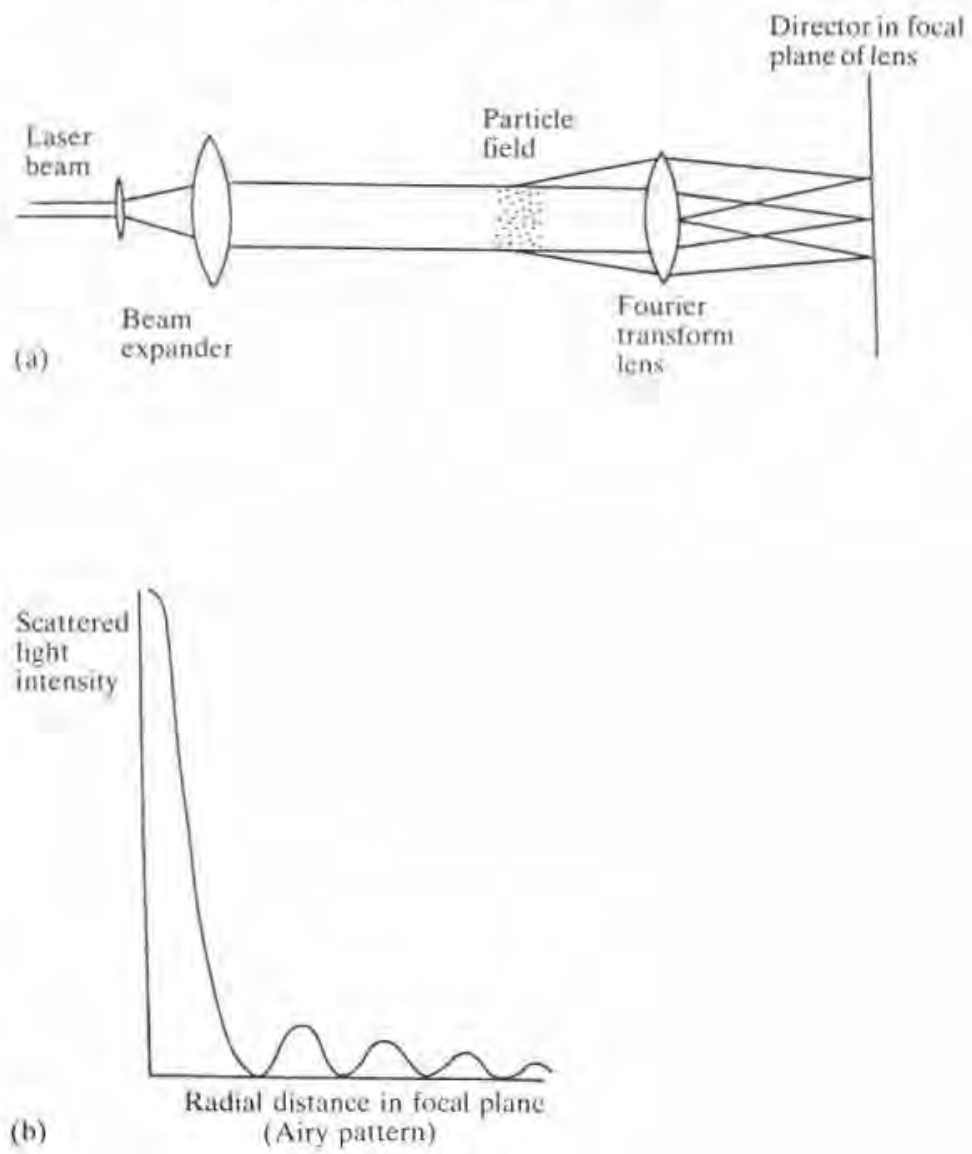

Fig. 34.2 Fraunhofer diffraction.

(a) The optical arrangement for the measurement of Fraunhofer diffraction.

(b) The intensity distribution (Airy pattern) of scattered light. The diameter of the diffraction patterns is inversely proportional to the diameter of the particles and the pattern obtained is independent of the position of the particle and therefore measurements can be made with particles moving at any speed.

and Saito 1983; Bayvel and Jones 1981; Bayvel et al. 1982).

\subsubsection{Dynamic light-scattering techniques}

The above text briefly describes a few examples of the various analytical methods based on static light scattering from macromolecules and particles. When, however, an ensemble or particles are suspended in a medium they will move under Brownian motion and the various elemental scatterings from 
each particle will, at some distance from the scatterers, add to some resultant intensity and phase. As the particles move randomly under Brownian motion, so the observed intensity at a distance fluctuates with time; the timescale of the intensity fluctuations carries information about the particles' size and size distribution. This is the basis of dynamic light scattering or intensity fluctuation spectroscopy (also known as quasi-elastic light-scattering spectroscopy or QELS). Examination of the timescales of intensity fluctuations is performed by electronic signal processors to yield particle size distribution, etc. When the processor is a photon-correlator, as is nowadays usual, then the technique is referred to as photon-correlation spectroscopy or PCS.

There is a second branch of dynamic light scattering which may be useful as a sensor technique, for example, in the measurement of fluid flow inventory. In this variation, known as laser Doppler velocimetry (LDV), one or more laser beams probe the liquid containing the macromolecular or particulate suspension. The geometry of the laser beams and the light-detection systems depend on the nature of the sample to be analysed and is discussed later. The timescales of intensity fluctuations from translating particles measures their velocity.

These new and powerful techniques, PCS and LDV, are potentially of great value to the study of biological macromolecules and particles. Although comparatively recent in their development and unfamiliar to most biotechnologists, there exist several commercially produced instruments that are beginning to be used in the biological sciences.

34.3.2.1 Photon correlation spectroscopy PCS is now a well developed and widely used research technique in which (Fig. 34.3) light from a continuous, visible laser beam is directed through an ensemble of macromolecules or particles in suspension and moving under Brownian motion. Some of the laser light is scattered by the particles and this scattered light is collected by a lens and detected by a photo-detector which generates an electrical signal proportional to the light intensity detected. The light intensity fluctuates on a timescale related to the time taken for a particle to diffuse a distance

Fig, 34.3 Photon correlation spectroscopy.

(a) Diagram showing the component parts of a PCS system.

(b) Graphical representation of the fluctuation in light intensity at the detector which contains information about the diffusion rates of the scattering particles.

(c) The fluctuating intensity is converted into a train of standardized photoelectron pulses in the photon counting detector. Peaks in intensity are thus observed as 'bunching' in the pulse train.

(d) The signal arising from the above is fed into a digital correlator and used to generate a correlation function. The diagram shows a normalized correlation function from which the background has been removed. See text for details. 

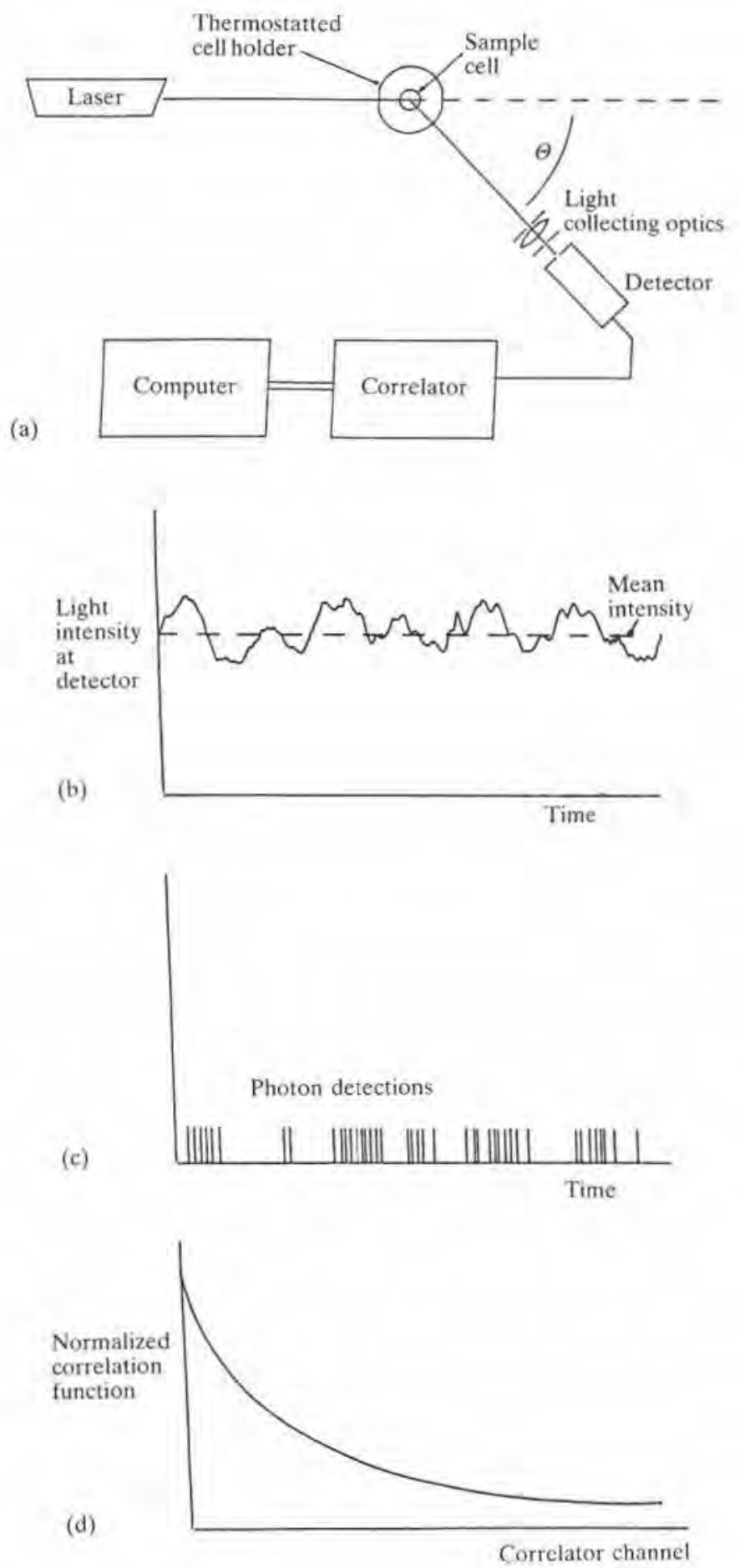
comparable with the light wavelength which in turn is related to the particle's hydrodynamic radius.

The electrical output signal of the photo-detector is analysed in a hardwired digital signal processor known as a photon correlator. The correlator computes averages of the signal compared (multiplied) with itself at different delay times, i.e. the autocorrelation function of the signal. The autocorrelation function formed is characteristically an exponentially decaying function of decay time. The decay time is related to the size of the macromolecules or particles that scattered the light. By examining in detail the multi-exponentiality of the decaying function, a particle size distribution, etc. can be constructed.

Mathematically we can briefly describe the above process in the following manner. For Brownian diffusing spherical macromolecules of identical size the electric field correlation function of the scattered light is

$$
g^{(1)}(\tau)=\mathrm{e}^{\left(-D_{1} K^{2} \tau\right)} \mathrm{e}^{\left(i \omega_{0} \tau\right)}
$$

where $D_{\mathrm{t}}$ is the translational diffusion coefficient of the macromolecules, $\tau$ is the delayed time, $K$ is the modulus of the scattering vector, related to the angle of observation, and $\omega_{0}$ is the angular frequency of the incident light, When large numbers of scatterers are illuminated, then the measured intensity autocorrelation function can be shown to be

$$
g^{(2)}(\tau)=1+B\left|g^{(1)}(\tau)\right|^{2}=1+B \mathrm{e}^{\left(-2 D_{1} K^{2} \tau\right)}
$$

which is an exponentially decaying function. But $D_{t}$ is related to the particles size by the Stokes-Einstein equation

$$
D_{\mathrm{t}}=\frac{k T}{6 \pi \eta R_{\mathrm{h}}}
$$

where $k$ is Boltzmann's constant, $T$ is temperature, $\eta$ is viscosity, and $R_{\mathrm{h}}$ is the hydrodynamic radius of the macromolecules. Thus $R_{\mathrm{h}}$ can be estimated from the autocorrelation function.

Of course, most particulate systems are polydisperse and thus $\left|g^{(1)}(\tau)\right|$ is the sum of many differing exponential decays. Formally $\left|g^{(1)}(\tau)\right|$ is given by

$$
\left|g^{(1)}(\tau)\right|=\int_{0}^{\infty} G(\Gamma) \mathrm{e}^{-\Gamma \tau} \cdot \mathrm{d} \Gamma
$$

where the linewidth $\Gamma=D_{t} K^{2}$ and $G(\Gamma)$ is the intensity weighted linewidth distribution. An estimate of $G(\Gamma)$ and hence the radius distribution can be obtained using Laplace inversion techniques (Bertero et al. 1985). Further details of this and the rigorous details behind the simple statements above can be found in Cummins and Pike $(1974,1977)$, Earnshaw and Steer (1983), Schulz-DuBois (1983), Dahneke (1983), Chen et al. (1981), Pusey and 


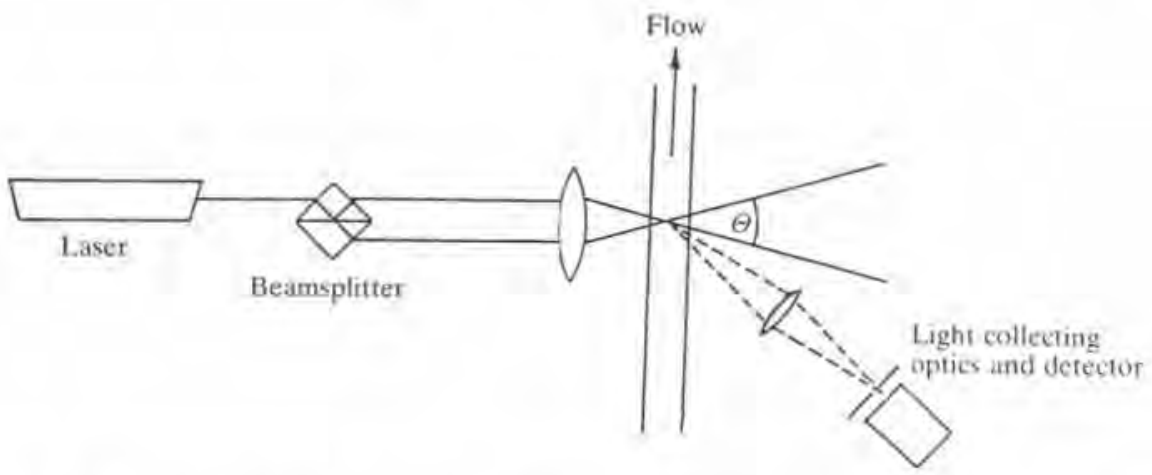

Fig. 34.4 Laser Doppler velocimeter. Schematic representation of a laser Doppler velocimeter (incoherent detection arrangement). See text for details.

Vaughan (1975), and Pecora (1985).

Typically PCS operates successfully with between $10^{4}$ and $10^{10}$ particles $/ \mathrm{ml}$ with particle sizes between a few nanometers and a few microns.

34.3.2.2 Laser Doppler velocimetry Laser velocimetry is a non-instrusive means of acquiring velocity information through analysis of light intensity fluctuations caused by the motion of macromolecules or particles through one or more laser beams. Velocities ranging from $\mu \mathrm{m} / \mathrm{min}$ to $\mathrm{km} / \mathrm{sec}$ may be studied with the same instrumentation. A schematic diagram of a typical experimental arrangement is shown in Fig. 34.4. Continuous, plane polarized monochromatic laser light of typically a few $\mathrm{mW}$ power is usually divided into two equally intense beams that are focused and made to intersect in the fluid flow at a position where the velocity is varying. Small particles embedded in the flow traversing the laser beam intersection region (or measuring volume) scatter laser light from each laser beam with a slightly different Doppler frequency due to their motion relative to the different laser beam directions. Some of the scattered laser light is collected by a lens placed at some convenient position and the light 'beats' at a photodetector. The electrical signal from the photodetector contains frequency information linearly related to the difference in the Doppler frequencies created from each laser beam and thus to the particles velocities. In the event of strong light signals, analogue signal processing such as frequency analysis may be employed to yield an estimate of the velocities of the particles; much weaker optical signals are processed by a photon-correlator as in PCS, to obtain the velocity statistics. With the optical geometry described the flow velocity component measured is that one perpendicular to the bisector of the angle between the propagating laser beams and lying in the plane that the laser beams define. It may be shown that for statistically independent 
orthogonal velocity fluctuations the form of the intensity autocorrelation function is

$$
g^{(2)}(\tau)=\int_{0}^{\infty} P(u) \exp \left(\frac{-u^{2} \tau^{2}}{r^{2}}\right)\left[1+\frac{m^{2}}{2} \cos \left(\frac{2 \pi u \tau}{s}\right)\right] \mathrm{d} u
$$

where $g^{(2)}(\tau)$ is as above, $r$ is the laser beam radius, $m$ is a measure of the signal contrast, $u$ is the velocity component measured, $P(u)$ its probability distribution, and $s(=\lambda / 2$ sin $(\theta / 2))$ is the fringe spacing. The correlogram takes the form of an exponentially decaying cosine oscillation, and when $u^{2} \tau^{2} \ll r^{2}$ :

$$
g^{(2)}(\tau)=1+\frac{m^{2}}{2} \times \int_{0}^{\infty} P(u) \cos \left(\frac{2 \pi u \tau}{s}\right) \mathrm{d} u .
$$

$P(u)$ and the velocity statistics may be recovered through a Fourier-cosine transformation (Cummins and Pike 1977; Schulz-DuBois 1983).

\subsection{Applications of dynamic light-scattering techniques in biology}

Although dynamic light-scattering techniques have yet to become widespread in research into the structure and behaviour of biological systems, a significant amount of work has been carried out that demonstrates their potential usefulness in a wide range of applications. Reviews of the impact and use of light scattering and dynamic light-scattering techniques in the biological sciences have been written by Cummins $(1973,1983)$ and Burchard and Cowie (1972). Bloomfield (1985) has discussed more recent studies in this area. Steer (1983b) has discussed aspects of biological samples that must be considered in light-scattering studies. Sample preparation (Tabor 1972) and the effect of choice of solvent and other factors such as temperature and pressure have been discussed (Huglin 1972) as have fundamental aspects of light scattering by biological polyelectrolyte macromolecules (Eisenberg 1973; Nagasawa and Takahashi 1972) and their study by QELS (Giordano and Micali 1983).

Light scattering from polymers is the subject of a comprehensive text (Huglin 1972) within which a variety of aspects pertinent to biological samples have been considered. The use of Raman and Brillouin scattering in the study of bulk polymers and solutions of polymers as well as amorphous polymers and polymer gels has been discussed by Vaughan (1982) and Patterson (1985).

The study, by light-scattering techniques, of polymerization, association, and aggregation of molecules and macromolecules has been concisely reviewed by Elias (1972). Similarly, interparticle interactions and their 
effect on particle-size determination by QELS has been discussed using micelles and charged proteins as examples (Nicoli and Dorshow 1983; Mazer 1985). Interestingly, in the analysis of the immunoassay reaction by dynamic light-scattering methods PCS has been shown to offer a 100 -fold increase in sensitivity compared with conventional assays (1000-fold when in the inhibition mode). It compares in sensitivity with radioimmunoassay but uses no radiochemicals and can be performed rapidly with no prior separation of bound and unbound antigen yet works with sample volumes as low as $1 \mu \mathrm{l}$ (Cohen and Benedek 1975; von Schulthess et al. 1976a,b). Rate nephelometry, using laser light has been employed in the measurement of the immunoprecipitin reaction (Sittampalam and Wilson 1984a) and lightscattering techniques in general for the immunoassay of specific proteins have been reviewed by Price et al. (1983). Other optical immunoassay techniques have been developed that employ light reflected at an angle close to the pseudo-Brewster angle off an antibody-coated silicon surface (Arwin and Lundstrom 1985).

The study of gels and gel formation has been carried out using dynamic light-scattering methods (Vaughan 1982; Tanaka, 1985). Sellen (1983) studied the diffusion of macromolecules within gels, in particular the movement of dextran fractions within calcium alginate and agarose gels. DNA has been studied using dynamic light-scattering techniques (Cummins and Pike 1974; Dahneke 1983; Earnshaw and Steer 1983). The changes in comformation of superhelical DNA following binding of intercalating agents was studied by Newman (1984). 'Splitting' of the diffusion coefficient of dinucleosomal-sized DNA (average 375 base pairs) following lowering of ionic strength has been demonstrated using QELS implying that the simple relating of hydrodynamic radius to particle size may lead to erronous conclusions regarding the conformation of the molecule (Schmitz and Lu 1984). Folding-unfolding transitions of ribonuclease A over a range of temperatures have been studied with dynamic light scattering (Wang et al. 1980) indicating that the technique should prove valuable in protein denaturation and DNA melting studies. The ability of PCS and other light-scattering techniques to give data as to the size and shape of particles has been shown to be of value in the determination of tertiary structure and behaviour of macromolecules of biological importance for instance, gramicidin (De La Torre $e t$ al. 1984) and bovine factor VIII (Gabriel et al. 1984).

The animal protein collagen has been studied by the use of PCS (Hwang and Cummins 1982) employing a new approach to determine simultaneously both the length of the molecule and the viscosity of the solution. Proteoglycan aggregates were studied using both static and dynamic light scattering (Shogren et al. 1983) and data obtained of the size of the molecule was shown to be in good agreement with that obtained by electron micro- 
scopy, indicating that the aggregates visualized by electron microscopy were also present in solution.

Studies on micelle structure and interaction are well suited to dynamic light-scattering techniques (Corti et al. 1983; Hirsch et al. 1984; Mazer 1985) and in particular casein micelles have been investigated (Griffin and Anderson 1983; Holt 1983). Vesicles have been studied using similar techniques (Steer 1983a; Yu 1983).

The ease with which it is possible to obtain a regular and monodisperse sample of the tobacco mosaie virus (TMV) has meant that it has become, like the polystyrene spheres, a model object for dynamic light-scattering studies. More realistically, polydisperse solutions of TMV virus were characterized by PCS (Wiltzius 1982), the technique demonstrating its ability to resolve a number of different sized particles simultaneously. A number of other virus particle suspensions have been characterized (Pusey et al. 1974; Camerini-Otero et al. 1974).

Typing of blood cells has been attempted (Arefyev et al. 1978) using the immunoprecipitation technique and was found to be an improvement on the methods employed routinely in forensic science. The chemotactic response of Escherichia coli cells has been followed using PCS and other light-scattering methods (Wang and Chen 1983). Similarly the phototactic response of unicellular motile algae was analysed by PCS (Ascoli and Frediani 1983). The motility of another type of cell, spermatozoa, has also received considerable attention, concise reviews of which may be found in Volochine (1983) and Earnshaw (1983).

The motility of cells and micro-organisms in general and its study by dynamic light-scattering techniques has been reviewed in detail by Boon (1983) and its biochemical significance discussed by Klein (1984). Carlson (1983) and Carlson and Fraser (1973) have shown PCS to be a powerful technique in the understanding of cellular movement of a very specific kind, muscle contraction.

The transient electrical birefringence of rodlike molecules when combined with QELS studies has been used to determine the length and diameter of such particles. Flexible molecules can be characterized in terms of their persistence length from deviations from rodlike behaviour (Eden and Elias 1983; Elias and Eden 1981).

Using these methods the electric polarizability and behaviour of DNA fragments and the filamentous virus Pf3 were studied. Jennings (1972) has reviewed electric field light-scattering and, more recently, Ware (1983) has discussed in some detail the application of LDV in electrophoretic light scattering and its use in studies on living cells, vesicles, and counterion condensation onto DNA. Automated instruments combining LDV and electrophoretic systems are now available and have been used to study, with high precision and rapidity ( $1-3 \mathrm{~min})$, the electrophoretic mobility of leuco- 
cytes and lymphocytes from several types of leukaemia (Steiner et al. 1985) and similarly, the electrophoretic mobility of erythrocytes (Spendley et al. 1982).

\subsection{The future}

The development of increasingly sophisticated and sensitive instrumentation in conjunction with advances in the understanding of the theoretical complexities underlying many light-scattering techniques have resulted in a large variety of instruments being made available to the researcher in the biological sciences. Whilst the routine analysis and study of more complex biological parameters would benefit from advances in instrument and component design and performance in general it is the very complexity and variability of biological systems that commonly results in poor reproducibility or ambiguous data. These problems are common to all of the light scattering and other light interaction techniques discussed above, and highlight the problem of attaining specificity from optical measurements of biological processes.

Methods of achieving specificity include the use of labels, either chemical (e.g. fluorescent) or biochemical (e.g. polystyrene spheres attached to monoclonal material), and the use of combinations of the various sensing techniques. One such multi-parameter light-scattering technique, a derivative of CIDS currently under development, (Salzmann and Gregg 1984) would appear to be a promising technique for differentiating between different species and types of microbial cell. As the technique is dependent on the analysis of the total cell genome it may prove problematical to ensure reproducibility of data from a given cell type in the face of the significant ef fects that variations in the cell growth medium and conditions and time of sampling can have on the conformation and composition of nuclear material. In addition, it remains to be seen whether the presence of cell wall and virus coat material which represent significant refractive index boundaries will obscure the genetic material within the cell.

When changes in the scattered light are used as a means of monitoring the formation of aggregates, e.g. the nephelometric or PCS determination of the immunoprecipitin reaction, the presence of significant amounts of scattering background material as is found in serum samples can seriously reduce the sensitivity of the techniques. Sensitivity can be enhanced by the attachment to the reactants of optical markers such as polystyrene spheres of relatively large size $(100 \mathrm{~nm})$ that scatter more than the macromolecules of interest or the background (Price et al, 1983). However, in monoepitopic antibody-antigen reactions only lower order aggregates are formed, thus with high background, more specific labelling (e.g. fluorescence) is required.

Light-scattering techniques may find future areas of new application in 
such industrial activities as fermentation process monitoring, immunoassays (as discussed earlier), down-stream processing and other areas where monitoring of small particles is required.

The techniques outlined above, have, in the most part, evolved from early, crude optical instruments and theories into the powerful analytical tools that exist today in the research laboratory. However, even these modern systems are restricted in their application to certain situations due to the bulky and inflexible nature of their component parts (e.g. lenses, mirrors, apertures, etc.). Most of the techniques suffer from a number of disadvantages of which the most obvious include high cost, large size, the requirement for frequent and careful calibration (except PCS), a high level of operator expertise and more often than not, a considerable amount of sample preparation and manipulation. Samples are usually analysed, with varying degrees of rapidity, one at a time and often only after some isolation or separation stage. Furthermore, it is usual for the sample in question to have to be taken from the site of interest to the created instrument.

Optical fibres offer a revolutionary alternative to existing optical designs. It may prove possible to adapt a large number of the above techniques to configurations possessing several major additional advantages. Modern fibre optics are capable of maintaining a particular polarization state or phase (a prerequisite for many optical analytical mechanisms), allow remote (many kilometres distant) points of analysis or measurement and are of increasingly low cost. Their small diameters enable very small sample volumes to be addressed (down to $1 \mu \mathrm{l}$ ) with a consequent saving in possibly expensive reagents. This small size makes possible in situ or in vivo measurements previously considered inaccessible and their inherent inertness and ruggedness makes optical fibres suitable for hostile environments.

The capacity for a large number of optical fibres to be addressed very rapidly by a single source and detector by means of optical multiplexers should reduce significantly the 'cost per channel' of a multi-analyser instrument. The simultaneous revolution in microelectronics and the advent of smaller, faster, and more powerful electronic 'chips', particularly through uncommitted array and VSLI technologies, will accelerate the development of true biosensor devices capable of employing the most sophisticated of current optical techniques.

One can therefore conceive of analysis equipment being centrally positioned within a laboratory or, more importantly, an industrial environment and coupled to perhaps hundreds of light scattering, parameter-gathering sensors remotely positioned. Examples of such a labour un-intensive philosophy are starting to emerge, e.g. I km distance remote laser Raman, to and from a measuring point, via multimode fibres and using material embedded into porous glass welded to the far (measurement) end of the fibre (Hirschfield 1984). 
Already PCS and LDV have been successfully demonstrated in millimetre dimension packages via optical fibres and we confidently expect demonstration of other light-scattering techniques via fibres also.

\section{References}

Arefyev, I. M., Barsegyanz, L. O., Eskov, A. P. and Alekseyeva, V. I. (1978), Species diagnosis of blood by light beating spectroscopy. Sudebno-Meditsinskaya Ekspertiza Moskva 21(1), 26-7.

Arwin, H. and Lundstrom, I, (1985). A reflectance method for quantification of immunological reactions on surfaces. Anal. Biochem. 145, 106-12.

Ascoli, C. and Frediani, C. (1983). The application of laser light scattering to the study of photo-responses of unicellular motile algae. In The application of laser light scattering to the study of biological motion (eds. J. C. Earnshaw and M. W. Steer), NATO Advances Science Institute Series, Vol. 59, pp. 669-679. Plenum Press, New York.

Barth, H. G. (1984), Modern methods of particle size analysis, Wiley, New York.

- and Sun, S.-T. (1985). Particle size analysis, Anal. Chem. 57, 151 R-175R.

Bayardelle, P. and Richet, H. (1984). Rapid screening for bacteriuria with a Jaser nephelometer. Can. J. Microbiol. 30, 927-9.

Bayvel, L. P. and Jones, A. R. (1981). Electromagnetic scattering and its application. Applied Science Publishers, Englewood, N.J.

- Eisenklam, P. and Jones, A. R. (1982). A light scattering instrument for measuring drop sizes in the range of 0.1 to $1000 \mu \mathrm{m}$. Proc. 2nd. Int. Conf. on Liquid Atomization and Spray Systems (ICLASS), June 1982, Wisconsin. pp. 329-34.

Benson, M. C., McDougal, D. C. and Coffey, D. S. (1984). The application of perpendicular and forward light scattering to assess nuclear and cellular morphology. Cytometry, 5, 515-22.

Bertero, M. and Pike, E. R. (1983). Particle size distributions from Fraunhofer diffraction. I. An analytical eigenfunction approach. Optica Acta 30(8), 1043-9.

- Brianzi, P., Pike, E. R., DeVilliers, G., Lan, K. H. and Ostrowsky, N. (1985). Light scattering polydispersity analysis of molecular diffusion by Laplace transform inversion of weighted spaces. J. Chem. Phys, 15, 1551-6.

Bloomfield, V. A. (1985). Biological applications. In Dynamic light scattering (ed. R. Percora), pp. 363-416. Plenum Press, New York.

Boon, J.P. (1983). Motility of living cells and micro-organisms. In The application of laser light scattering to the Study of Biological Motion (eds. J. C. Earnshaw and M. W. Steer), NATO Advanced Science Institute Series, Vo. 59, pp. 561-606. Plenum Press, New York.

Brenci, M., Falciai, R. and Scheggi, A.M. (1983). Multimode optical fibre sensors. Alta Frequenza, 52(3), 206-8.

Brown, D. C. (1980). Solid state lasers. In Applied optics and optical engineering. Vol. VI, Chapter 1. Academic Press, New York.

Bruls, W. A. G. and van der Luen, J. C. (1984). Forward scatter properties of human epidermal layers. Photochem, and Photobiol, 40(2), 231-42.

Burchard, W. and Cowie, J, M. G. (1972). Selected topics in biopolymeric systems, In 
Light scattering from polymer solutions (ed. M. B. Huglin), pp. 725-787. Academic Press, New York.

Butler, J.F. (1980). Semiconductor diode lasers. In Applied optics and optical engineering, Vol. VI, Chapter 3, pp. 53-88. Academic Press, New York.

Camerini-Otero, R. D., Pusey, P. N., Koppel, D. E., Schaefer, D. W, and Franklin, R.M. (1974). Intensity fluctuation spectroscopy of laser light scattered by solutions of spherical viruses: R17, Q $\beta$, BSV, PM2 and T7. I. Diffusion coefficients, molecular weights, solvation and particle dimensions. Biochemistry 13(5), 960-72.

Carlson, F. D. (1983). The application of quasi-electric light scattering to the study of muscular contraction. In The application of laser light scattering to the study of biological motion (eds. J.C. Earnshaw and M. W. Steer), NATO Advanced Science Institute Series, Vol. 59, pp. 405-58. Plenum Press, New York.

- and Fraser, A. (1973). Intensity fluctuation autocorrelation studies of the dynamics of muscle contraction. In Photon correlation and light beating spectroscopy (eds. H.Z. Cummins and E. R. Pike), NATO Advanced Study Institute Series B: Physics, Vol. 3, pp. 519-38. Plenum Press, New York.

Carter, T., Dahne, C. and Place, J.F. (1982). Method for the determination of species in solution with an optical waveguide, Eur. Pat. Appl. No. 0-075-353.

Charney, E. and Yamaoka, K. (1982). Electric dichroism of deoxyribonucleic acid in aqueous solutions: electric field dependence. Biochemistry 21, 834-42.

Chen, S-H., Chu, B. and Nossal, R. (eds.) (1981). Scattering techniques applied to supramolecular and non-equilibrium systems. Plenum Press, New York.

Cherin, A. H. (1983). An introduction to optical fibres. McGraw-Hill, New York.

Cohen, R.I. and Benedek, G. B. (1975). Immunoassay by light scattering spectroscopy. Immunochemistry 12, 349-51.

Corti, M., Minero, C. and Degiorgio, V. (1983). Light scattering from micellar solutions - proposal for a light scattering standard. In The application of laser light scattering to the study of biological motion (eds. J. C. Earnshaw and M. W. Steer), NATO Advanced Science Institute Series, Vol. 59, pp. 333-46. Plenum Press, New York.

Cramp, J. H. W, and Ried, R. F. (1982a). Sensitive optical fibre. Eur. Pat. Appl. No. 0-062-443.

— (1982b). Optical Fibre Sensor. Eur. Pat. Appl. No. 0-061-884.

Culshaw, B. (1983). Optical systems and sensors for measurement and control. I. Phys, E: Sci. Instrum, 16, 978-86.

Cummins, H.Z. (1973). Application of light beating spectroscopy to biology. In Photon correlation and light beating spectroscopy (eds, H. Z. Cummins and E. R. Pike), NATO Advanced Study Institute Series B: Physics, Vol. 3, pp. 285-330. Plenum Press, New York.

- (1983). Analysis of diffusion of biological molecules by quasi-elastic light scattering. In The application of laser light scattering to the study of biological motion (eds. J. C. Earnshaw and M. W. Steer), NATO Advanced Science Institute Series, Vol. 59, pp, 171-208. Plenum Press, New York.

- and Pike, E. R. (eds.) (1974). Photon correlation and light beating spectroscopy. NATO Advanced Study Institute Series B: Physics, Vol. 3. Plenum Press, New York. 
- (eds.) (1977). Photon correlation spectroscopy and velocimetry. Plenum Press, New York,

Dahneke, B. E. (1983). Measurement of suspended particles by quasi-elastic light scattering. John Wiley, New York.

Dakin, J. P. (1983), Optical fibre sensors - principles and applications. Fibre Optic '83, SPIE 374, 172-82.

Degiorgio, V. (1983). Physical principles of light scattering. In The application of laser light scattering to the study of biological motion (eds. J.C. Earnshaw and M. W. Steer), NATO Advanced Science Institutes Series, Vol. 59. Plenum Press, New York.

De La Torre, G., Martinez, M. C. L. and Tirado, M. M. (1984). Dimensions of short, rodlike macromolecules from translational and rotational diffusion coefficients. Study of the gramicidin dimer. Biopolymers 23, 611-15.

Drain, L.E., Smith, N. and Dalzell, W. (1982). Aspects of light scattering by spherical particles, Proc, Max, Born Conf., SPIE 369, 610-15.

Earnshaw, J.C. (1983). Laser doppler velocimetry in a biological context. In The application of laser light scaltering to the study of biological motion (eds. J.C. Earnshaw and M. W. Steer) NATO Advanced Science Institute Series, Vol. 59, pp. 123-142. Plenum Press, New York.

- and Steer, M.W. (eds.) (1983). The application of laser light scattering to the study of biological motion. NATO Advanced Science Institutes Series, Series A, Life Sciences, Vol. 59. Plenum Press, New York.

Eden, D. and Elias, J. G, (1983). Transient electrical birefringence of DNA restriction fragments and the filamentous virus $\mathrm{Pf} 3$. In Measurement of suspended particles by quasi-elastic light scattering (ed. B. E. Dahneke), pp. 401-38. John-Wiley, New York.

Eisenberg, H. (1973). Light scattering intensity studies in multicomponent solutions in biological macromolecules. In Photon correlation and light beating spectroscopy (eds. H. Z. Cummins and E. R. Pike), NATO Advanced Study Institute Series B: Physics, Vol. 3, pp. 151-68. Plenum Press, New York.

Elias, H.-G, (1972). The study of association and aggregation via light scattering. In Light scaitering from polymeric solutions (ed. M. B. Huglin), pp. 397-457. Academic Press, New York.

- and Eden, D, (1981). Transient electric birefringence study of the persistence length and electric polarizability of restriction fragments of DNA. Macromolecules 14, 410-19.

Ford, N.C. (1985). Light scattering apparatus. In Dynamic light scattering (ed. R. Pecora), pp. 7-57. Plenum Press, New York.

Forsyth, J.M. and Wilson, J. (1980). Gas lasers. Applied optics and optical engineering, Vol. VI, Chapter 2, pp. 29-52. Academic Press, New York.

Gabriel, D. G., Kirkland, J.A., Cooper, H. A. and Wagner, R. H. (1984). A light scattering study of bovine factor VIII. Arch. Biochem, Biophys. 231(1), 189-92.

Giallorenzi, T.G., Bucaro, J.A., Dandridge, A., Sigel, G.H., Cole, J.H., Rashleigh, S. C. and Priest, R. G. (1982). Optical fibre sensor technology. IEEE J. of Quantum Elect, QE18(4), 626-65.

Giordano, R. and Micali, N. (1983). Correlation spectroscopy and structural properties of macromolecular solutions. In The application of laser light scattering 
to the study of biological motion (eds. J. C. Earnshaw and M. W. Steer), NATO Advanced Science Institute Series, Vol. 59, pp. 221-6. Plenum Press, New York. Griffin, M.C.A. and Anderson, M. (1983). Laser light scattering study of the fractionation of casein micelles in skim milk by controlled pore glass chromatography. In The application of laser light scattering to the study of biological motion (eds. J. C. Earnshaw and M. W. Steer), NATO Advanced Science Institute Series, Vol. 59, pp. 347-52. Plenum Press, New York.

Hirsch, E., Candau, S, and Zana, R. (1984). Micellar structure and inter-micellar interactions in solutions in tetradecyltrimethylammonium bromide in the presence of 1-pentanol: light scattering and viscosity study. J. Coll. Interface Sci. 97(2), 318-26.

Hirschfield, T. (1984). Proc. ICO '84, Sapporo, Japan. Remote analysis by fibre optics.

Holt, C. (1983). Structural studies on bovine casein micelles by laser light scattering. In The application of laser light scattering to the study of biological motion (eds. J.C. Earnshaw and M.W. Steer), NATO Advanced Science Institute Series, Vol. 59, pp. 353-8. Plenum Press, New York.

Huglin, M. B. (ed.) (1972). Light scattering from polymer solutions. Academic Press, New York.

Hwang, J.S. and Cummins, H.Z. (1982). Dynamic light scattering studies of collagen. J. Chem. Phys. 77(2), 616-21.

Jeannesson, P., Manfait, M. and Jardillier, J. (1983). A technique for laser Raman spectroscopy studies of isolated cell populations. Anal. Biochem. 129, 305-9.

Jennings, B. R. (1972). Electric field light scattering. In Light scattering from polymer solutions (ed. M. B. Huglin), pp. 527-79. Acad. Press. New York.

Jones, M.N. and Midgley, P.J.W. (1984). Low angle laser light scatter from surfactant solubilised biological macromolecules. Proc. 607th Meeting ('Lasers in Biochemistry') Biochem. Soc. Trans. 12, 625-7.

Kerker, M. (1969). The scattering of light and other electromagnetic radiation, Academic Press, New York.

Klein, R. A. (1984). The measurement of motility in micro-organisms and its biochemical significance. Proc. 607th Meeting ('Lasers in Biochemistry') Biochem. Soc. Trans. 12, 627-30.

Koch, A. L. (1968). Theory of the angular dependence of light scattering by bacteria and similar-sized biological objects. J. Theoretical. Biol. 18, 133-56.

- and Ehrenfeld, E. (1968). The size and shape of bacteria by light scattering measurements. Biochim. Biophys. Acta 165, 262-73.

Korpel, A. (1980). Acousto-optics In Applied optics and optical engineering, Vol. VI, Chapter 4, pp. 89-109. Academic Press, New York.

Kreuger, R. (1984). The effect of low ionic strength on the circular dichroic spectrum of chromatin and nucleosomal subunits. Arch. Biochem. Biophys. 231(1), 183-8.

Latimer, P. (1982). Light scattering and absorption as methods of studying cell population parameters. Ann. Rev. Biophys, Bioeng. 11, 129-50.

Mazer, N.A. (1985). Laser light scattering in micellar systems. In Dynamic light scattering (ed. R. Pecora), pp. 305-46. Plenum Press, New York,

Milanovich, F. P., Hirschfeld, T. B. and Wang, F. T. (1984). Clinical measurements using fibre optics and optrodes. In Novel optical fibre techniques for medical applications. SPIE, Vol. 494, pp. 18-24. 
Moore, E. L. and Ramer, O. G. (eds.) (1983). Fiber optic and laser sensors. Proceedings of SPIE, Vol, 412, April 5-7, 1983, Arlington, Virginia, USA.

Nagasawa, M. and Takahashi, A. (1972). Light scattering from polyelectrolyte solutions. In Light scattering from polymer solutions (ed. M. B. Huglin), pp. 672-723, Academic Press, New York.

Nakadate, S. and Saito, H. (1983). Particle size distribution measurement using a Hankel transform of a Fraunhofer diffraction spectrum. Optics Letts. 8(11), 578-80.

Newman, J. (1984). Dynamic light scattering as a probe of superhelical DNA-intercalating agent interaction. Biopolymers 23, 1113-19.

Nicoli, D. F. and Dorshow, R. B. (1983). Effects of interparticle interactions on particle size determinations by QELS. In Measurement of suspended particles by quasi-elastic light scattering (ed. B.E. Dahneke), pp. 501-28. John-Wiley, New York.

Oakley, D. M., Jennings, B. R., Wateman, D. R. and Fairey, R. C. (1982). An electro-optic birefringence fine-particle sizer. J. Phys. E. 15, 1077-82.

O'Shea, D. C., Callen, W. R. and Rhodes, W. T. (1979). Introduction to Lasers and their applications. Addison-Wesley Mass, USA.

Patterson, G. D. (1985). Dynamic light scattering in bulk polymers. In Dynamic light scattering (ed. R, Pecora), pp. 245-76. Plenum Press, New York.

Pecora, R. (ed.) (1985). Dynamic light scattering; applications of photon correlation spectroscopy. Plenum Press, New York.

Plasek, J. and Marik, T. (1982). Determination of undeformable erythrocytes in blood samples using laser light scattering. Appl. Opt. 21(23), 4335-8.

Prescott, B., Steinmetz, W. and Thomas, G. J. (1984). Characterization of DNA structure by laser Raman spectroscopy. Biopolymers 23, 235-56.

Price, C. P., Spencer, K. and Whicher, J. (1983). Light scattering immunoassay of specific proteins: a review. Ann. Clin. Biochem. 20, 1-14.

Pusey, P.N. (1984). Detection of small polydispersities by photon correlation spectroscopy. J. Chem. Phys. 80(8), 3513-20.

_ and Vaughan, J.M. (1975). Light scattering and intensity fluctuation spectroscopy. In Dielectric and related molecular processes, Vol. 2, Chapter 2, 48-105. Chem. Soc. Specialist Periodical Report.

Koppel, D. E., Schaefer, D. W., Camerini-Otero, R. D. and Koenig, S. H. (1974). Intensity fluctuation spectroscopy of laser light scattered by solutions of spherical viruses: R17, Q $\beta$, BSV, PM2 and T7. I. Light scattering technique. Biochemistry 13(5), 952-60.

Randall, J. J. and Vaughan, J. M. (1979). Brillouin scattering in systems of biological importance. Phil. Trans. R. Soc. Lond. A293, 341.

Salzman, G, C, and Gregg, T. G. (1984). Current and experimental methods of rapid microbial identification. Biotechnology March, 243-8.

Schulz-DuBois, E. O. (ed.) (1983). Photon correlation techniques in fluid mechanics, Springer Verlag, Berlin.

Schmitz, K.S. and Lu, M. (1984). Quasielastic light-scattering studies on dinucleosomal-sized DNA: ionic-strength dependence. Biopolymers 23, 797-808.

Sellen, D. B. (1983). The diffusion of compact macromolecules through biological gels. In The application of laser light scattering to the study of biological motion 
(eds. J. C. Earnshaw and M. W. Steer), NATO Advanced Science Institute Series, Vol. 59, pp. 209-220. Plenum Press, New York.

Shogren, R. L., Blackwell, J., Jamieson, A. M., Carrino, D. A., Pechak, D. and Caplan, A. 1. (1983). Light scattering studies of chick limb bud proteoglycan aggregate. J. Biol. Chem. 258(24), 14741-4.

Sittampalam, G. and Wilson, G.S. (1984a). Experimental observations of transient light scattering formed during immunoprecipitin reactions. Anal, Chem. 56, 2170-5.

(1984b). Theory of light scattering measurements as applied to immunoprecipitin reactions. Anal. Chem. 56, 2176-80.

Spendley, D. G., Sones, D. P., Smith, A. T., Lloyd, D. S. and Cooke, E. D. (1982). Inexpensive laser Doppler instrument for cell electrophoresis. Proc Max Born Conf., SPIE Vol. 369, 174-177.

Steer, M. W. (1983a). Vesicles, In The application of laser light scattering to the study of biological motion (eds. J.C. Earnshaw and M. W. Steer), NATO Advanced Science Institute Series, Vol. 59, pp. 359-66. Plenum Press, New York.

- $(1983 \mathrm{~b})$. Applications of laser light scattering to biological systems. In The application of laser light scattering to the study of biological motion (eds. J. C. Earnshaw and M. W. Steer), NATO Advanced Science Institute Series, Vol. 59, pp, 43-52. Plenum Press, New York.

Steiner, R., Ottmann, O., Kaufmann, R., Light, P. A. and Hoffmann, W. (1985). Cell electrophoresis: automatic measurements by light scattering with Lazypher. Electrophoresis 6, 82-9.

Swithenbank, J., Beer, J. M., Taylor, D. S., Abbot, D. and McCreath, G. C, (1977). A laser diagnostic technique for the measurement of droplet and particle size distribution. Prog. Astronautics and Aeronautics 53, 421-47.

Tabor, B.E. (1972). Preparation and clarification of solutions. In Light scattering from polymer solutions (ed. M. B. Huglin) pp. 1-25. Academic Press, New York. Tanaka, T. (1985). Light scattering from polymer gels. In Dynamic light scattering (ed, R. Pecora), pp. 347-62. Plenum Press, New York.

Theophanides, T. (1979). Infrared Raman spectroscopy of biological molecules. NATO Advanced Study Institute Series. D. Reidel, Dordecht, Holland.

van de Hulst, H.C. (1957). Light scattering by small particles. Wiley, New York. (Also 1981, Dover Books,)

Vaughan, J.M. (1982). Quasi-elastic light scattering from polymer solutions. In Static and dynamic properties of the polymeric solid state (eds. R. A. Pethrick and R. W. Richards), pp. 305-347. D. Reidel, Dordecht, Holland.

Volochine, B. (1983). Light scattering studies of biological populations, In The application of laser light scattering to the study of biological motion (eds. J.C. Earnshaw and M. W. Steer), NATO Advanced Science Institute Series, Vol. 59, pp. 635-656. Plenum Press, New York.

von Schulthess, G.K., Cohen, R. J. and Benedek, G. B. (1976a). Laser light scattering spectroscopic immunoassay in the agglutination-inhibition mode for human chorionic gonadotropin (hCG) and human luteinizing hormone (hLH), Immunochemistry 13, 963-66.

- Sakato, N. and Bendek, G. B. (1976b), Laser light scattering spectroscopic immunoassay for mouse IgA. Immunochemistry 13, 955-62. 
Vurek, G. G. (1984). In vivo chemical sensors, In Novel optical fibre techniques for medical applications. SPIE, Vol, 494, pp. 2-6.

Walters, P. T. (1980). Practical applications of inverting spectral turbidity data to provide aerosol size distributions. Appl. Opt. 19(14), 2353-65.

Wang, C.-C., Holland Cook, K. and Pecora, R. (1980). Dynamic light scattering studies of tibonuclease. Biophysical Chem, 11, 439-42,

Wang, P. C. and Chen, S. H. (1983). Chemotaxis and band formation of Escherichia coli studied by light scattering. In The application of laser light scattering to the study of biological motion (eds, J.C. Earnshaw and M. W. Steer), NATO Advanced Science Institute Series, Vol. 59, pp. 607-28. Plenum Press, New York.

Ware, B. R. (1983). Electrophoretic light scattering: modern methods and recent applications to biological membranes and polyelectrolytes. In The application of laser light scattering to the study of biological motion (eds. J.C. Earnshaw and M. W. Steer), NATO Advanced Science Institute Series, Vol. 59, pp. 89-122. Plenum Press, New York.

Williams, D, J. (1984). Organic polymeric and non-polymeric materials with large optical nonlinearities. Angew. Chem. Int. Ed, Engl. 23, 690-703.

Wiltzius, P. (1982). Light scattering study on polydisperse TMV solutions. Appl. Opt. 21(11), 2022-6.

Wyatt, P.J. (1975a). Differential light scattering techniques for microbiology. In Methods in microbiology. (ed. J. R, Norris), Vol. 7A, Chapter 6, pp. 183-263. Academic Press, New York.

- (1975b). Atlas of the light scattering characteristics of microparticles. Science Spectrum Inc., Santa Barbara, Calif., USA.

Yamaoka, K. and Matsuda, K. (1980). Electric dipole moments of DNA in aqueous solutions as studied by the reversing-pulse electric birefringence. Macromolecules $13,1558-60$.

(1981). Electric dichroism study of a sonicated DNA and its complex with an acridine dye in aqueous solutions: Field-strength dependence and linear dichroic spectra. Macromolecules 14, 595-601.

— and Yueda, K, (1982). Reversing-pulse electric birefringence study of helical poly $(\beta$-I-glutamic acid) in $N, N$-dimethylformamide with emphasis on a new data analysis for the polydisperse system. J. Phys. Chem. 86, 406-13.

Yariv, A. (1975). Quantum electronics, Wiley, New York.

$\mathrm{Yu}, \mathrm{H}$. (1983). Structure and dynamics of disc membrane vesicles. In The application of laser light scattering to the study of biological motion (eds. J. C. Earnshaw and M. W. Steer), NATO Advanced Science Institute Series, Vol. 59, pp. 367-382. Plenum Press, New York. 

Applications of microprocessors 



\title{
The use of microprocessors for the evaluation of the analytical performance of enzyme- based sensors
}

\author{
DANIEL R. THÉVENOT, THIERRY TALLAGRAND, and \\ ROBERT STERNBERG
}

\subsection{Introduction}

Biosensors such as enzyme electrodes, using enzymatic membranes and electrochemical detectors, usually present a great specificity for a given metabolite such as sugar or amino acid (Thévenot et al. 1978, 1979; Sternberg et al. 1980). The operating properties and the analytical characteristics of these biosensors are dependent upon a large number of physical, chemicals and enzymatic parameters (Sternberg et al. 1983a) which are often difficult to discriminate. Programmable calculators and microcomputers have been used by several research groups for instrument development (Skogberg et al. 1979; Jaenchen et al. 1982; Kernevez et al. 1983; Wieck et al. 1984). Numerous Japanese patents on enzyme-based sensors refer to automation involving microcomputers or microprocessors (Kawana et al. 1980; Kyoto Daiichi Kagaku K.K. 1982; Tsuji et al. 1983; Mitsubishi Rayon Co. 1983). These instruments may alternatively be of great help in a more direct association with enzyme electrodes:

firstly, for the study and optimization of their analytical properties, especially their precision, repeatability, and extention of measurable concentration domain (De Laforcade 1980);

secondly, for the direct or indirect determination of some parameters which play an important role in the sensor response (Thévenot 1982; Dubois 1984).

This chapter presents attempts to use programmable calculators and microcomputers to evaluate the analytical performances of amperometric glucose electrodes (Tallagrand et al. 1983).

\subsection{Material and methods}

\subsubsection{Enzyme electrodes}

All the enzyme electrodes used in this study consisted of glucose oxidase 
(GOD) membranes maintained in close contact with a platinum disc. Enzymatic membranes were either prepared by acyl-azide activation of reconstituted collagen films (Thévenot et al. 1979) or by entrapment of enzyme in cellulose acetate films (Sternberg et al. 1983b). The latter membrane was fabricated manually, in steps, from a solution consisting of $5 \%$ cellulose diacetate, $91.5 \%$ acetone, $1 \%$ polyvinylpyrrolidone, $2.5 \%$ water, and an enzyme solution of $5 \mathrm{mg}$ glucose oxidase (EC 1.1.3.4 Boeringer grade II) in $3 \mathrm{ml}$ of acetate buffer; $3 \mathrm{ml}$ of cellulose acetate solution and $0.2 \mathrm{ml}$ of enzyme solution were mixed for $5 \mathrm{~min}$, then spread on a glass plate with the aid of a 5, 15, or 30 micron speader to produce a thin film. After drying for 2-5 min, the membrane was rinsed with distilled water and then stored in acetate buffer, $\mathrm{pH}$ 5.6.

Electrochemical measurements were made using a Solea PRGDEL potentiostat and current amplifier connected to a strip chart recorder. When a differential set-up was used i.e. when both an enzymatic and a non-enzymatic sensor were placed in contact with stirred or flowing samples, both working electrodes were connected to a Solea DELTAPOL differential current amplifier, linked to the potentiostat. This set-up (Thévenot et al. 1979) permitted the measurement of the difference between the current corresponding to the enzymatic reaction $\left(I_{1}\right)$ and of the background current $\left(I_{2}\right)$ i.e. calculation of $I_{1}-k . I_{2}$ with $k$ ranging between 0.5 and 2 . When the first and second derivative of current outputs were needed, an analogic derivative amplifier (Solea Derivol) and 1 second time-base (Solea GCMR) were used.

Electrodes housings were either modified gas electrodes dipped into a thermostatted stirred solution (Thévenot et al. 1979) or modified electrochemical cells for HPLC (Solea Tacussel type DEL-1). The latter consisted of two blocks of polyethylene separated by a Teflon spacer. One block contained the entry and exit holes for liquid, the other accommodated two platinum electrodes (working and auxiliary) and a reference electrode $(\mathrm{Ag} / \mathrm{AgCl}$, sat. $\mathrm{KCl})$. The Teflon spacer was hollowed out in the middle in order to permit circulation of liquid and to define the volume of the reaction chamber $(0.02 \mathrm{ml})$. A cellulose acetate membrane containing enzyme was placed in the ceil between the sensing electrodes and the Teflon spacer. Solution was circulated to the cell using a Gilson Minipuls 11 peristaltic pump at a flow rate ranging between 0.1 and $2 \mathrm{ml} / \mathrm{min}$.

\subsubsection{Programmable table calculator for an enzyme electrode}

In order to evaluate the linearity and repeatability of glucose sensors, we added to the previous equipment a Hewlett Packard $97 \mathrm{~S}$ programmable table calculator (De Laforcade 1980). Its binary coded decimal input and four outputs were interfaced to the potentiostat and to an electric buret through a Solea Ionomate 80 digital Millivoltmeter (range $\pm 2000 \mathrm{mV}$, 


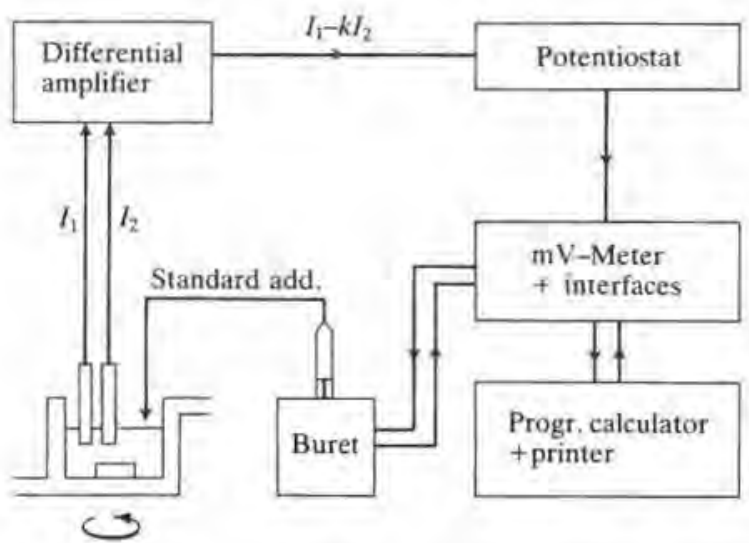

Fig. 35.1 Block diagram of electrodes and electronics using a programmable calculator for an automated enzyme-based electrode, (from Thévenot et al. 1982).

precision $0.1 \mathrm{mV}$ ) (Fig. 35.1). We have developed a program that performs two main functions:

a) determination of steady-state response by detecting stable output currents before and after sample addition into the reaction vessel where electrodes are dipped in, and print-out of steady-state current;

b) control of glucose standard additions by the electronic buret (Solea EBX with Solea EBX-INT interface) and statistical analysis of responses to several equal additions ( 1 to 50 ) with print-out of each response as well as mean, standard deviation, and coefficient of variation.

For determination of unknown glucose concentrations, this program has been modified, replacing the second step by the automatic calibration of the glucose sensor with two successive standard glucose additions and calculation of glucose level, in unknown samples, using the response to the second glucose standard. In order to check for interferences, a post-calibration procedure has been developed: after several sample additions, another glucose standard is added into the vessel and the steady-state response variation is printed.

\subsubsection{Microcomputer for an enzyme electrode}

The above-mentioned calculator does not allow storage of more than 26 variables and thus does not permit storage of the entire response curves, therefore an alternative set up was developed using an Apple II $64 \mathrm{~K}$ microcomputer (Fig, 35.2). The data acquisition interface connected to the potentiostat consisted of a 12 bit, 16 channel, 4 range A.D.C. (GD 16V 12B 4G) and a programmable offset 8 bit D.A.C. (GD Offset Prog). Data 


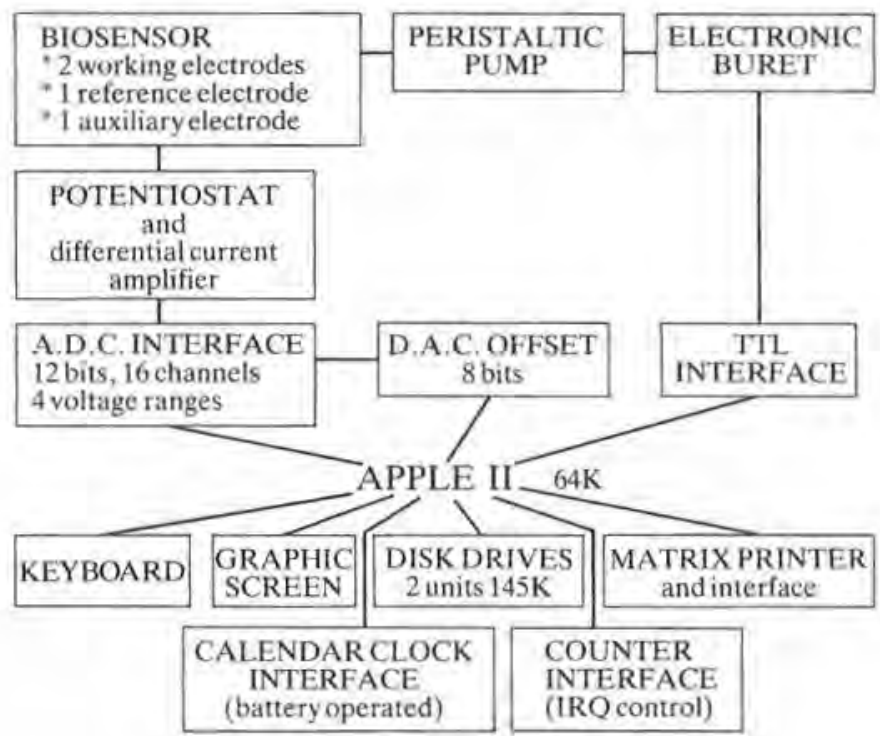

Fig. 35.2 Block diagram of electrodes and electronics using a microcomputer for an automated enzyme-based electrode.

acquisition was controlled by IRQ interrupts generated by a programmable timer module (CCS 7440A). A battery operated real-time calendar clock interface (Mountain Hardware Apple Clock) allowed identification of experiments by exact start time. The electronic buret Solea EBX was interfaced to the microcomputer by a 8 TTL input and 8 relay card (MID CR8C).

\subsubsection{Electrode responses}

When a glucose-containing sample is added to a solution of $0.2 \mathrm{M}$ acetate buffer, $0.1 \mathrm{M} \mathrm{KCl}$ ( $\mathrm{pH} 5.7$ ) into which both electrodes are dipped or circulating through modified HPLC electrode, several different current $v s$. time curves may be recorded:

a) $I_{2}$ is the output current of the non-enzymatic, compensating electrode $\mathrm{E}_{2} . I_{2}$ is the background response and is usually very low except if the sample contains electrochemical reducing species (ascorbate, urate, sulphites etc.) and if the GOD membrane presents no permselectivity (reconstituted collagen films).

b) $\left(I_{1}-k \cdot I_{2}\right)$ where $k$ is close to 1 , and corresponds to the detection of enzymatically generated hydrogen peroxide; it reaches a steady-state value after $2-3 \mathrm{~min}(0.3-0.5 \mathrm{~mm}$ thick collagen films) or $0.5-3 \mathrm{~min}$ (0.005-0.025 $\mathrm{mm}$ thick cellulose acetate films); this is the steady-state response of the sensor. 
c) $\mathrm{d}\left(I_{1}-k \cdot I_{2}\right) / \mathrm{d} t$ is maximum after $20-50 \mathrm{~s}$ (collagen) or $10-60 \mathrm{~s}$ (ccllulose acetate); the height of the peak is the dynamic response of the sensor. Thus 3 different current $v$ s. time curves are available and usually recorded on a 3 -channel Linear 395 recorder. When non-enzyme compensating electrode $\mathrm{E}_{2}$ is absent, only $I_{1}$ and $\mathrm{d} I_{1} / \mathrm{d} t$ signals are recorded. The calculator or microcomputer are linked to either $I_{1}-k \cdot I_{2}$ or to $I_{1}$ and $I_{2}$ current outputs.

\subsubsection{Analytical evaluation of sensors}

Methods of analytical evaluation of glucose electrodes are different for dipped-in and flow-through sensors.

In the first case, glucose oxidase and compensating electrodes are dipped in a $20 \mathrm{ml}$ thermostatted stirred buffer solution or incorporated into the wall of a special $1-5 \mathrm{ml}$ thermostatted vessel capable of supporting horizontal glass rods. All assays are performed by small volume additions of samples or standard glucose solutions into the vessel. Prior to the experiment, buffer solutions are saturated with air at the given experimental temperature, i.e. 30 or $37^{\circ} \mathrm{C}$. Background current is measured in buffer solution after $0.5-2 \mathrm{~h}$ polarization of the platinum working electrodes. Calibration, linearity, and repeatability assays are performed simultaneously by 10 to 50 additions of equal amounts of glucose standard into the reaction vessel. These additions are usually made by electronic buret when stable output current is detected by the calculator, i.e. when previous response to glucose addition has reached a steady state. Responses are calculated either by comparing steady-state current to background current $I_{\text {bg }}$ prior to any glucose addition or by subtracting the steady-state current corresponding to previous addition: thus either $I-I_{\mathrm{bg}}$ vs. $C$ or delta $I /$ delta $C$ vs. $C$ curves are plotted ( $C$ is the total glucose concentration in reaction vessel).

When the flow-through cell is used, either buffer solution or glucose-containing buffer solution is pumped through the sensor. In both cases these solutions are carefully thermostatted and saturated with air at the experimental temperature $\left(37^{\circ} \mathrm{C}\right)$ prior to experiment. Calibration, linearity, and repeatability assays are performed, as mentioned above, by recirculating the output solution into the storage vessel.

\subsection{Automation of glucose enzyme electrodes using programmable calculator}

Determination of analytical patterns of enzyme in standard solutions is usually performed by accurate tests of electronic equipment alone as well as careful study of the whole instrument, including biosensor, electronic equipment, and recording or display devices. Although the potentiostat, the differential curent amplifier, and the derivating current amplifier used in this 


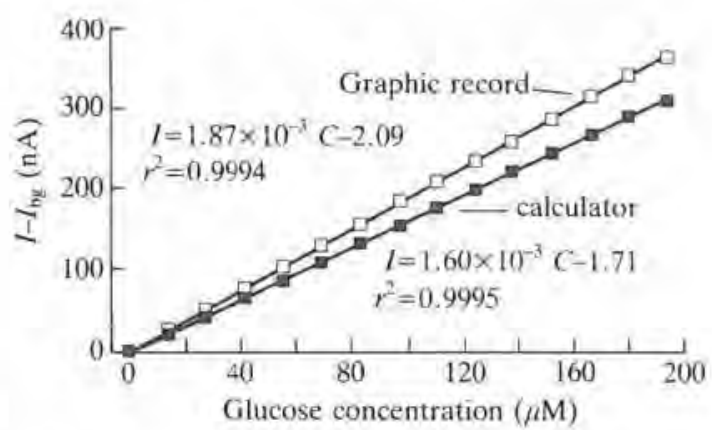

Fig. 35.3 Calibration curves of steady-state responses to 15 additions of a glucose standard: comparison of responses determined on chart recorder and with a programmable calculator. GOD collagen membrane, $30.0 \pm 0.1{ }^{\circ} \mathrm{C}, 50 \mu 1$ additions of $5.55 \mathrm{~mm}$ glucose into $20 \mathrm{ml}$ of acetate buffer (from De Laforcade 1980).

study gives good accuracy, linearity, repeatability, and very low drift, the 3-channel recorder offers poor reproducibility yielding a coefficient of variation $(\mathrm{CV})$ of $1.73 \%$ for 50 successive measurements on a dummy cell (De Laforcade 1980). On the contrary the programmable calculator, connected to the output signals by a BCD interface and a $\mathrm{mV}$-meter presents a much better repeatability ( $\mathrm{CV}=0.64 \%$ for 50 assays). Thus, it seems important to use both the calculator and the graphic recorder during repeatability assays of the whole instrument.

In order to determine simultaneously the background current $I_{\mathrm{bg}}$ and the calibration curve of the instrument, successive additions of glucose standard may be made and the steady-state current measured before all additions and after each addition. These steady-state currents are either directly measured on recorded response curve or determined using the calculator when signal drift is lower than a threshold value (usually $0.2 \mathrm{nA} / \mathrm{min}$ ). Besides this steady-state current determination, the calculator is also able to perform two other functions: it controls additions of glucose standards, by electronic buret into the vessel where the sensor is dipped, and it calculates and prints mean values and coefficients of variation for several equal additions of standard. Thus the whole experiment may be performed without interruption in a very accurate and reproducible way (Fig. 35.1).

Printouts and graphics curve responses may be plotted as calibration curves, i.e. as $I-I_{\text {bg }}$ vS. C. Fig. 35.3 presents typical results obtained with 15 successive $50 \mu \mathrm{l}$ additions of $5.55 \mathrm{~mm}$ glucose standard into $20 \mathrm{ml}$ acetate buffer. Linear regression of these plots gives the following equations:

for steady-state graphic responses:

$$
I(\mathrm{~A})=1.87 \times 10^{-3} \mathrm{C}-2.09 \text { with } r^{2}=0.99945,
$$


for steady-state printed responses:

$$
I(\mathrm{~A})=1.60 \times 10^{-3} \mathrm{C}-1.71 \text { with } r^{2}=0.99949,
$$

glucose concentration $C$ being expressed in mole/ $/$ in reaction vessel. As such plots do not allow an accurate determination of linearity, we prefer to plot the increase of steady-state current $v s$. the increase of glucose concentration in the vessel, i.e. delta $I v s$. delta $C$. In all experiments such plots give a better linearity or repeatability if they are measured using the calculator: coefficients of variation equal, for example, 6.5 and $8.0 \%$ for 15 successive steadystate calculator and graphic responses, respectively. It appears on almost all such assays that response to the first glucose addition is significantly smaller than to the following ones: indeed, when unknown glucose solutions are determined by addition of samples into the same buffer, we prefer to use the response to the second glucose standard as the reference for calculation of the calibration curve equation. When the derivative current amplifier is used, it is also possible to measure the maximum of the first derivative $\mathrm{d} I / \mathrm{d} t$. This dynamic response is proportional to the glucose concentration increase and usually presents a better reproducibility than the graphic steady-state response $(\mathrm{CV}=5.7 \%$ for 15 assays $)$.

The use of this automated glucose electrode enables rapid characterization of sensors prepared with various enzymatic membranes, electrode housings, and reaction vessels, avoiding time-consuming production and interpretation of graphs. For example, it appears that better stirring of buffer solution gives better reproducibility of steady-state responses (Fig. 35.4): an increase of the $1 \mathrm{~cm}$ stirring-bar rotation rate from 320 to $530 \mathrm{rpm}$ slightly decreases the steady-state calibration curve slope from 1.7 to $1.6 \mathrm{~mA} / \mathrm{M}$ and significantly decreases the steady-state coefficient of variation from 9.4 to $2.0 \%(n=8)$. Thus a better definition of hydrodynamic conditions in the enzymatic membrane vicinity is necessary; a flow-through cell derived from an electrochemical detector for HPLC has been tested. An alternative to such hydrodynamic cells is a rotating membrane electrode in which diffusion layer thickness may be defined with great accuracy; such a set-up is especially suitable for permeability and diffusion coefficients determination of the enzymatic membrane (Dubois 1984).

Since the noise level increases with total concentration and current levels, the absolute precision on delta $I /$ delta $C$ is better for the first standard additions than for the following ones: for example $\mathrm{CV}=2.0 \%$ for the first eight glucose standard additions, whether $\mathrm{CV}=10.9 \%$ for the following seven standard additions (Fig. 35.4 bottom, steady-state response on calculator). Thus, when the enzyme-based sensor is used in a stirred reaction vessel where both standard and samples are added, one should change the buffer solution and rinse the sensor often enough to keep the precision at a suitable level. 

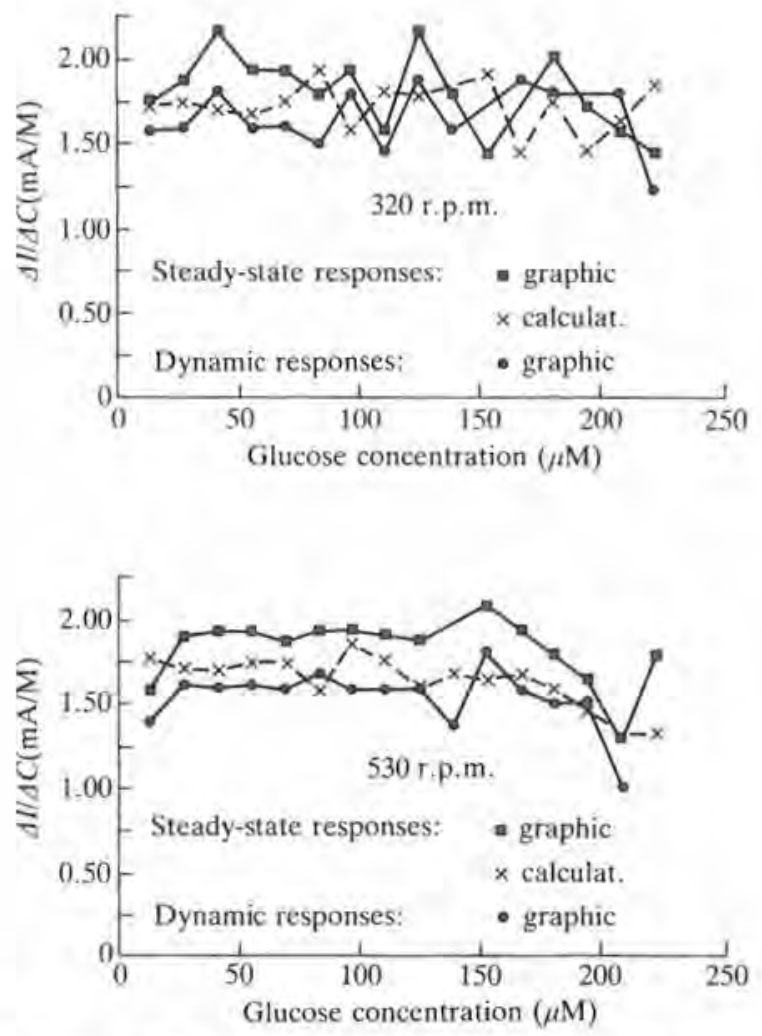

Fig. 35.4 Comparison of the reproducibility of response to 16 additions of glucose under different stirring conditions: (top) 320 and (bottom) $530 \mathrm{rpm}$ of a $1 \mathrm{~cm}$ stirring bar in $20 \mathrm{ml}$ buffer solution. (+) Graphic and ( $\bullet$ ) calculator steady-state, and ( $x$ ) dynamic responses; same conditions as Fig. 35.3.

Such a set-up is able to evaluate analytical performances of steady-state responses, but it is unable to characterize transient responses and to store a whole response curve for a more detailed study. Thus we developed a more powerful device which uses a microcomputer for data storage and treatment.

\subsection{Microcomputer for automation of glucose enzyme electrodes}

\subsubsection{Development of an automated device for enzyme electrode} evaluation

The use of personal microcomputers enables much wider possibilities for data storage and versatility than any table calculator. The previously 
described calculator and $\mathrm{mV}$-meter interface can be replaced by a commercially available 12 bit A.D.C. interface connected to a stable 8 bit D,A.C. used as programmable offset for increasing precision of analogue to digital conversion (Fig. 35.2). Whereas in the previous set-up the acquisition rate was fixed to the maximum possible value at $c a .0 .5 \mathrm{~Hz}$, this device allows a much more accurate and versatile control of acquisition rate: this is achieved with IRQ interrupts generated by a time-counter card containing three 16 bit chained counters. The buret is controlled by a TTL input, relay output card and may add given amounts of glucose standard to the reaction vessel.

Software has been developed for this application using two input channels respectively connected to the enzymatic $E_{1}$ and compensating $E_{2}$ electrodes. As shown in Fig. 35.5, this software is organized around a branching 'menu' and contains several programs linked together by a parameter file. Depending upon the speed necessary for each of these programs, either 6502 machine or compiled basic language is used. For example, the installation procedure during which all experimental parameters such as (a) identification numbers, (b) potential range ( $\pm 20, \pm 100, \pm 500$, or $\pm 2500 \mathrm{mV}$ ), (c) signal units ( $\mathrm{nA}$ or $\mathrm{mA}$ ), (d) actual ranges (ex: $200 \mathrm{nA}$ for $5000 \mathrm{mV}$ ), (e) acquisition frequency, and ( $f$ ) number of data (lower than 2000) is stored in a random access file during a conversational compiled basic program.

The actual experimental program consists of several linked compiled basic programs during which:

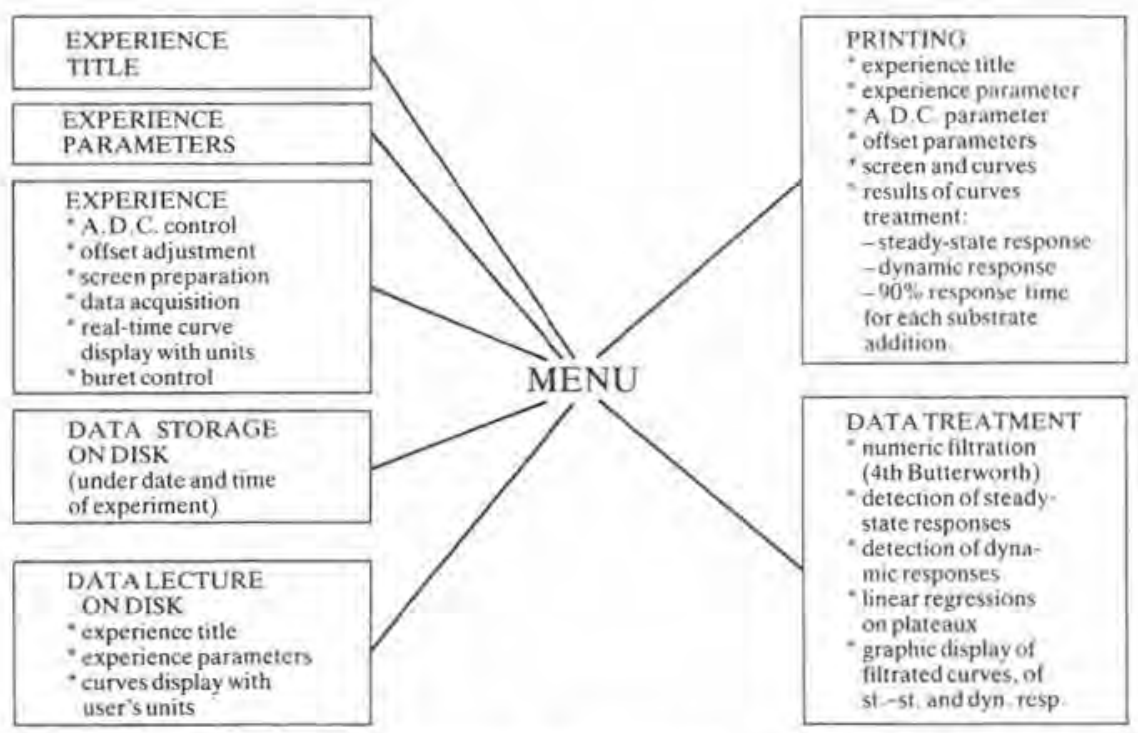

Fig. 35.5 Block diagram of software developed for the automated enzyme-based electrodes using an Apple II + microcomputer. 
a) the A.D.C. card is tested for zero drift,

b) the offsets for both channels are optimized for decreasing background signals prior to the experiment,

c) the high resolution screen is prepared and axes are graduated in user defined units,

d) the actual starting date and time is measured and stored on the parameter file,

e) the time counters are initiated for IRQ interrupts at the controlled frequency,

f) and the acquisition procedure is started under a manually operated switch.

Then several machine language programs

a) control data acquisition on both channels,

b) store numerical values as most significant and less significant bytes,

c) and display points corresponding to both channels on the high resolution screen.

Such a procedure of acquisition and real-time curve display is not limited by execution time of these programs but by response times of A.D.C. and D.A.C. With these commercially available devices a period of $30 \mathrm{~ms}$ is necessary when potential ranges and offset values are different for each channel. If the system is used with a single channel, the acquisition frequency reaches $2.5 \mathrm{kHz}$; it may ultimately reach $15 \mathrm{kHz}$ when curve display on the screen is performed at the end of the experiment. Finally, addition of given volumes of standard at a given periodicity may be achieved using a machine language program which controls the step motor of the buret and counts delivered volume as TTL signals.

Results may be stored on the user's disk as parameter and data files named under starting date and time. Alternatively, a previous experiment may be completely recalled by reading corresponding experimental parameters and data files and by displaying curves on the high-resolution screen in the user's unit.

In order to keep track of each experiment all parameters including A.D.C. zeros and offset values, actual screen displays, and untreated or treated curves and results of data treatment may be hard-copied on a dot-matrix printer (Fig. 35.6 top).

The last component of this automated device is a data treatment unit which is a set of linked compiled basic programs. These programs, placed on the second side of the program disk, perform several treatments on previously stored data:

a) a 4 th order Butterworth numeric filter attenuates the signal at a frequency larger than a threshold value, chosen by the user, 

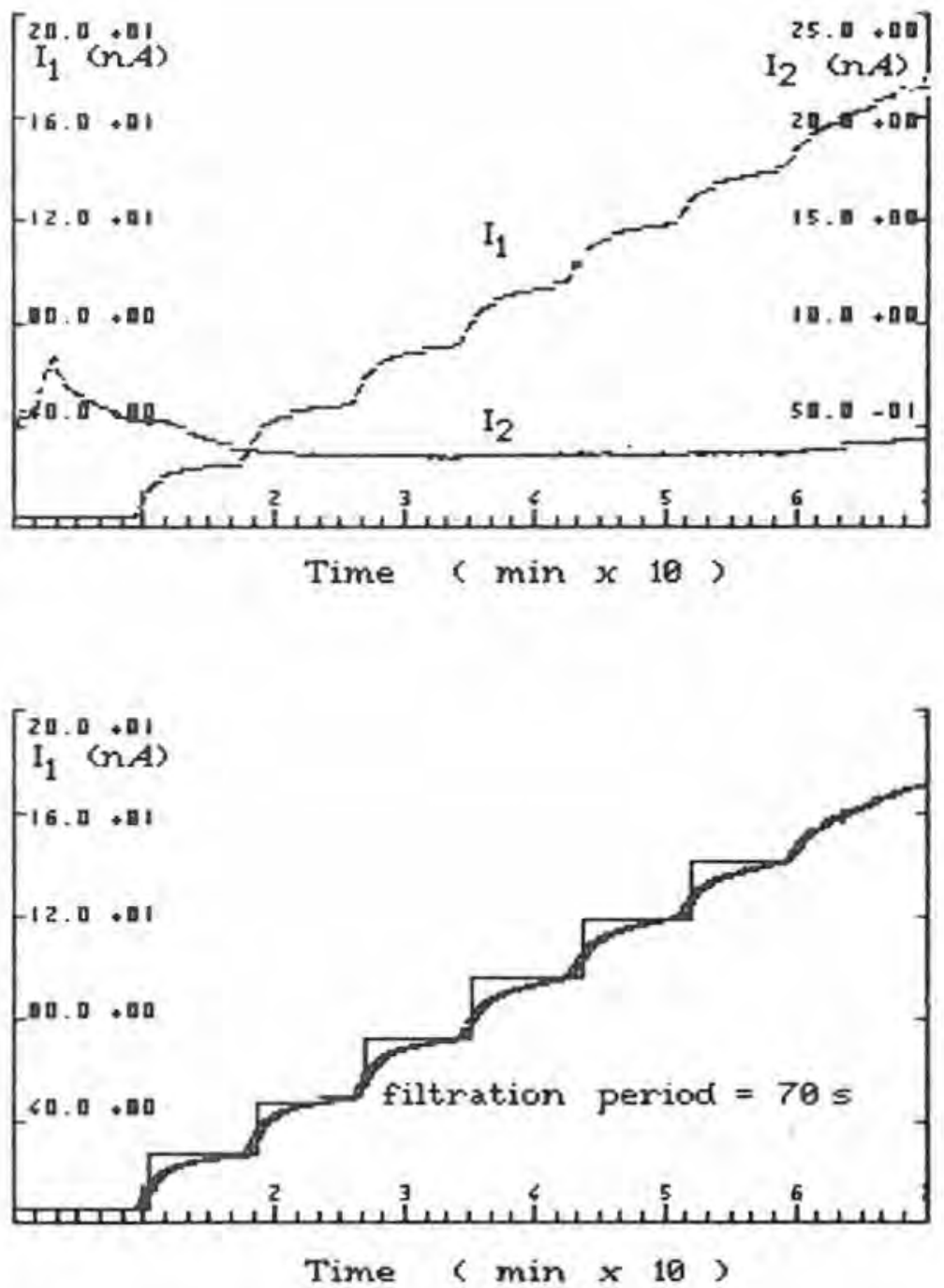

Fig. 35.6 Hard copy of high resolution screen during linearity assays of a flowthrough glucose electrode at high concentration: (top) experimental and (bottom) treated response curves to seven increases of glucose concentration by $5 \mathrm{~mm}$ steps. GOD cellulose acetate membrane, $37.0 \pm 0.1^{\circ} \mathrm{C}$, seven $30 \mu \mathrm{l}$ additions of $0.50 \mathrm{M}$ glucose into $3 \mathrm{ml}$ of acetate buffer placed in a closed loop (additions every $500 \mathrm{~s}$ ). 
b) steady-state signals are detected on filtered data when their slopes are smaller than a user chosen value, the equations of these almost horizontal parts of curves are calculated by linear regression and the corresponding steady-state response, i.e. delta $I$, are determined,

c) when the first derivative of the filtered data reaches a maximum, the corresponding dynamic responses $(\mathrm{d} I / \mathrm{d} T)_{\max }$ are determined,

d) all these operations are controlled by the user, since filtrated curves, plateaux and inflexion points are displayed on screen (Fig. 35.6 bottom).

Simuitaneously a file is created with all steady-state and dynamic responses, in users units, and all dynamic and steady-state response times; the user may either validate these data and store them on the corresponding parameter file, or discriminate undesirable values, or even resume the whole treatment procedure.

Finally, software has been created for reading all parameters and data on previously mentioned files and for storing both channels and time coordinates, in user's units, under the data-interchange format (D.I.F.) used by most commercial software such as worksheets: thus any systematic calculation may be performed on either all data or on a selection of them made by the experimenter.

\subsubsection{Use of the automated device for glucose electrode evaluation}

This set-up and software allows large number of repetitive experiments designed for comparison of various GOD membranes and glucose sensors to be performed. Figure 35.6 presents typical results of repeatability and linearity assays at high glucose concentration using a cellulose acetate enzyme membrane placed in a flow-through cell: seven increases of glucose concentration by $5 \mathrm{~mm}$ steps give steady-state responses (upper curve); the value of these responses may be determined using the data treatment programs (lower curve) as ranging between 21.9 and $23.7 \mathrm{nA}$ using a filtration period of $70 \mathrm{~s}$; corresponding dynamic response range between 21.7 and $24.4 \mathrm{nA} / \mathrm{s}$ demonstrating a good linearity of this glucose sensor response up to $30 \mathrm{mM}$ glucose.

During similar evaluation of glucose sensors we have tested their response to an artificial glucose concentration $v s$. time profile. Figure 35.7 presents such a profile (upper curve) for simulated intravenous glucose tolerance tests (IVGTT) of various kinetic patterns. It appears that such a device responds to increasing and decreasing glucose level but that its steady-state response time may be somewhat too large for such an application.

Such membranes are suitable for some in vivo glucose determination, as tested on an external blood shunt of conscious rats (Thévenot et al. 1985) but present relatively low response values (1 to $\left.10 \mu \mathrm{A} \times \mathrm{M}^{-1} \times \mathrm{mm}^{-2}\right)$ and sometimes excessive response values ( 0.4 to $3 \mathrm{~min}$ ). Further experiments are 

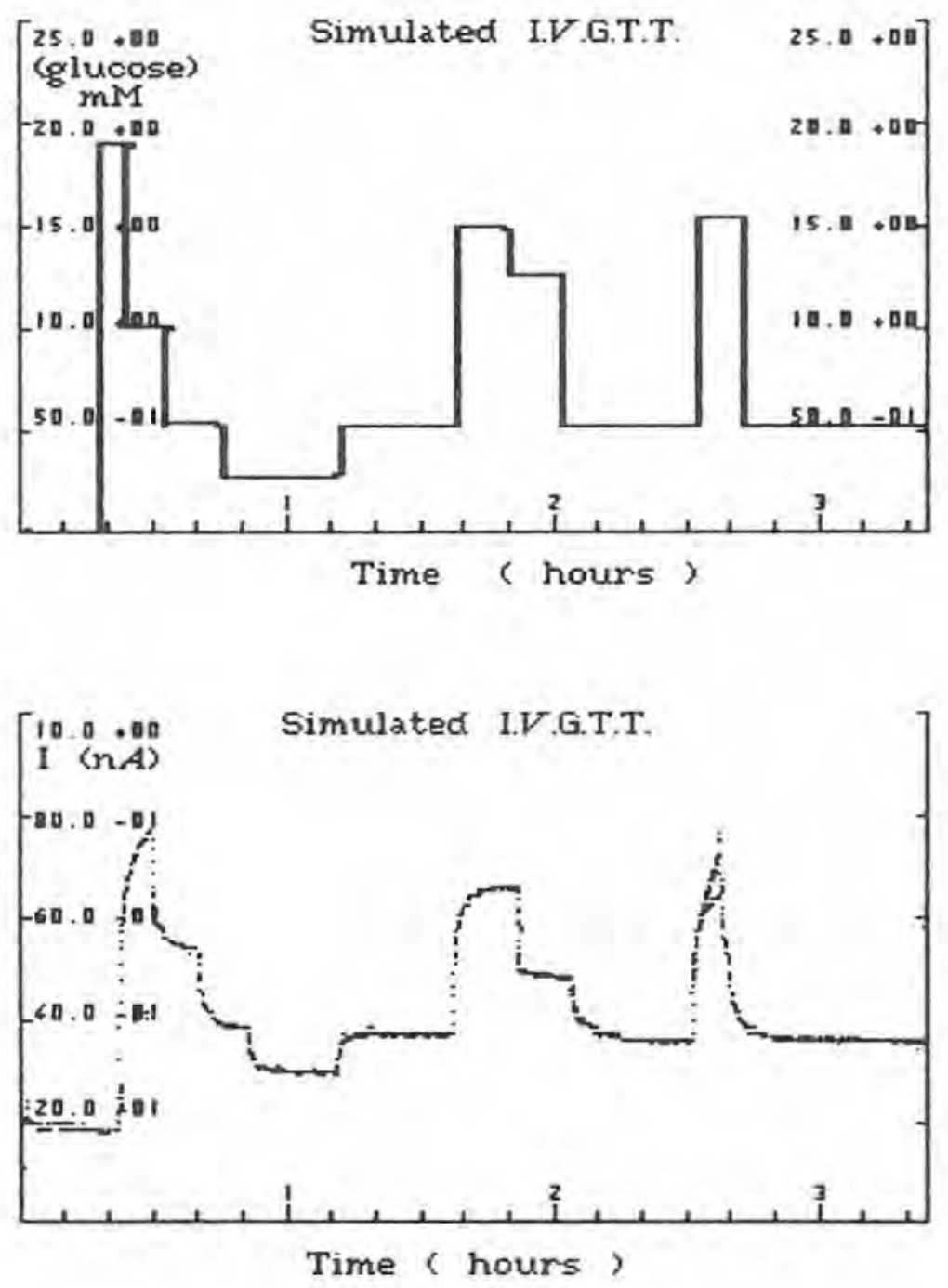

Fig. 35.7 Hard copy of high resolution screen during assays of a flow-through electrode to simulated intravenous glucose tolerance tests: (top) variations of glucose level simulating IVGTT under different physiological conditions and (bottom) response of the glucose electrode to this concentration profile. GOD cellulose acetate membrane, $37.0 \pm 0.1^{\circ} \mathrm{C}, 0.2$ to $19 \mathrm{~mm}$ glucose concentration profiles performed by addition of either glucose standard or buffer in acetate buffer placed in a closed loop. 
in progress to prepare glucose sensors with similar linearity for high concentrations of glucose but with larger response amplitudes and shorter response times,

\subsection{Conclusions}

Good characterization of any sensor and especially biosensors such as enzyme-based electrodes implies a large number of linear and repeatable assays, and the treatment of corresponding steady-state or dynamic responses. As a matter of fact, several thousands of such glucose assays have been performed using the same reconstituted collagen membrane for periods of up to four successive months of operation at $30^{\circ} \mathrm{C}$ and storage at room temperature (Thévenot et al. 1982). Such numerous assays would not have been possible without using automation of both the addition of 10 to 50 glucose standards in the reaction vessel and the detection of steady-state responses and their statistical treatment. This chapter presents two different set-ups for such automation: on the one hand, a simple programmable calculator carries out all these operations with a precision often better than a manual experiment, using a chart recorder; on the other hand, it may be of considerable interest to store the whole response curve and to analyse carefully its shape: such a situation is encountered when the substrate follows the non-step-like increase of concentration common in industrial or clinical processes. This second set-up, which exploits microcomputers and commercially available interface cards, enables both acquisition and real-time display on a high resolution screen, and various types of data treatments. It is currently used in our laboratory for comparing the principal analytical parameters of various enzyme membranes and reaction vessels, and also for recording and treatment of in vivo assays of flow-through glucose sensors under different physiological conditions of conscious animals (Thévenot et al. 1985).

\section{Acknowledgements}

The support of the Caisse Nationale de l'Assurance Maladie des Travailleurs salariés (France) Grant CNAMTS-INSERM 85.3.54.8.E, of National Institute of Health (US) Grant AM30718, and of Association des Jeunes Diabétiques (Paris, France) is grateful acknowledged.

\section{References}

De Laforcade, V. (1980). Study of a table set-up for glucose determination with an enzyme-based electrode. Research report, Université Paris-Val de Marne, 106 pp.

Dubois, C. (1984). Electrochemical characterization of membranes used in enzyme electrodes. Research report, Université Paris-Val de Marne, 42 pp.

Jaenchen, M., Scheller, F., Pfeiffer, D., Pittelkow, R., Wiegand, P. and Nentwig, J. (1982). GKM 01: a new measuring instrument for glucose using enzyme electrodes. Z. Med. Laboratoriumsdiagn. 23(1), 39-42. 
Kawana, S., Nihira, K., Miyashiro, H. and Takada, Y. (1980). Analyzer using immobilized enzymes. Jpn. Kokai Tokyo Koho JP 55/10523 (80/10523), 25 Jan 1980, 3 p. Appl. or Pr. 78/83098, 10 Jul 1978.

Kernevez, J. P., Konate, L. and Romette, J. L. (1983). Determination of substrate concentrations by a computerised enzyme electrode. Biotechnology and Bioengineering 25, 845-55.

Kyoto Daiichi Kagaku, K. K. (1982). Apparatus for automatic and continuous

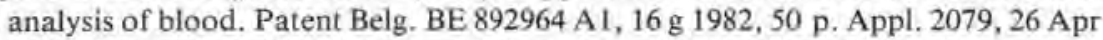
1982; JP Appl. 81/63160, 24 Apr 1981; JP Appl. 81/150924, 9 Oct 1981.

Mitsubishi Rayon Co. Ltd. (1983). Automatic electrode apparatus for determination of biological components. Jpn. Kokai Tokyo Koho JP 58/135950 A2 (83/135950),

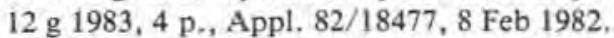

Skogberg, D., Richardson, T. and Blasczyk, T. (1979). Automatic sampling and monitoring of potentiometric electrodes: steady-state response by first and second derivative techniques. Analytical Chemistry 51(12), 2054-7.

Sternberg, R., Apoteker, A. and Thévenot, D. R. (1980). Trace glucose electrode for clinical, food and environmental determinations. In Analytical chemistry symposia Series Vol. 2 (ed. W. F. Smith), pp. 461-473. Elsevier, Amsterdam.

- Tallagrand, T. and Thévenot, D. R. (1983a). Experimental and theoretical studies of enzymatic membranes used in enzyme electrodes. Communication to the Electroanalysis International Symposium, Cardiff (Great Britain) abstract no. 82.

Tallagrand, T, and Thévenot, D. R. (1983b). Enzymatic membranes used in enzyme-based electrodes. Communication to 1st National colloquium of the French Bioelectrochemistry Group, Port-Leucate (France) abstract no. 24, pp. 93-96.

Tallagrand, T., Grunberg, M. and Thévenot, D. R. (1983). Microcomputers for specific electrodes using immobilised enzyme. Communication to 1st National Colloquium of the French Bioelectrochemistry Group, Port Leucate (France) abstract no. 25, pp. 97-101.

Thévenot, D. R. (1982). Use of electrochemical methods in the characterization of enzymatic membranes. Communication to the 33rd congress of the International Society of Electrochemistry, Lyon (France), abstract p. 784.

- Sternberg, R. and Coulet, P. (1982). A glucose electrode using high-stability glucose-oxidase collagen membranes. Diabetes Care 3, 203-6.

- Coulet, P. R., Sternberg, R. and Gautheron, D. C. (1978). A highly sensitive glucose electrode using glucose oxidase collagen film. Bioelectrochemistry and Bioenergetics 5, 548-53.

- Sternberg, R., Coulet, P. R., Laurent, J. and Gautheron, D. C. (1979). Enzyme collagen membrane for electrochemical determination of glucose. Analytical Chemistry 51, 96-100.

- Tallagrand, T., Velho, G, and Reach, G. (1985). Enzyme electrodes for use in vivo. 30th I.U.P.A.C, Meeting, Manchester, UK.

Tsuji, N., Nakamura, K., Endoh, K., Hamada, T. and Ishida, K. (1983). Blood sugar analyzing apparatus. US Patent 4407959 A, 4 Oct 1983, 11 p. Appl. 313666, 21 Oct 1981; JP Appl. 80/150756, 29 Oct 1980; JP Appl., 80/172665, 9 Dec 1980; JP Appl. 80/172666, 9 Dec 1980.

Wieck, H. J., Heider, G. H. and Yacynych, A. M. (1984). Simple approach to microcomputer-controlled electrochemistry. Anal, Chim. Acta 166, 315-19. 



\section{Commercialization and future prospects}





\title{
36
}

\section{Biosensors in medicine: the clinician's requirements}

\author{
P. D. HOME and K. G. M. M. ALBERTI
}

While the practice of modern medicine still depends heavily on the traditional skills of the doctor in observation, taking a clinical history, and examining the patient, the advent of effective therapy this century has been matched by a rapid growth in techniques of making objective determinations of measures of illness (pathology) and their response to treatment. Chemical and biochemical measurement in medicine has evolved into a specialty of its own ('chemical pathology', more lately 'clinical biochemistry'), generally as an offshoot of the more traditional laboratory-based pathology disciplines, tissue pathology and microbiology. This laboratory bias has led increasingly towards the use of more sophisticated and automated analytical devices, particularly under the pressure of increased clinical demand. The necessary batch processing makes the laboratory less able to respond flexibly to urgent demands, and removes local services at small hospitals in favour of a major installation in population centres.

A number of trends in medicine are seeking to reverse these pressures (Marks and Alberti 1985). It has always been the case that seriously ill patients, in intensive care units, could show rapid swings in biochemical variables, and that recognition and appropriate action were equally urgent. A rapid increase in open heart surgery and renal transplantation in particular has meant that many more people are requiring intensive life support for short periods. Furthermore it is now possible to of fer more successful treatment in cases of natural catastrophe, when associated with acute renal or hepatic failure or acute severe heart failure, for example. The need for a quick answer to the request for a particular biochemical measurement has often been met by the establishment of small ward-based laboratories, often at first using traditional analytical techniques, but later by the development of dedicated single specimen devices capable of giving rapid results. Measurement of blood gases, potassium, and glucose are familiar examples.

A second major pressure to move away from the 'routine laboratory' comes from the increasing pace and pressures of modern medicine, coupled to some extent to economic considerations. Both on the wards and in their offices clinicians find it increasingly less satisfactory to analyse a problem, 
implement investigations, and then have to arrange to reassess the problem at some later timepoint in the light of the results of those investigations. Patients, too, are not unnaturally keen to have their problems sorted out as rapidly as possible. The drive here, then, is to have the ability to perform some types of investigation in the doctor's of fice, hospital out-patient clinic, or on the ward. Examples are given below. In some cases instant investigation can be important even in the home, blood glucose in some diabetic states being the obvious example.

A further major pressure has come from the availability of reagent strips based on glucose oxidase for the determination of capillary blood glucose. Because subcutaneous insulin therapy in diabetes is a very erratic and unsatisfactory tool, knowledge of blood glucose levels both at home and on the ward is very helpful to both the patient and clinician. Capillary blood is easily collected from a finger-prick, and a widescale and reasonably successful implementation of blood glucose monitoring by this method (Tattersall 1979) has both opened clinicians' eyes to the possibilities of 'instant' biochemical determinations, and led to demands for even more convenient, robust, and operator proof blood glucose systems.

The erratic nature of the response to subcutaneous insulin therapy has also led to the drive to produce devices capable of physiologically appropriate intravenous insulin delivery. Because the amount of insulin required varies considerably with meals, exercise, stress, injury, and infection, and even the time of day, some kind of device which monitored blood glucose continuously, and whose output was used to control an insulin pump, has attracted considerable attention and speculation. Somewhat inevitably it has been the glamour of this 'artificial pancreas' (Fogt et al. 1978) which has proved a magnet for those interested in the application of biosensors to medicine, rather than the more mundane measurement-only devices.

This chapter will attempt to identify some of the areas where clinicians have a requirement that might be fulfilled by the use of a biosensor, and will then consider what is required in putting such a device to practical use in medicine.

\subsection{Openings for biosensors in medicine}

\subsubsection{The intensive care unit}

It is fairly easy to understand that the availability of continuous monitoring, or high frequency continual monitoring, in the intensive care unit can be a great aid to patient care. This is already achieved for many physical measurements (heart rate, blood pressure, temperature) and to some extent for a limited range of biochemical measurements (oxygen, $\mathrm{pH}$, glucose). It is also possible to define the range of biochemical parameters that would be useful in such circumstances (Table 36.1), but it is less easy to predict how these 
Table 36.1 Biochemical measurements which would be useful when monitored continuously (or at high frequency) in the intensive care unit

In blood

Oxygen, carbon dioxide, $\mathrm{pH}$

Lactate

(Amino acids, ketone bodies)

In blood or subcutaneous tissue

Glucose

Sodium, potassium

Calcium

Creatinine, urea

In urine

Sodium, potassium

Creatinine, urea

systems would be put into operation. The problem is not just one of clinical requirements, for the nature and ease of use of such devices will also determine their practical applicability. At present, even in units with access to blood-glucose-controlled insulin-infusion systems (artificial pancreas, Biostator), in practice blood glucose is often determined with a bedside reagent strip meter and the insulin infusion pump adjusted from experience or using written algorithms.

Blood gas (oxygen, carbon dioxide, $\mathrm{pH}$ ) and electrolyte (sodium, potassium) estimation will presumably continue to be by chemical sensors, but it is instructive to consider how different will be the clinical use of the information derived from these measurements. Blood oxygen tensions and saturation in the ill patient are largely determined by the condition of the lungs and circulation, and are not easily correctable by changing the inspired oxygen concentration or rate of administration. Furthermore rapid changes in oxygen tension can occur. The demand is therefore for true continuous monitoring, but with the response loop closed by the human brain. Electrolytes and water balance are much more directly affected by input and output however. In the situation where blood concentrations, urine concentrations, and fluid output can be measured it is relatively easy to conceive of an automatic 'fluid balance centre', controlling input in closed loop fashion, though here the lessons of the Biostator must be learnt. Note that in most circumstances hourly estimations would suffice, provided an option was retained for emergency override.

The requirements for blood glucose estimation are similar to the pattern for electrolytes. Thus a bedside device, operated manually by application of blood samples at 15 minute to 4 hour intervals, is the major advance from 
relying on laboratory-measured samples. Reagent-strip meters meet this market already to some extent, but their response time is inconveniently long ( $2 \mathrm{~min}$ ) and careful operator training is required. Continuous monitoring would be a worthwhile advance not only in diabetic patients on the unit, but also in those receiving high doses of inotropic agents (which can be diabetogenic) for cardiac support, and those receiving high osmolarity glucose solutions for intravenous nutrition. While it is desirable to move to blood-glucose-controlled insulin infusion in such circumstances, in practice the patients are additionally receiving glucose solutions, both to 'buffer' diabetic control and to provide free water. It may therefore be that when sophisticated blood-glucose-control systems are available for use in intensive care units, it will be in conjunction with fluid balance centres.

The role of the monitoring of other aspects of intermediary metabolites in the critically ill patient has yet to be explored, and indeed such research awaits the advent of biosensors for the continuous monitoring of blood lactate, alanine, and ketone bodies (Noy and Alberti 1981a, b). In these circumstances it is at present difficult to predict how the information might be used clinically, except in lactic acidosis, and at first it may merely allow the physician to monitor metabolic status more closely.

Intensive care units would also find instant and repeated measurements of renal function (creatinine, urea) extremely useful.

\subsubsection{Casualty/emergency rooms and general wards}

In the circumstance of seeing an acutely ill patient in whom the diagnosis is uncertain the clinician's primary need is for instant results to biochemical tests to complement the clinical history and examination, both of which already provide answers on which an immediate assessment can based. Whether the results are required for diagnostic purposes or as a guide to therapy is immaterial, except insofar as the latter are more likely to be repeated at intervals of hours.

The list of tests which account for $95 \%$ of such urgent investigations is not large, and they are listed (Table 36.2) irrespective of whether biosensors, physico-chemical sensors, reagent-strip meters or standard biochemical techniques are likely to form the basis of usable devices. Glucose and amylase would probably be in greatest demand in medical and surgical emergencies, followed sadly by estimations of paracetamol and salicylate levels in blood. Creatine kinase is an enzyme used to confirm the diagnosis of coronary thrombosis. The overall level of demand for electrolytes and creatinine (or urea) would be high, particularly when wards are included, but generally the urgency and importance of these requests will not be of such high priority. It will again be noted that there will be a requirement for urine based, as well as blood based, estimation of electrolytes and creatinine.

While the commonest need for emergency drug investigations is for para- 
Table 36.2 Biochemical tests for which instant results would be invaluable in emergency situations

Amylase
Glucose
Paracetamol
Salicylate
Creatine kinase
Aspartate aminotransferase
Ammonia
Sodium
Potassium
Calcium
Creatinine or urea
Oxygen, pH, carbon dioxide

cetamol and salicylate, emergency admissions for overdosage of other drugs is also common. Estimation of drug levels is performed not just to confirm the diagnosis, but also so that specific measures may be taken to counteract the deleterious effects of the drug (paracetamol) or to enhance its elimination (salicylate). Unfortunately neither of these is possible for most pharmacological agents, and treatment is therefore supportive (e.g. artificial ventilation) without the need for identification of specific drugs. Though exceptions do exist (phenobarbitone), and in other cases prognostic information can be helpful (paraquat), the local rate of poisoning with any individual agent will always be low.

If antigen-antibody interactions can be successfully harnessed into biosensors then detection of hepatitis B antigen or HTLV-III (AIDS) virus antigen in blood samples would be much in demand for protection of laboratory personnel. Similarly drugs can be detected by antibody interactions, and the heart drug digoxin is an obvious candidate where fast answers are often required.

\subsubsection{Physician's consulting rooms}

Again the need here is for instant information to aid diagnosis. The convenience of office-based measurements will vary from country to country, for unlike the UK many consulting rooms are not hospital based. Occasionally diagnostic tests are urgent even in the physician's of fice, and glucose, amylase, and creatine kinase would be most useful. Glucose is already widely measured in routine practice (reagent strips with or without meters), in diabetic clinics, as a diagnostic measure, and in the management of the ill diabetic patient. Blood cholesterol and triglycerides are likely to be more in demand as the results of the lipid intervention studies (Lipid Research Clinics 
Program 1984) become more widely appreciated, both for selective screening and to save the extra patient visits presently necessary to have a current result available at the time of consultation. Creatinine and potassium would also be useful investigations to have 'on tap' at the clinic.

The viral antigen and drug tests mentioned above would also be useful in this situation. Another example from current use is the measurement of anticonvulsant levels in neurology.

\subsubsection{Diabetes and the patient}

The attention of sensor engineers has focused upon diabetes for a number of reasons. Diabetes can be regarded as a disease of relative or absolute deficiency of the messenger/effector (hormone) insulin, a protein produced by one of the ultimate natural biosensors, the B cell of the Islets of Langerhans in the pancreas (Malaisse et at. 1979). Insulin has a very short half life in blood (4 to $5 \mathrm{~min}$ ) and hence its concentration, a major determinant of its action, depends on its rate of secretion. The $B$ cells are sensitive to a wide range of substances, as befits the many biochemical actions of insulin, the most important of these being blood glucose. The blood glucose concentration in blood is normally tightly regulated by this means (between 3.5 and $5.5 \mathrm{mmol} / \mathrm{l}$ ), but in diabetes insulin deficiency results in high blood glucose levels with consequent short-term ill health and long-term tissue damage.

The obvious treatment is to give insulin, and indeed before insulin became available patients usually died after a few months. Without the continuous monitoring of glucose (and other substances) it is not possible to give insulin in a normal physiological fashion however, and so diabetic patients still have periods of high blood glucose levels, and, through excessive and inappropriate insulin administration low blood glucose levels.

Improvements in control of diabetes, and the life style of the diabetic patient, came with the introduction of the glucose-oxidase-based reagent strips (Tattersall 1979), at first read by rather clumsy meters, which are however now much refined. A further major advance was the visually-read strip (by colour matching), effectively freeing the patient from needing to carry or buy a meter. To compete with visually-read reagent strips direct reading biosensors (working on a drop of blood) will need to be competitive on cost (say UK£0.25 per test), and use very light, portable, and reliable hardware. In its basic form the latter should retail at no more than UK£70, but it is increasingly likely that such devices will form the basis of more sophisticated recording devices, which will be able to advise on insulin dosage.

Patients will however continue to suffer from hypoglycaemic reactions due to low blood glucose concentrations on occasion, for reasons as diverse as exercise or alcohol consumption. Considerable effort has gone into attempting to build 'hypoglycaemia detectors' (Levandowski et al, 1983), but to date these have depended on changes in skin conductivity (due to perspiration). 
These changes are however very non-specific, and after a few false alarms the patient (or his parents) abandons its use. An evident way around this is to sense actual blood glucose levels, or a related measurement such as subcutaneous blood glucose concentration. This use of needle-type indwelling glucose sensors is likely to precede their use in controlling insulin delivery pumps, for much troublesome hypoglycaemia is at night and the sensor need

Table 36.3 Potential uses of biosensors in diabetes

\begin{tabular}{|c|c|c|}
\hline Type of device & Circumstance of use & Place of use \\
\hline \multicolumn{3}{|l|}{ Glucose sensors } \\
\hline \multirow{6}{*}{$\begin{array}{l}\text { Spot blood glucose } \\
\text { sensor }\end{array}$} & Routine measurement & At home (by patient) \\
\hline & & Diabetic clinics \\
\hline & & $\begin{array}{l}\text { Consulting rooms } \\
\text { Medical/surgical wards }\end{array}$ \\
\hline & Emergency measurement & $\begin{array}{l}\text { Medical/surgical wards } \\
\text { As for routine }\end{array}$ \\
\hline & & measurement \\
\hline & & $\begin{array}{l}\text { Casualty/emergency } \\
\text { rooms }\end{array}$ \\
\hline \multirow{4}{*}{$\begin{array}{l}\text { Needle sensors } \\
\quad \text { (subcutaneous) }\end{array}$} & Hypoglycaemia alarms & Overnight at home \\
\hline & Blood glucose profiles & Outpatient monitoring \\
\hline & & $\begin{array}{l}\text { Conventional insulin } \\
\text { delivery }\end{array}$ \\
\hline & Continuous monitoring & $\begin{array}{l}\text { Hybrid feedback insulin } \\
\text { delivery }\end{array}$ \\
\hline Intravenous sensors & Continuous monitoring & $\begin{array}{l}\text { Automatic insulin } \\
\text { delivery }\end{array}$ \\
\hline \multicolumn{3}{|l|}{ Ketone body sensors } \\
\hline \multirow[t]{2}{*}{$\begin{array}{l}\text { Spot blood ketone } \\
\text { sensor }\end{array}$} & $\begin{array}{l}\text { Diagnosis of keto- } \\
\text { acidosis }\end{array}$ & $\begin{array}{l}\text { Casualty/emergency } \\
\text { rooms }\end{array}$ \\
\hline & & Diabetes wards/units \\
\hline Skin, breath sensors & $\begin{array}{l}\text { Guard against keto- } \\
\text { acidosis }\end{array}$ & Home or hospital \\
\hline Subcutaneous sensors & $\begin{array}{l}\text { Adjunct to insulin infusion } \\
\text { systems }\end{array}$ & Outpatient use \\
\hline $\begin{array}{l}\text { Combined glucose/ } \\
\text { ketone sensor }\end{array}$ & Continuous monitoring & $\begin{array}{l}\text { Automatic insulin } \\
\text { delivery }\end{array}$ \\
\hline \multicolumn{3}{|l|}{ Lactate sensor } \\
\hline Intravenous sensor & Critically ill patients & Intensive care unit \\
\hline $\begin{array}{l}\text { Combined glucose/ } \\
\text { lactate sensor }\end{array}$ & Continuous monitoring & $\begin{array}{l}\text { Automatic insulin } \\
\text { delivery }\end{array}$ \\
\hline \multicolumn{3}{|l|}{ Amino acid sensor } \\
\hline $\begin{array}{l}\text { Combined glucose/ } \\
\text { amino acid sensor }\end{array}$ & Continuous monitoring & $\begin{array}{l}\text { Automatic insulin } \\
\text { delivery }\end{array}$ \\
\hline
\end{tabular}


only be worn for the period spent asleep. Aside from reassuring the patient at home, a few overnight profiles of blood glucose concentration would greatly help in adjusting insulin regimens.

The technical prerequisites for the use of sensors in medicine are discussed in some detail below. Evidently however the signal from a rapidly responding blood glucose sensor could be used to control an insulin delivery pump, given reliability, selectivity, and absence of signal drift. Combined with the necessarily sophisticated implantable insulin infusion pumps (which should be tested in patients in 1986), an effective replacement for the B cell could be made available. Subcutaneous sensors (Schichiri et al. 1982) offer obvious advantages in terms of access, but the signal at mealtimes is delayed compared to changes in blood glucose concentrations, a problem that may be difficult to resolve as physiological insulin concentrations rise very quickly after meals (Ahmed et al. 1976). Furthermore in order to control an implanted pump the signal will have to be transmitted back across the skin to any implantable pump. Early subcutaneous sensors are therefore likely to be used with conventional subcutaneous insulin administration by injection or infusion pump, with the human brain intervening to interpret the data, probably with the aid of dosage computers (Schiffrin et al. 1985).

It would be useful to measure other substrates in the diabetic patient (Table 36.3). With more physiological day-to-day insulin delivery the risks of acute deterioration of diabetes due to other illness (e.g. infection) appear to be increased, and a device warning of high blood ketone body levels (e.g. 3-hydroxybutyrate) would be welcome. Again skin or subcutaneous sensors (or acetone breath detectors) might be more feasible. Aside from the intensive care unit rapid rises in blood lactate concentrations may, in the absence of exercise, indicate an over-rapid increase in the glucose turnover rate, suggesting overinsulinization. Incorporating a lactate sensor into the glucose sensor controlling insulin delivery might be the first step to approaching the complexity of the natural $\mathrm{B}$ cell response, with amino acids being the next candidates.

\subsection{The application of biosensors in medicine}

The application of technology to medicine imposes special demands which inevitably vary with the proximity of any device to human tissues, and the importance of its output, whether information or action, to vital functions. In many situations detection and measurement devices, although capable of warning of abnormalities, only result in modifications to treatment through human intervention, so that for example, an abnormal cardiac rhythm does not result in the patient receiving direct-current cardioversion (DC shocks) without medical intervention. In more sophisticated applications this link may be missing and impossible to restore, the most familiar example being 
cardiac pacemakers, whose output can be life saving but also fatal.

These differences result in very different requirements in terms of reliability, accuracy, precision, bioavailability, patient acceptability, and patient interfacing. From the engineering point of view an external measurement device subjected to routine quality-control monitoring may be constructed to little better than ordinary consumer standards, while life-dependent devices used continuously at home will need to meet military/aerospace specifications.

\subsubsection{Accuracy/precision/sensitivity}

It is readily apparent that any measurement device must give a result that approximates to the real value (accuracy), and must do this with sufficient reproducibility (precision) for individual readings to be dependable. Accuracy will not generally be a problem with devices calibrated against known reference methods, except insofar as calibration drift occurs. Clinical medicine is surprisingly undemanding on precision when compared with mechanical or chemical engineering, with doctors generally unresponsive to differences of $\pm 10 \%$ in making spot decisions, though for serial measurements this kind of difference may be of value in detecting trends due to treatment. In some circumstances (measurement of serum uric acid, or high levels of blood amylase or paracetamol for example) even less precision is necessary.

There is a need however to be careful in interpreting clinical requirements in terms of current medical practice. Many patients currently read their blood glucose monitoring strips to $\pm 30 \%$ (being the steps on the colour chart), and present the data to their physicians, who find the results a satisfactory basis on which to adjust insulin dosage. Such imprecision would not however be acceptable if each individual measurement were used for adjustment of insulin dosage in an attempt to maintain constant physiological blood glucose concentrations, as might be the case if subcutaneous insulin delivery was therapeutically more effective. It can be anticipated that when more sophisticated use is made of blood glucose data (in computations of dose, absorption of food and insulin sensitivity, or for feedback control of intravenous insulin delivery) then much better precision will be required.

It is important to recognize that the effective range of a measurement device for use in clinical medicine will generally relate to the patho-physiological range for that parameter and not the physiological range. Blood glucose is normally maintained between 3.5 and $5.5 \mathrm{mmol} / \mathrm{I}$, as discussed above, but it is important to be able to distinguish $1.0 \mathrm{mmol} / 1 \mathrm{from}$ $2.0 \mathrm{mmol} / 1$, and to know that a recorded change from 50 to $40 \mathrm{mmol} / 1$ is real. The hormone TSH is important in monitoring low levels of thyroid gland activity (it rises under these circumstances), and endocrinologists have long used an assay that is incapable of distinguishing concentrations below 
the upper limit of the physiological range. The sensitivity of a device has therefore to be tailored to its role in medicine.

The availability of more portable, precise, and reliable measurement devices will inevitably be a further stimulus to the demands of the medical research establishment. Those involved in the development of biosensors will be approached for devices of high accuracy and precision for use in clinical and fundamental physiological research.

\subsubsection{Response time}

A blood sample sent to the routine hospital laboratory for analysis will generally produce a result anywhere between 30 minutes and several days later. During this time the clinician will proceed with therapy on the basis of clinical judgement. Paradoxically where bedside monitoring is undertaken the response time of the device becomes more important as there will usually be the intention of making an instant decision. As discussed above this is where biosensors should have an early impact on modern clinical management. While waiting for a result from a sensor-type device staff will not have time to complete another activity, so in general response times of over 120 seconds are unsatisfactory. This should include any calibration or preparative procedures. A suitable aim would be 30 seconds, and it is very important that no opportunity should be given of making a reading before the intended interval has elapsed.

\subsubsection{Calibration}

It is at present difficult to conceive of biosensors that will maintain their accuracy with repetitive or continuous usage over a period of time. The problem will not occur of course with single-use devices. Furthermore while physical devices such as reflectance meters can be calibrated using prepared dry strips or filters, biosensors may need calibration with fluid incorporating the substance to be measured. Such fluids may themselves need to incorporate fixed concentrations of interfering substances where necessary. Unless response times are very fast it would be unsatisfactory to perform calibration at each usage, but it is highly desirable that devices are preprogrammed to demand recalibration after a fixed number of tests and at fixed time intervals. External calibration against a laboratory based machine is inpracticable for routine purposes, and should be reserved for quality control schemes organized by clinical and laboratory personnel.

In vivo monitoring presents further problems, and drifts in accuracy will often be the limit to the useful lifetime of a sensor. In general any intravenous or subcutaneous needle should have a minimum expected lifetime of 24 hours before replacement is contemplated (and transcutaneous wires and cannulae generally must be changed after a maximum of 3 days). Within the hospital external calibration against a blood sample would usually be the method of 
choice, though care would be necessary where blood concentrations were to be related to tissue concentrations in ill patients. Equally, looking to the future, there is no good reason why external calibration from a capillary blood sample should not be used to reset an indwelling implanted sensor, probably through a dedicated external unit, but again this could not be done more than once every 24 hours by the patient at home.

\subsubsection{Interfering substances}

The problem of non-specificity of biosensors in relation to measurements in biological fluids is well recognized. It is important for bioengineers to realise that the range of interfering concentrations of any substance present in blood is not its physiological range. The very ill patient may have very abnormal blood levels of glucose, oxygen, organic acids, urate, etc., and it is in just such patients that in vivo monitoring may at first be contemplated. Furthermore multiple drug therapy is almost the rule in medicine, and these compounds and their metabolites are another potential source of interference.

\subsubsection{What sample}

Clinicians will use for measurement any fluid sample that they can obtain urine, cerebro-spinal fluid, saliva, sweat, effusions, and exudates - but most commonly blood. Blood is often the fluid most easily obtained, especially in an emergency, and is also highly representative of chemical disturbances in the body. Unfortunately blood poses two special problems. Firstly a variable proportion of it is compartmentalized into erythrocytes, themselves around $30 \%$ protein as haemoglobin, and whose internal composition is distinct to plasma. Secondly blood contains a series of agents purposefully designed by evolution to react with surfaces. Though centrifugation may be used to separate erythrocytes from plasma, it does take time and equipment, and can be difficult with smaller samples. Furthermore mechanical breakage of vessels can present a real health hazard through the formation of aerosols of plasma.

Sensors therefore need to be incorporated into systems which accept blood, and which estimate concentrations within the plasma compartment for these substances which do not equilibrate rapidly across the erythrocyte membrane.

\subsubsection{Safety and reliability}

It might seem logical to say that a device could be used in medicine if its chances of saving life were higher than its risks to life. This simple idea is unacceptable in practice, even if the risks were quantifiable, which is generally not the case. Furthermore many treatments in medicine are aimed at relieving morbidity (suffering) rather than reducing mortality, and setting 
risks to health (and life) against such treatments or means to treatment is fraught with philosophical and social difficulties. In general then the benefits from any new treatment or device have to far outweigh potential problems before being medically acceptable.

\subsubsection{False results Malfunction of any sensor causing a false result and} a subsequently inappropriate automatic or informed response to it must remain of major concern. Examples of a dangerous response might include an in vivo blood glucose monitoring device, controlling an insulin infusion system, generating a falsely high signal. Even a home blood glucose meter, making the same error, could result in a patient taking dangerous action to correct the perceived abnormality. Equally, failure to detect a real abnormality could result in clinicians not taking appropriate measures in potentially fatal circumtances. High ketone body levels in the blood (diabetic ketoacidosis) or paracetamol poisoning might be pertinent examples.

Sophisticated in vivo monitoring devices can be provided with circuits to monitor electronic malfunction, though there can be no guard against an incorrect sensor signal, unless duplication of sensors is used. Within certain limits devices can be programmed to monitor the rate of change of measured parameters and alert users when the result changes outside present limits, but this is heavily dependent on the error developing more quickly than changes might be expected to occur as a result of the disease. Thus the design of all parts of sensor devices needs continuing attention to reliability, and the use of the highest practicable engineering standards.

Bedside and patient-operated devices present a more difficult judgement, partly because to be useful they must be offerred at a sensible retail price. Part of the answer here lies in the hands of the doctors themselves, so that users are educated not to react to a single result that could cause a dangerous change in treatment without thought and perhaps cross-checks.

36.2.6.2 Toxicity It is self evident that in vivo monitoring devices must be made of materials that are non-toxic, and considerable commercial expertise is available for structural materials (metals and plastics) used in heart valves, pacemaker wires, and bone and vessel prostheses, Attention must also be paid to novel organic substances, including enzymes, that might leach from sensors or otherwise be picked up by the body's scavenger cells (macrophages). The anticipated problem would be activation of the immune system and production of antibodies, with the potential of organ damage through immune complex disease or amyloidosis. These risks are remote however, given the likely load of toxic or antigenic materials to the body, and the containment of most of them behind diffusion barriers.

\subsubsection{Mechanical dangers Every breach of the skin is a destruction of}


anatomical integrity, and every advance deeper into the body carries with it further risks of disturbing vital functions. Sensors placed in the subcutaneous tissue, and associated with a transcutaneous wire, provide a entry portal for infection. They must therefore be sterile and need changing every 1 to 3 days. The risk is higher for devices placed within the circulation (the infection is more serious), while the procedural risks of placing such devices in deep veins means that regular changing is not practicable outside the intensive care unit or operating theatre. Such devices should therefore be totally implantable and have a long life (over two years). Furthermore the risks of thrombosis are not fully understood, and it appears from experience with pacemaker wires that anything lying up against the vessel wall may be incorporated within it. This might obviate sensor function!

The other risk from intravascular devices is that of mechanical disintegration resulting from long-term placement in fluid at $37^{\circ} \mathrm{C}$ in an environment that never stops moving. It is for all these reasons that sensors intended for permanent intravascular placement in man will have to be proved over some years in dogs or pigs, and will only then be in a position for approval by the regulatory authorities.

\section{References}

Ahmed, M., Gannon, M. C. and Nuttall, F. Q. (1976). Postprandial plasma glucose, insulin, glucagon and triglyceride responses to a standard diet in normal subjects. Diabetologia 12, 61-7.

Fogt, E. J., Dodd, L. M., Jenning, E. M. and Clemens, A. H. (1978). Development and evaluation of a glucose analyser for a glucose-controlled insulin infusion system (Biostator). Clin. Chem. 24, 1366-72.

Levandowski, L. A., White, N. H., Popp, D. and Santiago, J. V. (1983). Teledyne sleep sentry - a possible aid for the detection of symptomatic nocturnal hypoglycaemia in insulin-dependent diabetes. In Artificial systems for insulin delivery (eds. P. Brunetti, K. G.M.M. Alberti, K.D. Hepp, A. M. Albisser, and M. Massi-Benedetti), pp. 353-6. Raven Press, New York.

Lípid Research Clinics Program (1984). The lipid research clinics coronary prevention trial results. J. Am. Med. Assoc. 251, 351-64.

Malaisse, W, J., Sener, A., Herchuelz, A. and Hutton, J. C. (1979). Insulin release: the fuel hypothesis. Metabolism 28, 373-86.

Marks, V. and Alberti, K. G. M. M. (eds.) (1985). Clinical biochemistry nearer the patient. Churchill Livingstone, Edinburgh.

Noy, G.A. and Alberti, K, G, M, M, (1981a), In vivo monitoring of intermediary metabolites. In Advances in clinical biochemistry (eds. K. G. M. M. Alberti and C. P. Price), Vol. 2, pp. 229-41. Churchill Livingstone, Edinburgh.

- $(1981 b)$. In vivo monitoring of metabolites using enzyme based analytical techniques. In Applied biochemistry and bioengineering (eds. L. B. Wingard, Jr, E. Katzin-Katalski, and L. Goldstein), Vol. 3, pp. 233-52. Academic Press, New York. 
Schichiri, M., Kawamori, R., Yamasaki, Y., Hakui, N. and Abe, H. (1982). Wearable artificial endocrine pancreas with needle-type glucose sensor. Lancet ii, 1129-31.

Schiffrin, A., Mihic, M., Leibel, B.S. and Albisser, A. M. (1985). Computer assisted insulin dosage adjustment. Diabetes Care 8, 545-52.

Tattersall, R. B. (1979). Home blood glucose monitoring. Diabetologia 16, 71-4. 


\title{
37 \\ Exploiting biosensors
}

\author{
JAMES MCCANN
}

Biosensors have attracted considerable attention in recent years as successors to a wide range of analytical techniques in process control, in the clinical laboratory, in veterinary health care, and in the food industry. Indeed, the potential applications of the technology are so widespread that a short chapter in this book can merely indicate some of the major trends affecting the exploitation of these devices over the new few years. Furthermore, one is faced with the problem of defining exactly what is a biosensor. For example, consider the clinical biochemistry market where analysers have been around for at least ten years. These analysers range from small hand-held models, costing a few hundreds of pounds, to vast automated systems costing many thousands. Clearly many of these demand the epithet biosensor, yet they have been rarely discussed as such.

Given the problems of definition, and the wide range of existing products that cleary fall into the category, I have decided to adopt a permissive definition of the word biosensor. Thus I make no apologies for the inclusion of items and technologies which are not normally classified under the title biosensor. Their inclusion is not merely to provide background material, but also to provide precedents for what may or may not happen to some of the new devices that are discussed elsewhere in this book. Moreover, these earlier analytical techniques can provide insight into those forces that can lead to the successful commercialization or demise of a biosensor - 'the invisible hand' of the market place so beloved of by eighteenth century economists.

In order to add structure to a discussion of how and where the biosensors are to be exploited I shall appeal to a conceptual framework well known to the doyens of Madison Avenue - the product life cycle. This concept gained widespread currency during the 1970s as an explanation of how markets behave and the reasons for this behaviour. Its importance arises from the fact that certain types of market behaviour are usually observed at different stages in the product life cycle, irrespective of the product involved. For example, the degree of competition, size of the market, advertising, and distribution methods can all be related to the stage of the product life cycle. For the purposes of this discussion the product in question is a biosensor and we shall examine the following markets for the devices: 
1) human health care,

2) veterinary health care,

3) fermentation and process control.

I have selected these three categories because they represent markets at different stages of the product life cycle, and therefore widely different opportunities for biosensors. Taken together, they also represent the major markets. But first, I shall digress for a short introduction to the product life cycle.

\subsection{The product life cycle}

The product life cycle is usually divided into four separate stages: introduction, growth, maturity, and decline. The introduction phase of the life cycle represents the initial sales following product introduction. As sales increase the market enters a growth phase characterized by increasing sales. When this growth slackens the market reaches a period of decline. Figure 37.1 shows a sketch of a typical product life cycle.

The introduction phase of a product is usually the result of many years of research and development by a single company. In the case of biosensor devices the product development will entail laboratory work, prototype testing, regulatory approval from appropriate bodies, and extensive clinical testing.

Once the product is introduced the company will also have to expend considerable resources in establishing distribution, marketing, and service networks and in educating consumers. The success of a product is crucially dependent upon the effectiveness of this programme. Thus relatively inferior products may enjoy success because of adept marketing and distribution, while better designed products may flounder. An analogy may be drawn here with computers, where IBM have always exploited their superior marketing,

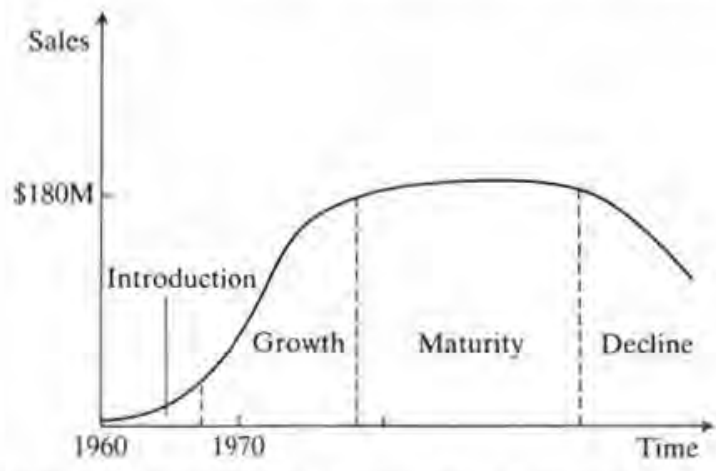

Fig. 37.1 The product life cycle: the market for radioimmunoassays in the USA. 
service and distribution to dominate the market, despite inferior product design.

In the case of medical products the introduction phase of a product is usually very long. There are a number of reasons for this including high complexity; the large number of people involved in adopting the new method (doctors, nurses, administrators); the risks associated in moving to a new method as opposed to staying with an established one; the expense involved in purchasing new equipment; and the innate conservatism of some parts of the medical profession. The cost of overcoming these hurdles and obtaining market acceptance for a medical product will be many times greater than the cost of the research and development effort. Thus many small innovative companies, which are adept at product invention and development, will fail to earn a return on their investment because they lack the resources to overcome these problems. During this introductory phase the pioneer company will typically be in a monopoly position, which will be reinforced by patents developed during the research period.

The growth phase is usually characterized by a rapid increase in product sales. However, the increase in sales and high prices usually charged by the monopoly supplier attracts new competition and products. These new products will typically be as close to the existing product in performance as possible, while, at the same time, avoiding patent infringement. In many cases these new competitors will be large health care companies that hope to expand primary demand for the product with their large advertising and marketing budgets, worldwide distribution, and sales forces. These new entrants usually employ large staffs of legal counsel and development teams to circumvent the advantages of the pioneering company. Their focus on development, rather than innovation, means they are unlikely to be as adept at product innovation as the pioneer.

Sales growth during the growth phase will be rapid due to increased consumer acceptance, a history of reliable product use, large marketing and advertising budgets, and widespread product availability. However, as the market reaches saturation, this sales growth will decline and the market will reach a period of maturity.

This stage is characterized by a large number of competitors all selling products using very similar technology, the novelty afforded by patent protection having been eroded by time, cross licensing agreements, and endless efforts at circumvention. Since the consumer is now faced by a plethora of products all performing in roughly the same way there will be intensive price competition, with widespread price cutting. Some competition may leave the market entirely or try to distinguish their product by means of 'product differentiation' - changing some of the product characteristics such that the consumer may be willing to pay a premium for it. As can be imagined, trying to enter a market at this stage is very difficult indeed, unless 
one has something that is clearly and demonstrably superior. Even then, the consumers may have invested large amounts in existing capital equipment and trained staff, and will thus be unwilling to change.

Eventually the market may begin to decline in real terms due to obsolescence, the introduction of radical new technology, or a substitute product.

Arguably the best example of this type of market behaviour related to biosensors is the market for radioimmunoassays.

This market was pioneered during the early sixties following the discovery that a radioisotope tag could be used to quantitate the reaction of a specific antibody with its specific antigen. At the time of its discovery this method was the only available means of carrying out many standard assays. As a result the market grew rapidly during the 1960 s and 1970 s with many companies entering the field. Technological improvement, although rapid at first, slowed and most competitors were selling a similar product by the middle of the 1970s. Since most of the products could be read on any available gammacounter, and most clinical chemistry laboratories had purchased one, switching between various suppliers became endemic. Purchase decisions became dominated by price concerns and the profitability of many of the participants fell correspondingly. The market displayed all the characteristics of maturity.

However, the market has now entered a period of slow decline because new analytical technologies, most notably those using an enzyme or fluorescent molecule to tag the antibody, have been developed. These new methods of fer ease of disposal, stability during storage and transport, and lower equipment costs. Nevertheless, the demise of radioimmunoassays will be very slow due to the large amount of capital invested in gamma counters and trained staff. Indeed, radioimmunoassays still account for at least $40 \%$ of the U.S. market for immunoassays, despite the advent of enzyme labels almost ten years ago. Table 37.1 lists the estimated market sizes of the various types of immunoassay reagents in the USA during 1984. The largest category was radioimmunoassay with annual sales of up to $\$ 250$ million. Table 37.2 breaks

Table 37.1 Estimated 1984 immunoassay reagent sales by product category and market share with USA

\begin{tabular}{lc}
\hline Category & Market size (\$ millions) \\
\hline Immunology & 40 \\
Blood banking & 80 \\
Serology (testing) & 80 \\
Radioimmunoassays & 250 \\
Non-isotopic assays (mostly enzyme-linked systems) & 160 \\
Total & $\$ 610$ million \\
\hline
\end{tabular}


Table 37.2 Estimated US Market shares of the ten largest-selling radioimmunoassay (RIA) tests in the USA in 1983

\begin{tabular}{lc}
\hline RIA test & Approximate market size (\$ millions) \\
\hline Hepatitis & 50 \\
$\mathrm{~T}_{4}$ & 30 \\
Digoxin & 20 \\
Human chorionic gonadotrophin & 20 \\
$\mathrm{~B}_{12} /$ folate & 12 \\
Thyroid stimulating hormone & 11 \\
Carcinogenic embryonic antigen & 10 \\
Cortisol & 8 \\
$\mathrm{~T}_{3}$ & 7 \\
Ferritin & 6 \\
& $\$ 174$ \\
\hline
\end{tabular}

The total market for RIA tests in the USA in 1983 was estimated to be \$250 million.

down the radioimmunoassay market still further into the ten largest-selling tests.

The second largest category of immunoassay was non-isotopic assay, the majority of which used an enzyme to replace the radioactive label. This segment is the most rapidly growing because it of fers the user a combination of ease of use, low equipment cost, simple protocols, and rapid response. Table 37.3 breaks the market for enzyme immunoassays down further and gives details of the six largest selling tests.

Table 37.3 Estimated US market shares of enzyme immunoassays in the USA in 1984

\begin{tabular}{lc}
\hline Test & Approximate market size (\$ millions) \\
\hline Theophylline & 30 \\
Phenytoin & 11 \\
Digoxin & 6 \\
Phenobarbital & 8 \\
Tobramycin & 9 \\
Gentamicin & $\frac{8}{}$ \\
& $\$ 72$ million \\
\hline
\end{tabular}

Total US market size in 1983 for enzyme immunoassays in the USA was estimated to be $\$ 120$ million, divided between approximately forty tests. The market was estimated to be growing at over $10 \%$ per annum. 
The remainder of the market for immunoassays comprises reagents used for immunology, blood banking, and serology. The immunology area comprises reagents for the characterization of immunoproteins. The blood banking segment comprises tests used for the screening of blood prior to transfusion, including blood typing. The serology segment comprises reagents used for the testing of bacterial and viral antigens, and for yarious metabolic diseases such as lupus and arthritis.

With this brief overview of the way that markets behave and the current US market for immunoassays, we shall now move on to examine the market for biosensors in each of our chosen categories.

\subsection{Human health care}

The traditional home of analytical chemistry in the health care field has been the centralized clinical laboratory. These laboratories are situated in the larger general hospitals and are charged with the analysis of patient samples from the entire hospital, or even from an entire hospital district. This structure has developed because the complex nature of the tasks to be carried out required the use of a small cadre of highly trained staff in one laboratory. With time, the ever increasing volume of samples led to the application of automated sample handling and dilution equipment. Thus instrument manufacturers have focused on the provision of large clinical chemistry analysers capable of handling hundreds of samples per day. The high cost of these machines has further increased centralization of testing in the clinical laboratory. Indeed, the high cost of the large analysers has become the primary reason why testing is concentrated in one centralized laboratory.

The clinical laboratory market now shows all the characteristics of maturity. In 1971 the market had approximately five competitors, a market for reagents and instruments of approximately $£ 600$ million and a growth rate of $15 \%$ per annum. By 1983 the market had grown to approximately $£ 1.4$ billion, but the number of competitors had grown to 17 and market growth was estimated at between 0 to $4 \%$. Some competitors, such as Eastman Kodak, were actually making substantial losses. Most of the assays on offer use exactly the same technology: typically a UV spectrophometric-based assay with complex automatic sample-handling techniques. Thus patent protection appears to be unimportant. More importantly, the recent analysers are virtually indistinguishable to the consumer; one just pushes a few buttons and gets a computer printout a few seconds later. The market is therefore becoming very price sensitive as consumers make their purchase decisions on price alone.

The potential for new biosensors in this market is very limited indeed. The existing analysers are rapid, easy to use, and accurate. Competition is well entrenched and is highly regarded by the medical profession. Moreover, even 
if new biosensor technologies allowed a reduction in the actual cost of reagents and the sensor element; the realizable reduction in the price of the whole machine would be minimal because most of the system cost is embedded in the sample and data handling functions.

The most promising market for new biosensors lies in areas other than the central clinical laboratory. These new markets have developed primarily because of legislative attempts to reduce the overall cost of supplying health care. The forerunner in the field is the USA, which was spending $10 \%$ of its gross national product on health care by 1983 , twice the current UK level.

During 1984 the USA introduced new methods of reimbursing hospitals so as to give them an incentive to reduce the cost of treating patients. In effect this induced hospitals to reduce the length of patient stay and move therapy to out-patient departments, hospices, and off-site delivery sites where the costs of treatment are considerably less. In many cases these changes require the development of new low-cost methods for diagnosis, for monitoring of patient condition, and for therapy. In short, these changes demand biosensors that can be employed in low technology environments outside the clinical chemistry laboratory.

The requirements for these devices are very different from the large clinical chemistry analysers. The medical profession will have to buy large numbers of them so that they can be situated in each ward, out patient department, or physician's office. They must therefore be low cost. They will be used in an unsophisticated environment and must therefore be easy to use and require minimal sample pretreatment. At the same time they must retain acceptable accuracy and precision. A comparison of instruments developed to measure blood glucose in the clinical chemistry laboratory and the 'off site' market serves to illustrate these points. Details of the two instruments are given in Table 37.4. On one side there is the Yellow Spring's Instrument, an amperometric system for measuring glucose that relies upon the oxidation of glucose according to:

$$
\text { Glucose }+\mathrm{O}_{2} \stackrel{\text { Glucose oxidase }}{\longrightarrow} \mathrm{H}_{2} \mathrm{O}_{2}+\text { Gluconolactone }
$$

$\mathrm{H}_{2} \mathrm{O}_{2}$ is then electrochemically reduced at a platinum electrode. The system is

Table 37.4 Clinical chemistry instrument versus off-site instrument

\begin{tabular}{lll}
\hline & Off site & Clinical chemistry instrument \\
\hline & Glucometer & Yellow springs instrument \\
Cost & $\$ 150$ & $\$ 6000$ \\
Weight & $1 \mathrm{~kg}$ & $30 \mathrm{~kg}$ \\
Sample & $50 \mu \mathrm{l}$ whole blood & $20 \mu \mathrm{l}$ plasma \\
\hline
\end{tabular}


commonly used in a centralized laboratory to measure emergency glucose samples.

In contrast the Glucometer is a small hand held instrument that uses a small disposable element to quantitate the amount of glucose present, according to:

$$
\begin{aligned}
& \text { Glucose }+\mathrm{O}_{2} \stackrel{\text { Glucose oxidase }}{\longrightarrow} \mathrm{H}_{2} \mathrm{O}_{2}+\text { Gluconolactone } \\
& \mathrm{H}_{2} \mathrm{O}_{2} \stackrel{\text { Horse-radish peroxidase }}{\longrightarrow} \mathrm{H}_{2} \mathrm{O}+\text { Oxidized } \\
& \text { chromogen }
\end{aligned}
$$

The oxidized chromogen then produces a colour change that is used to quantitate the amount of glucose present. Note the difference in cost, weight, and degree of sample pretreatment.

The 'off site' testing market displays all the characteristics of a market in the early growth phase. Sales are smaller than the clinical chemistry market, but are growing rapidly. Patent protection and proprietary technology appear to be important. Indeed, there are many technologies still under development and many specific opportunities for small innovative companies to fill.

\subsection{Veterinary health care}

Veterinary health care is concerned with the diagnosis, therapy, and treatment of animals used for commercial enterprises and as pets. The whole market is valued in the range of \$5-\$6 billion per annum in the USA, but this includes antibiotics, vaccines, vitamins, general veterinary services, feed additives such as amino acids, growth stimulants and hormones, and steroids. It is certain that diagnostic tests only comprise a very small proportion of the total - probably in the range $\$ 100-\$ 200$ million per annum. Thus the existing market is very small in comparison to human health care.

There clearly are a number of possible opportunities for biosensor type products in veterinary health care, particularly for those devices that are robust, easy to use, and amenable to a low technology environment. However many of the potential users are totally unfamiliar with diagnostic testing procedures. Furthermore, the unsanitary conditions of a farm or slaughterhouse compare very unfavourably to those of a domestic household or an out-patients department. Thus new devices will have to be particularly user friendly and robust. In addition, the considerable costs associated with consumer education, the establishment of new distribution channels, service and product support, and meeting the regulatory requirements must not be underestimated. The prognosis, therefore, is rather less favourable than 
some of the opportunities in the human health care sector. Indeed, the prohibitive costs of developing the market argues against commercial enterprises aiming for these applications in isolation. Rather, one will see companies developing products for human health care applications and then modifying them slightly for veterinary use. This model of development is already happening. For example the Ames division of Miles Laboratories Inc. of Ekhart, Indiana, now sells some of its human diagnostic tests to the veterinary market.

\subsection{Fermentation and process control}

Once again the ambit of the fermentation and process control market extends far beyond the space available to the writer. However, it is clear to say that there are a number of potential applications for biosensors in this area. These include the monitoring and control of industrial pollutants such as methane, carbon dioxide, carbon monoxide; the monitoring of microbial contamination in enclosed processes (e.g. heating and ventilation systems, water distribution networks); and the control of standard fermentation processes by monitoring substrate levels, microbial biomass or substrate formation. The biological sensing element of a biosensor may, furthermore, lend a biosensor device particular advantages over competing technologies. For example, enzymes or immunological components of fer a unique degree of specificity coupled with an ability to select a target molecule in a highly complex soup of other molecules. Biosensors may also of fer a high degree of sensitivity. On the other hand, they are highly sensitive to temperature, $\mathrm{pH}$, and osmotic pressure and deteriorate relatively rapidly with time.

Industrial processes may also have particular characteristics that make them singularly inappropriate for biosensors. For example, many processes require sensors at remote parts of the system where it is difficult to obtain access to replace delicate biological sensor elements at regular intervals. Many food processing applications demand a steam sterilization procedure to prevent microbial contamination. Clearly this is incompatible with a biosensor, unless one has a separate bleed off from the main process to a remote testing site. My own experiences tell me that individuals in the fermentation would be loath to compromise their well-established steam sterilization procedures with something as innocuous as a small line leading to a biosensor!

\subsection{Conclusions}

As you may have gathered the commercial potential of biosensors varies greatly from one application to another. The commercial future prophesied by many can be compromised by a whole range of unforeseen problems including product recalls, entrenched competition, lack of demand, technical 
difficulties, legislative or patent problems, poor marketing or distribution, and poor product design. The route to successful commercialization of a biosensor will require thorough market analysis, a good understanding of potential benefits, sound financial backing, comprehensive marketing and distribution, and tenacity. Provided that appropriate commercial decisions are made, the potential of these devices is very great indeed. However, the commercialization of biosensors is not a field for one who subscribes to the old Cromwellian dictum of he who goes furthest knows not whither he goes! 


\section{Index}

accumulation 485

accuracy 731

acetaldehyde 267

acetic acid 16-20, 23, 26, 28, 295

acetic acid sensor $16 \mathrm{ff}$

acetone breath detectors 730

acetone-treated E. Coli 23

acetylation 122

acetylcholine 581

ENFET 510

acetylcholinesterase 512

inhibitors 592

acid phosphatase 51,82

$\alpha$-acid glycoprotein 174

acidosis 324

Acinetobacter calcoaceticus 109

acoustic $v, 3$

impedance $549 \mathrm{ff}$

interferometry 560

microscopy 563 .

resonance densitometry $565 \mathrm{ff}$

wave propagation $559 \mathrm{ff}$

acoustical techniques $551 \mathrm{ff}$

acridinium esters 633

acridinium salts 625,626

active oxalate series 626

acupuncture 445

adenine 47

adenosine $31,37-41,47,545$

base nucleotide 37

deaminase $38,39,41,47,66,91,124$. 545

diphosphate (ADP) 38, 41

electrode 38

monophosphate (AMP) 31, 38-41, 44, 47

deaminase $39,40,43$

enzyme electrode $42 \mathrm{ff}$

sensor $42 \mathrm{ff}$

sensor $37 \mathrm{ff}$

triphosphate (ATP) 38, 41, 263, 586, 618, 620

analysis 621

calibration curve 475

detectors 631

sensor 474,475

admittance $428 \mathrm{ff}$

admittance spectroscopy $427 \mathrm{ff}$ adrenal disease 364

adsorbent 609

adsorption $92,211,265,305,667$

of saturated monomers 211

aequorin 622,632

aerobes 293

Aeromonas formicans 21

affinity constant $60,648,651$

affinity sensor 402

affinity pairs 641

agarose 90,127

aggregate cell populations 563

aggregation 690

AIDS virus 727

Airy pattern 683,684

alanine $34,201,541,726$

aminotransferase 327,340

dehydrogenase 541

albumin 586, 669

as interferent 474

Alcaligenes eutrophus 295

alcohol 9, 48, 141, 255

as a carbon source 18

dehydrogenase $121,125,267,328$

oxidase $90,91,329,331,586,587$

sensor 18ff, 292, 329

alcoholism 341

aldehyde vapours 541

alginate 87,305

algorithm 421

alkaline phosphatase $39-41,63,82,333$. 338,608

alternating current (AC)

ring disc technique 241

techniques 428

voltammetry 228

alumina 88

amines 27,542

amino acid 15, 19, 23, 27, 14I, 148, 150 , 725,730

deaminase 533

oxidase 332

sensor 729

D-amino acid 34,145

oxidase $34,201,202,204,227$

L-amino acid $48,135,144,255$

oxidase $148,201,202,205,227,253$ 
$\alpha$-aminobutyric acid (GABA) 219

6-[N-(4-aminobutyl)-N-ethyl] amino 2,3 dihydro-1, 4-phthalazine-1, 4-dione (ABEI) 628

$\gamma$-aminopropyltriethoxy silane (ATPS) 668 amino transferase 9

ammonia 26-28, 33, 44, 53, 65, 137, 141, $143,144,150,531,543,727$

in aqueous solutions 541

electrode $62,68,147$

gas sensing probe $30,31,37,38,42,44$. $45,50,54$

gas-sensitive FET 510,512

gas sensitivity 535

-sensitive Ir MOS capacitators 537

sensor $26 \mathrm{ff}, 31,45,53,535,557$

ammonia- $\mathrm{N}$ in blood 542

amniocentesis 79

amniotic fluid 79

amperometric, v, 26, 47, 48, 61

detector, flowthrough 174

enzyme-linked immunoassay $61,63 \mathrm{ff}$

hydrogen peroxide electrode 7

immunoassay $61 \mathrm{ff}$

sensors 165ff, 258, 267

ampicillin 540

susceptibility 539

amplification 581

amplifier 41

amygdalin 141, 145

amylase, $9,339,340,726,727,731$

amyloidosis 734

anaerobic conditions 23

anaerobes 293

anaesthetics 612

analytical performance, microprocessors for the evaluation of $705 \mathrm{ff}$

angler-fish 617

angular light scattering 683,684

animal husbandry vi

animal tissue $30 \mathrm{ff}$

animal tissue electrodes 54

animals 9

anion membrane 261

anistropy 555

anode 4,5

anodic generation of oxygen 10

anodic pulse stripping voltammetry 65

anomolous diffraction approximation 683

antenatal diagnosis 71

antithrombin-3 deficiency 80

antibiotic assay 620

antibiotics 23,291

antibody $v, 11,60,65,337,515,517,518$, $582,627-9,641,646,652,657,659$. $669-74,693,727$

-coated silicon 691 goat antihuman $\lg$ G 559

immobilization 667,668

antibody-antigen reactions 648

antibodies industry 591

antigens $11,60,517,518$

bacterial 60

labelled with electroactive species 64

antitrypsin 80

deficiency 78

apolipoproteins 80

application limits 384

apyrase 586

aquatic plants 56

aqueous humour of eye 367,403

arginine $30,34,47$

electrode 30,31

argument ('direction') 430

arterial oxygen tension 356

arthritis 742

Arthrobacter simplex 88

artificial beta-cell 404

artificial endocrine pancreas 322,327 , $390 f f, 398$

artificial pancreas 724,725

ascorbic acid $5,7,86,127,149,261,309$, $317,329,399,586,708$

ascorbic acid oxidase $90,584,586$

asparaginase $55,66,90,144,544$

asparagine $47,144,545$

determination 544

aspartase 545

aspartate $9,34,545$

Aspergillus 87

assimilable sugar sensor $13 \mathrm{ff}$

assimilable sugars 28

attenuated total reflection (ATR) 656, 660, $661,662,666,669,674$

auto-analysers 22,23

autocorrelation 452

automated enzyme electrode 707, 708, 712, 713

automated glucose electrode evaluation 716

automated sample handling 742

automated TELISA 583

automation 705,718

automatic calibration 707

autopower spectrum 452

autoradiography 75-7

avidin 82

azurin 254,270

back-scattered light 648

bacterial 37

activity 295 
antigens 60

bioluminescence 621

biosensors 30

collagen 16

contamination 34

electrode 36

infection 620,631

luciferase 632

luminescence 620

bacteriophages 103

bacteriuria 683

banana 31,51

band-gap 482,483

energy 482

barium titanate 553

basal insulin supplementation 421

battery 308

bedside-type artificial endocrine pancreas 414

benzoquinone $90,401,584$

1,2-benzophenoxazine-7-one 214

benzyl viologen 297

betaine aldehyde 201, 203

bienzyme sensors 333,340

bienzyme glucose electrodes 271

bifunctional reagents 91,95

bilirubin 86,650

binding constant $644,649,651,652$

binding protein 641

binding sites 641

biochemical fuel cell 291

biochip 547

biocompatibility 368,397

bioelectrochemistry $395,425 \mathrm{fr}$

biofuel cell $277 \mathrm{ff}, 291,298$

biological oxygen demand (BOD) 25,291 , 295

5-day BOD 25

sensor $25 \mathrm{ff}, 28$

bioluminescence $608 \mathrm{ff}, 617$

bioluminescent organisms 617

biomass determinations 620

biomedicine 3

bioreactors 453

bioreceptors $638 \mathrm{ff}, 641$

biosensor, definition v, $6,617,737$

biostator 725

glucose-controlled insulin infusion system 327,404

Biot number 168

biotin-avidin 629

biotin-binding 168

biotin-dUTP 72,82

bipyridyl 268

4,4'-bipyridyl 170, 237, 241, 254

blocked interface 157

blood $4,5,20,128,261,288,304,325,326$,
$329,333,340,544,733$

cells 692

gases $9,357 \mathrm{ff}, 723$

monitoring 359,361

glucose $10,158,174,317,318,321,322$, $356,367,370,392,404,414,590$, $651,724,725,729,730,731$

in dogs 370

L-lactate 324

off-site market 743

meters 726,728

samples 541

serum 542

transfusion 742

blood ethanol determination 587

bone diseases 333

borate 51

bovine factor VIII 691

bovine liver $30,31,47,48$

bovine serum albumin (BSA) 123, 157

BSA-dansyl chloride 656

Brevibacterium lactofermentum 14, 28

bridge methods 447

brilliant cresyl blue 297

Brillouin scattering 684

bromine titration of proteins 167

bromo-isoalloxazines 126

broth 13

Brownian motion $685,686,688$

bubble size 446,454

bubble size and velocity 446

bubble velocity 454

butanol 587

Butler-Vollmer equation 165

$\beta$-galactosidase 581,589

$\beta$-glucosidase 581,586

calcium 725,727

carbonate $\left(\mathrm{CaCO}_{3}\right) 89$

ion 622

-sensitive ISFETs 507

-sensitive membrane 504, 506

calculator $709,710,711,712$

calibration $354,369,694,710,732,733$

adjustment 398

drift 731

calorimetric sensors $575 \mathrm{ff}$

calorimetry $573 \mathrm{ff}$

cAMP, see cyclic adenosine monophosphate

cane molasses 13

capacitimetry 444

cardiovascular diseases 334

carbodiimide $285,287,306$

carbohydrates 19,303

carbon 89-91

dioxide $9,23,65,137,141,143,144,147$, $601,611,725$ 


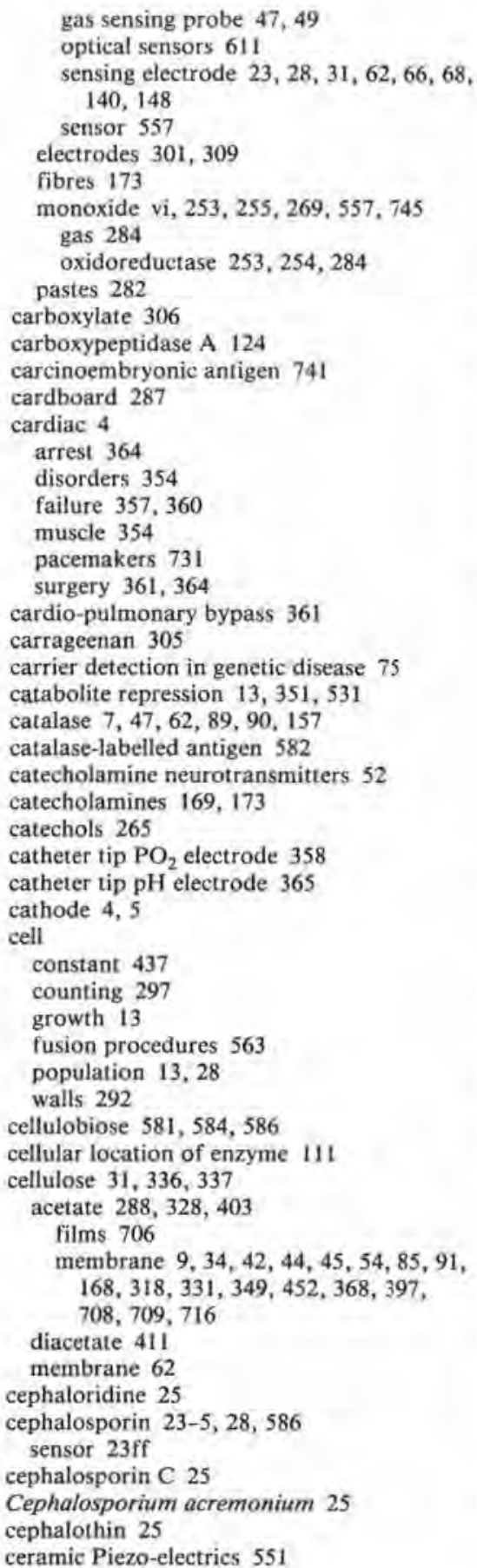

ceramics 287,582

cerebral oxygen deficiency 325

cerebrospinal fluid (CSF) 34, 325

cerebrospinalis 326

chamber-type electrodes $379,386,388 \mathrm{ff}$

channel 492

characterization of unknown cells 445

charge-transfer resistance 518,521

CHEMFET, see chemically sensitive fieideffect transistor

chemical transducers 3

chemiluminescence $608,617 \mathrm{ff}$

mechanisms 626

chemiluminescent immunoassays 627

chemiluminescent probes 60

chemical defence vii

chemical modification of electrodes 213,215

chemical modifications 121

chemical potential 503

chemically sensitive field-effect transistor (CHEMFET) 532, $481 \mathrm{ff}$

chemilithotrophs 293

chick fibroblasts 565

chip $496,498,694$

chloride 668

chloroperoxidase 66

chlorophenol 53

chlorohexidine diacetate 48

cholesterol $9,141,253,334,335,586,727$

esterase 586

esters 586

oxidase $253,334,576,586$

cholesterol metabolism/heart disease 8

choline 253

oxidase $201-3,206,253,336$

and catalase co-immobilized 585

chorionic villus sampling 79-81

chorionic villi 80

chromatographic eluents 446

chromatography 13,18

calorimetric monitoring 588

chromogen 276,744

chronic obstructive lung disease 357

chymotrypsin 91,123

CIDS, see circular intensity differential scattering

circular dichroism 684

circular intensity differential scattering (CIDS) 684, 693

Citrobacter freundii $20-4,28$

cladding 680

clam 624

Clark oxygen electrode $5,6,11,61 \mathrm{ff}, 90$, $173,327,333,357,361,370,396$. 398,403

clinical vi, $4,26,66,257,304,311,317$. $327,339,356 \mathrm{ff}, 409,588,642,737$

analysis $285,291,471,474,582,586$ 
biochemistry 723

chemistry $135,139,587$

analysers 742

market 744

diagnostics $316,339,675$

laboratory market 742

medicine 731

microbiology 684

samples 674

clinician's requirements. $723 \mathrm{fr}$

clogging 591

cloning of human DNA 72, 74

closed loop $393,394,725$

device 395,404

feedback control of insulin 366

glycaemic control 420,421

insulin 404

infusion 391ff, 403

system 370

Clostridium acetobutylicum 539

Clostridium butyricum 291, 295

Clostridium pasteuriamum 170

coelenterates 622,624

co-enzyme 294

attachment 125

recycling 582

regeneration 541

coal mining $2 \mathrm{I}$

codeine 64

cofactor 114

detector 399,400

recycling 332

regeneration 264

Cole-Cole 438

collagen $80,89,92,691$

bacterial 16

disorders 80

films 706, 708, 709

membrane $24,90,397,718$

collection efficiency 238

comb electrodes 456

commercial $287,339,404,684$

development 288

exploitation $737 \mathrm{ff}$

of biosensors $737 \mathrm{ff}$

in vivo glucose sensors 372

potential 745

commercialization $18,19,26,63,150,316$, $721 \mathrm{ff}, 746$

compensation $310,399,590$

method 350

compensated enzyme electrodes $347 \mathrm{ff}$

compensating electrode 708,709

competition for oxygen 398

competitive binding $607,638,646$

competitive inhibitor 51

complex collection efficiency 240

complex permittivity 437 complex plane 431

compound heterozygotes 71

computer algorithm for closed-loop insulin infusion 421

computer control 280,448

computers 738

con A, see concanavalin A

con-A-based biosensor 644

concanavalin A (con A) $65,90,93,371$, $402,607,643,645,648,652,668$

concentration range 586

conductimetric correlation functions $v, 3,6$, 453

conductimetry 444

in biosensors 446

conducting organic metals 266,270

conducting organic salt $171,180,199,204$

conducting organic salt electrodes $194 \mathrm{ff}$

conduction band $482-5$

conductivity $433,437 \mathrm{ff}$

fuctuations 454

congenital heart defects 3,361

conjugate 66

conjunctival lactate 9

conjunctival $\mathrm{PO}_{2}$ sensor 361

construction 369

of mediated amperometric biosensors $276 \mathrm{fr}$

constant drain current operation 500-1

constant gate voltage operation 499

consulting rooms 727

contamination, bacterial 34

continuous monitoring 417

control and measurements circuitry 498

controlled delivery 306

controlled pore glass (CPG) 579,590

controlled release of drugs 219

copy DNA probes 72

corn kernel $31,47,49$

coronary 4,335

thrombosis 726

cortisol 66,741

prednisolone transformation 88

Cottrell equation 229

Coulombic output 302, 303

Coulombic yield 296

coulter counter 444,445

covalent attachment 306

covalent attachment of redox mediators 211

covalent bonding 95,97

covalent coupling 667

covalent linkage 668

cows' milk 589

creatine $34,47,141,147,148,150,328,545$. $586,592,725,726,727,728$

amidohydralase $(\mathrm{CI}) \quad 147,328$

determinations 533,545 
iminohydrolase $531,545,586$

as interference 474,475

kinase $9,77,255,263,620,726$

kinase $\mathrm{MB} 9$

creatininase 533,545

critical angle 657

cross-correlation function 452

cross-linking 91,94

cross-power spectrum 452

cross-reactivity 62

cross-talk 381

cucumber 31,52

Cucumis saturis 53

culture broths 13,18

culture growth monitor 566

culture media 20,444

cuticle 53

cyanide $141,145,592$

cyanuric chloride 265

cyanuric chloride to couple redox groups 212

cyclic adenosine monophosphate (cAMP) $4 !$

cyclic hydrazides 627

cyclic voltammetry $247 \mathrm{ff}, 268,277,300$, 479,521

cyclic voltammogram $175,220,221,226$, 247,248

Cypridina 624

Cypridina hilgendorfii 618,622

cysteine 53

biosensor 53

L-cysteine 53

desulphhydrolase 53

cystic fibrosis 71

cytochrome $b_{2}$ (L-lactate ferricytochrome c oxidoreductase) 270

cytochrome c 20,123, 127, 169, 170, 237, $241,267,270,271$

oxidase 268

cytochrome c (551) 254

cytochrome oxidase 254

cytochromes 292

cytoplasm 292

cytoplasmic enzymes 307

daily glycaemic excursion 420

Damkoehler number 172

data handling 743

data treatment 718

data treatment programs 716

data treatment unit 714

deamination 39,40

Debye and Sears 560

deep sea fish 617

dehydrogenase $3,9,63,213,262,264,303$

depletion 486 deletion of DNA sequence 76

deoxyribonucleic acid (DNA) 71, 113 , $115-17,441,684,691$

blotting 72

cDNA probes 76,103

deletions 76

DNAase-1 73

fragments 75,77

polymorphisms 75,78

probes $71 \mathrm{ff}, 82$

sequencing 107,114

depolarization 647

depth of penetration 658,659

derivative action 421

design of bioactive layers 304

design of microbial sensors 303

desorption 305

detection limits 674

detergents 333

dextrans $124,643,644,645,648,651,652$, 691

diabetes vi, 317, 651, 728, 729,

diabetic $255,322,327,365,367,724$

diabetic ketoacidosis 333,724

diabetic patients $409,726,727,730$

dialysis fibre 643,644

dialysis membrane $32,36,44,49,52,53,90$, $142,283,591$

dialysis tube 647

diaphorase 336

diatomaceous earth 88

cis-dibenzo, 18-crown-6

dichlorophenol indophenol (DCPIP) 326

2,4-dichlorophenol 53

didodeclyamine 557

dielectric

constant 437

dispersion $437 \mathrm{ff}$

loss 437

relaxation time 438

spectroscopy $441 \mathrm{ff}$

spectrum 455

diethylenetriaminepentaacetic (DTPA) 6

differential electrode 175

differential measurements 310

differential pulse polarography $9,224,226$, 227

differential sensing 292

diffraction light scattering 684

diffusion 396

across membrane 280

barrier $94,158,168,318$

coefficient $215,511,711$

of oxygen 237

constants 511

controlled 165, 166, 283, 387

of glucose 348,648

layer 232 
limited currents 298

limiting 310

diffusion/mass transfer I66ff

diffusional limitations 300

diffusional transport 222

digoxin 68,741

dihyrofolate 120

reductase 122

dihydronicotinamide oxidations 126

dihydroxyacetone 10

dihydroxyphenylalanine 53

dilution 591

dimethylferrocene 255,370

1,1'-dimethylferrocene $261,284,285,320$

dimethylsuberimidate 124

dimethyl-trimethylferrocene methodiate 282

dinitrophenol 670

dioctadecylamine 557

dioxetans 627

Diplocardia longa 623

dipole moments 442

direct-current cyclic voltammetry 248

direct electron transfer $171,199,271,315$

direct oxidation or reduction 211

direct potential difference 65

disaccharide 307

dise transient 240

disposable cloth-type electrode 363

disposable fibre-optic 675

disposable optical fibre 674

dissipation factor 440

dissociation 608

rate 648

disulphides 169

dithiol oxidations 126

DNA, see deoxyribonucleic acid

doctor's office 724

dopamine $31,51,52,219$

sensor 5Iff

Doppler frequency 689

double-layer capacitance 227, 382

double-layer charging current 221

double-layer theory 515

downstream analysis 592

downstream processing 694

drain $489,490,492,496,499,500,508,522$ current $490,492,493,494,500,501,516$, 523,526

voltage 492

drift 368

base line 412

drinks 323

dropping mercury electrode $4,5,173$, 224

drugs 60

dry chemistry systems 276

Duchenne muscular dystrophy $76,78,80$

durohydroquinone oxidase 127 dwarfism 80

dynamic light scattering 685,690 ff

dynamic response $709,711,716$

E. coli, see Escherichia colt

ENFET, see, enzyme-based field-effect transistor

effluent 589,590

systems 161

electric field correlation function 688

electric field light scattering 682

electrical admittance spectroscopy $427 \mathrm{ff}$

electrical circuit analysis 433

electrical impedance $425 \mathrm{ff}$

electroactive proteins 169

electroactive species $13,62,64$

electrocatalytic glucose sensor 402

electrochemical

detection 61

immunoassay 68,338

mechanism 199

modulation 310

oxidation of NADH 63

polarization 212,23

potential 502,507

rate constants 198

rate equation 300

sensor 257

uric acid oxidation 329

electrochemically coupled enzymatic reactions $250 \mathrm{fr}$

electrochemistry 3

geometry 173

kinetics 198

materials 301

potentials of mediators 300

preconditioning 175

pretreatment 173

stability 199

surface chemistry 156

electrode-electrolyte impedance 382,383 , 384

electrode-electrolyte interface 381,434

electrogravimetric sensor 557

electron transfer $257 \mathrm{ff}$

electroless gold-plating 287

electrolysis 4, 384

current 350

of water 349

electromagnetic density 662

electron bridging 308

electron mobility 492,493

transduction 29

transfer 3

transition complexes 87,92

tunnelling 308

electrons 85 
electrophoresis 75

ellipsometry 662

emphysema 80

encapsulant 497, 498, 507, 508

encapsulation 397,528

end labelling 74

endogeneous ammonia- $\mathrm{N} 545$

energy band diagrams $482,485,486$, 493

energy production 13

Enterobacteriaceae susceptibility to ampicillin 539

entrapment $87,91,93$

environmental analysis 591

environmental monitoring 445

environmental control 582

enzymatic membranes 706

enzyme

activities 339

activity determination 587

calorimetry 575

components $v$

costs 22

electrodes v, 3ff, $11,12,36,135,140$. $141,143,146,149,180,705$

immobilization 92, 632

immunoassays (EIA) 60

market shares 741

-linked immunoassays 60,63

purification process 588

recycling 9

stability 294

thermistors $575 \mathrm{ff}$

transistor 531

enzyme-based amperometric biosensors $315 \mathrm{ff}$

enzyme-based CHEMFET 510

enzyme-based field-effect transistor (ENFET) 472-474

ENFET theory s10ff

enzymes $\mathrm{y}, 60$

epidermal layer 53

equilibrium 60,600

equivalent electrical circuit 431

ergodic systems 450

Erwinia carotovora 295

erythrocyte deformation 683

erythrocytes 693

Escherichia coli 20, 28, 106, 295, 539, 692

ampicillin susceptibility 539

B/r 297

K12 processes

ML-308 295

phage M13 108

esterases 3,581

ethanol 18-20, 27, 28, 205, 206, 267, 295, $302,331,541,586,587$

\section{dehydrogenase 204 \\ sensor 304}

ethanol-methanol fuel cell 303

ethylamine 543

ethylenediamine 122,123

eukaryotic cells 307

Euperget C 543

evanescent wave $641,645,657-62,664,669$, 670,674

ex vivo 362

exploitation of biosensors $737 \mathrm{ff}$

externally buffered enzyme electrode 352

externally buffered glucose electrode $\mathbf{3 5 2}$

extract of Methylosinus trichosporium OB3b 281

extrinsic sensors 680

fabrication of a CHEMFET 496

factor VII 80

factor VIII-related antigen 338

factor IX 80

FAD, see flavin adenine dinucleotide

false results 734

Faradaic current 248

Faradaic impedance 434

farm 744

feedback-controlled insulin administration 394

feedback-controlled systems 404

fermentation $3,11,13,18,22,291,329$, $331,333,474,478,540$

analysis $582,586,589,591$

broth 14-19, 23, 531, 590

control $311,316,322,323,332,339,347$. $350,738,745$

monitoring vi, $161,557,592,694$

process control 350

technology 453

fermenters $351,354,446,453,454$

Fermi level $484-7$

ferredoxin 20, 169, 170

ferricyanide $9,180,260,322,326,329$

method 13

K-ferricyanide 90

ferritin 741

ferrocene $9,64,85,87,91,180,249,254$, $255,260,261,282,298,310,322,40 \mathrm{I}$

derivatives 253

-mediated glucose sensor 370

modified electrode 261

monocarboxylic acid 249

morphine conjugate 64

fertilizers 333

FET, see, field-effect transistor

fetal blood 71

fetal DNA 79

$\alpha$-fetoprotein 628 
fibre-optic $364,599,631$

biosensors $638 \mathrm{ff}$

glucose sensor 371

glucose sensor in dogs 371

light guides 360

oximeter 360,364

spectrometer 403

fibrin deposition 417

Fick's first law 166

Fick's second law 228

field-effect transistor (FET) 531ff

FET-based sensors 471ff

FET chemical sensors, theory of $481 \mathrm{ff}$

fireflies $617,624,631,633$

firefly bioluminescence 618

-luciferase in ATP analysis 620

-luciferase stability 632

-luciferin 625

FITC

as label 671

-BSA 656

-labelled antibody 670

-labelled 672

flat band 489

flat band condition 488

flavin 271, 641

adenine nucleotide (FAD) $34,90,401$

mononucleotide 325

mononucleotide oxidoreductase 90

prosthetic group 200

flavins 169,227

flavoprotein electrochemistry 227

flavoproteins 169,199

flayopapain 126

flow cell $664,665,670$

flow cytometry 683

flow-through cell 716

flow-through glucose electrode 715

flow-through glucose sensor 718

now-through sensors 709

fluctuations of oxygen 398

fluid balance centres 725,726

fluid dynamics 453

fluorescein $602,611,614,629,644,675$, 646

fluorescein-labelled dextran 402, 607

fluorescence $602-4,607,610,611,613,618$, $626,627,639,640,642,645,649$, $650,656,660,661,664,670,672$, 693,740

depolarization 648

energy transfer 607-646

quenching $606,612,646$

yield 625

fluorescent

acceptor 629

antibody 82

complexes 613 dextran 644

emission 645

labels 641

probes 60

fluorescently labelled dextran 607,643

fluoride 51

electrodes 65

fluoro-immunoassays 641

fluorophor $604,612.613$

folate 741

food $22,311,316,317,323,329,337,557$

analysis 480

industry vi, 13

processing 745

quality 445

seasoning 478

foods 295

forced convention electrodes 232

forensic science 692

formaldehyde 282

formate 291

dehydrogenase 20

formic acid 17, 19-21, 28, 291, 295 sensor $20 \mathrm{ff}$

fouling $257,325,354,403,531,566$

of the electrode 265

protein 417

Fourier series 450

Fourier transform 450, 452, 558

Fraunhofer approximation 683,684

Fraunhofer diffraction 685

free-energy diagram 183, 184, 189

freeze-drying 288

frequency 427

frequency-domain methods 448

frequency-response analysis (FRA) $\mathbf{4 2 8}$

freshness testing 445

$\beta$-fructofuranosidase/mutarotase 90

fructose $9,10,14,15$

fruits 31

fuel 21

fuel cells $20,28,291 \mathrm{ff}, 300$

functionalized organisms 307

fungi 9

galactose $9,10,15,255,323,584,586$

oxidase $323,584,586$

D-galactose oxidase 90

B-galactosidase 323

$\beta$-D-galactosidase 90

gas bubbles 562

gas chromatography 21,22

GASFET, see gas-sensitive field effect transistor

gas-permeable Teflon membrane 536

gas-sensing electrodes 53

gas-sensitive field-effect transistor $522 \mathrm{ff}$ 
gastric juices 20

gastrointestinal disease 364

gate $492,494,495,498,502,507-9,522-5$

voltage $492,493,498-501,526$

gated fluorimeters 646

gel 305

filtration 588

formation 691

gelatín 87,89

gene probe $7,75,76$

generation of electricity 295

genetic disorders $71 \mathrm{ff}$

genetic engineering vi, 100ff, 115

genetic engineering of whole organisms 110

genome 693

gentamicin $586,642,741$

geophysical prospecting 445

germanium-liquid interface 669

glass 90

glass electrode $24,26,135,146$

$\alpha$-globin $73,76,80$

$\beta$-globin $71,73,80,81$

globin gene probe 76

glow-worms 617

glucagon infusion 421

glucamylase $87,88,337,340$

glucokinase 403

Glucometer 744

gluconate 8

gluconic acid 6

gluconolactone 8

glucose $48,51,63,135,144,253,265,270$, $272,277,283,285,292-6,300,302$, $309,317,321,322,324,359-2,365$, $512,583,584,586,607,621,644$, $652,707,708,715,723,724,726$, 727,744

analysers $318,319,592$

binding to con A 648

biosensor based on separation principle 643

in blood 644

controlled insulin infusion 726

controlled insulin infusion systems 725

dehydrogenase 109, 322

electrochemical sensors 398

electrode $149,158,180,261,271,283$, $348,353,398,705,709,711,717$

enzyme electrode 286

enzyme electrode in dogs 369

enzyme electrode in blood samples 475

infusion 327

as interference 474,475

oxidase (GOD) $6,8,11,31,51,63,89$, $90,91,109,115,140,144,149,155$. $157,159,171,175,180,194,199$. $227,240,253,258,259,261,263$,
$264,270-72,276-80,282,284,285$,

$287,293,317,318,319,322,337$.

$348,349,350,352,370,394,395$.

$401,409,512,576,581,586,705$,

$709,724,744$

cellulose acetate membrane 715

co-immobilized with catalase 584

collagen membrane 710

I, I'-dimethyferrocene 283

immobilization 476

injected 367

membrane $397,708,716$

platinum electrode 710

with catalase 584

oxidase-catalase 587

thermistor 589

-sensitive ENFET 510

sensors $317,405,409,608,648,649,651$, $706,707,716,718,729,730$

alternative approaches 402

based on a microelectrode 475

based on non-enzymatic approaches 402

in rhesus monkeys and rabbits 369

standards 710,718

for stimulation of insulin relcase 395

in subcutaneous tissue 633

transport 349

in whole blood 633

glucose-6-phosphate 51

dehydrogenase 582

deficiency 80

glucose isomerase 403

glucosidase 145

glutamate $25,31,47,48,219,352,480$

biosensor 48

decarboxylase $23,48,144,148$

oxidase 478

immobilized 479

sensor based on micro $\mathrm{O}_{2}$ electrode

t-glutamate

acid 478

decarboxylase 332

dehydrogenase 544

column 545

oxidase 341

glutamic acid $15,18,22,23,28,141,144$

sensor $22 \mathrm{ff}$

L-glutamic acid 478

glutaminase $31,33,35,36,54,56,144,148$

glutamine $22,23,31-7,47,54,141,148$

sensor $31 \mathrm{ff}, 34-6,54,56$

L-glutamine 144

glutathione 317

reductase $253,254,262,282$

glycan 307

glycerol

dehydrogenase 336 
kinase 336

oxidase 336

glycerophosphate $39-41$

oxidase $\mathbf{3 3 6}$

glycine 34,47

glycollate 253,255

oxidase 253

glycolysis 318

gold 157

goniometric techniques 683

Gram-negative bacteria 445

Gram-positive bacteria 445

Gram-positive and-negative organisms 294

gramicidin 691

graphite $89,157,159$

graphite foil 284

graphite paste enzyme sensor 283

gravimetric biosensors 556

gravimetric immunosensors 559

growth hormones 741

growth of a yeast 561

growth rate of micro-organisms 558

guanase 46

guanine $31,41,45,47$

biosensor $44 \mathrm{ff}$

guanosine 41

phosphorylase 47

$\mathrm{H}_{2} \mathrm{O}_{2}$, see hydrogen peroxide

haematology disorders 329

haemoglobin $8,669,670$

haemoglobinopathies $71,74,75,79,80$

haemophilia 78

haemophilia A 80

halide sensors 614

Hanes plots 192, 196

Hansenula anomala 295

hard copy 715,717

heart disease 339,620

heart-lung machine 362

heat production 539

by $E$, coli 540

heavy metal chelates 645

heavy metal 591

hepatitis 339,341

hepatitis B 11,93

antigen 727

surface antigen 174

herbicides vi

heterogeneous electron transfer $165,169 \mathrm{ff}$

heterogeneous membranes 505, 506

heterogeneous immunoassay 66

hexacyanoferrate $9,180,260,322,326,329$

hexakinase 318

hexamethylenediamine/glutaraldehyde 87

hexanucleotide priming. 74

hexokinase $263,276,576,583,586$ high-pressure liquid chromatography 25 , 167

high sugar 13

histamine 125

t-histidine 34

histologic changes 417

hollow dialysis fibre 648,651

hollow fibre 646

homoarginine 123

homochromatography 74

homogeneous electrochemical immunoassays 64

homogeneous energy transfer 631

homogeneous immunoassay 628,657

homogeneous techniques 646

homozygotes 71

hormones 60,583

hospital wards 726,743

host response 411

HPLC, see high-pressure liquid chromatography

HTLV-III (AIDS) virus 727

human blood 66 chorionic gonadotrophin 628,641

diabetes 415

gene library 73

genetic diseases $71,75 \mathrm{ff}$

growth organism 80

health care $738,742 \mathrm{ff}$

immunoglobin $G$ (IgG), 66, 671, 672

serum orosomucoíd 64,65

volunteers 414

Huntington's chorea 80

hybridization 117

hybrid sensor $50,51,333$

hybrid urea sensor $50,51,333$

hydrodynamics 396,711

hydrogen 20, 21, 23, 119, 291

biologically generated 277

evolution 539

gas determination 538

ion concentration 365

leak detector 538

monitoring 540

peroxide $5-8,31,47 \mathrm{ff}, 159,240,258,269$. $270,328,329,330,403,410,586$, $632,703,743$

detection 247,399

electrode 147

microelectrode 475,478

potentiometric detection 400

sensitive palladium gate IGFET 522

hydrogen-detecting PdMOS 533

hydrogen-sulphide-sensitivity of PdMOS sensors 547

hydrogenase $20,531,533,538,540$

hydrolases 127

hydrophobicity 305 
hydroquinones 265

2-hydroxyl-1, 4-naphthoquinone 298, 302

B-hydroxyl-1,3,6,-pyrentrisulfonic acid HPTS 610

hyperinsulinaemia 394

hyperlipidaemia 335

hypervitaminosis 333

hypoglycaemia $9,728,729$

alarm 366

hypoxaemia 359

hypoxanthine 9

identifying a protein 457

identifying cells by dielectric properties 444 immobilization vi, 3, 13, 18, 26, 31, 38, 49, $85 \mathrm{ff}, 114,127,668$

of enzyme 285,287

of enzyme co-factor 157

of enzyme and mediator 157

of glucose oxidase 411

of microorganisms 293, 305

of mouse small-intestinal cells 37

procedure 403

immobilized 14, 15, 20, 21, 48, 150

glucose oxidase 264

in alginate beads 539

concanavalin A 651

on CPG 540

E. coli 23

enzyme flow-enthalpimetric analyser 577

enzymes $140,141,227,240$

haemoglobin 613

hydrogenase 538

indicator 609

intact cells 592

mediators $171,185,213,247$

methane oxidizing bacteria 21

microorganisms $19,23,24,28,111$

riboflavin 401

tissue

urease 583

yeast 17

immune reaction 397

immune complex disease 739

immunoassay $60,93,338,604,627,628$. $641,646,655 f f, 641,664,665,667$. $669,673-5,691,694$

electrical 174

kits 647

market 741,742

sales 740

immunobiosensors 87

immunochemical analysis 582

immunochemically sensitive FET

(IMFET) $513 \mathrm{ff}$

immunochemicals 528 immunoclectrode 513,514

immunoenzymometric assays (IEMA) 60

immunoglobin $\mathrm{G}(\operatorname{IgG}) 66,93,174,39,659$, 671,673

immunoglobins 93

immunological activity 668

immunological analysis 586

immunoradiometric (IRMA) 60

immunosensors 11, 93, 337, 559

impedance $301,428 \mathrm{ff}$

bridge 447

diagrams 431

in electrical systems $433 \mathrm{ff}$

plethysmography 445

value 383

impedimetric systems 446

impedimetry 444

implantable 356,366

sensors vi, 4, 322, 475

implantable glucose sensors $391,394,401$, 403

implantable glucose oxidase sensor 395

implantable closed-loop artificial $\beta$-cell 409

improved yield of enzyme 109

improvement of enzyme properties 110

in-line detection $660,661,671$

in-line fluorescence 660

in-line geometry 672

in-line oxygen monitoring systems 363

in situ electron spin resonance 231

in situ fermentation biosensor 11

in situ separation 657

in vivo $8,161,285,326,327,329,331,531$, $674,680,694$

assays 718

blood glucose monitoring 734

calibration 175

characteristics of glucose sensor $413 \mathrm{ff}$

clinical use 409

electrochemical analysis $377 \mathrm{ff}$

ferrocene-mediated glucose sensor 370

glucose electrode characteristics 367

glucose sensors $390 \mathrm{ff}$

design 401

in humans 370

in humans and/or animals 369

lifetime 398

measurements 600

monitoring vi, $173,410,546,589,732$, 733

oxygen sensors 357

pH 365

potassium ion sensor 364

response time 402

tests using dogs 401

indicating electrode 155

indicator 605

indium $\left(\operatorname{In} 3^{+}\right) 64$ 
indium label 64

indole propionic acid 124

industrial waste water 26

industrial wastes 277

indwelling needle 414

infarction 9

infection 735

infusion pump 356

infusion systems 390

inhibitors 47,51

initial rate measurement 45

inosine 47

insects 56

insertional inactivation 103

insoluble mediators 282

instability 46

instrumentation for optical sensors 602

insulin $60,390,586,590,728$

aggregation 392

-dependent diabetes $366,391,393,419$, 421,422

delivery $161,404,729$

dosage 731

infusion $322,327,367,394,739,734$ pump 725

pumps 4

secretion in response to glucose 396

therapy $392,393,724$

insulated gate field-effect transistor (IG FET) 481, 482, 489ff, 522-5

intact bacterial cells 36

integrated circuit technology 476

integrated circuits 378,381

integrated opto-electronic chips 642

intensity auto-correlation function 688,690

intensity fluctuation spectroscopy 686

intensive care unit $724,725,726$

interface $257,434,706-8,713$

interference $38,41,46,48,49,51,64,114$, $149,317,324,327,329,331,612$, $633,645,657,661,674,707$

by chemicals $38,41,46,48,49,51,64$. $114,149,255,261,309,310,317$. $324,399,531,633,645,650,657$, $661,707,733$

effects 681

by metal ions 26

from oxygen 301

interfering 310

compounds 531

substances. $399,650,733$

internal reflection element (IRE) 670

intestinal mucosal cells 41

intracellular ATP-level 539

intracellular glucose 10

intracorporeal glucose sensor 409

intramolecular protein motions 454

intramolecular relaxations 456 intravenous glucose tolerance tests 716,717

intravascular 3

intrinsic level 484,487

intrinsic sensors 680

introns 103

inulase 87

invasive 9

inverse Fourier transform 452

inversion larger $487,490,498,516,517,525$ n-lype 490

invertase $323,584,586$

iodide $65,141,144$

selective electrode 148

sensor 149

iodoacetamide 34

ion gate field-effect transition (IGFET) 482

ion gates 219,220

ion-selective electrode (ISE) $62,65,66$, $135-40,153,364,387,446,481,501$, $503,504,506,510,513,514,527$

ion-selective membranes $501-5,507,50$, $510-13,520,527$

ion-sensitive field-effect transistor (ISFET) $364,471,472,481,501-6,510-13$, 527,732

theory 502

ionic strength 305,611

ionophore 66

IRE, see internal reflection element

IrMOSFET 538

IrMOS 538

IrMOS capacitator 537,545

for amines 543

ISE, see ion-selective electrode

ISFET, see ion-sensitive field-effect transistor

islets of Langerhans 366,728

isocitrate 332,333

dehydrogenase $254,332,333$

Jack bean meal 31,50

Japan $13,18,19,25,26,311,319,320$, $321,326,327,329,334,336,339$

Japanese 62,318

jaundice diseases 341

Johnson noise 384

junction potential 155

jugular vein, dogs 414

$\beta$-ketoapidic acid 66

ketoacidosis 367

ketone body $725,726,734$

levels 734

sensors 729

Ketschmann prism 661 
kidney cells 56 ammonia probe 34

kidney function $146,147,327,471$

kinetics immobilized enzyme 172

Klenow fragment enzyme 74

Khuyveromyces marxiamus 87

La Roche 339

labelled antibodies 60

labelling of probes 73

$\alpha$-lactalbumin 123

$\beta$-lactam, antibiotics 119

B-lactamase 119

lactate $3,4,8-10,48,324,325,326,327$. $582,586,725,726,730$

dehydrogenase $9,125,282,325,326$, 582

flavocytochrome 62,254

in fermentation broth 55

2-monooxygenase 585,586

oxidase $8,326,339,582,585$

sensor 729

D-lactate 326

D-L-lactate 295

L-lactate $269,270,339$

analysers 326, 339

dehydrogenase $254,339,576$

monooxygenase 325

oxidase 91,263

lactic acidosis 324,726

Lactobacillus arabinosis 28

Lactobacillus fermenti 28, 295

lactose $295,323,581,584,586$

in whey 589

Langevin function 442

Langmuir isotherms 649

laser light 683

laser light-scattering $679 \mathrm{fr}$

laser Doppler velocimetry (LDV) 686, 689, 695

lasers vi, 602, 639, 679, 680, 684, 686, 689, 691

LDV, see laser Doppler velocimetry

Lesch-Nyhan syndrome 80

leucine 253

leucocytes 692

migration 417

leukaemia 693

life expectancy 413

in vivo 415

lifetime 398

light guide 632

light-emitting diodes 360

light measuring techniques 630

light scattering $680,681 \mathrm{ff}$

techniques $683 \mathrm{ft}$

linear ranges 316 linear response range 352

linear scan 175

linearity $368,395,716,718$

of an impiantable glucose sensor 396 of in vivo response 395

linkage analysis $78-81$

linked DNA polymorphisms 78

lipase 88, 336

lipid analyser 334,336

lipoamide dehydrogenase $253,254,262$, 282

lipophilicity 298

lipoprotein lipase $584,585,586$

liquid chromatography 174

liquid ion-exchange electrodes 137,13

liquid-waveguide interface 664

lithium battery 413

liver diseases 339

liver microsomes 54

LOD, see lactate oxidase

luciferase $90,618-20,622,623,632$

luciferin $618,619,621-3,633$

luciferyl sulphate 622

luminescence 629,630

luminous bacteria 620

luminol $625,627,628,629,632,633$

lungs 357

lupus 742

luteinizing hormones 62

lymphocytes 693

lysine 47,332

monooxygenase 125

sensor 28

L-ysine 90, 149

alpha-oxidase 89

lysozyme 123, 683

M-value 422

magnesium 47

magnitude 430

malate 621

dehydrogenase 20

malfunction of sensor 734

mammalian $30,31,41,44,49,54$

mannose 15

manufacturing 287

market $737 \mathrm{ff}$

analysis 746

mask structure 380

mass production 287

mass-transfer limited 632

mass-transfer rate 182

mass-transport limitations 299

Maxam and Gilbert method 107

Maxwell's equations 658

MBG, see mean blood glucose

mean blood glucose (MBG) $\mathbf{4 2 2}$ 
measuring frequency 319

mechanical impedance $549 \mathrm{ff}$

mechanism of tissue biosensor response 54ff

mediation 9, 271, 292

mediated biosensors $247 \mathrm{rr}$

mediated electrochemistry $291 \mathrm{ff}$

mediated electron transport 169

mediated fuel cells 306

mediators $170,180,185,259,260,266-80$, $285,287,294,295-300,304,306-10$, $315,325,326$

anchored 306

immobilized 294, 333

medicine $4,723 \mathrm{ff}$

meldola blue 169

membrane $v, 32,173,288,410$

diffusion coefficient for oxygen 400

failure 405

material 403

more permeable to oxygen than glucose 410

oxygen solubility 323

particles 298

potential 364

meningitis 325

mercuric acetate 64

mercuric labelled 64

mercury 591

mercury electrodes 267

meridian points 445

messenger RNA 103

metabolite monitoring 592

metal ion sensors 613

metal insulator semiconductor FET

(MISFET) 472, 484, 487, 488

structure $484 \mathrm{ff}$

nitride oxide semiconductor field-effect transistor (MNOSFET) 482

methane 745

oxidizing bacteria 22

sensor $21 \mathrm{ff}, 557$

methanol $17,20,28,253,280,282,295$, 587

dehydrogenase 280, 282

methionine 148

$\mathrm{L}$-methionine 149

methotrexate 670

methyl mannotide 644

methylamine 543

methylene blue 297

methylomonas flagellata 21, 22, 28

methylomonas methylovora 295

fuel ceil 302,303

$O$-methylisourea

methylotroph 303

$N$-methylphenazinium (NMP +) 171, 180, 264; see also phenazine methosulphaste (PMS) 4-methylumbelliferone 610,611

methylpicolonimidate 121

micelles 692

Michaelis constant $\left(K_{\mathrm{M}}\right) 113,172,190$

Michaelis-Menten equation 113, 190

Michaelis-Menten kinetics 303

microbial

biomass 444

monitoring 565,566

contamination 745

cultures 561,562

electrode 14

fuel cells 291, 295, 296, 299

glucose sensor 16

growth 444

hydrogen production 539

process 591

sensor for alcohol 18

sensors $13,15,25,27,299,305$

micro-

ATP sensor 474

bioassay 232

biology 317

biosensors $471 \mathrm{ff}$

calorimeters 638

capsule 91

computer $332,420,705,707-9,712,213$. 718

electrode-based sensors 475 If

electrodes 10, 173, 377ff, 471

environment 86

fluorimeters 638

glucose sensor 476

glutamate sensor 480

organism-based sensors 13ff, 28

organisms $14,16,21,270,315$

as filters 309

numbers 444

processors $703 \mathrm{ff}$

urea sensor 471

Mie scattering 682, 683

Milk 295, 323

miniaturization 625,680

of enzyme sensors 471

miniaturized biosensors 532,638

miniaturized electrochemical cells 389

miniaturized multiple sensor arrays 379

miniaturized reference electrode 389

miniaturized sensor 387

miniaturized thin-film electrode $378 \mathrm{ff}$. 379

miniaturized thin-film multiple electrode probes $380 \mathrm{ff}$

mitochondria $31,36,37,307,308$

mitochondria-based biosensor 54

mitochondrial biosensors 37,54

mitochondrial electrode 36,54

mitochondrial fraction 54 
mixed potential 520,522

model 55,56

modified electrodes 169, 259ff, 263, 265 . 266

kinetics $214 \mathrm{ff}$

modulus 429

molar enthalpies of enzyme reactions 576

molasses 16

molecular biology 71

molecular cloning 100

molybdate 51

monoamine oxidase 125

monoclonal antibodies $62,82,93$

monooxygenase 125

morphine 64,670

morphology 683

motility 692

mouse

liver guanine biosensor 47

small intestine biosensor 38

small intestine mucosal cells $31,39,40$. 47

mRNA 71,72

mucosal cells 41

multicomponent analysis 562

multicomponent sensors 547

multi-dimensional dielectric spectrum 454

multiple electrode 377

probe $378 \mathrm{ff}$

multiple-internal reflection plate 665

multiple-internal reflections 674

multivalent binding behaviour 652

muscle contraction 692

muscle tissue 40

muscular function 328

mutagen 28

mutagenesis 116

mutants 294

mutarotase 324

mutation 71

myocardial disease 341

myocardial infarction $263,270,339$

myoglobin 126

n-type 496

$\mathrm{NAD}(\mathrm{H})$, see nicotinamide adenine dinucleotide (reduced)

$\operatorname{NADP}(\mathrm{H})$, see nicotinamide adenine

Nafion 168 dinucleotide phosphate (reduced)

natural gases 21

needle-type sensor $322,402,404$

in humans 370

needle-type glucose sensor 409ff, 729

needle-type hydrogen peroxide detector 370 nconatal medicine 359

nephelometry $683,691,693$

Nernstian behaviour 155

Nernstian response 48

nerve tissue 364

nick translation $73,74,82$

nick translation kits 73

nicotinamide adenine dinucleotide (NAD)

analogue 125

derivative 91

electrodes $186 \mathrm{ff}$

(reduced)(NADH) $125,169,186-8,253$, $258,263-6,270,298,303,325,403$. $540,582,618,620,621$

Jehydrogenase 576

spectrophotometric measurement 63

nicotinamide adenine dinucleotide phosphate (NADP) reduced (NADPH) 253, 254, $258,262-5,333,620,621$

nicotinic acid 28

nitrate $51,141,145$

electrode 139

reductase 145,270

nitrite 141,145

oxidizing 89

reductase 145

nitrifying bacteria 26,28

Nitrobactersp. 26

nitrogen dioxide 28

Nitrosomonas sp. 26

NMA ' (methylacridinium) TCNQ ${ }^{*} 270$

noble metal electrodes 155

Nocardia salmonicolor 295

noise filters 421

noise range 412

of in vivo measurement 414

nonactin $139,146,148$

non-competitive inhibition 51

non-invasiye glucose monitoring 367,403

non-invasive sensing 358

non-linear dielectric properties 455

non-radiative plasmons 661

non-radioactive labelling 72

non-radioactive DNA labelling $\mathbf{8 2}$

non-specific binding 65,671

non-specific heat 580

non-specific interactions 674

Northern blotting 107

nucleic acid hybridization 107

nucleic acids $\mathrm{v}, 100$

p-nucleotides 81

numerical aperture 639,643

nylon $31,32,45,58,89,90,148,403$

cloth 398

net $14,36,42,352$

tubing 579,591

-bound penicillinase 590

nystatin 28 
$\mathrm{O}_{2}$, see oxygen

$\mathrm{O}_{2}$ (Clark), see Clark oxygen electrode

$\mathrm{O}_{2}$ microelectrode 478

$\mathrm{O}_{2}$ probe $28,93,326$

$\mathrm{O}_{2}$ sensor 31,51

oestriol 64

oligonucleotide 116

probes 74,77

on-line control 13

on-line determination 22,589

on-line measurement 13,18

on-line monitoring 17,20

open heart surgery 723

open loop 393

optical $v, 3$

fibre $403,599,602-4,607,609-12$, $638-40,642-4,647,652,664,665$, $668,674,679,680,694,695$

indicator 611

material for IREs 666

oxygen sensor 612

with immobilized oxydase 614

pH sensor 611

reciprocity 660

sensors 599ff, 609, 611

optimization strategy for tissue biocatalysis 44

opto-electronic devices $276,638,652$

optode 599

optrode 599

organisms $v$

organelles $v, 315$

organic metal $267,271,326$

organic molecules $\mathbf{v}$

organic phosphates 51

organic pollution 25

ornithine 47

transcarbamylase deficiency 80

ovalbumin electrode 65

overpotential 266

overvoltage 265

фX174 I16

oxalate 9,253

analyser 592

decarboxylase 586

oxidase $91,256,282,586,587$

oxalic acid 586,587

oxaloacetate decarboxylase 341

oxidase $3,10,125,262,264,270,315$. 580

oxidation of NADH 266,267

oxidoreductases $8,9,10,258,261,315$

oxygen $5,7,10,11,52,64,86,109,255$, $260,270,318,322,329,330,332$, $369,534,580,581,600,601,604$, $638,725,727$ see also $\mathrm{O}_{2}$

based on fluorescence 602 consumption 321 as a co-substrate 172

detectors 398

diffusion 341

electrodes $8,10,15,17,18,21,22,26,47$, $51,140,143,258,291,300,325,349$, $352,409,415,385,587,612$

in contact with conjunctiva 361

-insensitive mediators. 261, 310

interference $64,180,262,280,287$

level, variations 396

limitation 347

optical sensors for 612

sensor 642

based on chemiluminescence 609

in the brain of animals 387

solubility 347,348

stabilization 352

-stabilized electrode 352

-stabilized glucose electrode 347,3481 f

status of blood 362

tension 9,412

effect in vivo 415

tensions of conjunctiva 362

transfer 300

transport 173

uptake 26

oxyluciferin 618,619

p-type 496

$P$, aeruginosa, see Pseudomonas aeruginosa

$P$. vulgaris, see Pseudomonas vulgaris

$\mathrm{pCO}_{2}$, see partial pressure of carbon dioxide

$\mathrm{pO}_{2}$, see partial pressure of oxygen

packaging for CHEMFET sensors 497

paediatric 4,9

samples 545

palladium 89

palladium metal oxide semiconductors (PdMOS) 535, 539

PdMOS-components 53I

PdMOS-structures 546

palladium metal oxide semiconductor fieldeffect transistor (PdMOSFET) 533 , $534,537,538,539$

pancreas 390

pancreatitis 340

papain 126

Papyex 286

paracetamol $726,727,731$

poisoning 734

Paracoccus deniitrificans 270

parametric plot 650

partial pressure of carbon dioxide $\left(\mathrm{pCO}_{2}\right) 357,362$

partial pressure of oxygen $\left(\mathrm{pO}_{2}\right) 9,10,357$. 362

$\mathrm{pO}_{2}$ of arterial blood samples 360 
$\mathrm{pO}_{2}$ of blood 364

$\mathrm{pO}_{2}$-independent $1 \mathrm{~L}$

particle size determination 690

particle sizing 683

partition coefficient for oxygen 397

partitioning 128

passive electrical properties 437

patent 746

pattern recognition 453

$\mathrm{pBr} 22119$

PCS, see photon correlation spectroscopy 691

penicillin $119,135,145,150,512,586$, 590

-sensitive ENFET $510,512,513,514$

sensor 471

penicillinase $145,150,471,512,576,586$, 591

penicillinase ( $\beta$-lactamase) 590

pentammeruthenium 126

peptidoglycan 307

percutaneous transluminal coronary angioplasty 365

permeability 300

permittivity $437 \mathrm{ff}$

phosphorus ( $\left.{ }^{32} \mathrm{P}\right)$

32P-labelled plasmid 80

32P-labelling 72, 81

photo-acoustic effect 360

Photobacterium phosphoreum 620

photo-crosslinked polymer 305

photodiodes 631

photolithographic production 379

photomultiplier tubes $630,632,681$

photomultipliers 633

photon-correlation spectroscopy (PCS) 686, $689,691,692-5$

photon correlator 639,688

photoprotein 622,632

photosensitive film 631

physical entrapment of organisms 305

physician's office 727,743

physiological glycaemic regulations 422

piezo-electric biosensors 567

piezo-electric ceramics 553

piezo-electric polymers 551

piezo-electric transducers S5Iff

pigs 368

blood glucose 368,371

PIMIA, see potentiometric ionophore modulation immunoassay

plant leaves 31

plant materials $48,52,53$

plant tissue 49

plants $9,30 \mathrm{ff}, 54$

plasma $8,66,324,336,339,364,674$

plasma-enhanced chemical vapour deposition 379 plasma $\mathrm{K}^{+} 364$

plasmid $73,86,100,109$

probe $72-4$

plasmons 661,662

plastics 287

plastocyanin 123,269

platinum $89,90,157,170$

oxide 160

surface pretreatment 159,160

PMS, see phenazine methosulphate

pneumography 445

poised potential configurations 280

polarization 299,563

polarographies $3,6,9$

permselective membrane $168,267,318$

pernicious anaemia 339

peroxidase $7,148,149,270,271,623,628$, 632

system 624

peroxide 7,8

periplasmic enzymes 307

pH $3,6,9,45,135,144,147,149,258,604$, $609,638,725$

blood 362

detectors 399

electrode $28,32,62,135,137,291,601$

extreme $\mathrm{pH} 352$

glass 155

indicators 611

optimum 114,120

-sensing, optical 611

-sensitive 507

ISFETs 512

sensor $328,601,603,605$

phage cloning vector 106

pharmaceuticals 13

phenazine ethosulphate 280,332

phenazine methosulphate (PMS) 325,333 ; see also $\mathrm{N}$-methylphenazinium (NMP)

phenol 8,63

red 609

phenobarbital 741

phenobarbitone 727

7-phenyl-acetylamidodesaceroxy-sporanic acid 25

phenylarsonic acid 670

phenyl phosphate 63

phenylketonuria 80

phenylalanine 148

L-phenylalanine $40,41,148$

phenylalanine hydroxylase 80

phenytoin $63,174,741$

Pholas dactylus 624

phosphate $18,20,39,40,41,47,51,333$

phosphate/fluoride 31

sensor 50

phospholipase D 336

choline oxidase 584 
phospholipid determination 584

phospholipids. 336

phosphor 613

pollution vi, 316

poly ( $\mathrm{N}$-vinylcarbazole) 308

polyacrylamide 305

electrophoresis 74

gel 94

polyaromatic hydrocarbons 609

polycarbonate 9,91

film 89

membrane 91

polyelectrolytes $60,441,690$

polyethylene membrane 5

polyethyleneimine/glutaraldehyde 88

polyethylenenephthalate film 89

polymer synthesis 306

polymers 552,690

polymeric get 91,93

polymerization 690

polypeptide chain 120

polyphenol oxidase 52

polysaccharides 336

polyurethane 368,411

polyvinyl ferrocene 253,254

polyvinylidene fluoride piezo-electric film 554

porcine kidney $31-4,47$

porcine kidney cells $31,36,37$

porcine kidney cortex cells 31,54

porcine kidney tissue 36,55

porous carbon electrodes 287

portable closed-loop insulin infusion device 394

potassium $40,41,65,66,68,723,725,727$. 728

dihydrogen phosphate $\left(\mathrm{KH}_{2} \mathrm{PO}\right) 19$

monitoring 364

-selective membrane 504

potato $31,50,51$

tissue slice 333

potential step chronoamperometry $228 \mathrm{ff}$

potentiometric $v, 3,6,33,47,61,68$

biosensors 153,156

electrode 53

electrode-linked immunoassays 65

enzyme electrode 257

enzyme-linked immunoassay $65 \mathrm{ff}$

immunoassays 62,65

ionophore modulation immunoassay

$$
\text { (PIMIA) 66-69 }
$$

sensors 140

potentiometry 26

potentiostatic 301

power-time curve 591

PQQ, see pyrolloquinoline quinone

practical mediator 260

precision $319,330,705,718,731$ pregnant diabetics 9

premature infant 323

premature neonates 357

prenatal diagnosis $76,77,79,80$

preservative 34,48

pressure sensors 388

probe configurations $282 \mathrm{ff}$

probe fabrication 379

process control vi, $317,347,586,589,590$. $592,737,738,745$

product development 738

product differentiation 739

product life cycle $737,738 \mathrm{ff}$

progesterone 629

programmable calculator $705 \mathrm{ff}, 710,718$

promoters 269

propanol 587

propagation of ultrasonic waves in inhomogeneous suspensions 562

proportional action 42 !

propionic acid 18,20

proteoglycan aggregates 691

proteases 338

protein dynamics 456

protein electrochemistry 292

protein engineering 113

Proteins vulgaris 295, 296, 300

fuel cell 297,302

protonation enthalpy 581

Pseudomonas aeruginosa 297

Psendomonas putida 295

Pseudomonas fluorescens 15, 28

pseudo-univalent binding constants 652

pullulan 336, 337

pullulanase 340

pulse echo technique 560

pulse polarography $9,224 \mathrm{ff}$

pulse voltammetric 427

purines 169

pyrenebutyric acid 612

pyridine aldebyde thiosemicarbozones (V) 269

pyridine nucleotides 292

pyridines III and IV 268

pyrolloquinoline quinone (PQQ) 109

pyruvate $9,13,47,49,253,295,541,582$

decarboxylate 49

oxidase $253,282,339,341$

Q-factor 440

$Q^{*}$ see quinolinium

quantum yield 619,624

quartz $553,667,669$

crystal(s) 556,558

IREs 666

optical fibres 671

waveguide 656 
quartz-liquid interface 670

quasi-elastic light-scattering spectroscopy $686,691,692$

QELS, see quasi-electric light-scattering spectroscopy

quench fluorescence 674

quenching $606,646,649$

of fluorescence 614,642

quinoline 171

quinolinium $\left(Q^{+}\right) \quad 195-7,271$

o-quinone 263

p-quinone $263,319,322$

quinone intermediates 292

quinones 260

quinoprotein glucose dehydrogenase 87

quinoproteins $262,282,287$

rabbit 31

liver $31,45,47$

guanine sensor 46

muscle $31,42-4,47$

radioactive nucleotides 72

radioactively labelled DNA probe 75

radioimmunoassay 60

market 740,741

radioisotopes 60

raffinose 10

Raman scattering 684

range 319,650

rapid methods vi

rare-earth chelate fluorophores 645

rat liver mitochondria 270

rats 716

rates of reduction of mediators 297

Rayleigh 682

scattering 681

Rayleigh-Gans-Debye (RGD) scattering 681,682

reactance 433

reactor 24

reagent strip $725,726,727,728$

real samples 315

recalibration 347

receptors $\mathrm{v}$

recombinant DNA technology 100

red blood cells 565

red cells 8,60

redox coupling reagents 292

redox dyes 295

redox polymer electrodes $153,156,264$

redox potential 300

redox proteins $267 \mathrm{ff}$

reduced sugars 13

reference electrodes $155,287,495,499-501$, $512,516,600$

reference field-effect transistor

(REFET) 472,473
REFET, see, reference field-effect transistor refractive index 659

reliability 733

renal 328

disease 364

failure 333

transplantation 723

Renilla 618

luciferin 621

reniformis 622

repeatability $705,716,718$

reproducibility 712

resazurin 295

residual current 412

resolution 368

resolved fluorimetry 645

resonance minimum 662

respiratory activity 14,294

respiratory chain 292

respiratory distress syndrome 357

respiratory insufficiency 359

respiratory quotient 297

response characteristics, models $648 \mathrm{ff}$

response mechanism 54

response times $300,349,368,395,412,602$, $632,716,718,732$

responsiveness 651

restriction endonucleases 74, 100

restriction enzyme 75

analysis 74

sites 73

restriction fragment length polymorphisms 78,79

retinopathy 359

retrolental fibroplasia 357

reversible binding 641,648

reverse transcription 103

Reye's syndrome 34

rhodamine 646

-labelled lgG 657

-labelled insulin 657

Rhodospirillum rubrum 20

riboflavin 401

ribonuclease 123, 124

ribonuclease A 691

right angle 672

detection 660,666

diffusion rate 647

fluorescence 671

geometry 664

Roche lactate analyser 326

rotating-disc electrode $166,168,172$

rotating ring disc 272

electrode $238 \mathrm{ff}, 272$

Saccharomyces cerevisiae 28

saccharose 15,19

safety 733 
salicylate 726,727

salinity of marine environment 445

sandwich ELISA 62

Sanger sequencing 108

Sarcina flava 36

sarcosine 253

oxidase $147,252,253,328$

saturated region of operation 492

scale-up 454

studies of bioreactors 539

scanning acoustic microscope 564

scanning acoustic microscopy 563

scanning electron-microscopic examination of glucose sensors 417

scattered light 648

scavenger column 544

Schiff linkage 476

seasoning 22

second subsirate oxygen 161

selectivity 46,146

coefficient 136

electrode 146

enhancement strategy $30,37,38$

for glucose 477

semiconductor 661

fabrication technology 471

gas sensors $531 \mathrm{ff}$

$n$-type 483

p-type 483

semi-synthesis 127

sensitive s;stems 674

sensitivity $294,318,650,731$

sensor 161

fabrication $496 \mathrm{ff}$

geometry 403

packaging 497

sequencing DNA 100

serine 47

L-serine 34

serum $8,86,323,334,339,340,544,545$, $583,587,674$

samples 693

Severinghaus carbon dioxide electrode 611 shrimp 617

sickle cell anaemia 71, 77, 78

signal amplification 541

analysis 449

disturbances 384

transduction 294

transfer 381

silastic drum 11

silastic membrane 367

silica 88,668

silicon 482, 483-90, 525

-based sensors 409ff

dioxide $472,481,503$

fabrication $471 \mathrm{ff}$

$n$-type $484,487,489,490$ nitride 504,507

surface 476

nitrite 472

p-type $484,487,489,490$

substrate 381,494

waters 496,668

silver reference electrode 5

silver/silver chloride reference electrodes 286

single base change 77

single-stranded DNA 73, 77

sinusoidal voltages 427

site-specific mutagenesis 108

slaughter house 744

SM ISFET, see suspended mesh ion-sensitive field-effect transistor

sodium 725,727

azide $34,38,45,48$

software 713,716

solid-state electrodes 137

solid-state lasers 652

solid-state membranes 503

solid-state pH membranes 506

source $489,490,496,499,508,522$

Southern blot 75-80

Southern-blotting method 74, 82, 107

soy sauce 332

spatial separation techniques 642

spate-charge region 492

specific measurement 673

specificity $294,368,395$

spectral analysis $449 \mathrm{ff}$

spectrophotometry 13

split-flow arrangement 580

spongy mesophyll 53

sports medicine 325

SPR, see surface plasmon resonance

spray coat 288

squid 617

stable biosensor 46

stable during storage 288

stability $128,318,319,330,354,601$

of glucose oxidase 397

stabilization $35,45,46,86,114$

stabilizing agents 45

starch 336,337

static light-scattering 683

steady-state response $707,708,711,712$, 716

sterile 735

sterile conditions 37

sterilizability 347

stirring 280

of analyte 168

Stokes-Einstein equation 688

storage conditions 47

strain selection 294

streptavidin 82 
strip devices 285

subcellular component 54

subcutaneous insulin infusion 391

subcutaneous tissue 414,417

of diabetic subjects 418

of dogs 418

implantation 367

oxygen tension 415

pH 365

substrate

channelling 128

diffusion 141

inhibition 18

recycling 582

subtilisin 120

succinate 295

succinylation 122

sucrose 13-15, 295, 324, 584, 586

sugar beet 31,52

suicide substrate 121

sulphokinase 622

superoxide ion 626

surface acoustic wave 559

surface modification 301

surface plasmon oscillation 661

surface plasmon resonance (SPR) 660, 662, $666,671,673,674$

surface potential 525,526

surface states 488

surgical implantation 11

suspended gate GASFET 525

suspended mesh ISFET $507,508,525$

suspended mesh ion-sensitive field-effect transistor (SM ISFET) 509

sweetener 323

synthetic oligonucleotide probes 73

T4 lysozyme 120

TCNQ, see tetracyanoquinodimethane

Tc $\mathrm{PO}_{2}$, see transcutaneous partial pressure of oxygen

Teflon 32, 357

membrane $16,17,20,21,26,32,44,62$, 557

telemetry glucose monitoring 417

telemetry monitoring 418

TELISA, see thermometric enzyme-linked immunosorbent assay

temperature sensors 388

temperature stability 120

TEP, see thermal enzyme probe

tertiary structure 691

testosterone 621

tetracyanoquinodimethane $171,266,271$, 272

tetrahydrofolic acid synthetase 20
$N, N, N^{\prime}, N^{\prime}$-tetramethyl-pphenylenediamine (TMPD) 279

tetramethyl rhodamine-BSA 657

tetrathiafulvalene (TTF) 171, 266, 271

TTF ${ }^{*}$ mediator 272

TTF + TCNQ $^{-195-8,266}$

$\alpha$-thalassaemia 72, 76

$\beta$-thalassaemia $71,73,77-81$

theophylline 63,741

theory of amperometrie enzyme electrode response 172

thermal enzyme probe (TEP) 575, 577

thermal bioanalysers 575

thermal stability 124

thermistor 361,578

thermogram 591

thermolysin 124

thermometric enzyme-linked immunosorbent

thin-film assay (TELISA) 582,586

gold electrodes 384

ion-selective electrodes 384

microelectrodes $377 \mathrm{ff}$

silver/silver chloride electrodes 385

thiol lactamase 119

thiols 169

thionine $296,297,298,302,306$

three-dimensional structure 115

threonine 47

threshold voltage $487,488,490-1,495$

thrombosis 735

thyroid 328

thyroid stimulating hormone (TSH) 731 . 741

TSH thyrotropin 628

time constant 433

time-domain methods 448

time response 506

time series 450

TIRF, see total internal-reflection fluorescence

tissue $\mathrm{v}, 30,31,36,44$

-based adenosine electrode 40

-based glutamine biosensor $30,34,37$

-based peroxidase sensor 48

damage 367

electrode 36

glucose concentration 370

glutamine sensor 35

reaction 368

response 397

slices 34,315

titanium tetrachloride 86

titamium wire 65

TMPD, see $N, N, N^{\prime}, N^{\prime}$-tetramethyl-pphenylenediamine

TNT, see trinitrotoluene 
tobacco mosaic virus (TMV) 692

tobramycin 741

total assimilable sugars 14

total internal reflection fluorescence

(TIRF) $656,660,666,670,671,672$, 674

TIRF immunoassays 675

Ioxicity 734

Toxi-guard 591

transaminases 340

transcription 103

transcutaneous glucose monitor 367

transcutaneous oxygen tension 358

transcutaneous partial pressure of oxygen $\left(\mathrm{TcPO}_{2}\right) 360$

$\mathrm{TcPO}_{2}$ monitoring 359

transcutaneous $\mathrm{PO}_{2}$ electrode 359

transducers $v, 7,56$

transfer junction 453

transfer of electrons 3

transfer of electrons across cell walls 295

transformation 103

translation 103

transmission 670

transport 55

mechanism 54, 55

process 56

Trichosporium cutaneum 25, 28

Trichosporon brassicae 17-19, 28

triglyceride determination 584

triglycerides $325,586,727$

trinitrobenzene sulphonate 125

trinitrotoluene (TNT) 621

reductase 621

trypsin 122, 576, 581

tryptophan 148, 642

$\mathrm{TSH}$, see thyroid stimulating hormone

TTF, see tetrathiafulvalene

tumour antigens 60

tunnelled fluorescence 660

turbidimetric methods 23,648

turbidimetry 683

turnover number 113

rwo-phase flows in bioreactors 553

two-site immunofluorometric assay 671,672

tyramine 125

tyrosinase 52

tyrosine $31,52,148,399$

biosensor $52 \mathrm{ff}$

t-tyrosine 144, 148, 149

tyrosy! tRNA 118

ultrasonic absorption spectroscopy 651

ultrasound fields 563

undiluted whole blood 3,10

unsaturated region of operation 492

urate 399, 708 urea $30,31,34,37,47,50,135,141,143$. $144,146,147,150,327,473,512$, $545,546,547,586,725,726,727$

biosensor 50

calibration curve 474

determination 543

as interference 475

-sensitive ENFET 472, 510, 512

sensor $50 \mathrm{ff}, 471$

in serum 583

urease $3,30,50,66,89,143,144,146,147$. $150,512,531,533,543,545,576$, 586,591

-containing electrodes 446

immobilized 472

probe based in IrMoS capacitor 546

uric acid $255,331,586,731$

uric acid analyser 329

uricase $90,329,576,586$

electrodes 330

urinary-tract infections 539

urine $20,27,148,333,339,340,544,545$, $587,683,725$

glucose $86,149,317,329$

USA spending on health care 743

vacuum deposition 287

vacuum level 482

valence band $482,473-7$

valine 34,47

valinomycin 138, 140, 364, 504

vapour deposition 476

variable angle prisms 664

variations of oxygen 401

vector quantity 429

velocity statistics 690

very high frequency audio signal 418

very high frequency methodologies 447

venous oxygen saturation 363,364

veterinary vi

health care $737,738,744 \mathrm{ff}$

Vibrio harveyi 620

viral DNA 82

virus 60,692

vitamin $B_{1} 28,295$

vitamin $C$ assays 584

vitamin D deficiency 333

volatile dehydrogenase substrates 541

volatile organoleptic compounds 557

voltammetric immunoassay 64

Beta-vulgaris altissima 52

wall jet electrode 167

Warburg impedance 435

wastewater $26,291,561$

wastewater treatment 13, 25 
waveguide-liquid interface 660,669 waveguide made of quartz 649 waveguides $638,656,657,667,671,672$ wearable artificial endocrine pancreas 409 , 420,421

whey 589

whole blood $8,86,255,674$ see also blood

whole cell sensors 291

whole cell stability 294

whole micro-organisms $291 \mathrm{ff}$

work function $484,488,495$ xanthine $9,125,253$

oxidase $253,263,271,282$

peroxidase 270

yeast cells 91

Yellow Spring's Instrument (YSI) 8, 9, 85,

$318,319,323,324,326,331,337$. $339,340,399,743$

Yellow Squash $31,47,48$

YS1, see Yellow Spring's Instrument

YSI, analyser 8 
Biosensors promise to revolutionize approaches to many analytical problems providing a powerful technology with the specificity and sensitivity of biological systems and the computing capabilities of the microprocessor. Tests that previously took days before a result was available and required elaborate laboratories may now be possible using extremely cheap and simple devices in the home. The implications for monitoring personal health and fitness, the food we eat, and the environment we live in are enormous, just as they are for the industries and health services which currently maintain large analytical facilities.

This is the first substantial and comprehensive book to be published in this field. It has many contributors, chosen to reflect the spread of disciplines from which the new techniques have emerged as well as those to which they may be applied and those which will be required to develop the devices. As is to be expected in a growing field, there are differences in opinion and approach; they are expounded here by their main proponents.

\section{From reviews of the hardback}

'In short this book is a must for anyone currently engaged in biosensor research or for those contemplating future involvement in this rapidly growing field.' and 'This book successfully provides readers with stateof-the-art technical information on a highly interdisciplinary subject.'

Analytical Chemistry

'A mine of useful information for practising analytical scientists.'

Times Higher Education Supplement

'This particular monograph stands out from other contributions on biosensors because of the contributions and the generally realistic appraisal of the subject. The book is well written and well produced, with a comprehensive index, and I would strongly recommend it as the best available text on the subject of biosensors.' Analytica Chemica Acta

'For those wanting a wealth of information at their fingertips and a picture of biosensor research at the present time, it is to be recommended.'

Journal of Molecular Electronics

'In conclusion, the book is of great value as an introduction for newcomers to the field or for those wanting to learn about more specific aspects of biosensors and their applications.'

Trends in Analytical Chemistry

OXFORD UNIVERSITY PRESS Also available in hardback

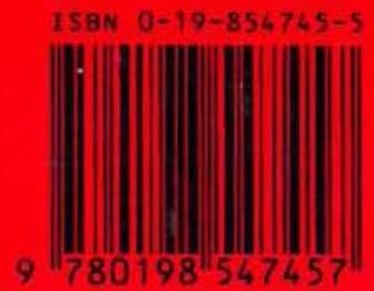

